



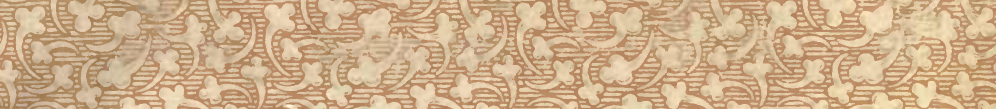

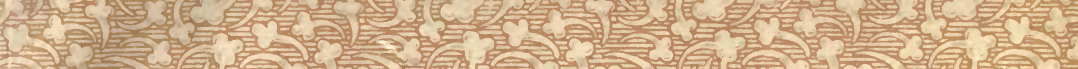
H. . (2)

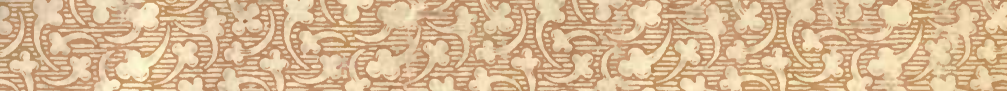

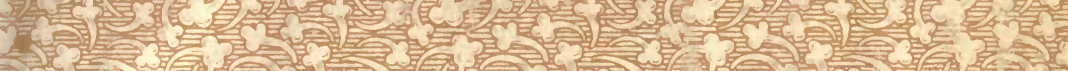

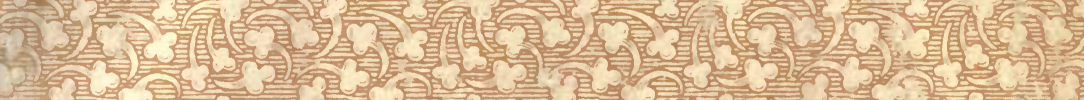
-

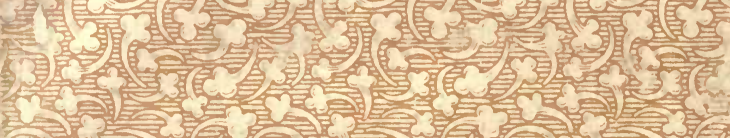
(10)

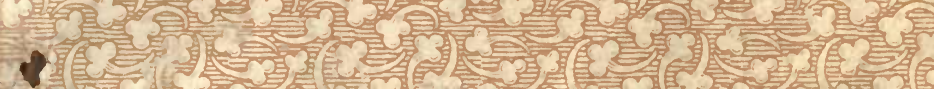

(i)

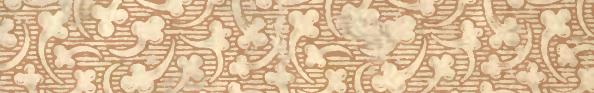

\section{(s)}

(- net

Her Hents 글

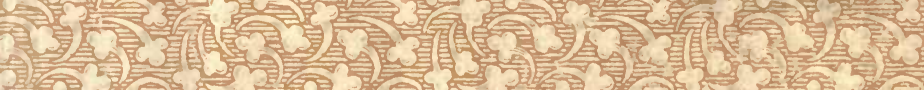
int 3.

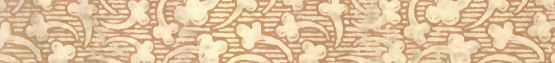

H. 6 . 罢

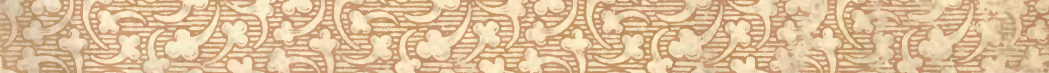

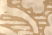





\section{A . TEXT-BOOK \\ OF ZOOLOGY.}

BY

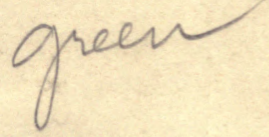

T. JEFFERY PARKER, D.Sc., F.R.S.

PROFESSOR OF BIOLOGY IN THE UNIVERSITY OF OTAGO, DUNEDIN, N.Z.

AND

WILLIAM A. HASWELL, M.A., D.Sc., F.R.S. PROFESSOR OF BIOLOGY IN THE UNIVERSITY OF SYDNEY, N.S.W.

IN TWO VOLUMES

VOL. I

WITH ILLUSTRATIONS

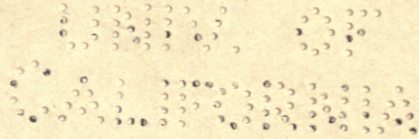

MACMILLAN AND CO., LIMITED

ST. MARTIN'S STREET, LONDON 


$$
\begin{gathered}
\text { ol.42 } \\
P 33 \\
1910 \\
r .1
\end{gathered}
$$

$$
\begin{aligned}
& \text { BIOLOGY } \\
& \text { LIBRARY }
\end{aligned}
$$

$$
409021
$$

Richard Clay and Sons, Limited, BREAD STREET HILL, E.C., AND BUNGAY, SUFFOLK.

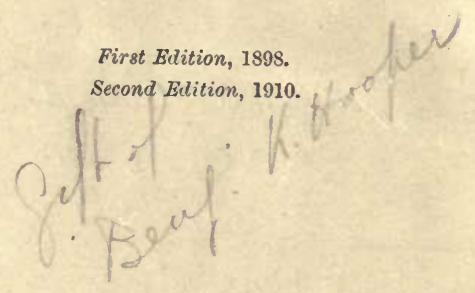




\section{PREFACE TO THE FIRST EDITION}

In spite of its bulk, the present work is strictly adapted to the needs of the beginner. The mode of treatment of the subject is such that no previous knowledge of Zoology is assumed, and students of the first and second years should have no more difficulty in following the accounts of the various groups than is incidental to the first study of a complex and unfamiliar subject.

There can be little doubt that the study of Zoology is most profitably as well as most pleasantly begun in the field and by the sea-shore, in the Zoological Garden and the Aquarium. In a very real sense it is true that the best zoologist is he who knows the most animals, and there can certainly be no better foundation for a strict and scientific study of the subject than a familiarity with the general appearance and habits of the common members of the principal animal classes. But Zoology as a branch of academical study can hardly be pursued on the broad lines of general natural history, and must be content to lose a little in breadth of view-at least in its earlier stages-while insisting upon accurate observation, comparison, and induction, within the limited field of Laboratory and Museum work.

A not uncommon method of expounding the science of Zoology is to begin the study of a given group by a definition, the very terms of which it is impossible that the student should understand; then to take a general survey of the group, illustrated by casual references to animals and to structures of which it is highly unlikely he has ever heard; and, finally, to descend to a survey of the more important forms included in the group. It will probably be generally agreed that, from the teacher's point of view, this method begins at the wrong end, and is hardly more rational than 
it would be to deliver a course on the general characteristics of English Literature, suitably illustrated by "elegant extracts," to a class of students who had never read a single English poet or essayist.

There can be no question as to the vast improvement effected in zoological teaching by the practice of preceding the study of a given group as a whole by the accurate examination of a suitable member of it. With the clear mental image of a particular animal, in the totality of its organisation, the comparison of the parts and organs of other animals of like build becomes a profitable study, and the danger of the comparative method - that the student may learn a great deal of the systems of organs in a group without getting a clear conception of a single animal belonging to it-is much diminished.

The method of "types" has, however, its own dangers. Students are, in their way, great generalisers, and, unless carefully looked after, are quite sure to take the type for the class, and to consider all Arthropods but crayfishes and cockroaches, and all Molluscs but mussels and snails, as non-typical. For this reason a course of Zoology which confines itself entirely or largely to "types," or, as we prefer to call them, ${ }^{1}$ examples, is certain to be a singularly narrow and barren affair, and to leave the student with the vaguest and most erroneous ideas of the animal kingdom as a whole. This is especially the case when the number of examples is small, each of the Phyla being represented by only one or two forms.

In our opinion every group which cannot readily and intelligibly be described in terms of some other group should be represented, in an elementary course of Zoology, by an example. We have, therefore, in the majority of cases, described, in some detail, an example of every important class, and, in cases where the diversity of organisation is very great-as in Crustacea and Fishes-two or more examples are taken. The student is thus furnished with a brief account of at least one member-usually readily accessible - of all the principal groups of animals.

By the time the example has been studied, a definition of the class and of its orders will convey some idea to the mind, and will

1 Following a suggestion for which we are indebted to 1)r. Alexander Hill, Master of Downing College, Cambridge. 
serve to show which of the characters already met with are of distinctive importance, and which special to the example itself In order to bring out this point more clearly, to furnish a connection between the account of the example and that of the class as a whole, and to give some idea of the meaning of specific, generic, and family characters, we have introduced, after the classification, a paragraph giving the systematic position of the example, sometimes in more, sometimes in less detail.

Following the table of classification with its brief definitions comes the general account of the group. This is usually treated according to the comparative method, the leading modifications of the various parts and organs being described seriatim. In a few cases this plan has been abandoned and the class described order by order, but this is done only when the deviations from the type are so considerable as to lead us to think the comparative method unsuitable for beginners. On the other hand, when all the classes of the phylum present a very uniform type of structure, the phylum is studied comparatively as a whole. The description of each group usually ends with some account of its ethology and distribution, and with a discussion of its affinities and of the mutual relationships of its various subdivisions.

We have done our best to make the space devoted to each group proportional to its complexity and range of variation, and to subdue the natural tendency to devote most attention to the more recently investigated classes, or to those in which we ourselves happen to be especially interested. A few lesser groups have been put into small type, partly to economise space, partly because they seem to us to be of minor importance to the beginner.

Following out the plan of deferring the discussion of general questions until the facts with which they are connected have been brought forward, we have placed the sections on Distribution, on the Philosophy of Zoology, and on the History of Zoology at the end of the book. We have, however, placed a general account of the structure and physiology of animals immediately after the Introduction, and one on the Craniate Vertebrata before the description of the classes of that division, but it will be obvious that these deviations from the strictly inductive method were inevitable in order to avoid much needless repetition.

After a good deal of consideration we have decided to omit all 
references to the literature of the subject in the body of the work. Anything like consistent historical treatment would be out of place in an elementary book; and the introduction of casual references to particular discoveries, while they might interest the more advanced reader by giving a kind of personal colouring to the subject, could hardly fail, from their necessarily limited character, to be misleading to the beginner, and to increase rather than diminish his difficulties. We have, therefore, postponed all reference to the history of the science to the concluding Section, in which the main lines of progress are set forth, and have given, as an Appendix, a guide to the modern literature of Zoology. The latter is intended merely to indicate the next step to be taken by the student who wishes to acquire something more than a mere text-book knowledge. ${ }^{1}$

The various Sections have been written by the authors in fairly equal proportions, but the work of each has been carefully read and criticised by the other, and no disputed point has been allowed to stand without thorough discussion. We are therefore jointly and severally responsible for the whole work.

A very large proportion of the figures have been specially drawn and engraved for the book. Those in which no source is named are from our own drawings, with the exception of Figs. 571, 572, $1017,1018,1019,1022,1059,1063$, and 1071, for which we are indebted to Mrs. W. A. Haswell. Figs. 1002 bis, 1005 bis, are from photographs kindly taken for us by Mr. A. Hamilton. ${ }^{2}$ Many blocks have been borrowed from well-known works, to the authors and publishers of which we beg to return our sincere acknowledgments. All the new figures have been drawn by Mr. M. P. Parker.

1 In this connection we cannot resist the pleasure of quoting two passages, exactly expressing our own views, from the preface to Dr. Waller's Human Physiology, which came under our notice after the above paragraph was in type:"I have given a Bibliography after some hesitation, feeling that references to original papers are of no use to junior students, and must be too imperfect to be satisfactory to more advanced students.... Attention has been paid to recent work, but $I$ have felt that the gradually-formed deposit of accepted knowledge must be of greater intrinsic value than the latest 'discovery' or the newest theory. An early mental diet in which these items are predominant is an unwholesome diet; their function in elementary instruction is that of condiments, valuable only in conjunction with a foundation of solid food."

2 The figures referred to are numbered 608, 609, 1080, 1081, 1082, 1085, $1128,1132,1140,1063$, and 1067 in the new edition. 
We have received generous assistance from Professors Arthur Dendy, G. B. Howes, Baldwin Spencer, and J. T. Wilson, and from Mr. J. P. Hill and Dr. Arthur Willey. Professor W. N. Parker has very kindly read the whole of the proof-sheets and favoured us with many valuable suggestions, besides acting as referee in numerous minor difficulties which would otherwise have cost a delay of many weeks.

It is a mere truism to say that a text-book can never really reflect the existing state of the science of which it treats, but must necessarily be to some extent out of date at the time of publication. In the present instance, the revises of the earlier pages, giving the last opportunity for any but minor alterations, were corrected in the latter part of 1895, and the sheets passed for press in the middle of 1896 . We are, therefore, fully alive to the fact that much of our work already needs a thorough revision, and can console ourselves only by reflecting that "to travel hopefully is a better thing than to arrive, and the true success is to labour."

We may mention, in conclusion, that, whatever may be the merits or demerits of the book, it enjoys the distinction of being unique in one respect. The two authors have been separated from one another, during the greater part of their collaboration, by a distance of 1200 miles, and the manuscript, proofs, and drawings have had to traverse half the circumference of the globe in their journeys between the authors on the one hand, and the publishers, printers, artist, and engravers on the other. It will, therefore, be readily believed that all persons concerned have had every opportunity, during the progress of the work, of exercising the supreme virtue of patience. 



\section{PREFACE TO THE SECOND EDITION}

A NEW edition of this Text-Book has been called for on somewhat short notice, and, had it not been for the assistance generously rendered by Professor. W. Newton Parker, who has helped me greatly in the revision of the proofs, and has made a large number of useful suggestions, it would have been impossible for me to have completed the work within the time prescribed. Fortunately, also, materials for the most important of the alterations and additions had been already, to a certain extent, prepared.

The original plan of the work has not been in any way altered, and, though all parts have been subjected to careful revision, there is a good deal, especially in the descriptions of many of the examples, which has not been materially changed. On the other hand, some parts have been to a great extent re-written, and a good many illustrations have been added, a fair proportion of which are new to text-books of this description.

I have the pleasure of acknowledging assistance on special points received from Professor J. P. Hill, Mr. S. J. Johnston, B.A., B.Sc., Mr. E. J. Goddard, B.A., D.Sc., and Mr. H. L. Kesteven, B.Sc., all of the University of Sydney. A good many of the new illustrations were re-drawn by W. Birmingham, Laboratory Assistant, Department of Biology.

W. A. Haswell. 



\section{CONTENTS}

Preface

Contents of Sections in Vol. I. . . . . . . . xiii

List of Illústrations iN Vol. I. . . . . . . . . xix

Table of the Classification of the Aninal Kingdon . . e xxx́́

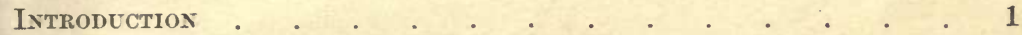

\section{SECTION I}

The General Structure and Physiology of Animals . . . 10

1. Amœba . . . . . . . . . . . . 10

2. The Animal Cell . . . . . . . . . . . 14

3. The Ovum : Maturation, Impregnation, and Segmentation : the

Germinal Layers . . . . . . . . . . . 19

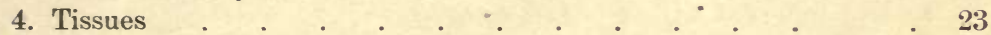

5. Organs . . . . . . . . . . . 31

6. The Reproduction of Animals . . . . . . . . 40

7. Symmetry . . . . . . . . . . . . 41

8. The Primary Subdivisions or Phyla of the Animal Kingdom . 43

\section{SECTION II}

Phylum Protozoa . . . . . . . . . . 45

Class I. Rhizopoda

1. Example of the Class-Amoeba proteus . . . . . 46

2. Classification and General Organisation . . . . . . . 47 Systematic Position of the Example . . . . . . 48 Appendix to the Rhizopoda . . . . . . . . 64

Class II. Mycetozoa

1. Example of the Class-Didymium difforme . . . . . 66

2. General Remarks . . . . . . . . 67

Class III. Mastigophora

1. Example of the Class-Englena viridis . . . . . . 67

2. Classification and General Organisation . . . . . . $\quad$. 69 Systematic Position of the Example . . . . . $\quad 70$

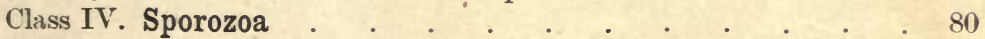

1. Example of the Class-Monocystis ayilis . . . . $\quad$. 80

2. Classification and General Organisation . . . . . 81

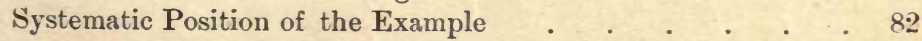


Phylum Protozon-continued.

PAGE

Class V. Infusoria . . . . . . . . . 88

1. Example of the Class-Paramocium caudatum . . . . 88

2. Classification and General Organisation . _ . . . . 91

Systematic Position of the Example $\quad$. . . . . . . 91

Further Remarks on the Protozoa . . . . . . . 101

\section{SECTION III}

Phylum and Class Porifera [Parazoa] . . . . . . . 105

1. Example of the Class-Sycon gelatinosum . . . . . . $10 \tilde{5}$

2. Distinctive Characters and Classification . . . . . . 111

Systematic Position of the Example . . . . . . . . 112

3. General Organisation . . . . . . . . . . 114

\section{SECTION IV}

Phylum Celenterata . . . . . . . . . . . 128

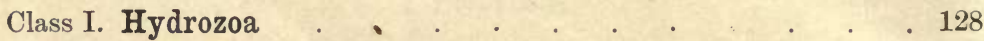

1. Example of the Class-Obelia . . . . . . . 128

2. General Structure and Classification . . . . . 140

Systematic Position of the Example . . . . . . 142

Additional Remarks . . . . . . . . . . . 167

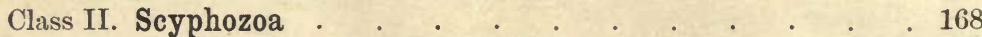

1. Example of the Class-Aurelia aurita . . . . . 168

2. General Structure and Classification _. . . . . . . 176

Systematic Position of the Example . . . . . . 177

Additional Remarks . . . . . . . . . . . . 184

Class III. Actinozoa . . . . . . . . . . . 185

1. Example of the Class-Tealia crassicornis . . . . 185

2. Distinctive Characters and Classification . . . . . 193

Systematic Position of the Example . . . . . . 196

3. General Organisation . . . . . . . . . 196

Class IV. Ctenophora $. \quad . \quad . \quad . \quad . \quad . \quad . \quad \ldots . . .211$

1. Example of the Class-Hormiphora plumosa . . . . . 211

2. Distinctive Characters and Classification . . . . . 220

Systematic Position of the Example . . . . . . 221

3. General Organisation . . . . . . . • . . 222

Appendix to Ctenophora-Ctenoplana and Coeloplana . . . 225

The Relationships of the Coelenterata . . . . . . 226

Appendix to the Coelenterata-The Mesozoa . . . . . 230

SECTION $Y$

Phylum Platyhelminthes

1. Examples of the Phylum . . . . . . . . . 236

i. Planaria or Dendrocolum . . . . . . . . . . 236

ii. Fasciola hepatica . . . . . . . . . . 240

iii. Tcenia solium . . . . . . . . . . . 245 


\section{Phylum Platyhelminthes-contimued.}

PAGE

2. Distinctive Characters and Classification . . . . . . 251 Systematic Position of the Examples . . . . . . . 253

3. General Organisation . . . . . . . . . . 254

4. Distribution, Mode of Occurrence, and Mutual Relationships . 283 Appendix to Platyhelminthes-Class Nemertinea . . . . 288

Distinctive Characters and Classification . . . . . . 295

\section{SECTION VI}

Phylum Nemathelminthes . . . . . . . . . . 297

Class I. Nematoda . . . . . . . . . . . 297

1. Example of the Class-Ascaris lumbricoides . . . . . 297

2. Distinctive Characters and Classification . . . . . 303

Systematic Position of the Example . . . . . . 304

3. General Organisation . . . . . . . . . 305

Class II. Acanthocephala . . . . . . . . . . 312

Class III. Chæ'tognatha . . . . . . . . . . 316

Appendix to Nemathelminthes . . . . . . . . 319

Family Chotosomidce . . . . . . . . . . 319

" Echinoderidae . . . . . . . . . . 319

, Desmoscolecidce . . . . . . . . . . 320

Aftinities and Mutual Relationships of the Nemathelminthes . 320

SECTION VII

Phylum Trochelminthes

Class I. Rotifera .

1. Example of the Class-Brachionus rubens . . . . . 323

2. Distinctive Characters and Classification . . . . . 327

Systematic Position of the Example . . . . . . 329

3. General Organisation . . . . . . . . . . 330

Class II. Gastrotricha . . . . . . . . . 335

Appendix to Trochelminthes-Dinophilea and Histriobdellea . 336

\section{SECTION VIII}

Phylum Molluscoida . . . . . . . . . . . 340

Class I. Polyzoa . . . . . . . . . . . 340

1. Example of the Class-Bugula avicularia . . . . . 341

2. Distinctive Characters and Classification . . . . . 347

Systematic Position of the Example . . . . . . 348

3. General Organisation . . . . . . . . . . 348

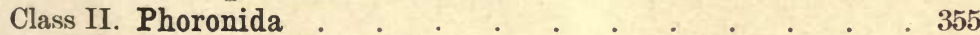

Class III. Brachiopoda . . . . . . . . . . 360

1. Example of the Class-Magellunia lenticularis . . . . 360

2. Distinctive Characters and Classification .. . . . . 366 Systematic Position of the Example . . . . . . 367

3. General Organisation . . . . . . . . . 367 Mutual Relationships of the Classes of the Molluscoida . . 372 


\section{SECTION IX}

Phylum Echinodermata

PAGE

1. Example of the Asteroidea-Asterias mibens or Anthenec flavescens. 375

2. Example of the Echinoidea-Strongylocentrotus or Echinus . . 393

3. Example of the Holothuroidea-Cucumaria or Colochirus _ . 401

4. The Crinoidea-Antedon rosacea. . . . . . . . 405

5. Distinctive Characters and Classification . . . . . . 410

Systematic Pusition of the Examples . . . . . . . 414

6. General Organisation . . . . . . . . . 415

\section{SECTION $\mathrm{X}$}

Phylum Annulata. . . . . . . . . . 439

Class I. Chætopoda . . . . . . . . . . . . 439

1. Examples of the Class . . . . . . . . . 440

i. Nereis dumerilii . . . . . . . . . 440

ii. Lumbricus . . . . . . . . . . . . 454

2. Distinctive Characters and Classification . . . . . 464 Systematic Position of the Examples . . . . . . . 466

3. General Organisation . . . . . . . . . 467

Appendix to the Chætopoda-Class Myzostomida . . . 489

Class II. Gephyrea . . . . . . . . . . . 491

1. Example of the Class-Sipunculus nudus . . . . . 492

2. Distinctive Characters and Classification . . . . . 495

3. General Organisation . . . . . . . . . . 496

Class III. Archi-annelida . . . . . . . . . . 503

Class IV. Hirudinea . . . . . . . . . . . 506

1. Example of the Class-Hirudo medicinalis and H. anstialis . 506

2. Distinctive Characters and Classification . . . . 515

Systematic Position of the Example . . . . . . . 517

3. General Organisation . . . . . . . . . . 517

4. General Remarks on the Annulata . . . . . . . 523

\section{SECTION XI}

Phylum Arthropoda

Class I. Crustacea . . . . . . . . . . 526

1. Examples of the Class . . . . . . . . . 526

i. Apus or Lepidumes . . . . . . . . . . . 526

ii. Astacus fluviatilis . . . . . . . . . . . 539

2. Distinctive Characters and Classification . . . . . 561

Systematic Position of the Examples . . . . . . . . 569

3. General Organisation . . . . . . . . . . 570

Affinities and Mutual Relationships . . . . . . . . 602

Appendix to Crustacea-Class Trilobita . . . . . 604

Class II. Onychophora $. \quad . \quad$. . . . . . . $\quad{ }^{6} 607$

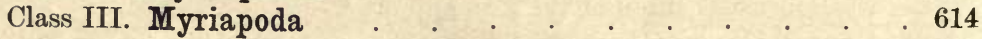

1. Distinctive Characters and Classification . . . . . 614

2. General Organisation . . . . . . . . . 615 
Phylum Arthropona-continued.

PAGE

Class IV. Insecta

1. Example of the Class-Periplaneta orientalis or P. americana . 619

2. Distinctive Characters and Classification . . . . . 631 Systematic Position of the Example . . . . . . 636

3. General Organisation . . . . . . . . . 636

Class V. Arachnida . . . . . . . . . . . 653

1. Example of the Class-Enscorpio or Buthus . . . . . 653

2. Distinctive Characters and Classification . . . . . 660

3. General Organisation . . . . . . . . . . 662

Appendix to the Arachnida-the Pycnogonida, Linguatulida, and Tardigrada . . . . . . . . . . . . 673

Relations of the Air-breathing Arthropoda . . . . . 676

\section{SECTION XII}

Phylum Mollusca . . . . . . . . . . . . 680

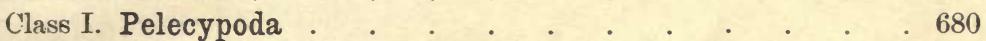

1. Example of the Class-Anodonta and Unio . . . . . 680

2. Distinctive Characters and Classification . . . . . 694

Systematic Position of the Examples . . . . . . . . . 696

3. General Organisation . . . . . . . . . . 696

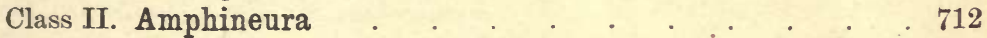

1. Distinctive Characters and Classification . . . . . 712

2. General Organisation . . . . . . . . . . 713

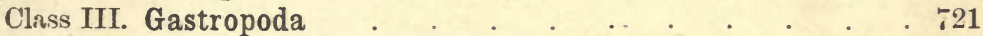

1. Example of the Class-Triton nodiferus . . . . . . 721

2. Distinctive Characters and Classification . . . . . 732 Systematic Position of the Example . . . . . . 734

3. General Organisation . . . . . . . . . 735

Appendix to the Gastropoda . . . . . . . 756

A. Class IN. Scaphoda . . . . . . . . . . 756

B. Rhodope . . . . . . . . . . . 758

Class V. Cephalopoda . . . . . . . . . . . . 759

1. Examples of the Class . . . . . . . . . 759

i. Sepia . . . . . . . . . . . . . . . 759

ii. Nautilus pompilius . . . . . . . . . . 776

2. Distinctive Characters and Classification . . . . $\quad 789$

Systematic Position of the Examples . . . . . $\quad 790$

3. General Organisation . . . . . . . . . . 790

General Remarks on the Mollusca . . . . . . . 804 


\title{
LIST OF ILLUSTRATIONS
}

\author{
VOL. I.
}

FIG.

1. Amœba proteus

PAGE

2. Amœba polypodia, fission

3. Alveolar theory of protoplasm

4. Reticular theory of protoplasm

5. Diagrams illustrating karyokinesis

6. Ovum of Sea-urchin . . . .

7. Maturation and fertilisation of ovum

8. Segmentation of ovum . . . . . . . . . . . . . . . . . .

9. Gastrulation . . . . . . . . . . . . . . 22

10. Gastrula . . . . . . . . . . . . . 23

11. Various forms of epithelium . . . . . . . . . 24

12. Diagram to illustrate structure of glands . . . . . . . . 25

13. Gelatinous connective tissue . . . . . . . . . . 26

14. Reticular connective tissue . . . . . . . . . . . 26

15. Fatty tissue . . . . . . . . . . . . 27

16. Hyaline cartilage . . . . . . . . . . . . . 27

17. Fibro-cartilage . . . . . . . . . . . . . 27

18. Bone . . . . . . . . . . . . . . . 28

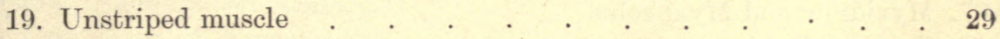

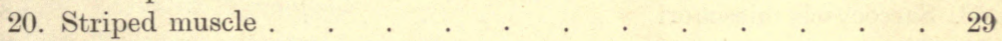

21. Nerve-cells . . . . . . . . . . . . 30

22. Nerve-fibres . . . . . . . . . . . . 30

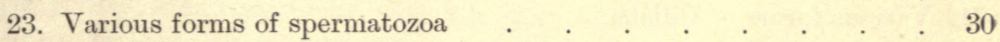

24. Viscera of Frog . . . . . . . . . . . . . 33

25. Bones of human arm with biceps muscle $\quad$. $\quad . \quad$. $\quad . \quad$. $\quad .37$

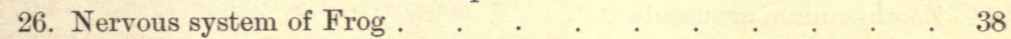

27. Hydra. . . . . . . . . . . . . . 41

28. Diagram of axes of body . . . . . . . . . . . 42

29. Radial symmetry . . . . . . . . . . . . . . . 42

30. Amœeba, various species . $\quad$. . . . . . . . . . . 47

31. Protamœba primitiva . . . . . . . . . . . . 49

32. Quadrula, Hyalosphenia, Arcella, and Difflugia . . . . . 49

33. Microgromia socialis . . . . . . . . . . 50 
FIG.

34. Platöum stercoreum

35. Various forms of Foraminifera

36. Shells of Foraminifera

37. Hastigerina murrayi

38. Dimorphism and altermation of generations in Polystomella

39. Actinophrys sol.

40. Actinosphærium eichhornii

41. Various forms of Heliozoa

42. Actinophrys sol, conjugation

43. Lithocircus annularis

44. Thalassoplancta brevispicula .

45. Aulactinium actinastrum

46. Actinomma asteracanthion

47. Collozoum inerme

48. Chlamydomyxa labyrinthuloides

49. Labyrinthula

50. Didymium difforme .

51. Euglena viridis .

52. Various forms of Flagellata

53. Trypanosome

54. Hæmatococcus pluvialis

55. Pandorina morum

56. Volvox globator

57. Heteromita rostrata .

58. Various forms of Choanoflagellata

59. Various forms of Dinoflagellata

60. Noctiluca miliaris

61. Monocystis .

62. Gregarina .

63. , development

64. Eimeria and Coccidium

65. Coccidium, life-history

66. Malaria parasite

67. Myxidium and Myxobolus . . . . . . . . . . 87

68. Sarcocystis miescheri . . . . . . . . . . . 88

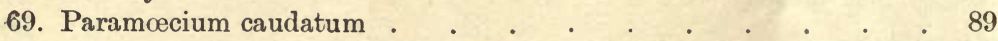

70.

conjugation

71. Various forms of Ciliata.

\section{2.}

73. Vorticella

74. Zoothamnium arbuscula

75. Opanna ranarum

76. Various forms of Tentaculifera

77. Diagram showing the mutual relationships of the Protozoa

78. Sycon gelatinosum

79 .

80.

magnified

81. , , 
FIG.

82. Sycon gelatinosum, pore-membrane

83. apopyle . .

84. External form of various Sponges .

85. Ascetta primordialis .

86. Diagrams of canal-system of various Sponges

87. Vertical Section of Spongilla .

88. Cells of ectoderm of Sponge .

89. Development of tri-radiate spicule

90. Skeleton of various Sponges

91. Various forms of Sponge spicules

92. Pheronema Carpenteri . .

93. Larva of Clathrina blanca

94. Development of Sycon raphanus

95. Obelia .

96. ", Vertical section of polype

97. Nematocysts of Hydra

133

98. Tentacle of Eucopella

134

99. Obelia, medusa .

135

136

137

138

140

144

145

146

147

148

150

110. Diagram illustrating formation of sporosac by degeneration of medusa

111. Early development of Eucope

112. Two Trachymedusse .

113. Two Narcomedusæ .

114. Aginura, tentaculocyst

115. Larva of Aginopsis .

116. Millepora alcicornis, skeleton

117. Millepora, diagram of structure

118. Stylaster sanguineus, skeleton

119. Halistemuna tergestinum

120. Diagram of a Siphonophore

121. Development of Halistemma . 
FIG.

129. Aurelia aurita, development

130. Tessera princeps

131. Lucernaria .

132. Pericolpa quadrigata

133. Nausithöe .

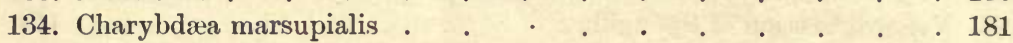

135. Pilema pulmo .

136. Pelagia noctiluca, development

184

137. Tealia crassicornis, dissection and transverse section _. . 186

138. Diagrammatic sections of Sea-anemone . . . . . . 188

139. Tealia crassicornis, section of tentacle . . . . . . . 190

140. Nematocysts of Sagartia . . . . . . . . . . . 190

141. Section of mesenteric filament of Sagartia . . . . . . 191

142. Transverse sections of embryos of Actinia . . . . . 193

143. Zoanthus sociatus . . . . • . . . . . . 197

144. Hartea elegans . . . . . . . . . . . . 197

145. Corallium rubrum . . . . . . . . . . . . . . 198

146. Astræa pallida . . . . . . . . . . . . . . . 198

147. Pennatula sulcata . . . . . . . . . . . 199

148. Tubipora musica . . . . . . . . . . . . . 199

149. Edwardsia claparèdii . . . . . . . . . . 200

150. Antipathes ternatensis . . . . . . . . . . 201

151. Parantipathes and Schizopathes . . . . . . . . 202

152. Minyas

202

153. Alcyonium palmatum

154. Gorgonia verrucosa

155. Structure of simple coral

203

204

156. Dendrophyllia and Madrepora . . . . . . . . 207

157. Adamsia palliata $. \quad . \quad$. . . . . . . . . . . 209

158. Hormiphora plumosa . . . . . . . . . . 211

159. " ", dissection and transverse section

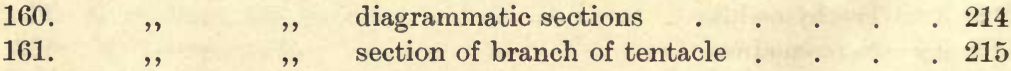

162.,$"$ sense-organ . . . . . . . 216

163. Ovum of Lampetia . . . . . . . . . . . 217

164. Segmentation of oosperm in Ctenophora . . . . . . 218

165. Development of Ctenophora . . . . . . . . . . 218

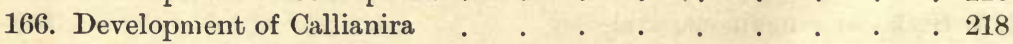

167. $" \quad$, (later stages) . . . . . . 219

168. Three Cydippida . . . . . . . . . . . . . 222

169. Deiopea kaloknenota . . . . . . . . . . . 223

170. Cestus veneris . . . . . . . . . . . . . . 223

171. Beröe forskälii . . . . . . . . . . . . 224

172. Ctenoplana kowalevskii . . . . . . . . . . 225

173. Sections of embryos of Actinia and Beröe . . . . . . 228

174. Diagram illustrating the mutual relationships of the Coelenterata . 229

175. Dicyema paradoxum with infusoriform embryos . . . . 230

176.

, ,, vermiform

230 
FIG.

177. Dicyema paradoxum, male

PAGE

178. Rhopalura giardii, male .

231

179.

female

232

180. Salinella, longitudinal section

232

181.

transverse

233

182. Planaria, digestive and excretory systems . . . . . . . 237

183., nervous system . . . . . . . . . 237

184., reproductive system . . . . . . . . . . 239

185. Transverse section of a Planarian . . . . . . . . . 240

186. Distomum hepaticum

187.

section of integument . . . . . 241

188.

intermal organisation

242

189. terminal part of reproductive apparatus

243

190. development

244

191. Tænia solium

246

192. ,, ,, head

247

193. ,, ,, transverse section

247

194

\section{proglottis}

248

195. ,, ,, ripe proglottis

250

196.

development

251

255

256

257

258

259

260

261

261

262

263

263

263

264

265

266

266

267

269

272

274

275

276

278

279

221. A Cysticercoid .

222. , with head eraginated . . . . . . 281

223. Cyst of Tænia echinococcus . . . . . . . . . . $\quad$. 282

224. Scolices

282 
225. Scolex of Tænia echinococcus

226. Process of budding in Microstomum

227. Diagram of the relationships of the Platyhelminthes and Nemertinea

228. Diagram of Nemertine

230. Tetrastemma

232. Proboscis of Hoplonemertean, retracted

235. Vascular and excretory systems of Nemertine

237. Ascaris lumbricoides

238.

transverse section

241. Nervous system of Nematoda

242. Ascaris lumbricoides, dissection of male organs

246. Oxyuris

247. Gordius, anatomy

249. Trichinella spiralis

250. Two species of Echinorhynchus (Gigantorhynchus) .

251. Echinorhynchus gigas, dissection of male
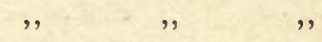

nephridia

255. Sagitta hexaptera

256. „, bipunctata, transverse sections . . . . . . 317

257. ", ,, heal

260. Chætosoma

261. Echinoderes

264. Brachionus rubens, female . . . . . . . . . . . . 324 
272. Chæetonotus maxinus

313. Asterina gibbosa, derelopment 
320. Echinus esculentus, peristome

321. Strongylocentrotus

322. Corona of Sea-urchin

324. Echinus, lantern of Aristotlo

325. Sea-urchin, anatomy, lateral view

326. Echinoid, transverse section of ambulacral zone

327. Sea-urchin, anatomy, oral view

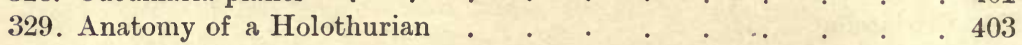

330. Antedon

331. Aboral view of Antedon .

332. Antedon disc

333. " transverse section of pinnule

334.,$\quad$ sagittal section . . . . . . . . 408

335. Anthenea, ventral view . . . . . . . . . . . 419

336. Ophioglypha lacertosa . . . . . . . . . . . . 420

337. Astrophyton arborescens . . . . . . . . . 421

338. Diagram of spine of Sea-urchin . . . . . . . . 422

339. Pedicellaria of Arbacia punctulata . . . . . . . 422

340. Hemipneustes radiatus . . . . . . . . . . 423

341. Clypeaster sub-depressus . . . . . . . . . 423

342. Metacrinus interruptus . . . . . . . . . . 424

343. Development of Echinoderms . . . . . . . . 431

344. ", Antedon . . . . . . . . . 432

345. Stalked larva of Antedon . . . . . . . . . 433

346. Diagram to illustrate the relationships of the classes of Echinodermata . . . . . . . . . . . 437

347. Nereis dumerilii . . . . . . . . . . . 440

348A. " , parapodium . . . . . . . . . 441

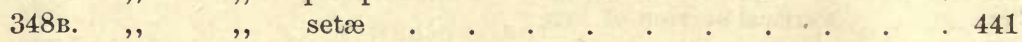

349. Nereis diversicolor, proboscis . . . . . . . . 443

350. Nereis dumerilii, anatomy . . . . . . . . . . 444

351.

transverse section

352. " " ",$\quad$ nervous system .

353.

,

eye

354. , , ,

nephridium

355. , , , development

356.

, ,

35̃7. Lumbricus herculeus 
366. Vermilia coespitosa

367. Chsetopterus

368. Setæ of various Polychrta

369. Section of setigerous sac of an Oligochæte

370. Polynöe extenuata, anterior end

471

371. Polychrta, various, heads

372. Tubifex

473

373. Terebella

474

374. Aphrodita, enteric canal .

475

375. Saccocirrus, transverse section . . . . . . . . . . . . 477

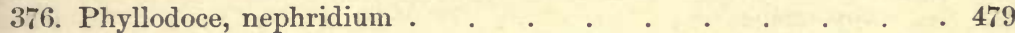

377. Nephridia and colomolucts . . . . . . . . . . . 480

378. Diagram illustrating development of gonad of Polychreta . . 482

379. Spirorbis lavis . . . . . . . . . 484

380. Eupomatus, development of trochophore . . . . . . 485

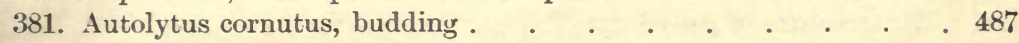

382. Syllis ramosa . . . . . . . . . . . . 487

383. Serpulæ with their tubes . . . . . . . . . . . . 488

384. Myzostomum . . . . . . . . . . . . 490

385. , anatomy . . . . . . . . . 491

386. Sipunculus nudus, anterior extremity . . . . . . . . 492

387. , , , tentacular fold . . . . . . . 493

388., ,, anatomy . . . . . . . . 494

389. , . , nervous system . . . . . . . . 494

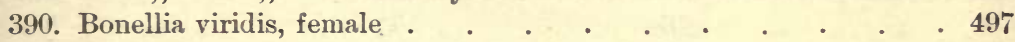

391. Echiurus . . . . . . . . . . . . . . . . . 497

392. Priapulus . . . . . . . . . . . . . . . . . . 498

393. Bonellia, anatomy . . . . . . . . . . . . . 499

394. Echiurus, ciliated funnel . . . . . . . . . . . . . 499

395. ,, anatomy . . . . . . . . . . 500

396. , nervous system . . . . . . . . . . 500

397. Bonellia, male . . . . . . . . . . . . . . 501

398. Echiurus, trochophore . . . . . . . . . . 501

399. Polygordius neapolitanus _ . . . . . . . . . 503

400. Protodrilus . . . . . . . . . . . . . 504

401. Polygordius neapolitanus, transverse section . . . . . 504

402. $", \quad$ trochophore . . . . . . 505

$403 . \quad, \quad, \quad$, later stage . . . 505

404. Hirudo medicinalis . . . . . . . . . . . 507

405. , , transverse section . . . . . . . 508

406.,$\quad$ " jaw . . . . . . . . . . 509

407. " australis, dissection from dorsal aspect . . . . 510

408. " australis, ," , left side . . . . . 511

409. ," medicinalis, nephridium . . . . . . . . . 512

410. " diagram of blood-channels . . . . . . . . 513

411.,$\quad$ section of eye . . . . . . . . . . . . . 514

412. $"$ cocoon . . . . . . . . . . . . 515

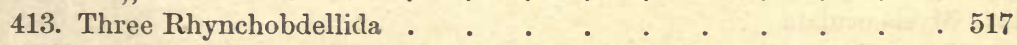


FIG.

414. Proboscis of Clepsine

415. Nephridium of Herpobdella

416. Pontobdella, nephridial system

417. Clepsine, development

521

418. Diagram of origin of metamerism .

419. Diagram illustrating the relationships of the Annulata and Trochelminthes

420. A pus cancriformis, dorsal aspect.

421. Lepidurus kirkii, side view

422. Apus glacialis, ventral aspect

423. , appendages

530

424. Lepidurus kirkii, sagittal section .

532

425. Apus, transverse section

534

426. , shell-gland

535

427. ,, cancriformis, nervous system

536

428. ,, structure of paired eye .

537

429. ,, development

538

4:30A.Astacus fluviatilis, male .

430в. ,,

transverse section of abdomen

540

431. , , appendages

543

432.

articulations and muscles of legr

545

433. Section of skin and exoskeleton of Lobster

546

434. Articulations and muscles of abdomen of Crayfish

435. Astacus fluviatilis, dissection from right side .

451. Cyclops and Calocalanus . 
F1G.

461. Gammarus

462. Asellus

585

463. Amphipoda

586

464. Isopoda

587

465. Shrimp and Prawn . . . . . . . . . . . . . . . . 588

466. Scyllarus arctus

589

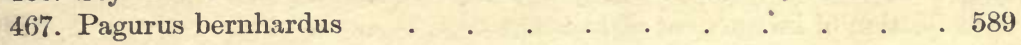

468. Cancer pagurus . . . . . . . . . . . . . . . . 590

469. Typical Brachyura . . . . . . . . . . . . . . 591

470. Squilla

592

471. Orchestia cavimana, anatomy . . . . . . . . . 594

472. Euphausia pellucida . . . . . . . . . . . . . . . 595

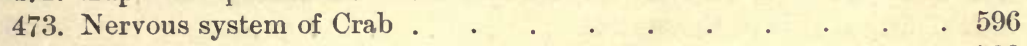

474. Cypris-stage of Lepas . . . . . . . . . . . . . . . . 598

475. Larvæ of Crabs : . . . . . . . . . . . . . . 600

476. Diagram illustrating the mutual relationships of the orders of

Crustacea . . . . . . . . . . . 604

477. Dalmanites and Phacops . . . . . . . . . . . . . . . . . . . . . . . . . 605

478. Triarthrus beckii . . . . . . . . . . . . 606

479. Peripatus capensis . . . . . . . . . . . . 607

480., , ventral view of head . . . . . 607

481., , anatomy : . . . . . . . . . . . . . 608

482., . tracheal pit . . . . . . . . . . 609

483., nephridium $. \quad . \quad . \quad . \quad . \quad . \quad . \quad . \quad . \quad .610$

484., novæ zealandiæ, development . . . . . . . 611

485., capensis . . . . . . . . . . . . . 613

486. Scolopendrella immaculata . . . . . . . . . . . . 61 . 610

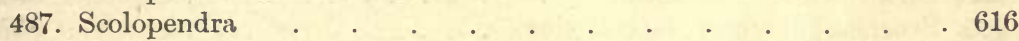

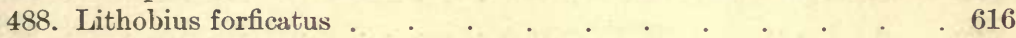

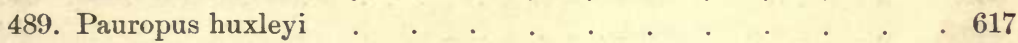

490. Strongylostoma, development . . . . . . . . . . . . 618

491. Periplaneta orientalis . . . . . . . . . . . . . . 620

492., mouth-parts . . . . . . . . . . . . 621

493. $\quad, \quad$ americana, lateral view of head $\quad . \quad$. 621

494., muscular system . . . . . . . . . . 624

495., anatomy $. \quad . \quad . \quad . \quad . \quad . \quad . \quad .625$

496., salivary glands . . . . . . . . . . . . 625

497. Trachea of caterpillar . . . . . . . . . . . . . . . . 626

498. Periplaneta, tracheal system . $. \quad . \quad . \quad . \quad . \quad . \quad 6627$

499.,$\quad$ nervous system . . . . . . . . . . . 627

500. $\quad, \quad$ male reproductive organs . . . . . . . 628

501., female reproductive organs $\quad . \quad \ldots \quad . \quad . \quad . \quad 628$

502. Segmentation of ovum of Insect . . . . . . . . . . 629

503. Ventral plate of embryo Cockroach . . . . . . . $\quad$. 630

504. Embryo Cockroach . . . . . . . . . . . . 630

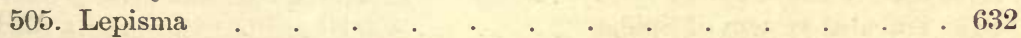

506. Podura . . . . . . . . . . . . . . 632

507. Locusta $\quad . \quad . \quad . \quad . \quad . \quad . \quad . \quad .633$

508. Ephemera . . . . . . . . . . . . . 633 
FIG.

509. Aphis rosæe

PAGE

510. Cicada .

633

511. Culex and larva .

512. Gastrophilus equi

513. Pieris

514. Crioceris

635

515. Section of integument of Insect

516. Mouth-parts of Honey-bee

520. Nervous, tracheal, and digestive systems of the Honey-bee

522. Heart of Cockchafer .

523. Nervous system of Diptera

524. Ocellus of Dytiscus larva

525. Chordotonal organ of Isopteryx

531. Apis mellifica, queen, worker, and drone

533. Euscorpio

ล34. Ventral surface of cephalothorax and pre-abdomen of Scorpion

535. Endosternite of Scorpion

536. Scorpion, anatomy, lateral view

539. Embryo of Scorpion

540. Chelifer bravaisii

541. Phrynus

542. Galeodes dastuguei

543. Epeira diadema .

544. , , , cheliceræ and pedipalpi of female

545. , , male.

546. Sarcoptes scabiæi

547. Trombidium fuliginosum .

548. Limulus

549. , ventral view 
558. Nymphon hispidum

559. Pentastomum tænioides

560. Macrobiotus hufelandi

561. Diagram to illustrate affinities of Arthropoda . . . . . 678

562. Anodonta cygnea

681

$563 . \quad$,

564. , ,

565.,

$566 . \quad$,

$567 . \quad$,

$568 .$,

$569 .$,

$570 . \quad$,

571. ,

572. , ,

$573 ., \quad$,

574. , ,

575. Anatomy of Pecten

interior of valve and animal removed from shell . section of shell and mantle cygnea, animal after removal of mantle-lobe ,, dissection from left side , structure of gills , transverse sections diagram of circulation statocyst early embryo later embryos .

682 683 685 686 687 688 690 691 692 692 693 694 697

576. Valves of Mya, Modiola, and Vulsella 698 577. Cardium edule 698 578. Venus gnidia 699 579. Scrobicularia piperata 699 580. Solecurtus strigillatus

581. Diagram of concrescence of mantle-lobes 700 700 582. Requienia and Hippurites 583. Teredo navalis

584. Aspergillum

585. Mytilus edulis

586. Nucula delphinodonta

703

587. Gills of Pelecypoda .

588. Gill-filaments of Mytilus

590. Donax, enteric canal

591. Nerrous system and auditory organs of Nucula . . . . . 707

592. Eye of Pecten

595. Embryos of Cyclas . • • • • • $\quad 710$

596. Diagram illustrating the mutual relationships of the Pelecypoda . 712

597. Chætoderma nitidulum . . . . . . . . . . . 713

598. Neomenia carinata . . . . . . . . . . . 714

599. Chiton, spinosus, dorsal view $\quad . \quad . \quad . \quad . \quad . \quad . \quad . .714$

600. ", ventral view . . . . . . . . . . 714

601. , $\quad$ valves of shell . . . . . . . . . . . 715

602. Chætoderma nitidulum, longitudinal section . . . . . 716

603. Chiton, longitudinal section . . . . . . . . . 717

604. Nervous system of Amphineura . . . . . . . . 717

605. Neomenia carinata, reproductive organs . . . . . . 718

606. Chiton, nephridial and genital systems _ . . . . . 719 
FIG.

607. Chiton, development

608. Triton nodiferus, shell

609. Triton ,

610. ,,

611. ,,

612. ,

613. , ,

614. ,

615. ,

616. ,,

617. , ,

618. ,,

shell, median section

operculum .

lateral view of body.

619. Diagrams of displacement of mantle-cavity, \&c.

731

620. Solarium perspectivum

621. Terebra oculata .

622. Cypræa moneta .

623. Doris tuberculata

624. Carinaria mediterranea

625. Limax .

626. Sigaretus lævigatus .

627. Aplysia

628. Shell-bearing Pteropoda .

629. Atlanta peronii .

630. Pterotrachea scutata

631. Helix nemoralis

632. Pleurophyllidia lineata

633. Patella vulgata .

634. Pulmonary cavity and related parts in Limax

635. Nervous system of Patella

636. Nervous system of Aplysia

637.

Limnæus .

638. Eyes of Gastropoda .

639. Osphradium of Murex

640. Reproductive organs of Helix

641. Orotestis of Gastropoda

642. Forms of egg-cases in Gastropoda .

643. Segmentation and formation of germinal layers in Gastropoda

644. Early development of Patella

645. Trochophore of Patella

646. Later trochophore of Patella.

652. Rhodope 
FIG.

656. Sepia, cultrata, cranial cartilage

PAGE

657. ,, ,, nuchal cartilage

763

763

658. ", , mantle-cavity

764

659. „, officinalis, jaws

765

660. , section of buccal mass .

766

661. „, officinalis, enteric canal . . . . . . . . . $\quad .766$

662. ", cultrata, dissection of male from posterior aspect . . . 767

663. " $\quad, \quad$ lateral dissection of male . . . . . . . 768

664.,$\quad$ officinalis, longitudinal section of ink-sac . . . . 769

665. ", cultrata, vascular system . . . . . . . 770

666. $, \quad, \quad$ cephalic ganglia . . . . . . 770

667. $, \quad, \quad$ pedal and pleuro-visceral ganglia . . . . 770

668. , section of eye . . . . . . . . . . 771

669.,$\quad$ cultrata, statolith . . . . . . . . . . 772

670. ,, officinalis, renal organs . . . . . . . . . . 773

671. $, \quad, \quad$ diagrammatic sagittal section of female . . . 774

672. ", male reproductive organs . . . . . . . . 775

673.,$\quad$ sperms and spermatophore . . . . . . . 775

674. Nautilus pompilius, section of shell . . . . . . . 776

675.,$" \quad$, female in shell . . . . . . . 778

676. Nautilus macromphalus, entire animal . $\quad . \quad$. . . . . 789

677. Nautilus pompilius, lobe of foot . . . . . . . . 780

678.,$\quad$, spadix . . . . . . . . . 781

679. $, \quad, \quad$ cephalic cartilage . . . . . . 781

680. $"$ ",$\quad$ mantle-cavity of male . . . . . 782

681. ", , 'dissection of male from left side . . . 784

682. $", \quad$ arteries . . . . . . . . 785

683. $" \quad \# \quad$ renal sacs, ctenidia, \&c. . . . . . . 786

684. $, \quad, \quad$ male reproductive organs . . . . . 788

685. $", \quad$ female,$\quad$. . . . . . . 788

686.,$\quad$ macromphalus, egg . . . . . . . . . 789

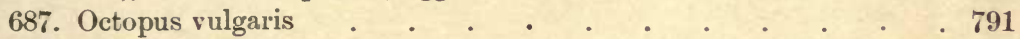

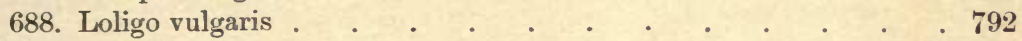

689. Argonauta argo . . . . . . . . . . . . . 793

690. Octopus lentus, male _ . . . . . . . . . . 793

691. Amphitretus pelagicus . . . . . . . . . . . . 794

692. Shell of Spirula . . . . . . . . . . . . . 794

693. Spirula peronii . . . . . . . . . . . . . 795

694. Ammonite . . . . . . . . . . . . . 795

695. Shell of Belemnite . . . . . . . . . . . . . . 796

696. $\quad$ Argonauta argo . . . . . . . . . . . 796

697. Segmentation of Loligo . . . . . . . . . . . . 798

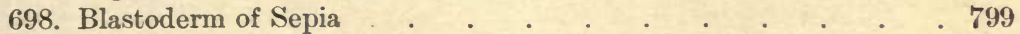

$699 . \quad$,, , sections . . . . . . . . . 799

700. Development of Loligo . . . . . . . . . . 800

$701 . \quad, \quad . \quad . \quad . \quad . \quad . \quad . \quad . \quad 801$

$702 . \quad, \quad$ "

703. $", \quad . \quad . \quad . \quad . \quad . \quad . \quad . \quad 802$

704. Diagram to illustrate the relationships of the Cephalopoda . . 804 



\section{CLASSIFICATION OF THE ANIMAL KINGDOM}

\section{IN THIS BOOK.}

\section{KINGDOM ANIMALIA.}

\section{Phylum I. PRotozoA.}

Class I. RHIZOPODA.

Order 1. Loвosa.

,2. ForaMINIFERA.

3. HeLIOZOA.

4. Radiolaria.

Class II. MYCETOZOA.

Class III. MASTIGOPHORA.

Order l. Flageliata.

,2. Сhod oflageliata.

„, 3. Divoflageldata.
Order 4. Crstoflagellata. Class IV. SPOROZOA.

Order 1. Gregarinida.

,2. Cocciditidia.

, .3. HeNOSPORIDIA.

, 4. MYXosporideA.

, 5. Sarcocystidea.

Class V. INFUSORIA.

Grder l. Ciliata.

, 2. TEXTACClifera.

\section{Phylui II. PORIfERA.}

Class PORIFERA.

Sub-class I. Calcarea.

Order 1. Howocela.
Order 2. Heterochela.

Sub-class II. Hexactinellida. , III. Demospongia.

Phylum III. Coelenterata.

Class I. HYDROZOA.

Order 1. LeptoliNe. Sub-order a. Anthomeduste.

Order 2. Trachylix. b. Leptomedusie.

Class II. SCYPHOZOA.

Order 1. Stauromedus.z.

,2. Corovata.

„3. Cubonedes.

"4. Discomedes.z.

Sub-order $a$. Semostomu. „b. Rhizostome.

Class III. ACTINOZOA.

vOL. I
Sub-elass I. Zoantharia.

Order 1. Acriviaria.

, 2. Madreporakia.

, 3. Axtipatharia.

Sub-class II. Alcyonaria.

Sub-orler a. Trachymerluste.

., b. Narcomedusie.

Order 3. HYDROCORALIINA.

, 4. SIPHOXOPHORA.

, 5. Graptolithida.

, 4. Alcronaces.

",. GorgoNacEa.

"6. Pexiatulacea. 


\section{Phylum III. CoLlenterata-continued.}

Class IV. CTENOPHORA.

Order 1. Cy DIPPIDA.

2. Lobata.
3. Cestida.
, 4. Reroid.

Appendix to Ctenophora-Ctenoplana and Coloplana.

Order 1. Platictenea.

Appendix (II) to Ccelenterata-Mesozoa.

\section{Phylum IV. PLATyhelminthes.}

Class I. TURBELLARIA.

Order 1. Policladida.

,2. Tricladida.

,, 3. RHABDOCELIDA.

Class II. TREMATODA.

Order 1. MoNociexetica.

, 2. DigeNetica.
Order 3. Aspidocotylea.

Class III. CESTODA.

,4. Temnocephalea.

Order 1. MosozoA.

,2. Polizoa.

Appendix to Platyhelminthes-Class NEMERTINEA.

\section{Phylum V. Nemathelminthes.}

Class I. NEMATODA.

Order 1. Nematoidea. „2. Nematomorpha.

Class II. ACANTHOCEPHALA.
Class III. CH ETOGNATHA.

Appendix to Nemathelminthes-Chat tosomide, Echinoderide, and Desmoscolecide.

\section{Phylum VI. TROChelminthes.}

Class I. ROTIF ERA.

Oriler 1. RHIzota. , 2. BDELLOÏDA. :

, 3. Ploïma.

Sub-order $\alpha$. Illoricata. , b. Loricata.

Order 4. Scirtopod.
Order 5. TRochospharid.. , 6. Seisoxida.

Class II. GASTROTRICHA.

Appendix to Trochelminthes-Dinophilea and Histriobdellea.

\section{Phylum VII. MOLluscoida.}

Class I. POLYZOA.

Sub-class I. Ectoprocta.

Order 1. Gymiol emata.

Sub-order $a$. Cyclostomata.

, b. Cheilostomata.

, c. Ctenostomata.
Order 2. Phylactolmiata. Sub-class II. Endoprocta.

Class II. PHORONIDA.

, III. BRACHIOPODA.

Order 1. Inarticulata.

, 2. Articulata.

\section{Physum VIII. ECHINODERMatA.}

Class I. ASTEROIDEA.

Order 1. Phanerozonia.

,2. Cryptozonia.

Class II. OPHIUROIDEA:

Order 1. LysophivR玉.

,2. 2. STREPTOPHIURE.
Order 3. Cladophicle.

,, 4. ZYGOPHIUKA.

Class III. ECHINOIDEA.

Order 1. Regularia.

,, 2. Clypeastridea.

, 3. Spatajgoidea. 


\section{Phylum VIII. ECHINOdERMATA-continued.}

Class IV. HOLOTHUROIDEA.

Order 1. Filasipoda.

$$
\text { ,2. Pedata. }
$$

3. APODA.

Class V. CRINOIDEA.

Sub-class I. Monocyclica.
Sub-class II. Dicyclica.

Class VI. CYSTOIDEA.

"VII. BLASTOIDEA.

„, VIII. EDRIASTEROIDEA.

, IX. CARPOIDEA.

\section{Phylum IX. ANNUlata.}

\section{ClasS I. CH ETOPODA.}

Sub-class I. Polychæta.

Order 1. ARCHI-Ch etopoda.

„2. Phanerocephata.

", 3. CRyptocephala.

Sub-class II. Oligochæta.

Order 1. Microdrili.

2. MEgadRILI.

Appendix to the Chretopoda-Class MYZOSTOMIDA.
Class II. GEPHYREA.

Order I. INERMIA.

,2. Armata.

Class III. ARCHI-ANNELIDA.

, IV. HIRUDINEA.

Order 1. RHYNCHOBdellida.

2. Arhyschobdellidi.

Sub-order 1. Gnathobdellida.

,, 2. Herpobdellida.

\section{Phylum X. AR'THROPODA. L}

Class I. CRUSTACEA.

Sub-class I. Branchiopoda.

Order 1. Axostraca.

,2. Notostraca.

, 3. Conchostraca.

, 4. Clladogera.

Sub-class II. Ostracoda.

, III. Copepoda.

Order 1. Eucopepona.

, 2. Branchitra.

Sub-class IV. Cirripedia.

Order 1. EUCIRRIPEDIA.

,2. RHIZOCEPHALA.

Sub-class V. Malacostraca.

Order 1. Mrsidacea.

2. Cumacea.

3. Tanaidacea.

4. ISOPODA.

5. ANPHIPODA. -

Sub-order 1. Macrura.

2. Anomura.

3. Brachyura.

Appendix to Crustacea-Class TRI-

\section{LOBITA.}

Class II. ONYCHOPHORA.

, III. MYRIAPODA.

Sub-class I. Progoneata.
Order 1. Pauropoda.

,2. DipLOPODA.

," 3. SyмphyLA.

Sub-class II. Opisthogoneata.

Order 1. Chilopoda.

Class IV. INSECTA.

Order 1. APtera.

, 2. ORthoptera.

, 3. NeUroptera.

, 4. Hemiptera.

" 5. Diptera.

"6. LEPIDOPTERA.

", 7. Coleoptera.

- ", 7. HYLEOPTERA.

Class V. ARACHNIDA.

Order 1. SCORPIONIDA.

, 2. Pseudoscorpionida.

„3. PEDipalpida.

," 4. Solptgida.

„5. Phalaygida.

"6. Araneida.

, T. ACARIDA.

", 8. Xiphosura.

„9. Eurtpterida.

Appendix to the Arachnida-The Pycrogonida, Livguatulida, and TarDIGRADA. 
Phylum Xi. MOLlúsCa.

\section{Class I. PELECYPODA.}

Order 1. Protobranchia.

2. Filibraxchia.

, 3. Pseudo-lamellibranchia

4. Eulamellibranchia.

Sub-order $a$. Integripalliata.

Order 5. Septibranchia.
,
b. Sinupalliata.

\section{Class II. AMPHINEURA.}

Order 1. Placophora.

,2. Aplacophora.

Class III. GASTROPODA.

Sub-class I. Streptoneura.

Order 1. AspidonRanchia.

Sub-order 1. Docoglossa.

, 2. Rhipidoglossa.

\section{Phylum XII. CHORDATA.}

SUB-PHylum I. ADELOCHORDA.

\section{Class ADELOCHORDA.}

Sub-PHylum II. UROCHORDA.

\section{Class UROCHORDA.}

Order 1. LARVACEA.

, 2. Thaliacea.

Sub-order $\alpha$. Cyclomyaria.

, b. Hemimyaria.

,, c. Pyrosomata.

Order 3. Ascidiacka.

Sub-order $\alpha$. Ascidice simplices.

, b. Ascidice composita.

SUb-PHYlum III. EUCHORDA.

Section I. Acrania.

Section II. Craniata.

Class I. cyclostomata.

Order 1. Petromyzontes.

Class II. PISCES.

,2. MrxinoIdeI.

Sub-class I. Elasmobranchii.

Order 1. Claddoselachir.

, 2. Pleuracantilei.

, 3. ACANTHOdeI.

, 4. Selachili.
Order 2. Pectivibkanchia. Sub-order 1. Platypoda.

2. Heteropoda.

Sub-class II. Euthyneura.

Order 1. OristhoвRANChi. Sub-order 1. Tectibranchia.

Order 2. Pulmonata.

Appendix to the Gastropoda-Class SCAPHODA and RHODOPE.

\section{Class V. CEPHALAPODA.}

Sub-class I. Dibranchiata.

Order 1. DuCaPoda.

,2. OCTOPODA.

Sub-class II. Tetrabranchiata.

Sub-order a. Protoselachii.

, b. Euselachii.

Section a. Squalida.

, B. Rajida.

Sub-class II. Holocephali.

,, III. Teleostomi.

Order 1. Crossopteryii.

,2. Chondrostei.

, 3. Holostej.

,4. Teleostei.

Sub-order a. Physostomi.

,, $\quad$ b. Anacanthini.

, c. Acanthopteri.

", d. Pharyngognathi.

, e. Plectognathi.

"f. Lophobranchii.

Sub-class IV. Dipnoi.

Order 1. Moxopxecmoxa.

2. DipsechoNa.

Appendix to Pisces-The Ostracodermi.

Class III. AMPHIBIA.

Order 1. URodela.

,2. Axura.

,, 3. Gruxophioxa.

," 4. Stegocephala. 


\section{CLASSIFICATION OF THE ANIMAL KINGDOM xxxix}

\section{Phylum XII. CHORDATA-continued.}

Class IV. REPTILIA.

Order 1. Squamata.

Sub-order $a$. Lacertilia.
b. Ophidia.
" c. Pythonomorpha.

Order 2. RhYNCHOCEPHALIA.
, 3. Chelonita.
4. THEROMORPHA.
5. Crocodilla.
6. SaUropiterygia.
7. ICHTHYOSAURIA.
8. Dinosauria.
9. Pterosiauria.

Class V. AVES. レ

Sub-class I. Archæornithes.

Sub-class II. Neornithes.

Division A. Ratitæ.

Order 1. Megistanes.

2. Apteryges.

3. Dinornithes.

4. RHEA.

5. StRUthiones.

6. EPYORNITHES.

7. Gastornithes.

Division B. Carinatæ.

Order 1. Stereornithes.

2. ODONTOLCE.

3. ICHTHYORNITHES.

4. Pygopodes.

5. ImpenNes.

6. Turbinares.

7. Steganopodes.

8. HERODIONES.

9. ANSERES.

10. Accipitres.

11. Crypturi.

12. Gallin 2.

13. Grallex.

14. Gaviz.

15. Limicole.
Order 16. Pterocletes.

,17. Columbe.

„18. Psittaci.

19. Striges.

20. PiCarize.

, 21. Passeres.

\section{Class VI. MAMMALIA.}

Sub-class I. Prototheria. L

II. Theria.

Section A. Metatheria (Marsupiat.IA).

Order 1. Polyprotodontia.

„2. Diprotodontia.

Section B. Eutheria. -

Order 1. Edentata.

„2. Cetacea.

Sub-order a. Mystacoceti. " b. Odontoceti

Order 3. Sirenia.

, 4. UngUlata.

Section 1. Ungulata vera.

Sub-order $a$. Perissodactyla.

b. Artiodactyla.

Section 2. Subungulata.

Sub-order a. Hyracoidea. ,

b. Proboscidea.

Order 5. Carnivora.

Sub-order $a$. Carnivora vera.

, b. Pinnepedia.

Order 6. Rodentia.

, 7. INSECTIVORA.

„8. Chiroptera.

Sub-order a. Megachiroptera.

, b. Microchiroptera.

Order 9. Primates.

Sub-order $a$. Prosimii.

" b. Anthropoidea 


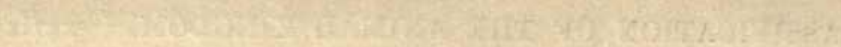

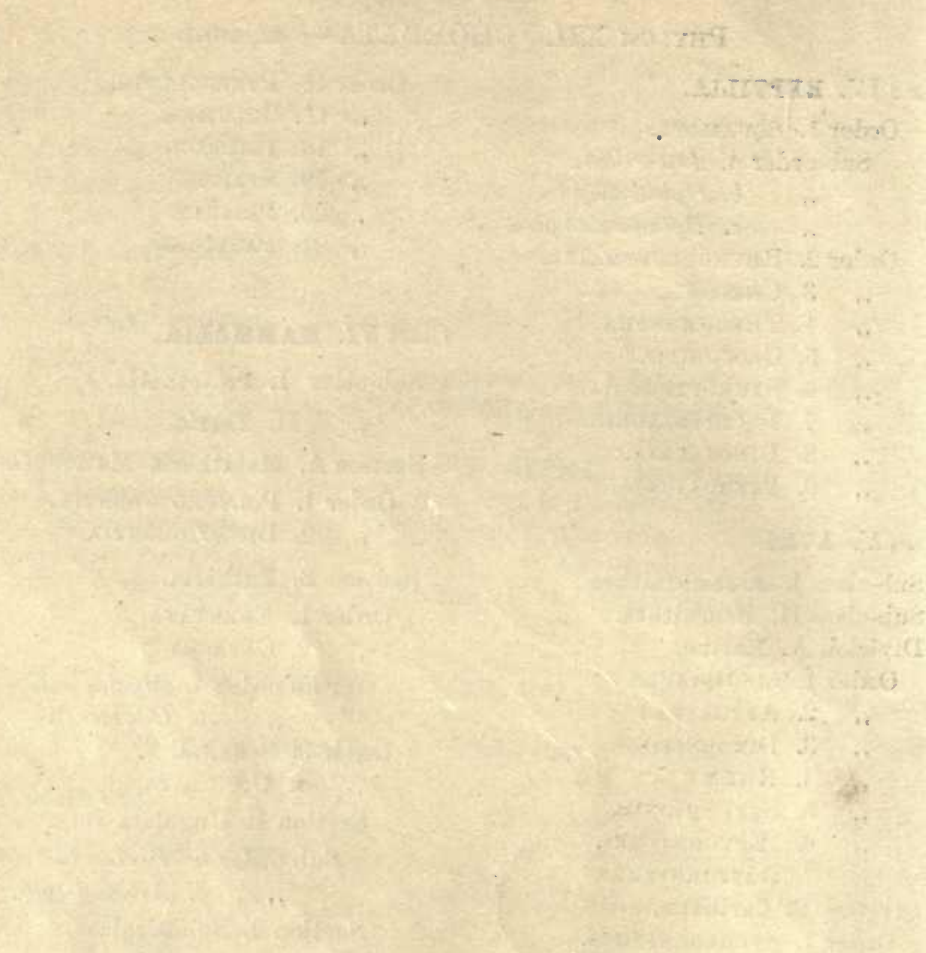




\section{Z OOLOGY}

\section{INTRODUCTION}

Zoology, the branch of Natural History which deals with animals, is one of the two subdivisions of the great science Biology, which takes cognisance of all organisms, or things having life, as distinguished from such lifeless natural objects as rocks and minerals. The second of the two subdivisions of Biology is Botany, which deals with plants.

The subject-matter of Zoology, then, is furnished by the animals which inhabit the land-surface, the air, and the salt and fresh waters of the globe: the aim of the science is to find out all that

\section{ERRATA.}

PAGE.

17, description of Fig. 5, for " atrosphere" read " astrophere."

52, description of Fig. 35, for " Rotalla" read " Rotalia."

71, description of Fig. 52, for "Astasiopis" read " Astasiopsis."

74, line 9, for "divison" read "division."

111, line 1, for " out" read "outer."

208, line 10, for "siphnozoids" read "siphonozooids."

272 , line 2 , for " prostrate" read " prostate."

402, line 43, for " periphæmal" read " perihaemal."

450 , line 7 , for "Fig. 346" read " Fig. 347." 


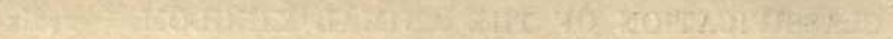

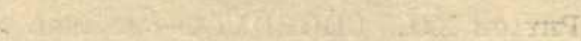

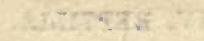

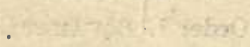

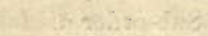




\section{Z OOLOGY}

\section{INTRODUCTION}

Zoology, the branch of Natural History which deals with animals, is one of the two subdivisions of the great science Biology, which takes cognisance of all organisms, or things having life, as distinguished from such lifeless natural objects as rocks and minerals. The second of the two subdivisions of Biology is Botany, which deals with plants.

The subject-matter of Zoology, then, is furnished by the animals which inhabit the land-surface, the air, and the salt and fresh waters of the globe: the aim of the science is to find out all that can be known of these animals, their structure, their habits, their mutual relationships, their origin.

The first step in the study of Zoology is the recognition of the obvious fact that the innumerable individual animals known to us may be grouped into what are called species, the members of which resemble one another so closely that to know one is to know all. The following example may serve to give the reader a fairly accurate notion of what Zoologists understand by species, and of the method of naming species which has been in use since the time of the great Swedish naturalist Linnæus.

The Domestic Cat, the European Wild Cat, the Ocelot, the Leopard, the Tiger, and the Lion are animals which agree with one another in the general features of their organisation - in the number and form of their bones and teeth, in the possession of retractile claws, and in the position and characters of their internal organs. No one can fail to see that these animals, in spite of differences of size, colour, markings, \&c., are all, in the broad sense of the word, "Cats." This is expressed in the language of systematic Zoology by saying that they are so many species of a single genus.

Aocording to the system of binomial nomenclature introduced by Linnæus, each kind of animal receives two names-one the generic 
name, eommon to all specres of the genus; the other the specific name, peculiar to the species in question. Both generic and specific names are Latin in form, and are commonly Latin or Greek in origin, although frequently morlern names of persons or places, with Latinised terminations, are employed. In giving the name of an animal, the generic name is always placed first, and is written with a capital letter, the specific name following it, and being written, as a rule, with a small letter. For instance, to take the examples already referred to, the Domestic Cat is called Felis domestica, the European Wild Cat $F$. catus, the Leopard $F$. pardus, the Tiger $F$. tigris, the Lion $F$. leo. Thus the systematic name of an animal is something more than a mere appellation, since it indicates the affinity of the species with other members of the same genus: to name an animal is, in fact, to classify it.

It is a matter of common observation that no two individuals of a species are ever exactly alike: two tabby Cats, for instance, however they may resemble one another in the general characters of their colour and markings, invariably present differences in detail by which they can be readily distinguished. Individual variations of this kind are of universal occurrence. Moreover, it often happens that the members of a species are divisible into groups distinguishable by fairly constant characters: among Domestic Cats, for instance, we find white, black, tabby, gray, and tortoiseshell Cats, besides the large long-haired Persian breed, and the tailless Manx Cat. All these are distinguished as varictics of the single species Felis domestica.

It is often difficult to decide whether two kinds of animals should be considered as distinct species or as varieties of a single species, and no universal rule can be given for determining this point. Among the higher animals mutual fertility is a fair practical test, the varieties of a species usually breeding freely with one another and producing fertile offspring, while distinct species either do not breed together or produce infertile hybrids or mules. Compare, for instance, the fertile mongrels produced by the union of the various breeds of Domestic Dog with the infertile mule produced by the union of the Horse and Ass. But this rule is not without exception, and in the case of wild animals is, more often than not, impossible of application: failing it, the only criterion of a "good species" is usually the presence of constant differences from allied species. Suppose, for instance, that a naturalist receives for description a number of skins of wild Cats, and finds, after an accurate examination, that in some specimens the tail is two-thirds the length of the body and the skin of a uniform reddish tint with a few markings on the head, while in the rest the tail is nearly half as long as the body, and the skin tawny with black stripes. If there are no intermediate gradations between these two sets of individuals, they will be placed without hesitation in distinct 
species: if, on the other hand, there is a complete series of gradations between them, they will be considered to form a single variable species.

As, therefore, animals have to be distinguished from one another largely by structural characters, it is evident that the foundations of a scientific Zoology must be laid in Morphology, the branch of science which deals with form and structure. Morphology may be said to begin with an accurate examination of the external characters; the divisions of the body, the number and position of the limbs, the characters of the skin, the position and relations of the mouth, eyes, ears, and other important structures. Next the internal structure has to be studied, the precise form, position, \&c., of the various organs, such as brain, heart, and stomach, being made out: this branch of morphology is distinguished as Anatomy. And, lastly, the various parts must be examined by the aid of the microscope, and their minute structure, or Histology, accurately determined. It is only when we have a fairly comprehensive knowledge of these three aspects of a given animal-its external characters, its rough anatomy, and its histology - that we can with some degree of safety assign it to its proper position among its fellows.

An accurate knowledge of the structure of an animal in its adult condition is not, however, all-sufficient. Nothing has been made more abundantly clear by the researches of the last halfcentury than that the results of anatomy and histology must be checked, and if necessary corrected, by Embryology-i.e. by the study of the changes undergone by animals in their development from the egg to the adult condition. A striking instance is afforded by the common Barnacles which grow in great numbers on ships' bottoms, piers, \&c. The older zoologists, such as Linnæus, grouped these creatures, along with Snails, Mussels, and the like, in the group Mollusca, and even the great anatomical skill of Cuvier failed to show their true position, which was made out only when Vaughan Thompson, about sixty years ago, proved, from a study of the newly hatched young, that their proper place is among the Crustacea, in company with Crabs, Shrimps, and Water-fleas.

Given a sound knowledge of the anatomy, histology, and embryology of animals, their Classification may be attempted-that is, we may proceed to arrange them in groups and sub-groups, each capable of accurate definition.

The general method of classification employed by zoologists is that introduced by Linnæus, and may be illustrated by reference to the group of Cats which we have already used in the explanation of the terms genus, species, and variety.

We have seen that the various kinds of true Cat-Domestic Cat, Lion, Tiger, \&c.- - together constitute the genus Felis. Now there 
is one nember of the cat-tribe, the Cheetah, or Hunting Leopard, which differs from all its allies in having imperfectly retractile claws and certain peculiarities in its teeth. It is therefore placed in a distinct genus, Cynolurus, to mark the fact that the differences separating it from any species of Felis are of a more fundamental character than those separating the species of Felis from one another.

The nearest allies of the Cats are the Hyænas, but the presence of additional teeth and of non-retractile claws - to mention only two points-makes the interval between Hyænas and the two genera of Cats far greater than that between Felis and Cynælurus. The varying degree of difference is expressed in classification by placing the Hyænas in a separate family, the Hyonida, while Felis and Cynælurus are placed together in the family Felida. Similarly, the Civets and Mongooses form the family Viverrida; the Dogs, Wolves, Jackals, Foxes, \&c., the family Canida; Bears, the family Ursida; and so on.

All the foregoing animals have sharp teeth adapted to a flesh diet, and their toes are armed with claws. They therefore differ fundamentally from such animals as Sheep, Deer, Pigs, and Horses, which have flat teeth adapted for grinding vegetable food, and hoofed feet. The differences here are obviously far greater than those between any two of the families mentioned above, and are emphasised by placing the flesh-eaters in the order Carnivora, the hoofed animals in the order Ungulata. In the same way gnawing animals, such as Rats, Mice, and Beavers, form the order Rodentia; pouched animals, such as Kangaroos and Opossums, the order Marsupialia; and so on.

Carnivora, Ungulata, Rodentia, Marsupialia, \&c., although differing from one another in many important respects, agree in the possession of a hairy skin and in the fact that they all suckle their young. They thus differ from Birds, which have a covering of feathers and hatch their young from eggs. The differences here are considerably more important than those between the orders of quadrupeds referred to, and are expressed by placing the latter in the class Mammalia, while Birds constitute the class Avcs. In the same way the scaly, cold-blooded Lizards, Snakes, Tortoises, \&c., form the class Reptilia; the slimy-skinned, scaleless Frogs, Toads, and Salamanders the class Amphibia; and the finned, water-breathing Fishes the class Pisces.

Mammals, Birds, Reptiles, Amphibians, and Fishes all agree with one another in the possession of red blood and an internal skeletonan important part of which is an axial rod or vertebral columnand in never having more than two pairs of limbs. They thus differ in some of the most fundamental features of their organisation from such animals as Crabs, Insects, Scorpions, and Centipedes, which have colourless blood, a jointed external skeleton, and 
numerous limbs. These differences-far greater than those between classes - are expressed by placing the backboned animals in the phylum or sub-kingdom Chordata, the many-legged, armoured forms in the phylum Arthropoda. Similarly, soft-bodied animals with shells, such as Oysters and Snails, form the phylum Mollusca, Polypes and Jelly-fishes the phylum Coelenterata. And finally the various phyla recognised by zoologists together constitute the kingdom Animalia.

Thus the animal kingdom is divided into phyla, the phyla into classes, the classes into orders, the orders into families, the families into genera, and the genera into species, while the species themselves are assemblages of individual animals agreeing with one another in certain constant characters. It will be seen that the individual is the only term in the series which has a real existence: all the others are mere groups formed, more or less arbitrarily, by man.

To return to the animal originally selected as an example, it will be seen that the zoological position of the Domestic Cat is expressed as follows:-

\author{
Kingdom-ANimalia. \\ Phylum-ChORdatA. \\ Class-Mammalia. \\ Order-CaRnivora. \\ Family-Felicta. \\ Genus-Felis. \\ Species-F. domestica.
}

The object of systematic zoologists has always been to find a nutural as opposed to an artificial classification of animals. Good instances of artificial classification are the grouping of Bats with Birds on the ground that they both possess wings, and of Whales with Fishes on the ground that they both possess fins and live in the water. An equally good example of a natural classification is the grouping of both Bats and Whales under the head of Mammalia because of their agreement, in all essential points of anatomy, histology, and embryology, with the hairy quadrupeds which form the bulk of that class.

With the older zoologists the difficulty was to find some general principle to guide them in their arrangement of animals-some true criterion of classification. It was believed by all but a few advanced thinkers that the individuals of each species of animal were descended from a common ancestor, but that the original progenitor of each species was totally unconnected with that of every other, having, as Buffon puts it, "participated in the grace of a distinct act of creation." To take an instance-all Wolves were allowed to be descended from a pair of ancestral Wolves, and all Jackals from a pair of ancestral Jackals, but the original pair in each case was supposed to have come into being by a supernatural 
process of which no explanation could or ought to be offered. Nevertheless it was obvious that a Jackal was far more like a Wolf than either of them was like a Tiger, and that in a natural system of classification this fact should be expressed by placing the Wolf and Jackal in one family, the Tiger in another.

All through the animal kingdom the same thing occurs: no matter what group we take, we find the species composing it resemble one another in varying degrees, or, as it is sometimes expressed, have varying degrees of relationship to one another. On the view that each species was separately created the word relationship was used in a purely metaphorical sense, as there could of course be no real relationship between two groups of animals having a totally independent origin. But it was assumed that creation had taken place according to a certain scheme in the Divine Mind, and that the various species had their places in this scheme like the bits of glass in a mosaic. The problem of classification was thus to discover the place of each species in the pattern of the unknown design.

The point of view underwent a complete change when, after the publication of Darwin's Origin of Species in 1859, the Doctrine of Descent or of Organic Evolution came to be generally accepted by biologists. A species is now looked upon, not as an independent creation, but as having been derived by a natural process of descent from some pre-existing species, just as the various breeds of Domestic Fowl are descended from the little Jungle-fowl of India. On this view the resemblances between species referred to above are actually matters of relationship, and species are truly allied to one another in varying degrees since they are descended from a common ancestor. Thus a natural classification becomes a genealogical tree, and the problem of classification is the tracing of its branches.

This, however, is a matter of extreme difficulty. Representing by a tree the whole of the animals which have ever lived on the earth, those existing at the present day would be figured by the topmost twigs, the trunk and main branches representing extinct torms. Thus the task of arranging animals according to their relationships would be an almost hopeless one but for two circumstances : one, that remains of many extinct forms have been preserved; the other, that the series of changes undergone by an animal in its development from the egg often forms an epitome of the changes by which, in the course of ages, it has been evolved from an ancestral type. Evidence furnished by the last-named circumstance is, of course, furnished by embryology : the study of extinct animals constitutes a special branch of morphology to which the name Palæontology is applied.

The solid crust of the earth is composed of various kinds of rocks divisible into two groups: (1) Igneous rocks, such as granite 
and basalt, the structure of which is due to the action of the internal heat of the globe, and which originate below the surface and are not arranged in layers or strata ; (2) Aqueous or sedimentary rocks, which arise by the disintegration, at the surface of the earth, of pre-existing rocks, the fragments or débris being carried off by streams and rivers and deposited at the bottom of lakes or seas. Being formed in this way by the deposition of successive layers or strata, the sedimentary rocks have a stratified structure, the lowest being in every case older than the more superficial layers. The researches of geologists have shown that there is a general order of succession of stratified rocks: that they may be divided into three great groups, each representing an era of time of immense but unknown duration, and that each group may be subdivided into more or fewer systems of rocks, each representing a lesser period of time. The following table shows the thirteen rock-systems usually recognised, arranged under the three great groups in chronological order, the oldest being at the bottom of the list.
III. Cainozoic or Tertiary. .
13. Quaternary and Recent.
12. Pliocene.
11. Miocene.
10. Eocene.
9. Cretaceous.
II. Mesozoic or Secondary .
8. Jurassic.
7. Triassic.
6. Permian.
5. Carboniferous.
4. Devonian.
I. Palæozoic or Primary ..
3. Silurian.
2. Cambrian.
1. Laurentian.

Imbedded in these rocks are found the remains of various extinct animals in the form of what are called fossils. In the more recent rocks the resemblance of these to the hard parts of existing animals is perfectly clear: we find shells hardly differing from those we pick up on the beach, bones easily recognisable as those of Mammals, Birds, or Fishes, and so on. But in the older rocks the fossils are in many cases so different in character from the animals existing at the present day as to be referable to no existing order. We find Birds with teeth, great aquatic Reptiles as large as Whales, Fishes, Molluscs, Crustacea, \&c., all of an entirely different type from any now existing. We thus find that the former were in many cases utterly unlike the present animal inhabitants of the globe, and we arrive at the notion of a succession of life in time, and are even able, in exceptionally favourable circumstances, to trace back existing forms to their extinct ancestors.

By combining the results of comparative morphology, embryology, 
and palæontology we get a department of Zoology called Phylogeny, the object of which is to trace the pedigrees of the various groups. There are, however, very few cases in which this can be done with any approach to exactness: most "phylogenies" are purely hypothetical, and merely represent the views at which a particular zoologist has arrived after a more or less exhaustive study of the group under discussion.

Animals may also be studied from the point of view of Distribution. One aspect of this study is inseparable from Palæontology, since it is obviously necessary to mention in connection with a fossil the particular system or systems of rocks in which it occurs : thus we distinguish geological distribution or distribution in time.

The distribution of recent forms may be studied under two aspects, their horizontal or geographical distribution, and their vertical or bathymetrical distribution. To mention the latter first, we find that some species exist only on plains, others-hence called alpine forms - on the higher mountains; that some marine shells, fishes, \&c., always keep near the shore (littoral species), others live at great depths (abyssal species), while others (pelagic species) swim on the surface of the ocean. Among aquatic animals, moreover, whether marine or fresh-water, three principal modes of life are to be distinguished. There are animals, such as Jelly-fishes, which float on or near the surface of the water, and are carried about passively by currents: such forms are included under the term Plankton. Most Fishes, Whales, and Cuttle-fishes, on the other hand, are strong swimmers, and are able to traverse the water at will in any direction; they together constitute the Nekton. Finally, such animals as Crabs, Oysters, Sponges, Zoophytes, \&c., remain permanently fixed to or creep over the surface of the bottom, and are grouped together as the Benthos.

Under the head of geographical distribution we have such facts as the absence of all Land-mammals, except Bats, in New Zealand and the Polynesian Islands, the presence of pouched Mammals, such as Kangaroos and Opossums, only in some parts of America and in Australia and the adjacent islands, the entire absence of Finches in Australasia, and so on. We find, in fact, that the fauna-i.e. the total animal inhabitants-of a country is to a large extent independent of climate, and that the faunæ of adjacent countries often differ widely. In fact, it is convenient in studying the geographical distribution of animals largely to ignore the ordinary division into continents, and to divide the land-surface of the globe into what are called zoo-geographical regions. The characteristics of these regions will be discussed in a future section; at present it is only necessary, for convenience of reference, to give their names and boundaries. 
1. The Holarctic Region includes the whole of Europe, Asia as far south as the Himalayas, Africa north of the Sahara, together with the corresponding portion of Arabia, and North America as far south as Mexico. For convenience of reference it is often customary to divide this region into two: its Eurasian portion is then called the Palcearctic, its American portion the Nearctic region.

2. The Ethiopian Region includes Africa south of the Sahara, Southern Arabia, and Madagascar with the adjacent islands.

3. The Oriental Region includes India, Ceylon, South China, the Malayan Peninsula, and what are known as the Indo-Malayan islands, i.e. those islands of the Malayan Archipelago which lie to the west of a line-called Wallace's line-passing to the east of the Philippines, between Borneo and Celebes and between Bali and Lombok.

4. The Australian Region includes Australia, Tasmania, and the Austro-Malayan islands, i.e. the islands of the Malayan Archipelago lying to the east of Wallace's line.

5. The New Zealand Region includes New Zealand and the adjacent islands, such as the Chatham, Auckland, and Campbell groups.

6. The numerous groups of islands lying between Australia and Southern Asia to the west, and America to the east, are conveniently grouped together as the Polynesian Region.

7. The Neotropical Region includes the whole of South and Central America and part of Mexico.

There are still two departments of zoological science to be mentioned. As it is impossible to have a right understanding of a machine without knowing something of the purpose it is intended to serve, so the morphological study of an animal is imperfect without some knowledge of its Physiology, i.e. of the functions performed by its various parts, and the way in which they work together for the welfare of the whole. It is hardly possible to give more than occasional references to physiological matters in a text-book of Zoology, but in order to pave the way for such references a brief account of the general principles of Physiology will be given in the next section.

Not only may we study the action of a given animal's organs, but also the actions of the animal as a whole, its habits, its relations to other animals - whether as friends, as enemies, or as prey, to the vegetable kingdom, and to its physical surroundings, such as temperature, humidity, \&c. In a word, the whole question of the relation of the organism to its envircnment gives us a final and most important branch of Natural History which has been called Ethology or Bionomics. 


\section{SECTION I.}

\section{THE GENERAL STRUCTURE AND PHYSIOLOGY OF ANIMALS}

\section{AMEBB.}

IF we examine under the microscope a drop of water containing some of the slimy deposit which collects at the bottom of pools of rain-water and in similar situations, we occasionally find it to abound in microscopic life ; and among the minute moving creatures in such a drop we frequently find examples of a remarkable organism-the Amoba or Proteus Animalcule (Fig. 1). This is

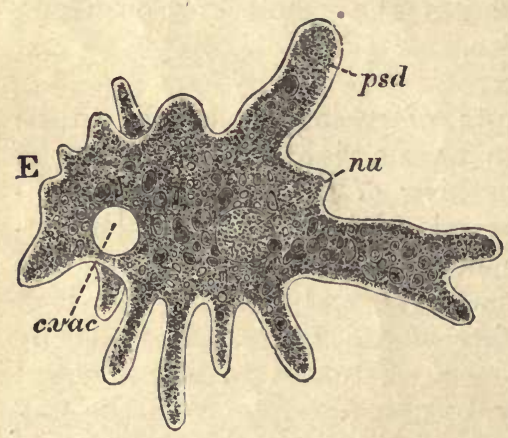

Fro. 1.-Amœba proteus, a living spẹcimen. c. vac. contractile vacuole; nu. nucleus; psd. pseudopods. (From Parker's Biology, after Gruber.)

a little particle of irregular shape, which we should be likely, on a cursory examination, to put down as motionless; it appears somewhat like an irregular particle of some colourless glass-like substance with a more granular central portion. If, however, we make an exact drawing of the outline of the Amœba, and, after an interval, compare the drawing with the original, we find that the drawing appears no longer to represent what we see; a change has taken place in the shape of the Amœba; and careful observation shows that this change is constantly going on: the Amoba is constantly varying in shape. This change is effected by the pushing out of projections or processes, called pseudopods $(p s d$.$) , which undergo various alterations of size$ and shape, and may become withdrawn, other similar processes being developed in their place. At the same time careful 
watching shows that the Amœba is also, with extreme slowness, changing its position. This it effects by a kind of streaming motion. A projection forms itself on one side, and the entire substance of the Amœba gradually streams into it; a fresh projection appears towards the same side, the streaming movement is repeated, and, by a constant succession of such movements, an extremely gradual locomotion, which it often takes very close watching to detect, is brought about. In these movements, it is to be noticed, the Amœba is influenced to some extent by contact with other minute objects; when the processes come in contact with small grains of sand or other similar particles their movements are modified in such a way that the Amœba, in its slow progress onwards, passes on one side of them, so that it might be said to feel its way among the solid particles in the drop of sediment.

Judging from the nature of these movements, we are obliged to infer that the substance of which this remarkable object is composed must be soft and semi-fluid, yet not miscible with the water, and, therefore, preserving a sharp contour. These and other characteristics to be mentioned subsequently enable us to conclude that we have to do with the substance of complex chemical composition termed protoplasm, which constitutes the vital material of all living organisms whether animals or plants. In Amœba the protoplasm is in many cases clearly distinguishable into two parts, an outer homogeneous, glassy-looking layer completely enclosing a more granular internal mass.

Examination of the Amœba with a fairly high power of the microscope reveals the presence in its interior of two objects which with a low power we should be likely to overlook. One of these is a small rounded body with well-defined contour, which preserves its form during all the changes which the Amœba as a whole undergoes. This is termed the nuclcus (Fig. 1, nu.); it is enclosed in an extremely delicate membrane, and consists of a protoplasmic material differing from that which forms the main bulk of the Amœeba in containing a substance which refracts the light more strongly and which has a stronger affinity for certain colouring matters. The other minute object to be distinguished in the interior appears as a clear rounded space (c. vac.) in the protoplasm. When this is watched it will be observed to increase gradually in size till it reaches a maximum of, let us say, a fifth of the total diameter of the Amœba, when, by a contraction of its walls, it suddenly disappears, to reappear presently and gradually grow again to its maximum size. This pulsating clear space is the contractile vacuole. Other clear spaces which do not pulsate are the non-contractile vacuoles.

By watching the Amceba carefully for some time we may be enabled to observe that the movements of the protoplasm of the body not only effect locomotion, but are connected also with the 
reception of certain foreign particles of organic nature-i.e. either entire minute animals or plants, or minute fragments of larger forms-into the interior of the protoplasm. A process of the protoplasm is pressed against such a particle, which becomes sunk in the soft substance, and passes gradually into the interior. Here it becomes enclosed in one of the non-contractile vacuoles, and by degrees partially or wholly disappears; the part, if any, which remains subsequently passes outwards from the protoplasm into the surrounding water. The matter which disappears evidently mixes with the protoplasm and adds to its bulk. All, in fact, of the matter of the foreign body that is capable of doing so, becomes digested and assimilated by the protoplasm. The fluid in the vacuole enclosing the food-particle (for such is the true nature of the foreign body) probably contains some ingredient of the nature of a ferment, which is able to act on certain substances and render them more soluble or capable of being more readily taken up by the protoplasm. This we infer mainly from what we know of the digestion and absorption of food in the higher animals; but the fact, which has been established by experiment, that the Amoba is able readily to digest certain classes of organic substances, while others, when taken into the interior of the protoplasm, remain unaltered, seems to indicate that some special property, similar to those possessed by the digestive ferments of the higher animals, is present in the watery fluid surrounding the food-particle.

The movements of the Amœba, slow and gradual though they are, must involve a certain expenditure of energy or working power; this can only be derived from the energy of chemical affinity which the protoplasm possesses in virtue of its complex chemical composition. The protoplasm loses some of this energy by its conversion into energy of movement. This loss implies the breaking up of the complex chemical ingredients of which protoplasm is composed into simpler ones; the protoplasm falls a grade in the scale of chemical compounds, and by its fall generates the force by means of which the Amœba moves. The energy of chemical affinity which the protoplasm possesses is thus analogous to the potential energy which the weight of a clock has when it is wound up. As the weight, by virtue of its position, is able as it falls to deal out working power so as to cause the movement of the machinery of the clock, so the protoplasm is able, by the degradation or decomposition of its complex compounds, to deal out working power enabling the Amœba to move. In the case of the clock-weight there comes a time when all the potential energy is expended; the weight reaches its lowest limit, and unless it is wound up again the clock stops. The like holds good of the Amœba ; the protoplasm is continually being used up-decomposed into compounds of a lower order-and, in course of time, the whole potential energy would become exhausted, were it not that a new 
supply is being constantly received. This new supply of energy is derived from the substance of the food-particles; and this at the same time maintains the bulk of the Amoba, which, if food particles are absent from the water, gradually diminishes.

Accompanying the degradation, or destructive metabolism as it is termed, of the protoplasm, and intimately connected with it, is the passage inwards of oxygen from the air dissolved in the water, and the passage outwards of carbonic acid gas. Oxygen is a necessary agent in the process of destructive metabolism, and

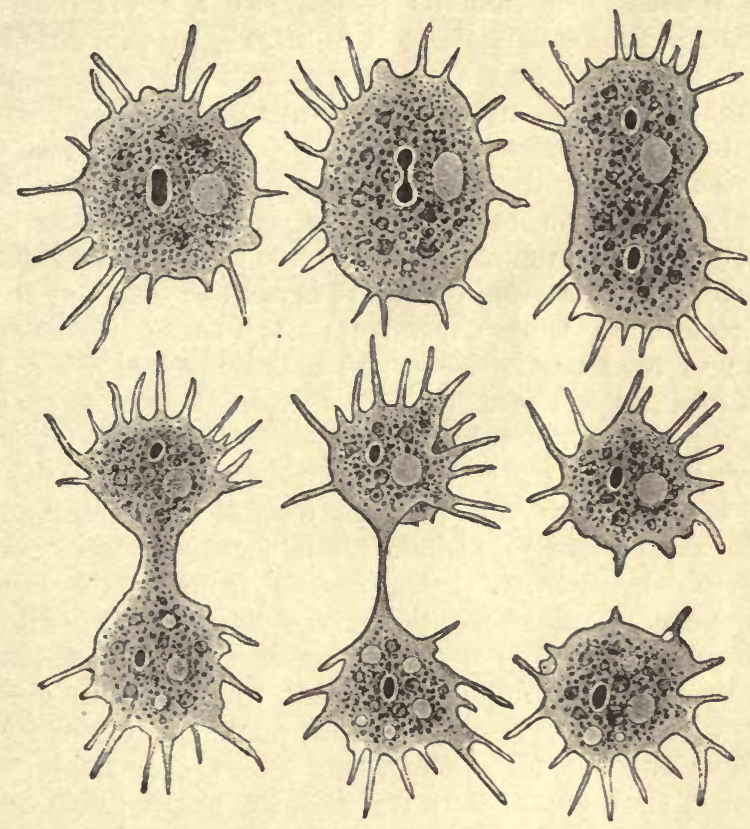

Fig. 2.-Amœba poly podia in successive phases of division. The light spot is the contractile vacuole; the dark the nucleus. (From Lang's Text-Book, after F. E. Schulze.)

carbonic acid is a constant waste-product of such action. This interchange of oxygen and carbonic acid is the essence of the process of respiration observable in all living things. In addition to the carbonic acid given off in this process, other waste-products are formed and have to be got rid of. In all probability the contractile vacuole already referred to has to do with this process - the process of excretion-since uric acid, which in higher animals is the typical form assumed by such waste-products, is said to have been detected in the interior of the contractile vacuole in the case of certain near relatives of Amøba.

When food is abundant the Amœba increases in bulk-more 
food being ingested than is required for simply maintaining the size unaltered-and soon a remarkable change takes place. The processes become withdrawn, and a fissure appears dividing the Amœba into two parts (Fig. 2). 'This fissure grows in wards, and the two parts become more and more completely separated from one another, till eventually the separation becomes complete, and we have two distinct Amœbæ resulting from the division of the one. While the protoplasm has been undergoing this division into halves the nucleus has also divided, and each of the two new Amobæ possesses a nucleus similar to the original one, and developed from it by division. It is mainly by this simple process of division into two, or binary fission as it is called, that reproduction or multiplication takes place in the Amœba.

In spite of the great simplicity of its structure, the Amœba thus carries on a number of different functions. The practically structureless particle of protoplasm is able to act on matter absorbed as food in such a way as to alter the chemical composition of the latter and to assimilate it ; it is able to carry on movements of locomotion, as well as movements-those involved in the taking in of food particles-which may be looked upon as movements of prehension; it exhibits a certain degree of sensitiveness or irritability, as shown by the modifications of its movements which result from contact with foreign bodies; it is able to respire; it carries on processes of excretion; and, finally, it is capable of reproducing its kind. It is these functions that characterise living beings as distinguished from non-living matter. What is specially characteristic of the living organism in general when compared with a non-living object is the capacity of the former to respond by changes in itself to influences operating on it from without. In the case of such an extremely simple organism as Amœba, these changes are also, necessarily, extremely simple; but they are of a quite definite character. In addition to the effects produced on its actions by mechanical obstacles and the presence of food-particles, it can be shown by experiment that Amoba responds by definite changes in itself to such external influences as changes in the amount of oxygen supplied, in the quantities of various salts present, in the temperature, and in the electric conditions of the water in which it lives. The power of locomotion, the capacity for assimilating organic substances, and the absence of two special cornpounds-chlorophyll and celluloseare specially characteristic of the animal as distinguished from the plant.

\section{The Animal Cell.}

In all but the lowest animals the various functions just enumerated are carried on by means of a more or less complex machinery 
of organs-muscles, alimentary or enteric canal, glands, heart and blood-vessels, gills or lungs, nervous system, organs of excretion, and organs of reproduction. But in all animals, however complex, the same substance, protoplasm, which in Amoba constitutes the bulk of the body, is the essential and active part. Wherever in the body active functions are being discharged and active changes are going on, there we find protoplasm present; where there is no protoplasm there is no vital activity. In the earliest stages of their existence all animals are formed entirely of protoplasm. Every animal consists at first of a single minute particle of protoplasm, not widely different from an Amœba. Soon this particle divides into a number of parts which, instead of separating completely from one another, like the parts of a divided Amœba, remain associated together, forming a clump of minute particles of protoplasm. Such minute protoplasmic particles are termed cells ; every animal consists, at first, of a single cell, and afterwards, in all higher animals, this single cell becomes converted by division and subdivision into a little cluster or clump of cells.

It is time that we should inquire more particularly into the meaning of these two terms-cell and protoplasm-evidently so important in the study of both plants and animals. Protoplasm, we have already seen, is a semi-fluid, gelatinous, clear or finely granular substance of complex chemical composition. It is known not to be a definite compound, but to be a somewhat varying mixture of chemical compounds, the most essential of which are bodies of the class of proteids - highly complex substances, into the composition of which the elements carbon, hydrogen, oxygen, nitrogen, and sulphur all enter. Living protoplasm always contains a large amount of water. It is soluble in weak acids or weak alkalies; and is capable of being coag:1lated - rendered firmer and more opaque - by the action of heat and of strong alcohol. Its reaction is slightly alkaline. As regards its minute structure, it is generally acknowledged that there are two kinds of sub-

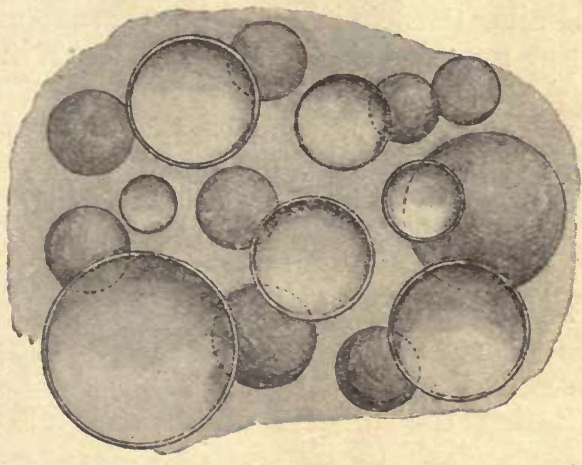

Fig. 3.-Diagram to illustrate the alveolar theory of protoplasm. (After Dahlgren and Kepner.) stance in the protoplasm, in some cases more, in others less, distinctly marked off from one another. One of these kinds of material is apparently of less fluid consistency than the other. According to one view (alveolar theory) the two kinds are intimately com- 
bined in the form of an emulsion or froth, the one forming the minute vesicles or bubbles in the froth, the other the ground substance in which the bubbles are embedded (Fig. 3). According to another view (reticular theory), one of these substances,

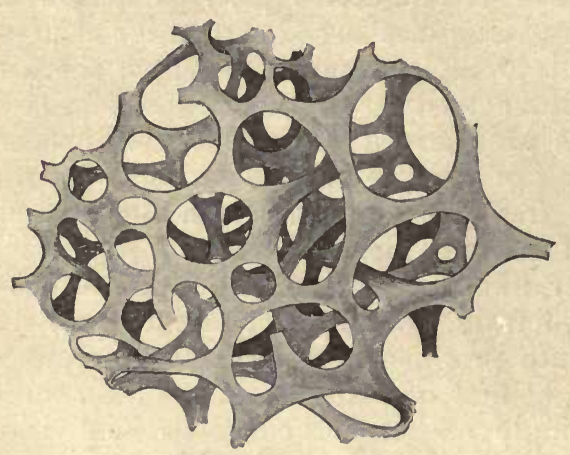

FIG. 4.-Diagram to illustrate the reticular theory of protoplasin. (After Dahlgren and Kopner.) the less fluid, appears to be arranged in the form of a network of threads, composed of numerous minute rounded granules enclosing the second, more fluid substance in its meshes (Fig. 4).

To a particle of protoplasm, typically containing a nucleus in its interior, constituting the entire body of such a simple organism as Amœba, and forming one of the constituent ele-

ments of which a higher plant or animal is made up, the term cell is applied. The word was first employed in reference to the microscopic structure of plants, in connection with which it is much more appropriate than in connection with the microscopic structure of animals ; for a plant-cell has, nearly always, a definite, firm, enclosing envelope or cell-wall (Fig. 5, I, c.w) - a structure which is only exceptionally present in the case of animals. In the interior of the cell-protoplasm, or cytoplasm, is a body termed the nucleus, similar to the nucleus of Amœba, and usually of rounded shape, with the appearance of being enclosed in a thin nuclear membrane. $(\mathrm{A}$, nu.m), perforated by numerous minute apertures. In the nucleus is a single coiled thread, or a network of threads, or one or more rounded clumps, of a substance-chromatin (chr.)-which differs from ordinary protoplasm in having a stronger affinity for most staining agents. A rounded body termed the nucleolus $\left(n \iota^{\prime}\right)$, which usually occurs in the interior of the nucleus, is formed either of a solid mass of chromatin, or of a substance differing somewhat from chromatin in its properties, and less strongly affected by staining agents. When the nucleus divides during the process of division of the cell, its contents, more particularly the chromatin, in many cases go through a remarkable series of changes, to which the term karyolinesis or mitosis is applied.

At the time when this mitotic division is about to be initiated, either one or two minute bodies (Fig. 5, A, c) are to be distinguished situated close together in the cytoplasin in the immediate neighbourhood of the nucleus. When only one of 
these bodies is present at the outset it subsequently becomes divided into two. These are the centrosomes-minute masses of a specially modified protoplasmic substance, capable of being rendered conspicuous by certain staining agents, : surrounded by a light zone. The centrosomes, at first close together, gradually separate from one another, a spindle-shaped bundle of very fine fibres of achromatic ${ }^{1}$ material-the nuclear spindle
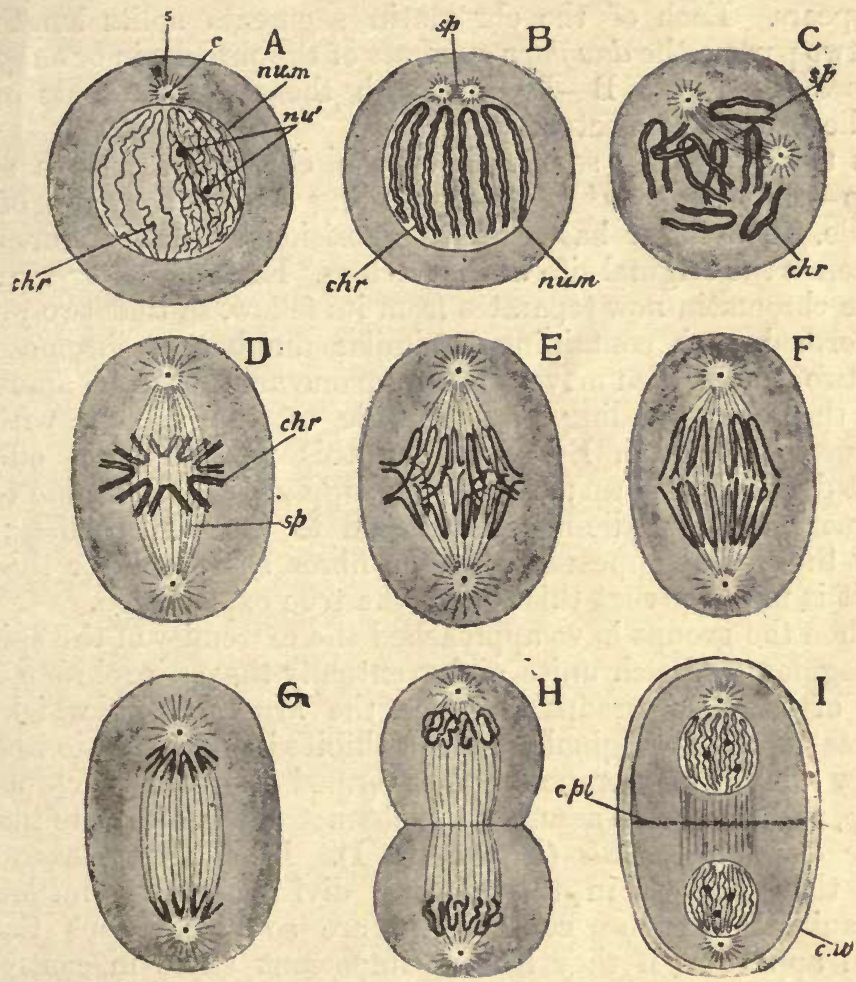

FIG. 5.-Diagrams illustrating karyokinesis. A, the resting cell ; B, C, D, successive phases in the formation and arrangement of the chromatin loops and of the nuclear spindle ; E, F , G, separation of the two sets of daughter-chromosomes and their passage towards the poles of the spindle; H, I, division of the cell-body and formation of the two new nuclei ; $c$. centrosome ; chr. chromatin ; cpl. cell-plate; $n u^{\prime}$. nucleoli ; $n u$. m. nuclear membrane ; s. atrospherc ; sp. spindle. (From Parker's Biology, after Flemming, Rabl, \&c.)

- extending between them (Fig. 5 , C). At the same time, or at an earlier stage, each centrosome has become the centre of a system of fine achromatin fibres (apparently made up, like the fibres of the spindle, of rows of granules) which are arranged round it in a radiating manner, forming a structure

I The term achromatin is usually applied to all the matter of the nuclevs that has not the special characteristics of chromatin; but it applies to cytoplasmic structures-i.e. structures belonging to the body of the cell-as well. 
termed the attraction-sphere or astrosphere (Fig. 5, A,s). Meantime important changes have been in progress in the nucleus. The chromatin first becomes arranged in a close tangle (spireme), and then becomes divided up into a number of parts - the chromatin segments or chromosomes - which frequently have the form of looplike threads (Fig. 5, B, C, chr), but often assume other forms. The number of chromosomes varies, but is constant throughout the cells of the same species of animal. The nuclear membrane disappears. Each of the chromatin segments splits lengthwise into two parts - the daughter-segments of the chromatin or daughterchromosomes (Fig. 5, B-D), and with these the filaments of the spindle become connected.

At this point the segments of the chromatin form a single group-the equatorial plate-extending across the axis of the spindle. The latter has shifted its position, so that its fibres now run across the original site of the nucleus. Each daughter-segment of the chromatin now separates from its fellow, so that two groups are formed, each containing a similar number of chromosomes. The two groups then move apart from one another, each approaching the corresponding end or pole of the spindle with its centrosome (Fig. 5, E-G). How this movement is effected is not definitely known; it has been supposed that it is due to the contraction of spindle-fibres attached to the centrosomes; but since there is no appearance of the fibres shortening or thickening, it is unlikely that this can be the true explanation.

When the groups have approached the extremity of the spindle, the segments of each unite, and eventually the entire chromatin of each of the two groups assumes the arrangement which the chromatin of the original nucleus exhibited before division began. A new nuclear membrane becomes formed around each chromatin group, and the whole assumes the character of a complete nucleus - the daughter-nucleus (Fig. 5, H, I). It is of importance to note that, though in this mitotic division of the nucleus of the animal cell the centrosomes are so conspicuous that it would appear as if they had an important share in controlling the process, yet mitosis takes place during cell-division of the higher plants on the same general lines as in animals though centrosomes have rarely, if ever, been observed in plants higher than the Mosses.

A furrow which appears on the surface of the cell-protoplasm (Fig. $5, \mathrm{H}, \mathrm{I})$, surrounding it in the form of a ring in a plane at right angles to the long axis of the spindle, deepens gradually so as to give rise to a cleft, eventually completely separating the substance of the cell into two halves. Each of these halves encloses one of the daughter-nuclei, and has assumed the character of a complete daughter-cell. During this process there is sometimes distinguishable along the line corresponding to the division line between the 
two cells a narrow septum; this is known as the cell-plate (I., c.pl.). But a cell-plate is not of general occurrence in the division of the animal cell.

In some instances the division of the nucleus is direct or amitotic, the nucleus simply becoming separated into two equal parts, without disappearance of the nuclear membrane and without any complicated re-arrangement of the chromatin.

\section{The Ovum : Maturation, Impregnation, and Segmentation : The Germinal Layers.}

Amœba is simply an independent animal cell; or, to express the same meaning in another way, is a unicellular animal, and as such it is a member of the phylum of the Protozoc or unicellular animals. All the rest of the animal kingdom, forming the division Metazoa, are multicellular in the fully developed condition; but each of these multicellular animals or Metazoa originates from a single cell-the ovum. The ovum is a typical cell (Fig. 6), usually spherical in shape, with one or more enclosing membranes, with cell-protoplasm enclosing a nucleus (germinal vesicle) in which are contained one or more rounded masses of chromatin (germinal spot or spots). The ovum may contain in addition to the protoplasm a quantity of non-protoplasmic nutrient material or yolk.

Before the process of impregnation or fertilisation which gives the impulse to development, the ovum undergoes a change which is termed maturation (Fig. 7, A). This consists, in essence, of the throwing out of portions of the nucleus. The latter approaches the surface and divides, mitotically, into two partsone coming to project on the surface and finally the projection being completely separated off from the ovum as a rounded particlethe first polar body ( $p o l$.$) . A second division of the nucleus$ results in the throwing off of a second polar body; and, after this has been formed, the portion which remains in the ovum resumes its central position and forms what is termed the female pronucleus ( $\mathrm{B}$, $q$ pron.). The essential ultimate result of maturation is the reduction of the number of chromosomes in the ovum by one-half.

In the process of impregnation a very minute body, the male 
SECT.

cell, sperm-cell, or sperm, penetrates into the interior of the female cell or ovum, and the nucleus which it contains-the male pronucleus (C, of pron.) coalesces with the female pronucleus to form a single nucleus called the segmentation nucleus (E, seg. nucl.). The
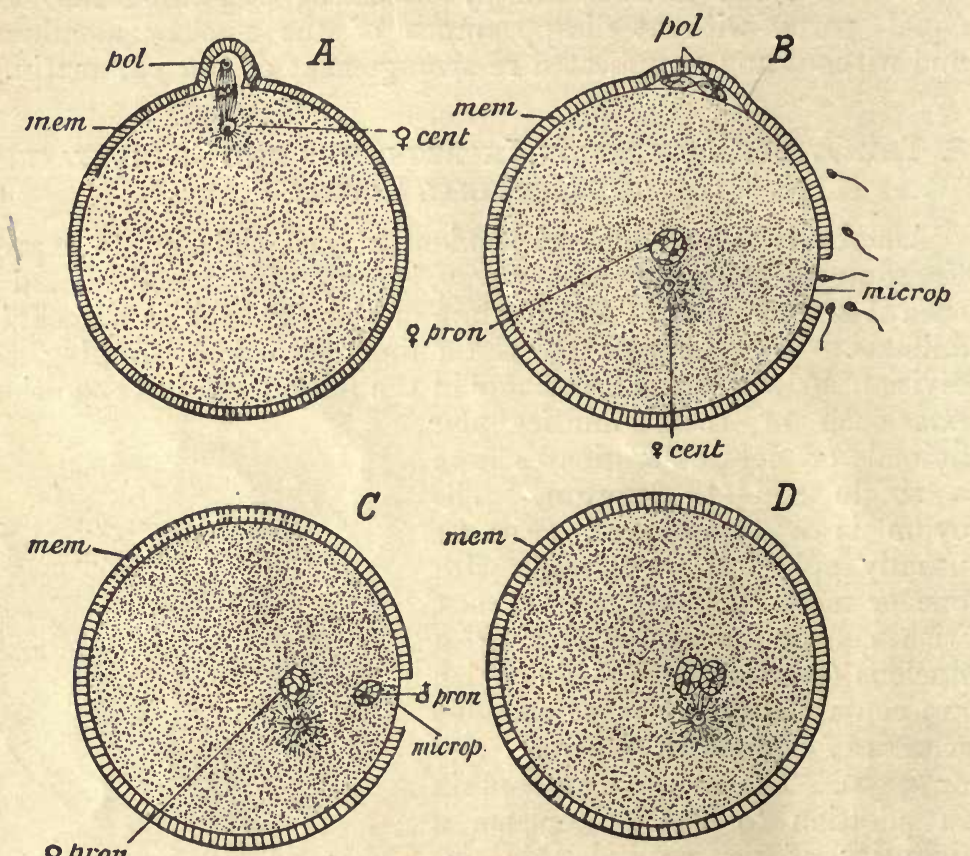

oprore

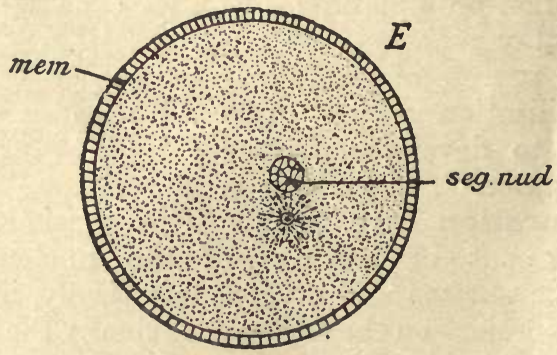

Fra. 7.-Diagram illustrating the maturation and fertilisation of the ovum. A, formation of first polar body ; B, beginning of fertilisation, sperms approaching the micropyle; $C$, formation

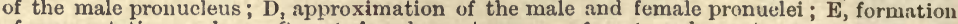
of segmentation-nueleus; $q$ cent. female centrosome; $\delta$ cent. male centrosome; mem. egg. membrane; microp. micropyle; pol. polar bodies; + pion. female pronucleus; $\delta$ pron. male pronucleus; seg. nucl. segmentation nucleus.

principal part in the process of fertilisation is thus played by the two nuclei. The female centrosome disappears: a male centrosome enters with the sperm.

Apparently in this process of fertilisation some attraction is 
operative between the male and female cells. In many instances a prominence (the receptive prominence) is pushed out by the ovum at the point where the sperm enters. The female pronucleus, leaving its former central position, approaches the male cell as it enters. In most cases a single sperm alone enters the ovum in impregnation. According to the older observers, as soon as a sperm enters the ovum, a membrane is formed around the latter hindering the penetration of additional sperms. But it has now been shown that such a membrane occurs only in certain cases, and is quite exceptional. That, as a general rule, only one sperm penetrates into the ovum appears to be due to the circumstance that, as a result of the entry of the one sperm, the peculiar attraction above referred to becomes in some way destroyed or diminished. But, though the entry of one sperm only is usual, cases of the entry of several-polyspermy, as it is termed-are by no means rare, and would appear to be quite normal in some groups of animals.

In some animals the ovum develops parthenogenetically-i.e. without any process of fertilisation by means of a male cell. This is a normal phenomenon in certain families of insects, for example. In a considerable number of marine invertebrate animals it has been shown that though gamogencsis, i.e. development as the result of fertilisation of ovum by male cell, is the normal process, yet parthenogenesis can be produced by various artificial means. By adding various salts to the water in which the ova are contained, by changes of temperature, or by subjection to the action of carbonic acid gas, the ova, in the absence of sperms, may be caused to give rise to normal embryos. Such experiments on artificial parthenogenesis, as it is termed, show that the entry of a male cell into the ovum is not necessary for the development of the embryo even in cases in which gamogenesis is normal; but that other exciting influences may bring about the same result.

Though, as stated above, the female pronucleus, under normal circumstances, plays so important a rôle in the development, it has been shown that it can be dispensed with. When unfertilised ova of a sea-urchin are .broken up, and fragments devoid of nuclei are placed in water along with sperms, the fragments may be fertilised; and, the nucleus of the sperm taking the place of the segmentation-nucleus, normal young, differing from those produced in the usual manner only in their smaller size, may be developed. This phenomenon is known as merogony.

The result of fertilisation is the formation of the impregnated ovum, or oosperm as it is called. The oosperm, it is to be noted, before development begins, consists in general of the primary ovum minus the portions of the substance of its nucleus removed 
in the polar bodies and also minus its centrosome, and plus the sperm with its nucleus and centrosome.

On impregnation follows shortly the process of division already briefly referred to, which is known as segmentation (Fig. 8). This either affects the entire substance (holoblastic or complete
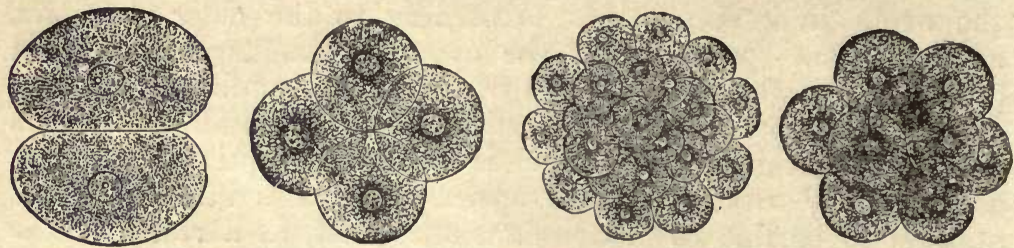

Fig. 8.- Various stages in the segmentation of the ovum. (From Gegenbaur's Comparative Anatomy.)

segmentation) or only a part (meroblastic or incomplete segmentation) of the oosperm. In the former case the ovum usually contains little or no food-yolk, consisting exclusively, or nearly so, of protoplasmic matter. The first stage in the process of segmentation is the mitotic division of the segmentation-nucleus, accompanied by the division into two parts of the substance of the protoplasm-the result being the formation of two cells, each with its nucleus (Fig. 8). Each of these two cells then divides -four cells being thus formed; the four divide to form eight; the eight divide to form sixteen, and so on ; until, by the process of division and subdivision, the oosperm becomes segmented into a large number of comparatively small cells which are termed the blastomeres. This mass of cells is spherical in shape, and the

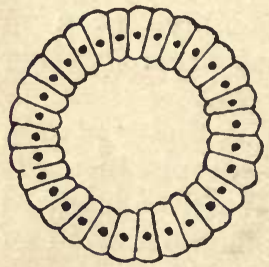

A

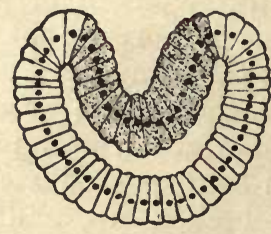

$B$

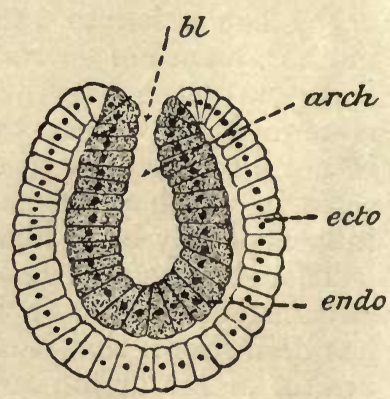

C

Fig. 9.-Gastrulation.

arch. archenteron; $b l$. blastopore ; ecto. ectoderm; endo. endoderm.

rounded blastomeres of which it is composed project on its surface so as to give it somewhat the appearance of the fruit of the mulberry, whence it is termed the mulberry body or morula stage. The blastomeres next become arranged regularly in a single layer - the embryo (Fig. 9, $A$ ) assuming the form of a hollow 
sphere, the blastosphere or blastula, with a wall composed of a single layer of cells enclosing a cavity-the segmentation cavity or blastorcele.

One side of the hollow blastula next becomes pushed inwards or invagimated (Fig. 9, $B, C$ ), as one might push in one side of a hollow india-rubber ball, the result of this process of invagination, or gastrulation as it is termed, being the formation of a cup-the gastrula (Fig. 10)-with a double wall. The cavity of the cup-shaped gastrula is the archenteron or primitive digestive cavity; the opening is termed the blastopore, the outer layer of the wall of the cup is the ectoderm (or epiblast), the inner the endoderm (or hypoblast). The ectoderm and endoderm are the primary germinal layers of the embryo; from one or both of them are developed the cells of a third layer-the mesoderm (mesoblast)-which is subsequently formed between them.

This mode of formation of the primary germinal layers in holoblastic oosperms by a process of gastrulation prevails in a number of different sections of the animal kingdom. In many animals, however, it becomes modi-

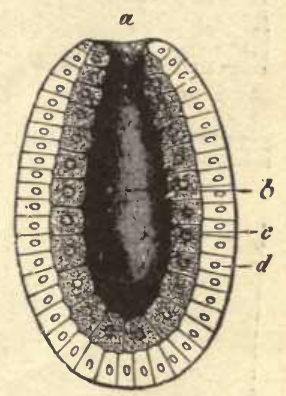

Fio. 10.--Gastrula in longitudinal section; $a$, blastopore; $b$, arch. enteron ; $c$, endoderm; $d$, ectoderm. (From Gegenbaur's Compara. tive Anatomy.) fied or disguised in various ways; and in many meroblastic oosperms it is doubtful if there occurs anything of the nature of true gastrulation.

The cells of the three germinal layers give rise to the various organs of the body of the fully-formed animal-each layer having a special part to play in the history of the development. As the various parts of the embryo become gradually moulded from the cells of the germinal layers, it becomes evident on comparison that their internal structure-the form and arrangement of their constituent cells-is undergoing gradual modifications, the nature of which is different in the case of different parts. A differentiation of the cells is going on in the developing organs, resulting in the formation of a variety of different kinds of tissues.

\section{Tissues.}

The cells of the tissues of the animal body differ greatly in form in different cases. Some are rounded, others cubical, others polygonal; some are shaped like a pyramid, others like a cone, others like a column or cylinder; others are flattened and tabular or scale-like. Cells situated on free surfaces are in many cases beset at their free ends with delicate, hair-like structures or cilia which vibrate to and fro incessantly during the life of the cell 
(Fig. 11, a); sometimes there is on each ceil a single, relatively long, whip-like cilium, which is then termed a flagellum $(f, g)$. Cells

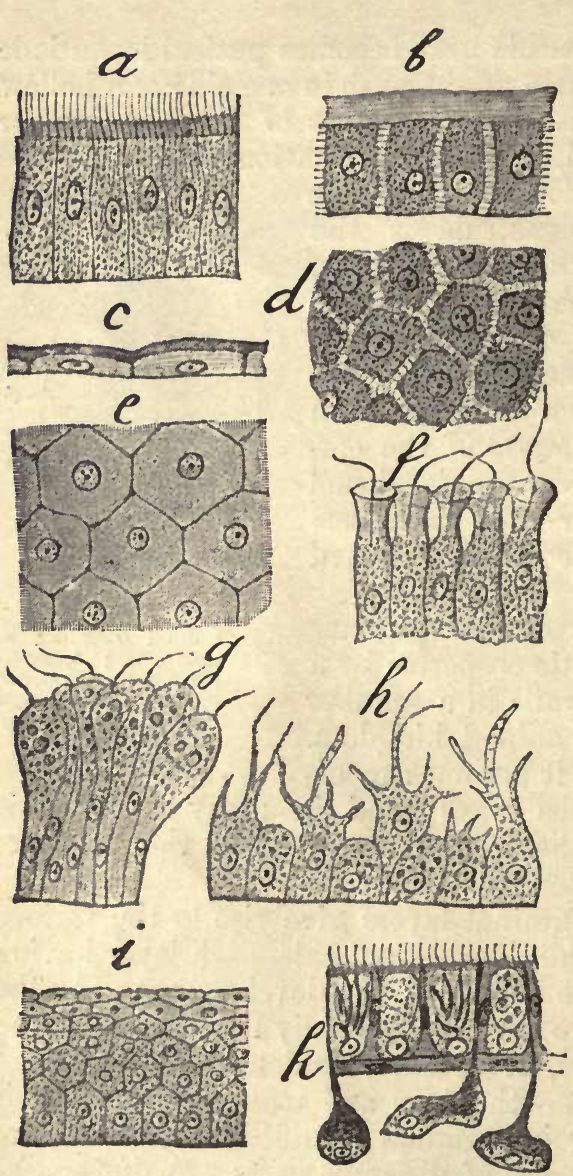

Fro. 11-Various forms of epithelium. $a$, ciliated epitheliurn; $b$, columnar; $d$, surface view of the same; $c$, tesselated ; $e$, the same from the surface ; $f$, flagellate epithelium with collars; $g$, flagellate epithelium without collars; $h$, epithelium of intestine with pseudopods; $i$, stratified epithelium ; $k$, deric epithelium of a marine planarian with pigment cells, rod-cells, and sub-epithelial glands. (From Lang's Comparative Anatomy.) provided with cilia are termed ciliated, such as bear flagella flagellate cells.

Some tissues are composed entirely of cells. Others, though originating from cells or by the agency of cells, consist in greater or less measure of non-protoplasmic matter formed between the cells. 'Tissues composed entirely of cells take the form, for the most part, of membranes covering various surfaces, external and internal. Such membranes are known under the general name of epithelia (Fig. 11); they may consist of a single layer of cells $(a-h)$ or may be many-layered (i); the former are termed non-stratified, the latter stratificd, epithelia. The cells of an epithelium may be flattened $(c, e)$, their edges being cemented together so as to form a continuous membrane; or they may be cubical or cylindrical or prismatic $(a, b)$; in the case of a stratified epithelium the cells may be of different forms in different strata (i). The epidermis, which covers the outer surface of the body of an animal, is an example of an epithelium; sometimes it is stratified, sometimes unstratified; its cells sometimes possess cilia, sometimes are devoid of them. Lining the internal cavities of the body are layers of cells, or epithelia, sometimes in a single layer, sometimes in several layers, sometimes ciliated, sometimes non-ciliated. 
Glands (Fig. 12) are formed for the most part by the modification of certain cells of epithelia. In many cases a single cell of the epithelium forms a gland, which is then termed a unicellular gland (Fig. 12, A). The secretion (or substance which it is the function of the gland to form or collect) gathers in such a case in the interior of the cell, and reaches the surface of the epithelium through a narrow prolongation of the cell which serves as the duct of the gland $(B)$. In other cases the gland is multicellular-formed of a number of cells of the epithelium lining a depression or infolding, simple or complex in form, of the latter $(D-G)$. In the central cavity of such a gland the secretion collects to reach the general surface or cavity lined by the epithelium through the passage or duct.

A series of tissues in which the cells are, in most instances, subordinate, as regards bulk, to substances formed between them, is the group known as the connective tissues, including gelatinous connective tissue, retiform connective tissue, fibrous connective tissuc, cartilage, and bone. In the majority of forms of connective tissue the cells lie embedded in an intermediate substance called the matrix or ground-substance of the connective tissue.

In the case of gelatinous connective tissue (Fig. 13) the groundsubstance $(g)$ is of a gelatinous character, sometimes supported by systems of fibres $(e f)$, and the
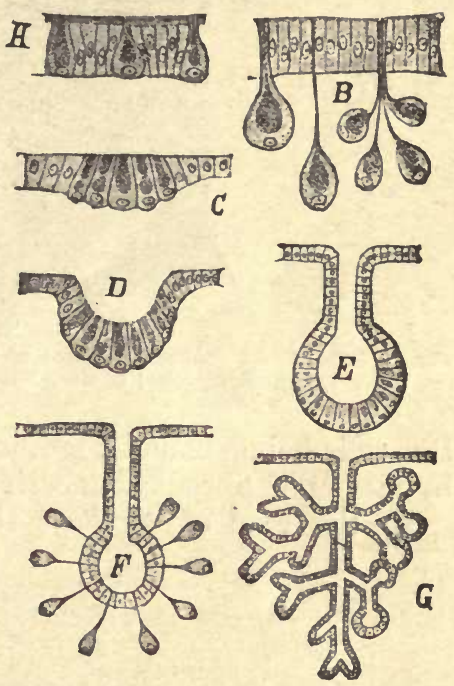

FIG. 12.-Diagram to illustrate the structure of glands. $A$, unicellular glands in an epithelium ; $B$, unicellular glands lying below epithelium and communicating with the surface by narrow processes (ducts); $C$, group of gland-cells ; $D$, group of gland-cells lining a depression; $B$ and $F$, simple multicellular gland; $G$, branched multicellular gland. (From Laug.) cells are usually stellate or starshaped with radiating processes. Retiform or reticulate connective tissue (Fig 14) consists of stellate or branching cells with processes which are prolonged into fibres-the fibres from neighbouring cells joining so as to form a network. In this form of connective tissue there is no true ground-substance-the interspaces between the cells being filled with other tissue elements.

Fibrous connective tissue, which is a very common form, has a ground-substance containing gelatin, consisting mainly of numerous fibres, usually arranged in bundles. Thicker yellow elastic fibres may be present among the others, and may be so numerous as to give the entire tissue an elastic character. Associated with fibrous 
tissue, and produced by modification of its cells, is adipose or fatty ttssue (Fig. 15), which consists of masses of large cells in which the protoplasm has more or less completely become replaced by fat,

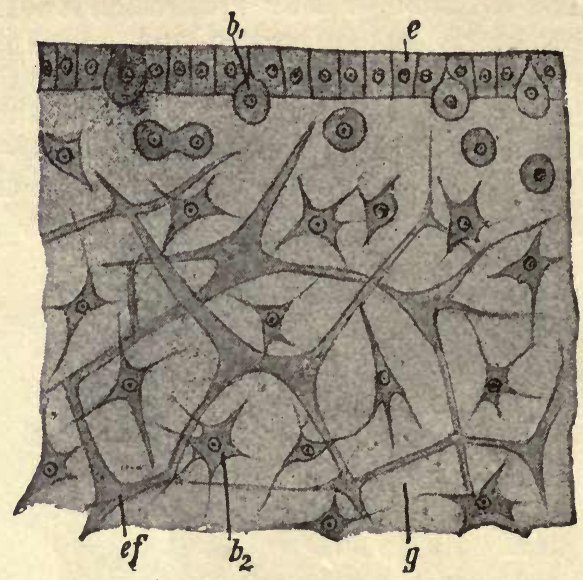

Fig. 13.-Gelatinnus connective tissue of a Jelly-fish; $e$, eplthelium; $g$, gelatinous matrix $\iota_{2}$, branching cells; $e f$, elastic fibres. (From Lang's Comparative Anatomy.)

the cells being bound together into groups and masses or lobules by means of fibrous connective tissue.

In the case of cartilage the matrix is of a firm but elastic

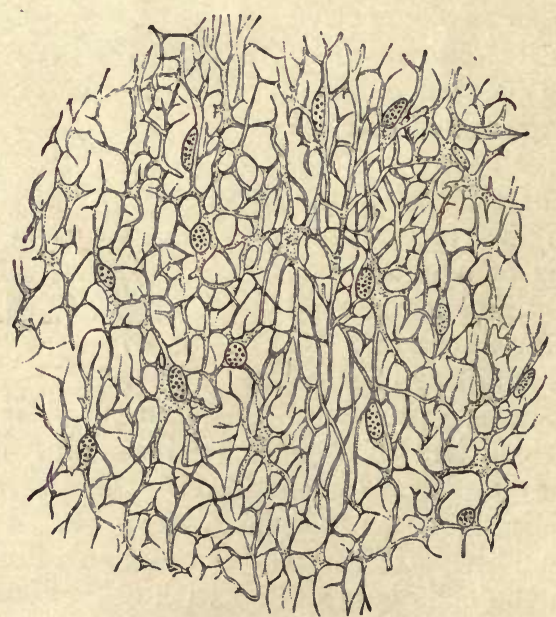

Fia. 14.-Reticular connective tissue. (From Lang.)

character, sometimes quite homogeneous in appearance (hyaline cartilage, Fig. 16), sometimes permeated by systems of fibres (fibrocartilage, Fig. 17), which may be of an elastic nature (yellow elastic 
cartilage). The cells are usually rounded, and as a rule several occur together in spaces scattered through the matrix; sometimes condensation of the matrix round each of the spaces in which the cells are contained forms a cell-capsule. The outer surface is

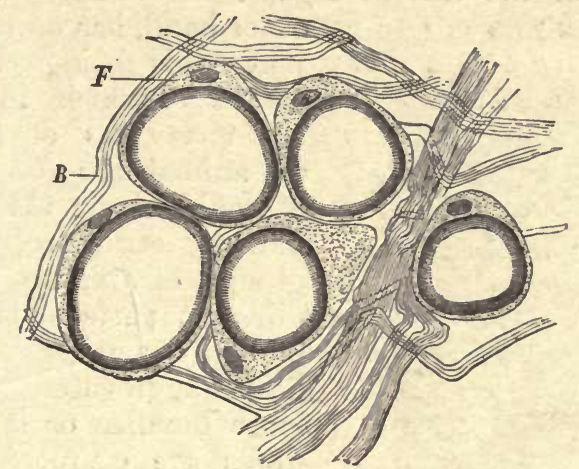

Fic. 15.-Fatty tissue; $F$, fat-cells; $B$, connective-tissue fibrils. (From Lang, after Ranvier.)

covered over by a fibrous membrane-the perichondrium. Cartilage is frequently hardened by the deposition in the matrix of salts of lime-and is then known as calcified cartilage.

In bone or osseous connective tissue (Fig. 18) the matrix is exceedingly dense and hard owing to its being strongly impregnated with carbonate and phosphate of lime. It consists typically of numerous thin lamellæ, which are arranged partly parallel with the surface, partly concentrically around certain canals-the Haversian canals (c)-in which blood-vessels lie. The cells, or bone-corpuscles, lie

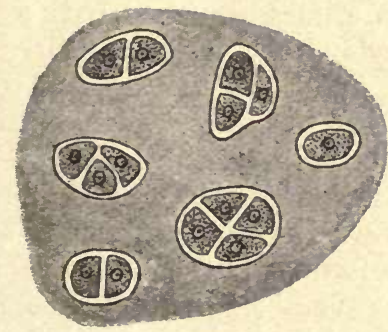

Fia. 16.-Hyaline cartilage.

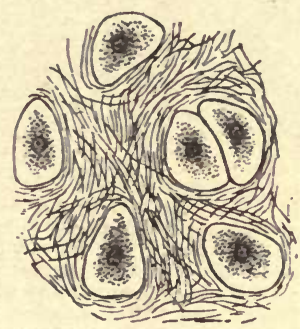

Fia. 17.-Fibro-cartilage.

in minute spaces-the lacuno-between the lamellæ, and a system of exceedingly fine channels - the canaliculi-extend from lacuna to lacuna, containing fine protoplasmic processes by means of which neighbouring cells are placed in communication with one another The outer surface of the bone is covered by a vascular fibrous 
membrane-the periosteum-which takes an active part in its growth and nutrition.

The connective tissues are all more or less passive in the functions which they perform, serving mainly for support and for binding together the various organs. Muscular tissue, on the

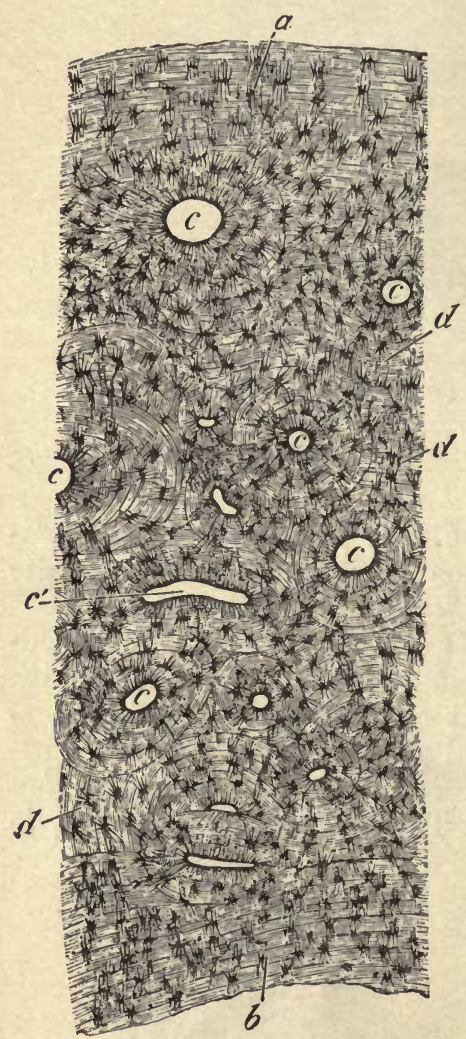

Fia. 18.-Transverse section of compact bone, $a$, lamellæ concentric with the outer surface; $b$, lamellæ concentric with the surface of the marrow cavity ; $c$, section of Haversian canals; $c^{\prime}$, section of a Haversian canal just dividing into two; $d$, interstitial lamellie. (From Huxley's Lessons in Physiology.) other hand, has an active part to play-this being the tissue by means of which, in general, all the movements of the body of an animal are broug'.lt about. Muscular tissue varies greatly in minute structure in different groups of animals, and even in different parts of the same animal. It consists of microscopic fibres aggregated together into large bundles or layers. These fibres are composed of a substance - the muscle-substance which when living has the special property of contractility, contracting or becoming shorter and thicker on the application of a stimulus. There are two principal varieties of muscular tissue to be distinguished, termed respectively non-striated and striated muscle. Each fibre of non-striated muscle (Fig. 19) is usually a single, greatly elongated cell, sometimes branched, with a single nucleus; it may contain a core of unaltered protoplasm, or all except the nucleus may be altered into muscle-substance; crossstriation is absent. A fibre of striated muscular tissue (Fig. 20) is formed by the close union of several cells which are represented by their nuclei $(n)$. Sometimes there is a core of protoplasm ; but more usually the entire fibre is composed of musclesubstance, with perhaps a remnant of protoplasm in the neighbourhood of each nucleus. The substance of the fibre is crossed by numerous transverse bands and striæ, the precise significance of which is a matter of controversy. The fibre is usually enclosed in a delicate sheath-the sarcolemma. Striated muscular 
tissue is specially characteristic of parts in which rapid movement is necessary.

The principal elements of nervous tissue are nerve-cells and nerve-fibres.

Nerve-cells (Fig. 21) vary greatly in form; they are relatively

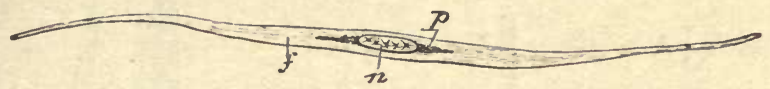

Fia. 19.-Non-striated muscle-cell ; $f$, substance of fibre ; $n$, nucleus ; $p$, unaltered protoplasm in the neighbourhoud of the nucleus. (From Huxley's Lessons in Physiology.)

large cells with large nuclei and one or several processes, one of which is always continuous with a nerve fibre.

The nerve-fibres (Fig. 22), which are to be looked upon as greatly produced processes of nerve-cells, are arranged for the most part in strands which are termed nerves. The fibres themselves vary greatly in structure in different classes of animals. In the higher animals the most characteristic form of nerve-fibre is that which is termed the medullated nerve-fibre. In this there is a central cylinder-the axis-cylinder or neuraxis $(A, a x)$-which is the
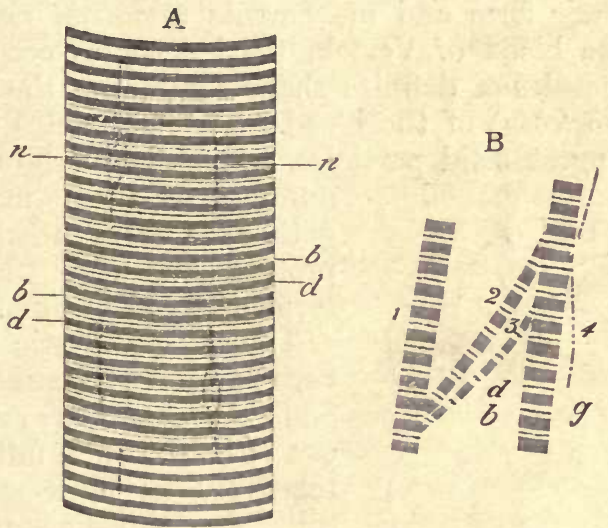

Fia. 20.-Striated muscle. $A$, part of a muscular fibre of a Frog; $B$, portion of striated muscle teased out to show separation into fibrillas. (From Huxley's Lessons in Physiology.) $b, d, g$, transverse bands and strix ; $n$, nuclei.

essential part of the fibre and is made up of numerous extremely fine primitive fibrilla; this is surrounded by a layer of a white glistening material - the white substance of Schwann or medullary shcath (med), enclosed in turn in a very delicate membrane-the neurilemma (ncur).

The blood, the lymph, and other similar fluids in the body of an animal may be looked upon as liquid tissues, having certain cells 
-the corpuscles-disseminated through a liquid plasma, which takes the place of the ground-substance of the connective tissues.

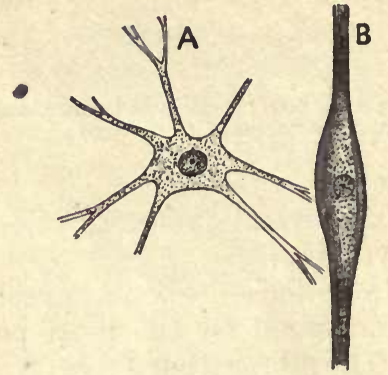

FIG. 21.-Nerve-cells. $A$, multipolar ; $B$, bipolar.

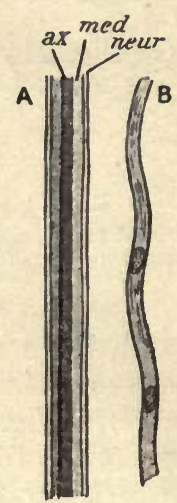

Fig. 22.-Nerve-fibres. $A$, medullated; $B$, non-medulated ; $a x$, neuraxis; med, medullary sheath; neur, neurilemma.

In a large proportion of cases such corpuscles are similar to Amœbæ in their form and movements (amoboid corpuscles, leucocytes). In the blood of Vertebrates leucocytes occur along with coloured corpuscles of definite shape containing the red-colouring matter (hcemoglobin) of the blood. The leucocytes are able, like Amœbæ, to ingest solid particles, and under certain conditions a

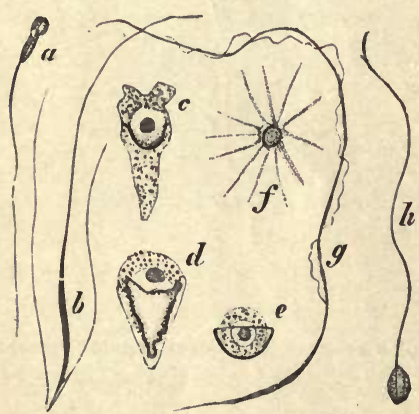

Fic. 23.-Various forms of spermatozoa. $a$, of a Mammal ; $b$, of a Turbellarian worm ; $c$, and $d$, and $e$, of Nematode worms; $f$, of a Crustacean ; $g$, of a Salamander; $h$, the eommonest form with oval head and long flagellum. (Frorn Lang's Comparative Anatomy.) number of them may unite together to form a single mass of protoplasm with many nuclei, termed a plasmodium.

The characteristic cells of the reproductive tissues are the ova and the spermatozoa or sperms. The ova (Fig. 6), when fully formed, are relatively large, usually spherical cells, sometimes composed entirely of protoplasm, but usually with an addition of nutrient food-yolk. Each ovum, as already mentioned, encloses a large nucleus (gcrminal vesicle) and in the interior of that one or more nucleoli or germinal spots. The sperms (Fig. 23) are extremely minute bodies, nearly always motile, usually slender and whip-like, tapering towards one extremity, and commonly with a rounded hcad at the other. 
The sperms are developed by a succession of cell-divisions from certain cells-the primitive male cells-similar in character to immature ova.

\section{Organs.}

The chief systems of organs of an animal are the integumentary, the skeletal, the muscular, the alimentary or digestive, the vascular, the respiratory, the nervous, the excretory, and the reproductive.

The skin or integument consists in the majority of animals of a cellular membrane-the epidermis-to which reference has already been made, with, superficial to it, in many animals, a noncellular layer the cuticle, and below it usually a fibrous layer which is known as the dermis. The epidermis may consist of a single layer or may be stratified; it is frequently ciliated, and some of its cells frequently assume the form of unicellular glands. Modification of its superficial layers of cells gives rise frequently to the formation of hard structures contributing to the development of an exoskeleton (vide infra).

The cuticle, when present, varies greatly in thickness and consistency. Sometimes it is very thin and delicate; in many animals it becomes greatly thickened and hardened so as to form a strong protecting crust, sometimes of a material termed chitin, somewhat akin to horn in consistency, sometimes soliditied by the deposition of calcareous salts. The cuticle is to be looked upon as a secretion from the cells of the epidermis; but the term is frequently applied in the case of the higher animals - in which a cuticle in the strict sense of the term is absent-either to a superficial part of the epidermis, in which the cells have become altered and horny, or to the whole of that layer.

The layer or layers of the integument situated beneath the epiderm consist of fibrous connective tissue and muscular fibres, constituting, as mentioned above, the derm or dermis.

The term skeleton or skeletal system is applied to a system of hard parts, external or internal, which serves for the protection and support of softer organs and often for the attachment of muscles. This system of hard parts may be external, enclosing the soft parts, or it may lie deep within the latter, covered by integument and muscles: in the former case it is termed an exoskeleton or external skeleton; in the latter an endoskeleton or internal skeleton. In many groups of animals both systems are developed. An exoskeleton is formed by the thickening and hardening of a part or the whole of one of the layers of the integument enumerated above; or more than one of these layers may take part in its formation. In many invertebrate animals, such as Insects, Crustaceans, and Molluscs, it is a greatly thickened and hardened 
cuticle which forms the exoskeleton. The horny scales of Reptiles, the feathers of Birds, and the fur of Mammals are examples of an exoskeleton derived from the epidermis, while the bony shell of Turtles and the bony scales of Fishes are examples of a dermal exoskeleton.

When an endoskeleton is present, it usually consists either of cartilage or bone or of both; but sometimes it is composed of numerous minute bodies (spicules) of carbonate of lime or of a siliceous material.

A skeleton, whether internal or external, is usually composed of a number of pieces which are movably articulated together, and which thus constitute a system of jointed levers on which the muscles act.

The alimentary or digestive system consists of a cavity or system of cavities into which the food is received, in which it is digested, and through the wall of which the nutrient matters are absorbed; together with certain glands.

In the lowest groups in which a distinct alimentary or enteric cavity is present it is not distinct from the general cavity of the body ; but in all higher forms there is an enteric canal which is suspended within the cavity of the body, and the lumen of which is completely shut off from the latter. It may have simply the form of a sac or bag with a single opening which serves both as mouth and anus; in other cases the sac becomes branched and may take the form of a system of branching canals. In most animals, however, the alimentary canal has the form of a longer or shorter tube beginning at the mouth and ending at the anal opening (Fig. 24). In most cases there are organs in the neighbourhood of the mouth serving for the seizure of food; these may be simply tentacles or soft finger-like appendages, or they may have the form of jaws, by means of which the food is not only seized, but torn to pieces or pounded up to small fragments in the process of mastication. The alimentary canal itself is usually divided into a number of regions which differ both in structure and in function.

In general there may be said to be three regions in the alimentary canal-the ingestive, the digestive and absorbent, and the egestive or efferent. The ingestive region is the part following behind the mouth, by which the food reaches the digestive and absorbent region. But, besides serving as a passage, it may also act as a region in which the food undergoes certain processes, chiefly mechanical, which prepare it for digestion. This ingestive region may comprise a mouth-cavity or buccal cavity, a pharynx, an osophagus or gullet, with sometimes a muscular gizzard which may be provided with a system of teeth for the further breaking up of the food, and sometimes a crop or food-pouch.

The digestive and absorbent region is the part in which the chemical processes of digestion go on, and from which takes place 
the absorption of the digested food-substances. Into this part are poured the secretions of the various digestive glands, which act on the different ingredients of the food so as to render them more soluble. Through the lining membrane of this part the digested nutrient matter passes, to enter the blood-system. This region may present a number of subdivisions; nearly always there are at least two-a wide sac, the stomach, and a narrow tube, the intestine.

The egestive or efferent region of the alimentary canal is the posterior part of the intestine, in which digestion and absorption do not go on, or only go on to a limited extent, and which serves

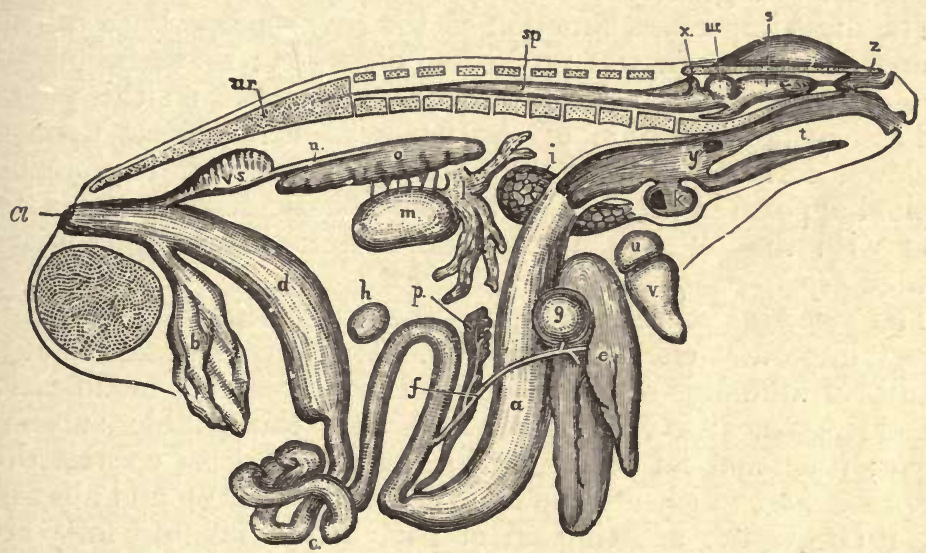

Fig. 24.-General view of the viscera of a male Frog, from the right side. $a$, stomach ; $b$, urinary bladder; $c$, small intestine; $c l$, cloacal aperture; $d$. large intestine; $e$, liver; $f$, bile-duct: $g$, gall-bladder $; h$, spleen; $i$, lung; $k$, larynx; $l$, fat-hody ; $m$, testis; $n$, ureter; o, kidney; $p$, pancreas; $s$, cerehral hemisphere; $s p$, spinal cord ; $t$, tongie $; u$, auricle; $u r$, urostyle ; vi, ventricle; $v s$, vesicula seminalis; $w$, optic lobe; $x$, cerebellum; $y$, Eustachian recess; $z$, nasil sac. (From Marshall.)

mainly for the passage to the anal opening of the foeces or unabsorbed effete matters of the food.

The whole of the interior of the alimentary canal is lined by a layer of cells - the alimentary or enteric epithelium. The form and arrangement of the cells of this epithelium vary greatly in different groups of animals. Usually, they are vertically elongated, prismatic or columnar, or pyramidal in shape ; frequently they are ciliated. In some lower forms, the cells lining the alimentary cavity have the power, like Amœba, of thrusting forth processes of their protoplasin (Fig. 11, $h$ ), and of taking minute particles of food into their interior to become digested and absorbed (intracellular digestion). Sometimes they are all more or less active in secreting a fiuid destined to act on the food and render it more soluble; sometimes this function is confined to certain of the cells, which have a specici form; very often the secreting cells 
line special little pouch-like, simple or branched glands, opening by a passage or duct into the main cavity of the alimentary canal. Besides these glands formed from specially modified cells of the enteric epithelium there are nearly always present certain large special glands, separate from the alimentary canal itself, but opening into it by means of ducts. Of these the most generallyoccurring are the glands termed salivary glands, liver, and pancreas. The salivary glands have the function of secreting a fluid called the saliva, which, in many cases at least, has a special action on starchy matters, converting them into sugar. The ducts of these glands open always, not into the digestive, but into some part of the ingestive region of the alimentary system.

The most important function of the liver-properly so calledis one distinct from the process of digestion; its secretion--the bile-has, however, at least a mechanical effect on this process, and assists the secretion of the pancreas in its effects upon fat. In lower forms the organ to which the term liver is commonly applied appears in many cases to combine the functions of a true liver with that of a pancreas, and is thus more appropriately termed hepato-pancreas or liver-pancreas.

The pancreas secretes a fluid-the pancreatic juice-which has a very important effect in digestion. It renders substances of the nature of albumins soluble by converting them into modifications termed peptones; it converts starch into the soluble substance sugar; it acts on fatty matters in such a way as to convert them into cmulsions which are capable of being taken up and absorbed, and it effects the splitting up of part of the fat into fatty acids and glycerine.

When the food has been acted on by the various digestive secretions, the soluble part of it is fitted to be taken up and absorbed through the wall of the alimentary canal into the blood (in animals in which a blood-system exists), or into the fluid which takes its place. In the higher animals a part of the soluble matter of the food passes directly into the blood contained in the blood-vessels; while another part is taken up by a set of special vessels, the lacteals - which are a part of the lymphatic system, and reaches the blood indirectly.

In some of the lower groups of animals there is no system of blood-vessels, and the nutrient matter of the food, absorbed through the alimentary canal, merely passes from cell to cell throughout the body, or is received into a space or series of spaces containing fluid intervening between the alimentary canal and the wall of the body. But in the majority of animals there is a system of branching tubes containing a special tluid-the blood, and it is into this that the nutrient matter absorbed from the food sooner or later finds its way. The bloed has for one of its principal functions the conveyance of the nutrient matters from the 
alimentary canal throughout the body, so that the various organs may select from it the material which they require for the carrying on of their functions. To carry out this office the blood is contained in a complicated system of branching tubes or blood-vessels.

The essence of the process of respiration, as we have alreadyseen, is an interchange of oxygen and carbonic acid which takes place between the tissues of an organism and the surrounding medium, whether air or water. During the vital changes which go on in the bodies of all animals, as in Amœba, oxygen is constantly being used up and carbonic acid being formed. The necessary supply of oxygen has to be got from the air, or, in the case of aquatic animals, from the air dissolved in the surrounding water. At the same time the carbonic acid has to be got rid of. In the lowest animals-as for instance Amœba, and many of higher organisation-the oxygen passes inwards and the carbonic acid outwards through the general surface of the body. But in the great majority of animals there is a special set of organs-the organs of respiration-having this particular function. In some animals these organs of respiration are processes, simple or branched, lined by a very delicate membrane, and richly supplied with blood-vessels. Such processes are called gills or branchice; they are specially adapted for the absorption of oxygen dissolved in water.

In other animals the oxygen is obtained directly from the air; and in such air-breathing forms the organ of respiration is very often a sac, either simple or compound, termed a lung. The interior of this sac is lined with an epithelium of extreme delicacy, immediately outside of which is a network of microscopic bloodvessels or capillaries with thin walls; and the oxygen readily passes from the air in the cavity of the lung through its lining and the thin wall of the blood-ressel into the blood. In other airbreathing forms the organs of respiration are trachec, which are ramifying tubes, by means of which the air is conveyed to all parts of the body. In such forms, of which the Insects are examples, the air is conveyed, by means of these tubes, from openings on the surface of the body to all parts, and respiration goes on in all the organs.

In order that the air or water in contact with the surface of the lungs or gills may be renewed, there are usually special mechanical arrangements. In many gill-bearing animals the gills are attached to the legs, and are thus moved about when the animal moves its limbs. In others certain of the limbs are constantly moving in such a way as to cause a current of water to flow over the gills. In air-breathing forms there is usually a pumping apparatus, by means of which the air is alternately drawn into and expelled from the lungs.

In a great number of animals there is in the blood a substance 
called hoemoglobin, which has a strong affinity for oxygen; and the oxygen from the air, when it enters the blood, enters into a state of loose chemical combination with it. In this state, or simply dissolved in the fluid plasma of the blood, the oxygen is conveyed throughout the body.

Thus the blood, besides receiving the solid and liquid food from the alimentary canal and carrying it throughout the body for distribution, receives also the oxygen or gascous food, and supplies it to the parts requiring it. In all parts of the body in which vital action is taking place chemical changes are constantly going on. These chemical changes in the tissues, having for their result the production of heat, motion, secretion, and nerve-action, are for the most part of the nature of oxidations, and involve a constant consumption of oxygen; while a product which becomes formed as à result of this action is carbonic acid gas.

To carry out all the functions which it has to perform as a distributor of nourishment and oxygen and a remover of carbonic acid, the blood has to be moved about through the vessels-to circulate throughout the various organs. In the lowest forms in which a definite blood-system is to be recognised, this movement is effected in great measure by the general movements of the body of the animal. In others certain of the vessels contract and drive the blood through the system; such contractions are of a peristaltic character, the contractions being of the nature of constrictions running in a definite direction along the course of the vessel, with an effect similar to that produced by drawing the hand along a compressible india-rubber tube.

In all higher forms the movement of the blood is effected by means of a special organ-the heart. The heart is a muscular organ which by its contractions forces the blood through the system of vessels. In its simplest form it usually consists of two chambers, both with muscular walls,- the one, called the auricle, receiving the blood and driving it into the other, which is called the ventricle. The latter, in turn, when it contracts, drives the blood through the vessels to the various parts of the body-the return of the blood backwards to the auricle from the ventricle being prevented by the presence of certain valves, which act like folding doors opening from the auricle towards the ventricle, but closing when pressure is exerted in the opposite direction. In the higher animals the heart becomes a more complex organ than this, with a larger number of chambers and a more elaborate system of valves.

Carbonic acid, as already mentioned, is a waste-product constantly being produced in the tissues and being carried off by the blood to pass out by the gills or lungs. Besides the carbonic acid, there are constantly being formed waste-substances of another class-viz., substances containing nitrogen, of which uric acid and urea are the principal ultimate forms. These are separated from 
the blood and thrown out of the body by a distinct set of organs called renal organs, or organs of urinary excretion. The form of these organs varies greatly in the different groups; in many cases they are more or less intimately connected with the genital system.

In place of the simple contractions and extensions of the protoplasm which constitute the only movements of Amœba, the higher animals are capable of complex and definite movements. These are brought about by the agency of a set of organs termed the muscles. A muscle is a band or sheet of muscular fibres endowed in the living state with the property of contractility, by virtue of which, when stimulated in certain ways, it contracts in the direction of its length, becoming shortened, and, at the same time, thickened (Fig. 25). The extremities of the muscle are

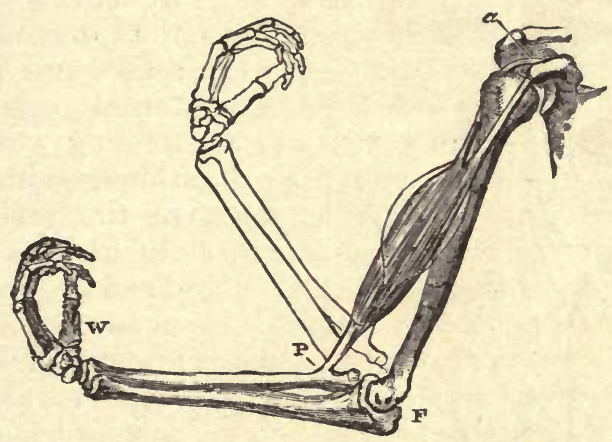

Fig, 25.-Bones of the human arm and fore-arm with the biceps muscle, showing the shortening and thickening of the muscle during contraction and the consequent change in the relative position of the bones-viz., flexion of the fore-arm on the upper arm. (From Huxley's Physiology.)

frequently composed, not of contractile muscular fibres, but of a form of strong fibrous connective tissue-the tendon of the muscle. The ends of the muscle are usually firmly attached to two different parts of the jointed framework or skeleton, external or internal; and, when the muscle contracts and becomes shortened, these two parts are drawn nearer to one another.

In all but the most lowly-organised animals there is a system of organs - the nervous system-by means of which a communication is effected between the various parts of the body, enabling them to work in harmony, and by means of which also a communication is established between the organism and the external world. The two essential elements of the nervous system-the nerve-cells and nerve-fibres-have a regular arrangement which varies in the different animal types both as regards structural details and the relations borne to the other systems of organs; but there are to be recognised two chief parts or sets of partsthe central and the peripheral. 
The central parts of the nervous system consist (Fig. 26) of certain aggregations of nerve-matter known as nerve-ganglia, containing a large number of nerve-cells; a relatively large mass of this matter may be

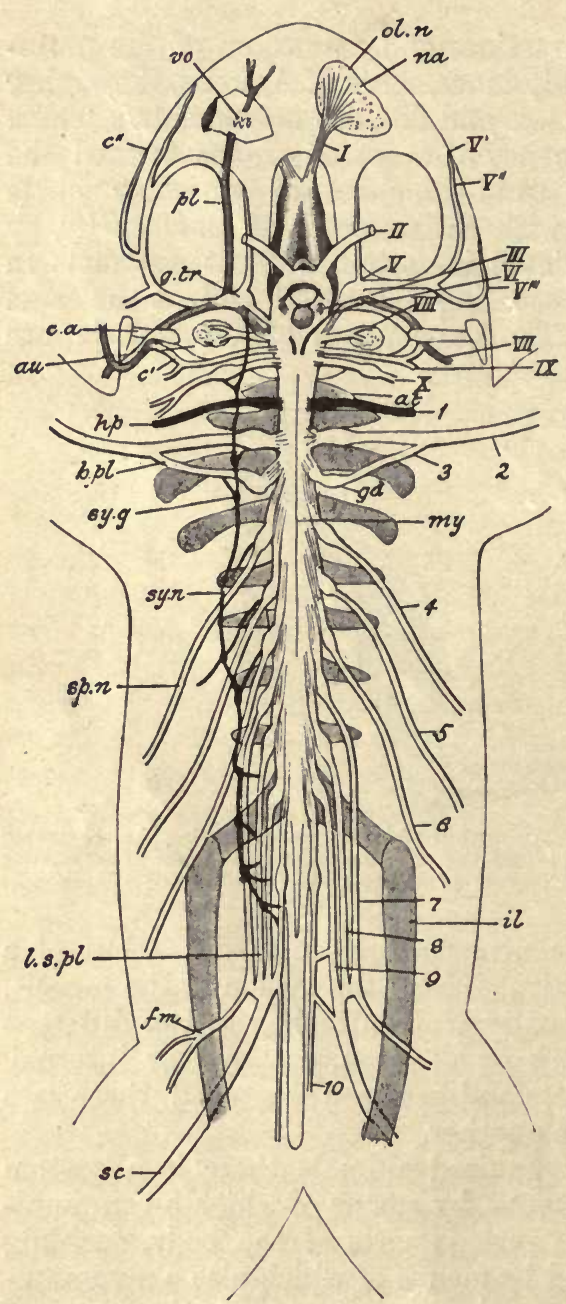

Fia. 26.-Nervous system of the Frog. (From Howes's Atlas.) collected together to form a brain. To or from these central parts pass all the systems of nerve-fibres, constituting the peripheral part of the system; the former have the office both of receiving impressions conveyed by the nerve-fibres from the surface, from the organs of special sense, and from the internal organs, and of sending off messages through similar channels to the various parts of the body - to muscles, to glands, to alimentary canal, and to vascular system. When a movement is to be effected a message passes from the nerve-centre along a nerve-fibre to a muscle and causes it to contract; when an organ requires the amount of blood supplied to it to be increased or diminished a message is conveyed along a nerve-fibre and causes the dilatation or contraction of the bloodvessels of the part; and a similar initiatory or controlling influence is exerted over the activities of all the organs.

In certain groups of animals all the impressions from the external world are received through the integument of the general surface, and this is the case in all animals with the general impressions of touch and of heat and cold. The sensitiveness of 
the integument to such general impressions may be increased by the presence in it of a variety of tactile papillæ or corpuscles having nerve-fibres terminating in them. In most animals, however, there are certain organs, the organs of special sense, adapted to receiving impressions of special kinds-eyes for the reception of the impressions produced by light, cars for the reception of those produced by the waves of sound, olfactory organs or organs of smell, and gustatory organs or organs of taste. The most rudimentary form of eye is little more than a dot of pigment which absorbs some of the rays of bright light-these producing a nerve-disturbance in certain neighbouring nerve-cells. To this may be added clear, highly-refracting bodies which intensify the effect. In the higher types of eye there are the same characteristic parts-the clear, highly-refracting substance, the pigment, and the nerve-cells; but each has undergone a development resulting in the construction of an organ adapted to the reception of lightimpressions of a very definite character. The highly-refracting body assumes the form of a lens for the focussing of the light-rays; the nerve-cells are arranged within a regular layer, the retina, from which nerve-fibres pass to the central part of the nervous system; the pigment is so arranged as to absorb the light-rays and prevent their passage beyond the retina, and in certain cases also lines a diaphragm, the iris, with a central aperture through which the rays of light are admitted to the central parts of the eye. In some animals (Insects, Crustacea) the eye consists of a very large number of independent elements, each with its refracting apparatus, its nervous element, and its absorbing pigment.

The ear in its simplest form is a membranous sac or otocyst with internally projecting stiff cilia, and containing a liquid in which there lie a number of particles of carbonate of lime. The soundwaves evidently set in vibration the liquid and its contained calcareous particles, and by means of these vibrations acting on the cilia, an impression of a definite character is produced in the cells of a neighbouring nerve-ganglion. In higher forms the apparatus for receiving the vibrations becomes extremely complex, and there is elaborated a nervous mechanism by which sounds of different pitch and intensity produce impressions of a distinct character. The organ of hearing usually possesses the additional function of an organ ministering to the sense of rotation, and thus has an important part to play in the maintenance of the equilibrium of the body.

The essential elements of the reproductive organs-the ova and spermatozoa-have already been briefly alluded to (p. 30). The ova are developed in an organ termed the ovary, and the sperms in an organ called the spermary or testis. Sometimes ovaries and testes are developed in the same individual, when the arrangement is termed monocious or hermaphrodite; sometimes 
the ovaries occur in one set of individuals - the females-and the testes in another set-the males, when the term unisexual or dicecious is employed. Very frequently the male differs from the female in other respects besides the nature of the reproductive elements-in size, colour, and the like; when such differences are strongly marked the animal is said to be sexually dimorphic. The ova and sperms are usually conveyed to the exterior by canals or ducts-the ovarian ducts or oviducts, and the testicular ducts, spermiducts, or vasa deferentia. In some instances the ova are impregnated after being discharged from the oviducts, and the development of the young takes place externally; in other cases the impregnation takes place in the oviduct, and the young become fully developed in the interior of a special enlargement of the oviduct termed the uterus. In the former case the animal is said to be oviparons, in the latter viviparous; but there are, numerous intermediate gradations between these two extremes.

\section{The Reproduction of Animals.}

In a limited number of groups of animals reproduction takes place by means of cells corresponding to ova developed in organs similar to ovaries, but without impregnation by means of sperms. This phenomenon is known as parthenogenesis ( $c f . p .21$ ).

Besides the sexual process of reproduction by means of ova and spermatozoa, there are in many classes of animals various asexual modes of multiplication. One of these-the process of simple fission - has been already noticed in connection with the reproduction of Amœba. The formation of sporcs is an asexual mode of multiplication which occurs only in the Protozoa, and will be described in the account of that group. Multiplication by budding takes place in a number of different classes of animals. In this form of reproduction a process or $b u d$ (Fig. $27, b d$ ) is given off from some part of the parent animal; this bud sooner or later assumes the form of the complete animal, and may become detached from the parent either before or after its development has been completed or may remain in permanent vital connection with the parent form.

When the buds, after becoming fully developed, remain in vital continuity with the parent, a sort of compound animal, consisting of a greater or smaller number of connected units, is the result. Such a compound organism is termed a colony, and the component units are termed zooids. In some cases such a colony is produced by a process which is more correctly termed incomplete fission than budding.

Alternation of generations ; heterogamy ; pædogenesis.In the life-history of a considerable number of animals, a stage in which reproduction takes place by a process of budding or fission 
alternates with a stage in which there occurs a true sexual mode of reproduction. Such a phenomenon is termed alternation of generations or metagenesis. The term heterogamy is applied to cases in which two different sexual generations-usually a true sexual and a parthenogenetic-alternate with one another. Padogenesis, or the development of young by a sexual process from

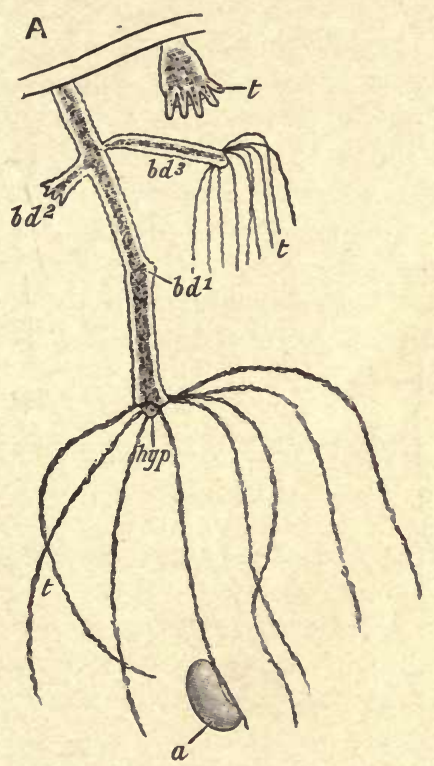

Fig. 27.-Fresh-water polype (Hydra), two specimens, the one expanded, the other contracted, showing multiplication by budding. bd.1 bu. ${ }^{2} b d_{0}{ }^{3}$ buds in various stages of growth. (From Parker's Biology.)

individuals that have not attained the adult condition, is a phenomenon which is to be observed in some groups of aninals.

\section{SYMMETRY.}

The general disposition or symmetry of the parts in an animal presents two main modifications-the radial and the bilateral. The gastrula (p. 23) is the simplest and most generalised form among multicellular animals or Metazoa; but no adult animal retains this simple shape. In the gastrula we may imagine a central primary axis (Fig. 28, $A B$ ) passing through the middle of the blastopore and of the archenteric cavity, and a series of secondary axes $(a b, c d$,$) running at right angles to this to the outer surface. In a$ symmetrical gastrula the secondary axes would be all equal. Many 
animals are in the adult condition similar in their symmetry to the gastrula, except that there are special developments along a series of regularly arranged radiating secondary axes; these radial developments may be in the form of tentacles or radially arranged processes (Fig. 29), or may assume the character of a radial arrangement of internal parts. Such an animal is said to be radially symmetrical. The body of a radially symmetrical animal is capable

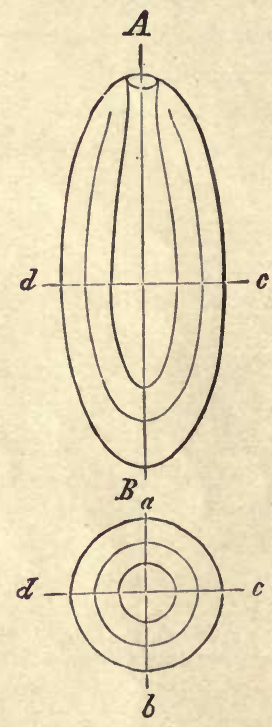

Fug. 28.-Diagram of the axes of the body. $A B$, primary axis; ab, cd, secondary axes. The lower figure is a transverse section of the upper one showing its two secondary axes. (From Gegenbaur.)
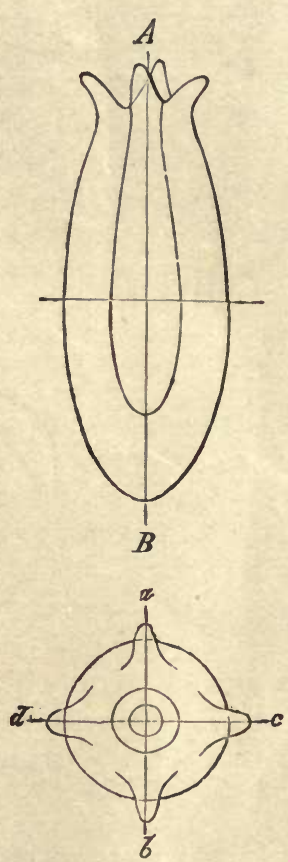

Fia. 29.-Radial symmetry. Letters as in Fig. 28. The processes at $\mathbf{A}$ are the tentacles; the lower figure represents the upper or oral surface. (From Gegenbaur.)

of being divided into a series of equal radial parts or antimeres, each of which is symmetrically disposed with regard to one of the secondary or radial axes.

In animals which are not permanently fixed, locomotion usually takes place in the direction of the primary axis of the body, and one side, habitually directed downwards, becomes modified differently from the other which is habitually directed upwards: lower or ventral surface becomes distinguishable from an upper or dorsal. Thus the radial symmetry is now disturbed; the secondary axes have become unequal; the dorso-ventral or vertical secondary axes 
are, to a greater or less extent, different from the transverse or horizontal secondary axes, and the body of an animal having such a disposition of the parts is divisible into two equal lateral halves or hemisomes by a median vertical plane passing through the primary axis. This is the bilateral symmetry observable in all but a few types of animals.

Sometimes the bilaterally symmetrical animal is unsegmented; sometimes it is divided into a series of segments or metameres. A distinct head may be present or absent. The head end or anterior end is that which, save in exceptional cases, is directed forwards in locomotion. It is towards this end that the organs of special sense are situated, as well as the opening of the mouth and the organs for the prehension and mastication of food. A head is developed when the anterior part bearing these structures is marked off externally from the rest. In segmented animals the head consists of a number of segments amalgamated together, and it contains the brain or the principal central ganglia of the nervous system.

\section{The Primary Subdivisions or Phyla of the Animal KINGDOM.}

The various systems of organ)-digestive, circulatory, nervous, excretory, etc.-present under one form or another in all the higher groups of animals, are variously arranged and occupy various relative positions in different cases, producing a number of widely different plans of animal structure. According as their structure conforms to one or another of these great plans, animals are referred to one or another of the corresponding great divisions or phyla of the animal kingdom. That animals do present widely differing plans of structure is a matter of common knowledge. We have only to compare the true Fish, such as Cod, Haddock, etc., in a fishmonger's shop with the Lobsters and the Oysters, to recognise the general nature of such a distinction. The first-named are characterised by the possession of a backbone and skull, with a brain and spinal cord, and of two pairs of limbs (the paired fins); they belong to the great vertebrate or backboned group-the division Vertebrata of the phylum Chordata. The Lobsters, on the other hand, in which these special vertebrate structures are absent, possess a body which is enclosed in a hard jointed case, and a number of pairs of limbs also enclosed in hard jointed cases and adapted to different purposes in different parts of the body-some being feelers, others jaws, others legs: their general type of structure is that which characterises the phylum Arthropoda. The Oysters, again, with their hard calcareous shell secreted by a pair of special folds of the skin constituting what is termed the mantle, and with a special arrangement of the nervous system and other organs which 
need not be described here, are referable to the phylum Moliuscc. Other familiar animals are readily to be recognised as belonging to one or other of these great phyla. A Prawn, a Crab, a Blue-bottle Fly, a Spider, are all on the same general plan as the Lobster: they are jointed animals with jointed limbs, and have the internal organs occupying similar positions with relation to one another: they are all members of the phylum Arthrcpoda. Again, a Mussel, a Snail, and a Squid are all to be set side by side with the Oyster as conforming to the same general type of structure: they are all members of the phylum Mollusca. A Dog, a Lizard, and a Fowl, again, are obviously nearer the Fish : they all have a skull and backbone, brain and spinal cord, and two pairs of limbs, and are members of the great group Chordata.

Altogether twelve phyla are to be recognised, viz.:-
I. Protozoa
II. Porifera
III. Coelenterata
IV. Platyhelminthes
V. Nemathelminthes
VI. Trochelminthes

VII. Molluscoida
VIII. Echinodermata
IX. Annulata
X. Arthropoda
XI. Mollusca
XII. Chordata

But these do not comprise all known animals. There are a number of smaller groups which are only very doubtfully to be associated with one or other of the phyla; and it is in some cases chiefly to avoid multiplication of the latter that such groups are not treated as independent. Such forms, until their places are more definitely fixed, are best dealt with as appendices to the phyla to which they appear most nearly related. 


\section{SECTION II}

\section{FHYLUM PROTOZOA}

IN the preceding section we learnt the essential structure of an animal cell, and it was pointed out that in the lowest organisms the entire individual consists of a single cell. All such unicellular animals are placed in the lowest primary subdivision of the animal kingdom-the phylum Protozoa.

We have also learnt that cells vary considerably in character. They may be amceboid or capable of protruding temporary processes of protoplasm called pseudopods; flagellate, or produced into one or more-always a small number-of threads having an intermittent lashing movement; ciliated, or produced into numerous rhythmically moving threads of protoplasm; or encysted, the protoplasm being enclosed in a cell-wall. Moreover, under certain circumstances, amœboid cells may fuse with one another to form a plasmodium.

These well-marked phases in the life of the cell allow us to divide the Protozoa into subdivisions called Classes. The same organism may be amœboid, Hlagellate, encysted, and plasmodial at various stages of its existence, but nevertheless we find certain forms in which the dominant phase in the life-history is amoboid, others which are characteristically flagellate or ciliated, others again in which the tendency to form plasmodia is a distinctive feature. In this way five well-marked groups of unicellular organisms may be distinguished.

Class 1. RHIzopodA.-Protozoa in which the amoboid form is predominant, the animal always forming pseudopods. Flagella are often present in the young, and occasionally in the adult. Encystation frequently occurs.

Class 2. MrcetozoA.-Terrestrial Protozoa in which the plasmodial phase is specially characteristic, as also is the formation of large and often complex cysts.

Class 3. Mastigophora.-Protozoa in which the flagellate form 
is predominant, although the amœboid and encysted conditions frequently occur.

Class 4. Sponozod.-Parasitic Protozoa without special locomotive parts in the adult. Encystation is almost universal, and the young may be flagellate or amœboid.

Class 5. InfusoniA.-Protozoa which are always ciliated, either throughout life or in the young condition.

\section{CLASS I.-RHIZOPODA.}

\section{Example of the Class-Amoba proteus.}

Amœba has been fully described in the preceding chapter; it will therefore be unnecessary to do more than recapitulate the most essential features in its organisation.

It is an irregular mass of protoplasm (Fig. 30, E) about $\frac{1}{6} \mathrm{~mm}$. in diameter, produced into irregular processes or pseudopods $(p s d)$ of variable size and form and capable of being protruded and retracted, often with considerable rapidity. The protoplasm is divisible into a granular internal substance or enciosarc and a clear outer layer or ectosarc; the difference between the two is hardly a structural one, but depends simply on the accumulation of granules in the central portion. The granules are, for the most part, various products of mietabolism-proteinaceous or fatty.

Imbedded in the endosarc is a large nucleus (nu), of spherical form, consisting of a clear achromatic substance, enclosed in a membrane, and containing minute granules of chromatin. The contractile vacuole (c. vac.), a very characteristic structure of the Protozoa, lies in the outer layer of the endosarc, and exhibits rhythmical movements, contracting and expanding at more or less regular intervals.

Amcba feeds by ingesting minute organisms (Fig. 30, c, f. vac.) or fragments of organisms - i.e., by enveloping them in its substance, retaining them until the proteids they contain are dissolved and assimilated, and then crawling away and leaving the undigested remnants behind.

Amœbæ are sometimes found to undergo encystation; the pseudopods are withdrawn and the protoplasm surrounds itself with a cell-wall or cyst $(\mathrm{D}, c y)$, from which, after a period of rest, it emerges and resumes active life. The cyst is formed of a chitinoid material-i.e., a nitrogenous substance allied in composition to horn and to the chitin of which the armour of Insects, Crayfishes, etc., is composed.

Reproduction takes place by simple or binary fission; direct or amitotic division of the nucleus is followed by division into two of the cell-body (I). Occasionally two Amœbæ have been observed to 
conjugate or undergo complete fusion, but nothing is known of the result of this process or of its precise significance in this particular case.

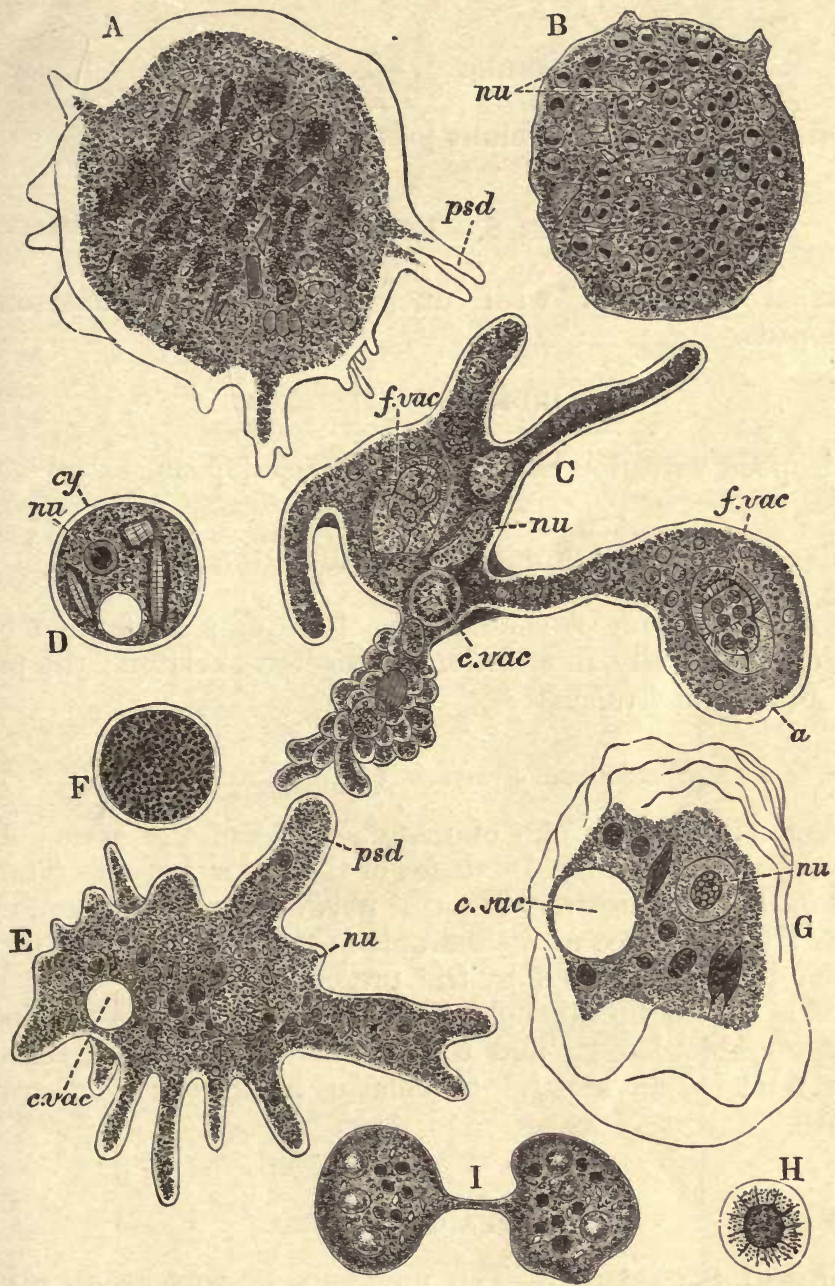

Fig. 30.-Amœiba. A, A. quarta; B, the same killed and stained; C, A. protens; D, encysted specimen; E, A. proteus ; F, nucleus of same, stained; G, A. verrucosa; $\mathrm{H}$, nucleus of same, stained ; I, A. proteus, undergoing binary fission; $a$, point of union of enclosing pseudopods; $c$. vac. contractile vacuole; cy. cyst; $f$. vac. food-vacuole; $n u$. nucleus (numerous in A. quarta); psd. pseudopod. (From Parker's Biology, after Leidy, Gruber, and Howes.)

\section{Classification and General Organisation.}

The Rhizopoda differ among themselves in the character of their pseudopods, which may be short and blunt or long and 
delicate; in the number of nuclei; and in the presence or absence of a hard shell within or around the protoplasm. The following four orders may be distinguished :-

\section{Order 1.-Lobosa.}

Rhizopoda with short, blunt pseudopods.

\section{ORDER 2.-FORAMINIFERA.}

Shelled Rhizopoda with fine, branched, and anastomosing pseudopods.

\section{Order 3.-HeLiozoA.}

Rhizopoda with fine, stiff, radiating pseudopods.

\section{ORDER 4.-RADIOLARIA.}

Rhizopoda having a shell in the form of a perforated central capsule, and usually, in addition, a siliceous skeleton: the pseudopods are long and delicate.

\section{Systematic Position of the Example.}

Amoba proteus is one of many species of the genus Amoba, belonging to the family Amobida, of the order Lobosa. The blunt pseudopods not uniting to form networks place it among the Lobosa: the absence of a shell, among the Amœbidæ. The genus Amœba is distinguished by the presence of one or more nuclei, and of a contractile vacuole. In A. proteus the pseudopods are of considerable length and sometimes branched, and there is a single nucleus, having its chromatin in the form of scattered granules.

\section{ORDER 1.-LoBOSA.}

General Structure.-The members of this group all agree with Amœba in essential respects, their most characteristic feature being the short, blunt pseudopods. The chief variations in structure upon which the genera and species are founded have to do with the number and character of the nuclei, the form of the pseudopods, and the presence or absence of a shell.

In Amœba itself there may be one (Fig. 30, E) or several (B) nuclei, the chromatin of the nucleus may be arranged in various ways $(\mathrm{F}, \mathrm{H})$, and the pseudopods may be prolongations of con- 
siderable relative size (c), or mere wave-like elevations of the surface $(G)$. Sometimes specimens are found in which neither nucleus nor vacuole is present; these are placed in the genus

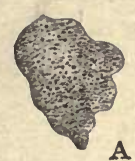

A

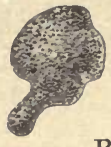

B

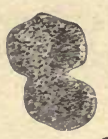

C

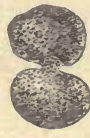

D

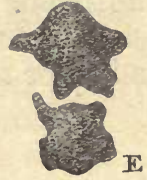

FIG. 31.-Protamœba primitiva. Showing changes of form and three stages in binary fission. (After Haeckel, from Parker's Biology.)

Protamaba (Fig. 31). Very probably, however, future investigation will show this and other non-nucleate forms to possess a potential nucleus in the form of minute scattered granules of chromatin.

The largest of the naked or shell-less Lobosa is Pelomyxa, which may be as much as $8 \mathrm{~mm}$. in diameter; it is multi-nucleate and is further distinguished by the presence of numerous non-contractile vacuoles in the endosarc.

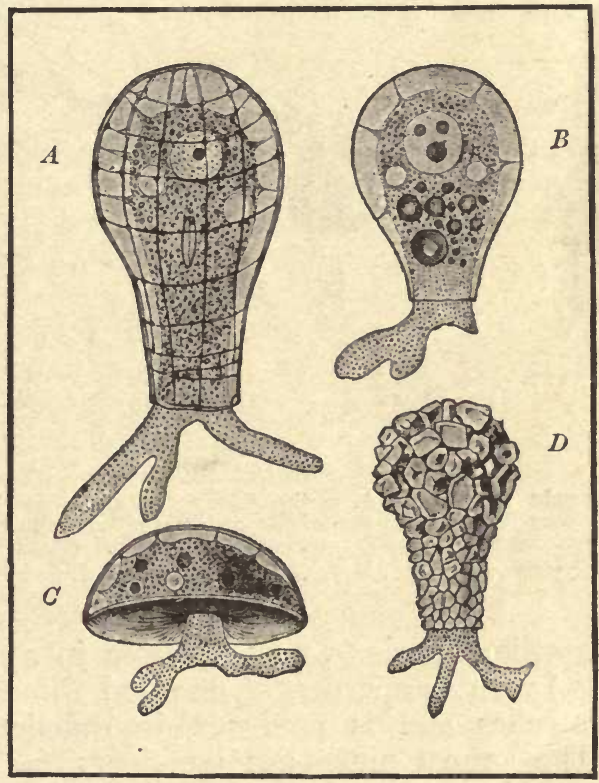

FIG. 32.-A, Quadrula symmetrica; B. IIyalosphenia lata; C, Arcella vulgaris ; D, Difflugia pyriformis. (From Lang's Comparative Anatomy.)

Skeleton.-We may understand the relation of the shelled to the shell-less Lobosa by supposing an Amœba to draw in the pseudopods from the greater part of its body, and to secrete, from that part only, a cell-wall ; such a cell-wall or capsule would differ 
from a cyst in having an aperture at one end to allow of the protrusion of pseudopods from a small naked area. This is exactly what we find in Arcella and its allies (Fig. 32, A-C), in which the shell is chitinoid. A different kind of shell is found in Difflugia (D), which secretes a gelatinous coating to which minute sandgrains and other foreign particles become attached.

\section{ORDER 2.-FORAMINIFERA}

General structure.-The members of this order differ from the Lobosa in the fact that their pseudopods are long and delicate and unite to form networks; moreover, with few exceptions, they agree with Arcella and its allies in possessing a shell. In the majority of cases this shell is formed of calcium carbonate.

One of the simplest members of the group is Microgromia (Fig. 33). It consists of a protoplasmic body (B), with a single nucleus
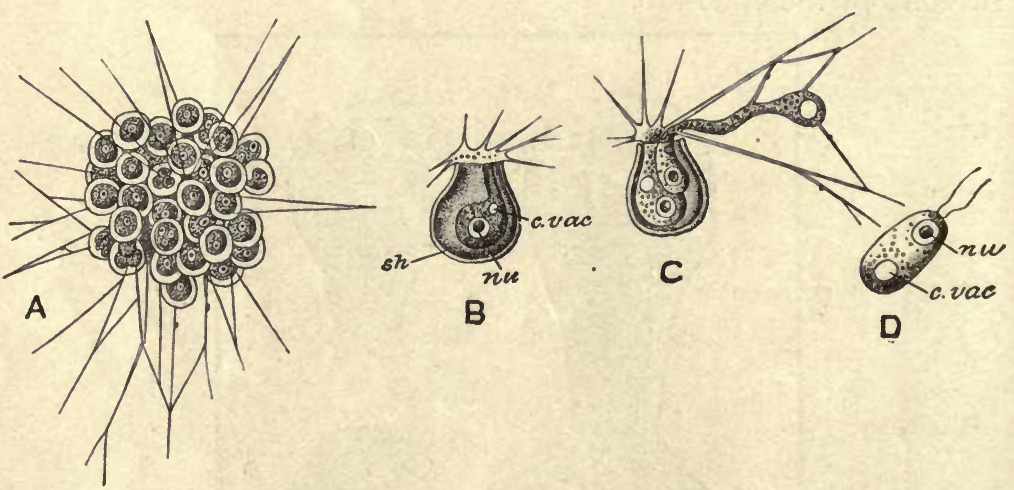

Fia. 33.-Microgromia socialis. A, entire colony; B, single zooid; C, zooid which has undergone binary fission, with one of the daughter-cells creeping out of the shell; D, flagellula; c. vac. contractile vacuole; $n u$. nucleus; sh. shell. (From Butschli's Protozon, after Hertwig and Lesser.)

(nu.) and contractile vacuole (c.vac.), enclosed in a chitinoid cellwall or shell $(s h$.) with an aperture at one end through which the protoplasm protrudes and is produced into delicate radiating pseudopods. The animal multiplies by binary fission, and the individuals or zooids thus produced remain united in larger or smaller clusters, or cell-colonies (A). Sometimes the cell-body of a zooid divides and one of the daughter-cells creeps out of the cellwall (C), and, after moving about for a time like an Amœba, draws in its pseudopods, assumes an oval form, and sends out two flagella by means of which it is propelled through the water (D). We shall find other instances in which the young of a Rhizopod is 
a flagellula, i.e. a cell provided with one or more flagella, which, if its history were not known, would be included among the Mastigophora.

Platoum (Fig. 34, A) is a form resembling Microgromia, but illustrating a very interesting type of colony. The protoplasm flows out of the mouth of the shell in the form of a long plate (B)

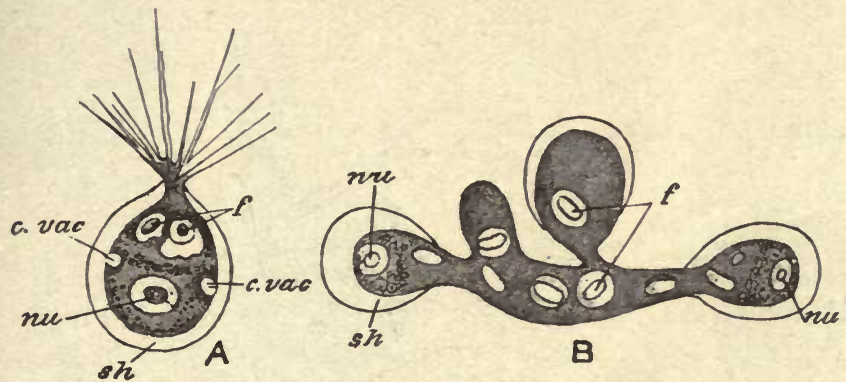

FiG. 34.-Platoum stercoret m. A, single zooid; $\mathrm{B}$, formation of colony ; $c$. vac. contractile vacuole; f. food particles; nu. nucleus; $s h$. shell. (From Bütschli's Protozow, after Cienkowsky.)

which sends off rounded side branches, and each of these, acquiring a cell-wall, becomes a zooid of the simple cell-colony.

Gromia (Fig. 35, 1) leads us to the more typical Foraminifera. The protoplasm of this form protrudes from the mouth $(a)$ of the chitinoid shell (sh.) and flows around it so that the shell becomes an internal structure. The pseudopods are very long and delicate and unite to form a complicated network, exhibiting a streaming movement of granules and serving, as usual, to capture prey.

Skeleton.-Squammulina (Fig. 35,3) differs from Gromia mainly in having the shell formed of calcium carbonate and possessing the character of a hollow, stony sphere, with an aperture at one end. It appears that all the calcareous Foraminifera begin life in this simple form; but in the majority of cases the adult structure attains a considerable degree of complexity. The protoplasm of the original globular chamber overflows, as it were, through the aperture; but, instead of forming an elongated plate from which side buds are given off, as in Platoum, the extended mass rounds itself off, and secretes a calcareous shell in organic connection with the original shell, and communicating with it by the original aperture. In this way a two-chambered shell is produced, and a repetition of the process gives us the many-chambered shell found in most genera. New chambers may be added in a straight line (Fig. 36,3$)$, or alternately on opposite sides of the original chamber.(5), or with each new chamber enclosing its predecessor (4), or in a flat spiral, each new chamber being larger than its predecessor $(7,8)$, or in a spire in which the newer chambers 
overlap the older $(9,10)$, or in an irregular spiral of globular chambers $(6)$, or in an extremely compact spiral in which the new chambers completely enclose their predecessors (11). In all cases

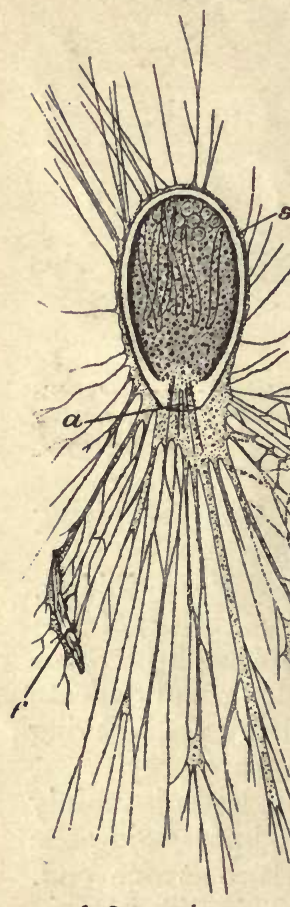

1. Gromia

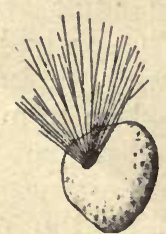

3. Squammulina
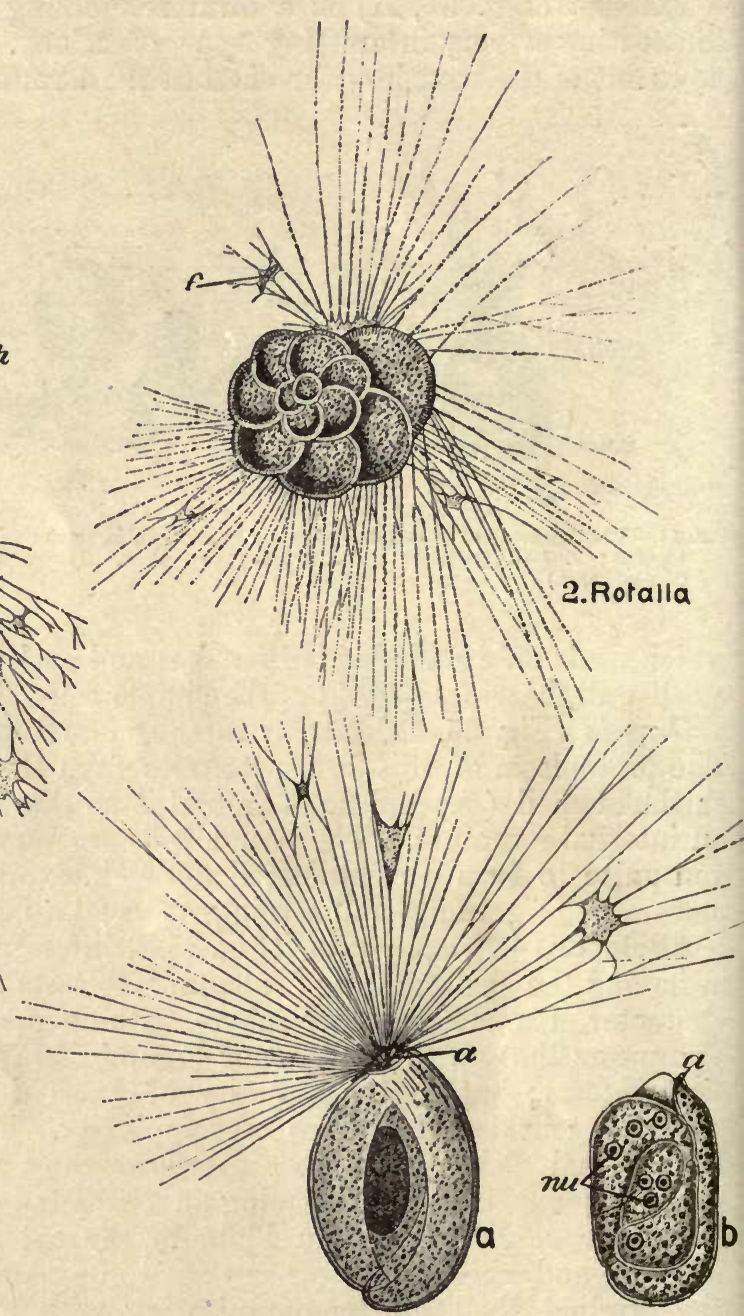

4.Miliola

Fia. 3\%-Various forms of Foraminifera. In 4, Miliola, $a$, shows the living animal; $b$, the same killed and stained ; $a$. aperture of shell; $f$. food particles; $n u$. nucleus; sh. shell. (From Bütschli's Protozoa and Claus's Zoology.)

adjacent chambers communicate with one another either by a single large hole or by numerous small ones: the protoplasm is thus perfectly continuous throughout the organism. With the 
increase in the number of chambers there is a multiplication of the nucleus (Fig. 35, 4, b, nu).

Not only does the shell increase in size by the formation of new
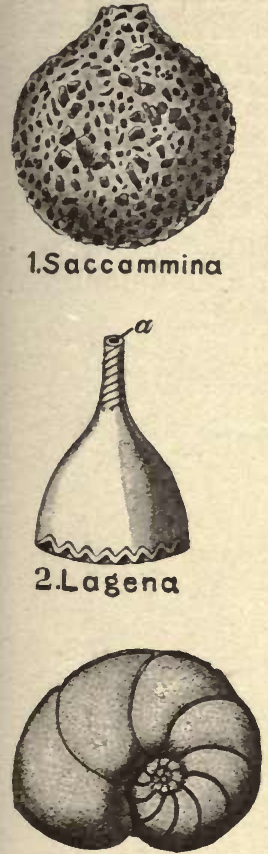

7. Discorbina
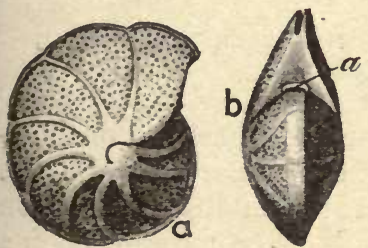

9.PIanorbulina

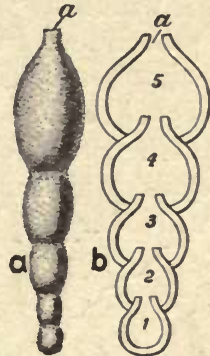

3. Nodosaria

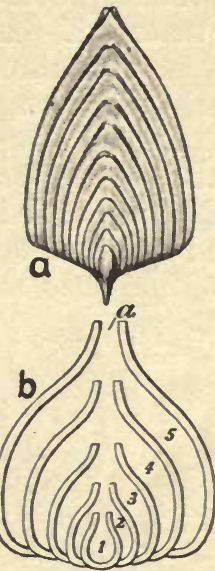

4.Frondicularia
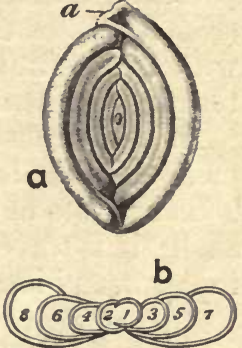

5. Spiroloculina

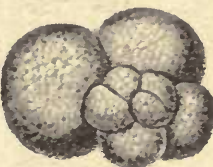

6. Globigerina

Frg. 36. - Shells of Foraminifera. In 3,4, and $5, a$ shows the surface view, and $b$ a section $8 a$ is a diagram of a coiled cell without supplemental skeleton; $8 b$ of a similar form with supplemental skeleton (s. sl..); and 10 of $\mathrm{a}$ form with overlapping whorls; in $11 a$ half the shell is shown in horizontal section; $b$ is a vertical section; $a$. aperture of shell; $1-15$, successive chambers, 1 being always the oldest or initial chamber. (After Carpenter, Brady, and Bütschli.)

chambers: individual chambers become larger. In this process layers of calcareous matter are added to the shell from without by the agency of a thin layer of protoplasm that extends over the 
surface, a corresponding thickness being, probably, removed by solution from the inner side at the same time.

The shell presents two leading types of structure apart from the form and arrangement of the chambers: either it is of a porcelain-like texture and provided with a single terminal aperture, (Fig. 35, 4), or the texture is glassy and the whole shell perforated with very minute apertures, through which, as well as through the terminal aperture, pseudopods are protruded (Fig. 35, 2).

In many cases additional complexity is attained by the development of what is called the supplemental skeleton (Fig. $36,8 b, s$. sk.). This consists of a deposit of calcium carbonate outside the original shell; it is traversed by a complex system of canals containing protoplasm, and is sometimes produced into large spines. Foraminifera

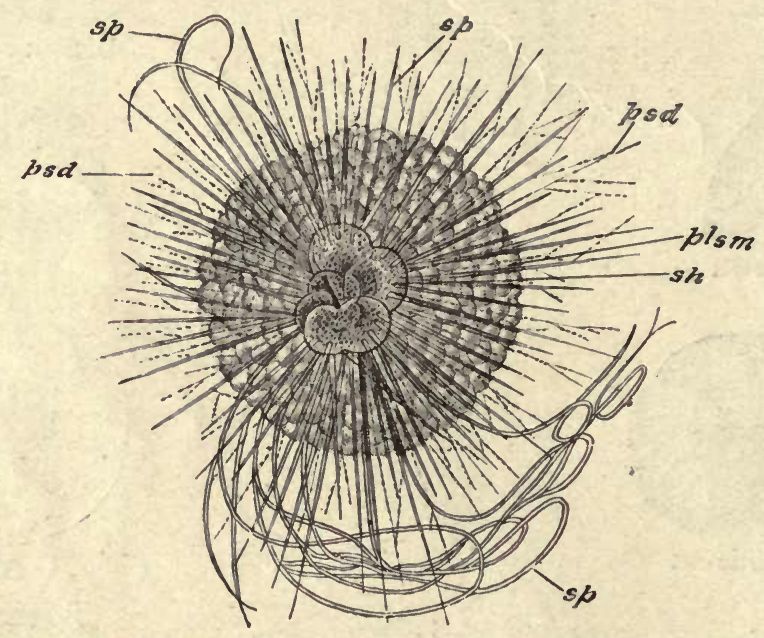

Fia. 37.-Hastigerina murrayi. plsm. vacuolated protoplasm surrounding shell: psi. pseudopods; sh. shell; sp. spines. (After Brady.)

in which this secondary skeleton occurs are sometimes of considerable size $-2-3 \mathrm{~cm}$. in diameter-and of extraordinary complexity.

Many Foraminifera resemble Difflugia in having a skeleton formed of sand-grains, sponge-spicules, and other foreign bodies cemented together by a secretion from the protoplasm (Fig. 36, 1). Some of these are formed on the imperforate type, having the protoplasm protruded from a single terminal aperture; others on the perforate type, small pseudopods being protruded between the particles forming the shell.

In many cases the pseudopods are the only portions of protoplasm outside the shell, whereas in Gromia, as we saw, the shell is invested with a layer of protoplasm, and is thus in strictness an internal structure. In one of the calcareous forms with 
perforated spiral shell, called Hastigerina (Fig. 37), a very remarkable modification of this condition of things obtains. The shell

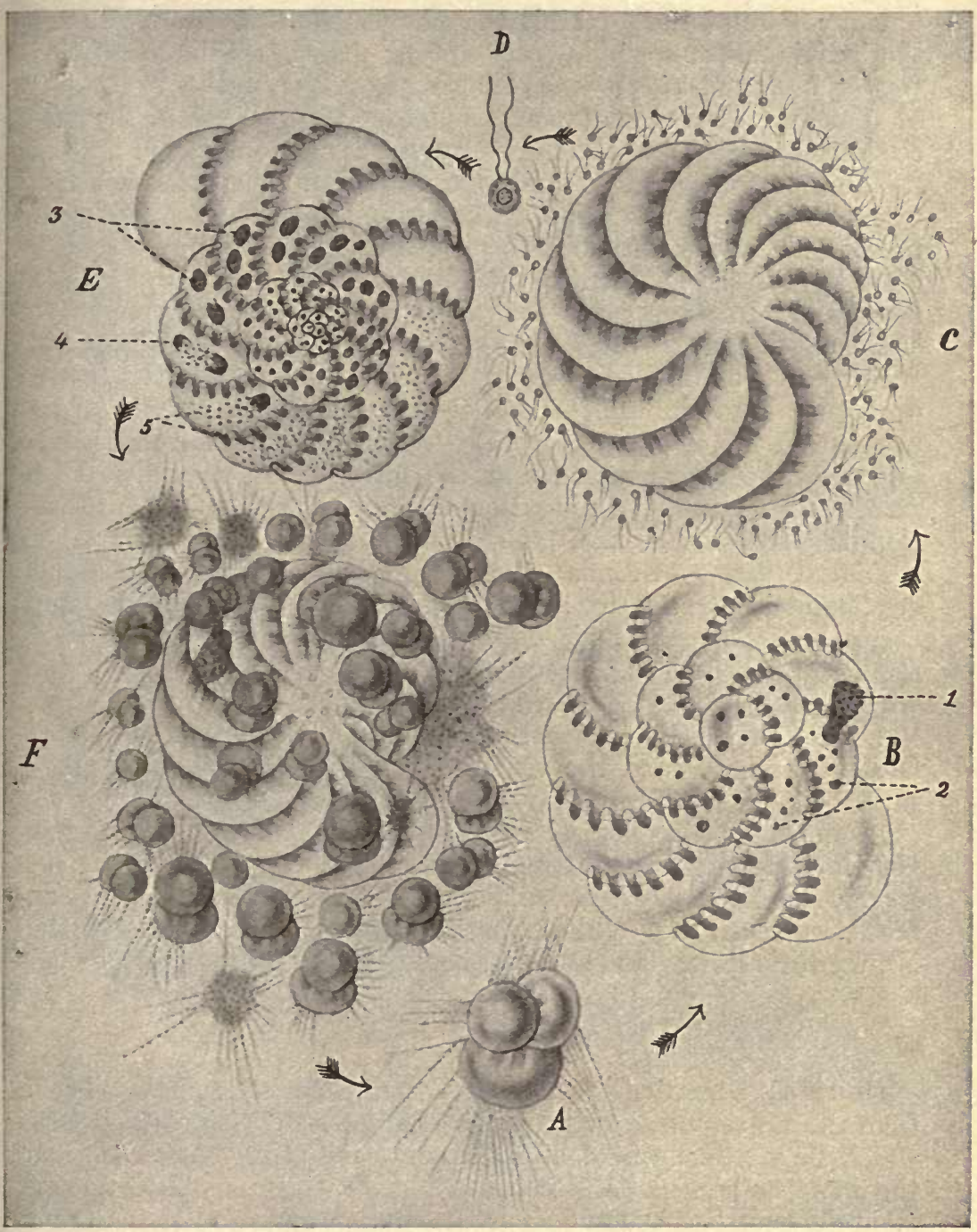

F1G. 38.-Dimorphism and alternation of generations in Polystomella crispa. The arrows indicate the direction of the life-cycle. $A$, young megaspheric individual; $B$, full-grown megaspheric individual, decalcified ; $C$, megaspheric individual in the act of spore-formation, the protoplasm leaving the shell in the form of flagellulæ; $D$, flagellula more highly magnified; $B$, microspheric individual developed from a flagellula; $F$, microspheric individual in the act of producing amoebuid embryas. (From Lang, after Schaudinn.)

$(s h$.$) is surrounded with a mass of protoplasm (\mathrm{plsm}$.$) many times$ its own diameter, and so full of vacuoles as to present a bubbly or 
frothy appearance. The shell itself, moreover, in this and allied forms is provided with numerous delicate, hollow, calcareous spines $(s p$.$) , which are only to be seen in perfect, freshly-caught specimens.$

Many Foraminifera exhibit the phenomenon of dimorphism: the individuals of a single species occur under two distinct forms (megaspheric and microspheric) differing from one another in the size of the central chamber, the shape and mode of growth of the succeeding chambers, and the number and size of the nuclei (Fig. 38).

The reproduction of Foraminifera is mainly by spore-formation, with or without conjugation. The protoplasm has been observed in some to divide into minute masses which may be amœboid or may be of the nature of flagellulie-each provided with a flagellum. In some cases the flagellulæ have been observed to conjugate in pairs. The young may develop shells while still within the shell of the parent or only after becoming free. In the dimorphic Foraminifera there is evidence of the occurrence of an alternation of generations (p. 41) - the megaspheric form alternating with the microspheric, and the latter being developed as a result of a process of conjugation, the former without it (alternation of sexual and asexual generations).

Distribution.-Gromia, Microgromia, and a few other forms are found in fresh-water: one species has been found in damp earth, but the great majority of the Foraminifera are marine, some being pelagic, i.e. occurring at or near the surface of the ocean, others abyssal, i.e. living at great depths. In the Atlantic, large areas of the sea-bottom are covered with a gray mud called Globigerina-ooze from the vast number of Globigerinæ contained in it.

From the palæontological point of view, the Foraminifera are a very important group. Remains of their shells occur in various formations from the Silurian period to the present day, certain rocks, such as the White Chalk (Cretaceous period) and the Nummulitic limestone (Eocene), being largely made up of them.

\section{ORDER 3.-HELIOZOA.}

General Structure.-The Heliozoa are at once distinguished from the preceding groups by the character of their pseudopods, which have the form of stiff filaments radiating outwards from the more or less globular cell-body, presenting very little movement beyond the characteristic streaming of granules, and not uniting to form networks.

One of the simplest forms is the common "Sun-animalcule," Actinophrys sol (Fig. 39). The body is nearly spherical, and contains a large nucleus and numerous vacuoles, some of which, near the surface, are contractile. Each of the stiff radiating pseudopods has a firm axis, apparently composed of protoplasm, 
which is traceable through the general protoplasm as far as the nucleus. Living organisins are devoured in much the same way as in Amœba: each is ingested along with a droplet of water, and is thus seen, during digestion, to lie in a definite cavity of the protoplasm, called a food-vacuole. 'If the organism be small, processes of the protoplasm are developed, and surround and engulf it. If it be larger, several pseudopods are applied to it, their axial fibres becoming absorbed, and their substance envelops it, enclosing it in a vacuole. The animal can fix itself by means of

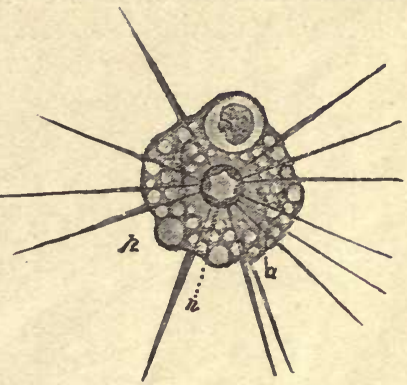

Fio. 32-Actinophrys sol. a. axial filaments of pseudopods ; $n$. nucleus; p. pseudopod. (From Lang's Comparativa Anatomy, after Grenacher.) its pseudopods, the ends of which become viscid, and it is able to crawl slowly by their means. Sometimes it floats freely in the

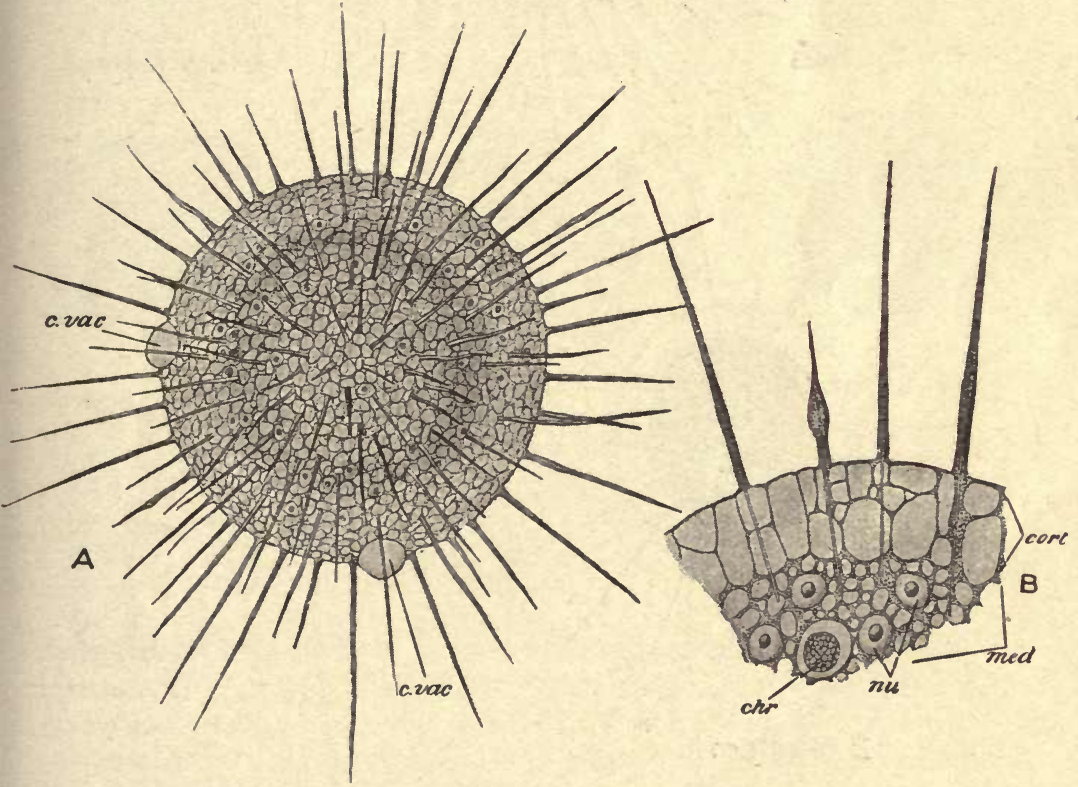

FIG. 40.-Actinosphærium eichhornii. A, the entire organism; B, a small portion highly magnified; chr. chromatophore; cort. cortex; $c$. vac. contractile vacuole; med. medulla ; nu. nuclei. (From Bütschli's Protozoa, after Hertwig and Lesser.)

water, and it possesses the power of rising or sinking by some unknown means.

Actinosphcerium (Fig. 40, A), another fresh-water form, is more 
complex. The protoplasm is distinctly divided into a central mass, the medulla or endosarc (B, med.), in which the vacuoles are small,

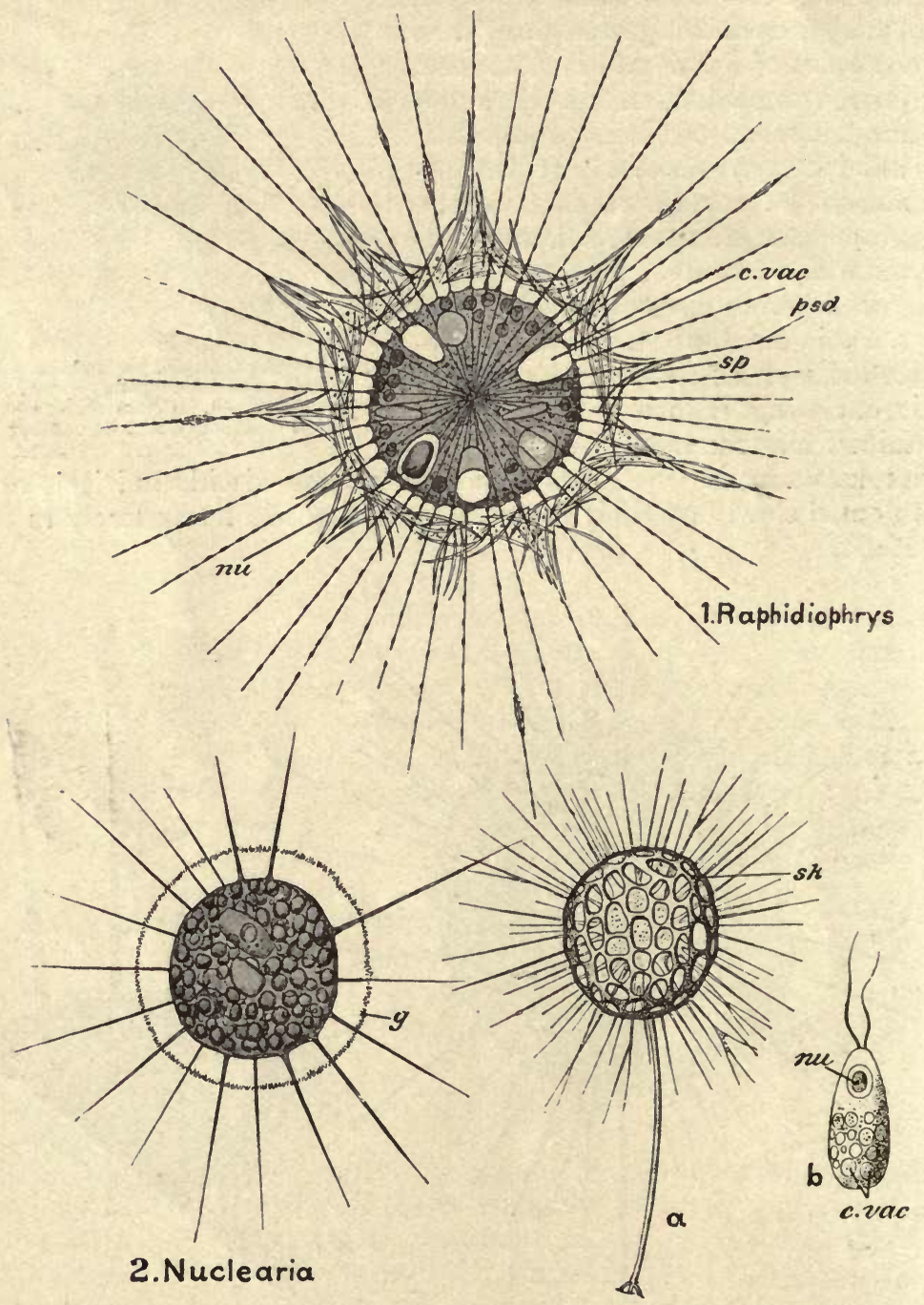

3. Clathrulina

Fig. 41.-Various forms of Feliozoa. $3 a$, the entire animal; $3 b$, the flagellula; $c$. vac. contractile vacuole; $g$. gelatinous investment; $n u$. nucleus psd. pseudopods; $s k$. siliceous skeleton; sp. spicules. (From Bütschli's Protozoa, after Schulze and Greeff.)

and an outer layer, the cortex or ectosarc (cort.), in which they are very large. There are numerous nuclei (nu.) and chromatophores $(c h r$.$) , the latter coloured green by chlorophyll, the characteristic$ pigment of green plants. 
Many genera form colonies. Numerous zooids may be united by bridges of protoplasm into an open network, or the connecting bridges may be shorter and the zooids more numerous, giving the colony a more compact appearance.

Transitional stages occur between the naked genera already referred to and forms with a distinct skeleton. Sometimes the body simply surrounds itself with a temporary gelatinous investment (Fig. 41, 2, g.), in other cases it is surrounded by a capsule of loosely woven fibres through which the pseudopods pass, thus reminding us of the state of things characteristic of perforate Foraminifera.

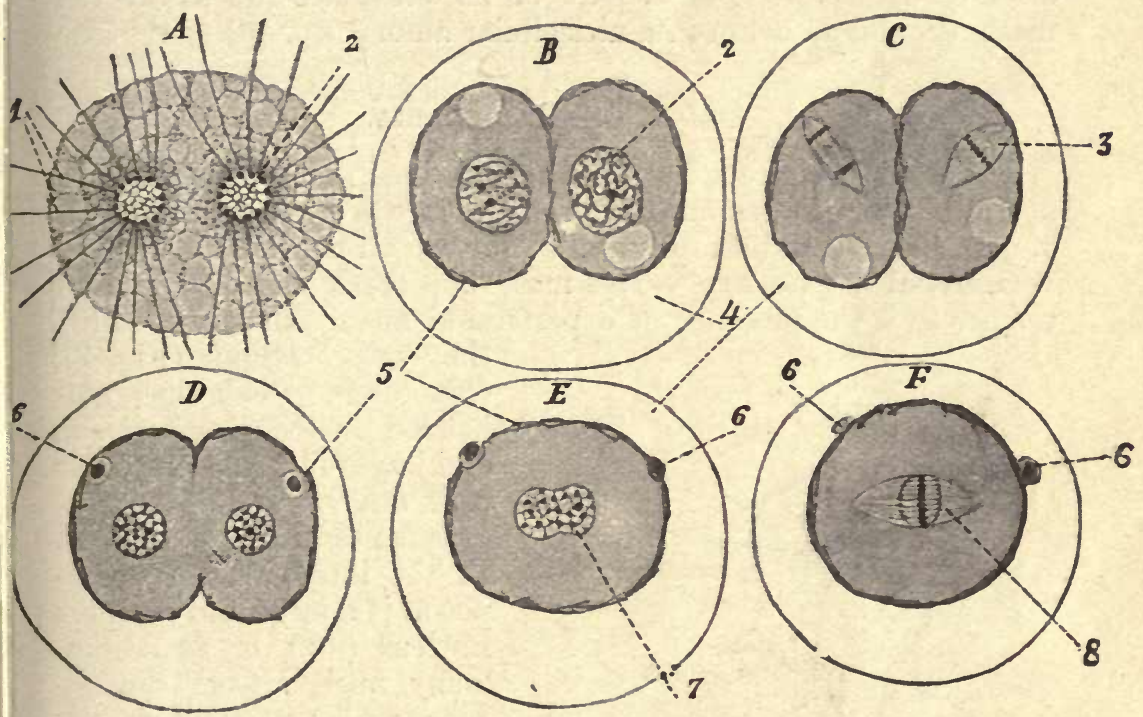

Fiu. 42.-Actinophrys sol. Conjugation with fusion of nuclei (karyogamy). A. two individuals in the first phase of conjugation; $B$, beginning of the encystation; $C$, maturation ; $D$, completion of maturation; $B$, coalescence of nuclei ; $F$, completion of the first spindle of the zygote resulting from the conjugation. 1, axial filaments of the pseudopods; 2 , nucleus ; 3 , spindles concerned in maturation; 4, 5, outer and inner layers of cyst; 6 , polar budies ; 7 , nucleus formed by the uuion of the two nuclei ; 8 , first (mitotic) division. (From Lang, after Schaudinn.)

One genus has a shell formed of agglutinated sand-grains; in another (Fig. 41,1) the skeleton consists of loosely matted needles of silica. Lastly, in the graceful Clathrulina (3) the body is enclosed in a perforated sphere of silica, quite like the skeleton of many of the Radiolaria (p. 61).

Reproduction ordinarily takes place by binary fission; a peculiar form of budding has been observed, and spore-formation also occurs, with or without encystation. Actinosphærium, for instance, encloses itself in a gelatinous cyst and undergoes multiple fission, forming numerous spores each enclosed in a siliceous cell-wall. These resting spores remain quiescent 
throughout the winter, and in spring the protoplasm emerges from each and assumes the form of the ordinary active Actinosphærium. In Clathrulina spore-formation takes place in the active condition, and the spores (Fig. $41,3 b$ ) are flagellulæ, each being an ovoid body provided with two flagella.

Conjugation has been observed in some instances between two or more individuals, which may separate again without any nuclear changes taking place; or the conjugation may be followed by a sexual process, comprising the coalescence of the protoplasm of the two individuals and the coalescence of the nuclei (Fig. 42) after each has given off a part of its substance (6), as in the maturation of an ovum in multicellular animals (p. 19).

\section{ORder 4.-RADIOLARIA.}

The Radiolaria are a large and well-defined group of Rhizopods, noticeable, in most instances, by the presence of a siliceous skeleton of great beauty and complexity. They are all marine.

General structure.-The most important characteristic of the group is the presence of a perforated membranous sac, called

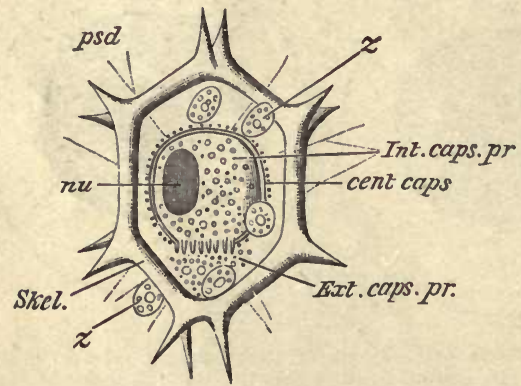

Fia. 43. - Lithocircus annularis. cent. caps. central capsule; ext. caps. $m$. extra-capsular protoplism; int. caps. pr. intra-capsular protoplasm ; $n u$. nucleus ; psel. pseudopods ; skel. skeleton; z. cells of Zoochlorella. (After Bitschli, from Parker's Biology.) the central capsule (Fig. 43, cent. caps.), which lies embedded in the protoplasm, dividing it into intra-capsular (int. caps. pr.) and extracapsular (ext.caps.pr.) regions. In the intra-capsular protoplasm is a large and complex nucleus (nu.), or sometimes many nuclei: from the extra-capsular protoplasm the pseudopods ( $p s d$.$) are given$ off in the form of delicate radiating threads, which in some cases remain free, in others, e.g. Lithociveus, anastomose freely, i.e. unite to form net-

works. In one large section-the Acantharia - the pseudopodia contain firm axial rods similar to those in the pseudopods of the Heliozoa. There is no contractile vacuole, but in many forms the extra-capsular protoplasm contains numerous large non-contractile vacuoles, which give it the frothy or bubbly appearance noticed previously in Hastigerina. The vacuolated portion of the protoplasm has a gelatinous consistency, and is distinguished as the calymma.

The central capsule may be looked upon as a chitinoid internal skeleton, reminding us of the shell of Gromia and of 
the perforated calcareous shell of Hastigerina with its investment of vacuolated protoplasm. It is found in its simplest form in Thalassoplancta (Fig. 44), in which it is spherical and uniformly perforated with minute holes. In other forms, such as Lithocircus (Fig. 43), it is more or less conical in form, and the apertures are restricted to the flat base of the cone. Lastly, in the most complex forms (Fig. 45), the membrane of the capsule is double, and there are three apertures-a principal one having a central position and provided vith a lid or operculum (op.), and two subsidiary ones on the opposite side. In relation with the principal or lidded aperture there is found in the extracapsular protoplasm a heap of pigmented matter called the phocodium (ph.), probably partly of the nature of excreta. The central capsule encloses, in addition to the nucleus or nuclei, oil-drops, vacuoles, proteid crystals, and pigment.

In some genera the central capsule is the only skeletal structure present, but in most cases there is in addition a skeleton - mainly external-formed, as a rule, of silica, but in one subdivision of the class of a substance called acanthin, composed of strontium sulphate, so transparent that it can only be distinguished from silica by chemical tests. The siliceous skeleton may consist of loosely woven spines (Fig. 44), but usually (and the acanthin skeleton always) has the form of a firm frame-work of globular, conical, stellate, or discoid shape, frequently produced into simple or branched spines. In the forms with an acanthin skeleton the spines frequently have inserted into them a number of contractile filaments arising from thè gelatinous extra-capsular layer. A very beautiful form of skeleton is exhibited by Actinomma (Fig. 46), in which there are three concentric perforated spheres (A, sk. 1, sk. 2, sk. 3) connected by radiating spicules. The outer of these spheres occurs in the extra-capsular protoplasm $(\mathrm{B}$, ex. caps. $p r$.$) , the middle one in the intra-capsular protoplasm,$ and the inner one in the nucleus ( $n u$.$) .$

Colonial forms are comparatively rare in this order, but occur in some genera by the central capsule undergoing repeated divisions while the extra-capsular mass remains undivided. In this way is produced-in Collozoum for instance (Fig. 47, A, B, C) 


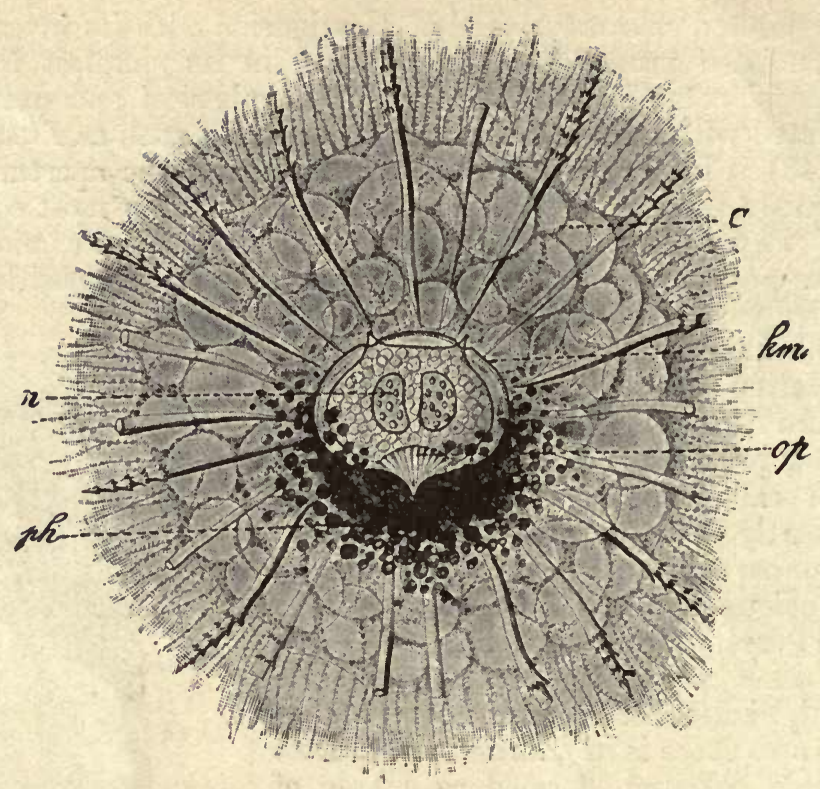

Fig. 45.-A ulactinium actinastrum. c. calymma; $k m$. central capsule; $n$. nucleus; op operculum ; $p h$. phæodium. (From Lang's Comparative Anatomy, after Hacckel.)

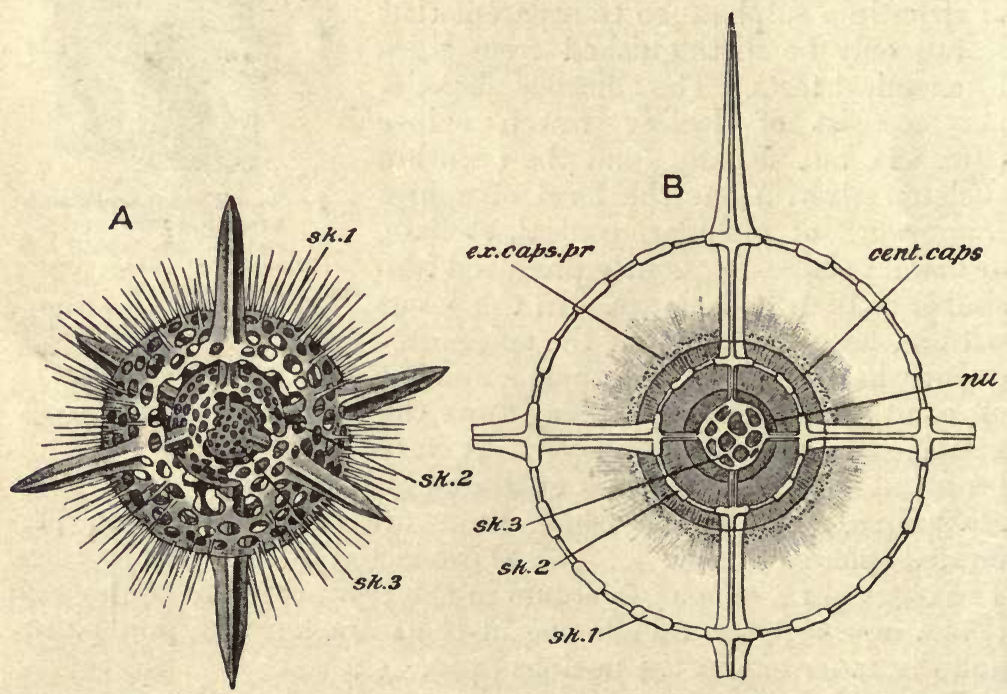

FIG. 46.-Actinomma asteracanthion. A, the shell with portions of the two outer spheres broken away; B, section showing the relations of the skeleton to the animal; cent. caps. central capsule ; ex. caps. pr. extra-capsular protoplasm; nu. nucleus ; sk. 1, outer.$s k$. 2, middle, sk. 3, inner sphere of skeleton. (From Butschli's Protozoa, after Haeckel and Hertwig.) 
-a firm gelatinous mass, the calymma or vacuolated extracapsular protoplasm $(\mathrm{D}, v a c$.$) conimon to the entire colony,$ having embedded in it numerous central capsules (c. caps.) each indicating a zooid of the colony. Collozoum may attain a length of 3 or $4 \mathrm{~cm}$.

Reproduction by binary fission has been observed in some cases, and is probably universal. The nucleus divides first, then the central capsule, and finally the extra-capsular protoplasm.

Spore-formation has been observed in Collozoum and some other genera: the intra-capsular protoplasm divides into small masses, each of which becomes a flagellula (Fig. 47, E, F) provided with a single flagellum. In some instances all the spores produced are

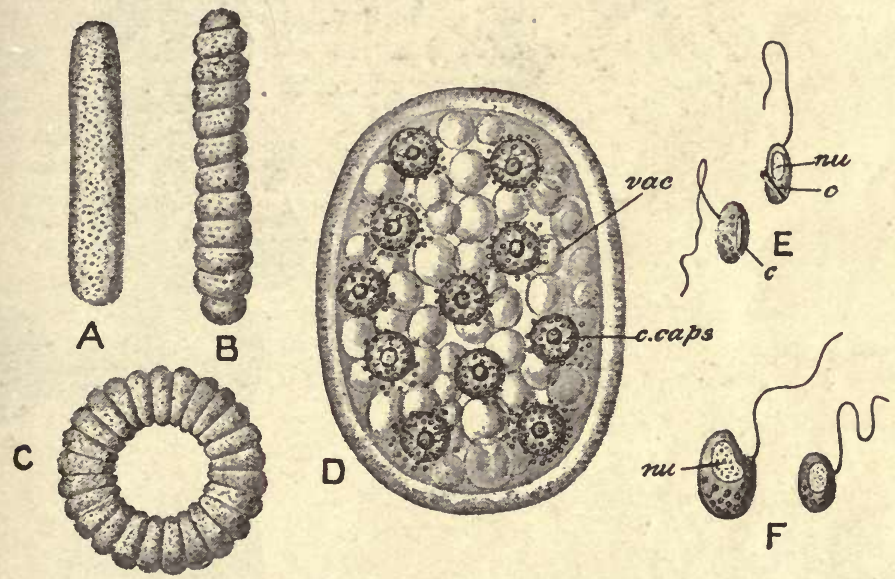

FIG. 47.-Collozoum inerme, A-C, three forms of the entire colony, nat. size; D, a small colony showing the numerous central capsules (c. caps.) and extra-capsular protoplasm with vacuoles $(v a c.) ; \mathrm{E}$, spores contrining crystals $(c.) ; \mathbf{F}$, mega- and microspore. (From Bütschli's Protozoa, after Hertwig and Brandt.)

alike (E), and each encloses a small crystal (c.): in other cases (F) in the same species-the spores are dimorphic, some being small (microspores), others large (megaspores). Their development has not been traced.

Symbiosis.-One most characteristic and remarkable feature of the group has yet to be mentioned. In most species there occur in the extra-capsular protoplasm (in the intra-capsular in some cases) minute yellow cells (Fig. $43, z$.) which multiply by fission independently of the Radiolarian. It has been proved that these are unicellular organisms, sometimes regarded as plants (Class Algæ), sometimes as animals (Class Mastigophora of the Protozoa), and named Zoochlorelloe. This intimate association of two organisms is called symbiosis : it is probably a mutually beneficial partnership, the Radiolarian supplying the Zoochlorellæ with carbon dioxide and nitrogenous waste matters, while the Zoochlorellæ 
give off oxygen and produce starch and other food-stuffs, some of which must make their way by diffusion into the protoplasm of the Radiolarian.

\section{APPENDIX TO THE RHIZOPODA.}

\section{Chlamydomyxa and Labyrinthula.}

Chlamydomyxa (Fig. 48), of which two species have been described, has been found living on Bog-mosses (Sphagnum) in Ireland and in Germany and
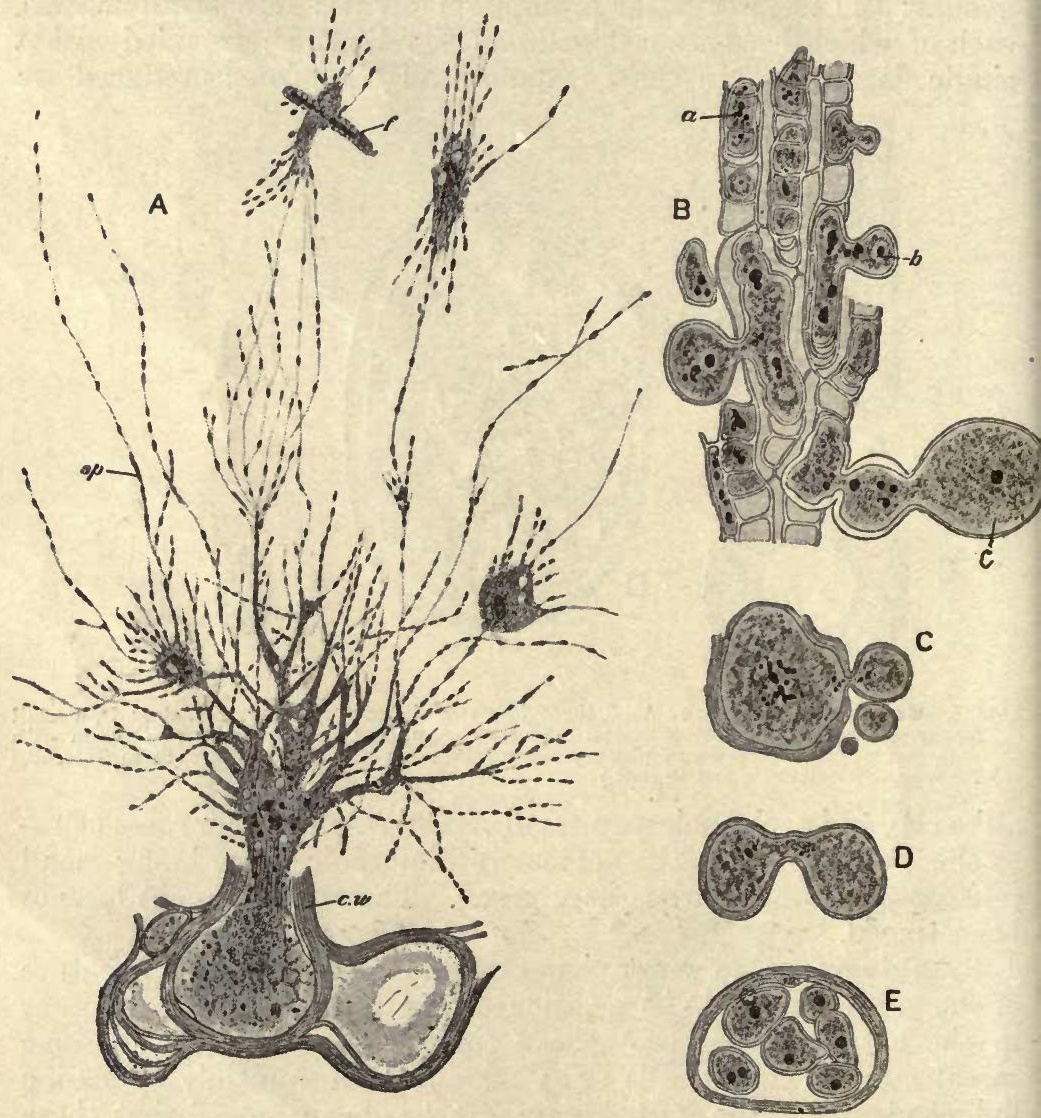

Fig. 48.-Chlamydomyxa labyrinthuloides. A, active phase; c.vo. cell-wall ; $f$. fragment of Alga ingested as food; $s p$. spindles in course of pseudopods; $\mathrm{B}$, resting-stageuumerous individuals in the cells of a fragment of Sphagnum; $a$, specimen enmpletely enclosed in cell ; $b$ and $c$, specimens which have emerged through the ruptured cell-wall ; C, specinen nultiplying by budding; $\mathrm{D}$, by binary fission; $\mathrm{E}$, by internal fission. E may represent a stage in spore-formation.' (A after Archer, B-E after Geddes.)

Switzerland. It may occur either in the active or in the resting condition. In the latter $(\mathrm{B}, a, b, c)$ it consists of a mass of protoplasm with a number of nuclei surrounded by a laminated wall of cellulose (p. 14). In the protoplasm are 
numerous non-nucleated protoplasmic bodies or chromatophores, containing chlorophyll and a green or brown colouring matter in varying proportions. There are also a number of minute rounded bodies of a bluish tint probably composed of reserve food-materials. In the young condition $(a)$ the resting cells are globular and microscopic, lying enclosed within the cells of the Sphagnnm, but as they grow in this confined space they become elongated and irregular, and finally burst through the wall of the moss-cell, forming masses $(b, c)$ quite visible to the naked eye. These may bud (C) or undergo binary fission (D); or the protoplasm, retreating from the cell-wall, may divide into nnmerous small uninucleated amceboid masses, each of which subsequently surrounds itself with a new cell-wall $(\mathbf{E})$.

During the whole of the resting stage there is nothing to distinguish Chlamydomyxa from a plant, and it would certainly be placed among the lower Algæ if the active phase of its existence were unknown.

In the active stage (A) the protoplasm protrudes from the ruptured cell-wall in the form of stiff pseudopods produced into a complex network of extremely delicate filaments, which are much branched and perhaps anastomose, and may unite to form larger masses of protoplasm at a considerable distance from the original cell. At the same time the bluish spheres (sp.) found in the resting stage take on a spindle shape and travel slowly along the filaments.

In one of the two known species the protoplasm entirely leaves the cyst wall and becomes free in the water.

The filaments are used to capture living organisms $(f$.$) which are digested by$ the protoplasm surrounding them, the products of nutrition being conveyed along the network to all parts of the organism. Thus in the active condition the nutrition of Chlamydomyxa is holozoic, i.e. strictly like that of an animal, the food consisting of living protoplasm. In the resting stage, on the other hand, uutrition is purely holophytic, i.e. like that of an ordinary green plant, the food

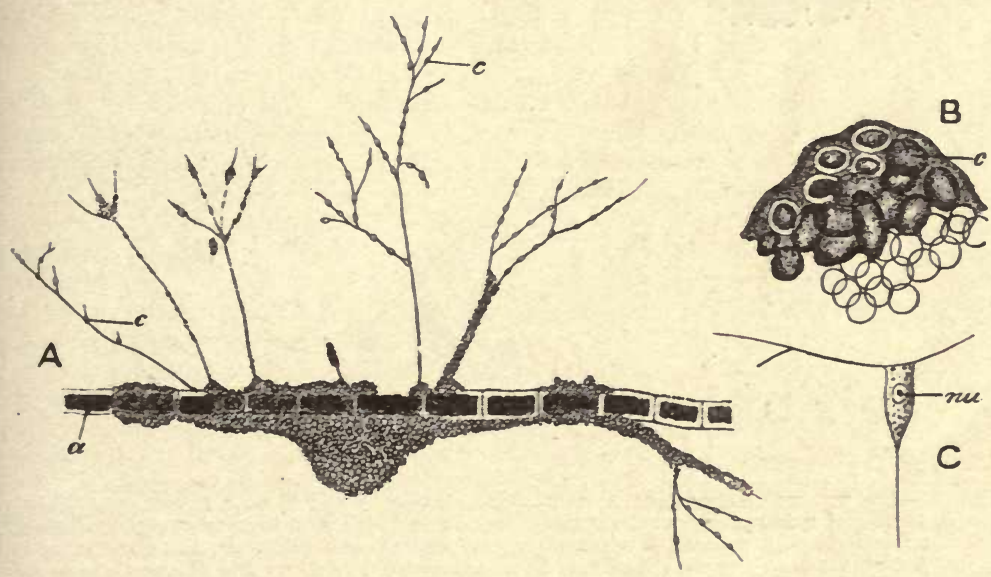

FIG. 49.-Zaby rinthula vitellina. A, specimen crawling on a fragment of Alga (a.); c. ceils travelling in the filaments. $B$, part of specimen in resting condition with heap of cells (c.); $\mathrm{C}$, a single cell from an actively moving specimen with connecting threads; $n u$. nucleus. (From Bütschli's Protozoa, after Cienkowsky.)

consisting of the carbon dioxide and various mineral salts dissolved in the water. Chlamydomyxa multiplies in the resting condition by the formation of spores each containing two nuclei. These give rise to flagellulæ, the further history of which has not been traced.

Labyrinthula (Fig. 49 ) in the resting stage $(\dot{B})$ consists of a heap of small 
nucleated cells $(c$.$) connected by a homogeneous substance. In the active condi-$ tion (A) it is produced into long delicate stiff filaments of pseudopodial character, along which the cells $(c$.$) travel, in the same manner as the spindles of Chlamy-$ domyxa. Labyrinthula has, therefore, the character not of a single cell, but of a cell-colony, formed of numerous cells connected together. Chlamydomyxa, on the other hand, has the character of a single multinucleate cell. There is thus no close connection between these two aberrant forms: but both may, perhaps, best be regarded as Rhizopoda with nearer relationships to the Foraminifera (Gromia in particular) than to any of the other orders.

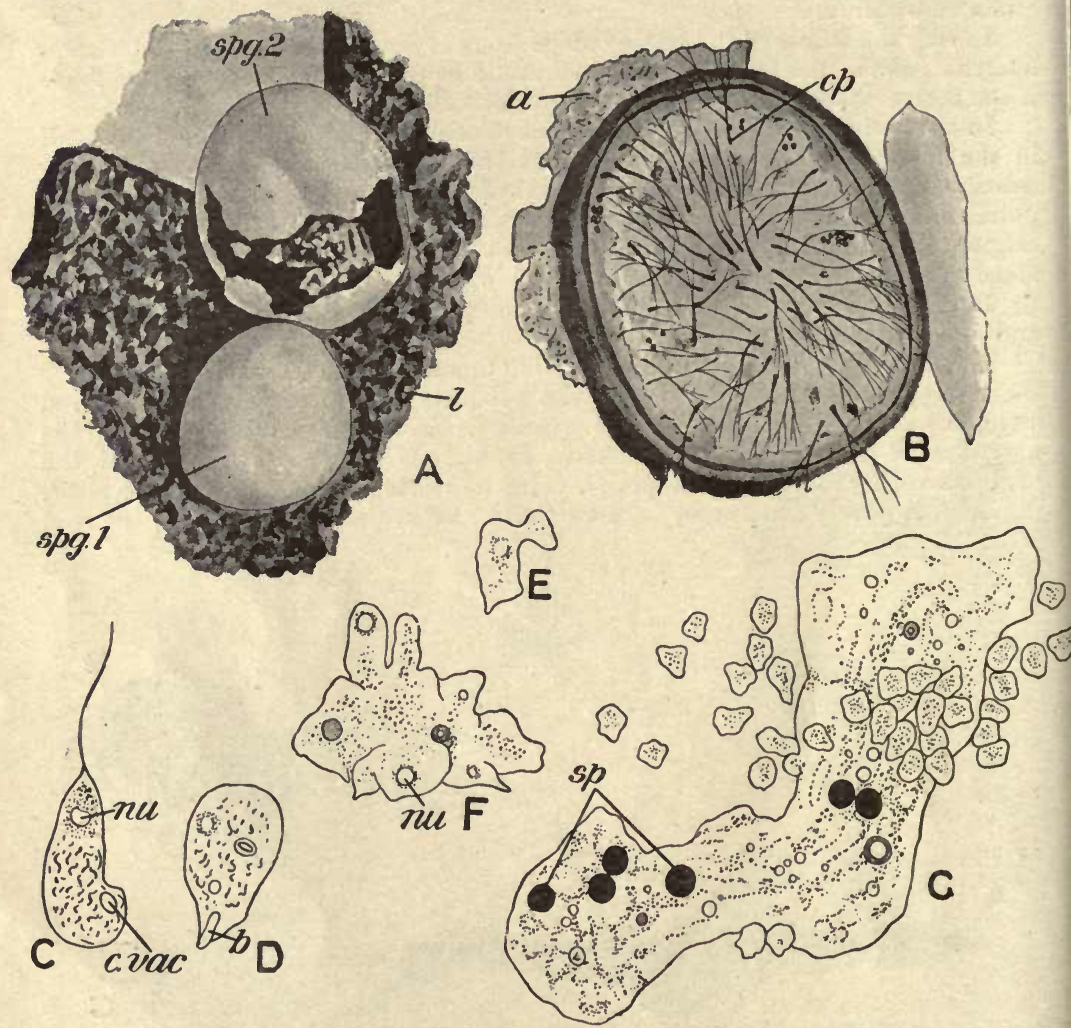

Fig. 50 -Didymium difforme. A, two sporangia (spg. 1 and 2) on a fragment of lcaf (l.). $\mathrm{B}$, section of sporangium, with ruptured outer layer (a.); and threads of capillitium (cp.). C, a flagellula with contractile vacuole (c. vac.) and nucleus (nu.). D, the same after loss of flagellum; $b$, an ingested Bacillus. E, an amobula. F, conjugation of amobulie to form a small plasmodium. G, a larger plasmodium accompanied by numerous amœbulæ; $8 p$. ingested spores, (3tfter Lister.)

\section{CLASS II.-MYCETOZOA.}

\section{Example of tae Class-Didymium diffurme.}

Didymium occurs as a whitish or yellow sheet of protoplasm (Fig. 50, G), often several centimetres across, which crawls, like a gigantic Amoba, over the surface of decaying leaves. It shows the characteristic streaming move- 
ments of protoplasm, and feeds by ingesting various organic bodies, notably the Bacilli which always occur in great numbers in decaying substances. Numerous nuclei are present.

After leading an active existence for a longer or shorter time, the protoplasm aggregates into a solid lump, surrounds itself with a cyst, and undergoes multiple fission, dividing into an immense number of minute spores. The cyst (Fig. 50, A, spg. 1, spg. 2) is therefore not a mere resting capsule, like that of Amœba, but a sporangium or spore-case. Its wail consists of two layers, an inner of a dark purple colour and membranous texture, formed of cellulose, and an outer of a pure white hue, formed of calcium carbonate. Thus the whole sporangium, which may attain a diameter of 3 or $4 \mathrm{~mm}$., resembles a minute egg. From the inner surface of the wall of the sporangium spring a number of branched filaments of cellulose, which extend into the cavity among the spores and together constitute the capillitium (B, $c p$.).

The spores consist of nucleated masses of protoplasm surrounded by a thick cellulose wall of a dark reddish-brown colour. After a period of rest the protoplasm emerges in the form of an amoboid mass which soon becomes a flagellula (C), provided with a single flagellum, a nucleus $\left(n u_{0}\right)$, and a contractile vacuole (c. vac.). The flagellulæ move freely and ingest Bacilli $(\mathrm{D}, b$.$) , and multiply by$ fission: then, after a time, they become irregular in outline, draw in the flagellum, and become amœboid (E). The amœbulæ thus formed congregate in considerable numbers and fuse with one another (F), the final result being the production of the great amœboid mass $(G)$ with which we started. There is no fusion of the nuclei of the amœbulæ. Thus Didymium in its active condition is a plasmodium, i.e. a body formed by the concresence of amcbulæ.

\section{General Remarks on the Mycetozoa.}

Speaking generally, the Mycetozoa differ from all other Protozoa in their terrestrial habit. They are neither aquatic, like most members of the phylum, nor parasitic, like many other forms, but live habitually a sub-aërial life on decaying organic matter. They are also remarkable for their close resemblance in the structure of the sporangia and spores to certain Fungi, a group of parasitic or saprophytic plants in which they are often included, most works on Botany having a section on the Myxomycetes or "Slime-fungi," as these organisms are then called. They are placed among animals on account of the structure and physiology of the flagellate, amœboid, and plasmodial phases, which exhibit automatic movements and ingest solid food. The Mycetozoa are sometimes included among the Rhizopoda, a course which their very peculiar reproductive processes appears to render inadvisable.

An interesting organism, called Protomyxa, probably belongs to this group. In its plasmodial phase it consists of orange-coloured masses of protoplasm, about $1 \mathrm{~mm}$. in diameter, which crawl over sea-shells by means of their long, branched pseudopods, and ingest living prey. No nuclei are known. The protoplasm becomes encysted and breaks up into naked spores, which escape from the cyst as flagellulæ, but soon become amœboid and fuse to form the plasmodium.

\section{CLASS III.-MASTIGOPHORA.}

\section{Example of the Class-Euglena viridis.}

Euglena (Fig. 51) is a flagellate organism commonly found in the water of ponds and puddles, to which it imparts a green colour. The body $(\mathrm{E}, \mathrm{H})$ is spindle-shaped, and has at the blunt anterior end a depression, the gullet $(\mathrm{F} ;$ as. $)$, from the inner surface of which 
springs a single long flagellum $(f$. $)$. According to recent observations the flagellum is not a simple thread, but is beset with delicate cilium-like processes. The organism is propelled through the water by the lashing movements of the flagellum, which is always directed forwards ; it can also perform slow worm-like movements of contraction and expansion (A-D), but anything like the free pseudopodial movements which charactcrise the Rhizopoda is precluded by the presence of a very thin membrane or cuticle which invests the body. Oblique and longitudinal lines in the outer layer of the protoplasm may be due to the presence of contractile fibrils. There is a nucleus (nu.) near the centre of the body, and at the anterior end a contractile vacuole $(\mathrm{H}, c . v a c$.$) , leading into$

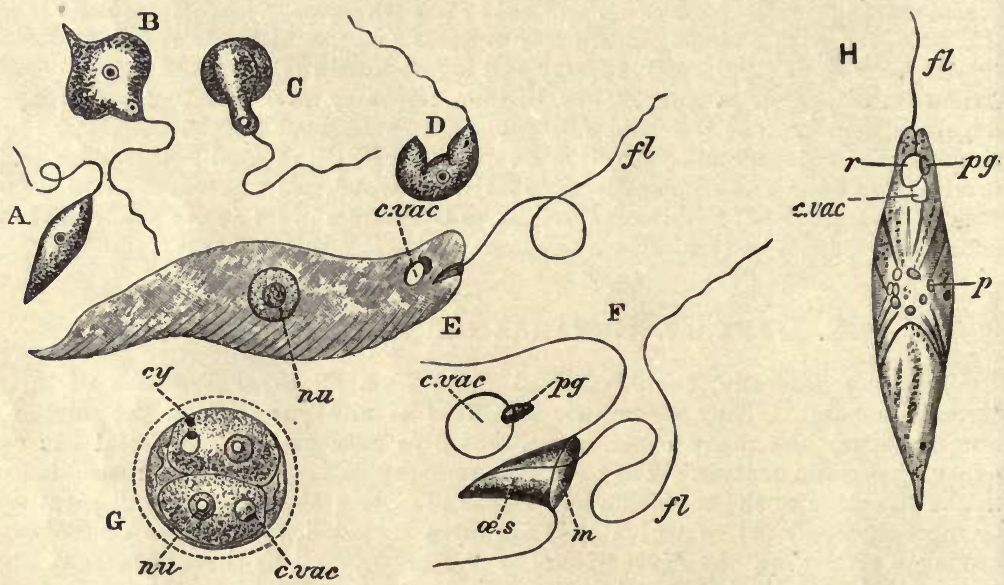

FIG. 51,-Euglena viridis. A-D, four views illustrating euglenoid movements; $\mathbf{E}$ and $\mathbf{H}$, enlarged views; F, anterior end further enlarged; G, resting form after binary fission ; $c$. vac. contractile vacuole in $\mathbf{H}$, reservoir in $\mathbf{E}$ and $\mathbf{F} ; c y$. cyst; $t$. flagellum ; $m$. mouth; nu. nucleus ; os, gullet; $p$. paramylum bodies; $p g$. pigment spot; $r$. (in $\mathrm{H}$ ), reservoir. (From Parker's Biology, after Kent and Klebs.)

a large non-contractile space or reservoir $(r$.$) which discharges into$ the gullet.

The greater part of the body is coloured green by the characteristic vegetable pigment, chlorophyll, and contains rod-shaped grains of paramylum $(H, p$.$) , a carbohydrate allied to starch. In$ contact with the reservoir is a bright red speck, the stigma $(p g$.$) ,$ formed of a pigment allied to chlorophyll and called 7ccrnatochrome. It seems probable that the stigma is a light-perceiving organ or rudimentary eye.

Euglena is nourished like a typical green plant: it decomposes the carbon dioxide dissolved in the water, assimilating the carbon and evolving the oxygen. Nitrogen and other elements it absorbs in the form of mineral salts in solution in the water. But it has 
also been shown that the movements of the flagellum create a whirlpool by which minute fragments are propelled down the gullet and into the soft internal protoplasm. There seems to be no doubt that in this way minute organisms are taken in as food. Euglena thus combines the characteristically animal (holozoic) with the characteristically vegetable (holophytic) mode of nutrition. But, in all probability, the Euglena is in large measure saprophytic, the products of the decay of organic matter dissolved in the water being absorbed through the general surface.

Sometimes the active movements cease, the animal comes to rest and surrounds itself with a cyst or cell-wall of cellulose $(G)$, from which, after a quiescent period, it emerges to resume active life. It is during the resting condition that reproduction takes place by the division of the body in a median plane parallel to the long axis $(G)$. Under certain circumstances multiple fission takes place, and flagellulæ are produced, which, sometimes, after passing through an amœboid stage, develop into the adult form.

\section{Classification and General Organisation.}

The Mastigophora form a very extensive group, the genera and species of which show a wonderful diversity in structure and habit. The only character common to them all is the presence of one or more flagella. Some approach plants so closely as to be claimed by many botanists; others are hardly to be distinguished from Rhizopods; while the members of one order present an interesting likeness to certain peculiar cells found in Sponges.

The class is divisible into four orders as follows :-

\section{Order 1.-Flagellata.}

Mastigophora having one or more flagella at the anterior end of the borly.

\section{ORDER 2.-ChOANOFLAGELLATA.}

Mastigophora having a single flagellum surrounded at its base by a contractile protoplasmic collar.

\section{Order 3.-Dinoflagellata.}

Mastigophora having two flagella, one anterior, the other encircling the body like a girdle.

\section{Order 4.-Cystoflagellata.}

Mastigophora having two flagella, one of which is modified into a long tentacle, while the other is small and contained within the gullet. 


\section{Systematic Fosition of the Example.}

Euglena viridis is one of several species of the genus Englena, and belongs to the family Euglenidce, sub-order Euglenoidea, and order Flagellata.

The presence of an anterior flagellum and the absence of a collar, transverse flagellum, or tentacle, indicate its position among the Flagellata. It is placed among the Euglenoidea in virtue of possessing a single flagellum and a small gullet into which the reservoir opens. The genus Euglena is distinguished by its centrally placed nucleus, green chromatophore, red stigma, and euglenoid movements. E. viridis is separated from other species of the genus by its spindle-shaped body with blunt anterior and pointed posterior end, and by the flagellum being somewhat longer than the body.

\section{Order 1.-Flagellata.}

The cell-body is usually ovoid or flask-shaped (Fig. 52, 6, 7, 9, \&c.), but may be almost globular (1), or greatly elongated (3). Anterior and posterior ends are always distinguishable, the flagella being directed forwards in swimming, and, as a rule, dorsal and ventral surfaces can be distinguished by the presence of a mouth or by an additional flagellum on the ventral side. They are, therefore, usually bilaterally symmetrical, or divisible into equal and similar right and left halves by a vertical antero-posterior plane.

Some of the lower forms have no distinct cuticle, and are able, under certain circumstances, to assume an amœboid form (2). The curious genus Mastigamoba (4) has a permanently amœboid form, but possesses, in addition to pseudopods, a single long flagellum. It obviously connects the Mastigophora with the Rhizopoda, and indeed there seems no reason why it should be placed in the present group rather than with the Lobosa. Similarly, Dimorpha (5) connects the Flagellata with the Heliozoa: in its flagellate phase $(\alpha)$ it is ovoid and provided with two flagella, but it may send out long stiff radiating pseudopods, while retaining the flagella, or may draw in the latter and assume a purely helizoan phase of existence provided with pseudopods only $(b)$.

Nuclei of the ordinary character are universally present. In addition there is present in the cytoplasm near the base of the flagellum a much more minute, deeply-staining body, which is termed the blepharoblast (Fig. 53). This has sometimes been taken for a micronucleus such as is general in the Infusoria, but it is not of nuclear origin, and does not take an active part in any reproductive processes.

The number of flagella is subject to great variation. There mav be one (Fig. 52, 1-3), two $(9,10)$, three $(6)$, or four (7). Sometimes the flagella show a differentiation in function; in 
Heteromita, e.g. (Fig. 57) the anterior flagellum ( $f$. 1) only is used in progression, the second or ventral flagellum $(f .2)$ is trailed

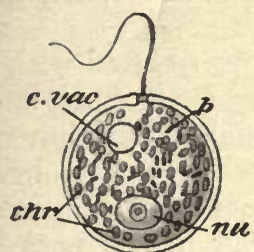

1.Trachelomonas

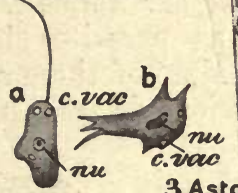

2. Oikomonas (?)

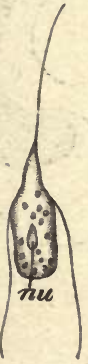

6.Dallingeria

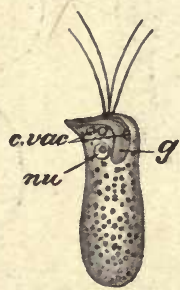

7. Tetramita

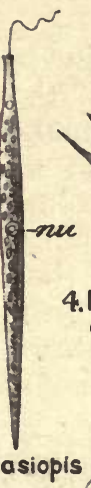

siopis

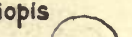

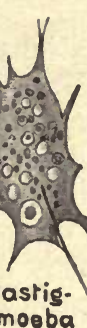
amoeba
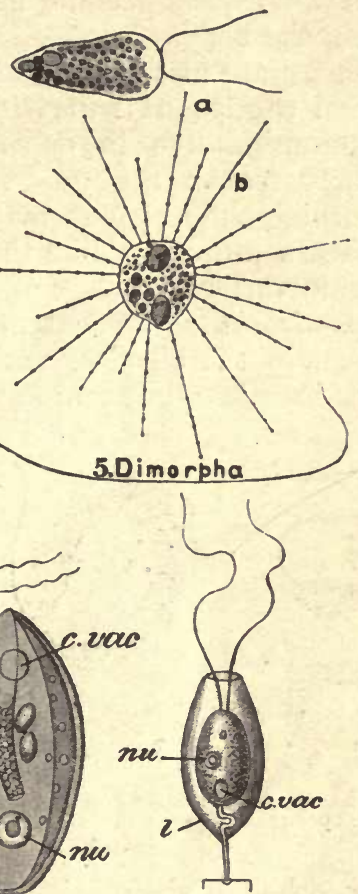

8.Oikomonas

9.Cryptomonas

10. Diplomita

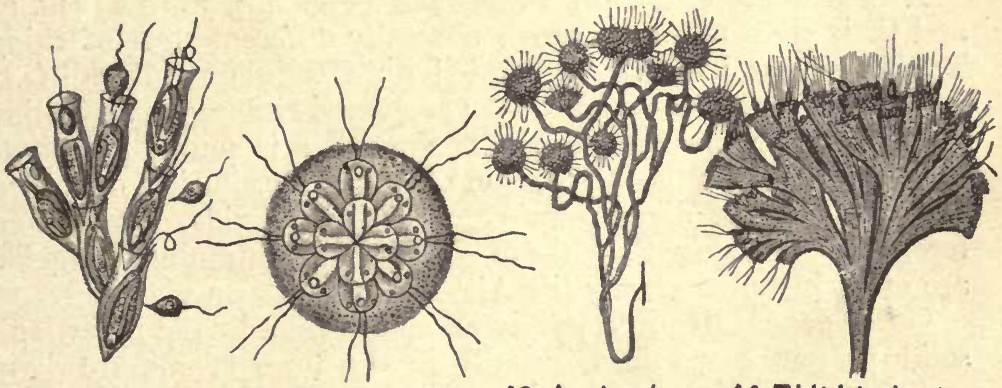

11. Dinobryon 12.Syncrypla 13. Anthophysa 14.Rhipidodendron

Fig. 52.-Various forms of Flagellata.-In 2, flagellate $(a)$ and amoeboid $(b)$ phases are shown; in 5 , flagellate (a) and heliozoan (b) phases; in 8 are shown two stages in the ingestion of a food-particle $(f$.$) ; chr. chromatophores; c$. vac. contractile vacuole; $f$. food particle $g$. gullet; $n u$. nucleus; $l$. lorica ; $p$. protoplasm; $p e r$. peristome ; $v . i$. vacuole of ingestion. (Mostly from Biitschli's Protozoa, after various authors:)

behind when the animal is swimming freely, or is used to anchor it to various solid bodies. In some (Trypanosomes, Fig. 53) the 
flagellum (or one of them, if two are present) is attached throughout its length, or in the greater part of its length, to the edge of a wavy protoplasmic flange, or undulating membrane, running along the body.

There are also important variations in structure correlated with varied modes of nutrition. Many of the lower forms, such as Heteromita, live in decomposing animal infusions: they have neither mouth nor gullet and take no solid food, but live by absorbing the nutrient matters in the solution; their nutrition is, in fact, saprophytic, like that of many fungi. A few live as parasites in various cavities of the body of the higher animals. The Homoflagcllata, an extensive group, live as parasites in the plasma of the blood of various vertebrates. Most of these appear to be harmless, but some are the causes of serious diseases in Man
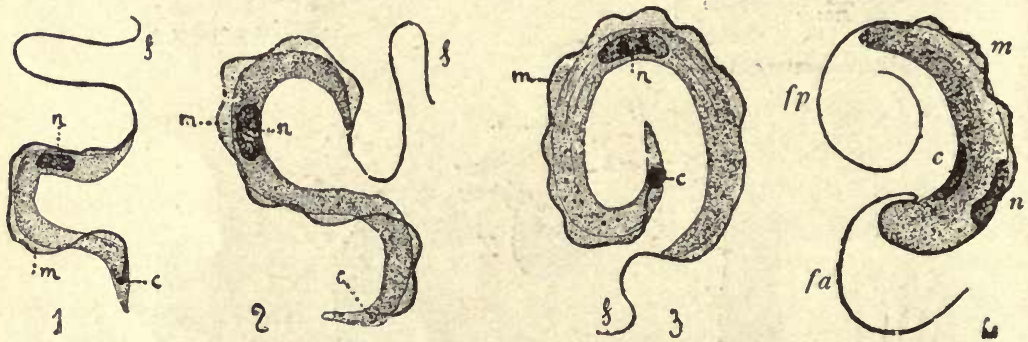

Fic. 53.-Trypanosomes of Fishes. c. blepharoblast; $f$. flagellum; fu, and $f p$. (in 4 ) anterior and posterior flagella; $m$. undulating membrane; $n$. nueleus. (After Iaveran and Mesnil.)

and other higher animals. One Euglena-like form lives as an intra-cellular parasite within the cells of one of the lower worms.

Howatococcus (Fig. 54), Pandorina (Fig. 55), Volvos (Fig. 56), and their allies present us with a totally different state of things. The mouthless body is surrounded by a cellulose cell-wall (c.v:), and contains chromatophores (chr.) coloured either green by chlorophyll or red by hæmatochrome. Nutrition is purely holophytic, i.e. takes place by the absorption of a watery solution of mineral salts and by the decomposition of carbon dioxide. It is, therefore, not surprising that these chlorophyll-containing Flagellata are often included among the Algæ or lower green plants.

Other genera live in a purely animal fashion by the ingestion of solid proteinaceous food, usually in the form of minute living organisms: in these cases there is always some contrivance for capturing and swallowing the prey. In Oikomonas (Fig. 52, 8), we have one of the simplest arrangements: near the base of the flagellum is a slight projection containing a vacuole $(v . i$.$) ; the$ movements of the flagellum drive small particles (f.) against this region, where the protoplasm is very thin and readily allows the particles to penetrate into the vacuole, where they are digested. 
In Euglena, as we have seen, there is a short, narrow gullet, and in some genera $(9, g)$ this tube becomes a large and well-marked structure.

Skeleton.-While a large proportion of genera are naked or covered only by a thin cuticle, a few fabricate for themselves a delicate chitinoid shell or lorica $(10, l$.$) , usually vase-shaped and$ widely-open at one end so as to allow of the protrusion of the contained animalcule. In the chlorophyll-containing forms there is a closed cell-wall of cellulose (Fig. 54, c.2v.). One group of

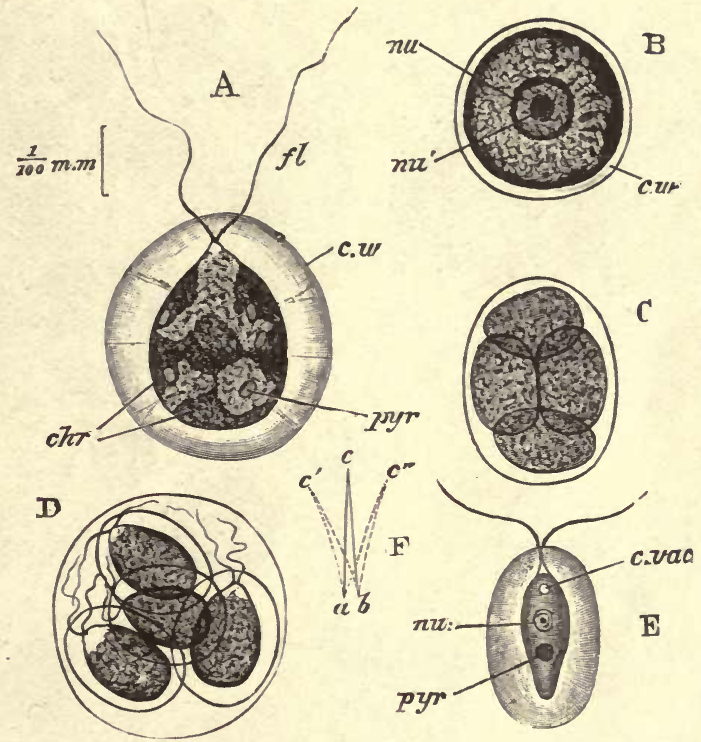

Fia. 54.-Hæmatococcus pluvialis. A, motile stage; B, resting stage; C, D, two modes of fission; E, Hcematococcus lacustris, motile stage; $\mathbf{F}$, diagram of movements of flagellum ; $c h r$. chromatophores; $c$. rac. contractile vacuole; c.e. cell-wall ; $n u$. nucleus; $n u$ '. nucleolus; pyr. pyrenoids. (From Parker's Biology.)

marine Flagellates have siliceous skeletons similar to those of the Radiolaria, with which they were originally classed.

In many genera colonies of various forms are produced by repeated budding. Some of these are singularly like a zoophyte (see Sect. IV.) in general form (Fig. 52,11), being branched colonies composed of a number of connected monads, each enclosed in a little glassy lorica; or green (chlorophyll-containing) zooids are enclosed in a common gelatinous sphere, through which their flagella protrude (12); or tufts of zooids, reminding us of the flower-heads of Acacia, are borne on a branched stem (13). In Volvox (Fig. 56) the zooids of the colony are arranged in the form of a hollow sphere, and in Pandorina (Fig. 55) in that of a solid sphere enclosed in a delicate shell of cellulose. Lastly, in Rhipido- 
dendron (Fig. 52, 14) a beautiful branched fan-shaped colony is produced, the branches consisting of closely adpressed gelatinous tubes each the dwelling of a single zooid.

Binary fission is the ordinary mode of asexual multiplication, and may take place either in the active or in the resting condition. Hæmatococcus (Fig. 54) and Euglena (Fig. 51), for instance, divide while in the encysted condition; Heteromita (Fig. 57)

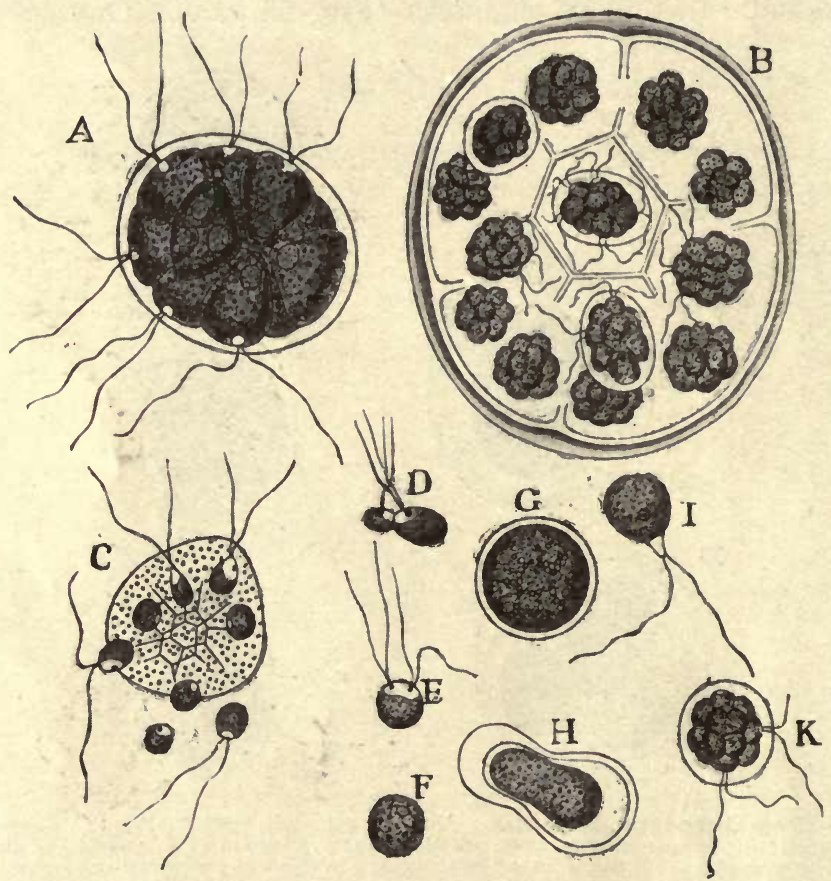

Fia. 55.-Pandorina morum. A, entire colony; B, asexual reproduction, each zooid dividing into a daughter-colony ; $\mathrm{C}$, liberation of gametes; D-F, three stages in conjugation of gametes; G, zygote; H--K, development of zJgote into a ncw colony. (From Parker's Biology, after Goebel.)

and other saprophytic forms while actively swimming: in the latter case the divison includes the almost infinitely fine flagellum.

In correspondence with their compound nature, the colonial genera exhibit certain peculiarities in asexual multiplication. In Dinobryon (Fig. 52, 11) a zooid divides within its cup, in which one of the two products of division remains; the other crawls out of the lorica, fixes itself upon its edge, and then secretes a new lorica for itself. In Pandorina (Fig. 55) each of the sixteen zooids of the colony divides into sixteen (B), thus forming that number of daughter-colonies within the original cell-wall, by the rupture of 
which they are finally liberated. In Volvox (Fig. 56), certain zooids, called parthenogonidia $(\mathrm{A}, \boldsymbol{a})$, have specially assigned to them the function of asexual reproduction: they divide by a process resembling the segmentation of the egg in the higher animals $\left(D^{1}-D^{5}\right)$, and form daughter-colonies which become detached and swim freely in the interior of the mother-colony.

A very interesting series of stages in sexual reproduction is found in this group. In Heteromita two individuals come together
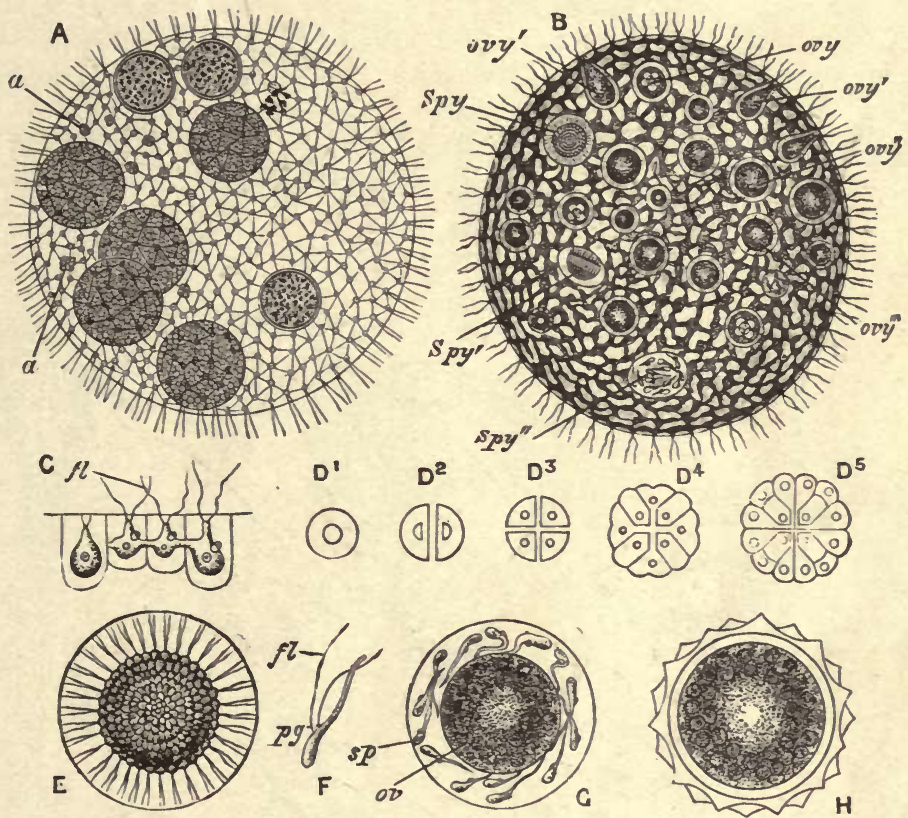

Fra. 56.-Volvox globator. A, entire colony, enclosing several daughter-colomies; $\mathrm{B}$, the swme during sexual maturity; $\mathbf{C}$, four zooids in optical section; $\mathrm{D}^{1}-\mathrm{D}^{5}$, derelopment of parthenogonidium; E, ripe spermary; F, sperm; G, ovary containing ovum and sperms; H, osperm; $a$, parthenogonidia; $t$. flagellum; ov. ovum; ory. ovaries; $p g$. pigment spot; spy. spermaries. (From Parker's Biology, after Cohn and Kirchner.)

(Fig. 57, $\mathrm{E}^{1}$ ) and undergơ complete fusion $\left(\mathrm{E}^{2}-\mathrm{E}^{4}\right)$ : the result of this conjugation of the two gametes or conjugating cells is a thinwalled sac, the zygote $\left(\mathrm{E}^{5}\right)$, the protoplasm of which divides by multiple fission into very minute spores. These, when first liberated by the rupture of the zygote $\left(\mathbf{E}^{6}\right)$, are mere granules, but soon the ventral or trailing flagellum is developed, and afterwards the anterior flagellum $\left(\mathrm{F}^{1}-\mathrm{F}^{4}\right)$. In Pandorina (Fig. 55) the cells of the colony escape from the common gelatinous envelope (C) and conjugate in pairs (D, E), forming a zygote $(F, G)$, which, after a period of rest $(\mathrm{H})$, divides and forms a new colony $(\mathrm{K})$. 
In some cases the conjugating cells are of two sizes, union always taking place between a large cell or megagamete and a small cell

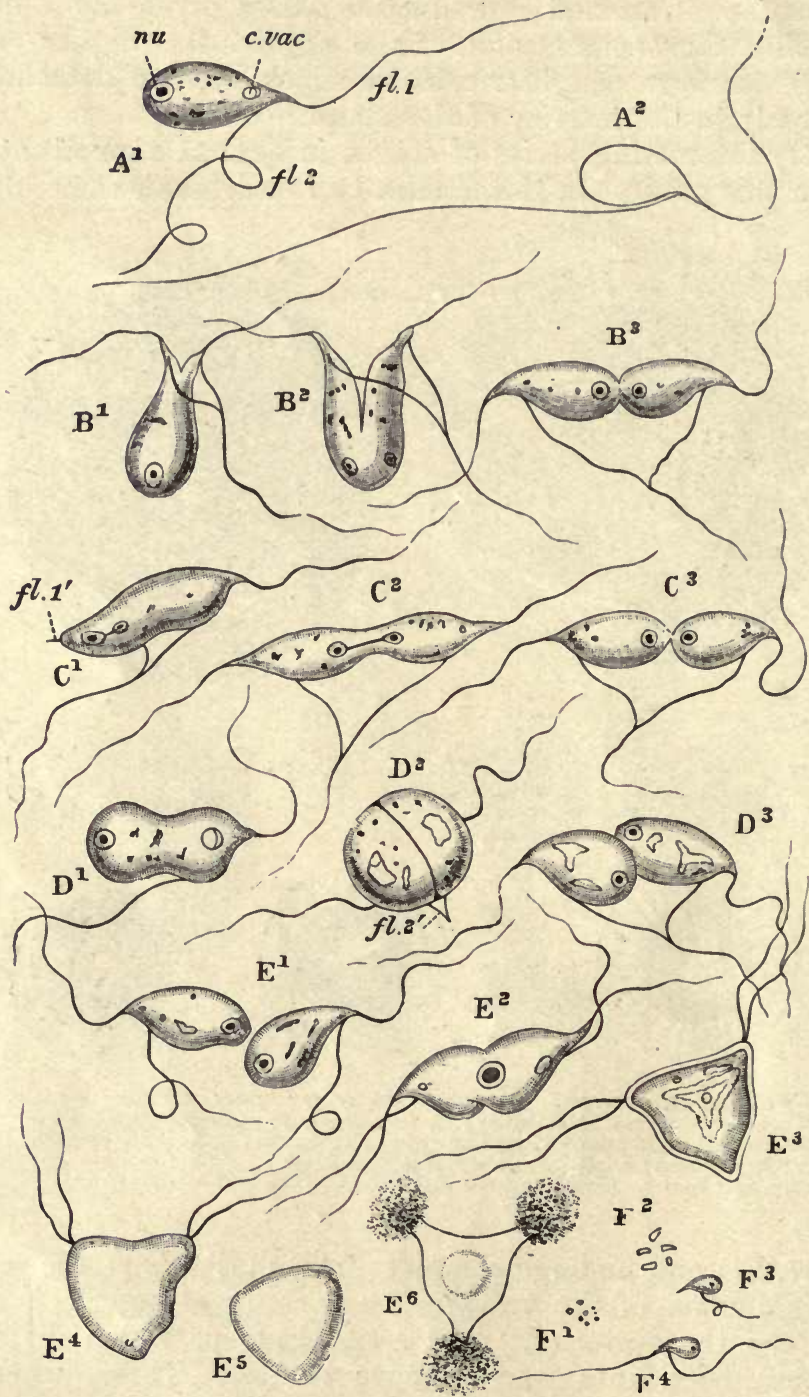

FIG. 57 . - Eeteromita rostrata. A, the positions assumed in the springing movements of the anchored form; B, longitudinal fission of anchored form; C, transverse fission of the same; D, fission of free-swimming form ; $\mathbf{E}$, conjugation of free-swimming with anchored form ; $\mathbf{E}^{5}$, zygote ; $\mathbf{E} 6$, emission of spores from zygote : F, development of spores; fl.1, ante. rior; fl. , ventral flagellum. (From Parker's Biology, after Dallinger.)

or microgamete. In Volvox (Fig. 56) this dimorphism reaches its extreme, producing a condition of things closely resembling what 
we find in the higher animals. Certain of the zooids enlarge and form megagametes $(B, v v y$.$) , others divide repeatedly and give rise$ to groups of microgametes $(\mathrm{B}$, spy. E, F), each in the form of an elongated yellow body with a red pigment-spot and two flagella. These are liberated, swim freely, and conjugate with the stationary megagamete $(G)$, producing a zygote $(H)$, which, after a period of rest, divides and reproduces the colony. It is obvious that the megagamete corresponds with the ovum of the higher animals, the microgamete with the sperm, and the zygote with the oosperm or impregnated egg.

It should be noticed that in the more complex cases of reproduction just described we meet with a phenomenon not seen in cases of binary fission, viz., development, the young organism being far simpler in structure than the adult, and reaching its final form by a gradual increase in complexity.
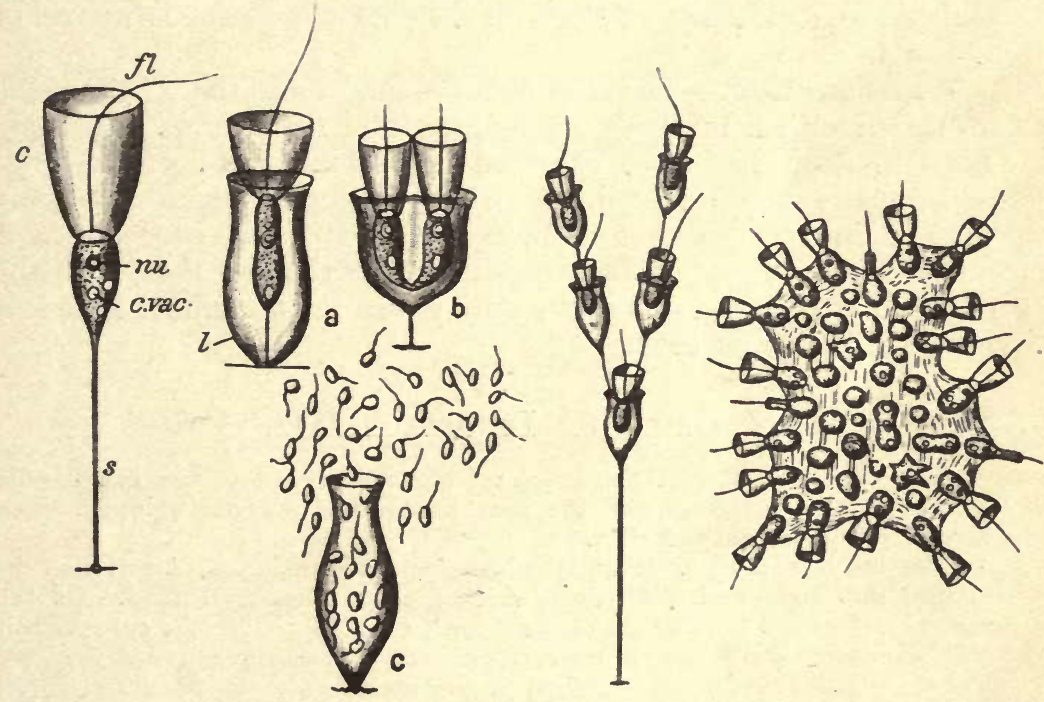

1.Monosiga.

2.Salpingoeca.

3.Polyoeca.

4. Proterospongis.

Fig. 5S.-Various furms of Choanofiagellata. 26 illustrates longitudinal fission ; $2 c$, the production of flagellula; $c$. collar; $c$. vac; contractile vacuole; $l_{\text {. }}$ flagclium; $l$. lorica; $n u$. wucleus ; s. stalk. (After Saville Kent.)

\section{ORDER 2.-ChOANOFLAGELLATA.}

General Structure.-The members of this group are distinguished by the presence of a vase-like prolongation of the protoplasm, sometimes double, called the collar (Fig. 58, 1,c.), surrounding the base of the single flagellum $(f l$.). The collar is contractile, and, although its precise functions are not yet certainly known, there is 
evidence to show that its movements cause vorticesin the water which draw in small bodies towards the outside of the collar to which they adhere. By degrees such bodies are drawn towards the base, and each is received into a vacuole which moves back into the interior of the protoplusm, another vacuole taking its place. The animalcule may draw in both collar and flagellum and assume an amœboid form.

The nucleus (nu.) is spherical, and there are one or two contractile vacuoles (c. vac.), but no trace of mouth or gullet. Some forms are naked (1), others (2) enclosed in a chitinoid shell or lorica of cup-like form. A stalk (s.) is usually present in the loricate and sometimes also in the naked forms.

The genera mentioned in the preceding paragraph are all simple, but in other cases colonies are produced by repeated fission. In Polyoeca (3) the colony has a tree-like form, which may reach a high degree of complexity by repeated branching. A totally different mode of aggregation is found in Proterospongia (4), in which the zooids are enclosed in a common gelatinous matrix of irregular form.

Reproduction.-The "collared monads," as these organisms are often called, multiply by longitudinal fission (2b). In some cases multiple fission of encysted individuals has been observed (2c), small simple flagellulæe being produced which gradually develop into the perfect form.

The order is especially interesting from the fact that, with the exception of Sponges, it is the only group in the animal kingdom in which the collar occurs.

\section{ORder 3.-DiNoflagellata.}

The leading features of this group are the arrangement of the two flagella - which they always possess, and the usual presence of a remarkable and often very beautiful and complex shell.

The body (Fig. 59, 1) is usually bilaterally asymmetrical, i.e. it may be divided into right and left halves, which are not precisely similar. On the ventral surface is a longitudinal groove $(l . g r$.$) , extending along the anterior half$ only, and meeting a transverse groove $\left(t . g r_{\text {. }}\right)$, which is continued round the body like a girdle. From the longitudinal groove springs a large flagellum $(f l .1)$, which is directed forwards and serves as the chief organ of propulsion; a second flagellum $(f l .2)$ lies in the transverse groove, where its wave-like movements formerly caused it to be mistaken for a ring of small cilia.

The body is covered with a shell (2) formed of cellulose, and often of very complex form, being produced into long and ornamental process, and marked with stripes, dots, \&c. Besides a nucleus and a contractile vacuole, the protoplasm contains chromatophores $(1, c h r$.$) coloured with chlorophyll or an allied$ pigment of a yellow colour, called diatomin. Nutrition is holophytic or holozoic.

The foregoing description applies to all the commoner genera. Prorocentrum (3) is remarkable for the absence of the transverse groove, while Polykrikos (4) has no fewer than eight transverse grooves and no shell. The latter genus also has stinging-capsules or nematocysts $(a, b)$ in the protoplasm, resembling those of Zoophytes (see Sect. IV.), and has numerous nuclei of two sizes, distinguished as meganuclei (nu.), and micronuclei (nu'.). 
Reproduction is, as usual, by binary fission, the process taking place sometimes in a free-swimming individual, sometimes in one which has lost its flagella and come to rest.

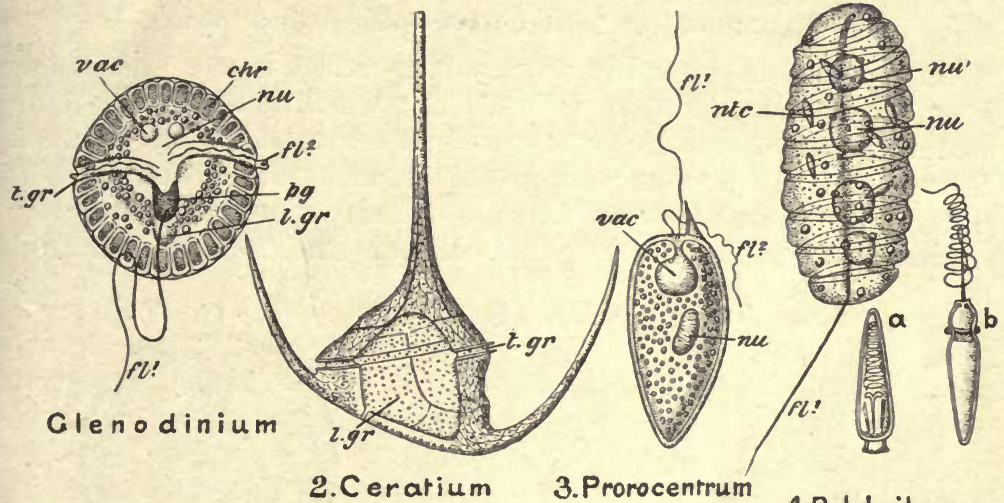

2. Ceratium

3.Prorocentrum

4.Polykrikos

F1G. 59.-Various forms of Dinofiagellata. 2 shows the shell only; $4 a$ is an undischarged, and $b$ a discharged stinging-capsule; chr. chromatophores; $f$. 1, longitudinal flagellum; f. 2 , transverse flagellum; $l$. gr. longitudinal groove; ntc. nematocyst; $n u$. meganucleus; $n u^{\prime}$. micronucleus; $p g$. pigment spot; $t$. gr. transverse groove. (From Bütschli's Protozoa.)

The Dinoflagellata are mostly marine. Some are phosphorescent. Certain kinds occasionally occur in such abundance in bays and estuaries as to cause a deep brownish or red discoloration of the sea-water.

\section{- Order 4-Cystoflagellata.}

This group includes only two genera, Noctiluca and Leptodiscus. A description of Noctiluca miliaris, the organism to which the diffused phosphorescence
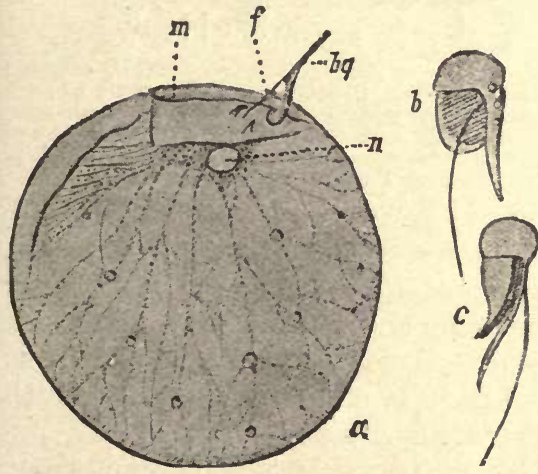

FIG. 60.-Noctiluca miliaris. $a$. the adult animal; $b, c$. flagellulæ; $b g$. tentacle; $f$. flagel. lum ; $m$. mouth; $n$. nucleus. (From Lang.) of the sea is largely due, will serve to give a fair notion of the leading characteristics of the order.

Noctiluca (Fig. 60) is a nearly globular organism, about $\frac{1}{2} \mathrm{~mm}$. in diameter. It is covered with a delicate cuticle, and the medullary protoplasm is greatly vacuolated. On one side is a groove from which springs a very large and stout flagellum or tentacle (bg.), noticeable for its transverse striation. Near the base of this flagellum is the mouth $(m$.$) , leading into a short$ gullet in which is a second flagel$\operatorname{lum}(f$.$) , very small in proportion$ to the first. On the side opposite to the mouth is a strongly marked superficial ridge. The light-giving region is the cortical protoplasm.

Reproduction takes place by binary fission, the nucleus dividing indirectly. Spore-formation also occurs, sometimes preceded by conjugation, sometimes not. 
The spores $(b, c)$, formed by the breaking up of the protoplasm of the parent, escape as flagellula.

\section{CLASS IV.-SPOROZOA.}

1. Example of the Class-Monocystis agilis.

One of the most readily procured Sporozoa is the microscopic worm-like Monocystis agilis (Fig. 61, A), which is commonly found leading a parasitic life in the vesiculæ seminales of the common Earthworm. It is flattened, greatly elongated, pointed at both ends, and performs slow movements of expansion and contraction, reminding us of those of Euglena. In this, the trophozoite or adult

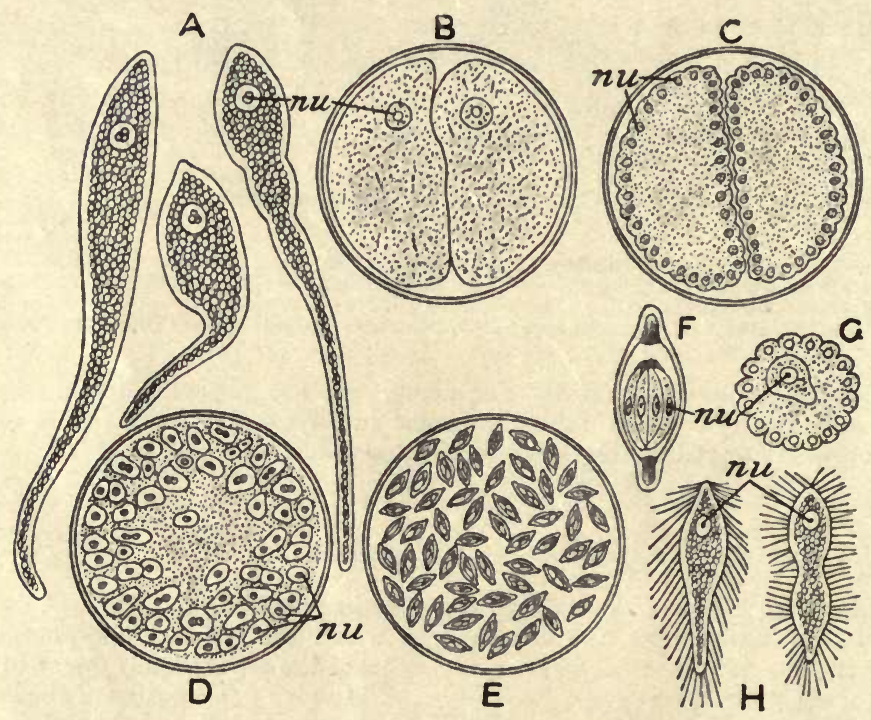

F1G. 61.-Monocystis. A, Trophozoites in different stages of contraction. B, encysted gametocytes. C, division of gametoeytes into gametes. D, conjugation of gametes to form zygotes. E, Cyst enclosing ripe spores formed from the zygotes. F, single spure, showing the (8) sporozoites in its interior. G. group of developing sperm-cells of the earthworm, enclosing a sporozoite in the centre. $\dot{H}$, young trophozites still surrounded with the tails of the degenerated sperms. nu, nuelei. (From Parker's Practical Zoology.)

condition, the protoplasmic body is covered with a firm cuticle, and is distinctly divided into a denser superficial portion, the cortex, and a central semi-fluid mass, the medulla. There is a large clear nucleus (nu.) with a distinct nucleolus and nuclear membrane, but the other organs of the protozoan cell-body are absent: there is no trace of contractile vacuole, of flagella or pseudopods, of mouth or gullet. Nutrition is effected entirely by absorption.

Reproduction takes place by a peculiar and cilaracteristic process of spore-formation. Two individuals come iogether, and become rounded off and enclosed in a common cyst (B). The nucleus of each divides repeatedly, until a large number of nuclei are formed (C). Each of the nuclei becomes surrounded by a thin layer of protoplasm. The minute cells thus formed, after 
moving to and fro actively for a time, unite in pairs after the substance of the two individuals has become coalescent (D). From each of the cells or zygotes that are formed by the union of two of the original small cells or gametes, a spore is formed, so that the cyst now comes to contain numerous small spores $(\mathrm{E})$. These are spindle-shaped bodies, each enclosed in a strong chitinoid case $(\mathrm{F})$, and thus differing in a marked manner from the naked spores of the Rhizopoda and Mastigophora. The protoplasm and nucleus of each spore then undergo fission, becoming divided into a number of somewhat sickle-shaped bodies which are arranged within the spore-coat somewhat like a bundle of sausages. By the rupture of the spore-coat these falciform young or sporozoites are liberated, and at once begin active movements, the thin end of the body moving to and fro like a clumsy flagellum. The falciform young appear, in fact, to be greatly modified flagellulæ. They make their way to the clumps of developing sperms, bore their way in, and are thus found surrounded by sperm-cells in various stages of development (G). After thus living an intracellular life for a time, they escape $(\mathrm{H})$ into the cavity of the vesicula and grow into the adult form.

\section{Classification and General Organisation.}

The Sporozoa are exclusively parasitic, being the only group of Protozoa of which this can be said. They have no organs of locomotion and always multiply by spore-formation. The class is divisible into the following five orders:-

Order 1.-Gregarinida.

Sporozoa in which the trophozoite is free and motile.

\section{ORder 2.-CocCIDIIDEA.}

Sporozoa in which the trophozoite is a minute intracellular parasite.

\section{Order 3.-HeMosPoridia.}

Sporozoa in which the trophozoite is amœboid, and lives as a parasite in the coloured blood-corpuscles of Vertebrates.

\section{ORDER 4.-MYXOSPORIDEA.}

Sporozoa in which the trophozoite is amœboid, but not intracellular.

\section{ORDER 5.-SARCOCYSTIDEA.}

Elongated Sporozoa, usually found in muscle. 


\section{Systematic Position of the Example.}

Monocystis agilis is a species of the genus Monocystis, belonging to the family Monocystida, of the order Gregarinida. It is placed in the Gregarinida on account of being free and motile in the trophozoite state. The absence of partitions dividing the protoplasm into segments indicates its position among the Monocystidx. Monocystis is distinguished by its elongated form, by the absence of any special apparatus in the cyst for the liberation and dispersal of the spores, and by its spindle-shaped spores with thickened ends, each producing $4-8$ falciform young. The differences between the species of Monocystis depend largely upon size.

\section{ORDER 1.-GREGARINIDA.}

All the more typical members of the class belong to this group. With the exception of Monocystis, already described, the only genus to which it will be necessary to draw attention is Gregarina (Figs. 62 and 63), the various species of which are parasitic in the intestines of Crayfishes, Cockroaches, Centipedes,
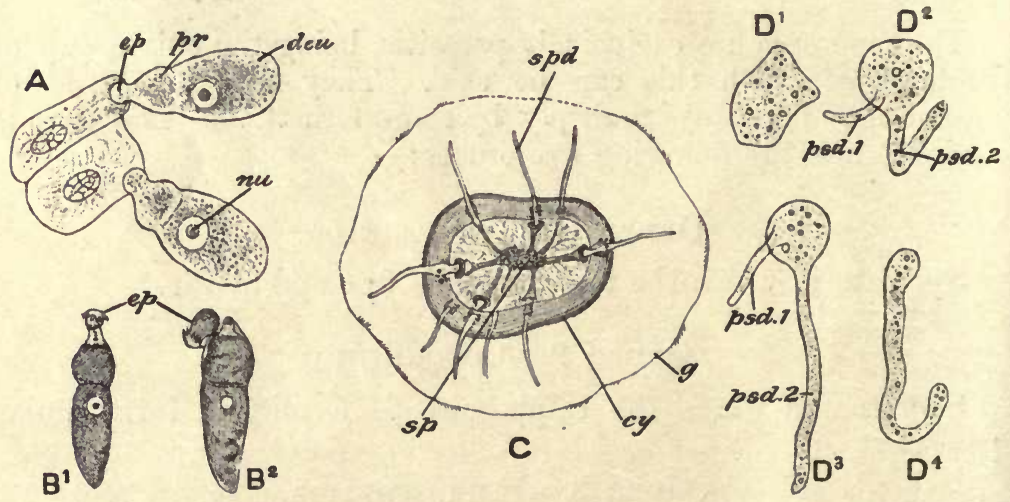

Fig. 62.-Gregarina. A, two speeimens of G. blattarem partly embedded in enteric epithelial cells of Cockroach; $\mathrm{B}^{1}, \mathrm{~B}^{2}$, two speeimens of $G$. dujarlini; in $\mathrm{B}^{2}$ the epimerite $\left(e p^{3}\right)$ is cast off ; $\mathbf{C}$, eyst of $G$. blattarum, from which most of the spores have been discharged; $D$, four stages in the development of $G$. gigantea. cy. cyst; deu. deutomerite ; ep. epimerite; $g$. gelatinous investment of cyst; $n u$. nueleus; $\mathrm{pr}$. protomerite; $p s d$. 1 , short pseudopod; p2si. \&, long pseudopod; sp. mass of spores; spd. sporoducts. (From Butschli's Protozoa.)

and other articulated animals. It differs from Monocystis in having the medullary protoplasm of the adult divided into two sections, an anterior, the protomerite $(p r$.$) , and a posterior, the$ deutomerite (deu.), in which the nucleus is situated. Anteriorly 
to the protomerite there is sometimes found, especially in young individuals, a third division, the epimerite (ep.), which may be provided with hooks $\left(\mathrm{B}^{1}\right)$, serving to attach the parasite to the epithelium of the intestine of its host, by becoming embedded in the substance of one of the cells. As maturity is reached the epimerite is thrown off $\left(\mathrm{B}^{2}\right)$, and the parasite then lies freely in the cavity of the intestine.

The cysts of Gregarina (C) are often very complex and provided with delicate ducts $(s p d$.) in the thickness of the wall,

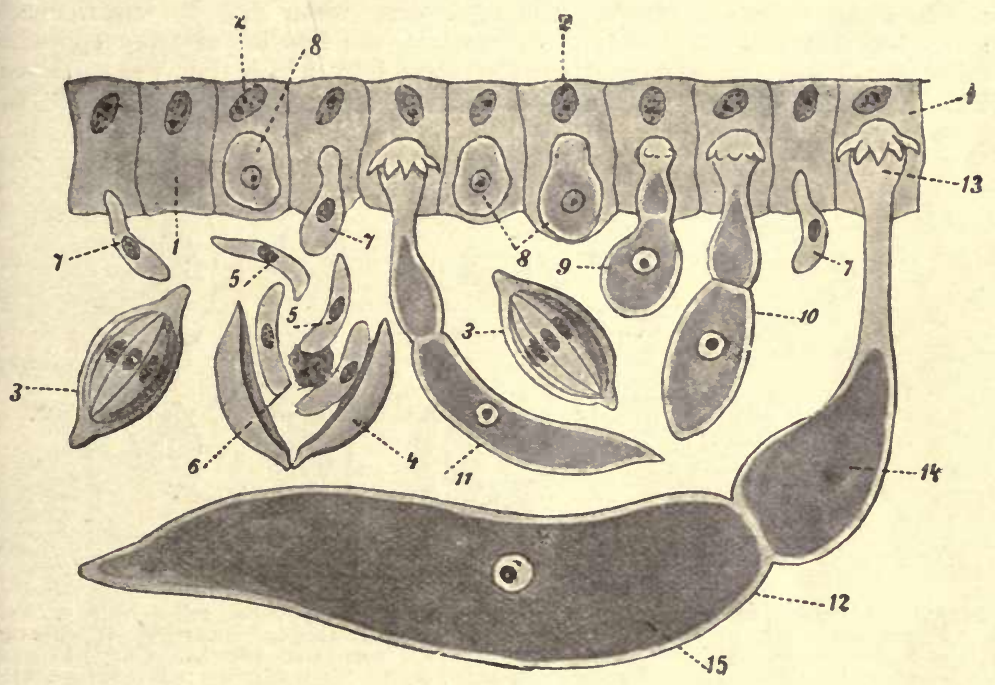

FIG. 63.-Gregarina. Developinent from the sporuzuite. 1, cells of the digestive epithelium of the host; 2 , nuclei of the same; 3 , spore; 4, spore discharging sporozoites (5) leaving residual mass $(6) ; 7$, sporozoites in the act of entering epithelial cells; 8 , the same as intracellular parasites; $9-12$, different stages in the growth of the young Gregarines into the lumeu of the intestine; 13, epimerite ; 14. protomerite ; 15, deutomerite. (After Lang.)

through which the spores escape. In Gregarina gigantea of the Lobster, the young (sporozoite) is liberated from the spore in the form of a non-nucleated amœbula $\left(\mathrm{D}^{1}\right)$, with one long and one short pseudopod $\left(D^{2}\right)$; this divides by the long pseudopod ( $\left.p s d .2\right)$ becoming separated off, and each product of fission, developing a nucleus, passes into the adult (trophozoite) form $\left(\mathrm{D}^{3}, \mathrm{D}^{4}\right.$.) In other species of Gregarina the sporozoites do not divide, but each develops directly into the trophozoite (Fig. 63).

\section{ORDER 2.-COCCIDIIDEA.}

Coccidium (Figs. 64, 65) and allied genera are parasites in the interior of cells, both in Vertebrates and Invertebrates. They live in the cells of various 
orgyans, most frequently in those of the epithelium of the digestive canal. They never-inhabit blood-corpuscles. A few are intra-nuclear parasites. Two distinct modes of multiplication occur-by schizogony, a kind of multiple fission, and by sporogony, a process of spore-formation preceded by conjugation between male and female cells. The trophozoile, or adult phase, as we may term it, of the parasite, grows to a certain size within the cell without destroying its vitality - the nucleus murely being pushed on one side. So far, in fact, from impairing the nutrition of the cell, the presence of the parasite seems, in some cases, for a time, rather to stimulate it At a certain stage of growth schizogony (Fig. 65, $b-c$ ) takes place. The nucleus divides to form a number of nuclei. These migrate towards the surface, and each becomes surrounded by protoplasm, with the result that a number of small cells are formed. Each of these gives rise to a club-shaped merozoite. The merozoites, when they become free, are active bodies, which are able to penetrate into the interior of other epithelial cells and develop into trophozoites like those from which they were derived. This multiplication may take place on such an extensive scale that the

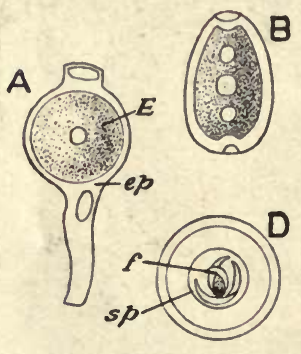

1. Eimeria
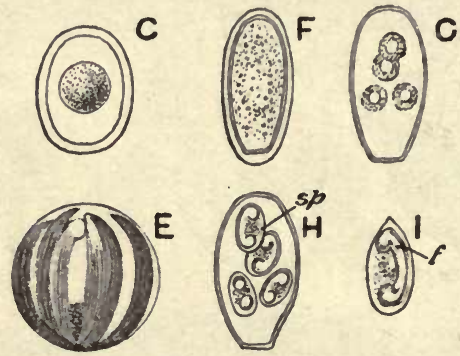

2. Coccidium

F1G. 64.-Coccidiidea. A, adult Eimeria (E) in entcric epithelial cell (ep.) of mouse; B, encysted form; C, encysted form, the protoplasm contracting to form a spore; D, formation of falciform young $\left(f_{*}\right)$ in interior of spore $(8 p.) ; \mathbf{E}$, spore with falciform young; $\mathbf{F}$, adult encysted form of Coccidium from liver of rabbit; $G$, division into spores; $H$, cyst containing ripe spores $(s p$.$) , each with a single falciform young; I, single spore with falciform young (f$.$) .$ (From Butschli's Protozoa, after Leuckart and Eimer.)

epithelium may be partially or completely destroyed. It is only, apparently, when such extensive damage has been done, or is threatened, that multiplication by sporogony takes place-the invasion of a new host being by this process rendered probable, and the continuance of the race being thus provided for in the event of the death of the host in which the epithelium has become destroyed. In this process certain of the merozoites, instead of developing into trophozoites, grow more slowly $(d)$, and become converted into either micro- or megagametocytes. Each of the former $(h, j)$ gives rise by division to a number of narrow biflagellate microgametes or sperms. Each of the megagametocytes $(e, f)$, after a process of the nature of maturation, forms a single rounded megagamete (ovum). When this becomes fertilised by the penetration into it of a single microgamete, the resulting body (zygote or oosperm) divides to form a varying number of cells each enclosed in a resistant cyst $(k)$. These give rise to spores with a firm, chitinous spore-membrane, each containing two or more falciform young or sporozoites (l). The cyst destroys the cell as it grows, and thus becomes free in the cavity by which the epithelium is lined. The spores may thus pass out to the exterior, and, if taken into the digestive canal of a new host, may liberate the now active sporozoites, which may penetrate into epithelial cells $(a)$ to become the trophozoites with which the cycle began. 
In some of the Coccidiidea this life cycle is modified in various ways, as, for example, by the omission of schizogony-the trophozoites in such a case developing directly into gametocytes.

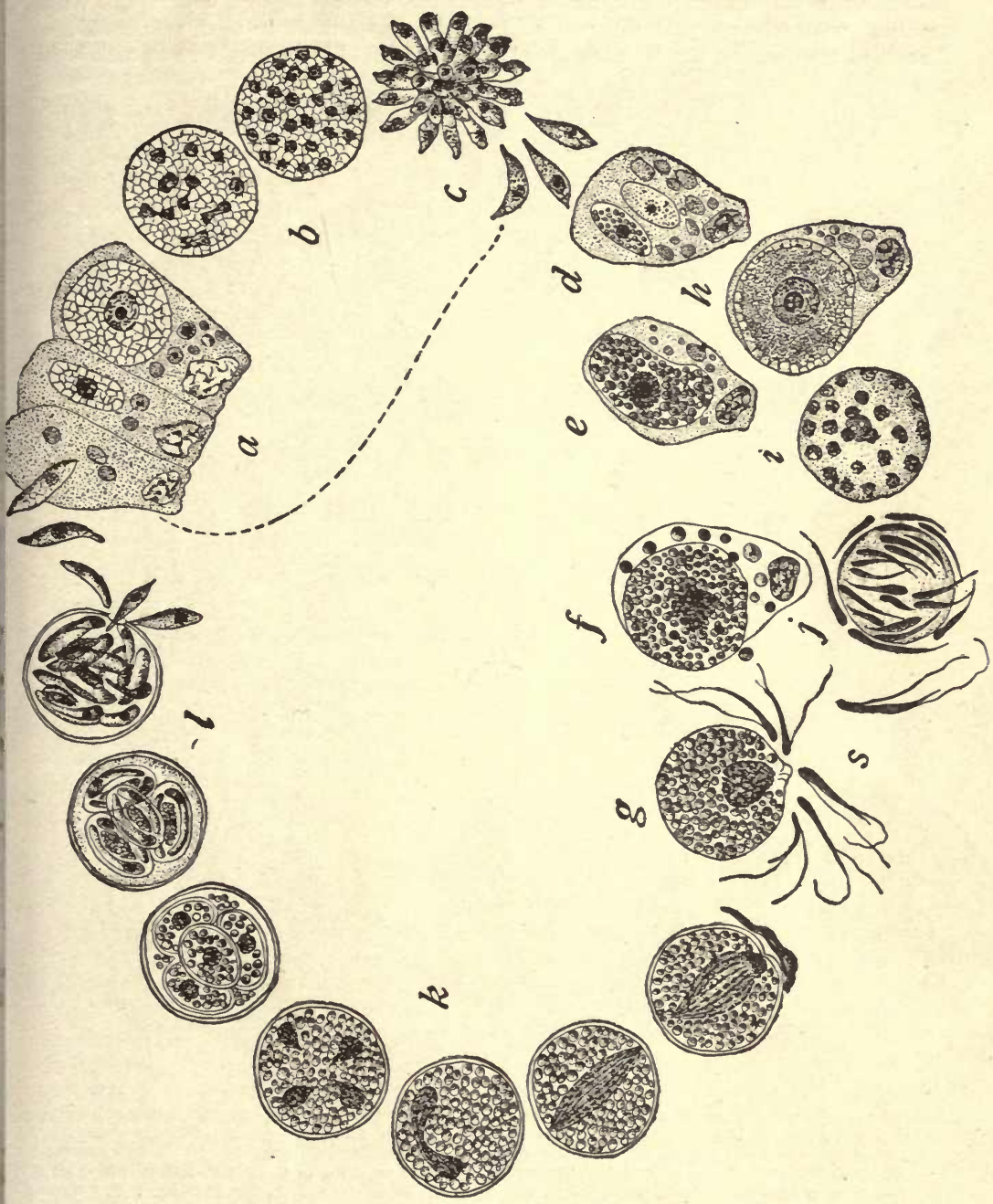

FIG. 65.-Life-History of Coccidium schubergi, a. penetration of epithelium cell of host by sporozoite; $b-c$, stages of ruultiple fission (schizogony); $d$, gametocyte; $e, f$, furmation of megagamete (ovum) $: g$, fertilisation; $h, j$, formation of microgametes (sperms); $k$, development of fertilised ovum into four spores ; $l$, formation of two sporozoites (falciform young) in each spore. (From Calkins, after Schaudinn.)

\section{ORDER 3.-HÆMOSPORIDEA.}

These are Sporozoa which in the trophozoite condition live as parasites in the interior of the coloured blood-corpuscles of all classes of Vertebrates, but are occasionally found in other cells. In Man and in some other mammals and 
in certain birds it has been found that their presence is the cause of various feverish affections. The various forms of malaria in man have been proved to be due to the presence in the blood-corpuscles of the patient of parasites belonging to this order. The malaria-parasites, the history of which has been carefully worked out, pass through a life-cycle comparable to that of Coccidium described above. In the trophozoite stage (Fig. 66, $A-G$ ) they live as amœboid

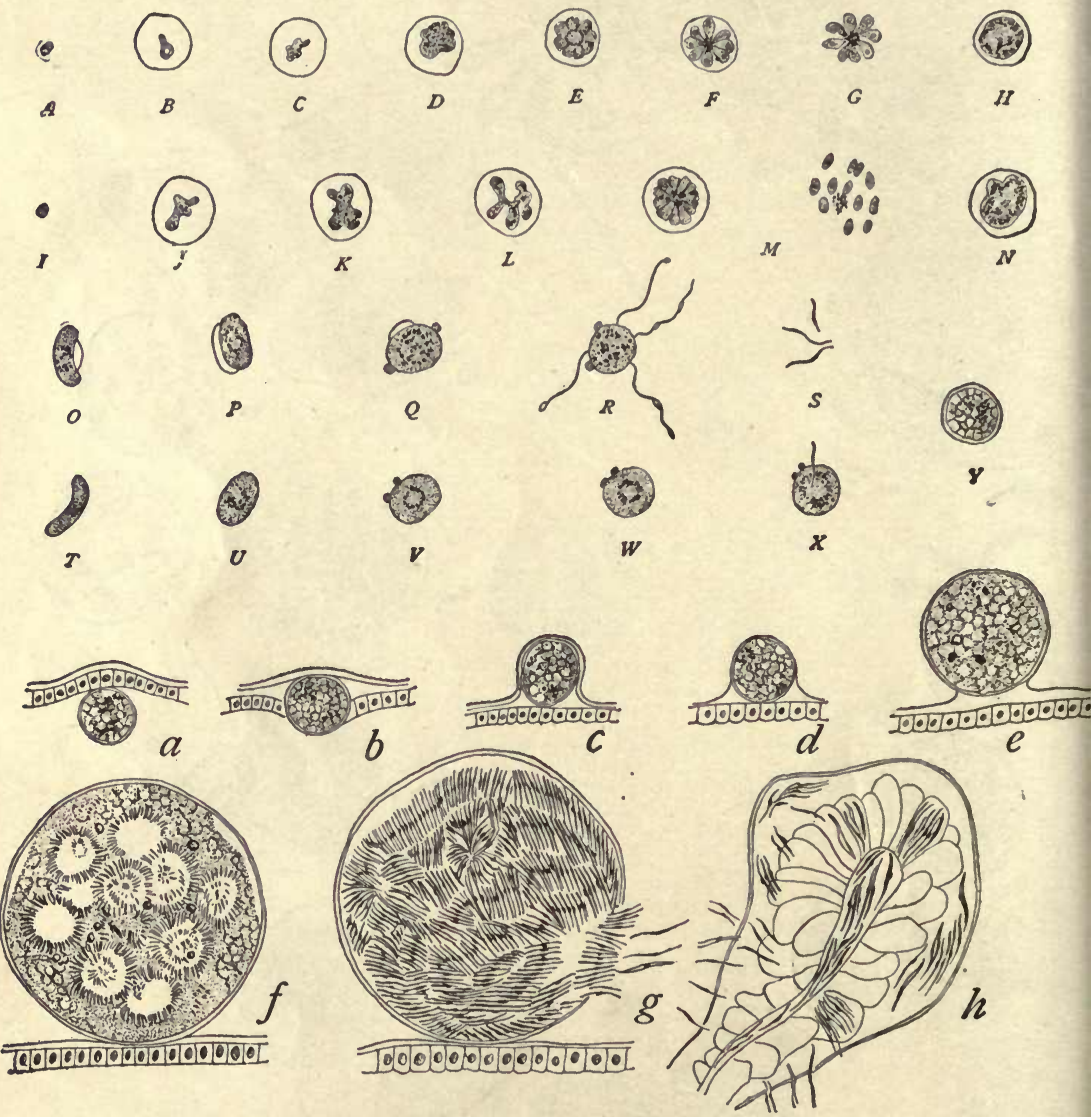

Fia. 66.-Life-History of Malaria Parasites. $A-G$, parasite of quartan fever, showing development of trophozoite in a blood-eorpuscle and the formation of merozoites; $H$, gametocyte of the same; $I-M$, parasite of tertian fever to the formation of the merozoites; $N$, gametocyte; $O-T$; crescentie gametocytes of Laverania; $P-S$, formation of microgametes or sperms; $U-W$, maturation of megagamete or ovum; $X$, fertilisation ; $Y$, zygote. $a$, zygote enlarging in stomach of mosquito; $b-e$, passing into the body-cavity ; $f, g$, development of the contents into a mass of sporozoites; $h$, sporozoites passing into the salivary glands. (From Calkin's Protozoa, after Ross and Fielding Ould.)

intracellular parasites in the interior of the coloured corpuscles of their host. Here they multiply by schizogony -the products (merozoites) entering other corpuscles. Some of the merozoites when they become established in the interior of the corpuscles develop into rounded or crescentic bodies which become the gametocytes $(H, N, O, T)$. In order that the life-cycle may be completed, it is necessary that the parasite at this stage should be taken into the interior of a 
second or intermediate host. In the case of the parasite of human malaria the intermediate host is a mosquito of the genus Anopheles. On the mosquito drawing up a drop of the blood of a malaria patient, all stages of the parasite that occur in it are destroyed by the digestive juices of the insect with the exception of the gametocytes; these survive and form gametes in the stomach of the mosquito. Each male gametocyte gives rise to a number of slender filamentous microgametes (sperms, $P, S$ ) and each female gametocyte forms a single megagamete (ovum). After maturation $(U-W)$ the megagamete is fertilised $(x)$ by one of the actively-moving microgamates, the result being the formation of an active spindle-shaped ookinete. This perforates the stomach wall and comes to rest in the subjacent tissues. It then becomese encysted and increases greatly in size, bulging out into the body-cavity $(b-e)$. The contents of the cyst eventually become divided up $(f, g)$ into a large number of long, narrow sporozoites. When the cyst becomes ruptured into the bodycavity, these find their way to the salivary glands $(h)$, and thence they may readily be transferred to the blood-system of a human being when the mosquito bites. Penetrating into the interior of coloured corpuscles they reach the trophozoite condition.

The Hæmogregarines, which may most conveniently be referred to here, are Sporozoa which live, like the malaria parasites, in the coloured blood-corpuscles of all classes of Vertebrates; but which in the mature or trophozoite condition are not amœboid, retaining the Gregarina-like form, and are therefore to be regarded as belonging to the Gregarinida.

\section{ORDER 4.-MYXOSPORIDEA.}

This group includes a small number of genera which are anœboid in the trophozoite phase, and which reproduce continuously by spore-formation during that phase (Fig. 67, A). Many nuclei are present

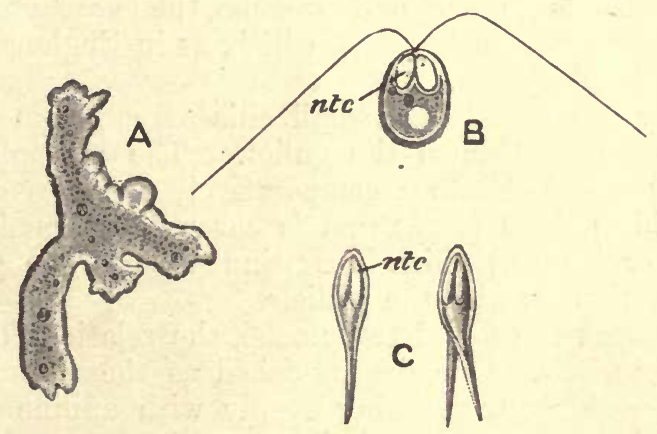

FIG. 67.-A, Myxidium lieberkühnii, amoboid phase; B, Myxobolus mülleri, spore with discharged nematocysts (ntc.); C, spores (psorosperms) of a Myxosporidian; ntc. nematocysts. (From Bütschli's Protozoa.)

in the amœboid body, which may be of comparatively large size. The spores $(B)$ produced within the protoplasm of the trophozoite are provided each with one or more bodies like the nematocysts of zoophytes and jelly-fish [See Section IV]. Myxosporidea occur as parasites mainly of fishes and amphibians, but very many occur in various groups of Invertebrates. "Pébrine," the destructive silk-worm disease, is due to the presence of a Sporozoan belonging to this order. A good example of the order is Myxidium, found in the urinary bladder of the pike. 


\section{ORDER 5.-SARCOCYSTIDEA.}

The best known form of this order is Sarco'ystis (Fig. 68), which occurs in the flesh of mammals, each parasite having the form of a long spindle embedded

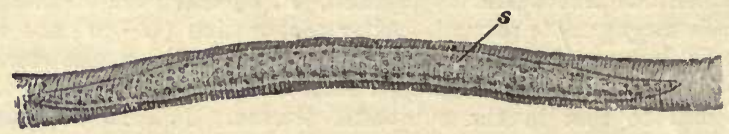

Fia. 68. - Sarcocystis miescheri, adult form (8) in striped muscle of pig. (From Buitschli's Protozoa, after Rainey.)

in a striped muscular fibre. They are often known as Rainey's or Miescher's corpuscles. The protoplasm divides into spores from which falciform young are liberated.

\section{CLASS V.-INFUSORIA.}

\section{Example of the Class-Paramocium caudatum.}

Structure.-Paramocium, the "slipper-animalcule," is tolerably common in stagnant ponds, organic infusions, \&c. The body (Fig. 69) is somewhat cylindrical, about $\frac{1}{4} \mathrm{~mm}$. in length, rounded at the anterior and bluntly pointed at the posterior end. On the ventral face is a large oblique depression, the buccal groove (buc. gr:), leading into a short gullet (gul.), which, as in Euglena, ends in the soft internal protoplasm.

The body is covered with small cilia arranged in longitudinal rows and continued down the gullet. The protoplasm is very clearly differentiated into a comparatively dense cortex (cort.) and a semi-fluid medulla (med.), and is covered externally by a thin pellicle or cuticle (cuv.) which is continued down the gullet. The cilia are continuous with the pellicle.

In the cortex are found two nuclei, the relations of which are very characteristic. One, distinguished as the meganucleus (nu.), is a large ovoid body staining evenly with aniline dyes, which, when it divides, does so directly by a simple process of constriction. The other, called the micromucleus ( $p a . n u$.), is a very small body closely applied to the meganucleus; when it divides it goes through the complex series of stages characteristic of mitosis (p. 16).

The contractile vacuoles $(c . v a c$.) are two in number, and are very readily made out. Each is connected with a series of radiating spindle-shaped cavities in the protoplasm which serve as feeders to it. After the contraction of the vacuole these cavities are seen gradually to fill, apparently receiving water from the surrounding 
protoplasm: they then contract, discharging the water into the vacuole, the latter rapidly enlarging while they disappear from
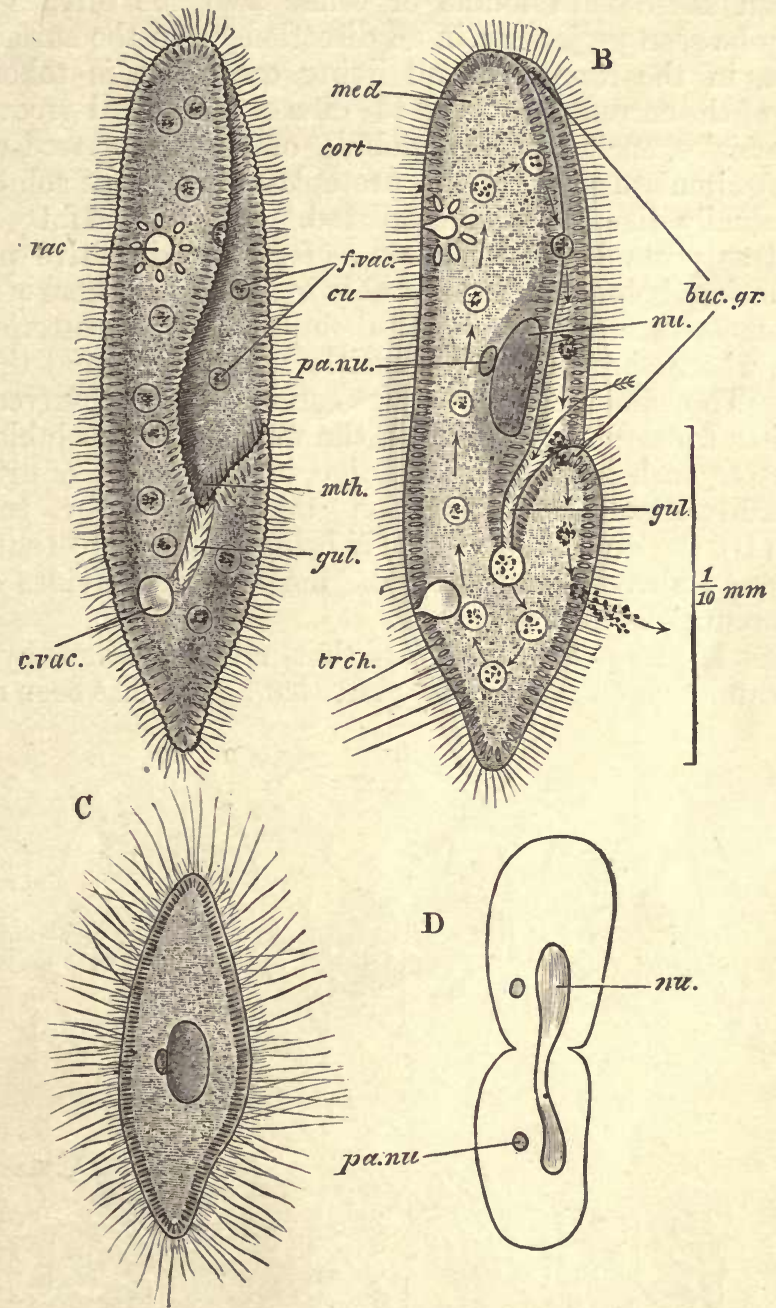

Fia. 69-Paramœcium caudatum. A, the living animal from the ventral aspect; B, the same in optical section: the arrow shows the course taken by food-particles; C a specimen which has discharged its trichocysts; D, diagram of binary fission; buc. gr. buccal groove; cort. cortex; $c u$. cuticle; $c$. vac. contractile vacuole; $f$. vac. food vacuole; gul. gullet; med. medulla; nu. meganucleus; $p a . n u$. mieremucleus ; trch. trichocysts. (From Parker's Biology.)

view; finally the vacuole contracts and discharges its contents externally.

The cortex contains minute radially arranged sacs called trichocysts (trch.). When the animal is irritated, more or fewer of 
these suddenly discharge a long delicate thread, which, in the condition of rest, is very probably coiled up within the sac. In a specimen killed with iodine or osmic acid the threads can frequently be seen projecting in all directions from the surface $(C)$.

Food, in the form of small living organisms, is taken in by means of the current caused by the cilia of the buccal groove. The food-particles, enclosed in a globule of water or "food-vacuole" (f. vac.), circulate through the protoplasm, when the soluble parts are gradually digested and assimilated. Starchy and fatty matters, as well as proteids, are available as food, the digestive powers of Paramœcium being thus considerably in advance of those of Amœba. Effete matters are egested at a definite anal spot posterior to the mouth, where the cortex and cuticle are less resistent than elsewhere. The whole feeding process can readily be observed in this and other Infusoria by placing in the water some insoluble colouring matter, such as carmine or indigo, in a fine state of division.

Reproduction.-Multiplication takes place by transverse fission (D), the division of the body being preceded by that of both nuclei. As already mentioned, the meganucleus divides directly, the micronucleus indirectly.

It has been proved, however, that multiplication by binary fission cannot go on indefinitely; but that after it has been repeated

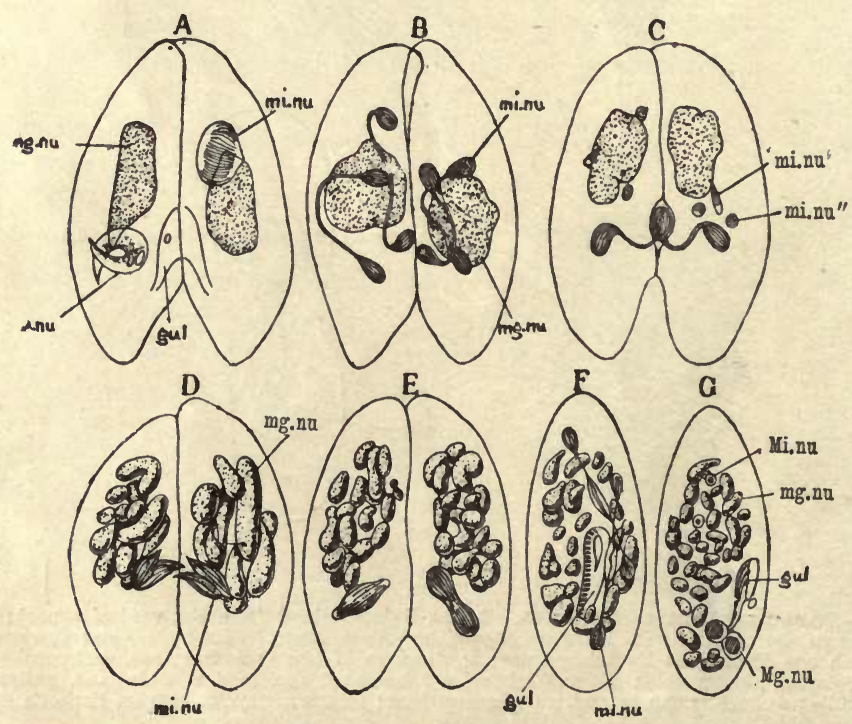

FIG. 70. - Paramœeium caudatum, stages in conjugation. gul. gullet; $m g . n u$. meganucleus; $m i$. nu. micronucleus; $M g$. nu. reconstructed meganucleus; Mi. nu. reconstructed micronucleus. (From Parker's Biology, after Hertwig.)

a certain number of times it is interrupted by conjugation. In this very remarkable and characteristic process two Paramœcia 
become applied by their ventral faces (Fig. 70, A), but do not fuse. The meganucleus ( $m g . n u$.) of each breaks up into small masses, which disappear, being apparently absorbed into the protoplasm. At the same time the micronucleus ( $m i$. $n u$.) of each divides, each product of division immediately dividing again, so that each gamete or conjugating body is provided with four micronuclei (B). Two of these (mi. nu.', mi. nu.") disappear; of the remaining two one is distinguished as the stationary pronucleus, the other as the active pronucleus. The active pronucleus of each Infusor now passes into the body of the other and fuses with its stationary pronucleus (D), each individual thus coming to possess a single nuclear body derived in equal proportions from the two conjugating cells $(\mathrm{E})$. The animalcules then separate from one another, and the nucleus of each divides and gives rise to the permanent mega- (G, Mg. nu.) and micronuclei (Mi. nu..).

\section{Crassification and General Organisation.}

In the majority of the Infusoria the body is ciliated throughout life, but in certain forms cilia are present only in the immature condition, the adult being provided with peculiar organs of prehension or tentacles. We thus get two orders, viz.:-

\section{Order 1.-Ciliata.}

Infusoria provided with cilia throughout life.

\section{Order 2.-Tentaculifera.}

Infusoria possessing ailia in the young condition, tentacles in the adult.

\section{Systematic position of the Example.}

Paramœcium aurelia is one of several species of the genus Paramcecium, belonging the family Parmocida, of the sub-order I'richostomata, and order Ciliata. The presence of cilia in the adult condition places it among the Ciliata: the presence of a permanently open mouth into which food particles are swept by the movement of the cilia, among the Trichostomata. The Paramœcidæ are free-swimming, asymmetrical, uniformly ciliated, with a ventrally placed mouth. P. caudatum is about $\frac{1}{4}-\frac{1}{5} \mathrm{~mm}$. in length, its length about four times its breadth, rounded in front, and bluntly pointed behind, and a single micronucleus is present.

\section{Order 1.-Ciliata.}

This order presents a wider range of variations-some of them of a truly extraordinary character-than any other group of Protozoa. 
The form of the body is very varied: it may be ovoid (Fig. $71,1)$, kidney-shaped (2), trumpet-shaped (3), vase or cup-shaped $(4,9)$; produced into a long, flexible, neck-like process $(5)$, or into large paired lappets $(6)$; flattened from above downwards, or elongated and divided into segments reminding us of those of a segmented worm (8).

Most species are free-swimming, but some are attached to weeds, stones, \&c., by a stalk. This may be a purely cuticular structure (9), or may contain a prolongation of the cortex in the form of a delicate contractile axial fibre (Figs. 73 and 74, ax. f.), which serves to retract the Infusor, its contraction causing the stalk to coil up into a close spiral.

The arrangement of the cilia is also subject to great variation, and presents four chief types. In the holotrichous type, of which Paramœcium is an example, the cilia are all small, equalsized or nearly so, and arranged in longitudinal rows (Fig. 69, Fig. $71,1)$. The second or heterotrichous type is seen in its simplest form in Nyctotherus (Fig. 71, 2), in which the left side of the peristome is bordered by a row of specially large adoral cilia, the rest of the body being covered with small cilia. In Stentor (3) the peristome is situated on the broad distal end of the trumpetshaped body, and the adoral band of cilia takes a spiral course. This leads us to the peritrichons type of ciliation: in Vorticella (Fig. 73) the vase-shaped body is, for the most part, quite bare of cilia, but around the thickened edge of the peristome passes one limb of a spiral band of large cilia united at their bases, the other limb being continued round a raised lid-like structure, or disc, into which the distal region is produced. This arrangement of cilia reaches its greatest complexity in Epistylis plicatilis (Fig. 71,9), in which the ciliary spiral makes no fewer than four turns.

But it is in the hypotrichous type that the most extraordinary modifications are found. The flattened body bears on its dorsal surface mere vestiges of cilia in the form of very minute processes of the cuticle, while on the ventral surface the cilia take the form of large hooks, fans, bristles, and plates with fringed ends (Fig. 71, 7). The hooks and plates do not vibrate rhythmically like ordinary cilia, but are moved as a whole at the will of the animal, thus acting as legs. The hypotrichous Ciliata, in fact, in addition to swimming freely in the water, creep over the surface of weeds, \&c., very much after the manner of Woodlice. One of the most extraordinary forms in this group is Diophrys ( 7$)$, the size and arrangement of its polymorphic cilia giving it a very grotesque appearance. In another genus (10) the distal end of the flaskshaped body bears a circlet of large fringed cilia, giving the animal the appearance of a Rotifer (vide Section VII.).

In addition to cilia, many genera possess delicate sheets of protoplasm or undulating membranes in connection with the 
peristome. They contract so as to produce a wave-like movement which aids in the ingestion of food. In some cases (Fig. 71, 11) the undulating membrane $(u, m b$. $)$ is a very large and obvious structure.

Certain peculiar forms have yet to be mentioned. Multicilia (Fig. $71,12)$ has an irregular body of varying form, and bears a small number of very long flagellum-like cilia. Another genus in which the cilia approach to flagella is Lnphomonas (13), the ovoid body of which bears a tuft of close-set cilia at its anterior end. Actinobolus (14) is remarkable for the possession, in addition to cilia, of long retractile tentacles used for attachment. In Didinium (15) the barrel-shaped body is encircled by two hoops of cilia.

As we have seen, the meganucleus in Paramccium is ovoid: in other genera it may be elongated and band-like $(3, m g . n u$.), horseshoe-shaped (9), very long and constricted at intervals so as to look like a string of beads (16), or much convoluted and branched (17). In some genera the meganucleus undergoes repeated divison, forming at last a very great number of small bodies only discoverable by staining: this process of fragmentation of the nucleus may proceed so far that the protoplasm of a stained specimen has the appearance of being strewn with granules of chromatin. The discovery of this phenomenon has tended to throw doubt on the reported total absence of a nucleus in some Rhizopods.

In nearly all species one or more micronuclei are present, the number sometimes reaching nearly thirty. In Opalina (Fig. 75) numerous nuclear bodies (nu.) are present, some of which on account of their mitotic mode of division are to be regarded as micronuclei, while the rest are meganuclei.

In Vorticella and other peritrichous genera there is a single contractile vacuole (Fig. 73,c. vac.), which, like that of Euglena, opens through the intermediation of a reservoir into the vestibule. In the remaining Ciliata there may be one, two, or many--sometimes a bundred-contractile vacuoles. They may be scattered all over the cortex (Fig. 71, 18), or arranged in one or two rows (8). The star-like arrangement of radiating canals, described in Paramœcium, occurs in several genera: or there may be two long canals, or the number of these channels in the protoplasm may reach thirty $(19, c)$. In some instances the protoplasm is hollowed out by numerous non-contractile vacuoles $(18, \tau a c$.) so as to have a reticulate appearance, reminding us of the extra-capsular protoplasm of Radiolaria.

Trichocysts, like those of Paramœcium, are found in many holotrichous forms, but are rarely present in the other subdivisions of the order. In the peritrichous Epistylis umbellaria, however, there are found numerous minute capsules (Fig. 71, 9, ntc.) arranged in pairs, each containing a coiled thread. They are 
Most of the Ciliata are naked, having no shell or other form of skeleton; but in a few forms the body is provided with a shell or lorica, formed of a chitinoid material, and reminding us of the

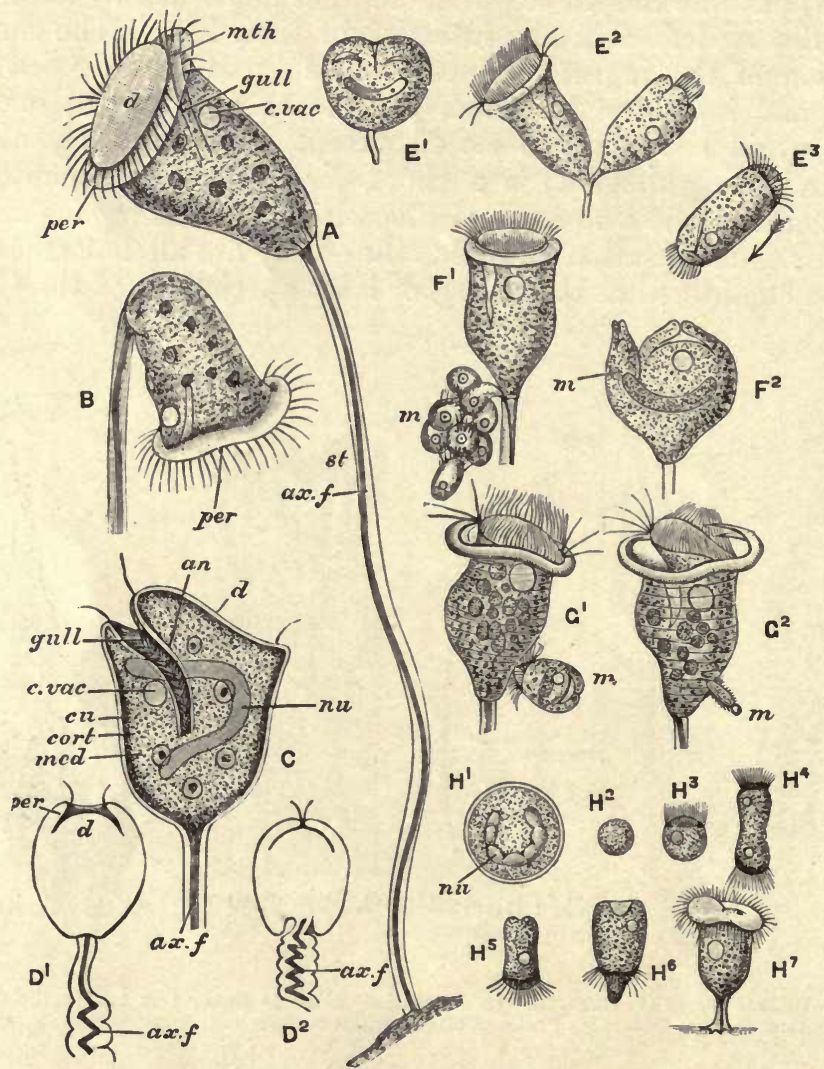

F1G. 73. -Vorticella. A, B, living specimens in different positions, C, optical section ; D1, D2, diagrams illustrating coiling of stalk; $\mathrm{E}^{1}, \mathrm{E}^{2}$, two stages in binary fission; $\mathrm{E}^{3}$, free zooid; $F^{1}, F^{2}$, division into mega- and microzooids; $\mathbf{G}^{1}, \mathrm{G}^{2}$, conjugation; $\mathrm{H}^{1}$, multiple fission of encysted form; $\mathrm{H}^{2}, \mathrm{H}^{3}$, development of spores; $a x$. $f$. axial fibre; cort. cortex ; cu. cuticle ; c. vac. contractile vacuole; $d$. disc; gull. gullet; m. micruzooid; $n$ th. mouth; nu. meganucleus; per. peristome. (From Parker's Biology.)

similar structure found in so many of the Mastigophora. Some (Fig. 71, 4) have bell-like shells, variously ornamented, and in others (Fig. 72, 1) the similarly shaped shell is perforated and resembles the skeleton of some of the Radiolaria. A chitinoid plate or operculum (Fig. 72, 2,op.) may be fixed to the edge of the peristome, and, when the animal is retracted in its case accurately closes the mouth of the latter, or a similar operculum (3) is 
attached to the interior of the tube, and is closed by a contractile thread of protoplasm $(m$.), which acts as a retractor muscle.

Compound forms or colonies are common among the Peritricha, rare in the other subdivisions. Many peritrichous forms occur as branched, tree-like colonies, often of great complexity (Fig.71, 9a; Fig. 74). The stem of these may be a purely cuticular structure and non-contractile (Fig. 71,9, b), or may contain an axial fibre or muscle, like that of Vorticella (Fig. 73, ax.f.). In Ophridium (Fig. 72,4 ) the colony is an irregular mass, sometimes $3-4 \mathrm{~cm}$. in diameter, consisting of a gelatinous substance in which a delicate, branching stem is embedded, each branch terminating in a zooid. Some genera (Fig. 72,5) secrete a hollow, brown, gelatinous tube, branched dichotomously; the end of each branch is the habitation of one of the zooids.

Reproduction.-Transverse fission is the universal method of reproduction, the entire process taking from half an hour to two

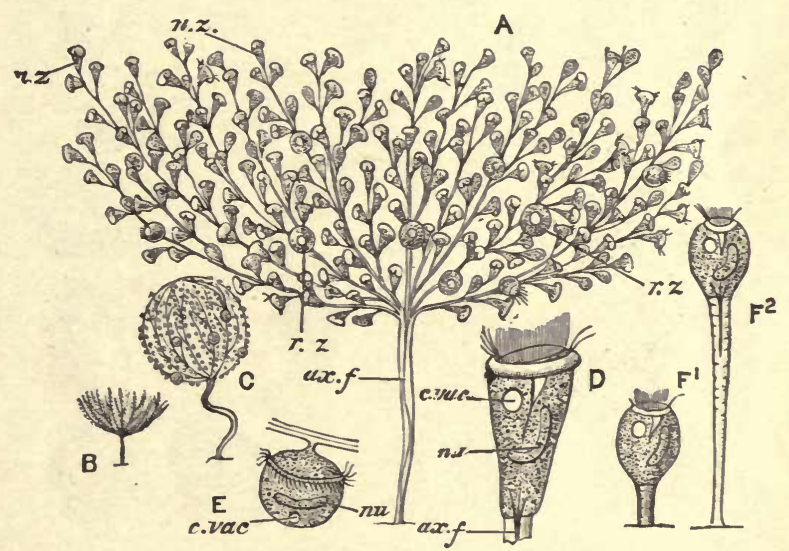

Fro. 74.-Zoothamnium arbuscula. A, entire colony; B, the same, natural size; C, the same, retracted ; D, nutritive zooid ; E, reproductive zooid; $F^{1}, F^{2}$, development of reproductive zooid; ax,f. axial fibre; $c$. vac. contractile vacuole; nu. nucleus; n.z. nutritive zooid; r.z. reproductive zooid. (From Parker's Biology, after Saville Kent.)

hours in different species. In Vorticella (Fig. 73, E) and other Peritricha the plane of division is parallel to the long axis of the bell-shaped body, but as the distal surface probably corresponds with the dorsal surface of such forms as Paramœeium, fission is really transverse in this case also. In such simple Peritricha as Vorticella division proceeds until two zooids are produced on a single stalk; one of the two then acquires a second circlet of cilia near its proximal end, becomes detached $\left(\mathrm{E}^{3}\right)$, and, after leading a free-swimming life for a time, settles down and develops a stalk: in this way the dispersal of the non-locomotive species is ensured. In many species of Zoothamnium (Fig. 74) the zooids 
are dimorplic: the ordinary bell-shaped forms (n.z.) divide in the usual way, but as they remain attached, the process results only in the increased complexity of the colony, not in the development of a new one. The larger zooids $(r . z)$ are globular and mouthless : they become detached, swim off, and, after a short free existence, settle down, develop a stalk (F), divide, and so form a new colony.

In Vorticella multiplication by ludding also occurs: a small process is given off from one side (Fig. 73, F), develops a basal circlet of cilia, and swims off as a microzooid, the parent individual

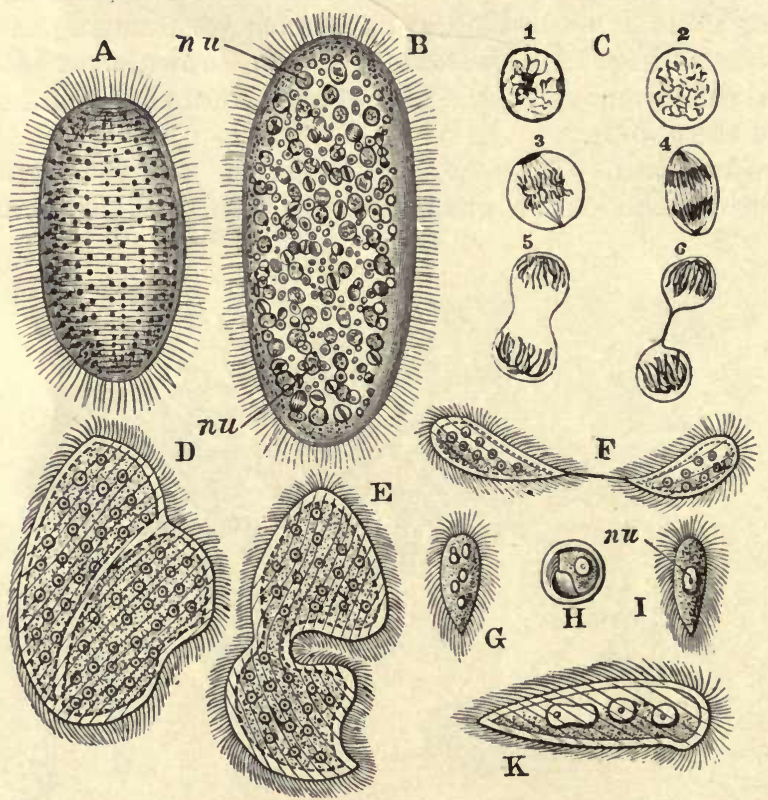

FIG. 75.-Opalina ranarum. A, living specimen; $B$, stained specimen showing nuclei; C, stages in nuclear division; D-F, stages in fission; $G$, final product of fission; $H$, encysted form; I, yeung form liberated from cyst; $\mathbf{K}$, the same after multiplication of the nucleus has begun; nu. nucleus. (From Parker's Biology, after Saville Kent and Zeller.)

or megazooid being left attached to the stalk. Obviously this process is simply a modification of binary fission, the products of division being of very different dimensions instead of equal-sized as is the more usual case.

Spore-formation take place in Colpoda. The Infusor becomes encysted, and divides into two, four, and finally eight masses, each of which, becoming surrounded by a special investment, becomes a spore. A somewhat similar process has been described in Vorticella (Fig. 73, $H$ ) and others.

A peculiar kind of spore-formation, specially adapted to the requirements of an internal parasite, takes place in Opalina 
(Fig. 75), a parasite in the intestine of the Frog. Binary fission takes place (D, E, F), and is repeated again and again so rapidly that the daughter-cells are unable to grow to the adult size before the next division. The final results of the process are small bodies $(\mathrm{G})$, each with only two or three nuclei instead of the large number characteristic of the adult. These become encysted (H), and in this passive condition are passed out of the Frog's intestine with its fæces, frequently being deposited on water-weeds. All this takes place during the Frog's breeding season : the tadpoles or Froglarvæ feed upon the water-plants, and in doing so frequently take in the spores or encysted Opalinæ along with their food. When this occurs the cyst is dissolved by the digestive juices of the host, and the protoplasm of the spore is set free as a rounded body with a single nucleus (I), which rapidly grows into an adult Opalina (K).

Conjugation, in the form of a temporary union accompanied by interchange of micronuclei, has been described in Paramœcium (p. 90), and takes place in many Ciliata. In others (e.g. Stylonychia histrio) there is a complete union of the two gametes. In Vorticella union is also permanent, and takes place, not between two ordinary forms, but between one of the ordinary stalked individuals, or megagametes, and a free-swimming, small form, or microgamete, produced, as described above, by budding $\left(\mathrm{G}^{1}, \mathrm{G}^{2}\right)$. The essence of conjugation is the reception of nuclear material derived from another individual : its effect appears to be a renewal of vitality, usually manifesting itself in increased activity in multiplication by fission.

\section{Order 2.-Tentaculifera.}

Judged from the adult structure alone, the members of this order would certainly be placed in a separate class of the Protozoa: it is only in virtue of the facts of development that they are united in a single class with the Ciliata.

The body may be globular (Fig. 76, 1a), ovoid (1b), or cupshaped $(2 a)$, but presents nothing like the variety of form met with among the Ciliata. The distinguishing feature of the group is furnished by the tentacles which are always present in greater or less number, and which, in some cases at least, are the most highly differentiated organs found in the whole group of Protozoa. The characters of the tentacles vary strikingly in the different genera.

In the common forms Acineta (2), and Podophrya (1), the tentacles spring either from the whole surface, or in groups from the angles of the somewhat triangular body. Each tentacle is an elongated cylindrical structure (1c), capable of protrusion and retraction, and having its distal end expanded into a sucker. It is, moreover, practically tubular, the axial region consisting of a semi-fluid 
protoplasm, while the outer portion is tolerably firm and resistant. When partially retracted, a spiral ridge is sometimes observable

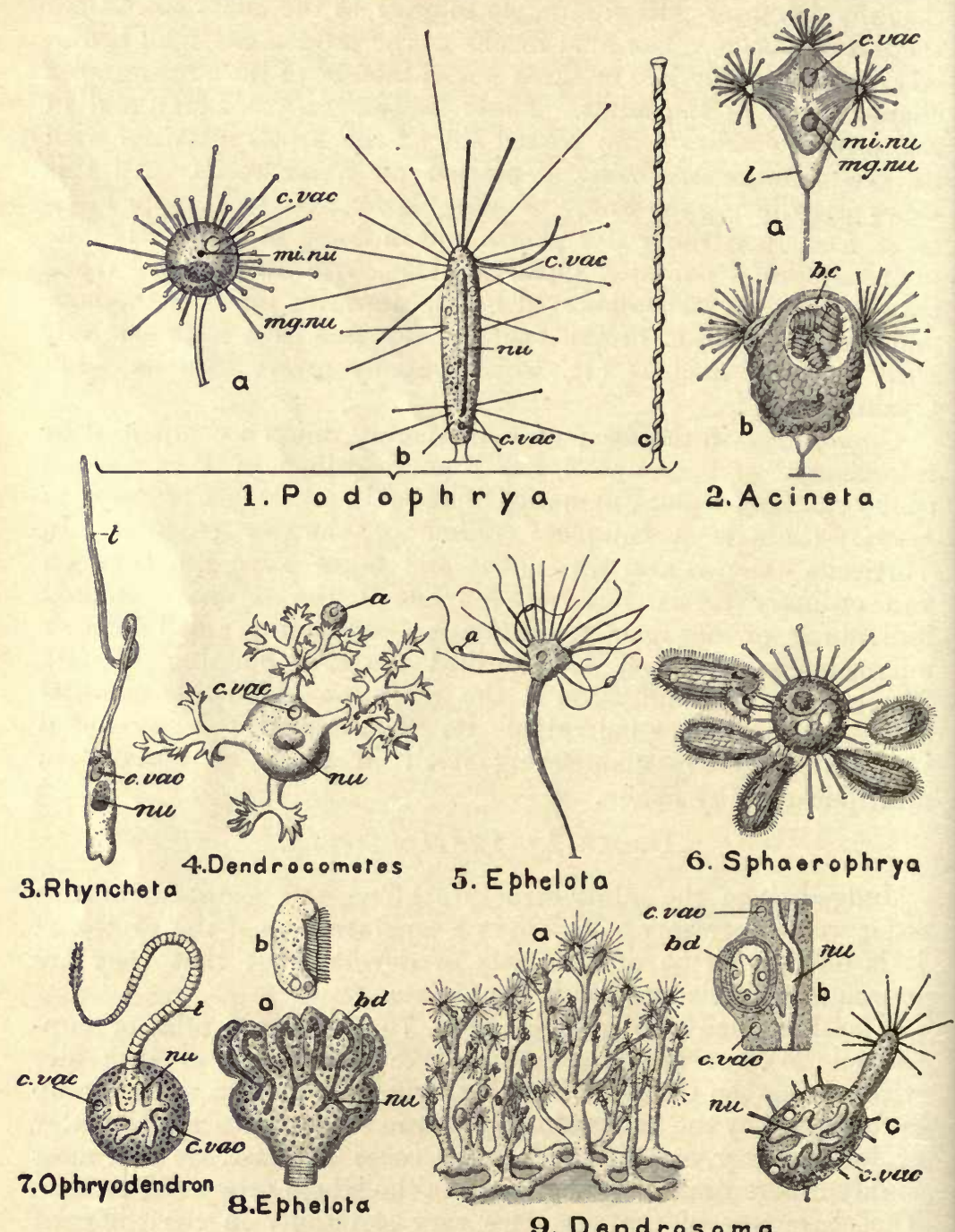

\section{Dendrosoma}

FIa. 76.-Various forms of Tentaculifera, $1 a$ and $b$, two specics of Podophrya; $c$, a tentacle much enlarged; $2 a$, Acineta jolyi; $2 b, \mathbf{A}$. tuberosa; in 6 the animal has captured several small Ciliata; $8 a$, a specimen multiplying by budding; $8 b$, a free ciliated bud; $9 a$, the entire colony ; $9 b$, a portion of the stem; $9 c$, a liberated bud; $a$, organism captured as food; b.c. brood-cavity; bd. bud; c. vac contractile vacuole; l. lorica; $n g$. nu. meganucleus; mi. $n u$. micronucleus; $t$. tentacle. (After Bitschli and Saville Kent.)

around the tentacle: this may indicate the presence of a band of specially contractile protoplasm, resembling the axial fibre in the 
stalk of Vorticella. Infusors and other organisms are caught by the tentacles $(4,6)$, the cuticle of the prey is pierced or dissolved where the sucker touches it, and the semi-fluid protoplasm can then be seen flowing down the tentacle into the body of the captor. A single tentacle only may be present (3), or the tentacle may be branched (4), the extremity of each branch being suctorial. In some forms there are no terminal suckers (5), and the tentacles are waved about to catch the prey instead of standing out stiffly as in Acineta. In other cases there are one or more long, striated tentacles with tufted ends ( 7$)$.

The nucleus may be ovoid (1a), horseshoe-shaped, or branched $(8,9)$ : in many cases a micronucleus $(1 \mathrm{a}, m i$. nu. $)$ has been found and it probably occurs in all. There are one or more contractile vacuoles (c. vcce.).

Some genera are naked (1): others form a stalked shell or lorica (2a) like that met with in many of the Mastigophora.

The only colonial form is the wonderful Dendrosoma (9), in which the entire colony attains a length of about $2 \mathrm{~mm}$., and bears an extraordinary resemblance to a zoophyte (vide Sect. IV.). It consists of a creeping stem from which vertical branches spring, and the various ramifications of these are terminated in Podophrya-like zooids with suctorial tentacles. The nucleus is very remarkable, extending as a branched axis throughout the colony (b, nu.). Micronuclei of the ordinary character are present as well.

Reproduction by binäry fission takes place in many species. In Ephelota gemmipara (8) a peculiar process of budding occurs: the distal end of the organism grows out into a number of projections or buds, into which branches of the nucleus extend. These become detached, acquire cilia on one surface, and swim off (b). After a short active existence tentacles appear and the cilia are lost. In this case budding is external, but in Acineta tuberosa $(2 \mathrm{~b})$ the buds become sunk in a depression, which is finally converted into a closed brood-cavity (b.c.): in this the buds take on the form of ciliated embryos, which finally escape from the parent. In Dendrcsoma the common stem of the colony produces internal buds $(b, b d$.).

Further Remarks on the Protozoa.

The majority of the Protozoa are aquatic, the phylum being equally well represented in fresh and salt water. They occur practically at all heights and depths, from 8,000 to 10,000 feet above"sea-level, to a depth of from 2,000 to 3,000 fathoms. Some forms, such as species of Amœba and Gromia, live in damp sand and moss, and may therefore be almost considered as terrestriai organisms. In accordance with their small size and the readiness with which they are transported from place to place a large pro- 
portion of genera and even of species are universally distributed, being found in all parts of the world where the microscopic fauna has been investigated.

Numerous parasitic forms are known. Besides the entire class of Sporozoa, species of Rhizopoda, Mastigophora, and of Infusoria occur both as internal and external parasites. Species of Amœba are common in the intestines of the higher animals, and one species has been found in connection with a cancerous disease in Sheep. A ciliate Infusor, Ichthyophthirius, is found in the skin of freshwater Fishes, where it gives rise to inflammation and death.

Many instances have been met with in our survey of the Phylum of compound or colonial forms, the existence of which seems at first sight to upset our definition of the Protozoa as unicellular animals. But in all such cases the zooids or unicellular individuals of the colony exhibit a quasi-independence, each, as a rule, feeding, multiplying, and performing all other essential animal functions independently of the rest, so that the only division of labour is in such forms as Zoothamnium and Volvox, in which certain zooids are incapable of feeding, and are set apart for reproduction. In all animals above Protozoa, on the other hand, the body is formed of an aggregate of cells, some of which perform one function, some another, and none of which exhibit the independent life of the zooid of a protozoan colony. It cannot, however, be said that there is any absolute distinction between a colony of unicellular zooids and a single multicellular individual: Proterospongia and Volvox approach very near to the border-land from the protozoan side, and a similar approach in the other direction is made by certain animals known as Mesozoa, which will be discussed hereafter (Sect. IV.). Moreover, the Mycetozoa, the plasmodia of which are formed by the fusion of Amœbulæ, the nuclei of the latter remaining distinct and multiplying, are rather non-cellular than unicellular. This point will also be referred to at the conclusion of the section on Sponges (Sect. III.).

In each division of the Protozoa we have found comparatively low or generalised forms side by side with comparatively high or specialised genera. For instance, among the Rhizopoda, there can be no hesitation in placing the Lobosa, and especially Protamœba, at the bottom of the list, and the Radiolaria at the top. Similarly, among the Mastigophora, such simple Flagellata as Oikomonas (Fig. 52,2 and 8) and Heteromita are obviously the lowest forms, Noctiluca and the Dinoflagellata the highest. But whether the Rhizopoda, as a whole, are higher or lower than the Flagellata, is a question by no means easy to answer. A flagellum certainly seems to be a more specialised cell-organ than a pseudopod, and some of the Mastigophora rise above the highest of the Rhizopoda in the possession of a firm cortex and cuticle, 
and the consequent assumption of a more definite form of body than can possibly be produced by the flowing protoplasm of a Foraminifer or a Radiolarian. On the other hand, the nucleus of the Radiolaria is a far more complex structure than that of the Mastigophora; and in Foraminifera, Radiolaria, and Heliozoa the organism frequently begins life as a flagellula, a fact which, on the hypothesis that the development of the individual recapitulates that of the race, appears to indicate that these orders of Rhizopoda are a more recently developed stock than at any rate the lower Flagellata. These circumstances, and the fact that Mastigamœba might equally well be classed as a lobose Rhizopod with a flagellum or as a Flagellate with pseudopods, seem to indicate that the actual starting-point of the Protozoa was a form

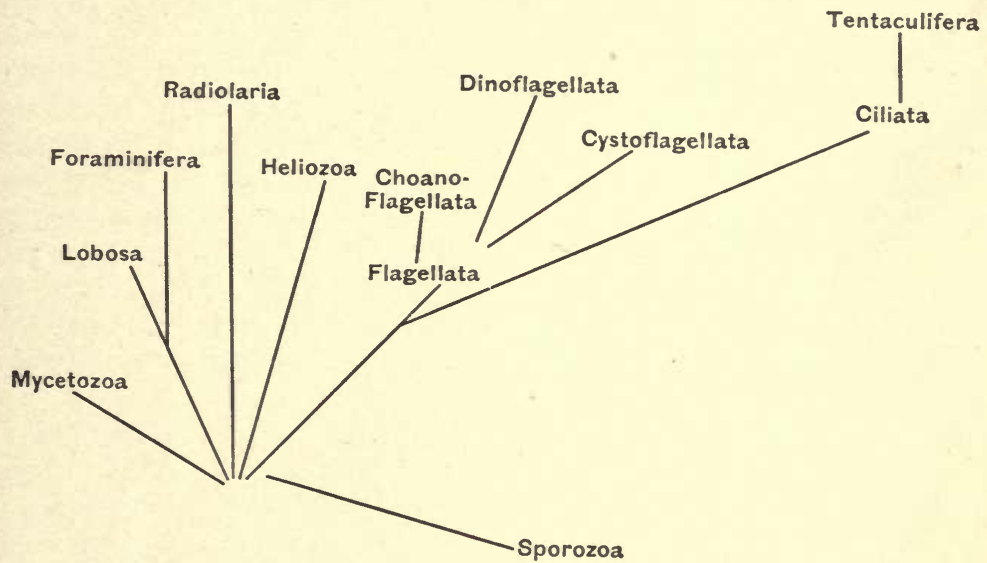

Fia. 77.-Diagram showing the mutual relationships of the chief groups of Protozoa.

capable of assuming either the amœboid or the flagellate phase. From such a starting-point the Lobosa, Foraminifera, Heliozoa, Radiolaria, and Flagellata diverge in different directions, the first four keeping mainly to the amœboid form, but assuming the flagellate form in the young condition, in the case of Foraminifera, Heliozoa, and Radiolaria.

The Choanoflagellata, Dinoflagellata, and Cystoflagellata are obviously special developments of the Flagellate type along diverging lines.

As to the Ciliata, Multicilia and Lophomenas (Fig. 71, 12 and 13) appear to indicate the derivation of the order from the Flagellate type, since their cilia are long and flageilum-like; but the evidence is not strong and no other is at hand. The derivation of the Tentaculifera from a ciliate type appears to be clear. The Tentaculifera and the hypotrichous Ciliata are undoubtedly the highest develop- 
ment of the Protozoan series, since they show a degree of differentiation attained nowhere else by a single cell.

The Mycetozoa appear to have been derived from the common amœboid-flagellate stock, since they are all predominantly amœboid in the adult condition, flagellate when young. The Sporozoa probably had a similar origin, but the characters of this class have evidently been profoundly modified in accordance with their parasitic mode of life.

The diagram on the previous page is an attempt to express these relationships in a graphic form. 


\section{SECTION III}

\section{PHYLUM AND CLASS PORIFERA [PARAZOA]}

The microscopic animals described in the preceding section are, as already repeatedly pointed ont, characterised by their unicellular character, and in this respect stand in contrast to the remainder of the animal kingdom. The animal kingdom is thus capable of division into two great subdivisions, the Protozoa or unicellular animals, and the Metazoa or multicellular forms - the latter comprising all the groups that remain to be dealt with. In the earliest stage of their existence all the multicellular animáls or Metazoa are, as already pointed out (p. 19), in a unicellular condition, originating in a single cell, the fertilised ovum or oosperm. By the process of segmentation or yolk-division the unicellular oosperm becomes converted in all the Metazoa into a mass of cells from which the body of the adult animal is eventually built up. Of the Metazoa, the group which approximates most closely to the Protozoa is that now to be dealt withthe Porifera or Sponges. With all the other multicellular groups the Sponges are so strongly in contrast that the Metazoa may be regarded as falling into two main divisions-the Porifer'a or Parazoa, on the one hand, and all the rest of the Metazoa, grouped together as Enterozoa, on the other.

\section{Example of the Class-Sycon gelatinosum.}

\section{General External Appearance and Gross Structure.-} Sycon gelatinosum, ${ }^{1}$ one of the Calcareous Sponges, has the form of a tuft, one to three inches long, of branching cylinders (Fig. 78),all connected together at the base, where it is attached to the surface of a rock or other solid body submerged in the sea. It is flexible, though of tolerably firm consistency; in colour it presents various shades of gray or light brown. To the naked eye the surface appears smooth, but when examined under the lens it is found to exhibit a pattern of considerable regularity, formed by the presence of

1 This species is an inhabitant of southern seas. In all essential respects the account of it given above will apply to $S$. ciliatum, a common European species which differs chiefly in the absence of the pore-membranes. 
innumerable elevations of a polygonal shape, which cover the whole surface and are separated off from one another by a system of depressed lines. In these depressions between the elevations are to be detected, under the microscope, groups of minute pores - the ostia or inhalant pores. At the free end of each of the cylindrical branches is a small but distinct opening, surrounded by what appears like a delicate fringe. When the branches are bisected longitudinally (Fig. 79), it is found that the terminal openings $(0)$ lead into narrow passages, wide enough to admit a stout pin, running through the axes of the cylinders; and the passages in the

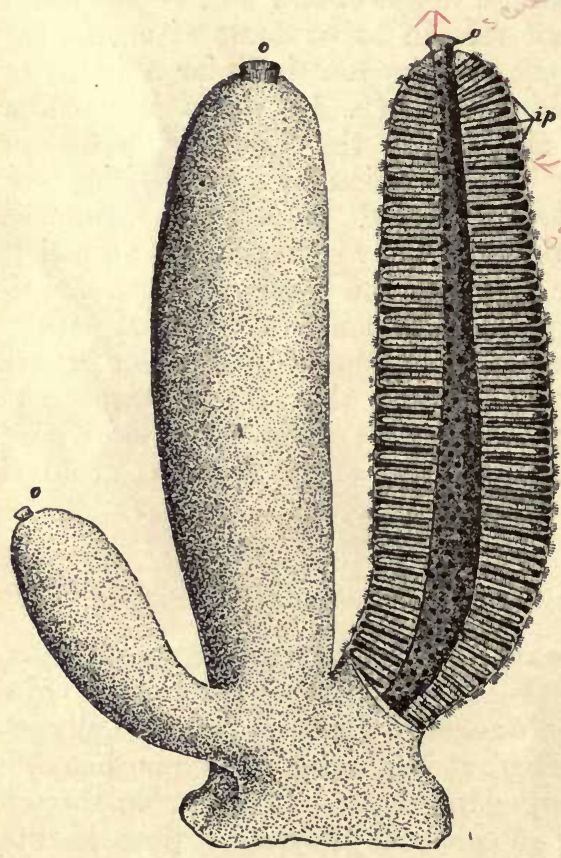

Fia. 79.-Sycon gelatinosum.-A portion slightly magnified; one cylinder (that to the right) bisected longitudinally to show the central paragastric cavity opening on the exterior by the osculum, and the position of the incurrent and radial canals; the former indicated by the black bands, the latter, dottedsip. marks the position of three of the groups of inhalant pores at the outer ends of the incurrent canals ; 0 . osculum.

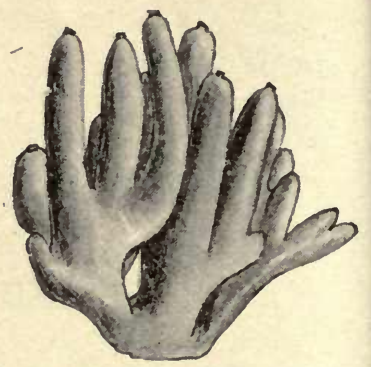

Fig. 78.-Sycon gelatinosum. -Entire sponge, consisting of a group of branching cylinders (natural size).

interior of the various branches join where the branches join-the passages thus forming a communicating system. On the wall of the passages are numerous fine apertures which require a strong lens for their detection. The larger apertures at the ends of the branches are the oscula of the sponge, the passages the paragastric cavitics. If a living Sycon is placed in sea-water with which has been mixed some carmine powder, it will be noticed that the minute particles of the carmine seem to be attracted towards the surface of the sponge, and will often be seen to pass into its substance through the minute inhalant pores or ostia already mentioned as occurring in groups between the elevations on 
the outer surface. This would appear to be duc to the passage of a current of water into the interior of the sponge through these minute openings dotted over the surface; and the movement of the floating particles shows that a current is at the same time flowing out of each of the oscula. A constant circulation of water would thus be seen to be carried on-currents moved by some invisible agency flowing through the walls of the sponge to the central paragastric cavities, and passing out again by the oscula.

If a portion of the Sycon is firmly squeezed, there will be pressed out at first sea-water, and then, when greater pressure is

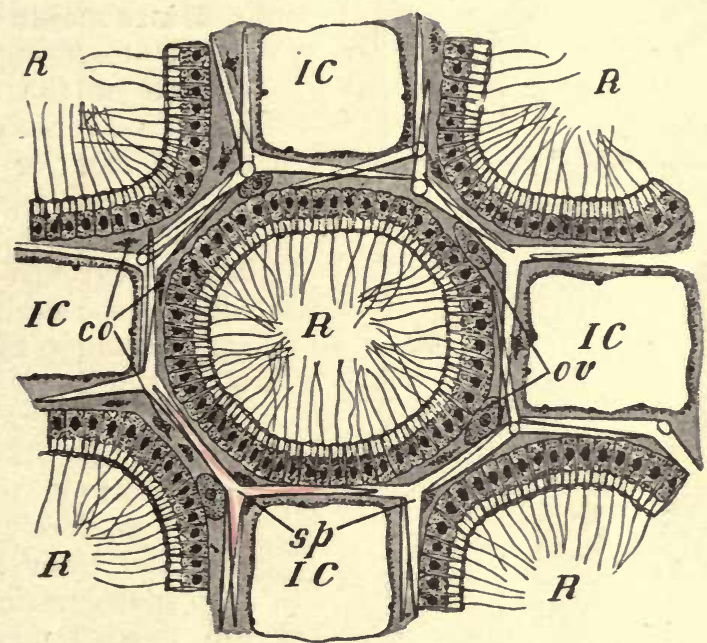

Fig. 80.-Sycon gelatinosum. Section through the wall of a cylinder taken at right angles to the long axes of the canals, highly magnified; co, collencytes; $I C$, incurrent canals; ov. young ova; $R$, radial canals; $8 p$. triradiate spicules.

exerted, a quantity of gelatinous-looking matter, which, on being examined microscopically, proves to be partly composed of a protoplasmic material consisting of innumerable, usually more or less broken cells with their nuclei, and partly of a non-protoplasmic, jelly-like substance. When this is all removed there remains behind a toughish felt-like material, which maintains more or less completely the original shape of the sponge. This is the skeleton or supporting framework. A drop of acid causes it to dissolve with effervescence, showing that it consists of carbonate of lime. When some of it is teased out and examined under the microscope, it proves to consist of innumerable, slender, mostly three-rayed microscopic bodies (Figs. 80 and 81, sp) of a clear glassy appearance. These are the calcareous spicules which form 


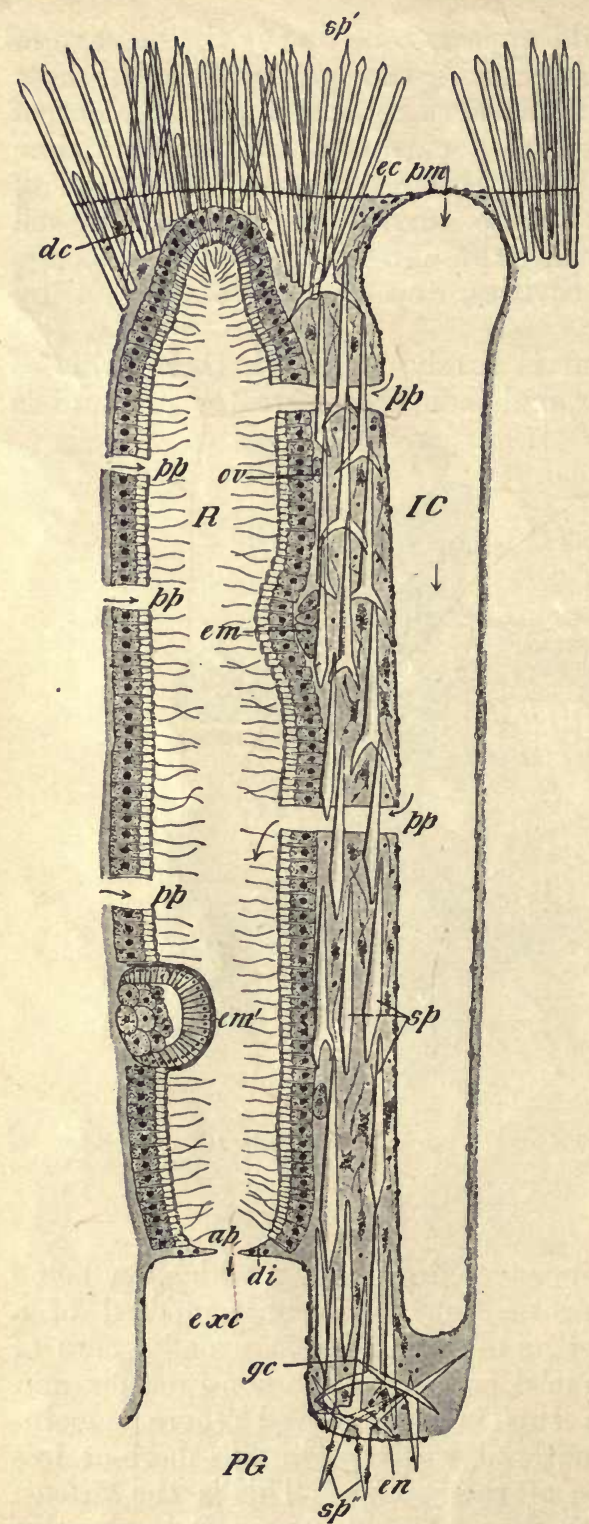

Fig. 81.-Sycon gelatinosum Transverse section through the wall of a cylinder (parallel with the course of the canals), showing one incurrent (IC), and one radial $(R)$ canal throughout their length; $s p$. triradiate spicules ; $s p^{\prime}$. oxeotc spicules of dermal cortcx $(d c$.$) ; s p^{\prime \prime}$. tetraradiate spicules of gastral cortex $(g c$.$) ; ec. ectoderm; en. layer of flattened cells$ lining the paragastric cavity ; pin. pore-membrane; $p p$. prosopyles; ap. apopyle ; di. diaphragm; exc. excurrent passage; P.G. paragastric cavity; em. early embryo; $\mathrm{em}^{\prime}$. late embryo. The arrows indicate the course of the water through the sponge. the skeleton of the Sycon.

The arrangement of the spicules, their relation to the protoplasmic parts, and the structure of the latter, have to be studied in thin sections of hardened specimens (Figs. 80 and 81). An examination of such sections leads to the following results.

\section{Microscopic struc-} ture.-Covering the outer surface of the sponge is a single layer of cells - the dermal layer or ectoderm ${ }^{1}$ (Fig. 81, ec)-through which project regularlyarranged groups of needlelike and spear-like spicules $\left(s p^{\prime}\right)$, forming the pattern of polygonal elevations on the outer surface. The cells of the ectoderm are in the form of thin scales, which are closely cemented together by their edges. The paragastric cavities are lined by a layer of cells (en) which are, like those of the ectoderm, thin flattened scales. Running radially through the thick wall of the cylinders are a large number of regularlyarranged straight passages. Of these there are two sets, those of the one set-the incurrent canals (Figs. 80

1 The terms ectoderm and endoderm are here used as convenient terms for the outer and inner layers of the Sponge, though, as will appear later, these layers differ completely in their mode of formation from the layers so named in the higher phyla. 
and $81 I C$ ) -narrower, and lined by ectoderm similar to the ectoderm of the surface; those of the other set-the radial or flagellate canals $(R)$-rather wider, octagonal in cross-section, and lined by endoderm continuous with the lining of the paragastric cavity. The incurrent canals end blindly at their inner extremities-not reaching the paragastric cavity; externally each becomes somewhat dilated, and the dilatations of neighbouring canals often communicate. These dilated parts are closed externally by a thin membranethe pore-membrane (Fig. $81 \mathrm{pm}$, and Fig. 82), perforated by three or four small openings (Fig. $82, p$ ) - the ostia already referred to. The flagellate canals are blind at their outer ends, which lie at a little distance below the surface opposite the polygonal projections referred to above as forming a pattern on the outer surface; internally, each communicates with the paragastric cavity by a short, wide passage - the excurrent canal (Fig. 81 exc). Incurrent

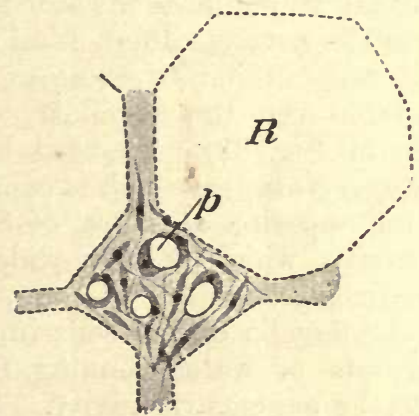

Fio. 82.-Sycon gelatinosum. Surface view of a pore-nembrane highly magnified : $p$. ostium; $k$. position of the outer end of a radial canal.

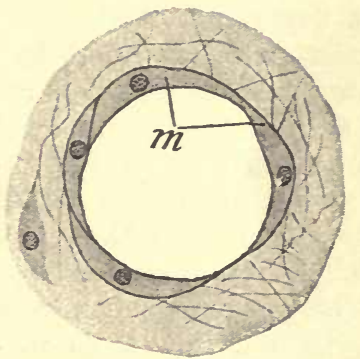

Fio. 83.-Sycon gelatinosum. An apopyle surrounded by its dia. phragm ; $m$. contractile cells.

and flagellate canals run side by side, separated by a thin layer of sponge substance except at certain points, where there exist small apertures of communication-the prosopyles $(p p)$,- - uniting the cavities of adjacent incurrent and flagellate canals. Each prosopyle is a perforation in a single cell termed a porocyte.

The ectoderm lining the incurrent canals is of the same character as that of the outer surface. The endoderm of the flagellate canals, on the other hand, is totally different from that which lines the paragastric cavity. It consists of cells of columnar shape ranged closely together so as to form a continuous layer. Each of these flagellate cndoderm cells, or collared cells, or choanocytes, as they are termed, is not unlike one of the Choanoflagellate Protozoa (p. 77); it has a nucleus, one or more vacuoles, and, at the inner end, a single, long, whip-like flagellum, surrounded at its base by a delicate, transparent, collar-like upgrowth, similar to that which has already been described as occurring in the Choanoflagellata. If a portion of a living specimen of the sponge 
is teased out in sea-water, and the broken fragments examined under a tolerably high power of the microscope, groups of these collared cells will be detected here and there, and in many places the movement of the flagella will be readily observed. The flagellum is flexible but with a certain degree of stiffness, especially towards the base, and its movements resemble those which a very supple fishing-rod is made to undergo in the act of casting a long line-the movement being much swifter and stronger in the one direction than in the other. The direction of the stronger movement is seen, when some of the cells are observed in their natural relations, to be from without inwards. It is to these movements that the formation of the currents of water passing along the canals is due. The collars of the cells in specimens teased in this way become for the most part drawn back into the protoplasm.

The short passage or excurrent canal, which leads inwards from the flagellate canal to the paragastric cavity, differs from the former in being lined by flattened cells similar to those of the paragastric cavity; it is partly separated from the flagellate canal by a thin diaphragm (Fig. 81, di, and Fig. 83), perforated by a large circular central aperture-the apopyle $(a p)$-which is capable of being contracted or dilated: its opposite aperture of communication with the paragastric cavity, which is very wide, is termed the gastric ostium of the excurrent canal.

The effect of the movement of the flagella of the cells in the flagellate canals is to produce currents of water running from without inwards along the canals to the paragastric cavity. This causes water to be drawn inwards through the prosopyles from the incurrent canals, and, indirectly, from the exterior through the perforated membranes at the outer ends of the latter.

Between the ectoderm of the outer surface and of the incurrent canals, and the endoderm of the inner surface and of the flagellate canals, are a number of spaces filled by an intermediate layerthe mesogloca-in which the spicules of the skeleton are embedded. Each spicule is developed from cells termed scleroblasts, which migrate inwards from the ectoderm. Each ray is formed by the agency of a separate scleroblast, so that there are three at least of the latter for each triradiate, and four for each tetraradiate spicule. The spicules (Figs. 80 and $81, s p$ ) are regularly arranged, and connected together in such a way as to protect and support the soft parts of the sponge. Most are, as already noticed, of triradiate form. Large numbers, however, are of simple spear-like or club-like shape $\left(s p^{\prime}\right)$; these, which are termed the oxeote spicules, project on the outer surface beyond the ectoderm, and are arranged in dense masses, one opposite the outer end of each of the ciliated canals, this arrangement producing the pattern already referred to as distinguishable on the 
out surface. The thick outer layer in which the bases of these oxeote spicules lie embedded, is termed the dermal cortex $(d c)$. A thick stratum at the inner ends of the canals and immediately surrounding the paragastric cavity is termed the gastral cortex $(g c)$. It is supported by triradiate and also by tetraradiate spicules, one ray of each of which $\left(s p^{\prime \prime}\right)$ frequently projects freely into the paragastric cavity, covered over by a thin layer of flattened endoderm cells.

The mesoglœa itself, as distinguished from the spicules which lie embedded in it, consists of a clear gelatinous substance containing numerous nucleated cells of several different kinds. Most of these are small cells of stellate shape, with radiating processes-the conncctive-tissue cells or collencytes (Fig. ১0, co); others are fusiform; a good many-the amœboid wandcring cellsare Amœba-like, and capable of moving about from one part of the sponge to another.

Around the inhalant pores and the apopyles are elongated cells (Figs. 82 and 83), sometimes prolonged into narrow fibres. These are contractile-effecting the closure of the apertures in question,and are therefore to be looked upon as of the nature of muscular. fibres. In the case of the inhalant pores they are ectudermal; in that of the apopyles they are endodermal. A band of similar fibres surrounds the osculum-the oscular sphincter.

The sexual reproductive cells - the ova (Figs. 80 and 81,ov) and sperms - are developed immediately below the flagellate endoderm cells of the flagellate canals, and in the same situation are to be found developing embryos $\left(\mathrm{cm}, \mathrm{em}^{\prime}\right)$, resembling in their various stages those of Sycon rophanus, as described below.

\section{2.-Distinctive Characters and Classification.}

Sponges are plant-like, fixed, aquatic Metazoa, all, with the exception of one family, inhabitants of the sea. The primary form is that of a vase or cylinder, the sides of which are perforated by a number of pores and in the interior of which is a single cavity; but in the majority of Sponges a process of branching and folding leads to the formation of a structure of a much more complex character. The surface of the Sponge is covered by a single layer of flattened cells - the ectodcrm ${ }^{1}$-and the internal cavities, or a part of them, are lined by a second single layer-the cndodermpart or the whole of which consists of a single layer of choanocytes, i.e. columnar collared cells, each provided internally with a long flagellum. Between these two layers is a quantity of tissue usually of a gelatinous consistency-the mesogloca-containing a number of cells of various kinds. The wall of the Sponge is pierced by a number of apertures. The skeleton or supporting

I See footnote on p. 108. 
framework, developed in the mesuglœea from cells derived from the ectoderm, consists in some cases of fine, flexible fibres of a material termed spongin; in others of spongin-fibres supplemented by microscopic siliceous spisules; in others of siliceous spicules alone; in others of spicules of carbonate of lime. Reproduction takes place both asexually by the formation of gemmules, and sexually by means of ova and sperms. The ovum develops into a ciliated free-swimming larva, which afterwards becomes fixed and develops into the plant-like adult Sponge.

The Sponges are sufficiently far removed in structure from the rest of the Metazoa to justify us in louking upon them as constituting one of the great divisions or phyla of the animal kingdom. At the same time there is so much uniformity of structure within the group that a division into classes is not demanded; the phylum Porifera contains a single class.

The class Porifera is classified as follows :-

\section{Sub-Class I.-Calcarea.}

Sponges with a skeleton of calcareous spicules, and with comparatively large collared cells.

\section{ORDER 1.-HOMOCELA}

Calcareous Sponges in which the internal lining membrane consists throughout of flagellate collared cells.

\section{Order 2.-Heteroccela.}

Calcareous Sponges in which the paragastric cavity is lined by flattened cells, the collared cells being restricted to flagellate canals or chambers.

\section{Sub-Class II.-Hexactinellida.}

Sponges with six-rayed, tri-axon, siliceous spicules, and simple canal system represented by unbranched or branched flagellate chambers.

\section{Sub-Class III.-Demospongia.}

Sponges either devoid of skeleton or with spongin fibres alone, or a combination of spongin fibres and siliceous spicules, the latter, when present, never six-rayed; the canal system of the Rhagon type (p. 118), usually complicated.

\section{Systematic Position of the Example.}

Sycon gelatinosum is one of many species of the genus Sycon. Sycon is one of several genera of the family Sycettidoe; and the 
family Sycettidae is one of several families of the order Heterocoela of the class Calcarea. Among the families of the Heteroccela, that of the Sycettide is distinguished by the following features, which characterise all its members:-

"The flagellate chambers are elongated, arranged radially around a central paragastric cavity, their distal ends projecting more or less on the dermal surface, and not covered over by a continuous cortex. The skeleton is radially symmetrical."

Of the genera into which the Sycettidae are divided, Sycon is characterised as follows:-

"The flagellate chambers are not intercommunicating; their distal ends are provided each with a tuft of oxeote spicules."

The members of one of the other genera of the family-Sycettawhile possessing the general characteristics of the family, differ from those of the genus Sycon in wanting the tufts of oxeote spicules; those of a third-Sycantha-have the flagellate chambers united in groups; the chambers of each group intercommunicating by openings in their walls, and each group having a single common opening into the gastric cavity. The members of this genus resemble Sycon, and differ from Sycetta, in the presence of tufts of oxeote spicules at the distal ends of the flagellate chambers.

These distinctions between classes, orders, families, and genera are of an entirely arbitrary character. No such divisions exist in nature; and they are merely established as a convenient way of grouping the sponges and facilitating their classification. But a classification of this kind, if carried out on sound principles, should nevertheless have something corresponding to it in nature, inasmuch as the grouping of the various divisions and subdivisions aims at expressing the relationships of their members to one another. The members, for example, of the family Sycettidce are all regarded, on account of the features which they possess in common, as being more nearly related to one another than to the members of the other families, and as having been derived from a common ancestor which also possessed those features-the divergences of structure which we observe in the different genera and species being the result of a gradual process of change.

Within the limits of the genus Sycon, S. gelatinosum is distinguished from the rest as a group of individual Sponges all possessing certain specific characters which it will be unnecessary to detail here. But the individual Sponges referable to this species frequently differ somewhat widely from one another: there are numerous individual variations. If we compare a number of specimens all possessing the species-characters of Sycon gelatinosum, we find that they differ in the number of branches, in the shape of the cylinders-some being relatively narrow, some relatively wide - in the degree of development of the oscular crown of spicules, in the ratio of the thickness of the wall to the width 
of the contained paragastric cavities, and in many other more minute points; in fact, we find as a result of the comparison that no two specimens are exactly alike. These differences are so great that some very distinct races or varietics of $\mathrm{S}$. gelatinosum have been recognised, and some have received special names. Here again, as in the case of the families and orders, the distinctions are of an arbitrary character-some writers on Sponges setting down as several species what others regard merely as varieties of one species. It is impossible, in fact, to draw a hard and fast line of distinction between species and varieties. In the higher groups of animals the attempt is made to establish a physiological distinction; all the members of a species are regarded as being fertile inter se, and capable of producing fertile offspring as a result of their union; but such a mode of distinguishing species is impossible of application among lower forms such as the sponges. In these lower groups, accordingly, a species can only be defined as an assemblage of individuals which so closely resemble one another that they might be supposed to be the offspring of a parentform similar to themselves in all the most essential features. And, according to the view taken of the relative importance of different points of colour, shape, and internal structure, the conceptions of the species and their varieties and mutual relationships formed by different observers must often differ widely from one another.

\section{General Organisation.}

General Form and Mode of Growth.-The simplest Sponges are vase-shaped or cylindrical in form, either branched or unbranched; and, if branched, with or without anastomosis or coalescence between neighbouring branches. But the general form of the less simple Sponges diverges widely from that of such a branching cylinder as is presented by Sycon gelatinosum (Fig. 78).

From the point to which the embryonic sponge becomes attached it may spread out horizontally, following the irregularities of the surface on which it grows, and forming a more or less closely adherent encrustation like that of an encrusting lichen (Fig. 84,A). The surface of such an encrustation may be smooth; more commonly it is raised up into elevations-rounded bosses, cones, ridges or lamellæ; and the edges may be entire or lobed. In other cases the sponge grows at first more actively in the vertical than in the horizontal direction, and the result may be a long, narrow structure, cylindrical or compressed, and more or less branched (Fig. 84, B'). Sometimes vertical and horizontal growth is almost equal, so that eventually there is formed a thick, solid mass of a rounded or polyhedral shape (Fig. 84, C), with an even, or lobed, or ridged surface. Very often, after active vertical growth 
has resulted in the formation of a comparatively narrow basal part or stalk, the Sponge expands distally, growing out into lobes or branches of a variety of different forms, and frequently anastomosing. Sometimes, after the formation of the stalk with rootlike processes for attachment, the Sponge grows upwards in such a way as to form a cup or tube with a terminal opening. Such a

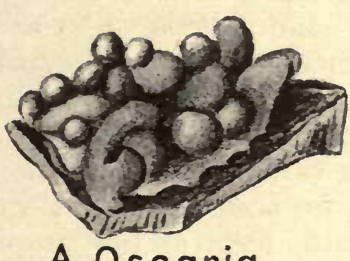

A. Oscaria

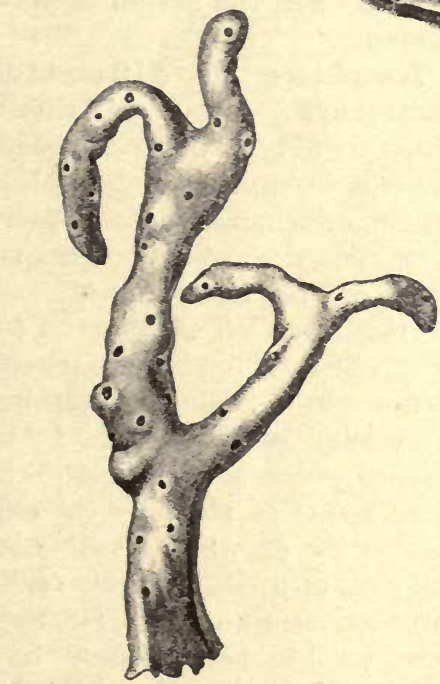

B.Psammoclema
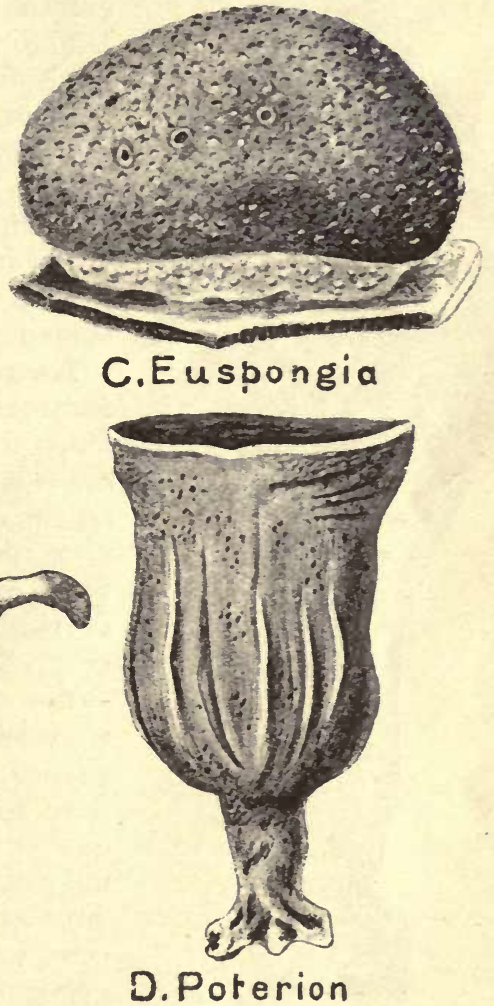

Fig. 34. - External form of various Sponges. A, Oscaria, an encrusting form, with the upper surface raised up into a number of rounded prominences; $B$, Psammoclema, a ramifying subcylindrical Sponge; $C$, Euspongia (toilet sponge), a massive form with a broad base; D, Poterion (Neptune's Cup), an example of a complex Sponge assuming the form of a vase. (After Vosmaer.)

cup-shaped Sponge, exemplified in the gigantic Neptune's Cup (Poterion, Fig. 84, D), is not to be confounded with the simple vase or cup referred to above as the simplest type of Sponge, being a much more complex structure with many oscula. Sometimes the Sponge grows from the narrow base of attachment into a thin flat plate or lamella; this may become divided up into a number of parts or lobes, which may exhibit a divergent arrange- 
ment like the ribs of an open fan. Often the lamella becomes folded, and sometimes there is a coalescence between the folds, resulting in the development of a honey-comb-like form of sponge.

Sponges resemble plants, and differ from the higher groups of animals, in the readiness with which, in many cases, their form

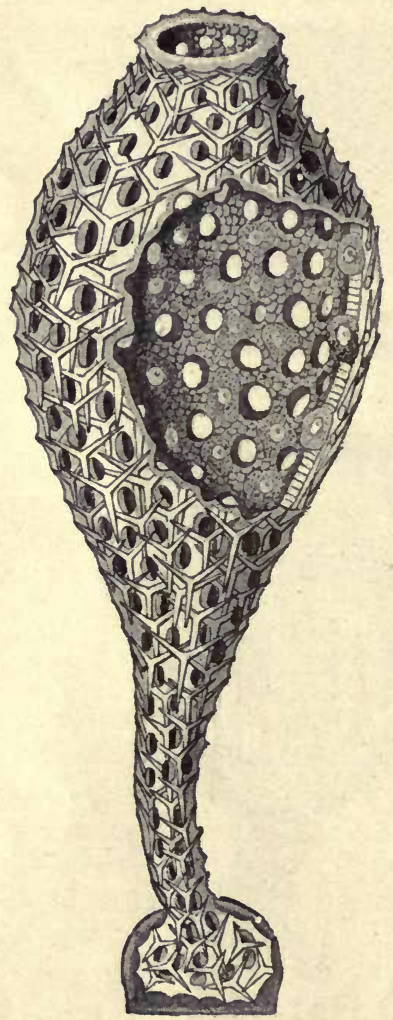

Fı. 85.-Olynthus stage of a simple calcareous Sponge (Clathrina). A portlon of the wall of the vase-like sponge removed to show the paragastric cirvity. (After Hacckel.) becomes modified during growth by external conditions (environment). Different individuals of the same kind of Sponge, while still exhibiting the same essential structure and the same general mode of growth, may present a variety of minor differences of form, in accordance with differences in the form of the supporting surface or in the action of waves and currents.

\section{Leading Modifications of} Structure.-Sycon gelatinosum belongs to a type of Sponges intermediate between the very simplest forms on the one hand, and the more complex on the other. The simplest type of Sponge-structure is that of the so-called Ascetta or Olynthus (Fig. 85). This is not a mature form -no adult Sponge retaining such simplicity of structure. It is vaseshaped, contracted at the base to form a sort of stalk by the expanded extremity of which it is attached; at the opposite or free end is the circular osculum. So far there is a considerable resemblance to Sycon gelatinosum; but the structure of its wall in Ascetta is extremely simple. Regularly arranged over the surface are a number of small rounded apertures, the inhalant pores; but, since the wall of the Sponge is very thin, these apertures lead directly into the central or paragastric cavity (Fig. $86 \mathrm{~A}$ ), the long passages or canals through which the communication is effected in Sycon being absent. The wall consists of the same three layers as in Sycon, but the middle one, though it contains a small number of spicules, is very thin. The ectoderm is a thin layer of flat cells; the paragastric cavity is lined throughout by choanocytes similar to those of the flagellate canals of Sycon. 
A somewhat more complex type of structure than that of Ascetta is exhibited by those sponges in which the wall becomes thickened and perforated by radially-arranged canals, which open directly on the outersurface by means of inhalant porcs or ostia, and lead directly into the paragastric cavity by means of "popylesthe whole inner surface as well as the radial canals being lined with flagellate endoderm cells. In forms which may be regarded as representing the next stage of development (Fig. $86, B$ : see also the figures of Sycon gelatinosum), there are formed by infolding of the surface, in the intervals between the radial canals, canallike spaces, the incurrent cancels, lined by ectoderm and communicating with the exterior on the one hand, either by a wide opening or by pores (ostia) perforating a pore-membrane, and on the other by means of small openings, the prosopyles (the equivalents of the inhalant pores of the Olynthus), with the radial canals. Sponges similar to Sycon gelatinosum,
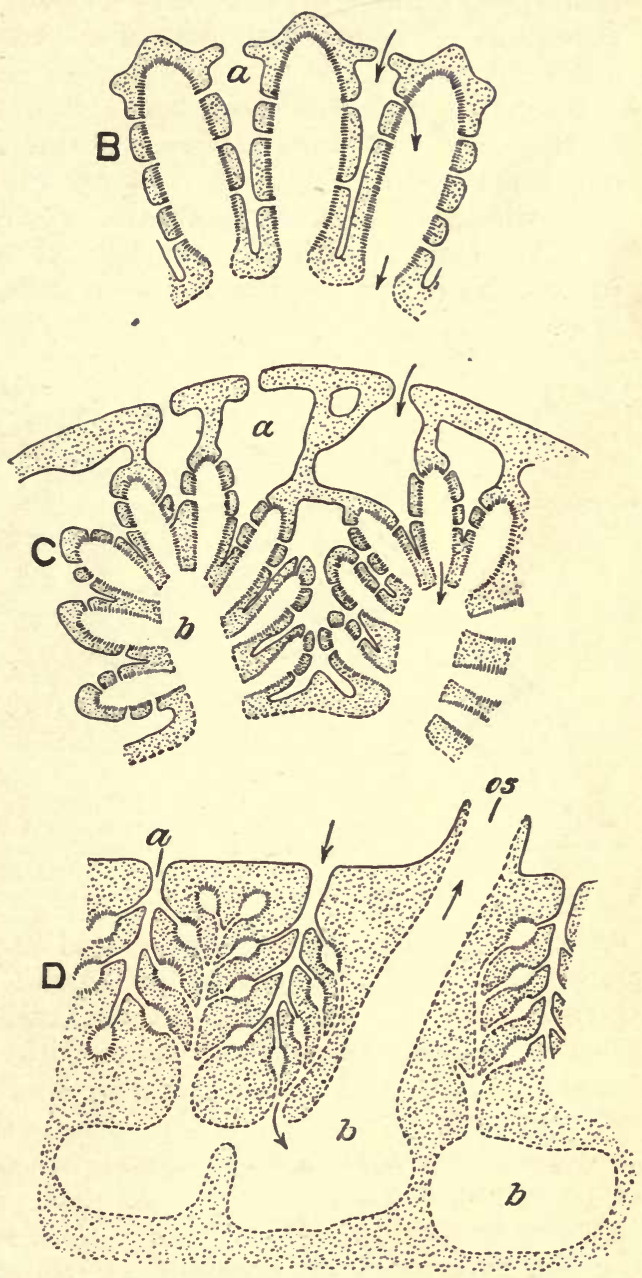

FiG. 86.-Diagram of the canal system of various sponges, the ectoderm denoted by a continuous narrow line; the flattened endoderm by an interrupted line; the flagellate endoderm by short parallel strokes. $A$, cross-section through a part of the wall of an Ascon; $B$, cross-section through a part of the wall of a Sycon; $C$, cross-section through a part of the wall of Leucilla convexa; $D$, vertical section through Oscarella: $a$, spaces of the incurrent canal system ; $b$, spaces of the excurrent canal system ; os. osculum. (After Korschelt and Heider.) 
but with flagellate canals arranged in groups, each group centred round a main excurrent canal (Fig. 86, C) afford us the next grade of advancing complexity. In these the incurrent canals may form a branching system. In all the higher groups of Sponges (Fig. 86, D and Fig. 87) the flagellate cells are confined to certain special enlargements of the canals-the so-called "ciliated chambers" $(C)$-and the rest of the canals are lined by flattened cells.

Special names have been applied to the main types of canalsystem briefly sketched above. Forms in which the paragastric cavity is lined by flagellate cells are said to belong to the Ascon type, whether the paragastric cavity communicates directly or by flayellate canals with the exterior. Forms in which there is a paragastric eavity lined by flattened cells, and a system of radially

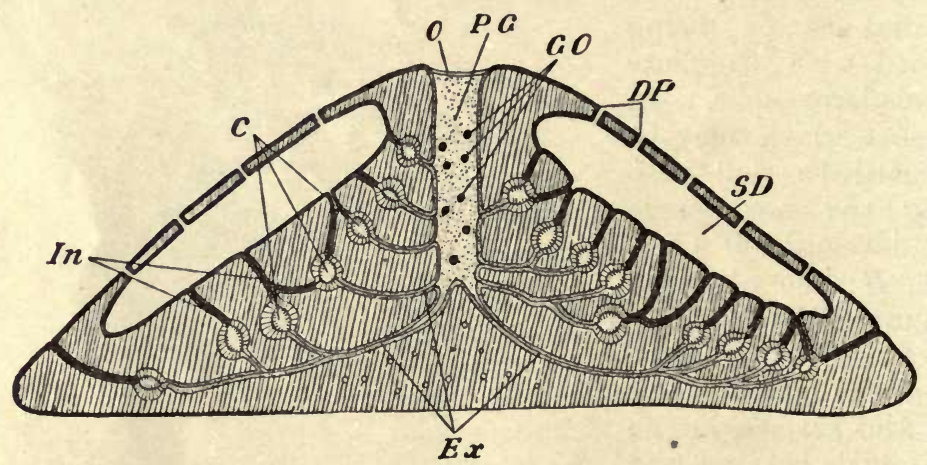

Fia. 87.-Vertical section of a fresh-water sponge (Sponzilla), showing the arrangement of the canal-system. $C$. ciliated chambers; $D P$. dermal pores; $E x$. excurrent canals; $G O$. openings of the excurrent canals; $P G$. paragastric cavity; $S D$. subdermal cavities; 0 . osculum. (Modified from Lcuckart and Nitsche's diagrams.)

arranged flagellate chambers, are said to possess the Sycon type of structure. Such Sponges as have small rounded flagellate chambers ("ciliated chambers"), communicating in most cases by narrow branching incurrent canals with the exterior (directly or indirectly) on the one hand, and by similar excurrent canals with the paragastric cavity on the other-the flagellate cells being confined to the flagellate chambers - are said to possess the Rhagon type of canal-system. In the liliagcn proper the arrangement $o_{1}$ parts is very simple. The Sponge has a paragastric cavity opening on the exterior by an osculum. Opening into this central cavity by wide apopyles are a number of rounded chambers each communicating with the exterior by an inhalant pore (prosopyle).

The development of branches from the originally simple Sponge, and the coalescence of neighbouring branches with one another, greatly obscure the essential nature of the Sponge as a colony or zooids similar to the branches of Sycon gelatinosum; and this effect 
is increased by the development of a-variety of infoldings of the ectoderm which appear in the higher forms. The oscula distributed over the surface of the mass may indicate the component zooids, but these are not always recognisable, being carried inwards by the infoldings or closed up altogether.

A thicker or thinner specialised outer layer-the dcrmal cortex - situated immediately below the superficial ectoderm, is present in many Sponges. This is a layer of mesogloea with special skeletal clements, usually containing spaces and canals lined by ectoderm-(subdermal cavitics, Fig. 87, SD) -which communicate directly with the exterior, and, internally, usually with more deeply situated spaces (subcortical cavilics), from which the incurrent canals lead to the ciliated chambers. This dermal cortex is present, though not highly developed, in Sycon gelatinosum (Fig. 81, dc), and the enlarged outer ends of the incurrent canals lying in the dermal cortex and closed externally by the porebearing membrane, may be regarded as representing dermal cavities. In most higher sponges a special inner layer is developed; this is the gastral cortex, represented in a rudimentary form in Sycon gelatinosum (Fig. 81, gc.) as the internal layer with special spicules, in which the excurrent canals are situated.

Histology.-In the protoplasmic elements or cells of the various groups of Sponges there is little variation, except in minor points. The cells of the ectoderm (Fig. 88) are flattened, and very rarely assume other forms; in some cases each flattened ectodermal cell is provided with a flagellum. Lining the paragastric cavities and canals is a layer of flattened cells similar to those of the ectoderm, or of flagellate collared cells. In the gelatinous substance of the mesoglœa are embedded connective-tissue cells, amœboid wandering cells, and, in certain positions (around orifices), muscle-cells. Unicellular glands (see p. 25) are present in some sponges, both calcareous and siliceous; also cells containing the pigment to which the bright colour of many sponges is due, though in most cases the pigment is not confined to special cells, but occurs scattered through the connective-tissue cells and flagellate cells. Fresh-water Sponges are green, owing to the presence of chlorophyll, the colouring matter to which the prevailing green colour of plants is due. 
The elements of the skeleton differ in character in the different classes. In the Calcarea they consist of calcareous spicules, usually tri-radiate in form. Each of these spicules is developed from special

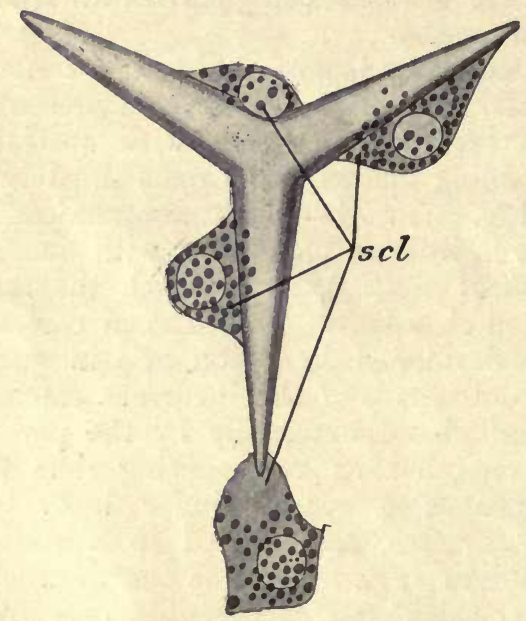

Fia. 89.-Development of a tri-radiate spicule of

Clathrina. $8 c l$, scleroblasts. (After Minchin.) cells - the sclcroblasts (Fig. 89). In the remaining groups of Sponges the skeleton either consists of spongin fibres alone (Fig 90, A), or of siliceous spicules alone, or of a combination of spongin fibres with siliceous spicules (B): in some Demospongia (the Myxospongia) skeletal parts are altogether absent. Spongin is a substance allied to silk in chemical composition: the fibres are exceedingly fine threads, consisting of a soft granular core and an outer tube of concentric layers of spongin. These threads branch and anastomose, or are woven and felted together in such a way as to form a firm, elastic, supporting structure. They are secreted by the activity of certain cells in the mesogluea which are called the sponginblasts, derived from the ectoderm. In certain exceptional cases the spongin assumes the form of spicules. The siliceous spicules (Fig. 91) are much more varied in shape than the spicules of the Calcarea, and in a single kind of Sponge there may be a number of widely differing forms of spicules, each form having its special place in the skeleton of the various parts of the Spongebody. In most forms siliceous spicules and spongin fibres combine to form the supporting franework, the relative development of these two elements varying greatly in different cases. But in certain groups, including the common Washing-sponges (Fig. $90 \mathrm{~A}$ ), spicules are completely absent, and the entire skeleton consists of spongin. In some forms which are devoid of spicules, the place of these is taken by foreign bodiesshells of Radiolaria, grains of sand, or spicules from other sponges (Fig. 90, C). In others, again, such as the Venus's Flower-Basket (Euplectella), the Glass-Rope Sponge (Hyaloncma), and Phermema (Fig. 92), the skeleton consists throughout of siliceous spicules bound together by a siliceous cement.

Reproduction in the Sponges is effected either sexually or asexually. The process by which, in all but the simplest forms of Sponges, a colony of zooids is formed from the originally simple 
cylinder or vase, may be looked upon as an asexual mode of reproduction by budding. In some cases asexual multiplication also takes place by the production of external buds; in others of internal buds in the shape of groups of cells called gemmules, which eventually become detached and develop into new individuals. In the Fresh-

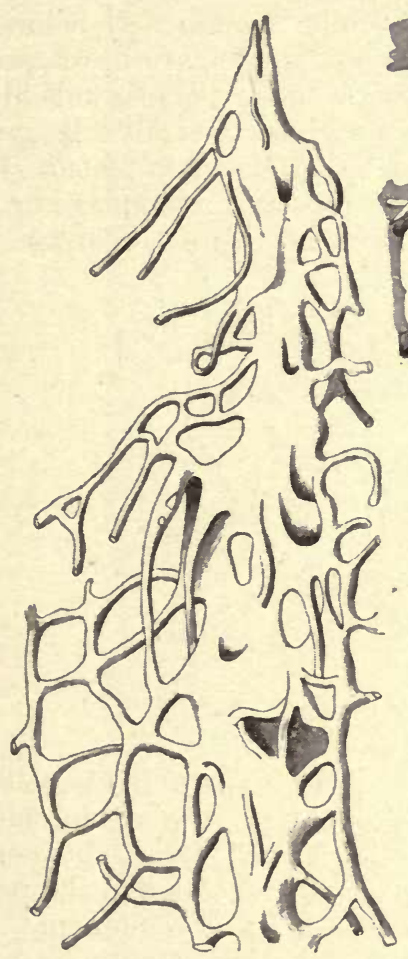

A.Euspongia

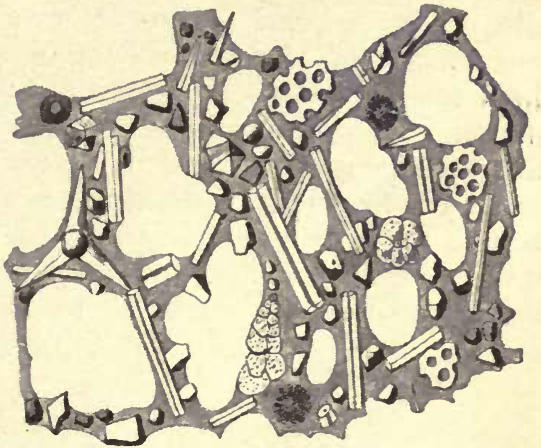

C. Spongelia

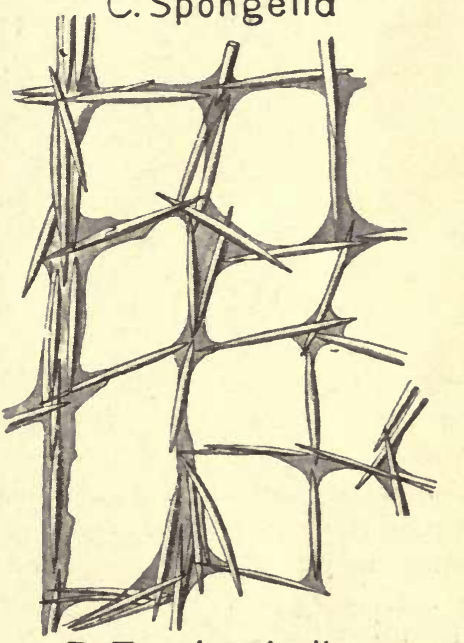

B.Pachychalina

Fig. 90 -Microscopic structure of the skeleton in various sponges. $A$, Euspongia, network of spongin fibres; $B$, Pachychalina, spongin strengthened by siliceous spicules; $C$, Spongelia, spongin strengthened by various foreign siliceous bodies, fragments of spicules of other sponges, \&c. (After Vosmaer.)

water Sponges (Spongillida) multiplication takes place very actively by means of such gemmules, each of which is a spherical group of cells enclosed in an envelope composed of peculiarly shaped siliceous spicules, termed amphidiscs (Fig. 91, right side). These gemmules are formed in the substance of the Sponge towards the end of the 
year; they are set free by the decay of the part of the parent sponge in which they are developed, and fall to the bottom. In spring the contained mass of protoplasmic matter reaches the exterior through an aperture in the wall of the gemmule, and develops into the adult form.

All Sponges multiply by a sexual process-by means of male cells, or spcrms, and fernale cells, or ova. These are developed from certain of the amœboid wandering cells of the mesoglœa, which take up a special position, usually immediately below the collared cells of the endoderm. Ova and sperms are developed in the same Sponge, but rarely at the same time. The anюboid cell destined to form sperms divides into a number of small cells, giving rise to a rounded mass of sperms. The latter, when mature, have oval or pear-shaped heads and a long tapering appendage or tail. Each amœboid cell destined to form an ovum enlarges, and

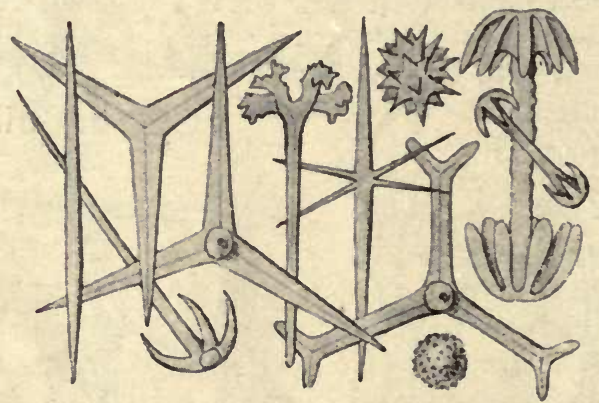

Fig. 91.-Various forms of sponge spicules. (From Lang's Text-Book.)

eventually assumes a spherical form. After a sperm has penetrated into its interior and effected impregnation, the ovum usually becomes enclosed in a brood-capsule formed for it by certain neighbouring cells, and in this situation, still enclosed in the parent Sponge, it undergoes the earlier stages of its development. The boring Sponge, Cliona, is the only one, so far as known, in which the early stages of development are passed through externally.

In all known cases there is a free-swimming ciliated larval stage; but the form assumed by the larva differs profoundly in different Sponges. Of the simpler types of calcareous sponges with a structure resembling that of the Uiynthus, the development has been followed out in the case of Clathrina blanca. In this sponge segmentation is followed by the formation of an oval blastula, the wall of which consists of a single layer of cells all alike in character--elongated, columnar, and flagellate. At one pole of the blastula is seen a pair of cells which are of a different character, being large, rounded, and granular. These are destined to give rise to the archococytes, some of which form the repro- 
ductive cells. Certain of the flagellate cells then withdraw their flagella and pass into the internal cavity, becoming amœboid. Soon

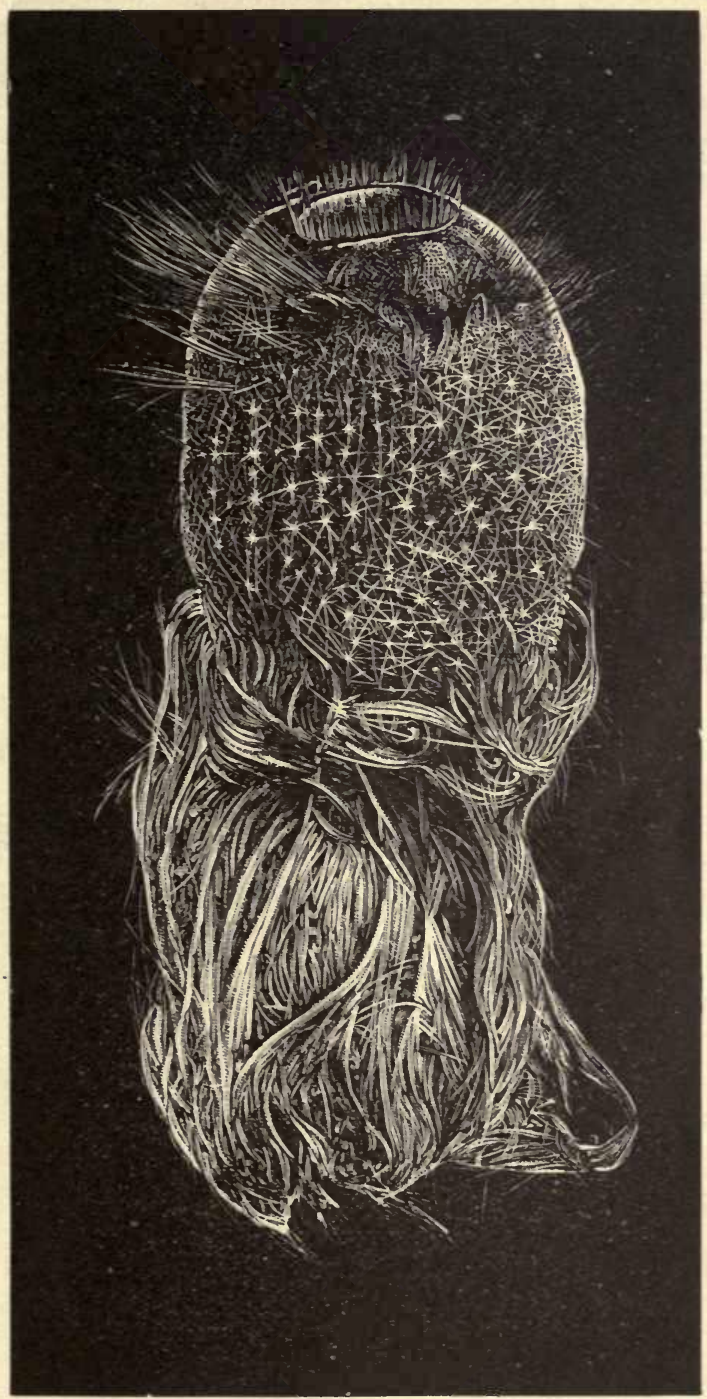

Fig. 92.-Pheronema carpenteri, nne of the Hexactinellida. (From Wyville Thomson.)

a large number of these amœboid cells come to fill up a great part of the cavity of the larva, which now passes into a stage corresponding to the planula larva of the Colenterates (Sect. IV). This is the 
larval form known as the parenchymula. The parenchymula (Fig. 93) consists of three kinds of cells:-(1) an external layer of flagellate cells; (2) an inner mass of amoboid cells; (3) the two posterior granular cells. In this condition it becomes fixed, and develops into the form of a flat plate

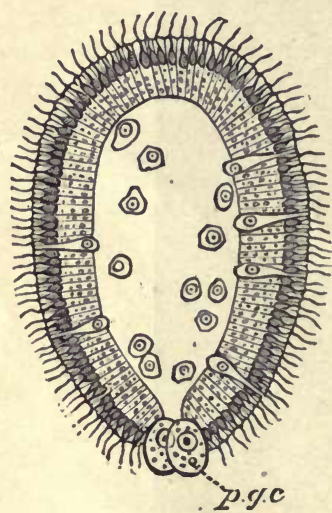

F1a. 93.-Median lougitudinal section of the parenchymula larva of Clathrina blanca. p.g.c., posterior granular cells which give rise to the archaocytes. (From the Cambridge Natural History, after Minchin.) with an irregular outline. Most of the amceboid cells now migrate to the outer surface, passing between the flagellate cells and then becoming arranged outside them to form the ectoderm. The flagellate cells now form an irregular mass together with a number of non-flagellate cells derived from the ectoderm, which are destined to give rise to the porocytes. A cavity appears in the mass, and becomes surrounded by a layer of porocytes. The cavity increases in size, and is soon seen to be bounded not by the porocytes alone, but in part also by flagellate cells. Subsequently the flagellate cells come to form the entire boundary of the cavity, the porocytes passing outwards to become perforated by apertures - the inhalant apertures-in the wall of the sponge. Among the flagellate cells and porocytes there are also amœboid cells derived from the two original granular cells; some of these give rise to the reproductive cells. The scleroblasts are formed of certain ectoderm cells which migrate inwards, and at an early stage arrange themselves in threes to give rise to the tri-radiate spicules. The development of the sponge becomes completed by the enlargement of the internal cavity (paragastric cavity) which is now lined by flagellate cells, and by the development of the osculum.

In Sycon the early stages (Fig. 94, $a-c$ ) differ somewhat from those in Clathrina blanca, and the embryo leaves the parent sponge in the peculiar stage to which the name of rmphiblastula is applied. When the blastula is formed the greater part of its wall consists of clear cells, with a number of granular cells-the archæocytes-at the posterior pole. The clear cells become elongated and flagellate. The archæocytes pass into the internal (segmentation) cavity and become completely enclosed by the flagellate cells (stage of so-called pseudogastrula).

The cells at the posterior end then lose their flagella and become large rounded granular cells, so that after a time the wall of the embryo comes to be composed in one half of the flagellate cells that have remained unaltered, and in the other half of the large granular cells. It is in this stage-terined the amphi- 
blastula (e)-that the larval sponge becomes free. At a later stage the Hagellate cells become partly overgrown by the granular cells, the latter eventually giving rise to the ectoderm of the adult, while the former become the flagellate collared cells. The larva becomes fixed by one side, and soon assumes a cylindrical
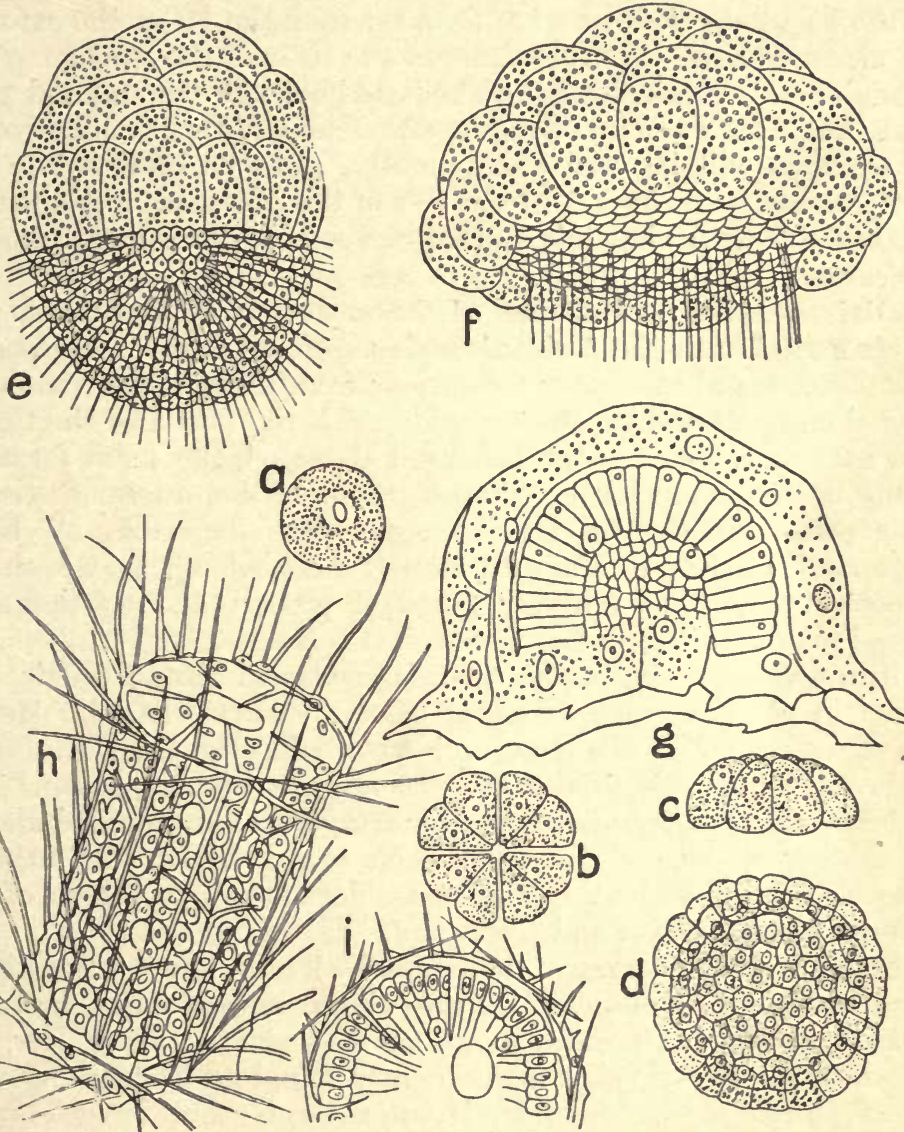

Fig. 94.-Development of Sycon raphanus. $a$, ovum ; $b, c$, ovum segmented- $b$, as seen from above, $c$, lateral view; $d$, blastula; $e$, amphiblastula ; $f$, commencement of invagination ; $g$, larva attached by its oral face; $h, i$, young sponge- $h$, lateral view; $i$, as seen from above. (From Sollas, after Schulze.)

form (Fig. 94, $h, i$ ). An aperture which is developed at the free end becomes the osculum, and small perforations in the sides of the cylinder form the inhalant apertures. As the wall of the cylinder increases in thickness by the growth of the mesoglœa, the radial canals are formed, the endoderm extending into them and its cells becoming flagellate. 
The amphiblastula type of larva is characteristic of the Calcarea, and is probably universal in that sub-class except in such primitive forms as Clathrina.

In the Silicispongiæ, on the other hand, the typical larva is a solid body with a superficial layer of ciliated cells, and an internal mass of granular cells. From the former, apparently, the collared cells of the flagellate chambers are formed: from the latter the external ectoderm and the other elements of the body of the Sponge. The granular cells break through the ciliated cells at one end and grow over the latter as an investing layer. This is a remarkable reversal of what, as will be seen subsequently, is to be observed in the Cœelenterata - and in fact in the rest of the Metazoa, but is readily reconcilable with what takes place in Sycon and the more complex - Calcarea.

Distribution and Mode of Occurrence of Sponges, and their Position in the Animal Series.-Fossil remains of Sponges have been found in various formations from those of the Cambrian period onwards, the greatest abundance being found in the Chalk. No extinct class or order has been detected, the fossil forms all being members of existing groups. Some of the orders of existing Sponges-such as the Myxospongix-are incapable of being preserved as fossils, and the fossil forms belong, as we should expect, to the more highly silicified groups and to the more complex groups of the Calcarea.

Fresh-ivater Sponges (Spongillida) occur in rivers, canals, and lakes in all the great divisions of the earth's surface. Marine Sponges occur in all seas, and at all depths, from the shore between tide-marks to the deepest abysses of the ocean. The Calcarea and the true horny sponges (Ceratosa) are most abundant in shallow water, and have not been found below 450 fathoms. The Sponges found at the greatest depths are members of the groups Hexactinellida and Choristidu.

Sponges do not appear to be edible by Fishes or even the higher Crustaceans or Molluscs. Countless lower animal forms, however, burrow in their substance, if not for food, at least for shelter, and the interior of a Sponge is frequently found to be teeming with small Crustaceans, Annelids, Molluscs, and other Invertebrates. None of the Sponges are true parasites. The little Boring Sponge, Cliona, burrows in the shells of Oysters and other bivalves, but for protection and not for food. But a Sponge frequently lives in that close association with another animal or plant to which the term messmateism, or commensalism, is applied, associations which benefit one or both. Thus some species of Sponge are never found growing except on the backs or legs of certain Crabs. In these cases the Sponge protects the Crab and conceals it from its enemies, while the Sponge benefits by being carried from place to place and thus obtaining freer oxygenation. Certain Cirripede Crustaceans 
(members of the order to which the Barnacles and Acorn-shells belong) are invariably found embedded in certain species of Sponge. Frequently a Sponge and a Zoophyte grow in intimate association, so that they seem almost to form one structure. Thus the GlassropeSponge (Hyalonema) is always found associated with a Zoophyte (Palythoa), and there are many other instances. Sponges often also grow in very close association with certain low forms of plants (Algæ).

The position of the Porifera in the animal series is unquestionably among the Metazoa. The view that they are compound Protozoa is now no longer maintained since the significance of the facts of their development has been fully recognised. A Sponge is to be regarded as a colony of Protozoa only in the sense in which the same may be said of one of the higher animals. It consists of a complex of cells, some of which have a considerable degree of independence, and some of which have a close resemblance to certain Protozoa; but the same is true of one of the higher animals, the difference being one of degree and not of kind. Like the rest of the Metazoa, the Sponge develops from the oosperm by a process of yolk-division.

But the Porifera are perhaps somewhat nearer the Protozoa than are any of the other types of Metazoa; and among the Protozoa they appear to approach nearest to certain colonial Flagellata. The genus Proterospongia (Fig. 58), already referred to (p. 78), appears to be the member of the latter group which of all known forms most closely resembles a sponge. Proterospongia consists of a colony of collared Flagellates (Choanoflagellata) embedded in a mass of gelatinous substance, in which there are also amœboid zooids similar to the amœboid wandering cells of Sponges.

But, while the Porifera are clearly Metazoa, and not Protozoa, there is some room for difference of opinion as regards their relationships to the Coelenterata, with which great phylum they have been sometimes amalgamated. The reasons for and against such an arrangement will be discussed in considering the general relationships of the Coelenterata. 


\section{SECTION IV}

\section{PHYLUM COELENTERATA}

THE possession of an interval cavity lined by a special internal layer of cells - the endoderm-in which the digestive and absorptive functions are centred, distinguishes all the remaining groups of Metazoa from the Parazoa or Sponges. The former are grouped together under the comprehensive title of Enterozoa, or animals with enteric cavity. The simplest Enterozoa have an internal cavity in which there is no separation between the enteric or digestive cavity and the colome or body-cavity-one continuous space representing both and opening on the exterior by the aperture of the mouth. These constitute the phylum Coelenterata. They are all animals of a low type of organisation with a conspicuous radial symmetry, disguising, in some cases, a more obscure bilateral arrangement, which may be more primitive.

The most familiar examples of Cœlenterata are the horny, seaweed-like "Zoophytes,"-or, as they are sometimes called, "Corallines," to be picked up on every sea-beach-Jelly-fishes, Sea-anemones, and Corals. The phylum is divided into four classes as follows :-

Class 1. HydrozoA, including the Fresh-water Polypes, Zoophytes, many Jelly-fishes-mostly of small size,-a few Stony Corals, and the peculiar Palæozoic fossils known as Graptnlites.

Class 2. ScyphozoA, including most of the large Jelly-fishes.

Class 3. Actinozon, including the Sea-anemones, and the vast majority of Stony Corals.

Class 4. CTENophora, including certain peculiar Jelly-fishes known as "Comb-jellies."

\section{CLASS I.-HYDROZOA.}

1. Example of the Class-Obelia.

General Structure.-Obelia is a common zoophyte occurring in the form of a delicate, whitish or light brown, almost fur-like 
growth on the wooden piles of piers and wharfs. It consists of branched filaments about the thickness of fine sewing-cotton: of these, some are closely adherent to the timber, and serve for attachment, while others are given off at right angles, and present at intervals short lateral branches, each terminating in a bud-like enlargement.

The structure is better seen under a low power of the microscope. The organism (Fig. 95) is a colony, consisting of a common stem or axis, on which are borne numerous zooids. The axis consists of a horizontal portion (hydrorhiza) resembling a root or creeping stem, and of vertical axes, which give off short lateral branches in an alternate manner, bearing the zooids at their ends. At the proximal ends of the vertical axes the branching often becomes more complex: the offshoots of the main stem, instead of ending at once in a zooid, send off branches of the third order on which the zooids are borne. In many cases, also, branches are found to end in simple club-like dilatations $(B d .1, \mathscr{2})$ : these are immature zooids.

The large majority of the zooids have the form of little conical structures (P. 1-P. 4), each enclosed in a glassy, cup-like investment or hydrotheca $(h . t h)$, and produced distally into about two dozen arms or tentacles $(t)$ : these zooids are the polypes or hydranths. Less numerous, and found chiefly towards the proximal region of the colony, are long cylindrical bodies or blastostyles (bls), each enclosed in a transparent case, the gonotheca (g.th), and bearing numerous small lateral offshoots, varying greatly in form accerding to their stage of development, and known as medusc-buds $(m . b d)$. By studying the development of these structures, and by a comparison with other forms, it is known that both blastostyles and medusabuds are zooids, so that the colony is trimorphic, having zooids of three kinds.

To make out the structure in greater detail, living specimens should be observed under a high power. A polype is then seen to consist of a somewhat cylindrical, hollow body, of a yellowish colour, joined to the common stem by its proximal end, and produced at its distal end into a conical elevation, the manubrium or hypostome $(m n b)$, around the base of which are arranged the twentyfour tentacles in a circle. Both body and manubrium are hollow, containing a spacious cavity, the enteron (ent), which communicates with the outer world by the mouth $(m t h)$, an aperture placed at the summit of the manubrium. The mouth is capable of great dilatation and contraction, and accordingly the manubrium appears now conical, now trumpet-shaped. Under favourable circumstances small organisms may be seen to be caught by the polypes and carried towards the mouth to be swallowed.

The hydrotheca (h.th) has the form of a vase or wine-glass, and is perfectly transparent and colourless. A short distance from its 
narrow or proximal end, it is produced inwards into a sort of circular shelf $(s h)$, perforated in the centre: upon this the base of the polype rests, and through the aperture it is continuous with the common stem. When irritated-by a touch or by the addition of alcohol or other poison - the polype undergoes a very marked contraction: it suddenly withdraws itself more or less completely into the theca, and the tentacles become greatly shortened and curved over the manubrium (P. 2).

The various branches of the common stem show a very obvious distinction into two layers: a transparent, tough, outer membrane, of a yellowish colour and horny consistency, the perisarc $(p)$, and an inner, delicate, granular layer, the conosare (cœ), continuous by a sort of neck or constriction with the body of each hydranth. The cœnosare is hollow, its tubular cavity being continuous with the cavities of the polypes, and containing a fluid in which a flickering movement may be observed, due, as we shall see, to the action of cilia. At the base of each zooid or branch the perisare presents several annular constrictions, giving it a ringed appearance: for the most part it is separated by an interval from the cœenosarc, but processes of the latter extend outwards to it at irregular intervals, and in the undeveloped zooids $(B d .2)$ the two layers are in close apposition.

In the blastostyle both mouth and tentacles are absent, the zooid ending distally in a flattened disc: the hydrotheca of a polype is represented by the gonotheca $(g . t h)$, which is a cylindrical capsule enclosing the whole structure, but ultimately becoming ruptured at its distal end to allow of the escape of the medusabuds. These latter are, in the young condition, mere hollow offshoots of the blastostyle: when fully developed they have the appearance of saucers attached by the middle of the convex surface to the blastostyle, produced at the edge into sixteen very short tentacles, and having a blunt process, the manubrium, projecting from the centre of the concave surface. They are ultimately set free through the aperture in the gonotheca as little medusæ or jelly-fish (B-D), which will be described hereafter.

The microscopical structure of a polype (Fig 96) reminds us, in its general features, of that of such a simple sponge as Ascetta, but with many characteristic differences. The body is composed of two layers of cells, the ectoderm (ect) and the endoderm (end): between them is a very delicate transparent membrane, the mesogloea or supporting lamella $(\mathrm{msgl})$, which, unlike the intermediate layer of sponges, contains no cells and is practically structureless. The same three layers occur in the manubrium, the ectoderm and endoderm being continuous with one another at the margin of the mouth. The tentacles are formed of an outer layer of ectoderm, then a layer of mesogloea, and finally a solid core of large endoderm cells arranged in a single series. The 
conosarc, blastostyles, and medusa-buds all consist of the same layers, which are thus continuous through the entire colony.

The perisarc or transparent outer layer of the stem shows no cell-structure, but only a delicate lamination. It is, in fact, not a cellular membrane or epithelium, like the ectoderm and endoderm, but a cuticle, formed, layer by layer, as a secretion from the ectoderm cells (see p. 31). It is composed of a substance of chitinoid or hornlike consistency, and, like the lorica of many Protozoa, serves as a protective external skeleton. When first formed it is of course in contact with the ectoderm, but when the full thickness is attained

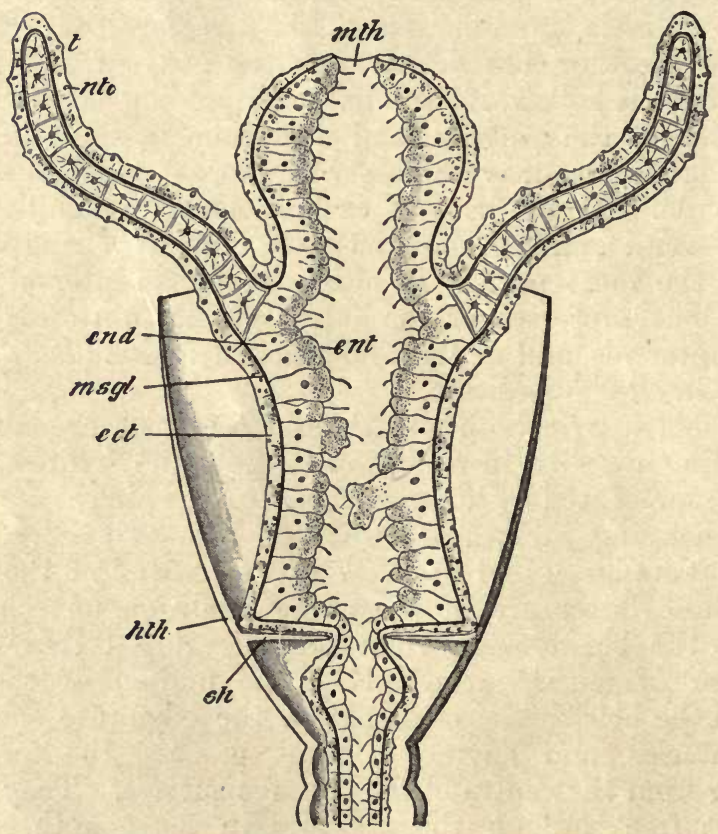

FIG. 96.-Obelia sp. Vertical section of a polype, highly magnified; ect. ectoderm; end. endoderm; ent. enteric cavity; h.th. hydrotheca; msgl. mesogloea; mth. mouth; ntc. nematocysts; $s h$. shelf-like prolongation of hydrotheca; $t$. tentacle.

the latter retreats from it, the connection being maintained only at irregular intervals. In the same way the hydro- and gonothecæ are cuticular products of the polypes and blastostyles respectively: in the young condition both occur in the form of a closely fitting investment of the knob-like rudiment of the zooid (Fig. 95, $B d 1$, 2).

The ectoderm has the general character of a columnar epithelium (see p. 24), but exhibits considerable differentiation of its component cells. It is mainly composed of large conical cells with their bases outwards, and having between their narrow inner ends clumps of small rounded interstitial celts, and occasional large branched nerve- 
cells (Fig. 98, nv.c). The tentacles and the manubrium contain, in addition, a layer of unstriped muscle-fibres between the ectoderm and the mesogloea: they are arranged longitudinally, and serve for the rapid shortening of the tentacles (Fig. 98, $m f$ ). This muscular layer is a derivative of the ectoderm, and may be looked upon as a rudimentary mesoderm.

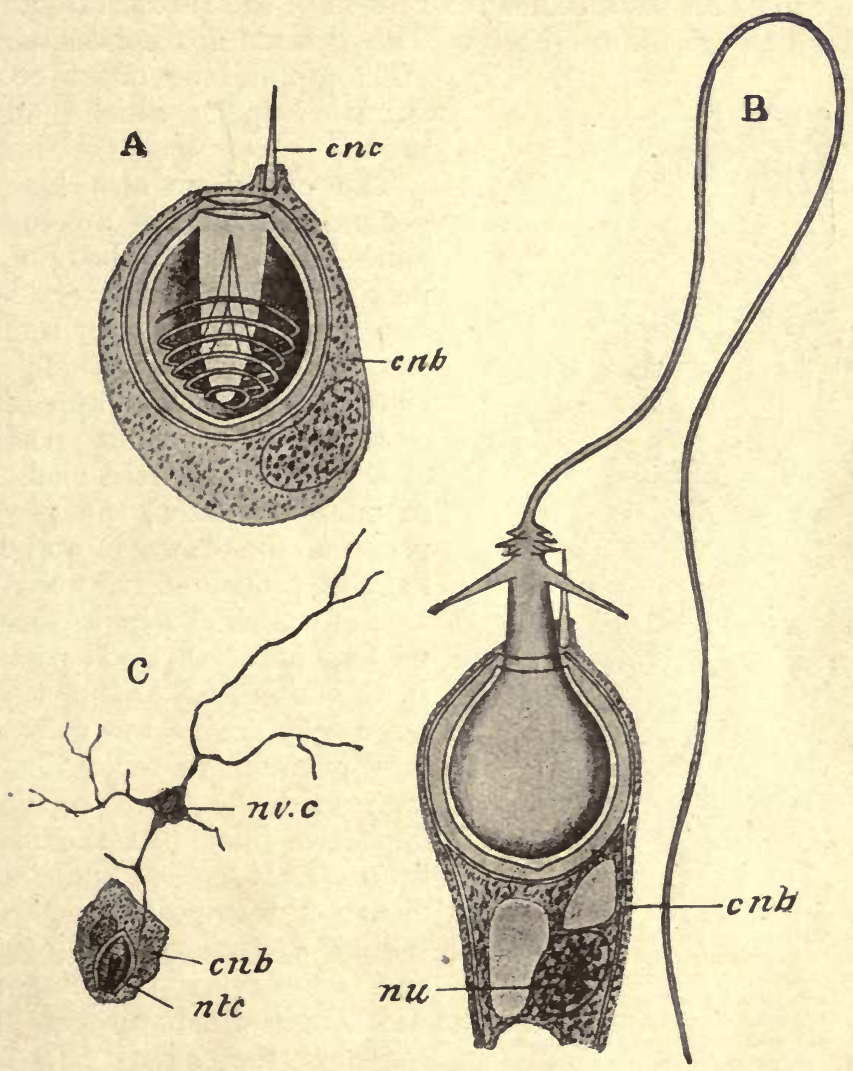

FIG. 97.-Nematocysts of Fydra. A, undischarged ; B, discharged ; C, nerve-supply ; $c n b$, cnidoblast ; cnc. cnidocil ; nu. nucleus ; ntc. nematocyst; nv.c. nerve-cell. (From Parkcr's Biology, after Schneider.)

Embedded in the ectoderm are numerous clear ovoid bodies, the stinging-capsules or nematocysts (Figs. 96-98 ntc), organs closely resembling those of Epistylis umbellaria (p. 93), and like them, serving as weapons of offence. Each consists (Fig. 97, A) of a tough ovoid capsule, full of fluid, and invaginated at one end in the form of a hollow process continued into a long, coiled, hollow thread. The whole apparatus is developed into an interstitial cell called a cnidoblast (cnb), which, as it approaches maturity, migrates towards 
the surface and becomes embedded in one of the large ectoderm cells. At one point of its surface the cnidoblast is produced into a delicate protoplasmic process, the cnidocil or trigger-hair (cnc): when this is touched - for instance by some small organism brought into contact with the waving tentacle-the cnidoblast undergoes a sudden contraction, and the pressure upon the stinging capsule causes an instantaneous eversion of the thread (B), at the base of which are minute barbs. The threads are poisonous, and

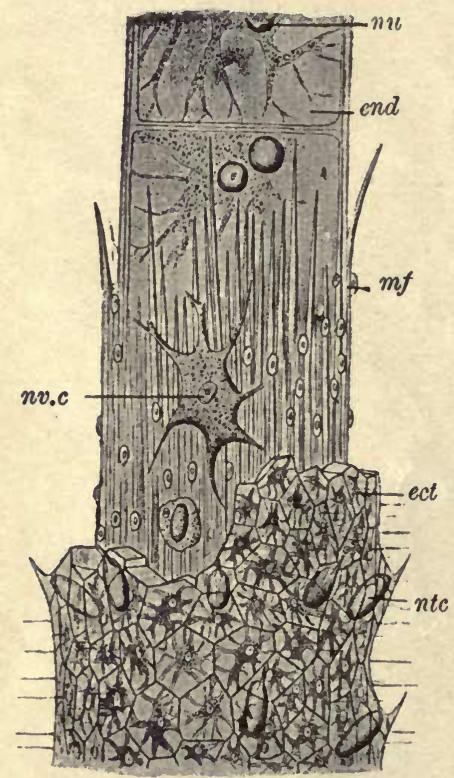

Fio. 98.-Tentacle of Eucopella. The lower part of the figure shows the ex. ternal surface, in the middle part the ectoderm is removed and the muscular and nervous layer exposed, in the upper part these latter are removed so as to show the core of endoderm cells; ect. ectoderm ; end.endoderm; $m f$. muscle. fibres; ntc. nematocyst; nu. nucleus ; nv.c. nerve-cell. (After von Lendenfeld.) exert a numbing effect on the animals upon which Obelia preys.

The endoderm also has the general character of a columnar epithelium. In the body of the polype the cells are very large and have the power of sending out pseudopods at their free ends (Fig. 96), which apparently seize and ingest minute portions of the partly-digested food. As in many Protozoa, the pseudopods may be drawn in and long flagella protruded, the contraction of which causes a constant movement of the food particles in the enteron. Amongst these large cells are narrow cells with very granular protoplasm : they are gland-cells, and secrete a digestive juice. In the manubrium a layer of endodermal muscle-fibres has been described taking a transverse direction, and so serving to antagonise the longitudinal muscles and contract the cavity. In the tentacles (Figs. 96 and 98) the endoderm (end) consists of a single row of short cylindrical cells, nearly cubical in longitudinal section: their protoplasm is greatly vacuolated and their cell-walls so thick that they may be considered as forming a sort of internal skeleton to the tentacles.

The structure of the medusæ-formed, as we have seen, by the development of medusa-buds liberated from a ruptured gonotheca -yet remains to be considered. The convex outer surface of the bell or umbrella (Fig. 95, B-D) by which the zooid was originally attached to the blastostyle is distinguished as the ex-umbrella, the 
concave inner surface as the sub-umbrella. From the centre of the sub-umbrella proceeds the manubrium $(m n b)$, at the free end of which is the four-sided mouth ( $m t h)$. Very commonly, as the medusa swims the umbrella becomes turned inside out, the subumbrella then forming the convex surface and the manubrium springing from its apex (Fig. 95, C, and Fig. 99, A).

The mouth (Figs. 95, 96, 99, and 100, $\mathrm{mth}$ ) leads into an enteric cavity which occupies the whole interior of the manubrium, and from its dilated base sends off four delicate tubes, the radial canals (rad. c), which pass at equal distances from each other through the substance of the umbrella to its margin, where they all open into a circular canal (circ. c), running parallel with and close to the margin. By means of this system of canals the food, taken

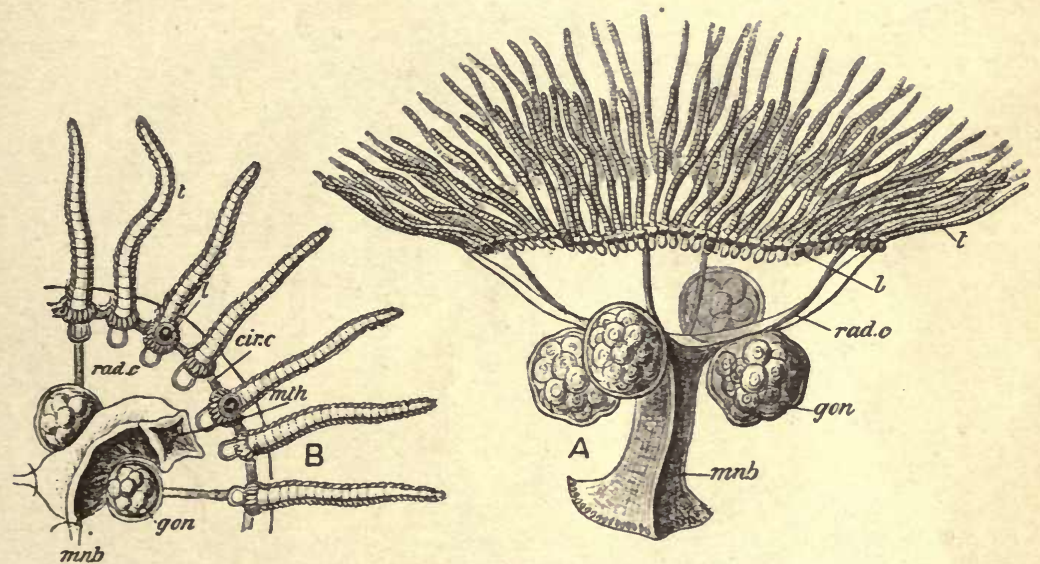

Fig. 99.-Obelia sp. A, mature medusa swimming with everted umbrella; B, one quarter of the same, oral aspect; circ.c. circular canal; gon. gonad; $\iota$. lithocyst; mub. manubrium; mth. mouth; roul. c. radial canal; $t$. tentacle. (After Haeckel.)

in at the mouth and digested in the manubrium, is distributed to the entire medusa.

The edge of the umbrella is produced into a very narrow fold or shelf, the velum (Fig. 100,vl), and gives off the tentacles $(t)$, which are sixteen in number in the newly-born medusa (Fig. 95), very numerous in the adult (Fig. 99). At the bases of eight of the tentacles-two in each quadrant-are minute globular sacs (l), each containing a calcareous particle or lithite. These are the marginal sense-organs or lithocysts : they were formerly considered to be organs of hearing, and are hence frequently called otocysts: in all probability their function is to guide the medusa by enabling it to judge of the direction in which it is swimming. The marginal organs, in this case, may therefore be looked upon as organs of the sense of direction.

The manubrium (Fig. 100, mnb) of the medusa consists of 
precisely the same layers as that of the hydranth-ectoderm, mesogloea, and endoderm. The ectoderm is continued on to the sub-umbrella, and then round the margin of the bell on to the ex-umbrella, so that both surfaces of the bell are covered with ectoderm. The endoderm is continued from the base of the enteric cavity into the radial canals and thence to the circular canal, so that the whole canal-system is lined by endoderm. In the portions of the bell between the radial canals there is found, between the outer and inner layers of ectoderm, a thin sheet of endoderm, the endoderm-lamella (end. lam), which stretches between adjacent radial canals and between the circular canal and the enteric cavity. In the bell, as in the manubrium, a

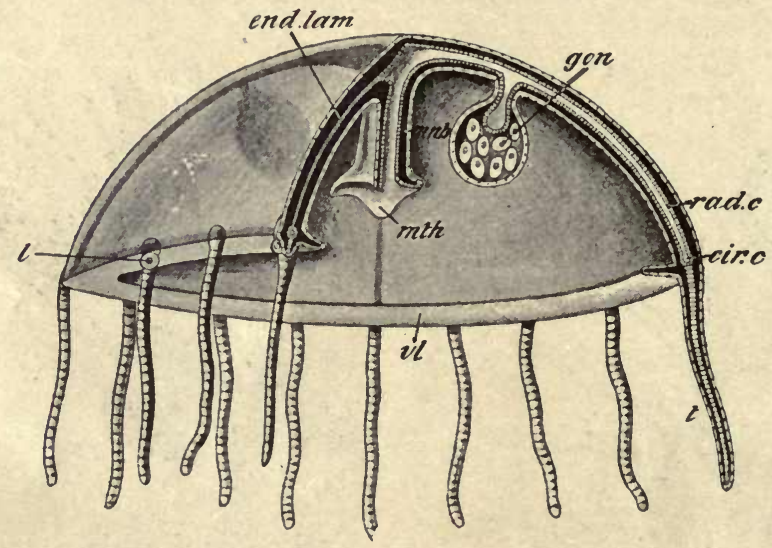

Fig.100,-Dissection of a medusa with rather more than one-quarter of the umbrella and manubrium cut away (diagrammatic). The ectoderm is dotted, the endoderm striated, and the mesogloea black. circ. $c$. circular canal; end. lam. endoderm lamella; gon. gonad; $l$. lithocyst; mnb. manubrium; $m t h$. mouth; rad. c. radial canal; $v l$. velum.

layer of mesoglœa everywhere intervenes between ectoderm and endoderm.

The velum $(v l)$ consists of a double layer of ectoderm and a middle one of mesogloa: there is no extension of endoderm into it. The tentacles, like those of the hydranth, are formed of a core of endoderm covered by ectoderm, the cells of the latter being abundantly supplied with stinging-capsules.

Comparison of Polype and Medusa.-Striking as is the difference between a polype and a medusa, they are strictly homologous structures, and the more complex medusa is readily derivable from the simpler polype-form. It is obvious, in the first instance, that the apex of the umbrella corresponds with the base of a hydranth (Fig. 101, A and D), being the part by which the zooid is attached in each case to the parent stem: the mouth with the manubrium are also obviously homologous structures in 
the two cases. Suppose the tentacular region of a polype to be pulled out, as it were, into a disc-like form (B), and afterwards to be bent into the form of a saucer (C) with the concavity distal,

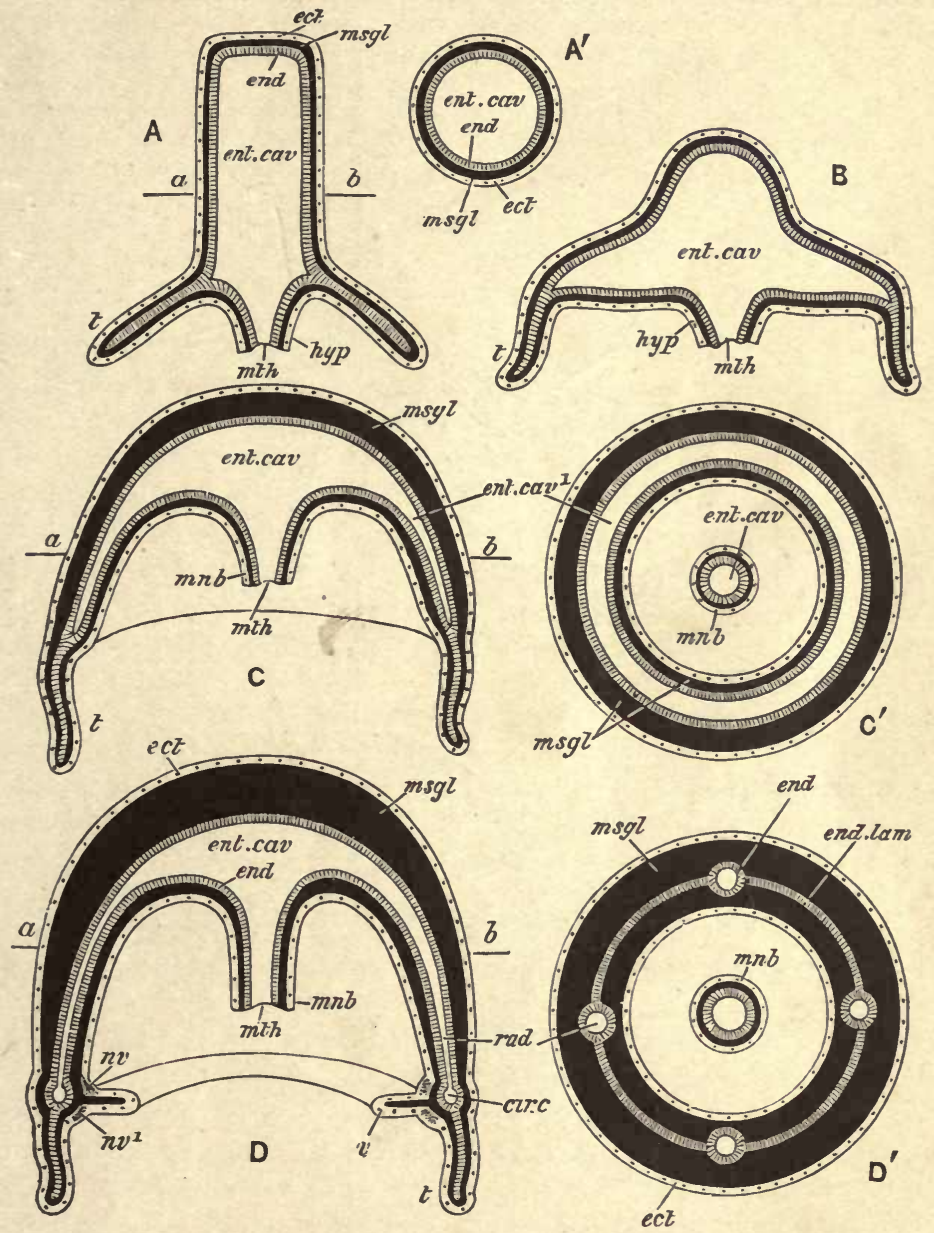

Fig.101.-Diagrams illustrating the derivation of the medusa from the polype. A, longitudinal, and $\mathbf{A}^{\prime}$, transverse section (along the line $a b$ ) of polype-form; $\mathrm{B}$, polype-form with extended tentacular region ; $C$, vertical, and $\mathbf{C}^{\prime}$, transverse section (along the line $a b$ ) of form with tentacular region extended into the form of a bell; $D$, vertical, and $D^{\prime}$, transverse section (along the line $a b$ ) of medusa. The ectoderm is dotted, the endoderm striated, and the mesogløea black. cir. $c$. circular canal ; ect. ectoderm; end endoderm; end. lam. endoderm lamella; ent. cav. enteric cavity; hyp. hypostome or manubrium; mnb. manubrium; msgl. mesogloea ; mth. mouth ; $n v . n v^{\prime}$, nerve-rings; $t$. tentacle; $v$. velum. (From Parker's Biology.)

i.e. towards the manubrium. The result of this would be a medusalike body $\left(\mathrm{C}, \mathrm{C}^{\prime}\right)$ with a double wall to the entire bell, the narrow space between the two layers containing a prolongation of the 
enteron (ent. cav') and being lined with endoderm. From such a form the actual condition of things found in the medusa would be produced by the continuous cavity in the bell being for the most part obliterated by the growing together of its walls so as to form
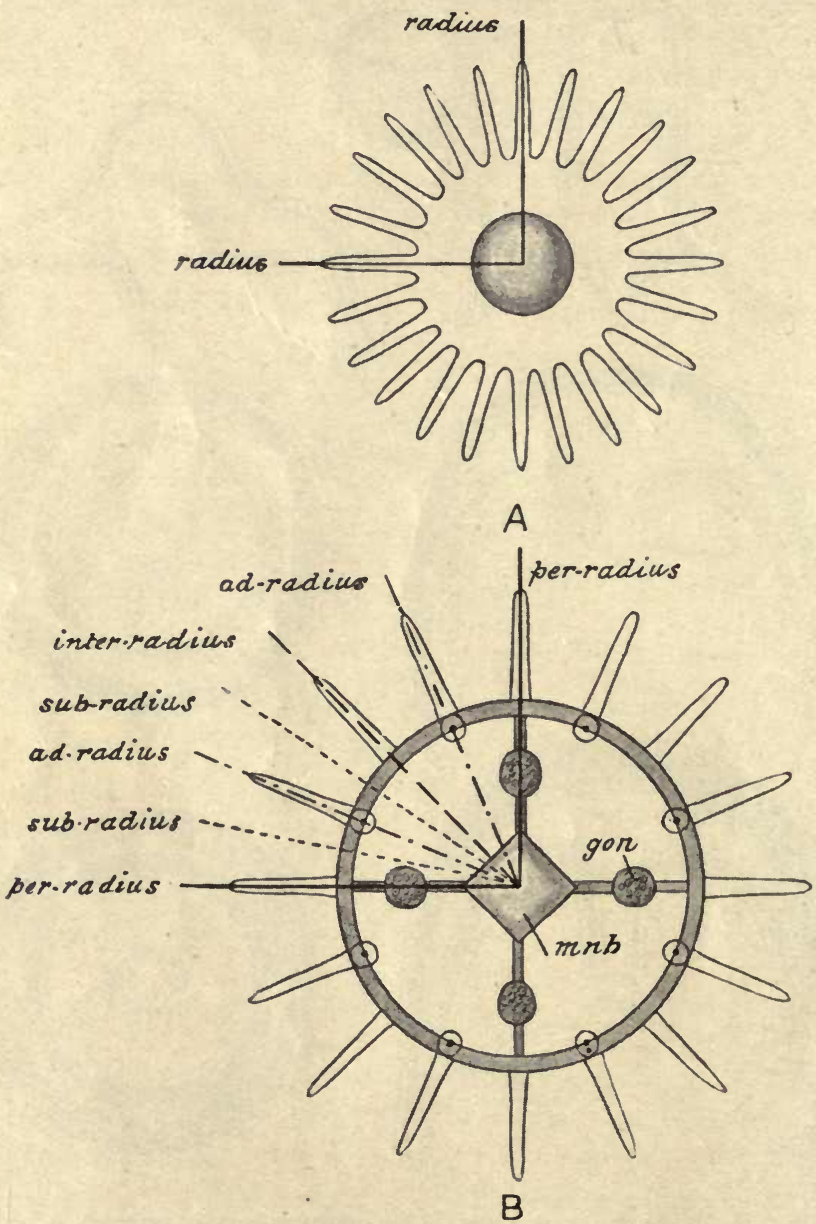

FIG 102-Projections of polype (A) and medusa (B), showing the various orders of radii; gon. gonad; $m n b$. manubrium.

the endoderm-lamella ( $\mathrm{D}^{\prime}$, cnd. lam), and remaining only along four meridional areas-the radial canals $(\mathrm{rad} . \mathrm{c})$, and a circular area close to the edge of the bell-the circular canal (cir. c).

While both polype and medusa are radially symmetrical, the increase in complexity of the medusa is accompanied by a differentiation of the structures lying along certain radii. If a polype is projected on a plane surface (Fig. 102, A), 
taken at right angles to its long axis, a large number of radii-about twentyfour - can be drawn from the centre outwards, all passing through similar parts, i.e. along the axis of a tentacle and through similar portions of the body and manubrium. But in the medusa (B) the case is different. The presence of the four radial canals allows us to distinguish four principal radii or per-radii. Half way between any two per-radii a radius of the second order, or inter-radius, may be taken; half way between any per-radius and the inter-radius on either side a radius of the third order, or ad-radius, and half way between any ad-radius and the adjacent per- or inter-radius, a radius of the fourth order, or sub-radius. Thus there are four per-radii, four inter-radii, eight ad-radii, and sixteen sub-radii. In Obelia the radial canals, the angles of the mouth, and four of the tentacles are per-radial, four more tentacles are inter-radial, and the remaining eight tentacles, bearing the lithocysts, are ad-radial. The sub-radii are of no importance in this particular form.

Reproduction.--In the description of the fixed Obelia-colony no mention was made of cells set apart for reproduction, like the ova and sperms of a sponge. As a matter of fact, such sexual cells are found only-in their fully developed condition at leastin the medusæ. Hanging at equal distances from the sub-umbrella, in immediate relation with the radial canal and therefore perradial in position, are four ovoid bodies (Figs. 99 and 100, gon), each consisting of an outer layer of ectoderm continuous with that of the sub-umbrella, an inner layer of endoderm continuous with that of the radial canal and enclosing a prolongation of the latter, and of an intermediate mass of cells which have become differentiated into ova or sperms. As each medusa bears organs of one sex only (testes or ovaries, as the case may be), the individual medusæ are diocious. It will be noticed that the gonad has the same general structure as an immature zooid-an outpushing of the body-wall consisting of ectoderm and endoderm, and containing a prolongation of the enteric cavity.

Development. - When the gonads are ripe, the sperms of the male medusæ are shed into the water and carried by currents to the females, impregnating the ova, which thus become oosperms or unicellular embryos. The oosperm undergoes complete segmentation (Fig. 103, A-F), and is converted into an ovoidal body called a planula $(\mathrm{G}, \mathrm{H})$, consisting of an outer layer of ciliated ectoderm cells and an inner mass of endoderm cells in which a space appears, the rudiment of the enteron. The planula swims freely for a time $(\mathrm{H})$, then settles down on a piece of timber, seaweed, \&c., fixes itself by one end (K), and becomes converted into a hydrula or simple polype (L, M), having a disc of attachment at its proximal end, and at its distal end a manubrium and circlet of tentacles. Soon the hydrula sends out lateral buds, and, by a frequent repetition of this process, becomes converted into the complex Obelia-colony with which we started.

This remarkable life-history furnishes the first example we have yet met with among the Metazoa of alternation of generations, or 
metagenesis (see p. 41). The Obelia-colony is sexless, having no gonads, and developing only by the asexual process of budding; but certain of its buds - the medusæ-develop gonads, and from

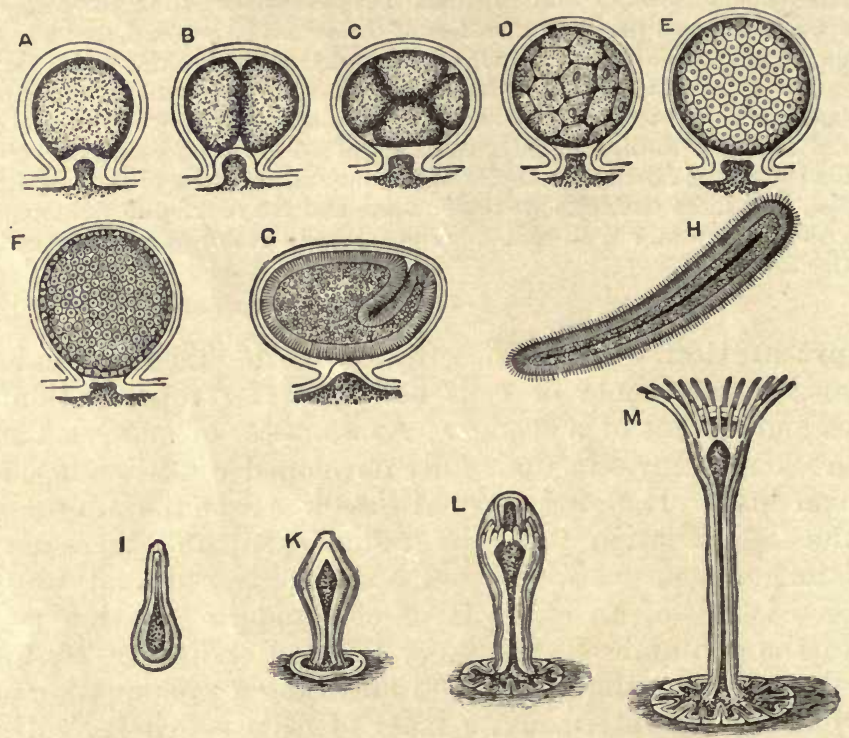

FiG. 103.-Stages in the development of two Zoophytes (A-H, Laomedea, I-M, Eudendrium) allied to Obelia; $\mathbf{A}-\mathbf{F}$, stages in segmentation; $\mathrm{G}$, the planula enclosed in the maternal tissues; $H$, the free-swimming planula; I-M, fixation of the planula and development of the hydrula. (From Parker's Biology, after Allman.)

their impregnated eggs new Obelia-colonies arise. We thus have an alternation of an asexual generation, or agamobium - the Obeliacolony, with a sexual generation, or gamobium - the medusa.

\section{General Structure and Classification.}

The Hydrozoa may be defined as multicellular animals in which the cells are arranged in two layers, ectoderm and endoderm, separated by a gelatinous, non-cellular mesoglœa, and enclosing a continuous digestive cavity which communicates directly with the exterior by a single aperture - the mouth - and is lined throughout by endoderm. The ectoderm consists of epithelial cells, interstitial cells, muscle-fibres, and nerve-cells. Certain of the interstitial cells give rise to characteristic organs of offence-the stinging-capsules. The endoderm consists of flagellate or amœboid cells, gland-cells, and sometimes muscle-fibres. There are two main forms of zooids, polypes or nutritive zooids, which are usually sexless, and medusæ or reproductive zooids. In correspondence with its locomotive habits, the medusa attains a higher 
degree of organisation than the polype, having more perfect muscular and nervous systems, distinct sense-organs, and a digestive cavity differentiated into central and peripheral portions, the latter taking the form of radial and circular canals. The reproductive products are discharged externally, and are very commonly, though not always, of ectodermal origin.

Many Hydrozoa agree with Obelia in exhibiting alternation of generations, the asexual generation being represented by a fixed, more or less branched hydroid colony, the sexual generation by a free-swimming medusa. In other forms there are no free medusæ, but the hydroid colony produces fixed reproductive zooids. In others, again, there is no hydroid stage, the organism existing only in the medusa-form. Then, while in most instances the only skeleton or supporting structure is the horny perisarc, there are some forms in which the cœnosarc secretes a skeleton of calcium carbonate, forming a massive stony structure or coral. Lastly, there are colonial forms which, instead of remaining fixed, swim or float freely on the surface of the ocean, and such pelagic species are always found to exhibit a remarkable degree of polymorphism, the zooids being of very various forms and performing diverse functions.

Thus we have zoophyte colonies known to produce free medusæ, zoophyte colonies known not to produce free medusæ, and medusæ known to have no zoophyte stage. Moreover, there are many medusæ of which the life-history is unknown, so that it is uncertain whether or not a zoophyte stage is present. It is also found that in some cases closely allied zoophytes produce very diverse medusæ, while similar medusæ, in other cases, may spring from very different zoophytes. For these reasons a sori of double classification of the Hydrozoa has come about, some zoologists approaching the group from the point of view of the zoophyte, others from that of the medusa. On the whole the following scheme seems best adapted for bringing before the beginner the leading modifications of the class.

\section{ORDER 1.-LEPTOLINE.}

Hydrozoa in which there is a fixed zoophyte stage, and in which the sense-organs are exclusively ectodermal.

\section{Sub-Order a.-Anthomedusce.}

Leptolinæ in which the polypes are not protected by hydrothecæ or the reproductive zooids by gonothecæ : the medusæ bear the gonads on the manubrium and have no lithocysts.

\section{Sub-Order b.-Leptomedusa.}

- Leptolinæ in which hydro- and gonothecæ are present : the medusæ bear the gonads in connection with the radial canals and usually have lithocysts. 


\section{ORDER 2.-TRACHYLIN}

Hydrozoa in which no fixed zoophyte stage is known to occur, all members of the group being locomotive medusæ, some of which have been proved to develop directly from the egg. The senseorgans are formed partly of endoderm.

$$
\text { Sub-Order a.-Irachymedusa. }
$$

Trachylinæ in which the tentacles spring from the margin of the umbrella, and the gonads are developed in connection with the radial canals.

\section{Sub-Order b.-Narcomedusa.}

Trachylina in which the tentacles spring from the ex-umbrella, some distance from the margin, and the gonads are developed in connection with the manubrium.

\section{Order 3.-Hydrocoralisina.}

Hydrozoa in which a massive skeleton of calcium carbonate is secreted from the cœnosarc, the dried colony being a coral.

\section{ORDER 4.-SIPHONOPHORA.}

Pelagic Hydrozoa in which the colony usually exhibits extreme polymorphism of its zooids.

\section{ORder 5.-Graptolithida.}

An extinct group of Hydrozoa, found only in rocks of Palæozoic age, in the form of the fossilised perisare of the branched colonies.

\section{Systematic Position of the Example.}

Obelia, in virtue of the possession of gono- and hydrothecæ, and of gonads formed in connection with the radial canals, belongs to the sub-order Leptomedusæ. It is placed in the family Campanulariida, distinguished by having cup-shaped thecx borne at the ends of distinct branchlets: the gerius Obelia is distinguished from other genera of the same family by the fact that the reproductive zooids are free-swimming medusæ.

\section{ORDER 1.-LEPTOLINA.}

The more typical members of this group agree in all essential respects with Obelia, consisting of branched colonies bearing two principal forms of zooids, which serve for nutritive and reproductive purposes respectively.

General Structure.-The form and size of the colonies are subject to great variation: they may be little insignificant tufts growing on shells, sea-weeds, \&c., or may take the form of complex trees three feet in height, and containing many thousand 
zooids. The hydranths may be colourless and quite invisible to the naked eye, or, as in some Tubulariæ (Fig. 105, 5) may be brilliantly coloured, flower-like structures, nearly an inch in diameter. The medusæ may be only just visible to the naked eye, or, as in Aquorea, may attain a diameter of $38 \mathrm{~mm}$., or about 15 inches: they are often seen with great difficulty owing to the bubble-like transparency of the umbrella; but frequently the manubrium is brightly coloured, or brilliant dots of colour - the ocelli or eye-spots - may occur around the margin of the umbrella. They are also frequently phosphorescent, the phosphorescence of the ocean being often due to whole fleets of medusæ liberated in thousands from the hydroid colonies beneath the surface.

The two sub-orders of Leptolinæ are distinguished by the arrangement of the perisarc. In the Anthomedusæ, of which Bougainvillea (Fig. 104) is a good example, the cuticle stops short at the bases of the hydranths, and the reproductive zooids are not enclosed in gonothecr. It is for this reason that, in classifications founded on the zoophyte stage, the Anthomedusæ are called Gymnoblastea or naked-budded zoophytes (see also Fig. 105, 1, 4, 5). In the Leptomedusæ the cuticle is usually of a firmer consistency than in the first sub-order, and furnishes hydrothecæ for the hydranths and gonothecæ for the reproductive zooids: they are hence often classified as Calyptoblastea or covered-budded hydroids. To this group belong the commonest species of hydroids found on the seashore, and often mistaken for seaweeds - the "Sea-firs" or Sertularians.

The medusæ also exhibit characteristic differences in the two sub-orders. In the Anthomedusæ the umbrella is usually strongly arched, and may even be conical or mitre-shaped (Figs. 104; 105, 7 ; 109,1 and 2): its walls are thick, owing to a great development of the gelatinous mesoglœa of the ex-umbrella, that of the sub-umbrella remaining thin; and the velum is considerably wider than in Obelia. But the most important characteristics are the facts that the gonads $(g \circ n)$ are developed on the manubrium and that lithocysts are absent. Sense-organs are, however, present in the form of specks of red or black pigment at the bases of the tentacles. These ocelli (oc) consist of groups of ectoderm cells containing pigment, and it has been proved experimentally that they are sensitive to light: they are, in fact, the simplest form of eyes. In the Leptomedusæ the umbrella is usually less convex, thinner, and of softer consistcncy than in the Anthomedusæ, the gonads are developed as buds formed in connection with the radial canals and projecting from the sub-umbrella, the velum is feebly developed, and sense-organs take the form sometimes of ocelli, but usually of lithocysts.

In the majority of Leptolinæ the cœnosarc, as in Obelia, consists of a more or less branched structure attached to stones, timber, seaweeds, shells, \&c., by a definite root-like portion (hydrorhiza). The 
curious genus Hydractinia (Fig. 105, 1) is remarkable for possessing a massive conosarc, consisting of a complex arrangement of branches which have undergone fusion, so as to form a firm brownish crust on the surfaces of dead gastropod shells inhabited by Hermit-crabs. The constant association of Hydractinia with

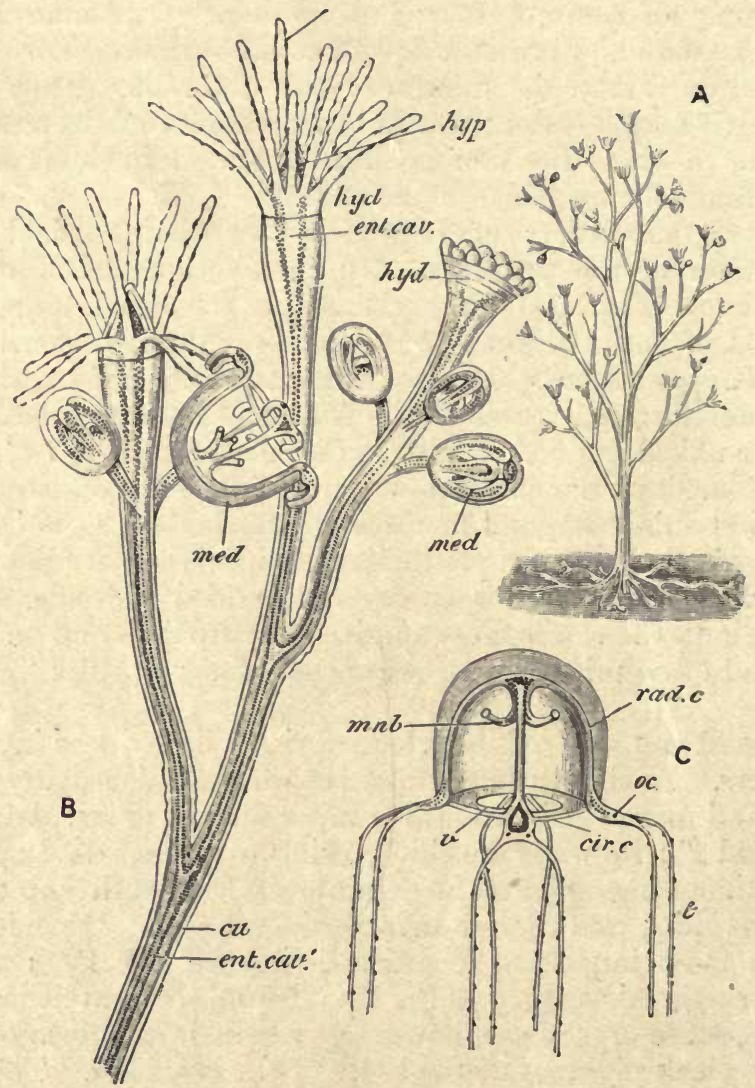

Fra.104.-Bougainviliea ramosa. A, entire colony, natural size; B, portion of the same magnified; C, immature medusa cir. $c$. circular canal; cu. cuticle or perisarc ; ent. cav. enteric cavity; hyd. polype or hydranth; hyp. hypostome or manubrium; med. medusa ; mnb. manubrium; rad. c. radial caual; $t$. tentacle; $v$. velum. (From Parker's Biology, after Allman.)

Hermit-crabs is a case of commensalism: the hydroid feeds upon minute fragments of the Hermit-crab's food, and is thus its commensal or messmate; and the Hermit-crab is protected from its enemies by the presence of the inedible, stinging hydroid. Hydractinia belongs to the Anthomedusæ: the Leptomedusan 


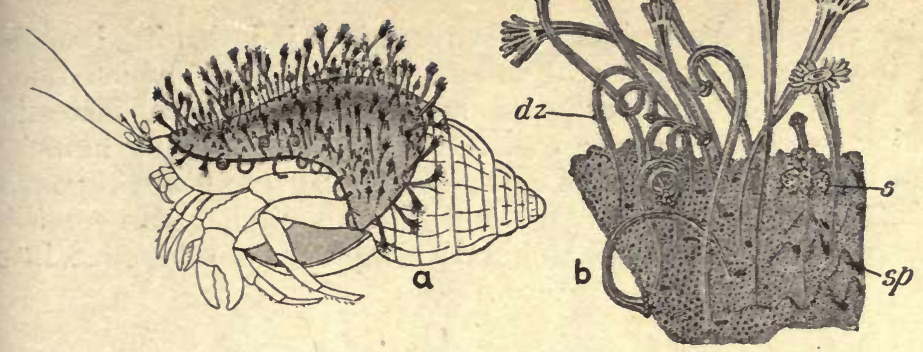

1. Hydractinia

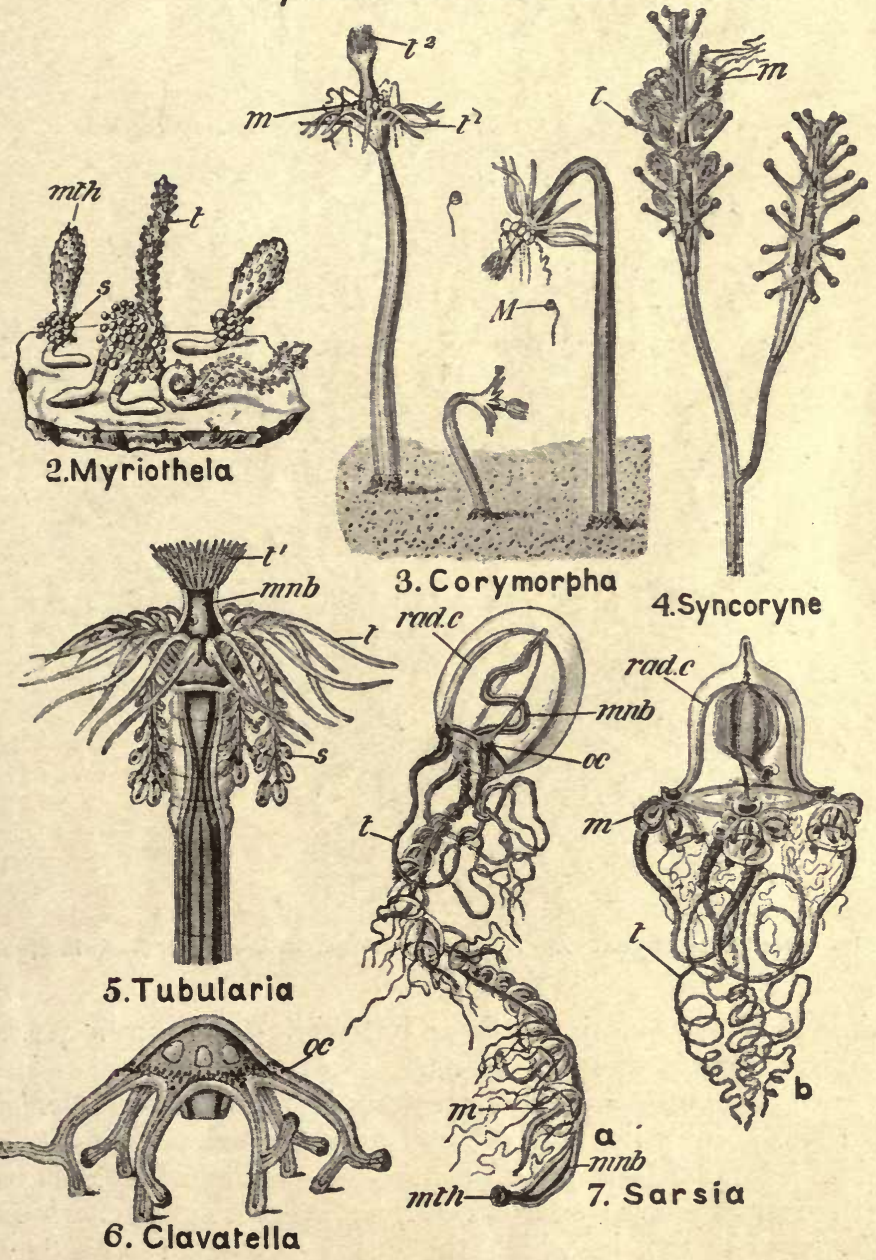

FiG. 105.-Various forms of Ieptolina. In 1, $a$ shows the entire colony, $b$ a portion highly magnified; in $7, a$ is a species producing medusa-buds from the manubrium, $b$ from the bases of the tentacles; $d z$. dactylozooids; $m$. and M. meduse; mnb. manubrium; meth. mouth; oc. eje-spots; rad. $c$. radial canals; s. sporosacs; sp. spines; $t, t 1, t^{2}$, tentacles. 
Clathrozoon, an Australian genus, resembles it in having branched and intertwined cœnosarcal tubes, the perisarc of which undergoes fusion; but the complex mass thus produced, instead of forming an incrustation on a shell, is a large, abundantly branched, tree-like structure, resembling some of the fan-corals or Gorgonacea (vide infra). Ceratclla (Fig. 106) has a similar fan-coral-like appearance, with a branching axis composed of numerous inter-

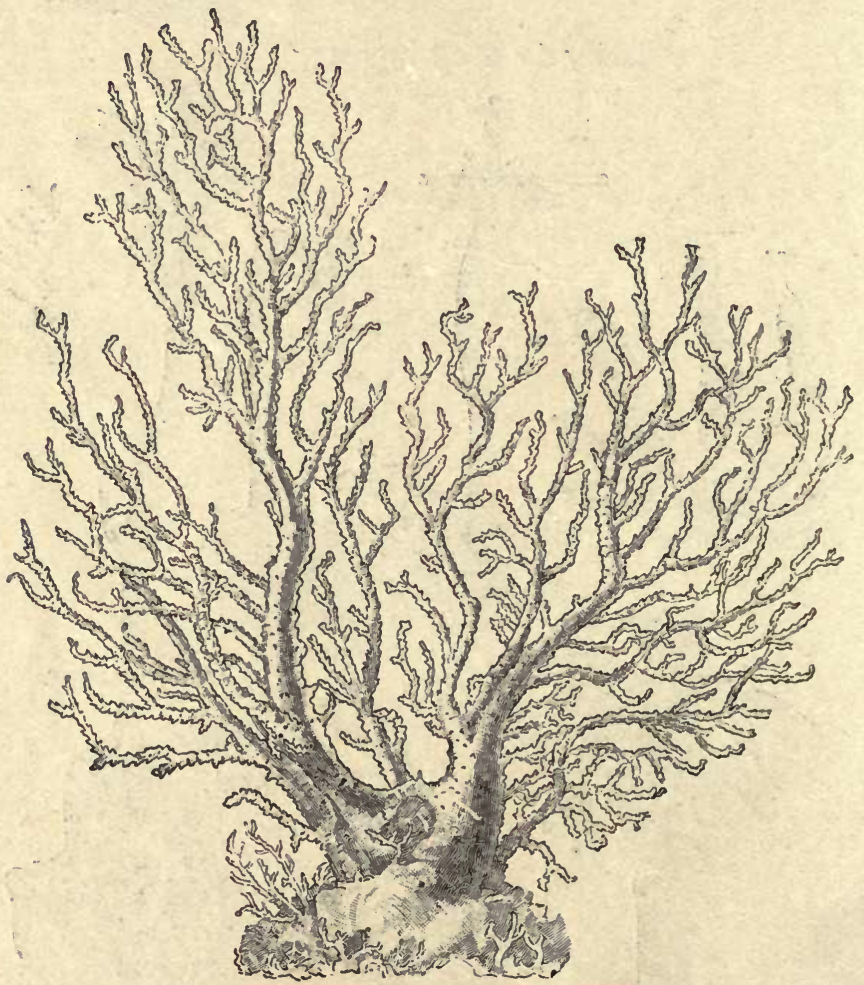

FIG. 106.-Ceratella fusca. About nat. size. (From Hickson, after Baldwin Spencer.)

twining and anastomosing tubes; but while Clathrozoon possesses thecæ, in Ceratella they are absent.

A great simplification of the colony is produced in Myriothela (Fig. 105 2), in which the short cœnosarc bears a single large terminal hydranth, and gives off numerous slender branches which bear the reproductive zooids $(s)$. Even greater simplicity is found in Corymorpha (3), in which the entire organism consists of a single stalked polype, from the tentacular region of which the medusæ $(m)$ arise. 
But the simplest members of the whole class, with the exception of one or two imperfectly known forms which will be referred to

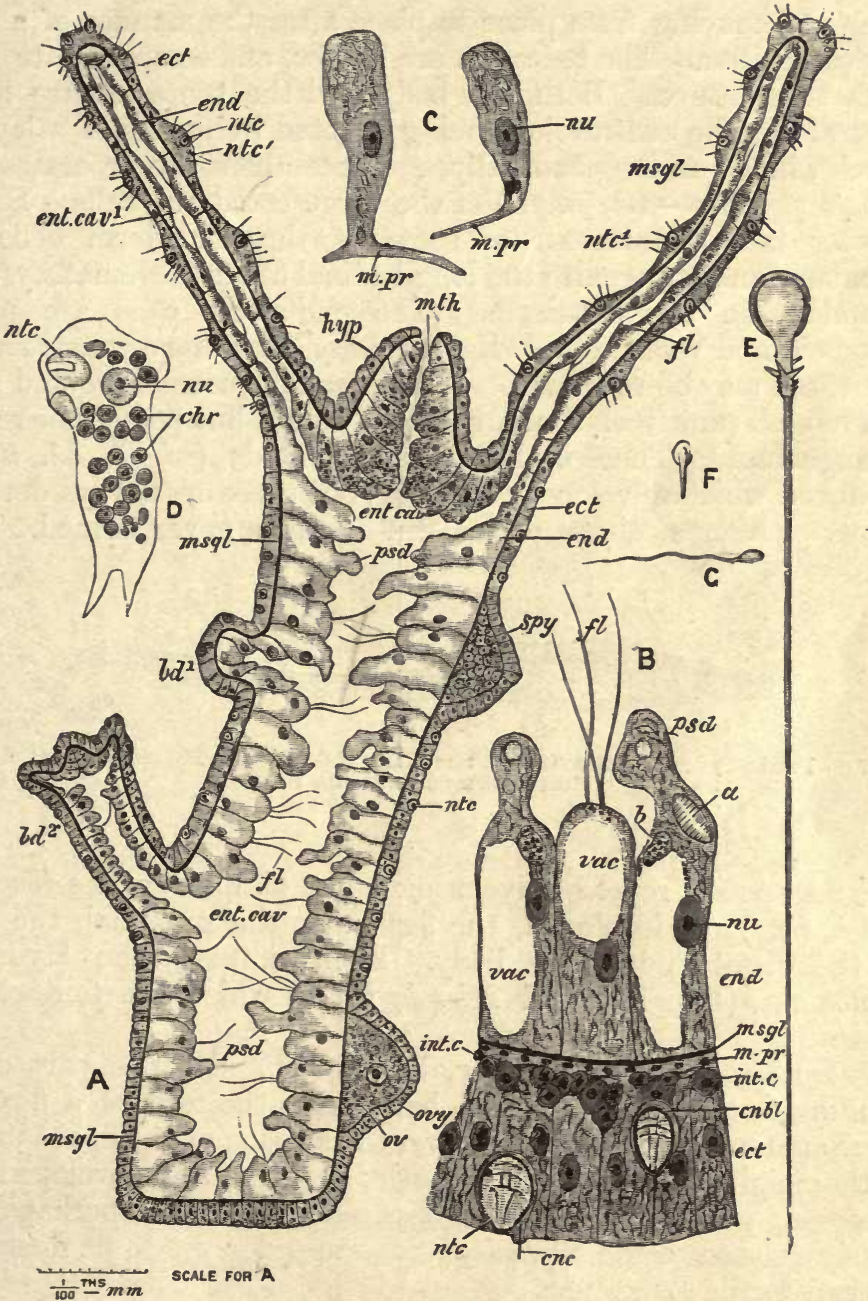

Fig.107.-Hydra. A, vertical section of entire animal; B, portion of transverse section, highly magnified; C, two large ectoderm cells; $\mathrm{D}$, endoderm cell of $H$. viridis; $\mathrm{E}$, large nematocyst : F, small nematocyst; G, sperm. $a$, ingested diatom ; bd. 1, bd. 2, buds ; chr. chromatophores ; cnbl. cnidoblast; enc. cnidocil ; ect. ectoderm; end. endoderm; ent. cav. enteric cavity; ent. $c a v^{\prime}$. its prolongation into the tentacles; $f$. flagellum; hyp. hypostome or manubrium; int. $c$. interstitial cells; $m$. pr. muscle-processes ; mth. mouth : msgl. mesogloea : ntc. large, and ntc'. small nematocysts; nu. nucleus; ov. ovum; ovy. ovary; psd. pseudopods ; sp. spermary ; vac. vacuole. (From Parker's Blementary Biology, after Lankester and Howes.)

below, are the Fresh-water Polypes of the genus Hydra. The entire organism (Figs, 27 and 107) consists of a simple cylindrical 
body with a conical hypostome and a circlet of six or eight tentacles. It is ordinarily attached, by virtue of a sticky secretion from the proximal end, to weeds, \&c., but is capable of detaching itself and moving from place to place after the manner of a looping caterpillar. The tentacles are hollow, and communicate freely with the enteron. Both the body and the tentacles are highly contractile, the contractions being effected by means of a layer of fibres which run longitudinally. These fibres are processes-the muscle processes-(C, m. pr.) of the large ectoderm cells. Similar shorter muscle-processes of some of the endoderm cells run circularly and antagonise the longitudinal fibres. Nematocysts are abundant in the ectoderm. The endoderm cells are mostly amœboid and vacuolated. Each usually bears one or more flagella, but these may be retracted. Glandular cells occur here and there. Nerve-cells (multipolar) occur in both layers, but present no regular arrangement. There is no perisarc. Buds (bd. 1, $b d$. 2) are produced which develop into Hydræ, but these are always detached sooner or later, so that a permanent colony is never formed. There

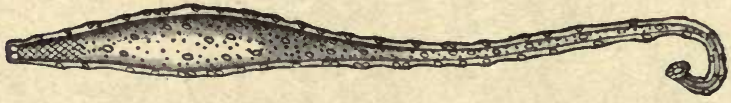

Fig.108.-Protohydra leuckartii. (From Chun, after Greeff.) The mouth is to the left, the disc of attachment to the right.

are no special reproductive zooids, but simple ovaries (ovy) and testes (spy) are developed, the former at the proximal, the latter at the distal end of the body. Even simpler than Hydra are Protohydra (Fig. 108) and Microhydra, in which the tentacles are absent.

Pelagohydra is also solitary, but is pelagic. The part corresponding to the base in Hydra here takes the form of a float, and there are tentacles distributed over the surface of the float as well as in the neighbourhood of the mouth; medusæ are developed from processes on the float. Pelagohydra, however, is perhaps more nearly related to the Siphonophora-an order yet to be dealt with - than to the Leptolinæ.

The polypes are usually cylindrical, as in Obelia, but in some genera they are widened out into a vase-like form (Fig. 105, 5), in others elongated into a spindle-shape (4). The tentacles may be disposed in a single circlet, as in Obelia and Hydra, or there may be an additional circlet round the hypostome $(3,5)$, or at the base of the polype, or they may be scattered irregularly over the whole surface (4). In Myriothela (2) they are short, and so numerous as to have the appearance of close-set papillæ. In some forms 
they are knobbed at the ends, the knobs being loaded with stingingcapsules (4).

In some species a dimorphism of the hydranths obtains, some of them being modified to form protective zooids. In Hydractinia (I) these are simply mouthless hydranths with very short tentacles abundantly supplied with nematocysts, capable of very active movements, and called dactylozooids $(d z)$. In Plumularia there are small structures called "guard-polypes," resembling tentacles in structure, with very numerous nematocysts, and each enclosed in a theca. In Hydractinia the cœnosarc is also produced into spines $(s p)$, which may be much modified zooids.

But the most remarkable modifications occur in the reproductive zooids. In a large proportion of genera, both of Anthomedusæ and Leptomedusæ, these take the form of locomotive medusæ, agreeing in general structure with the descriptions already given. Each appears at first as a hollow bud-like process of the blastostyle, or of an ordinary polype, or, more exceptionally, of the cœnosarc. This becomes constricted at the junction and rounded off. The ectoderm at its free extremity becomes thickened, and this thickening, as it grows, pushes the endoderm before it, producing a sort of involution. In the interior of the mass of ectoderm a cavity appears: this is destined to form the sub-umbrellar cavity. The ectodermal partition that at first separates the cavity from the exterior, becomes perforated and most of it is absorbed, what remains round the edge going to form the velum. The endoderm is reduced to a thin layer except along four radial lines where it gives rise to the four radial canals, the thin parts between going to form the endoderm lamella.

In different families and genera the medusæ exhibit almost endless variety in detail. As to size they vary from about $1 \mathrm{~mm}$. in diameter up to $400 \mathrm{~mm}$. (16 inches). The number of tentacles may be very great (Fig. 109, 2) or these organs may be reduced to two (Fig. 109, 1), or even to one (Fig. 105, 3); in the last-named cases it will be noticed that the medusa is no longer radially, but bilaterally symmetrical, i.e. it can be divided into two equal and similar halves by a single plane only-viz., the plane passing through the one or two tentacles. With the increase in the number of the tentacles a corresponding increase in that of the radial canals often takes place (Fig. 109, 3).

Some medusæ creep over submarine surfaces, walking on the tips of their peculiarly modified tentacles (Fig. 105,6) but the majority propel themselves through the water in a series of jerks by alternately contracting and expanding the umbrella, and so, by rhythmically driving out the contained water, moving with the apex foremost. In correspondence with these energetic movements there is a great development of both muscular and 


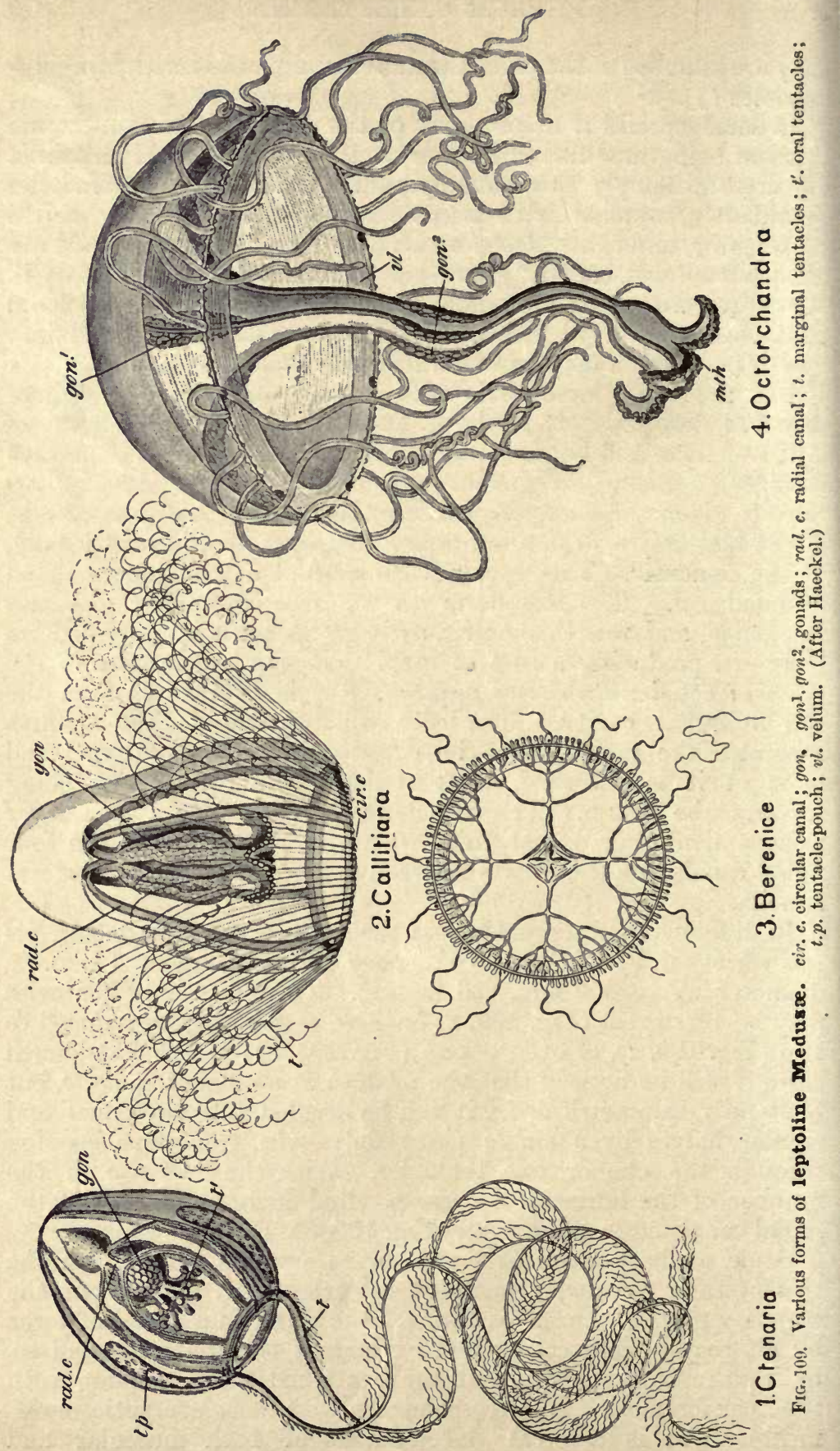


nervous systems. The velum and the sub-umbrella possess abundance of muscle-fibres, presenting a transverse striation, and round the margin of the umbrella is a double ring of nervecells and fibres, one ring being above, the other below the attachment of the velum (Fig. 101, D, nv, uv'). The medusæ thus furnish the first instance we have met with of a ceratral nemous system, i.e. a concentration of nervous tissue over a limited area serving to control the movements of the whole organism. It has been proved experimentally that the medusæ is paralysed by removal of the nerve-ring. Over the whole sub-umbrella is a loose network of nerve-cells and fibres connected with the nervering, and forming a peripheral nervous system.

In some medusæ the circular canal communicates with the exterior by minute pores placed at the summits of papillæ, the
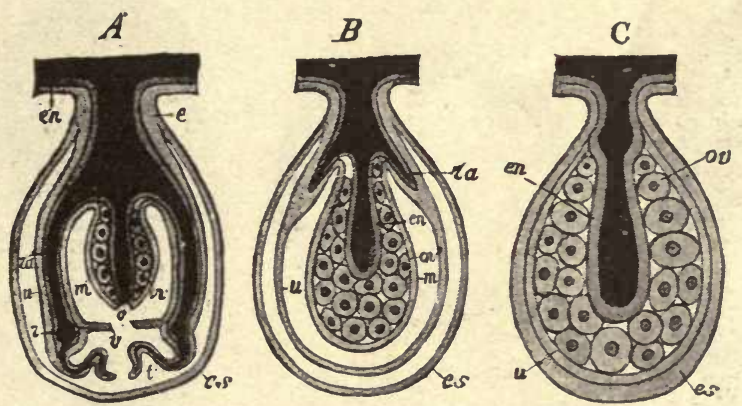

FIG. 110-Diagram illustrating the formation of a sporosac by the degradation of a medusa. $A$, medusa enclosed in ectodermal envelope $(e s) ; B$, intermediate condition with vestiges of umbrella $(u)$ and radial canals $(r a) ; C$, spurosac. ec. ectoderm ; $e n$, endoderm ; $m$, nuanubrium ; $o v$, ovary ; $t$, tentacle ; $v$, velum. (From Lang's Comparative Anatointy.)

endoderm cells of which contain brown granules. There seems to be little doubt that these are organs of excretion, the cells withdrawing nitrogenous waste-matters from the tissues and passing them out through the pores. If we except the contractile vacuoles of Protozoa, this is the first appearance of specialised excretory organs in the ascending series of animals.

Besides producing gonads, some medusæ multiply asexually by budding, the buds being developed either from the manubrium (Fig. 105, 7a), or from the margin of the umbrella $(7 b)$ or the base of the tentacles. The buds always have the medusa form.

In many Leptolinæ the reproductive zooids undergo a degradation of structure, various stages of the process being found in different species. Almost every gradation is found, from perfect medusæ to ovoid pouch-like borlies called sporosacs (Fig. 105, 1b, $5, s$ ), each consisting of little more than a gonad, but showing an indication of its true nature in a prolongation of the digestive cavity 
of the colony, representing the stomach of the manubrium (Fig. 110). We thus have a reproductive zooid reduced to what is practically a reproductive organ. It is obvious that a continuation of the same process might result in the production of a simple gonad like that of Hydra: there is, however, no evidence to show that the Fresh-water Polype ever produced medusæ, and the probabilities are that its ovaries and testes are simply gonads, and not degenerate zooids. The case is interesting as showing how a simple structure may be imitated by the degradation of a complex one. It is quite possible, on the other hand, that the reproductive organs of the Leptomedusæ (Fig. 100) are sporosacs, i.e. reproductive zooids, not mere gonads. In some rare cases the
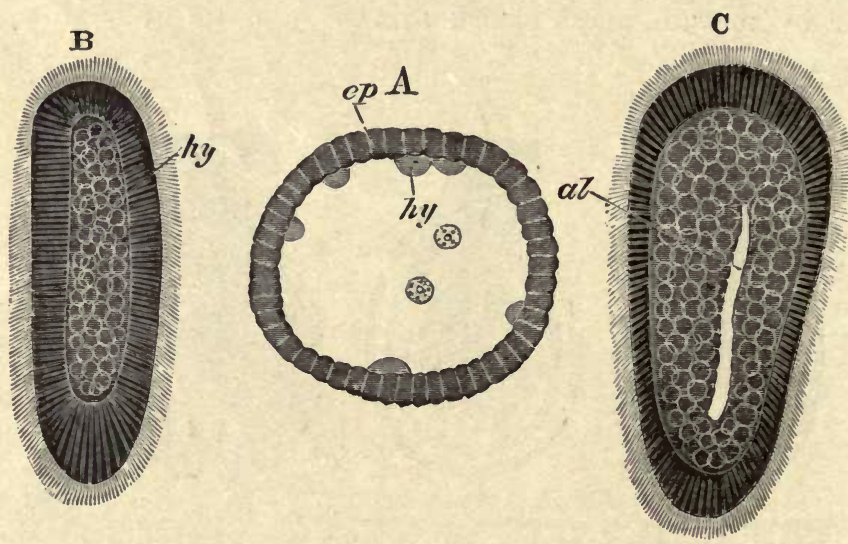

Fra.111.-Early development of Eucope. A, blastularstage; B, planula with solid endoderm ; C, planula with enteric cavity; al. enteric cavity; ep. ectoderm; hy. endoderm. (From Balfour's Enbryology, after Kowalevsky.)

sexual cells are not developed either in medusæ or in sporosacs, but are formed directly in the blastostyles.

In Obelia we found the medusæ to be budded off from peculiarly modified mouthless zooids - the blastostyles. This arrangement, however, is by no means universal: the reproductive zooids - whether medusæ or sporosacs-may spring directly from the coenosarc, as in Bougainvillea (Fig. 104), or from the ordinary hydranths (Fig. 105, 4 and 5). The primitive sex-cells, from which ova or sperms are ultimately developed, are sometimes formed from the endoderm or (more usually) ectoderm cells of the reproductive zooid; but in many cases originate in the coenosarc, and slowly migrate to their destination in the ectoderm of the gonad, where they metamorphose in the usual way into the definitive reproductive products, which when mature pass into the space below the ectoderm of the gonad.

The development of the Leptolinæ frequently, but not always, 
begins within the maternal tissues, i.e. while the oosperm or impregnated egg-cell is still contained in the gonad of the medusæ or in the sporosac. The oosperm divides into two cells, then into four, eight, sixteen, \&c. Fluid accumulates in the interior of the embryo, resulting in the formation of a blastula or hollow globe formed of a single layer of cells (Fig. 111, A). The blastula elongates, and the cells at one pole undergo division, the daughtercells passing into the cavity, which they gradually fill (B). At this stage the embryo is called a planula: it consists of an outer layer of cylindrical cells - the ectoderm - which acquire cilia, and an inner mass of polyhedral cells-the endoderm. In some cases the planula arises by a different process : a solid morula is formed, the superficial cells of which become radially elongated and form ectoderm, the central mass of cells becoming endoderm. By means of its cilia the planula swims freely, and before long a cavity appears in the middle of the solid mass of endoderm, the cells of which then arrange themselves in a single layer around the cavity or enteron $(\mathrm{C}, a l)$. The planula then comes to rest, fixes itself at one end to some suitable support, and becomes converted into a simple polype or hydrula by the attached end broadening into a disc and the opposite extremity forming a manubrium and tentacles. The hydrula soon begins to send off lateral buds, and so produces the branched colony.

In Tubularia the oosperm develops, while still enclosed in the sporosac, into a short hydrula, which, after leading a free existence for a short time, fixes itself by its proximal end, buds, and produces the colony. In Hydra development begins in the ovary, and is complicated by the fact that the ectoderm of the morula gives rise to a sort of protective shell: in this condition the embryo is set free, and, after a period of rest, develops into the adult form.

\section{ORDER 2.-TRACHYLINA}

General Structure.-The members of this order are all medusæ: no zoophyte stage is certainly known in any of them, and several species have been proved to develop directly from the egg. They thus differ from the members of the preceding order in the fact that no alternation of generations ordinarily occurs in their life-history.

Most species are of small or moderate size, the largest not exceeding $100 \mathrm{~mm}$. ( 4 inches) in diameter. The gelatinous tissue or mesoglœa of the ex-umbrella is usually well developed, giving the medusa a more solid appearance than the delicate jelly-fish of the preceding order: this is well shown in Fig. 112, in which the apical region of the umbrella has a comparatively immense thickness. The tentacles are also stiff and strong, and are always solid in the young condition, although they may be replaced in the adult by hollow tentacles. 
But the most characteristic anatomical feature of the group is the structure of the sense-organs, which are club-shaped bodies (Figs. 112 and 113,tc) consisting of an outer layer of ectoderm
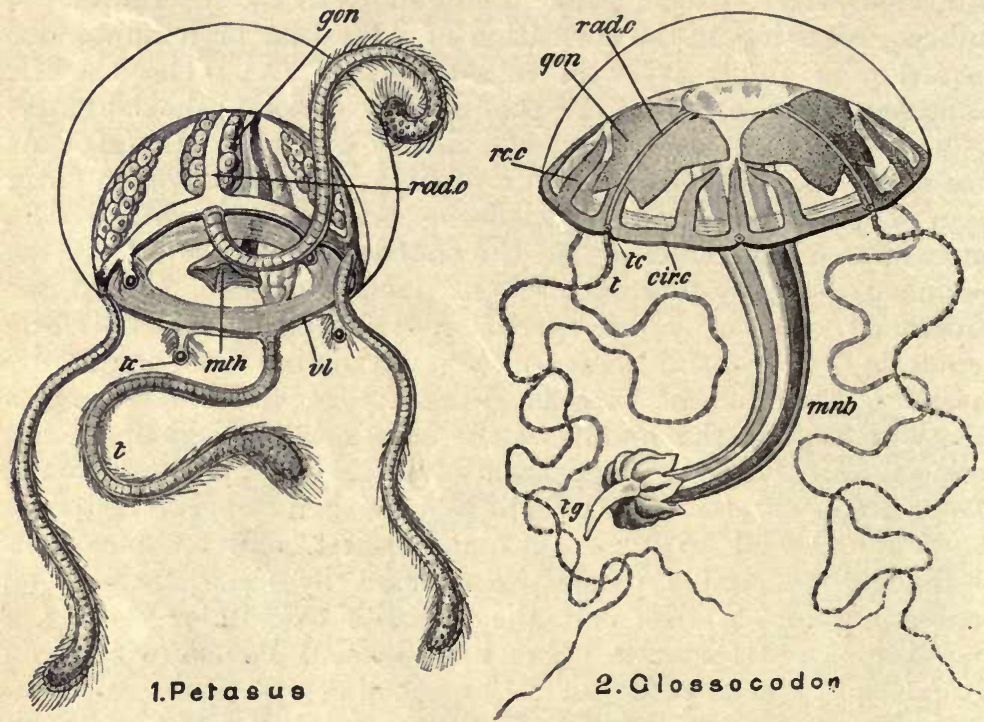

FIG. 112,-Two Trachy medusæ. cir. c. circular canal; gon. gonad; mnb. manubrium; mith. mouth; rad. $c$. radial canal; rc. $c$. recurrent canal; $t$. tentacle; $t$. tentaculocyst; $t g$. tongue; vl. velum. (After Haeckel.)

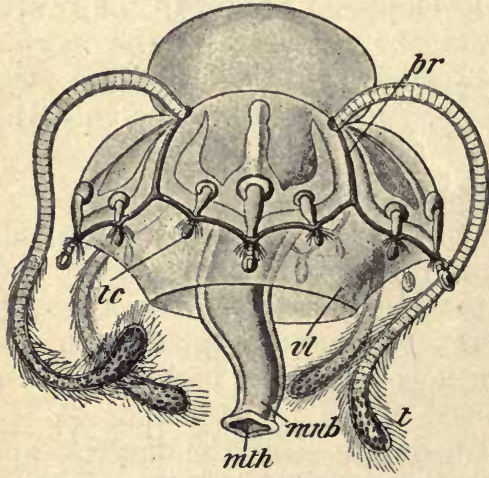

1. Cunarcha

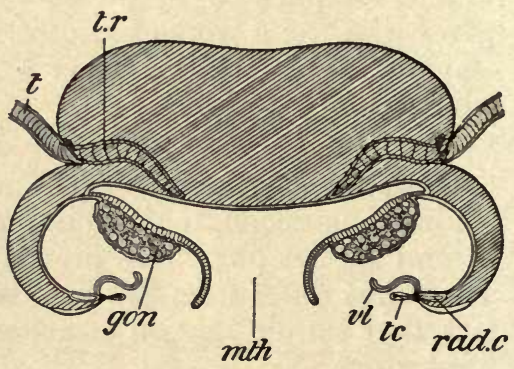

2.Polycolpa

FIG. 113.-Two Narcomedusx, 2 in vertical scetion. gon. gonad; mnb. mauubrium; mth. mouth ; $p r$. peronium ; rad.c. radial canal ; $t$. tentacle ; tc. tentaculocyst ; $t . r$. tentacle-root ; v.l. velum. (After Haeckel.)

enclosing a central axis of endoderm cells (Fig. 114): they have, therefore, the structure of tentacles. They contain one or more lithites, which are always derived from the endoderm. To 
distinguish them from the lithocysts of Leptomedusæ, and to mark the fact that they are modified tentacles, they are called tentaculocysts. They may either project freely from the margin of the umbrella, or may become enclosed in a pouch-like growth of ectoderm and more or less sunk in the tissue of the umbrella. Eyes occur in some, and are always of simple structure.

The two sub-orders of Trachylinæ are characterised by the mode of origin of the tentacles.

In Trachymedusæ, as in the preceding order, they arise near the edge of the umbrella (Fig. 112), but in the Narcomedusæ they spring about half-way between the edge and the vertex (Fig. 113), and are continued, at their proximal ends, into the ielly of the ex-umbrella in the form of "tentacle-roots" (t.r).

As to the position of the reproductive organs, there is the same difference between the two sub-orders of Trachylinæ as between the two sub-orders of Lepto-

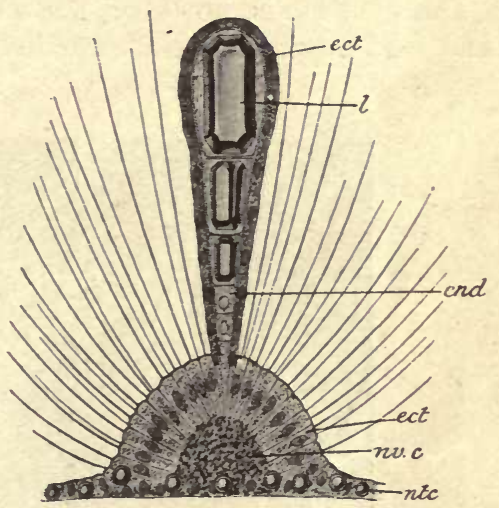

Fig. 114.-Fginura myosura, a tentaculocyst highly magnified. ect. ectoderm; end. endoderm; $l$. lithites; ntc. nematocysts ; nc.c. group of nerve-cells. (After Haeckel).

linæ. In the Trachymedusæ the gonads (Fig. 112, gon) are developed in the course of the radial canals : in the Narcomedusæ (Fig. 113) they lie on the manubrium, sometimes extending into the pouch-like offshoots of its cavity.

There is always a well-developed velum, which, as in Fig. 113, 1, may hang down vertically instead of taking the usual horizontal position. In the Narcomedusæ the manubrium is short; in the Trachymedusæ it is always well developed, and is sometimes (Fig. $112,2)$ prolonged into a long, highly contractile peduncle, having its inner surface produced into a tongue-like process $(t g)$ which protrudes through the mouth. In some the gastric cavity is situated in the manubrium, which in such a case is looked upon as partly of the nature of a process of the sub-umbrella (pseudomanubrium).

The simplest case of the development of Trachylinæ is seen in Aginopsis, one of the Narcomedusæ. The oosperm gives rise to a ciliated planula, which forms first two (Fig 115), then four tentacles, and a mouth, hypostome, and stomach. The larva of Eginopsis is thus a hydrula, closely resembling the corresponding stage of Tubularia. After a time the tentacular region grows out, carrying the tentacles with it, and becoines the umbrella of the 
medusa. Thus the actual formation of the medusa from the hydrula of Eginopsis corresponds precisely with the theoretical derivation given above (p. 136). It will be seen that in the present case there is no metagenesis or alternation of generations, but that development is accompanied by a metamorphosis-that is, the egg gives rise to a larval form differing in a striking manner from the adult, into which it becomes converted by a gradual series or changes.

Metagenesis is, however, not quite unknown among the Trachylinæ. In a parasitic Narcomedusa (Cunina parasitica) the planula

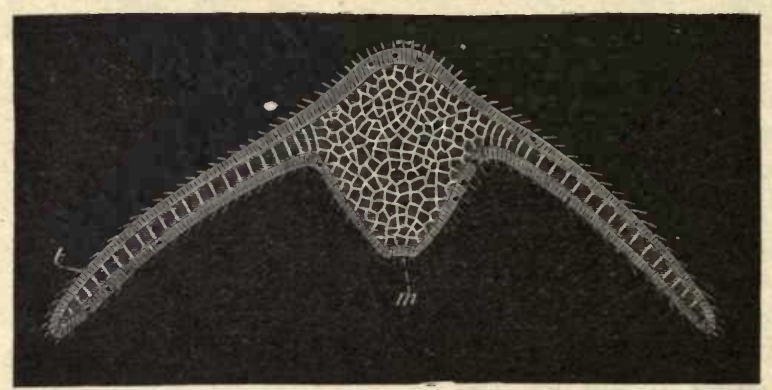

FiG. 115. -Larva of Fginopsis. m. mouth; $t$. tentacle. (From Balfour, after Metschnikoff.)

fixes itself to the manubrium of one of the Trachymedusæ which serves as its host, and develops into a hydrula. But the latter, instead of itself becoming metamorphosed into a medusa, retains the polype form and produces other hydrulæ by budding, these last becoming converted into medusæ in the usual way.

\section{ORder 3.-HydROCORALlina.}

The best-known genus of Hydroid Corals is Millepora, one species of which is the beautiful Elk-horn Coral, M. alcicornis. The dried colony (Fig. $116 \mathrm{~A}$ ) consists of an irregular lobed or branched mass of carbonate of lime, the whole surface beset with the numerous minute pores to which the genus owes its name. The pores are of tivo sizes: the larger are about 1 or $2 \mathrm{~mm}$. apart, and are called gastropores ( $\mathrm{B}, 9 . p)$; the smaller are arranged more or less irregularly round the gastropores, and are called dactylopores (d.p). The whole surface of the coral between the pores has a pitted appearance. Sections (C) show that the entire stony mass is traversed by a complex system of branched canals, which communicate with the exterior through the pores. The wide vertical 
canals in immediate connection with the gastropores are traversed by horizontal partitions, the tabulce $(t b)$.

In the living animal each pore is the place of origin of a zooid: from the gastropores protrude polypes (Fig. 117, $P$ ) with hypostome and four knobbed tentacles; from the dactylopores long, filamentous, mouthless dactylozooids or feelers $(D . Z)$, with irregularly disposed tentacles: the function of these latter is probably protective and tactile, like that of the guard-polypes of Plumularia and the dactylozooids of Hydractinia. The bases of the zooids are connected with a system of delicate tubes, which ramify through the

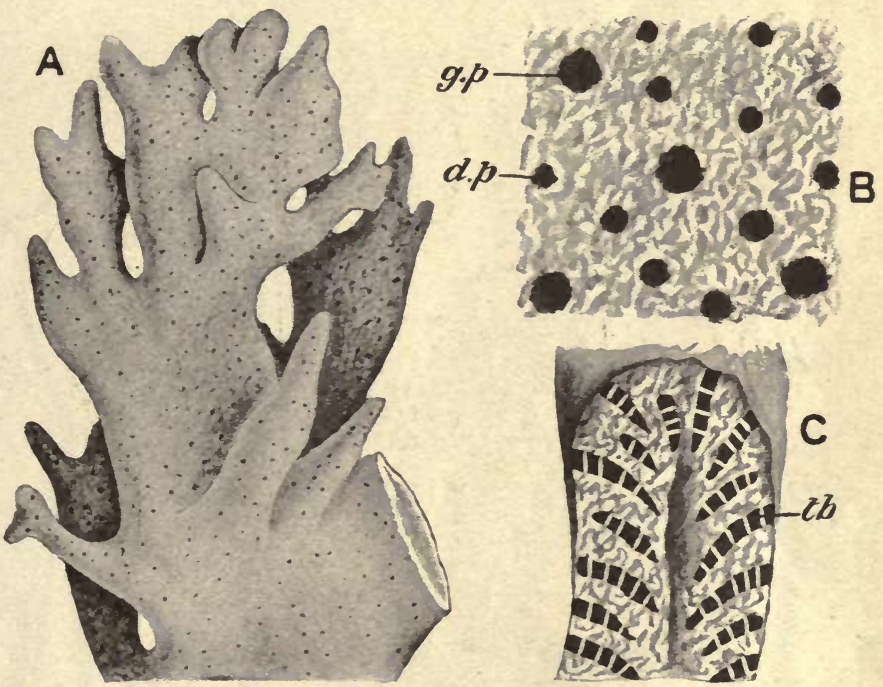

FiG. 116-IMillepora alcicornis. A, part of skeleton, natural size; B, portion of surface, magnified; C, vertical section, magnified; $d . p$. dactylopores; g.p. gastropores; $t b$. tabula. (After Nicholson and Lydekker.)

canals of the coral and represent a much-branched cœnosarc, recalling that of Hydractinia (p. 144).

The cœnosarcal tubes have the usual structure, consisting of ectoderm and endoderm with an intervening mesoglœe. From the relative position of the parts it will be obvious that the calcareous skeleton is in contact throughout with the ectoderm of the colony: it is, in fact, like the horny perisarc of the Leptolinæ, a cuticular product of the ectoderm.

The only other genus to which we shall refer is Stylaster (Fig. 118), which forms a remarkably elegant tree-like colony, abundantly branched in one plane, and of a deep pink colour. On the branches are little cup-like projections with radiating processes passing from the wall of the cup towards the centre, and thus 
closely resembling the true cup-corals belonging to the Actinozoa (vide infra). But in the case of Stylaster each "cup" is the locus, not of one, but of several zooids-a polype projecting from its centre, and a dactylozooid from each of the compartments of its peripheral portion. A calcareous projection, the style, the presence of which is the origin of the generic name, rises up from the tabula at the bottom of each pore.

The gonophores in most species of Millepora are developed in certain of the pores in dilatations or ampulla; in one species at

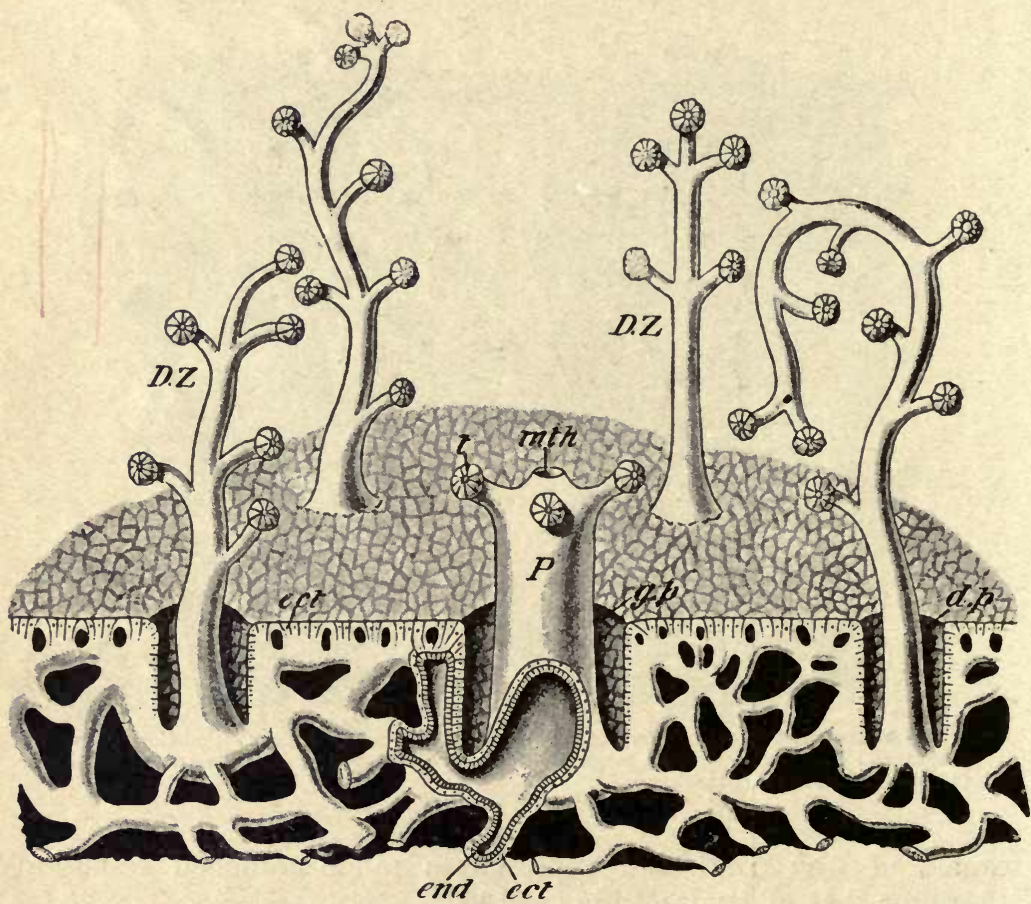

Fra. 117.-IMillepora. Diagrammatic view of a portion of the living animal, partly from the surface, partly in vertical section. In the sectional part the ectoderm is dotted, the endoderm striated, and the skeleton black. ect. ectoderm; end. endoderm; d.p. dactylopore ; D.Z. dactylozooid; g.p. gastropore; $m t h$. mouth; $P$. polype; $t$. tentacle. (Altered from Moseley.)

the apices of the dactylozooids. They are medusæ, but never have the complete medusa-form, being devoid of velum, mouth, radial canals and tentacles. Both male and female medusæ become free, but the period of free existence is very short.

In Stylaster the medusoid character is much more completely lost, and the gonophores are more of the nature of sporosacs or 
degraded reproductive zooids lodged in special chambers ( $a$ ) of the coral.

The Hydrocorallina occur only in the tropical portions of the Pacific and Indian Oceans, where they are found on the "coral-

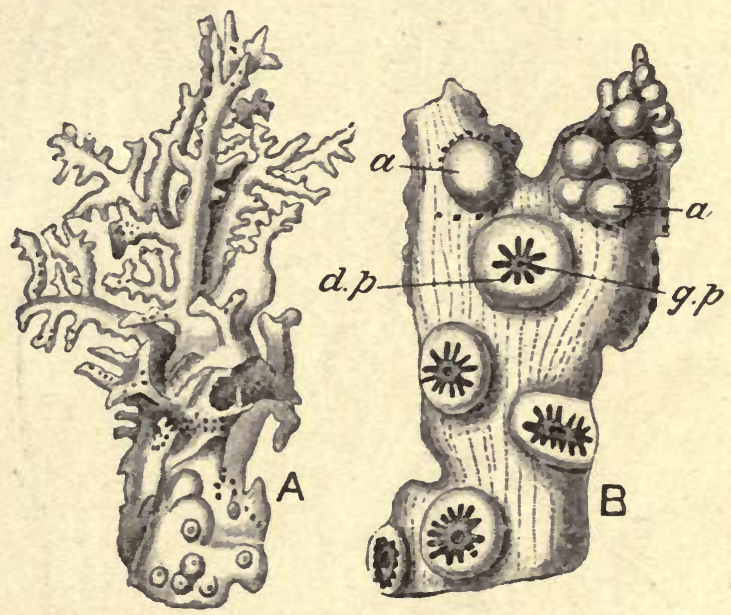

Fin. 119,-Stylaster sanguineus. A, portion of skeleton, natural size ; B, small portion, magnified; $a$. ampullie; $d . p$. dactylopores; g.p. gastropores. (After Nicholson and Lydekker.)

reefs" partly or entirely surrounding many of the islands in those seas. Fossil forms are found as far back as the Triassic epoch.

\section{ORDER 4.-SIPHONOPHORA.}

The diversity of form exhibited by the members of this order is so great that anything like a general account of it would only be confusing to the beginner, and the most satisfactory method of presentation will be by the study of a few typical genera.

Halistemma (Fig. $119 \mathrm{~A}$ ) occurs in the Mediterranean and other seas, and consists of a long, slender, floating stem, to which a number of structures, differing greatly in form, are attached. At one - the uppermost-end of the stem is an ovoid, bubble-like body containing air-the float or pneumatophore $(p n)$. Next come a number of closely set, 'cransparent structures $(n c t)$, having the general characters of unsymmetrical medusæ without manubria, each being a deep, bell-like body, with a velum and radiating canals. During life these swimming-bells or nectocalyces contract rhythmically-i.e. at regular intervals - drawing water into their cavities, and immediately pumping it out, thus serving to propel the entire organism 


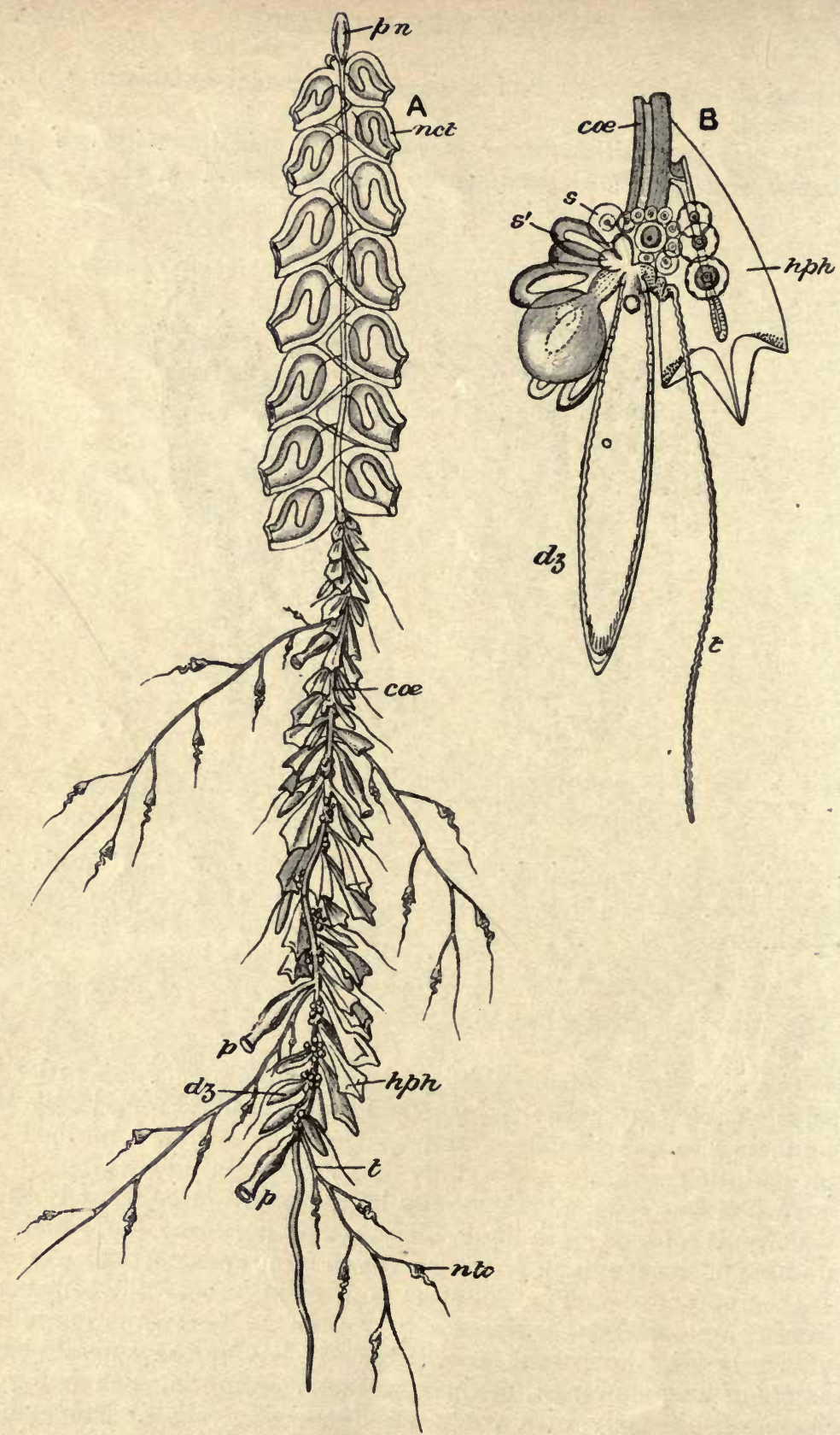

Fig. 119.-Halistemma tergestinum, A, the entire colony; B, a single group of zooids. $c œ$. conosarc; $d z$. dactylozooid; $h p h$. hydrophyllium or bract; nct. nectocalyx or swimmingbell ; ntc. battery of nematocysts; $p$. polype; $p n$. pneumatophore or float; $s, s^{\prime}$. sporocysts ; t. tentacle. (After Claus.) 
through the water. Below the last nectocalyx the character of the structures borne by the stem changes completely: they are of several kinds, and are arranged in groups which follow one another at regular intervals, and thus divide the stem into segments, like the nodes and internodes of a plant.

Springing from certain of the "nodes" are unmistakable polypes $(p)$, differing however from those we have hitherto met with in having no circlet of tentacles round the mouth, but a single long branched tentacle $(t)$ arising from the proximal end, and bearing numerous groups or " batteries" of stinging-capsules (ntc). In the remaining nodes the place of the polypes is taken by dactylozooids or feelers $(d z)$-mouthless polypes, each with an unbranched tentacle springing from its base. Near the bases of the polypes and dactylozooids spring groups of sporosacs $\left(\mathrm{B}, s, s^{\prime}\right)$, some male, others female; and finally delicate, leaf-like, transparent bodiesthe bracts or hydrophyllic (hph) - spring from the "internodes" and partly cover the sporosacs.

It is obvious that on the analogy of such a hydroid polype as Obelia, Halistemma is to be looked upon as a polymorphic Hoating colony, the stem representing a cœnosarc, and the various structures attached to it zooids-the polypes nutritive zooids, the feelers tactile zooids, the sporosacs reproductive zooids, the bracts protective zooids, and the swimming-bells locomotory zooids. The float may be looked upon as the dilated end of the stem, which has become invaginated or turned-in so as to form a bladder filled with air, its outer and inner surfaces being furnished by ectoderm, and the middle portion of its wall by two layers of endoderm, between which the enteric cavity originally extended (Fig. 120, $p n$ ). The upper or float-bearing end is proximali.e. answers to the attached end of an Obelia-stem: it is the opposite or distal end which grows and forms new zooids by budding.

In some Siphonophora the bracts contain indications of radial canals, so that these structures, as well as the swimming-bells and sporosacs, are formed on the medusa-type, while the hydranths and feelers are constructed on the polype-type.

It will be noticed that the radial symmetry, so characteristic of most of the Hydrozoa previously studied, gives way, in the case of Halistemma, to a bilateral symmetry. The swimming-bells are placed obliquely, and the mouth of the bell is not at right angles to the long axis, so that only one plane can be taken dividing these structures into two equal halves: the same applies to the polype and feelers with their single basal tentacle. When first formed the various zooids are all on one side of the stem, but the latter becomes spirally twisted during growth, and so causes them to arise irregularly.

VOL. I 
The egg of Halistemma gives rise to a ciliated planula resembling that of the other Hydrozoa. At one pole the ectoderm becomes invaginated to form the float (Fig. 121, ep), the opposite extremity is gradually converted into the first polype $\left(p_{0}\right)$, and

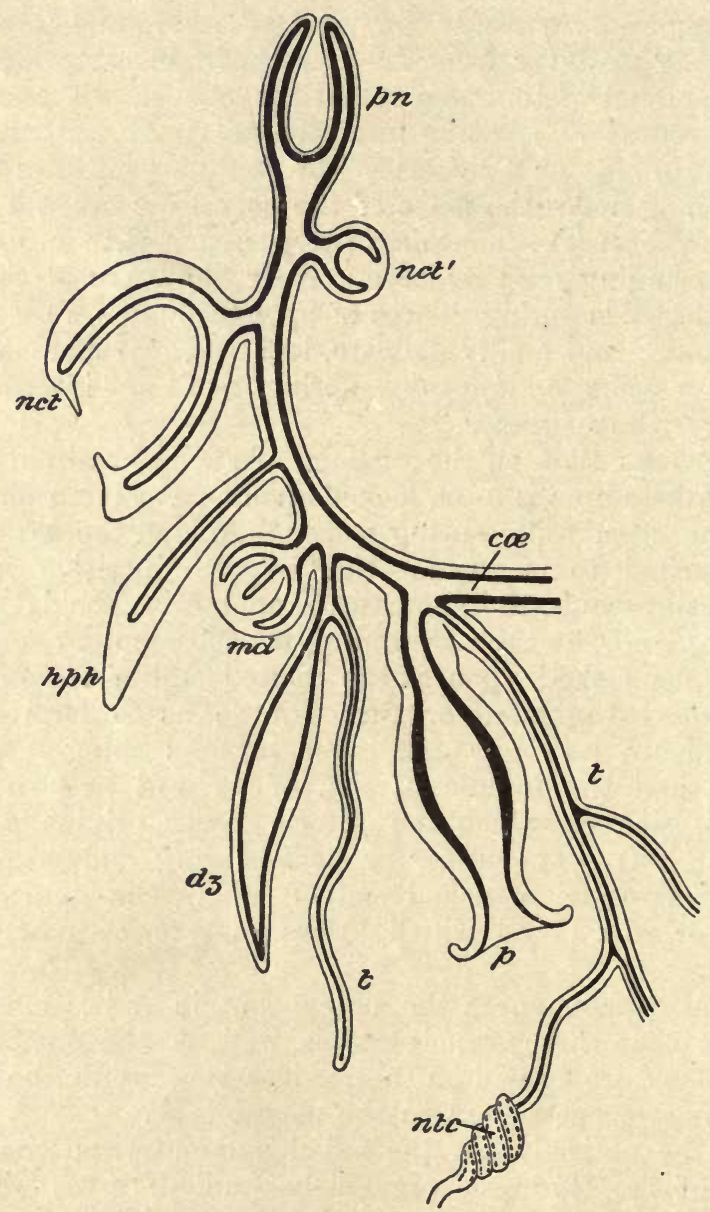

F1G. 120.-Diagram of a Siphonophore: the thick line represents endoderm; the space external to it, ectoderm; the interual space, the enteric cavity. co. copnosarc; $d z$. dactylozooid; hph. hydrophyllium; mul. sporosac ; nct, nct'. nectocalyces; ntc. battery of nematocysts ; p. polype ; $p n$. pneumatophore; $t$. tentacle. (After Claus.)

a bud appears on one side which becomes the first tentacle $(t)$. By gradual elongation, and the formation of new zooids as lateral buds, the adult form is produced; the various zooids are all formed between the first polype and the float, so that the two 
become further and further apart, being always situated at the distal and proximal ends of the colony respectively.

In an allied form (Agalma) the first structure to appear in the embryo is not the float, but the first bract, which grows considerably and envelops the growing embryo in much the same way as the umbrella of a medusa envelops the manubrium. On this and other grounds some zoologists look upon the Siphonophorecolony as a medusa the manubrium of which has extended immensely and produced lateral buds after the manner of some Anthomedusæ (Fig. 105, $7 a$ ).

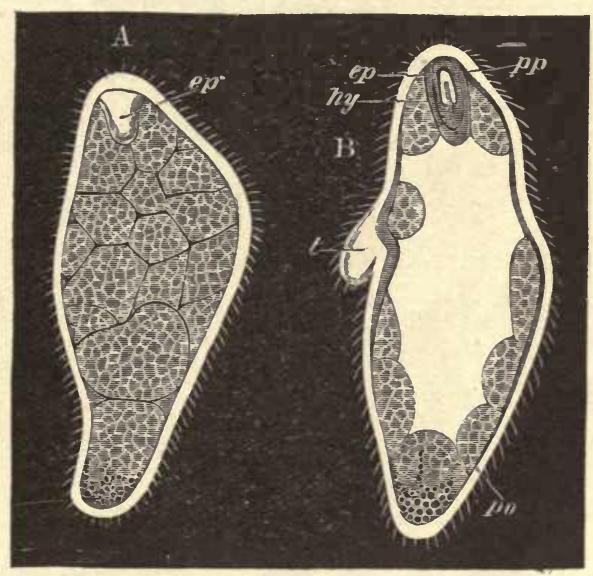

Fig. 121. -Two stages in the development of Halistemma : the endoderm is shaded, the ectoderm left white. ep. pneumatocyst or air-chamber of pneumatophore; hy. endoderm surrounding pneumatophore; po. polype; $p p$. pneumatophore; $t$. tentacle. (From Balfour, after Metschnikoff.)

On this theory the entire cœnosare is an extended manubrium, and the first or primary bract is the umbrella. But frequently-as in Halistemma-a primary bract is not formed, and when present there appears to be no reason against regarding it as a lateral bud of the axis, of quite the same nature as the remaining zooids.

In the well-known "Portuguese man-of-war" (Physalia) there is a great increase in proportional size of the float and a corresponding reduction of the rest of the cœnosarc. The float (Fig. $122, p n)$ has the form of an elongated bladder, from 3 to $12 \mathrm{~cm}$. long, pointed at both ends, and produced along its upper edge into a crest or sail $(c r)$ : as a rule it is of a brilliant peacock-blue colour, but orange-coloured specimens are sometimes met with. At one end is a minute aperture communicating with the exterior. There are no swimming-bells, but from the underside of the float hang gastrozooids $(p)$, dactylozooids, branching blastostyles (gonodendra) with groups of medusoids looking like bunches of grapes of a deep blue colour, and long retractile tentacles $(t)$, 
sometimes several feet in length and containing batteries of stinging-capsules powerful enough to sting the hand as severely as a nettle. The male reproductive zooid remains attached, as in

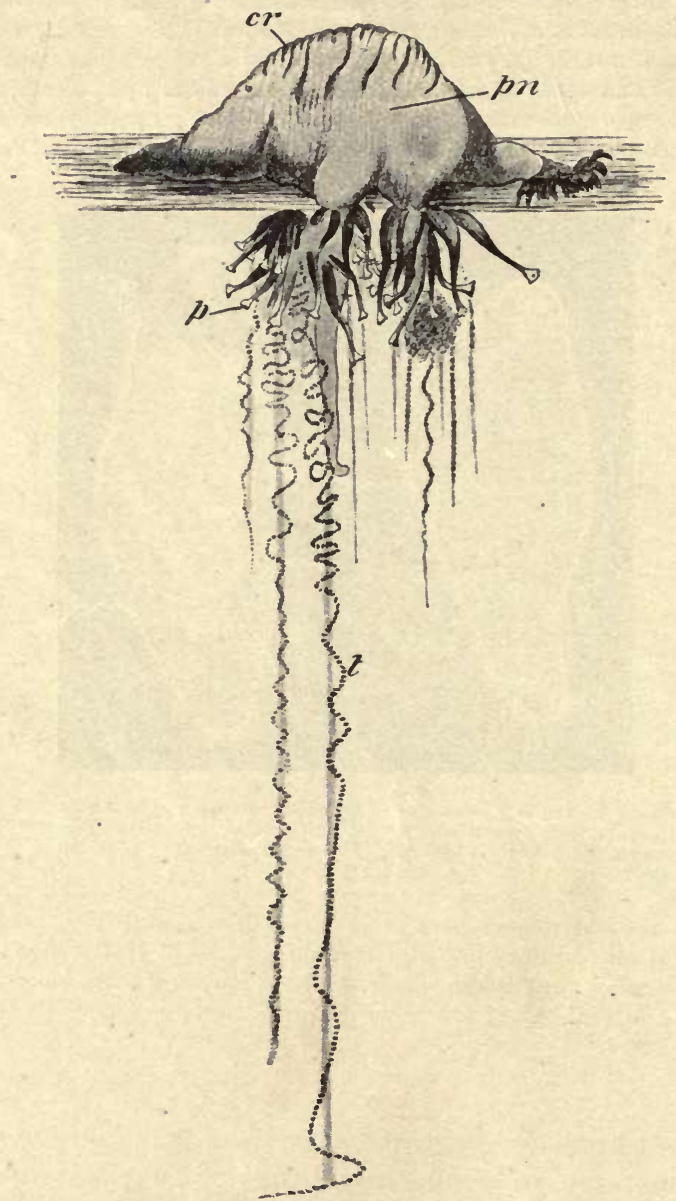

Fig. 122.-Physalia : the living animal floating on the surface of the sea. $c r$. crest; $p$. polype ; pn. pneumatophore: $t$. tentacle. (After Huxley.)

Halistemma, but the female apparently becomes detached as a free medusa.

In Diphyes the float is absent. Two swimming-bells (Fig. 123 A,m) of proportionally immense size are situated at the proximal end of the cœnsarc, and are followed by widely-separated groups of cooids $(B)$, each group containing a polype $(n)$ with its tentacles $(i)$, 
a meduzoid $(y)$, and a large enveloping bract $(t)$. The stem often breaks at the internodes, and the detached groups of zooids then swim about like independent organisms.

Porpita is formed on a different type, and has a close general resemblance to a medusa. It consists (Fig. 124) of a discoid

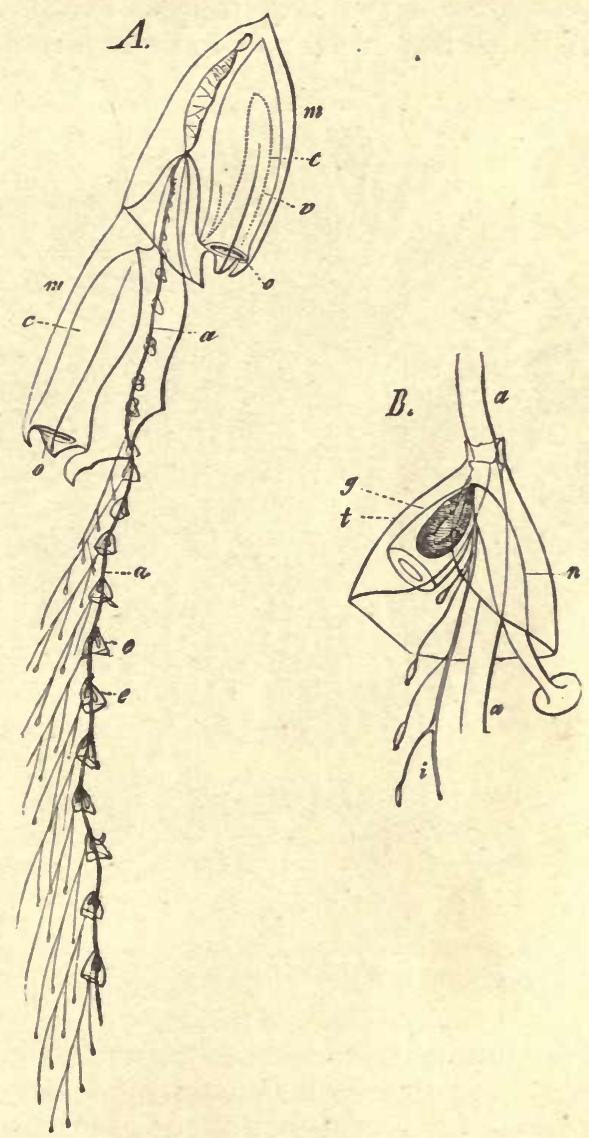

FiG. 123. - Diphyes campanulata A, the entire colony; B, single group of zooids. $a$, conosarc; $c$, cavity of swimming-bell ; $e$, groups of zooids; $g$, medusoid ; $i$, grappling line or tentacle; $m$, swimming-bell; $n$, polype; $o$, mouth of swimming-bell; $t$, bract. (From Parker's Biology, after Gegenbaur.)

body, enclosing a chambered chitinoid shell (sh) containing air, and obviously corresponding with the float of Physalia. The edge of the disc is beset with long dactylozooids $(t)$ and from its lower surface depend numerous closely set blastostyles provided with mouths and bearing medusæ, while in the centre is a single large 
gastrozooid (hy). The closely allied genus Velella is of rhomboidal form, and bears on its upper surface an oblique sail.

The reproductive zooids are liberated as free medusæ. The eggs give rise to young which have a close resemblance to flat medusæ with manubrium, marginal tentacles, and an air-chamber or float developed in the ex-umbrella. Thus it is quite possible that the Siphonophora of the Porpita-type may be medusæ the sub-umbrella of which has given rise to buds forming the feelers
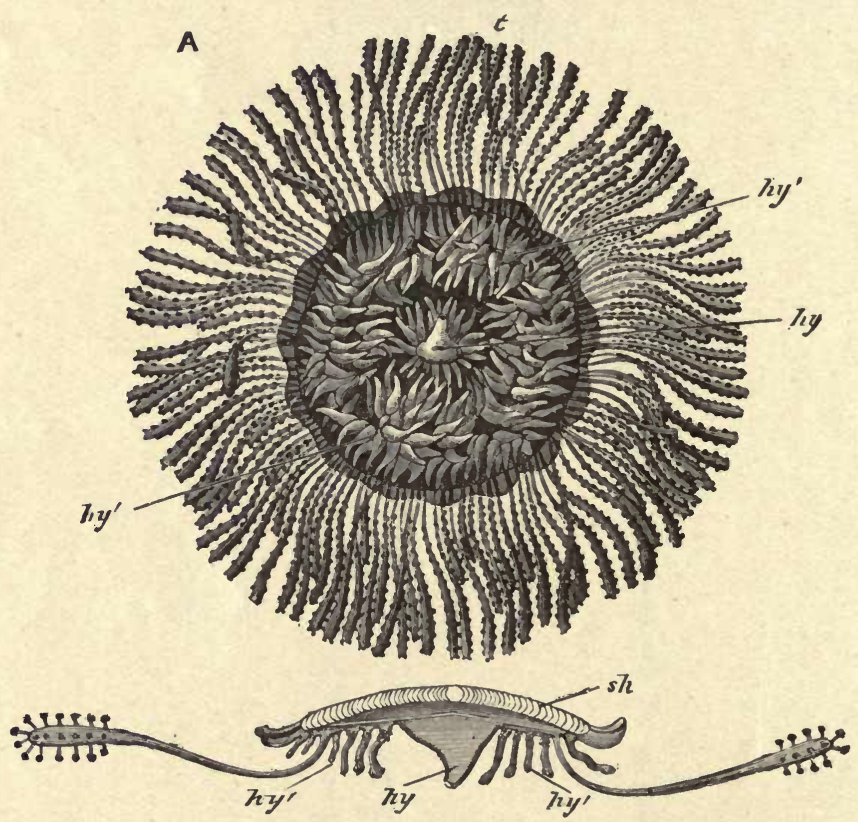

Fig. 124.-Porpita pacifica. A, from beneath; B, vertical section. hy, lirge central gastro. zooid; $h y^{\prime}$. blastostyles; sh, chambered shell; $t$, dactylozooids. (From Parker's Biology, after Duperry and Koelliker.)

and blastostyles. But, as their early development is not known, it is still quite legitimate to describe them in the same terms as the other Siphonophora-i.e. to consider them as hydroid colonies in which the conosarc is represented by the discoid or rhomboid body with its contained air-chamber.

\section{ORDER 5.-GRAPTOLITHIDA.}

The "Graptolites" are fossil Hydrozoa found in the Upper Cambrian and Silurian rocks. They are known only by their fossilised chitinoid skeleton, all trace of the soft parts having, as in the majority of fossils, disappeared.

With oue doubtful exception they are compound, consisting of an elongated tube-the perisarc of the common stem, having attached to it, either in a single 
or a double row, numerous small projections, the hydrothecæ (Fig. 125, h.th). The cœnosarcal skeleton is strengthened by a slender axis, the virgula $(v)$, the proximal end of which is connected with a small dagger-shaped body, the sicula $(s)$, supposed to be the skeleton of the primary zooid by the budding of which the colony was produced. In connection with some species oval or cup-like capsules have been found: these may be of the nature of gonothecæ. But it must be added that the evidence in favour of associating the Graptolites with the Hydrozoa is by no means conclusive, and reasons have been adduced for regarding them as connected with groups much higher in the scale.

\section{Additional Remarks on the Hydrozoa.}

The vast majority of Hydrozoa are marine, the only exceptions being Hydra, found all over the world; Microhydra, at present known only in North America; Cordylophora, one of the Anthomedusæ, found in Europe, America, Australia, and New Zealand; Polypodium, also an Anthomedusa, found in the Volga, where in one stage of its existence it is parasitic on the eggs of a Sturgeon; Limnocodium, a doubtful Trachymedusa, hitherto found only in a tank in the Botanical Gardens, Regent's Park, where it was probably introduced from the West Indies; and Limnocnida, found in Lake Tanganyika, Africa.

The oldest known Hydrozoa are the Graptolites, found first in the Cambrian rocks; Hydractinia occurs in the Cretaceous epoch, and Hydrocorallinæ from the Cretaceous bonwards.

Parasitism, although rare, is not unknown in the class. Polypodium, one of the Anthomedusæ, is parasitic during part of its existence, in the ovary of the Sturgeon; and Cunina, one of the Narcomedusæ, is parasitic on a Trachymedusa.

In the section on the Protozoa we saw that while the majority of species are independent cells, each performing alone all the essential functions of an animal, others, such as Pandorina, Volvox, and Proterospongia, consist of numerous unicellular zooids associated to form a colony in which a certain division of labour obtains, the function of reproduction, for instance, being assigned to certain definite cells and not performed by all alike. Thus the colonial Protozoa furnish an example of individuation, numerous cells combining to form a colony in which the several parts are dependent one upon another, and which may therefore be said to constitute, from the physiological point of view, an individual of a higher order than the cell. 
This is still more notably the case in the lower Metazoa, such as Ascetta and Hydra, in which we have numerous cells combined to form a permanent two-layered sac with a terminal aperture, some of the cells having digestive, others tactile, others reproductive functions. Thus while an Amœba or a Paramœcium is an individual of the first order, Hydra and Ascetta are individuals of the second order, each the equivalent of an indefinite number of individuals of the first order.

In the Hydrozoa we see this process carried a step further. Budding takes place and colonies are produced, the various zooids of which-each the equivalent of a Hydra-instead of remaining all alike, become differentiated both morphologically and physiologically, so as to differ immensely from one another both in form and function. In Obelia, for instance, reproduction is made over exclusively to the medusæ, while in Halistemma we have zooids specially set apart, not only for reproductive, but for tactile and protective purposes. Thus in Halistemma and the other Siphonophora there is a very complete subordination of the individual zooids to the purposes of the colony as a whole, the colony thus assuming, from the physiological point of view, the characteristics of a single individual, and its zooids the character of organs. In this way we get an individual of the third order, consisting of an aggregate of polymorphic zooids, just as the zooid or individual of the second order is an aggregate of polymorphic cells or individuals of the first order.

\section{CLASS II.-SCYPHOZOA.}

\section{Example of the Class-The Common Jelly-fish (Aurelia aurita).}

Aurelia is the commonest of the larger jelly-fishes, and is often found cast up on the sea-shore, when it is readily recognisable by its gelatinous, saucer-shaped umbrella, three or four inches in diameter, and by having near the centre four red or purple horseshoe-shaped bodies-the gonads-lying embedded in the jelly.

External Characteristics.-The general arrangement of the parts of the body is very similar to what we are already familiar with in the hydrozoan jelly-fishes. Most conspicuous is the concavo-convex umbrella, the convex surface of which, or exumbrella, is uppermost in the ordinary swimming position (Figs. 126 and $127, \mathrm{~A})$. The outline is approximately circular, but is broken by eight notches, in each of which lies a pair of delicate processes, the marginal lappets (mg. lp.): between the pairs of lappets the edge of the umbrella is fringed by numerous close-set marginal tentacles $(t)$. 

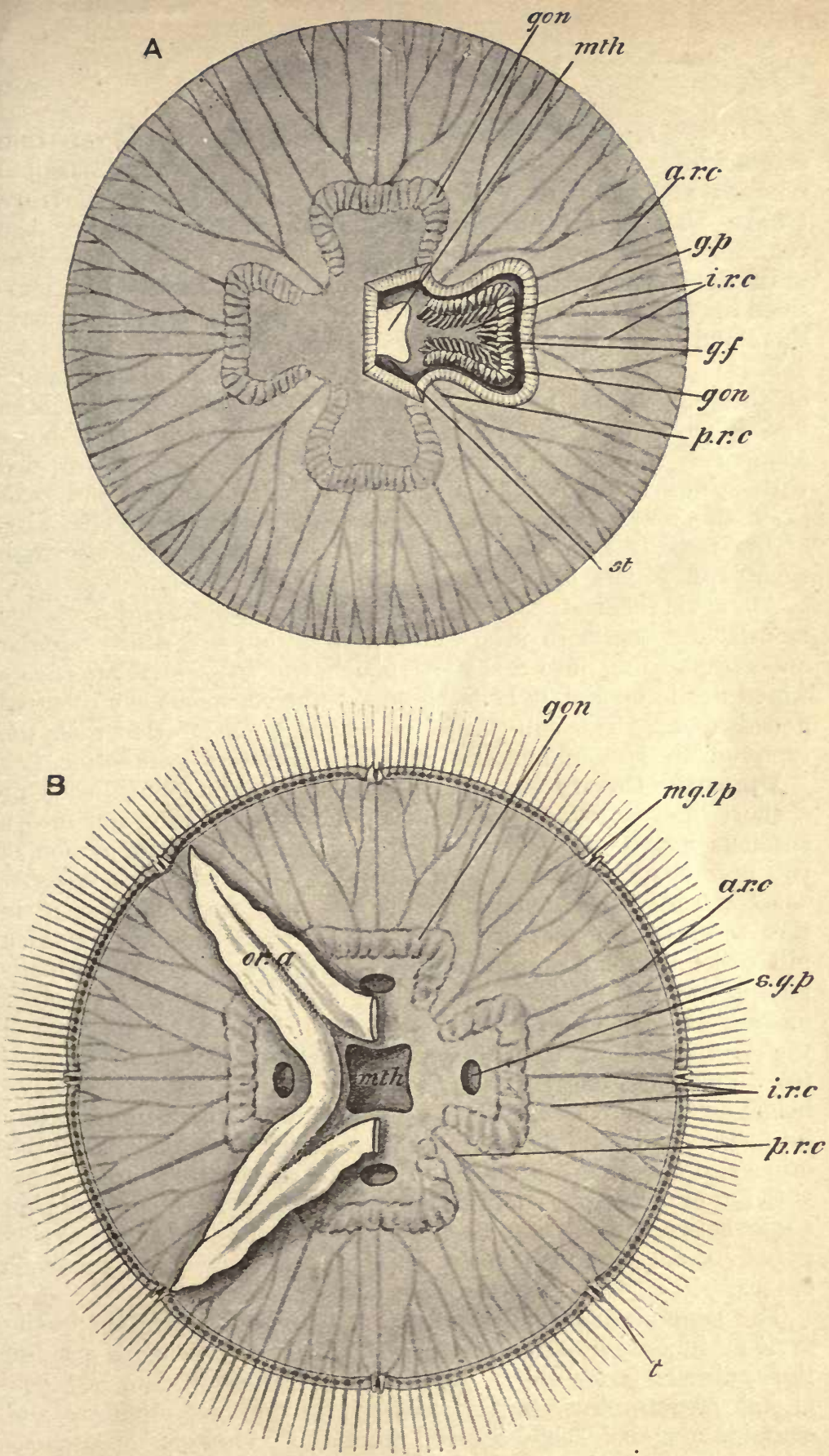

Fig. 126.-A urelia aurita. A, dorsal view, part of the ex-umbrella eut away to show part of the stomach and one of the four gastric pouches; $B$, ventral view - two of the oral arms are removed, a.r. e. adradial canal; g. $f$. gastric f̧ilaments; gon. gonads; $g .1$. gastric pouch; i.r. c. inter-radial canal ; $m g . l p$. marginal lappet; $m$ th. mouth; or. $a$. oral arm; p.r.c. perradial can ; s.g. p. sub-genital pit; st. stomach; $t$. tentacles. 
A narrow region of the umbrella adjoining the edge is very thin and flexible: the structure thus constituted, with its marginal notches and the fringe of marginal tentacles, is the velarium. Unlike the true velum of the medusæ of the Hydrozoa the velarium contains endodermal canals.

In the centre of the lower or sub-umbrellar surface is a foursided aperture, the mouth ( $m$ th), borne at the end of an extremely short and inconspicuous manubrium : surrounding it are four long delicate processes, the oral arms (or. a), lying one at each angle of the mouth and uniting around it. Each arm consists of a folded membrane, tapering to a point at its distal end, beset along its edges with minute lobules, and abundantly provided with stinging-capsules. The angles of the mouth and the arms lie in the four per-radii, i.e. at the end of the two principal axes of the radially symmetrical body: of the marginal notches with their lappets, four are per-radial and four inter-radial.

At a short distance from each of the straight sides of the mouth, and therefore inter-radial in position, is a nearly circular aperture leading into a shallow pouch, the sub-genital pit (s.g.p). which lies immediately beneath one of the conspicuously coloured gonads (gon). The sub-genital pits have no connection with the reproductive system, and are probably respiratory in function.

Digestive Cavity and Canal-System.-The mouth leads by a short tube or gullet ( $g u l)$, contained in the manubrium, into a spacious stomach (st), which occupies the whole middle region of the umbrella, and is produced into four wide inter-radial gastric pouches $(g . p)$, which extend about half way from the centre to the circumference, and are separated from one another by thick pillar-like portions of the umbrella-jelly. In the outer or peripheral wall of each gastric pouch are three small apertures, leading into as many radial canals, which pass to the edge of the umbrella and there unite in a very narrow circular canal (circ. c). The canal which opens by the middle of the three holes, is of course inter-radial (i.r.c): it divides immediately into three, and each division branches again: the canals from the other two holes are ad-radial (a.r.c), and pass to the circular canal without branching. There is also an aperture in the re-entering angle between each two gastric pouches: this leads into a perradial canal $(\gamma \cdot r \cdot c)$, which, like the inter-radial, branches extensively on its way to the edge of the umbrella.

The general arrangement of the cell-layers in Aurelia is the same as in a hydroid medusa (Fig. 127, B). The main mass of the umbrella is formed of gelatinous mesogloea, which, however, is not structureless, but is traversed by branching fibres and contains amoeboid cells derived from the endoderm. Both exand sub-umbrellæ are covered with ectoderm, and the stomach and canal system are lined with endoderm, which is ciliated through- 
out. Some observations seem to show that the short tube described above as a gullet and a pair of the gastric pouches are lined, not by endoderm, but by an in-turned portion of the ectoderm, but this matter cannot be considered as definitely settled.

It was mentioned above that in the free medusa the gonads appear through the transparent umbrella as coloured horseshoe-

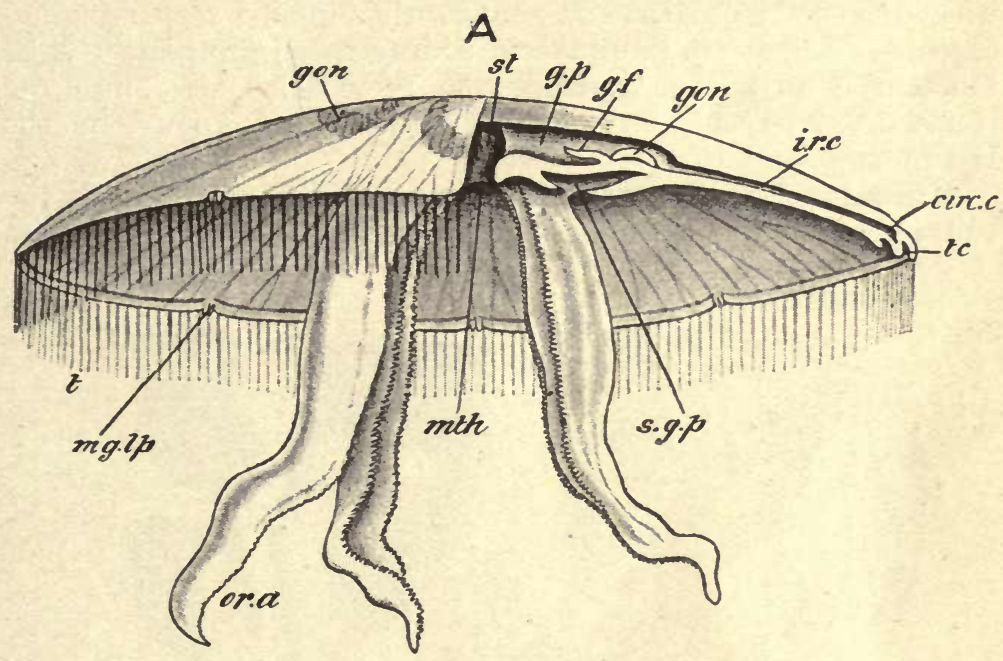

B

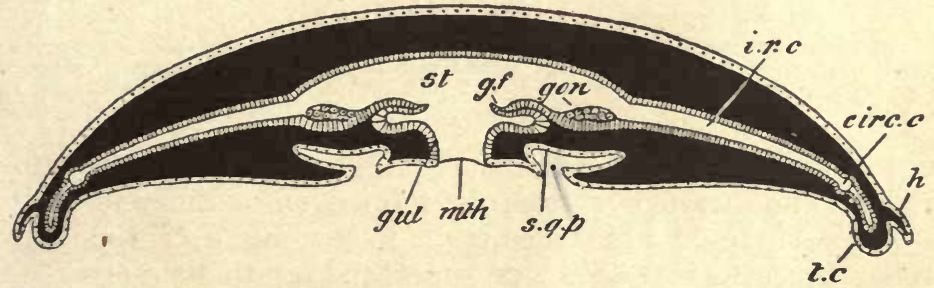

FIG. 127.-Aurelia aurita. A, side view, one-fourth of the umbrella cut away; B, diagrammatic vertical section, ectoderm dotted, endoderm striated, mesogloea black. circ. c. circular canal ; g.f. gastric filaments; gon. gonad; g. p. gastric pouch; gul. gullet; $h$. hood; $i . r . c$. inter-radial canal; mg. lp. marginal lappet; mth. mouth; or. a. oral arm; s.g. p. sub-genital pit; st. stomach.

shaped patches. Their precise position is seen by cutting away a portion of the ex-umbrella so as to expose one of the gastric pouches from above (Fig. 126, A). It is then seen that the gonad (gon) is a frill-like structure lying on the floor of the pouch and bent in the form of a horse-shoe with its concavity looking inwards, i.c. towards the mouth. Being developed from the floor of the enteric cavity, the gonad is obviously an 
endodermal structure: when mature, its products-ova or sperms - are discharged into the stomach and pass out by the mouth. Here, then, is an important difference from the Hydrozoa, in which the generative products are usually located in the ectoderm, and are always discharged directly on the exterior. The sexes are lodged in distinct individuals.

Lying parallel with the inner or concave border of each gonad is a row of delicate filaments (Fig. 126, 127, g.f), formed of endoderm with a core of mesoglœa and abundantly supplied with stingingcapsules. These are the gastric filaments or phacellæ: their function is to kill or paralyse the prey taken alive into the stomach. No such endodermal tentacles are known in the Hydrozoa.

Muscular and Nervous Systems.-The contractions of the bell by which the animal is propelled through the water are
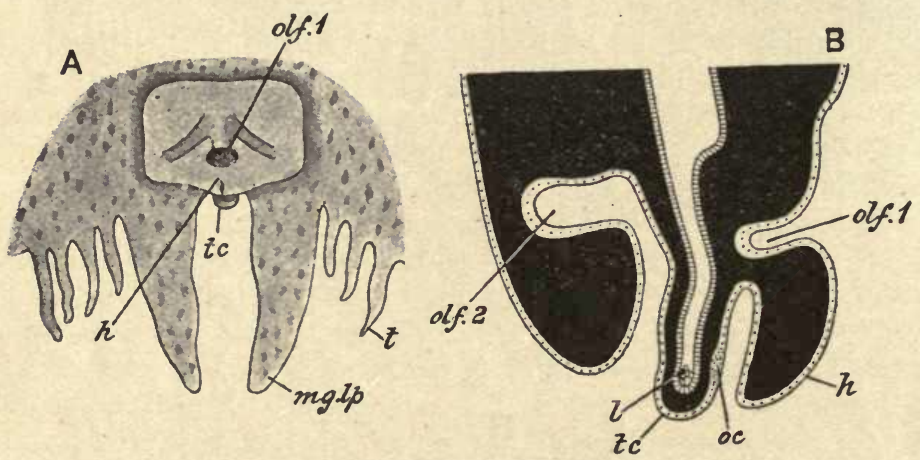

Fig. 128. - Aurelia aurita. A, small portion of edge of umbrella, showing the relations of the tentreuloeyst; $\mathrm{B}$, vertical section of the same region (diagrammatie), $h$, hood; $l$, lithite; $m g . l p$, marginal lappet; oc, ocellus ; olf. 1, olf. 2, olfactory pits. (Altercd from Lankester.)

effected by means of a muscular zone round the edge of the subumbrella. The nervous system is formed on a different plan from that of the hydroid medusæ. Extending over the subumbrellar surface between the superficial epithelial layer of ectoderm and the muscular layer is a plexus of simple nerve-fibres. This presents radial thickenings, most strongly developed externally in the per-radii and inter-radii, corresponding to the position of the marginal notches and sense-organs. About the base of each of the latter are special groups of nerve-cells. A slight ring-like thickening of the plexus extends round the margin in the neighbourhood of the marginal canal.

The sense organs (Fig. 128) are lodged in the marginal notches in close relation with the nerve-patches: like the latter, therefore, four of them are per-radial and four inter-radial. Each consists of a peculiar form of sense-club or tentaculocyst, containing 
a prolongation of the circular canal, and thus representing a hollow instead of a solid tentacle. At the extremity are calcareous concretions or lithites (l) derived from the endoderm, and on the outer side is an ectodermal pigment-spot or ocellus $(c c)$. The tentaculocysts are largely hidden by the marginal lappets ( $m g . l p$ ) and by a hood-like process $(h)$ connecting them; and in connection with each are two depressions, one on the ex-umbrella (olf. 1), the other immediately internal to the sense-club (olf. 2): these depressions are lined with sensory epithelium and are called olfactory pits.

The development and life-history of Aurelia present several striking and characteristic features. The impregnated egg-cell or oosperm divides regularly and forms a morula, which, by accumulation of fluid in its interior, becomes a blastula-a closed sac with walls formed of a single layer of cells. One end of this sac becomes invaginated to form the gastrula. The blastopore or gastrulamouth does not completely close, the resulting two layered planula (Fig. 129) differing in this respect, as well as in its mode of formation, from the corresponding stage of a Hydrozoan.

The planula swims about by means of the cilia with which its ectodermal cells are provided, and, after a brief free existence, settles down, loses its cilia, and becomes attached by one pole. At the opposite pole a mouth is formed, the process taking place by a sinking-in or invagination of the surface so as to produce a depression lined with ectoderm $(\mathrm{B}, s t)$, the bottom of which becomes perforated so as to communicate with the enteric cavity $(\mathrm{C}, s t)$ : the depression is the stomodoum, a structure of which there is no trace in the Hydrozoa. On two opposite sides of the mouth hollow processes grow out, forming the first two tentacles: soon two others appear at right angles to these, the organism thus being provided with four per-radial tentacles. Subsequently four inter-radial and eight adradial tentacles appear. At the same time the attached or proximal end is narrowed into a stalklike organ of attachment (E), and the endoderm of the enteric cavity is produced into four longitudinal ridges, inter-radial in position, and distinguished as the gastric ridges or tanioles (D $t n$.). The mouth (E, mth.) assumes a square outline, and its edges become raised so as to form a short manubrium (mnb.); and, finally, the ectoderm of the distal surface-i.e. the region lying between the mouth and the circlet of tentacles-bccomes invaginated in each inter-radius so as to produce four narrow. funnel-like depressionsthe septal funnels or infundibula (E and F, s. f.) - sunk in the four gastric ridges.

The outcome of all these changes is the metamorphosis of the planula into a polype (E), not unlike a Hydra or the hydrula-stage of the Leptolinæ, but distinguished by a pronounced differentiation of structure, indicated by the sixteen tentacles developed in 
SECT.

regular order, the stomodæum, and the four gastric ridges with their septal funnels. The Scyphozoon-polype is called a scyphula or scyphistoma.

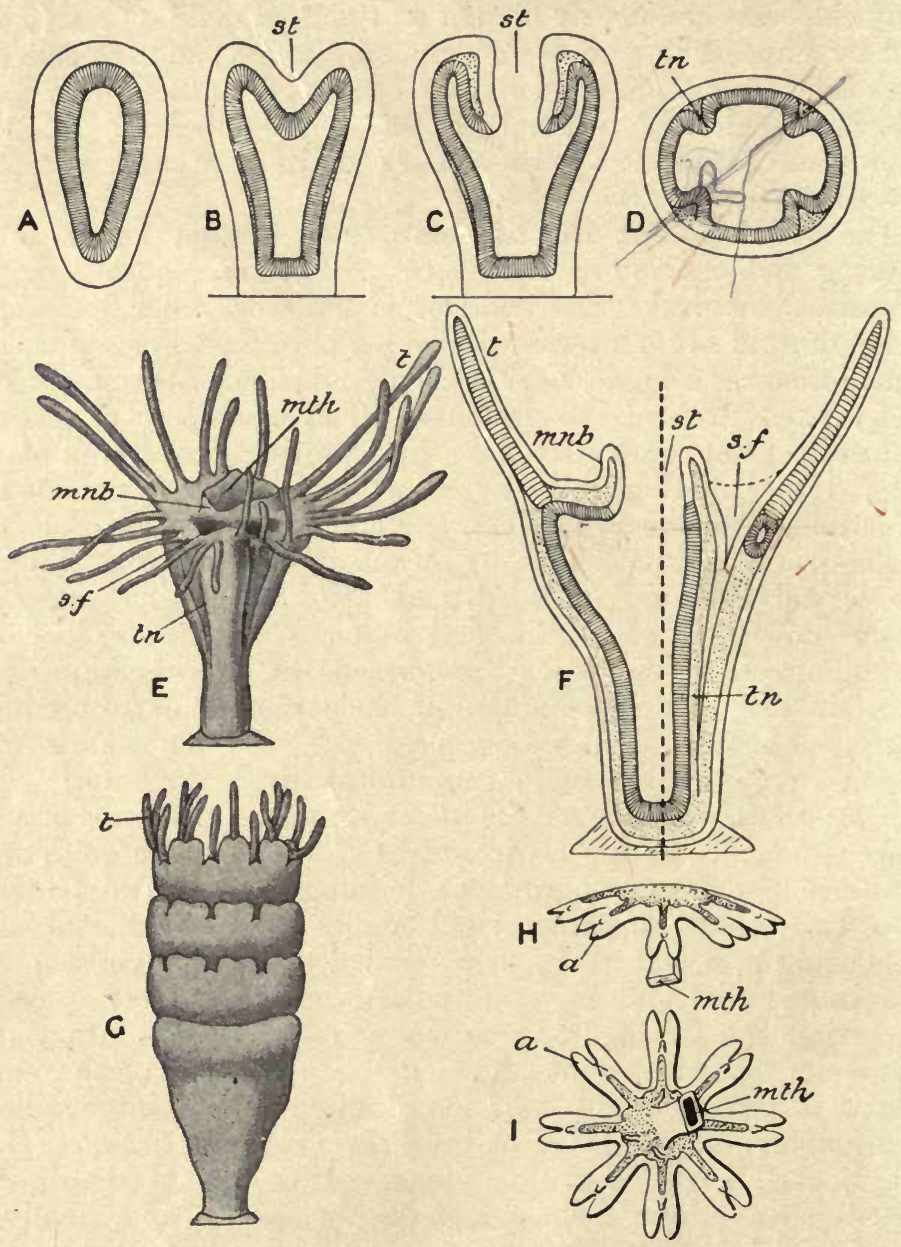

FIG. 129.-Aurelia aurita, development. A, planula, erroneously represented as eompletely closed; B, C, formation of stomodæum; D, transverse section of young scyphula; E, scyphula; F, longitudinal section of same: the section passes through a per-radius on the left of the dotted line, through an inter-radius on the right; $G$, division of scyphula into ephyrula; $\mathrm{H}$, ephyrula from the side; $\mathrm{L}$, the same from bencath. In $\mathrm{A}-\mathrm{D}$ and F the cctoderm is unshaded, the endoderm striated, and the mesogloea dotted. $a$. lobes of umbrella ; mnb. manubrium ; mth. mouth ; s.f. scptal funnel ; st. stomodxum ; $t$. tentaele ; in. tænioles. (From Korschelt and Heider's Embryology.)

The scyphula may grow to a height of half an inch, and sometimes multiplies by budding. After a time it undergoes a process 
of transverse fission $(\mathrm{G})$, becoming divided by a series of constrictions which deepen until the polype assumes the appearance of a pile of saucers, each with its edge produced into eight bifid lobes, four per- and four inter-radial. Soon the process of constriction is completed, the saucer-like bodies separate from one another, and each, turning upside down, begins to swim about as a small jelly-fish called an ephyrula $(H, I)$. The umbrella of the ephyrula is divided into eight long bifid arms (a) with deep (per-radial or inter-radial) notches: it has of course carried away with it a segment of the stomach with the gastric ridges of the scyphula: during the process of constriction this becomes closed in on the proximal or ex-umbrellar side, while on the sub-umbrellar side it remains open, and its edges grow out to form a manubrium. Round the margin there are the bases of eight per-radial and inter-radial tentacles, each in the notch of one of the arms, and eight ad-radial tentacles in the intervals between the lobes: the latter disappear completely; the former may persist as the tentaculocysts. On each gastric ridge appears a single gastric filament, soon to be followed by others, and in the notches at the extremities of the eight arms tentaculocysts are now recognisable. In the meantime the spacious enteric cavity is continued into the eight arms in the form of wide radiating canals.

As the ephyrula grows the adradial regions-at first deeply notched-grow more rapidly than the rest, the result being that the notches become gradually filled up, and the umbrella, from an eight-rayed star, becomes a nearly circular disc. Four oral arms are developed and numerous marginal tentacles, and the ephyrula gradually assumes the form of the adult Aurelia. It seems probable that the sub-genital pits of the medusa are formed from sections of the septal funnels of the scyphula.

Thus the life-history of Aurelia differs in several marked respects from that of any of the Hydrozoa. There is; in a sense, an alternation of generations as in Obelia, the gamobium being represented by the adult Aurelia, the agamobium by the scyphula. But instead of the medusa being developed either as a bud on a branched colony, as in Leptolinæ, or by direct metamorphosis of a polype, as in Trachylinæ, it is formed by the metamorphosis of an ephyrula developed as one of several transverse segments of a polype; so that the life-history might be described as a metamorphosis complicated by multiplication in the larval (scyphula) condition, rather than a true alternation of generations.

It has been shown that, under exceptional circumstances, the egg of Aurelia develops into scyphulæ which do not undergo transverse division, the entire scyphula becoming metamorphosed into a single adult. 


\section{General Structure and Classification.}

The Scyphozoa may be defined as medusoid Cœlenterata, having the same general structure and arrangement of the layers as the medusoid Hydrozoa, but differing from them in the possession of endodermal gastric tentacles; in having gonads the sexual cells of which are lodged in the endoderm and which discharge their products into the digestive cavity ; in the absence of a true velum, and in nearly all cases, in the presence of sense-organs in the form of hollow sense-clubs or tentaculocysts. Whether a stomodæum or ectodermal gullet occurs is uncertain. As in the Hydrozoa, the medusa develops directly from the egg in some Scyphozoa, while in others there is a sort of alternation of generations, a polypeform (agamobium) giving rise to the medusa-form (gamobium) by a process of transverse fission. In the majority, however, nothing is known of the life-history, the process of development having been worked out only in a few cases.

As far as is known, the segmenting embryo gives rise to a gastrula by invagination in all with the exception of Luccrnaria and its allies: by the partial or complete closure of the blastopore a planula is produced, at one end of which a second invagination takes place, forming the stomodæum.

The Scyphozoa are divisible into four orders, as follows :-

\section{Order 1.-STauromedusa (LUCERNarida).}

Scyphozoa having a conical or vase-shaped umbrella, sometimes attached to external objects by an ex-umbrellar peduncle: no tentaculocysts.

\section{Order 2.-Coronata.}

Scyphozoa having the umbrella divided by a horizontal coronary groove: four to sixteen tentaculocysts.

\section{ORDER 3.-CuBomedUSA.}

Scyphozoa with a four-sided cup-shaped umbrella: four perradial tentaculocysts.

\section{ORDER 4.-DISCOMEDUSA.}

Scyphozoa with a flattened saucer- or disc-shaped umbrella: not fewer than eight tentaculocysts-four per- and four interradial. 


\section{Sub-Order $a$-Semostomce.}

Discomedusæ in which the square mouth is produced into four long oral arms.

\section{Sub-Oider b-Rhizostomce.}

Discomedusæ having the mouth obliterated by the growth across it of the oral arms: the stomach is continued into canals which open by funnel-shaped apertures on the edges of the arms.

\section{Systematic Position of the Example.}

Aurelia aurita is one of several species of the genus Aurelia, and is placed in the family Ulmarida, the sub-order Semostoma, and the order Discomedusce.

Its saucer-shaped umbrella and eight tentaculocysts place it at once among the Discomedusæ: the presence of a distinct mouth surrounded by four oral arms places it in the first sub-order or Semostomæ. This group contains six families, characterised mainly by differences in the canal system: the Ulmaridæ are distinguished by narrow branched radial canals opening into a circular canal. Of the eight genera in this family, Aurelia stands alone in having its tentacles attached on the dorsal or ex-umbrellar side of the margin, and in the oral arms showing no trace of bifurcation. Eight species of Aurelia are recognised, $A$. aurita being distinguished by having the oral arms slightly shorter than the radius of the umbrella, and by possessing a trichotomous inter-radial canal and two unbranched adradial canals springing from each gastric pouch.

\section{Order 1.-STauromedus (LuCERnarida).}

Tessera (Fig. 130), formerly regarded as the simplest member of this group, is now looked upon as probably not a mature form. It is described as a small medusa about $4 \mathrm{~mm}$. in diameter having the same general characters as the scyphula-stage of Aurelia, except that the bell-shaped body is free-swimming. The edge of the umbrella is surrounded by eight tentacles, four per-radial (p.r.t.) and four inter-radial (i.r.t.), and movement is effected by a well-developed system of circular and radial muscles.

Lucernaria (Fig. 131), a genus not uncommon on the British coasts, is in one respect even more like a scyphula, since it is attached by a peduncle developed from the centre of the ex-umbrella. The margin of the umbrella is prolonged into eight short hollow adradial arms, bearing at their ends groups of short adhesive tentacles $\left(t_{.}\right) . \quad$ As in the scyphula, each gastric ridge contains an infundibulum, lined with ectoderm and opening on the sub-umbrella. The gastric filaments $\left(g . f_{.}\right)$are very numerous - a distinct advance on Tessera-and the gonads (gon.) are band-like. There are no sense-organs in Lucernaria, but in an allied genus, Halicystus, there are eight per-radial and inter-radial marginal bodies (anchors) of the nature of reduced and modified tentacles, each surrounded at its base by a cushion-like thickening containing many adhesive cells. Internal to each anchor on the sub-umbrellar side is a pigment spot 
(rudimentary eye). Stenocyphus is an allied form which probably is able to move by creeping (looping) movements like those of a leech. Capria has no

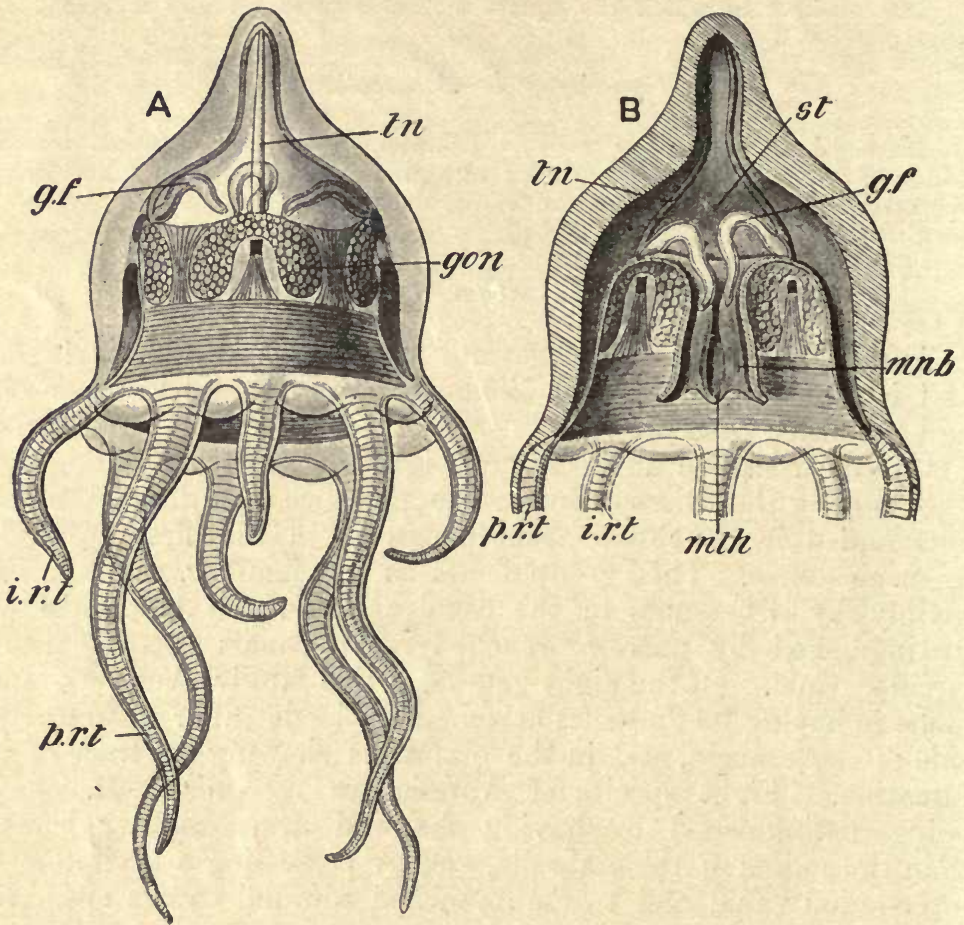

Fio. 130.-Tessera princeps. A, external view; B, vertical section. g. f. gastric filament; gon. gonad; i.r. $t$. inter-radial tentacle ; mnb. manubrium; $m t h$. mouth; $p . r$. $t$. per-radial tentrale; st. stomach ; $t n$. treniole. (After Haeckel.)
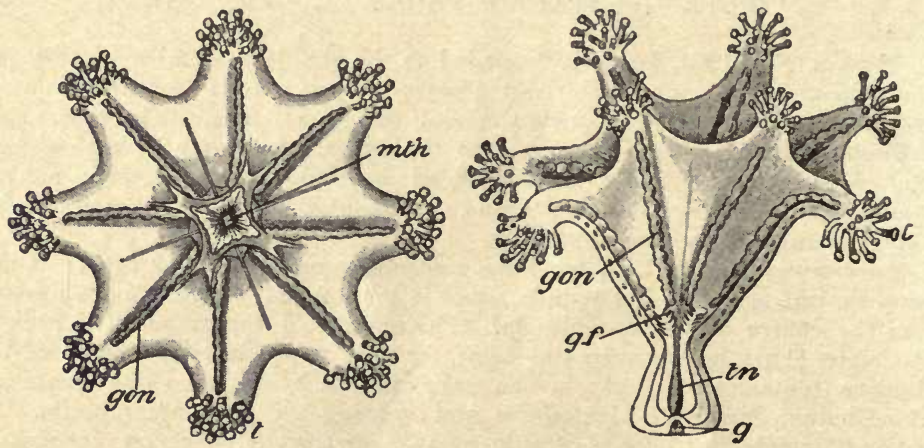

Fig. 131.-Iucernaria. A, oral aspect; B, from the side, $g$. foot-gland; $g . f$ gastric filaments gon. gonad; mith. mouth; $t$. tentacles; $t n$. trenioles. (After Claus.)

tentacles. The Depastridce have an almost entire margin fringed with tentacles. 


\section{ORDER 2.-CORONATA.}

This group includes a number of rare and beautiful Medusæ of curiously complex structure, of which Pericolpa may be taken as an example. The umbrella (Fig. 132) is usually conical, and is divided by a horizontal furrow (coronary groove) into an apical region or cone $(c n$.$) and a marginal region or$

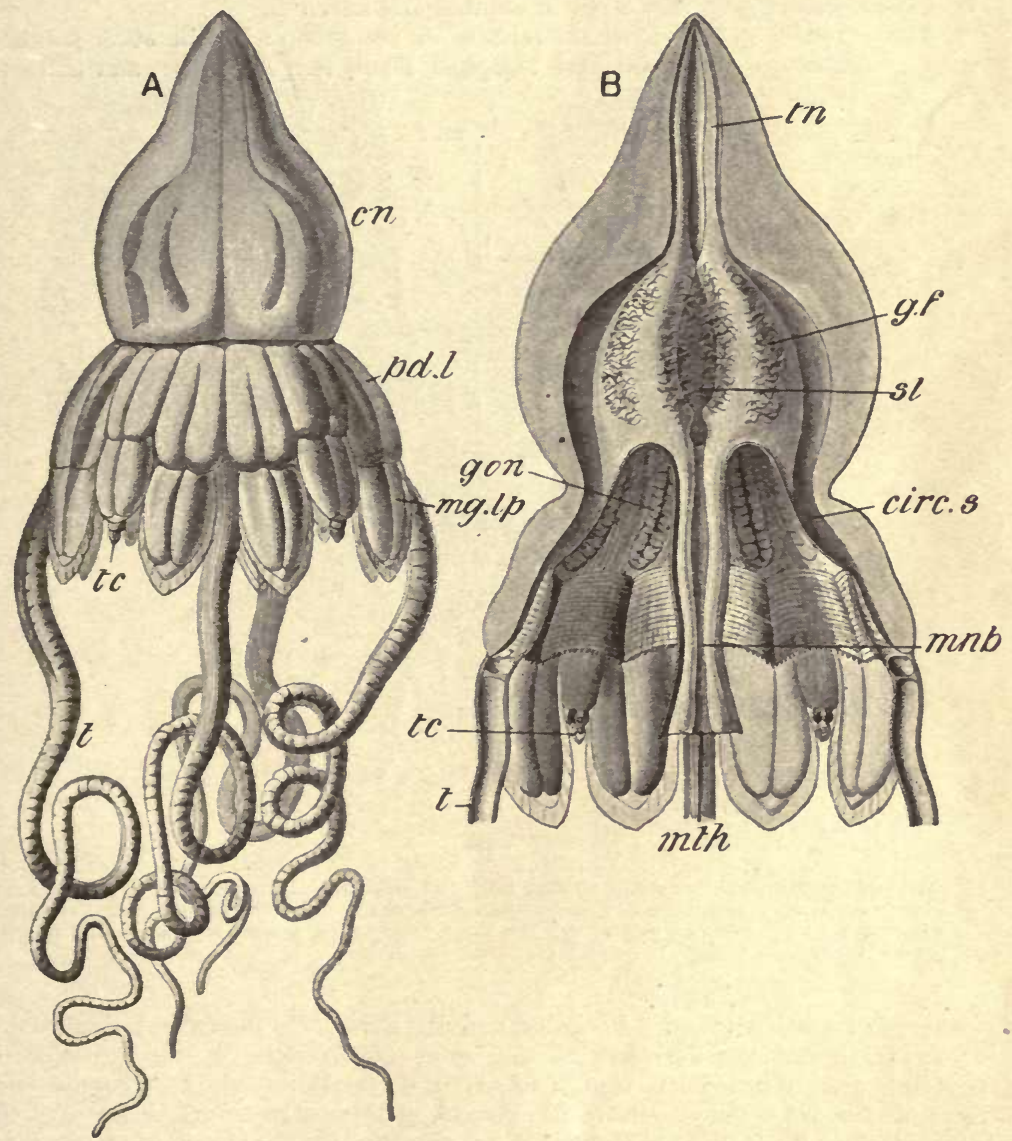

Fic. 132. - Pericolpa quadrigata. A, external view; B, vertical section. circ. s. circular sinus; $c n$. cone; $q$. f. grastric filaments; gon. gonads; $m g$. lp. marginal lappets; mn $b$ manubrium; mth. mouth; $p l$. $l$. pedal lobes; st. stomach; $t$. tentacles; $t c$. tentaculocysts; $t n$. trenioles. (After Haeckel.)

crown; the crown is again divided by a second, rather irregular horizontal furrow into a series of pedal lobes ( $p d . l$.$) , adjacent to the cone, and a series of$ marginal lappets $(m g . l p$.$) , forming the free edge of the bell. In some of the$ Coronata, such as Pericolpa, the pedal lobes and marginal lappets correspond (i.e. are in the same radii); in others (Periphylla, Ephyropsis) they alternate. 
In Pericolpa four of the pedal lobes, inter-radial in position, bear tentaculocysts $(t c$.$) ; four others, per-radially situated, give origin to long,$ hollow tentacles $(t$.$) . In the more complex genera there are eight additional$ adradial tentacles.

The mouth ( $m$ th. $)$ is very large, and leads by a wide manubrium (mnb.) into a spacious stomach (st.), which is continued quite to the apex of the cone. In the wall of the stomach are four wide per-radial slits, leading into an immense circular sinus (circ. s.). As in Lucernaria, there are four wide inter-radial imfundibula. The gastric filaments $\left(g . f_{.}\right)$are very numerous, and the elongated $\mathrm{U}$-shaped gonads (gon.) are eight in number and adradial.

The coronary groove is characteristic of the group : but in other points such as the number of pedal and marginal lobes, tentaculocysts, and tentacles

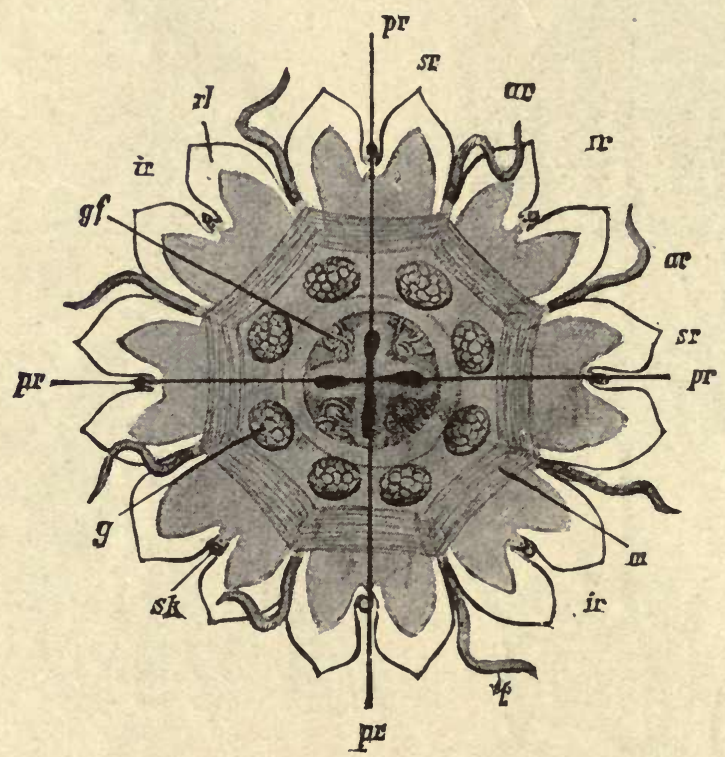

FIG. 132.-Nausithoe. The entire animal from the oral aspect. ar. adradii ; g. gonads; g.f. gastric filaments ; $i r$. inter-radii ; $m$. circular muscle of sub-umbrella; $p r$. per-radii ; $r l$. tentaculocysts ; $8 r$. sub-radii ; $t$. tentacles. The black cross in the centre represents the mouth. (From Lang's Comparative Anatomy.)

-there is great variation. Pericolpa and its allies (Peromedusa) resemble the Lucernarida and the members of the order Cubomedusa in the presence of tænioles and inter-radial septa: Ephyropsis and its allies (Cannostoma) resemble the order Discophora in the absence of these structures. The scyphula larva of Nausithö̈ (Fig. 133) lives as a parasite in the interior of a horny sponge.

\section{Order 3.-CuBomeduse.}

The Jelly-fishes forming this order are, as the name implies, of a more or less cubical form, resembling a deep bell with somewhat flattened top and square transverse section. They resemble the hydrozoan Medusæ more than any of the other Scyphozoa. The best known species, Charybdoea marsupialis (Fig. 134), is about $5 \mathrm{~cm}$. in diameter and of very firm consistency. 
As in the lower Coronata, the margin of the umbrella bears four tentacles $(t$.$) and four tentaculocysts (t c$.$) but the position of these organs is reversed, the$ tentaculocysts being per-radial, the tentacles inter-radial. The tentaculocysts are set in deep marginal notches, and the tentacles spring from conspicuous gelatinous lobes $(l$.$) , which probably answer to the pedal lobes of the preceding$ order. These pedal lobes sometimes bear a number of supplementary tentacles.

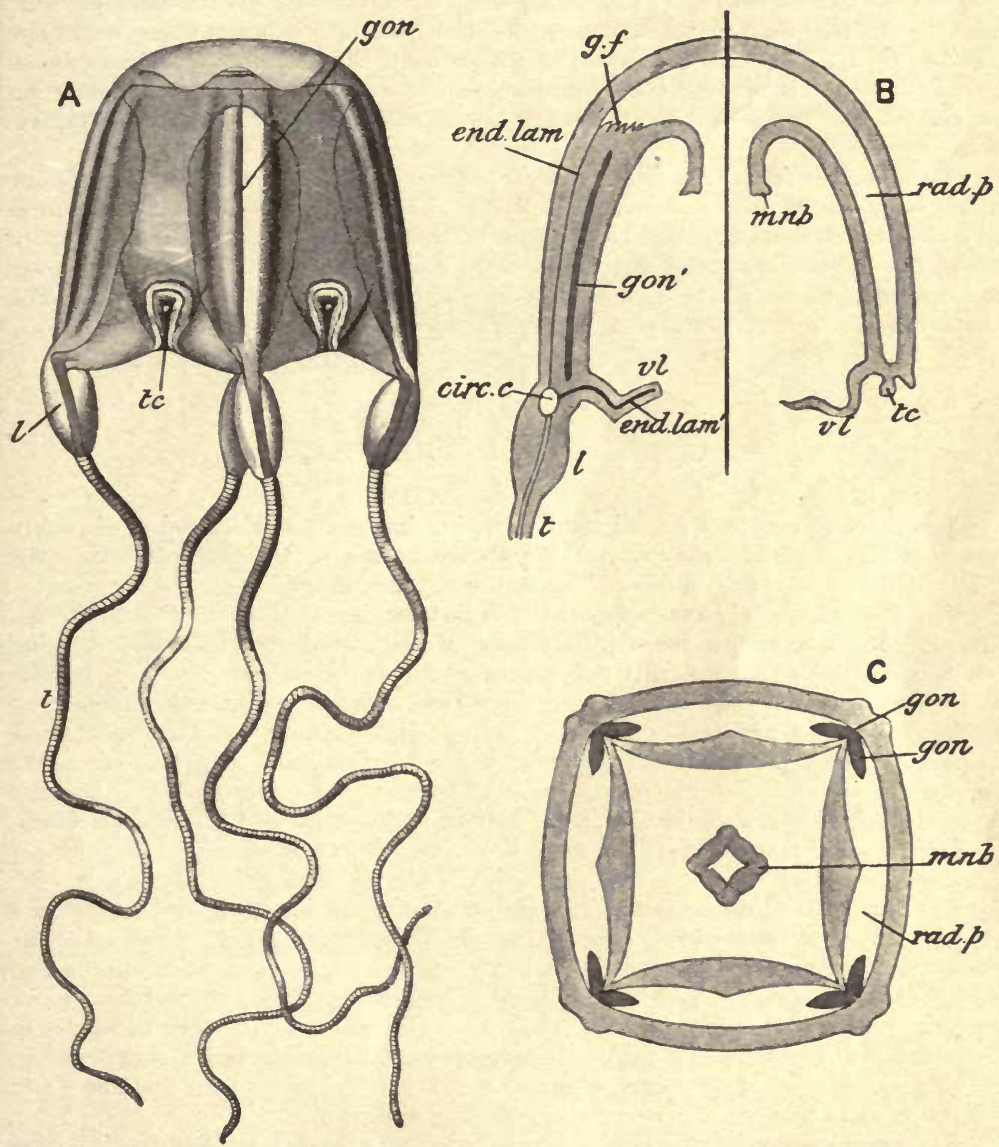

FiG. 134.-Charybdæa marsupialis. A, side view of the entire animal ; B, vertical section passing on the left side through an inter-radius, on the right through a per-radius; C, transverse section. circ. $c$. cireular canal; end. lam. endoderm lamella; end. lam'. its prolongation into the velarium; $g . f$. gastric filaments; gon. gonad; gon'. septum separating gonads; $i$. lappet; mnb. manubrium; rad. $p$. radial pouch; $t$. tentacle; $t c$. tentaculocjst ; vl. velarium. (After Claus, somewhat altered.)

The margin of the umbrella is produced, in most cases but not in all, into a horizontal shelf $(v l$.$) , resembling the velum of the hydroid Medusæ, but differing$ from it in containing a series of branched vessels (end. $\mathrm{lam}^{\prime}$.) continuous with the canal-system and of course lined with endoderm. In the Hydrozoa, it will be remembered, the velum is formed simply of a double layer of ectoderm with a 
supporting layer of mesogløa. Such a false velum, like the produced thin edge of the umbrella in Aurelia, is known as a velarium.

The mouth is situated at the end of a short manubrium ( $m n b$.) leading into a wide stomach, from which go off four very broak shallow per-radial pouches (rad. p.), occupying the whole of the four flat sides of the umbrella, and separated from one another by narrow inter-radial septa or partitions (mesenteries) placed at the four corners. These pouches are equivalent to wide radial canals, and the partitions between them to a poorly developed endoderm lamella (end. lam.). At the margin of the umbrella the pouches communicate with one another by apertures in the septa, so that a kind of circular canal is produced (circ. c.), which is divided into chambers by the mesenteries. Near the junction of the gastric pouches with the stomach are the usual four groups of gastric filaments $(g . f$.$) .$

The gonads (gon.) are four pairs of narrow plate-like organs, attached one along each side of each inter-radial septum. The nervous system takes the form of a sinuous nerve-ring round the margin of the bell, bearing a distinct group of nerve-cells at the base of each tentaculocyst and tentacle. The Cubomedusa are the only Scyphozoa which, like the Hydrozoa, have a complete nerve-ring. The tentaculocysts are very complex, each bearing a lithocyst and several eye-spots.

\section{ORDER 4.-DiscomedUSA.}

The preceding orders are all small ones, i.e., include a small number of genera and species. The vast majority of Scyphozoa belong to the present order-the "Disc-jellies" or "Sea-blubbers" as ordinarily understood.

The umbrella is always comparatively flat, having the form of an inverted saucer. The edge is produced primitively into eight pairs of marginal lappets, but in some of the more highly differentiated forms the number both of lappets and of tentaculocysts becomes greatly increased. Most of the Semostomæ and Rhizostomæ are large, and one of the former group-Cyanea arctica-may attain a diameter of 2 metres and upwards, while its marginal tentacles reach the astonishing length of 40 metres or about 130 feet. But in spite of their size and apparent solidity, the amount of solid matter in these great Jelly-fishes is extraordinarily small; some of them have been proved to contain more than 99 per cent. of sea-water.

The marginal tentacles are hollow and often of great length in the Semostomæ (Fig. 126), and altogether absent in the Rhizostomæ (Fig. 135). In the Semostomæ there are four oral arms (Fig. 126, or. $\boldsymbol{a}_{\text {. }}$ ), each resembling a leaf folded along its midrib, and having more or less frilled edges : in the Rhizostomæ each of the original four arms (Fig. 135, or. a.) becomes divided longitudinally in the course of development, the adult mombers of the group being characterised by the presence of eight arms, often of great length, and variously lobed and folded so as to present a more or less root-like appearance.

The arrangement of the enteric cavity and its offshoots presents an interesting series of modifications. In no case are there any tænioles or inter-radial septa (mesenteries). In the Semostomæ (Fig. 126) the stomach-lobes give off well-defined radial canals, which are frequently more or less branched, often unite into complex networks, and sometimes open into a circular canal round the margin of the umbrella.

In the Rhizostomæ (Fig. 135, B) a similar network of canals is found in the umbrella, but an extraordinary change has befallen the oral or ingestive portion of the enteric system. Looking at the oral or lower surface of one of these Jellyfishes, such as Pilema, no mouth is to be seen, but a careful examination of the oral arms shows the presence of large numbers-hundreds, or even thousands in soma cases-of small funnel-like apertures (B, C, s.mth.) with frilled margins. 
Rhizostomes have been found with prey of considerable size, such as fishes, embraced by the arms and partly drawn into these apertures, which are therefore called the suctorial mouths. They lead into canals in the thickness of the arms $(B, c$.$) , the lesser canals unite into larger, and then finally open into the stomach$ (st.). We thus get a polystomatous or many-mouthed condition which is practically unique in the animal kingdom, the only parallel to it being furnished by the Sponges, in which the inhalant pores are roughly comparable with the suctorial mouths of a Rhizostome.

It has been found that this characteristic arrangement is brought about by certain changes taking place during growth. The young Rhizostome has a single mouth in the usual position, and more or less leaf-like arms, folded along the midrib so as to enclose a deep groove, from which secondary grooves pass, like

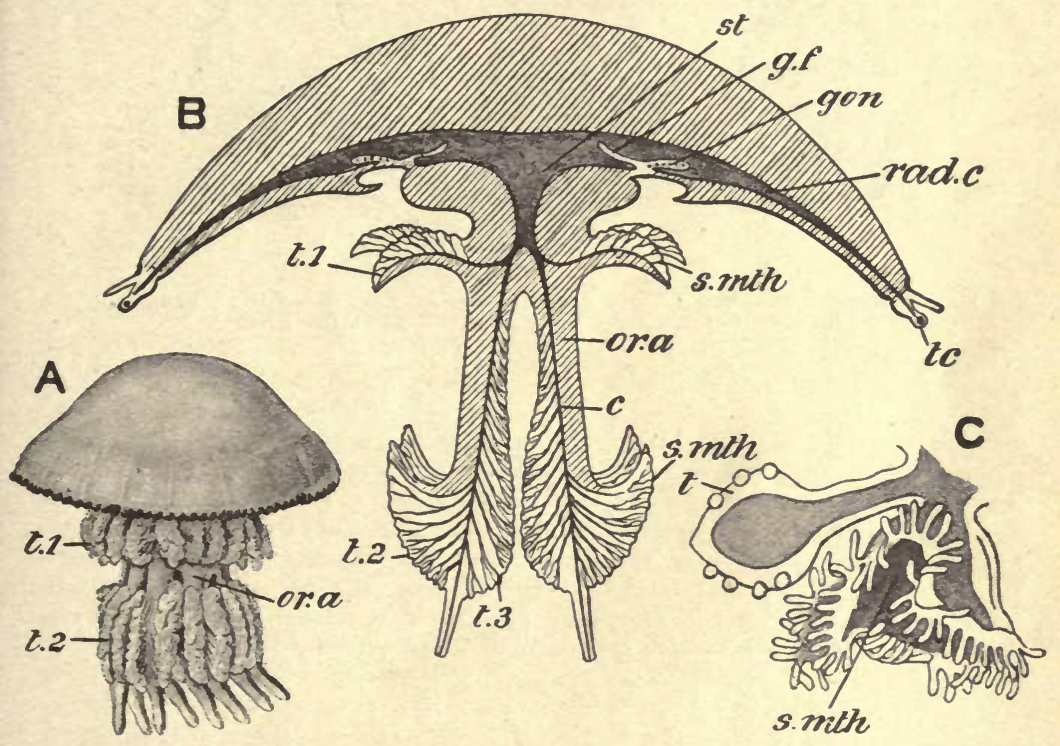

FiG.135. - Pilema pulmo. A, side view of the entire animal; B, vertical section, diagrammatic; C, one of the suctorial mouths, magnified. $c$. arm canal; $g . f$. gastric filaments; gon. gonads; or. a. oral arms; ral. c. radial canal; s. mth. suctorial mouths; st. stomach; $t 1, t 2, t 3$, tentacles on oral arms. (After Cuvier, Claus, and Huxley.)

the veius of a leaf, towards the edge of the arm. As development proceeds, these grooves become converted into canals by the union of their edges, thus forming a system of branching tubes opening proximally into the angles of the mouth and distally by small apertures-the suctorial mouths-on the edges of the arms. At the same time the proximal ends of the arms grow towards one another and finally unite across the mouth, closing it completely, and forming a strong horizontal brachial disc, which in the adult occupies the centre of the subumbrellar surface.

The gastric filaments are usually very numerous. In the higher Rhizostomæ a remarkable modification is produced in connection with the sub-genital pouches; the four pouches approach the centre and fuse with one another, forming a single spacious chamber, the sub-genital portico, which lies immediately below the floor of the stomach and above the brachial disc. 
In many of the Discomedusæ development takes place in the same general way as in Aurelia, i.e. the impregnated egg gives rise to a scyphula or asexual polype stage, which, by transverse division, prodnces sexual medusæ. In Cassiopeia the scyphula arising from the fertilised ovum gives off buds which become detached as free-swimming planulæ, and these, coming to rest, develop into scyphulæ. But in other cases there is no alternation of generations, and development is direct. For instance, in Pelagia (Fig. 136)-one of the Semostomæ-a blastula is formed which becomes invaginated at one end,
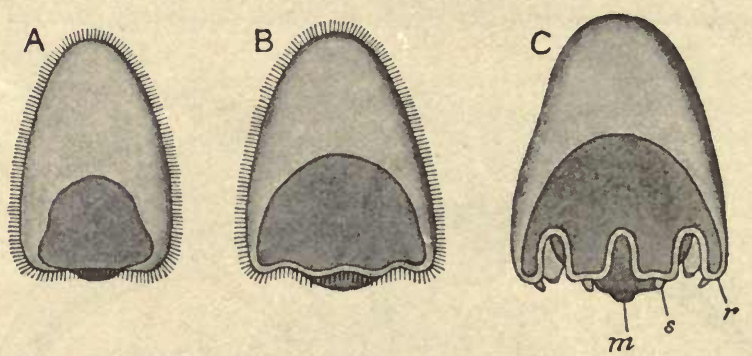

FiG. 136.-Pelagia noctiluca : Three developmental stages. $m$. mouth; $r$ marginal lappet; s. tentaculocyst. (From Korschelt and Heider, after Krohn.)

forming a gastrula. The blastopore or gastrula-mouth remains open, and a considerable space is left between the invaginated endoderm and the ectoderm. Next the mouth region becomes elevated, forming a manubrium, and around this a circular depression appears - the rudiment of the sub-umbrellar cavitysurrounded by a raised ridge, the umbrella margin, which soon becomes divided into lobes, the marginal lappets. Up to this time the embryo is ciliated externally, but soon the cilia disappear, and the little creatures assume somewhat the form of an ephyrula, which gradually develops into the adult Pelagia.

\section{Additional Remarks on the ScyphozoA.}

The Scyphozoa are all marine, and the majority are pelagic, i.e. swim freely on the surface of the ocean. A few inhabit the deep sea, and have been dredged from as great a depth as 2,000 fathoms. Nearly all are free-swimming in the adult state: some, however, live on coral-reefs or mud-banks, and are found resting, in an inverted position, on the ex-umbrella ; and a few, such as Lucernaria, are able to attach themselves at will by a definite exumbrellar peduncle.

Many of the Scyphozoa are semi-transparent and glassy, but often with brilliantly coloured gonads, tentacles, or radial canals. In many cases the umbrella, oral arms, \&c., are highly coloured, and some species, e.g. Pelagia noctiluca, are phosphorescent. They are all carnivorous, and although mostly living upon small 
organisms, are able, in the case of the larger species, to capture and digest Crustaceans and Fishes of considerable size. In many cases small fishes accompany the larger forms and take shelter under the umbrella.

Considering the extremely perishable nature of these organisms, and the fact that many of them contain not more than 1 per cent. of solid matter, it is not to be expected that many of them should have left traces of their existence in the fossil state. Nevertheless, in the finely grained limestone of Solenhofen, in Bavaria, belonging to the Upper Jurassic period, remarkably perfect impressions of Jelly-fishes have been found, some of them readily recognisable as Discomedusæ.

\section{CLASS III.-ACTINOZOA.}

\section{Example of the Class.-A Sea-Anemone (Tealia crassicornis).}

Sea-anemones are amongst the most abundant and best known of shore-animals. They are found attached to rocks, sea-weeds, shells, \&c., either in rock-pools or on rocks left high and dry by the ebbing tide. Usually their flower-like form and brilliant colour make them very conspicuous objects; but many kinds cover themselves more or less completely with sand and stones, and contract so much when left uncovered by water, that they appear like soft shapeless lumps stuck over with stones, and thus easily escape observation. Any of the numerous species will serve as an example of the group: the form specially selected is the "Dahlia Wartlet" (Tealia crassicornis), one of the commonest British species.

External characters.-Tealia (Fig. 137, A) has the form of a cylinder, the diameter of which slightly exceeds its height. It is often as much as 3 inches $(8 \mathrm{~cm}$.) across, is of a grcen or red colour, and habitually covers itself with bits of shell, small stones, \&c. It is attached to a rock or other support by a broad sole-like base, sharply separated from an upright cylindrical wall or column, the surface of which is beset with rows of adhesive warts or tubercles: at its upper or distal end the column passes into a horizontal plate. the disc or peristome. In the middle of the disc, and slightly elevated above its surface, is an elongated slit-like aperture, the mouth ( $m$ th.), from which streaks of colour radiate outwards. Springing from the disc and encircling the mouth are numerous short conical tentacles $(t$.$) , which appear at first sight to be$ arranged irregularly, but are actually disposed in five circlets, of which the innermost contains five, the next five, the third ten, 

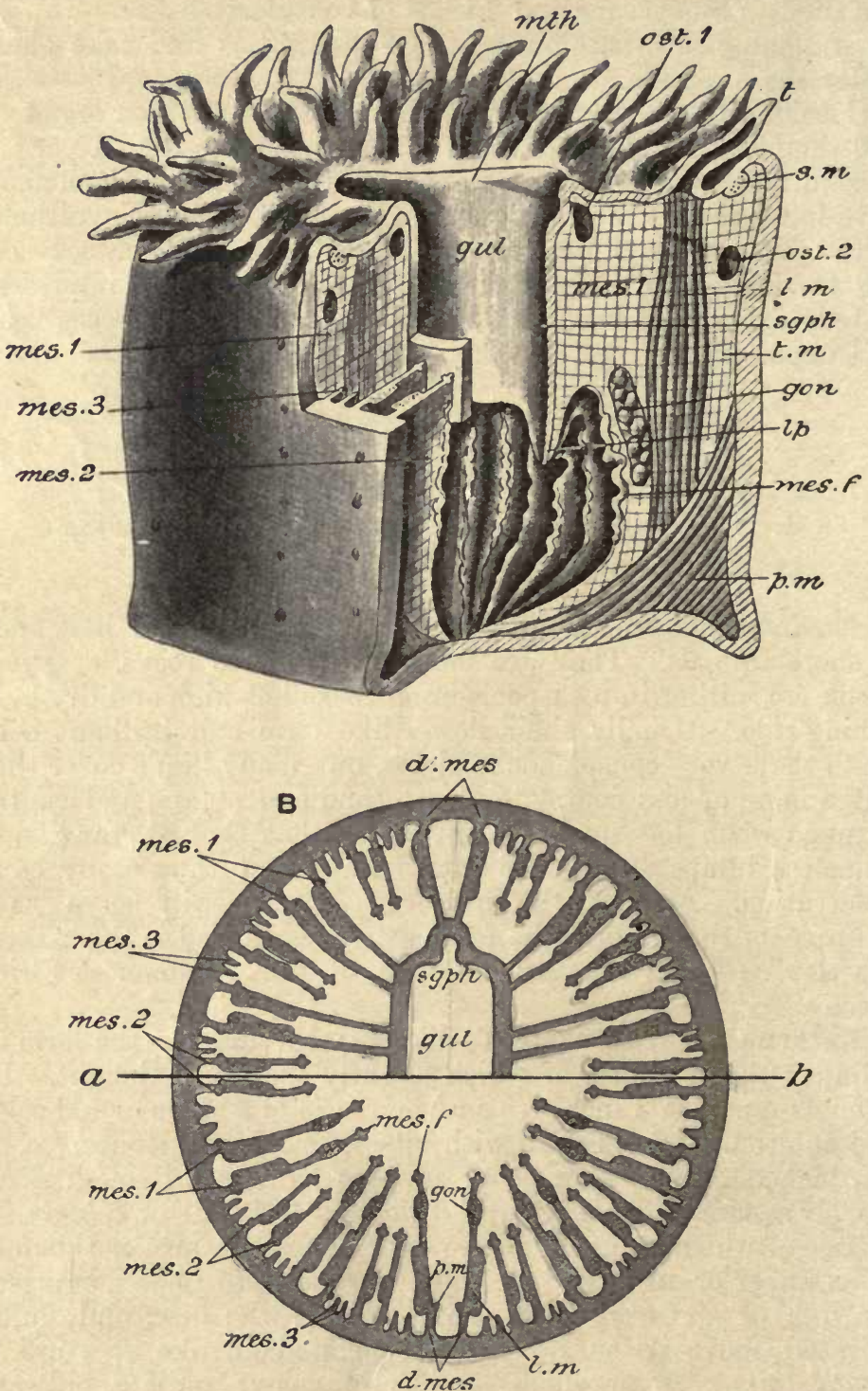

Fig. 137.-Tealla crassicornis. A, dissected specimen; B, transverse section, the half above the line $a b$ through the gullet, the lower half below the gullet. $d$. mes. directive mesenteries; gon. gonads; gul. gullet; ; . m. longitudinal muscle; lp. lappet; mes. 1 , primary, mes. 2, secondary, mes. 3, tertiary mesenteries; mes. $f$. mesenteric filaments; mih. mouth; ost. 1, ost. 2, ostia; $p . m$. parietal muscle; $s g p h$. siphonoglyphe; 8.12 . sphincter muscle ; $t$. $m$. transverse muscle. 
the fourth twenty, and the fifth or outermost forty, making a total of eighty.

Obviously the Sea-anemone is a polype, formed on the same general lines as a Hydra or a scyphula, but differing from them in having numerous tentacles arranged in multiples of five, and in the absence of a hypostome, the mouth being nearly flush with the surface of the disc. Its great size and bulk, and the comparative firmness of its substance, are also striking points of difference between Tealia and the polypes belonging to the classes Hydrozoa and Scyphozoa.

Enteric System.-Still more fundamental differences are found when we come to consider the internal structure. The mouth does not lead at once into a spacious undivided enteric cavity, but into a short tube ( $g u l$.), having the form of a flattened cylinder, which hangs downwards into the interior of the body, and terminates in a free edge, produced at each end of the long diameter into a descending lobe or lappet (lp.). This tube is the gullet or stomiodoum, a structure we have already met with in the Scyphozoa, but which here attains a far greater size and importance. Its inner surface is marked with two longitudinal grooves (A and B, sgph.), placed one at each end of the long diameter, and therefore corresponding with the lappets : they are known as the gullet-grooves or siphonoglyphes.

The gullet does not simply hang freely in the enteric cavity, but is connected with the body-wall by a number of radiating partitions, the complete or primary mesenteries (mes. 1): between these are incomplete sccondary mesenteries (mes. 2), which extend only part of the way from the body-wall to the gullet, and tertiary mescnteries (mes. 3), which are hardly more than ridges on the inner surface of the body-wall. Thus the entire internal cavity of a Sea-anemone is divisible into three regions: (1) the gullet or stomodaum, communicating with the exterior by the mouth, and opening below into (2) a single main digestive cavity, the stomach or mesenteron, which gives off (3) a number of radially arranged cavities, the inter-mescnteric chambers or metenter $a$. It is obvious that we may compare the gullet and stomach with the similarly named structures in the scyphula-stage of Aurelia, and the mesenteries with the gastric ridges; indeed, there seems to be little doubt that these structures are severally homologous. A further correspondence is furnished by the presence of an aperture or ostium (ost. 1) in each mesentery, placing the adjacent intermesenteric chambers in direct communication with one another: in Tealia a second ostium (ost. 2) is present near the outer edge of the mesentery. Moreover, the free edge of the mesentery below the gullet is produced into a curious twisted cord, the mesenteric filament (mes. $f$.), answering to a gastric filament of the Scyphozoa. In many Sea-Anemones the mesenteric filaments 
are produced into slender threads-the acontia-which may be protruded through the mouth or through special apertures (cinclides) of the body-wall (Fig. 138, A.)

The general arrangement of the cell-layers is the same as in the two preceding classes. The body-wall (Fig 138)-base, column, and disc - consists of a layer of ectoderm outside, one of endoderm within, and between them an intermediate layer or mesogloea, which is extremely thick and tough. The gullet (gul.), which, like that of the scyphula, is an in-turned portion of the body-wall, is lined with ectoderm, and its outer surface-i.e. that facing the inter-mesenteric chambers - is endodermal. The mesenteries (mes.) consist of a supporting plate of mesoglœea, covered on both sides by
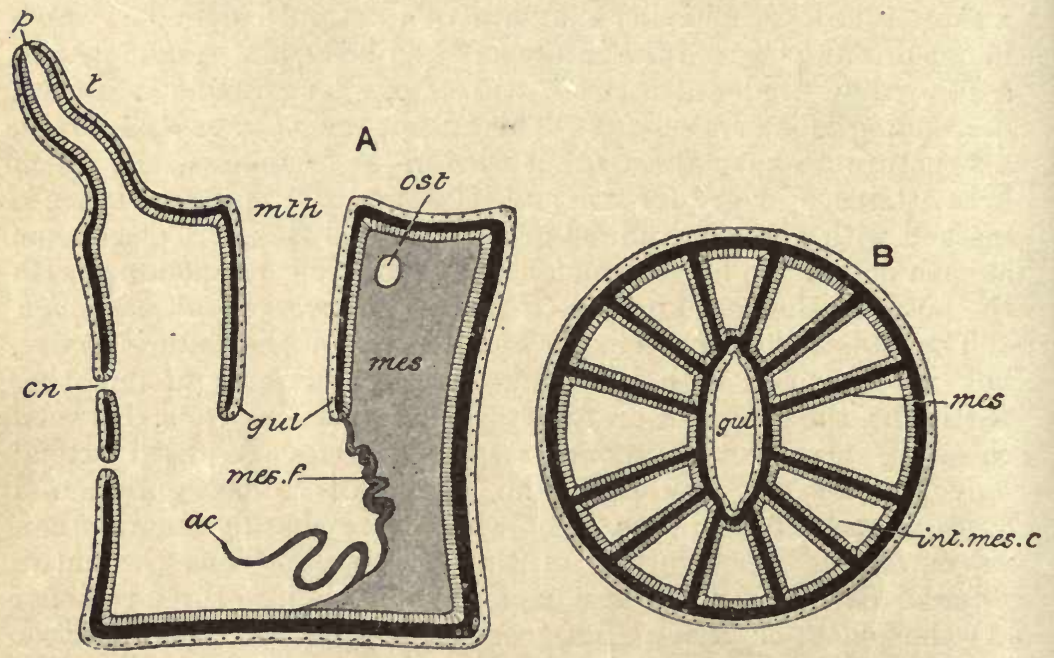

FIG. 138.-Diagrammatic vertical (A) and transverse (B) sections of a Sea-anemone. The ectoderm is dotted, the endoderm striated, the mesogloeablack. ac. acontium; $\mathrm{cn}$. cinclis; gul. gullet; int. mes. $c$. inter-mesenterie chamber; mes. mesentery; mes. f. mesenteric filament; $m$ th. mouth; ost. ostium; $p$. pore ; $t$. tentacle.

endoderm. The tentacles $(t)$ are hollow out-pushings of the disc, and contain the same layers.

Muscular System.-Sea-anemones perform various characteristic movements: the column may be extended or retracted, the tentacles extended to a considerable length, or drawn back and completely hidden by the upper end of the column being folded over them like the mouth of a bag; the gullet, and even the mesenteries, may be partially everted through the mouth; and lastly, the whole animal is able, very slowly, to change its position by creeping movements of its base.

These movements are performed by means of a very welldeveloped set of muscles. A mesentery examined from the surface 
is seen to be traversed by definite fibrous bands, the two most obvious of which are the longitudinal or retractor muscle (Fig. 137, l.m.), running as a narrow band from base to disc, and the parietal muscle (p.m.), passing obliquely across the lower and outer angle of the mesentery. Both these muscles are very thick, and cause a projection or bulging on one side of the mesentery, specially obvious in a transverse section (B. $l . m$.) : a third set of fibres, forming the trausverse muscle $(t . m$.), crosses the longitudinal set at right angles, but is not specially prominent. The longitudinal muscles shorten the mesentery, and draw the disc downwards or towards the base, thus retracting the tentacles; the parietal muscles approximate the column to the base, and the transverse fibres produce a narrowing of the mesentery and thus, opposing the action of the longitudinal muscles, act as extensors of the whole body. The withdrawal of disc and tentacles, during complete retraction, has been compared to the closure of a bag by tightening the string, and is performed in much the same way, the string being represented by a very strong band of fibres, the circular or sphincter muscle (s.m.), which encircles the body at the junction of the column and disc.

The foregoing muscles can all be seen by the naked eye, or under a low magnifying power. They are supplemented by fibres, only to be made out by microscopic examination, occurring both in the body-wall and in the tentacles. The latter organs, for instance, are able to perform independent movements of extension and retraction by means of delicate transverse and longitudinal fibres.

It was mentioned above that the thickness of the longitudinal and parietal muscles produces a bulging on one surface of the mesenteries. A transverse section shows that the arrangement of the mesenteries and of their muscles is very definite and characteristic (Fig. 137, B). At each end of the gullet, opposite the siphonoglyphe, are two mesenteries (d. mes.), having their longitudinal muscles turned away from one another: they are distinguished as the directive mesenteries, and, in the case of Tealia, there are two couples of directive mesenteries, one at each end of the long axis of the gullet. Of the remaining complete or primary mesenteries there are four couples on each side (mes. 1), differing from the directive couples in having the longitudinal muscles turned towards one another. The secondary and tertiary mesenteries (mes. 2, mes. 3) are also arranged in couples, and in all of them the longitudinal muscles of each couple face one another.

Symmetry.- It will be noticed that Tealia, unlike the typical hydrozoan and scyphozoan polypes, presents a distinct bilatercil symmetry, underlying, as it were, its superficial radial symmetry. It is divisible into equal and similar halves by two planes only, viz. a vertical plane taken through the long diameter of the gullet, and a transverse plane taken through its short diameter. 
SECT.

The general microscopic structure of a Sea-anemone is well shown by a section through a tentacle (Fig. 139). Both ectoderm (ect.) and endoderm (encl.) consist mainly of very long columnar, ciliated, epithelial cells, and the mesoglœa ( $m s g l$.) is not only extremely thick, but has the general characters of connective tissue, being traversed by a network of delicate fibres with interspersed cells. The middle layer has, in fact, ceased to be a mere gelatinous supporting lamella or mesogloca, and has assumed, to a far greater

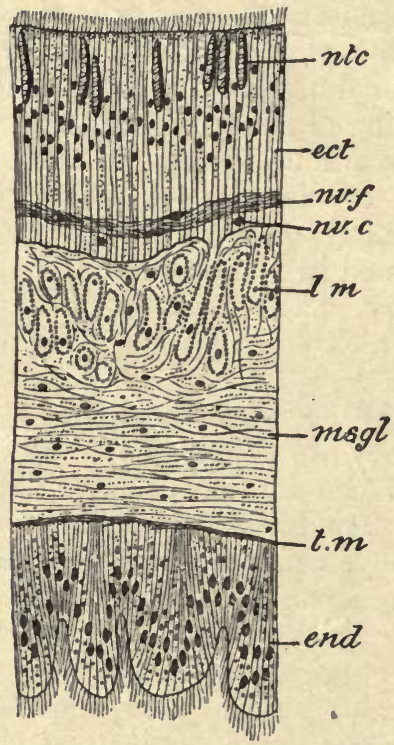

F16. 139.-Tealia crassicornis. Trans. verse section of tentacle. ect. ectoderm ; end. endoderm; $l . m$. longitudinal muscles ; msgl. mesogloes; nv.c. nerve-cells; nv.f. nerve-fibres; ntc. nematocysts; $t$. $m$. transverse muscles. (After Hertwig.)

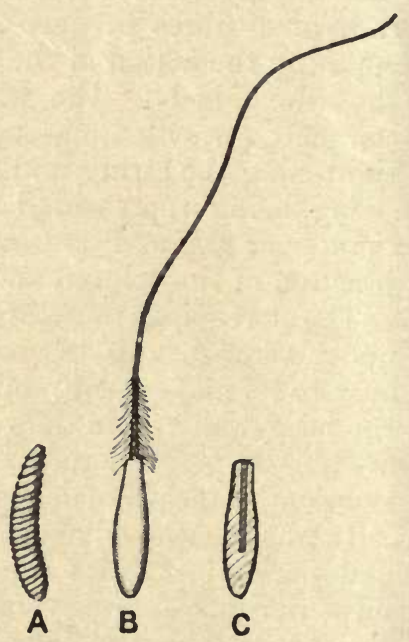

Fio. 140.-Three nematocysts of

Sagartia. (After Hertwig.)

extent than in any of the lower groups, the characters of an intermediate cell-layer or mesoderm.

Stinging-capsules occur in the ectoderm, and are also very abundant in the mesenteric filaments. They (Fig. 140) resemble in general characters the nematocysts of Hydrozoa, but are of a more elongated form, and the thread is usually provided at the base with very numerous slender barbs (B). Very frequently the coiled thread is readily seen in the undischarged capsule (A). Gland-cells (Fig. 141, gl.) are very abundant in the ectodermal lining of the gullet and in the mesenteric filaments: the latter are trilobed in section, and the glandcells are confined to the middle portion, the lateral divisions 
being invested with ordinary ciliated cells (c.). In virtue of possessing both stinging-capsules and gland-cells, the mesenteric filaments perform a double function. The animal is very voracious, and is able to capture and swallow small Fishes, Molluses, Seaurchins, \&c. The prey is partly paralysed, before ingestion, by the nematocysts of the tentacles, but the process is completed, after swallowing, by those of the mesenteric filaments. Then as the captured animal lies in the stomach, the edges of the filaments come into close contact with one another and practically surround

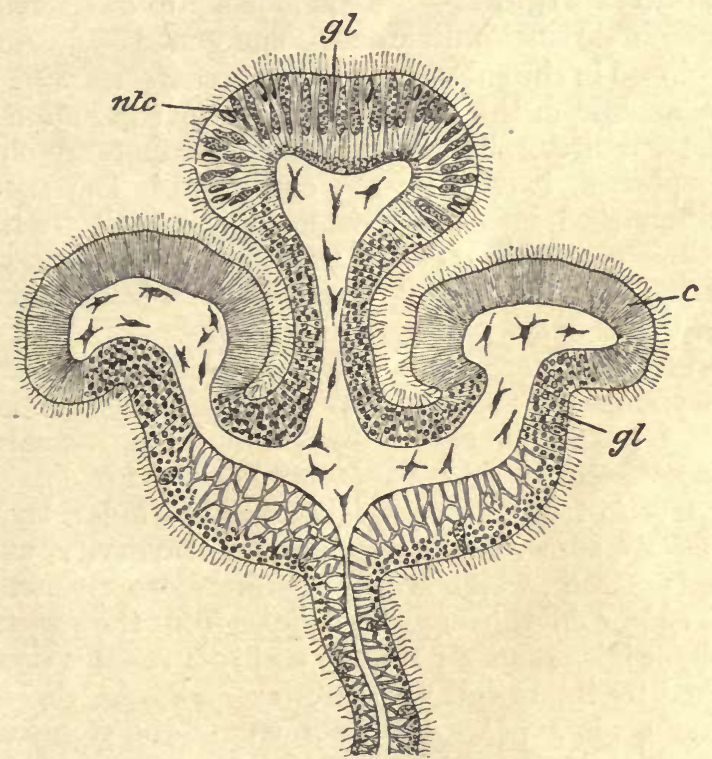

Frg. 141.-Transverse section of mesenteric filament of Sagartia. c. ciliated cells ; gl. glandcells; ntc. nematocysts. (After Hertwig.)

it, pouring out, at the same time, a digestive juice secreted by their gland-cells.

The muscles described above consist partly of spindle-shaped nucleated fibres, and partly of muscle-processes, like those of Hydra: the latter occur chiefly in the transverse muscular layer of the tentacles and are endodermal, the longitudinal layer is formed of distinct fibres of ectodermal origin : the great muscles of the mesenteries are of course endodermal. Although always derived either from the ectoderm or endoderm, many of the muscle-fibres of Tealia undergo a remarkable change of position by becoming sunk in the mesoglœa, and thus appearing to belong to that layer (Fig. 139 l.m.). This fact is significant from the circum- 
stance that, as we shall see, the muscles of all animals above Cœlenterata are mesodermal structures.

The nervous system is very simple. It consists of a layer of delicate fibres lying between the epithelial and muscular layers of the ectoderm. Among the fibres are found nerve-cells (Fig. 139, nv.c.), often of large size, and occurring chiefly in the disc and tentacles. Thus, as in the polype-forms previously described, the nervous system is in a generalised condition, and shows no concentration into a definite central nervous system such as occurs in Medusæ.

Reproductive organs. - Sea-anemones are diœcious, the sexes being lodged in distinct individuals. The gonads-ovaries or testes - are developed in the substance of the mesenteries (Fig. 137, gon.), a short distance from the edge, and, when mature, often form very noticeable structures. The reproductive products are obviously, as in the Scyphozoa, lodged in the endoderm. The sperms, when ripe, are discharged into the stomach and escape by the mouth: they are then carried, partly by their own movements, partly by ciliary action, down the gullet of a female, where they find their way to the ovaries and impregnate the eggs.

The development of Sea-anemones resembles, in its main features, that of Scyphozoa. The oosperm undergoes more or less regular division, the details differing considerably in individual cases, and becomes converted into a planula, an elongated ovoidal body with an outer layer of ciliated ectoderm, and an inner layer of large endoderm cells, surrounding a closed enteric cavity, usually filled with a mass of yolk, which serves as a store of nutriment.

In this condition the embryo escapes from the parent, through the mouth, swims about for a time, and then settles down, becoming attached by its broader or anterior end. At the opposite or narrow end a pit appears, the rudiment of the stomodæum; this deepens, and its lower or blind end becoming perforated, effects a communication with the enteron.

The mesenteries are developed in regular order, but in a way which would certainly not be suspected from their arrangement in the adult. First of all, a single pair of mesenteries (Fig. 142; A, 1) grow from the body-wall to the gullet, being situated one on each side of the vertical plane, at right angles to the long diameter of the stomodæum, and near one end of that tube. The enteron thus becomes divided into two chambers, a larger or dorsal and a smaller or ventral, and the embryo acquires a distinct bilateral symmetry. Next a pair of mesenteries (2) appear in the dorsal chamber, dividing it into a median and two lateral compartments ; then a third pair $(3)$ in the ventral chamber, producing a similar division; then a fourth pair (4) in the middle compartment of the dorsal chamber; then a fifth pair $(B, 5)$ in the lateral compartments of the dorsal chamber; and a sixth (6) in the lateral compartments of the ventral chamber. Soon the longitudinal muscles are developed, and the fate of these primitive pairs of mesenteries can be seen. The third and fourth pairs become the two directive couples of the adult ; another couple of primary mesenteries is constituted, on each side of the vertical plane, by one of the mesenteries of the first 
and one of the sixth pair ; a third couple is similarly formed by a mesentery of the second and one of the fifth pair. Thus it is only in the case of the directive mesenteries that an adult couple-coincides with an embryonic pair: in other instances the two mesenteries of a couple are of different orders, belonging to distinct embryonic pairs. The mesenteric filaments of the first cycle of
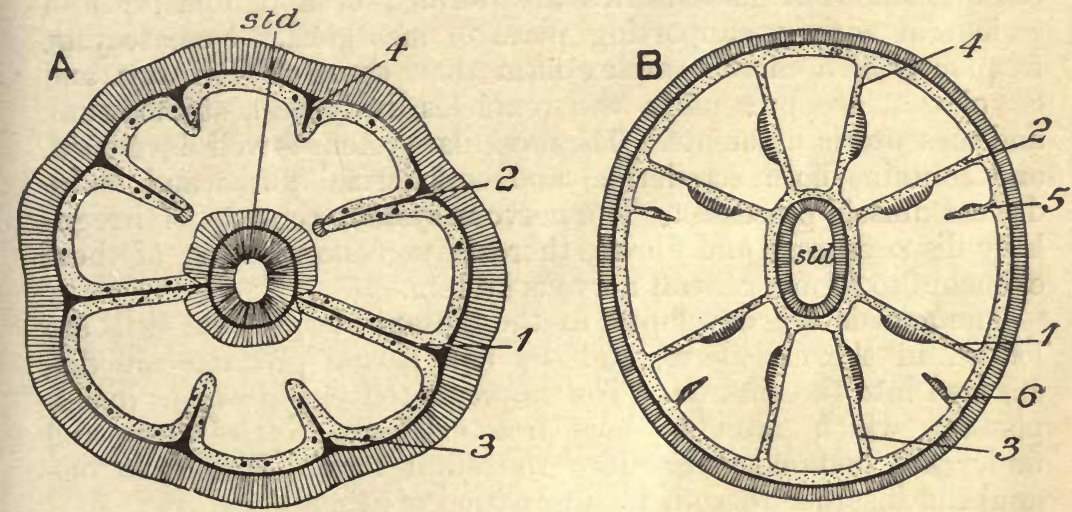

Fig. 142,-Transverse sections of early (A) and later (B) stages of an embryo Sea-anemone (Actinia.) The mesenteries are numbered in the order of their development; std. stomodæum. (After Korschelt and Heider.)

mesenteries are partly ectodermal, partly endodermal in origin, those of the remainder entirely endodermal.

The tentacles are developed in a somewhat similar order to that of the development of the mesenteries. The first to make its appearance is connected with the larger or dorsal enteric chamber mentioned above : for some time it remains much longer than any of its successors, and thus accentuates in a marked degree the bilateral symmetry of the embryo.

It will be noticed that the development of the Sea-anemone is accompanied by a well-marked metamorphosis, but that there is no alternation of generations. In this respect its life-history offers a marked contrast with that of Obelia.

\section{Distinctive Characters and Classification.}

The Actinozoa are Coelenterata which exist only in the polypeform, no medusa-stage being known in any member of the class. The actinozoan differs from the hydrozoan polype mainly in possessing a stomodæum : it differs from the hydrozoan and many scyphozoan polypes in the possession of mesenteries or vertical radiating partitions, which extend inwards from the body-wall and some of which join the stomodæum. The free margins of the mesenteries bear coiled mesenteric filaments, which appear to answer to the gastric filaments of Scyphozoa, but may be partly ectodermal in origin. The mesenteries are developed in pairs, 
symmetrically on each side of a vertical plane: their final radial arrangement is secondary.

The body-wall consists of ectoderm and endoderm separated by a stout mesoglœa containing fibres and cells. The stomodæum consists of the same layers reversed-i.e. its lining membrane is ectodermal. The mesenteries are formed of a double layer of endoderm with a supporting plate of mesoglœa. Nematocysts, frequently of a more complex form than those of Hydrozoa and Scyphozoa, are present in the tentacles, body-wall, stomodæum, and mesenteric filaments. The muscular system is well developed, and contains both ectodermal and endodermal fibres and endodermal muscle-processes. The nervous system consists of irregularly disposed cells and fibres; there is no concentration of these elements to form a central nervous system.

'I'he gonads are developed in the mesenteries, the sex-cells are lodged in the endoderm, and the ripe sexual products are discharged into the enteron. The impregnated egg develops into a planula, which, after a short free existence, settles down and undergoes metamorphosis into the adult form. Except in one doubtful instance there is no alternation of generations.

In some Actinozoa the animal remains simple throughout life, but in most members of the class an extensive process of budding takes place, the result being the formation of colonies of very various form and often of great size. Sorne kinds, again, resemble Tealia in having no hard parts or skeletal structures of any kind; but the majority possess a skeleton, formed either of carbonate of lime or of a horn-like or chitinoid material, and developed, in most cases though not in all, from the ectoderm.

The Actinozoa are classified as follows :-

\section{Sub-Class I.-Zoantharia.}

Actinozoa in which the tentacles and mesenteries are usually very numerous and are frequently arranged in multiples of five or six. The tentacles are usually simple, unbranched, hollow cones. There are commonly two siphonoglyphes and two pairs of directive mesenteries: the remaining mesenteries are usually arranged in couples with the longitudinal muscles of each couple facing one another.

\section{ORDER 1.-ACTINIARIA.}

Zoantharia which usually remain simple, but. in a few instances form small colonies. The tentacles and mesenteries are numerous, and there is no skeleton. This order includes the Sea-anemones.

\section{ORDER 2.-MADREPORARIA.}

Zoantharia which resemble the Actiniaria in the general structure of the soft parts, but which usually form colonies, and 
always possess an ectodermal calcareous skeleton. This order includes the vast majority of Stony Corals (Figs. 146 and 156).

\section{ORDER 3.-ANTIPATHARIA.}

Compound, tree-like Zoantharia in which the tentacles and mesenteries are comparatively few $(6-24)$ in number. A skeleton is present in the form of a branched chitinoid axis, developed from the ectoderm, which extends throughout the colony. This order includes the "Black Corals" (Fig. 150).

\section{Sub-Class II.-Alcyonaria.}

Actinozoa in which the tentacles and mesenteries are always eight in number. The tentacles are pinnate, i.e. produced into symmetrical branchlets. There is never more than one siphonoglyphe, which is ventral in position, i.e. faces the proximal end of the colony. The mesenteries are not arranged in couples, and their longitudinal muscles are all directed ventrally, i.e. towards the same side as the siphonoglyphe.

\section{Order 4.-Alcyonacea.}

Alcyonaria in which the skeleton usually consists of calcareous spicules or small irregular bodies occurring in the mesogløea, but probably originating from wandering ectoderm cells. The common "Dead men's fingers" (Alcyonium, Fig. 153) has a skeleton of this type. In some cases the spicules become aggregated so as to produce a coherent skeleton, which may form a branched axis to the whole colony, as in the precious Red Coral (Corallium, Fig. 145), or a series of connected tubes for the individual polypes, as in the Organ-pipe Coral (Tubipora, Fig. 148). In the "Blue Coral" (Heliopor $a$ ) the skeleton is a massive structure resembling that of the Madreporaria. Most genera are compound; a few, such as Hartea - which, however, is probably a larval form (Fig. 144) are simple.

\section{ORder 5.-Gorgonacea.}

Compound tree-like Alcyonaria, with a calcareous or horny skeleton of ectodermal origin forming a branched axis throughout the colony. Spicules are present in the mesoglœa. There is no siphonoglyphe. The beautiful "Sea-fans" belong to this group (Fig. 154).

\section{Order 6.-Pennatulacea.}

Alcyonaria in which the colony is usually elongated, and has one end embedded in the mud at the sea-bottom, while the opposite or distal end bears the polypes, usually on lateral 
branches. The stem is supported by a calcareous or horny skeleton. The polypes are dimorphic. The "Sea-pens" (Pennatula) are the commonest members of this group (Fig. 147).

\section{Systematic Position of the Example.}

Tealia crassicornis is one of several species of the genus Tealia: it belongs to the family Tealide, which, with several other families, make up the tribe Hexactinia, of the order Actiniaria, of the sub-class Zoantharia.

The presence of numerous tentacles, arranged in multiples of five, places it at once among the Zoantharia. The fact that it is simple and devoid of a skeleton causes it to be assigned to the Actiniaria. This order is divided into tribes characterised by differences in the arrangement of the mesenteries, especially by the presence of one or two couples of directive mesenteries, and by the direction in which the longitudinal muscles face. In the Hexactiniæ the mesenteries are all arranged in couples with the longitudinal muscles of each couple facing one another, except in the case of the two directive couples. 'The mesenteries are in multiples of five, and the stomodæum has two siphonoglyphes and two lappets.

The family Tealidæ is characterised by the possession of numerous mesenteries, of tentacles of moderate length which are completely covered by the closed-in disc during retraction, and by the presence of a large endodermal sphincter muscle. The genus Tealia is distinguished from other members of the same family by being broader than high, by having numerous retractile, equal-sized tentacles, and by the presence of longitudinal series of warts on the column. The species crassicornis is distinguished from other species of the genus by the warts being of approximately equal size.

\section{General Organisation.}

The chief variations in the external form of the Actinozoa are due to the diverse modes of budding: as we shall see, the structure of the individual polypes or zooids is remarkably uniform-at least as regards all the essentials of their organisation.

Nearly all the Actiniaria or Sea-anemones are simple, and, in the few instances where colonies are formed, these are usually small, and contain a very limited number of zooids. In Zoanthus (Fig. 143), for instance, the original polype sends out a horizontal branch or stolon (st.), from which new polypes arise. Besides the Sea-anemones the only simple forms are certain Madreporarian corals, such as Flabellum (Fig. 155, A, B), and three genera of Alcyonacea, of which Hartea (Fig. 144) may be taken as an example. 
The simplest mode of budding is that just described in Zoanthus, in which new zooids are developed from a narrow band-like

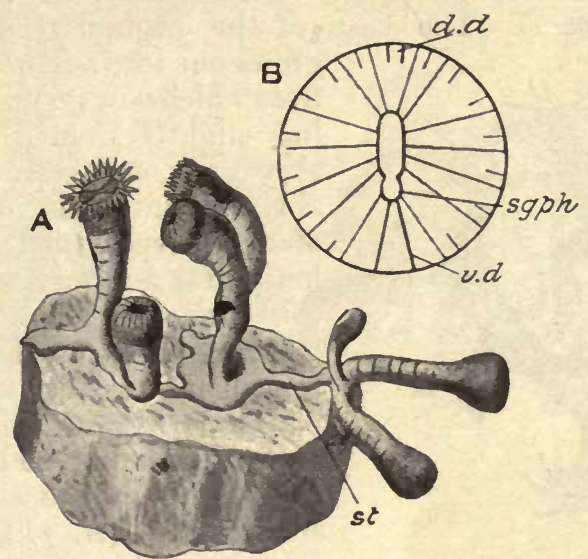

Fio. 143. -Zoanthus sociatus. A, entire colony; st. stolon. B, transverse section, sgph. siphomoglyphes; $d . d$. dorsal, and $v$. $d$. ventral directive mesenteries. (After McMurrich and Korschelt and Heider.)

or tubular stolon (Fig. 143,st). A more usual method resembles that with which we are already familiar in Hydrozoa, new buds being

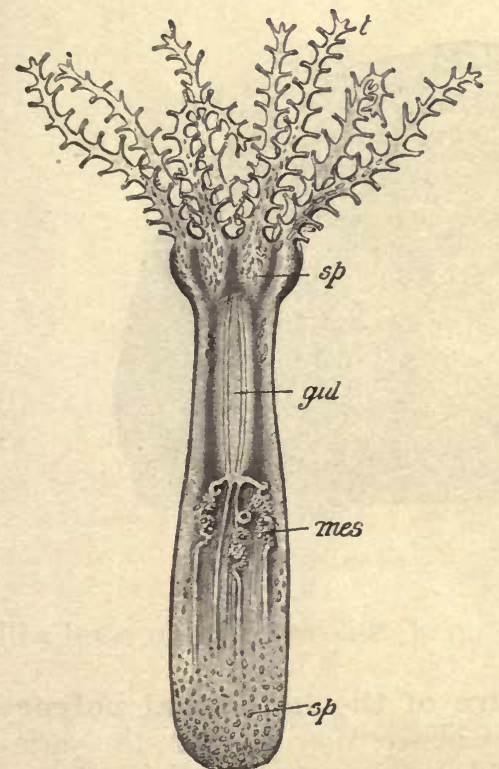

FiG. 144.-Hartea elegans. gul. gullet; mes. mesentery; sp. spicules; $t$. tentacles. (After Perceval Wright.) formed as lateral outgrowths, and a tree-like colony arising with numerous zooids springing from a common stem or coenosarc. Corallium and Gorgonia (Figs. 145 and 154) are good examples of this type of growth. In other cases the buds grow more or less parallel with one another, producing massive colonies either of closeset zooids or of zooids separated by a solid cœnosarc. As examples of this type we may take Palythoa, the most complex of the Actiniaria, and many of the common Madreporaria, such as Astrcea (Fig. 146). In the Sea-pens (Pennatulacea) the proximal end of the elongated colony (Fig. 147) is sunk in the mud, and the distal end bears zooids springing either directly from 
the conosarc or, as in Pennatula itself, from flattened lateral branches. The stem itself is the equivalent of a polype.

A very peculiar mode of budding occurs in the Organ-pipe Coral ('Tutipora). The base of the original polype (Fig. 148)

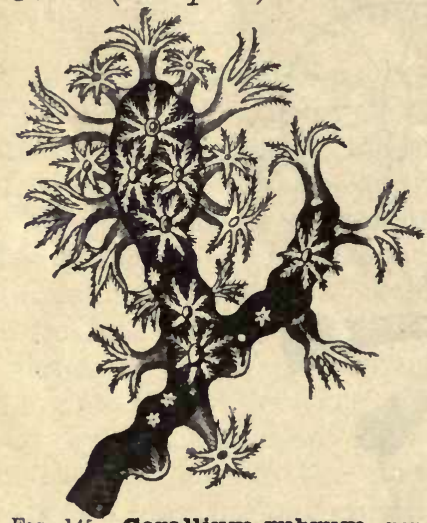

FIa. 145.-Corallium rubrum, portion of a branch. (From Claus, after Lacaze-Duthiers.) grows out into a flattened expansion from which new polypes arise, diverging slightly from one another as they grow, and separated by tolerably wide intervals. The distal ends of the polypes then grow out into horizontal expansions or platformis $(p l$.$) , formed at first of ectoderm and$ mesogloea only, but finally receiving prolongations of the endoderm. The platforms extend, come in contact with one another, and fuse. In this way platfor ms of considerable extent are formed $(\mathrm{A}, p l$.), uniting the polypes with one another. From the upper surfaces of the platforms, between the older polypes, new buds arise, and in this way the colony tends to assume the form of an inverted pyramid, the number of zooids, and consequently the diameter of the colony, increasing pari passu with the vertical

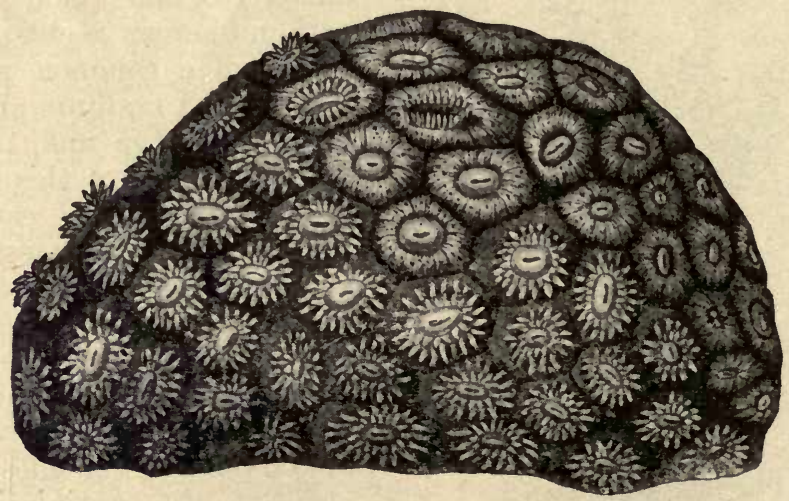

FIG. 146,-Astræa pallida, the living colony. (After Dana.)

growth of the latter. The skeleton of this remarkable coral will be referred to hereafter.

Although the general structure of the individual polypes of the Actinozoa is, as mentioned above, very uniform, the variations in detail are numerous and interesting, especially among the Actiniaria. One of the most important points to consider 

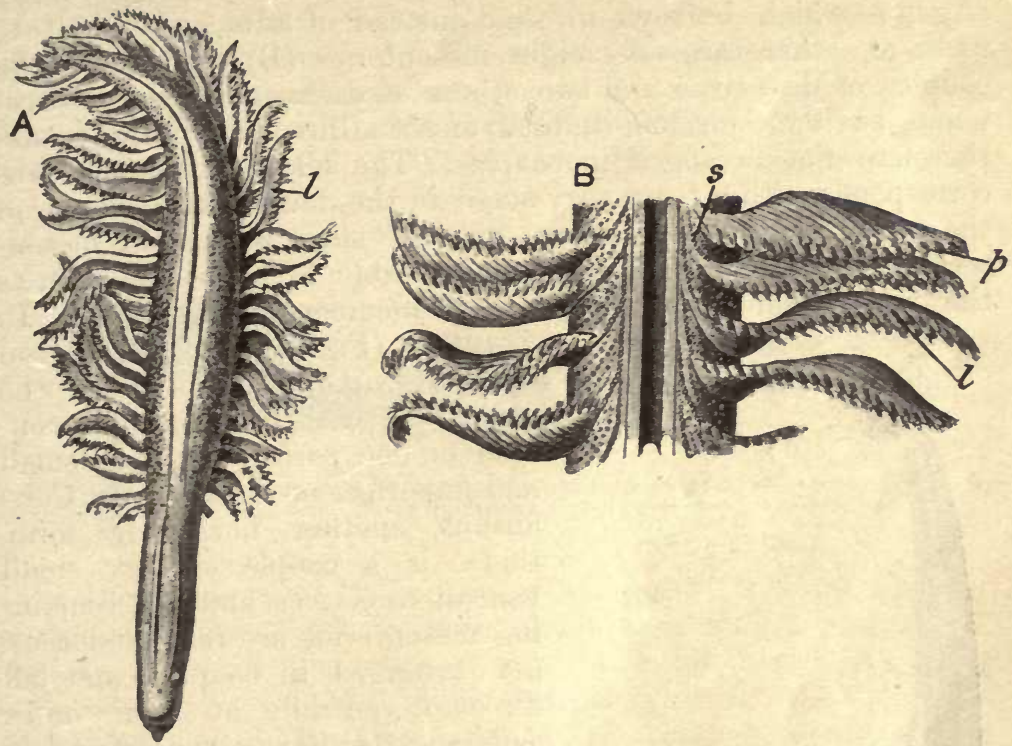

Fig. 147. - Pennatula sulcata. A, entire colony; B, portion of the same magnified. $l$. lateral branch; $p$. polype ; s. siphonozooid. (After Koelliker.)
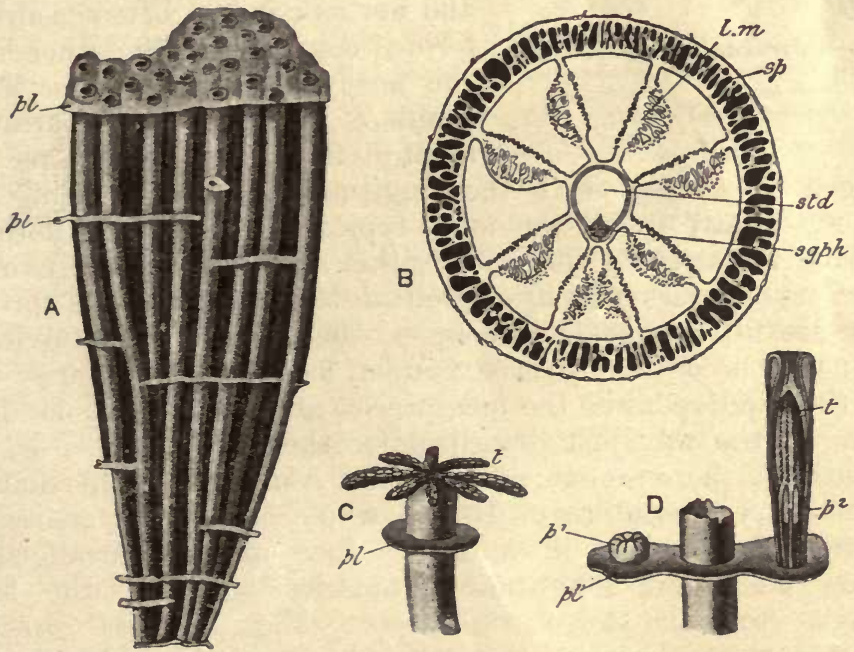

FIG. 148. - Tubipora musica. A, skeleton ol entire colony ; B, transverse sections of polype ; C. single polype with tube and commencement of platform; D, growth of new polypes from platform, l.m. longitudinal muscles; $p^{1} . p^{2}$. polypes; $p l$. platform; $g, p h$. siphonoglyphe; $s p$. spicules; std. stomodrum. (After Cuvier, Quoy and Gaimard, and Hickson.) 
is the arrangement of the mesenteries. In Edwardsia (Fig. 149), a genus which burrows in sand instead of attaching itself to rocks, \&c., there are only eight mesenteries (B) - the usual two couples of directives, and two others on each side of the vertical plane, having their longitudinal muscles directed ventrally, and therefore not arranged in couples. The adult Edwardsia thus corresponds with a temporary stage in the development of one of the more typical sea-anemones, viz., the stage with eight mesenteries shown in Fig. 142, A.; it is probably to be looked upon as the most primitive or generalised member of the order. In

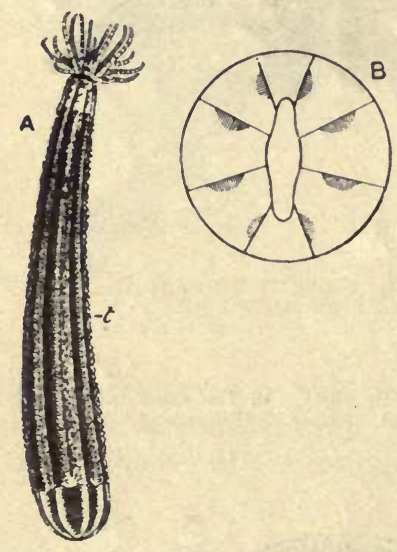

Fic. 149.-Edwardsia claparèdi1. $A$, the entire animal; $t$. tube. B, transverse section. (After Andres, and Korschelt and Heider.)

Zoanthus (Fig. 143, B) the dorsal directives (d.d.) do not reach the gullet, and each lateral couple consists of one perfect and one small and imperfect mesentery. In Cerianthus, another burrowing form, there is a couple of very small ventral directives, and the remaining mesenteries are very numerous, not arranged in couples, and all directed ventrally at their outer ends, so as to have a very obviously bilateral arrangement: in this genus, as growth proceeds, new mesenteries are added on the dorsal side, and not, as is usual, between already formed couples. On the other hand, the newly discovered Gyiactis exhibits a perfectly radial arrangement: the mesenteries are all arranged in couples with the longitudinal muscles facing one another. Lastly, in all the more typical Sea-anemones (forming the tribe Hexactinice) there are either six, eight or ten pairs of perfect mesenteries, which, as well as the secondary and tertiary cycles, are all arranged in couples, the longitudinal muscles of all but the one or two directive couples facing one another.

In the Madreporaria the mesenteries are arranged, so far as is known, in the way just described for the Hexactiniæ. In the Antipatharia there are six primary, and sometimes either four or six secondary mesenteries. In the whole of the Alcyonaria the mesenteries are eight in number: they are not arranged in couples, and their longitudinal muscles all face the same way, viz., towards the ventral aspect (Fig. 148, B). In this whole sub-class, therefore, the resemblance to Edwardsia is very close, the main difference being that the longitudinal muscles of the ventral directives face inwards in the Alcyonaria, outwards in Edwardsia. 
The tentacles in Zoantharia are usually very numerous, and in nearly all cases have the form of simple glove-finger-like outpushings of the disc. In Edwardsia, however, they may be reduced to sixteen, and in some genera of Sea-anemones they are branched. In the Antipatharia (Fig. 150) they vary in number from six to twenty-four. When more than six are present, six of them are larger than the others.

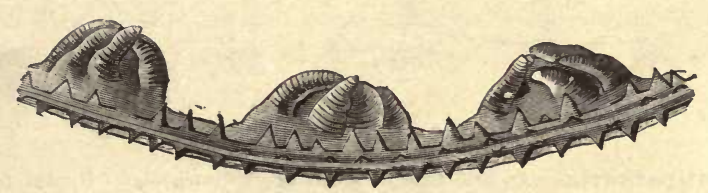

FIG. 150.-Antipathes ternatensis, portion of a branch, showing three zooids and the herny axis beset with spines. (From the Cumbridge Natural History, after Schultze.)

In the Alcyonaria, on the other hand, the tentacles, like the mesenteries, are eight in number and are always pinnate, i.e. slightly flattened and with a row of small branchlets along each edge (Fig. 144). Many Actiniaria have the tentacles perforated at the tip (Fig. 138, A, p.); and in some species these organs undergo degeneration, being reduced to apertures on the disc, which represent the terminal pores of the vanished tentacles and are called stomidia.

Many Sea-anemones possess curious organs of offence called acontia (Fig. 138, A, and Fig. 157, ac.). These are long delicate threads springing from the edges of the mesenteries: they are loaded with nematocysts, and can be protruded through minute apertures in the column, called "port-holes" or cinclides ( $c n$.$) .$

Enteric System.-The gullet in the Actiniaria presents some remarkable modifications. It is usually a compressed tube with two siphonoglyphes, but in Zoanthus and some other genera the ventral gullet-groove alone is present (Fig. 143, B), and in Gyractis both grooves are absent, and the tube itself is cylindrical with a circular mouth. The ordinary compressed form of gullet often assumes, in the position of rest, an $\infty$-shaped transverse section, owing to its walls coming together in the middle and leaving the two ends wide open. In most of the Antipatharia the zooid is drawn out in the direction of the long axis of the branch (Fig. 151), and in some it becomes constricted into three parts $(B)$ which may have the appearance of separate zooids, the central part containing the gullet with the mouth. while the lateral parts each contains a gonad; each of these apparent zooids bears two of the six tentacles; the median one has all six mesenteries attached internally to the gullet; in each lateral part there is only the outer portion of one of the 
transverse mesenteries. In such a form as Schizcpathes (Fig. 151, B) there is thus recognisable an arrangement of the parts which might
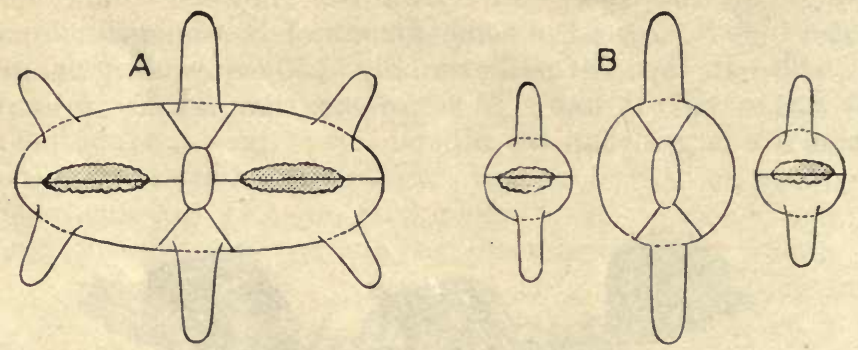

Fia. 151.-Antipatharia. A, oral face of zooid of Parantipathes. B, oral face of zooid of
Schiznpathes. (After Delage et Hérouard.).

be interpreted as a dimorphism of the zooids, one set - the parts containing the mouth and gullet-being regarded as gastrozooids, and the others containing the gonads as gonozovids.

Fixed and Free Forms. - A large proportion of Actinozoa are permanently fixed, such, for instance, as most of the Stony Corals, the Sea fans, Black Corals, \&c. Most Sea-anemones are temporarily attached by the base, but are able slowly to change their position: some forms, such as Edwardsia (Fig. 149) and Ceriventhus, usually live partly buried in sand enclosed in a tube formed of discharged stinging-capsules, the oral end with its crown of tentacles alone being exposed: others, such as Peachia, live an actually free life, habitually lying on the sea-bottom with the longitudinal axis horizontal like that of a worm: a few, such as Minyas (Fig. 152), have the aboral end dilated into a sac containing air and serving as a float; by ita means these animals can swim at the surface of the sea, and are thus, alone among the Actinozoa, pelagic.

Dimorphism.-With the exception of one genus of Stony Corals, the Zoantharia are all homomorphic, i.e. there is no differentiation of the zooids of a colony. But in the Alcyonaria dimorphism is common : the ordinary zooids or polypes are accompanied by smaller individuals, called

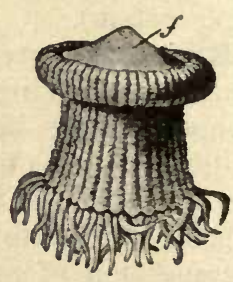

FIG. 152. - Minyas. f. float. (After Andre?.) siphonozooids (Fig. 147, s.), having no tentacles, longitudinal muscles, or gonads.

None of the Actiniaria have a true skeleton: in some, however, there is a thick cuticle, and several kinds enclose themselves in a more or less complete tube (Fig. 149), which may be largely formed of discharged nematocysts. The simplest form of skeleton is found in the solitary Alcyonarian genus Hartea (Fig. 144), already 
referred to, in which minute irregular deposits of calcium carbonate, called spicules (sp.), are deposited in the mesoglœe. A similar spicular skeleton occurs in the "Dead-men's finger" (Alcyonium, Fig. 153), where spicules of varying form are found distributed throughout the mesoglœa of the cœnosarc. In Tubipora (Fig. 148), the "Organ-pipe Coral," the mesoglœal spicules become closely fitted together, and form a continuous tube for each polype, the tubes being united by horizontal culcareous platforms $(p l$. $)$ formed by deposits of spicules in the expansions of the same name already referred to. The skeleton of Tubipora is, therefore, an internal

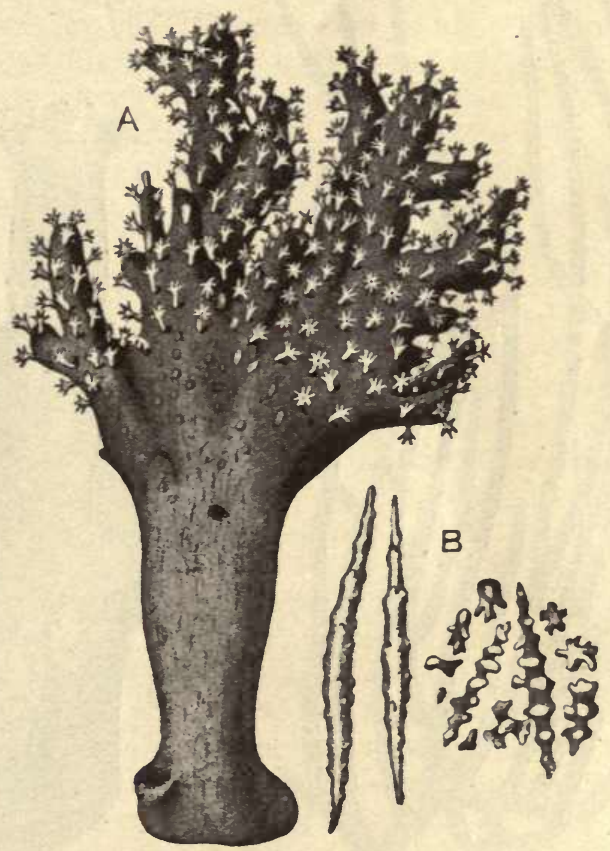

Fic. 153.-Alcyonium palmatnm, A, entire colony ; B, spicules (After Cuvier.)

skeleton, and in the living state is covered by ectoderm. In the Red Coral of commerce (Corallium, Fig. 145) the originally separate spicules are embedded in a cement-like deposit of carbonate of lime, the result being the production of an extremely hard and dense branched rod, which extends as an axis through the cœnosarc. In the Blue Coral (Heliopora), on the other hand, the stony calcareous skeleton is not made up of fused spicules, but is solid from the first.

Another type of skeletun is found in the Antipatharia (Fig. 150) and in the Gorgonacea (Fig. 154). It also consists of an axial rod, extending all through the colony and branching with it, but is 
SECT,

formed of a flexible horn-like material. Moreover it is not mesoglœal, but ectodermal in origin: in close contact with it is an epithelium, from the cells of which it is produced as a cuticular secretion, and this epithelium is formed as an invagination of the base of the colony. In addition to its axis, Gorgonia contains numerous spicules in the mesogløa of the cœnosarc. In some

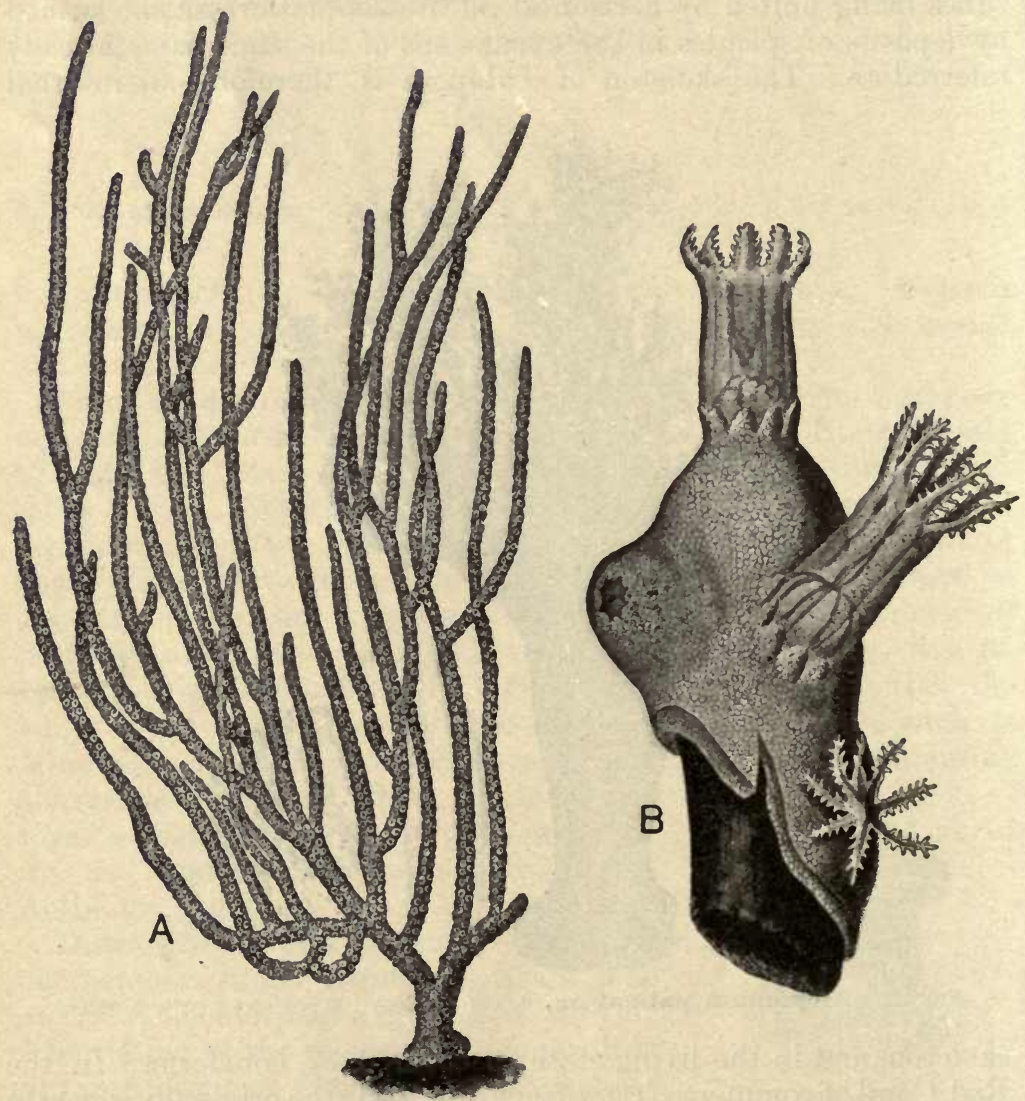

Fia. 154.-Gorgonia verrucosa A, entire colony; B, portion of the same magnified, $c$ conosare; $p$. polype. (After Koch and Cuvier.)

of the Gorgonacea the axial skeleton is partly horny, partly calcareous.

In the Sea-pen (Pennatula, Fig. 147) and its allies the stem of the colony is supported by a horny axis which is unbranched, not extending into the lateral branches. In this case the axis is contained in a closed cavity lined by an epithelium, the origin of 
which is still uncertain. Spicules occur in the mesoglœea, some of them microscopic, others readily visible to the naked eye.

In the Madreporaria we have a skeleton of an entirely different type, consisting, in fact, of a more or less cup-like calcareous structure, secreted from the ectoderm of the base and column of the polype. When formed by a solitary polype, such a "eupcoral" is known as a corallite: in the majority of species a large number-sometimes many thousands-of corallites combine to form a corallum, the skeleton of an entire coral-colony.

The structure of a corallite is conveniently illustrated by that of the so!itary genus Flabellum (Fig. 155, A, B). It has the form of a short conical cup, much compressed so as to be oval in section. Its wall or thecr (th.) is formed of dense stony calcium carbonate, white and smooth inside, rough and of a brownish colour outside, except towards the margin, where it is white. Its proximal or aboral end is produced into a short stalk or peduncle, by which the Coral is attached in the young state, becoming free when adult: in many other simple Corals there is no stalk, but attachment to the support is effected by means of a flattened proximal surface or basal plate $(\mathrm{C}, b . p l$.). From the inner surface of the theca a number of radiating partitions, the septa (sep.), proceed inwards or towards the axis of the cup, and, like the mesenteries of a polype, are of several orders, those extending furthest towards the centre being called primary septa, the others secondary, tertiary, and so on. Towards the bottom of the cup the primary septa meet in the middle to form an irregular central mass, the columella (col.). In some Corals the columella is an independent pillar-like structure arising from the basal plate $(\mathrm{D}, \mathrm{col}$.).

In many Corals there is a distinct calcareous layer investing the proximal portion of the theca, and called the epitheca $(\mathrm{C}$, e.th.). Some species have the inner portions of the septa detached so as to form a circlet of narrow upright columns, the pali. In others there are horizontal partitions or dissepiments passing from septum to septum, and in others, again, complete partitions or tabuloc, like those of Millepora (p. 157), extending across the whole corallite. In the Mushroom-coral (Fungia), the corallite is discoid, the theca is confined to the lower surface, and small calcareous rods, the synapticula, connect the septa with one another.

In the living condition the polype fills the whole interior of the corallite and projects beyond its edge to a greater or less degree according to its state of expansion (C). The proximal part of the body-wall is thus in contact with the theca, which has the relation of a cuticle, and is, in fact, a product of the ectoderm. The free portion of the body-wall is frequently, in the extended state, folded down over the edge of the theca so as to cover its distal portion. The septa alternate with the mesenteries, each lying in the space between the two mesenteries of one couple, and each being in- 
vested by an in-turned portion of the body-wall ( $\mathrm{E}, \mathrm{F})$. Thus the septa, which appear at first sight to be internal structures, are really external: they lie altogether outside the enteric cavity, and are in contact throughout with ectoderm.

The ectudermal nature of the entire corallite is further proved by its development. The first part to appear is a ring-shaped

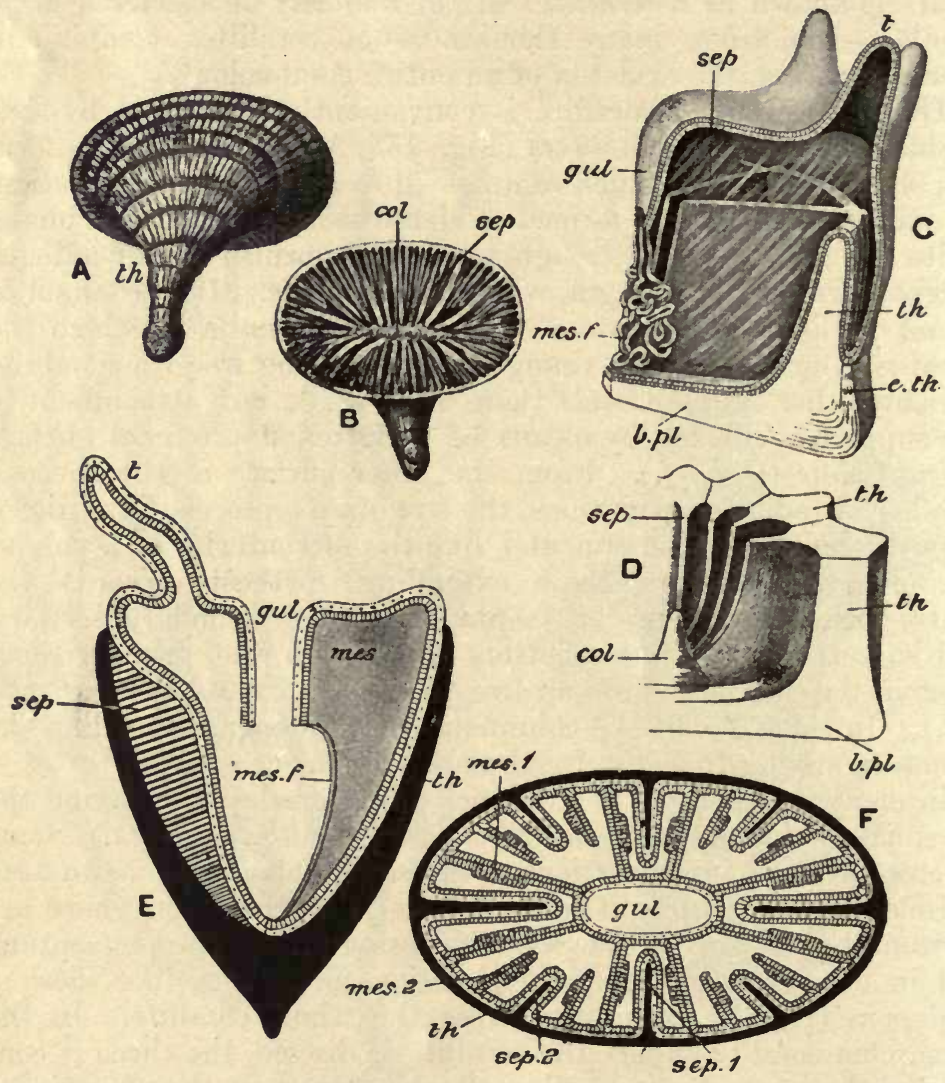

Fra. 155.-A, B, two vicws of Flabellum curvatum. C, semi-diagrammatic vicw of a simplo coral; D, portion of a corallite ; E, F, diagram of a simple coral in longitudinal and transverse section; ectoderm dotted, endoderm striated, skeleton black. b. pl. basal plate; col. columella ; e. th. epitheca ; gul. gullet; mes, mes. 1, mes. 2, mesenteries ; mes. $f$. mesenteric filaments ; sep. septa ; $t$. tentacle; $t h$. theca. (A and B after Moseley; C and D after Gilbert Bourne.)

deposit of carbonate of lime between the base of the polype and the body to which it adheres: sections show this ring to be formed by the ectoderm cells of the base. The ring is soon converted into a disc, the basal plate, from the upper surfaces of which a number of ridges arise, arrayed in a star-like fashion: these are the rudiments of the septa Here, again, sections show that each septum corre- 
sponds with a radial in-pushing of the base, and is formed as a secretion of the invaginated ectoderm. As the septa grow they unite with one another at their outer ends, and thus form the theca. In some cases, however, the theca appears to be in independent structure.

The almost infinite variety in form of the compound corals is due, in the main, to the various methods of budding, a subject which has already been referred to in treating of the actinozoan colony as a whole. According to the mode of budding, massive Corals are produced in which the corallites are in close contact with one another, as in Astr:ea (Fig. 146); or tree-like forms, such
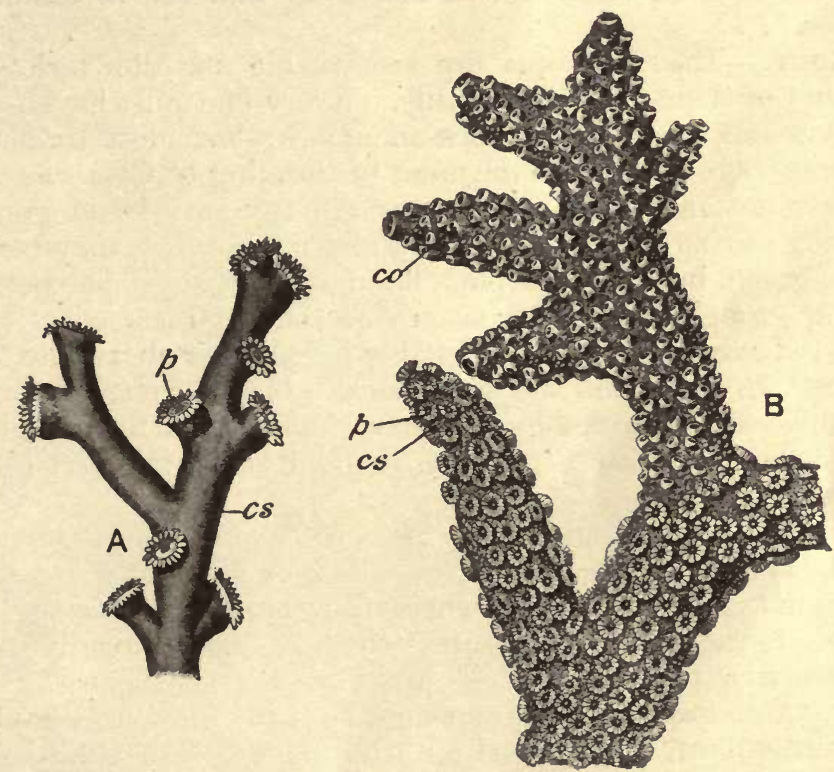

FIG. 156.-Dendrophylla nigrescans, B, Madrepora aspera. co. corallites ; cs. conosarc; $p$. polypes. (After Dana)

as Dendrophyllia (Fig. 156, A), in which a common calcareous stem, the coenenchyma, is formed by calcification of the conosarc (cs.), and gives origin to the individual corallites. It is by this last-named method, the cœnosarc attaining great dimensions and the individual corallites being small and very numerous, that the most complex of all Corals, the Madrepores (Madrepora, Fig. 156, B) are produced.

The microscopic structure of corals presents two main varieties. In what are called the aporose or poreless corals, such as Flabellum, Astræa, \&c., the various parts of the corallite are solid and stony, while in the perforate forms, such as Madrepora, all parts both of 
the corallites and of the connecting conenchyma, have the characters of a mesh-work, consisting of delicate strands of carbonate of lime united with one another in such a way as to leave interstices, which in the living state are traversed by a network of interlacing tubes, representing the coenosarc, and placing the polypes of the colony in cominunication.

The Blue Coral (Iteliopora), one of the Alcyonacea, has a massive corallum the same general appearance as a Madreporarian. The lobed surface bear's apertures of two sizes, the larger being for the exit of the ordinary polypes, the smaller for the siphnozoids. 'Tabulæ are present, and septum-like ridges, which, however, have no definite relations to the mesenteries and are inconstant in number.

Colour.-The Actinozoa are remarkable for the variety and brilliancy of their colour during life. Every one inust have noticed the vivid and varied tints of Sea-anemones; but most dwellers in temperate regions get into the habit of thinking of Corals as white, and have no conception of their marvellously varied and gorgeous colouring during life. The Madrepores, for instance, may be pink, yellow, green, brown, or purple: Tubipora has green polypes, contrasting strongly with its crimson skeleton; and the effect of the bright red axis of Corallium is greatly heightened by its pure white polypes. In Heliopora the whole coral is bright blue; the tropical Alcyonidæ are remarkable for their elaborate patterns and gorgeous coloration; and Pennatula, in addition to its vivid colours, is phosphorescent.

In most cases the significance of these colours is quite unknown. In some species, however, "yellow-cells" or symbiotic Algæ have been fuund in the endoderm, where they probably serve the same purpose as the similar structures which we have already studied in Radiolaria (p. 63).

Many Actinozoa, like many sponges (p. 126), furnish examples of commensalism, a term used for a mutually beneficial association of two organisms of a less intimate nature than occurs in symbiosis. An interesting example is furnished by the Sea-anemone Adamsia palliata (Fig. 157). This species is always found on a univalve shell - such as that of a Whelk-inhabited by a Hermit-crab. The Sea-anemone is carried from place to place by the Hermit-crab, and in this way secures a more varied and alundant food-supply than would fall to its lot if it remained in one place. On the other hand, the Hermit-crab is protected from the attack of predaceous Fishes by retreating into its shell and leaving exposed the Seaanemone, which, owing to its toughness, and to the pain caused by its poisonous stinging-capsules, is usually avoided as an article of food.

Other Sea-anemones-such as the gigantic Discosoma of the great Barrier-Reef-are found associated with Small Fishes or 
Crustacea, which have their abode in the enteric cavity. In this case the Fish secures shelter in a place where it is very unlikely to be disturbed, and the two animals are strictly commensals or "messmates" since they share a common table. A somewhat similar instance is furnished by the Blue Coral (Heliopora), already referred to more than once. 'The corallum contains, not only the apertures for the polypes and siphonozooids, but also tubular cavities of

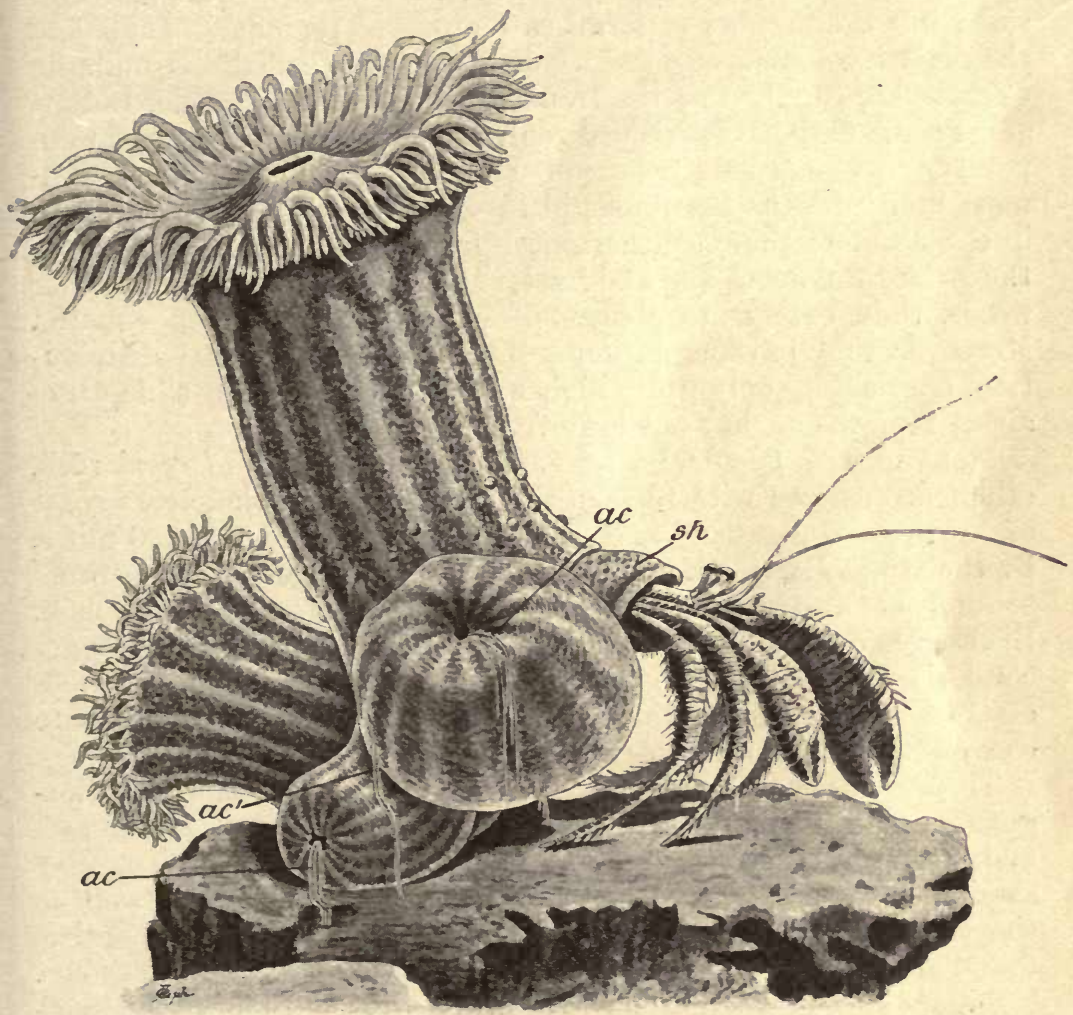

Fio. 157.-Adamsia palliata, four individuals attached to a Gasteropud shell inhabited by a Hermit-crab. $a c$. $a c^{1}$. acontia ; $s h$. shell of Gasteropod. (After Andres.)

an intermediate size, in each of which is found a small chætopod Worm, belonging to the genus Lencodore. As the polypes are frequently found retracted at a time when the Worms are protruded from their holes in search of food, it is not surprising that the latter should have been credited with the fabrication of the coral. Trapezia, a genus of Crabs, always lives in interstices of a particular species of Madrepore.

The distribution of the Actiniaria is world-wide, and in many cases the same genera are found in widely separated parts 
of the world. They are, however, larger, and of more varied form and colour in tropical regions, for instance on coral-reefs. The largest reef-anemone, Discosoma, found also in the Mediterranean, attains a diameter of 2 feet. Most members of the order are littoral, living either between tide-marks or at slight depths, but a few are pelagic, and several species have been dredged from depths of from 10 to 2,900 fathoms.

The Madreporaria, taken as a whole, have also a wide distribution; but the number of forms in temperate regions is small, and the majority-including the whole of what are called reef-building Corals-are confined to the tropical parts of the Atlantic, Indian, and Pacific Oceans, flourishing only where the lowest winter temperature does not sink below $68^{\circ} \mathrm{F} .\left(20^{\circ} \mathrm{C}\right.$.). Thus their northernmost limits are the Bermudas in the Atlantic, and Southern Japan in the Pacific; their southernmost limits, Rio and St. Helena in the Atlantic, Queensland and Easter Island in the Pacific : in other words, they extend to about $30^{\circ}$ on each side of the equator. Moreover, they have a curiously limited bathymetrical distribution, flourishing only from high-water mark down to a depth of about 20 fathorns, but not lower.

Many of the Pacific Islands are formed entirely of coral rock, others are fringed with reefs of the same, and the whole east coast of Northern Queensland is bounded, for a distance of 1,250 miles, by the Great Barrier Reef, a line of coral rock more or less parallel to and at a distance of from 10 to 90 miles from the land. Such reefs consist of gigantic masses of coral rock fringed by living coral, the latter growing upon a basis of dead coral, the interstices of which have been filled up with débris of various kinds, so as to convert the whole into a dense limestone.

The Antipatharia, and many of the Alcyonaria, such as the Gorgonacea and Pennatulacea, have also a world-wide distribution, and, even in temperate regions, Black Corals and Sea-fans may attain a great size: the members of both these groups, as well as the Sea-pens, are found at moderate depths. The Red Coral is found only in the Mediterranean, at a depth of 10 to 30 fathoms. Tubipora and Heliopora have the same distribution as the reefbuilding Corals.

From the palæontological point of view, corals are of great importance: they are known in the fossil condition from the Silurian epoch upwards, and in many formations occur in vast quantities, forming what are called coral limestones. The majority of fossil forms are referable to existing families, but in the Palrozoic era the dominant group was the Ringosa, the affinities of which are still very obscure. In these the corallites are usually bilaterally symmetrical, the septa are arranged in multiples of four, and the cup presents on one side a pit, the fossula, where the septa are greatly reduced. 


\section{CLASS IV.-CTENOPHORA.}

1. Example of the Class-Hormiphora plumosa.

External Characters.-Hormiphora is a pear-shaped organism about 5-20 mm. in diameter, and of glassy transparency (Figs. 158 and 159). The species H. plumosc is found in the Mediterranean; allied forms belonging either to the same genus (often called ('ydippe) or to the closely allied genus Pleurobrachia are common pelagic forms all over the world.

From opposite sides of the broad end depend two long tentacles $(t$.$) , provided with numerous little tag-like processes, and springing$

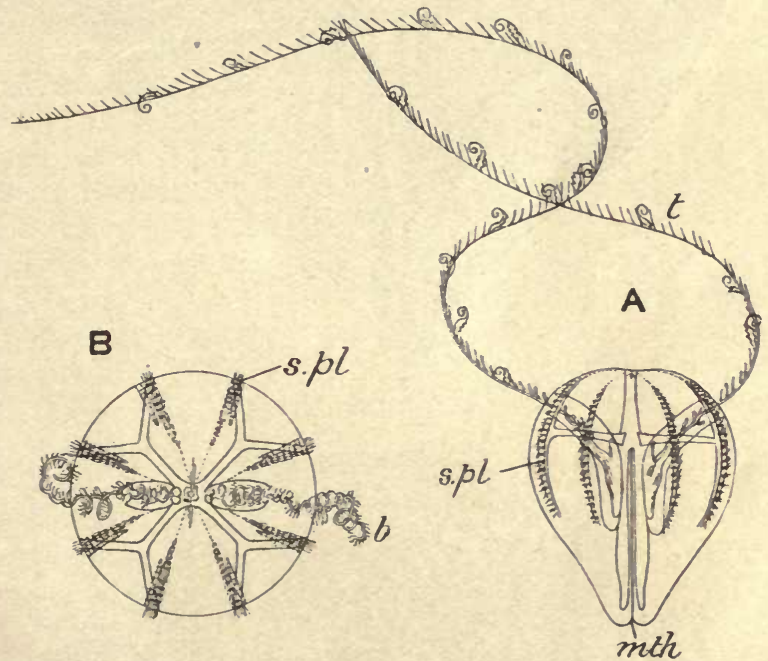

Fig. 158.-IIormiphora plumosa. A, from the side, B, from the aboral pole. mth. mouth ; 8. pl. swimming plates; $t$. and b. tentacles. (After Chun.)

each from a deep cavity or sheath, into which it can be completely retracted (Fig. 159, t.sh.). At the narrow end-where the stalk of a pear would be inserted-is a slit-like aperture, the moulh (mth.): this end is therefore oral. At the opposite or aboral pole is a slight depression, in which lies a prominent sense-organ (s.o.), to be described hereafter.

But the most striking and characteristic feature in the external structure of Hormiphora is the presence of eight equidistant meridional bands (s.pl.), starting from near the aboral pole, and extending about two-thirds of the distance towards the oral pole. Each band is constituted by a row of transversely arranged comb-like structures, consisting of narrow plates frayed at their outer ends. During life the frayed ends are in constant movement, lashing to and fro, and so propelling the animal through the water. The combs 

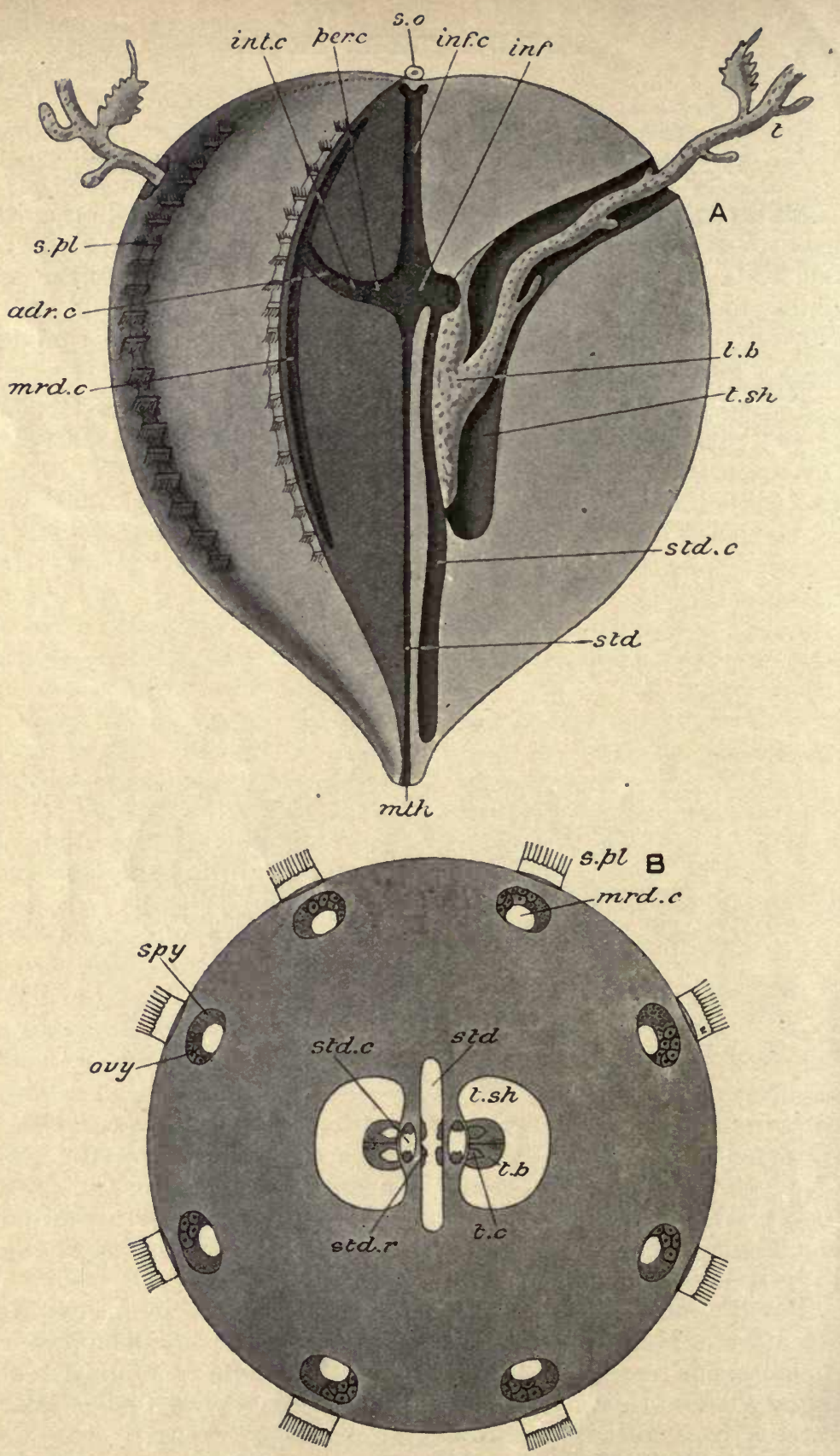

Fig. 159.- Formiphora plumosa. A, dissected specimen having rather more than one quarter of the boly cut away. B, transverse scetion; diagrammatic. all. $c$. adradial canal; inf. infundibulum; inf. $c$. infundibular canal; int. $c$. inter-radial canal; mrel. $c$. meridional canal ; $m t h$. mouth ; ovy. ovary; per. $c$. per-radial canal; s. o. sense-organ; s. $p l$. swimming-plate; spy. spermary ; std. stomodæum ; std. c. stonodreal canal ; std. $r$ stoniodieal ridges; $t$. tentacle; $t$. $l$. base of tentacle; $t$. $r$. tentacular caulal; $t$. $8 h$. tentacular slieath. 
are, in fact, rows of immense cilia, fused at their proximal ends : their presence and mode of occurrence-arranged in meridional comb-ribs or swimming-plates-are strictly characteristic of the class, and indeed give it its name.

It will be seen at once that-apart from all considerations of internal structure-Hormiphora presents a similar combination of radial with bilateral symmetry as in some Hydrozoa, such as Ctenaria (Fig. 109, 1), and as in the majority of Actinozoa. The swimming-plates are radially arranged, and mark the eight adradii, but the slit-like mouth and the two tentacles indicate a very marked and characteristic bilateral symmetry. A plane passing through the longitudinal axis of the body, parallel with the long axis of the mouth, is called, as in Actinozoa (see p. 189), the vertical plane: it includes two per-radii, which are respectively dorsal and ventral. A plane at right angles to this, passing through both tentacles, and including right and left per-radii, is called the transverse plane.

Enteric System.-The mouth leads into a flattened tube (Fig. $159, s t d$.$) , often called the stomach, but more correctly the gullet or$ stomodacum. It reaches about two-thirds of the way towards the aboral pole, and its walls are produced internally into ridges (std. $\imath^{\circ}$.), which increase the area for the absorption of digested food. Living prey is seized by the tentacles, ingested by the aid of the mobile edges of the mouth, and digested in the stomodæum, which is thus physiologically, though not morphologically, a stomach. The products of digestion make their way into the various parts of the canal-system, presently to be described, and indigestible matters are passed out at the mouth.

Towards its upper or aboral end the stomodæum gradually narrows and opens into a cavity called the infundibulum (inf.), which probably answers to the stomach of an Actinozoon or a medusa, and is flattened in a direction at right angles to the stomodxum-i.e. in the transverse plane. From the infundibulum three tubes are given off: one, the infundibular canal (inf.c.), passes directly upwards, and immediately beneath the aboral pole divides into four short branches, two of which open on the exterior by minute apertures, the excretory pores (Fig. 160, A, ex. p.). The two other canals given off from the infundibulum are the per-radial canals (per.c.): they pass directly outwards, in the transverse plane, and each divides into two inter-radial canals (int.c.), which in their turn divide each into two adradial canals ( $a d r . c$.$) . These succes-$ sive bifurcations of the canal-system all take place in a horizontal plane (Fig. 160, B), and each of the ultimate branches or adradial canals opens into a meridional cancl ( $\mathrm{mrd}$. $c$.), which extends upwards and downwards beneath the corresponding swimming-plate. Furthermore, each per-radial canal gives off a stomodacal canal (stc. c.), which passes downwards, parallel to and in close contact 

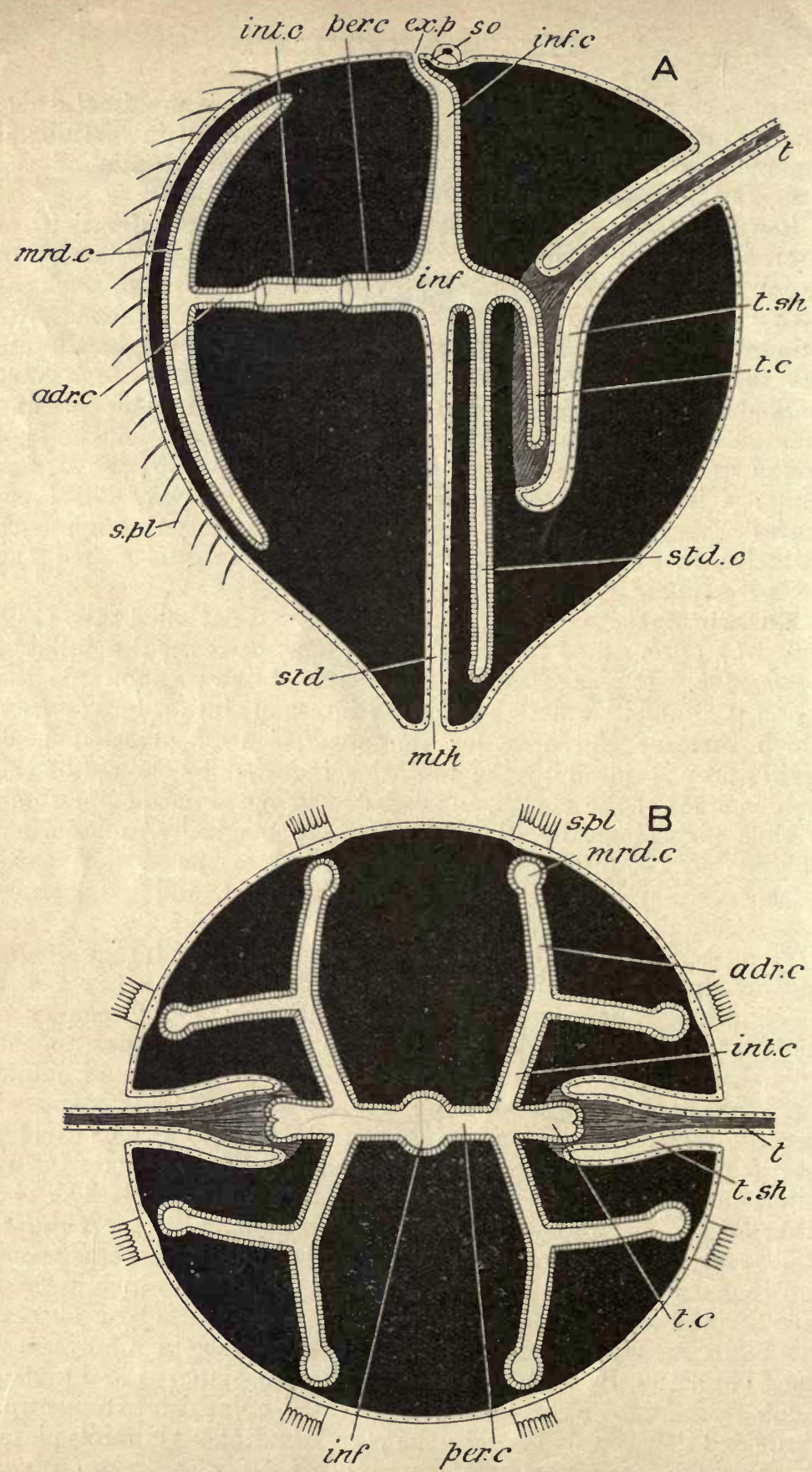

F1G. 160.-Hormiphcra plumcsa, diagrammatic longitudinal (A) and transverse (B) sections. The ectoderm is dotted, the endoderm striated, the mesogloea black, and the muscular axis of the tentacles gray. Lettering as in Fig. 159, exeept ex. $p$. excretory pore. 
with the stomodæum, and a tentacular canal ( $t$. $c$.) which extends outwards and downwards into the base of the corresponding tentacle. Each tentacle presents a thickened base ( $t$. b.), closely attached to the wall of the sheath, and giving off a long flexible filament, beset with processes of two kinds-one simple and colourless, the other leaf-like, beset with branchlets, and of a yellow colour.

Cell-layers.-The body is covered externally by a delicate ectodermal epithelium (Fig. 160), the cells from which the combs arise being particularly large. The epithelium of the stomodæum is found by development to be ectodermal, that of the infundibulum and its canals endodermal : both are ciliated. The interval between the external ectoderm and the canal-system is filled by a soft jellylike mesoglœa. The tentacle-sheath is an invagination of the ecto-
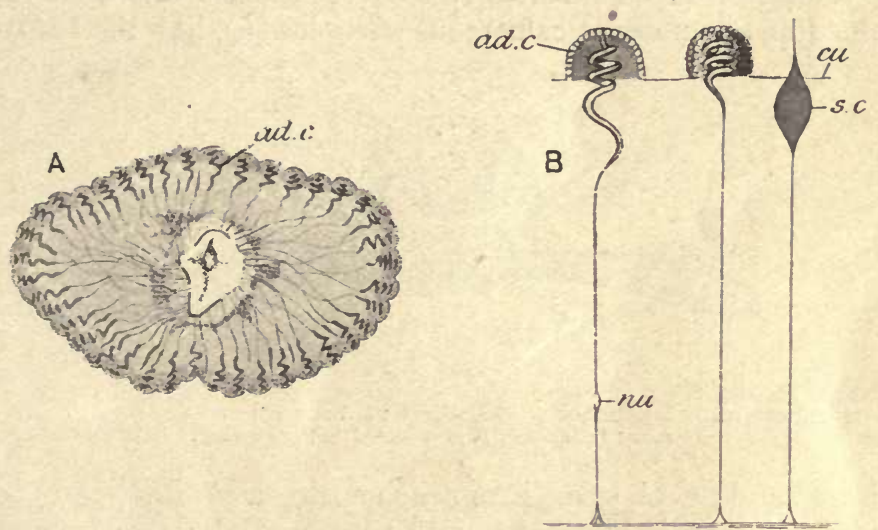

Fig. 161. Iormiphora plumosa. A, transverse section of one of the brauches of a tentacle ; B, two adhesive cells ( $a d . c_{\text {. }}$ ) and a sensory cell (s. c.) highly magnified. cu. cuticle ; nu. nucleus. (After Hertwig and Chun.)

derm, and the tentacle itself is covered by a layer of ectoderm, within which is a core or axis formed by a strong bundle of longitudinal muscular fibres, which, as we shall see, are of mesodermal origin, and which serve to retract the tentacle into its sheath.

Delicate muscle-fibres lie beneath the external epithelium and beneath the epithelium of the canal-system, and also traverse the mesogloea in various directions. The feeble development of the muscular system is, of course, correlated with the fact that the swimming-plates are the main organs of progression, the Ctenophora differing from all other Colenterata in retaining cilia as locomotory organs throughout life.

A further striking difference between our present type and the Coelenterata previously studied is the absence, in Hormiphora, of stinging-capsules. The place of these structures is taken by the peculiar adhesive-cells with which the branches of the tentacles 
are covered. An adhesive-cell (Fig. 161, ad.c.) has a convex surface, produced into small papillæ, which readily adheres to any object with which it comes in contact and is with difficulty separated. In the interior of the cell is a spirally coiled filament, the delicate inner end of which can be traced to the muscular axis of the tentacular branch. These spiral threads act as springs, and tend to prevent the adhesive-cells being torn away by the struggles of the captured prey.

Both the central nervous system and the principal senseorgan are represented by a peculiar apparatus situated, as already mentioned, at the aboral pole. In this region is a shallow depression (Fig. 162, c. ๆ.) lined by ciliated epithelium and produced in the transverse plane into two narrow ciliated areas, the polar plates $(p . p l$.$) . From the depression arise four equidistant groups$ of very large S-shaped cilia (sp.), united to form as many springs (sp.), which support a mass of calcareous particles $(l$.), like the lithites of

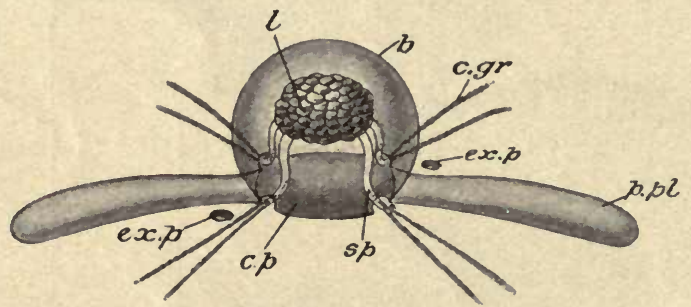

Fig. 162.-Formiphora plumosa, Sense-organ : $b$. bell ; $c . p$. ciliated plate; $c$. gr. ciliated groove; $e x . p$. cxcretory pore; $l$. lithites; $p . p l$. polar plate; $s p$. spring. (Modified from Cluun.)

Hydrozoa and Scyphozoa. From each spring a ciliatcd groove (c. gr). proceeds outwards, bifurcates, and passes to the two swimmingplates of the corresponding quadrant. The lithitic mass, with its springs, is enclosed in a transparent case or bell (b.), formed of coalesced cilia. It appears that the whole apparatus acts as a kind of steering-gear, or apparatus for the maintenance of equilibrium. Any inclination of the long axis must cause the calcareous mass to bear more heavily upon one or other of the springs: the stimulus appears to be transmitted by the corresponding ciliated groove to a swimming-plate, and results in a vigorous movement of the combs. Thus the sensory pit acts as a central nervous system, and the ciliated grooves as nerves. A sub-epithelial plexus of nerve-fibres with nerve-cells extends all over the surface of the body.

Reproductive Organs.-The animal is hermaphrodite, the organs of both sexes being found in the same individual. The gonads are developed in the meridional canals (Fig. 159, B), each of which has an ovary (ovy.) extending along the whole length of one side, a spermary (spy.) along the whole length of the opposite side. 
The organs are so arranged that in adjacent canals those of the same sex face one another. It will be seen that the reproductive products have, as in Scyphozoa and Actinozoa, the position of endoderm-cells: whether they are developed, in the first instance, from that layer is uncertain. When ripe, the ova and sperms are discharged into the canals, make their way to the infundibulum, thence to the stomodæum, and finally escape by the mouth. Impregnation takes place in the water.

Development. - The process of development has been traced in several genera closely allied to Hormiphora, so that there is every reason to believe that, in all essential particulars, the following description will apply to that genus.

The egg (Fig. 163) consists of an outer layer of protoplasm ( $p l s m$. containing the nucleus ( $m u$.), and of an internal mass of a frothy or vacuolated nature $(y k)$ : the vacuoles contain a homogeneous substance which serves as a store of nutriment to the growing embryo, and apparently corresponds with the yolk which we shall find to occur in a large proportion of animal eggs. Enclosing the egg is a thin vitelline membrane (v.m.), separated from the protoplasm by a considerable space, filled with a clear jelly.

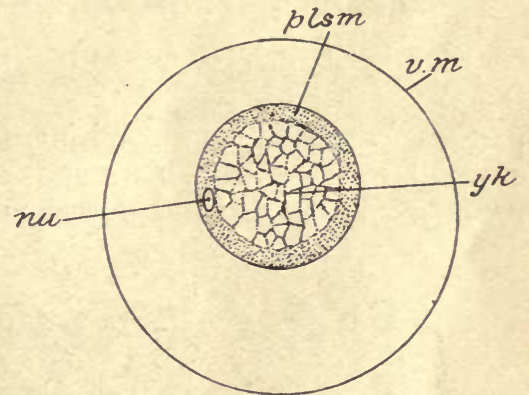

FiG. 163.-Ovum of Iampetia nu. nuelens; $p l s m$. protoplasm; $v$. $m$. vitelline membrane; yl. jolk. (After Chum.)

After impregnation the oosperm segments, but the details of the process are very different from those we are familiar with in the other Cœlenterata. The protoplasmic layer accumulates on the side which will become dorsal, and the oosperm divides along a vertical plane, forming two cells each with a sort of protoplasmic cap (Fig. 164, A, p/sm.). A second division takes place at right angles to the first, producing a four-celled stage (B), and each of the four cells divides again into daughter-cells of unequal size, the result being an eight-celled embryo, each cell with a protoplasmic cap at its dorsal end (C, D). Next a horizontal division takes place, dividing off the protoplasmic caps as distinct cells, and so producing a sixteen-celled stage $(\mathrm{E}, \mathrm{F})$ in which we can distinguish eight large, ventral, yolk-containing cells or megameres (mg.), and eight small, dorsal, protoplasmic cells or micromeres (mi.).

The micromeres increase rapidly in number by division, and are further added to by new, small cells being budded off from the megameres (Fig. 164, G, H, and Fig. 165, А). The result of this increase is that the micromeres gradually overspread the megameres 
(Fig. 165, C), the final result being the production of an embryo consisting of a central mass of large yolk-containing cells (ma.),
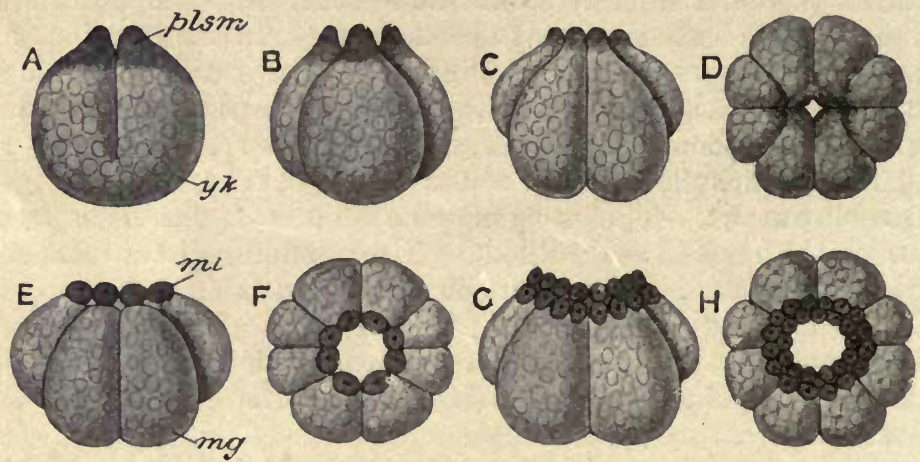

Fig. 164.-Segmentation of the oosporm in Ctenophura. mg. megameres; mi. mieromeres ; plsm. protoplasm; $y k$. yolk. (Modified from Korschelt and Heider.)

partly surrounded by an epithelium-like layer, incomplete below, of small cells $(m i$.$) . This stage corresponds with the gastrula of$ preceding types, the micromeres forming the ectoderm, the mega-
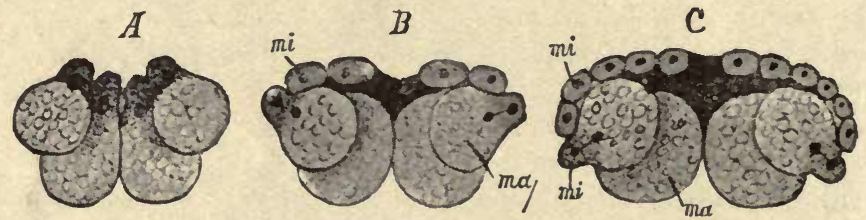

Fra. 165.-Three stages in the development of Ctenophora. ma. megameres; mi. micromeres. (From Lang's Comparatice Anutomy.)

meres the endoderm, and the ventral edge of the ectodermal investment representing the blastopore. There is, however, no archenteron or gastrula-cavity, and the stage has been produced,
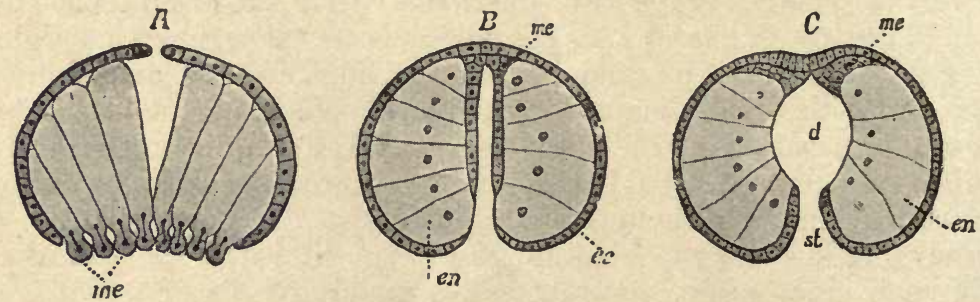

FrG. 166. - Three stages in the development of Callianixa. $d$. infundibulum; ec. ectoderm; en. endoderı ; me. mesoderm ; st. stomodsum. (From Ling's Comparative Anatomy.)

not by a process of invagination or tucking-in, but by one of epiboly or overgrowth.

The endoderm-cells increase in number, and become much elongated and arranged obliquely, their long axes radiating, 
upwards and outwards, from the long axis of the entire embryo (Fig. 166, A). Their lower (ventral) ends then become divided off, forming a number of small cells, which constitute the rudiment of a true middle cell-layer or mesoderm (A, me.). A kind of invagination of the megameres with their mesoderm cells then takes place, resulting in the formation of a cavity - the infundibulum $(\mathrm{B}, \mathrm{d}$.) - bounded below by the megameres, now placed horizontally, and above by the mesoderm. The mesoderm gradually retreats to the dorsal surface (C), finally spreading out between the dorsal ectoderm and the infundibulum. At the same time the ectoderm cells bounding the aperture of the infundibulum grow into it so as to line its ventral portion : in this way the stomodrum (st.) is produced. The remainder of the cavity widens out and becomes the definite infundibulum (d.), and before long sends off four adradial pouches, the rudiments of the canal-system. At the same time a gelatinous layer (Fig. 167,g.), the mesoglœa, makes its appearance between the ectoderm and endoderm.

The later processes of development may be described very briefly. The canal-system gradually assumes its adult complexity and the swimming-plates appear. A thickening of the ectoderm on each side of the body gives rise to the epithelium of the tentacle and of its pouch. The inuscle-fibres forming the axis of the tentacle $\left(\mathrm{B}, \mathrm{me}_{\mathrm{.}}\right)$ are derived from the mesoderm, which also gives rise to the contractile fibres of the mesoglœa $\left(m e_{0_{1}}\right)$. The lithites are formed in the ectoderm-cells of the apical pole, but gradually make their way on to the free surface of the cells, and become supported on four groups of fused cilia. Four outer groups of cilia unite with one another to form the bell (sk.).

The most noteworthy points in this somewhat complex process of development are the following:-

1. The distinction between a purely protoplasmic part of the egg and a yolkcontaining portion. In the Hydrozoa and Actinozoa the yolk-material is small in amount and evenly distributed, the egg being described as alceithal or yolkless. In the present instance the yolk is at first accumulated in the centre of the egg, which is thus centrolecithal 
or mid-yolked, but soon the protoplasm accumulates at one end and the yolk at the opposite end of the developing embryo, producing a telolecithal or end-yolked condition.

2. The fact that segmentation is unequal, there being a distinction into large cells or megameres, containing yolk, and purely protoplasmic small cells or micromeres.

3. The formation of a peculiar type of gastrula by epiboly or overgrowth, the ectoderm cells (micromeres) growing over and partly enclosing the endoderm cells (megameres).

4. The presence, for the first time in the ascending animal series, of a true middle embryonic layer or mesoderm. In the other Cœlenterata, as well as in the Sponges, two embryonic layers only are formed, and the intermediate layer of the adult is formed by the comparatively late separation of muscle-cells and connective-tissue fibres either from ectoderm or endoderm. In the present case a definite layer of mesoderm cells becomes separated from the endoderm during the gastrula stage.

\section{Distinctive Characters and Classification.}

The Ctenophora are pelagic Colenterata in which the formation of colonies is entirely unknown. No indication of a polype-stage, so characteristic of the remaining Crelenterata, can be detected either in the adult or in the embryonic condition. Ciliary movement, instead of being a merely embryonic form of locomotion as in the preceding classes, is retained throughout life, the cilia being fused to form comb-like structures, which are arranged in eight meridional rows or swimming-plates. Tentacles, when present, are usually two in number, situated in opposite (right and left) per-radii, and retractile into pouches. The enteron communicates with the exterior by a large stomodæum which functions as the chief digestive cavity. From the enteron is given off a system of canals, the ultimate branches of which are adradial and have a meridional position, lying beneath the swimming-plates; a single axial canal is continued to the aboral pole, where it commonly opens by two excretory pores. There are no gastric filaments. The central nervous system is represented by a ciliated area on the aboral pole, and is connected with a single sensory organ, having the character of a peculiarly modified lithocyst. The gonads of both sexes are lodged in the same individual, the ovaries and testes being formed on opposite sides of the meridional canals. The oosperm undergoes unequal segmentation, the gastrula is formed by epiboly or overgrowth, and a definite mesoderm is established during the gastrula stage. There is no alternation of 
generations: but in some cases development is accompanied by a well-marked metamorphosis.

The Ctenophora are divisible into four orders as follows:-

\section{ORDER 1.-CYDIPPIDA.}

Ctenophora having two tentacles, retractile into sheaths, and unbranched meridional and stomodral vessels. The body is either circular in section or is slightly compressed in the transverse plane (Figs. 158 and 168).

\section{Order 2.-Lobata.}

Ctenophora having numerous non-retractile lateral tentacles contained in a groove: the bases of the two principal tentacles are also present, but have no sheaths. The stomodæal and meridional vessels unite with one another. The body is compressed in the transverse plane, and is produced into two large oral lobes or lappets and into four pointed processes or auricles (Fig. 169).

\section{Order 3.- Cestida.}

Ctenophora having a band-like form, owing to the extreme compression of the body in the vertical plane. The bases of the two principal tentacles are present, enclosed in sheaths, and there are also numerous lateral tentacles contained in a groove. Union or anastomosis of the meridional and stomodxal vessels takes place (Fig. 170).

\section{ORDER 4.-BEROÏDA.}

Ctenophora having no tentacles. The mouth is very wide, and the gullet occupies the greater part of the interior of the body. The meridional vessels are produced into a complex system of anastomosing branches (Fig. 171).

\section{Systematic Position of the Example.}

Hormiphora plumosa is a species of the genus Hormiphora, belonging to the family Pleurobrachiida and to the order Cydippida.

T'he presence of two tentacles, retractile into sheaths, and of unbranched meridional canals places it in the order Cydippida. In this order there are three families, amongst which the Plevi०obrachiidae are distinguished by the absence of any compression of the body, the transverse section being circular. The genus Hormiphora is distinguished by having a rounded body somewhat produced at the oral pole, and by the aperture of the tentacle- 
sheath being on a higher level than the funnel. In the species plumosa the stomodreal ridges are of a brown colour, and the leaflike branchlets of the tentacles yellow.

\section{General Organisation.}

Compared with the two former classes of Colenterates, the Hydrozoa and Actinozoa, the organisation of the Ctenophora is remarkably uniform. This is due to the fact that all the species are pelagic, none are colonial, and none form skeletons. Nevertheless a very great diversity of form is produced in virtue of differences in proportion and modifications of the tentacular and canal systems.

The Cydippida agree in all essential respeets with Hormiphora, the most important deviation from the type-form being the compression of the body in the transverse plane in some genera, e.g. Euchlora (Fig. 168, 2), the result being an

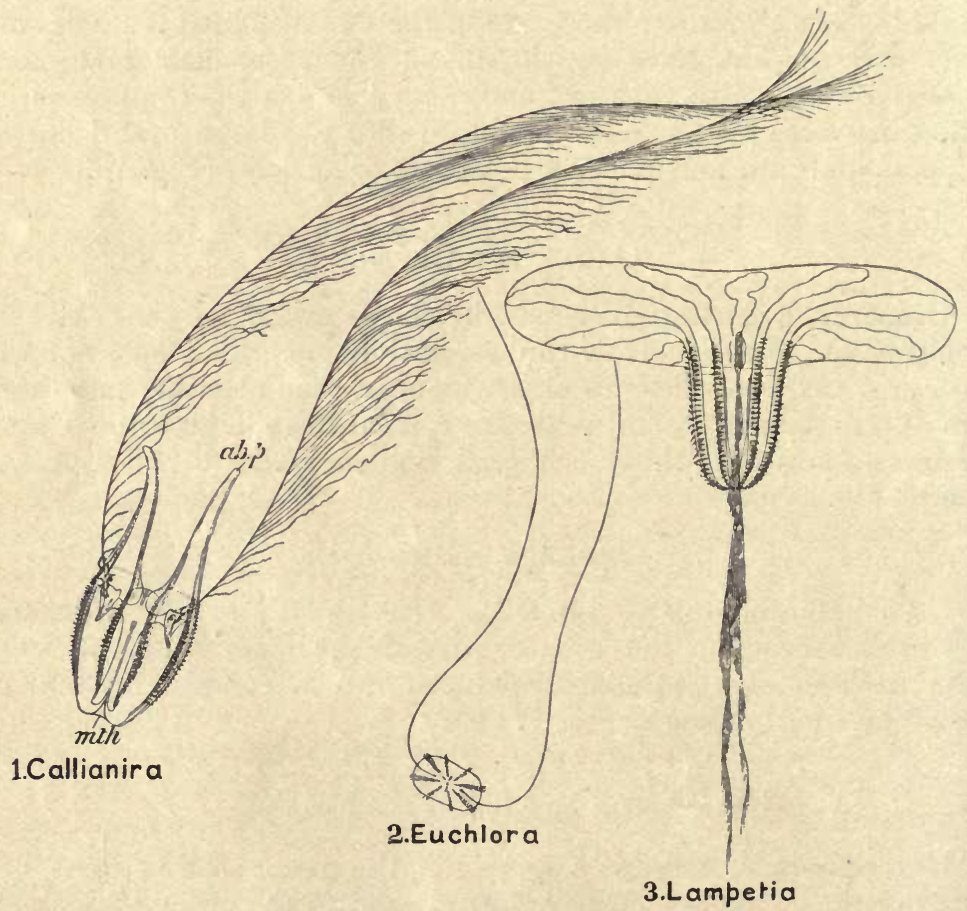

FIG. 168.-Three Cydippida. $a b . p$. aboral process; mth. mouth. (After Chun.)

oval instead of a circular transverse section, with the tentacles at the end of the long axis. The aboral pole may be produced into wing-like appendages, as in Callianira (1), and in Lampetia (3) the mouth is so dilatable as to form, when expanded, a sole-like plate by which the animal retains itself on the surface of the water or creeps over submarine objects. In Euchlora rubra minute nematocysts have been found, and there is reason to believe that it was by the modification 
of these characteristic cœlenterate organs of offence that the adhesive cells of Ctenophora were evolved.

The Lobata, for instance Deiopea, are distinguished, as their name implies, by the presence of a pair of large lappets (Fig. $169 \mathrm{~A}, \mathrm{lp}$. ), into which the oral

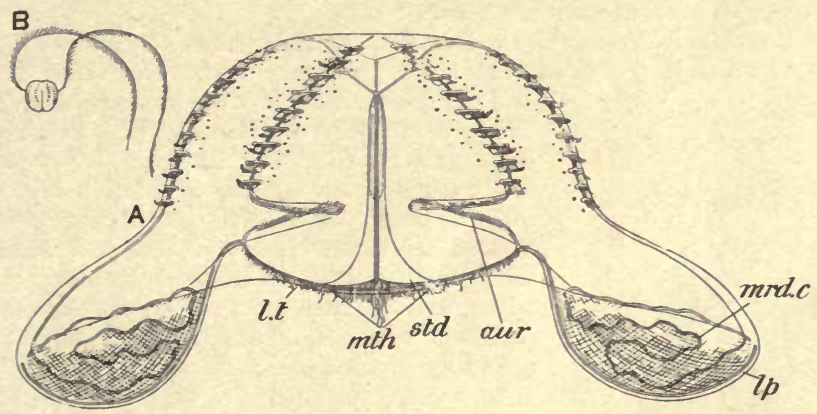

FIG. 169.-Deiopea kaloknenota. A, adult; B, young. aur. auricle; $l p$. lappet ; $l$. $t$. lateral teutacles; mrd. c. meridional canal ; mth. mouth. (After Chun.)

surface is produced at either end of the vertical plane. Four of the swimming plates are shorter than the others, and at their bases arise elongated processes called auricles (aur.), which bear swimming-plates. The meridional canals (mdr.c) unite with one another, and, with the osophageal canals, are continued into the lappets, where they become curiously coiled. The principal tentacles are usually absent in the adult, but are represented by their basal portions, which are small, situated at the oral end, and devoid of sheaths. From each tentaclebase grooves are continued aloug the oral surface to the auricles, and from the grooves depend numerous small lateral tentacles (l.t.). In the young condition the Lobata resemble such compressed Cydippida as Euchlora, having a pair of long principal tentacles, no lappets, and unbranched ressels (B).

The Cestida are represented by the remarkable "Venus's Girdle" (Cestus veneris), a band-shaped Ctenophore (Fig. 170) which sometimes attains a length

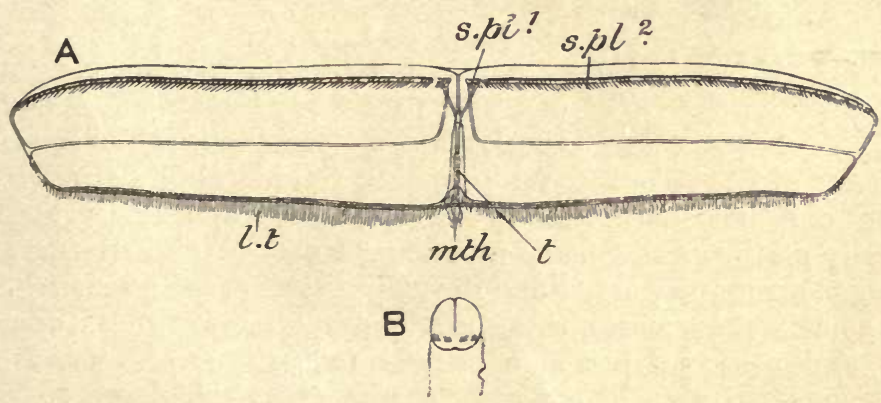

Fia. 170.- Cestus veneris. A, adılt; B, joung. $l$. $t$. lateral teutacles; mth. mouth; s. pl.1, 8. $p l^{2}$ swimming-plates; $t$. tentacle. (After Chum.)

of $1 \frac{1}{2}$ metre, or nearly five feet. The body is greatly elongated horizontally in the vertical, and compressed in the transverse plane, so as to have the form of a ribbon, which progresses by undulations of the whole body as well as by the action of its swimming-plates. Four of the swimming-plates $\left(s \cdot p^{1} .^{1}\right)$ are very 
small ; the other four $\left(s . p l .^{2}\right)$ are continued all along the aboral edge of the body. The bases of the two principal tentacles ( $t$. ) are large and are enclosed in sheaths, and, as in Lobata, numerous small lateral tentacles (l.t.) spring from grooves which, in the present case, are continued the whole length of the oral edge. The young of Cestus (B) resembles a compressed Cydippid which undergoes gradual elongation in the median plane.

Berö, the prineipal genus of the Beroïda, has the form of a cylinder (Fig. 171), one end of which is rounded and bears the sense-organ, the other truncated

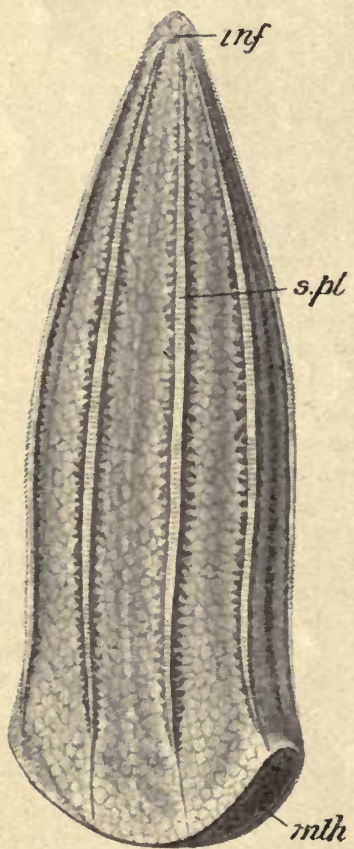

FIG. 171.-Bercë fors kalii. inf. infundibulum; mth. mouth; 8. pl. swimming-plates. (After Chun.)

diameter of $25-40 \mathrm{~mm}$. and occupied entirely by the immense mouth $(m t h$.$) .$ The greater part of the body is taken up by the huge gullet; the infundibulum (inf.), per-radial and infundibular canals, \&c., all being crowded into a small space at the aboral pole. The meridional canals send off branches which unite with one another, forming a complex network of tubes, and at their oral ends the four meridional canals of each (right and left) side and the corresponding stomodxal canal unite into a horizontal tube, which runs parallel with the margin of the mouth. There is no trace of tentacles either in the adult or in the embryonic condition.

The Ctenophora are usually perfectly transparent, and quite colourless, save for delicate tints of red, brown, or yellow in the tentacles and stomodæal ridges. Cestus has, however, a delicate violet hue, and when irritated shows a beautiful blue or bluish-green fluorescence. Beroë is coloured rose-pink.

Ctenophora are found in all seas from the Arctic regions to the tropics. As is to be expected from their perishable nature, there is no trace of the group in the fossil state.

A very remarkable fact has been made out with regard to Bolina hydatina, one of the Lobata, a Ctenophore which attains a dition and not more than $0.5-2 \mathrm{~mm}$. in diameter, it becomes sexually mature, the gonads producing ripe ova and sperms; and the eggs are impregnated and develop in the usual manner. Soon the gonads degenerate, the larva metamorphoses into the adult form, and a second period of sexual maturity supervenes. This precocious ripening of sex-ceils, occurs as we shall see in other animal groups, and is called predogenesis. 


\title{
APPENDIX TO CTENOPHORA
}

\author{
Ctexoplana and Coloplaxa.
}

Before leaving the Ctenophora mention must be made of two remarkable organisms which have been supposed to connect the present class with the Turbellaria Polycladida, or Planarians, a group of worms to be described in the following section.

Ctenoplana (Fig. 172) is a small marine animal, nearly circular in outline, flattened dorso-ventrally, and abont $6 \mathrm{~mm}$. in diameter. It has hitherto been
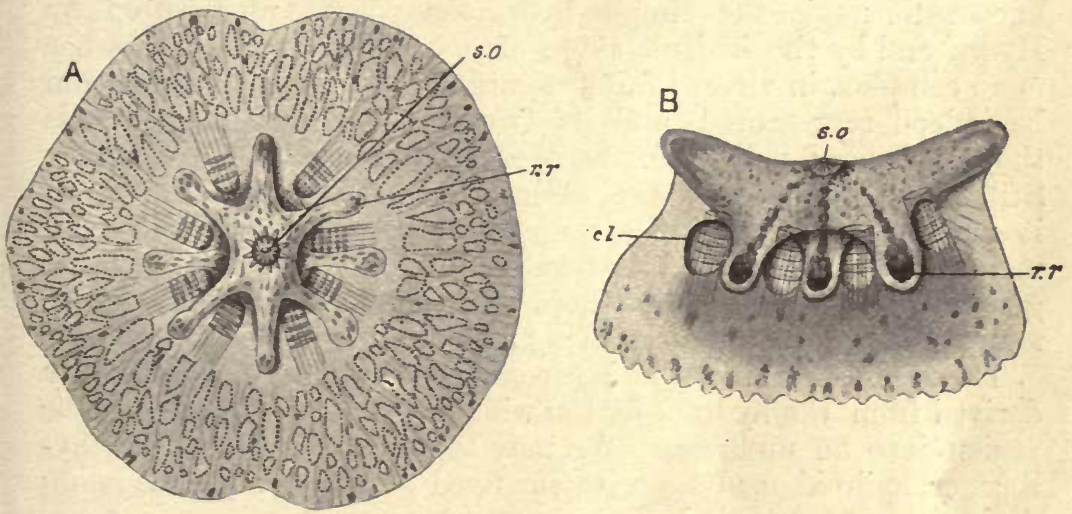

Fif. 172.-Ctenoplana kowalevskii. A, from above; B, froin the side. cl. clefts; $r$. $r$. radiating ridges; s. o. sense-organ. (After Korotneff.)

found only twice-once in the Indian Ocean and once in New Britain. Instead of swimming freely, like a Ctenophoran, it crecps on its ventral surface, like a worm. In the centre of the dorsal surface is a vesicle (s.o.) containing a mass of lithites surrounded by eight radiating ridges $(r . r$.$) , alternating with which$ are as many clefts $(c l$.$) , each containing a protrusible row of stiff processes,$ resembling the swimming-plates of Ctenophora. The mouth is in the centre of the ventral surface, and leads into a stomach, from which are given off numerous anastomosing canals, as well as a vertical canal which passes upwards and ends beneath the sense-organ. In diverticula of this system are found the testes, which have independent ducts opening on the exterior. There are two solid tentacles contained in sacs, and a nerve-centre lies beneath the sense-organ (s.o.). Beneath the ectoderm is a basement-membrane, which acts as an organ of support, and the muscular system is complex. Near each tentacle is an aperture leading into a branched canal which is probably excretory, like the nephridial tubes of Flat-Worms. (See Section V.)

Coloplana is found in the Red Sea. It is also flattened dorso-ventrally, but is oval instead of circular in outline, its dimensions being about 6 by $4 \mathrm{~mm}$. It resembles Ctenoplana in its ventral mouth, dorsal sense-organ, paired retractile tentacles, and complex system of anastomosing canals from the stomach. There are, however, no swimming-plates, and the ectoderm is ciliated.

Nothing is known of the development of either genus. 
Ctenoplana and Coloplana are perhaps best looked upon as forming an additional, somewhat aberrant, order of the Ctenophora, viz.-

\section{Order 5.-Plat'TCTENea.}

Flattened Ctenophora of creeping habit, with a pair of retractile lateral tentacles. The costæ (swimming-plates), when present, are retractile. There are no meridional canals, but a system of anastomosing peripheral vessels.

\section{-THE RELATIONSHIPS OF THE CELENTERATA.}

There can be little doubt that the lowest cœlenterate form known to us is the simple hydrozoan polype, represented by Hydra and by the hydrula stage of many Hydrozoa. Somewhat more complex, in virtue of its stomodæum (if a true stomodæum be indeed represented) and its gastric ridges and filaments, is the scyphozoan polype, represented by the scyphula of Aurelia. Still more complex is the actinozoan polype, or actinula, as it may be called, with its large stomodxum, mesenteries and mesenteric filaments, and elaborate muscular system. Speaking generally, one may say that these three polype-forms represent as many grades of organisation along a single line of descent.

The medusa-form in the Hydrozoa is, as we have seen, readily derived from the hydrula by the widening out of the tentacular region into an umbrella. We may thus conceive of the Trachylinæ, or hydroid medusæ with no fixed zoophyte stage, as being derived from a pelagic hydrula.

The Leptolinæ may be considered to have arisen in consequence of the adoption of asexual multiplication, by budding, during the larval or hydrula stage. Instead of the hydrula giving rise directly to a medusa, we may suppose it to have formed a temporary colony by budding, after the manner of the Hydra, the individual zooids being ultimately set free as medusæ. The next stage would be the establisment of a division of labour, in virtue of which a certain proportion only of the zooids became medusæ, the rest retaining the polype-form, remaining permanently attached, and serving for the nourishment of the asexual colony.

The Hydrocorallina appear to be a special development of the leptoline stock, the nearest affinities of the order being with such forms as Hydractinia.

The Siphonophora may be conceived as having originated from a hydrula specially modified for pelagic life by the conversion of the basic disc into a float-something after the fashion of Minyas (Fig. 152). In such a form extensive budding, accompanied by division of labour, would give rise to the complex siphonophoran colony.

The lowest Scyphozoa are the Lucernarida, some of which, 
however, show evidence of degeneration, so that it is quite possible to conceive them as having been derived from more highly organised forms, instead of springing directly from simple polypes of the scyphula type. The Semostomæ, Cubomedusæ, and Rhizostomæ clearly represent three grades of increasing complexity along the same general line of descent, the Coronata diverging somewhat. It is to be noted, however, that such a supposed line does not lead towards the simpler Actinozoa, but towards a type which diverges from the latter-as well as from the Lucernarida, Cubomedusæ and Peromedusæ-in the absence of septa or mesenteries in the adult condition.

The close similarity of Edwardsia and the Alcyonaria in the number and arrangement of the mesenteries seems to indicate the derivation of both Zoantharia and Alcyonaria from a common ancestor in the form of a simple actinozoan polype or actinula. Edwardsia clearly leads us to the Hexactiniæ or typical Seaanemones, and the Madreporaria are undoubtedly to be looked upon as skeleton-forming Hexactiniæ.

The relationships of the Ctenophora to the other Cœlenterata are very doubtful. Ctenaria, one of the Anthomedusa (Fig. 109, 1), presents some remarkable resemblances to a Cydippid, such as Hormiphora. It has two tentacles, situated in opposite per-radii, and each having at its base a deep pouch in the umbrella resembling the sheath of Hormiphora. There are eight radial canals formed by the bifurcation of four inter-radial offshoots of the stomach, and corresponding with them are eight bands of nematocysts diverging from the apex of the ex-umbrella. If these striking reseriblances indicate true homologies, we must compare the whole sub-umbrellar cavity of Ctenaria with the stomodæum of Hormiphora, the margin of the bell of Ctenaria with the mouth of Hormiphora, and the mouth of Ctenaria with the aperture between the stomodæum and the infundibulum of Hormiphora. But, as we have seen, the gullet of Ctenophora is a true stomodæum developed as an in-pushing of the oral ectoderm, and has therefore a totally different origin from the sub-umbrella of a medusa. Moreover, the tentacles of Ctenaria have no muscular base contained in the sheath, but spring from the margin of the umbrella as in other Hydrozoa: its gonads are developed in the manubrium, not in the radial canals, and there is no trace of an aboral sense-organ.

Of Hydroctena, which has also been supposed to afford us a connecting link between the Hydrozoa and the Ctenophora, almost the same inay be said. Hyclroctenc is bell-like, and provided with a velum. At its apex is an ampulla bearing two lithites supported on spring-like processes of the epithelium. From the apex of the gastric cavity a canal is given off which extends to the senseorgan, where it terminates blindly, and from the sides a pair of 
short canals, each of which terminates blindly at the base of the corresponding tentacular sheath. Only two tentacles are present, with sheaths at their bases: these are situated, not on the margin of the bell, as in a medusæ, but between it and the apex. There are no traces of swimming plates, and, so far as the evidence at present forthcoming goes, there is not sufficient evidence to establish Ctenophoran affinities.

On the other hand, the resemblance between transverse sections of an embryo Ctenophore (Fig. 173, B) and of an embryo Actinian
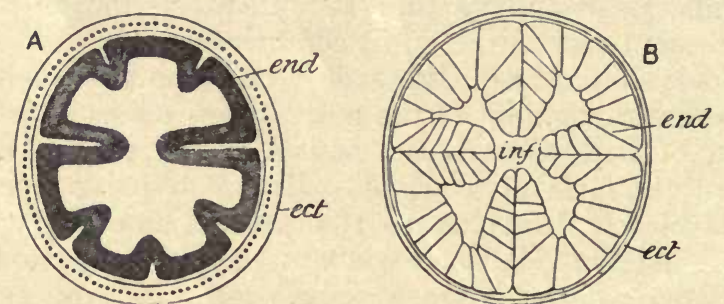

Fig. 173.-Transverse seetion of embryos of Actinia (A) and Beroe (B), ect. ectoderm ; end. endoderm; inf. infundibulum. (After Chun.)

(A) is very striking, and the presence of a well-developed stomodæum, and of gonads developed in connection with the endoderm and discharging their products through the mouth, may be taken as further evidences of affinity between the Ctenophora and the Actinozoa.

The special characteristics of the Ctenophora are, however, so numerous and so striking, and their development so utterly unlike that of any of the other Colenterata, that in our present state of knowledge it is impossible to determine their affinity with the other classes with any degree of certainty.

As to the orders of Ctenophora, it seems tolerably clear that both Lobata and Cestida are derived from cydippid forms, since they both pass through, in the course of development, a stage closely resembling the lower Cydippida. The Beroïda are more highly organised in certain respects, e.g. in the details of their histology, than the other Ctenophora, and it seems quite possible that they may be derived from tentaculate forms. Whether the Platyctenea are primitive or specially modified, remains doubtful, especially in the absence of data regarding their development; but the latier appears the more probable conclusion.

These relationships are expressed in the diagram on the opposite page.

By many authors the Sponges have been looked upon as so closely related to the Coelenterata that they may be regarded as members of the same great phylum. The points of resemblance are readily to be recognised: the simple structure, with the large central cavity into which a wide opening - the mouth or the osculum, 
as the case may be-leads ; the absence of a well-developed mesoderm, the fixed mode of life, and associated with it, the tendency to form compound structures or colonies by a process of budding. In addition, the occurrence of larval stages which have at least a superficial correspondence in the liwo phyla would appear to constitute an important connecting link. But a closer examination of the subject shows that some of these apparent points of resemblance are superficial only, and establishes a number of differences between Sponges and Coelenterates too important to allow us to suppose that a close relationship exists. One of these differences stands out beyond the others as the most radical. The osculum of a sponge is found, when we trace the development of

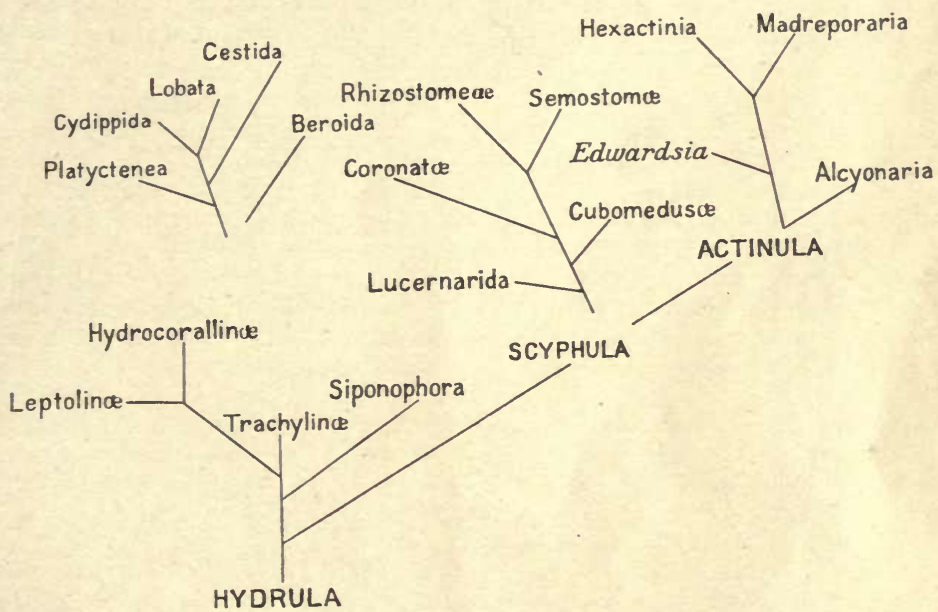

F1G. 174.-Diagram illustrating the mutual relationships of the Coelenterata.

the larva, to correspond in no sense with the mouth of the Colenterate. This alone, apart from important differences in the adult structure, such as the presence in the wall of the Sponge of the system of inhalant apertures, the presence of the peculiar collared endoderm cells, and the absence of stinging capsules, would suffice to remove the Sponges from the Colenterata, and place them in a phylum apart. But not only is the grouping of Sponges and Coelenterates in one phylum thus rendered impossible by important differences in their structure and development; a comparison of the mode of formation of the embryonic layers in the two groups shows such radical dissimilarity that it is scarcely possible to find sufficient evidence for regarding them as having been derived from the same metazoan ancestors, and there is much to be said in favour of the view that they have originated separately from the Protozoa. 


\section{APPENDIX (II.) TO THE CGLENTERATA.}

\section{The MesozoA.}

Under the designation MEsozoA have been comprised certain lowly organised animal forms, formerly supposed to afford us something of the nature of a connecting link between the Protozoa and the Metazoa, but now more generally looked upon as degenerate members of the latter subdivision. It has been proposed to term them the Moruloidea, from the resemblance which they bear to the morula stage in embryonic development.

They are all multicellular, with an ectoderm composed of a single layer of cells ciliated in whole or in part, and an endoderm either composed of a single elongated cell or of several cells : a mesoglea is not represented. The Mesozoa comprise at least three families, the Dicyemida, the Heterocyemida, and the Orthonectida, all the members of which are internal parasites.

The Dicyemida are parasities in the kidneys of various Cuttle-fishes and Octopods (Cephalopoda). Dicyema (Fig. 175), the length of which is between

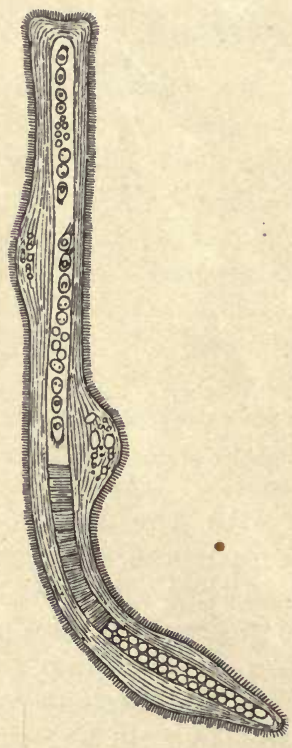

FiG. 175.-Dicyema paradoxum, with infusoriform embryos (males). (From Bronn's Thierreich, after Kölliker.)

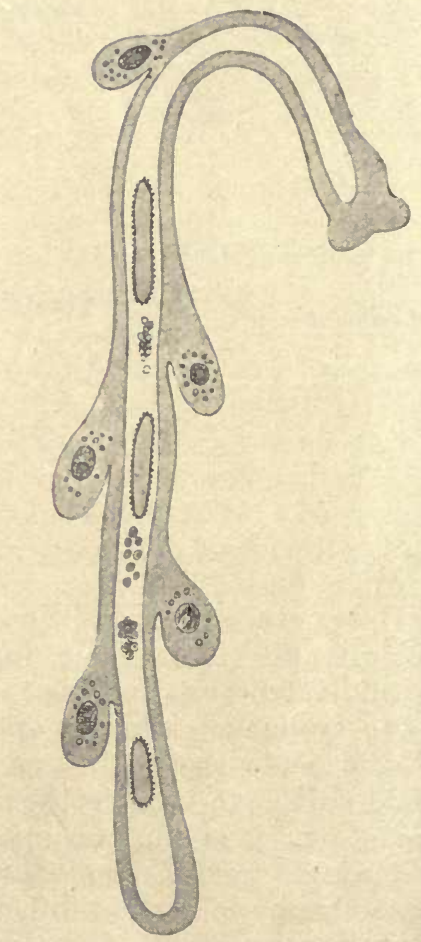

FIG. 176.-Dicyema paradoxum, with vermiform embryos. (From Bromn's Thierreich, after Kölliker.)

0.75 and 6 or 7 millimetres, consists of a head-part or calotte, and an elongated body. The form of the calotte varies a good deal, according to age; in young specimens it is isotropic (i.e. symmetrical around the long axis); in the adult 
condition ventral and dorsal sides are distinguishable. It consists of a swollen disc of four cells and a ring of four or five pole cells. The cells of the head all bear cilia, which are shorter and thicker than those of the body-cells.

The body consists of a single large axial cell, and of a single layer of outer cells which completely invest the axial cell. The outer cells which follow immediately on the head are distinguishable from the rest by their granular contents, and by their being dilated internally in such a way that the apex of the axial cell is constricted.

The axial cell is either almost completely cylindrical or spindle-shaped, and is covered in its entire extent by the outer cells. It presents a differentiated cortical layer, beneath which the finely granular gelatinous contents are at first homogeneous, but afterwards become vacuolated. In the middle of the cell is a large oval or ellipsoidal nucleus.

The life-history is a true alternation of generations. The primitive nucleus of the axial cell divides mitotically to form a smaller asexual germ-nucleus and a larger nucleus - the definitive nucleus (somatic nucleus) of the axial cell. Further germ-nuclei result from subsequent divisions. The germ-cells undergo a process similar to segmentation. Of the cells thus formed one gives rise to the axial cell of the embryo : the others, increasing in numbers and becoming smaller, gradually grow over the axial cell until they at length completely enclose it. The embryo increases greatly in length, and escapes from the interior of the parent, which it completely resembles, by perforating the bodywall. This phase of the parent animal (Fig. 176) is the phase to which the term nematogene is applied : the asexually developed young are the so-called vermiform embryos. The latter swim about for a time in the fluid of the kidney of the host; afterwards they attach themselves by means of the head to certain appendages - the venous appendages-of the walls of the cavity. A number of generations of these asexually developed forms succeed one another until, when the venous appendages of the kidney have become thickly infested with the parasites, a change takes place, and a sexual process of multiplication becomes initiated. In the interior of the nematogene a change is observable, and female sexual individuals are formed instead of vermiform embryos. Unlike the latter the former do not leave the body of the parent; they also differ in the nondevelopment of an enclosing layer. In their development, as in that of the vermiform embryos, the first nucleus of the axial cell of the parent divides into two. One of these becomes the permanent somatic nucleus of the axial cell : the other becomes the nucleus of the primitive ovum, and surrounds itself with protoplasm. This divides to give rise to a number of ova, which become more numerous till they come to fill the axial cell. Then the first generation of these ova are discharged into the protoplasm of the parent axial cell: this first generation of ova are derived from the cells representing the outer layer. Later further generations of ova, which are the descendants of the primitive ova, make their escape. These all eventually wander away into the protoplasm of the axial cell of the parent, increase in size, undergo a process of maturation, and become fertilised. Fertilisation is effected by means of typical tailed sperms developed in a second set of sexual individuals, the males (Fig. 177), which were formerly known as the infusoriform embryos. In its mature form the male is approximately pear-shaped, the narrower end being posterior. Several axial cells are present : these form the testes, in which the sperms are developed. They are surrounded by the outer cells, which at the posterior end take the form of a flat ciliated epithelium. The complete development of the sperms only takes place when the young male leaves the

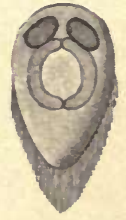

FiG. 177. - Male of Dicyema para. doxum. (From Broun's Thierreich, after Kölliker.)

host in which it was formed and seeks a new one; thus it is only by the sperms of a male from another host that the ova can be fertilised. The males 
are developed from the fertilised ova and subsequently escape, their development being similar to that of the vermiform embryos : the phase of the parent form to which they are developed is that known as the rhombogene. After a number of generations of males have been formed in this way, the rhombogene undergoes modification, and the last generation of fertilised ova gives rise, not to males, but to vermiform embryos-i.e., to an asexual generation-and with these the eycle begins anew.

The Heterocyemida, which are also parasites of the Cephalopoda, resemble the 1)icyemidre in most respeets, but the head is wanting.

The family Orthonectida comprises only two genera-Rhopalura and Stoecharthrum - which live as parasites in a Polyclad (Leptoplana), a Nemertine (Lineus), an Annelid, and a Brittle-Star (Amphiura). In the stage that represents the asexual form of the Dieyemidre the Orthonectid assumes the character of a plasmodium, or mass of finely granular protoplasm containing many nuclei, and is eapable of active amoboid movements. In the interior of the plasmodia the sexual forms are devcloped. A nucleus of the plasmodium surrounds itself "with protoplasin and gives rise to a germ-ecll,

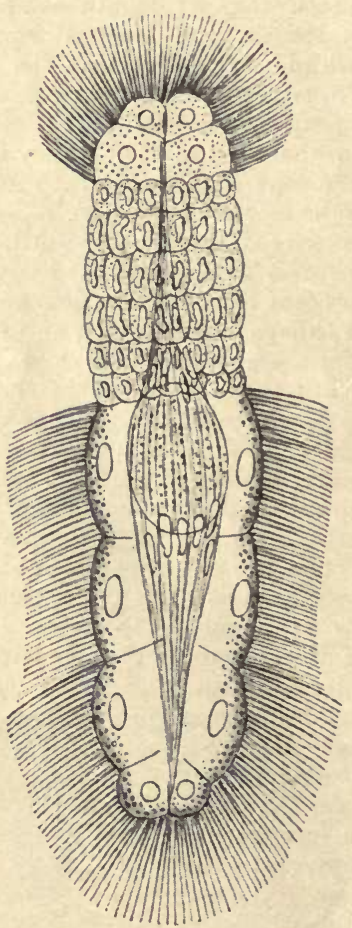

Fig. 178.-Rhopalura Giardii, male. (From Bronn's Thierreich, after Julin.)

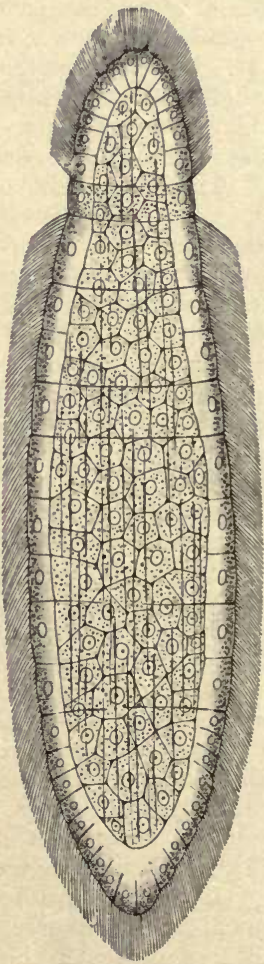

Fig. 179.-Rhopalura Giardii, female. (From Bronn's Thierreich, after Julin.

which by a process of segmentation develops into the sexual stage. In some cases only males are developed in one plasmodium and females in another : in others both sexes are formed together in the same plasmodium. In some forms the sexes are united. The sexual animals, especially the females (Fig. 179), 
bear a considerable resemblance to the Dicyemidæ, but instead of the axial cell there are a number of cells, the ova or sperm-cells. The outer cells are arranged in segments or rings. In front is usually a region composed of a few rings in which the outer cells bear cilia which are directed forwards : then comes a shorter region devoid of cilia, and behind that is the longest region, having cilia directed backwards. In shape the body is usually spindle-like-the males (Fig. 178) differing somewhat from the females. In about the middle of the internal space of the male is the compact oval testis containing small tailed sperms. Beneath the outer layer in the male, but not in the female, is a layer of fibres sometimes regarded as muscular. The plasmodia multiply by fragmentation. The development of the embryos either goes on in the intact plasmodium, or the latter breaks up and the embryos are to be found at various stages free in the host.

In the development of a male from the germ-cell the first segmentation is unequal. The further segmentation results in the formation of a solid morula. The outer cells become differentiated into two distinct groups, the one giving rise to the external layer of the anterior region, the other to that of the posterior region of the body. The inner cells multiply and give rise to the inmerous small spermatocytes of the testis. The formation of the layer of fibres only takes place later.

In the case of the female the segmentation appears to be equal from the first, and results in the formation of a blastula-like stage, which becomes converted into a solid morula-like body by the passing inwards of a number of cells. As in the male, the central cells multiply to form the sexual cells, and the outer cells form the external layer with its segments. In all probability, though this has not been actually proved, the mature sexual animals become free from the plasmodia, and the females, after fertilisation, find their way to another host where they become transformed into plasmodia, the germ-cells of which are the fertilised ova.

To be mentioned in connection with the Dicyemidæ and Orthonectidæ, as perhaps allied with them, are the remarkable parasites Amobophrya and Lohmanella - the former living in certain Radiolarians (Protozoa) the latter in the body-cavity of a Fritillaria (Urochorda). These both resemble the groups described above, and differ from the other Metazoa, in the presence of only a single body-layer. This remarkable simplicity of body-structure occurs also in Salinella, though too little is known with regard to this animal to provide adequate data for determining its affinities with certainty.

Salinella (Figs. 180 and 181), which has only been found on one occasion in water in which some salts from the Argentine Republic had been dissolved, is a

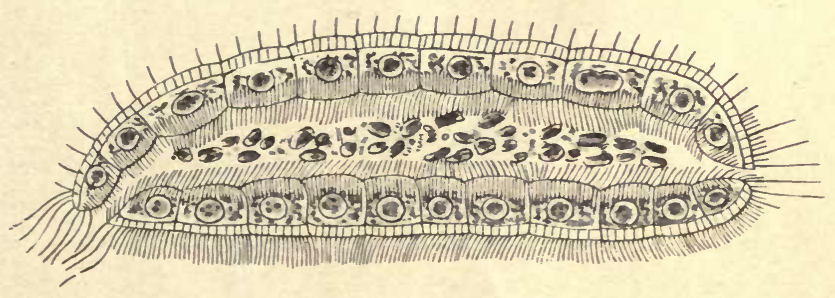

Fic. 180.-Salinella, longitudinal section. (After Frenzel.)

minute animal in the form of a somewhat depressed cylinder, open at both ends, and with a wall composed of a single layer of cells. The anterior end is somewhat pointed; around the anterior opening or mouth, which is ventrally directed, is a circlet of from fifteen to twenty long whip-like cilia. The posterior aperture (anus), which is usually closed, is surrounded by a few stiff setæ. The 
ventral surface is flattened, and is covered with fine vibratile cilia, while on the dorsal surface and the sides are regularly arranged rows of straight setæ (nonmotile cilia). The internal cavity (enteron) is found to contain sand, plant

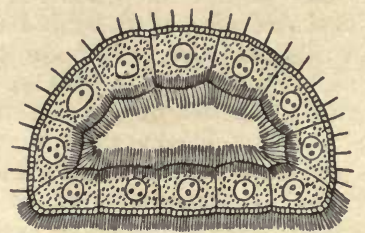

Fic. 181.-Salinella, transverse section. (After Frenzel.)

fragments, and Bacteria ; its sturface is beset with long cilia. Multiplication is said to take place by transverse fission; and a process of conjugation followed by encystation has also been observed.

Trichoplax and Treptoplax, which have been supposed to be Mesozoa, appear to be merely special modifications of developmental phases of Hydrozoa. 


\section{SECTION V}

\section{PHYLUM PLATYHELMINTHES}

A number of classes of Metazoa, some a little, others very decidedly, higher in organisation than the Coelenterata, were formerly regarded as constituting one great sub-kingdom or phylum-the Virmes or Worms. The groups ordinarily referred to the Vermes differ, however, very widely from one another: points of agreement, except such as are merely negative, are, in fact, frequently hardly recognisable: and rather than group together under one common designation such a heterogeneous assemblage of forms, it is usually considered to be more expedient to avoid the term Vermes altogether, and to endeavour to divide the "Worms" into phyla the members of which shall have points of positive resemblance to one another. The four phyla Platyhelminthes, Nemathelminthes, Trochelminthes, Molluscoida, and Annulata, with their appendices, all consist of forms which are or have been comprised in the Vermes. They differ from the Coelenterata in the presence of three well-developed body-layers - of which the middle one, or mesoderm, is of relatively predominant importance; and for the most part, in the much higher stage of complexity attained by the various systems of organs. The first four phyla present no metameric segmentation (p.43): in the Annulata, metamerism is more or less strongly pronounced.

The Platyhelminthes or Flat-Worms are a group of soft-bodied, bilateral, usually flattened animals, which are devoid of true metameric segmentation. With a sufficient degree of uniformity of structure to render the phylum a fairly compact and well-defined one, there is yet a considerable range in complexity, from the simplest forms - certain of which have been supposed to be nearly connected with the Ctenophora among the Coelenterata-to the highest, which have all the various systems of organs very much more highly developed. The body is built up from three embryonic layers-ectoderm, mesoderm, and endoderm - as in all higher groups of animals. An excretory vascular system of 
a peculiar kind-the vater-vascular or protonephridial system-is present in nearly all members of the phylum. A body-cavity (see following Sections) is not present, the spaces between the various organs and the wall of the body being filled up with a peculiar form of connective-tissue termed the parenchyma. The egg is in most instances composite, the egg-shell enclosing not only the oosperm or impregnated ovum, but a quantity of nutrient material or food-yolk, derived, in most instances, from a special set of glands, the yolk or vitelline glands.

The main features which distinguish the Platyhelminthes from the Coelenterata are-the pronounced bilateral symmetry with the many secondary features which it involves, the presence of a middle embryonic layer or mesoderm, and the non-occurrence of fixed colonies formed by budding.

\section{Examples of the Phylum.}

\section{i. A Fresh-water Triclad (Planaria or Dendrocolum). ${ }^{1}$}

General Features.-Species of fresh-water Planarians of the genera Planaria and Dendroccelum are common in the mud at the bottom of ponds of fresh-water in all quarters of the globe. They are small, thin, flattened worms a few millimetres in length, broader at one end, the anterior, than at the other, the posterior, which is more or less pointed. The animal (Figs. 182-184) is very readily recognised to be bilaterally symmetrical, with an upper or dorsal and a lower or ventral surface, right and left borders, and anterior and posterior ends. The colour varies in different species and in different individuals; but is usually gray, red, brown, or black. Movements of locomotion in the direction of the long axis of the body are recognisable in the living animal. Sometimes this is a steady gliding movement, which is brought about by the action of a coating of vibratile cilia on the surface; sometimes the worm moves along somewhat after the fashion of a Leech, the ventral surface of the anterior end of the body being of a sticky adhesive character, and performing the part of the anterior sucker of the Leech.

Close to the anterior extremity on the dorsal surface are two rounded black spots, the eyes (Fig. 183). On the ventral surface, a considerable distance behind the middle of the body, is the opening of the mouth (Fig. 182, mo.), and further back still, near the posterior pointed end, is a smaller median opening, the genital aperture (Fig. 184).

Digestive System.-The mouth (Fig. 183, mo.) leads through

1 The account is sufficiently general to apply to species of either of these genera. 
a short mouth-cavity into a cylindrical thick-walled chamber, the pharynx (ph.), which is highly mobile, and is capable of being thrust out as a proboscis through the mouth, beyond which it

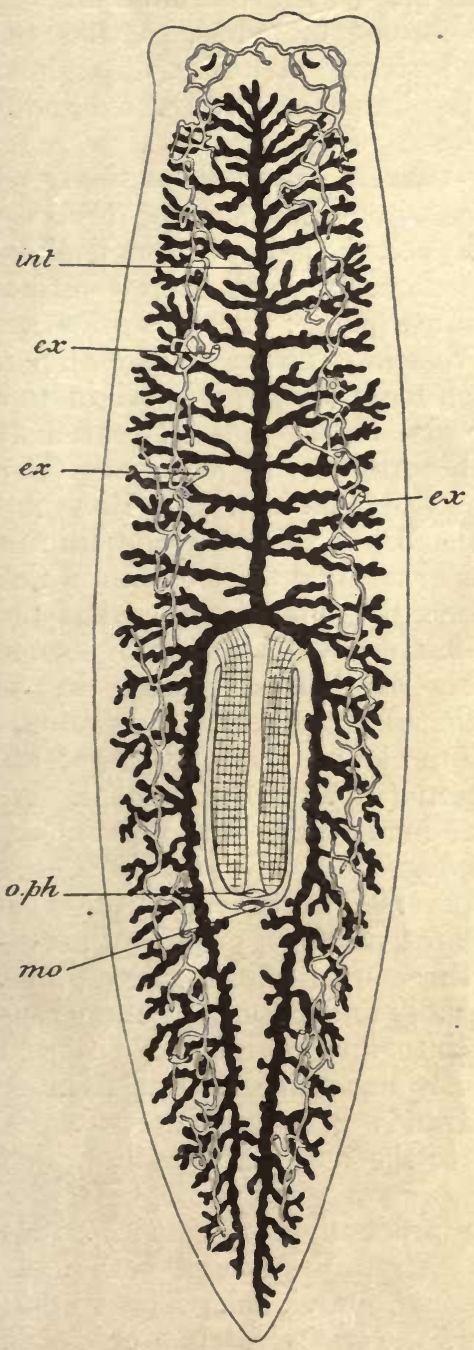

FIG. 182.-Planaria. Digestive and excretory systems. ex. openings of excretory system; int. intestine; mo. mouth; o. ph. opening of pharynx. (After Jijima and Hatschek.)

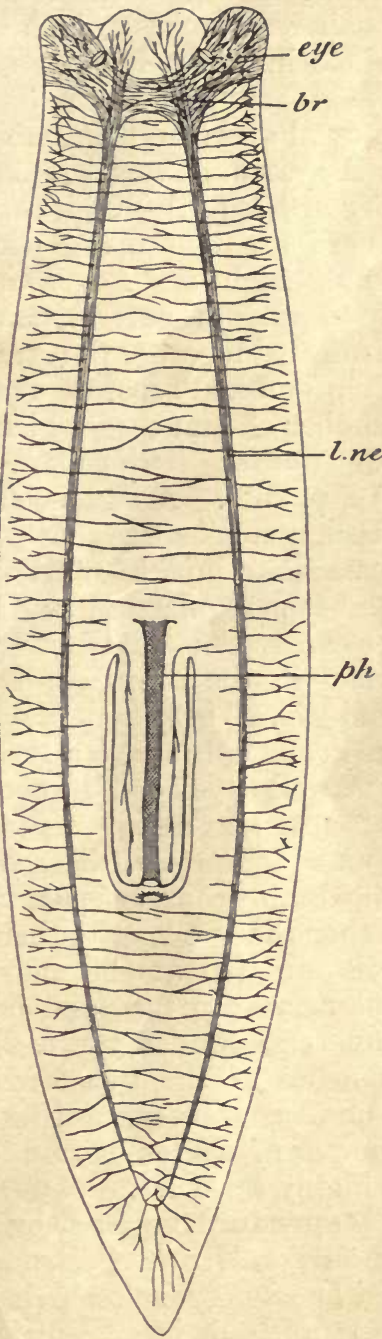

F16.183.-Planaria. Nervous system. n. brain ; exe, eye ; l. ne. longitudinal nerve; $p h$. pharynx. (After Jijima and Hatschek.)

may then be extended to a relatively considerable distance. When retracted it lies within an enclosing muscular sheath. The 
cavity of the pharnyx opens in front into the intestine (int.), which almost immediately divides into three narrow main branches, one running forward in the middle line, the other two running backwards. Each of these three main branches gives off numerous smaller branches, which in turn become branched, so that the whole intestine forms a ramifying system, extending throughout the greater part of the body; all the branches terminate blindly, an anal aperture being absent.

A system of vessels - the water-vessels or vessels of the excretory system (ex.)-sends ramifications through all parts of the body. There are two main, considerably coiled, pairs of longitudinal trunks, right and left, which open externally on the dorsal surface by means of several pairs of minute pores; in front they are connected together by a transverse vessel. The vessels of each pair often join and separate again. Each main trunk gives origin to a number of branches, which in turn give off a system of extremely fine capillary vessels, many of which terminate in flame-cells (Fig. 214 , p. 269). A flame-cell is a nucleated cell having in its protoplasm a small space into which one of the capillaries leads; in this space lies a bundle of ribratile cilia, or a single thick cilium, which performs regular undulating movements, giving it somewhat the appearance of a flickering candle-flame. Cilia are said also to occur in the course of some of the capillaries, though this is doubtful. This system of vessels is usually regarded as excretory; but it may also have a respiratory function.

A well-developed nervous system (Fig. 183) is present. At the anterior end is a central knot of nerve-matter, the orciin $(I . \gamma)$, from which proceed backwards two longitudinal ncr've-cords (l. ne.). The brain consists partly of transverse fibres connecting together the two longitudinal nerve-cords, partly of groups of nervecells situated at the ends, or in the course of, the nerve-fibres. The nerve-cords give off both internally and externally numerous transverse "anches, which divide into finer twigs; the internal branches of the two cords frequently anastomose, thus forming commissures or connecting nerve-strands between the two. A number of nerves extend forwards to the anterior margin, which is highly sensitive.

Reproductive System.-The reproductive organs (Fig. 184) are hermaphrodite or monocious in their arrangement, both male and female organs occurring in the same individual. The genital aperture leads into a small chamber, the genital atrium or cloaca, which is common to both the male and the female reproductive systems.

The male part of the apparatus consists of testes, rasa deferentia, and penis. The testes (tes.) are numerous rounded glands, situated near the right and left borders. Two ducts, the right and left vasa deferentia (v.d.), run backwards from the neighbourhood 
of the testes and unite in the middle line posteriorly. The median duct formed by the union of the two vasa deferentia traverses a protrusible muscular organ, the penis $(p)$, to open into the genital cloaca. At the base of the penis, where the vasa deferentia meet, the median canal is slightly enlarged to form a rounded dilatation, the vesicula seminalis. Into the mediain canal open the narrow ducts of a number of unicellular glands, the prostate glands $(p r)$.

The female part of the reproductive apparatus consists of ovaries (germaria), oviducts, vitelline glands, uterus, and muscular sac. The germaria (ov.) are two in number-small rounded bodies situated near the anterior end, each connected with an elongated duct, the oviduct. The two oviducts (od.) unite posteriorly to form a short median common oviduct opening into the genital atrium. With this cavity are connected also the uterus (ut.), a median rounded chamber, and a thick-walled muscular body, the muscular sac (m.). Numerous branching tubes - the vitelline glands (vit.)-open into the oviducts.

Reproduction is entirely sexual. The oosperm is enclosed within a protecting case or shell, which contains also a quantity of foodyolk derived from the vitelline glands. When the larva has reached a certain stage it develops a temporary larval mouth and gullet (see Fig. 218), and swallows the food-yolk, by the aid of which it grows rapidly.

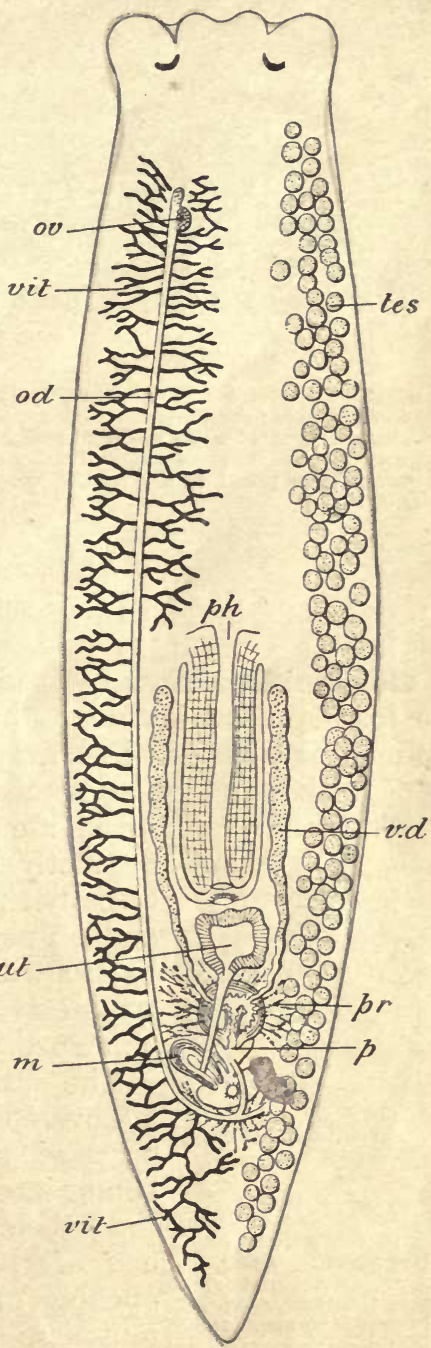

Fig. 184.-Planaria. Reproductive system. m. muscular sac; ov. germarium; $p h$. pharynx; $p$. penis ; $m$. prostate; tes. testes; $u t$. uterus; $v$. $d$. vas deferens ; rit. vitelline glanås. (After Jijima and Hatschek.)

The larval mouth disappears, and a new one-the permanent mouth-is developed in its place. When the embryo leaves the shell, it has assumed the characteristic shape of the parent. 


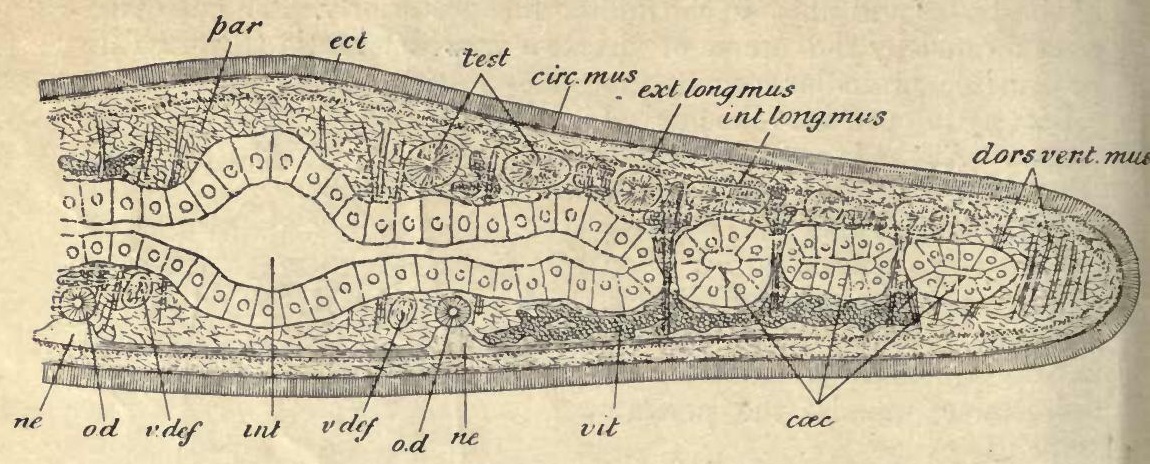

FIa. 185.-Transverse section of a Planarian. civc. mus. circular muscular fibres; coec. intestinal cæca; dors. vent. mus. dorso-ventral muscular fibres; ect. ectoderm ; ext. long. mus. external layer of longitudinal muscular fibres; int. central limen of the intestine; int. long. mus. internal layer of longitudinal muscular fibres; ne. nerve-cords; 0 . $d$. oviducts; pav. parcnchyma; test. testes; $v$. def. vas deferens; vit. vitelline glands. (From Hatschck's Lehrbuch.)

ii. The Liver-Fluke (Fasciola hepatica).

General Features.-The Liver-Fluke of the Sheep, which is to be found in the interior of the larger bile-ducts of the infested animal, is a soft-bodied worm of flattened leaf-like shape (Fig. 186), with a triangular process, the head-lobe, projecting from the

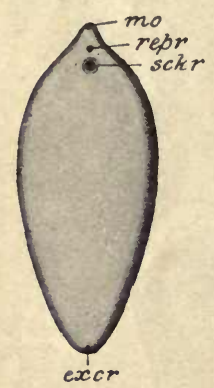

Fig. 186.-Fasciola hepatica, natural size. excr. excretory pore ; mo.mouth ; repr. reproductive aperture; $s c k \%$. posterior sucker.

lobe, is a second much larger posterior sucker (sckr.). Between the two suckers, but rather nearer the posterior one, is a median aperture, the genital opening (repr.), through which a curved muscular process, the cirrus or penis may be protruded. In the middle of the posterior end of the body is a minute opening, the excretory pore (excr.). 
Body-wall.-The body-wall (Fig. 187) is found on section to comprise three layers:-(1) a homogenous cuticle (cut.) of which the spinules (sp.) are special developments; (2) a layer of circularly disposed muscular fibres (circ. mus.); (3) a layer of longitúdinal muscular fibres (lomg. mus.). A cellular epidermis is wanting. Beneath the muscles are numerous unicellular glands ( $g l$.), the ducts of which, in the form of processes of the cells, open on the outer surface. Internally, the interspaces between the organs are

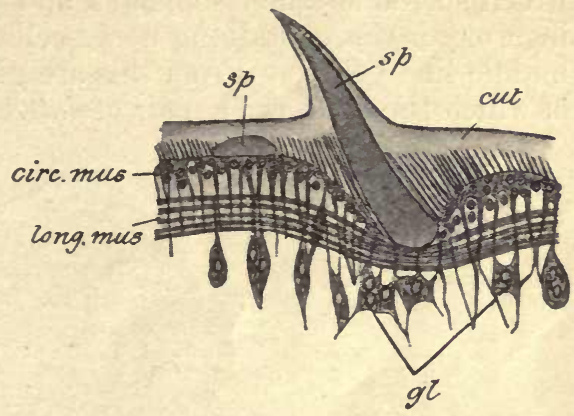

FIG. 187.- Fasciola hepatica. Section of the integument. circ. mus. layer of circular muscular fibres; cut. cuticle; gl. unicellular glands; long. mus. layer of longitudinal muscular fibres; $s p$. spinules. (After Braun.)

filled by a peculiar form of connective-tissue, the parenchyma.

Digestive System.-The mouth (Fig. 188) leads to a small rounded bulb-like body, the pharnyx (ph.), with thick muscular walls and a small cavity. From this a short passage, the oesophagus, opens into the intestine. The latter (int.) is frequently a very conspicuous structure, owing to its being filled with the dark biliary matter mixed with blood on which the Fluke feeds. It divides almost immediately into two main limbs, right and left, and from each of these are given off, both internally and externally, a number of blind branches or coca, those on the inner side being short and simple, while those on the outer side are longer and branched. The two limbs of the intestine with their branches thus form, as in the Planarian, a complicated system, the ramifications of which extend throughout the whole of the body. There is no aperture of communication between the intestine and the exterior, the only external opening of the alimentary system being through the mouth.

A branching system of vessels - the water-vessels or vessels of the excretory system-ramify throughout the body. A longitudinal main trunk opens behind by means of the excretory pore already mentioned as occurring at the posterior end. In front it gives off four large trunks, each of which branches repeatedly, the branches giving off smaller vessels, and these again still smaller twigs, until we reach a system of extremely fine microscopic vessels or capillaries. Each of these ends internally in a slight enlargement situated in the interior of a large cell, an excretory cell or flamecell, similar to a flame-cell of the Planarian.

The Liver-Fluke has a well differentiated nervous system, 
which shares in the prevailing bilateral arrangement of the parts. The central part of this system consists of a ring of nerve-matter which surrounds the osophagus and presents two lateral thickenings, or ganglia, containing nerve-cells, and a single ganglion in the middle line below. From this are given off a number of nerves, of which the chief are a pair of lateral cords running back to the

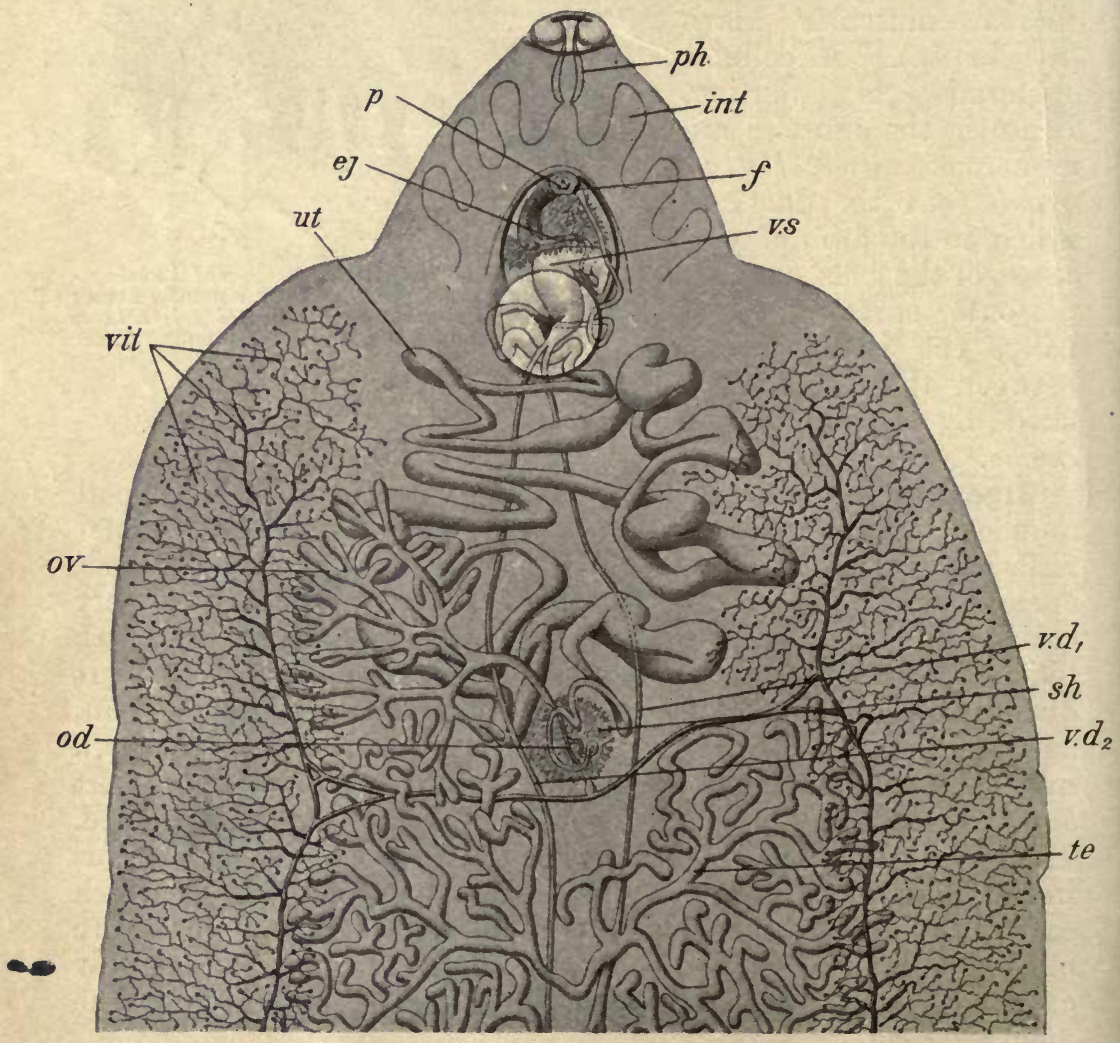

Fig. 188.-Fasciola hepatica. Internal organisation. General view of the anterior portion of the body, showing the various systems of organs as seen from the ventral aspect. $e j$. ejaculatory duct ; $f$. fernale reproductive aperture; int. anterior portion of the intestine (the rest is not shown); od. commencement of oviduct; ov ovary (germarium); $p$. cirrus; $p h$. pharynx; $s h$. shell-gland: te. testes; $u t$. uterus; $v d l$. left vas deferens; vel'. right vas deferens; vit. lobes of vitelline glands; $v 8$. vesicula seminalis. (After Sommer.)

posterior end and giving off numerous branches. There are no organs of special sense.

The reproductive organs (Fig. 188) are constructed on the hermaphrodite plan, i.e. both male and female organs occur in the same individual. The male part of the apparatus consists of testes, vasa deferentia, and cirrus. The testes (te.) are two greatly 
ramified tubes, which occupy the middle part of the body, one situated behind the other. From each testis there runs forwards a duct, the vas deferens, the two vasa deferentia (v.d.) opening anteriorly into an elongated sac, the vesicula seminalis (v.s.), from which a narrow tube-the ejaculatory duct (Fig. 189, ej.d.)-leads to the male aperture at the extremity of the cirrus. The female part of the reproductive apparatus consists of a single ovary (germarium), an oviduct, a uterus, an ootype, vitelline glands, vitelline ducts, and shell-glands. The germarium (Fig. 188, ov.) is a branched tube situated on the right-hand side in front of the testes; the branches open into a common narrow tube, the oviduct (od.). The vitelline glands (vit.) consist of very numerous, minute, rounded follicles, which occupy a considerable zone in the lateral regions of the body. On each side are two large ducts, anterior and posterior, uniting to form a single main lateral duct, right or left; and these run nearly transversely inwards to open into a

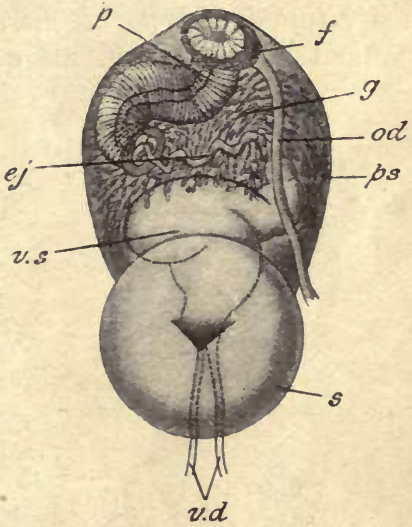

FIG. 189.-Fasciola hepatica. Terminal part of the reproductive apparatus. $e j$. cjaculatory duct; $f$. female aperture; $g$. uniccllular glands ; od. terminal part of oviduct ; p. cirrus; ps. cirrus-sheath ; 8 . sucker; $v$. $d$. vasa deferentia; $v$. $s$. vesicula seminalis. (After Sommer.) small sac, the yolk reservoir. From this a single median vitelline duct runs backwards for a short distance to join the oviduct. Around the junction are grouped a mass of unicellular shell-glands (sh. $g l$.$) , each of which is produced into a narrow process or duct$ opening into the end of the oviduct in the region of the latter to which the term ootype is applied. The uterus (ut.) is a wide convoluted tube, formed by the union of the oviduct and median vitelline duct; in front it opens close to the base of the cirrus. When the cirrus is withdrawn, a small cavity, the genital atrium or cloaca, is formed common to the external apertures of both male and female ducts. A canal, termed the canal of Laurer, leads from the junction of the oviduct and median vitelline duct to open externally on the dorsal surface.

Development.-Each ovum on impregnation becomes surrounded by a mass of vitelline matter or yolk derived from the yolk-glands. It then becomes enclosed, while passing through the ootype, in a chitinous shell, the substance of which is usually said to be derived from the shell-glands. ${ }^{1}$ The completed egg remains

1 The agency of the shell-glands in producing the material of the egg-shell is not universally acknowledged. This point will be referred to in the section dealing with the general organisation. 
for a little time in the uterus; eventually it is discharged, and, passing down the bile-ducts of the Sheep into the initestine, reaches the exterior with the fæces. Active development only begins at this stage, and, three to six weeks later, a portion of the egg-shell at one end becomes separated off as a sort of lid or operculum, and gives exit to the contained embryo. This, the ciliated embryo or miracidium (Fig. 190, A), is a somewhat conical body covered all over with vibratile cilia, and with two
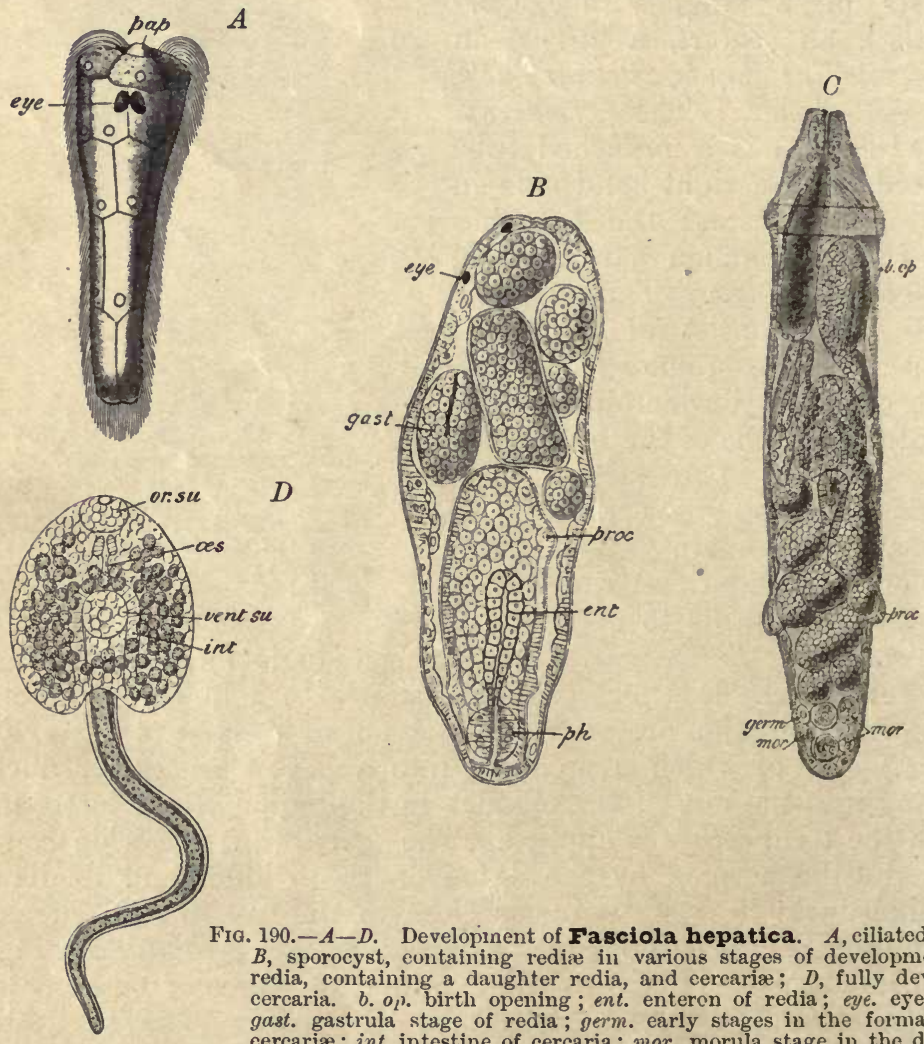

Fig. 190. $-A-D$. Developinent of Fasciola hepatica. $A$, ciliated larva ; $B$, sporocyst, euntaining rediæ in various stages of development; $C$, redia, containing a daughter redia, and cercariæ; $D$, fully developed cercaria. b. o $\%$. birth opening; ent. enteron of redia; eye. eje-spots ; gast. gastrula stage of redia; germ. early stages in the formation of cercaria ; int. intestine of cercaria; mor. morula stage in the development of eercariæ ; ces. œesophagus ; or. su. oral sucker; pap. head-papilla of ciliated embryo; ph. pharynx; proc. processes of redia; vent. su. ventral sucker. (After Thomas.)

spots of pigment, the eye-spots (eye), near the broader or anterior end, which is provided with a triangular head-lobe ( ap. .). There is an imperfectly developed intestine and a pair of flame-cells, each with a fine canal opening on the surface. The rest of the interior is filled with a mass of germ-cells. The ciliated larva swims about in water, or moves over damp herbage for a time, and perishes unless it happens to reach a Pond-snail, 
(Lymnaus), as a parasite of which it is alone able to enter upon the next phase in its life-history. When it meets with the Snail, the embryo bores into it by means of the head-lobe, coming to rest in the pulmonary sac or some other organ of the mollusc. Established in the interior of the Snail, it loses its ectoderm and grows rapidly into the form of an elongated sac, the sporocyst (Fig. $190, B)$, with an internal cavity containing germ-cells and lined by a layer of cells, with remnants of the eye-spots, and with flame-cells. The sporocyst may divide into two similar bodies by a process of transverse fission, but this is exceptional. Eventually cells are budded off from the layer that lines the internal cavity of the sporocyst or from the germ-cells, and these undergo a process of segmentation similar to the holoblastic segmentation of the impregnated ovum, resulting in the formation of a morula, which becomes converted into a stage resembling a gastrula. The gastrula elongates and gives rise to a body called a redia $(C)$, which begins to move about, and, eventually forcing its way out of the interior of the sporocyst, finds its way to some other part of the Snail, usually the liver. When fully formed, the redia is a cylindrical body with a pair of short processes (proc.) near the posterior end, and with a circular ridge near the anterior end. It possesses a mouth leading to a pharynx and simple sac-like intestine, and there is a system of excretory vessels. In the interior of the redia cells are budded off and develop into gastrulæ, exactly as in the case of the sporocyst; these gastrulæ either develop into a fresh generation of rediæ if the season should be winter, or, if it should be summer, give rise to bodies termed cercario. The latter $(D)$ are provided with long tails : they have anterior and posterior suckers, and a mouth and pharynx, followed by a bifid intestine. An opening, the birth-opening $(C, b . o p$.$) , is formed in the wall$ of the redia near the circular ridge, and through this the cercarix escape ; they move actively by means of their tails, and force their way out of the body of the Snail. They then, losing the tail, become encysted, attached to blades of grass or leaves of other herbage. The transference of the larval Fluke in this stage to its final host, the Sheep, is effected if the latter swallow the grass on which the cercaria has become encysted. The young Fluke then escapes from the cyst and forces its way up the bile-ducts to the liver, in which it rapidly grows, and, developing reproductive organs, attains the adult condition.

\section{iii. The Common Tape-Worm of Man (Toenia solium).}

General Features.-Tonnia solium occurs as a parasite in the intestine of man. It has the form of a narrow ribbon (Fig. 191), which may attain a length of several yards, attached at one end to 
the wall of the intestine, the remainder hanging freely in the interior. Towards the attached end the ribbon becomes very much narrower than it is towards the opposite end; and at this narrower

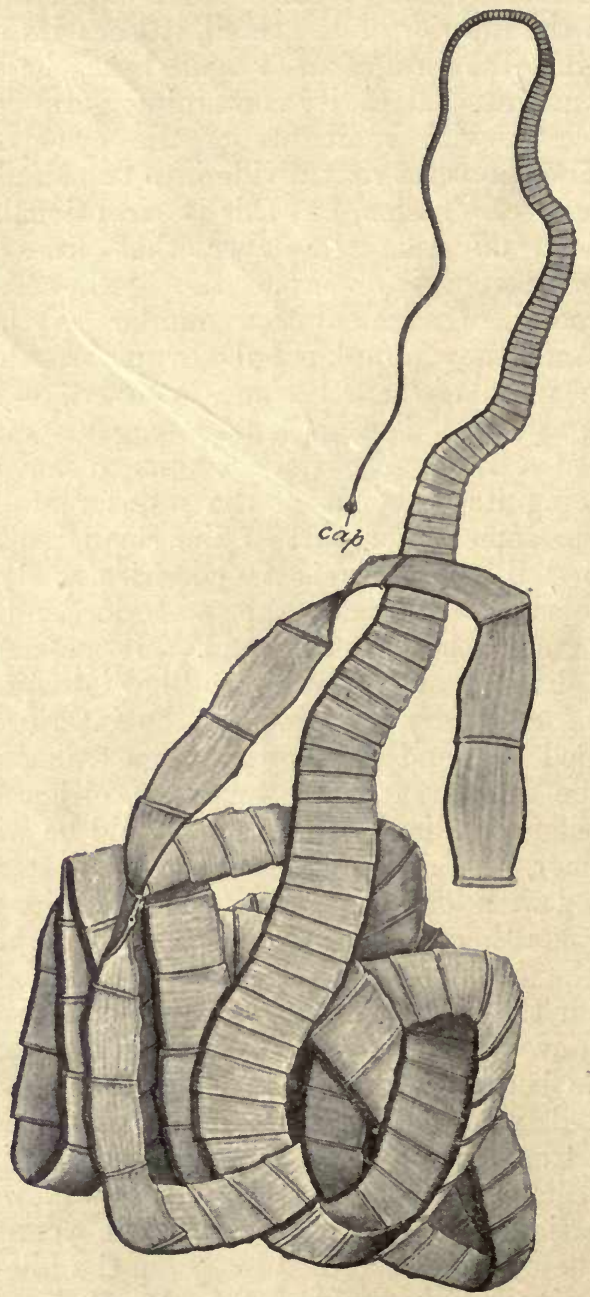

FIG. 191.-Tænia solium. Entire specimen, reduced; cap. head. (After Leuckart.)

extremity is a small, rounded, terminal knob, which is known as the head or scolex; ${ }^{1}$ the rest of the animal is termed the body or strobila; the narrow part immediately behind the head is some-

1 Though very probable, it is not certain that this end of the Tape-Worm actually corresponds to the anterior end in the Liver-Fluke, as will be explained later. 
times called the neck. The attachment of the Tape-worm to the wall of the intestine is slight and temporary; it is effected by certain organs of adhesion, the hooks and suckers on the head.

The head (Fig. 192) may be roughly described as pear-shaped, but becomes four-sided at the broader end. In the middle of this broader, anterior end is a rounded prominence, the rostellum, round the base of which there is a double row of usually about twenty-eight curved and pointed chitinous hooks. The rostellum is capable of being protruded and retracted to a slight extent, and the position of the hooks varies accordingly: when the rostellum is fully retracted the points of the hooks are directed forwards, and may even meet in the centre; as the rostellum is protruded the hooks become rotated until their apices come to be directed backwards. Four cup-shaped suckers project slightly from the surface behind the circlet of hooks.

The body or strobila has a jointed appearance, owing to its being made up of a string of segments, or proglottides-about 850 alto-

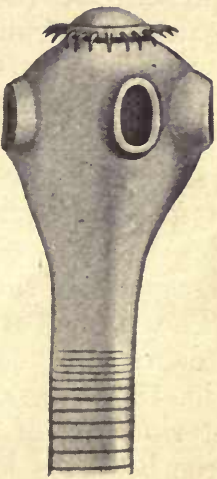

FIG. 192.-Head of Tæenia solium, magnified. (After Leuckart.)

gether. These are narrower and shorter in front, gradually increasing in size towards the posterior free extremity. The neck or part immediately following the head is devoid of any trace of segmentation. The two surfaces of the proglottides are not to be distinguished by any differences visible to the unassisted eye; but that side towards which the female reproductive organs are

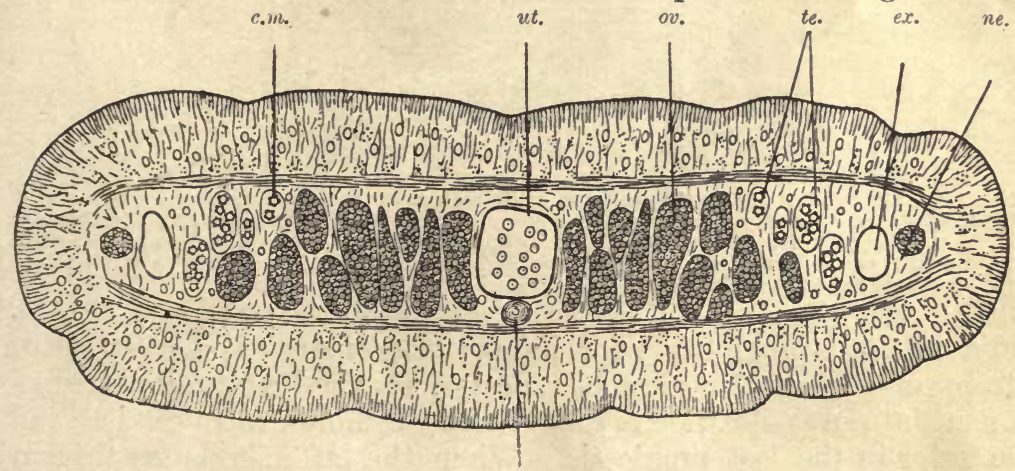

o.d.

Fig. 193.-Transverse section of Tænia solium. c.m. circular layer of muscle ; ex. longitudinal excretory vessel; ne. longitudinal nerve; o.d. oviduct; ov. uvary; $u t$. uterus. (After Shipley.)

more nearly approximated is regarded as the ventral, the opposite as the dorsal surface. On one border, alternately on the right and left, of each proglottis, is a little prominence, the genital 
papilla, on which is the opening of a chamber, the genital cloaca, into which both the male and female reproductive ducts open.

An examination of entire living and of preserved and stained Tape-Worms under the microscope shows (1) that an alimentary system is not present; (2) that nervous and excretory systems are represented; (3) that there is a complete set of reproductive organs, constructed on the same general plan as those of the Liver-Fluke, present in each of the proglottides.

The nervous system consists of two not very well-defined ganglia - united by a broad transverse commissure-in the head; of slender nerves passing from these to the suckers, and of two longitudinal nerves which run backwards through all the proglottides to the posterior end of the body. The ganglia seem to correspond to the ganglia on the nerve-ring of the Liver-Fluke.

The excretory organs consist of a richly branched system of excretory vessels. There are four main longitudinal trunks (Fig. 194, can. excret.), two near each lateral margin in the more anterior part of the strobila; in the more posterior region one of these becomes lost on each side. The two pairs of longitudinal vessels are connected together in the head by a ring-like vessel

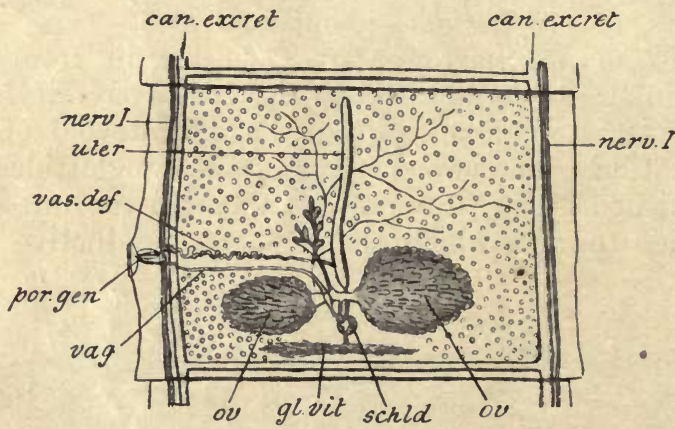

FIG. 194.-A proglottis of Tænia solium with mature reproductive apparatus. can. excret. longitudinal excretory canals with transverse connecting vessels; gl. vit. vitelline glands; nerv. $l$. longitudinal nerves; ov, ov. ovaries (germaria); por. gen. genital pore ; schld. shell. glands; uter. uterus; vag. vagina; vas. def. vas deferens. The numerous small round bodies are the lobes of the testes. (After Leuckart.)

and in each proglottis near its posterior margin by a straight, transverse, connecting branch. Posteriorly the longitudinal trunks open into a pulsatile caudal vesicle, communicating with the exterior in the last proglottis. When the latter becomes thrown off, the vesicle is lost with it, and, subsequently, the longitudinal vessels have their separate openings on the exterior. These main trunks of the excretory system give origin to a number of branches, and these in turn give off numerous fine.canalicules, or capillaries, terminating in flame-cells similar to those of the Fluke. 
The reproductive organs (Fig. 194), repeated in each fully formed proglottis, are in essential respects very similar to those of the Liver-Fluke. In the most anterior proglottides they are not developed; it is only at about the 200 th proglottis that they first appear: at first the male parts of the system are alone differentiated; then in the succeeding proglottides, till we approach near the posterior extremity of the body, the female organs are likewise developed. In the most posterior segments modifications and reductions of some of the parts take place, owing to the great increase in size of the uterus. The male portion of the apparatus consists of the testes with their efferent ducts, the vas deferens (vas. def.), and the cirrus, with its sac. The testes consists of numerous rounded lobes situated nearer the dorsal than the ventral surface, and extending throughout the greater part of the length and breadth of the proglottis. With each lobe is connected a fine efferent duct; the ducts of neighbouring lobes unite together to form somewhat larger ducts; and the larger ducts, receiving numerous tributaries, eventually open into the inner extremity of the vas deferens, or main duct of the testis. The vas deferens is a convoluted tube which extends outwards towards the lateral margin (right or left as the case may be) of the proglottis.

The terminal part of the vas deferens, which is somewhat narrower than the rest, traverses a narrow protrusible process, the cirrus, and opens at its extremity by the male genital aperture in the genital atrium or cloaca. The cirrus is enclosed at the base by a muscular sac, the cirrus-sac.

The ovary (germarium) (ov.) differs from that of the LiverFluke in being a paired organ, consisting of two approximately equal, right and left, halves. It is situated towards the posterior border of the proglottis. Like that of the Liver-Fluke, it consists of a number of branching tubes, in the interior of which the ova are developed. From opposite sides these tubes converge towards the median line, where they open into the oviduct. A yolk-gland (gl. vit.), of less relative extent than in the Liver-Fluke, consists of a number of minute lobules; a duct, the yolk-duct, which runs forward from it, opens into the oviduct. The numerous lobules of a rounded shell-gland (schld.) surround the yolk-duct where it passes forward to join the oviduct; and the many shell-gland ducts open into the oviduct near its junction with the yolk-duct: this part of the oviduct is the ootype-the part in which the egg becomes completed. In front this passes into the uterus. The female genital pore, situated in the genital atrium, leads into a narrow passage which runs inwards and backwards towards the middle line of the proglottis, where it ends in a dilatation usually filled with sperms-the receptaculum seminis. From this a narrow duct - the fertilising duct or spermatic duct-runs to join the oviduct. 
The uterus, in the segments in which it first makes its appearance, is a simple cylindrical diverticulum of the oviduct; it retains its simple form as far back as about the 600th proglottis, where it begins to branch, the ramifications increasing in extent and volume in the posterior segments. It has no opening on the exterior.

Masses of sperms (probably from the same proglottis) pass in the act of copulation along the vagina to the receptaculum seminis; through the fertilising duct they pass to the oviduct to fertilise the ova. As in the case of the Liver-Fluke, the oosperm proper becomes surrounded by a quantity of food-yolk developed in the yolk-glands, and is then enclosed in a firm chitinous shell formed for it by the secretion of the shell-gland. It then passes into the uterus. The first completed eggs are found in the uterus in some proglottis between the 400th and the 500th. From this point backwards they rapidly accumulate, until the cavity of the uterus, which now becomes branched, is filled and distended with them. Eventually in the most posterior, so-

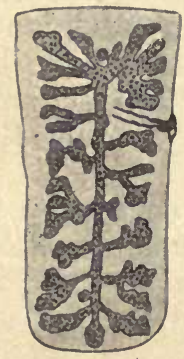

Fig. 195.- "Ripe" proglottis of Tænia solium. (After Leuckart.) called "ripe," proglottides (Fig. 195), the uterus, with its contained accumulation of eggs, becomes so large as to fill the greater part of the interior of the proglottis, the remainder of the reproductive apparatus meanwhile having become absorbed.

Development.-When the ripe proglottides are detached they pass to the exterior with the fæces of the host. For a time they exhibit movements of contraction. The embryos contained within the eggs have meantime assumed the form of rounded bodies, each armed with six chitinoid hooks - the six-hooked or hexacanth embryo (Fig. 196, A) enclosed within two membranes. If the proglottides, or the eggs which have escaped from them, should now be taken into the alimentary canal of the Pig, which forms the ordinary second host of the parasite, the hooked embryos, becoming freed from their coverings, bore their way with the aid of their hooks through the wall of the alimentary canal, and reach the voluntary muscles. Here they increase greatly in size, and develop into rounded cysts with a large cavity filled with watery fluid-the proscolex stage (B). On the wall of the proscolex, at one side, is formed a hollow ingrowth, or invagination $(\mathrm{C})$; and on the inner surface of this are developed the hooks and suckers characteristic of the head or scolex of the adult (D). When these are fully formed the hollow ingrowth becomes everted (E), the suckers and hooks thus coming to be situated on the outer surface (F). The whole embryo has now the form of a bladder or vesicle, with which is connected at one point a process having all the characters of the head and neck of the mature Tænia solium; this is the bladder-worm stage, 
or cysticercus. If a portion of Pig's muscle containing cysticerci which have not been killed by cooking is taken into the stomach

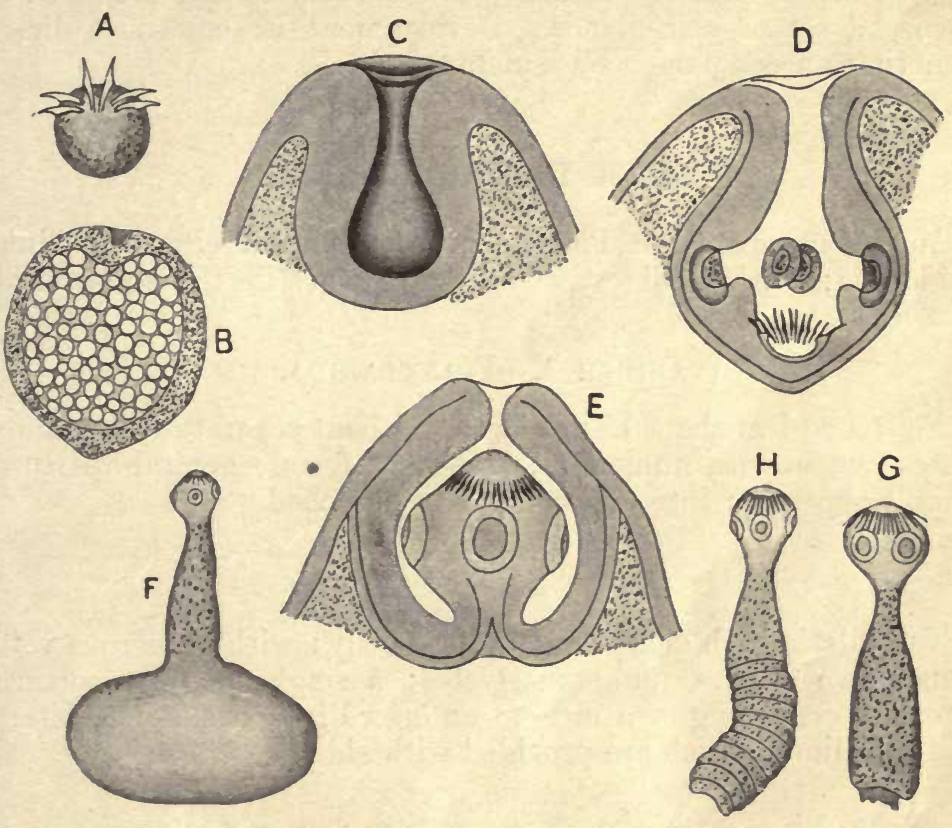

FIG. 196.-Development of Tapeworm. A, hexacanth embryo; $B$, proscolex of Tcenia saginata; $C-B$, stages in the formation of the scolex of the same; $C$, the invagination before the hooks and suckers have become developed; $D$, after the appearance of the hooks and suckers ; $E$, partly evaginated ; $F$, fully evaginated scolex of $T$. solium with caudal vesicle ; $G$, scolex of $T$. serrata with remains of the vesicle ; $H$, young tapeworm of $T$. serrata. (After Lcuckart.)

of Man, the bladder is thrown off, the scolex attaches itself to the wall of the intestine by its hooks and suckers, and develops the series of proglottides of the adult Tape-Worm.

\section{Distinctive Characters and Classification.}

The Platyhelminthes are bilaterally symmetrical, usually dorsoventrally compressed animals, devojd of hard supporting skeletoneither external or internal, and also of metameric segmentation; with three embryonic layers-ectoderm, mesoderm, and endoderm - entering into the formation of the body. A body-cavity is not present. There is a system of excretory vessels, communicating in the majority of cases with the exterior, and furnished with ciliary flames. There is no blood-vascular system. An enteric cavity may be absent, may be rudimentary, or may be highly developed; 
it is never provided with an anal aperture. The completed egg contains, in addition to the oosperm, a quantity of yolk-matter, usually in the form of definite yolk-cells, and usually produced by a special set of yolk-glands. Development is sometimes direct, sometimes accompanied by a metamorphosis.

\section{CLASS I.-TURBELLARIA.}

Mostly non-parasitic Platyhelminthes with a ciliated cellular epidermis; with a digestive cavity (except in the sub-division Acola).

\section{ORder 1.-PolyCladida.}

Flattened leaf-shaped Turbellaria, without separate yolk-glands; testes and ovaries numerous; male and female genital apertures usually separate; intestine complexly branched.

\section{ORDER 2.-TRICLADIDA.}

Turbellaria with elongate depressed body; with numerous yolkglands, two ovaries, numerous testes; a single genital aperture; intestine consisting of a median anterior division and two lateral posterior limbs which are provided with side branches.

\section{Order 3.-Rhabdocalida, incl. Accela.}

Comparatively small Turbellaria, with the body usually elongate and cylindrical or compressed: with simple, or nearly simple, sac-like intestine; with or without yolk-glands; with one or two ovaries and two or many testes.

\section{CLASS II. -TREMATODA.}

Ecto- or endo-parasitic Platyhelminthes devoid of cilia, ${ }^{1}$ or of a cellular epidermis $;^{2}$ with a well-developed digestive apparatus.

\section{Order 1.-Monogenetica (Heterocotylea).}

Mostly ectoparasitic Trematodes; with direct development.

\section{Order 2.-Digenetica (Malacocotylea).}

Endoparasitic Trematodes with complicated life-history.

1 Except in certain species of Temnocephala.

2 Except in the Temnocephalea and Actinodactylella. 


\section{ORdER 3.-ASPIDOCOTYLEA.}

Endoparasitic Trematodes with direct development; adhesive apparatus in the form of a large sucker, which is divided by septa into compartments, and occupies nearly the entire ventral surface.

\section{ORDER 4.-TEMNOCEPHALEA.}

Trematodes with direct development, which live on the outer surface or in the respiratory cavities of various animals-e.g., Crustaceans; most non-parasitic as regards their nutrition, with organs of adhesion in the form of a simple posterior sucker and a system of anterior or marginal tentacle-like appendages.

\section{CLASS III.-CESTODA.}

Endoparasitic Platyhelminthes without cilia and without digestive cavity, the animal consisting in most cases of a rounded head bearing organs of adhesion in the form of suckers and hooks, and an elongated compressed body consisting of a string of similar proglottides, each containing a complete set of hermaphrodite reproductive organs.

\section{Order 1.-MONOZOA.}

The body not divided into proglottides.

\section{Order 2.-PolyzoA (Merozoa).}

The body consisting of head or scolex, and string of proglottides.

\section{Systematic Position of the Examples.}

Planaria and Dendrocolum are genera of the family Planarida or fresh-water Planarians, which is one of the two families of the order Tricladida, differing from the other family, the Gcoplanida or Land Planarians, mainly in having the body less elongated and more dorso-ventrally compressed.

The genus Fasciola, to which the Liver-Fluke belongs, is a . member of the family Distomida of the Monogenetic Trematodes. The Distomida are characterised by the following features:-They have a cylindrical or more or less flattened body, always provided with two suckers - the anterior terminal or nearly so, the posterior ventral and either terminal, or in a varying position on the ventral surface. A pharynx may be present or absent. The intestine is always forked, the limbs simple or branched. The genital pore is 
ventral, either median or lateral, sometimes at the posterior end. There are two testes, sometimes fused into one, sometimes broken up into more or less numerous follicles, but always provided with only two vasa deferentia. There is a single germarium, not uncommonly lobed or divided up into a number of separate parts. A receptaculum seminis, or a Laurer's canal, or both, are present. The vitelline glands are, in most instances, paired, more or less richly branched, extending towards the lateral borders of the body.

The genus Fasciola is a member of the sub-family Fasciolince of the Distomidce, and this is distinguished from the other sub-families by the following characteristics. The Fasciolince are broad, leaflike Distomida, with the integument spinose or scaly. They have a well-developed pharynx. The intestinal limbs are simple or branched. The genital aperture is median, and situated in front of the posterior sucker. The testes are situated one behind the other, directly or obliquely: they are either simple, divided into lobes, or branched. The ovary is immediately in front of the testes, the uterus in front of the ovary. A Laurer's canal is present. The receptaculum seminis is absent or small. Among the many genera into which this sub-family is now divided the genus Fasciola presents the following distinctive features:-The anterior end is distinctly differentiated into a head-lobe; the intestinal limbs have long branched diverticula on the outer side, short on the inner; the gonads are all richly branched; there is no receptaculum seminis.

Tania solium is one of the many species of the genus Tcenia, of the family Toniadce, which is distinguished from the other families of Cestodes by the possession of four suckers, with or without a circlet of hooks, and by the development of well-defined proglottides which become separated off when mature.

\section{General Organisation.}

General External Features.-As the name of the phylum denotes, the body in the Platyhelminthes is, in the great majority of cases, much compressed in the dorso-ventral direction; very thin, so that when very short it may be described as leaflike, or, when more elongated, as ribbon-like; or thickish in the middle and becoming thinner towards the margin. Some, however, have the body comparatively thick, usually with a certain amount of dorso-ventral compression; a few are approximately cylindrical or fusiform. The symmetry is always bilateral (p. 43), the radial arrangement of parts so prevalent in the Coelenterata and primarily, as we have seen, associated with a fixed or stalked condition, never being observable. A Flat-Worm has dorsal and 
ventral surfaces, right and left sides or borders, and anterior and posterior ends. The anterior end is that which is directed forwards in ordinary locomotion: it usually has some of the features which distinguish a head-end; but a distinct head is rarely developed, and the mouth, when present, is usually placed some distance back on the ventral surface.

In the Turbellaria (Fig. 197) the leaf-form is the prevailing one, a shape resembling that described for Planaria being very common. In many, however, the body is greatly elongated, and it may assume the shape of a thin ribbon with puckered edges, as in some marine forms; or may be thickened and band-like, as in the Land Planarians; or it may approach the shape of a cylinder, as in some Rhabdocoeles. A head-region is not usually distinct; but there is always something to mark off the anterior from the posterior end-a difference in shape, the presence of eyes, and, sometimes, of a pair of short tentacles; in some a slight constriction separates off an anterior lobe, on which the eyes are borne, from the rest of the body. In others the anterior end is retractile, and may be everted as a proboscis. The mouth is never at the extreme anterior end, but always ventrally placed, sometimes behind the middle. In some Polycladida there is a small

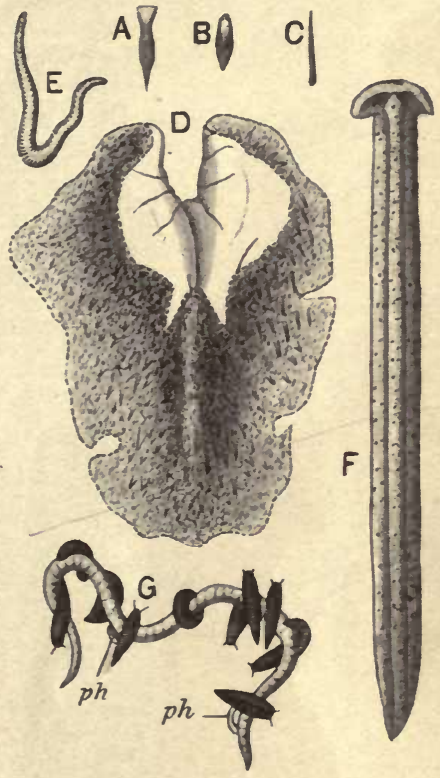

Fig. 197.-Various Planarians. $A$, Convoluta; $B$, Vortex; $C$, Monotus; $D$, Thysanozoon; $B$, Rhynchodemus; $F$, Bipalium ; $G$, Polycelis. All natural size. (After Von Graff.) ventral sucker, probably with a copulatory function; and in some Rhabdocœles both the anterior and posterior ends, though not provided with suckers, are adhesive, so that the animal can loop along like a Hydra or a Caterpillar. There is never any external appearance of segmentation, though in at least one exceptional instance (Gunda segmentata, Fig. 198) the internal parts may be so disposed as to approximate to the metameric arrangement (pseudo-metamerism). In such a case a number of transverse muscular septa are present, imperfectly dividing the body internally into a series of segments; and various internal organs-intestinal cæca, gonads, transverse commissures of the nervous system-are arranged in pairs following this division. A few 


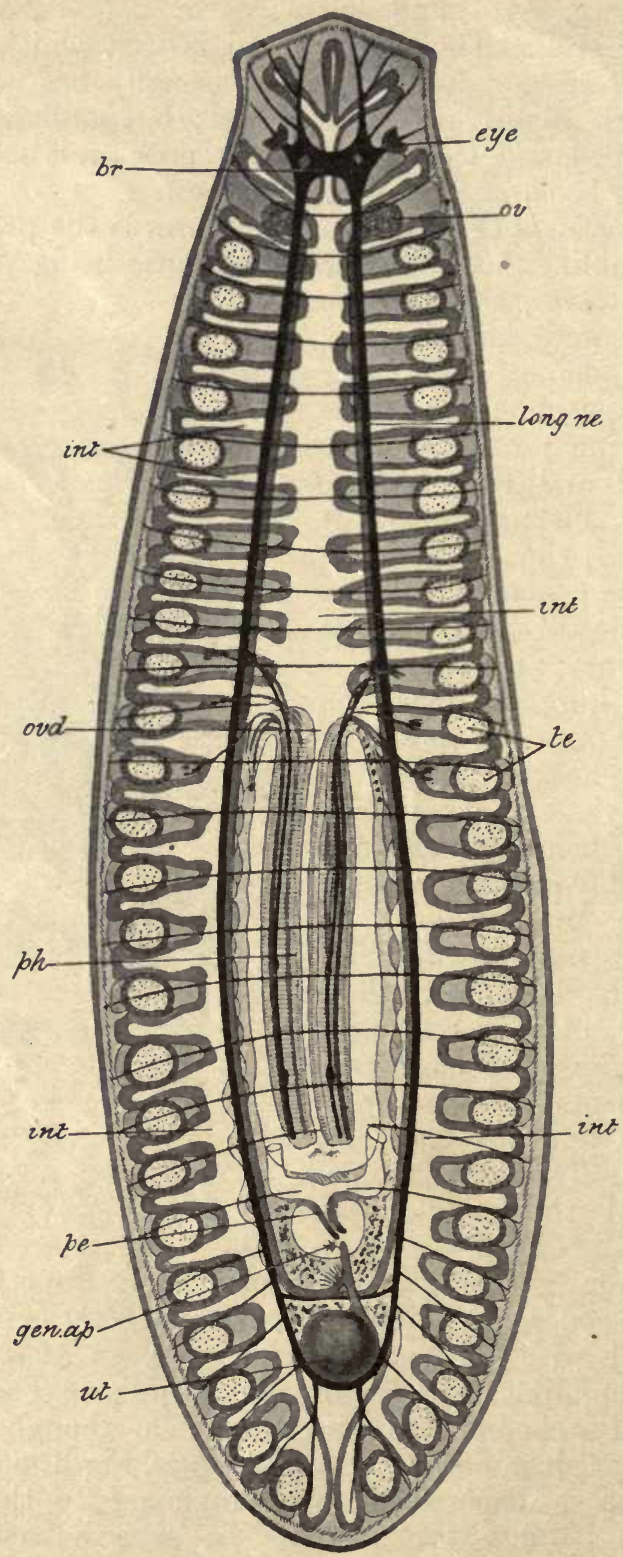

FTG. 198. Gunda segmentata. General view of the organisation. br. brain; eye eye; gen. ap. genital aperture; int. intestîne with its creca; long. ne. longitudinal nerve-cord; ov. ovary; ovd. oviduct: pe. penis ; ph. pharynx ; te. testes ; $u t$. uterus. (Aftcr lang.) 
Turbellaria multiply by budding, and these form long chains, having something in common with the string of proglottides of a Cestode, but differing radically, as will be shown later, in the mode of development. Colour is very general in the Turbellarian, though some are transparent and colourless. The most vivid coloration characterises some of the marine Polyclads, the Rhabdocoles being comparatively obscure. The surface is covered with a coating of fine vibratile cilia, the vibration of which subserves respiration as well as (in the smaller forms) locomotion. Among the ordinary cilia are frequently disposed longer whip-like cilia or flagella, likewise motile; and sometimes non-motile (sensory) cilia may occur here and there.

The Trematodes (Figs. 186, 199, 200, 201), nearly related to the Turbellarians in internal organisation, resemble them also in
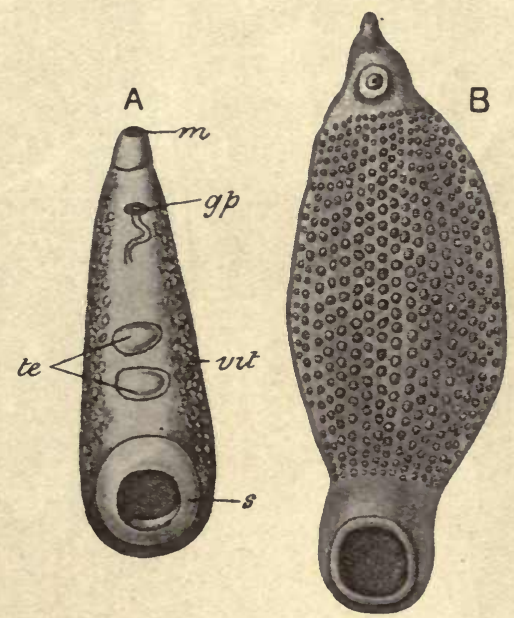

Fra. 199.-Digenetic Trematodes. A, Amphistomum; B, Homalogaster. g. p. genital aperture; m. mouth; s. posterior sucker; te. testes; vit. vitelline glands. (After M. Braun.)

external form, with certain modifications connected with a parasitic mode of life. As in the latter class, the leaf-shape prevails; an elongated form also occurs, though more rarely. The body is usually thicker and more solid than in most Turbellaria. The anterior end is distinguished from the posterior by its shape, by the arrangement of the suckers, and, in many of those Trematodes that are external parasites, by the presence of eyes. Suckers, present in the Turbellaria only in some of the Polycladida and a few Tricladida, are universal in their occurrence. They are always ventrally placed, their chief function being to fix the parasite to the surface of its host in such a way as to facilitate the taking in by the mouth of animal juices and epithelial débris; their number and arrangement vary considerably. There are nearly 
always present an anterior set of suckers (or a single anterior sucker surrounding the mouth) and a posterior set, or a single
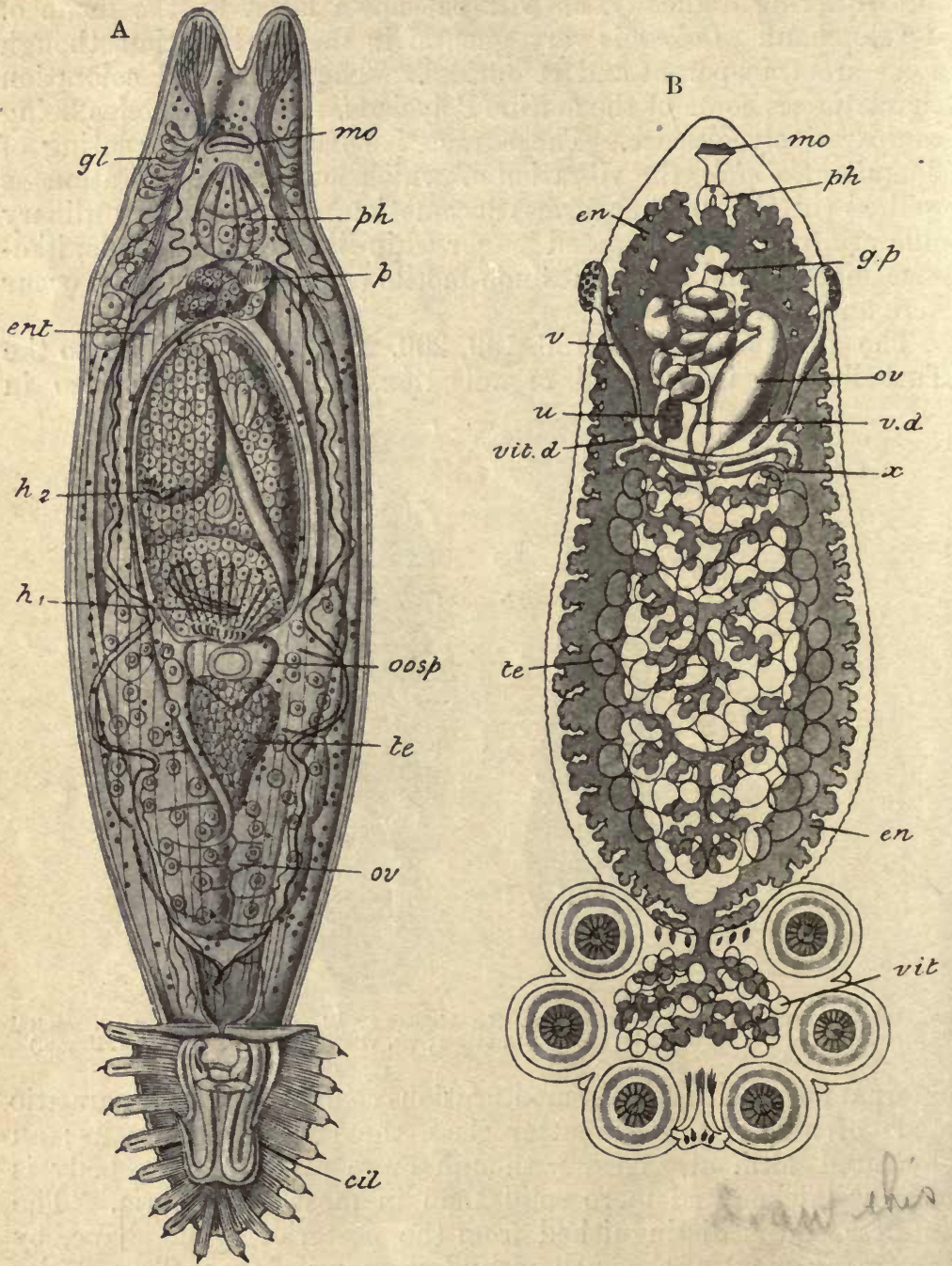

FiG. 200.-Monogenetic Trematodes. A, Gyrodactylus. cil. disc bearing hooks und processes at the posterior end ; ent. intestine; $g l$. unicellular glands whose ducts open on the surface about the anterior end $; h^{1}$. caudal disc of the first embryo; $h^{2}$. caudal disc of the second embrro; mo. mouth; oosp. oosperm; ov. ovary; $p$. penis ; $p h$. pharynx; te. testes.

B, Polystomum. en. intestine ; g. p. genital porc; mo. mouth; ph. pharynx; ov. ovary; te. testes; $u$. uterus; $v_{\text {. }}, v_{\text {. }} d$. vas deferens; vit. vitelline glands ; vit. $d$. vitelline ducts; $x$. marks the position of the genito-intestinal canal connecting the oviduct with the intestine. (Froin M. Braun.)

large posterior sucker. The arrangement.already described as characterising the Liver-Fluke is that which is typical in the 
digenetic forms - a single anterior and a single posterior sucker ; but in some of the Digenctica the posterior sucker is wanting. Adhesive papillæ on the dorsal or ventral surface may supplement the adhesive action of the suckers (Fig. 199, B.). In the Monogenetica the suckers are often more numerous; in the family Gyrodactylidoe

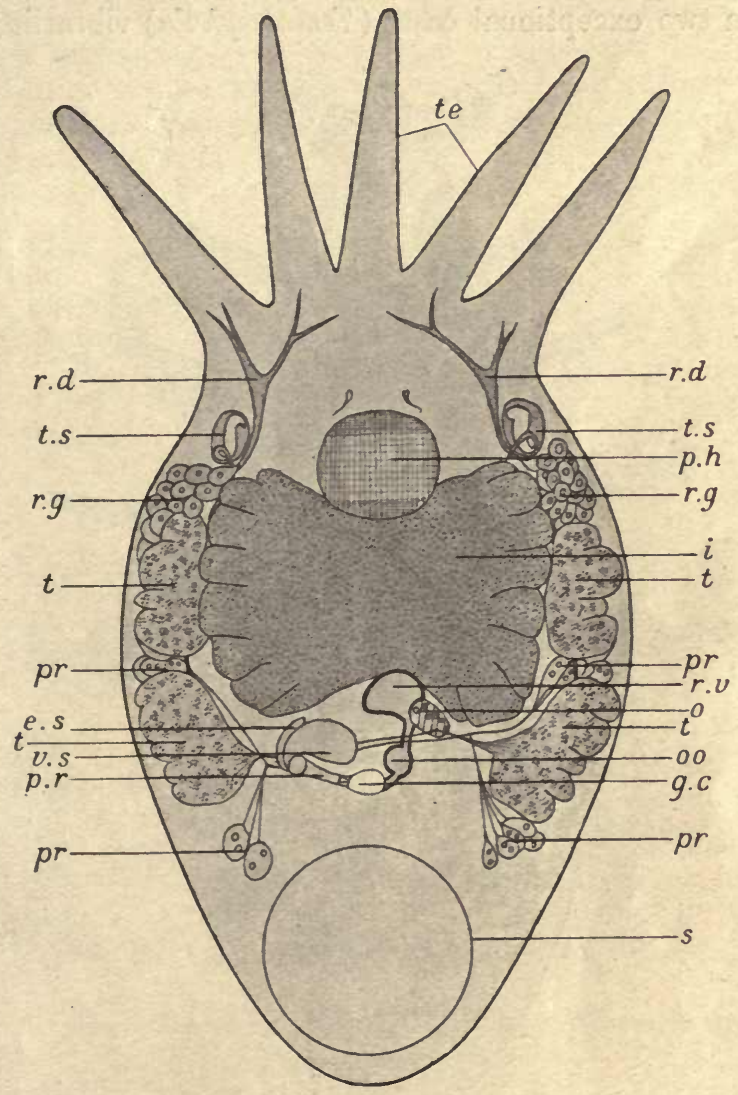

Fif. 201.-Temnocephala minor, general view of the organisation. c. cirrus; e. $\delta$. ejaculatory sac; g. c. genital atrium; $i$. intestine; o. germarium; oo. ootype; ph. pharynx; $p r$. prostate glacds; $r . d$. strands of ducts of integumentary glands running forwards to the tentacles; $r . g$, groups of integumentary (rhabdite-forming) glands; $r . v$. receptaculum; s. sucker; $t$. testes; te. tentacles; $t$. s. terminal sacs of excretory system; $v$. s. vesicula seminalis.

(Fig. 200, A) there is no anterior sucker, but at the posterior end one or two discs armed with hooks; in the Polystomece (Fig. 200, B) there is also a posterior disc on which are six suckers with several hooks; in the Temnocephalea (Fig. 201) there is no anterior sucker, but the anterior end develops a row (two only in Scutariella) of adhesive tentacles, while in Actinodactylella 
(Fig. 202) a series of marginal tentacles are present in addition to both anterior and posterior suckers. In the Aspidocotylea there is only a single sucker; but it extends over nearly the whole of the ventral surface, and is complicated in structure owing to its cavity being divided into a number of compartments by a system of partitions.

Save in two exceptional cases (Temnocephala) vibratile cilia are

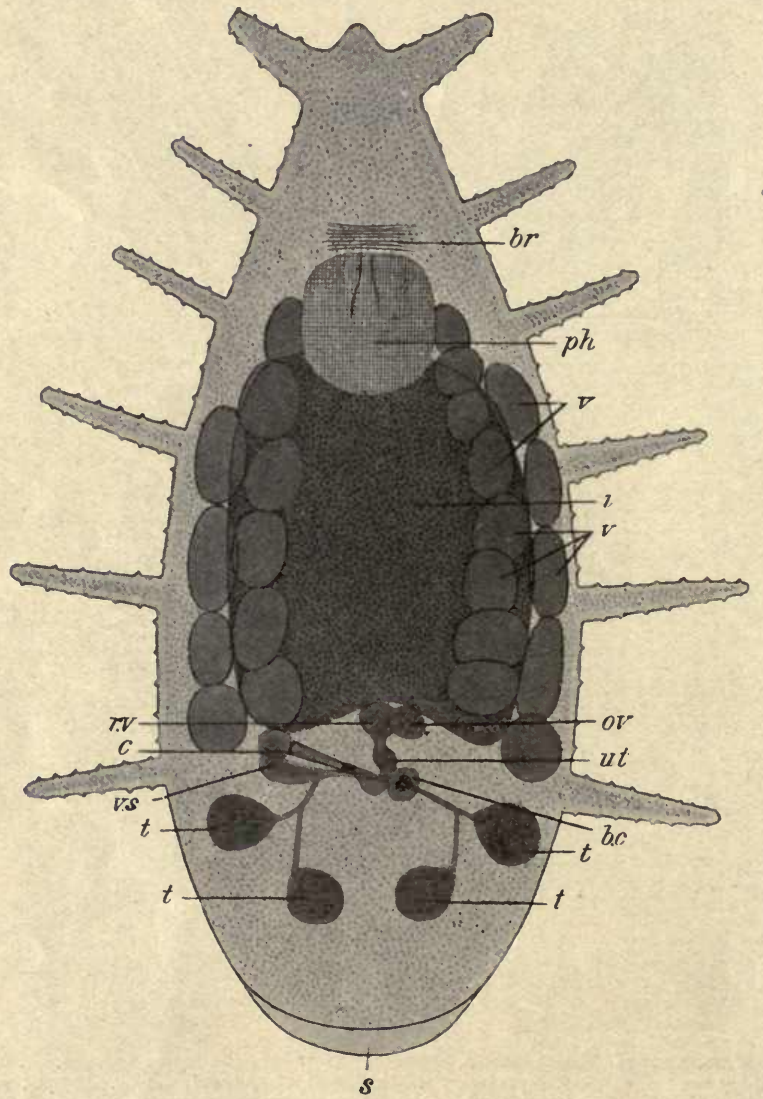

FIG. 202.-Actinodactylella. b. c. bursa copulatrix; br. brain; $c$. penis; $i$. intestine ; ov. ovary; $p h$. pharynx; $v$. v. receptaculum; $s$. sueker; $t$., $t$. testes; $u t$. uterus; $v$. vitelline glands; $v$. $s$. vesicula seminalis.

not known to occur on the surface in the adult condition; in some there are groups of non-motile cilia, situated on little conical elevations - the tactile cones. Pigment is rare in the endoparasitic Digenetica, save in a few that live in the interior of transparent animals; though many appear coloured variously by the internal organs shining through the translucent body-wall, or are stained 
by some fluid derived from their host. Pigment occurs in some of the ectoparasitic forms.

The relationship of the Cestoda to the Trematoda is, as will be subsequently shown, fairly close; but though there are connecting forms between the two classes, the shape of the average Cestode is very different from that of such an average Trematode as the Liver-Fluke. The body of an ordinary Cestode is of great lengthsometimes extending even to a good many feet-and relatively narrow, being compressed into the form of a ribbon. One end, which it will be convenient to designate anterior (though it may

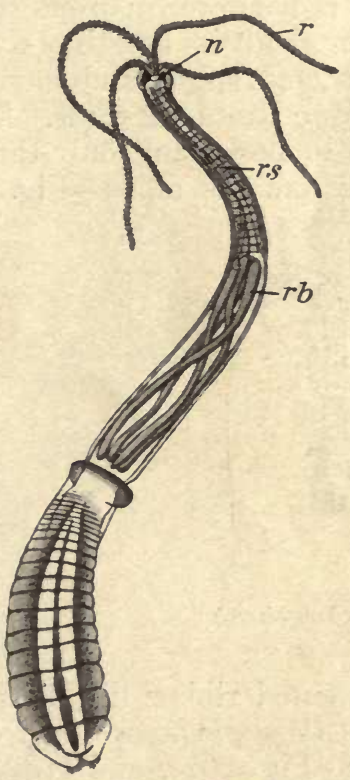

Fig. 203.-Tetrarhynchus. n. nervous system ; $r$. proboscides; $r$. sheaths, with their muscles (vb.). (From Leuckart, after Pintner.)

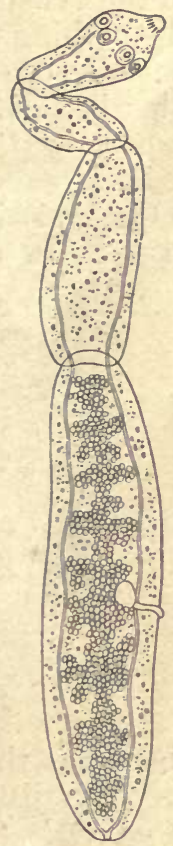

FIG. 204.-Tænia echinococcus. (After Cobbold.)

not, perhaps, correspond to the anterior end in a Trematode or a Turbellarian), is, in most cases, attached to the host by means of suckers and hooks placed on a rounded lobe, the head or scolex, connected with the body by a narrow part or neck. The head is usually rather radially than bilaterally symmetrical, with four suckers and a circlet of hooks. The hooks, when present, are borne on a longer or shorter retractile process, the rostellum, the long axis of which is in line with the long axis of the body. In Bothriocephalus and allied forms a pair of longitudinal grooves take the place of suckers, and there are no hooks. In many Cestodes para. 
sitic in Fishes the head bears four prominent thin folded flapsthe bothridia, which are exceedingly mobile, and are used more as creeping organs than as organs of fixation. In relation to each of these bothridia, which, by coalescence, may appear to be reduced to two, may be a small sucker of the ordinary kind. In T'etrarhynchus (Fig. 203) there are four very long and narrow rostella, or "proboscides," covered with hooklets, and capable of being retracted into sheaths.

The Cestoda are devoid of mouth, and in most of them the genital apertures are marginally placed, so that, externally, there is-except in the case of a few in which the genital apertures are not marginal-nothing to distinguish the dorsal surface from the ventral. The body, or strobila, which is narrower in front than it becomes further back, is made up throughout its length of a series of segments, or proglottides, which become larger and more distinctly marked off from one another as we pass backwards. Tonia cchinococcus (Fig. 204) is exceptional in possessing only three or foůr proglottides. In a few (Ligula and its allies-Fig. 205),

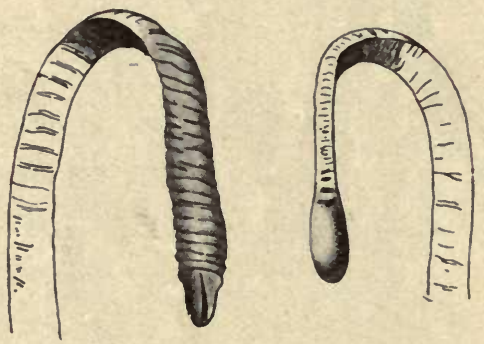

FiG. 205.-Iigula. (After Leuckhart.)

though the body has the normal elongated ribbon-like form, the segments are not distinct, and in Caryophyllows (Fig. 206), Amphilina, Gyrocotyle (Amphiptyches-Fig. 207), and Archigetes (Fig. 208)-(Monozoa), segmentation is entirely absent, the whole body in these genera consisting of a single proglottis. The surface in the Cestodes is devoid of cilia, and there is no pigment.

Integument and Muscular Layers. - In the Platyhelminthes in general there are integiumentary layers and underlying layers of muscle, which are more highly differentiated than in the Colenterates. But considerable differences exist in this respect between the members of the three classes. In the Turbellaria (Fig. 209) there is, as already noticed in the account given of the Planarian, a distinct epidermis (ep.) in the form of a layer of cells, most of which are ciliated. A delicate cuticle is usually, though not always, distinguishable, investing the epidermis externally. In one family the cuticle is developed, along the margin of the body, into a series 
of chitinous bristles. Among the ordinary epidermal cells there are in the Polycladida numerous cells containing short rod-like bodies - the rhabdites $(r h$.$) ; in the other orders of the Turbellaria$ these rhabdite-forming cells are sunk deeply within the parenchyma, and, in the Rhabdocœla, have very long ducts, formed of processes of the cells, by means of which the rods, together with a viscid matter, reach the exterior at certain points of the surface,

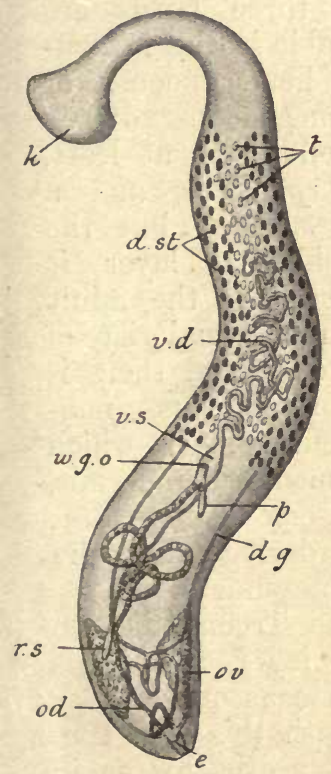

FiG. 206. - Caryophyllæus. d. $g$. vitelline duct; $d$. st. vitelline glands; e. excretory pore; $k$. mobile organ; od. oviduct; $o v$. germarium; $p$. cirrus; $r$. 8 . rceeptaculum seminis; $t$. lobes of testes ; $v . d$. vas deferens; $v$. 8 . vesicula seminalis ; v.g.o. female uperture. (After Leuckhart.)

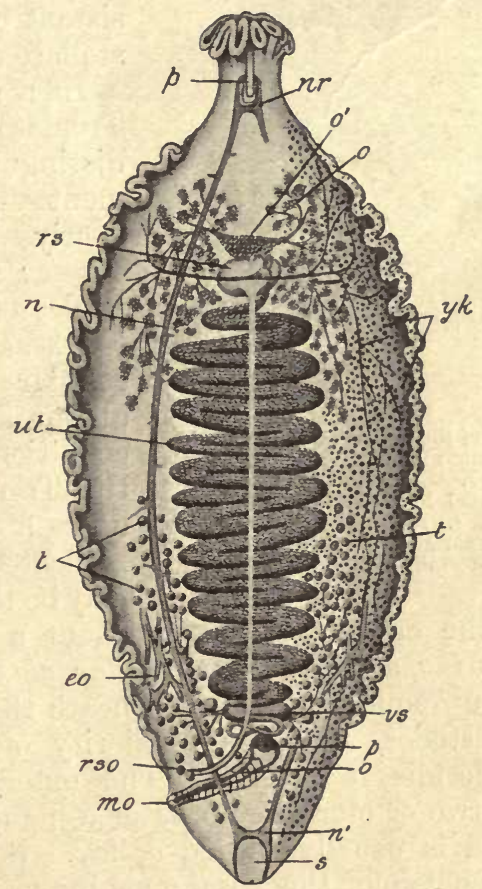

FIG. 207.-Gyrocotyle (Amphiptyches). e. o. excretory opening ; m. o. male opening; $n$. longitudinal nerve; $n^{\prime}$. anterior nervering ; n. r. posterior nerve-ring; o. opening of uterus; 0 . ovary ; $o^{\prime}$. receptaculum ovorum ; $p$. base of cirrus; $r$. 8 . receptaculum seminis; $r$. 8 . 0 . opening of vagina; 8 . sucker; $t$. testes ; ut. uterus; $v$. s. vesicula seminalis; $y k$. vitelline glands. (After Spencer.) The end here directed downwards represents the scolex-cnd of other Cestodes.

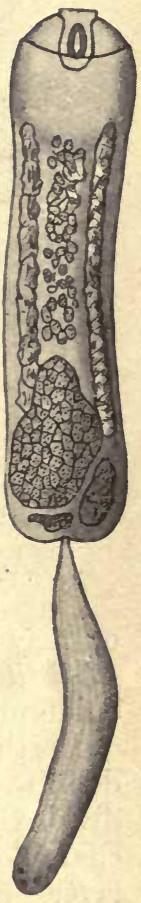

FiG. 208.-

Archigetes. (After Leuckhart)

chiefly around the anterior extremity. The function of these rhabdites is not in all cases certain; they have been supposed to add to the sensitiveness of the parts in which they are situated after the fashion of hairs or nails, or to have a skeletal function. In the Rhabdocola and Tricladida they undoubtedly aid in adhesion, and probably have the function of assisting in the entanglement and capture of food. . In certain of the Turbellaria 
stinging capsules occur similar to those of the Colenterata, and transition-forms between rhabdites and stinging capsules occur in

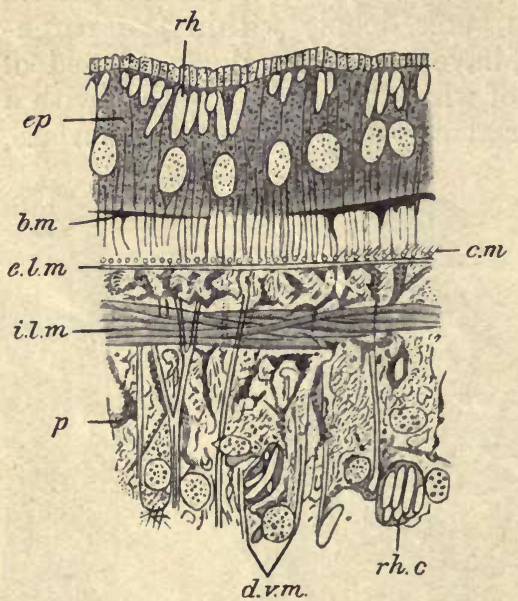

FrG. 209.-Section of the body-wall of a Triclad. $b$. $m$. basement membrane; $c$. $m$. circular muscles; $d$. v. m. dorso-ventral muscles; e. $l$. m. external longitudinal muscles; ep. epidermis ; $i . l$. $m$. internal longitudinal muscles; $p$. parenchyma; $r$. rhabdites; $r h$. $c$. rhabdite-forming cells. (After Jijima.)

some cases. Adhesive cells with processes also frequently occur in the epidermis. Beneath the epidermis is a basement membrane $(b . m$.), which in the Polycladida is of a thick resistent character, and contains stellate cells.

In a small number of the Trematoda three layers are distinguishable in the integument - a homogeneous, or nearly homogeneous, outer cuticle; a cellular, or at least, nucleated, epidermis, and a basement membrane; but the cellular epidermal layer is absent as such in the adult condition in the majority of the Trematodes, and there is only a homogeneous, nonnucleated outer layer, which may be the modified epidermis, or may be the cuticle, with or without a basement-membrane. Rhabdite-forming, and other unicellular glands derived from the epidermis, are frequently present beneath the integument.

In the Cestodes, as in the majority of the Trematodes, no definite epidermis is present. The external layer, sometimes divided into two or more strata, is of a homogeneous non-cellular character, and is usually termed cuticle. Beneath this is a thin layer of parenchyma, the basal membrane. Beneath this again is a layer of fusiform cells, narrow prolongations of which pass to the cuticle, into the inner part of which they penetrate and spread out into a thin layer. These cells are by some authors regarded as the cells that secrete the cuticle; but they may be concerned in the absorption of nutrient matter, and some of them are undoubtedly of the nature of nerve-cells and have nerve-fibres connected with them.

The muscular layers of the body-wall vary somewhat in their arrangement in the different groups of Platyhelminthes. Most commonly there is an external layer of circularly arranged, and an internal layer of longitudinally arranged fibres; frequently layers of fibres running in a diagonal direction are present also.

Characteristic of the Flat-worms is a peculiar form of connectivetissue, the parenchyma (Fig. 210)-mention of which has already 
been made in the descriptions of the examples-presenting many varieties, filling up the interstices between the organs and leaving only, in some instances, very small spaces-sometimes regarded as representing the body-cavity, or ccelome, which we shall meet with in other groups of worms. Sometimes the parenchyma appears to

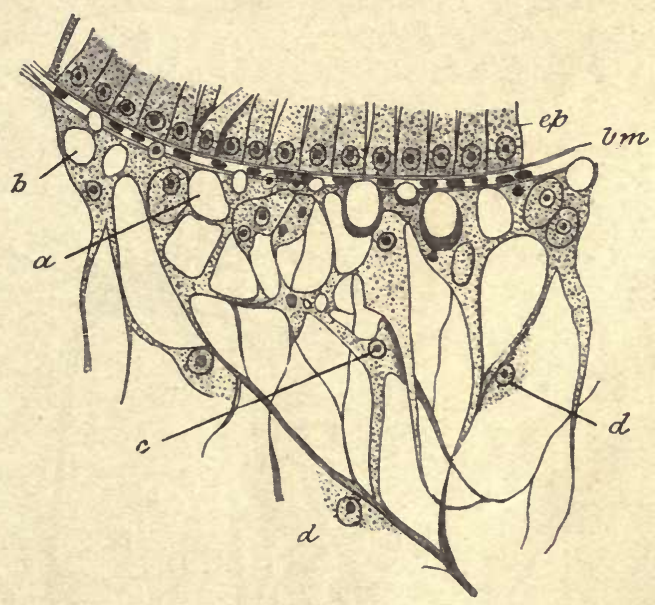

Fig. 210.-Parenchyma of Distomum. $a, b$. intercellular spaces ; $b \mathrm{~m}$. basement membrane ; $c$. nuclei; $d$. nuclei ; ep. epidermis. (After Braun.)

consist of distinct large cells with greatly vacuolated protoplasm, with interspaces here and there in which groups of rounded cells are enclosed. Sornetimes the constituent cells run together, and the parenchyma then appears as a nucleated, finely fibrillated, vacuolated mass in which the boundaries of the cells are not recognisable. Pigment occurs in the parenchyma in some Rhabdocole Turbellarians and a few Monogenetic Trematodes. In some Turbellaria-species of Convoluta and Vortex - the parenchyma contains numerous cells enclosing chlorophyll or xanthophyll corpuscles; these are symbiotic unicellular Algæ, similar in their mode of occurrence to the yellow cells which have been referred to as found in the Radiolaria. Running through the body, for the most part in a dorso-ventral direction, are numerous slender muscular fibres, the fibres of the parenchyma muscle; many of these become inserted externally into the basement membrane.

Great differences exist between the various groups of Platyhelminthes as regards the development of the alimentary system, differences which are, broadly, to be correlated with differences in the mode of nutrition. Some of the Flat-worms - the Turbellaria and some of the Monogenetic Trematodesprocure their food, in the shape of small living animal or vege- 
SECT.

table organisms, or floating organic débris, by their own active efforts. Others - the Digenetic Trematodes and the Cestodeshaving reached a favourable situation in the interior of their host, remain relatively or completely passive. An alimentary canal is

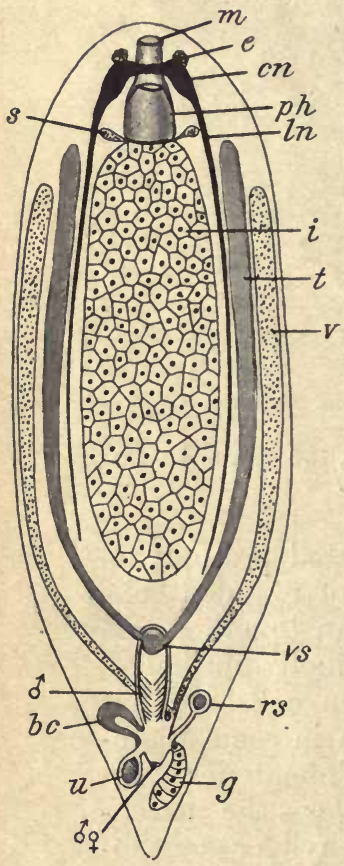

Fig. 211.-General plau of the structure of a Rhabdocole Turbellarian. b. c. bursa copulatrix ; $c n$. brain; e. eyc ; g. germarium; i. intestine; ln. longitudinal nerve; $m$. mouth; pl. pharynx; r.s. receptaculum seminis ; 8 . unicellular glands ; $t$. testis; $u$. uterus; $v$. vitellarium ; $v s$. vesicula seminalis; $\delta$ ejacu. latcry duct; $\delta$ o common genital aperture. (After Von Graff.)

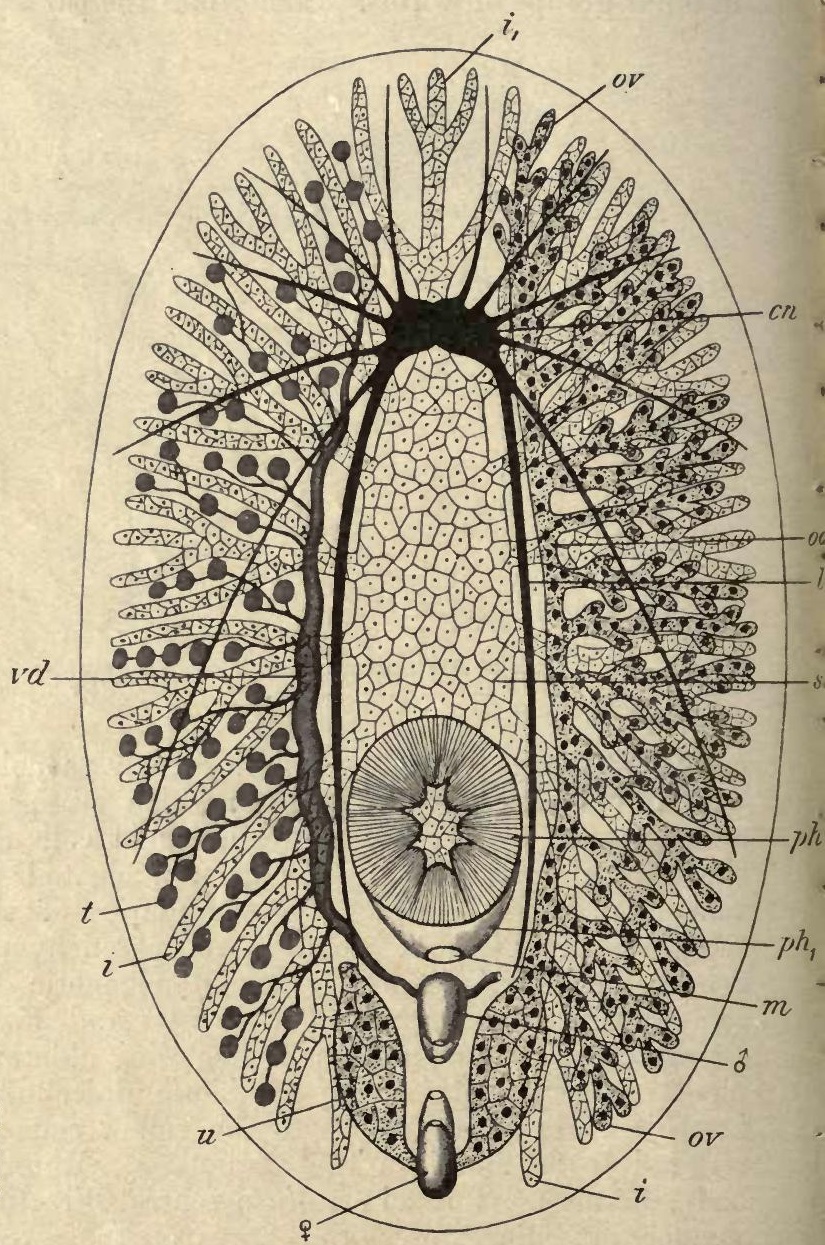

Fig. 212.-General plan of the structure of a Polyclad. cn. brian ; $e$. eye ; $i$. st. intestine; $l n$. longitudinal nerve cord; $m$. mouth ; ov. ovary; ph. pharyux; $p h l$. sheath of pharynx; $t$. testes; $u$. uterus $v d$. vas deferens; $v s$. vesicula seminalis; $\delta$ male aperture; $q$ female aperture. (After Vou Graff.)

completely absent in the last-named group, nutrition being effected by the absorption of digested matter from the interior of the animal in which the Cestode lives. In all the rest of the Platyhelminthes there is an alimentary canal, which never opens on the 
exterior by an anal aperture. All the Turbellaria (except some Acoela) and Trematoda have an alimentary apparatus consisting of two well-defined parts-a muscular pharynx and an intestine. The pharynx is usually a rounded muscular bulb, but is sometimes (some Turbellaria) of a cylindrical shape; it is usually capable of eversion and retraction. Actinodactylella (Fig. 202) is exceptional in having in addition to a large muscular pharynx, an extersile proboscis with a pin-shaped style, which becomes retracted within the opening of the mouth. Unicellular glands open into the pharynx in most cases.

The mouth is always ventral, but varies greatly in its position on the ventral surface, being sometimes central, sometimes situated behind, sometimes in front of, the middle of the length of the body. In the most lowly organised group of Turbellaria (the Accela) the intestine is represented merely by a vacuolated, nucleated mass of protoplasm without, or with only an irregular, lumen. In the others it is sometimes a simple sac (Rhabdocœle Turbellaria-Fig. 211, a few Trematoda), with or without short lateral diverticula. In the majority of the Trematodes it consists of a pair of simple canals; but in some, as in the Liver-Fluke, there is a pair of canals which give off numerous branches. In the Polycladida (Fig. 212) there is a central cavity from which numerous branching canals are given off. In the Tricladida (Fig. 213) one median canal passes forwards from the pharynx, and a pair of canals backwards from it, all three giving off branches which again branch. In some Polycladida there are minute pores, by means of which certain of the canals are placed in communication with the exterior. A number

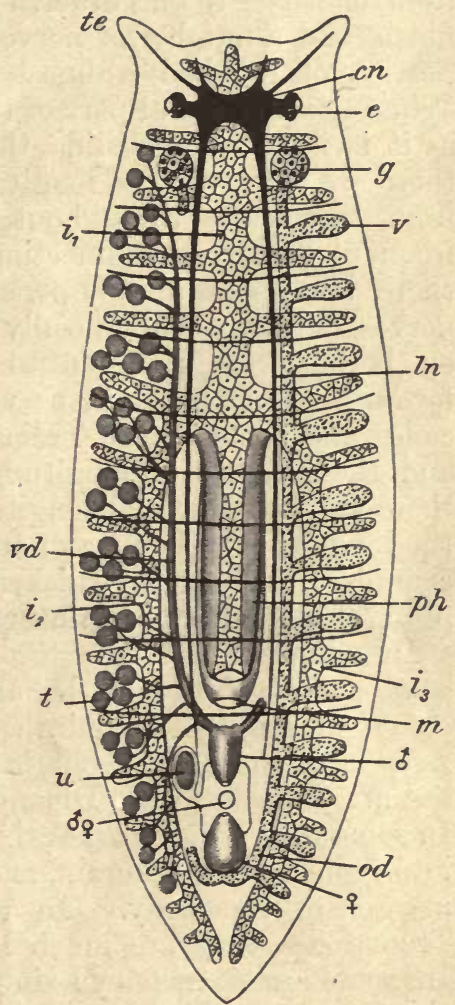

FIG. 213.-General plan of the structure of a Triclad. cn. braiu; e. eye; $g$. germarium: $i$. median limb of the intestine; $i_{2}$. right limb; $i_{3}$. left limb; ln. longitudinal nerve-cord; $m$. mouth; $o d^{\prime}$ oviduct; $p h$. pharynx; $t$. testes ; te. tentacles; $v$. vitellaria ; vd. vas deferens ; $u$. uterus; $\delta$ ejaculatory duct; $q$ vagina ; $\delta$ common genital aperture. (After Von Graff.) of unicellular glands, which probably produce a digestive secretion, open in many Trematodes and Rhabdocoeles at the junction of pharynx and intestine.

A bilateral nervous system is developed in all the Platy- 
helminthes. Its elements are nerve-fibres and nerve-cells. The nerve-cells, which are usually bipolar, more rarely uni- or multipolar, lie in the course of these fibres, with which the substance of the cells is in continuity. The degree of development of a central part of the nervous system, or brain, varies in the different groups; it is best developed in some Polycladida and some Monogenetic Trematodes. It consists of numerous nerve-fibres which here converge from the various parts of the body and pass across from one side to the other, together with a central mass of fine fibrils, and a number of nerve-cells. It is situated in the anterior portion of the body, almost invariably in front of the mouth. When the peripheral part of the nervous system is best developed, as it is in the Polycladida, the Tricladida, and some Trematodes, there are three pairs of longitudinal nerve-cords running backwards from the brain throughout the body, connected together by frequent transverse connecting nerves, or commissures. To these there are sometimes superadded fine net-works or plexuses of nerves, situated superficially under the dorsal integument, or on both dorsal and ventral surfaces. Sometimes nerves run forwards from the brain as well as backwards. In the Rhabdocœles and some of the Trematodes the whole system is simpler, and the number of longitudinal cords fewer. In the Cestodes there are two principal longitudinal trunks which run throughout the length of the body, and are connected together in the head by commissures, variously thickened to form ganglia representing the brain of other Platyhelminthes.

In addition to the tactile cones of some Trematodes and the sensory cilin of the Turbellaria, already referred to, the sensory organs of the Platyhelminthes are the eyes and the statocysts. E'yes occur in the Turbellaria and some Monogenetic Trematodes, but are wanting in the Digenetic Trematodes and in the Cestodes. In some of the Polycladida they are extremely numerous, collected into groups over the brain, and frequently arranged also round the margin of the body. In the Rhabdocœeles and Monogenetic Trematodes they are much less numerous-usually two to four. In some cases each eye simply consists of a pigment spot; to this may be added a refractive body. When most highly developed the eye is still of very simple structure, consisting of a cup formed of one or more pigment-cells enclosing refractive bodies $(r o d s)$, and having nerve-cells in close relation to it with processes (nerve-fibres) passing to the brain. The statocysts are sacs containing statoliths of carbonate of lime. The function of these bodies, which occur only in a small number of the Turbellaria, is unknown; there is no sufficient evidence that they are organs of hearing; it is more likely that they are organs connected with the maintenance of the equilibrium. Ciliated pits which appear to be sensory are developed in some Rhabdocoles in the head region. 
The only vascular system present in the Platyhelminthes is the system of water-vessels (protonephridia) which are commonly regarded as performing an excretory function. The arrangement of these, the mode of ending internally of the finest branches, and the way in which the system communicates with the exterior, vary greatly in the different groups. A series of main longitudinal trunks give off branches which subdivide to form a system of minute interlacing branches or capillaries. In little spaces at the ends of the capillaries are a number of highly characteristic structures-the ciliary flames. Each ciliary flame consists of a bundle of vibratile cilia; typically each is situated in the interior of a cell-the flame-cell (Fig. 214) -terminating one of the capillary branches. But there are some cases in which there are several flames in each flame-cell. The finer branches, and in some cases the larger trunks also, are intra-cellular, and are to be looked upon as perforations in linear rows of elongated cells. In the Cestoda, at least the larger trunks are inter-cellular, being lined by an epithelium of small cells. This system of water-vessels opens on the exterior in a variety of different ways : sometimes it opens by a number of minute pores; sometimes, as in the Liver-Fluke, there is a single posterior aperture; frequently there are two. In the Tricladida there are two longitudinal canals which open on the exterior through special branches by a series of pores.

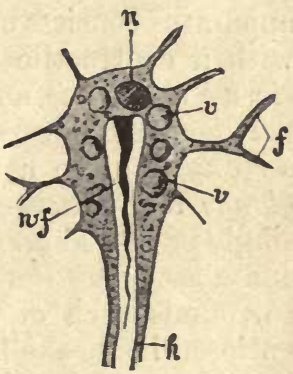

Fig. 214.-Flame-cell of a Turbellarian. $f$. processes ; $k$. termination of capillary ; n. nucleus; $v$. vacuoles ; vof. ciliary flame. (After Lang.)

In the Rhabdocœlida there are either two longitudinal main vessels or a single median one; the communication with the exterior in the former case may be by a pair of ventral apertures, or indirectly through the pharynx; or there may be a common short passage in which the two trunks unite, opening by a posterior median aperture. When a single main trunk is present it opens at the posterior end of the body. In the Trematodes there are usually two principal longitudinal trunks, which either unite behind to open at the posterior end of the body, or (Monogenetica) remain separate and open independently on the dorsal surface, each having, where it opens, a contractile excretory sac. In Temnocephala each dorsally opening excretory sac has ramifying through its wall-which consists mainly of a single large cell-a system of capillary vessels containing ciliary flames.

In the Cestodes there are usually four longitudinal trunks, which open through a contractile excretory sac at the posterior end of the body. In many cases it has been shown that the main trunks communicate with the exterior at intervals by means of fine canals. The excretory sic is thrown off when the last proglottis becomes 
separated off and does not in most cases become renewed, though in at least one species of Tape-worm (Tonia cucumerina), a new vesicle is developed again and again at the end of the body as a fresh segment is thrown off. The main trunks are connected together by a ring-vessel in the head and in some cases by a transverse branch in each proglottis, and where the latter originate from the main trunks are valves formed by folds of the wall of the vessel. In the posterior region only two of the longitudinal trunks (one on each side) may be retained.

The sexes are united in all the Platyhelminthes with only one or two exceptions, and the reproductive organs are sometimes somewhat complicated-presenting a remarkable advance on those of the Colenterata. The male part of the apparatus consists of testes, with their ducts, the vasa deferentia, often with a contractile terminal enlargement or vesicula seminalis, a cirrus ${ }^{1}$ or a penis, and often prostate or granule-glands. The female part comprises ovary or ovaries, receptaculum seminis, oviduct, uterus, an ootype, often a bursa copulatrix, shell-glands, vitelline or yolkglands, and cement-glands. In most, though not in all, there is a single or paired germarium (ovary), in which the ova are formed, and a set of vitellaria or vitelline glands, producing material which surrounds each of the mature impregnated ova before it becomes enclosed in its shell. In some, on the other hand, ova and vitelline matter are formed in the same organ-the germ-vitellarium. The shell-glands are so named because they are usually supposed to secrete the chitinoid substance of the egg-shells; but the share which they take in this process is uncertain. The cement-glands secrete a viscid material for causing the eggs to adhere together, enclosing them in a cocoon or fastening them to some foreign body. The oviduct is the passage by which the ova reach the exterior from the ovary; but an enlarged part of this passage, into which ducts of the shell glands open, is distinguishable as the ootype, while a terminal part, leading to the female aperture may be modified as a vagina. In some cases (Heterocotylean Trematodes) there is a vagina or a pair of vaginæ in the shape of a passage, or a pair of passages, distinct from the oviduct and opening independently on the exterior. A uterus in the form of an enlarged part of the oviduct or of an outgrowth from the latter or from the atrium is very usually developed for the reception of the completed eggs. A special sac or bursa copulatrix, lined with spines, acts as the female copulatory organ. A sac, the receptaculum, opening into the oviduct or into the atrium (Figs. 201, 202, r.v.), may serve as a reservoir for the semen received in copulation or for the vitelline matter or yolk, or for surplus reproductive

1 The term cirrus is here restricted to cases in which the terminal part of the male duct, often provided with spines and other chitinous structures, is involuted within a sheath when at rest. 
material. Male and female ducts sometimes have separate and independent openings; but very commonly there is a common chamber or genital atrium into which both lead, opening on the exterior by a single aperture.

In the Polyclad Turbellaria (Fig. 212) the testes are numerous, and there are a corresponding number of fine tubes which combine to form the two vasa deferentia, leading to the male aperture with its penis. The latter is sometimes multiple. The ovaries consist of numerous small rounded masses of cells, and there are no separate yolk-glands. Numerous narrow oviducts lead from the ovaries, and unite to form larger ducts; these, in turn, open into elongated uteri, in which numerous eggs collect. The uteri open into a median egg-duct, with which the ducts of the shell glands communicate (ootype), and in which the eggs receive their chitinoid investment. This leads to the female aperture, a part of it being, in some cases, surrounded by a muscular sheath to form a bur'sa copulatrix.

In many cases the egg-duct gives off posteriorly a narrow duct which usually terminates behind in a vesicle known as the accessory sac or receptaculum seminis, which may be double. In a few Polyclads this duct opens on the exterior on the ventral surface some distance behind the main female aperture, in one instance on the dorsal surface. A genito-intestinal canal connecting this duct with one of the intestinal cæca has been found in one Polyclad. In most cases male and female apertures are distinct from one another, the former being situated in front of the latter. But sometimes, though rarely, both lead into a common chamber or atrium with a single opening on the exterior.

In the Tricladida (Fig. 213) there are also numerous testes, but the fine tubes connecting them with the two rasa deferentia are absent. There are two germaria, situated far forwards, and numerous yolk-glands. Two oviducts, into which the yolk is discharged from the yolk-glands by a series of lateral apertures, lead from the ovaries to unite in a median ootype or vagina, receiving the ducts of glands which may secrete the substance of the cocoon. The condition is thus intermediate between that observable in most of the Rhabdocœles and that which characterises the Polyclads. Though germaria and vitellaria are separate, they have a common duct, and might be regarded as distinct lobes of one germo-vitellarium. A uterus is present, formed as an outgrowth of the vagina or of the atrium, or as an independent sac or pair of sacs opening independently on the exterior. There may be a receptaculum seminis, and in some there is a duct of communication between this and the intestine (genito-intestinal canal). A common genital atrium with a single external aperture receives the ducts of both sexes.

In the Rhabdocceles (Figs. 211 and 215) there are usually only 
two compact testes and two vasa deferentia leading to the unpaired male aperture at the extremity of the cirrus. The prostrate or granule glands-a set of unicellular glands, which secrete round,

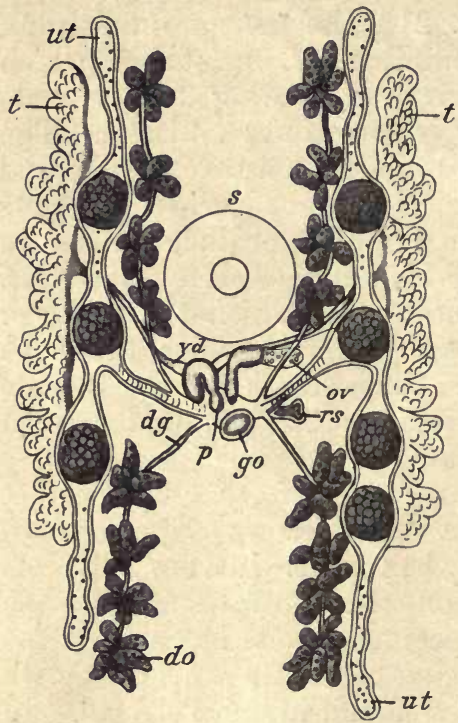

FIG. 215.-Reproductive organs of Mesostomum Fhrenbergii. $d g$. duct of vitelline glands; do. vitelline glands; go. common reproductive aperture ; ov. ovary; p. cirrus; $r s$ receptaculum seminis; 8 . pharynx; $t$., $t$. testes ; $u t$. uterus; $v d$. vas deferens. (From Claus, after von Graff and Schneider.) bright granules destined to mix with the sperms-are specially well developed in the Rhabdocœles, and are present in some other Turbellaria and in certain Trematodes. Ovaries (germvitellaria) alone occur in some, separate germaria and vitellaria in others; there are either two germaria or one only. A receptaculum seminis may be present as a swelling or diverticulum of the main female duct, or of the atrium. The terminal part of this duct may form a muscular vagina, or there may be a muscular bursa copulatrix developed from the wall of the atrium. A uterus is present in most cases as an outgrowth from the wall of the atrium. Male and female ducts have a common chamber or genital atrium with a single external opening.

In the Acola there are in nearly all cases separate male and female apertures. The two testes are divided into numerous small lobes. There are no vitellaria in most cases - the two ovaries producing large ova containing abundant food-yolk. Oviducts are absent in most cases. Into the main female genital passage opens a peculiar single or double sac or bursa, usually provided with chitinous structures.

The Trematodes nearly all have two testes, usually compact, sometimes branched; in a few instances there are four. The vasa deferentia unite into a median duct, which is dilated at the base of the cirrus to form a vesicula seminalis. There is a single oval or branched germarium, and two sets of vitelline glands. A canal termed Laurer's canal in some Malacocotyleans, such as some species of Distomum, leads from the exterior to the oviduct or vitelline duct. 'This may be replaced by a receptaculum seminis, or both structures may co-exist. The distal part of the oviduct is enlarged to act as a uterus. In the Heterocotylea there is a vagina, which is sometimes paired, opening 
on the surface independently of the uterus: internally it communicates with the oviduct through the main vitelline duct. In some of the Heterocotylea there is a genito-intestinal canal occupying a corresponding position to Laurer's canal, but opening into the intestine. In the Aspidocotylca this is replaced by a stalked yolk-rcceptacle. There is nearly always a genital atrium common to the ducts of both sexes.

In the Temnucephalea (Figs. 201, 202) there is a genital atrium and a single genital aperture. There are two pairs of compact testes; the right and left vasa deferentia unite in a vesicula seminalis, and granule-or prostate glands are well developed. The cirrus has a chitinous tube and a variety of eversible spines. There is a single compact ovary; the oviduct has connected with it a large receptaculum, and dilates posteriorly to form an ootype into which the shell-glands open. Actinodaclylella (Fig. 202) alone has a bursec copulatrix (b. c.).

In the ordinary Cestodes each segment or proglottis contains a set of reproductive organs similar to those of a Trematode. There may be a single genital aperture leading into a genital cloaca, into which both male and female ducts open; or the male and female apertures may be distinct. The testis is divided into numerous minute lobes, from which proceed a number of fine canals joining together to form the vas deferens, at the extremity of which is the chitinous cirrus. There are two germaria, and either a single vitelline gland, or two. The oviduct has its origin in a sort of isthmus connecting the two germaria. It receives a narrow fertilising duct from the receptaculum seminis and the vitelline ducts and becomes surrounded by a rounded mass of shell-glands to form the ootype, which is not definitely enlarged. Further forward it gives off the uterus. The latter is at first a simple cylindrical outgrowth from the oviduct, but it usually becomes large and may be extensively ramified. It has no external opening in most instances, so that the eggs only escape from it by the breaking down of the proglottis or by dehiscence. But in some (e.g., Dibothriocephalus), it has an independent external opening. The female aperture leads into a narrow canal - the vagina-which ends in a receptaculum seminis from which the narrow fertilising duct conveys the sperms to the oviduct.

The development of some of the Platyhelminthes (Rhabdocœla, Monogenetic Trematodes) is direct-i.e., not complicated by the occurrence of a metamorphosis; in the Digenetic Trematodes, the Cestodes, and some of the Planarians a metamorphosis occurs.

The eggs of the Polyclads, each of which consists merely of the fertilised ovum (oosperm) usually enclosed in an egg-shell, are, in most instances, laid in large numbers embedded in a plate of slimy secretion. The ovum (Fig. 216) divides first into two equal parts, then into four. From each of these four cells is then 
separated off a small cell. The embryo at this stage consists of eight cells, four large-the megameres, and four small-the micromercs. The four micromeres increase rapidly by division, and extend over the embryo, forming a layer, the cetoder'm, completely covering it in all parts except for a median fissure, the blastoport, which runs along what is destined to become the middle ventral line: this soon closes up. The ectoderm cells develop a coating of cilia. The four megameres have previously given off

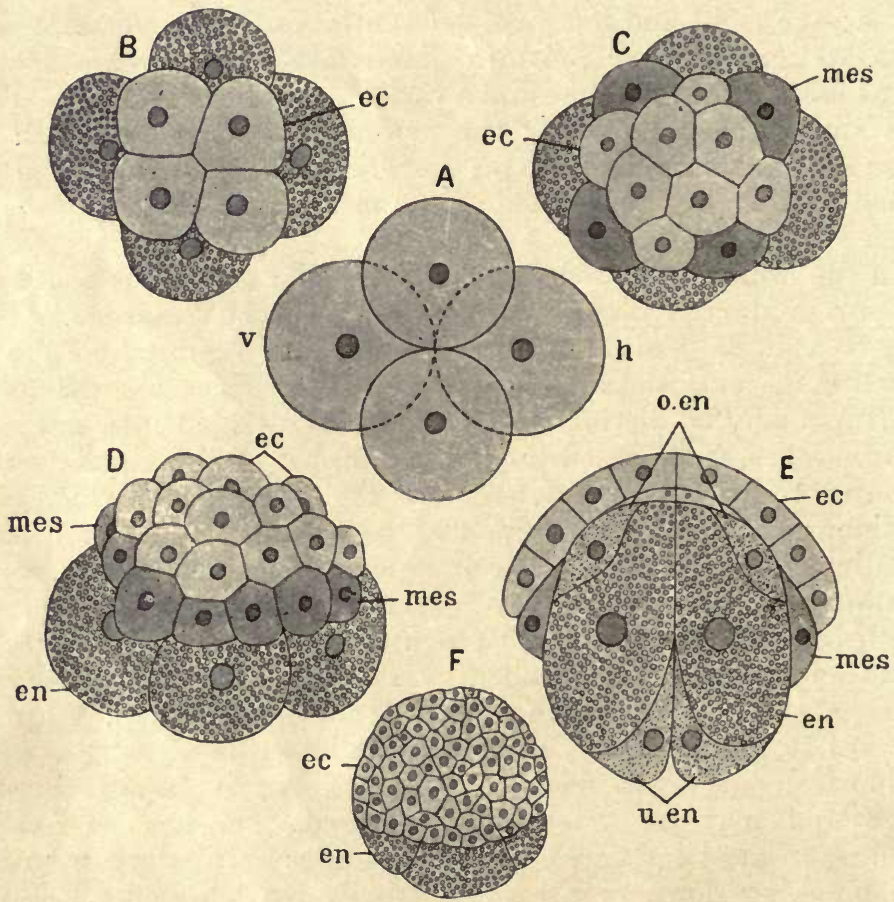

Fia. 213.- Early stages in the development of a Polyclad. A, stage of four acells, of which those lettered $v$ and $h$ correspond to the anterior and posterior portions of the body; $B$ to $D$, later stages; $B$ and $C$, seen from above; $D$, from the side; $E$, earlier, and $F$, later stage of epibolic gastrula, lateral view. ec. ectoderm; en. endoderm; mes. mesoderm; o. en. and $u . e n$. upper and lower endoderm. (From Korschelt and Heider, after Lang.)

four more small cells or micromeres, which increase in number by division, and eventually form the middle layer or mesoderm of the embryo. These extend over the surface below the ectoderm as four mesodermal bands which subsequently fuse together to form a continuous layer. The megameres give off a number of additional micromeres which form the endoderm layer, giving rise to the epithelium of the intestine ; finally the megameres become disintegrated, and their substance goes to nourish the cells of the developing embryo. The process by which the germinal layers have 
become formed is, as in the Ctenophora (p. 218), a process of epibolic gastrulation. The brain is developed from a pair of thickenings of the ectoderm : these unite into a common mass from which the longitudinal nerves are formed as backward outgrowths. The mouth is developed as an ingrowth from the ectoderm in the position of the former blastopore, the involuted epidermal cells giving rise to the epithelium of the pharnyx and pharyngeal sac, while the muscular tissues of the wall of the pharnyx are formed from surrounding mesodermal elements. The intestine is at first simple in form; the cæca are developed as a result of the formation of vertical mesodermal septa which, growing inwards, constrict the enteric wall and the enclosed mass of nutrient material. The embryo, which has assumed an ellipsoidal shape, becomes flattened in the dorso-ventral direction, and, having absorbed the greater part of the nutrient matter, escapes by rupture of the egg-shell.

In many cases the embryo develops into a characteristic larval form, such as that known as Müller's larva (Fig. 217). It assumes
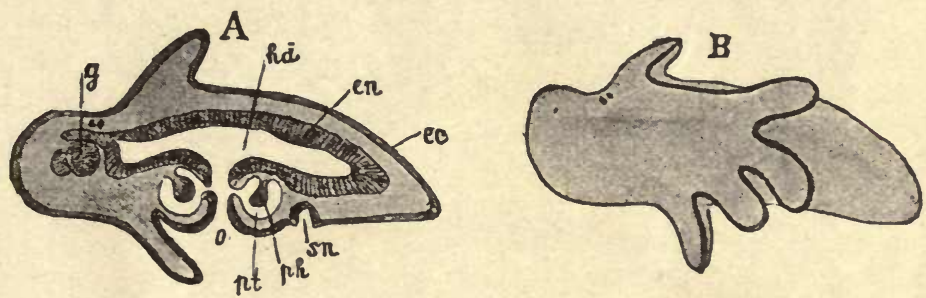

FIG. 217.-Muiller's larva. $A$, longitudinal section; $B$, lateral view. ec. ectoderm; en. endoderm; g. brain; hu. enteron; o. mouth; $p h$. pharynx; $p t$. pharyngeal pouch; $8 n$ sucker. (Erom Lang.)

an oval shape, with a series of eight elongated processes, covered with long cilia, and connected together by a ciliated band. There are eye-spots at the anterior end and a mouth in the middle of the ventral surface. The form of the body alters after a time, becoming gradually longer and flatter, and the arms are gradually reduced in length, till, eventually, they become completely absorbed.

The development of the Triclads is very different from that of the Polyclads. Each egg-capsule or cocoon encloses a number of oosperms and a quantity of yolk-cells. After segmentation a blastoderm is formed composed of a rounded mass of cells surrounded by the yolk-material, the cells of which become more or less fused. Around the periphery of the blastoderm a layer of cells form a thin membrane-the ectoderm. About the middle appears a rounded group of cells which passes to the periphery and becomes connected with the ectoderm; a cavity is formed in its interior and it becomes converted into the 
embryonal pharynx. Meanwhile a group of four cells enclosing a cavity are modified to form the foundation of the endoderm, and increasing in number give rise to the embryonal intestine, into which the embryonal pharynx soon opens, the latter opening on the exterior by a mouth aperture.

The embryonal pharynx (Fig. 218, pli) has the function of swallowing the yolk-matter with which the embryonal intestine becomes greatly distended. At a subsequent stage the embryonal pharynx and intestine are aborted, and the former comes to be represented merely by a mass of cells. In this a cavity arises - the cavity of the permanent pharynx. 'The permanent intestine

A

B

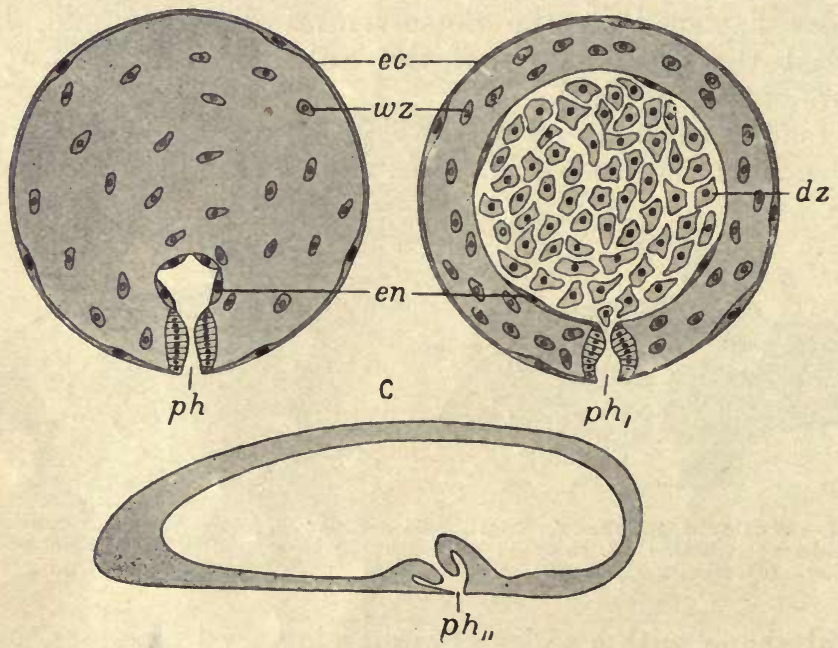

Fra. 218. - Sections through embryos of Dendrccœlum la cte um (somewhat diagrammatic). $d z$, yolk-eells ; $e c$, ectoderm ; en, endoderm ; ph', enlbryonal pharynx ; ph", permanent pharynx : wz, wandering cells. (From Korschelt and Heider, after Hallez.)

becomes formed and the cavity of the pharynx opens into its lumen. Subsequently the permanent mouth makes its appearance. The brain is formed in the thickness of the blastoderm, and thus appears to be of mesodermal origin, not of ectodermal, as in the Polyclads.

In some Rhabdocœles (certain species of Mesostoma) two distinct kinds of eggs are formed-summer and winter eggs. The oosperms are deposited singly, and each, together with a mass of yolk-cells, is enclosed in a chitinous, usually stalked, shell. Segmentation takes place very much as in the Polyclads. No embryonal pharynx is formed, the permanent pharynx performing the function of swallowing the yolk-material: it appears to be of endodermal derivation. The intestine arises from a group of cells-the 
primitive endoderm-among which a cavity appears. But in some Rhabdocœles no definite intestinal epithelium is developed, and the syncytial mass which represents it is only to be distinguished from the surrounding mesodermal syncytium by its enclosing the remains of the yolk. No metamorphosis is known to occur.

An account has already been given (p. 244, Fig. 190) of the development and metamorphosis of the Liver-Fluke (Fasciola hepatica) which may be looked upon as typical of the Digenetic Trematodes in general. There is thus to be recognised in the Digenetic Trematodes an alternation of generations comparable to that which has been described as so general in the Cœlenterata. In the Trematoda, however, it is to be observed, it is an alternation of a sexual, not with an asexual, but with a parthenogenetic generation (the sporocyst), the ova of which develop into a second parthenogenetic generation (the rediæ); and these finally produce larvæ (the cercariæ) capable of developing into the sexually mature form. The term heterogeny is applied to a life-history of this kind, in which several distinct generations succeed one another in a regular series.

In some of the Distomidæ the eggs, instead of becoming free as in the case of the Liver-Fluke, are taken directly into the digestive canal of the intermediate host, and there hatched out. The sporocyst stage may take the form of a branching tube in the interior of which cercaria are developed-the redia stage being omitted. Sometimes the sporocyst becomes directly developed into a redia instead of giving rise to a generation of the latter by such a process of internal development as that described in the case of the Liver-Fluke. The cercariæ in most Digenetic Trematodes only develop further if they succeed in establishing themselves in a second intermediate host instead of merely becoming encysted on the surface of herbage, as in the case of the Liver-Fluke. The cercariæ of different Trematodes differ greatly, particularly with regard to the nature of the tail. In some forms the cercaria is tail-less: such cercariæ do not become free, but are taken directly - with the intermediate host in which they have been developedinto the digestive canal of the final host.

Among the Heterocotylea, Gyrodactylus (Fig. 200, A) is viviparous, and the remarkable phenomenon is observed that the embryo $\left(h^{\prime}\right)$, while still within the body of the parent worm, develops another embryo $\left(h^{2}\right)$ in its interior, and this again develops a third. The rest of the Heterocotylea deposit eggs each of which, within a chitinous shell, contains an oosperm and a number of yolk-cells. Usually there is a stalk and often an operculum. In general the development appears to be direct; but Polystomum passes through a larval stage with five rows of cilia, and in Diplozoon paradoxum, a parasite on the gills of certain 
fresh-water fishes, in which there is also a ciliated larval'stage, the young animals do not become sexually mature until two of them have permanently united with one another.

Temnocephala produces relatively large eggs, stalked or sessile, with a thick chitinous shell, enclosing a single oosperm and a mass of yolk-cells, which later become fused into a continuous mass. Segmentation results in the formation of an irregular, massive blastoderm composed of cells of several sizes. In this collects a rounded group of larger cells, in the middle of which a space appears (Figs. 219, 220). The space (endocole) increases greatly in size, the boundary cells becoming spread out to form a thin layer, and approaches the periphery of the egg. A part of the

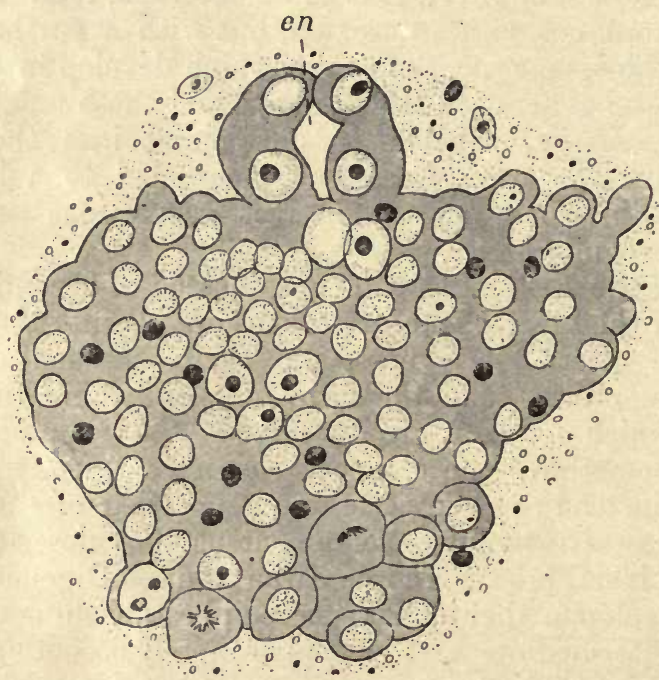

Fia. 219.-Section through the blastoderm of Temnocephala, showing early stage of endoccele (en).

endocœle becomes subsequently rounded off to form the internal lining membrane of the pharynx, the cavity of which remains cut off from the exterior by a partition, which is the outer part of the wall of the endocœle, until the young worm is ready to leave the egg. A short prolongation backwards from this cavity ends blindly in a mass composed mainly of yolk, still containing degenerate nuclei and cells which have wandered into it from the blastoderm. Only at a late stage, when the yolk has become taken up, is an arrangement of cells recognisable in the form of an intestinal epithelium (endoderm) enclosing a lumen (intestinal lumen). An ectoderm likewise does not appear as an embryonic layer, the epidermis only appearing late, and extending over the surface evidently by modification in situ of cells that have become 
separated from the main body of the blastoderm. The rudiments of the brain are formed in the blastoderm near the wall of the endocœle, and thus have no connection with an ectoderm. The excretory sacs and main vessels are formed from a small number of large cells connected with the wall of the endocœle: subsequently these rudiments shift their position to the dorsal surface on which the sacs form their permanent apertures piercing the epidermis. There is no metamorphosis of any kind-all the organs, including even the male part of the reproductive apparatus, being well advanced towards full development before the young animal leaves the egg.

The egg of a Cestode is similar in essential respects to that of a Trematode: there is a tough, chitinoid membrane or egg-shell, which encloses not only the ovum but a number of yolkcells. The result of segmentation is the formation of a superficial layer of cells (ectoderm) and a central mass, all enclosed in a membrane composed of a single layer of cells thrown off when the embryo escapes from the egg. The ectodermal cells become ciliated, so far as is known, only in Bothriocephalus; in the others they are thrown off or ultimately absorbed with-

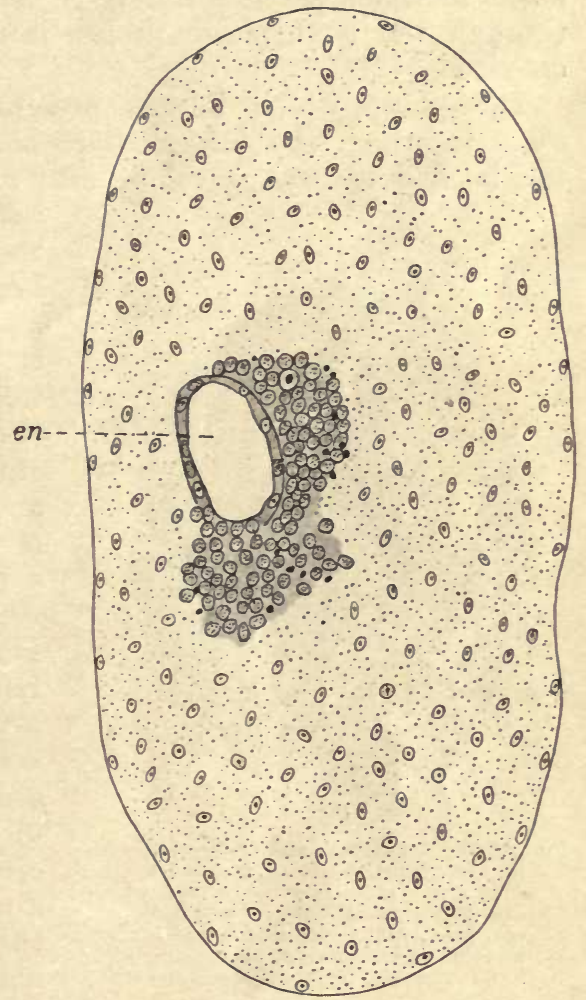

FIG. 220.- Longitudinal section through the entire egg of Temnocephala with the shell removed, showing blastnderm with developing endocoele $(e n)$.

out developing cilia. The central mass of cells alone forms the embryo. The embryo, while still consisting of a small number of cells, develops a series of six chitinous hooks. These early changes all take place in the majority of Cestodes while the egg is still in the uterus of one of the most posterior of the proglottides of the parent worm. When the proglottis in question becomes separated off, and has passed out from the body of the final host, the eggs are discharged.

In order that development may proceed further, the embryo 
must in most cases reach the interior of a second or intermediate host. This is a passive migration, since the embryo of the ('estode is still confined within the egg-shell, and the transference has to take place in the water or food. The digestive fluids of this intermediate host dissolve the egg-shell and set free the contained sixhooked or hexacanth embryo, which bores its way by means of its hooks to some part of the body in which it is destined to pass through the next phase in its life-history, and there becomes encysted.

The phase which follows presents two main varieties. In cases in which the intermediate host is an invertebrate animal the hooked embryo develops into a form to which the name of

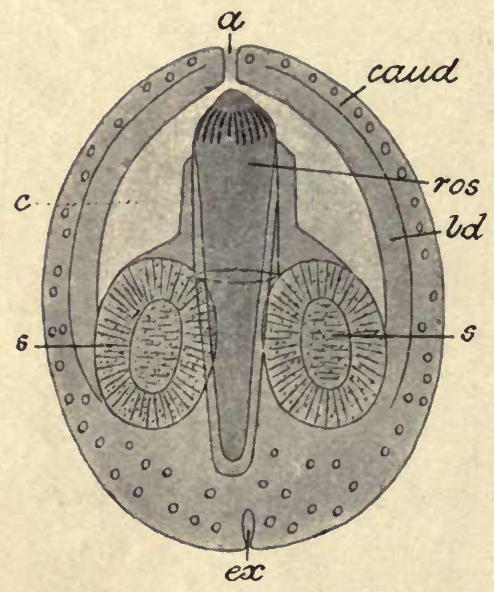

FiG. 221.-A Cysticercoid (Polycercus) with the head and rostellum enclosed by the caudal vesicle. $a$. aperture through which evagination takes place; brl. body; $c$. cavity of cyst; crud. caudal vesicle ; $e x$. aperture of excretory system ; ros. rostellum ; s. sucker. (After Haswell and Hill.) cysticercoid is given; when, on the other hand, the intermediate host is a vertebrate, the form assumed is nearly always that termed cysticcrcus, or bladderworm. The cysticercoid form (Figs. 221 and 222) is to be regarded as the more primitive and less modified. Cysticercoids of various tape-worms occur in a great variety of different invertebrates-e.g., Insects of all kinds, Water-fleas, Centipedes, Earthworms. The hooked embryo loses its hooks and develops into the cysticercoid in some part of the invertebrate intermediate host. 'The cysticercoid consists of three partsa tape-worm head or scolex with the hooks and suckers of the mature worm, a so-called body, and a caudal vesicle. Sometimes there is a tail recalling to some extent the tail of a cercaria. Sometimes the caudal vesicle is absent: when present, either from the first, or as a result of later changes, it encloses the head as well as the body after the manner of a cyst. While undergoing these changes the cysticercoid is usually enclosed in an adventitious cyst formed for it by the tissues of its host, but it often lies free in the body-cavity. The transference to the final host is effected by the intermediate host, or the part of it containing the cysticercoid, being taken into the alimentary canal of the final host. Sometimes, if the intermediate host is a relatively small animal, such as a water-flea, this may take place "accidentally" ; in other cases the invertebrate intermediate host actually forms the food 
of the final host. Thus a cysticercoid having as an intermediate host an Earthworm is taken with the latter into the alimentary canal of a Sea-Gull-its final host. In this way the cysticercoid is set free in the alimentary canal of the final host, the head becomes pushed out from the enclosing caudal vesicle and body (probably owing to the stimulus of the higher temperature), so that the suckers and hooks come into play and attach the young tape-worm to the wall of the alimentary canal.

The cysticcrcus or bladder-worm differs from the cysticercoid mainly in its much greater size and in the development of a relatively large caudal vesicle or caudal bladder. When the hooked embryo has reached that part of the vertebrate host in which it is destined to develop into the cysticercus it undergoes a remarkable change; it becomes greatly enlarged, and a cavity, filled with fluid or with a very loose form of connective-tissue, appears in its interior, so that it assumes the appearance of a relatively large bladder. On one side of this bladder appears a small invagination with a cavity opening freely on the exterior. On the bottom of this is formed an elevation projecting into its interior; this is the rudiment of the rostellum on which the hooks are borne; at its base, on the inner surface of the side walls of the invagination, appear the suckers. When inverted this invagination corresponds closely

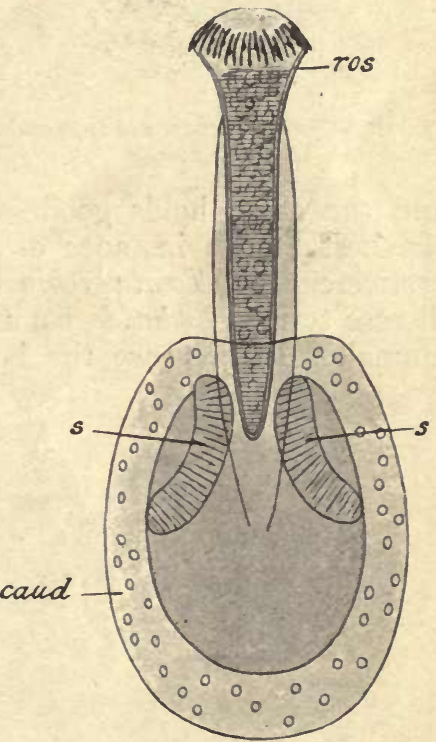

FIG. 222.-A Cysticercoid with the rostellum evaginated. ros. rostellum; s., s. suckers; caud. caudal vesicle. (After Haswell and Hill.) with the head and body of the cysticercoid; the bladder corresponds to the caudal vesicle. Thus the chief difference between a cysticercus and a cysticercoid is that in the former the caudal vesicle is relatively very large and that the order of development of the parts is somewhat modified.

A very small number both of cysticercoids and cysticerci multiply by proliferation-by the formation of more than one tape-worm - head from one embryo. In the few instances in which this occurs among the cysticercoids the hooked embryo gives rise, not directly to a cysticercoid, but to a mass of cells from which are given off a number of buds, each developing into a cysticercoid with the three parts already described. One such form occurs 
in certain Earthworms, another in a Myriapod (Glomeris limbatus).

Tania conurus of the Dog has a bladder-worm stage in the Sheep and Rabbit which gives rise to several tape-worm heads,

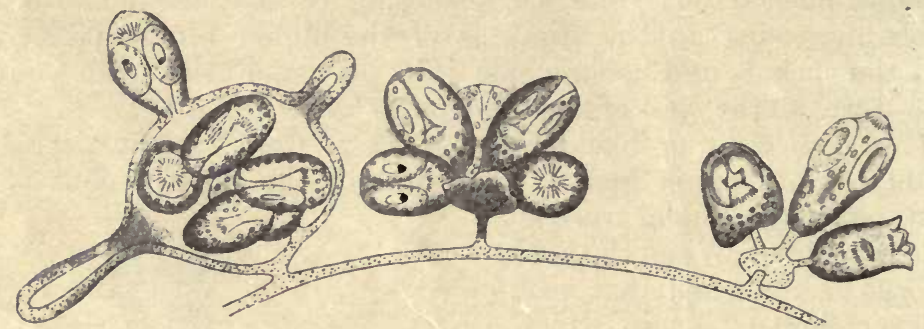

FIG. 223.-Cyst of Trenia echinococcus with the developing daughter-eysts and scolices. (After Leuckart.)

and the same holds good of Tonia serialis from the Fox. But the best known instance of multiple production of scolices in a cysticercus is Tania echinococcris-well known as cause of the disease termed hydatids, common in Man and in various domestic animals. In this case the hooked embryo develops into a large

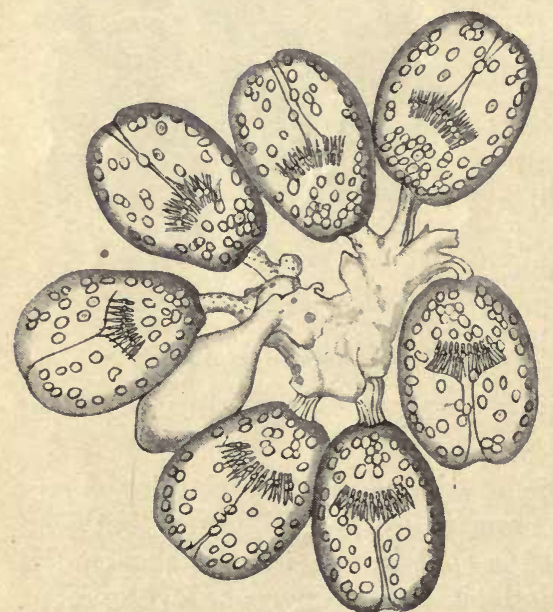

Fia. 224.-Seolices of T. echinococcus. (After Cobbold.) mother-cyst, from the interior of which daughter-cysts are budded off (Fig. 223). Eventually from the walls of these daughter-cysts there are formed numerous tape-worm heads, or scolices (Figs. 224

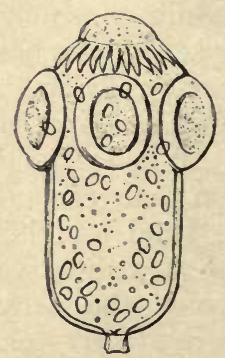

Fif. 225.-Separate seolex of T. echinococcus. (After Cobbold.)

and 225), which, when fully formed, assume the appearance of cysticercoids withont the caudal vesicle. These are readily detached, and, should the organ in which the cyst has been developed be devoured by a Dog-which is the final host of the parasite-some of these scolices become attached to the wall 
of the intestine and develop into the adult Tania echinococcus - which are very small as compared with the size of the cyst and as compared with other tape-worms. The eggs, passing out with the fæces of the Dog, may be taken into the digestive canal of Man or of one of the domestic animals, and the minute embryos escaping, reach some organ, such as the liver or lung, in which they are capable of developing into a comparatively enormous cyst.

Asexual reproduction also occurs in some Platyhelminthes. In some Rhabdocœle Turbellaria (Microstomum) a process of budding (Fig. 226) results in the formation of strings of sexual individuals which may eventually separate; the new bud is always formed from the posterior end of the last individual of the string.

The sporocyst stage in the Trematodes may, as already mentioned, multiply by budding or fission. The formation of new proglottides in the Tape-worm may be looked upon either simply as growth accompanied by segmentation, or as asexual multiplication, according as we regard the proglottides as segments of a simple animal or as zooids of a colony. There is this essential difference between the formation of proglottides and the asexual multiplication by budding in Microstomum, that in the former case the proglottides, when they have been formed by segmentation of the undivided part behind the head, do not in turn give rise by budding to new proglottides. Spontaneous transverse fission

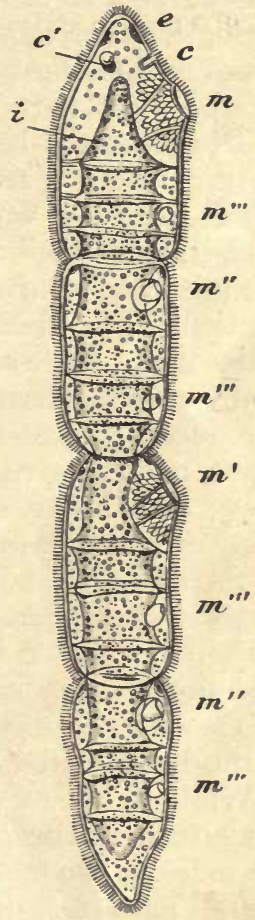

FIG. 226.-Process of budding in Microstomum. $c_{\text {., }} c^{\prime}$. ciliated groove; e. eje-spot; i. intestine; $m ., m^{\prime}, m$. $^{\prime \prime}, m_{.}{ }^{\prime \prime \prime}$ mouth. (After Von Graff.)

has been observed in certain Tricladida, and is often followed by the regeneration of the lost portion.

\section{Distribution, Mode of Occurrence, and Mutual RELATIONSHIPS.}

Of all the great groups of the animal kingdom above the Protozoa the Platyhelminthes are the widest in their distribution. Members of the phylum occur on land, in fresh-water down to the bottom of some of the deepest lakes, on the sea-shore, in the deep sea, and on the surface of the ocean; and parasitic Flat-worns live, in one phase or another, in animals of nearly every class of the Metazoa. 
As regards their mode of life, they present almost every possibl, gradation between free-living forms which procure their food-consisting of minute animals and plants - by their own exertions, an forms that are only capable of living in a special part of the interior of a certain other animal, and are quite incapable of procuring food for themselves, living by the passive absorption of the juices of their host or of its digested food. The Turbellaria are for the most part free living, and their food consists of sinal Crustacea or the larvæ of larger forms, Insect larvæ, Water-mite. Rotifers, small Worms, and the like; or sometimes of Diatoms anc minute Algæ of various kinds. Some, however, live a life of truc parasitism. Such are certain Rhabdocaeles which are parasitic i the alimentary canal of various Holothurians and Gephyreans ( $v$. Sections IX. and X.). In these there is correlated with the inactive mode of life a tendency to degradation of structure, a degradation which is characteristic of parasites in general: the pharnyx is reduced in size as compared with that of non-parasitic allier forms, not being required for the capture and swallowing of livin prey; and the eyes, useless to an animal living in complete darkness, are absent. Some of the Turbellaria, though not parasitic in the strict sense, live in a state of commensalism with another, larger animal : that is to say, are more or less constantly associated with it, living on its surface or in one of its cavities that open freely on the exterior, and often sharing its food. An example of this mode of life is the Triclad Bdelloura, which lives on the surface of the King-Crab (Limulus).

While a free existence is the rule in the Turbellaria, trut parasitism is the rule in the Trematodes, and is universal in the Cestodes. The majority of the Monogenetic Trematodes are external parasites, living on a part of the outer surface of a larger animal: and feeding on mucus and other secretions of the integument. Many are parasites on the gills of Fishes. A few however, inhabit the interior of various organs, and are trut internal parasites: one, for example (Polystomum), lives in the urinary bladder of the Frog; another (Aspidogaster) lives in the pericardial cavity of a Fresh-water Mussel. At least one family of Trematodes (the Temnocephalea) are not parasites at all in the strict sense of the term, living on the surface of the "host" animal, depositing their eggs there, and being carried about by it, but subsisting on minute living animals captured in the water.

The Digenetic Trematodes are all internal parasites, and in the adult condition inhabit, in nearly all cases, the alimentary canal, liver, or lungs of some vertebrate animal, swallowing the digested food or various secretions of their host. But, as mentioned before in the account given of their development, they are internal parasites, not only in the adult condition, but throughout the greater part of their life. After a short period of freedom as 
ciliated larvæ, they again enter into a state of parasitism as sporocysts or redix in a second host; and, after a second free interval as cercariæ, may enter the body of a third host to become encysted. The second host is, very generally, a Mollusc, and the cercaria may become encysted in the same animal.

The Cestodes are, of all the Platyhelminthes, those that are most modified in accordance with the condition of internal parasitism in which they remain throughout life. The adult Cestode is lmost always an inhabitant of the alimentary canal of a verterate. The intermediate host is frequently also a vertebratecommonly of a kind which is liable to become the prey of the final host. In the case of Tcenia crassicollis of the intestine of A $i$ domestic Cat, for example, the cysticercus-stage occurs in the livers of Rats and Mice; the cysticercus of T'cnia serrata of the Dog is found in Hares and Rabbits. But in many cases the intermediate host is an invertebrate. In either case the passage from one host to another is a passive translation, not an active igration as in the Trematodes.

A few human parasites belong to the Trematoda, but none that are of very common occurrence among Europeans. Fasciola hepatica has occasionally been found in the human liver; Distomum rathousii is a common intestinal parasite in China; Opisthorchis sinensis occurs in the liver of Man in China and Japan; Dicrolium lanceolatum and various other species of the genus occasionally occur in the human subject. Schistosomum homebibium and S.japonicum, which differ from most other Trematodes in being unisexual, are found in the human portal system of veins various parts of Africa, in Arabia, the Philippines, and Japan. Eggs with contained larvæe are voided with the urine, and if they reach water, the larvæ may gain access to the human host by being swallowed in drinking water or by perforating the skin.

The commonest human Cestode parasites among Europeans are T'cenia solium and 'T'. saginata (othervise called 'T'. mediocanellata). The cysticercus stage of $T$. solium (Cysticercus cellulosa) occurs, as already stated, chiefly in the muscles of the Pig, that of ' $T$ '. saginala in the muscles of the $\mathrm{Ox}$; and the relative prevalence in different countries of these two Tape-Worms varies with the habits of the people with regard to flesh-eating: where more swine's flesh is eaten in an imperfectly cooked state Tania solium is the more prevalent; where more beef, T. saginata. Bothrioceplualus latus, a very large tape-worm without hooks, and with a pair of longitudinal sucking-grooves on the head instead of ordinary suckers, is a common human parasite in eastern countries. Its cysticereus, which is elongated and solid, occurs in the Pike and certain other fresh-water Fishes.

Of all the Cestode parasites of man, however, the most formidable is one which occurs in the human body, not in the sexually 
mature or strobila condition, but in that of the cysticercus. This is Tania echinococcus, the presence of which produces what is termed hydatid disease (p. 282). The adult 'T'ania echinococcus is a very small tape-worm with only three or four proglottides, occurring in the intestine of the domestic Dog. The eggs passing out with a liberated proglottis in the foeces, may reach the alimentary canal of Man uninjured in drinking-water, on the surface of salad vegetables, and the like; and, the egg-shells becoming dissolved, the contained hooked embryos bore their way to the liver or the lungs or some other organ. Arrived at its final destination, the embryo develops into a cyst, which may become of enormous size. In the interior of the primary or mother-cyst are developed a number of sccondary or daughter-cysts, and from the walls of these, both internally and externally, are formed very numerous scolices in the way already described (p. 282). Hydatid cysts are very common in some domestic animals (Oxen, Sheep), as well as in Man. Various other Cestodes occur in the bladder-worm stage occasionally in Mane.g., the Cysticercus cellulosa of T'ania solium.

The most primitive of the Platyhelminthes are, without doubt, some of the simplest Turbellaria, and it is among these that we must look for the nearest existing relatives to the Coelenterata. In none, however, is the relationship very close. Caloplana and Ctenoplana (p. 225) are probably rather to be looked upon as Ctenophores specially modified in accordance with a creeping mode of progression than as intermediate forms between Ctenophores and Turbellaria. The relationship with the Cœlenterata is shown, perhaps, most strikingly when we take into account the development of the Turbellaria, in the earlier stages of which there is to be recognised a marked tendency towards a radial symmetry. In their development the Turbellaria, that is to say the Planarians, show some special points of resemblance to the Ctenophora; the ectoderm cells are formed and spread over the rest in a similar way, and the bands of cilia have a disposition and mode of movement that strongly bring to mind the ciliary swimming plates of the Ctenophora. But though there is much to be said in favour of the view that the Turbellaria and the Ctenophora were derived from a common, not very distant stock, the latter are too specially modified to be looked upon as the direct ancestors of the former.

The connection between the T'urbellaria and the Monogenetic Trematodes is very close-so much so that it is difficult to give any characters of universal occurrence distinguishing all the members of the two classes. The Trematodes are, in fact, to be looked upon as Turbellaria some of whose external characteristics -and, in the case of the Digenetica, whose life-history - have been specially modified in accordance with a parasitic mode of life. It is not unlikely that the Trematodes may be a polyphyletic group- 
i.e., that different families may have become developed from different families of Turbellaria altogether independently, some of them appearing to be nearer the Rhabdocœles, others nearer the Polyclads, and others, again, nearer the Triclads, in the majority of their characters.

The remarkable life-history of the Digenetic Trematodes is, as already pointed out, to be looked upon as a special form of alternation of generations - the alternation of a sexual with a poedogenetic and parthenogenetic generation (heterogeny). The sporocyst and redia are to be regarded as intercalated stages-as cercarixe which exhibit pædogenesis. The cercaria is the characteristic larval stage of the Trematodes, and corresponds to the cysticercus or cysticercoid of the Cestode. The most important difference between these is in the presence in the former of an enteric cavity, and its absence in the latter. There seems to be something to be said in favour of the view that the enteric cavity of the cercaria is represented by the frontal sucker of some scolices, and by the rostellum of the majority.

Between the adult Cestodes and the Trematodes an intimate relationship is traceable. Caryophyllaus (Fig. 206) is a Cestode
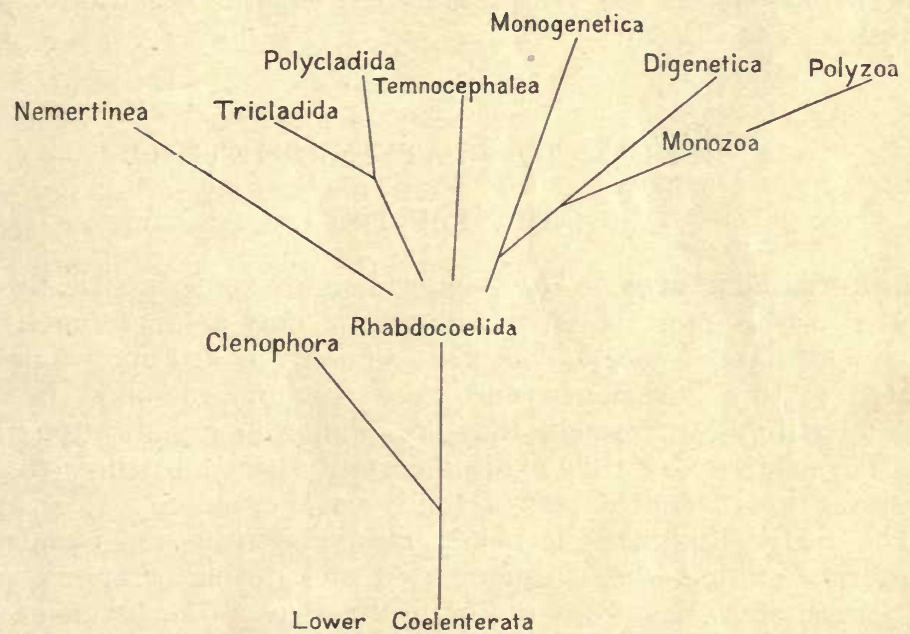

FIG. 227. - Diagram of the relationships of the Platyhelminthes (together with the Nemertinea).

which, but for the absence of an enteric cavity and the want of organs of adhesion at the posterior end, is not far distant from the Trematodes; and the same might be said of Gyrocotyle (Fig. 207), Amphilina, and Archigetes (Fig. 208). ${ }^{1}$ The most

1 It is possible, however, that in the last two forms we have to do with larval Cestodes which have failed to reach the mature stage, and have undergono a precocious development of the sexual apparatus. 
important differences between a Cestode and a Trematode, in addition to the absence of an enteric cavity in the former and its presence in the latter, is the occurrence in the Cestodes of strobilation. Ligula in a certain sense forms a connecting link in this respect between the Trematode and the ordinary Cestode, the bedy being elongated and the reproductive organs repeated as in the normal Tape-Worm, but there being no corresponding division of the body into a string of definitely separated proglottides.

Of importance in connection with the subject of the relationship of Trematodes and Cestodes is the question whether the scolex of the latter is at the end corresponding to the anterior end of the former, or whether it is the free end of the strobilia that is ir reality anterior. In favour of the latter conclusion is the facc that the hooks of the hexacanth larva, developed at its anterior end, are found in the cysticercoid to lie in the tail region, i.e., th region most remote from that which develops the scolex, and th at the end which should represent the free extremity of strobila. On the other hand, the specialisation of the nervous system to form quite definite and comparatively elaborate nerve centres (brain) in the scolex of some Cestodes (c.g., Moniezia) tells in favour of the view that the scolex is anterior and corresponds to a head.

\section{APPENIIX TO PLATYHELMINTHES.}

\section{Class Nemertinea.}

General Features.-The Nemerteans are non-parasitic, unst mented worms, most of which are marine, only a few forms living on land or in fresh-water. They are commonly looked upon as nearl. related to the Turbellaria and were formerly included in that class; but in some respects they are higher in organisation than the Turbellaria, and they exhibit certain special features distinguishing them from the rest of the lower Worms.

The body (Fig. 228) is riearly always narrow and elongated, cylindrical or depressed, unsegmented and devoid of appendages. In length it varies from a few millimetres to as much as ten metres. In some cases there is a short narrower posterior region or "tail"; a head is rarely marked off from the body proper. The entire surface is covered with vibratile cilia, and frequently the integument is vividly coloured. Gland-cells of the epidermis secrete a mucous matter, which may serve as a sheath or tube for the animal. The mouth $(\mathrm{m}$.$) is at or near the anterior extremity$ on themeral aspect. Near it in front (rarely united with it) there ppening through which can be protruded a very long musculw, jsan, the proboscis $(p r$.$) , the possession of which is one$ 
of the most characteristic features of this class of Worms. The proboscis is hollow; when extended to its utmost, a part still remains which is not capable of being everted. This hollow tube (Fig. 229) is open in front, where its edges are continuous with the body-wall, and closed behind. Its wall in the eversible part consists of an epithelium (internal when at rest) continuous with the epidermis and similar to the latter, a basement-membrane, and either two or three layers of muscle, circular and longitudinal, with an external thin epithelium of flat cells. The circular muscular fibres are not continued back on the non-eversible part, but the longitudinal fibres pass backwards (mo form the retractor muscle, by Heans of which the proboscis is uached to the sheath in which it is enclosed, and by means of which also it is retracted. The internal epithelium of the proboscis develops variously formed and arranged papillæe, and in most cases its cells form rods of a similar character to that of the rods or rhabdites of Turbellaria. Exceptionally the cells contain nematocysts similar to those of Colenterates. In the part besiveen the eversible and noneversible regions, a part which inay itself become elongated and complicated in structure, is developed in many Nemerteans (Metanemertini) - a median calcareous stylet (Figs. 232, 233) with groups of smaller accessory stylets at the sides. In the everted proboscis these are borne at the free anterior extremity, and are thus capable of being

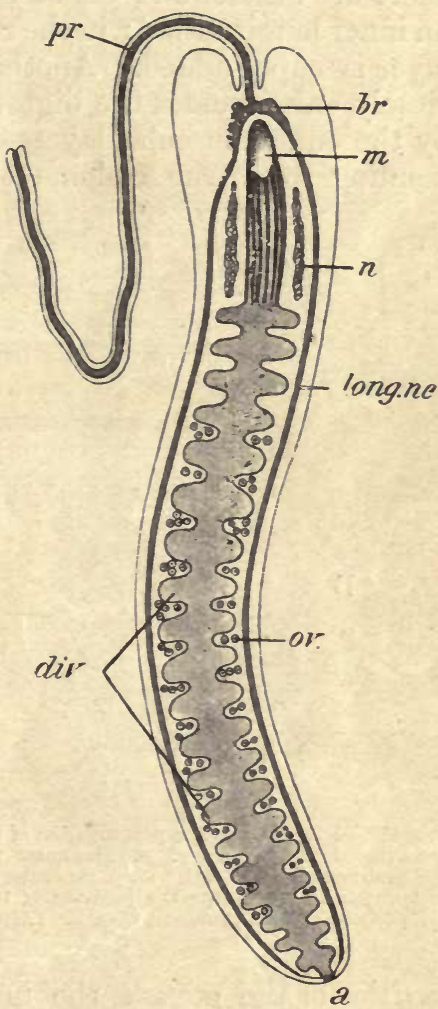

FiG. 228.-Diagram of the organs of a Nemertine, from below. $a$. anus; $b r$. brain ; div. creca ; long. ne. longitudinal nerve-cords ; m. mouth; $n$. nephridia ; ov. ovaries; $p r$. proboscis. (After Hubrecht.) used as weapons. In Drepanophorus there are a number of small stylets supported on a narrow curved plate, together with accessory stylets. In the rest of the Nemerteans stylets are not developed. It is by contraction of the muscular walls of the sheath, the cavity of which ( $r$ hynchocoele) contains a corpuscur, that the proboscis becomes everted. The abundant the proboscis points to its ${ }^{2}$ eing used partly as ascisi gan. 
The outermost layer of the body-wall is an epidermis of columnar cells many of which are ciliated, while others are unicellular glands, some of which are arranged in groups; these secrete the mucus with which the surface is usually covered, and which may form a gelatinous tube. Beneath the epidermis is a basement membrane, very thin in most cases, followed by the muscúlar layers. In some Nemerteans (whence called Dimyaria) there are only two layers of muscular fibres, an outer circular and an inner longitudinal; in the rest (Trimyaria) a third (longitudinal) layer is super-added. Another circular layer of muscular fibres closely encompasses the digestive canal. The interspace enclosed by the outer muscular layers does not comprise any cavity corresponding to a true colome or body-cavity, except, perhaps, the
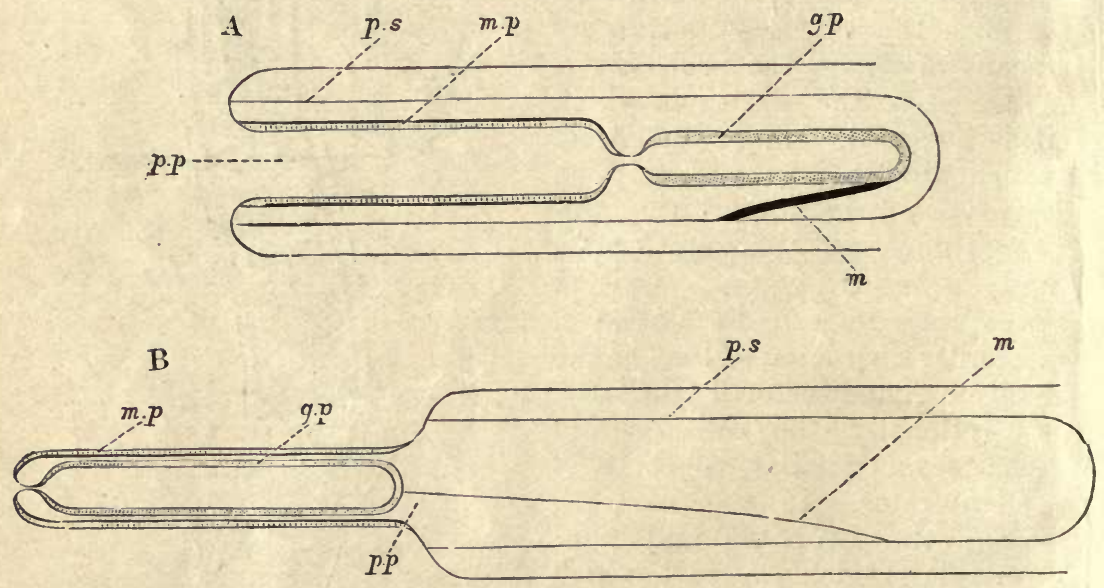

FIG. 229.-Diagrammatic representation of proboscis: (A) in the retracted condition, (B) in the everted condition. g. p. glandular portion of the proboseis; $m$. muscle attaching the proboscis to its sheath; $m$. p. muscular portion of the proboscis; $p . p$. in $\mathrm{A}$, proboscis pore ; $p$. $p$. in B represents the position of the provoscis-pore in the retracted condition of the proboscis; $p$. s. proboscis sheath. (After Sheldon.)

cavities of the gonads, the interspaces between the organs being filled with parenchyma (Fig. 234).

The digestive canal consists of a tube which extends throughout the length of the body from the mouth-situated near the anterior extremity on the ventral side, to the anus at the posterior extremity. ${ }^{1}$ The mouth is usually placed some distance behind the proboscis pore, but may be shifted forwards so as to lie close to the latter, or to be incorporated with it. The first part of the digestive canal is usually a simple tube-cesophagus (stomodaum) - but may be more complicated, and divided into various regions. Posteriorly it opens into the intestine. The latter may

1 When a tal present the intestine m-y, or may not, be continued through it. 
(Metanemertini) project forwards below the oesophagus as a ventral coccum, which may give off paired lateral diverticula. The intestine, constituting by far the greater part of the length of the canal, may be a simple unconstricted tube, or may be only slightly constricted at intervals by the paired gonads. In most cases the constrictions corresponding to the gonads are very deep, so that the intestine comes to be provided with two rows of lateral diverticula or cæca, which may be branched. The creca are separated from one another by incomplete transverse septa of dorso-ventral muscular fibres-the arrangement of the creca and septa with the alternately arranged gonads bringing about an appearance of imperfect metamerism such as is observable in some of the Platyhelminthes (Gunda, species of Temnocephala).

The Nemerteans possess a system of vessels usually regarded as representing a blood - vascular

system (Figs. 230 and 235), with well-defined walls consisting of a layer of epithelium surrounded by a thin layer of muscular fibres arranged circularly. There are three principal longitudinal trunks -a median dorsal (dors. ves.) and two lateral (lat. ves.). The blood is, in most cases, colourless, and contains rounded or elliptical, usually colourless, corpuscles.

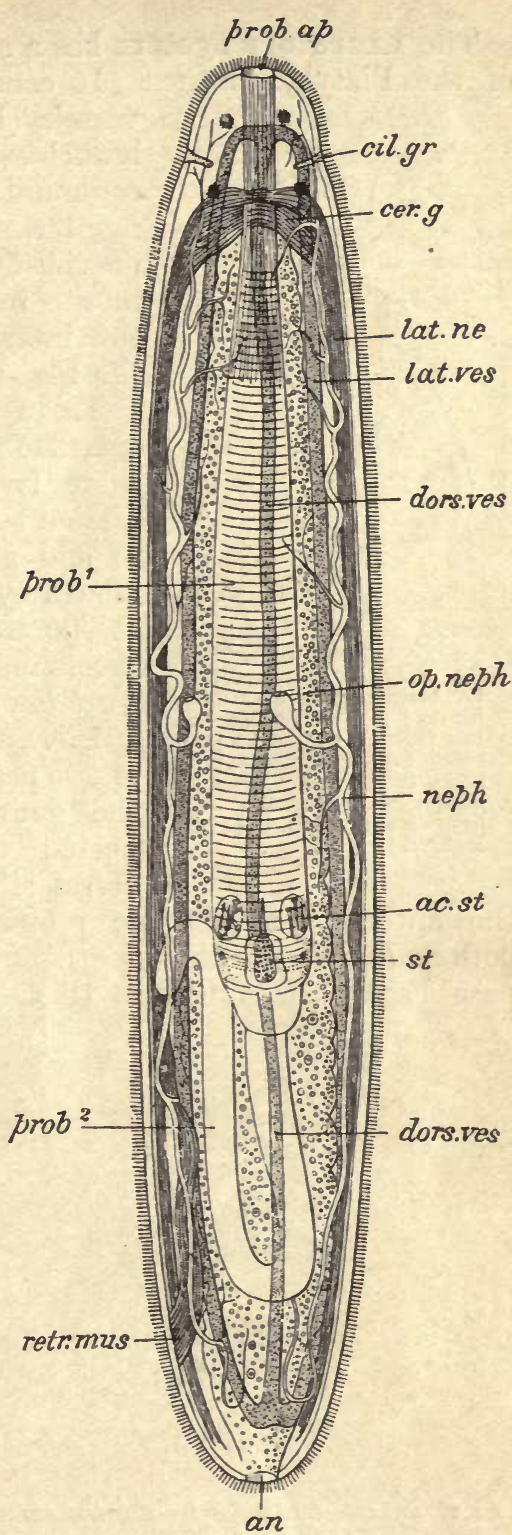

FiG. 230.-Tetrastemma. General view of the internal organs. an. anus; ac. st. accessory stylet; cer. g. brain ; cil. gr. ciliated groove of cerebral organ; dors. ves. dorsal vessel; lat. ne. lateral nerve; lat. ves. lateral vessel; neph. nephridium; op. neph. nephridial aperture; probl. eversible part of proboscis; $p^{2} b^{2}$. noneversible part of proboscis; prob. ap. aperture for the protrusion of the proboscis; retr. mus. retractor muscle of the proboscis; st. stylet. (From Hatschek's Lehrbuch.) 
- The excretory system has a considerable resemblance to that of the Platyhelminthes. It consists of a pair of longitudinal

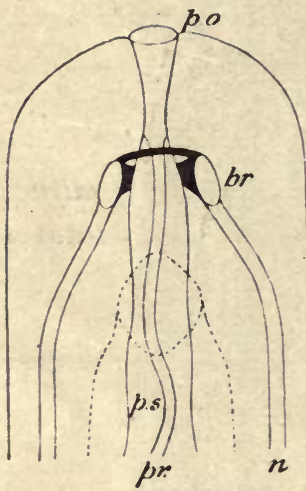

Fig. 231.-Anterior portion of the body of a Nemertine. r. brain-lobes; $n$. lateral nerves ; $p$. o. extermal opening through which the proboscis is everted; $p$. 8 . proboscis-sheath; $p r$. proboscis. Esophagus and mouth shown by dotted lines. (After Hubrecht.) vessels (Fig. 235, neph.) which give off branches, by one or several of which each communicates with the exterior. The fine terminal branches of the system are provided with ciliary flames, each situated in the mirlst of a group of cells, not in the interior of a single flame-cell as in the Flat-worms.

There are no special organs of respiration in any of the group. But there is evidence that this function is carried out, in part at least, by the taking in and giving out of water through the mouth by the œsophagus.

The nervous system is in some respects more highly developed than in the Turbellaria. The brain (Figs. 228 and 231, br., and Fig. 230 cer. g.) is composed of two pairs of ganglia, dorsal and ventral, the ganglia of each pair being connected together by commissures, the dorsal situated above, the ventral below, the anterior part of the proboscis and proboscis sheath, and both being above the mouth and oesophagus. From the brain pass backwards a pair of thick longitudinal nerve-cords which
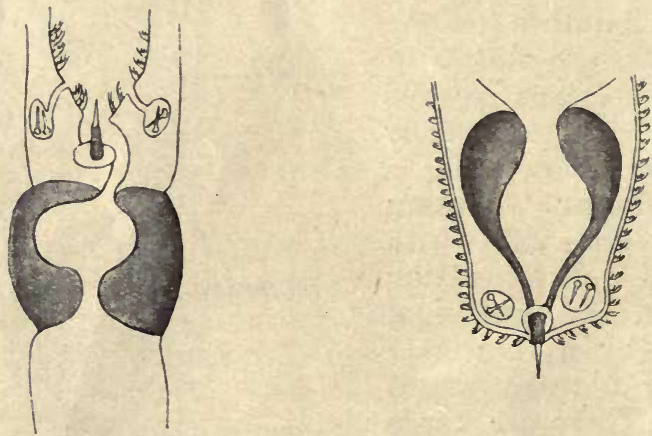

FIGS. 232 and 233.-Proboscis of a Foplonemertean, with stylet reserve-sacs and muscular bulb. Fig. 232 retracted, Fig. 233 evertcd. (After Hubrecht.)

run throughout the length of the body. Usually these are lateral in position, sometimes approximated dorsally, sometimes ventrally. Usually the lateral nerve-cords meet posteriorly in a commissure usually situated above, but in one genus below, the 
anus. A third median dorsal nerve of smaller size than the lateral cords extends backwards from the dorsal commissure of the brain. Associated with the nerve-cords in the Protonemertini and

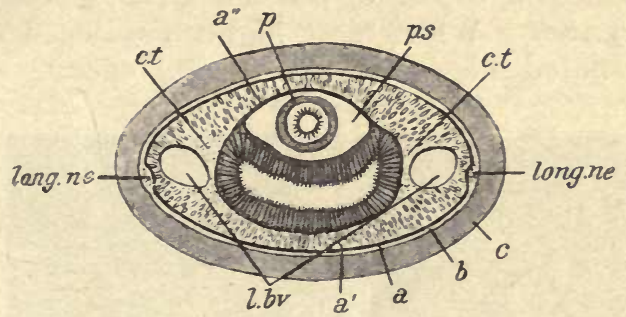

FIG. 23t.-Diatgrammatic transverse section of a Iremertean (Carinella). $\iota, b, c$. layers of body-wall; c. t. connective tissue between body-wall and enteron; $l$. bv. lateral blood-vessuls ; long. ne. longitudinal nerves; $p$. proboscis; $p$. s. proboscis-sheath. (After Hubrecht.)

the Heteronemertini is a nerve-plexus extending all over the body. In the Metanemertini, instead of a nerve-plexus there is a series of slender transverse connectives running across at short intervals between the lateral nerve-cords, and from each cord are given off numerous branches arranged with some regularity.

The position of the brain and lateral nerve-cords and the nerve-plexus, or the system of commissures and nerve-branches,

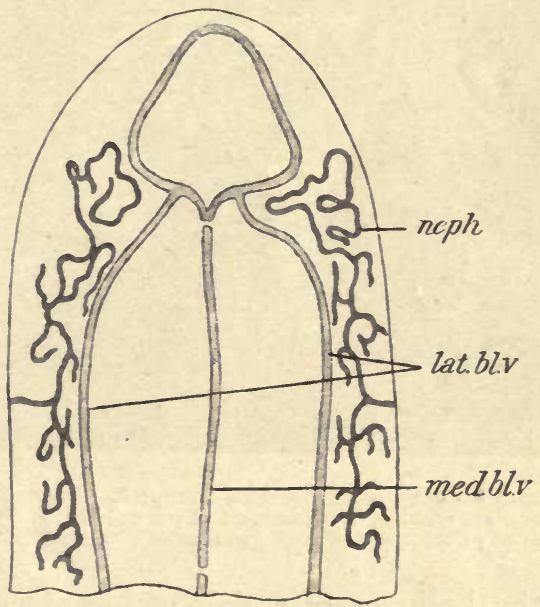

Fig. 235.-Anterior portion of a Nemertean (Drepanophorus), showing the blood-vascular and excretory systems. lat. $b l . v$. lateral blood-vessels; med. bl. $v$. median bloud-vessels; ne $p h$. nephridial (excretory) tubes. (After Oudemans.)

varies in the different groups. In the Protonemertini (Fig. 234) they occupy the most primitive position, being quite superficially situated at the bases of the epidermal cells. In the rest they are deeper: in the Metanemertini they lie in the parenchyma within 
the muscular layers. The median cord is always, except in the Heteronemertines, superficially placed.

A remarkable apparatus connected with the nervous system is that composed of a pair of peculiar structures known as the cerebral organs. When most highly developed these consist of a pair of ciliated tubes (Fig. 230, cil. gr.), opening externally in

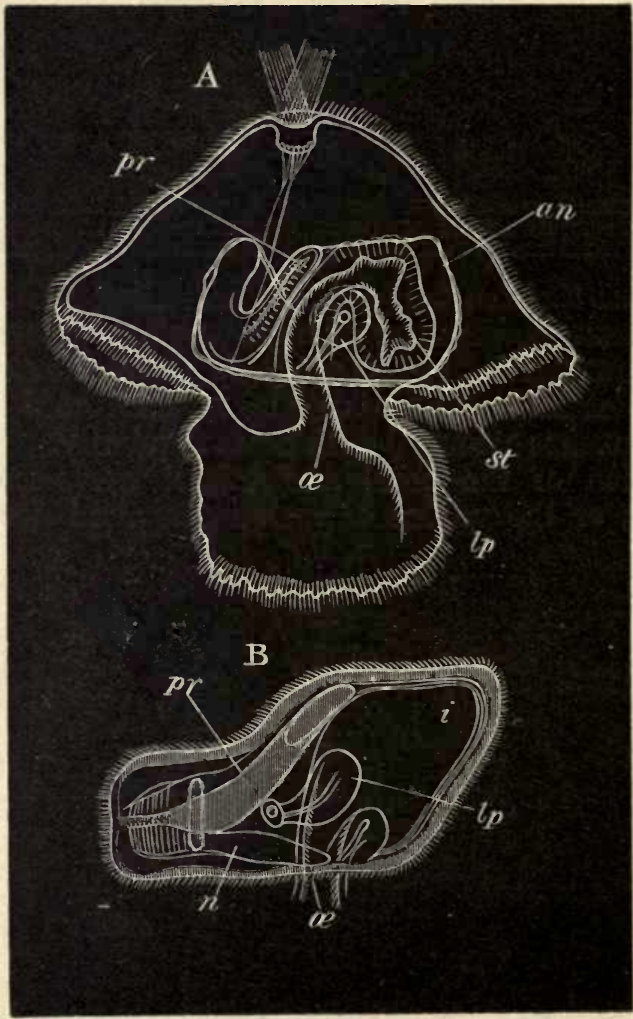

Fig. 236. - $A$, Pilidium with advanced Nemertine worm ; $B$, ripe embryo of Nemertes from interior of pilidium, an. amnion, or investment of the embryo; $i$. intestine ; $l p$. lateral pit ; $n$. nervous system; $\propto$. gullet; $p r$. proboscis; st. stomach. (From Balfour, after Butschli.)

the region of the brain or of a pair of lobes separate from the latter. This apparatus may have a respiratory function, more especially for the oxygenation of the substance of the brain, but perhaps it has also a sensory function. It has some resemblance to the ciliated pits developed in certain Turbellaria.

Eyes are present in the majority of Nemerteans, and in the more highly organised species occur in considerable numbers. Sometimes they are of extremely simple structure; in other cases 
they are more highly developed, having a spherical refractive body with a cellular "vitreous body," and a "retina" consisting of a layer of rods enclosed in a sheath of dark pigment, each rod having a separate nerve-branch connected with it. Statocysts containing statoliths have been found in only a few of the Nemerteans.

Reproductive System.-Most species are diocious. The ovaries (Fig. 228), ov.) and testes are situated in the intervals between the intestinal crea. The ovary or testis is a sac the cells lining which give rise to ova or spermatozoa; when these are mature each sac opens by means of a narrow duct leading to the dorsal, rarely to the ventral surface, on which it opens by a pore. In all probability the cavities of these hollow gonads are all that represent the coelome of higher forms.

Development.-Some of the Nemerteans go through a metamorphosis; in the others the development is direct. The characteristic larval form is the pilidium (Fig. 236). This is a helmetshaped body with side lobes like ear-lappets, and a bunch of cilia representing a spike. In the metamorphosis two pairs of ectodermal invaginations, growing inwards around the intestine, fuse together and form the integument and body-wall of the future worm, which subsequently frees itself from its investment and develops into the adult form. In others there is a ciliated ereeping larva called the "larva of Desor," in the interior of which the larval worm is developed much as in the case of the pilidium.

Though none of the Nemerteans exhibit metameric segmentation, yet in some of them there is, as in Gunda segmentata (p. 255) among the Turbellaria, a serial repetition of the internal parts (pseudo-metamerism, associated with the presence of regularly arranged transverse partitions of dorso-ventral muscular fibres). Transverse fission is of frequent occurrence.

\section{Distinctive Characters and Classification.}

The Nemertinea are ciliated, unsegmented worms with elongated body, without distinct cœlome. There is an eversible proboscis enclosed in a sheath and capable of being protruded to a great length through an aperture situated usually in front of and above the mouth. The intestine usually has distinct lateral diverticula, and there is a posteriorly situated anus. There is a blood-vascular system and also a system of excretory vessels with ciliary flames.

\section{Order 1. Protonemertini.}

Dimyarian Nemertines with the lateral nerve-cords situated outside the muscular layers. The mouth is situated behind the brain. The proboscis is devoid of stylet. 


\section{Order 2. Mesonemertini.}

Dimyarian Nemertines having the lateral nerve-cords withdrawn within the musculature of the body-wall. The mouth is situated behind the brain. There are no stylets.

\section{Order 3. Metanemertini.}

Dimyarian Nemertines, in which the lateral nerve-cords lie inside the muscular layers in the parenchyma. The mouth is situated in front of the brain. The proboscis is provided with stylets. A ventral cacum is present.

\section{Order 4. Heteronemertini.}

Trimyarian Nemertines, in which the lateral nerve-cords are in the muscle-layers, between the outer longitudinal and the circular layers. The mouth is situated behind the brain. The proboscis has no stylet.

The Nemerteans are almost exclusively marine; and the greater number live between tide-marks or at moderate depths; a few have been obtained from considerable depths. The comparatively small number of terrestrial and fresh-water forms are all Metanemertini. The Nemerteans progress for the most part by slow crawling movements, leaving a track of slime behind them. Some burrow freely in mud or sand, the proboscis being made use of to help in the process. Some are able to swim by means of undulating movements of the body. Nearly all are carnivorous, and either capture living prey in the shape of small invertebrates of various kinds, or feed on dead fragments. The chief function of the proboscis is the capture of living prey, around which it becomes coiled and then draws the prey towards the mouth. One Nemertean lives in the interior of a Crustacean, and is probably a true parasite. Others, live, apparently as commensals or messmates, in the pharynx or atrial cavity of Ascidians, or within the mantle cavity of bivalve Mollusca.

A striking feature of the Nemerteans is the readiness with which, on being irritated by handling or by the action of some chemical agent, they break up transversely into fragments. This takes place most freely when the body is highly charged with sexual products, but is by no means confined to that condition. The process probably takes place spontaneously under certain circumstances. The broken-off fragments may remain alive for a considerable time, and under suitable conditions regeneration of the lost parts is readily effected, so that it is possible to look upon the entire process as a form of asexual reproduction. 


\section{SECTION VI \\ PHYLUM NEMATHELMINTHES.}

THE members of the preceding phylum are characterised, as a whole, by a marked dorso-ventral flattening. In the Worms included in the present group the body is elongated and cylindrical, whence their general name of Round- or Thread-worms. The phylum includes the following classes:-

Class 1. Nematoda.- The Round-worms in the strict sense of the term. The best known forms are internal parasites, but many genera and species are extremely abundant in fresh and salt water.

Class 2. Acanthocephala.-The "Hook-headed Worms," a group of formidable internal parasites.

Class 3. Chetognatha.-The "Arrow-worms," a small group of pelagic organisms.

The affinities of the Acanthocephala and Chætognatha with the Nematoda are somewhat doubtful, and the association of the three classes is largely a matter of convenience.

\section{CLASS I.-NEMATODA.}

1. Example of the Class-The Common Round-worm of Man. (Ascaris lumbricoides.)

Ascaris lumbricoides is a common parasite in the human intestine : a closely allied if not identical form ( $A$. suilla) occurs in the Pig, and another (A. megalocephala)-in the Horse. The following description will apply to any of these. The female A. lumbricoides is about $20-40 \mathrm{~cm}$. (8-16 inches) long, and about $6-8 \mathrm{~mm}$. (1 inch) in diameter; the male is considerably smaller.

External Characters. - When fresh the animal is of a light yellowish-brown colour: it is marked with four longitudinal streaks, two of which, very narrow and pure white in the 
living Worm, are respectively dorsal and ventral in position, and are called the dorsal (Fig. 237, d.l.) and ventral (v.l.) lines : the other two are lateral in position, thicker than the former, and brown in colour, and are distinguished as the lateral lines. The mouth is anterior and terminal in position, and is bounded by three lobes, or lips, one median and dorsal $(d . l p$. $)$, the other two ventro-lateral $(v . l p$.$) . A very minute aperture on the ventral side, and about 2$ $\mathrm{mm}$. from the anterior end, is the cxcretory pore (ex.p.). At about the same distance from the pointed and down-turned posterior end is a transverse aperture with thickened lips, the anus (an.), which in the male serves also as a reproductive aperture and gives exit to a pair of needle-like chitinoid bodies, the penial seta (pn. s.). In the female the reproductive aperture or gonopore is separate from the anus, and is situated on the ventral surface about onethird of the length of the body from the anterior end (Fig. 240, $g n p$.). The sexes are also distinguished externally by the form of

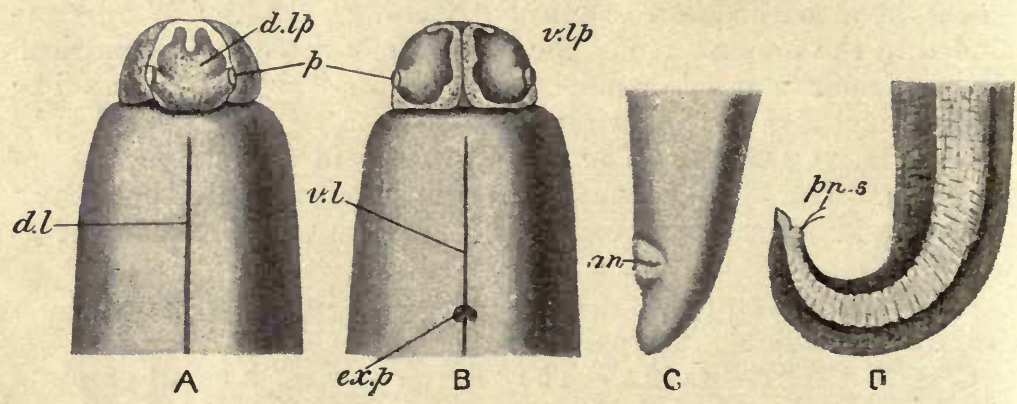

Fig. 237.-Ascaris lumbricoides. A, anterfor end from above; B. the same from below; C, posterior end of female, D, of male, side view. an. anus ; $d . l p$. dorsal lip ; $d . l$. dursal line ; $e x . p$. excretory pore; $p$. papillæ; pn. 8 . penial setæ; $v . l$. ventral line; $v$. $l p$. ventral lip. (After Leuckart.)

the short tail, or post-anal portion of the body, which in the male is sharply curved downwards (Fig. 237, D), while in the female $(C$ ) its ventral contour is nearly straight.

Body-wall. - The outer surface of the body is furnished by a delicate, transparent, elastic membrane, of a firm material of albuminoid composition, the cuticlc (Fig. 238, cu.). It is divisible into several layers, and is wrinkled transversely, so as to give the animal a segmented appearance. Beneath the cuticle is a protoplasmic layer (der.epthm.) containing scattered nuclei and longitudinal fibres, and representing a syncytial ectoderm-i.e., an ectoderm is which the cell-boundaries are not differentiated, and whose cellular nature is recognisable only by the nuclei. The cuticle is, as usual, a secretion of the ectoderm.

Beneath the ectoderm is a single layer of muscular fibrcs (m.), arranged longitudinally, and bounding the body-cavity. The structure of the muscles is very peculiar: each (Fig. 239, A) has 
the form of a spindle, striated longitudinally, and produced on its inner face (i.e. towards the body-cavity) into a large and almost bladder-like mass of protoplasm $(p)$ containing a nucleus (nu.). Apparently the whole of this structure is derived from a single cell, part of which has become differentiated into contractile substance $(c)$, the rest remaining protoplasmic. In transverse section the contractile portion (B. c) has the form of a plate bent upon itself so as to be, as it were, wrapped round the protoplasmic portion $(p)$. The protoplasmic processes project to a greater or less extent into the body-cavity, sometimes practically obliterating it, and are produced into delicate filaments $(f$.) which take a transverse

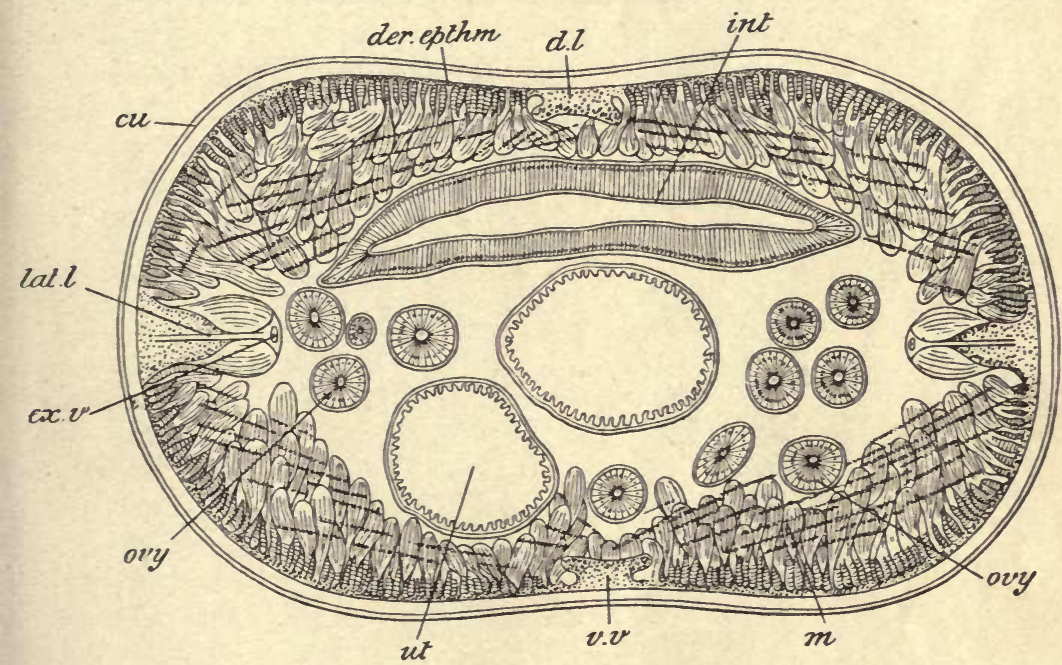

FIG. 23S.-Ascaris lumbricoides, transverse section. cu. cuticle ; d. l. dorsal line ; der. epthm. deric epithelium or epidermis ; $e x . v$. excretory vessel ; int. intestine ; lat. $l$. lateral line; $m$. muscular layer; ovy. ovary ; ut. uterus; v. v. ventral line. (After Vogt and Yung.)

direction, and are mostly inserted into the dorsal and ventral lines.

The muscular layer is not continuous, but is divided into four longitudinal bands or quadrants, two dorso-lateral and two ventrolateral, owing to the fact that at the dorsal, ventral, and lateral lines the ectoderm undergoes a great thickening and projects inwards, between the muscles, in the form of four longitudinal ridges (Fig. 238, d.l., v.v., lat.l.). The ridges are composed of fibres continuous with the fibres of the ectoderm. It is this arrangement that gives rise to the lines seen externally. The ridges forming the lateral lines are much more prominent than the other two.

Digestive Organs.-The mouth leads into the anterior division of the enteric canal, the pharynx or stomodocum (Fig. 240, 
ph.): its walls are very muscular, its cavity is three-rayed in crosssection, and it is lined by a cuticle secreted from the epithelial layer and continuous, at the mouth, with that of the body-wall. Posteriorly the pharynx opens into the intestine (int.), a thinwalled tube, flattened from above downwards, and formed of a layer of epithelial cells bounded both internally and externally by a delicate cuticle: it has no muscular layer (Fig. 238, int.). Posteriorly the intestine narrows considerably to form the short rectum, which has a few muscular fibres in its walls and opens externally by the anus (Fig. 240, an.). The food, consisting of the semi-fluid contents of the intestine of the host, is sucked in by movements of the pharynx, and is then absorbed into the system
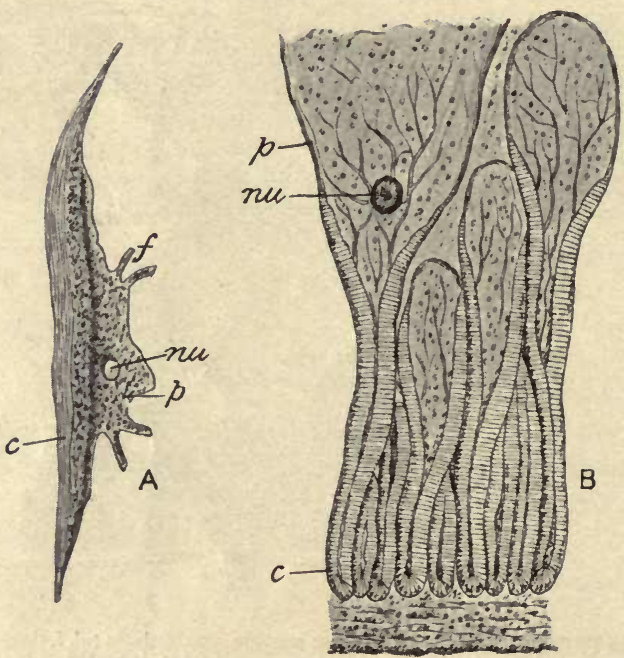

Fig. 239.-A scaris lumbricoides. A, a single muscle fibre; B, several fibres in transverse section with portion of ectoderm (below). $c$, contractile substance; $f$. fibrous proccsses; $n u$. nucleus ; $p$. protoplasmie portion. (After Leuckart.)

through the walls of the intestine. The food being already digested by the host, there is no need of digestive gland-cells, such as occur in animals which prepare their own food for absorption.

It will be noticed that in the above description the pharynx is also called stomodæum. This must not be taken to indicate that the two terms are synonymous, but that, in the present instance, the epithelial lining of the pharynx is derived from the ectoderm, being formed as an in-turned portion of the outer layer of the body-wall. The epithelium of the intestine, on the other hand, is endodermal, this portion of the canal being derived from the archenteron of the embryo. 
Between the enteric canal and the body-wall is a distinct space, the body-cavity, containing a clear fluid and more or less encroached upon by the protoplasmic processes of the muscle-cells. The cavity is bounded externally by these processes, internally by the outer cuticle of the intestine: there is no trace of epithelial lining such as occurs in most of the higher animals The body-cavity of the Nematode, in fact, does not exactly correspond to the cœlome to be met with in most higher phyla. It is not to be derived, directly or indirectly, from the archenteron of the embryo, and it does not lie, like a true cœlome, between layers of the mesoderm.

The excretory system presents a certain resemblance to that of Platyhelminthes. It consists of two longitudinal canals (ex. v.), one in each lateral line. Anteriorly these pass to the ventral surface, unite with one another, and open by the minute excretory pore (ex.p.) already noticed. The system is not ciliated, and contains no flamecells. Both canals are excavations in a small number of enorinously elongated and branched cells, each cell having a single nucleus.

The nervous system consists of a ring $(n v, r$.) surrounding the pharynx and giving off six nerves forwards and six backwards (Fig. 241). Of the latter two are of considerable size, and run in the dorsal and ventral lines respectively $(d l n, v l n$.). They are connected with one another by transverse commissures (c.), and the ventral nerve swells into a ganglion just in front of the anus. The pharyngeal nerve-ring contains nerve-cells, and

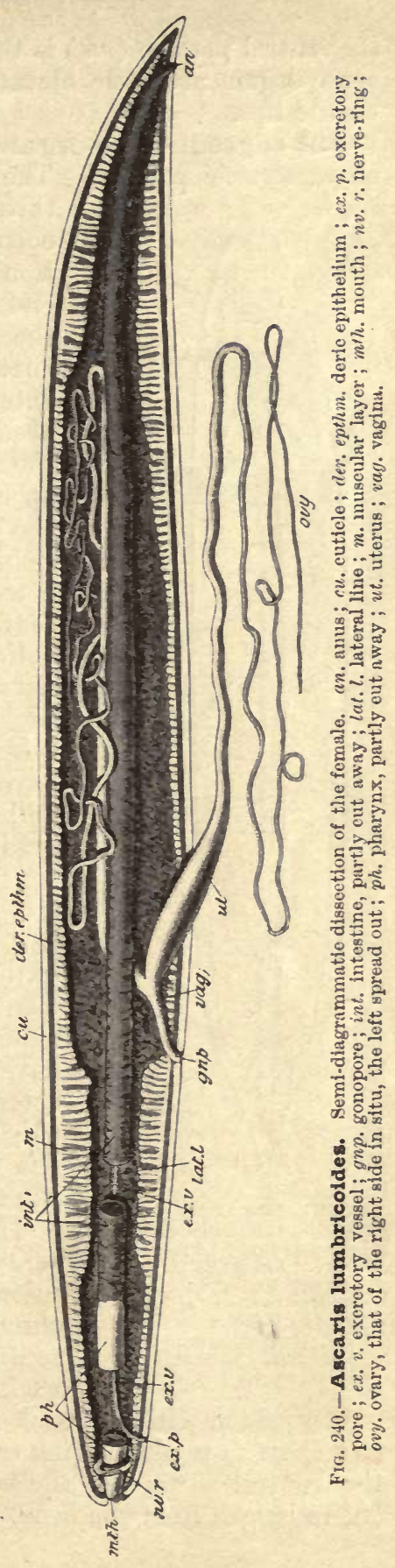


its ventral portion (un.) is thickened and ganglion-like. The only sense-organs are little elevations, the scnsory papillce (Fig. 237,p.), on the lips.

The reproductive organs are formed on a peculiar and very characteristic pattern. The testis (Fig. 242,ts.) is a long, coiled thread, about the thickness of fine sewing-

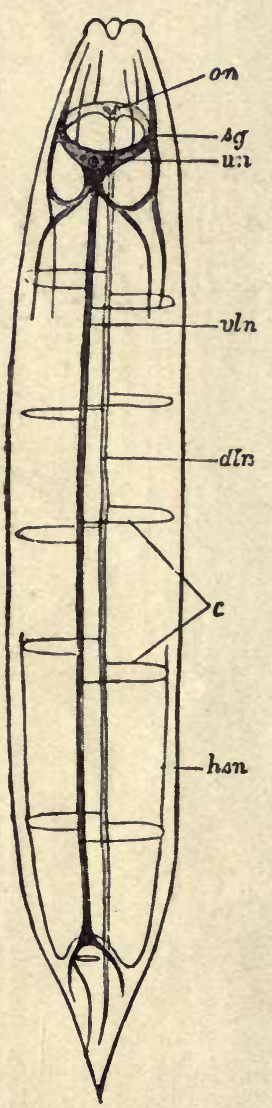

FIG. 241.-Diagram of nervous systen of Nematoda. c. commissures; dln. dorsal nerve; hsn. posterior lateral nerve; on. upper and $u$. under portion of nervering; s.g. lateral swellings ; $v l n$. ventral nerve. (From Lang, after Butschli.) cotton, and occupying a considerable portion of the body-cavity. At its posterior end it is continuous with the vas deferens, the two passing insensibly into one another so that the junction is not visible externally. The vas deferens, in its turn, becomes continuous with a wide canal, the vesicula seminalis (vs. sem.), which opens by a short, narrow muscular tube, the ductus ejaculatorius, into the rectum. Behind the rectum, and opening into its dorsal wall, are paired muscular sacs (s.), containing the penial setoe ( $p n$. s.) already noticed. The anterior end of the testis consists of a solid mass of sex-cells ; passing backwards there is found a cord or rachis occupying the axis of the tube and having the sperm-cells attached to it; still further back the sperms become gradually differentiated, and are finally set free in the vas deferens. The sperms are peculiar rounded cells (Fig. 23, p. 30, c. d. e.); when transferred into the body of the female they exhibit amoboid movements, but as long as they remain in the male ducts they are non-motile: they have no trace at any stage of the characteristic tail of the typical sperm. In this connection it may be mentioned that the tissues of Ascaris are remarkable for the total absence of cilia.

The organs of the female (Fig. 240) resemble those of the male, but are double instead of single. There are two coiled, thread-like ovaries (ovy.), each passing insensibly into a uterus (ut.). In the ovary, as in the testis, the eggs are developed in connection with an axial cord or rachis. The two uteri unite in a short muscular vagina (vag.) which opens, as already seen, on the ventral surface of the body (gnp.) at about one-third of the entire length from the head. 
Development.-The eggs are produced in immense numbersat the rate, it has been reckoned, of about 15,000 a day. They are fertilised in the upper part of the uterus, each becoming enclosed in a chitinoid egg-shell, and are passed out of the body of the host with its frees. Segmentation is complete, but the details of development are not known in this species. The results of experiments render it probable that infection is direct, without intermediate host, the embryo-containing eggs being taken, in

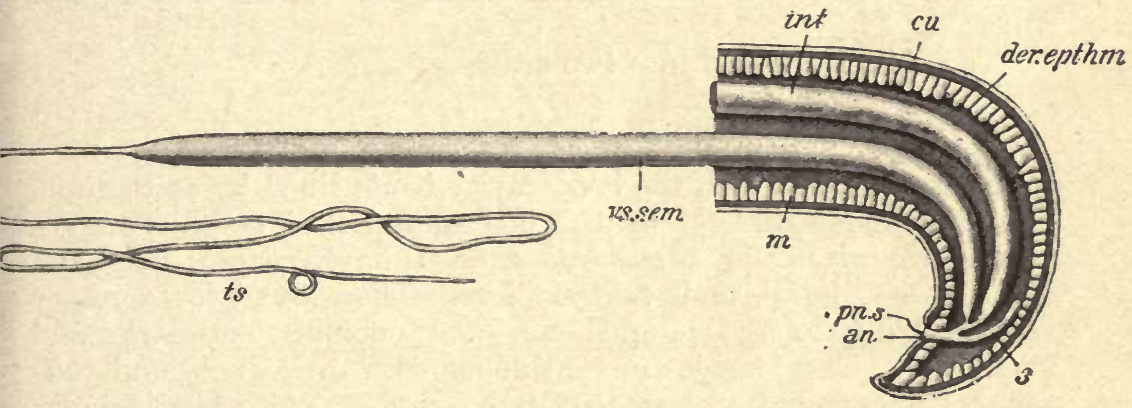

FIG. 242.-Ascaris lumbricoides, posterior extremity of male, dissected. an. anus ; $c u$. cuticle; der. epthm. epidermis; $m$. muscular layer; p. 8. penial seta; 8 . sac containing penial seta; $t s$. testis; $v 8.8 \mathrm{em}$. vesicula seminalis.

water, or in soil accidentally swallowed, into the intestine of a new human host, in which the embryos, escaping from the eggs, become mature.

\section{Distinctive Characters and Classification.}

The Nematoda are Nemathelminthes having a cylindrical body of great length in proportion to its diameter, and pointed at both ends. The body-wall consists of a tough external cuticle, an ectoderm in the form of a syncytium or protoplasmic layer containing nuclei and rarely exhibiting cell-structure, and a single layer of longitudinal muscular fibres which are interrupted along one or more (dorsal, ventral, and lateral) lines. The body-wall encloses a body-cavity containing a clear fluid, and more or less encroached upon by processes of the muscle-cells or other mesodermal tissues. The enteric canal is straight, and consists of pharynx, intestine, and rectum: the pharynx is a stomodrum. The mouth is anterior and terminal, the anus ventral and situated a short distance from the posterior end. Excretory canals, running in the lateral lines, are usually present. The nervous system consists of a pharyngeal ring containing nerve-cells and giving off nerves forwards and backwards : of these there is either a single ventral-cord, or there are two cords, respectively dorsal and ventral, 
of considerable size and extending to the posterior end of the body. The Nematoda are in nearly all cases diøecious: eggs are produced in immense numbers, and are impregnated within the body of the female. The sperms are non-motile, or perform amoboid movements only after entering the female organs. Cilia are wholly absent.

A large proportion of Nematoda are free-living, spending their whole life in fresh or salt water, damp earth, decaying matter, \&c. ; the remainder are parasitic during the whole or a part of life.

The class is divided into two orders.

\section{Order 1.-Nematoidea.}

Nematoda in which the body-cavity is not lined by epithelium, but is bounded directly by the body-muscles. Two chief nervecords are given off backwards from the pharyngeal ring and lie in the dorsal and ventral lines. There are two excretory canals lying in the lateral lines and opening anteriorly and ventrally. The gonads are continuous with their ducts, and consist of long, more or less convoluted cords. This order includes the whole of the free-living Nematodes as well as the large majority of parasitic forms.

\section{ORDER 2.-NEMATOMORPHA.}

Nematoda in which the body-cavity is lined by a distinct epithelium. The pharyngeal nerve-ring sends off a single large ventral nerve-cord well supplied with nerve-cells. 'The gonads, or' at least the ovaries, are arranged metamerically, and the reproductive products are discharged into the body-cavity and pass thence into the gonoducts. This order includes a small number of greatly elongated, thread-like worms (species of the genus Gordius), which are parasitic in the asexual, free-living in the sexual stage; and also the genus Necturus, which has only been found sivimming in the sea.

\section{Systematic Position of the Example.}

Ascaris lumbricoides is one of many species of the genus Ascaris, and belongs to the family Ascaridoe of the order Nematoidea.

The absence of an epithelial lining to the body-cavity, and the presence of elongated gonads continuous with their ducts, indicate its position as one of the Nematoidea. Among the numerous families constituting this order, the Ascaridæe are distinguished by the possession of three lips furnished with papillæ, and by the body of the male being curved ventrally and being provided with penial setæ. Ascaris is distinguished from the other 
genera of the family by the absence of a bulb-like enlargement at the posterior end of the pharynx, by the posterior extremity of the body having the form of a short blunt cone, and by the presence of two penial setæ in the male.

\section{General Organisation.}

External Characters. - The Nematoda vary much in size : the little Anguillula, one of the commonest of aquatic animals, does not exceed $1 \mathrm{~mm}$. in length, while the dreaded parasite known as the Guinea-worm (Filaria medinensis) is sometimes as much as 2 metres (6 feet) long. The length is always great in proportion to the diameter, and the body is always bluntly pointed at the anterior end and either pointed or forked posteriorly. One of the most striking cases of disproportion between length and breadth is exhibited by the free, sexual form of Gordius, one of the Nematomorpha; it is found in earth or water and resembles a tangle of brown string, the length being frequently as much as 15 or $16 \mathrm{~cm}$. while the diameter does not exceed $0.5 \mathrm{~mm}$.

Body-wall.-The body is always covered by a cuticle secreted by the deric epithelium or external ectoderm: the latter usually takes the form of a protoplasmic layer with scattered nuclei, but in the Nematomorpha it consists in part of a true epithelium-a single layer of distinct cells. Beneath the ectoderm is a muscular layer, which in many genera has the same structure as in Ascaris, i.e. consists of a single layer of longitudinal fibres, interrupted at the dorsal, ventral, and lateral lines, each fibre being spindle-shaped and produced into a protoplasmic process which projects into the body-cavity. But in many forms (e.g., Strongylus) the muscle-cells are flat rhomboidal plates (Fig. 243), and each quadrant contains only two rows, the total number in a transverse section

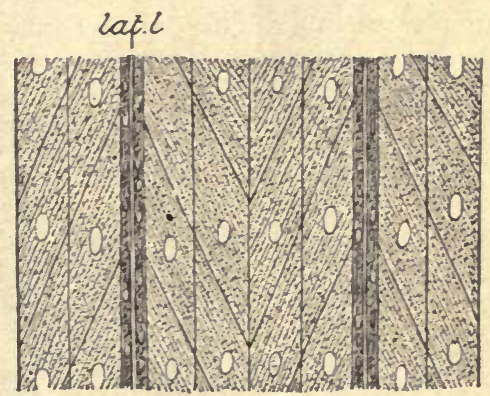

FIG. 243.-The body-wall of a platymyarian Nematode, spread out. lat. l. lateral lines. (After Leuckart.) being therefore eight. In the Nematomorpha the muscles are interrupted along the ventral line only, the dorsal and lateral lines being absent (Fig. 245). Moreover the muscular layer in this order is lined by a layer of epithelial cells which bounds the body-cavity.

Enteric Canal. - The mouth is frequently armed with spines (Fig. 244, C), by means of which the worms draw blood from the 
intestinal blood-vessels of their host. Many free-living forms have a sharp stylet for piercing the tissues of the plants on which they feed, and a suctorial apparatus for absorbing the juices. The

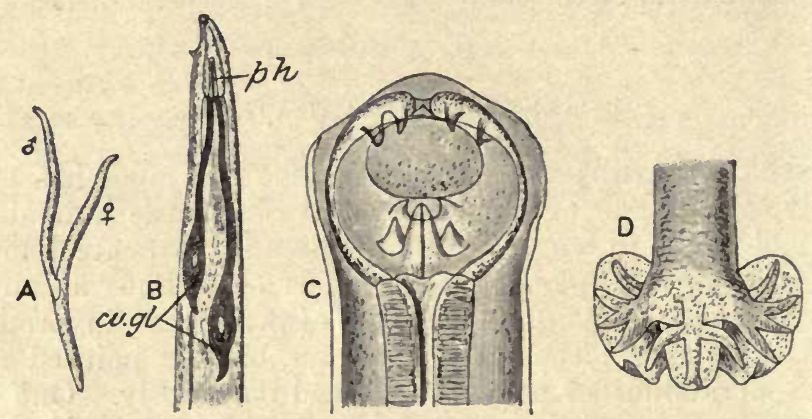

FIG. 244.-Ankylostoma duodenale. A, male and female in coitu. B, anterior end, showing-cv. gl. cervical glands; ph. pharynx. C, mouth with spines; D, posterior end of male, with bursa. (After Ieuckart.)

posterior end of the pharynx is often dilated to form a globular chamber with muscular walls, the gizzard (Fig. 246,gz.). The only specially interesting variation in the structure of the intestine is that occurring in Trichinella, one of the Nematodes parasitic in Man, in which this part of the enteric canal consists of a single row of perforated cells : the lumen is therefore not inter-cellular but intra-cellular, like the gullet of an Infusor. In the sexual stage of Gordius the enteric canal undergoes more or less complete degeneration. The alimentary canal in some rare cases has

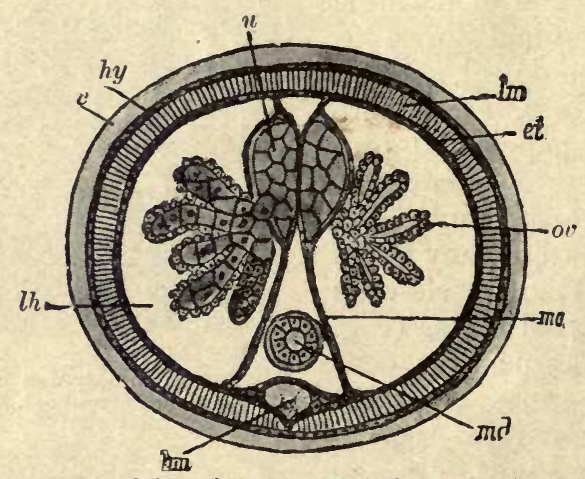

Fig. 245.-Transverse section of Gordius, $b m$. ventral nerve-cord; $c$. cuticle; et. epithelium linirg body-cavity; $h y$. epiderm; $l h$. borly-cavity; $l m$. muscular layer; md. intestine ; mes. mesentery; ov. ovary; $u$. uterus. (From Lang, after Vejdovsky.)

hollow appendages in the form of œsophageal glands or intestinal cæca. In Dochmius a pair of pear-shaped bodies of unknown function, the cervical glands (Fig. 244, $B, c v . g l$.), lie one on each side of the pharynx and probably open externally near the mouth. 
In Nematoidea the body-cavity is always a single continuous chamber crossed in various directions by delicate fibres, but in Gordius certain partitions or mesenteries (Fig. 245, mes.) extend longitudinally through it, dividing it into several compartments. The most important of these are a median ventral compartment containing the intestine and the nerve-cord, a pair of large lateral compartments containing the ovaries, and a pair of small dorsomedian canals which act as oviducts. It is stated that the median ventral compartment acts as an excretory canal and opens posteriorly along with the oviducts: in the Nematomorpha there are no lateral excretory canals like those of Ascaris and the other typical Nematodes.

In the Nematoidea, when definite excretory organs are developed, they take the form of longitudinal canals similar to those described as occurring in Ascaris. Sometimes only one canal is present. In some cases it is stated that the canal or canals open into the bodycavity.

In the Nematoidea the nervous system has the structure already described in Ascaris; it is, however, apparently absent in some free-living forms. But in Gordius it is much more highly developed: the pharyngeal ring is of great thickness and is continued into a single ventral cord (Fig. 245, bm.) containing nerve-cells. Fye-spots have been described in the sexual form of Gordius.

The reproductive organs in all the Nematoidea resemble those of Ascaris, the only important variation depending upon the fact that in the smaller forms the entire genital tube (gonad plus gonoduct) is short and not coiled (Fig. 246,ts. and $v . d f$.). A few forms are hermaphrodite,

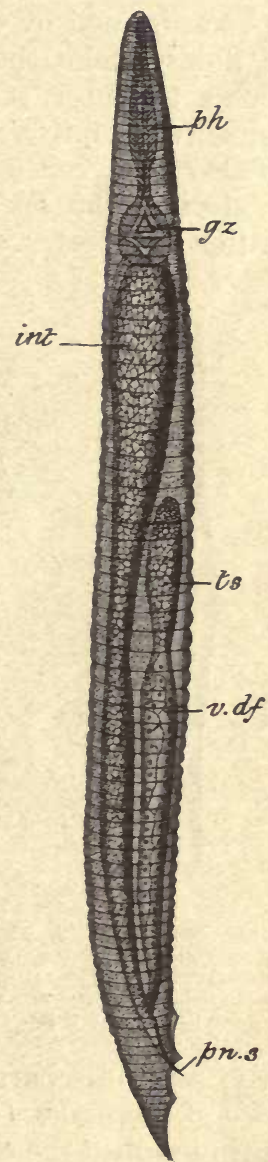

Fic. 246.-Oxyuris, from the right side. $g z$. gizzard; int. intestine; $p h$. pharynx pn. s. penial setre; ts. testis ; $v$. $d f$. vas deferens. (From Shipley, after Galeb.) but, instead of having a double set of reproductive organs, as in Platyhelminthes, organs of the ordinary female nematodetype are present, and the gonads produce first sperms and afterwards ova. Such animals are said to be protandrons (male products ripe first), and self-impregnation is as effectually 
prevented as if the organs of the two sexes were distinct. A totally different arrangement is met with in the Nematomorpha, the female having numerous pairs of ovaries (Fig 247, A, ovy.) arranged segmentally and attached to one of the partitions (mes.) of the body-cavity. The ripe eggs are discharged into large egg-sacs, formed by the lateral compartments of the body-cavity, and finally make their way into the medio-dorsal compartments which act as uteri $(\mathrm{C}, u t$.) and are continued posteriorly by short vaginæ (va.g.) into a median chamber. The latter opens externally, and also receives the duct of a large spermotheca (spth.) or chamber for storing the sperms received in copulation. In the male Gordius the testes are not known: they seem to disappear very
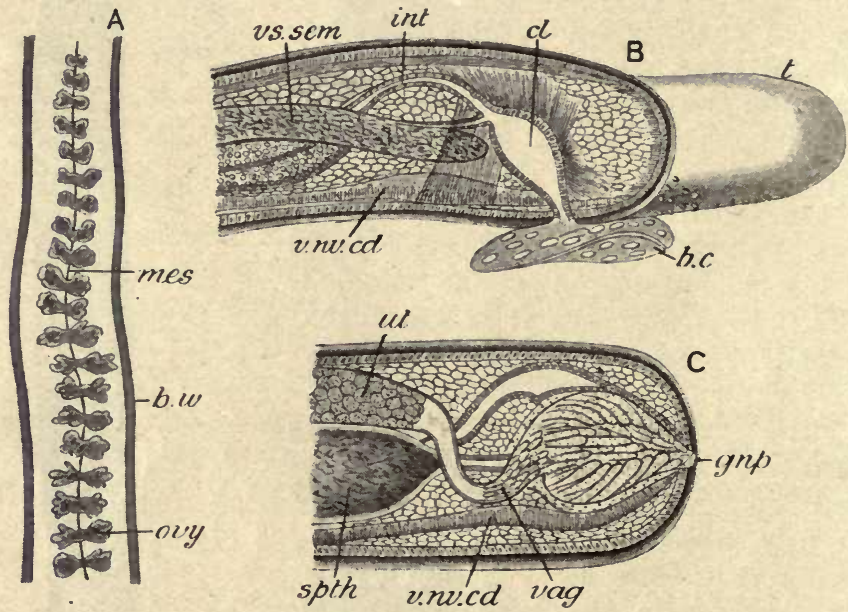

Frg. 247.-Gordius. A, hurizontal section of female, showing ovaries (ovy) attached to mesentery (mes.); b. 20. body-wall. B, posterior extrcmity of male, sagittal section, $b . c$. bursa copulatrix; $c l$. cloaca ; int. intestine ; $t$. tail ; $v$. nv.cd. ventral nerve-cord ; vs. sem. vesicula seminalis. C, posterior extremity of female, sagittal section. gnp. gonopore; spth. spermatheca ; ut. uterus; vag. vagina; v. nv. cd. ventral nerve-cord. (After Vejdowsky.)

early, after discharging their contents into large reservoirs or vesiculo seminales $(\mathrm{B}$, vs. sem.): from these, vasa deferentia are continued into the cloaca $(\mathrm{cl}$.$) or dilated extremity of the intestine,$ part of which can be everted as a bursa copulatrix (b.c.).

In the development of Nematodes segmentation may be unequal from the outset, or equal at first, becoming unequal after the first two or three divisions. There may be an invagination (embolic gastrulation), or, as in Rhabdonema nigrovenosum (Fig. 248) a kind of epiboly, or a process of an intermediate character. The blastopore always disappears, taking no part in the formation of the apertures of the adult. The archenteron also becomes obliterated, and the lumen of the intestine has no connection with it, but is formed anew by the development of a fissure between the endoderm cells 
which have become arranged in two rows. The epithelium of the pharynx is formed by an involution of the ectoderm, so that this division of the enteric canal forms a stomodoum (Fig. 248, I, stdm.), and the rectum appears also to be lined by ectodermal cells and thus to be of the nature of a proctodoum. The middle layer is formed from a pair of cells (D, mes.) of the inner layer which enlarge and multiply to form a layer of cells between the
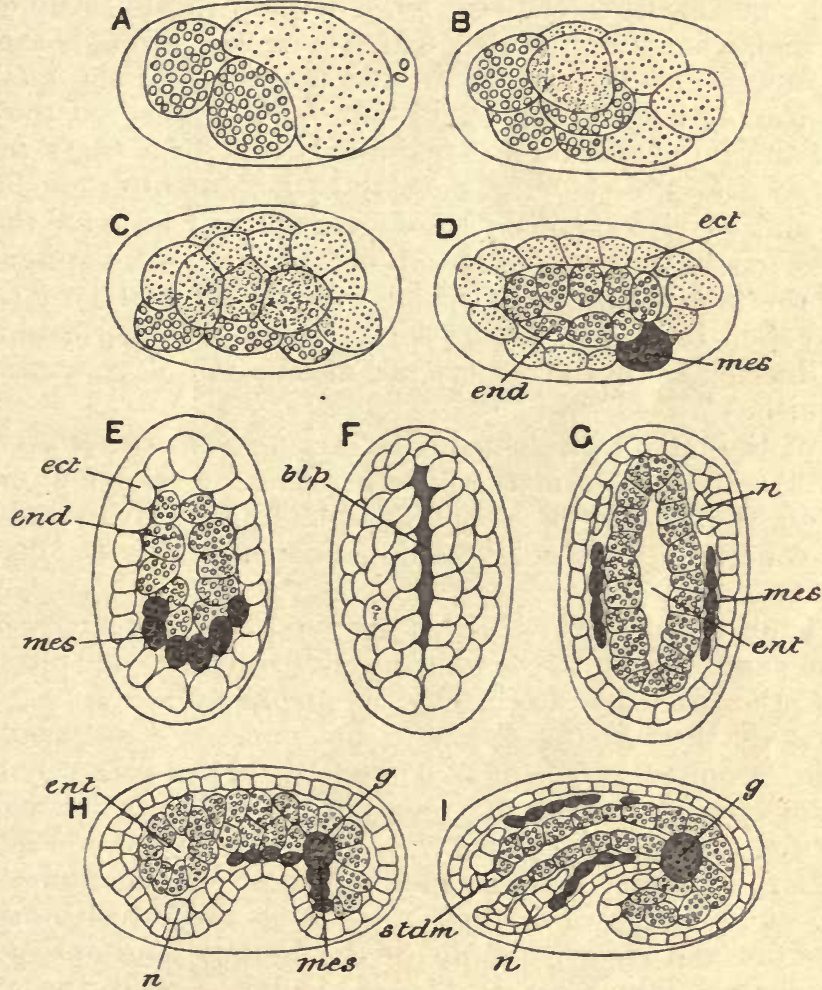

FiG. 248.-Development of Ascaris nigrovenosa. blp. blastorore; ect. ectoderm ; end. enduderm ; ent. enteron; g. genital cell; mes. mesoderm; n. nervous system; stdm. stomodæum. (From Korschelt and Heider, after Goette.)

ectoderm and the endoderm (E-H.) The body-cavity is not a true colome (i.e., a cavity formed in the interior of a mass of mesoderm or arising as an outgrowth from the archenteron), but is derived from the primitive blastula- or segmentation-cavity. The nervous sytem is developed from certain cells (G-I,n.) which bud off from the ectoderm at the anterior end of the body. The reproductive cells originate from a pair of the inner cells $(H, I, g$. which become differentiated from the rest at an early stage. 
Many of the Nematoda have a curious and complex lifehistory : a few examples will be selected for description.

Rhabdonema nigrovenosum lives, in the sexual condition, in the lungs of Frogs and Toads : it is remarkable among members of the class in being hermaphrodite. The eggs are laid and the embryos pass from the lungs inta the enteric canal of the host, are expelled with its fæces, and develop in water into a sexual Nematode, called the Rhabditis-form, in which the sexes are separate: in this the fertilised eggs develop in the body of the female, and, when fully formed, make their way through the wall of the uterus and proceed to devour the whole of the maternal tissues, leaving nothing but the cuticle. Being set free, they live in mud until they succeed in gaining access to a frog's mouth, when they pass into the lung, develop hermaphrodite reproductive organs, and so re-commence the cycle. It will be seen that we have here a peculiar form of alternation of generations, distinguished not by the alternation of a sexual with an asexual form (metagenesis) as in Hydrozoa, but by the alternation of a hermaphrodite with a diœcious form. This type of alternation of generations is distinguished as heterogeny.

One of the most terrible parasites of man is Trichinella spiralis (Fig. 249), a minute worm, the male (C) a little over $1 \mathrm{~mm}$. ( $\frac{1}{1} \mathrm{in}$.) in length, the female (B) about $3 \mathrm{~mm}$. ( $\frac{1}{8}$ in.). In the adult or sexual condition it lives in the intestine of Man, the Pig, and other Mammals.

The adult females, which are viviparous, leave the cavity of the intestine and bore into its wall, usually reaching the interior of one of the lacteal vessels of the lymphatic system. Here they deposit their young $(B, e)$ to the number of as many as a thousand or more at a time. These are carried passively in the stream of lymph, perhaps ultimately in the blood-stream, and thus distributed throughout the body. Eventually they travel into the system of voluntary muscles, such as those of the limbs, back, tongue, etc. Each worm then penetrates the sarcolemma of a muscle-fibre and coils itself up in the muscle substance (A); a spindle-shaped cyst (cy.) is formed round it, and the muscle undergoes more or less degeneration. This process gives rise to various morbid symptoms in the host, but, after some months the cysts become calcified, and the danger to the infected individual is over. The flesh of a "trichinised" human subject has been estimated to contain $100,000,000$ encysted worms, and that of an infected pig 85,000 to the ounce. In order that further development of the encysted and sexless Trichinæ should take place, it is necessary for the infected flesh of the host to be eaten by another animal in which the worm is capable of living, e.q. that of Man by a Pig or Rat, or that of a Pig by Man. When this is done the cysts are dissolved by the digestive juices, the worms escape, 
develop reproductive organs, and copulate, the young migrating into the muscles and producing the disease as before. The result of eating an ounce of "trichinised" or "measly" pork,

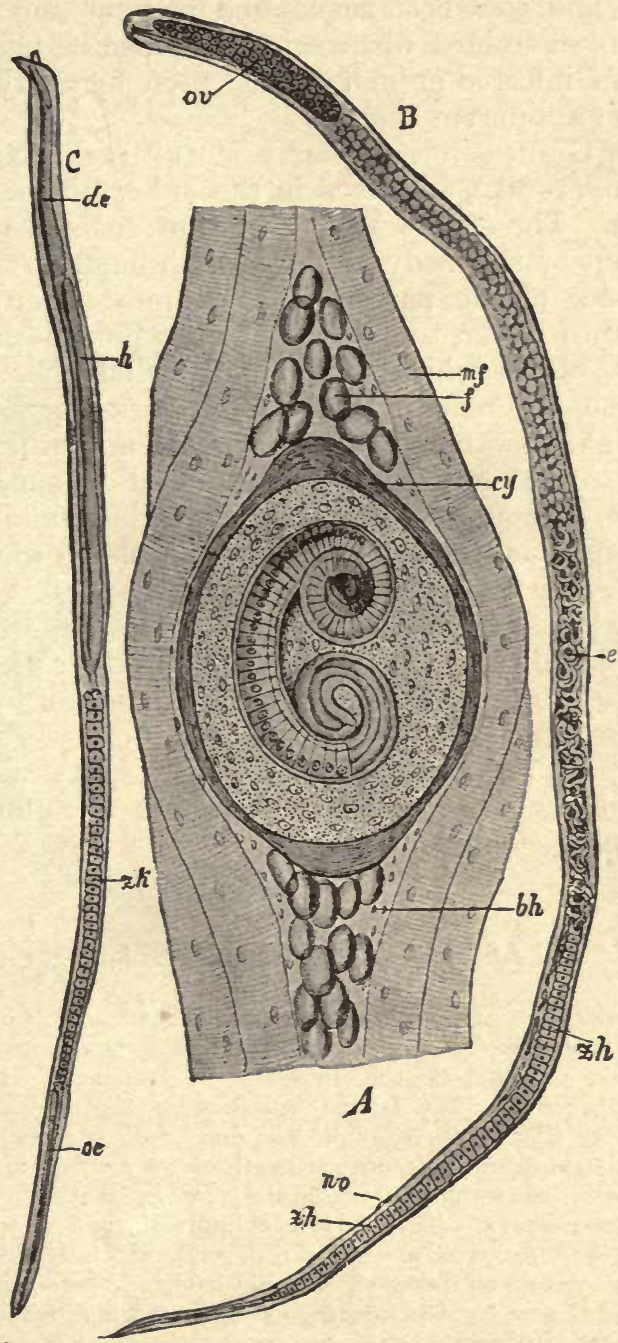

F10. 249.-Trichinella spiralis. A, encysted form, in muscle of host; B, female ; C, male. bh. connective-tissue envelope; cy. cyst; de ejuculatory duct; $e$ embryos, f rat-globules : $h$. testis; $m$. f. nuscle-fibre; oc. pharynx; ov. ovary ; 200 . gonopore ; $z h$. cell-masses. in intestine. (From Lang's Comparative Anatomy, after Claus.)

improperly cooked, might be the liberation in the human intestine of perhaps 80,000 worms; and, if half of these were females, each producing 1,000 embryos, some 40,000,000 worms 
would shortly begin to migrate into the muscles, and produce the various symptoms of "trichiniasis."

It will be noted that in this case the parasite is able to exist in various hosts, and that both sexual and asexual stages are passed through in the same host, dispersal of the species taking place by the flesh of an infected animal being eaten by another, either of the same or of a different species.

The female Guinea-worm (Filaria medinensis) attains a length of $30-200 \mathrm{~cm}$. (1-6ft.), and lives in the subcutaneous connectivetissue of Man. The eggs develop in the uterus, and the new-born young pass out of the body of the host through abscesses caused by the presence of the parasite. If, as must often be the case, they escape into water, they make their way into the body of a Water-flea (Cyclops), which is the intermediate host, and in this condition probably reach their human host once more in his unfiltered drinking-water. Filaria bancrofi $i$ and other species are, in the larval condition, parasites in the blood of man. The adult females of $F$. bancrofti live normally in the lymphatic vessels. They are viviparous, and the young when they escape reach the blood and are thus distributed. Normally they are to be found in the peripheral vessels only at night-time, when the superficial vessels are more dilated and thus permit of their passage. They are transmitted from one human host to another by the agency of mosquitoes, which act as intermediate hosts. $F$. bancrofti is very widely distributed in tropical countries, and is the cause of a disease called filariasis, with a variety of symptoms-such as anæmia, lymphatic tumours, elephantiasis.

\section{CLASS II.-ACANTHOCEPHALA.}

This class contains a number of genera of parasitic worms, of which Echinorhynchus is the chief. The present section will be devoted to this genus, a not uncommon parasite in the intestine of Mammals, Birds, Reptiles, Amphibians, and Fishes. The largest species, E. (Gigantorhynchus) gigas, is found in the Pig (Fig. 250, A), and has once been recorded in the human subject : it may attain, in the female, a length of $50 \mathrm{~cm}$, or more than half a yard. Most species are small, not exceeding $1 \mathrm{~cm}$. in length,

External Characters. - The body is cylindrical, and ends in front in a protrusible portion, the proboscis $(\mathrm{A}, p ., \mathrm{B}, p r$.$) , which is cylindrical and is$ covered with many rows of recurved shitinoid hooks. The worm lies with the proboscis sunk in the wall of the intestine of its host, which is sometimes riddled with holes formed in this way. In some species there is a distinet neck $(B, n$. between the proboscis and the trunk, and there may be a globular dilatation at the anterior end of the neck. At the hinder end of the body is a single aperture, the gonopore or reproductive aperture $(g n p$.$) : connected with this, in$ the male, is a protrusible, bell-like structure, the bursa $(b$.$) , which acts as a$ copulatory organ, like the somewhat similar organ in certain Nematoda. There is no trace of mouth, anus, or excretory pore.

The body-wall is covered with a stout cuticle, beneath which is a striated protoplasmic layer, probably representing the ectoderm. Then comes a layer of 
transverse, and then one of longtitudinal muscles. The body-wall thus constituted encloses a spacious body-cavity or cœlome containing a clear fluid.

In correspondence with the absence of mouth and anus there is no trace of enteric canal, the Acanthocephala resembling in this respect the Cestoda, the only other class of Metazoa which is entirely anenterous. Food is thus, as in tapeworms, taken entirely by absorption by the general surface of the body.

The proximal end of the proboscis is contained in a muscular sheath sunk in the anterior end of the trunk, and is provided with four retractor muscles (Fig. 250, r.m.). The muscles of the sheath are circular and act as protractors, At the sides of the base of the proboscis two club-shaped organs, the lemnisci
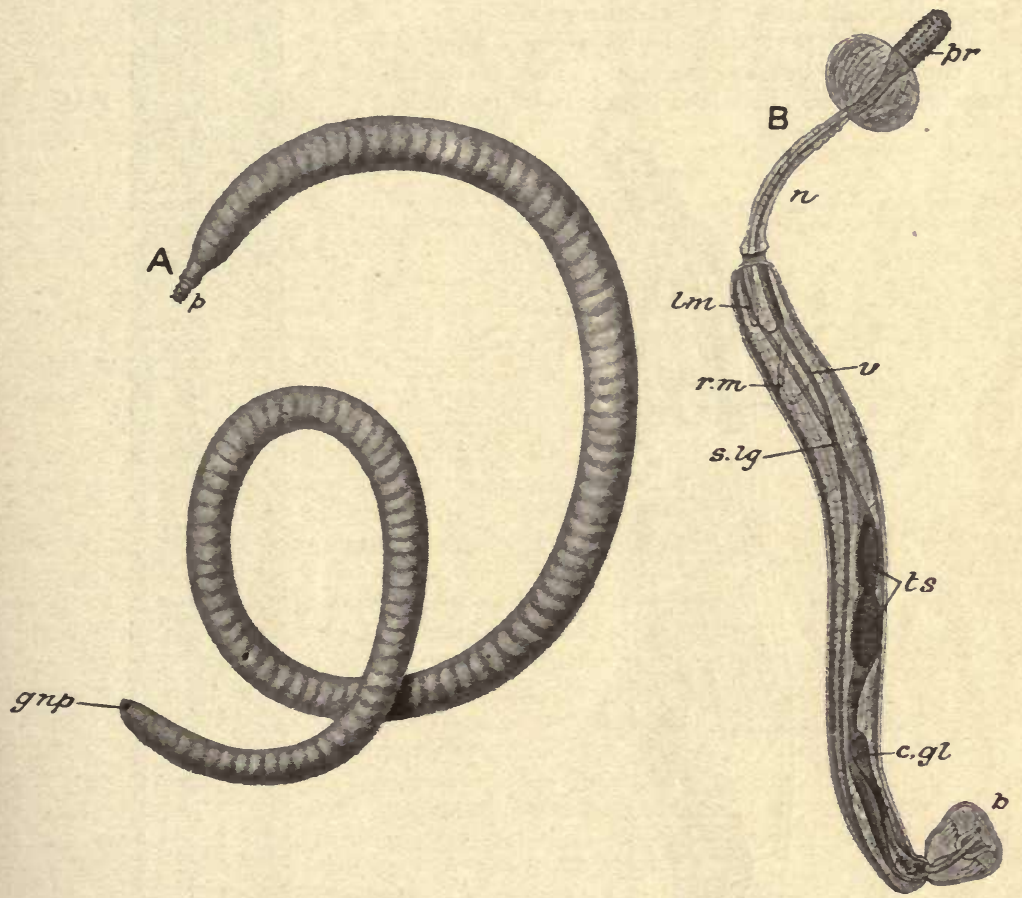

FIG. 250.-A, Pchinorhynchus (Gigantorhynchus) gigas, female from the Pig (nat. size; B, 2. lesiniformis, male from the edible Frog (magnified). b. bursa; c.gl. cement glands; gnp. gonopore ; lm. lemnisci ; $n$. neck; $p$. or $p r$. proboscis; $r$. $m$. retractor muscle of proboscis; 8 . lg. suspensory ligament; $t$. testis ; v. vessel.

(lm.), hang down into the body-cavity. Their function is quite unknown, but they have been compared with the cervical glands of Nematodes (p. 306).

In the body-wall run two longitudinal vessels ( $v$.) containing a granular fluid, and connected with a network of fine canals in the proboscis, bursa, \&c. The function of these vessels is not known with certainty : they may have to do with the absorption and circulation of nourishment.

The central nervous system (Fig, $251, n v$.) is represented by a single large ganglion placed at the base of the proboscis, and sending off nerves in various directions. In the male there are also two ganglia supplying the reproductive organs. Organs of sense are wholly absent.

A pair of remarkable excretory organs or nephridia (Fig. 253) have been found to occur in Echinorhynchus gigas. These consist of a pair of ramified 
protoplasmic masses situated in the body-cavity at the posterior end, near the genital aperture. In the interior is a system of branching canals, the terminal branches of which, each contained in one of the terminal lobes of the tree-like nephridium, are provided with ciliary flames; at the end of each lobe are a number of fine perforations placing the contained canal in communication with the body. cavity. The stalk of each nephridium contains a single main canal ; these unite to form a wide median dorsal channel which opens behind in the female into the unpaired portion of the oviduct and in the male into the ejaculatory duct.

The greater part of the body-cavity is occupied by the reproductive organs. The sexes are separate, and the female is larger than the male.

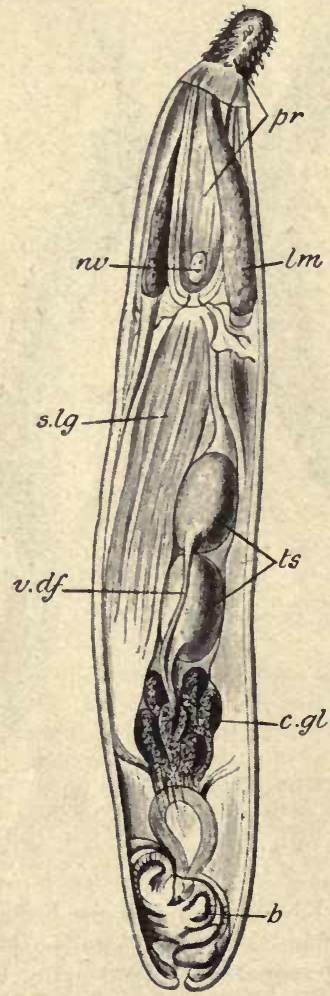

FIG. 251.-Echinorhynchus gigas. Dissection of male. b. bursa ; $c$. gl. cement glands ; lm. lemnisci; $n v$. nerve-ganglion; $p r$. proboscis ; s. lg. suspensory ligament; ts. testis ; $v$. $d f$. vas deferens. (After leuckart.)

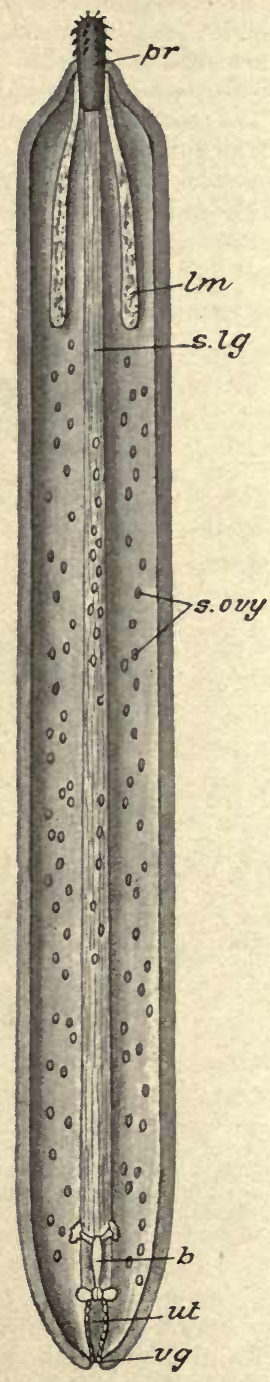

Fig. 252.-Echinorhynchus gigas. Dissection of female (semi-diagrammatic). b. bell ; $l m$, lemnisci ; $p r$. proboscis ; 8. ovy. swimming ovaries; $u t$. uterus; $v g$. vagina.

In both sexes the gonads and their ducts are connected with a great suspensory ligament (s.lg.), which extends backwards from the end of the proboscis-sheath.

In the male there are two ovoidal testes (Fig. $251, t s$. ) connected with the 
suspensory ligament. From each a vas deferens $(v . d f$.$) , furnished with several$ vesicula seminales or sacs for the storage of spermatic fluid, passes backwards
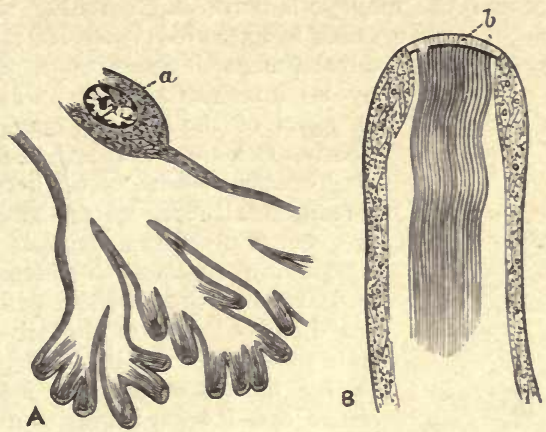

FIG. 253.-A, longitudiual section through the terminal twigs of the nephridia of Ichinorhynchus gigas; highly magnified. a, nucleus. B, a terminal twig more highly magnified. $b$, the porous membrane. (From Shipley, after Kaiser.)

and unites with its fellow to form an ejaculatory duct, with which are connected about half a dozen cement glands $\left(c . g l_{\circ}\right)$. The ejaculatory duct opens into the bursa or bell-like copulatory organ (b), and has at its opening a small papilla acting as a penis.

In the female the ovary is connected with the suspensory ligament (Figs. 252 and 254 , s.lg.). When ripe, groups of ova-known as the "swimming ovaries" (s.ory.)-become detached and swim freely in the borlyeavity, where they are impregnated. The ducts are very peculiar. Connected with the end of the suspensory ligament is a muscular uterine bell $(b)$, widely open anteriorly (Fig. 254, x) into the colome, and having towards its posterior end a small aperture, also communicating with the colome $(y)$. The bell is connected with a narrow double passage leading to a uterus (ut.), which itself opens by the genital aperture at the posterior end of the body. The uterine bell performs rhythmical swallowing movements, and as the eggs-containing partly developed embryos-float in the colome they are swallowed by the bell. The immature eggs, which are globular, are passed back into the cœlome through the posterior aperture $(y)$ of the bell; but the mature eggs, which are spindle-shaped and covered with a chitinous investment, make their way from the bell to the uterus through the narrow passages, and so to the vagina.

The early stages of development take place in the colome. Segmentation is regular, and a peculiar form of gastrula is produced, having neither archenteron nor blastocole-- in other words the ectoderm and endoderm are in close contact with one another, and no central cavity is enclosed by the latter. The ectoderm layer, which is devoid of cell-limits, secretes a cuticular membrane investing the embryo, then a second membrane is formed within the first, and a third within the second; the embryo thus comes to be enclosed in a triple case, which differs from an egg-shell in being

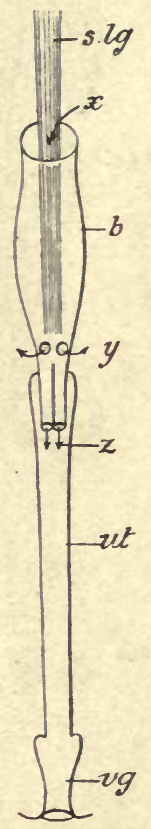

Fig. 254.-Female organs of Echinorhynchus. b. uterine bell; 8 . Ig. suspensory ligament ; ut. uterus ; vg. vagiua ; $x . y$. aperture of bell; $z$. apertures leading from bell to uterus. (After Hertwig.) 
formed by the developing ectoderm. At what will become the anterior end chitinoid hooks appear.

At about this period the embryo is born, and reaching the intestine of the

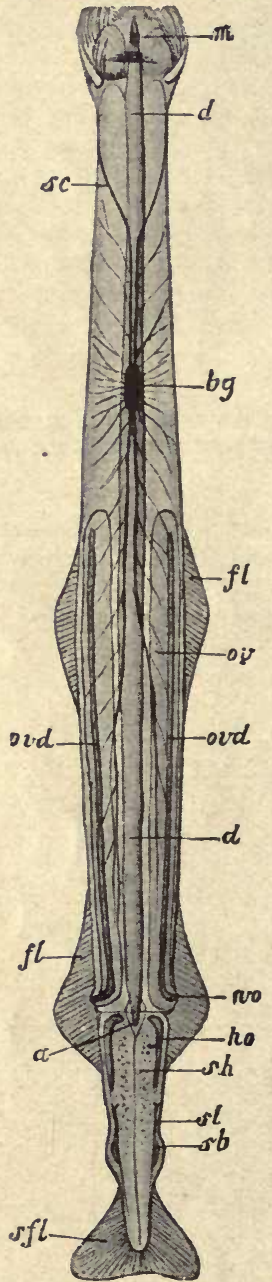

FIG. 255.-Sagitta hexaptera, from the ventral aspect. $a$. anus; bg. ventral ganglion; $d$. intestine; $f$. lateral fins; ho. testis ; m. mouth; ov. ovary; ord. oviduct; $8 c$. oesophageal connective; $s b$. vesicula seminalis; s. $f$. tail fin; sh, tailcavity ; sl. spermiduct. (From Lang's Comparative Anatomy, after Hertwig.)

preceding classes, is formed of host, is extruded with its fæces. Its further development depends upon its being swallowed by an intermediate host, which, in the case of E. gigas of the Pig is a maggot, the larva of a Beetle, Cetonia aurata. The Echinorhynchi of fresh-water Fish have for their intermediate host certain small fresh-water Crustacea belonging to the genera Gammarus and Asellus.

Having reached the intestine of the intermediate host, the chitinoid embryonic membranes are dissolved by its digestive juices, and the embryo either fixes itself to the wall of the intestine or makes its way into the colome; in either case it soon begins to undergo further development. The endoderm, hitherto a solid mass of cells, undergoes a process of splitting, becoming divided into an outer layer in contact with the ectoderm and a solid central axis. The latter gives rise to the reproductive organs and the suspensory ligament, the outer layer to an epithelium, from which the body-muscles arise; the cavity formed by the splitting of the endoderm is the cœlome. Part of the proboscis and its sheath are also of endodermal origin. The ectoderm gives rise to the protoplasmic layer of the body-wall, to the whole system of vessels, and to the lemnisci. The larval cuticle is thrown off and a new one formed. The larva reaches adult proportions and attains sexual maturity only if the intermediate host is eaten by the permanent host.

\section{CLASS III.-CH ETOGNATHA.}

The present group, like that just discussed, is a very small one, containing only three genera (Sagitta, Spadella and Krohnia) of curious arrowshaped worms, all but one species of which are pelagic.

External characters. - The body (Fig. 255) is elongated and nearly cylindrical, and is divided into head, trunk, and tail, the head being marked off by its somewhat rounded form, while the junction of trunk and tail is indicated by the ventrally placed anus $(a)$. The tail bears a horizontal expansion, or caudal fin $(s . f$.), and there are also horizontal lateral fins $(f$.$) - a single pair in$ Spadella, two pairs in Sagitta.

Body-wall. - There is no cuticle, but the outer layer of the body-wall is formed by an epidermis or deric epithelium (Fig. 256, d. epthm), which, instead of being syncytial as in the two delicate basement membrane, and then a layer of muscles $\left(m_{0}\right)$, the fibres of 
which are striated and disposed longitudinally in four bands-two dorso-lateral and two-ventro-lateral - an arrangement which recalls that of the corresponding layer in Nematoda.

Enteric Canal. - The mouth (Fig. 255, m.) is a longitudinal slit-like aperture on the ventral surface of the head; on either side of it are several sickle-shaped

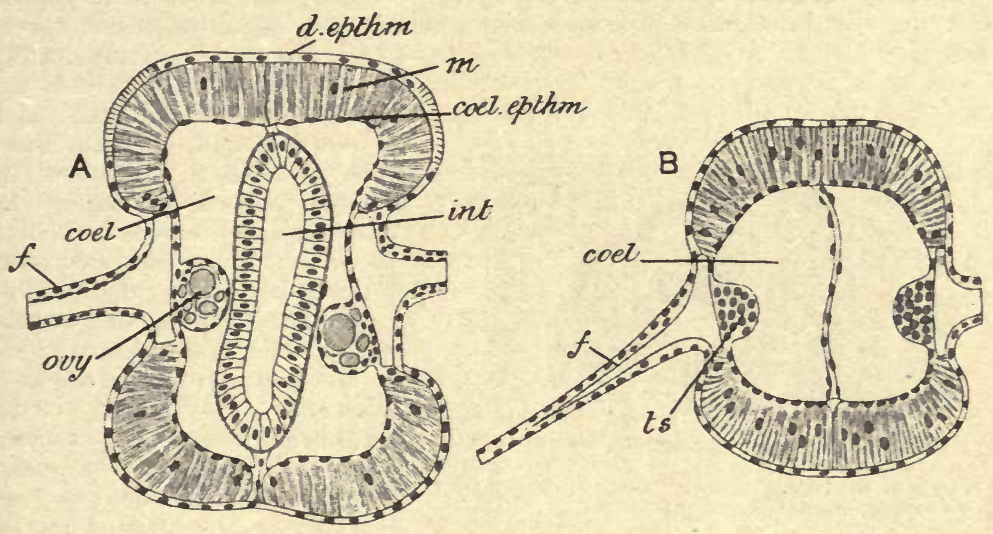

FIG. 256.-Sagitta bipunctata. Transverse sections, A, of trunk ; B, of tail. coel. coelome ; coel. epthm. layer of nuclei of the muscle-cells formerly regarded as a colomic epithelium; d. epthm. deric epithelium ; $f$. fin ; int. intestine ; $m$. muscles ; ovy. ovary; $t 8$. testis. (After Hertwig.)

chitinoid hooks (Fig. $257 \mathrm{gh}$.) which are moved by muscles in a horizontal plane and serve as jaws. The anterior region of the head also bears spines, and is strengthened by chitinoid plates and partly covered by a hood-like fold of the integument.

The mouth leads by a muscular pharynx or stomodæum into a straight intestine $(d)$, which extends through the trunk and opens by the anus $(a)$ at the junction of trunk and tail.

Cœlome.-At the junction of the head with the trunk, and of the trunk with the tail, are transverse partitions or septa, dividing the cœlome into compartments. The trunk region of that cavity is further sub-divided by two longitudinal partitions, the dorsal and ventral mesenteries, which respectively connect the dorsal and rentral surfaces of the intestine with the body-wall : the tail-region of the cœlome is similarly divided into right and left chambers by a longitudinal vertical partition (Fig. 256, $\mathrm{A}$ and $\mathrm{B}$ ).

There is no trace of vascular system or of excretory canals. The nervous system, on the other hand, is much

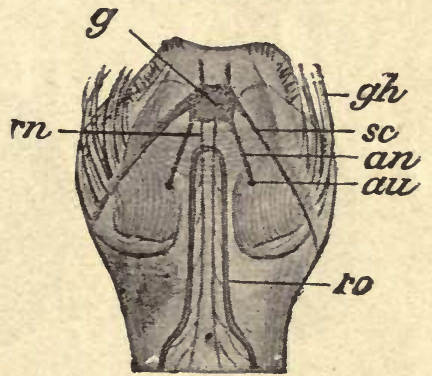

FiG. 257-Head of Sagitta bipunctata, from above. an. optic nerve; au. eye; $g$. brain : gh. hooks; $r n$. olfactory nerve ; ro. olfactory organ; sc. oesophageal connective. (From Lang's Comparative Anatomy, after Hertwig.) better developed than in either of the preceding classes, in accordance with a free life and active movements. On the dorsal side of the pharynx is a comparatively large brain (Fig. 257,g), which sends off on each side a long nerve-cord, the oesophageal connective (sc.). The two connectives sweep round the enteric canal and unite on the ventral surface, not 
far from the middle of the trunk, in an elongated ventral ganglion (Fig. 255, bg.), from which numerous nerves are given off. The brain senils nerves to the eyes (Fig. 257, an.) and to the olfactory organs (ro.), and is also connected with two pairs of ganglia in the head, which lie deeply sunk in the mesoderm: all the rest of the nervous system retains its primitive connection with the ectoderm.

sensory Organs.- On the surface of the body are numerous little papillæ carrying stiff bristle-like processes, and probably serving as organs of touch.

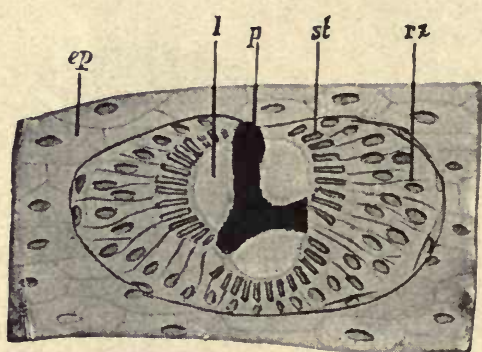

FIG. 258.-Section of eye of Sagitta hexaptera. ep. epiderm; $l$. lens; $p$. pigınent ; $r z$. visual cells; st. ruds. (From Lang's Comparative Anatomy, ufter O. Hertwig.)

There are two eyes (Fig. 258), situated one on each side of the dorsal surface of the head: each is globular and contains three biconvex lenses $(l$. $)$, separated by pigment $(p$.$) and surrounded by$ rod-like sensory cells $(r z$.). Behind the head is a ring-like structure, of the nature of an annular ridge of peculiarly modified and in part ciliated cells (Fig. 257, ro.): to this an olfactory function has been assigned.

Reproduction. - The Chrtognatha are monœeious. The ovaries (Fig. 255, ov., Fig. 256, ovy.) are elongated organs situated one on each side of the trunk-region of the colome, and opening by a narrow oviduct just in front of the posterior septum. The testes (Fig. 255, ho., Fig. 256, ts.) are similarly situated in the tailregion of the cœlome, and have the form of narrow ridges from which immature seminal cells are given off and develop into sperms in the cœlome. The spermiducts or vasa deferentia are delicate tubes $(s l$.) opening at one end into the colome by a ciliated funnel-like extremity, and at the other end dilating into a reservoir or vesicula seminalis $(s b$.), which opens externally in the posterior region of the tail.

Development.- Internal impregnation takes place, and the oosperm, segmenting completely and regularly, forms a typical gastrula by invagination (Fig. $259, \mathrm{~A})$. Two endoderm cells $(g$. $)$ at the anterior end of the archenteron, i.e. the
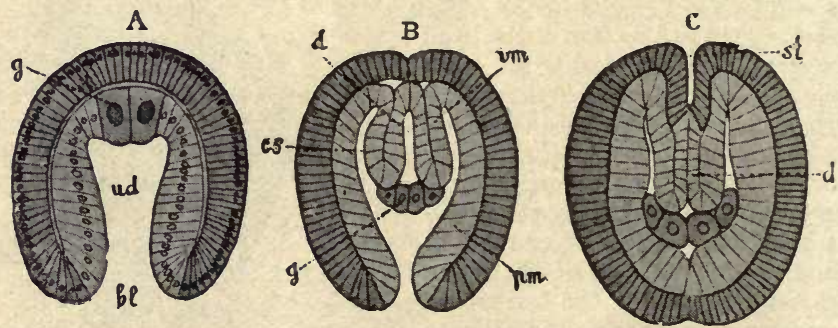

FIG. 259. - Three stages in the development of Sagitta. bl. blastopore; c8. colomic sacs; $d$. mesenteron ; $g$. sexual cells ; pm. parietal layer of mesoderm ; st. stomodæum ; vm. visceral layer of mesuderm. (From Lang's Comparative Anatomy, after O. Hertwig.)

end opposite to the blastopore, soon increase greatly in size, and are the rudiments of the gonads. This precocious differentiation of the sex-cells is a point of considerable importance, as will be seen hereafter. Before long these cells migrate into the archenteron and divide, forming a group of four cells $(B, g$.$) , two of which$ subsequently become the ovar and two the testes. At the same time two folds 
of endoderm grow into the archenteron from its anterior end, partly dividing the cavity into three parts - a middle division or mesenteron $(d)$-the rudiment of the intestine, and two lateral divisions - the metentera, or cœlomic sacs (c.s.)-which give rise to the right and left compartments of the colome of the trunk. From the latter are given off in froni a pair of small head-cavities. Owing to the rapid elongation of the embryo in the stages following, all the cavities become for a time obliterated: subsequently the cavities of the enteric canal and cœlomic sacs re-appear; the tail-region of the body-cavity is formed from the posterior, undivided portion of the archenteron. The blastopore (bl.) now closes and an invagination of ectoderm-the stomodæum (st.)-takes place at the anterior end, and finally communicates with the mesenteron.

From this it will be seen that the ectoderm of the gastrula gives rise to the deric epithelium of the adult and to the epithelium of the pharynx, which is therefore a stomodæum ; from the same layer the nervous system arises at a later stage. The epithelium of the intestine arises from the mesial (inwardly-turned) layers of the two endodermal ridges. The muscular layer of the body-wall arises from the rest of the endoderm, i.e. that portion of it which remains in immediate contact with the ectoderm. Thus in Sagitta the mesoderm is entirely derived from the endoderm of the gastrula.

\section{APPENDIX TO NEMATHEL- MINTHES.}

\section{Family Chatosomida.}

This family includes three genera of small worms, Chatosoma (Fig. 260), Tristicochata, and Rhabdogaster, which are sometimes included among the Nematoda.

The body is elongated, its anterior region being sometimes dilated to form a head. Either the whole body, or the dorsal surface only, is beset with fine setæ, and there may be a double row of movable chitinoid hooks round the head, reminding us of the "jaws" of Sagitta. The ventral surface bears curious locomotor rods $(f)$, either hooked or with knobbed ends: by these the animals crawl. The mouth is anterior and terminal, the anus posterior and ventral, and there is a muscular pharynx. The sexes are separate. The male has a single testis: the vas deferens opens along with the anus: there are two penial setæ. The female has paired ovaries and a single vagina opening near the middle of the body on the ventral side.

\section{Family Echinoderidae.}

Echinoderes is a minute marine worm of cylindrical form with a flattened rentral surface. The body is segmented, or divided into rings, eleven or twelve in number, all strongly cuticularised, and most of them bearing spines (Fig. 261). The mouth is placed at the anterior, the anus at the posterior end of the body : 
the former opens into a sac, which can be everted so as to form a proboscis or introvert, and is armed with spines. The enteric canal consists of a muscular pharynx and a straight intestine. A pair of sacs opening by ciliated ducts on the tenth segment appear to be excretory organs. The sexes are separate : the gonads are paired sacs opening at the posterior end of the body.

\section{Family Desmoscolecida.}

Desmoscolex is also a minute marine worm, having a globular head and a variable number of segments (Fig. 262). The head bears four movable chitinoid rods or setæ, and a pair of

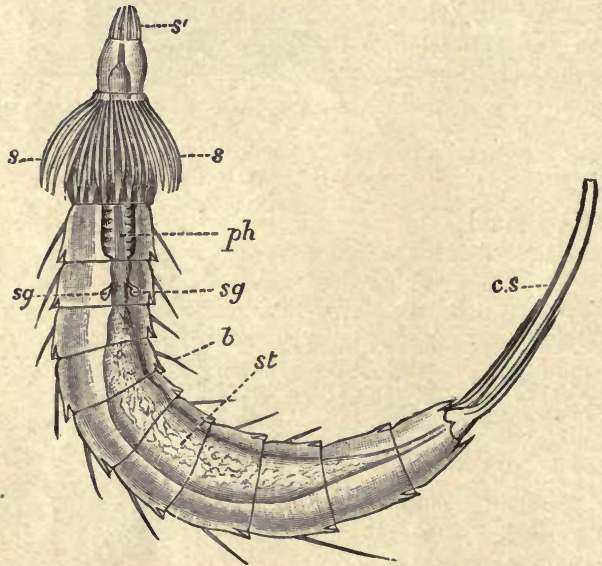

FIG 261.-Echinoderes, $x$ about 210. $b$, spine c.8. caudal spine; $p h$. pharynx; 8 . and $s^{\prime}$. spines on the proboscis; 8.9 . salivary glands; 8 . stomach. (After Hartog.)

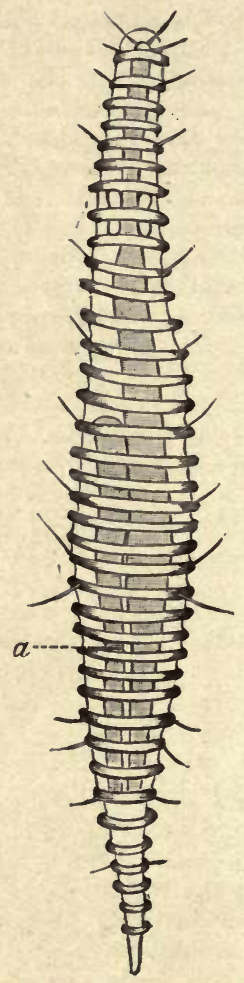

FiG. 262.-Female Desmoscolex ventral view, $\times 260$. $a$, ovary. (From Shipley, after Panceri).

similar structures occurs on many of the other segments. The terminal mouth leads by a muscular pharynx into a straight intestine : the anus is dorsal in position. The animal is diceious ; the gonads have the form of simple sacs, the testis opening along with the anus, the ovary on the ventral surface anterior to the anus. The male has a pair of penial setæ.

\section{AFFINITIES AND MUTUAL RELATIONSHIPS OF THE NEMATHELMINTHES.}

The affinities of all the classes of Nemathelminthes are very obscure, and the propriety of grouping them into a single phylum is extremely doubtful. They all agree in being elongated, cylindrical worms with a body-cavity, which is sometimes of the nature of a true cœlome; there is a certain resemblance 
between Nematoda and Chrtognatha in the muscular system; and the lemnisci of Acanthocephala have been compared with the cervical glands of Nematoda. Beyond these points there is little to unite the three classes; and, on the other hand, the proboscis of Acanthocephala recalls the rostellum of Cestoda.

Very various views have been put forward as to the affinities of the Chætognatha. But, in the absence of adequate evidence of any near relationship with higher phyla, they may be regarded as having their nearest known relatives, even if very remote, in the Nemathelminthes. In connection with this question, the Chrtosomidæ, briefly described in the Appendix (p. 319), seem to require consideration. Other possible relationships suggested by the mode of development of the coelome from hollow diverticula of the archenteron and by other features will be referred to in later sections.

The three families placed as an Appendix to the phylum present some undoubted resemblance to the Nematoidea : this is especially the case in the reproductive organs of the Chætosomidæ, and still more in those of Desmoscolex. But the segmentation of the body in both Desmoscolecidæ and Echinoderidæ and the presence of setæ show a certain resemblance to higher worms or Annulata, which will be more fully appreciated when that phylum has been studied. 


\section{SECTION VII}

\section{PHYLUM TROCHELMINTHES}

ThE typical larval form of a number of the groups which have yet to be studied is a form which is known as the trochospluere or, more usually, trochophore. It is necessary that a clear idea should be formed at this stage of this important larva, reference to which will very frequently be made in the sections that follow. The general shape of a typical trochophore is oval or pear-like (Fig. 263) with a broader and a narrower end and distinct bilateral

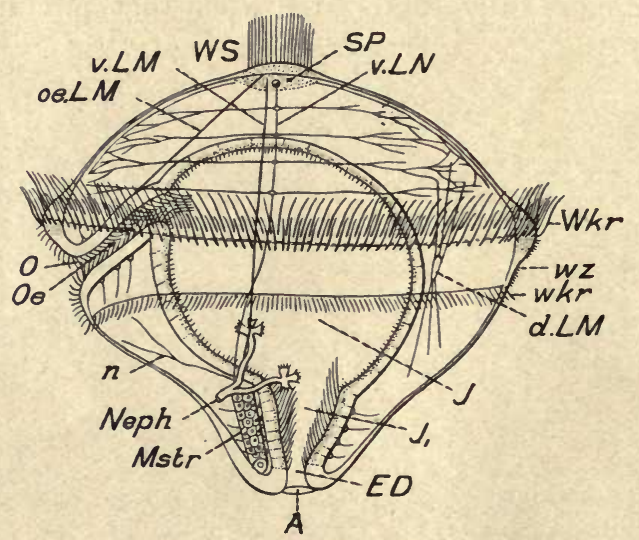

FIG. 263.-Trochophore. A. anus; $d . l M$. dorsal muscles ; $E D$. rectum; $J$. stomach ; $J 1$. intestine; Mstr. Inesoderm-band ; $n$. nerves; Neph. nephridia; 0 . mouth; Oe. cesophagus ; $a L M$. osophageal longitudinal muscle; $S P$. apical plate; $v . L M$. ventral nuscle; $v . L N$. lateral nerve ; $W k r$, vckr., pre- and post-oral bands of cilia ; WS. apical cilia ; vz. adoral cilia. (From Hertwig's Zoology, after Hatschek.)

symmetry. Encircling the body about the middle, or rather nearer the broad than the narrow end, is a double circlet of strong cilia, the pre-oral circlet ( $W k r$. ) or prototroch, situated on a corresponding ring-like thickening of the ectoderm; behind the mouth is often a second circlet of cilia, the post-oral circlet $(w k r$. ) and a ciliated groove or ciliated streak usually runs backwards from it along the middle of the ventral surface. The mouth, situated just 
behind the pre-oral circlet, leads into an alimentary canal, which at first runs nearly transversely, and then bends round so as to extend back towards the narrow end, near which it opens on the exterior by an anal aperture. About the middle of the broader (anterior) end of the trochophore is a thickening, the apical plate (SP.), projecting from which are usually a number of sensory cilia ( $W S$.); and in many trochophores eye-spots and a pair of short tentacles occur in close relation with the apical plate, which is the nerve-centre of the larva. A pair of ciliated tubes-the excretory organs or nephridia (Neph.) - may be present.

In the higher groups in which this form of larva occurs, the adult condition is attained by modifications and new developments of so radical a nature that the transition from larva to adult is of the nature of a metamorphosis. Sometimes the narrow part of the larva elongates and becomes divided into a series of sections foreshadowing the metameres of the adult animal ; in other cases, in which no metamerism occurs, radical changes of other kinds lead to the adult form. But in all these higher groups, whatever the nature of the changes involved, there is a metamorphosis, and the adult animal is totally unlike the larva. In a small number of forms now to be dealt with, however, there is no such radical change, and the adult may be looked upon as a somewhat modified trochophore. The groups thus associated together may not be be genetically related: they may have become independently developed from trochophore-like ancestors, but the possession of the general characters which have been referred to above renders it convenient to group them together and regard them as constituting a small but well-marked phylum. The groups referred to are the Rotifera or Wheel-animalcules, together with the Gastrotricha. Associated with these, though scarcely to be included in the same phylum, are the Dinophilea and Histriobdellea.

\section{CLASS I.-ROTIFERA.}

The Rotifers or "Wheel-animalcules" are microscopic creatures, very abundant in pools, gutters, \&c., and formerly classed with the Infusoria, to which several of them bear a superficial resemblance. But in spite of their minute size they are multicellular animals, having an enteric canal, a spacious body-zavity, nephridial tubes, gonads, a nervous system, and sense-organs, and have therefore no real relationship with the Protozoa.

\section{Example of the Class-Brachionus rubens.}

External Characters.-Brachionus (Fig. 264) is one of the commonest members of the class, being frequently found in abundance in ponds, ditches, \&c. The female is about $\frac{1}{3} \mathrm{~mm} \cdot\left(\frac{1}{75} \mathrm{in}\right.$.) in 
length, and is divisible into two distinct parts - a broad anterior region, the trunk, and a slender movable tail $(t$.$) . The trunk is$ enclosed in a glassy cuirass or lorica $(l r$.$) , formed by a thickening$ of the cuticle and produced into several spines: the tail is wrinkled superficially and ends in two slender processes, together forming a kind of forceps. One surface of the trunk is flattened, and owing to the position of the mouth, is considered as ventral,
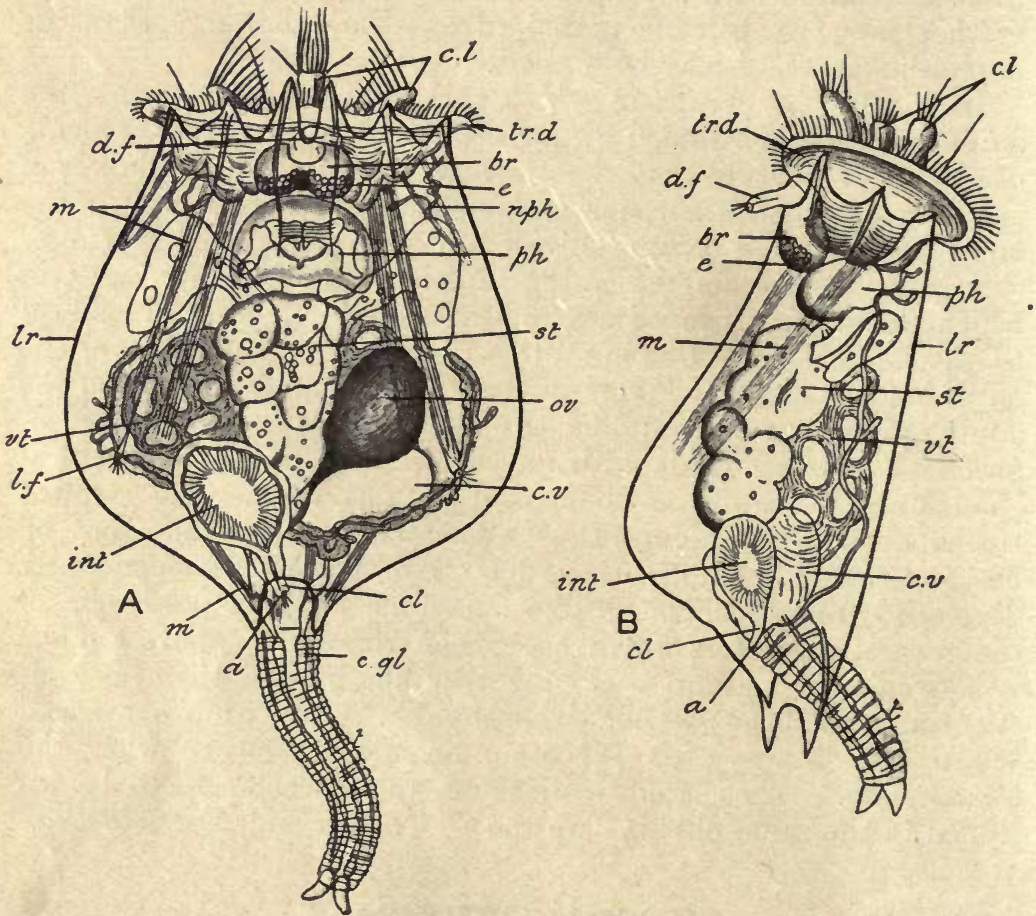

Fio. 264.-Brachionus rubens, female. A, from the dorsal aspect; $B$, from the right side. $a$. anus ; $b r$. brain ; $d . f$. dorsal feeler; $c . g l$. cement-gland; $c l$. cloaca ; $c . l$. ciliary lobes; $c . v$. contractile vesicle ; e. eye-spot; int. intestine; $l r$. lorica; $l$. $f$. lateral feeler; $m$. muscular bands; $n p h$. nephridial tubes; ov. germarium; $p h$. pharynx; st. stomach; $t$. tail ; tr. $d$. trochal dise; $v t$. vitellarium. (After Hudson and Gosse.)

the opposite or dorsal surface is convex both from before backwards, and from side to side.

The anterior portion of the body projects from the lorica in the form of a transverse disc (tr.d.) with a prominent edge fringed with cilia: this is the trochal disc, and is one of the most characteristic organs of the class. By the action of the cilia the animal is propelled through the water, and, as in Vorticella, their successive flexion gives an appearance of rotation to the disc or "wheelorgan" whence the name of the class is derived. Within the circlet of cilia arise three prominences (c.l.) covered with cilia of large 
size. The trochal disc is not perfectly symmetrical, but has at one part of its circumference a depression in which the mouth lies: this marks the ventral surface. The anus $(a$.$) is dorsal in position,$ and is placed at the junction of the tail with the trunk.

The body-wall consists of an epidermal layer, without celllimits, covered by a chitinoid cuticle : it is by a thickening of the latter in the region of the trunk that the lorica is produced. There is no continuous muscular layer, but several bands of unstriped muscle $\left(m_{0}\right)$ pass from the lorica to the trochal disc in front and to the tail behind, and act as retractors of those organs.

Digestive Organs.-The mouth (Fig. 267, mth.) lies, as already mentioned, in the ventral region of the trochal disc, anterior to the ciliary circlet but posterior to the three ciliated lobes; it leads by a short buccal cavity into a pharynx (ph.) of peculiar structure known as the mastax, and constituting one of the most characteristic organs of the class. The mastax is a muscular chamber (Fig. 265) of rounded form, and contains, as a thickening of its cuticular lining, an elaborate apparatus for triturating the food. In the middle line is a forked structure, the incus, consisting of a small base or fulcrum $(j$.$) and$ of two branches or rami $(r$.$) .$ On either side of the incus is a hammer-like structure, the malleus, consisting of a handle or manubrium $(m$.) and of a

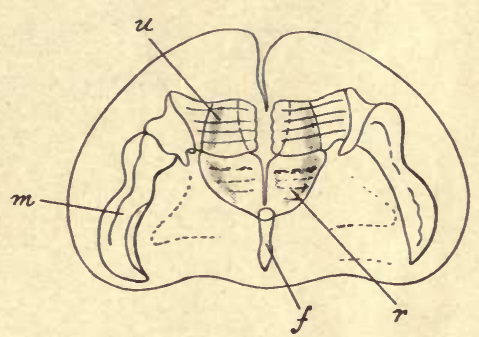

Fig. 265 - Pharynx of Brachionus rubens. $f$. fulcrum; $m$. manubrium; $u$. uncus; $r$. ramus. (After Hudson and Gosse.) toothed head or uncus (u.). By means of the muscular walls of the chamber the heads of the mallei are worked backwards and forwards upon the forked uncus, and thus reduce the organisms taken as food to a fine state of division.

The pharynx leads by a short gullet into a spacious stomach (st.), having a wall composed of very large epithelial cells, ciliated internally: with it are connected paired digestive glands. The stomach opens into a rounded intestine (int.), also ciliated internally, which communicates, by means of a short clorca $(c l$.), with the exterior. The stomach and intestine are formed from the archenteron of the embryo and are therefore lined by endoderm: the rest of the enteric epithelium is ectodermal, the pharynx being derived from the stomodæum, the cloaca from the proctodæum. Between the body-wall and the enteric canal is a spacious body-cavity containing a fluid which serves the purpose of blood and contains minute granules. 
The excretory system consists of paired nephridial tubes (Figs. 264 and 267, nph.) resembling those of the Platyhelminthes. Their general direction is longitudinal, but they are a good deal coiled and give off little tag-like processes ending in flame-cells. With the end of each tag, projecting into the body-cavity, is a long flagellum. The lumen of the tubes is intra-cellular: it is uncertain whether or not the cavities of the flame-cells communicate with the body-cavity by apertures in their walls. Posteriorly the nephridial tubes open into a bladder or contractile vesicle (c.v.), the contents of which are discharged, by periodical contractions, into the cloaca.

Nervous System and Sense Organs.-There is a single ganglion or brain (Figs. 264 and 267, br.), of proportionally large
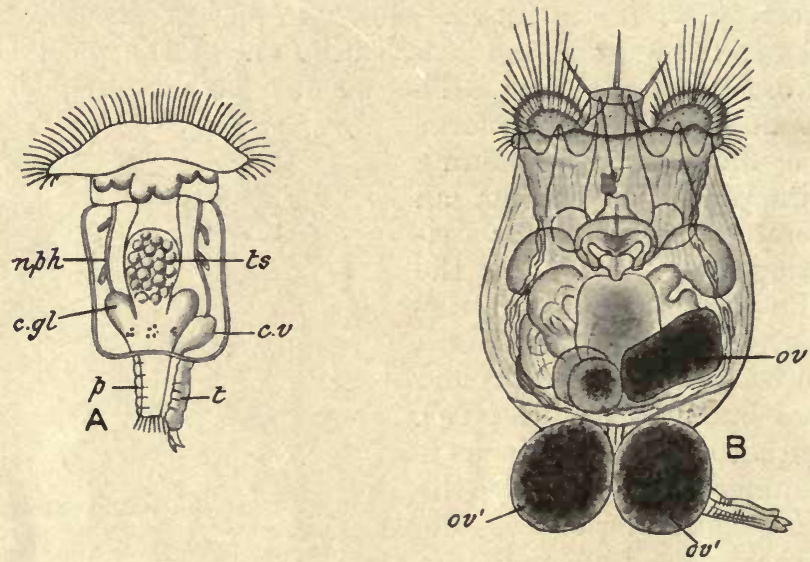

Fig. 266.-Brachionus rubens. A, male; B, female, with attached eggs; $c$. gl. cementglands; $c v$, contractile vesicle; $n p h$. nephridial-tube; ov. ovum in body; ov 1 . ova attached to base of tail ; $p$. penis ; $t$. tail ; $t$ s. testis. (After Hudson and Gosse.)

size, situated at the anterior end of the body, above (dorsal to) the mouth and pharynx. On the dorsal surface of the brain, where it comes into contact with the body-wall, is a small red eye-spot (e.). The only other organs which can be considered as sensory are three structures known as tactile rods or feelers; one of these $(d . f$.) is a small cylindrical process tipped with stiff hair-like bodies, which projects from the dorsal surface just behind the trochal disc : the other two $(l . f$.) are paired, situated on the dorsal surface of the lorica and not prominent.

The tail contains a pair of cement glands $(c . g l$.), by the secretion of which the animal is able temporarily to attach itself.

Reproduction and Development.-The sexes are lodged in distinct individuals, which present a striking degree of sexual dimorphism. The preceding description applies to the female, 
which is the form most commonly met with. In addition to the organs already mentioned, it has a germavium (ov., ovy.), connected with a large viteilarium (rt.) and opening by an oviduct into the cloaca.

The male (Fig. 266, A) is a very minute creature, not more than one-fourth the size of the female, and is strangely degenerate in structure. The enteric canal is absent, the trochal disc simple in structure, the nervous system and nephridial tubes greatly reduced, and the greater part of the body occupied by a large testis ( $t s$. which opens by a duct at the extremity of a protrusible, dorsally placed penis $(p$.$) .$

After extrusion the eggs are attached to the base of the tail of the female $\left(\mathrm{B}, o v^{\prime}.\right)$, where they undergo development: they are

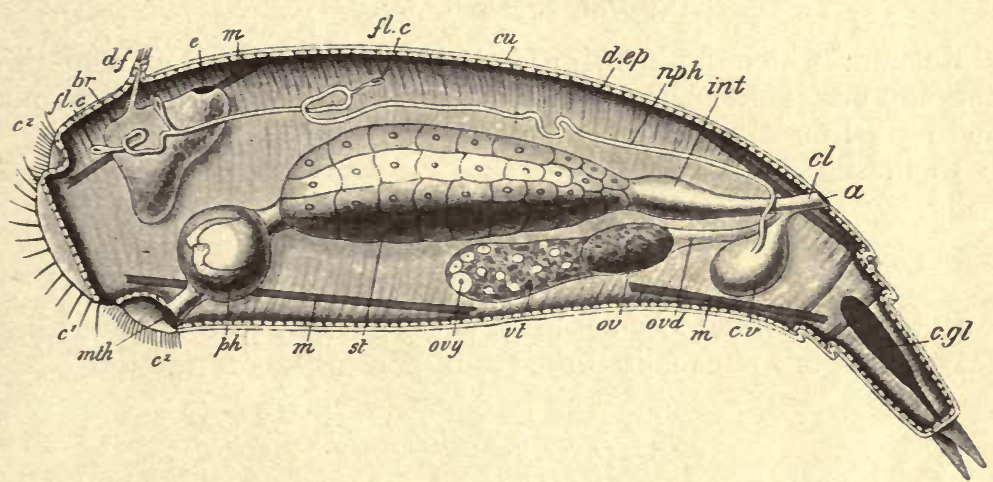

FIG. 267.-Diagram of a Rotifer. $a$. anus ; $b r$. brain; $c^{1}$. pre-oral, and $c^{2}$. post-oral circlet of cilia ; $c$. $g l$. cement gland; $c l$. cloaca ; $c u$. cuticle ; $d$. ep. deric epithelium ; $d$. $f$. dorsal feeler ; e. eye ; f. $c$. flame-cells; int. intestine; m. muscles; mth. mouth; $n$ ph. nephridial tube; ov. ovum; ovil. oviduct; ovy. germarium. ; ph. pharynx ; st. stomach; $v t$. vitellarium.

of two sizes, the larger giving rise to females, the smaller to males. Probably both kinds develop parthenogenetically, but in the autumn thick-shelled winter eggs are produced which appear to require fertilisation. These remain quiescent during the winter, and in the spring develop into females.

\section{2.-Distinctive Characters and Classification.}

The Rotifera are Trochelminthes of microscopic size. The anterior end is modified into a retractile trochal disc, with variously arranged cilia; the posterior end usually forms a mobile and often telescopically jointed tail. The mouth is anterior and more or less ventral in position, the pharynx contains a chitinous masticatory apparatus, and the anus is placed dorsally at the junction of the trunk with the tail. There is a spacious body-cavity devoid of epithelial lining. The excretory organs are a pair of nephridial tubes provided with flame-cells. The central nervous 
system consists of a single dorsal ganglion, with, in a few cases, a smaller ventral or sub-œsophageal ganglion. The sexes are separate, and the males are, in nearly all cases, smaller than the females and degenerate in structure.

The class is divided into five orders as follows :-

\section{Order 1.-Rhizota.}

Rotifera which are fixed in the adult state by the truncated end of the non-retractile tail.

Including Flloscularia, Stephanoceros, Melicerta, \&c.

\section{ORDER 2.-BDELLOÏDA.}

Rotifera which both swim freely by means of the cilia of the disc and creep after the manner of a Leech. The tail is telescopic and forked distally.

Including Rotifer, Philodina, \&c.

\section{ORder 3.-PloÏMA.}

Rotifera in which locomotion is performed by the ciliated disc only. The tail is usually forked and more or less retractile.

\section{Sub-order a.-Illoricata.}

Ploïma in which the trunk is not covered by a lorica.

Including Hydatina, Polyarthra Asplanchna, \&c.

$$
\text { Sub-order b.-Loricata. }
$$

Ploïma in which a lorica is present.

Including Brachionus, Euchlanis, \&c.

\section{ORDER 4.-SCIRTOPODA.}

Rotifera provided with setose appendages moved by striped muscles : skipping movements are performed by the aid of these, as well as swimming movements by the trochal disc. The tail is either absent or is represented by a pair of ciliated processes.

Including Pedalion and Hexarthra.

\section{ORDER 5.-TROCHOSPHARIDA.}

Globular Rotifera having the trochal disc represented by an equatorial circlet of cilia ; tail absent.

Including Trochosphcera only. 


\section{ORDER 6. -SEISONIDA.}

Marine parasitic Rotifers (Fig. 268), with the trochal disc reduced, the body long, narrow, and ringed, with a long slender

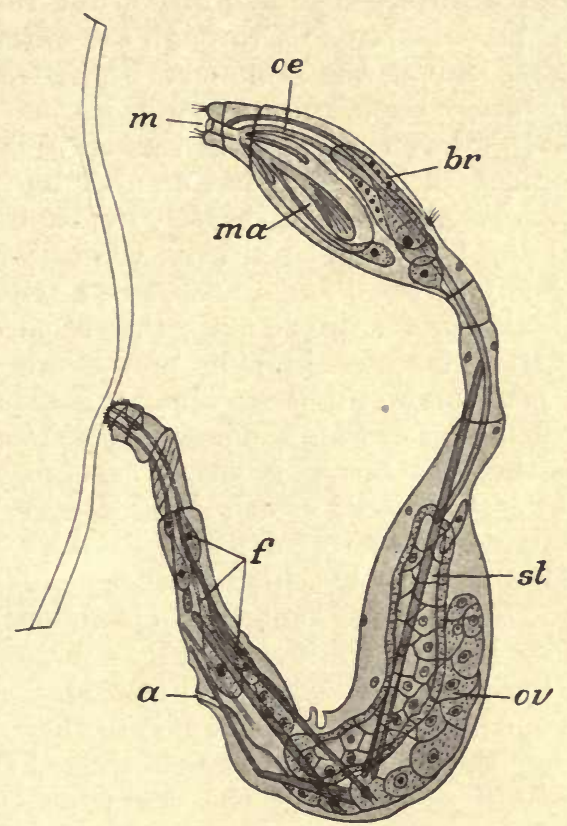

Fig. 268.-Paraseison asplanchnus, female $\times 230 . a$. genital aperture; $b r$. ganglion; $f$. foot-glands ; $m$. mouth ; ma. mastax; oe. oesophagus ; ov. ovary ; st. stomach. (After Plate.)

neck-region, and an elongated foot provided at its extremity with a perforated disc.

\section{Systematic Position of the Example.}

Brachionus rubens is one of the several species of the genus Brachionus: it belongs to the family Brachionidae, and to the sub-order Lovicata of the order Ploïma.

It is placed in the order Ploïma in virtue of its active swimming habits and the absence of looping or skipping movements. The presence of a distinct lorica places it in the sub-order Loricata. The family Brachionidæ is distinguished by having a box-like lorica open at both ends, and a long, flexible, retractile tail with wrinkled surface and forceps-like termination. In the genus Brachionus the lorica is not marked with ridges, and the tail is 
very long and perfectly retractile. In B. rubens the anterior edge of the lorica is produced dorsally into six spines and is sinuous ventrally.

\section{General Organisation.}

External Characters. - The majority of the Rotifera are freeswimming, being propelled rapidly through the water by the action of the trochal disc. But in the Bdelloida (Fig. 269, 5), in addition to this mode of progression, the animal performs looping movements like those of a leech: the tail in this order is freely jointed, the various segments fitting into one another like the tubes of a telescope, and the body is fixed alternately by it and by the anterior end, the trochal disc being kept retracted while the animal moves in this way. Many of the Ploïma also have a telescopic tail, but in some, e.g., Asplanchna (Fig. 269, 6), this organ is absent. In Pedalion (Fig. 270,1) curious skipping movements are performed by the aid of six hollow, limbs or appendages, one dorsal, one ventral, and two on each side. These curious organs are terminated by feathered setæ, and closely resemble the limbs of some of the lower Crustacea: each is moved by two opposing muscles which extend into its cavity $(1, \mathrm{~B}, m)$. Three pairs of similar appendages are present in the other genus of Scirtopoda, Hexarthra (Fig. 270, 2), the resemblance of which to the nauplius larva of Crustacea is very striking (see Fig. 429), and four genera of unarmoured Ploïma, e.g. Polyarthra (Fig. 269, 8) possess simple or fringed setæ moved by muscles attached to their bases.

In the Rhizota the adult is permanently fixed (Fig. 269, 1-4). The end of the tail is devoid of the characteristic fork, and is attached to plants or other supports. Moreover the animal is surrounded by a tube into which it can retract itself completely, protruding the anterior end with the trochal disc when undisturbed. In most instances, as for example in Floscularia (1) and Stephanocerus (2), the tube is formed of a delicate, transparent, gelatinous secretion of the epidermis; but in Melicerta (3) it is built up of rounded pellets, which the animal moulds in a cup-like depression on the dorsal surface and places in position one by one. The pellets are usually formed of foreign particles, but in some species are made of the animal's own frees.

The ciliation of the trochal disc is subject to considerable variation. In its simplest form the disc is surrounded by a single circlet of cilia, within which lies the mouth. A modification of this type may be produced by the prolongation of the ciliary crown into long arm-like processes fringed with cilia, as in Stephanoceros (2), or, as in Floscularia (1), into blunt elevations bearing long stiff cilia like those of the Heliozoa. The single circlet may be folded upon itself, or a second type may be produced by the addition of a second circlet within and parallel to 


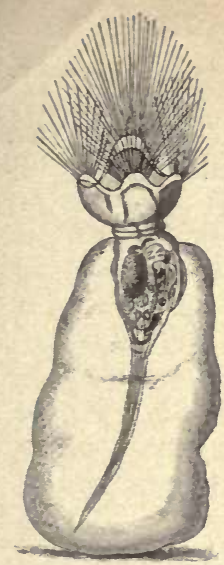

1.Floscularia

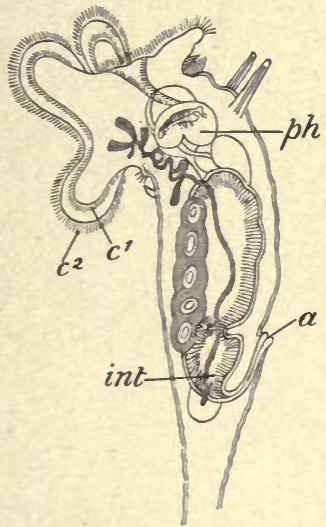

4. Melicerra

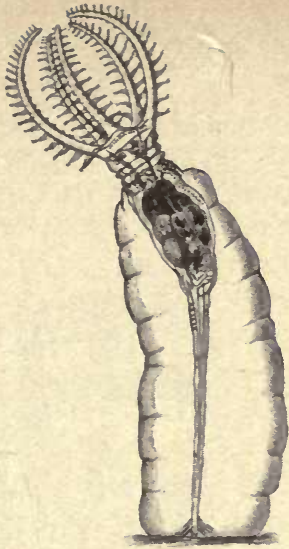

2. Srephanoceros

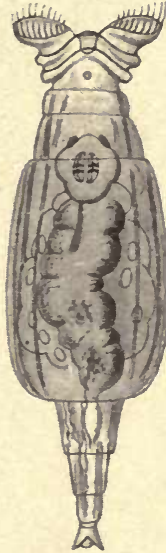

5.Philodina

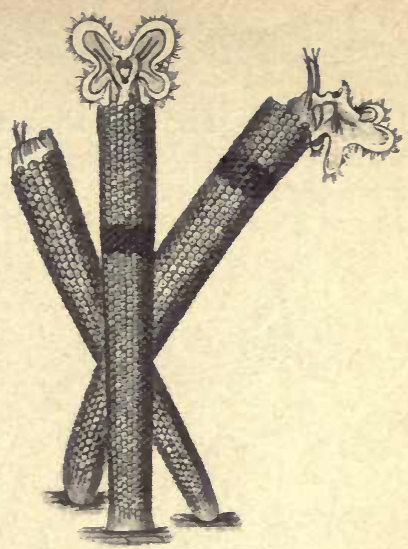

3. Melicerta

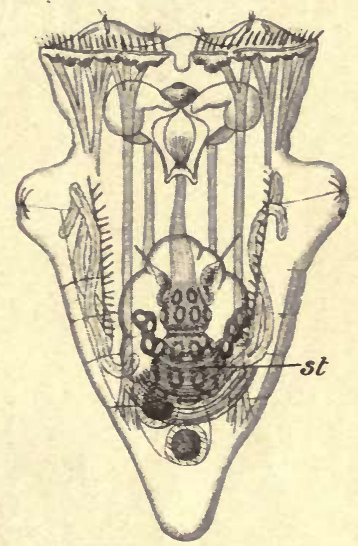

6. Asplanchna

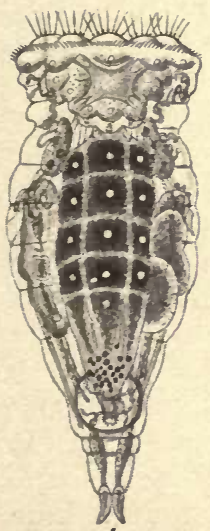

7. Hy.darina

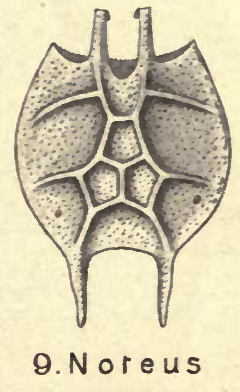

9. Noreus
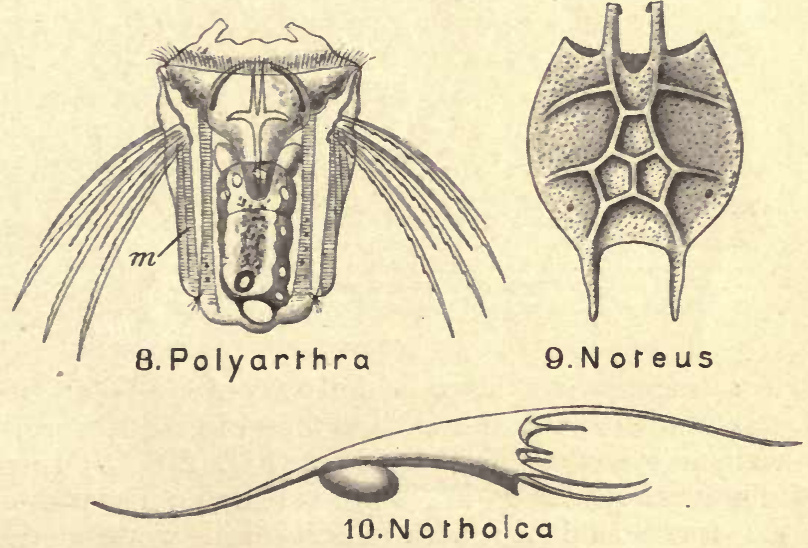

Fig. 269.-Typical forms of Rotifera :-9 and 10 show the lorica only. $a$. anus; $c^{1}$. $c^{2}$. ciliary circlets; int. intestine ; $m$. muscle; $p h$. pharynx. (After Hudson and Gosse.) 
the first. The mouth in this case is always placed between the two circlets on the ventral side (Fig. 2(i7), so that the inner or anterior circlet is pre-oral and corresponds with the chief ciliary band
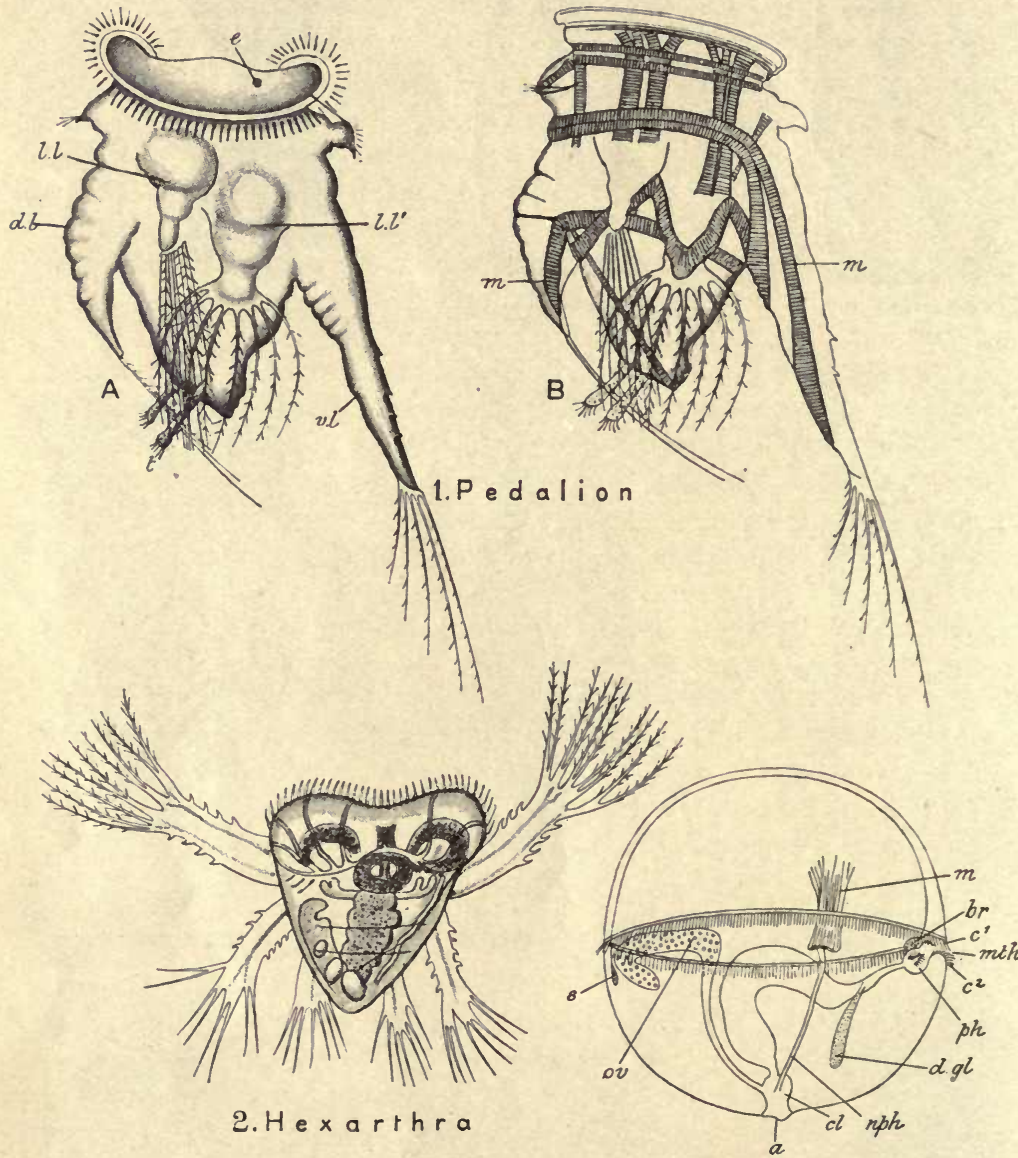

3.Trochosphaera

Fig. 270.-Typical forms of Rotifera. In 1, $a$ shows the outer form, $b$ the muscular system. $a$. anus; $b r$. brain; $c^{1} . c^{2}$. ciliary circlets; $c l$. cloaca; $d . g l$. digestive gland ; $d . l$. dorsal limb; e. eye-spot; $l$. $l ., l$. $l$ '. lateral limbs; $m$, muscles; $m t h$, mouth; $n p h$. nephridial tube ; ov. ovary; $p h$. pharyux; s. sense-organ; $v$. $l$. ventral limb. (After Hudson and Gosse (1 and 2) and Korschelt and Heider (3).)

of a trochosphere larva, while the outer or posterior circlet corresponds with the post-oral band found in many worm-larvæ. In the curious globular Trochosphnera.(Fig. 270, 3) there is a single equatorial circlet, which is pre-oral, and a few post-oral cilia: here the correspondence with the typical worm-larva is singularly 
close. Lastly, both the pre- and post-oral circlets may be produced into more or less complex lobes, as in Melicerta (Fig. 269, 4), or may be interrupted as in Brachionus, in which the pre-oral circlet is represented by three distinct lobes, or as in Pedalion, in which both circlets are divided into right and left moieties. In one genus the trochal disc is absent.

Digestive Organs.-The typical form of mastax or pharyngeal mill is that described in Brachionus (Fig. 265). There is an unpaired incus consisting of a short stem or fulcrum $(f)$ and of two broad branches or rumi $(r)$, and a pair of mallei, each consisting of a stout handle or manubrium $(m)$ and a broad, toothed head or uncus $(u)$. In some forms all the parts of the apparatus become very slender, the incus assuming the form of forceps (Fig. 27i, A). Or the mallei may be absent and the two rami movable upon one another so as to convert the incus into a pair of forceps (B)

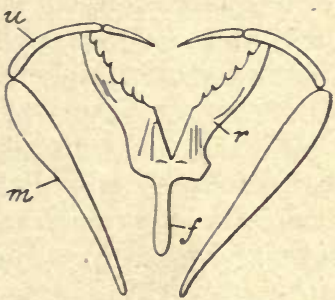

A
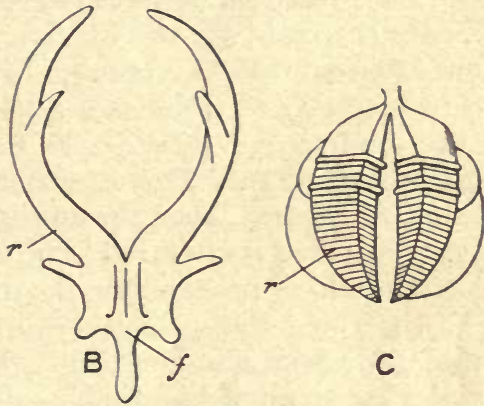

C

FiG. 271.-Typical forms of mastax. A, forcipate type; B, incudate type ; C, ramate type. $f$. fulcrum ; $m$. manubrium; $r$. ramus ; $u$. uncus. (After Hudson and Gosse.)

used to seize prey, the mastax being in this case protrusible. Lastly, the fulcrum and manubrium may be absent, and the unci and rami very strong and massive $(\mathrm{C})$. Glands, supposed to be salivary, open into the mastax or œsophagus.

The stomach is always large, and usually has a pair of digestive glands opening into it: it may pass insensibly into the intestine, or the latter may be a distinct chamber of more or less globular form. In the Rhizota the intestine turns forwards so as to allow of the anus being brought over the edge of the tube in defæcation (Fig. 269, 4, a). In Asplanchna (6) the stomach ends blindly, the intestine, cloaca, and anus being absent.

The excretory system is very uniform in structure. It consists of a pair of more or less coiled nephridial tubes, placed longitudinally and giving off lateral branchlets which end in flame-cells. The outer surface of each flame-cell usually bears one or sometimes two flagella, which lie free in the body-cavity. 
Frequently, but not always, the two tubes open posteriorly into a contractile vesicle or bladder which discharges into the cloaca.

Nervous System and Sense Organs. - The nervous system usually consists of a single ganglion (Fig. 267, br) towards the dorsal aspect of the anterior part of the body, and representing the brain or supra-œesophageal ganglion of the higher Worms : it sends nerves to the muscles, trochal disc, and tactile organs. In some cases a smaller ventral or infra-œesophageal ganglion is present as well, connected with the first by a pair of slender œsophageal connectives. Connected with the dorsal ganglion are a pair of lateral longitudinal nerves which run backwards to the tail, giving off branches in their course. One or more cyes (e) are usually present in close relation with the brain, and are sometimes mere spots of pigment, but may be provided with a refractive body or lens. The only other organs of sense are the tactile rods $\left(d . f ., l_{f}.\right)$, of which there is usually one on the dorsal surface near the anterior end of the body, and frequently two others, one on each side of the trunk. They are more or less rod-like structures, tipped with delicate sensory hairs and receiving nerves from the brain.

Reproduction and Development.-In most cases the female reproductive organs have the same general character as in Brachionus, i.e. the gonad is unpaired (Fig. 264), consists of germarium and vitellarium, and is provided with an oviduct (Fig. 267). But in some of the Bdelloïda, such as Philodina, there are two ovaries, not divisible into germ-gland and yolk-gland, and the oviduct is absent. The males are smaller than the females and degenerate in structure, the enteric canal being atrophied (Fig. 266, A). There is a large testis $(t)$ with a duct opening at the end of a protrusible penis $(p)$, which is dorsal in all but Asplanchna, in which it, as well as the cloacal opening of the female, appear to be ventral. Apparently hypodermic impregnation sometimes takes place, i.e. the body-wall of the female may be perforated at any place for the entrance of the sperms.

Three kinds of eggs are produced: large and small summer eggs, which always develop parthenogenetically, the larger giving rise to females, the smaller to males; and thick-shelled winter eggs, which probably require impregnation, and remain in an inert condition all through the winter, finally developing in the spring. Most Rotifers are oviparous, but some (Philodina, \&c.) bring forth living young, which are born by breaking through the body-wall or through the cloaca, thus causing the death of the parent.

Segmentation is total and irregular, the oosperm dividing into megameres and micromeres. An epibolic gastrula is formed, the blastopore closes, and invaginations of ectoderm give rise to the stomodæum and proctodæum. The tail is formed as a prolongation 
of the postero-ventral region of the embryo, and contains at first an extension of the endoderm. No metamorphosis is known to take place in any member of the class.

Ethology.-A few Rotifers live in the sea, but the majority are fresh-water forms, occurring in lakes, streams, ponds, and even in puddles, the water of which is rendered foul and opaque by mud and sewage. Frequently the water in which they live is dried up, and the thick-shelled winter eggs may then be widely dispersed by wind. It is even stated that the adult animals may survive prolonged desiccation and resume active life when again placed in water. They are able to survive prolonged exposure to temperatures far below the freezing point of water. Many forms cling to the bodies of higher animals in order to obtain a share of their food, thus leading a kind of commensal existence. Others go a step further and become true external parasites, like Drilophaga on a fresh-water Oligochæte (vide Section X), or Seison on the little Crustacean Nebalia (Fig. 457). Others, again, are internal parasites, such as Albertia in the cœlome of Earthworms and the intestines of fresh-water Oligochætes (Nais), and Notommata uerneckii in the cells of the fresh-water Alga Vaucheria.

Affinities.-The affinities of the Rotifera are very obscure. Their-general resemblance to the free-swimming larvæ of Annelids (phylum Annulata) is extremely close, and, in particular, the curious Trochosphare is, to all intents and purposes, a sexually mature trochosphere with a mastax. The excretory organs recall those of the Platyhelminthes, and also resemble the provisional nephridia or head-kidneys of Annulate larvæ. Lastly, the hollow muscular appendages of Pedalion and Hexarthra give those genera a certain resemblance - which is probably, however, merely adaptive - to the nauplius or free-swimming larva of Crustacea.

\section{Class II.-Gastrotricha.}

The Gastrotricha (Figs. 272 and 273) are a small group of minute fresh-water animals, which are apparently allied, though certainly not very closely, to the Rotifera, and are on that account placed in the present phylum. The body is spindle-shaped with flattened ventral surface. The ventral surface bears two longitudinal bands of cilia ; the dorsal is non-ciliated, but in some forms bears a number of longitudinal rows of slender, pointed, cuticular processes. The aboral end is narrow and usually bifurcated.

On the head are four tufts of flagella, which are partly sensory, partly vibratile. The mouth, situated at the anterior end, leads by a narrow tube into the thick-walled œsophagus. At the beginning of the latter are a number of small chitinous denticles, and in front of them a circlet of setæ. The œsophagus leads to a wide elongated stomach followed by a short intestine which terminates in an anal aperture at the posterior extremity. The nephridia are a pair of unbranched coiled tubes each opening on the ventral surface and terminating 
internally in a flame-cell. The nervous system consists of a large dorsally and anteriorly situated cerebral ganglion or brain giving off a pair of ventro-lateral longitudinal nerves. The sexes are united, and there is no metamorphosis.

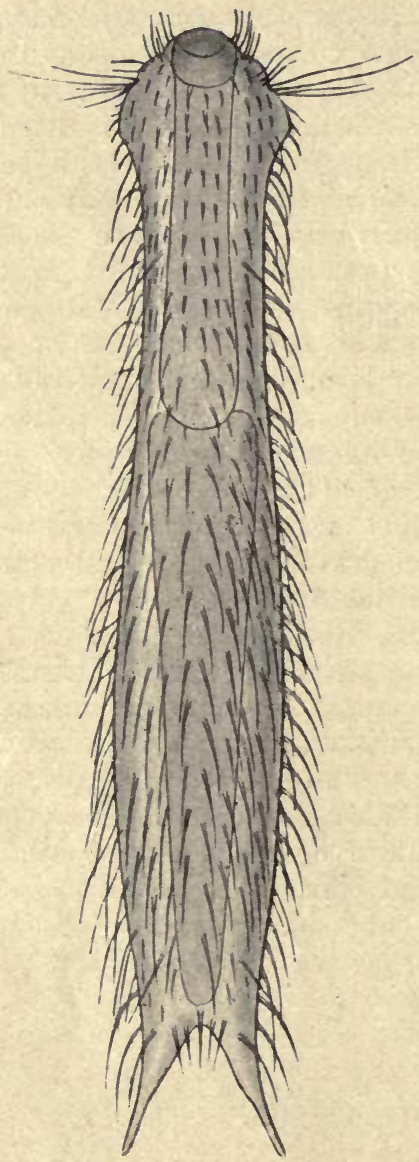

FIG. 272.-Chaetonotus maximus. Highly magnified. (After Zelinka.)

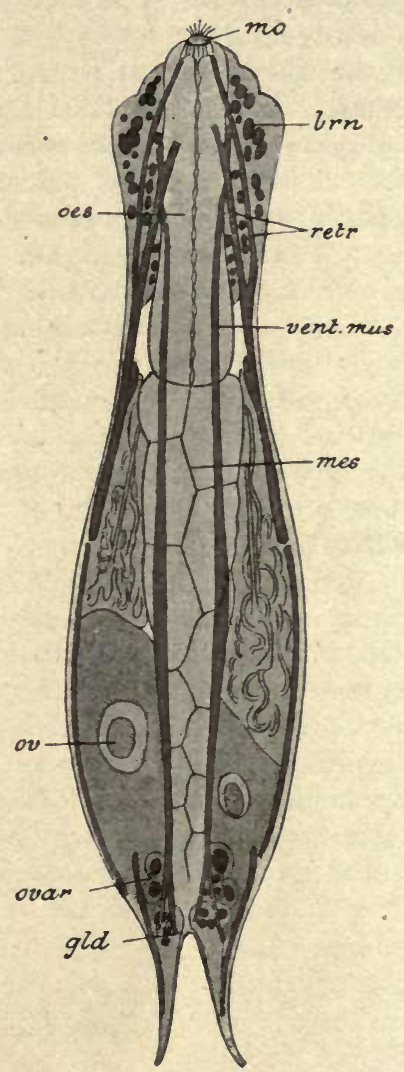

FIG. 273.-Chaetonotus maximus (or. ganisation). brn. brain; gld. adhesive gland; mes. mesenteron; mo. mouth ; oes. œesophagus; ov. ovum; ovar. ovary ; retr. retractor muscles ; vent. mus. ventral muscle. (After Zelinka.)

\section{APPENDIX TO THE TROCHELMINTHES.}

\section{The Dinophilea and Histriobdellea.}

These are two isolated groups of minute animals which may most conveniently be dealt with in association with the Trochelminthes, since they bear certain striking resemblances, now to one, now to another, member of that phylum; but they differ from all of them in the assumption of a simple kind of metamerism (p. 43), by virtue of which they have claims to association with the 
Annulata - a phylum to be treated of later. The Dinophilea are free-living animals, mostly marine, one species living in brackish water. The Histriobdellea are parasitic or commensal, and live on the European lobster and the Australian fresh-water crayfishes.

Dinophilus (Fig. 274) is a minute worm-like animal with a head or prostomium, a body compozed of from five to eight segments separated from one another by constrictions, and a short ventral tail. The prostomium bears two eye-spots and some sensory hairs : it is either covered uniformly with cilia, or bears two or three annular ciliated bands apparently representing the prototroch of the trochophore. The body is in some of the species uniformly ciliated; in others the cilia are disposed in rings corresponding to the segments, except on
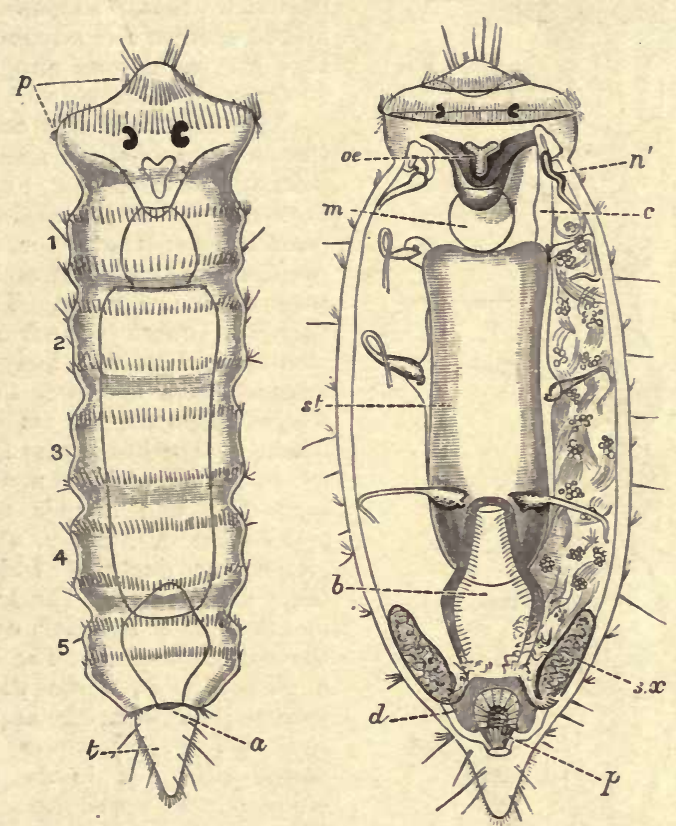

Fig. 274.-Dinophilus taeniatus. The left figure represents the dorsal surface of a young individual, $\times 76$; the mouth and alimentury tract are seen by trausparency. The right figure shows the anatomy of the male, $x 35$. $a n$. anus; $b$. rectum; $c$. body-cavity; $d$. vas deferens; $m$. pharjnx; $n^{\prime}$. the first nephridium; $\alpha$. entrance to the aesophagus; $p$., in left fig., prostomium; p., in right fig., penis; $8 t$. stomach; 8 . $x$. vesicula seminalis. (From Sheldou, after Harmer.)

the ventral surface, where the ciliation is always uniform. The mouth, which is situated on the ventral aspect of the prostomium, leads into an alimentary canal consisting of œosophagus, stomach, and intestine, all of which are ciliated; the anus $(a n)$ is placed dorsally over the tail. A protrusible muscular proboscis lies, when retracted, in a recess opening close to the mouth. There is an imperfectly developed cœlome which is crossed by strands of connective tissue. A nervous system is present, and consists of a large dorsal ganglion in the prostomium, giving off two anterior, and two posterior nerves or ventral cords (sometimes segmented into a series of ganglia connected in each segment by commissures), all situated in the epidermis.

The excretory system consists of a series of metamerically arranged pairs of 
tubes $\left(n^{\prime}\right)$. The inner ends of these do not open into the body-cavity, but are provided with peculiarly modified flagellate cells known as solenocytes, so that these paired excretory tubes resemble closely the nephridia of some of the Poly. chæta (phylum Annulata; see Section X.). The sexes are separate. In the male there is a conical ventral penis ; the last pair of nephridia act as vesicula seminales. In the ovary two sets of ova are developed, the larger destined to give rise to females, and the smaller destined to form males. They pass into the body-cavity and reach the exterior by an aperture on the ventral surface in front of the anus.

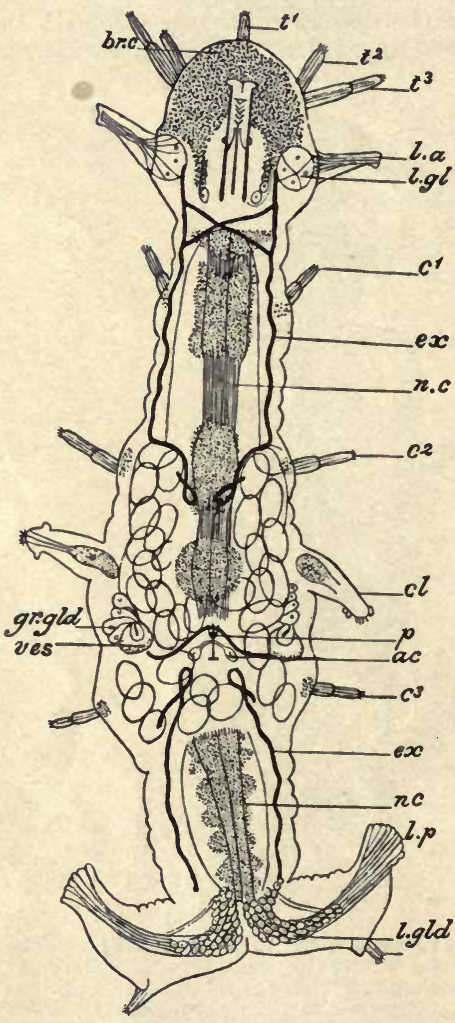

Fig. 275.-Stratiodrilus tasmanicus, male. ac. accessory gland of male apparatus; br. c. brain; $c^{1} \cdot c^{2} \cdot c^{3}$. cirri; $c l$. claspers (appendages peculiar to the male); $e x$. excretory tubes; gr. gla. granule-gland ; $l$. a. anterior limb; $l$. gl. gland at base of anterior limb; $l$. gld. gland at base of posterior limb; l. p. posterior limb; $n$. c. nerve-cord; $p$. penis; $t^{1} . t^{2} . t^{3}$. tentacles; ves. vesicula scminalis. A process of unequal segmentation is followed by the formation of an epibolic gastrula. What is known of the development is in favour of the view that Dinophilus is to be looked upon as a trochophore-like form that has made some progress in the evolution of metamerism.

The Histriobdellea comprise only the two nearly-allied genera Histriobdella and Stratiodrilus (Fig. 275) - the former found on the eggs of the European lobster, the latter in the gillcavities of Australian and Tasmanian fresh-water crayfishes. The animal is narrow, almost cylindrical, with a well-marked head, a body of six segments, and a narrower tail-region in which segmentation is not clearly marked. The head bears five tentacles $\left(t^{1}, t^{2}, t^{3}\right)$ tipped with non-motile sensory cilia, and a pair of retractile appendages or limbs $(l, a)$, with basal glands the ducts of which open at their extremities. The head has the mouth at its anterior extremity on the ventral aspect. The body bears, in Stratiodrilus, three pairs of twojointed non-retractile appendages or cirri $\left(c^{1}, c^{2}, c^{3}\right)$ tipped with nonmotile cilia, and in the male a pair of retractile appendages or claspers $(c l)$. At the end of the tail is a pair of large freely movable appendages or legs $(l p)$, which are the organs of locomotion: at the end of each of these open the ducts of a mass of unicellular glands. The anus is situated posteriorly between the bases of the legs. Opening from the mouthcavity on its ventral aspect is a muscular sac in which are enclosed, when retracted, a system of chitinous jaws reducible to the same general type as the mastax of the Rotifera, but with the relative position of malleus and incus inverted. There is a highly developed nervous system consisting of a large brain (br. c.) situated dorsally in the prostomium, a pair of œsophageal connectives, and a ventral nerve cord $(n c)$ with a series of ganglia which have a distinctly metameric 
arrangement. The excretory system takes the form of ciliated tubes $(e x)$, closed internally, and showing a tendency to metamerism : these extend into the head. The sexes are distinct: the male has a protrusible penis, directed ventrally. There is no metamorphosis.

There seems to be some reason for believing that Dinophilus and the Histriobdellea may help to bridge over the interval between the Trochelminthes and the higher segmented worms or Annulata. In this connection the Echinoderidæ, which were noticed in an appendix to the last Section (p. 319), have also to be kept in view. 


\section{SECTION VIII}

\section{PHYLUM MOLIUSCOIDA ${ }^{1}$}

THE phylum Molluscoida comprises three classes-the Polyzoa (including, provisionally, the Endoprocta), the Brachiopoda, and the Phoronida. The members of these three classes are tolerably widely divergent, so that it is somewhat difficult to frame a general account of the entire phylum; but the following are the most important common features :-

There is, except in the Endoprocta, a body-cavity (cœlome), lined in most cases with a coclomic epithelium, within which the alimentary canal is suspended by means of mesenteries or by means of funicular strands taking their place. The dorsal region of the body is abbreviated, being represented only by a short space between the mouth and anus, which are closely approximated. There is a lophophore or tentacle-bearing ridge, usually of a horseshoe shape, containing a special compartment of the cœlome, and overhanging the mouth on its anal side there is in most cases a sensitive process-the epistome - also containing a special compartment of the body-cavity. The central part of the nervous system consists of a single ganglion (supra-cesophageal), or of two ganglia (supra-œsophageal and infra-œsophageal), or of a nervering. The nephridia when present are in nearly all cases a single pair of ciliated tubes, which act also as gonoducts.

\section{CLASS I.-POLYZOA.}

The Polyzoa form colonies known as "Sea-mats," or "Corallines," which in many cases bear a close general resemblance to

1 This and all the remaining phyla of the animal kingdom are characterised by the possession of a true coelome, i.e. of a cavity interposed between the wall of the body and that of the enteron, and developed either directly by outgrowth from the archenteron, or formed from clefts that appear in solid masses of mesoderm cells. The only group hitherto dealt with in which a definite cœlome is present is the Chætognatha. In some of the groups which are here comprised in the cœlomate phyla, however, as will be seen, the cœlome is reduced, or entirely absent, or not typically developed. 
Hydroid Zoophytes, and only on a more minute inspection are found to differ totally from the latter and to exhibit a very much higher type of structure.

\section{Example of the Class.-Bugula avicularia.}

Bugula avicularia, the common Bird's-Head Coralline (Fig. 276), occurs in brown or purple bushy tufts, two or three inches long, on rocks, piles of jetties, and similar situations on the sea-shore in all parts of the world. On a naked-eye examination it presents a considerable resemblance to a $\mathrm{Hydroid} \mathrm{Zoophyte,} \mathrm{and} \mathrm{might} \mathrm{readily}$ be taken for a member of that group. It consists of dichotomously branching narrow stems, which are rooted by a number of slender root-filaments. Each stem is found, when examined with a lens, to be made up of a number of elements, the zoocia of the colony, which are closely united together and arranged in four longitudinal rows. The zoccia are approximately cylindrical in shape, but broader distally than proximally, four or five times as long as broad, and have, near the distal end, a wide crescentic aperture-the " mouth" of the zoupcium-on either side of which is a short blunt spine. A rounded structure-the occium-in many parts of the colony lies in front of each zoœcium (Fig. 276, ocec.). On each zoœcium, except a few at the extremities of the branches, is a remarkable appendage, the avicularium (avic), having very much the appearance of a bird's head supported on a very short stalk: if the Bugula is examined under the microscope in the living condition, the avicularia will be found to be in almost constant movement, turning from side to side; and a movable part, comparable to the lower jaw of the bird's head, will often be seen to be moved in such a way that the mouth of the avicularium is opened very widely and then becomes closed up with a quick "snap." All the parts hitherto mentioned can be shown, by using appropriate tests, to be composed of some material akin to chitin in composition. The chitinous wall of the zoœcia is the hardened and thickened cuticle of the zooids, having beneath it the soft bodywall. ${ }^{1}$ The anterior region of the body of the zooid forms an introvert, i.e. is capable of being involuted like the finger of a glove within the more posterior part: the cuticle covering this, continuous behind with the thick ectocyst, is quite thin and flexible. When the introvert is everted it is seen to bear at its anterior end a circlet of usually fourteen long, slender filiform tentacles (tent) on a circular ridge or lophophore surrounding the mouth of the zooid. The tentacles are densely ciliated except along their outer surfaces: the cilia vibrate actively in such a way as to drive currents of water,

1 The terms ectocyst and endocyst are commonly applied respectively to the hardened cuticle of the zooid and its soft body-wall. 
and with them food-particles, towards the mouth $(m o)$ : they are also capable of being bent in various directions. In the interior of

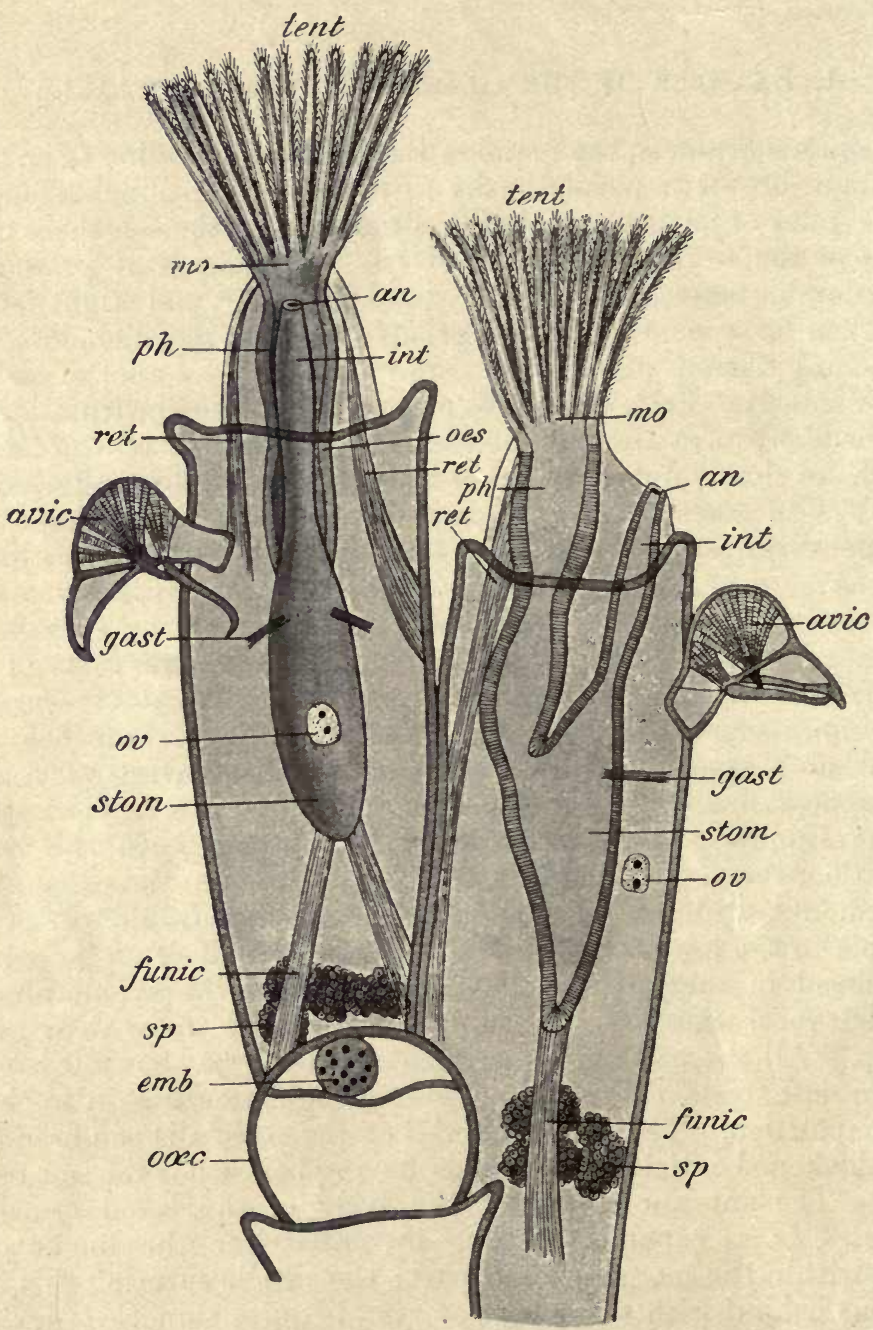

Fig. 276.-Bugula avicularia. Two zooids, magnified. an. anus; avic. avicularia; emb. embryo enclosed in the onecium; 'funic. funiculus; gast. muscular bands passing from the stomach to the body-wall ; int. intestine ; mo. mouth ; ooe. ooecium; aes. nesophagus; ov. ovary ; ph. pharynx; ret. parieto-vaginal muscles; sp. spermatidia ; stom. stomach; tent. tentacles. The ganglion, which is not indicated, lies just below the middle of the stroke from mo.

each is a narrow prolongation of the colome. In all probability, besides bringing minute particles of food to the mouth of the zooid by the action_of their cilia, the tentacles are prehensile as well as 
tactile, and also act as organs of respiration. When retracted they become enclosed by the walls of the introvert as by a sheaththe tentacle-sheath. A pair of bands of muscular fibres-the parietovaginal muscles (ret.) - passing to the introvert from the body-wall, serve to retract the introvert and tentacles.

The body-wall consists, in addition to the cuticle, of an epidermis composed of a single layer of large flattened cells, two muscular layers, the outer circular and the inner longitudinal, and a layer of an irregular cellular tissue, or parenchyma.

The cœlome is extensive; it is lined externally by the parietal layer of parenchyma forming the innermost layer of the body-wall, and internally by a visceral layer of the same tissue, ensheathing the alimentary canal. Across the cavity between the parietal and visceral layers of the parenchyma pass numerous strands of spindleshaped cells. A large double strand (funic) passes from the proximal or aboral end of the alimentary canal to the aboral wall of the zoœcium; this is the funiculus. A transverse partition cuts off (though not completely) a small anterior compartment of the colome from the rest. The former surrounds the basis of the tentacles, the narrow internal cavities of which are in communication with it: this is known as the circular canal. The colomic fluid contains a number of colourless corpuscles or leucocytes.

Alimentary Canal. - The mouth (mo) leads into a wide chamber - the pharynx $(p h)$ - just behind the bases of the tentacles; from this a somewhat narrower short tube, separated by a constriction from the pharynx, leads to the stomach (stom) from which it is also separated by a constriction. The stomach gives off a long conical prolongation or ccecum passing towards the aboral end of the zoøeium, to which it is attached by the funiculus. The intestine (int) comes off from the oral aspect of the stomach, not far from the œsophagus, with which it lies nearly parallel : it terminates in a rounded anal aperture (an) capable of being distended to a considerable size, situated not far from the mouth, but outside the lophophore. The entire alimentary canal is lined by an epithelium, which is ciliated throughout except in a portion of the stomach: the cells of the epithelium, which are arranged in a single lafer, vary in length in different regions, being longest in the pharynx, which is comparatively thick-walled. A pair of slender muscles (gast) passing from the body-wall to the stomach act as retractors of the alimentary canal when the introvert is drawn back.

There are no blood-vessels.

The nervous system consists of a small round ganglion situated between the mouth and the anus, giving off nerves to the various parts; organs of special sense are absent. Definite excretory organs do not occur in Bugula, the function of excretion 
(i.e. the collection of the nitrogenous waste-matters) being apparently carried on by the leucocytes and the cells of the funicular tissue.

Reproductive Organs.-Ovary and testis are found to occur together in the same zooid. They are both formed from specially modified cells of the parenchyma, either of the funiculus or of the body-wall. The testis, developed from the cells of the funicular tissue, gives origin to spherical masses of cells-the spermatidia $(s p)$ - which develop into sperms with very long motile tails. These become free from one another and move about in the body-cavity or in its prolongations into the tentacles. There is no spermiduct, and it is doubtful if the sperms pass to the exterior. The ovary $(o v)$ is a small rounded body formed from the parietal layer of the parenchyma about the middle of the zoœcium; it consists of only a small number of cells of which only one at a time becomes a mature ovum, certain smaller cells forming an enclosing follicle. The mature ovum is perhaps fertilised in the cœlome; it passes into the interior of a rounded outgrowth of the zoccium-the oocium (ooc) -lined with parenchyma, and forming a sort of broodpouch in which it undergoes development.

Development.-Segmentation (Fig. 277) is complete and nearly regular. A blastula is formed having the shape of a bi-convex lens. In the interior of the blastocole or cavity of the blastula, four cells (end) - the primitive endoderm cells - become distinguishable : these increase in number by division, and form a mass of free cells which almost completely fill the blastocœle; this mass apparently represents both endoderm and mesoderm. Small cavities which appear in it subsequently unite together to form the primitive colome. A very broad ring-shaped thickening - the corona $(G$, cor.)is formed round the equator of the embryo and becomes provided with cilia. A circular pallial groove arises on the oral side of the corona. A sac-like, afterwards beaker-shaped invagination of the ectoderm on what is destined to become the oral side of the ciliated ridge, forms a larval structure, termed the sucker (Fig. 278, suck), which afterwards serves to fix the larva. A second depression of the ectoderm in the region of the corona on the oral side forms the ectodermal groove. At the aboral pole is developed, also from the ectoderm, a second larval structure-the calotte or retractile disc (disc), on which motionless sensory cilia appear. In close relation to the ectodermal groove is formed a mass of cells, the pyriform organ $(p)$.

An alimentary canal is absent in the larva of Bugula when it escapes from the oœcium. After an interval of free existence as a ciliated larva, certain changes appear which lead to a very complete metamorphosis. The sucker becomes everted by a strong contraction of the body, and fixes the larva to some foreign 
object. The aboral side of the larva becomes greatly extended, so that almost the entire integument of the primary zooid is developed from this part (i.e. from the region occupied by the retractile disc and pallial groove). Accompanying the extension of the aboral surface are the obliteration of the pallial groove and the bending down of the corona towards the oral side. Thus the stage of the larva termed the umbrella-shaped stage is reached. The sucker is everted, and by means of it the larva becomes attached. The edge of the "umbrella" becomes bent downwards, and
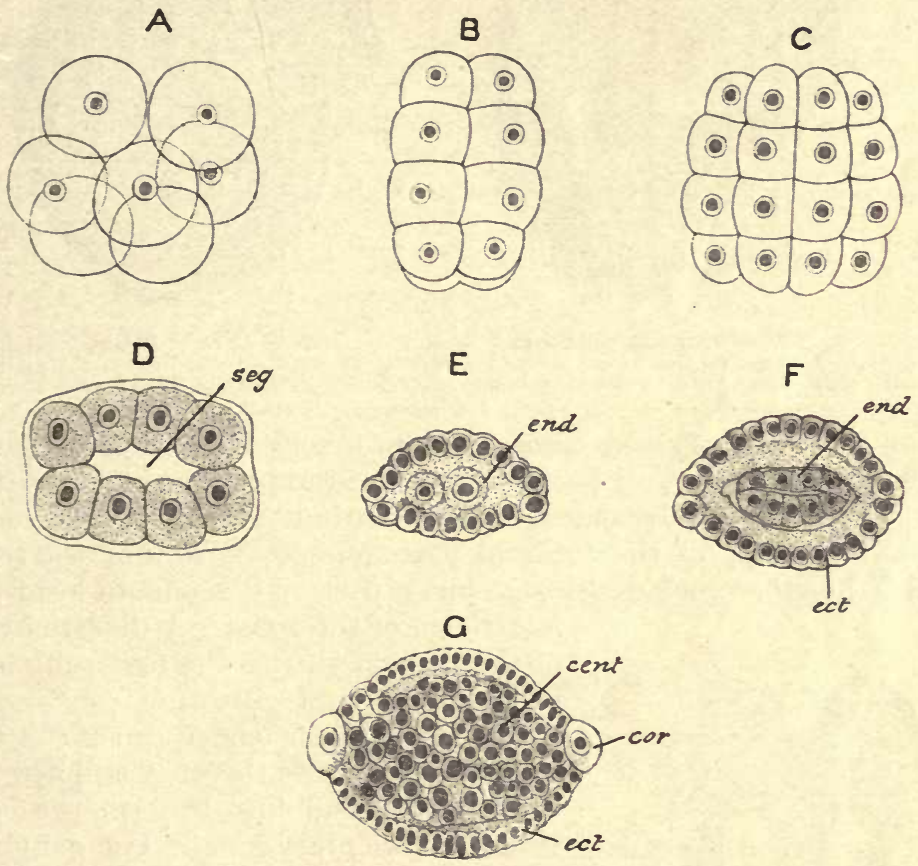

FIG. 2\% \%.-Early stages in the development of Bugula. cent. central mass of cells; cor. corona ect. ectoderm; end. endoderm ; seg. segmentation-cavity. (After Vigelius.)

fused with the broad plate into which the sucker has expanded, thus enclosing a circular cavity, the so-called vestibule (Fig. 279,v). The walls of this, consisting of the coronal cells and a portion of the original sucker, become broken up and the cavity is merged in the general cavity in the interior of the larva. All the larval structures have now disappeared with the exception of the basal plate of the sucker and the retractile disc. The former gives rise to the basal part of the wall of the primary zoøecium. From the latter, which becomes invaginated, or from a sac which is developed to replace it, are developed both the ectodermal and endodermal structures of the primary zooid. 
Occupying the interior of the larva at this stage in addition to this sac, there is only a mass of undifferentiated tissue derived from the original central tissue together with that derived
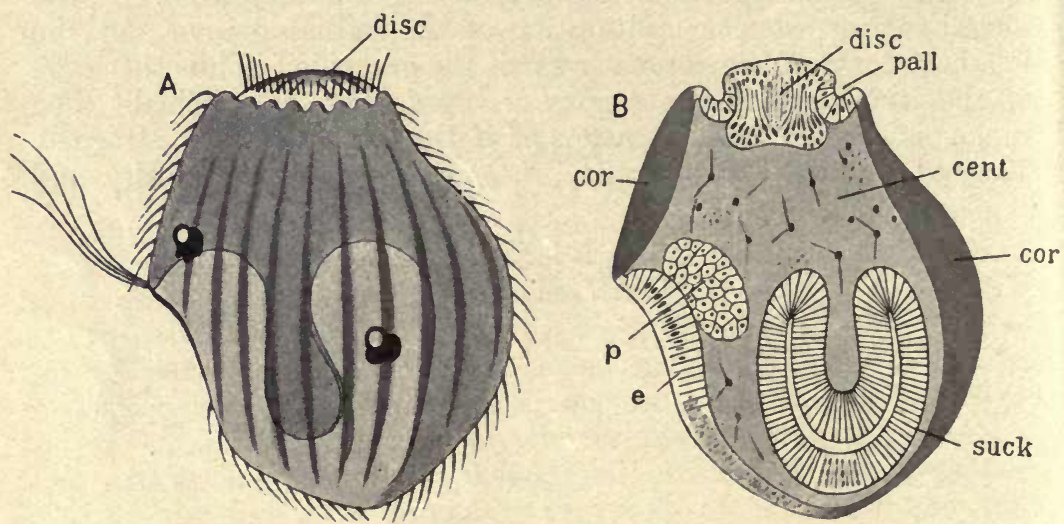

FIG. 278.-A, Larva of Bugula plumosa ; B, Sagittal section of larva of Bugula (diagrammatic). cent. central tissue ; cor. corona ; disc. retractile dise ; e. cctodernal groove ; $p$. pyriform organ; pall. pallial groove; suck. sucker. (From Korschelt and Heider, after Barrois.)

from the disintegrated corona, pyriform organ, and part of the sucker. The outer wall forms the wall of the primary zoœcium, the surface of which becomes covered with a chitinous cuticle or ectocyst. Most of the internal mass goes to form a brown body, which now becomes developed, but a part of it seems to form the

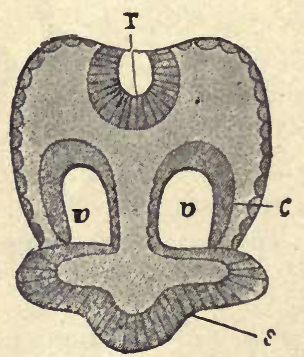

FIG. 279.-Longitudinal section of attached larva of Bugula. $c$. cells of corona; $r$ rudiment of the zooid in the form of a sac; 8. basal plate of everted sucker; v. vestibule. (From Korschelt and Heider, after Barrois.) mesoderm of the zooid. A diverticulum of the sac constitutes the first rudiment of stomach and intestine; a second diverticulum forms the rudiment of the œsophagus; these become applied to one another and fuse to form the continuous alimentary canal. The ganglion arises as an invagination of the ectoderm in the space between mouth and anus. The upper part of the cavity of the primitive sac, after the rudiment of the alimentary canal has been separated off, forms a space termed the atrium; the walls of this become converted into the tentacle sheath, while on its base appear the rudiments of the tentacles and lophophore. During the development of the organs of the adult zooid the brown body becomes closely applied to the stomach and gradually absorbed.

The primary zooid thus formed gives rise asexually by a process of repeated budding to the branching structure which has been 
described. In many of the zoœcia of a fully-developed colony no zooid is found to be present, but, instead, there is a dark brown body similar to that which occurs in the primary zoøecium. This is a zooid that has undergone degeneration - the lophophore, tentacles, and alimentary canal having become absorbed. Such degenerated zooids are capable of regeneration, the organs becoming re-developed and the brown body re-absorbed.

\section{Distinctive Characters and Classification.}

The Polyzoa are Molluscoida which, with one exception, form colonies of zooids connected together by a common organic substance. There is a lophophore bearing a series of slender, cilated, post-oral tentacles. The anterior part of the body forms, in the majority, a short introvert, within which the lophophore and the tentacles are capable of being withdrawn. In some the prostomium is represented by a small lobe-the epistome. The alimentary canal is U-shaped, and the anus is anterior, within, or just outside, the tentacular circlet. In most the nervous system is represented only by a small ganglion between the mouth and the anus. A cuticle, sometimes gelatinous, sometimes horny, sometimes calcified, forms a firm exoskeletal layer for the support of the colony. Nephridia (corresponding to the head-nephridia of the trochophore) occur only in the Endoprocta. There is no vascular system. The sexes are usually united. The majority of Polyzoa occur in the sea; a limited number are inhabitants of fresh water.

\section{Sub-Class I.-Ectoprocta.}

Colonial Polyzoa with the anus outside the lophophore, with a well-developed introvert and a spacious cœlome.

\section{Order 1.-GyMNOLEMATA.}

Almost exclusively marine Ectoprocta, with a circular lophophore, and without an epistome.

\section{Sub-order a.-Cyclostomata.}

Gymnolæmata with tubular calcareous zoœcia having circular apertures devoid of closing apparatus.

Including Crisia, Idmonea, \&c.

\section{Sub-order b.-Cheilostomata.}

Gymnolæmata with calcareous or chitinous zoœcia usually provided with opercula.

Including Bugula, Flustra ("Sea-mat") Membranipora, Cellepora, Selenaria. 


\section{Sub-order c.-Ctenostomata.}

Gymnolæmata with chitinous or gelatinous zoœcia provided with a series of tooth-like processes closing the aperture when the tentacles are retracted.

Including Alcyonidium, Serialaria, Paludicella.

\section{Order 2.-Phylactolmmata.}

Fresh-water Ectoprocta with horse-shoe-shaped lophophore and with an epistome.

Including Cristatella, Plumatella, Fredericella.

\section{Sub-Class II.-Endoprocta.}

Colonial or solitary Polyzoa multiplying by the formation of buds, which in Loxosoma soon become separated off, while in Pedicellina they remain connected together by a creeping stolon. The anus, as well as the mouth, is internal to the lophophore. The introvert is slightly or not at all developed. A pair of ciliated nephridial tubes are present.

\section{Systematic position of the Exanple.}

Bugula avicularia is an example of the sub-order Cheilostomata of the Gymnolæmata. It is a member of the family Bicellariidæ, which is characterised by the erect plant-like colony, with narrow compressed branches, and attached by root-like fibres; by the avicularia, when present, being stalked and bird's-head shaped; and by the wide oblique apertures of the zoccia all facing in the same direction. Bugula differs from the other genera of the family in the arrangement of the zoœcia in double or multiple rows, in their close union, and in the avicularia, when present, being on the side on which the mouth is situated. The various species differ in the exact shape of the zoœcia and of the avicularia.

\section{General Organisation.}

\section{Sub-Class I.-Ectoprocta.}

The Ectoprocta and the Endoprocta differ so considerably from one another that it is advantageous to deal with them separately. The Ectoprocta are all colonial-the colonies being capable, in most cases, like the colonies of hydroid zoophytes, of increasing in size to an apparently indefinite extent by continuous budding. The thickened cuticle which forms the support of the colony is sometimes gelatinous, sometimes chitinous, sometimes chitinous with sand-grains affixed, sometimes calcareous. The form of the 
colony varies in different families and genera in accordance with differences in the shape of the constituent zocecia, and differences in their mode of budding and consequent arrangement. The zoceia are sometimes tubular, sometimes ovoid, sometimes polyhedral. In some cases the buds are so developed that the colony assumes the form of a thin, flat expansion, which may be encrusting, and consist of a single layer of zoceia in close contact with one another or connected together by tubular processes; or may be erect, and with the zoccia either in one or two layers: sometimes the lamellar colony thus formed may be fenestrated or divided into lobes; sometimes it is twisted into a spiral. In other cases the colony, instead of being lamellar, has the form of an erect, shrublike structure, consisting of numerous cylindrical, many-sided, or

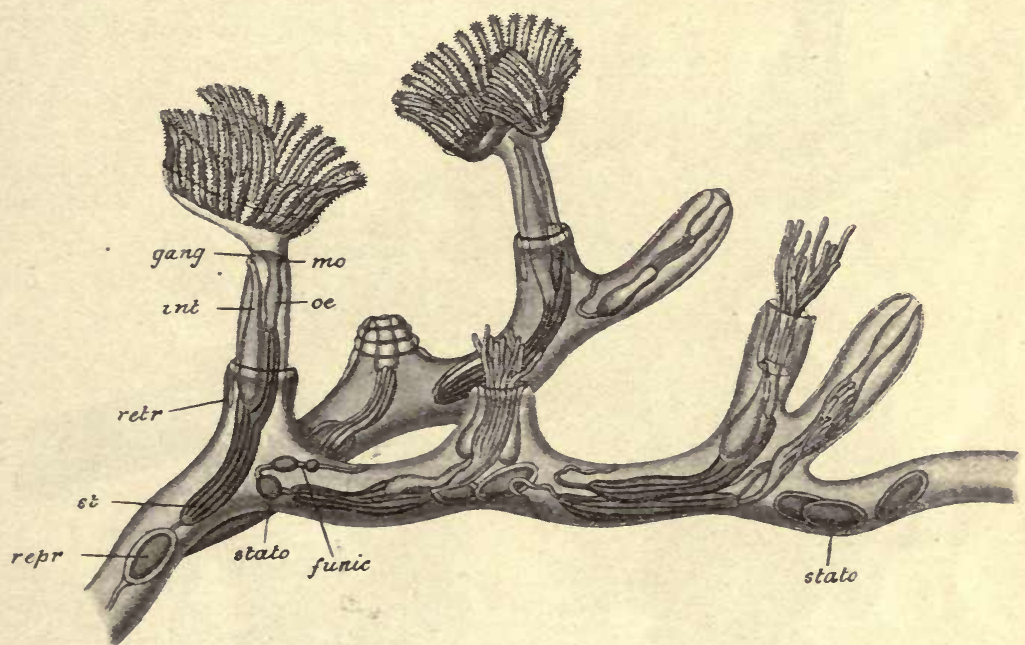

Fig. 250.-Plumatella. Portion of a colony, magnified. funic. funiculus; gang. ganglion; int. intestine; mo. mouth; $\alpha$. œesophagus; repr. gonad; retr. retractor muscle; st. stomach ; stato. statoblasts. (After Allman.)

strap-shaped branches arising from a common root. Sometimes there is a creeping cylindrical stolon, simple or branched, having the zooids arranged along it in a single or double row. The colony is free only in Cristatella (Fig. 281)-in which it performs creeping movements, in some other (American) forms of Phylactolæmata (in the younger stages of the colony), in one family of the Cheilostomata-the Selenariida, (in which it moves along with the aid of certain peculiar appendages-the vibracula-to be described subsequently), and in one or two other cases.

The zoœcia open on the exterior by means of circular, semicircular, or crescentic apertures, which in the Phylactolæmata and the Cyclostomata among the Gymnolæmata are devoid of any special closing apparatus; while in the Cheilostomata there is a movable 
lid or operculum closed by a pair of occlusor muscles when the introvert is retracted; and in the Ctenostomata there is a series of lobes or teeth which close in together over the opening. The cavities of the neighbouring zoœcia are in some forms completely cut off from one another by a continuation of the chitinous or calcareous exoskeleton; in others there is free communication; in others, again, there is communication through a number of minute perforations.

The oral (anterior) part of the body of each zooid is, as already described in the case of Bugula, covered only with a thin and

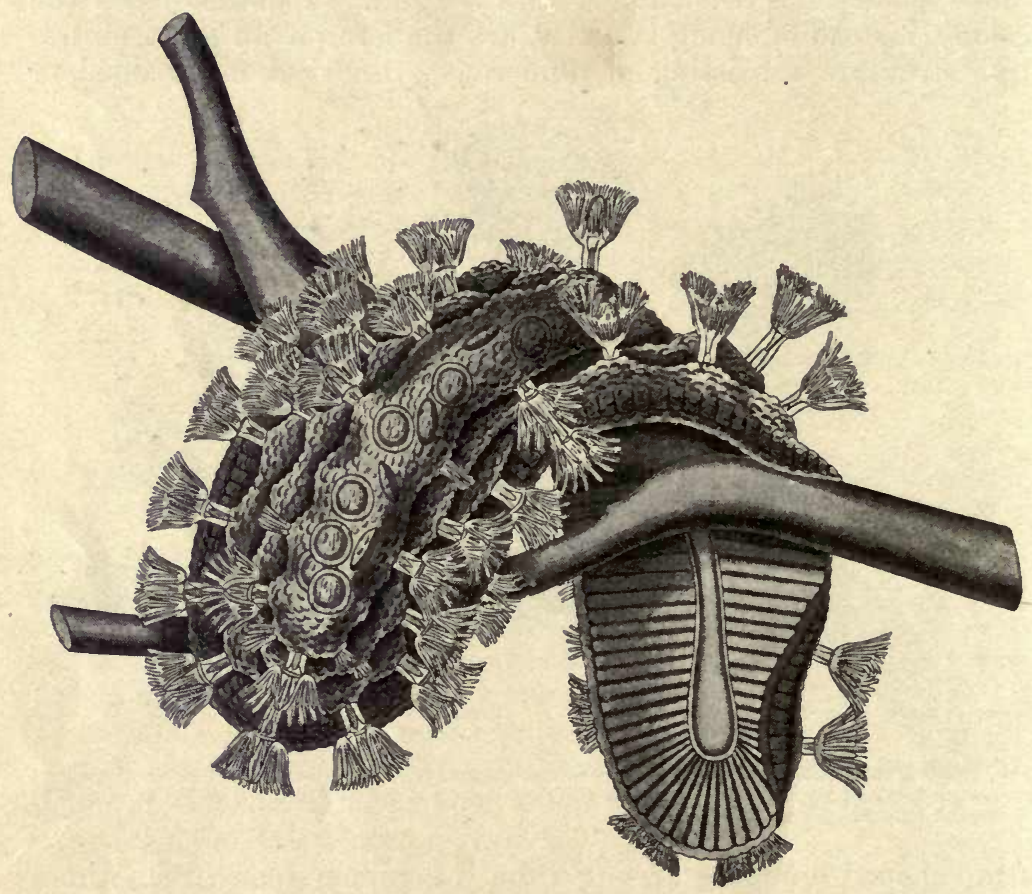

Fig. 281.-Cristatella mucedo. Entire colony. (After Allman.)

flexible cuticle, and forms an introvert capable of being retracted into the interior of the zoœcium. At the free end of the introvert is the mouth surrounded by a lophophore bearing tentacles. The tentacles are always simple, filiform, and hollow, each containing a narrow diverticulum of the circular canal or anterior compartment of the colome. They are beset with vibratile cilia by means of which currents are created subserving alimentation and respiration. They are also highly sensitive, and are capable of being bent about in various directions by the contraction of muscular fibres in their walls, so that they can be 
used for prehension. In the Phylactolæmata (Fig. 280) the lophophore is horse-shoe-shaped, in the Gymnolæmata (Fig. 276) circular : in the former, but not in the latter, there is a ciliated lobe, the epistome (Fig. 282, $e p$ ) - which may have a sensory function-overhanging the mouth on the anal side. The retraction or the introvert is effected by a pair of bands of muscular fibres, the parieto-vaginal muscles, passing to it from the body-wall, and by a pair of retractor muscles passing from the latter to the alimentary canal.

Structure of body-wall.-Beneath the cuticle is an epidermis, consisting of a single layer of flattened polygonal cells, firmly united together by their edges. Beneath this there is usually, but not always, a layer of muscle, which is arranged in two strata-an external composed of circular, and an internal of longitudinal fibres. There is an extensive cœlome lined in some forms (Phylactolæmata) by a definite colomic epithelium, in part ciliated; while in others there is no such definite epithelium, but its place is taken by thin parietal and visceral layers of an irregular cellular tissue-the parenchyma. Crossing the colome are strands, in some instances very numerous, of spindleshaped cells. In some cases two mesenteric bands suspend the alimentary canalan anterior attached near the mouth and a posterior passing

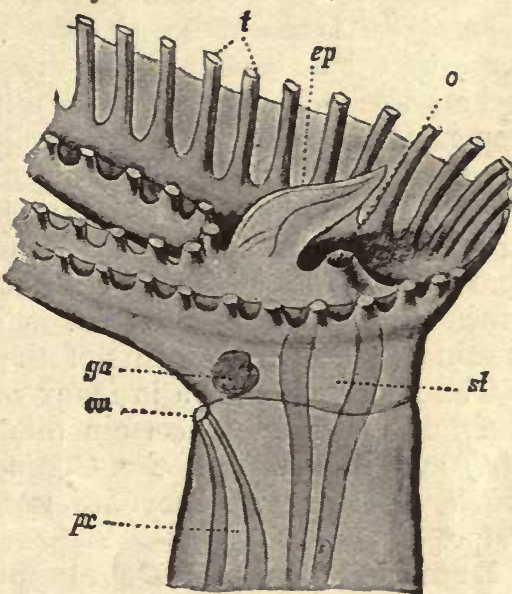

FIG. 282.-Anterior portion of the body of Lophopus, from the right side. an. anus; $e p$. epistome ; ga. ganglion; $o$. mouth ; $p r$. intestine; st. oesophagus; $t$. tentacles, cut off near the base. (From Lang's Comparative Anatomy. After Allman.)

from the cæcum to the aboral end of the zoœcium; in most cases the latter, to which the special name of funiculus is given, is alone present.

The alimentary canal has in all species the parts that have been already described in the case of Bugula. In some of the Cheilostomata it is stated that the cells of the œsophagus bear numerous striated muscle-fibre processes. In some Ctenostomata there is in addition a thick-walled chamber-the gizzard -with chitinous teeth, between the œsophagus and stomach.

The nervous system consists of a single, sometimes bilobed, ganglion (Fig. 280, gang, and Fig. 282, ga) placed between the mouth and the anal aperture, and of nerves passing from it to the various parts. There are never any organs of special 
sense, unless the epistome of the Phylactolæmata be of that nature.

Nephridia are not known with certainty to exist in any of the Ectoprocta. In some there is a pore through which water enters the body-cavity, or a ciliated intertentacular tube opening at the base of the tentacles. Excretion appears to be performed by certain cells of the funicular tissue and of the parenchyma or cœlomic epithelium. These become loaded with the products of excretion, and are set free as leucocytes in the colome, whence they may pass out through the intertentacular tube or may accumulate in the cells of the brown body.

In many Ectoprocta the colony bears a series of remarkable appendages - the avicularia - which are of the nature of modified zooids. In typical cases the avicularium has the bird's-head-like form that has been already described in the case of Bugula; sometimes it is completely sessile. A second set of movable appendages found in some forms are the vibracula; these are long tapering whip-like appendages which execute to-and-fro movements. The avicularia are frequently found to have seized in their jaws minute Worms or Crustaceans, and it is probable that their function, as well as that of the vibracula, is defensive; in the case of the Selenariido, which form unattached colonies, it is said that the movements of the vibracula subserve locomotion.

The impregnated ova in many cases undergo the early stages of their development in certain dilatations of the colony (Fig. 276, ooc.), and in many of the Gymnolæmata (Cheilostomata) these ovicells or occia, as they are termed, take on a very definite shape.

Reproduction and Development.-As a general rule the Ectoprocta are hermaphrodite. Both ovary and testis are derived from the layer lining the cœlome (parenchyma or cœlomic epithelium as the case may be), or from the funicular tissue. The testis may be single or double. The spermatidia, as in Bugula, or the mature sperms, become free in the colome. The ovary is very generally situated towards the oral end or about the middle, the testis towards the base. The mature ova escape into the colome, and in some forms there become impregnated apparently by the spermatozoa of the same individual. The development of the larva may take place in the coelome or in a special diverticulum of it ; in the Cheilostomata the fertilised ova pass into the ovicells; in some cases, both among the Phylactolæmata and the Gymnolæmata, they are received into a sheath formed by the tentacles of an imperfectly-developed zooid formed in a zoccium in which the original zooid had undergone degeneration.

In those cases in which the early stages of development are passed through in the body-cavity of the parent, the ciliated embryos may either escape through the zoœcial aperture after the 
zooid has undergone degeneration, or through a special opening formed for them in the wall of the zoccium. In some the fertilised ova pass out through the intertentacular tube. In Crisia and other Cyclostomata each of the ripe oœcia is found to contain a large number of embryos, developed from one ovum. The ovum in this genus segments to form a mass of cells from which finger-like processes arise, the end of each of these becoming constricted off to form an embryo.

Segmentation is total and approximately equal. The form of the free-swimming larva varies considerably, but in most there is a circular band with very long cilia, the corona, which may represent the tentacular crown of the adult; this divides the surface into two regions-oral and aboral. The larva may or may not be provided with a digestive canal. The aboral portion of the body presents a ciliated retractile disc or calotte; on the oral side is the sucker by which the larva afterwards becomes fixed. By a metamorphosis similar to that which has been described in the case of Bugula (p. 344), a primary zoœcium with a primary zooid is developed from the previously free ciliated larva. In the Cyclostomata the larva is barrel-shaped, with the mouth at one end, and at the other a prominence corresponding to the retractile disc. In the Phylactolæmata the larva is in the form of a ciliated hollow cyst from which the colony is formed by gemmation. A special form of asexual multiplication by means of bodies termed statoblasts (Fig. 280, stato) is observable in the Phylactolæmata. The statoblasts are internal buds formed from the funiculus and enclosed in a chitinous shell; they are set free eventually by the death and decay of the parent colony, and in spring each gives rise to a small zooid which fixes itself and develops into a colony.

Ethology and Distribution.-None of the Ectoprocta are parasites in the strict sense of the term, but very many of them live in intimate association with other organisms, often growing over and through them so as to form with them one complex structure. Certain genera are able by some means to excavate minute burrows in the shells of bivalves.

The majority of Ectoprocta are marine; but all the Phylactolæmata, together with Paludicella of the Ctenostomata, are inhabitants of fresh water. The fresh-water forms inhabit both running and stagnant waters; they occur at all elevations and are represented in all the great regions of the earth's surface. The marine forms are most abundant at moderate depths; but representatives of the group have been dredged from as great a depth as over 3,000 fathoms. In certain localities the larger kinds grow in great luxuriance, so as to form miniature forests.

Geologically the Ectoprocta are a very ancient group, being VOL. I 
represented in the Cambrian and later Palæozoic formations by forms which appear to have belonged mainly, if not exclusively, to the Cyclostomata. In the later formations of the Mesozoic period the Cheilostomata are also abundantly represented, and in the Tertiary the latter sub-order greatly outnumbers the Cyclostomata. The Tertiary Polyzoa flourished in certain localities in such luxuriance that their remains form calcareous deposits of very great extent.

\section{Sub-Class II.-Endoprocta.}

While the sub-class of the Ectoprocta comprises a large number of genera, that of the Endoprocta includes only Pedicellina (Fig. 283), Loxosoma, Urnatella, Myosoma, Gonopodaria and Ascopodaria, with one or two other less completely known forms. They are all marine except Urnatella-an American fresh-water genus. The feature indicated by the name of the sub-class - viz. the position of the anus within the circlet of the tentacles, is an important point of difference from the rest of the class ; but there are others of as great or greater importance.

In none of the Endoprocta is there is a distinct introvert. The body is cup-shaped, with a rim which is capable of being inverted over a cavity - the vestibule - within which the tentacles can be withdrawn, and which contains both mouth and anus. An epistome overhangs the mouth. The colome is almost or quite obliterated, the space between the alimentary canal and the wall of the body being filled, more or less completely, with a gelatinous hyaline matrix. A pair of nephridia are present. In Loxosoma they lie one on each side of the cesophagus and open separately on the exterior; they are ciliated intra-cellular tubes, each of which probably begins in a flame cell. In Urnatella the two nephridial tubes unite to open into the cloaca-a diverticulum of the vestibule. The ganglion (Fig. 283, gang), situated between mouth and anus as in the Ectoprocta, is bilobed in Loxosoma. Testes and ovaries occur in the same individual in some, but appear to mature at different times : they are provided with special ducts; in others the sexes are separate.

Pedicellina and Urnatella are colonial, Loxosoma solitary. In Pedicellina (Fig. 283) there is a creeping stolon with which a number of zooids are connected; a diaphragm separates the body of each zooid from the stalk. Gonopodaria ramosa has a branching stalk. Urnatella has a disc of attachment with one to six, jointed, branching stems. In Loxosoma, which is found attached to various Annulata, two parts are distinguishable-the calyx or body and the stalk. In the base of the latter is the so-called foot-gland, consisting of a small number of granular cells arranged around a central space opening on the exterior. Buds are formed, but 
become detached before reaching maturity. Segmentation of the ovum is complete, and a gastrula is formed by invagination.

Certain differences in the larval history have sometimes been regarded as separating very widely the Endoprocta from the Ectoprocta. The former, like the latter, have a free-swimming ciliated larva, provided with a corona and a ciliated disc. This develops directly into the primary zooid after becoming attached by means of the oral surface. The ectoproct larva also, as stated previously (p. 344), becomes attached by the oral surface; but any rudiments of a zooid-such as an alimentary canal-which may

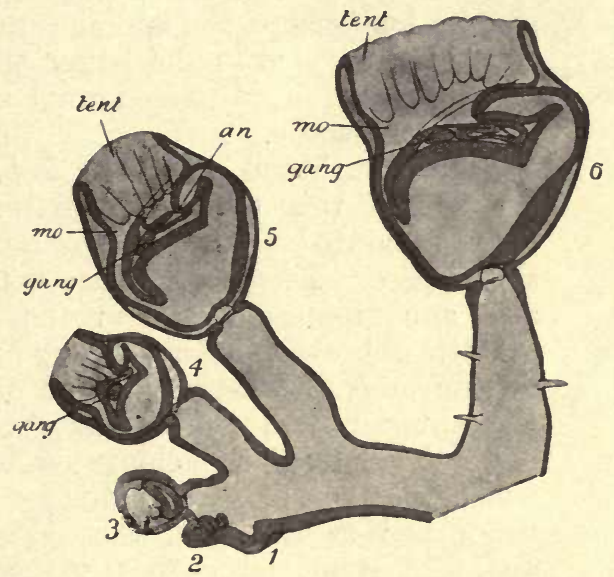

FIG. 283.-Pedicellina. Showing successive stages (numbered 1 to 6 ) in the development of zooids by budding. an. anus; gang. ganglion; mo. mouth; tent. tentacles (retracted). (After Hatschek.)

have been developed, become absorbed, and the primary zooid is developed at the free or aboral end of the larva, with its oral surface directed upwards, away from the base of attachment. The difference, however, is not so important as it may at first appear, for the parts of the larval Endoproct do not remain in the reversed position in which they are situated when attachment first takes place, with the vestibule, mouth, and anus directed downwards. Very soon a rotation is observed to take place, by virtue of which the vestibule and developing tentacles, with the mouth and anus, become carried to their permanent position on the free-surface of the animal.

\section{CLASS II.-PHORONIDA.}

The position of Phoronis, a worm-like marine animal, is a matter on which widely divergent views are held. On account of certain strong resemblances to the Polyzoa, and, more particularly, 
to the Phylactolæmata, it is most commonly looked upon as related to that class and to the Brachiopoda, and the Phoronida may thus conveniently be dealt with as a class of the Molluscoida.

Phoronis (Fig. 284) lives in associations consisting of a number of individuals, all of which are developed from ova, there being no process of asexual formation of buds. Each worm is enclosed in a membranous or leathery tube, within which it is capable of being completely retracted. The body is cylindrical, elongated, and unsegmented. At one end there is a crown of numerous slender, ciliated tentacles borne on a horse-shoeshaped lophophore, the lateral cornua of which are spirally coiled in the larger species; these are supported by a mesodermal skeleton and are non-retractile.

Both mouth and anus (Fig. 285, mo, an) are situated at this tentacular extremity of the body, separated from one another by only a short space. This short space between mouth and anus represents, as in the Polyzoa, the greatly abbreviated dorsal surface; but it will be convenient to term this end of the animal the anterior, and the opposite the posterior end: the side of the elongated body towards which the mouth is approximated may be distinguished as the oral, the opposite as the anal. A small lobe-the epistome (ep)-overhangs the mouth and lies between it and the anus. Near the anus open two ciliated nephridial tubes (neph) of mesodermal origin, which open internally each by two apertures into the posterior chamber of the cœlome.

The colome, which is lined with a cœlomic epithelium, consists of three main parts of very unequal extent. The first (prosocoele) is a narrow cavity in the epistome.. The second (mesoccele), which is in communication with the first, lies in front of a transverse septum or mesentery extending between the mouth and anus, and perforated by the œesophagus but

FIG. 2S4.-Phoronis australis, natural size.

not by the rectum; it is prolonged round the lophophore and gives off narrow diverticula to the hollow tentacles. The third, and by far the most extensive part of the cœlome (metacrele), occupies the whole of the length of the body behind the transverse septum. It is subdivided into two by a median longitudinal mesentery (Fig. 287, $m, m$.), which extends from the oral to the anal surface and supports both limbs of the alimentary 
canal; and each of these is further subdivided by a longitudinal mesentery extending from the body-wall to the œsophagus ( $\propto)$ in the one compartment (usually termed the right), and to the rectum $(r)$ in the other (left). The alimentary canal is bent on itself to form a loop, as in the Polyzoa: it is distinguishable into osophageal, gastric and intestinal regions. There is a closed system of bloodvessels with contractile walls containing red blood-corpuscles. The nervous system lies immediately below the cells of the epidermis. Nerve-

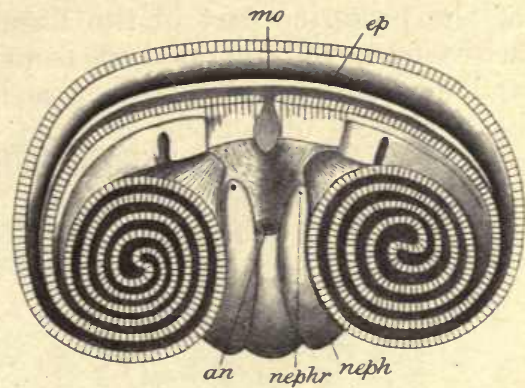

FIG. 255.-Phoronis australis, free end, maguified. an. anus; ep. epistome ; mo. mouth; nephr. nephridial aperture ; neph. nephridium; (After Benham.)

elements are generally distributed over the surface, but are specially concentrated in the form of a ring surrounding the body just behind the mouth, but not enclosing the anus,

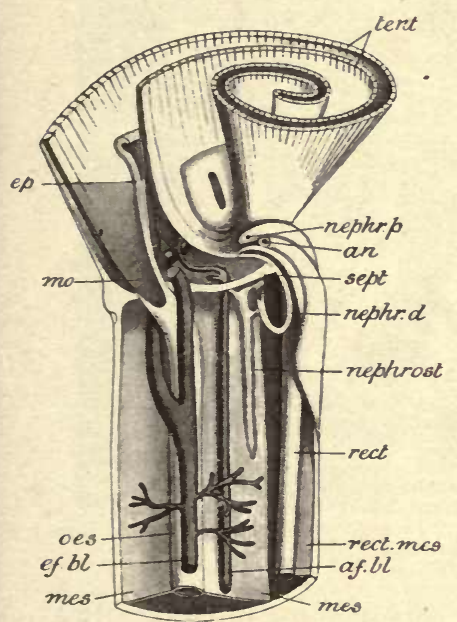

FiG. 286.-Fhoronis australis, internal organisation. af. bl. afferent blood vessel ; $u n$. anus; $e j . b l$. efferent blood vessel ; $e p$. epistome; mes. mesentery; mo. mouth ; nephr p. nepliridiopore; nephr.d. duct of nephridium; nephrost. nephrostome (internal opening of nephridium ; acs. aesophagus ; rect. rectum; rect. mes. rectal mesentery; sept. septum; tent. tentacles (cut short). (After Benham.) thickened into a ganglion between mouth and anus, and giving off nerves to the tentacles. There are no organs of special sense.

Phoronis is hermaphrodite. Ova and sperms are developed in the cœlome towards the posterior end from cells on the wall of one of the large blood-vessels. When mature these pass out through the nephridia to the spaces enclosed by the tentacles, where the ova are impregnated (- according to another account, fertilisation takes place in the colome-), and they go through the early stages of development fixed to the tentacles. The segmentation is complete and slightly unequal : when four blastomeres are formed two larger, darker endoderm and two smaller, clearer ectoderm cells are to be distinguished. A blastula is formed with clearer ectoderm cells on one side; invagination takes place; and, as the embryo elongates, the blastopore is 
drawn out into a slit which eventually becomes closed up behind, the anterior portion alone remaining open to form the mouth. The anus is developed later as an invagination in the position of the posterior part of the former blastopore. The mesoderm arises from cells budded off from the endoderm. The prosocole and mesocœle arise by the formation of fissures; the metaccle by a process of folding off from the archenteron. A large preoral lobe is formed, and the anus becomes surrounded by a circlet of cilia (Fig. 288, A). The part of the body on which the anus

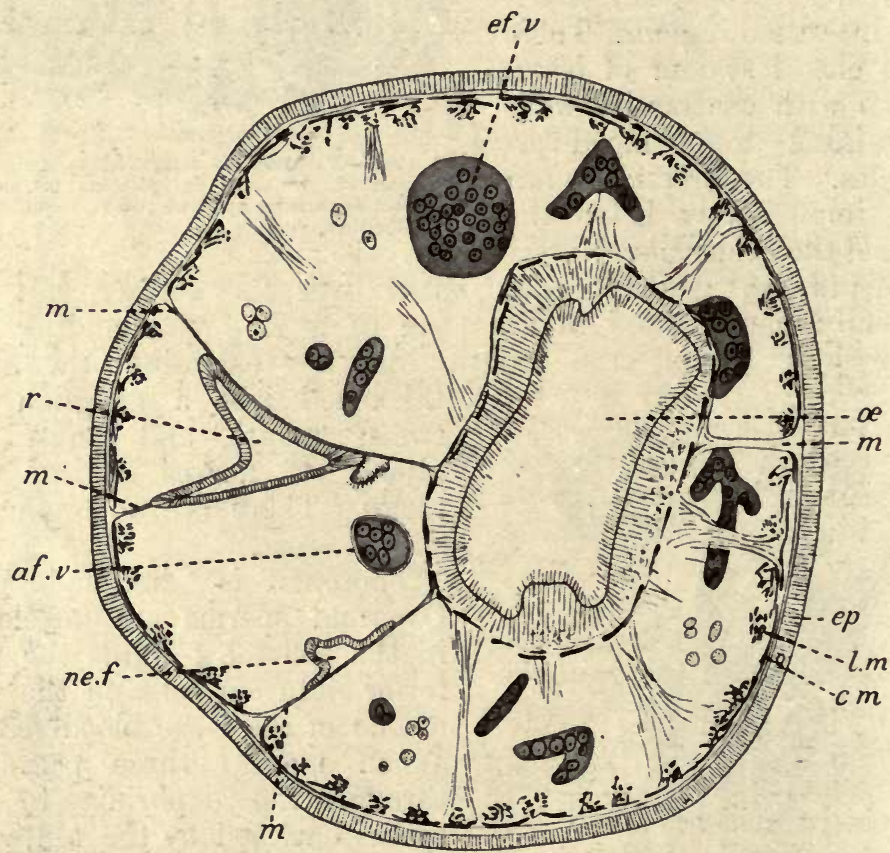

Fig. 287.-Fhoronis, transverse section towards the anterior.end. $a f . v$. afferent blood-vessel ; c. $m$. circular layer of muscular fibres; ef. $v$. efferent blood-vessel ; ep. epidermis; $c$. m. circular layer of muscle; $m, m$. mesenteries; ne. $f$. funnel-like opening of nephridium; $\propto$. asophagus; $r$. rectum. (After Benham.)

is situated becomes elevated into a conspicuous process. Behind the mouth there is a circlet of cilia, and from this region grow out a circlet of processes - the rudiments of the larval tentacles (B). The larva has now reached the stage to which the term actinotrocha is applied. It has a large hood-like lobe overhanging the mouth and a circlet of ciliated larval tentacles; the anus is situated on a prominent process.

There is a pair of larval excretory organs corresponding to those of the trochophore larva (p. 322): these apparently do not become converted into the nephridia of the adult. A thickening 
of the ectoderm of the pre-oral lobe, sometimes bearing eyespots, appears to represent the apical plate of the trochophore. At the point where the œsophagus opens into it, the gastric region of the alimentary canal gives off forwards in one species a pair of hollow diverticula, the cells of which contain vacuoles like those of the neighbouring parts of the stomach itself.

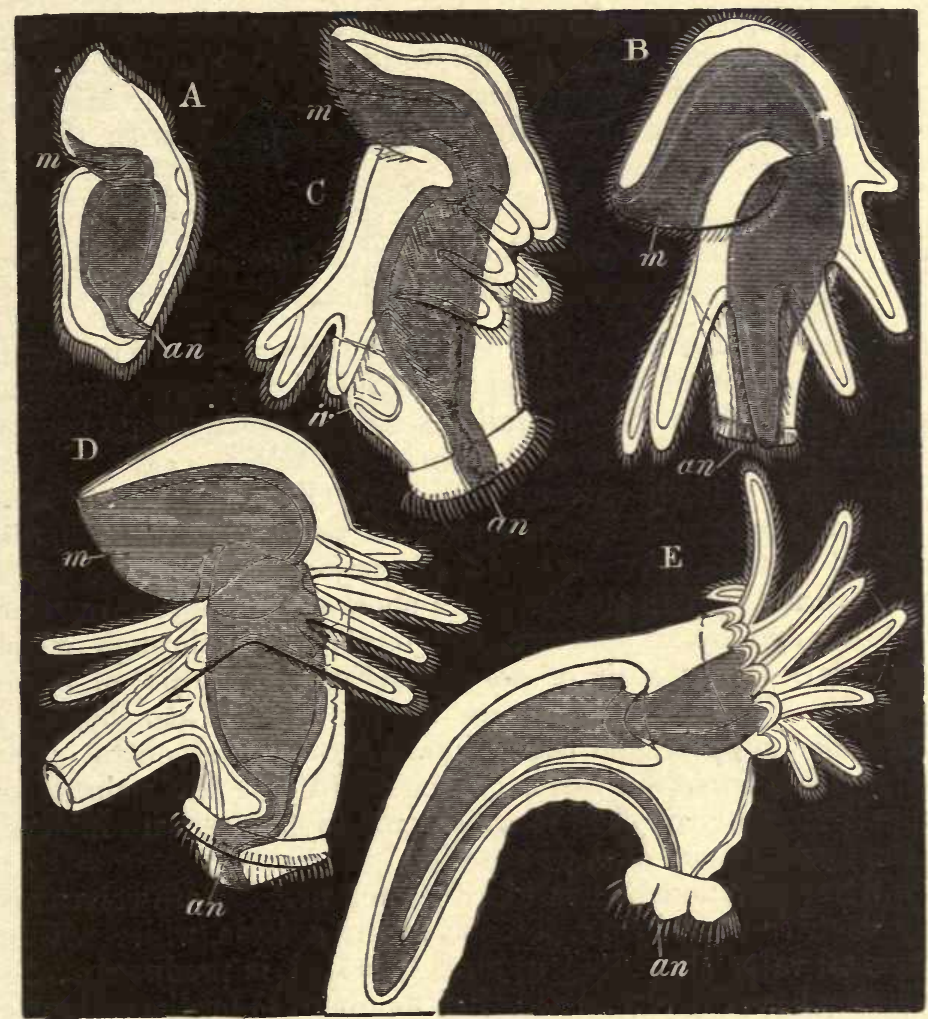

Fig. 2Ss.-Phoronis, development. A, young larva ; B, larva after the formation of the postoral circlet of tentacles; C. larva with commencing pit-like involution; D, larva with invagination partly everted; E. invagination completely everted. m. mouth; an. anus; iv. involution to form body. (From Balfour's Bmbryology.)

The ectoderm of the process on which the anus is situated subsequently becomes involuted to form a deep pit $(C, i v)$, and rudiments of the adult tentacles are formed as a ring of processes at the bases of the larval tentacles. The metamorphosis from this point is completed with great rapidity. The larva sinks to the bottom; the pit at the side of the anal elevation becomes everted $(D)$, and the alimentary canal of the larva is drawn into it $(E)$, the projection thus formed, which grows out at right angles with the 
long axis of the larva, becoming the body of the future animal; the larval tentacles and pre-oral lobe become thrown off, and the lophophore is developed.

\section{CLASS III.-BRACHIOPODA.}

The Brachiopoda are the fabricators of the well-known "Lampshells" found in most parts of the world. They occur in the sea at various depths, and were formerly classed under the Mollusca, their characteristic bivalved shell being compared with that of oysters, mussels, \&c.

\section{EXaMPle of the Class-Magellania (Waldheimia) lenticularis or $M$. flavescens.}

Magellania lenticularis is found in great numbers, at moderate depths, off the coast of New Zealand. An allied species, $M$. flavescens, is equally common in the Australian seas, and several other species are known in various parts of the world.

The body is entirely covered by a shell (Fig. 289) of oval form and pink colour, composed of two pieces or valves, one of which, distinguished as the ventral valve $(v . v)$, projects beyond the other or dorsal valve (d. v), in the form of a short conical beak $(b)$ perforated at the end by an aperture, the foramen $(b)$, through which passes a dark brown stalk or peduncle (Fig. 290, B, pd) of horny consistency. In the natural state the peduncle is attached to a rock or other support, and the animal lies with the ventral valve uppermost and with the valves gaping slightly. The pointed or peduncular end of the shell is considered to be posterior in position, the opposite end or gape anterior.

It will be convenient to consider the shell first. Both valves are deeply concavo-convex, of a pinkish colour outside, white within. The ventral valve (Fig. 289), as already stated, is produced posteriorly into a beak $(b)$, terminating in a foramen $(f)$ for the peduncle. The distal margin of the foramen is left incomplete by the shell proper, but is closed by a small double plate, the deltidium (d). Immediately anterior to the beak is the curved hinge-line along which the valve articulates with its fellow, and just anterior to the hinge-line the inner surface of the shell is produced into a pair of massive, irregular hinge-teeth $(t)$. On the inner surface of the valve, towards its posterior end, are certain shallow depressions marking the attachments of muscles $(a d . m, d . m)$.

The dorsal valve (D) has no beak, but its posterior edge forms a hinge-line which is produced in the middle into a strong cardinal process $(c . p)$ with a curiously folded surface: when the two valves are in position this process fits between the hinge-teeth of the 
ventral valve, the hinge-teeth in their turn being received into depressions $(s)$ placed on each side of the cardinal process. The inner surface of the dorsal valve is produced into a median ridge or septum ( $s p$ ), continuous posteriorly with the cardinal process, and attached on either side of the base of the latter are the two ends of a delicate calcareous ribbon, the shelly loop (s. l), which projects
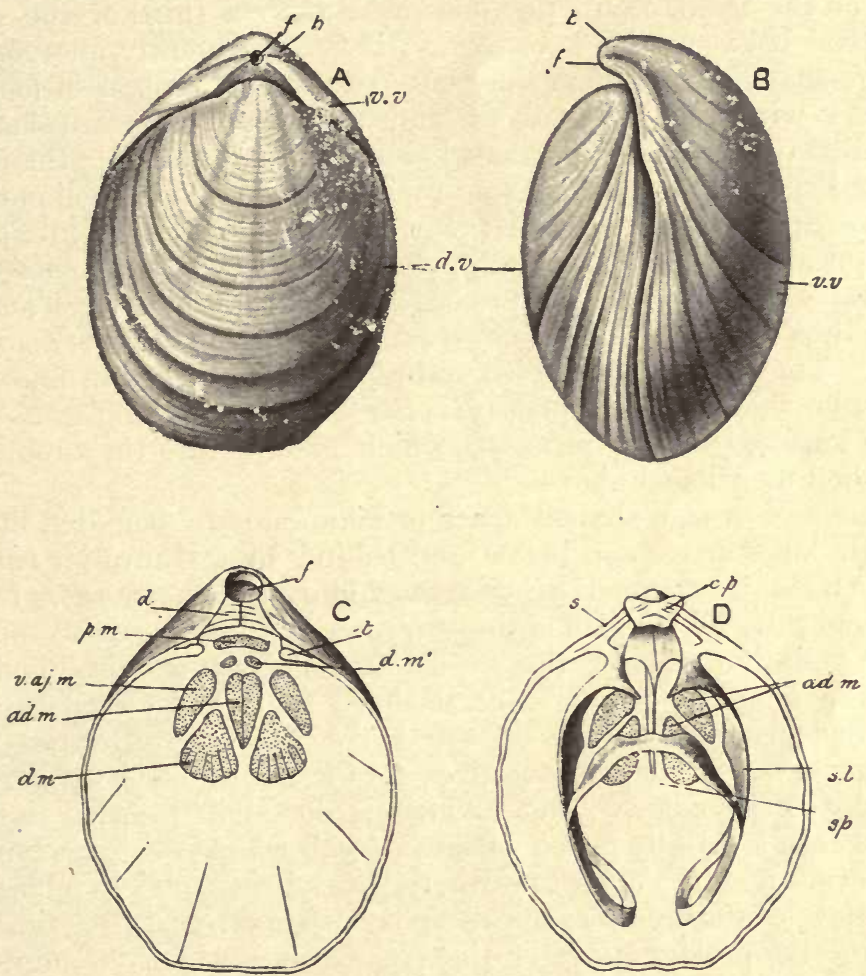

FIG. 289.- Magellania flavescens. A, the entire slıcll from the dorsal aspect, and B, from the left side; $C$, interior of ventral valve, and $D$, of dorsal valre. ad. $m$. adductor impressions; $b$. beak; c. p. cardinal process; $d$. deltidium; $d$. $m$. divaricator impressions; $d . v$. dorsal valve; $f$. foramen; $p$. $m$. protractor impressions; s. tooth-socket; $8 . l$. shelly loop; $s p$. septum; $t$. hinge-tooth; $v$. $a j . m$. adjustor impressions; $v$. $v$. ventral valve. (After Davidson.)

freely into the cavity enclosed between the two valves, and has the form of a simple loop bent upon itself. The inside of the dorsal valve also has muscular impressions.

Externally both valves present a series of concentric markings parallel with the edge or gape: these are lines of growth, the shell being built up by new layers being deposited within those previously formed, and projecting beyond them so as to form a series of outcrops. 
Microscopically the shell consists of prismatic rods or spicules of carbonate of lime, placed obliquely to the surface and separated from one another by a thin layer of membrane. It is also traversed, perpendicularly to the surface, by delicate tubules which begin on the inner surface in microscopic apertures and extend to within a short distance of the outer surface.

The actual body of the animal (Fig. 290, B) lies at the posterior end of the shell, occupying, not more than a third of the space enclosed between the two valves: it is consequently more or less wedge-shaped in form, and presents dorsal and ventral surfaces in contact with the two valves, and an anterior surface looking towards the gape. The dorsal is of greater extent than the ventral surface, so that the anterior surface is placed obliquely.

The dorsal and ventral regions are continued each into a flat reduplication of the body-wall, closely applied to the corresponding valve and containing a prolongation of the coelome. The two flaps thus formed are the dorsal $(d . m)$ and ventral $(v . m)$ mantlelobes. They are fringed with minute setæ $(s)$ lodged in muscular sacs, like those of Chætopods (vide Sect. X.), and give off from their outer surfaces hollow processes which extend into the tubules of the shell mentioned above.

The large wedge-shaped space or mantle-cavity, bounded by the mantle-lobes above and below, and behind by the anterior surface of the body, is occupied by a huge and complex lophophore (Figs. 290 and $291, l p h$ ), which springs from the anterior surface of the body, and, like that of the fresh-water Polyzoa and of Phoronis, has the general form of a horse-shoe. It is, however, peculiarly modified: the two limbs of the horse-shoe curve towards one another so as to adapt themselves to the mantle-cavity; and the middle of the concave edge, which is dorsal in position, is produced into a spirally coiled offshoot $\left(l p h^{\prime}\right)$ which lies between the two arms and is coiled towards the dorsal side. The lophophore is hollow, containing a spacious cavity or sinus: its two main arms also receive prolongations of the cœlome into which the digestive glands project: it is fringed throughout its whole extent with long ciliated tentacles which form the outer boundary of a ciliated food-groove, bounded on the inner side by a wavy ridge or lip $\left(l p, l p^{\prime}\right)$. By the action of the cilia microscopic particles are swept along the food-groove to the mouth.

Digestive Organs. - The mouth (mth) is a narrow crescentic aperture situated in the middle of the lophophore, towards its convex or ventral edge, and is bounded dorsally by the lip. It leads into a V-shaped enteric canal which consists of a gullet passing upwards from the mouth, an expanded stomach (st), and a straight intestine (int.) which extends from the stomach downwards and backwards towards the ventral surface and ends blindly, there being no anus. On each side of the stomach, and opening 
into it by a duct, is a large, branched digestive gland $(d . g l)$. The whole canal is lined with ciliated epithelium.
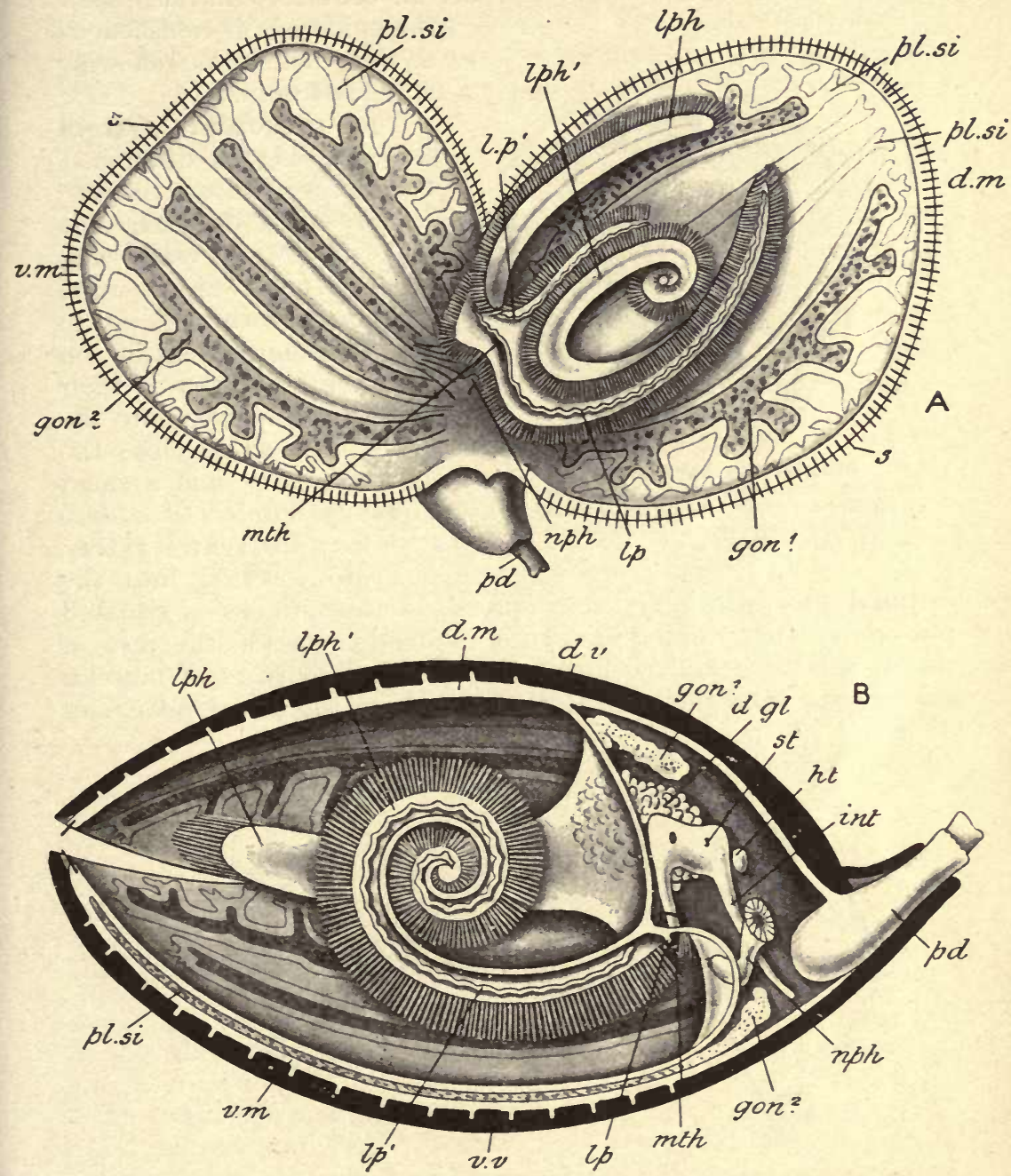

Fig. 290.-A, body of Magellania lenticularis, removed from shell; B, sagittal section of the entire animal. Both semi-diagrammatic, the lophophore being represented as of smaller proportional size than in the actual animal (cf. Fig. 291). d.gl. digestive gland; $d . m$. dorsal mantle-lobe ; $d . v$. dorsal valve of shell; gonl, gon'. gonads ; $h t$. heart ; int. intestine ; $l p, l p 1$. lip; lph. lophophore; lphl. its coiled process; mth. mouth; $n p h$. in $\mathbf{B}$, nephridium, in $\mathbf{A}$, nephridial aperture; $p d$. peduncle; $p l$. si. pallial sinuses; s. setæ; st. stomach; $v$. m. ventral lobe of mantle; $v$. $v$. ventral valve of shell.

The body-wall consists externally of an epidermis formed of a single layer of cells, then of a layer of connective tissue, of a 
cartilaginous consistency in many parts, and finally of a ciliated cœlomic epithelium lining the body-cavity. On the outer surfaces of the mantle-lobes, where they are in contact with the shell,

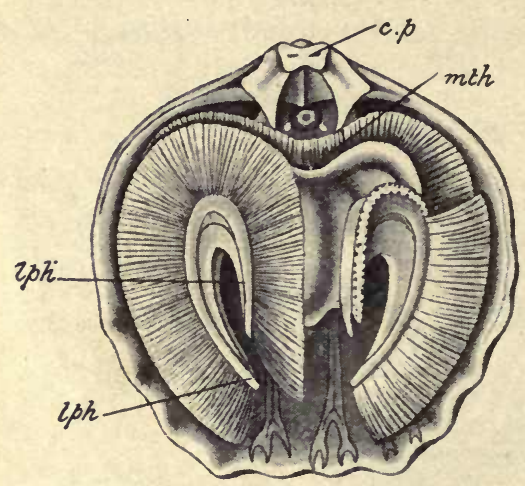

FiG.291.-Magellania flavescens, the ventral valve removed. c. p. cardinal process; $l p h$. arm of lophophore; lphl. its coiled process, with the tentacles removed on the right side; $m$ th. mouth. (After Davidson.) the epidermis is replaced by a thin membrane showing no cell-structure.

The muscular system (Fig. 292) is well developed. Two large adductor muscles (ad. $m$ ) arise on each side from the dorsal valve, and passing downwards, unite with one another so as to have a single insertion on the ventral valve: their action is to approximate the valves and so to close the shell. A large and a small pair of divaricators $\left(d . m, d n \iota^{\prime}\right)$ arise from the ventral valves, and are inserted into the cardinal process, which they depress : as this process is situated posteriorly to the hinge-line, its depression raises the rest of the dorsal valve and so opens the shell. Two pairs of muscles arising, one from the ventral, the other from the dorsal valve, and

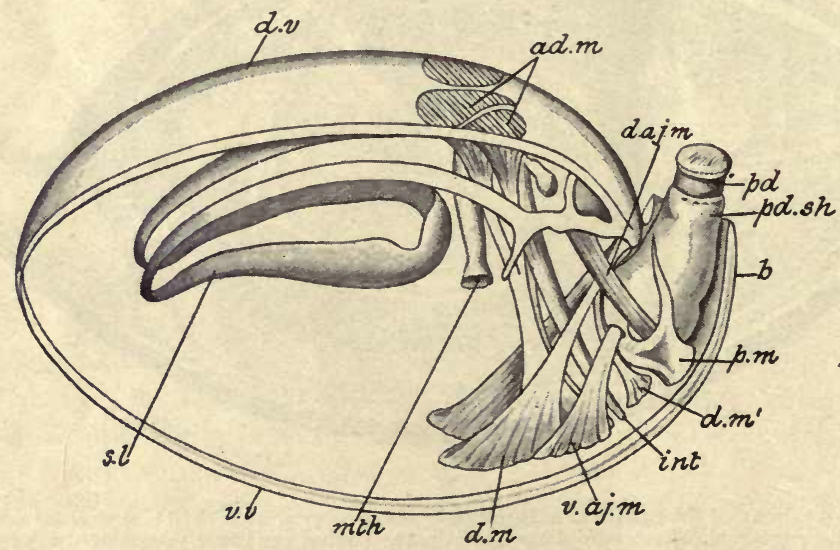

Fıg. 292.-Muscular system of Magellania. ad. m. adductors; $b$. beak ; $d . a j . m$. dorsal adjustors; $d . m ., d$. $m^{\prime}$. divaricators; $d . v$. dorsal valve; int. intestine; mth. mouth; pd. peduncle; $p d .8 h$. sheath of peduncle; $p . m$. protractor ; $8 . l$. shelly loop; $v$. $a j$. $m$. ventral adjustors; $v$. $v$. ventral valve. (After Hancock.)

inserted into the peduncle, are called adjustors $(a j . m)$ : the peduncle being fixed, they serve to alter or adjust the position of the animal as a whole by turning it in various directions. 
The cœlome is a spacious cavity more or less encroached upon by the muscles and other organs, and traversed by sheets and bands of membrane which connect the enteric canal with the body-wall, and thus act as mesenteries. The coelome is continued into each of the mantle-lobes in the form of four canals or pallial sinuses (Fig. 290, pl. si), the two outer of which are extensively branched.

Blood-System.-Attached to the posterior region of the stomach is a small, almost globular sac $(h)$, which has been proved to be contractile and is to be considered as a heart. Vessels have been traced from it to various parts of the body, but the relations

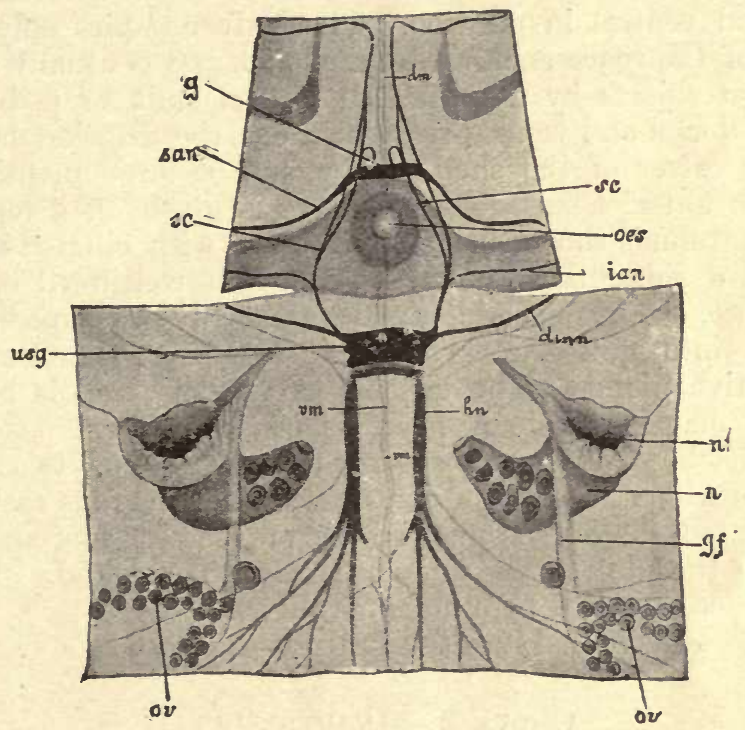

FIG. 293.-Anterior body-wall of Terebratula, to show nervous system, \&c. Im. dorsal mesentery ; g. brain; gf. genital folds; $n$. nephridium; nt. nephrostome; oes. gullet; ov. ovary ; $s c$. cesophageal connective; $u$ g. infra-cesophageal ganglion; $v m$. ventral mesentery; $d m n, h n$, ian, san. nerves. (From Lang's Comparative Anatomy, after van Bemmelen.)

of the whole circulatory system and the course of the circulation are very imperfectly known.

The excretory organs consist of a pair of very large nephridia $(n p h)$ lying one on each side of the intestine. Each is funnelshaped, having a wide inner opening or nephrostome, with plaited walls, opening into the cœlome, and a narrow, curved, outer portion which opens into the mantle-cavity not far from the mouth. As in many cases which have already come under our notice, the nephridia act also as gonoducts.

The nervous system (Fig. 293) is a ring round the gullet presenting supra- $(g)$ and infra- (usg) œesophageal swellings or ganglia, of which the infra-œsophageal is the larger. Nerves are given off 
to the inantle, lophophore, \&c. No special sense-organs are known.

Reproductive Organs.-The sexes are separate. There are two pairs of gonads (Fig. 290, gon), one dorsal and one ventral, in the form of irregular organs sending off branches into the pallial sinuses.

\section{Distinctive Characters and Classification.}

The Brachiopoda are Molluscoida in which the body is enclosed in a shell formed of two parts or valves which are respectively dorsal and ventral in position. The body occupies only a small portion of the space enclosed by the shell, and is usually attached to foreign objects by a posteriorly placed stalk or peduncle: it gives off dorsal and ventral reduplications, the mantle-lobes, which line the valves of the shell and enclose a large mantle-cavity. From the anterior surface of the body is given off a lophophore which surrounds the mouth, and is beset with ciliated tentacles. There is a ridge-like pre-oral lip which is continued on to the lophophore. The enteric canal is usually V-shaped, and is divisible into gullet, stomach, and intestine: there is a pair of digestive glands. The colome is spacious, and is continued into the mantle-lobes. A heart is usually present, attached to the stomach. The excretory organs are one or two pairs of nephridia which act also as gonoducts. The nervous system is a ganglionated circum-œsophageal ring: sense-organs are usually absent in the adult. The sexes are separate or united. Development is accompanied by a metamorphosis.

The class is divided into two orders :-

\section{ORDER 1.-INARTICUlata.}

Brachiopoda in which the shell is not composed of oblique prisms : the valves are not united by a hinge, and there is no shelly loop for the support of the lophophore. An anus is present.

Including Lingula, Crania, Discina, \&c.

\section{Order 2.-Articulata.}

Brachiopoda in which the shell is formed of oblique prisms or spicules of calcium carbonate: the two valves unite by a definite hinge, and there is usually a shelly loop, for the support of the lophophore, developed in connection with the dorsal valve. The intestine ends blindly.

Including Magellania, Terebratula, Rhynchonella, Cistella (Argiope), \&c. 


\section{Systematic position of the Example.}

The genus Magellania, of which there are several species, belongs to the family Terebratulidæ, and to the order Articulata.

The dissimilar valves of the shell articulated by teeth and sockets, and the absence of an anus, place it among the Articulata. The Terebratulidæe are distinguished by an oval or rounded shell, the structure of which is punctate, the dots corresponding with blind tubes receiving processes of the mantle; the beak of the ventral valve is prominent, and has a foramen partly bounded by a deltidium of one or two pieces: there is a shelly loop springing from the hinge-line of the dorsal valve. The genus Magellania is characterised by having the shelly loop fully half as long as the shell itself, and by the presence of a median septum on the inner face of the dorsal valve.

The specific differences between M. lenticularis and M. flavescens are largely matters of detail, depending upon the precise form of the shell and loop. More obvious differences are seen in the shell, which is pink, evenly-rounded, and short-beaked in M. lenticularis, while in M. flavescens it is horn-coloured, almost pentagonal, and has a prominent beak.

\section{General Organisation.}

The shell presents two distinct types: in the Articulata. the order to which Magellania belongs, the dorsal and ventral valves are dissimilar, the dorsal valve having a cardinal process and usually a shelly loop, the ventral a spout-like beak for the peduncle ; while in the Inarticulata, of which Lingula is a good example (Fig. 294, A), the two valves are nearly alike, and there is no shelly loop and no beak. These differences are accompanied by differences in microscopic structure; in the Articulata the shell is dense and stony, and is formed of obliquely placed calcareous prisms, while in the Inarticulata it has no prismatic structure, but usually consists of a chitinoid material more or less strengthened by calcareous spicules. Among the Articulata the loop may be absent; when present, it varies greatly in form and size, being sometimes very small and simple (Fig. 294, C, D), sometimes bent upon itself, as in Magellania, sometimes attached to the septum or to the interior of the dorsal valve (E), sometimes, as in the extinct Spirifera, represented by a complex double spiral $(\mathrm{F})$, sometimes reduced to short, paired rods springing from the septum $(G)$.

The majority of both orders are attached by a longer or shorter peduncle which passes between the proximal ends of the valves in Lingula (Fig. 294, A), through a perforation in the ventral valve in Discina (C), and through a foramen in the spout-like posterior end 
of the ventral valve in the Articulata. Crania (B) has the ventral valve fixed directly to foreign objects, the peduncle being absent.

The lophophore is found in its simplest form in Cistella (Fig."295, A), in which it is a horse-shoe-shaped disc with very short arms, attached to the dorsal mantle-lobe and surrounded with flexible tentacles which project between the valves. From this the lophophore of Magellania, which may be considered as typical for the Articulata, is easily derived by an increase in size, and by the prolongation of the middle region of the concave edge into a coiled offshoot. In the Inarticulata (C), and in Rhynchonella (B) among the Articulata, each arm of the horse-shoe is
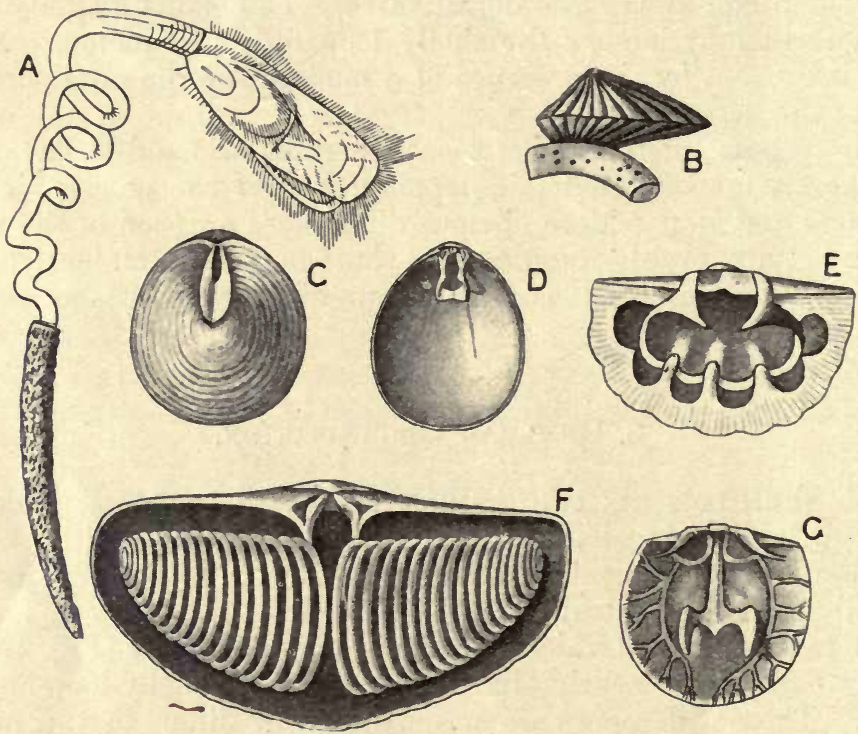

FIc, 294.-Typical Brachiopoda. A, Iingula ; B, Crania ; C, Discina ; D, Terebratula ; E, Cistella ; F, Spirifera ; G, Kraussina. (After Bronn.)

coiled into a conical spiral, which in some cases can be protruded between the valves.

The most noteworthy point about the muscular system is the fact that the shell is both opened and closed by muscular action. The dorsal valve may be taken to represent a lever of which the hinge-line is the fulcrum, the cardinal process the short arm, and the main portion of the valve the long arm. The muscles all arise from the ventral valve, the adductors being inserted into the inner face of the dorsal valve, which they depress, the divaricators into the cardinal process, their action depressing it and thus elevating the valve itself. In Lingula there is a very complex muscular system by means of which the valves can be rubbed upon one another, or moved laterally as well as opened and shut. 
In the Articulata the enteric canal is $\mathrm{V}$-shaped, as in Magellania, the intestine being straight or nearly so, and ending blindly. In the Inarticulata, on the other hand, the intestine is usually coiled, and always ends in an anus (Fig. 295, C, a), which generally opens into the mantle-cavity, but in one genus (Crania) into a pouch or sinus at the posterior end of the body between the valves.

A heart is usually present, but the function of blood is performed mainly by the cœlomic fluid, which is propelled by the cilia lining that cavity, and circulate both in the colome itself and

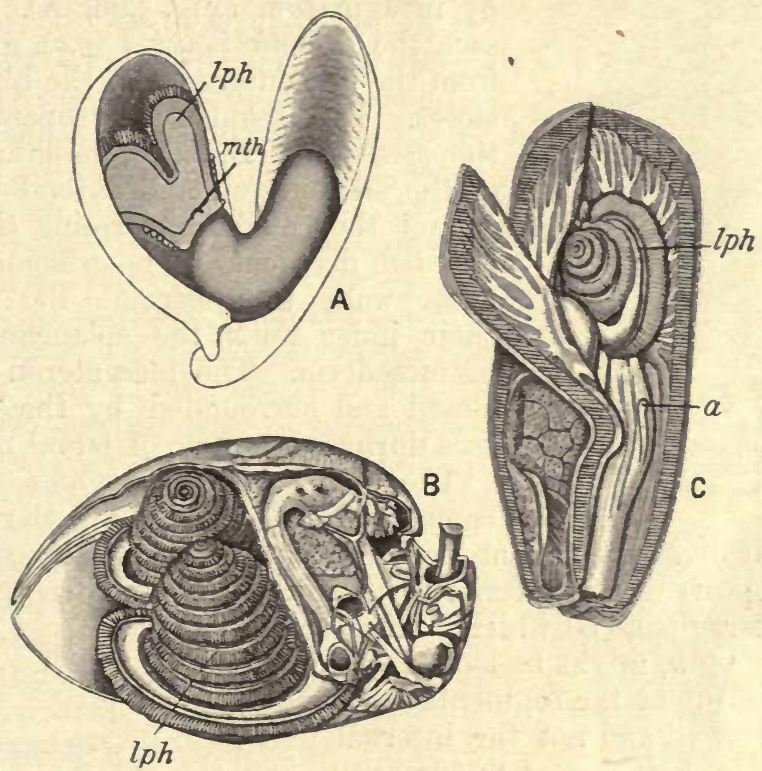

Fig. 295.-Dissections of A, Cistella ; B, Rhynchonella ; and C, I.ingula. a. anus ; Iph. lophophore; mth. mouth. (After Schulgin and Hancock ) $^{2}$

in the pallial sinuses, each sinus presenting-in Lingula at least - both an outgoing and an ingoing current.

A single pair of nephridia, resembling those of Magellania, occurs in all known genera except Rhynchonella, in which there are two pairs, one dorsal and one ventral. Besides discharging an excretory function they act as gonoducts.

The nervous system always takes the form of a circum-œsophageal ring with ganglionic enlargements, the largest of which is ventral or sub-œsophageal in position. Otocysts have been described in Lingula, rudimentary eyes in Megerlia, and patches of sensory epithelium in Cistella: with these exceptions sensory organs are unknown.

There are usually four gonads, two dorsal and two ventral, 
sending prolongations into the pallial sinuses. Some genera are diœcious, others hermaphrodite, the epithelium of the gonads producing, in the latter case, both ova and sperms.

The development of the Brachiopoda is best known in Cistella, in which the first stages of development are passed through
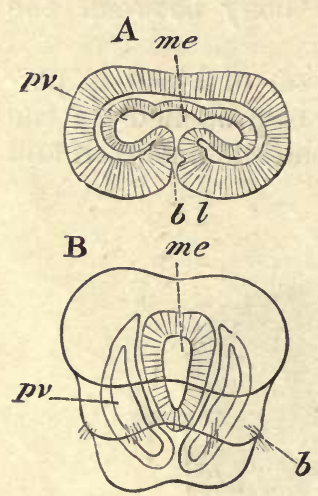

FiG. 296. - Two stages in the development iof Cistella (Argiope). b. provisional seta; bl. blastopore; me. mesenteron; pv. colomic pouches. (From Balfour's Embryology, after Kowalevsky.) in a pair of cavities, the brood-pouches, situated at the base of the lophophore. Segmentation is regular and complete, and results in the formation of a blastula, which is converted into a gastrula by invagination (Fig. 296, A). Paired sacs, the coelomic pouches $(p \cdot v)$, grow out from the archenteron, and the blastopore closes. The cœlomic sacs separate from the mesenteron $(\mathrm{B}, m e)$ or middle portion of the archenteron, and extend between it and the ectoderm, forming the right and left divisions of the colome: their outer walls thus become the somatic, their inner walls the splanchnic layer of mesoderm. The mesenteron remains closed and surrounded by the colomic sacs during the whole of larval life.

The embryo now elongates and becomes divided by an annular groove into two divisions, an anterior and a posterior: a second groove soon appears in the anterior division, the embryo then consisting of three regions (B), which, from a superficial point of view, might be looked upon as metameres. But as the segmentation affects only the body-wall and not the internal parts, the process is not one of metamerism, and the three apparent segments are called respectively the head-region, the body-region, and the peduncular region (Fig. 297).

Next the head-region grows out into an umbrella-like disc surrounded with cilia and bearing four eye-spots (Fig. 298, A), and on the body-region a backwardly-directed annular fold $(m)$ appears, bearing four groups of provisional setæ. In Cistella, which has no setæ in the adult condition, the provisional setæ are subsequently lost, and are

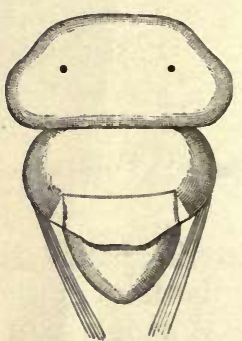

Fig. 297.- Young larva of Cistella, with the three segments, two eye-spots, and two bundles of setre (From the Cambridge Naturul History, after Kowalevsky.) not replaced. In forms which possess setæ in the adult condition the provisional setæ are likewise lost, but are replaced by the permanent setæ. Soon this mantle-fold divides into dorsal and ventral lobes, which, being directed backwards, cover the peduncular region. 
In this condition the larva swims freely like a trochophore. After a time it comes to rest and fixes itself by its peduncular segment (B). The two lobes of the mantle-fold $(m)$ become reflexed so as to point forwards instead of backwards, thus leaving the peduncular region exposed and covering the head-region: by this process the outer surface of the larval mantle becomes internal, and vice versa. A stomodæum is formed on the head-region, and, communicating with the mesenteron, establishes the enteric canal. The umbrella-like head-region decreases in size, and perhaps forms the lip, which is at first confined to the part immediately dorsal to the mouth. The lophophore appears at first on the inner surface of the dorsal mantle-lobe, but gradually extends and surrounds the mouth; in its earlier stages it is circular, but afterwards assumes the horse-shoe form by sending out paired extensions. In genera with a complex lophophore, like Magellania, this organ has at first a simple horseshoe form (Fig. 299, lph). A shell is secreted by the mantle-lobes, and the peduncular region becomes the peduncle of the adult.

Distribution.-The Brachiopoda are all marine. They are widely distributed geographically, and live at various depths-from between tide-marks to 2,900 fathoms. At the present day the class includes only about 20 genera and 100 species, but in past times the case was very different. Brachiopods appear first in the lower Cambrian rocks, where the existing genera

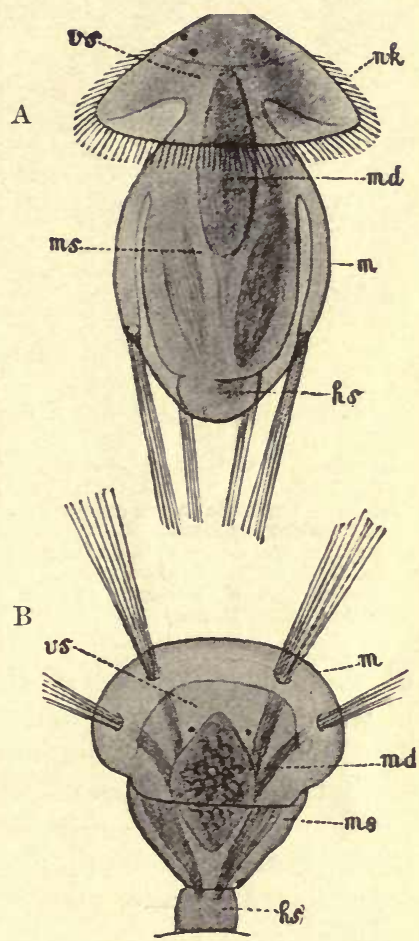

FIG. 298.-Two later stages in the developmient of Cistella. A, free-swimming ; B, after fixation. hs. peduncular region; $m$. mantle ; ms. body-region; $m d$. mesenteron ; $v c k$. ciliated ring; $v s$. head-region. (From Lang's Comparative Anatomy, after Kowalevsky.) Lingula and Discina are found. No more striking examples can be adduced of persistent types-organisms which have existed almost unchanged for the vast period during which the whole of the fossiliferous rocks have been in process of formation. Altogether 106 genera are known from the Palæozoic rocks, 34 from the Mesozoic, and 21 in the Cainozoic and Recent periods. Obviously the group is tending, though slowly, towards extinction.

Researches on fossil and recent forms have shown the 
Brachiopoda to illustrate, in a remarkable manner, the recapitulation theory already referred to: the theory, that is, that ontogeny or individual development is a more or less moditied

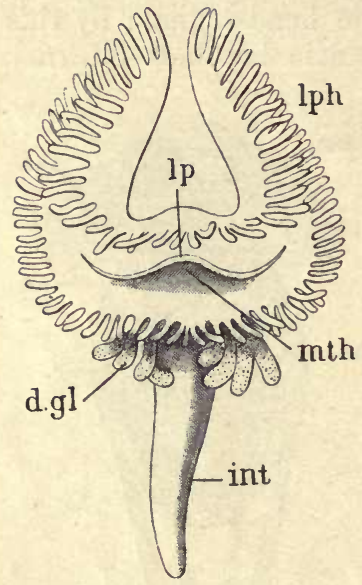

FiG. 299.--Lophophore of embryo of Terebratulina. $d . g l$. digestive gland; int. intestine; lp. lip ; lph. lophophore; mth. mouth (From Korschelt and Heider, after Morse.) recapitulation of phylogeny or ancestral development. It has been shown that there is a striking and almost complete parallelism between the stages in the development of the shelly loop in such highly organised forms as Magellania, and the entire series of articulated Brachiopods from those with the simplest to those with the most complex loop.

\section{Mutual Relationships of the Classes of the Molluscoida.}

In adult structure Phoronis exhibits marked resemblances to the Ectoprocta, more especially to the Phylactolæmata-resemblances which will be rendered clear by a comparison of the diagrams A and B in Fig. 300. In both, the ventral side of the body is greatly produced and elongated, and, by the approximation of the mouth and anus, the dorsal surface is reduced to a very short space between those two apertures. The form of the lophophore, the presence of an epistome having similar relationships in the two groups, and the fact that the coelome is similarly developed in both, point in the same direction. Some points which are supposed to indicate relationships with the Annulata and with the Chordata are referred to at a later stage.

The resemblances between the Brachiopoda and the other two classes of the phylum are somewhat disguised by the development of the shell, but are very obvious-more particularly when we take into account certain features of the development. One of the most striking points of resemblance between the three classes is the presence of the lophophore with its tentacles; in the earlier stages of its development in the Brachiopod, as we have seen, this structure (Fig. 299) has the horse-shoe shape which it retains in the adult Phoronida and Phylactolæmata, and a lobe-the armfold or lip $(l p)$ _comparable to the epistome, is present overhanging the mouth. The end of the body of the Brachiopod with which the peduncle is connected must correspond to the aboral extremity in the Polyzoa, since this represents the part by which the larval Polyzoan becomes fixed, the everted "sucker" of the latter being 
evidently homologous with the foot-segment of the larval Brachiopod. The end of the body of the Brachiopod from which the peduncle proceeds is thus the ventral portion. From the position of the epistome and lophophore, it follows that the dorsal valve of the Brachiopod, being on the same side of the mouth as the epistome, lies on the side of the body corresponding with the anal side of the Polyzoan, though the intestine is bent round in the

B
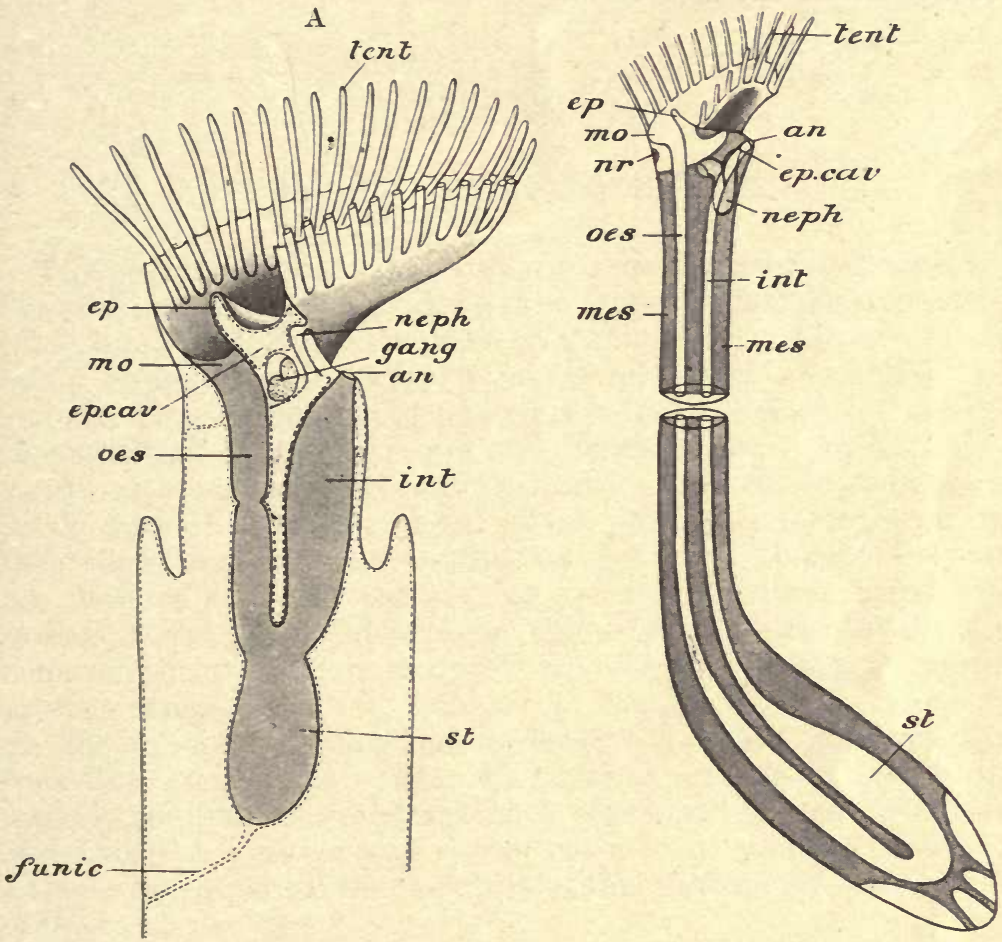

FIG. 300.-A, Diagrammatic median section of a phylactolrmatous Polyzoan. an. anus; ep. epistome ; ep. cav. epistome-cavity; funic. funiculus ; gang. ganglion ; int. intestine : mo. mouth ; neph. nephridium ; ars. cesophagus ; st. stomach ; tent. tentrcles. B, diagrammatic median section of Phoronis. mes. mesentery; $n r$. nerve-ring. Other letters as in A. (From Korschelt and Heider, after Cori.)

opposite direction and directed towards the ventral valve. The supra-œsophageal ganglion of the Brachiopod represents the single ganglion of the Polyzoa, though it is subordinate in importance to the infra-œsophageal ganglion - not represented in the latter group. Other important points of resemblance between the Brachiopoda and the Phoronida are seen in the character of the nephridia and the presence in both of larval forms which may very well be looked upon as modified trochophores. 
The setæ of Brachiopods, sunk in muscular sacs, are marks of annulate affinities, since such organs are found elsewhere only among Chrtopoda and Gephyrea (Sect. X.). The form of the larva tells in the same direction, the eye-bearing head region or prostomium and the provisional setæ being very striking characters. But the segmentation of the Brachiopod is quite different from that of the annulate larva, in which new segments are always added behind those previously formed, and in which metamerism always affects the mesoderm. 


\section{SECTION IX}

\section{PHYLUM ECHINODERMATA}

THE phylum Echinodermata comprises the Starfishes (Asieroidea), Sea-urchins (Echinoidea), Brittle-stars (Ophiuroidea), Feather-stars (Crinoidea), and Sea-cucumbers (Holothuroidea). All exhibit a radial arrangement of parts, which is recognisable as well in the globular Sea-urchins and elongated Sea-cucumbers as in the starshaped Starfishes, Brittle-stars and Feather-stars. Another universal feature is the presence of a calcareous exoskeleton, sometimes in the form of definitely shaped plates, which may fit together by their edges so as to form a continuous shell; sometimes merely in the form of scattered particles or spicules. In very many the surface is beset with tubercles or spines, from which feature the name of the phylum is derived. The various systems of organs attain a comparatively high degree of complexity. An extensive celome is present, developed in the embryo from hollow outgrowths from the archenteron. The Echinoderms are rarely capable of rapid locomotion, and are sometimes permanently fixed by means of a stalk; they never give rise to colonies by budding. Without a single exception, all the members of this phylum are inhabitants of the sea.

\section{Example of the Asteroidea.}

A Starfish (Asterias rubens or Anthenea flavescens).

General External Features of Asterias rubens.-The body of the Starfish is enclosed in a tough, hard integument, containing numerous plates, or ossicles as they are termed, of calcareous material. This exoskeleton is not completely rigid in the fresh condition, but presents a certain limited degree of flexibility. The body (Fig. 301) is star-shaped, consisting of a central part, the central disc, and five symmetrically arranged processes, the arms or rays, which, broad at the base, taper slightly towards 
their outer extremities. There are two surfaces-one, the aboral or abactinal, directed upwards in the natural position of the living animal ; the other, the oral or actinal, directed downwards. The aboral surface is convex, the oral flat; the colour of the former is much darker than that of the latter.

In the centre of the oral surface (Fig. 301) is a five-rayed aperture, the actinostome, and running out from this in a radiating manner are five narrow grooves, the ambulacral grooves, each extending along the middle of the oral surface of one of the arms to its extremity. Bordering each of the ambulacral grooves there are either two or three rows of movable calcareous spines, the ambulacral spines. At

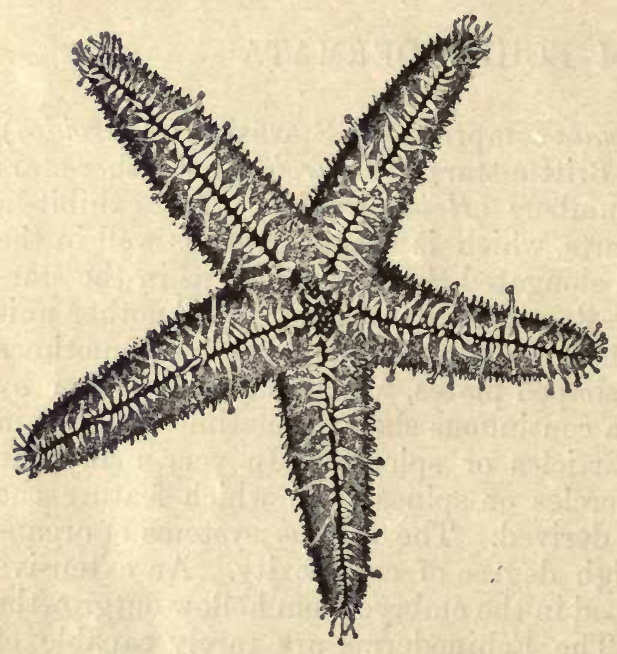

FrG:-301.-Starfish (Asterias vubens). General view of the oral or actinal surface, showing the tube-feet. (From Leuckart and Nitsche's Diagrams.) the central ends of the grooves the ambulacral spines of contiguous sides of adjacent grooves form five groups, the mouth papilloe, one at each angle of the mouth. External to the am. bulacral spines are three rows of stout spines which are not movable; and a third series runs along the border separating the oral from the aboral surface.

On the convex aboral surface there are a number of short stout spines arranged in irregular rows parallel with the long axes of the rays. These are supported on irregularly-shaped ossicles buried in the integument. In the soft interspaces between the ossicles are a number of minute pores, the dermal pores, scarcely visible without the aid of a lens. Through each of these pores projects a very small, soft, filiform process, one of the dermal branchice or papuloe (Fig. 305, Resp. cœ), which is capable of being entirely retracted.

Very nearly, though not quite, in the centre of the aboral surface is an aperture, the anus (Fig 310), wide enough to admit of the passage of a moderately stout pin. On the same surface, midway between the bases of two of the rays, is a flat, nearly circular plate, the surface of which is marked by a number of radiating, narrow, straight, or slightly wavy grooves; this is the madreporite (mad.). The presence of this structure interferes to some 
extent with the radial symmetry of the Starfish, two of the antimeres (p. 42), viz. those between which the madreporite is placed, being different from the rest. There thus arises a bilateral symmetry, there being one vertical plane, and only one-that passing through the middle of the madreporite and through the middle of the opposite arm-along which it is possible to divide the Starfish into two equal-right and left-portions. ${ }^{1}$ The two rays between which the madreporite lies are termed the bivium, the three remaining the trivium.

Attached to the spines of the oral surface, in the intervals between them, and in the intervals between the spines of the dorsal surface, are a number of very small, almost microscopic bodies, which are termed the pedicellarice (Fig. 305, Ped). Each of these is supported on a longer or shorter flexible stalk, and consists of three calcareous pieces-a basilar piece at the extremity of the stalk, and two jaws, which are movably articulated with the basilar piece, and are capable of being moved by certain sets of muscular fibres so as to open and close on one another like the jaws of a bird. In some of the pedicellariæ the jaws, when closed, meet throughout their entire length, while in the case of others, mostly arranged in circles round the spines on the aboral surface, one jaw crosses the other at the end like the mandibles of a Crossbill.

In a well-preserved specimen there will be seen in each of the ambulacral grooves two double rows of soft tubular bodies ending in sucker-like extremities; these are the tube-feet (Fig. 301). In a living specimen they are found to act as the locomotive organs of the animal. They are capable of being greatly extended, and when the Starfish is moving along, it will be observed to do so by the tube-feet being extended outwards and forwards (i.e. in the direction in which the animal is moving), their extremities becoming fixed by the suckers, and then the whole tube-foot contracting so as to draw the body forwards; the hold of the sucker then becomes relaxed, the tube-foot is stretched forwards again, and so on. The action of all the tube-feet, extending and contracting in this way, results in the steady progress of the Starfish over the surface. With the aid of the tube-feet the Starfish is also able to right itself if it is turned over on its back.

At the extremity of each of the ambulacral grooves is to be distinguished a small bright red speck, the eye (Fig. 305, A, oc), over which is a median process, the tentacle $(t)$, similar to the tubefeet, but smaller and without the terminal sucker. The tentacles have been ascertained by experiment to be olfactory organs, the Starfish being guided to its food much more by this means than by the sense of sight.

1 The slightly eccentric position of the anal aperture introduces a correspondingly slight inequality between the right and left portions. 
Transverse Section of an Arm.-If one of the arms be cut across transversely (Fig. 302 and Fig. 305, B) and the cut surface examined, the aboral part of the thick, hard wall of the arm will present the appearance of an arch (with its convexity upwards), and the oral part the form of an inverted $\mathrm{V}$, the ends of the limbs of which are connected with the oral ends of the aboral arch by a very short, flat, horizontal portion. Enclosed by these parts is a space, a part of the calome or body-cavity, and below, between the two limbs of the $\mathrm{V}$, is the ambulacral groove. The aboral arch is supported by a number of irregular ossicles and is perforated by the numerous small dermal pores, through which the

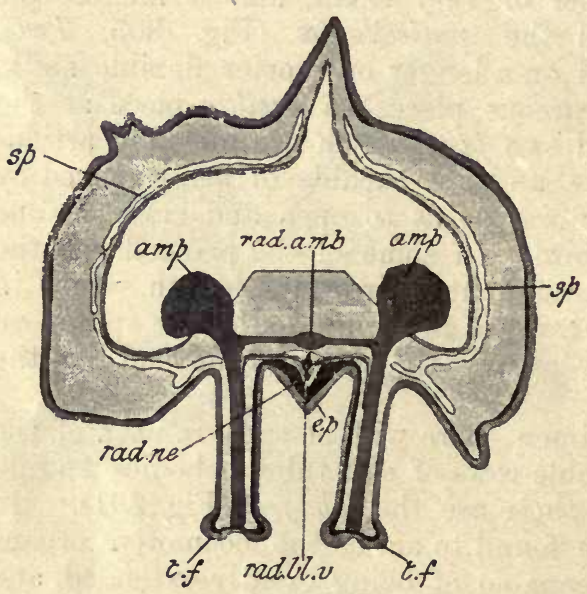

Fig. 302.-Starfish. Vertical section through an arm. $a m p$, ampullæ; $e p$. epidermis; rad. amb. radial vessel of the ambulacral system; rad.bl.v. points to the septum dividing the perihremal vessel into two parts; rad. ne. radial nerve of the epidermal system; $8 p$. spaces in mesoderm of body-wall ; $t$. $f$. tube-feet. (From Leuckart, after Hamann.) dermal branchiæ project. The V-shaped oral part of the body-wall-i.e the walls of the ambulacral groove-is supported by two rows of elongated ossicles, the ambulacral ossicles (Fig. 305, Amb.os), which meet together at the apex or summit of the groove like the rafters supporting the roof of a house, but with a movable articulation allowing of separation or approximation of the two rows so as to open or close the groove. At the end of the ray the ambulacral ossicles end in a median terminal ossicle. At the edges of the groove a

row of ossicles support the ambulacral spines and prominent tubercles. Between the ambulacral ossicles of each row are a series of oval openings, the ambulacral pures, one between each contiguous pair of ossicles, and so arranged that they form two rows on each side, one row higher than the other, the pores of the higher row alternating with those of the lower. In the ventral groove lie the contracted tube-feet $\left(T . H^{\prime}\right.$.): each tubefoot is found to correspond to one of the ambulacral pores, so that the former, like the latter, are arranged in a double alternating row on each side of the groove. When the tube-foot is drawn upon, it is seen to be continuous with one of a series of little bladder-like bodies, which lie on the other side of the ambulacral ossicles, i.e. in the cavity of the arm. These-the ampulloc 
(Figs. 302 and 305, amp.; Fig. 303, ap) -are arranged like the tubefeet, in a double row on each side, a higher row and a lower, there being one opposite each ambulacral pore. When one of them is squeezed, the corresponding tube-foot is distended and protruded, the cavities of the tube-foot and ampulla being in communication by means of a narrow canal running through the ambulacral pore and provided with a valve. It is in this way that the foot is protruded in the living animal : the corresponding ampulla being contracted by the contraction of the muscular fibres in its walls, the contained fluid is injected into the tubefoot and causes its protrusion, the return of the water backwards through the canal being prevented by the closing of the valve.

\section{Vascular and Nervous} system.-Running along the ambulacral groove, immediately below where the ambulacral ossicles of opposite sides articulate, is a fine tube, the radial ambulacral vessel (Fig. 302, rad. amb, Fig. 303, r), which appears in the transverse section as a small rounded aperture. From this short side-? branches (Fig. 303, $r^{\prime}$ ) pass out on either side to open into the bases of the tube-feet. Below the radial ambulacral vessel is a median thickening of the integument covering the ambulacral groove: this marks the position of the radial nerve

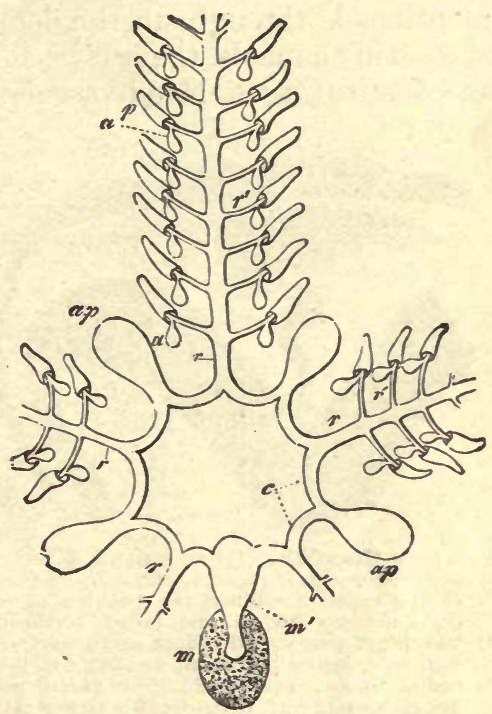

Fig. 303.-Ambulacral system of a Starfish. a. ampullæ; ap. Polian vesicles ; $c$. circular canal ; $m$. madreporite ; $m$ '. madreporic canal; $t$. tube-feet; $p$. radial vessels; $r^{\prime}$. branches to ampullæ. (After Gegenbaur.) (Fig. 302, rad. ne) of the cpidermal nervous system, and is traceable as a narrow thickened band running throughout the length of the groove, and terminating in the eye at its extremity, while internally it becomes continuous with one of the angles of a pentagonal thickening of a similar character, the nerve-pentagon, which surrounds the mouth. In thin sections (Fig. 304) the ventral median thickening, or radial nerve (rad. nerv.), as well as the nervepentagon, are seen to be thickenings of the epidermis, consisting of numerous vertically-placed, fibre-like cells, with their nuclei at their outer (lower) ends, intermixed with longitudinal nerve-fibres and with nerve-cells. Above this, on each side of the epidermal nerve-thickening constituting the radial nerve, is a band of cells 
(d. nerv.) also of a nervous character. These more deeply placed nerve-bands are the radial parts of the deep nervous system: like the epidermal, the deep nervous system has a central part in the form of a pentagon, which in this case is double, surrounding the mouth. A third set of nerve elements (the colomic nervous system) extends along the roof of the arm superficial to the muscles.

The two radial nerve-bands of the deep nervous system are thickenings of the lining membrane of a space overlying the radial nerve and underlying the radial ambulacral system. This space $(r a d . b l . v)$, extending, like the other parts that have been mentioned, throughout the length of the arm, forms part of a system of channels, the perihcemal system, which have been regarded as constituting a blood-vascular system. This radial perihoemal vessel or sinus, as it is termed,

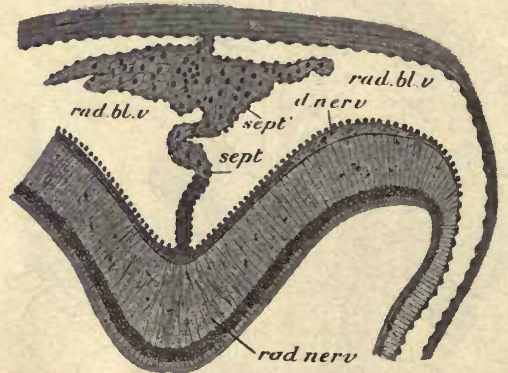

FiG. 304.-Starfish. Iower part of a vertical section through the arm, to show the structure of the radial nerve and the position of the deep nervous system and radial perihæmal vessels. $d$. nerv. strand of deep nervous system; rad. $b l . v$. radial perihæmal vessel; rad. nerv. radial nerve; sept. septum of radial perihæmal vessel; sept'. radial lacunar strand of the hæmal system (here represented as solid). (After Cuénot.) is divided longitudinally by a vertical septum (sept.) into two lateral halves. Internally it communicates with an orcul ring-vessel surrounding the mouth and likewise divided into two by a septum. The inner division of the ringvessel is connected with the axial sinus referred to on p. 384 .

In the septum dividing the radial perihremal sinus into two runs a strand of a kind of gelatinous connective tissue containing many leucocytes and perforated by irregular channels or lacunce: this is the radial strand of the lacunar or hoemal system. Like the radial vessels of the perihæmal system, the radial strands of the lacunar system are connected internally with an oral ring.

Structure of the Disc.-When the aboral wall of the central disc is dissected away, the remainder of the organs come into view (see Fig. 308). The rows of ambulacral ossicles appear in this view as ridges, the ambulacral ridges, one running along the middle of the oral surface of each arm to its extremity, and extending inwards to the corresponding angle of the mouth. At the sides of each of these ridges appear the rows of ampullæ. Within the pentagonal actinostome is a space, the peristome, covered with a soft integument, and in the centre of this is a circular opening, the true month, the size of which is capable of being greatly increased or diminished. 
Body-wall and Cœlome.-The entire outer surface is covered with a layer of ciliated epithelium, the epidermis or deric epithelium (Fig. 305, Der. Epithm), which is continued over the various appendages and processes - the tubercles aud spines, the pedicellariæ, the dermal branchiæ, and the tube-feet. Beneath it is a network of nerve-fibrils with occasional nerve-cells. The mesoderm (Derm) of the wall of the body beneath this consists of two layers, between which are a number of spaces: the ossicles (os)

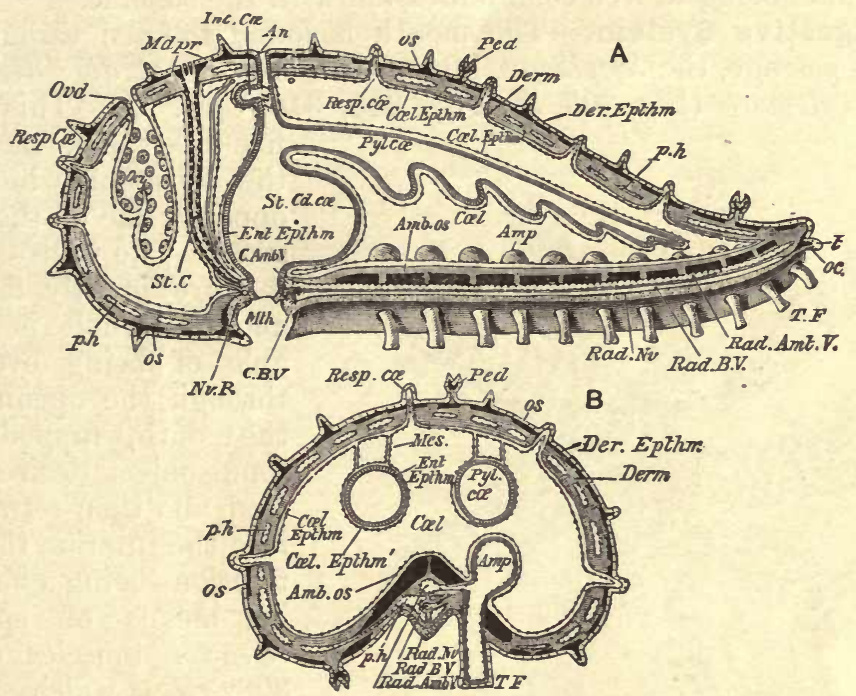

Fig. 305.-Diagrammatic sections of a Starfish. A, vertical section passing on the right through a radius, on the left through an inter-radius. The off-side of the ambulacral groove with the tube-feet (T. F.) and ampullæ (Amp.) are shown in perspective. B, transverse section through an arm. The ectoderm is coarsely dotted, the nervous system finely dotted, the ectoderm radially striated, the mesoderm evenly shaded, the ossicles of the skeleton black, and the coelomic epithelium represented by a beaded line. Amb. os. ambulacral ossicles; $A m p$. ampullæ; $A n$. anus; $C$. $A m b . V$. circular ambulacral vessel : $C . B$. V. septum of ring perihæmal; vessel; Cl. ca. cardiac caca; Cel. colome; Cal. Epithm. coelomic epithelium; Der. Epithm. deric epithelium ; Derm. mesoderm ; Ent. Epthm. enteric epithelium : Int. ca. intestinal cæca. $M d p r$. madreporite ; Mes. mesentery ; Mth. mouth; $N v$. R. nerve-ring; oc. eye; os. ossicles of body-wall; Ovd. oviduct; Ped. pedicellaria ; $p h$. perihwmal spaces; Pyl. caec. pyloric cæca; $R a d$. $a m b . v$ radial ambulacral vessel; $R a d . B$. $V$. points to septum in the radial perihæmal vessel; Rail. Nv. radial nerve; Resp. coe. dermal branchire; St. stomach; St. c. stone-canal; t. tentacle; T. F. tube-feet. (From Parker's Biology.)

are all, except the ambulacral ossicles and the inter-radial partitions, developed in the outer of these two layers. Each ossicle consists of a close network of calcareous rods. Between contiguous ossicles extend bands of muscular fibres.

The interior of the coelome (Col.) or body-cavity is lined by a ciliated epithelium, the colomic epithelium (Col. Epithm.), which not only covers the inner surface of the body-wall as the parietal layer, but also forms an investment for the contained organsthe various parts of the alimentary canal and its appendages, the gonads, the madreporic canal, ampullæ, etc. In addition 
to this visceral layer of the peritoneum, the wall of the alimentary canal and its cæca consists of a muscular layer and an internal lining, the enteric epithelium or endoderm (Ent. Epthm). The colome is filled with a fluid, the colomic fluid, consisting mainly of sea-water, but containing a number of amœboid corpuscles (amobocytes) containing a brown pigment. The dermal branchiæ consist of a muscular layer, an external epidermal layer, and an internal peritoneal layer, the internal cavities of the hollow branchiæ being in free communication with the colome.

Digestive System.-The mouth is found to open through a short passage, the oesophagus, into a wide sac, the cardiac division of the stomach (Fig. 305, St, Figs. 308, 310, card. st). This is a

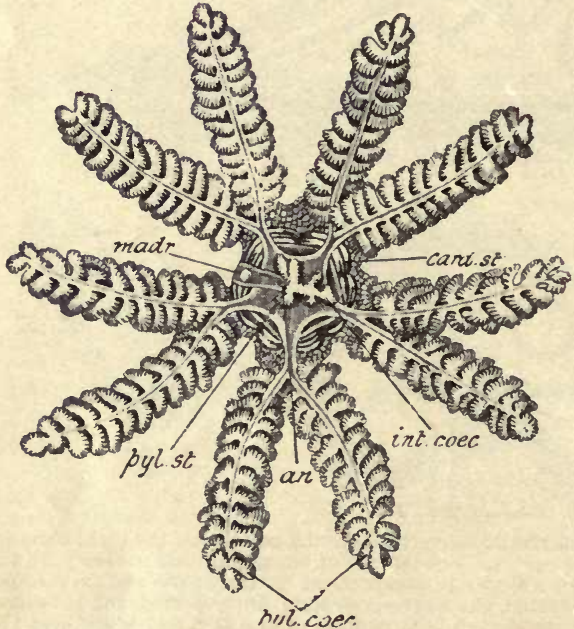

Fia. 306.-Asterias rubens, Digestive system. an. anus ; card. st. cardiac division of the stomach; int. coc. intestinal crea; madr. madreporite; pyl. coce. pyluric crea ; pyl. st. pyloric division of the stomach. (From Leuckart.) five-lobed sac, each of the lobes of which is opposite one of the five arms. The walls of the sac are greatly folded, and the whole is capable of being everted through the opening of the mouth, wrapped over some object desired as food, and then retracted into the interior, the retraction being effected by means of special retractor muscles (Fig. 308 , retr) which arise from the sides of the ambulacral ridges. This cardiac division of the stomach communicates aborally with a much smaller chamber, the pyloric division of the stomach, and this in turn opens into a very short conical intestine, which leads directly upwards to open at the anal aperture. The pyloric division of the stomach is pentagonal, each angle being drawn out to form a pair of large appendages, the pyloric coeca (Figs. 305, 306, 308, 310, pyl. coec). Each pair of pyloric cæca commences as a cylindrical canal or duct, the lumen of which is continuous with the cavity of the pyloric chamber. This soon bifurcates to form two hollow stems, extending to near the extremity of the cavity of the arm, and giving off laterally two series of short branches, each having connected with it a number of small bladder-like pouches. The walls of the pyloric cæca are glandular: they secrete a digestive fluid, and are therefore to be 
looked upon as digestive glands. It is found by experimenting with this digestive fluid that it has an action on food-matters similar to that exerted by the secretion of the pancreas in the Vertebrata, converting starch into sugar, proteids into peptones, and bringing about the emulsification of fats. While the pouches of the cardiac division of the stomach are attached to the oral wall of the body, the pyloric cæca are connected with the aboral wall. From the short intestine are given off inter-radially two hollow appendages, the intestinal coeca (Figs. 306 and 308, int. cæc), each with several short branches of irregular shape.

Ambulacral System.-Running downwards from the madreporite to near the border of the mouth is an S-shaped cylinder, the madreporic or stone-canal (Figs. 303, $m^{\prime} .310$, mad. can). The walls of this canal are supported by a series of calcareous rings, and projecting into it is a ridge which bifurcates to form two spirally rolled lamellæ occupying a considerable part of the lumen of the canal. In some Starfishes, such as Astropecten (Fig. 307), the internal structure is more complicated owing to the branching of the lamellæ. The interior of the madreporic canal communicates above with the exterior through the grooves of the madreporite. At the bottom of each of the grooves is a row of pores leading into a sac, the ampulla, which in turn leads into the madreporic canal. Below, the latter opens into a wide, fivesided, ring-like canal, the ring-

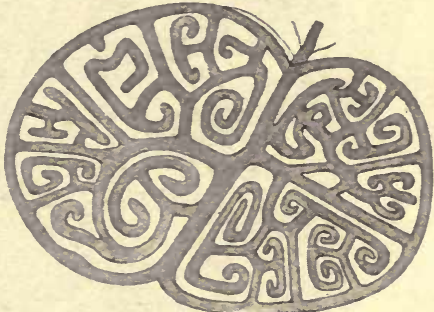

FIG. 307.-Transverse section through the madreporic canal of a Starfish (Astropecten). (From Gegenbaur, after Teuscher.) vessel of the ambulacral system. From this are given off the five radial ambulacral vessels, passing to the extremities of the arms. From the pentagonal canal are given off also in most Starfishes, but not in Asterias, a series of five pairs of appendages, the Polian vesicles (Fig. 303, ap ; Fig. 308, pol. ves)-pear-shaped, thin-walled bladders with long narrow necks - which are placed inter-radially. At the sides of the neck of each Polian vesicle (except in the inter-radius containing the madreporic canal, where there is one on one side only) project inwards a pair of little rounded glandular bodies, the racemose or Tiedemann's vesicles (Fig. 309, T), the cavity in the interior of each of which, opening into the ring-vessel, is divided into a number of chambers.

The various parts of the ambulacral system of vessels have a museular wall and an internal lining epithelium in addition to the coverings which they may derive, according to their situation, either from the external epidermis or the internal colomic epithelium. The muscular layer is most strongly developed on the 
tube-feet, where it consists of two strata, and is also well developed on the ampullæ and Polian vesicles.

The stone-canal is enfolded in the wall of a wider canal, the axial sinus (Fig. 309, ax. s), which forms a part of the perihæmal system already referred to. The axial sinus runs nearly vertically. At its oral end it opens into the internal division of the oral ring

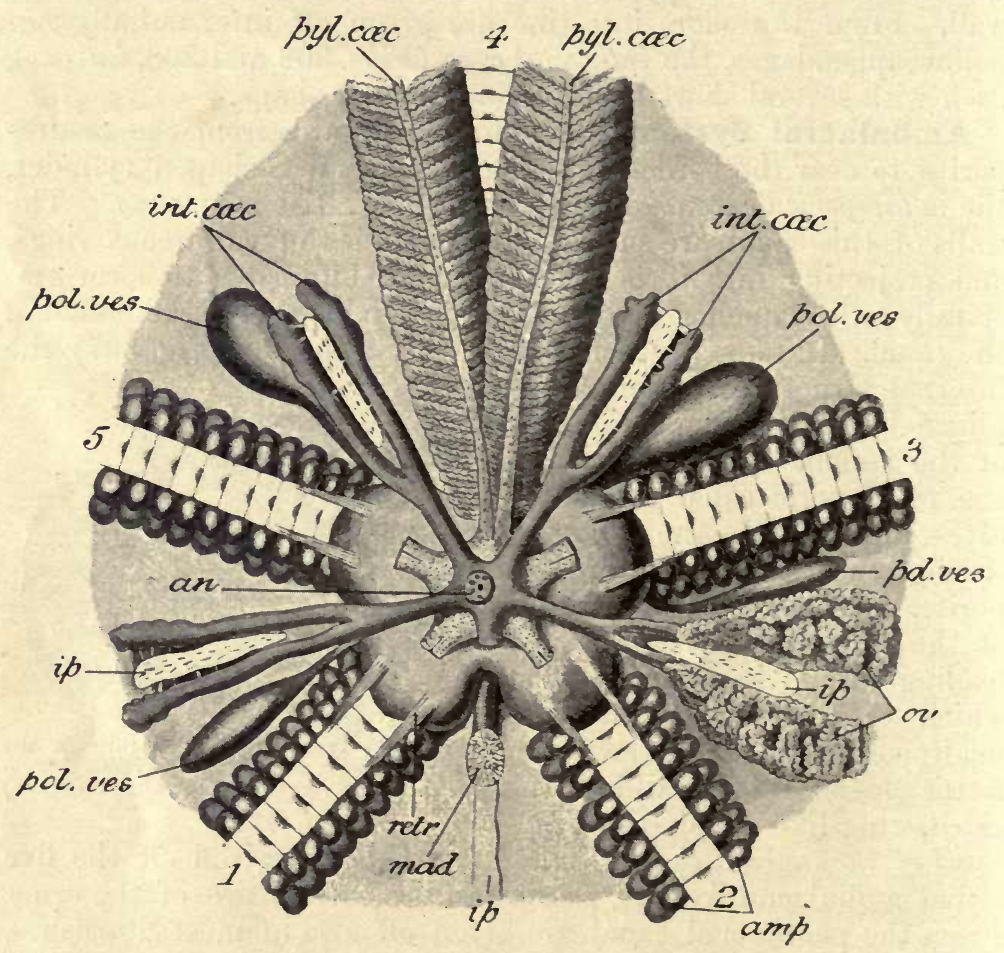

FIG. 308.-Anthenea flavescens. Upper view of a dissection of the internal organs. The aboral wall of the body, with the exception of a small portion round the anus and the madrepurite, has been completely removed. One of the five intcstinal crea has been removed with the exception of its proximal part. All the ovaries have been removed except one pair, and four of the pairs of pyloric creca have been cut away close to their bases. $1-5$, the five rays with their ambulacral ridges; amp. ampullæ; an. anus ; int. cœe. intestinal creca ; $i . p$. cut ends of the inter-radial partitions; mad.madreporite with the madreporic canal ; ov. ovaries; pol. ves. Polian vesicles; pyl. cac. pyloric creca; retr. retractor muscles inserted into the cardiac division of the stomach.

sinus; aborally it approaches close to, if it does not actually open into, an aboral ring sinus: it also communicates aborally with the stone-canal, and perhaps opens on the exterior through certain of the pores in the madreporite.

Accompanying the madreporic canal and also enfolded in the wall of the axial sinus there is an organ-the axial organ (Fig. 309, g. stol) - the relationships and function of which have given rise 
to a considerable amount of difference of opinion. It is a fusiform body, the interior of which assumes an appearance of complexity largely due to both its inner surface (i.e., that turned towards the axial sinus) and its outer (that facing the colome) being folded in a complicated manner. The axial organ contains strands of lacunar tissue, i.e. of the same tissue that composes the

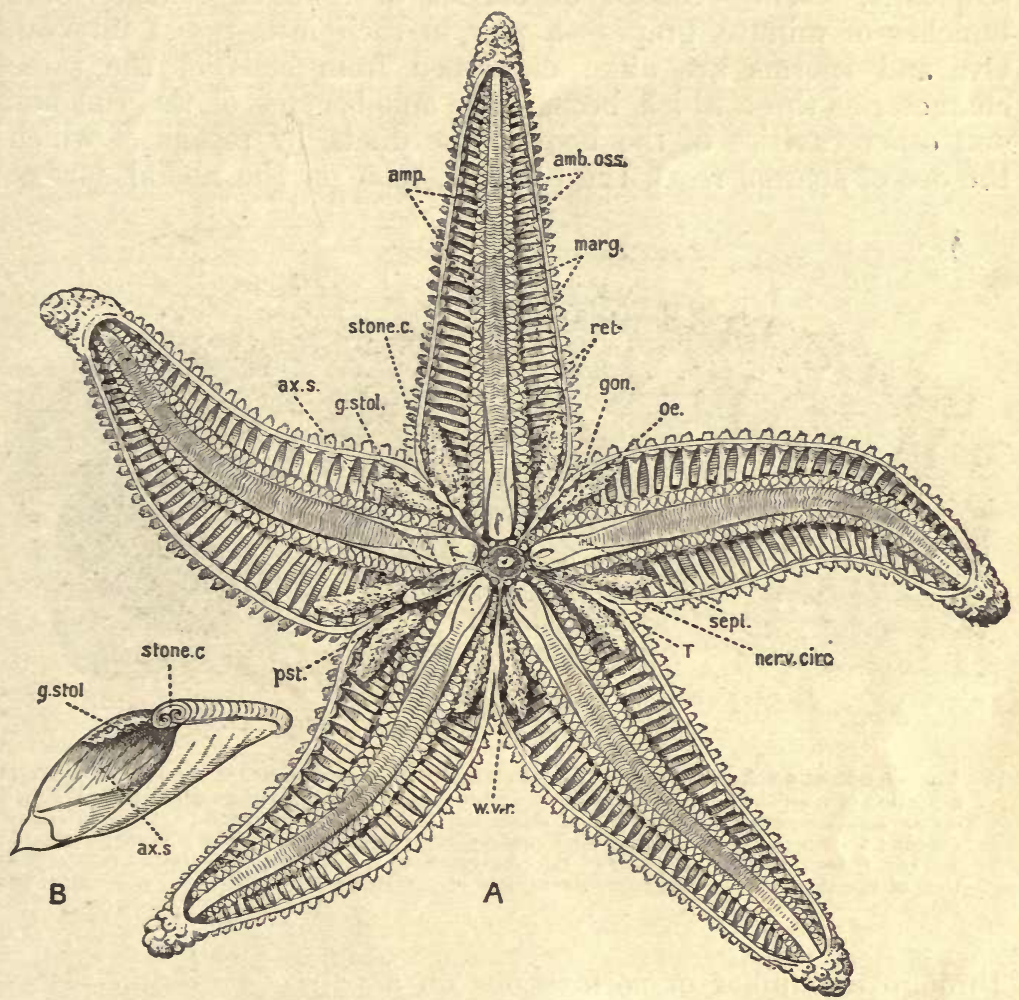

FIG. 309.-A, view of the under part of a specimen of Asterias rubens, which has been horizontally divided into two nearly equal portions. $B$, enlarged view of the axial sinus, stone-canal and genital stolon cut across. arib. 08s. ambulacral ossicle; $a$ mp. ampulla of the tube-feet; $a x .8$. axial sinus; gon. gonad; g. stol. genital stolon or axial organ ; marg. marginal ossicle ; nerv. circ. nerve-ring ; oe. cut end of cesophagus ; pst. peristome ; ret. retractor muscle of the stomach; sept. inter-radial septum; stone. $c$. stone-canal; $T$. Tiedemann's vesicle; vo. $v$. $r$. water-vascular ring-canal. (After MacBride.)

so-called hæmal system, and is intimately related with the latter. Its essential morphological character, however, appears to be that of a genital slolon. At its aboral end it is continuous with a genital rachis, which, in the form of a ring, runs in the aboral perihæmal sinus, and gives off branches to the gonads. There is evidence that the sexual cells originate in the aboral end of the axial organ, and travel through the genital rachis and its branches

VOL. I 
to the gonads, which are to be looked upon as the greatly expanded extremities of the latter. Strands of the lacunar tissue accompany the genital rachis and its branches to the gonads.

Reproductive System.-The Starfish is unisexual, each individual possessing either ovaries (Figs. 308, 309, and 310, ov) or testes, which appear very similar until they are examined microscopically. They consist of masses of rounded follicles, like bunches of minute grapes-a pair in each inter-radial interval. Ova and sperms are alike developed from cells of the same character as those which become the amobocytes of the colomic and other cavities of the body. The ducts, by means of which the ova or sperms reach the exterior, open on the aboral surface

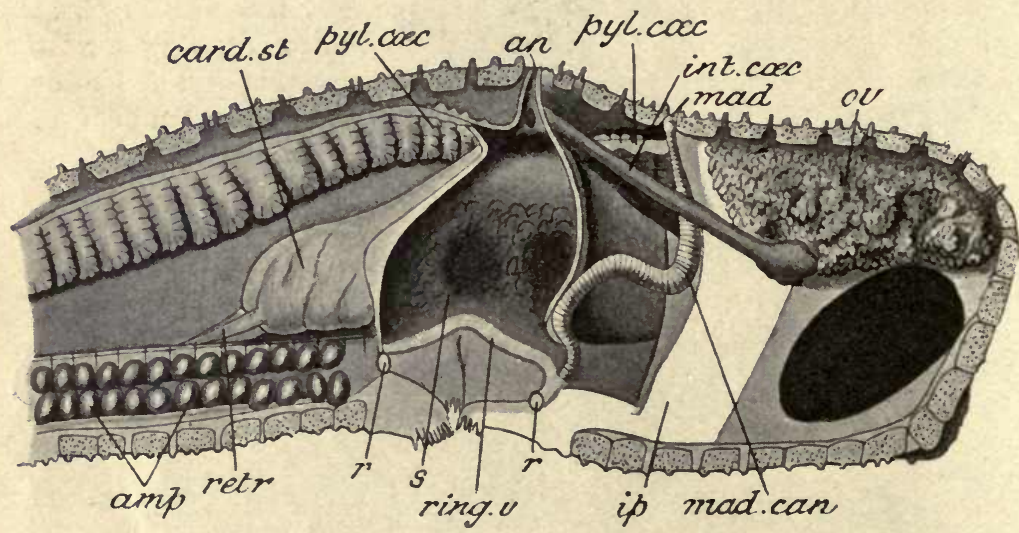

FrG. 310.-Anthenea flavescens. Lateral view of a dissection in which one of the rays and a portion of a second have been removed, and in which the alimentary canal has been laid open. amp. ampulle; an. anus ; card, st. cardiac pouch of the stomach; int. coec. intestinal cæcum ; ip. inter-radial partition ; mad. madreporite; mad. can. madreporic canal ; ov. ovary : pyl. coec. pyloric cæca; $r$. cut ends of the ring-vessel of the ambulacral system; ring $v$. position of the ring-vessel; retr. retractor muscle of cardiac pouch of stomach; 8 . cavity of the stomach.

through a number of perforations on a pair of sieve-like plates, situated inter-radially close to the bases of the arms.

Anthenea flavescens (Figs. 308, 310, 311, 312), a common Australian Starfish, which may be taken as an example instead of Asterias rubens, differs from the latter in the following main points.

The animal consists of a relatively large central disc and five relatively short arms, which taper rapidly towards their extremities. On the oral surface the comparatively broad, flat surfaces between the ambulacral grooves are roughish, owing to the plate-like ossicles being beset with a number of minute rounded tubercles, which, in the immediate neighbourhood of the ambulacral grooves, assume the character of short, blunt spines. Here and 
there among the tubercles, usually one in the middle of each ossicle, are pedicellarice, which differ widely from those of Asterias. Each pedicellaria in Anthenea is a small, narrow, oblong, calcareous body, consisting of two parallel narrow valves or jaws: these, instead of being supported on a flexible stalk, are articulated with the edges of a slit-like depression on the surface of the flat ossicle, and are thus on a level with the general surface. The term valvulate is applied to pedicellariæ of this description. In a living Anthenea many of the pedicellariæ will be found to have their valves widely open; when they are touched the valves close together, gradually open-

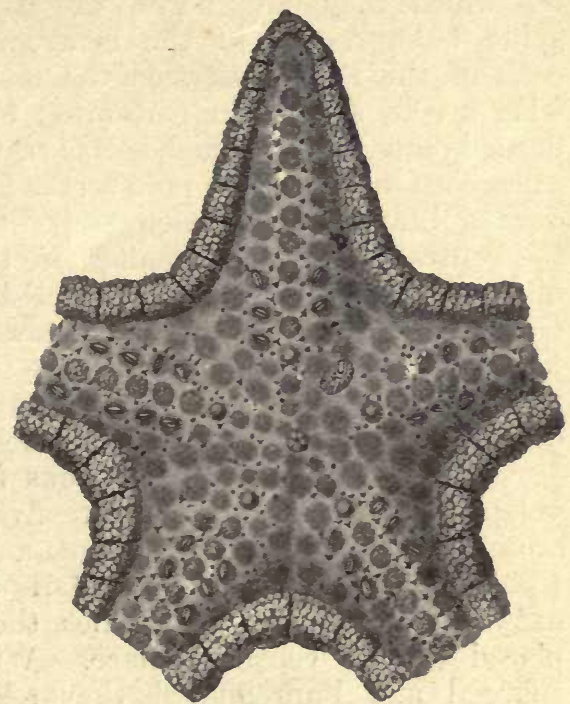

Fig. 311.-Anthenea, view of aboral surface. (After Sladen.)

ing again after a little time. The ambulacral spines bounding the ambulacral grooves are flattened and blunt, and arranged

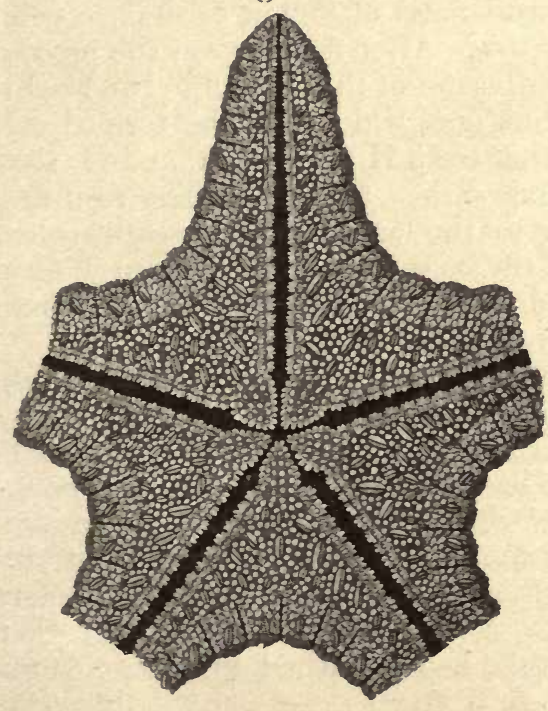

FIG. 312.-Anthenea, view of oral surface. (After Sladen.) in fan-like fasciculi. Round the border separating the aboral and oral surfaces the plates are arranged in two somewhat irregular rows.

The aboral surface is strongly convex, but not uniformly so, there being a more or less distinct depression in the form of a shallow open groove, the inter-radial depression, opposite each of the intervals between the arms. The surface is dotted over with numerous small rounded tubercles, arranged in somewhat irregular radiating lines. These aboral tubercles, though fewer than 
those on the oral surface, are for the most part more prominent, so that they assume the character of short spines. The ossicles on which they are borne are star-shaped with six rays, a spine being borne in the centre of each ossicle, and one on each of the rays. Between the ossicles the surface is covered with a soft, slimy skin, perforated by a large number of minute dermal pores, each of which is enclosed by a minute irregular ring of calcareous matter; each pore serves for the lodgment of one of the dermal branchiæ. Numerous pedicellariæ, similar to those on the ventral surface, but smaller, are borne on the ossicles, usually taking the place normally occupied by the central spine. The tube-fcet are arranged in a single row on each side of each ambulacral groove; but the ampulloe are in two rows, an upper and a lower, and each tube-foot has two ampullæ connected with it, one of the upper row and one of the lower row.

Anthenea has vertical calcareous inter-radial partitions not developed in Asterias. There are five bifid intestinal coca, which are narrow tubes slightly enlarged and lobed at the extremities.

\section{Development of a Starfish (Asterina gibbosa or A.} exigua ${ }^{1}$ ). - In these Starfishes the reproductive apertures are placed on the ventral surface. When the ova have been discharged and impregnated, they adhere by means of a viscid investment to the surface (rock or stone) on which they are laid, and gu through all the stages of their development in this position, never passing through a free pelagic stage. The eggs are about half a millimetre in diameter, and of a spherical shape. Each consists of a perfectly opaque central mass of yellow or orange yolk, and of a glassy layer enclosing this. After fertilisation the process of segmentation begins by the division of the ovum into two blastomeres alinost equal in size, but one, which may be termed cell I., slightly smaller than the other (cell II.). Both I. and II. soon afterwards divide, I. somewhat earlier than II. The resulting four cells again divide, leading to the formation of an eight-celled stage (Fig. 313, A), in which the four cells derived from I. form an incomplete ring not closed below, and the four derived from II. form an incomplete ring open above.

The eight cells then divide by meridional fissures into sixteen, and a further division results in the formation of thirty. two. The thirty-two cells become arranged in such a way as to enclose a central cavity which had been present in the four-celled stage: this stage $(B)$ is the blastula; the cavity is the segmentation-cavity or blastocole. The number of cells in the wall of this cavity increases by further divisions, and the whole surface becomes covered with vibratile cilia. A process of invagination then follows, one side of the blastula being pushed inwards to form

1 The development of these has been described in preference to that of the examples, as it is more completely known. 
a double-walled cup or gastrula $(C)$ opening on the exterior by an opening, the blastopore, which, at first very wide, gradually becomes narrowed. At the same time the shape of the larva alters, so as to be somewhat elongated, the blastopore, lying at first midway between the two poles, afterwards gradually drawing nearer to what becomes the posterior end.

Of the two layers of the gastrula ( $D$ and $E$ ), the outer is the cetoderm, the inner the endoderm; between them is a space, at first filled with gelatinous matter, in which cells soon appear, giving

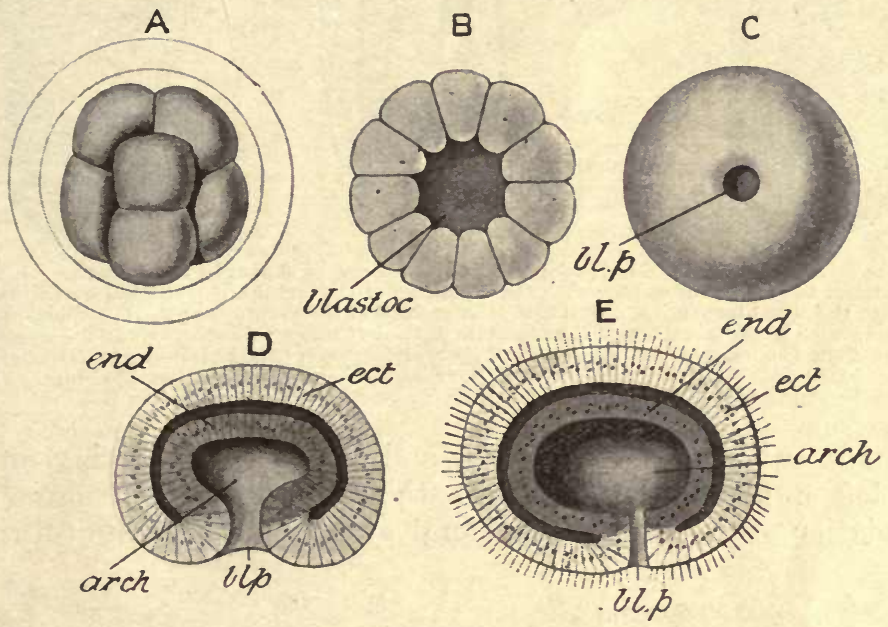

FiG. 313.-Early stages in the development of a Starfish (Asterina gibbosa). A, eight-celled stage ; $B$, stage of about thirty-two cells seen in section; $C$, gastrula stage $; D$, section of early gastrula; $E$, section of later gastrula. arch. archenteron ; blastoc. blastoccele; blp. blastopore; ect. ectoderm ; end. endodern. (Modified after Ludwig.)

rise subsequently to an intermediate mass of tissue, the mesenchyme.

The cavity in the gastrula is early distinguishable into two parts (Fig. 314, B) - that part into which the blastopore leads (arch), and a wider terminal part (ent); the former becomes the stomach and intestine of the larva, the blastopore giving rise to the larval anus; the latter is termed the enterocole (colome). The wall of the enterocœle becomes thinner, and it gives off two lateral swellings, the right and left enterocclic pouches (C, ent), which are closely applied to the sides of the larval alimentary canal: the left pouch is soon seen to be larger than the right. The enterocoele is subsequently completely closed off from the enteric canal. It now consists of three parts, an anterior undivided part, and the two pouches, right and left. Of the latter the left grows more rapidly than the right: both extend posteriorly in the space between the enteric canal and the body-wall to coalesce posteriorly 
in such a way as to give rise to the coelome of the adult. The anterior undivided part (anterior colome) forms the coelome of a conspicuous larval structure, the pre-oral lobe, and it eventually
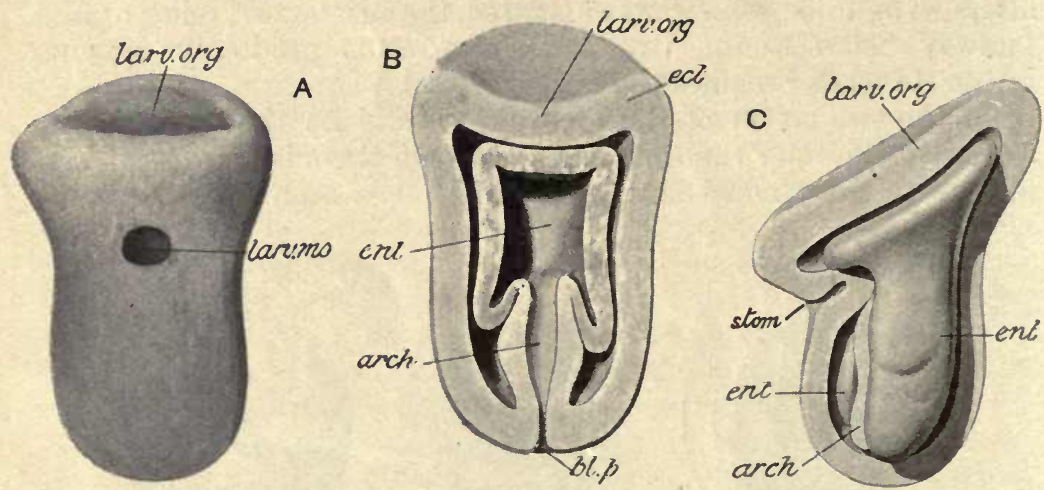

F1G. 314.-Iater stages in the development of the larva of Asterina gibbosa. A, newly hatched larva, ventral surface with the beginning of the larval organ at the anterior end and with the larval mouth. $B$, dorsal half of an embryo of the same age as $A$. $C$, somewhat older larva with larger larval organ, the ectoderm of the left side removed to expose the alimentary canal.and the walls of the body-cavity. arch. archenteron; $b l . p$. blastopore ; ect. cctoderm ; ent. enterocole; larv. mo. larval mouth; larv. org. pre-oral lobe ; stom. stomodæum. (From Ziegler's models.)

becomes cut off from the right and left pouches, giving off on the left a five-lobed outgrowth, the hydroccele, which forms the foundation of the entire ambulacral system of the adult: a right
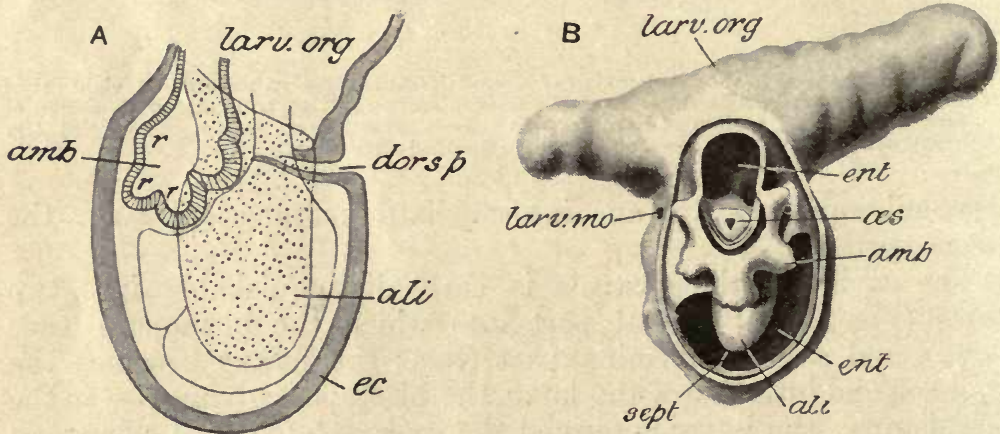

Fig. 315.-Larva of Asterina gibbosa. A, diagrammatic lateral view; the alimentary canal dotted, the ambulacral system striated, the ectoderm shaded. $B$, Larva seen from the left as an opaque object, the body-wall of the left side removed; hydrocoele separated off from left enteric sac and partly surrounding osophagus. ali. alimentary canal ; amb. ambulacral system or hydrocoele; dors. $p$. dorsal pore ; ent. enteric sacs and colome; lavv. mo. larral mouth; larv. org. pre-oral lobe; aes. œesophagus of adult; $r, r$. lobes of hydroccele ; sept. septum between the enterocolic sacs. ( $A$, after Ludwig; $B$, from Ziegler's models.)

hydrocœle is only represented by a small vesicle which in normal embryos undergoes no further development. Before the hydrocole is developed and before the right and left colomic pouches have become cut off, two apertures make their appearance 
on the surface of the larva: one, on the ventral side, is the opening of the stomodceum or larval mouth; the other, on the dorsal side, is the dorsal pore. The mouth subsequently opens into the larval stomach, and for a time the enteric canal of the larva opens on the exterior both by mouth and anus: soon, however, the larval anus becomes closed up. The dorsal pore is developed as an outgrowth of the anterior part of the enterocœle, a little to the left of the middle line, meeting a thickening of the ectoderm about the middle of the dorsal surface, where an aperture is formed.

The pre-oral lobe appears at an early stage as a dilatation at the anterior end of the larva. This

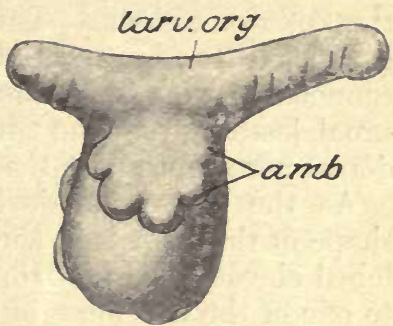

Fig. 316.-Larva of Asterina, view of the left side, showing the fivelobed prominence ( $a m b$.) formed by the developing ambulacral system on what is destined to become the ventral surface of the body of the Starfish; lare. org. larval organ. takes an antero-posterior direction, and assumes the character of an elongated, almost cylindrical, hollow appendage at the anterior end of the larva, consisting of a shorter, anterior, and a

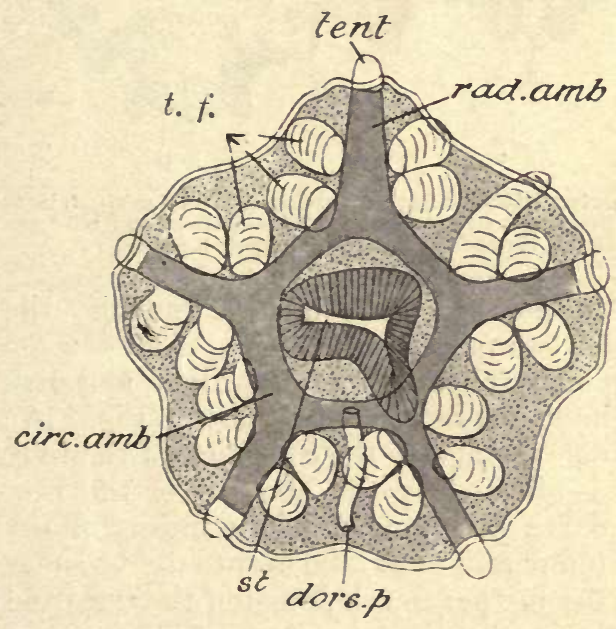

F1G. 317.-Asterina exigua. Young Starfish shortly after the metamorphosis has b ven completed, viewed from the oral side. circ. amb. circular ambulacral vessel ; dors. p. dorsal pore and madrcporic canal; rad. $a m b$, radial ambulacral vessel ; st. stomach ; tent. tentacle ; t. f. tube-feet.

more especially the cilia of the larval organ. longer, posterior, part. On the anterior surface of the pre-oral lobe a flattened area appears surrounded by a raised rim, which is beset with specially large cilia: this is the larval organ. In the middle of the larval organ appears an elevation, the rudiment of a sucker by means of which the larva becomes attached when the metamorphosis is about to begin. At this stage the larva (Fig. 316) is able to creep by contractions of the pre-oral lobe, and also to swim by the action of the cilia,

The hydrocoele, at first a five-lobed outgrowth of the enterocœle, grows into the form of a horse-shoe with five lobes, each of 
which represents one of the radial parts of the ambulacral system, the horse-shoe itself representing the ring-vessel. The rudiment of the madreporic canal arises as a groove on the posterior wall of the anterior cœlome. This develops into a canal leading from the hydrocœle to the anterior cœlome, and eventually entering into connection with the dorsal pore, forms a tube, the madreporic canal, leading from the ring canal to the madreporite, of which the dorsal pore represents the first-formed aperture.

As the hydrocœle develops, its form influences the external shape of the larva; on the left-hand side there grows out a fivelobed elevation (Fig. 316, $a \mathrm{mb}$ ), each of the lobes corresponding to one of the five lobes of the hydrocœle. Each of the latter then becomes divided, first into three rounded processes (Fig. 315, B, $a m b)$, and then into five, and these project freely on the
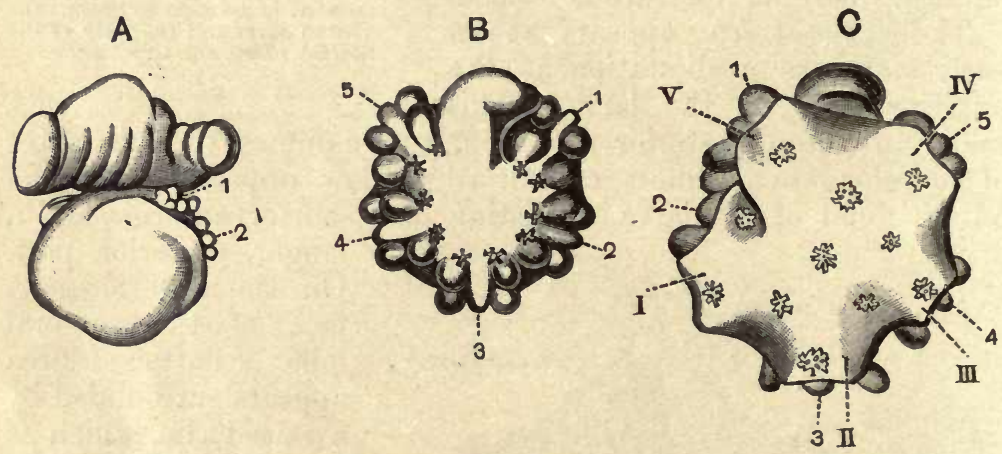

Fic. 318.-Views of the larva of Asterina gibbosa in the course of metamorphosis. $A$, larva of eight days, from the right; $B$, left, and $C$, right view of the larva of nine days; $1-5$, lobes of hydrocole; I-V, rudiments of arms. (From MacBride, after Ludwig.)

surface; the middle one is the rudiment of the tentacle, the lateral processes are the first two pairs of tube-feet. At the same time five elevations of the opposite wall become evident, and give rise to the beginnings of the dorsal regions of the arms (Fig. 318).

The transition from the larval stage to the condition of the fiverayed Starfish (Fig. 317) is effected by the abortion of the preoral lobe-(which, on the larva becoming fixed by means of the sucker, degenerates into a temporary stalk and eventually becomes completely absorbed) - by the further development of the arms and tube-feet, and by certain changes which take place in the internal organs. Of these, one of the most important is the formation of a new mouth and œesophagus (Fig. $315, B$, os), the larval mouth and cesophagus becoming abolished during the metamorphosis. Round this new mouth grows the ring-vessel of the ambulacral system. From the stomach, diverticula grow out radially into the developing arms to give rise to the cæca; and later the permanent anal opening is formed on the dorsal surface. 
When the first ossicles are definitely formed they present the following arrangement (Fig. 319). In the middle of the abactinal surface is a single central plate (dors). Around this are five basals (bas) one of which becomes merged into the madreporite. External to these, five radials ( $\mathrm{rad}$ ) appear somewhat later. At the end of each developing arm is a single terminal or ocular plate (term), which is carried outwards as the ambulacral and adambulacral ossicles of the arm are developed, supporting the corresponding eye and tentacle. A ring of secondary radials or infra-basals (sec. $\mathrm{rad}$ ) is developed between the radials and the central. In the

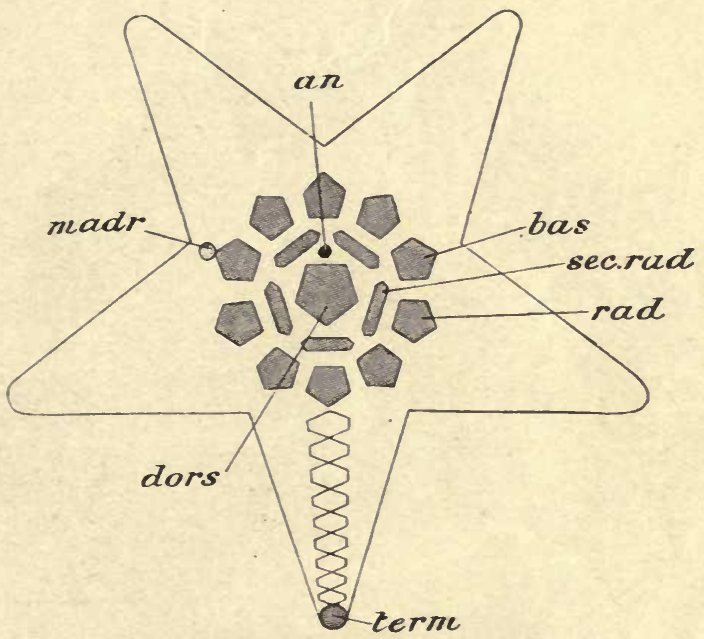

FIG. 319.-Diagram showing the relations of the chief plates of the apical system in the young starfish. an. anus; bas. basals; dors. central; madr. madreporite ; rad. radials ; sec. rad. secondary radials (infra-basals).

adult, by the intercalary development of numerous additional ossicles, these primary plates of the apical system, as it is termed, lose their original arrangement, and become no longer recognisable.

\section{Example of the Echinoidea.}

\section{A Sea-Urchin.-(Strongylocentrotus or Echinus.)}

General External Features.-The Sea-Urchin (Figs. 321 and 322 ) is globular in shape, but somewhat compressed in one direction, so that two poles are distinctly recognisable. At one of these the degree of flattening is greater than at the other; this is the oral pole, the opposite pole being termed the anal or aboral. At the oral pole is a rounded aperture, the mouth, through which may be seen projecting five hard white points, the extremities of the 
teeth, surrounding the mouth is a thin, soft membrane known as the peristome or peristomial membrane (Fig. 320). At the anal pole is a much smaller aperture, the anus, the space immediately surrounding which is termed the periproct (Fig. 322).

The entire surface, with the exception of the peristome and periproct, is bristling with spines-cylindrical, pointed, solid appendages, the surface of which is longitudinally fluted. These are movably articulated with the body so that they may be turned about in all directions. When one of them is removed (see Fig. 338, p. 422), it is found that the joint is of the character of a ball

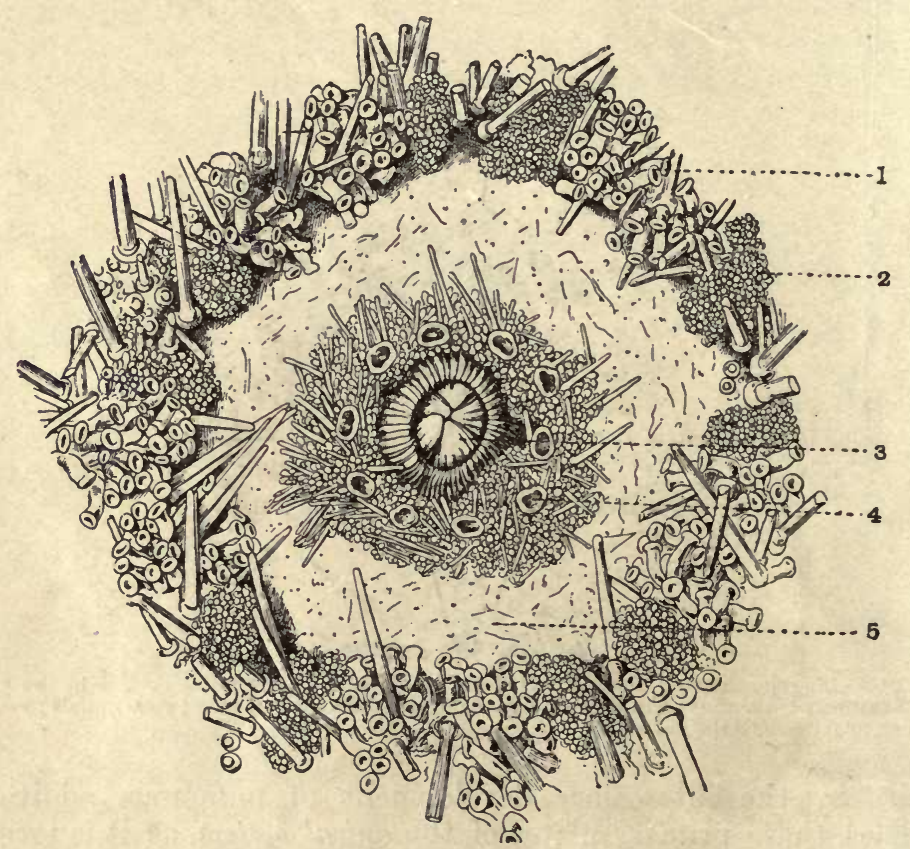

Fig. 320.-Fchinus esculentus; peristome. 1, tube-feet of the lower ends of the radii ; 2 , branchia; 3 , teeth ; 4 , buccal tube-foot; 5 , peristomial membrane. (From MacBride, after Kukenthal.)

and socket, a concavity on the base of the spine fitting over a hemispherical elevation on the surface of the Sea-urchin, and the spine being retained in place and caused to move by means of a capsule of muscular fibres enclosing the joint. Around the bases of the large spines are a number of very small spinules. Here and there among the spines are to be observed minute pedicellarice (see Fig. 340, p. 423), which are comparable to the stalked pedicellariæ of Asterias; but each has three jaws instead of two, and a relatively long stalk, which is supported by a slender calcareous rod. Here and there are to be found also small 
rounded bodies termed the sphceridia, which are perhaps, like the pedicellariæ, to be looked upon as modified spines: they contain ganglion-cells and are apparently organs of special sense, having possibly the function of detecting changes in the composition of the water.

Projecting from the surface among the spines all the way from the peristome to the periproct will be observed five double rows of tube-feet (Fig. 321), which in a living specimen will be found to be capable of great extension. These are similar to the tube-

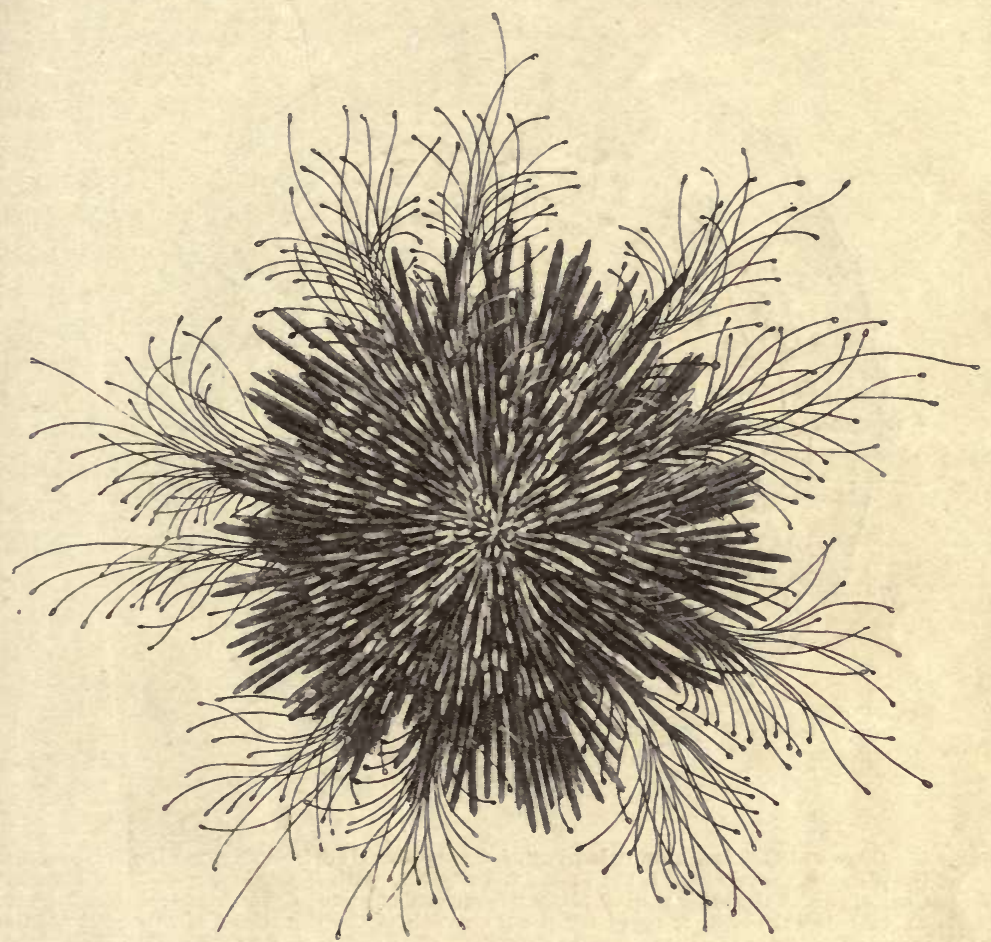

FIG. 321.-Strongylocentrotus, entire animal with the tube-feet extended. (From Brehm's Tierleben.)

feet of the Starfish, and have similar functions: the sucker-like extremity of each is supported by a perforated sieve-like plate of calcareous matter. Each double row of tube-feet occupies a meridional zone of the surface, termed the ambulacral area, corresponding to the ambulacral groove of the Starfish : the intermediate zones are termed the inter-ambulacral areas. At the oral end of each ambulacral area on the peristome (Fig. 320) is a pair of appendages similar to tube-feet, but shorter, and termed tentacles. Ten shrub-like appendages, the dermal branchice, are situated in 
the peripheral part of the peristome, a pair opposite each interambulacral area.

When the spines are removed, the body is found to be enclosed in a rigid globular shell, or corona (Fig. 322) as it is termed, formed of a system of plate-like ossicles, the edges of which fit accurately and firmly together, and the surfaces of which are ornamented with the rounded elevations or tubercles for the articulation of the spines. These plates are arranged in ten zones, each consisting of two rows, running in a meridional direction from the

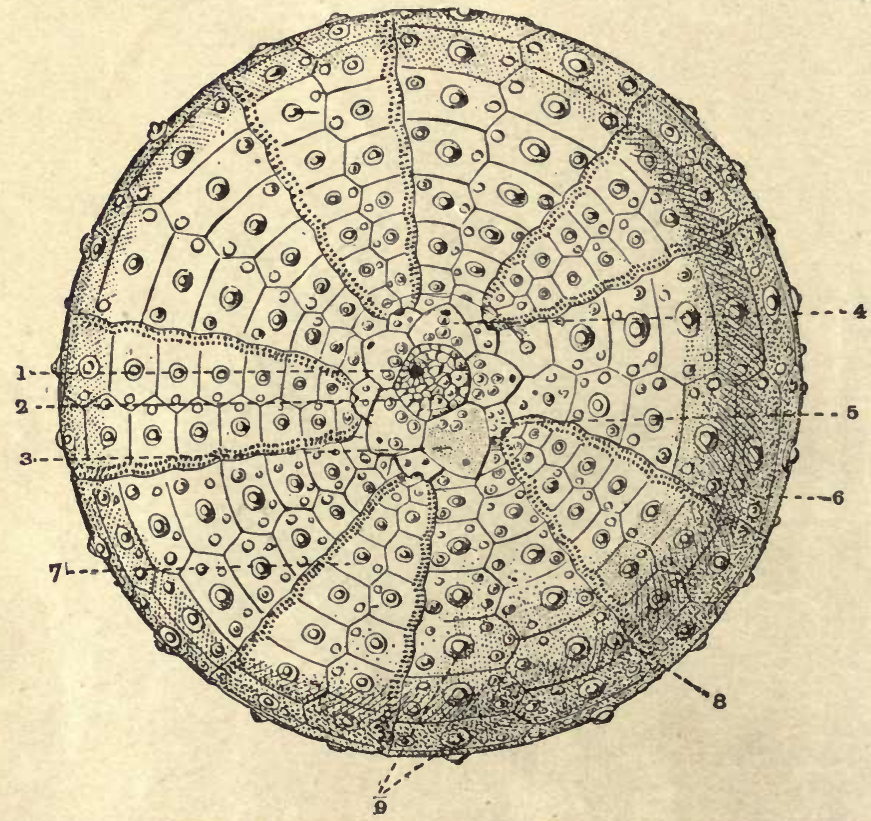

FiG. 322.-Corona of Ichinus esculentus, from the aboral surface, showing the arrangement of the plates of the corona. 1 , the anus ; 2 , periproct, with irregular plates; 3 , the madreporite; 4, one of the other genital plates; 5 , an ocular plate ; 6 , an inter-ambulacral plate; 7 , an ambulacral plate; 8 , pores for the protrusion of the tube-feet; 9 , tubercles. (After MacBride.)

edge of the peristome to the neighbourhood of the periproct. Of the zones of plates there are two sets, each consisting of five, the members of which alternate with one another. In the case of one of these sets of zones - the ambulacral zones or ambulacral areas already referred to-each of the plates is perforated towards its outer end by two minute pores, the ambulacral pores, for the protrusion of the tube feet. In the other five zones, the inter-ambulacral zones or areas, the plates are not perforated. At its anal end each area, ambulacral or inter-ambulacral, ends in a single apical plate, so that the periproct is surrounded by a ring of 
ten plates, the apical system of plates (Fig. 323). Of these, the five that are situated at the ends of the ambulacral areas are termed the ocular plates (oc), owing to the fact that each of them bears a structure once supposed to be a rudimentary eye, but now known to be a tentacle; while the five opposite the inter-ambulacral areas are termed the genitral plates (gen), each of them being perforated by an opening which is the aperture of one of the five genital ductsthe ducts of the ovaries or testes as the case may be. One of these genital plates (madr) has a swollen and spongy appearance, which distinguishes it from the others: this

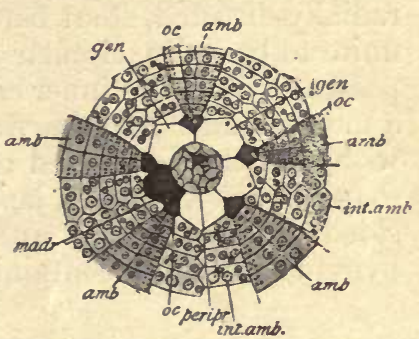

FiG. 323.-Apical system of plates and aboral extremities of zones of the shell of a Sea-urchin. amb. ambulacral zones; gen. genital plates; int. amb. inter-ambulacral zones ; mad $r$. madreporite ; oc. ocular plates; peripr. periproct. (Aftcr Leuckart.) is the madreporite, through which, as in the case of the structure of the same name in the Starfishes, the madreporic canal communicates with the exterior. The two ambulacral areas between which the madreporite lies constitute the bivium, the remaining three the trivium.

On the inner surface of the shell, close to the edge of the peristome, there project inwards five processes, the auricles (Fig. 325, aur), one opposite each ambulacral area. Within the ring of auricles lies a complex structure termed Aristotle's lantern (Fig. 324). This consists of the five teeth (e), the apices of which are to be
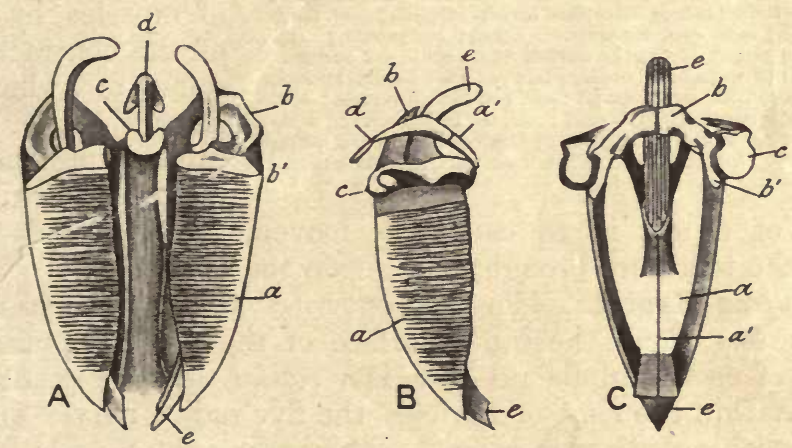

FIG. 324.-Lantern of Aristotle of Echinus. A, two of the five chief component parts apposed id viewed laterally. $B$, lateral, and $C$ internal view of a single part. $a$. alveolus; $a^{\prime}$. suture of rley's Invertebrates, after irilin-1 ted nrovimallv each 
Firmly united to the base of the alveolus is a stout bar, the epiphysis (b). Adjacent epiphyses are in close contact with one another, and running inwards from their points of union are five radially-directed, stout bars, the rotulce $(c)$, the inner ends of which unite to bound a circular aperture through which the oesophagus passes. With the inner end of each rotula is movably articulated a more slender bar, the radius $(d)$, which runs outwards, parallel with, and closely applied to, the rotula, to end in a free, bifurcated extremity. Aristotle's lantern as a whole is in the shape of a fivesided pyramid, at the apex of which project the five teeth; the pyramid is hollow, containing a passage which is the beginning of

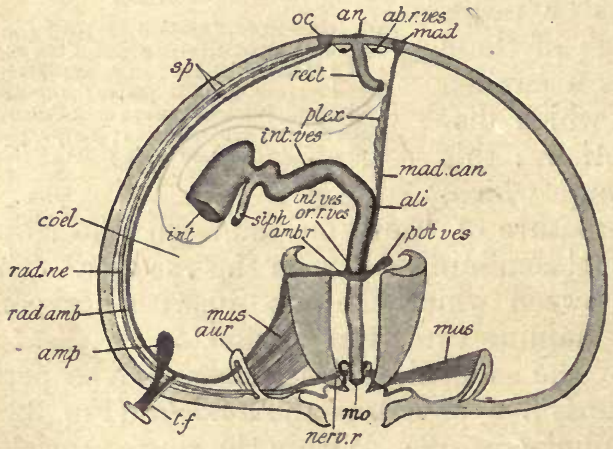

FIG. 325. - Lateral view of the internal organs of a seaurchin as seen or the removal of a half of the shell. $a b . r$. ves. hæmal strand, aboral ring; amb. $r$ ambulacral ring. canal ; amp.ampullæ; an. anus ; aur. auricle ; col. colorpe ; int. intestine ; int.ves.intestinal hæmal strands ; mad. madreporite; mad. can. madreporic canal; mo. mouth; mus. muscles passing from the auricles to Aristotle's lanitern nerv. $r$. nerve-ring; oc. ocular plate ; or.r.ves. hæmal strand, oral ring; ptex. ovoid gland; pol. ves. Polian vesicle; rud. $a m b$. radial ambulacral vessel; rad. ne. radial nerve; siph. siphon $; s p$. radial extension of the coelome surrounding the nerve ; $t$. $f$. tube-feet. (From Leuckart, after Hamann.) the œesophagus. The base has the appearance of a wheel, the tyre of which is represented by the five epiphyses, the spokes by the five rotula with the five radii in close contact with them, and the hub by the rounded central aperture. Passing between the various ossicles of the lantern, and from them to the auricles, : are systems of muscles by means of the contractions of some of which the lantern as a whole can be protruded or retracted, while the action of others is to cause the movements of the alveoli by which the teeth are brought to bear on the food.

Nervous System.-Passing outwards through each auricle, and running along the inner surface of the corona opposite the middle of each ambulacral area, is a rudial nerve (Fig. 32k, rad. ne). Within the ring of auricles the five radial nerves are connected with a nerve-ring (nerv. $r$ ) surrounding the mouth. $f_{\Delta}$ t its distal end each radial nerve is connected with the so-called eye (oc), borne by the corresponding ocular plate. These parts corres spond to the epidermal nervous systr ng to the ambulacral grooves hay 
Ambulacral System.--Internal to each radial nerve, and pursuing a corresponding course, runs a radial ambulacral vessel (Figs. 325 and $326^{\circ}$ ). From this are given off on each side a series of short branches to the tube-feet, with each of which is connected one of a series of compressed sacs, the ampulloe ( $a m p$ ), by two canals, one passing through each of the two pores. At their oral extremities the five radial ambulacral vessels unite with a ring-vessel surrounding the asophagus. Appended to the ring-vessel are five Polian vesicles (pol. ves.) in the form of small mammillated bodies. A madreporic canal ( $m a d$. can.), corresponding to that of the Starfish, but with soft membranous walls devoid of ossicles,

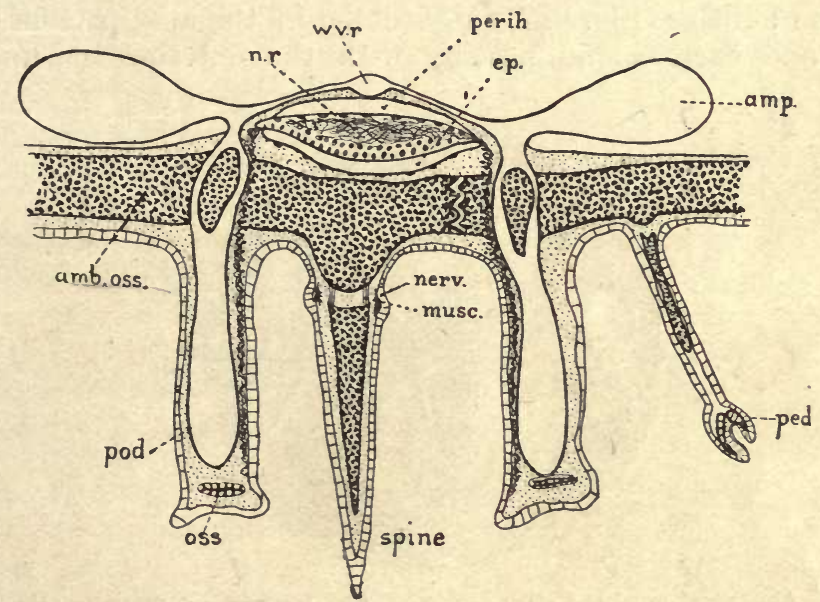

FIG. 326.-Diagramnatic transverse section of the ambulacral zone of an Fchinoid. $a \mathrm{mb}$. 08s. ambulacral ossicle; anı. ampulla of \& tube-foot; ep. epineural canal; musc. muscles attaching spine to its tubercle ; nerv. nervous ring in base of spinc; $n$. $r$. radial nerve-cord ; 088. ossicle in the sucker of the tube-foot; ped. pedicellaria; perih. radial perihæmal canal; pod. tube-foot; wv. r. radial ambulacral vessel. (After MacBride.)

runs from the madreporite at the side of the periproct to the ring-canal.

The enteric canal (Fig. 327, alt) is devoid of the radial cæea which it presents in the Starfish: it is a wide, soft-walled tube, which winds round the interior of the corona in its passage from the mouth to the anus, held in place by a band of threads, the mesentery, passing out from it to the inner surface of the shell. It gives off a short blind diverticulum, the siphon (siph); this, together with the intestine itself, probably acts as an organ for the respiration of the coelomic fluid.

The cœlome contains a fluid in which, as in the Starfish, there are numerous corpuscles. Of these there are two kinds - amcboid corpuscles (amcbocytes) with long pseudopodia, and vibratile corpusiles, which closely resemble sperms, having a rounded 
head and a slender vibratile tail : the latter aid in bringing about a constant circulation of the colomic fluid.

The part of the coelome containing Aristotle's lantern is completely cut off from the rest by the arrangement of the membrane enclosing the lantern, and the function of the branchiæ on the peristome is evidently the oxygenation of the colomic fluid enclosed in this compartment, which is known as the lanterncolome.

The perihæmal and hæmal or lacunar systems, as well as the axial organ, will be referred to in the account of the general structure of the phylum.

The reproductive organs consist of five masses of minute rounded follicles (Fig. 327, ov) situated in the anal portion of the shell, and each communicating with the exterior by its duct,

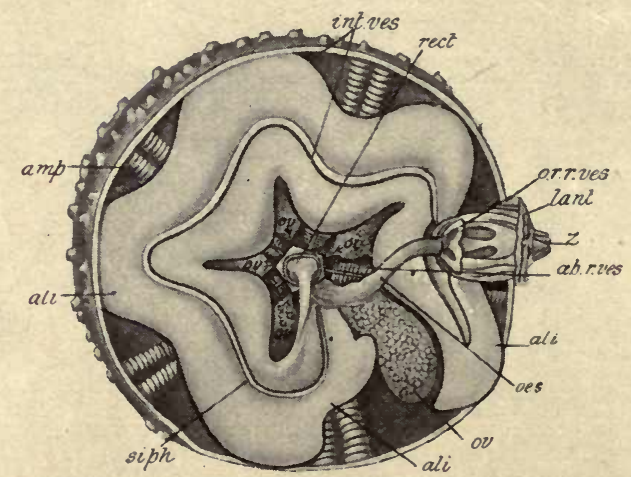

Fig. 327.-Alimentary canal and other organs of Sea-urchin as secn when the ora half of the corona has been removed. $a b . r$. ves, aboral ring of the hæmal system; ali. alimentary eanal; amp. ampullæ; int. ves. intestinal blood-vessels; lant. lantern of Aristotle ; os. cesophagus ; or. $r$. $v$. oral ring-vessel of the hæmal system; ov. ovary ; rect. rectum ; siph. siphon; z. teeth. (From Leuekart, partly after Cuvier.)

which perforates the corresponding genital plate. The sexes are distinct; as in the Starfish, there is little difference to be observed between the ovaries of the female and the testes of the male until we come to examine their microscopic structure. The genital rachides which in the Starfish connect the gonads with the genital stolon (p. 385) are aborted in the adult Seaurchin.

The early stages in the development of the Sea-urchin are very similar to the corresponding stages in the development of the Starfish described on page 388. The bilateral larva of the Sea-urchin, which is termed a pluteus, is provided with a number of elongated arms or processes supported by delicate calcareous rods. A metamorphosis, in which the bilateral larva becomes converted into the radial adult, takes place as in the Starfish. 


\section{Example of the Holothuroidea.}

\section{A Sea-cucumber.-Cucumaria or Colochirus.}

General External Features.-The body (Fig. 328) is elongated, in shape not unlike a miniature cucumber, somewhat irregulirrly five-sided, with an opening at each end. One end is somewhat thicker than the other, and the opening at this thicker (oral or anterior) end is the mouth, that at the opposite (aboral or posterior) end is the anus. The body is five-sided, and along each side there extends a double row of tube-feet. In Colochirus there is a very distinct ventral surface, into which three of the five sides enter, distinguished by the absence of the rows of tubercles that occur on the dorsal portion of the surface, and by the presence of three distinct bands of tube-feet. This ventral part of the body with its three ambulacral areas is the equivalent of the trivium of the Starfish, the rest representing the bivium. On the dorsal surface, instead of typical tube-feet, there are papilla devoid of sucking extremities, and similar appendages take the place of tubefeet at the ends of the three ventral bands. In Cucumaria the ventral surface is less distinctly defined, but its position is to be determined by reference to the

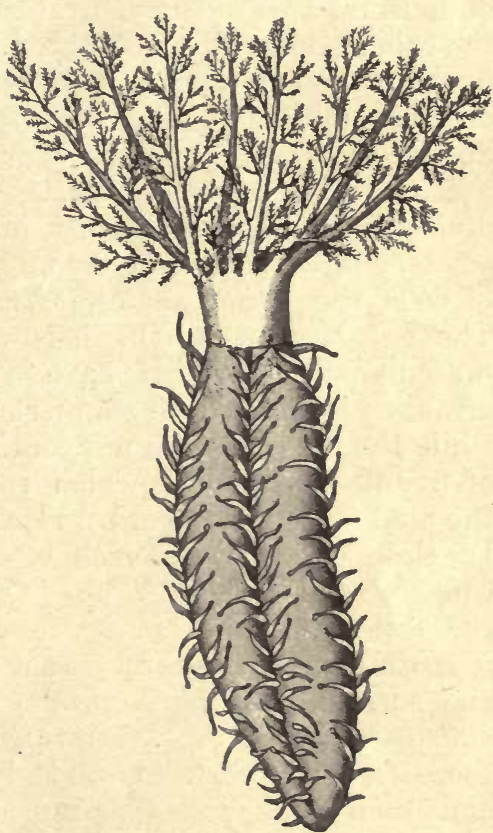

FIa. 328.-Cucumaria planci. Entire animal seen from the ventral surface. (From Hertwig's Lehrbuch, after Ludwig.) tentacles (vide p. 402); there are no papillæ. The ventral surface is, it is to be noticed, parallet with the axis joining mouth and anus, and the body, when compared with that of the Starfish or Sea-urchin, is greatly drawn out in the direction of the line joining mouth and anus.

There are no definite calcareous plates; but the integument is tolerably hard, owing to the presence in its substance of innumerable microscopic calcareous spicules, very variable in shape in different species of Cucumaria, and in Colochirus having the form of sieve-like or lattice-like plates, some of which are to be found 
even in the walls of the tube-feet. The tube-feet are, like those of the Starfish, used in locomotion, progression being effected by creeping with the ventral surface applied to the ground. In a Sea-cucumber living undisturbed under natural conditions there will be found protruding through the mouth a circlet of ten tentacles, which are to be looked upon as greatly developed and specially modified tube-feet. These are tree-like in shape-a central stem giving off a number of short branches, which may in turn be branched-and they are highly sensitive and contractile. Two of these tentacles will be seen to correspond to each of the ambulacral areas. The pair situated opposite the middle ambulacral area of the ventral surface are very much smaller than the others, and will be observed to perform the special function of pushing the food-particles into the mouth. All the tentacles are drawn completely back within the mouth when the animal is disturbed.

Structure of Body-wall.-When the wall of the body is divided, it is found to consist, in addition to the hardened integumentary layer, of two layers of muscle in addition to a thin layer of cells, the peritoneum or colomic epithelium, lining the colome. The outer layer of muscle is a complete, continuous layer of muscular fibres which have a circular arrangement, i.e. are arranged in a ring-like manner around the long axis of the body ; while the inner layer is not continuous, consisting, in fact, merely of five flattened bands which run longitudinally from the oral to the anal extremities, each underlying one of the ambulacral areas. In close contact with each of these bands, on its inner surface, runs a radial ambulacral vessel (Fig. 329, rad. amb) together with a radial nerve.

Ambulacral system.- Just behind the bases of the tentacles, and surrounding the beginning of the osophagus, is a circular ambulacral vesscl (ring. ves) which gives off the five radial vessels; these first run forwards and give off branches to the tentacles, and then backwards, passing along the ambulacral areas and giving off branches to the tube-feet, each of which is provided with its ampulla. From the ring-vessel also arises a large pearshaped Polian vesicle (pol.ves), and a short sinuous canal, the madreporic canal (mad.can), which ends in a perforated extremitynot situated, like the madreporite of the Starfish or Sea-urchin, on the outer surface of the body, but in the interior of the colome.

A nerve-ring surrounds the mouth and gives off the five radial nerves.

Both periphæmal and hæmal systems are well developed. The latter comprises a ring-like strand ( $r i . b l$. ves) situated close to the nerve-ring and sending off five radial strands, as well as dorsal and ventral strands (int. ves) accompanying the enteric canal, and a plexus surrounding the left respiratory tree (p. 404). 
The cœlome contains a fluid in which float numerous amœbocytes, similar to those of the Starfish, and also a number of

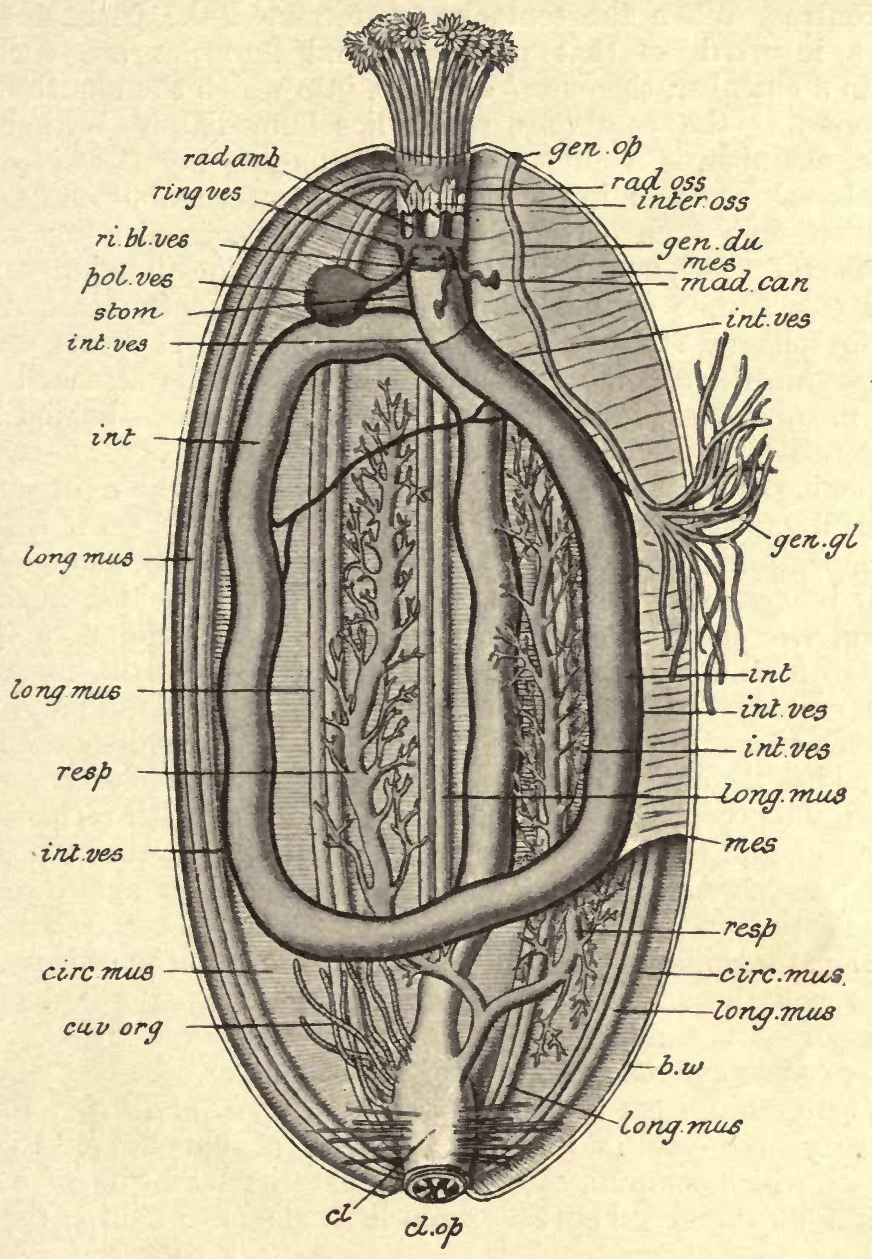

Fig. 329.-Internal organs of a Folothurian as seen when the body-wall is divided along the middle of the dorsal surface. $b . v$. body-wall; circ. mus. circular layer of muscle; cl. cloaca; $c l$. op. cloacal opening; Cuv. org. Cuvieran organs; gen. ap. genital aperture ; gen. du. genital duct; gen. gl. gonad; int. Intestine; inter. oss. inter-ambulacral ossicles; int. ves. intestinal hæmal strands; long. mus. longitudinal band of inuscle; mad. can. madreporic canals; mes. mesentery; pol. ves. Polian vesicles; rad. amb. radial ambulacral vessel ; rad. 0ss. ambulacral ossicles; $r i$ bl. ves. ring strand of hæmal system i resp. respiratory trees ; ring-ves. ring-vessel of the ambulacral system ; stom. stumach. (After Leuckart.)

flattened nucleated corpuscles containing a red colouring matter - hæmoglobin-almost identical with that which gives the red colour to the blood of the higher animals. 
The enteric canal is, as already mentioned, surrounded at its oral extremity by the circlet of tentacles, and within these, when they are fully exserted, is a narrow peristome with the mouth in the centre. When the tentacles are retracted the peristome becomes inverted, so that peristome and tentacles are enclosed within a chamber, the buccal chamber, into which the mouth leads. Surrounding the cesophagus, which lies immediately behind the buccal chamber, is a circlet of ten circum-cesophageal ossicles, five ambulacral (rad. oss) in position, and five inter-ambulacral (inter. oss). Through each of the former pass the corresponding radial ambulacral vessel, hæmal strand, and nerve. The alimentary canal itself is a simple cylindrical tube, only indistinctly marked out into œsophagus, stomach (stom), and intestine. It forms several coils within the cœlome, to the wall of which it is attached by a thin membranous dorsal mesentery, and terminates behind in a comparatively wide chamber, the cloaca $(c l)$.

Opening into the cloaca is a pair of remarkable organs of doubtful function, the so-called respiratory trees (resp). Each of these, beginning behind in a single tubular stem, becomes elaborately branched in front, some of the branches reaching nearly to the anterior end of the body-cavity. Each of the terminal branches ends in a small enlargement or ampulla. Besides having to do, most probably, with the respiration of the cœlomic fluid and with the excretion of waste-matters, these organs have a hydrostatic function; it is through them also that, when the tentacles are withdrawn, the overplus of fluid which would impede their retraction is got rid of, and by their means, in like manner, that the quantity is again increased when the tentacles are protruded again. In all probability it is through the permeable walls of these organs that additional supplies of sea-water are received into the colome, and thus reach the ambulacral system through the perforated end of the madreporic canal.

Reproductive Organs. - The Sea-cucumber, like the Starfish and Sea-urchin, has the sexes separate. Ovaries and testes (gen. gl) are very like one another, and consist of bunches of tubular follicles, which communicate with the exterior by means of a duct opening on the dorsal surface some little distance behind the oral end (gen. ap.).

The early stages of development are very similar to those of the Starfish (p. 388). The bilateral, however, assumes a shape somewhat different from that of the Asteroidea, and is termed the auricularia (Fig. 343): it has a number of short processes developed in the course of the ciliated bands. The larval mouth and œsophagus, instead of being abolished as in the case of the Starfish, persist to the adult condition. 


\section{The Crinoidea.}

\section{A Feather-Star.-Antedon rosacea.}

General External Features.-In the Feather-Star (Fig. 330), as in the Starfish, there are to be recognised a central dise and a series of five radiating arms. In the natural position of the animal the side of the disc which corresponds to the oral or actinal surface of the Starfish is directed upwards, and the aboral or abactinal surface downwards. The five arms are bifurcated at their bases; they are feather-like and highly flexible, acting as the locomotive organs of the animal, their alternate flexions and extensions resulting in a slow movement through the water. On

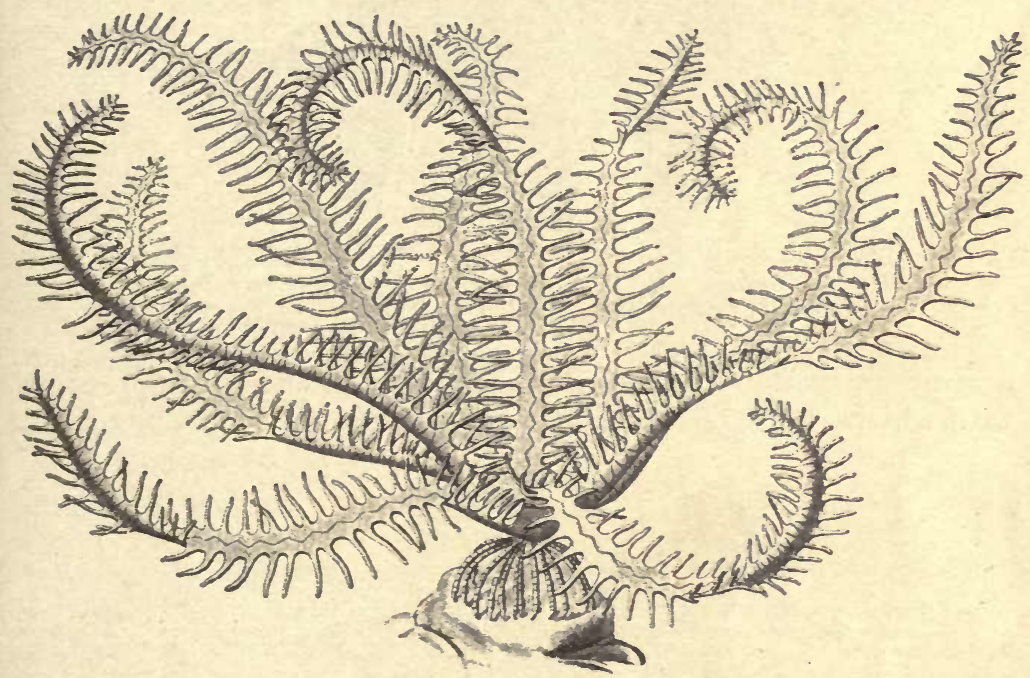

Fig. 330.-Antedon. Side view of "entire animal. (From Leuekart and Nitsche's Diagrams.)

the aboral side of the dise are whorls of slender, curved, cylindrical appendages, the cirri (Fig. 331), by means of which the Feather-star is enabled to anchor itself temporarily to a rock or a sea-weed.

On the oral side of the disc the body-wall is soft and flexible, containing only scattered irregular spicules of calcareous matter; and nearly, but not quite, in the centre of this surface is an opening, the mouth (Fig. 332, mo). From the mouth five very narrow grooves, the ambulacral grooves, radiate outwards towards the bases of the arms, near which they bifurcate, so that ten grooves are formed, one passing along the oral surface of each of the ten arm-branches to its extremity. The anal opening (an) is likewise on the oral surface, being situated on a papilliform elevation in the interspace between two of the radiating canals. 
The aboral side of the disc is occupied by a large, flat, pentagonal ossicle, the centro-dorsal ossicle (Fig. 331, $c$; and Fig. 334, CD),

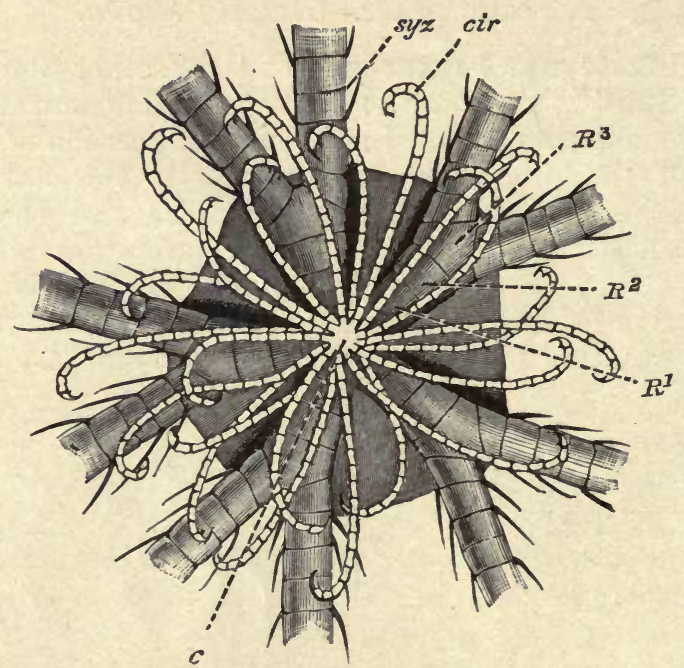

F16. 331.-Aboral view of Antedon. $c$. centro-dorsal ossicle ; cir. cirrus ; $R,{ }^{1} R,{ }^{2} R,{ }^{3}$ the three radial plates of one column ; syz. syzygy or articulation. (After MacBride.)

bearing on its outer surface a number of little cup-like depressions, with which the bases of the cirri are connected. The cirri (cirr)

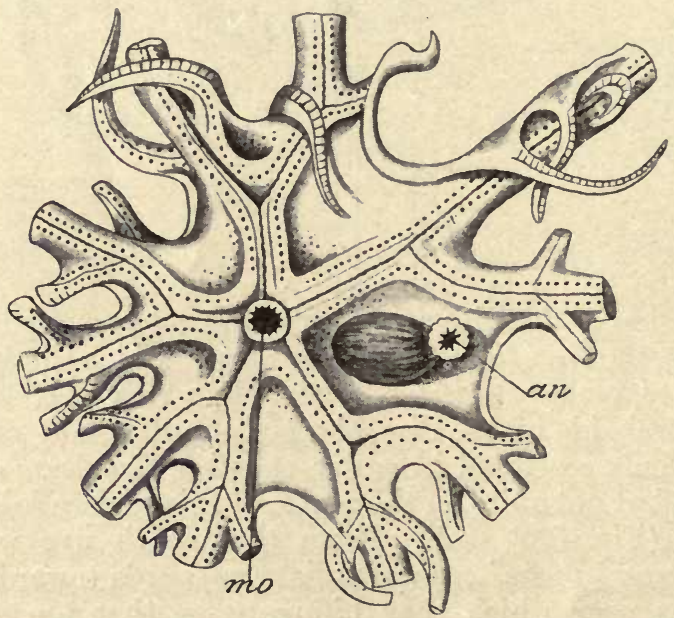

Fig. 332-Antedon, oral (upper) surface of the central disc. an. anus; mo. mouth. (From Vogt and Jung.)

consist each of a row of slender ossicles, covered, like all the rest of the animal, with epidermis, and connected together by means 
of muscular fibres. Concealed from view by the centro-dorsal ossicle is a thin plate termed the "rosette" (ros), formed by the coalescence of the basals of the larva. At the sides are five first radial ossicles $\left(R^{1}\right)$, also concealed by the centro-dorsal ossicle: with each of these articulates a second radial $\left(R^{2}\right)$, which is visible beyond the centro-dorsal. With each of the second radials articulate two third radials $\left(R^{3}\right)$, each forming the base of the corresponding arm-branch.

The ossicles of the arms-brachials $\left(B r .{ }^{1}, B r \cdot{ }^{2}\right)$-are arranged in a single row in each arm. They are somewhat elongated in the direction of the long axis of the arm, strongly convex on their aboral surfaces, longitudinally grooved on the oral surface, and connected together by the investing epidermis and by bundles of muscular fibres, by the contractions of which the movements of the arms are brought about. Fringing the sides of each arm are two rows of sidebranches, or pinnules, each supported by its row of connected ossicles, and each grooved along its oral surface.

The cœlome contains numerous strands of connectivetissue which serve to suspend the various organs.

Extending through the arms and pinnules between the supporting ossicles and the ambulacral grooves are three canals which are prolongations

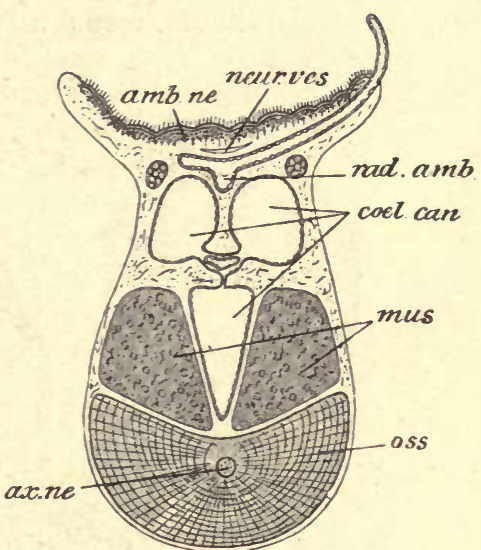

FIG. 333.-Antedon, transverse section of a pinnule. $a m b$. ne. radial nerve of the superficial (ambulacral) nervous system; $a x$. ne. axial nerve; col. can. sub-tentacular and coliac canals ; mus. muscles; neur. ves. radial sinus of the perihrmal system; rad. amb. radial ambulacral vessel giving off branches to the tentacles. Between the paired subtentacular and unpaired coeliac canals is the genital rachis. The small round bodies above the line from rad. $a \mathrm{mb}$. are the sacculi. (After Teuscher.)

of the cœlome (Fig. 333, coel. can). Two of these-the subtentacular canals-form a pair separated from one another by a median septum underlying the ambulacral groove. The otherthe coeliac canai-runs between these and the supporting cssicles (oss). The sub-tentacular canals and the cœliac canal communicate with one another at the extremity of each arm.

The enteric canal begins with a wide, funnel-shaped cesophagus leading to a spacious stomach which gives off a number of short, blunt diverticula and a pair of longer, narrower, "hepatic" cæca, which are slightly branched at the ends. Distally the stomach becomes contracted and opens into a wide intestine, which winds round the cœlome, becoming narrower where it passes upwards to 
open on the exterior, the terminal part, or rectum, projecting as a tubular papilla on the surface. In the living animal the rectal tube is observed to undergo frequent movements of contraction and dilatation, by means of which water is drawn into and expelled from the intestine; so that here, as in the Sea-urchin, there would appear to be a process of intestinal respiration.

The ambulacral system consists of a ring-vessel surrounding the mouth, and a series of radial vessels (Fig. 333, rad. amb.) which run in the ambulacral grooves, giving off branches to the pinnules. Connected with the radial vessels and their branches are a series of minute tubular appendages, the so-called tentacles (Fig. 334, tent.), which are homologous with the tube-feet of the Starfishes

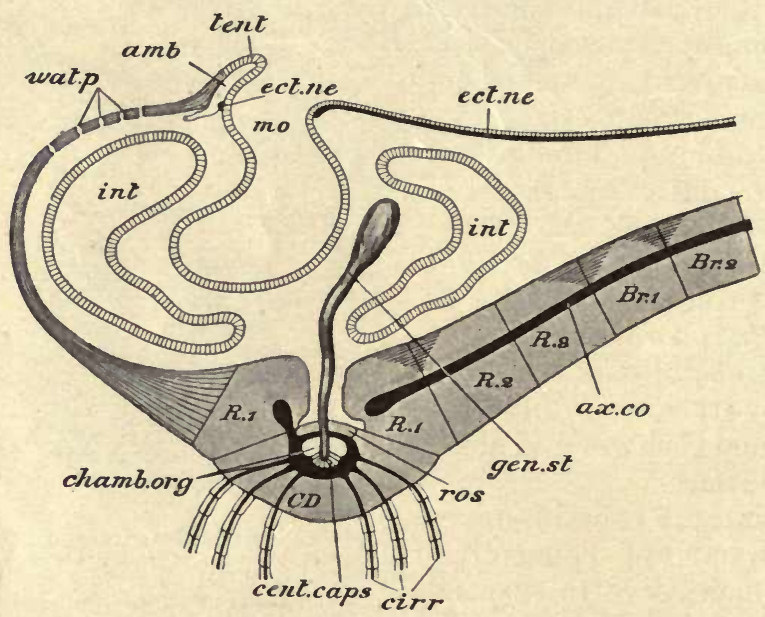

FIG. 334.-Antedon. Diagrammatic view of a median vertical section through the disc, passing through one radius and one inter-radius. $a m b$. ambulacral vessels; $a x$. co. axial nerve-cord passing through the ossicles of the arm; $B r_{0}^{1} \mathrm{Br}^{2}$ brachial ossicles; $C D$. centro-dorsal ossicle ; cent. caps. central capsule; chamb. org. chambered organ ; cirr. cirri; ect. ne. ambulacral (epidermal) nerve-ring and radial nerve; gen. st. genital stolon; int. intestine ; mo. mouth; . $^{1} R^{2}{ }^{2}{ }^{3}$ radials; ros. rosette; tent. tentacles; wat. p. water-pores. (Aftcr Milnes Marshall.)

and Sea-urchins, but are devoid of terminal suckers. These are not organs of locomotion : they bear numerous sensory papillæ, and are therefore to be looked upon as tactile organs, but they probably also have a respiratory function. Connected with the ring-vessel are a number of ciliated, branched, tubular diverticula, the watertubes, which are suspended within the cœlome, and may open freely into it at their extremities. A large number of vessels with minute ciliated openings-the water-pores (wat. p)-lead through the actinal wall of the disc: these and the ciliated tubes are to be considered as together representing the madreporic canal and its openings in the Star-fish and Sea-urchin.

The nervous system consists of three perfectly distinct partssuperficial, deep, and axial or aboral. A superficial radial nerve- 
ring (ect. ne) surrounds the mouth, and from it are given off a series of nerves-thickenings of the epidermis of the ambulacral grooves and their offsets-which extend throughout the length of the arms and pinnules. The deep nervous system follows the same general arrangement as the superficial. In the axis of the supporting ossicles of the arm is an axial nerve (ax.co), which gives off branches (Fig. 333, ax. ne) running through the axes of the ossicles of the pinnules. The axial nerves are connected internally, not with the circum-oral nerve-ring, but with a central body situated below the rosette, in the interior of the centro-dorsal ossicle. This, the central capsule (Fig. 334, cent. caps), forms the investment of a body termed the five-chambered organ (chamb. org), divided into five parts by radial septa, and continuous with the aboral end of the genital stolon. Processes from the five angles of the central capsule combine to form a pentagonal ring from which pass outwards the axial nerves of the arms. Aborally the central capsule gives off nerves to the cirri.

A system, corresponding to the perihæmal system of the Starfish is present, though reduced, and there is a highly developed and complicated lacunar or hæmal system.

Numerous bodies termed the sacculi, the character of which has given rise to much discussion, occur regularly arranged along the ambulacral grooves and also in other parts. They are small, spherical bodies which become vividly coloured when treated with staining agents. They are sometimes supposed to be parasitic Algæ; but the regularity of their arrangement is opposed to such a view. It has been suggested with more appearance of probability that they may be masses of reserve materials, stored up for the nutrition of the animal, or may consist of excretory matters.

The reproductive organs-ovaries or testes, as the case may be-are lodged in the dilated bases of the pinnules, which become considerably enlarged as the ova or sperms mature, those next to the bases of the arms alone remaining sterile. When mature, the sexual elements escape by means of short ducts. Each gonad is one of the terminal parts of a system of tubes lined by an epithelium, and extending from a central part or genital stolon (gen. st)-lodged in the vascular plexus that surrounds the œesophagus and connected dorsally with the chambered organ-outwards through the arms; the terminal portions, lying in the pinnules, are dilated to form the reproductive organs, and the cells of their epithelium become developed into ova or sperms, while the rest constitute a non-fertile connecting rachis. This system is enclosed throughout by a plexus of hæmal lacunæ.

Like the rest of the Echinoderms, the Feather-star undergoes a metamorphosis (Figs. 344 and 345). It passes through a freeswimming ciliated larval stage, which is followed by a fixed 
stalked stage known as the "pentacrinoid" larva on account of the resemblance which it bears to the adult Pentacrinus, one of the permanently fixed members of the same class. This fixed pentacrinoid larva passes into the adult free-swimming Featherstar by the development of the dorsal cirri, the elongation of the arms, and the absorption of the stalk.

\section{Distinctive Characters and Classification.}

The Echinodermata are radially symmetrical animals, the radial arrangement of whose parts imperfectly conceals a more obscure bilateral symmetry. The surface is covered with an exoskeleton of calcareous plates or ossicles, which usually support a system of movable or immovable calcareous spines. There is a large bodycavity or cœlome, and well-developed alimentary, nervous, and vascular systems. A characteristic system of vessels, the ambulacral system, is connected with the locomotion of the animal, as well as with other functions : the organs of locomotion are in most cases elastic and contractile tubular bodies, the tube-feet, which are appendages of the ambulacral system. Nearly all the systems of organs of the animal partake to a greater or less extent of the general radial form of the body. Reproduction is entirely sexual. In the course of its development from the egg the Echinoderm passes through a peculiar larval stage, in which the symmetry of parts is bilateral, instead of radial as in the adult animal. All the Echinodermata are marine.

The Echinodermata are classified as follows:-

\section{SUB-PHYLUM I.-ELEUTHEROZOA.}

Echinodermata devoid of a stalk, and always freely locomotive in the adult condition: with a system of radial ambulacra in the form of grooves or areas radiating out from the mouth, and containing a double series of tubular appendages of the ambulacral system, the tube-feet, usually employed in locomotion, and in the majority of cases provided with terminal suckers: the anus usually aboral; the mouth on the surface that is habitually directed downwards, or at the end habitually directed forwards in locomotion.

\section{CLASS I.-ASTEROIDEA.}

Free Echinoderms with star-shaped or pentagonal body, in which a central disc and usually five arms are more or less readily distinguishable, the arms being hollow, and each containing a prolongation of the cœlome and of its contained organs. There are distinct oral and aboral surfaces, on the former of which the anus and the madreporite are situated, and on the latter the 
mouth and five narrow ambulacral grooves lodging the tube-feet. The larva has the form either of a bipinnaria or of a brachiolaria. This class includes the Starfishes.

\section{Order 1.-Phanerozonia.}

Asteroidea with large marginal ossicles. The dermal branchiæ are present only on the aboral surface. The ambulacral ossicles not closely crowded. Pedicellariæ sessile.

\section{Order 2.-Cryptozonia.}

Asteroidea with the marginal ossicles inconspicuous. Dermal branchiæ not restricted to the aboral, but often present on the oral surface. Ambulacral ossicles crowded together. Pedicellariæ stalked or sessile.

\section{CLASS II.-OPHIUROIDEA.}

Star-shaped free Echinoderms, with a central disc and five arms, which are more sharply marked off from the disc than in the Asteroidea and which contain no spacious prolongations of the colome. There are distinct oral and aboral surfaces. The anus is absent; the mouth, as well as the madreporite, on the oral surface. Except in one fossil order there are no ambulacral grooves. The larva is a pluteus. This class includes the Sandstars and Brittle-stars (Figs. 336 and 337).

\section{ORDER 1.-LysOPHIURA.}

Extinct Ophiuroids with ambulacral grooves.

Silurian and Devonian.

\section{ORDER 2.-STREPTOPHIURE.}

Ophiuroids in which the ambulacral ossicles articulate with one another by simple ball-and-socket joints.

\section{Order 3.-Cladophiure.}

Ophiuroids in which the ambulacral ossicles articulate with one another by means of hour-glass-shaped surfaces. The arms may be branched.

\section{Order 4.-ZYGOPHIURA.}

Ophiuroids in which the movement of the ambulacral ossicles on one another is restricted by the presence of lateral processes and pits. 


\section{CLASS III.-ECHINOIDEA.}

Free Echinoderms with globular, heart-shaped, or disc-shaped body enclosed in a shell or corona of close-fitting, firmly united calcareous plates. The mouth is nearly always polar; the anus usually at the opposite (aboral) pole; the madreporite is close to the latter. There are no ambulacral grooves; but the surface is divided into alternating ambulacral and inter-ambulacral zones or areas, which usually run from pole to pole. The larva is a pluteus. This class includes the Sea-urchins, with the Heart-urchins and Cake-urchins.

\section{ORDER 1.-REgularia.}

Echinoidea with globular corona containing, in most cases, twenty meridional rows of plates. Mouth and anus polar. A lantern of Aristotle is present. This order includes the Seaurchins.

\section{Order 2.-Clypeastridea.}

Echinoidea with more or less flattened corona, with the mouth central, the anus excentric. A lantern of Aristotle is present. This order includes the Cake-urchins (Fig. 341).

\section{Order 3.-Spatangoidea.}

Heart-shaped Echinoidea with the mouth and anus excentric. No lantern of Aristotle. This order includes the Heart-urchins (Fig. 340).

\section{CLASS IV.-HOLOTHUROIDEA.}

Free Echinoderms with elongated, cylindrical or five-sided body, having the mouth and anus at opposite extremities. The bodywall is usually only supported by scattered ossicles or spicules. There is no external opening to the madreporic canal (except in some Elasipocia). The surface usually exhibits five ambulacral areas; but these may be absent. There is a circlet of large oral tentacles. The larva is an auricularia. This class includes the Sea-cucumibers and "Bêche-de-mer."

\section{Order 1.-Elasipoda.}

Holothuroidea with well-marked bilateral symmetry, with tubefeet on the ventral surface (which is flattened) and papillw on the dorsal. Confined to the deep sea. 
Order 2.-Pedata.

Holothuroidea with tube-feet either in longitudinal rows or scattered irregularly over the surface.

\section{ORDER 3.-APODA.}

Holothuroidea devoid of tube-feet and of radial ambulacral vessels.

\section{SUB-PHYLUM II.-PELMATOZOA.}

Echinodermata which are usually fixed at the base, and usually supported on a stalk composed of a row or rows of ossicles (Fig. 342): the mouth on the free surface, near or in the centre, and having extending out from it on the oral surface a radially arranged system of narrow, ciliated ambulacral grooves, having the function of food-grooves, which may run between the plates of the theca, on the surface of the theca, or along the oral surfaces of a system of radial processes or arms given off from it. The tube-feet of other Echinoderms, when represented, take the form of small, tubular, strongly ciliated appendages (tentacles) without suckers: the anus usually on the oral surface.

\section{CLASS I.-CRINOIDEA.}

Mostly fixed, stalked Pelmatozoa in which there is a theca comprising five regularly arranged radial and five basal plates, giving off five, usually branched, jointed processes or arms ; with food-grooves radiating out from the mouth along the oral surfaces of the arms, and extending along their branches: the central parts of the ambulacral, nervous, and reproductive systems, and of the cœlome lodged in the theca, send extensions through the arms.

This class comprises, together with many extinct forms, the only living Pelmátozoa.

\section{Sub-Class I.-Monocyclica.}

Crinoidea in which the base of the theca comprises basals only.

\section{Sub-Class II.-Dicyclica.}

Crinoidea in which the base comprises basals and infra-basals.

\section{CLASS II.-CYSTOIDEA.}

Fixed, stalked, or sessile Pelmatoza, with the plates of the theca sometimes irregular, sometimes arranged in a regular radial system, with food-grooves extending for a longer or shorter distance over the surface of the theca, sometimes on special plates lying above those of the latter, their terminal parts 
extending on to a varying number of unbranched arms or "fingers"; the theca perforated completely or partially by numerous pores which are supposed to have lodged respiratory processes.

Lower Silurian to Carboniferous.

\section{CLASS III.-BLASTOIDEA.}

Fixed Pelmatozoa with well-developed stalk, and theca with a regular system of plates; with five, rarely four, food-grooves radiating out from the central mouth, and each borne on a special "lancet plate," the inter-radial intervals between which are occupied by a corresponding number of oral or "deltoid" plates. The grooves are bordered by a series of side plates bearing small branches or "fingers" to which side branches of the grooves extend. In the intervals between the grooves on the aboral sides of the deltoids are a whorl of plates perforated by the apertures of groups of internally situated respiratory folds (hydrospires). The anus is eccentrically situated on the oral surface.

Upper Silurian to Carboniferous.

\section{CLASS IV.-EDRIASTEROIDEA.}

Fixed (or sometimes free?) Pelmatozoa, usually sessile, rarely with a short stalk ; with sac-like, cushion-shaped or disc-sliaped theca made up of numerous plates devoid of auy regular arrangement and without any appendages; with central mouth and five straight or curved radiating food-grooves bordered by covering plates : anus and madreporite on oral side.

Cambrian to Carboniferous.

\section{CLASS V.-CARPOIDEA.}

Pelmatozoa with a well-developed stalk, with the body laterally compressed, with only two food-grooves running out from the mouth. Theca composed of numerous small irregular plates with larger lateral plates forming a framework along the margins.

Cambrian and Silurian.

\section{Systematic Position of the Examples.}

Asterias rubens is a species of the genus Asterias, which, with several others, constitutes the family Asteriida of the order Cryptozonia. The family Asteriidce is characterised among the families of the Cryptozonia by the following distinctive features:The ossicles of the aboral surface are small, unequal, reticulate plates, bearing isolated or grouped spinelets (paxilloe). The margin of the actinostome is defined by the ambulacral plates. The pedicellariæ are of two forms, forceps-like and scissors-like. The tube-feet are in four rows. Asterias differs from the other genera of the family in having well-developed reticulate dorsal ossicles bearing definite spines.

The Sea-urchins of which a short description has been given are the genera Strongylocentrotus and Echinus, but the description is sufficiently general to apply to any member of the family Echinidoe, to which these genera, with a number of others, belong. 
The family Echinidce is one of about five families of the sub-order Ectobranchiata, the members of which all differ from the other sub-order-Entobranchiata-of the Regularia, or regular Seaurchins, in the possession of dermal branchiæ, and in having the auricles in the form of complete arches.

The Sea-cucumber (Cucumaria or Colochirus) is a member of the Stichopoda - one of the families of the sub-order Dendrochiroto of the Pedata, or foot-bearing Holothurians. The Dendrochirotce differ from the Aspidochiroto-the other sub-order-mainly in having arborescent instead of shield-shaped tentacles, and the Stichopoda differ from the rest of the Dendrochirotce in having the tube-feet arranged in five regular zones. The genus Cucumaria is distinguished from the rest by having ten tentacles with the two ventral smaller than the others. Colochirus is closely allied to Cucumaria, the principal distinction being the presence in the former of papillæ taking the place of tube-feet in certain situations, as already noted.

The Feather Star (Antedon rosacea) is a member of the family Comatulidae, which is distinguished from the four other living families comprised in the class Crinoidec of the Pelmatozoa, by the absence of a stalk in the adult condition.

\section{General Organisation.}

General Form and Symmetry.-Like the Cœlenterata, the Echinodermata are radially symmetrical, the body being capable of division into a series of sub-equal antimeres along a series of radiating planes at right angles to the principal axis. In the majority of existing forms (Asteroidea, Ophiuroidea, and Crinoidea) the radial symmetry is expressed in the external form of the body, which is produced into a number of radially disposed parts, the arms or rays, arranged around a sinaller or larger central dise. But in the Echinoidea the body is sub-spherical, and in the Holothuroidea sub-cylindrical, the radiate arrangement being in these classes indicated externally only by the distribution of the tube-feet, and internally by that of certain of the systems of organs.

Although, however, the general external form and the arrangement of some of the internal organs in the Echinodermata indicates a radial symmetry, it is invariably found that this radial arrangement serves to hide a more primitive and more fundamental bilateral symmetry. This is best marked in the larva, which has pronounced bilateral instead of radial symmetry, but is quite recognisable in the adult. In all Echinoderms there is, passing through the primary axis, a plane-the median plane-along which, and along which alone, the body is capable of being divided into two equal-or, to speak more correctly, approximately equalright and left halves. The existence of such a single median 
plane is, as already explained ( $p .377$ ), indicative of the bilateral form of symmetry.

The body is most usually five-rayed (Ophiuroidea, most Asteroidea, Crinoidea), cylindrical (most Holothuroidea) or globular (most Echinoidea), the surface in the two last cases being marked by five bands or zones of tube-feet, which divide it into five ambulacral and five inter-ambulacral areas. In the Ophiuroidea and Asteroidea two of the rays-constituting the bivium-have between them the madreporite, marking the position of the madreporic canal of the ambulacral system; the remaining three rays form the trivium. The median plane passes through the madreporite, and thus midway between the two rays of the bivium, and bisects longitudinally the middle ray of the trivium. A corresponding disposition of the parts is traceable also, as will be subsequently shown, in the cylindrical and globular Echinoderms.

In all the Echinodermata aboral or abactinal, and oral or actinal surfaces are more or less distinctly recognisable. In the Asteroidea, Ophiuroidea, and Echinoidea, the actinal surface is that in the middle of which the mouth is situated, and which is, in the natural position of the animal, directed downwards or towards the surface to which it is clinging. The opposite abactinal surface is, in the majority of the Asteroidea and Echinoidea, marked by the presence of the anal aperture: in the Ophiuroidea and some Asteroidea the anus is absent; in some Echinoidea it is situated on the border between the two surfaces, or even on the oral surface. In the Crinoidea the oral surface, which is habitually directed upwards in the natural position of the animal, bears both mouth and anus, the former central, the latter eccentric and interradial. In the fixed Crinoids the abactinal or aboral surface has attached to its centre the distal end of the stalk; in the free forms it has connected with it whorls of slender curved appendages, the dorsal cirri, by means of which temporary attachment is effected. In the Holothurians, owing to the elongation of the body in the direction of the line joining mouth and anus, oral and aboral surfaces corresponding to those of the other classes are not distinguishable; but in mary, as for example in Colochirus, there is a marked difference between one surface - the dorsal, which is habitually directed upwards, and another-the ventral, which is habitually directed downwards.

In considering the general external form in the various classes of Echinoderms, we have to take into account the arrangement of the tube-fect-the organs of locomotion-as these have important relations to the other parts and to the whole plan of organisation of the animal. These organs, as previously explained, are tubular appendages with highly elastic and contractile muscular walls, capable of being stretched out so as to extend a long way from the surface of the body. In the majority of cases the tube-foot has at 
its extremity a sucking-disc, by means of which it can be attached; in a few, however, this sucking-disc is absent.

The epidermis is ciliated in all but Holothuroidea. In the subjacent dermal layers there are always present calcareous bodies or ossicles, varying very greatly in form and arrangement in the different groups. Movable or immovable calcareous spines or tubercles projecting on the surface are very general. Peculiarly modified. spines, termed pedicellaria, are commonly, though not universally, present in certain parts in the Echinoidea and Asteroidea. A pedicellaria consists in essence of two or three calcareous jaw-like pieces or valves, movably articulated together, and capable of being separated or approximated by the contraction of bundles of muscular fibres; sometimes there is a long stalk; sometimes (as in the case of Anthenea, p. 387) a stalk is absent; during life the jaws or valves keep opening and closing. That such specialised structures have some important function to perform there can be no doubt, but there is some uncertainty as to what their special purpose is. According to some observers. the pedicellariæ of the Sea-urchin have been seen passing from one to another the particles of freal matter discharged from the anus, and their function would thus appear to be a cleansing one. On the other hand, it is stated that when a Sea-urchin is attacked, the spines may be bent aside from the assailed portion of the surface so as to allow of the pedicellariæ being brought to bear as defensive weapons on the assailant, and from these and other observations that have been recorded, both on Asteroids and on Echinoids, it is concluded that the main function of these appendages is to act as defensive organs. Pedicellariæ are absent in the Ophiuroids, but in the Euryalida there are peculiar hook-like organs of adhesion, most abundant on the oral surface and towards the extremities of the arms. The sphceridia, which have already been referred to as occurring in the Sea-urchin, are only doubtfully to be regarded as modified spines; they are confined to the Echinoidea. Also confined to that class are the clavulocslender spines covered with strong cilia, which occur in bands on the surface of the Spatangoids. Larger spines, resembling the clavulæ in being covered with strong cilia, occur also in the Clypeastroids and some Asteroids. The currents produced by the action of their cilia serve to keep constantly renewed the water in the neighbourhood of the anus and of the branchiæ.

There are two principal systems of plates to be recognised, an oral and an apical; the former corresponding with the oral or actinal, and the latter with the aboral or abactinal surface. The former vary considerably in the different classes: the constant clements are five orals, which may or may not be recognisable in the adult. The apical system consists (1) of a central plate; (2) of five basals which are inter-radial in position; (3) of five radials 
which are radial in position. In the Asteroidea (Fig. 319) the radials are late in making their appearance; before they are developed five terminal plates have become distinct, one at the end of each rudimentary arm; these are carried outwards by the extension of the arm, and each supports the corresponding tentacle. As a rule these plates of the apical system are only distinct in the young condition. In the Ophiuroidea the arrangement resembles that observable in the Asteroidea. In the Echinoidea (Fig. 323) the basals (genitals) are perforated by the ducts of the reproductive organs ; the radials (oculars) are perforated for the tentacle : the central (anal) rarely persists as a single plate in the adult, usually becoming broken up into a series of irregular plates. In the stalked Crinoidea the term central has been applied to a plate which is transformed into the disc of attachment at the base of the stalk, but the correspondence between this and the similarly named plate in the other classes is very doubtful; the ossicles of the stalk intervene between it and the basals. In the free forms the uppermost segment of the larval stalk, uniting with the central and the infra-basals, is transformed into a centro-dorsal plate, and the basals nearly always unite into a rossette-plate, which is concealed from view by the centro-dorsal and the radials. 'The apical system of plates is apparently not represented in the Holothuroidea.

Modifications of Form in the Five Classes.-The general shape in the Asteroidea is, as already pointed out, that of a star. There is a central part, or central disc, from which proceed a series of radially disposed arms or rays. The central disc and the rays are usually compressed in the vertical direction, as in Anthenea and Asterina, but in some Starfishes the rays are approximately cylindrical; they nearly always taper distally. In the majority of Starfishes, as in the examples described, the arms are five in number, except in malformed individuals; but in some they are six, in others seven, eight, or more. The proportions borne by the arms to the central disc are subject to considerable variation. In some, as in Asterias, the arms are long, and the central disc appears as little more than their point of union; in others, again, owing to coalescence of the arms, the whole Startish has the form of a five-sided disc, in which the arms are represented only by the five angles; while between these two extremes there are numerous intermediate gradations. The Brisingidoe differ from all the rest of the class in having the arms almost as sharply separated off from the central disc as in the Ophiuroids.

The abactinal or aboral, and the actinal or oral surfaces are always distinctly marked off from one another. In the middle of the latter (Fig. 335) is the mouth, running out from which are five or more narrow ambulacral grooves, one of which is continued along 
the oral surface of each arm to its extremity. Near to, but not quite in the middle point of the aboral surface is the anal aperture, absent in a few instances; and on the same surface, nearer the margin, between the two rays of the bivium in the five-rayed Starfishes, is the madreporite, a finely grooved calcareous plate perforated by a number of minute apertures. In some fossil Starfishes it is situated on the oral surface. Sometimes instead of one madreporite there are several.

The wall of the body in the Starfishes contains a number of calcareous ossicles, movably articulated together and connected by bands of muscle, so that, though the body is firm, and in the dried condition often quite rigid, the arms are capable during life of slow movements of flexion and extension, enabling the animal to creep through comparatively small fissures and crannies. A special system of ossicles-the ambulacral ossicles-are arranged in a double row along each ambulacral groove, the ossicles of the two rows articulating movably with one another at the apex of the groove. At the end of the arm the two rows of ambulacral ossicles end in a terminal ossicle which supports the unpaired tentacle. Spines are invariably present, but are sometimes confined to

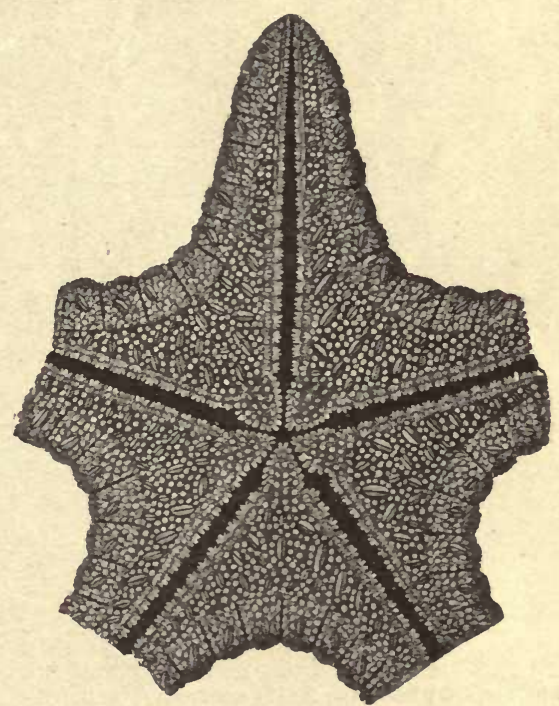

Fir. 335.-Anthenea. View of oral surface. (After Sladeu.) the margins of the ambulacral grooves, in which position they are movably articulated with the underlying assicles. Tubercles take the place of spines over most of the surface in many forms. In Astropecten the ossicles of the aboral surface take the special form to which the term paxillee is applied. Each paxilla is a plate which is produced into a short rod, divided at its extremity into a number of radiating processes.

The tube-feet are arranged in a double row along each of the ambulacral grooves, each connected through an aperture between the ambulacral ossicles with an ampulla, or, exceptionally, with two ampullæ, situated in the colome. Each double row of tube-feet terminates at the extremity of the arm in an unpaired appendage, the tentacle, which is tactile and olfactory, and not locomotive in 
function. The tube-feet are provided (except in Astropecten) with terminal suckers.

In the Ophiuroidea (Fig. 336) the central disc is much more sharply marked off from the arms than in the Asteroidea. The arms, which are five in number, are comparatively slender, and cylindrical, tapering towards the free extremities; in one group, the Euryalida (Fig. 337), they are branched. The mouth is in the middle of the oral surface of the disc, as in the Asteroidea, but there are no ambulacral grooves, and there is no anal aperture.
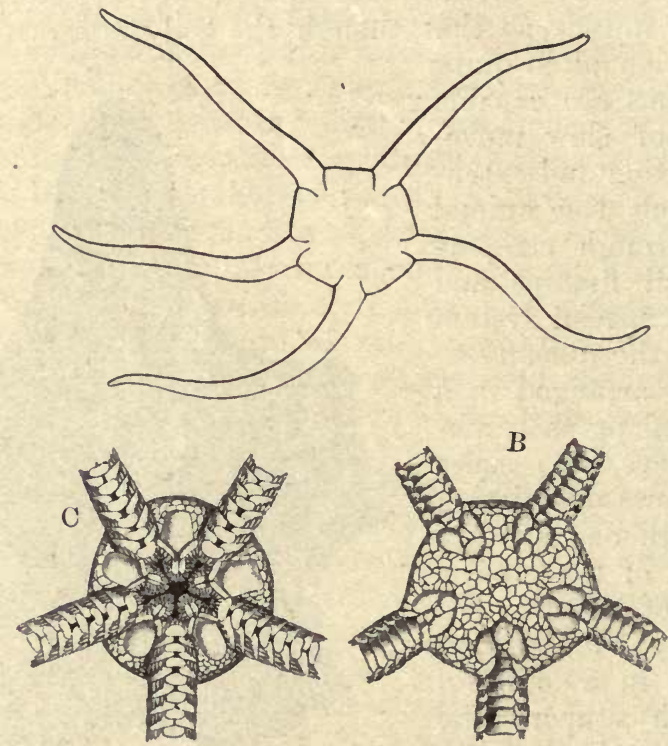

FiG. 336.-Ophioglypha lacertosa. $A$, outline, of the natural size. $B$, central disc, aboral surface. $C$, the disc, oral surface showing the mouth and genital fissures. (From Nicholson and Lydekker's Palaontology.)

Five pairs of slits on the oral surface (Fig. 336, C) lead into the genital bursæ, which receive the sperms and ova from the gonads, and which appear also to act as organs of respiration and perhaps also of excretion. The surface is covered with thin plate-like ossicles, usually beset along their edges with longer or shorter spines; sometimes irregular calcareous granules take the place of plates. Hook-like organs of adhesion are present only in the Euryalida. Each of the arms is supported by a row of internally situated ambulacral ossicles. Tube-feet are present and are protruded at the sides of the arms between the lateral plate-like ossicles; but they have no sucking-discs and no ampullæ, and locomotion is effected in the majority of the Ophiuroids by active flexions and extensions of the arms. In one genus there is a pair 
of fin-like appendages, supported by slender spines, on each joint of the arms. The madreporite is situated inter-radially on the oral, and not on the aboral surface as in the Asteroidea. In the Euryalida there are five madreporites and five madreporic canals.

In the Echinoidea the body is either globular, or heart-shaped, or flattened and disc-like. The exoskeleton is in the form of a

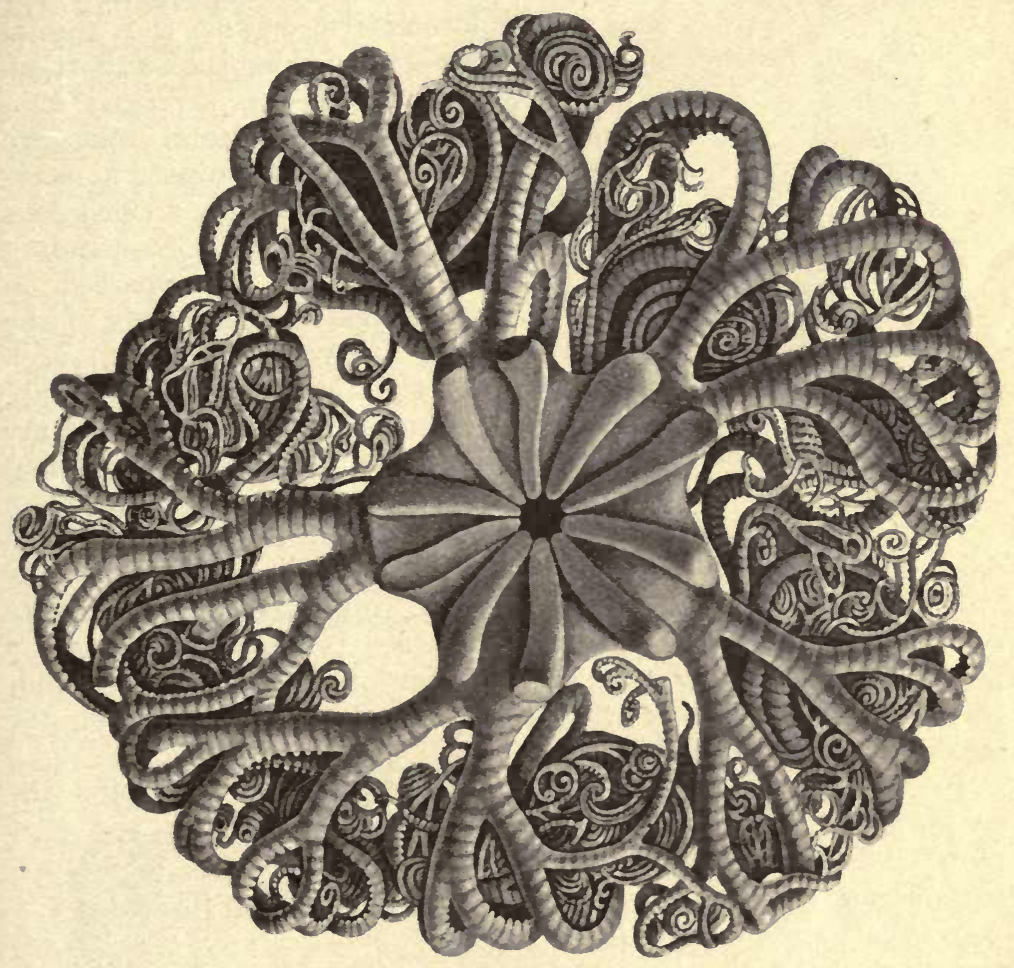

F16. 337.-Astrophyton arborescens, aboral surface. (After Ludwig.).

rigidly articulated system of calcareous plates, fitting closely together by sutures, so as to form a continuous shell or corona. Asthenosoma and allies, deep-sea forms, differ from all the rest in having a corona possessing a certain degree of flexibility and performing movements which are brought about by the contractions of five longitudinal bands of muscle running along the ambulacral areas on the inner surface.

In the globular forms, or regular Sea-urchins, the mouth is situated at the oral pole of the globe, the anus at the aboral, and the plates of the corona are in twenty regular meridional rows, 
arranged in ten zones, five ambulacral and five inter-ambulacral, as described in the account of Echinus, with peristome, periproct, ocular and genital plates, and madreporite. Spines (Fig. 338), pedicellarioe (Fig. 339), and sphocridia are present, as already described (p. 394), the last-named appen-

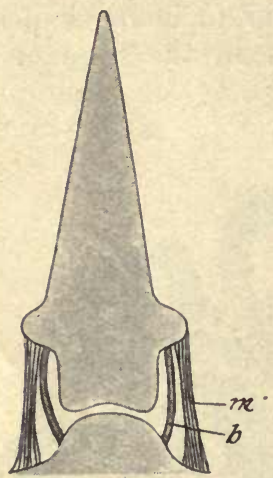

FIG. 338.-Diagram of spine of Sea-urehin showing mode of articulation. $m$. muscle; $b$. ligament. (From Leuckart.) dages, however, being absent in one group. The spines are usually defensive organs simply, but in some Sea-urchins they act also as the locomotive organs, the animal moving by their agency along the sea-bottom.

The tube-feet, which are arranged in a double row in each ambulacral zone, are extremely extensible, and terminate in sucking-membranes strengthened by a calcareous rosette. An unpaired tentacle, corresponding to that of the Asteroidea, is supported on each of the ocular plates at the ends of the ambulacral zones. Two tube-feet in each double row, situated on the peristome, are likewise of the nature of tentacles, and are sometimes devoid of sucking-membranes. Corresponding to the dermal branchioe of the Asteroidea are, in the majority, five pairs of branched, hollow appendages surrounding the peristome.

Surrounding the mouth are five teeth, supported by an elaborate system of ossicles (Aristotle's lantern, see p. 397), and a ring of processes, the auricles, from the interior of the corona, surrounds this and gives attachment to some of the muscles by which the ossicles are moved.

In the heart-shaped forms or Heart-urchins (Fig. 340) the corona is heart-shaped, the mouth is usually more or less eccentrically placed on the oral surface, and the peristome is usually transversely elongated; the anus is on or near the border beween the two surfaces. The ambulacral areas do not run continnously, but stop short at the margin (petaloid ambulacra); one of them, the anterior, is usually unlike the others and frequently devoid of pores. The genital and ocular plates are in the middle of the aboral surface, where the ambulacra converge, and are thus widely

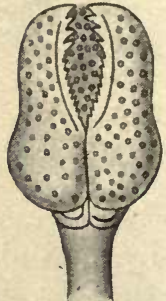

F1G. 339.-Pedicellaria of Arbacia punctulata. (From Leuckart.) separated from the anus; there are usually only four genital plates, and the genital apertures may be reduced to two. Slender spines beset the entire surface and are the chief organs of locomotion. Modified spines, the clavula, surround the anus in a ring and are distributed elsewhere. A few pedicellarix are present in the 
neighbourhood of the mouth, and sphæridia also occur. A series of tree-like dermal branchiæ surround the peristome. The "lantern of Aristotle," with its teeth, is not represented.

In the Clypeastridea or Cake-urchins the whole corona (Fig. 341) is usually greatly compressed so as to assume the form of a disc, sometimes notched at the edges or pierced by fenestræ. The mouth is in the middle of the flat or concave oral surface, the anus eccentrically situated near the margin. The ambulacra are petaloid. The genital and ocular plates are usually more or less - fused together at their edges, and the genital apertures are often not in the genital plates, but in the corresponding ambulacral zones. The spines are exceedingly fine and hair-like. Sphæridia are present, but pedicellariæ and clavulæ are absent. An "Aristotle's lantern" with teeth is present, as in the globular forms.

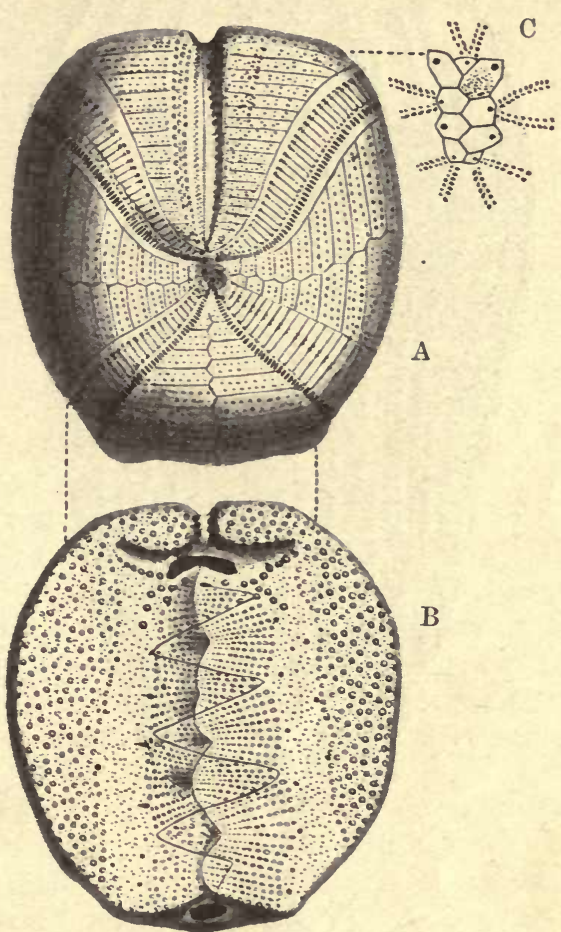

FIG. 340.- Femipneustes radiatus, $A$, aboral, and $B$, oral surface. $C$, apical plates. (From Broun's Tierrecich.)

In the Holothuroidea the body is more or less elongated in the direction of the axis joining mouth with anus, which are placed

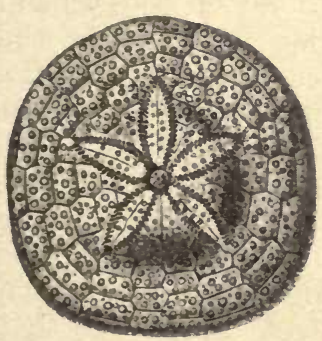

Fig. 341.-Clypeasterisubdepressus, view of aboral surface showing the petaloid ambulacra. (From Hertwig's Lehrbuch.) at opposite (anterior or oral, and posterior aboral or anal) extremities of the body. The shape is sometimes completely cylindrical, sometimes five-sided; in many there is more or less dorso-ventral compression, and the dorsal and ventral surfaces may differ greatly from one another. A flattened sole-like ventral surface bearing the three rows of tube-feet of the trivium is, as already stated, often distinguishable: it is most distinctly developed in Psolus and allied genera. In some Holothuroids the surface is enclosed 


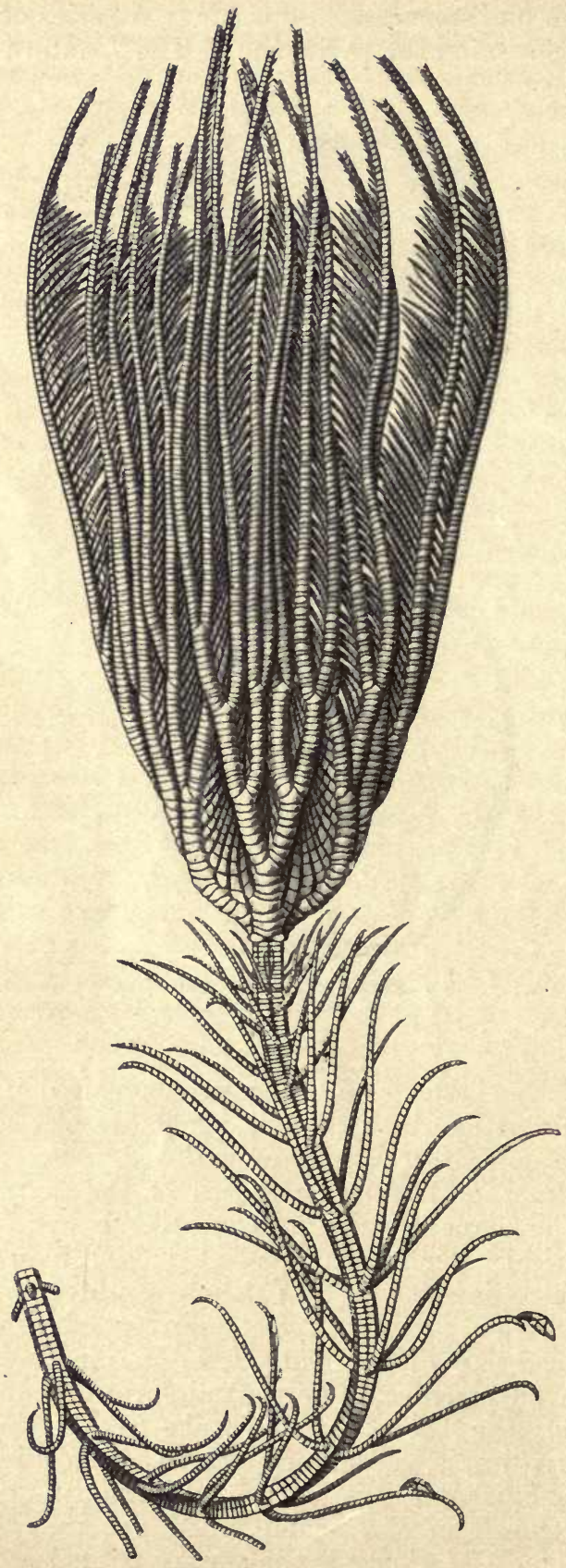

FiG. 342.-Metacrinus interruptus, (After P. H, Carpenter.) in an armour of closefitting plates; but in the vast majority the body-wall is comparatively soft, being strengthened merely by a great number of minute ossicles of a variety of shapes. In Synapta (Apoda) numerous minute anchor-like spicules, each connected with a latticed plate, project from the surface, and cause the animal to adhere to soft bodies with which it comes in contact. Around the mouth is a whorl of tentacles pinnate, shield-shaped, or arborescent. The tubefeet are sometimes entirely absent. When present they are usually uniform in character throughout, and may be arranged in five regular longitudinal rows, or scattered over the entire surface. Sometimes, as has already been stated in the account of Colochirus, the tube-feet of the dorsal and even some of those of the ventral surface may assume the form of papillæ. In the Elasipoda the tube-feet of the dorsal surface are remarkably modified, taking the form of greatly elongated processes.

In the Crinoidea the general shape is that which has been described in the case of the Feather-star-star-like, with a central disc and 
a series of radiating arms, which usually branch dichotomously. In the stalked forms (Fig. 342) a stalk, consisting of a row of elongated ossicles connected together by bundles of ligamentous fibres, attaches the animal to the sea-bottom. Along some of the joints of the stalk are usually arranged a number of slender, many-jointed appendages-the cirri. At its base the stalk usually breaks up into a number of root-like processes; distally it becomes continuous with the central disc. The ossicles forming the skeleton of the central disc are the basals and the radials: with the latter articulate externally the brachials, a single row of which gives support to each of the arms and its branches, while similar rows of smaller ossicles support the pinnules-the lateral appendages which fringe the arms in a double row. In the free forms the stalk is absent in the adult condition, though present in the larva, and from its terminal ossicle and other neighbouring plates is formed by coalescence a plate-the centro-dor:sal ossicle of the disc. To the centro-dorsal ossicle are attached whorls of many-jointed, slender, curved cirri.

The mouth in all the Crinoidea, with one exception (Actinomstra), is situated in the centre of the oral (upper) surface, and the anus in all, with the same exception, is eccentric and inter-radial. Running outwards from the mouth are a series of very narrow ambulacral grooves, one of which extends along the oral surface of each arm, giving off branches to the arm-branches and to the pinnules. Bordering the ambulacral grooves and their branches are a pair of rows of short tubular tentacles, which correspond morphologically with the tube-feet of the other classes, but are devoid of the terminal suckers, and are not locomotor, but probably sensory and respiratory in function.

The cœlome in the Echinoderms is a wide cavity lined by a ciliated colomic epithelium and containing a corpusculated fluid. Prolongations of it pass out into the rays, and, in the Ophiuroidea and Asteroidea, between the layers of the body-wall. In the Crinoidea it contains numerous strands of connective tissue. Special organs providing for the respiration of this fluid are the dermal branchioe or papula, the Stewart's organs, and the respiratory trees. The first of these, which are confined to the Asteroidea and Echinoidea, have been described in the accounts of the Starfish and Sea-urchin. In most Asteroidea they occur only on the dorsal surface, but in some forms they are present on the ventral surface as well. In some of the Echinoids the place of dermal branchiæ in providing for the respiration of the compartment of the cœlome enclosing Aristotle's lantern (lantern-cœlome) is taken by Stewart's organs, arborescent bodies which project inwards from the peristome. The respiratory trees are referred to below in connection with the enteric canal.

Some reference has already been made, in deseribing the general form of the body, to the ambulacral system of vessels. A 
ring-like circum-oral vessel (ring-vessel) in nearly all cases sends off a series of radicl branches, one passing along each of the rays or ambulacral areas and giving off branches to the ampullæ of the tube-feet or to the tentacles. In most of the Holothuroidea branches pass forwards to the circlet of shield-shaped or branched oral tentacles, and in some cases there are vesicles or ampulloe at their bases. In the Apoda, in which tube-feet are wanting, radial vessels are also absent, and the vessels to the tentacles come off directly from the ring-vessel. In all the classes, except Crinoidea, one or more bladder-like appendages - the Polian vesicles-are in most cases connected with the ring-vessel. The racemose vesicles, or Tiedemann's vesicles (p. 383), are characteristic of the Asteroidea. In all, except the Crinoidea and the majority of the Holothuroidea, there is a communication between the ring-vessel and the surrounding water through the madreporic canal. In the Asteroidea, and in Cidaris among the Echinoidea, the wall of this tube is strengthened by numerous calcareous ossicles. In the Asteroidea, Ophiuroidea, and Echinoidea, the communication with the exterior is through the madreporite. The fine pores perforating the madreporite and placing the madreporic canal in communication with the exterior, and the madreporic canal itself, are lined with strong cilia which move so as to drive a strong current inwards - the effect being to keep all parts of the ambulacral system in a condition of turgidity. In the few Holothuroids in which such a communication exists (Elasipoda) there is usually a simple opening, but sometimes a number of pores crowded together. In the remainder of the Holothuroidea the distal end of the madreporic canal, or canals, lies free in the interior of the body-cavity, with which it is placed in communication by a number of perforations. In the Crinoidea there is no madreporic canal; but the ring-vessel is placed in communication with the cœlome by means of a system of ciliated vater-tubes, while the colome communicates with the exterior through a number of minute water-pores, which perforate the oral body-wall. The fluid contained in the ambulacral system is similar to that in the cœlome, and contains similar corpuscles. In one Ophiuroid, however, the ambulacral system contains corpuscles coloured red with hæmoglobin. Tiedemann's vesicles appear to have the function of manufacturing the corpuscles.

It cannot be definitely stated that a blood-vascular system exists in the Echinoderms. But two systems have been regarded as playing the part of blood-vessels-the perihomal system and the homal system. Neither of these systems comprises vessels with contractile walls, and there is no definite circulation of the contained fluid. The perihæmal, or, as it is sometimes termed, pseudohæmal system, is present in all the classes of the phylum. When typically developed (Asteroidea, Ophiuroidea) it consists of a ring-like circum-oral vessel or sinus and five radial 
vessels given off from it, together with an axial sinus and aboral ring-vessels. These "vessels" are channels with a definite epithelial lining, and are of the nature of specialised parts of the cœlome, from which they are developed. In Asteroidea and Ophiuroidea the radial and ring-vessels, which lie between the corresponding parts of the ambulacral and epidermal nervous systems, are divided into two parts by a longitudinal septum, vertical in the radial, oblique in the ring-vessel. The axial sinus is nearly vertical in direction and partly encloses the axial organ in the way already described (p. 384). At its oral end it opens into the inner division of the circum-oral vessel : at its aboral end it opens into, or becomes closely applied to, the aboral vessel, which is in the form of a ring giving off radial branches towards the gonads : it also may communicate aborally with several of the pore-canals of the madreporite, and opens into the madreporic canal itself. In the Echinoidea the arrangement of the parts is modified in certain important respects. An oral ring-sinus is absent unless it be represented by the lantern-cœlome. The radial vessels of the system do not open orally into the lantern-cœlome : aborally they also terminate blindly, not opening into the aboral ring-sinus. The axial sinus is largely filled by the axial organ : it terminates blindly at the oral end; aborally it communicates with the madreporic canal and is not connected with the aboral sinus. In the Holothuroidea there are five radial sinuses extending through the ambulacral areas between the superficial radial nerve and the radial ambulacral vessel, ending blindly aborally and opening orally into an oral ring-sinus. There is no axial sinus. In the Crinoidea the perihæmal system is greatly reduced, though representatives of the radial siruses are present in the same situation as in the other classes.

The general disposition of the lacunar or so-called hæmal system in the Asteroidea has been described in the account given of the structure of the Starfish (p. 380). Save for certain minor alterations which are involved in the change in the position of the madreporite, the system is arranged in the Ophiuroidea on the same plan as in the Asteroidea. In the Echinoidea there is an oral ring giving off five radial strands which in the greater part of their course occupy the typical position between the superficial radial nerve and the radial ambulacral vessel; aborally they terminate blindly. A gastro-intestinal system given off from the oral ring is highly developed, and there is an axial plexus in the axial organ and an aboral ring, with strands passing to the gonads, as in the Asteroidea. In the Holothuroidea there is an oral ring with radial strands, and a well-developed gastro-intestinal system. In the Crinoidea this system of lacunæe is highly developed and complicated in arrangement.

Whatever be its functions, this system is not a system of blood- 
vessels. It is made up of strands of a kind of gelatinous connective tissue, with many leucocytes, permeated in a very irregular way by minute lacunæ without definite walls. The great development of the gastric and intestinal branches of this system in soine (Echinoids, Holothuroids), lends support to the view that its main functions are connected with the absorption and distribution of nourishment.

The axial organ (genital stolon) of the Echinodermata is closely connected both with the perihæmal and hæmal systems. Its general structure and relations in the Asteroidea have already been described (p. 384). In the Ophiuroidea there is a close correspondence with the Asteroidea, the chief differences being such as are involved in the change in the position of the madreporite from the aboral to the oral surface, and the resulting change in the direction of the madreporic canal and associated axial sinus and axial organ. In the Echinoidea the essentials are the same; but the axial organ has grown round the axial sinus so as to enclose it completely.

The enteric canal varies in the five classes more than any of the other systems of organs. It is a simple tube in the Holothurians and Echinoids, passing spirally through the body from the mouth at the oral pole to the anus at the opposite pole. In most of the latter group a complex masticatory apparatus with five teeth-the so-called "lantern of Aristotle" -is situated at its anterior extremity; the corresponding region in the Holothurians is surrounded by a circlet of ossicles, which protect the nervous and vascular rings and into which the longitudinal muscles of the body-wall are inserted.

In the Echinoidea there is a tubular cæcum, the siphon, connected with the intestine. In the Holothurians the so-called "respiratory trees" (absent in the Elasipoda and the Apoda) are branched appendages-usually two in number, sometimes single - of the cloaca or posterior wider portion of the intestine, and the "Cuvierian organs" are simple filiform glandular tubes, also connected with the cloaca.

The functions of the siphon and of the respiratory trees have already been referred to in the accounts of Echinus and Cucumaria. The Cuvierian organs, which occur only in a limited number of Holothurians, correspond to undivided basal branches of the respiratory trees: they are defensive organs, the animal when attacked throwing out numbers of these long filaments, which are very viscid and have the effect of entangling and hampering the assailant; but they may also have an excretory function.

In the Crinoidea the alimentary canal is simply a coiled tube with both mouth and anal opening on the same (actinal) surface of the body. In the Ophiuroids the central mouth leads into a 
simple sac giving off short diverticula, and there is no anal aperture. In the Asteroidea the alimentary canal is more complex than in the other classes. The stomach is divided, as already described in the account of the examples, into two portions, the cardiac and the pyloric, the former giving off five large rounded radial diverticula - the cardiac pouches or cardiac cæea, and the latter five pairs of very long branched diverticula-the pyloric or hepatic cæca. The intestine is short and conical, and opens, in all but a few, by an anal aperture. In some Asteroidea (as in Anthenea, Figs. 308 and 310) the intestine has connected with it a system of five elongated bifurcated inter-radial intestinal cæca; in others (as in Asterias, Fig. 306) these are represented only by two or three lobed diverticula. In one member of the class there are also ten cæca connected with the œsophagus.

In the nervous system of the Echinodermata three distinct parts, the relative development of which differs in the different classes, are to be recognised. These are the epidermal or superficial, the deep, and the aboral. The epidermal system is well developed in all the classes: its principal parts are a circumoral nerve-ring and radial branches, but a plexus of nerve-fibres with occasional nerve-cells extends from it through the epidermis. In the Ophiuroids the radial nerves and the ring nerve are similar in their arrangement to what is to be observed in the Asteroids, but are more deeply placed, being covered over by the investing calcareous plates. The deep-lying nervous system is absent in the Crinoidea, very feebly developed in the Echinoidea, but well developed in the Asteroidea, Ophiuroidea, and Holothuroidea. Its general arrangement has already been described in the account of the Starfish. The aboral system is best developed in the Crinoidea and is absent altogether in the Holothuroidea.

The sexes are distinct in all the Echinoderms, with one or two exceptions; but there is very rarely any trace of sexual dimorphism. Asterina gibbosa, the Starfish the development of which has been described (p. 388), is one of the exceptional hermaphrodite forms ; the young animals of this species are male, producing sperms, but at a later stage they become female and produce only ova. In the family Synaptidæ of the Apoda there are also numerous examples of hermaphroditism, the animal at first producing ova, later only sperms. In Amphiura squamata, an Ophiuroid, both ovaries and testes are present at once. The gonads, ovaries or testes as the case may be, are branching bodies, inter-radial in position, and usually in pairs. In the Asteroidea there are five pairs, the ducts from which open usually on a special plate on the aboral surface, but in one or two species on the oral surface. In the Echinoidea there are five ovaries or testes, the five ducts of which open on the genital plates of the apical system. In the 
Ophiuroidea there are five pairs of gonads, a pair in the walls of each of five genital burse, which open on the exterior by slits on the oral surface close to the mouth. In the Holothuroidea there is only a single branched gonad, sometimes imperfectly divided into two, with a duct opening on the dorsal surface not far from the mouth. In the Crinoidea the ovaries and testes occupy a remarkable position, being situated in the dilated bases of the pinnules; but, as in the other classes, they are conwected by means of a genital rachis running through the arm with a centrally situated genital stolon.

Development and Metamorphosis.-A few of the members of each class of Echinoderms are viviparous, in the sense that the development of the young takes place in some sheltering cavity, or brood-pouch, on the surface of the body of the parent. But in most, development takes place externally, and the larvæ are freeswimming. The ovum in all undergoes regular and nearly equal segmentation, resulting in the formation of a ciliated blastula, which becomes invaginated so as to form a typical gastrula, like that of some Coelenterata (p. 173). The invaginated cells form the lining membrane (the endoderm layer) of an internal cavity-the primitive alimentary cavity or archenteron; the enclosing cells form the ectoderm; between the endoderm and ectoderm, and derived from the former, appear the cells of the mesoderm or middle layer. From the archenteron is given off a hollow outgrowth, the enterocale, from which are derived the body-cavity with its enclosing peritoneal membrane, and the vessels of the ambulacral system with their various appendages. In the Crinoidea the vesicle destined to form the ambulacral system is developed independently of the colomic vesicles destined to form the bodycavity. A canal opening on the exterior by a dorsally situated opening, the dorsal pore (sometimes double), is formed by invagination from the surface ectoderm, and comes into relation with a canal arising as an outgrowth from the rudimentary ambulacral system to form the foundation of the madreporic canal of the adult. In the Crinoidea five dorsal pores and five canals are developed, but the two sets of structures do not enter into direct communication (see p. 408).

The part of the enterocole (hydrocole) destined to give rise to the ambulacral system, at first rounded, becomes compressed, and subsequently divided round the border into five lobes. Each of these lobes grows outwards to become developed subsequently into one of the five radial ambulacral vessels of the Echinoderm; the central part of the hydrocnele gives rise to the ring-vessel surrounding the osophagus.

The cilia, which at first (in the gastrula stage) covered the surface of the larva uniformly, become restricted to a peri-oral band (Fig. 343, por) surrounding a concave area on which the mouth 
opens. A smaller adoral band (aor) in the interior of the mouth has the function of attracting nutrient particles. The
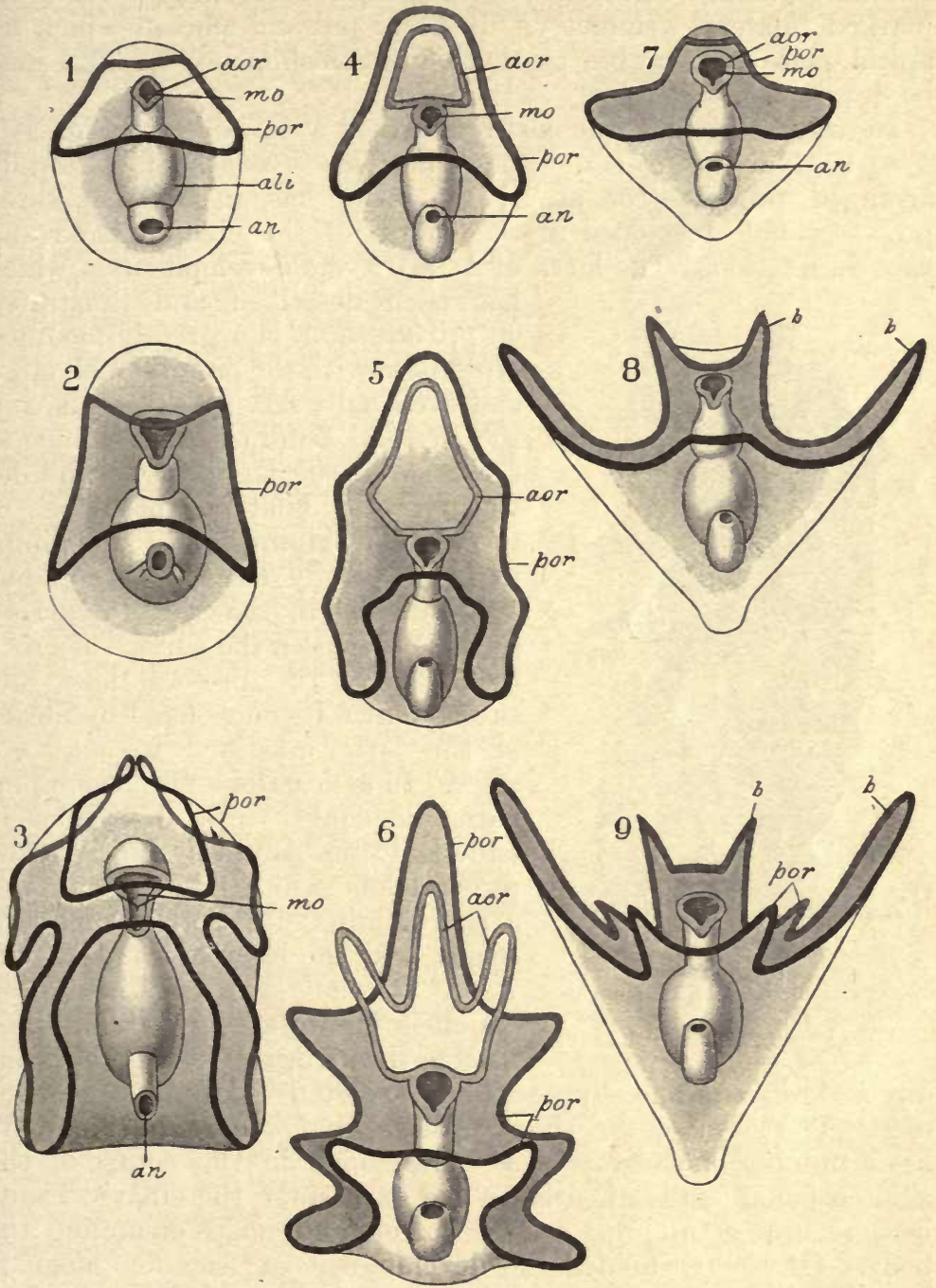

Fig. 343.-Diagrams of the development of the larvæ of Fchinoderms. 1, Primitive form of Echinoderm larva; 2 and 3, Development of an auricularia (Holothuroidea); 4, 5, and 6, Development of a bipinnaria (Asteroidea); 7,8 , and 9, Development of a pluteres (Echinoidea and Ophiuroidea). aor, adoral band of cilia ; ali. alimentary canal; $a n$. anus; $b, b$. processes or arms; mo. mouth; por. peri-oral ciljated band and processes. (From Leuckart and Nitsche's Diagrams.)

peri-oral band undergoes characteristic changes in the different classes, and the form of the larva at the same time becomes 
modified by the formation, except in the Crinoidea, of variously arranged processes along the course of the peri-oral band. The resulting larva, echinopocdium or dipleurula, always exhibits marked bilateral symmetry. It has a pre-oral lobe on which an apical plate comparable to that of the trochophore (p. 322) may be developed.

In the Asteroidea the larva is either a bipinnaria (Fig. 343, 4 to 6 ) or a brachiolaria. The former has a series of bilaterally arranged processes or arms; the latter has, in addition, three processes not developed in the course of the ciliated band and used for fixation. The larva of Asterina, the development of which

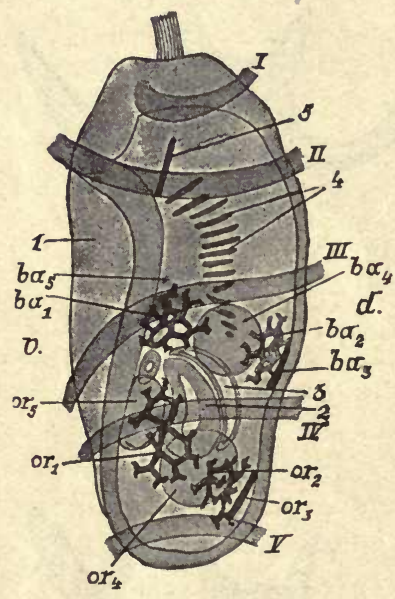

Fia. 344.-Free-swimming larva of Antedon, from the left side. $I-V$, ciliated bands; $b a_{1}$ to $b a_{5}$, the five basals; or 1 to or ${ }_{5}$, orals ; 1 , vestibule; 2 , intestinal vesicle; 3 , right enterocole; 4 , calcareous joints of the stalk; 5 , pedal plate. (From Lang, after Sceliger.) has been described and illustrated on pp. 388-393, is a greatly modified bipinnaria with the pre-oral lobe large and eventually serving as a stalk, and the pre-oral band of cilia confined to the edge of the larval organ and devoid of the bilateral processes of the normal bipinnaria. The bipinnaria is usually free-swimming, but sometimes, as in the case of Asterina (p. 391), creeps on the surface of a rock by means of the pre-oral lobe, and subsequently becomes fixed by means of the latter modified to act as a stalk. In at least one form the bipinnaria, developed in a brood-pouch, adheres to the parent by means of the pre-oral lobe which takes the form of a short stalk. In both the Ophiuroidea and the Echinoidea (Fig. 343, 7 to 9) the larva has the form which is known as the pluteus. The pluteus has a series of slender arms directed forwards and supported by a skeleton of delicate calcareous rods. The larva of the Holothuroidea, the auricularia (2 and 3), has a number of short processes developed in the course of the ciliated bands; subsequently, in the pupa stage, the ciliated bands become broken up into a series of ciliated hoops encircling the body. Of the Crinoidea the development of Antedon alone is known. Blastula and gastrula stages occur as in the Starfish, but the history of the archenteron and its diverticula is widely different, though the outcome is the same-viz., the differentiation of a primitive enteric canal, an anterior cœlome, from which a hydrocole becomes separated off, and a pair of cœlomic sacs. The larva (Fig. 344) becomes barrel-shaped, and the pre-oral lobe, which is not very conspicuous, develops an ectodermal thickening 
with a tuft of sensory cilia. The vibratile cilia on the surface are arranged in five transverse bands $(\mathrm{I}-\mathrm{V})$. Between the second and third of these is a wide shallow depression, the vestibule or stomodæum (1), which does not communicate with the mouth. After remaining in the free condition for a short time, the larva (Fig. 345) fixes itself by means of the pre-oral lobe, which elongates into a stalk (11), the cilia meanwhile being lost, and the apical plate absorbed. The vestibule becomes closed, and a solid rudiment of the adult cesophagus arises in close apposition with it. Round the resophagus the hydrocœele grows in the form of a ring. The vestibule (5) with the œsophagus and hydrocœle are rotated so as to come to lie at the free extremity. The radial canals first appear as five tentacles which at first project into the cavity of the vestibule, and subsequently-when the latter opens out, as it soon doeson the exterior. The œsophagus (8), meanwhile, has become completed, and the mouth pierces the bottom of the now open vestibular cavity. The arms appear as five processes which soon bifurcate: the five radial canals become applied to them and undergo a corresponding division. The first plates are formed while the larva is still in the free condition; in the fixed condition they undergo further development, and extend into the arms as they grow. After about six months this pentacrinoid larva becomes free by the absorption of the stalk and develops into the adult Antedon.

In the transition from the bilateral larva-pluteus, bipinnaria,

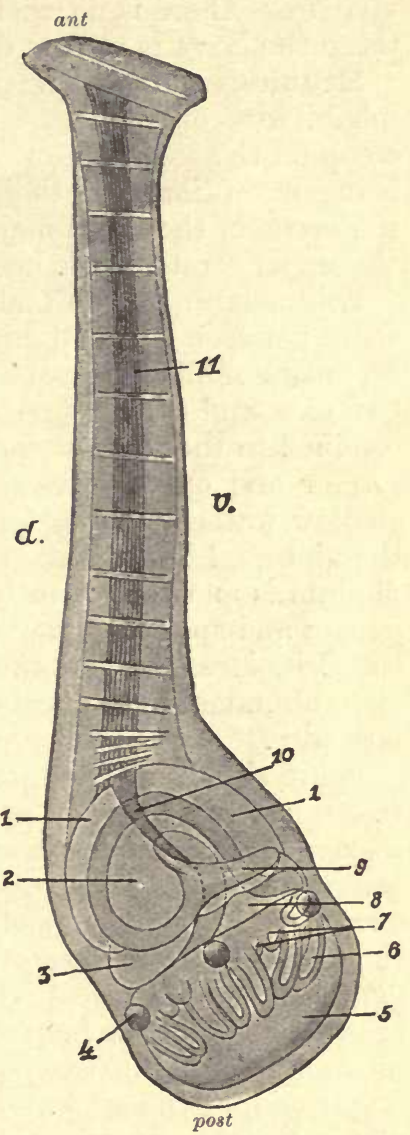

Fig. 345.-Stalked larva of Antedon, from the right side; calcareous plates not represented. 1, right coelomic sac ; 2, enteric cavity ; 3 , left coelomic sac; 4, sacculi ; 5 , vestibule, still closed; 6, primary tentacles; 7 , secondary tentacles; 8 , œesophagus ; 9 , rectum ; 10, axial organ; 11, fibrous strands in the stalk.' (From Lang, after Seeliger.) brachiolaria, or auricularia - to the radial adult there is a marked metamorphosis. As the adult form is developed on one side of the larva, with its principal axis at right angles to that of the latter, the larval arms or processes become absorbed. In the Holothuroidea and Ophiuroidea all the organs of the larva are 
carried on into the adult; in the Asteroidea and Echinoidea the larval mouth and oesophagus are abolished and a new permanent mouth and osophagus formed as a fresh invagination from the surface. In the very limited number of Echinoderms which are viviparous there is no such marked metamorphosis; but even in these the larva is at first distinctly bilateral in its symmetry.

Ethology, etc.-The Echinodermata are without exception, ${ }^{1}$ inhabitants of the sea. In the adult condition the majority creep on the sea-shore or on the sea-bottom, the stalked Crinoids being exceptional in their permanently attached condition; but the larvæ of the great majority are pelagic-i.e. live swimming in the upper strata of the ocean.

Echinoderms inhabit all depths of the sea, ranging from the shore between low and high-water limits to the greatest depths. Members of all the classes are found at all depths; but the stalked Crinoids, and the Elasipoda among the Holothuroidea are virtually confined to the deepest waters of the ocean, only one genus of the former and one species of the latter occurring in comparatively shallow water. Echinoderms are found in the seas of all parts of the globe. Like the majority of marine invertebrate groups, the phylum is more abundantly represented, as regards the number of genera and species as well as of individuals, in the warmer regions; the Crinoidea, the Holothuroidea and the Echinoidea are all much more abundant in tropical and warm temperate seas than in colder latitudes.

Echinoderms are of gregarious habits, large numbers of the same species frequently being found closely associated together in a comparatively narrow area. The movement of locomotion in the Starfishes is, as previously described (p. 377), a slow creeping one, through the agency of the tube-feet: the same holds good of the Echinoidea and those of the Holothuroidea that possess tube-feet (Pedata). The footless Holothurians (Apoda, such as Synapta) creep along with the help of the tentacles. Most of the Ophiuroids move by lateral flexions, sometimes sluggish, sometimes remarkably rapid, of the arms. The Comatulæ, on the other hand, swim along by the flexion and extension of the pinnate arms propelling them through the water. Many Asteroids, Ophiuroids, and Echinoids bury themselves in sand or mud; others creep into narrow fissures in rock or coral. Movements of manducation are performed by the tentacles in the Holothurians: in the Starfishes the mouth papillæ are separated from one another and the cardiac part of the stomach everted in order to enfold the prey, often of relatively large size. In those Echinoidea that possess a lantern of Aristotle there are very powerful and efficient movements of mastication. On the whole, as might be expected from the comparatively highly developed muscular and nervous systems,

\footnotetext{
1 One species of Synapta is said to inhabit brackish water.
} 
the co-ordination of movement is very much more complete in the Echinodermata than in the groups already dealt with.

A remarkable characteristic of the Echinoderms is the faculty of self-mutilation which many of them possess, together with the capacity for replacing parts lost in this way or by accidental injury. This is most marked in many Ophiuroids, some Asteroids, and some Holothurians, and does not occur at all among the Echinoids. Many Brittle-stars and some Starfishes, when removed from the water, or when molested in any way, break off portions of their arms piece by piece until, it may be, the whole of them are thrown off to the very bases, leaving the central disc entirely bereft of arms. A central disc thus partly or completely deprived of its arms is capable in many cases of developing a new set; and a separated arm is capable in some instances of developing a new disc and a completed series of arms. In some Starfishes (Ophiuroids and Asteroids) a process of separation of the arms and their development into complete individuals frequently occurs altogether independently of injury, and seems to be a regular mode of reproduction in these exceptional cases. Many Crinoids, also, readily part with their arms when touched and are able to renew them again; and some, at least, are capable of renewing the visceral sac of the central disc when it has become accidentally removed.

In the case of many Holothurians it is the internal organs, or rather portions of them, that are capable of being thrown off and replaced - the œesophagus, or the cloaca with the Cuvierian organs, or the entire alimentary canal, being ejected from the body by strong contractions of the muscular fibres of the body-wall, and in some instances, at least, afterwards becoming completely renewed.

Four out of the nine classes of the phylum Echinodermatathe Cystoidea, Blastoidea, Edriasteroidea, and Carpoidea-are represented only by fossil forms; and these are found only in rocks of the older (Palæozoic) formations, no representatives having survived to more recent times. Of the five classes that have living members, one, the Crinoidea, was very much more abundantly represented in the older geological periods than it is at the present day, the remains of stalked Crinoids forming great beds of limestone of Silurian to Carboniferous age: the free Comatulæ only appeared at a much later period. The other classes, or at least the Echinoidea, Asteroidea, and Ophiuroidea, were represented at a very early period by forms not very widely different from those now living; but the earliest Echinoids were peculiar in having the number of rows of plates variable, and in the plates overlapping one another. The Holothuroidea, owing to their comparatively soft integument, were less fitted to leave any remains in the form of fossils, and it 
is not till we come to the Mesozoic Period that undoubted traces of their existence are found.

Affinities. - The presence of radial symmetry was formerly regarded as involving a near relationship with the Coelenterata, which were grouped with the Echinodermata under the comprehensive class-designation of Radiata (see section on the History of Zoology). But, leaving out of account the presence of a bilateral symmetry underlying and partly concealed by the radial, we are led by a study of the anatomy of the various systems of organs to the conclusion that the Echinoderms are in no way closely or directly related to the Cœlenterates. One very great and very important difference between the two phyla consists in the presence in the Echinodermata of an extensive cœlome or body-cavity lined by mesodermal epithelium between the alimentary canal and the body-wall. In addition to this the Echinoderms are characterised by the possession of highly elaborated systems of organs-alimentary, vascular, and nervous-such as occur in none of the Colenterates, all of which exhibit extreme simplicity in their internal structure. A further point of difference, not perhaps of so much importance, is the absence in the Echinoderms of any tendency to form colonies of zooids by asexual multiplication by means of buds: all Echinoderms are simple, i.e. non-colonial, animals, and each of them is developed, save in certain very exceptional cases, as a result of a sexual process from an impregnated ovum. In spite, then, of the radial symmetry, we are forced to the conclusion that the Echinodermata are not more nearly related to the Colenterata than to some of the groups of Worms. They are, in fact, a singularly isolated group, and we look in vain ainong the known members, living and fossil, of other phyla, for any really close allies. The intermediate forms-whatever they may have been like-between the Echinoderms and other groups have become extinct, and have left no remains in the form of fossils, or such remains have not yet been discovered. So difficult has it been found to connect the Echinoderms with other animal types that it has even been proposed to regard an Echinoderm as a radially arranged colony of zooids connected together centrally, each ray being a zooid equivalent to an entire simple worm-like animal. But the history of the development is entirely at variance with such a view.

Whatever may have been the group of animals from which the Echinodermata were developed, there is every probability that it was a group with bilateral and not radial symmetry. The radial symmetry is evidently, as has already been pointed out, of a secondary character; it is only assumed at a comparatively late period of development, and even in the adult condition it does not completely disguise a more primitive bilateral arrangement of the parts. Accordingly, within the phylum itself, it is reasonable 
to regard those classes as the more ancient which have the radial symmetry less completely developed. Again, the free condition which characterises all existing Echinoderms with the exception of a few Crinoids, is probably less primitive than the attached, since in other phyla the radial symmetry is co-ordinated with, and seems to be developed on account of, a fixed, usually stalked condition. Probably, then, stalked Echinoderms were the progenitors of the existing free forms, and these werc preceded by primitive free forms with pronounced bilateral symmetry. It appears to be most probable that this ancestral form possessed the most essential features of the dipleurula larva (p. 432); i.e., that it was a bilaterally symmetrical form with a pre-oral lobe, simple alinentary canal with mouth on ventral surface and anus at posterior end ; that it had a colome, originally developed from the archenteron of the gastrula; and that it had a band of strong cilia running around the concave ventral surface. Such a dipleurula-like form became converted, it is supposed, into a fixed form, such as that represented by some of the extinct class of the Cystoidea. The fixation must be supposed to have become effected through the medium of the pre-oral lobe, and further changes must have involved the shifting of the mouth to about the middle of the free surface. From this primitive Cystoid, thus regarded as the most primitive of all known Echinoderms, the remaining classes, both fixed and free, might have been derived by some such order of succession as that indicated in the diagram below (Fig. 346).

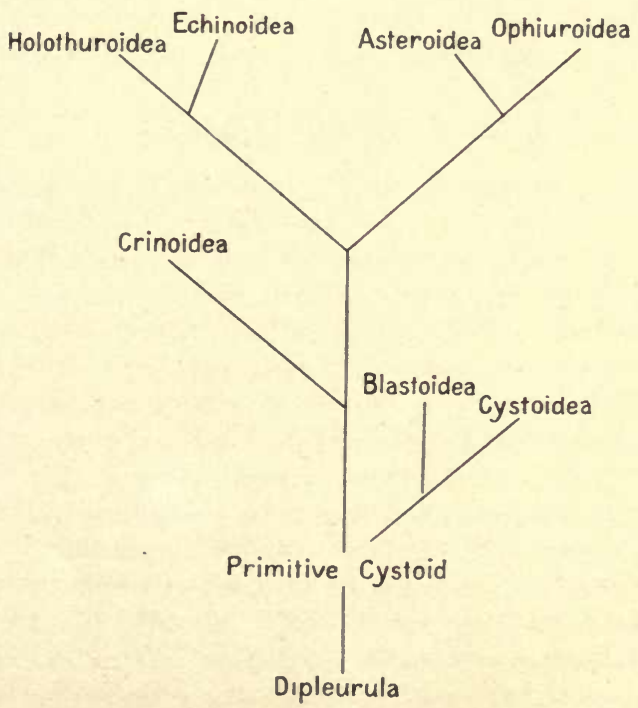

FIG. 346.-Diagrain to illustrate the relationships of the classes of the Echinodermata. 
According to another view, however, the most primitive of existing Echinoderms are Synapta and its allies (Holothuroidea apoda). The other Holothuroids are supposed, according to this conception of the relationships of the various classes, to have been derived from a Synapta-like ancestor. From the primitive stock of the Holothuroids is supposed to have been derived a form which gave origin to all the stalked classes. From this ancestral stalked Echinoderm, again, the remainder of the free classes-the Echinoidea, Asteroidea and Ophiuroidea-are regarded as having been descended.

Possible relationships between the Echinodermata and the Chordata will be referred to in the discussion of the affinities of the latter phylum. 


\section{SECTION $\mathrm{X}$}

\section{PHYLUM ANNULATA}

THE phylum Annulata comprises four classes of Worms-the Chotopoda or Earthworms and marine Annelids, the Archi-Annclida, the Gephyrea, and the Hivudinea or Leeches. All of these, except the Gephyrea, have the elongated body divided externally into a number of rings, which represent a division of the internal parts into a series of segments or metameres. There is usually an extensive ccelome, and there is in most a system of blood-vessels. The nervous system consists of a cerebral ganglion, œsophageal connectives, and a double ventral nerve-cord, which in all but the Gephyrea is segmented into a series of ganglia. The organs of excretion are in the form of metamerically arranged pairs of tubes, the nephridic or ssgmental organs, closed internally or leading from the colome to the exterior; and united with these, or distinct from them, are a series of paired ducts, the colomoducts, for the passage outwards of the reproductive elements.

\section{CLASS I.-CH ETOPODA.}

The Chætopoda, comprising the Earthworms, Fresh-Water Worms, and Marine Annelids, are Worms the body of which, unlike that of a Flat-worm or a Round-worm, is made up of a series of more or less completely similar segments or mctameres, each containing a chamber or compartment of the body-cavity and a section of the alimentary canal and other organs. At the sides of each are typically a pair of muscular processes, the parapodia, which do duty as limbs, bearing bundles of sel ce (chcetce) or bristles and usually also certain tactile appendages, the cirri. There is an extensive coelome, incompletely divided into a series of chambers corresponding to the segments by a series of muscular partitions which act also as mesenteres, being attached internally to the alimentary canal. The latter extends throughout the length of the body; the intestine is usually constricted, the constrictions being either segmental i.e. opposite the middle of the segments, or inter-segmental i.e. opposite the intervals between 
the segments. There is a well-developed blood-vascular system in the majority of the Chrtopoda, and organs of respiration in the shape of gills or branchiæ are usually developed. 'The excretory organs are in the form of segmentally arranged pairs of tubes, the nephridia. The nervous system consists of a bilateral principal ganglion or brain situated in the prostomium, and a double chain of ganglia extending throughout the body. The sexes are in some distinct, in others united. When a definite larval form occurs it is a trcchophore (cf. p. 322).

\section{Examples of the Class.}

\section{a. Nereis dumerilii. ${ }^{1}$}

General External Features. - Various species of Nereis occur abundantly between tide-marks on the sea-shore, under stones, and

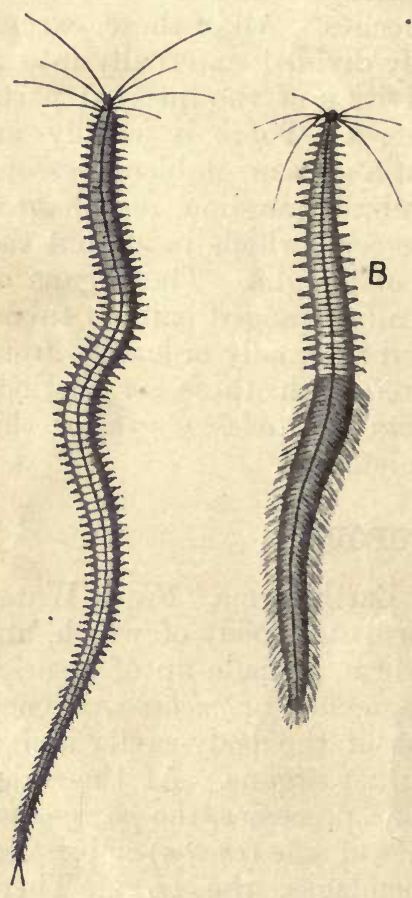

FIG. 347.-Nereis dumerilii, natural size. A, Nereis phase ; B, Heteronereis ph ase. (After Claparède.) among sea-weed, in all parts of the world. The worm varies considerably in colour even in the same speeies, the differences being partly due to differences in the stage of development of the sexual elements. In $N$. dumerilii the prevailing colour is some shade of violet, with a blush of red in the more vascular parts due to the.bright red colour of the blood. In shape (Fig. 347) the body, which may be about 7 or 8 centimetres in length, is long and narrow, approximately cylindrical, somewhat narrower towards the posterior end. A very distinct head, bearing eyes and tentacles, is recognisable at the anterior end; the rest is divided by a series of ring-.like narrow grooves into a corresponding series of segments or metameres, which are about eighty in number altogether; and each of these bears laterally a pair of movable muscular processes called the parapodia, provided with bundles of bristles or setoe (cholce). The head (Fig. 350) consists of two parts, the prostomium (prost) and the peristomium (perist). The former bears

1 Though Nereis dumerilii is here named as the example, and the majority of the figures refer specially to that species, the description given would apply almost equally well to a considerable number of species of the genus. 
on its dorsal surface four large rounded eyes, in front a pair of short cylindrical tentacles $(t \in n t)$, and further back a pair of somewhat longer stout appendages or palpi $($ palp). The peristomium, which has some resemblance to the segments of the body, though wanting the parapodia, bears laterally four pairs of long, slender, cylindrical tentacles (pcrist. lent): on its ventral aspect is a transversely elongated aperture, the mouth. The segments of the body differ little in external characters from one another

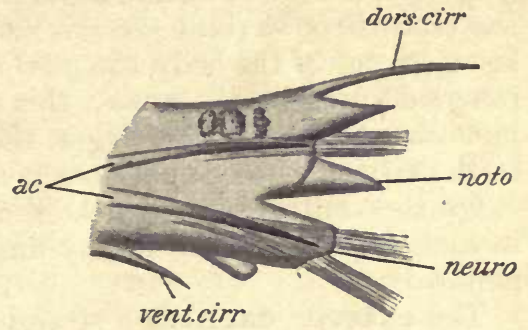

FIG. 348, A.- Nereis dumerilii. A single parapodium, magnified : ac. aciculum; don's. cirr. dorsal eirrus: neuro. neuropodium; noto. notopodium; vent. cirr. ventral cirrus. (After Claparèdc.) throughout the length of the worm. Each bears laterally a pair of parapodia, which in the living animal are usually in active movement, aiding in creeping, or acting as a series of oars for propelling it through the water. When one of the parapodia (Fig. 348, A) is examined more attentively it is found to be bircumous, or to consist of two distinct divisions-a dorsal, which is termed the notopodium (noto), and a ventral, which, is called the neuropodium (neuro). Each of these is further subdivided into several lobes, and each bears a bundle of setæ. Each of the bundles of setæ is lodged in a sac formed by invagination of the epidermis-the sctigerous sac-and is capable of being protruded or retracted and turned in various directions by bundles of muscular fibres in the interior of the parapodium. In each bundle there is, in addition to the ordinary setæ, a stouter, straight darkcoloured seta ( $\alpha c$. ), the pointed apex of which projects only a short distance on the surface; this is termed the aciculum. The ordinary setæ (Fig. 348, B) are exceedingly fine, but stiffish, chitinous rods, of which two principal kinds are recognisable: both have a terminal blade articulating with the main shaft of the seta by a distinct joint; but in the one variety the shaft of

Fir. 348, B.-Nereis dumerilii. Setre highly magnified. (After Claparede.)

the seta is finer than in the other, and the terminal blade long, slender, and nearly straight, whereas in the other variety it is 
short and slightly hooked. On the dorsal side of the parapodium is a short cylindrical, tentacle-like appendage, the dorsal cirrus (Fig. 348, A, dors. cirr), and a similar, somewhat shorter appendage, the ventral cirrus (vent. cirrr), is situated on its ventral side. The last segment of the body, the anal segment, bears posteriorly a small rounded aperture, the anus; this segment is devoid of parapodia, but bears a pair of appendages, the anal cirri, similar in character to the cirri of the ordinary segments, but considerably longer.

On the ventral surface, near the bases of the parapodia, there is in each segment a pair of very fine apertures, the openings of the nephridia.

The enteric canal is a straight tube running throughout the length of the body from the mouth to the anus. Between the outer surface of this tube and the inner surface of the wall of the body is a considerable space-the cœlome, body-cavity, or perivisceral cavity-filled with a fluid, the colomic fluid, containing amœboid corpuscles. The walls of the cœlome (Fig. 351) are lined with a thin membrane, the pcritoneum or colomic exithelium, of which the outer layer-that lining the body-wall-is the parietal layer (par. peri), that covering the outer surface of the alimentary canal the splanchnic or visceral layer (visc. peri). The space is divided by a series of transverse partitions or septa passing inwards from the body-wall to the wall of the alimentary canal opposite the grooves between the segments, and thus dividing the coelome into a series of chambers, each of which corresponds to one of the segments. These partitions are not complete, spaces being left around the alimentary canal and elsewhere through which neighbouring chambers communicate.

The mouth leads into a wide cavity, the buccal cavity, continued back into a pharynx (Fig. $350, p h$ ). These two chambers extend through the peristomium and the first to the fourth segments of the body. They are lined with a tolerably thick cuticle, continuous with a similar layer lining the outer surface of the body, and in the buccal cavity are a number of very small dark brown chitinous denticles, which are very regularly arranged. The posterior part of the pharynx (dentary region) has very thick walls composed of bundles of muscular fibres, which are concerned in the movements of a pair of laterally placed chitinous jaws. Each jaw is elongated in the direction of the long axis of the body, rounded at the posterior end or base where it is embedded in muscle, pointed at the apex, which is strongly incurved; the inner edge is divided into a number of strong serrations or teeth: the whole jaw might be compared to a pruning-hook with its cutting edge deeply serrated (Fig. $349, B$ ).

Behind the pharynx the alimentary canal narrows considerably to form a tube, the oesophagus (ces), which runs through about five segments to open into the intestine. 
Running backwards and inwards from the wall. of the peristomium to the wall of the buccal cavity and pharynx are a number of bands or sheets of muscle, the protractor muscles, by the contraction of which, and the pressure of the cœlomic fluid, this anterior part of the alimentary canal can be everted so as to form a prouoscis (Fig. 349), and thus the jaws are thrust forth and rendered capable of being brought to bear on some small living animal or fragment of animal matter, to be seized and swallowed as food. The eversion is arrested at a certain point by means of a muscular diaphragm passing from the wall of the buccal cavity to that of the first body-segment. The proboscis is withdrawn again by a retractor sheet of muscle, which passes inwards and forwards to be
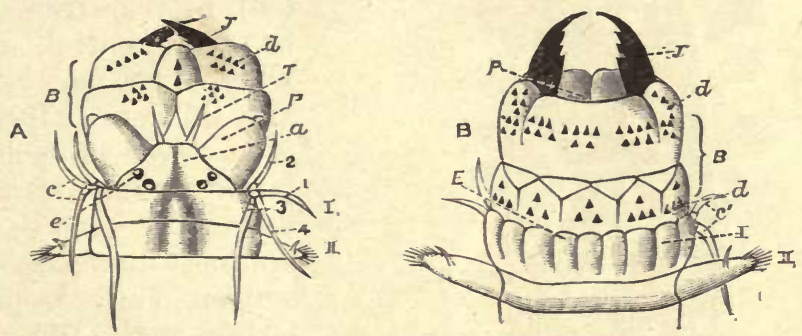

FiG. 349.-Nereis diversicolor, $\times 4$. Head with buccal-region everted. A, dorsal view ; $\mathbf{B}$, ventral view. $a$, prostomium; $B$, everted buceal region; $c, c^{\prime}$, peristomial tentacles, $1,2,3,4$; $\vec{d}$, denticles; $e$, eyes; $\boldsymbol{B}$, lower lip; $P$, palp in $\mathbf{A}$, entrance to pharynx in $\mathbf{B} ; J$, jaw ; $T$, prostomial tentacle ; $I$, peristomium; $I I$, parapodium of first body-segment. (From the Cambridge Natural Histony.)

inserted into the wall of the alimentary canal at the junction of the pharynx and œsophagus.

Into the esophagus open a pair of large unbranched glandular pouches, or caca (Fig. 350, gl), which probably are of the nature of digestive glands. The intcstine (int) is a straight tube of nearly uniform character throughout, regularly constricted in each segment - the constrictions becoming much deeper towards the posterior end of the body. The part of the intestine which lies in the last segment is termed the rectum.

The wall of the alimentary canal (Fig. 351) consists (1) of the visceral layer of the colomic epithelium (visc. peri); (2) of a layer of longitudinal muscular fibres (long. mus); (3) of a layer of circular muscular fibres (circ. mus); and (4) of the enteric epithelium (ent. ep), consisting of close-set, long, narrow cells. To these layers is superadded in the buccal cavity and the pharynx an internal chitinous cuticle, continuous with that of the general outer surface.

Developmentally the buccal cavity and the pharynx constitute the stomodoum, the rectum the proctodoum, the rest of the alimentary canal the mesenteron.

The wall of the body consists of a cuticle, an epidermis or 
deric epithelium, muscular layers, and the parietal layer of the

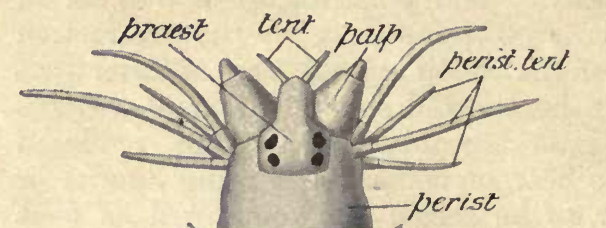
coelomic epithelium ( $p a r$ peri). The cuticle (cut) is a thin chitinous laye which exhibits an iridescent lustre due to the presence of two intersecting systems of fine lines it is perforated by numerous minute openings, the openings of the epidermal glands. The epidermis $(c p)$ is very thin, except on the ventral surface. where it becomes considerably thickened. It consists of a layer of cells containing numerous twisted unicellular glands, which are most abundant on the ventral surface, particularly near the bases of the parapodia; on the dorsal surface the epidermis contains plexuses of fine blood-vessels. The muscular layers are two in number-an external, in which the fibres run circularly (circ. mus), and an internal, in which they run longitudinally. The latter is not a continuous layer, but consists of four bundles of fibres, two dorso-lateral (dors. long. mus) and two ventrolateral (vcnt. long. mus).

Nereis has a well-developed system of vessels filled with blood of a bright red colour. A main dorsal vessel (Figs. 350 and 351, dors. vess) runs from one end of the

body to the other above the alimentary canal, and is visible in places through the body-wall in the living animal. It, as well as 
the majority of the vessels, undergoes contractions which are of a peristaltic character-waves of contraction passing along the wall of the vessel so as to cause the movement of the contained blood. These peristaltic contractions are more powerful in the case of the dorsal vessel than in that of any of the others, and run with great regularity from behind forwards, so as to drive a current of blood in that direction. The contractions are brought about partly by, a series of muscular fibres which are arranged in

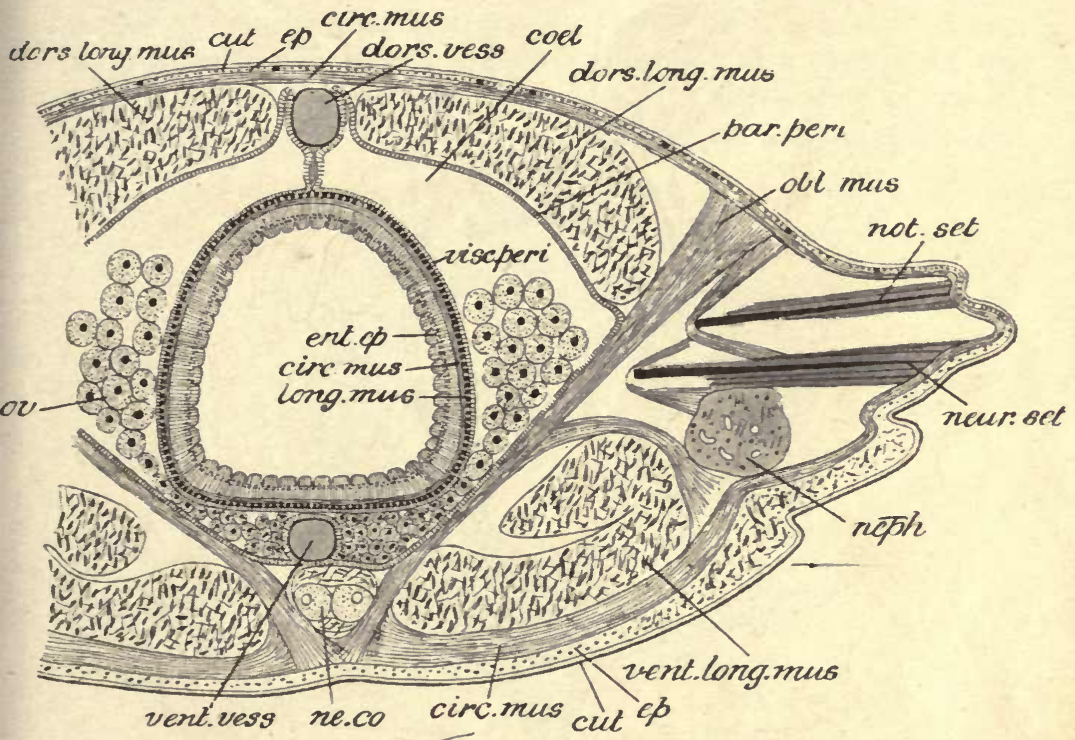

FIG. 351.- Nereis dumerilii. Semi-diagrammatic transverse section of the middle region of the bods. circ. mus. (external), circular layer of muscle of boty-wall ; circ. mus. (internal), circular layer of muscle of wall of enteric canal; col. coelome; cut. cuticle; dors. long. mus. dorsal longitudinal muscles of body-wall ; dors. vess. dorsal vessel ; ent. $c p$. euteric cpithelium ; ep. epidermis; long. mus. longitudinal muscle of wall of enteric canal; ne. co. nerve-cord; neph. nephridium; neur. set. neuropodial setæ and aciculum with their muscles; not. set. notopodial setæe and aciculum; obl. mus. oblique muscle; ov. ovary; par. peri. parietal layer of colomic epithelium; vent. long. mus. ventral longitudiual muscle; vent. vess. ventral vessel ; visc. peri. visceral layer of coelomic cpithelium. (The entire extent of the ccelomic epithelium is not represented.)

rings round the wall of the vessel at short intervals; but the wall of the vessel is itself contractile.

Along the middle of the ventral surface below the alimentary canal runs another large longitudinal vessel, the ventral vessel (vent. vess), in which the current of blood takes a direction from before backwards. Connecting the dorsal and ventral vessels, there are in each segment two pairs of loop-like transverse vessels which give off branches to the parapodia, the alimentary canal, and neighbouring parts. Some of these branches communicate with plexuses of fine vessels in the interior of the lobes of the parapodia and in the integument of the dorsal surface, and with dilatations or sinuses 
situated in the bases of the parapodia. A delicate longitudinal neural vessel accompanies the nerve-cord.

Nereis is devoid of any branchice; but there can be little doubt that the lobes of the parapods with their rich blood-supply, and the areas of integument occupied by plexuses of blood-vessels, subserve the function of respiration.

There is a well-developed nervous system (Fig. 352), which is bilateral and metameric in its arrangement, like the other systems

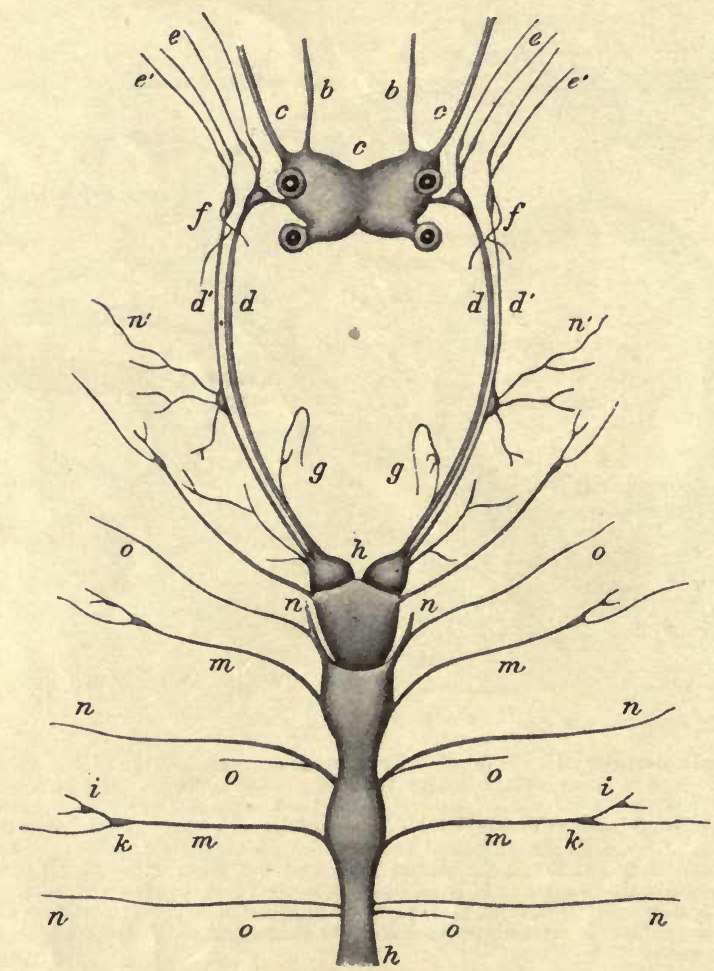

Fia. 352.-Nereis.-Anterior portion of nervous system, comprising the brain, the oesophageal connectives, and the anterior part-of the ventral nerve-cord. (After Quatrefages.)

or organs. Situated in the prostomium is a large bilobed mass of nerve-matter containing numerous nerve-cells, the cerelval ganglion or bruin (c). This gives off tentacular nerves to the tentacles and palpi, and two pairs of short thick optic nerves to the eyes. Behind, two thick nerve-strands, the ocsophageal connectives $(d)$, curve round the mouth in the peristomium to meet on the ventral aspect behind the mouth and below the pharynx. The osophageal connectives with the cerebral ganglion thus form a ring around the anterior part of the enteric canal. From them are 
given off nerves to the two anterior pairs of peristomial tentacles. Running backwards from the point of union of the resophageal connectives along the entire length of the body of the worm, on the ventral aspect, is a thick cord of nerve-matter, the ventral nerve-cord $(h)$. In each segment this cord presents a little dilatation from which nerves are given off to the various parts of the segment : and each of these enlargements is really double, consisting of a pair of closely-united ganglia. The intermediate parts of the cord, between successive pairs of ganglia, are also double, consisting of a pair of longitudinal connectives enclosed in a common sheath. Given off behind from the cerebral ganglion is a system of fine nerves with occasional small ganglia, the stomatogastric or visceral system, distributed to the anterior part of the alimentary canal.

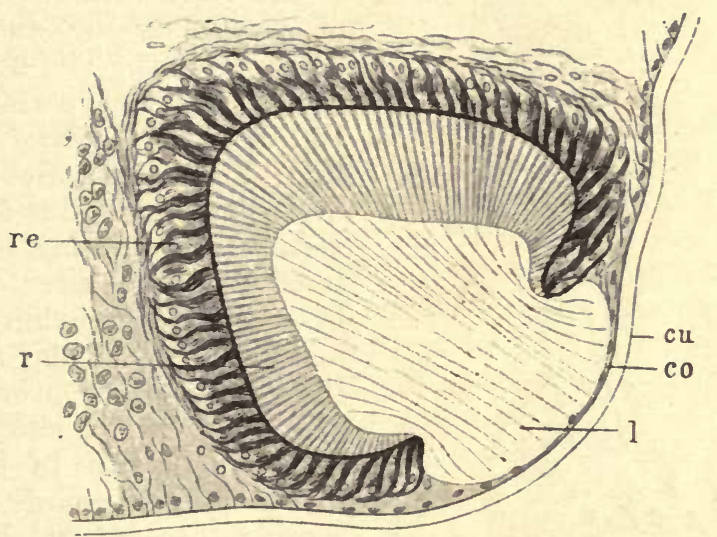

F19. 353.-Nereis.--Section through one of the cyess co. cornea; $c u$. cuticle; $l$. lens; $r$. layer of rods; re. retina. (After Andrews.)

The first ganglion of the ventral cord, which is situated in the third segment, represents at least two double ganglia which have coalesced, as is shown by the fact that it gives off nerves to the two posterior pairs of peristomial tentacles and to the first pair of parapodia.

The tentacles and palpi, as well as the cirri, are probably organs of the sense of touch. The only other sense-organs are the four eyes and the two nuchal organs, all situated on the prostomium. The eye (Fig. 353) consists of a darkly pigmented cup, the retina (re.), with a small rounded aperture, the pupil, and enclosing a mass of gelatinous matter, the lens $(l$.) The wall of the cup is composed of numerous long and narrow cells lying parallel with one another in a radial direction. The outer end of each cell narrows into a nerve-fibre forming part of the optic nerve; near this end is a nucleus; the main body of the cell is densely 
pigmented; the inner part projects towards the lens as a clear hyaline rod $(r)$. The cuticle of the general surface passes over the eye, and a continuation of the epidermis with its cells somewhat flattened, constitutes the cornea $(c o)$. The nuchal organis consist of a pair of pits lined by a special ciliated epithelium with gland-cells, situated in close contact with the posterior part of the brain near the posterior part of the prostomium on the dorsal side. They are regarded as olfactory in function.

The organs which are supposed to perform the function of excretion are a series of metamerically arranged pairs of tubes,

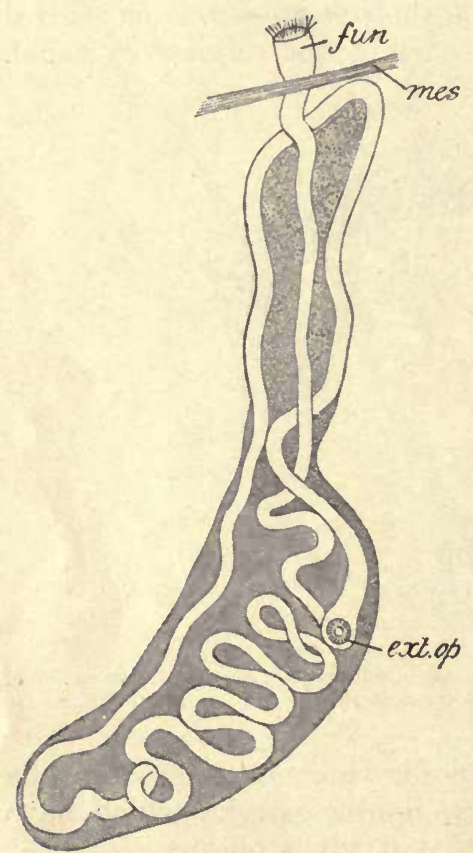

FIG. 354.-IVereis dumerilii. One of the nephridia. ext.op. external opening or nephridiopore; fun. internal funnel or nephrostome opening into the coelome; mes. transverse mesentery or septum. the segmental organs or nephridia (Figs. 350 and 351, neph, Fig. 354 ) occurring in all segments of the body with the exception of several at the anterior and posterior ends. The nephridium consists of two parts-a body and a narrow anterior prolongation. The body is of an irregular oval shape directed nearly transversely, but slanting somewhat; the outer end, situated in the base of the parapodium near its middle, is much the narrower; the inner end is continuous with a narrow prolongation about equal in length to the body, which runs forwards and inwards to become attached to the mesentery. The external opening or nephridiopore (ext. op) is a fine circular pore capable of being widened or contracted, situated on the ventral surface not far from the base of the ventral cirrus. This leads into a canal, ciliated except in its most external part, which runs through the anterior prolongation to its extremity, where it bends sharply back again and runs to the body, through which it pursues an extremely tortuous course to the outer end, and then bends back again and runs in the anterior prolongation to the extremity of the latter, where it opens into the colome through a ciliated bell or funnel ( $f u n$ ), the nephrostome, projecting through the septum into the cavity of the segment next in front of that in which the body of the organ lies. The edge of the nephrostome is produced into a number 
of narrow ciliated processes not represented in the figure. Throughout its course the canal is excavated in a mass of nucleated material of a granular character not distinguishable into cells.

On the dorsal side of each segment, in close relation to the longitudinal muscular bundle, is a specially developed ciliated tract of the colomic epithelium of the nature of a short funnel without external aperture, the dorsal ciliated organ. It is possible that at the time of sexual maturity an aperture is formed through the body-wall opposite this funnel, and that thus a genital duct of a temporary character becomes formed: but no such opening has ever been observed.

Nereis is unisexual. The sexual elements, ova or sperms, are formed from temporary masses of cells, cvaries or testes, which are developed towards the breeding season by a proliferation of the cells of the membrane (cœlomic epithelium) lining the cœlome and the structures it contains. In Nereis dumerilii there is in the male only a single pair of these proliferating masses of cells (testes), situated in one of the segments between the nineteenth and the twenty-fifth. But in other species of Nereis they are much more numerous. These, during the season of their active development, give off groups of cells which become disseminated throughout the cœlomic fluid. The original cells (mother-cells) undergo division into smaller cells, each of which develops into a sperm with a minute rod-shaped head and a long vibratile flagellum or tail. In the female the ovaries (Fig. 351, ov), formed by a similar process of proliferation, take the form of rounded masses of cells, metamerically arranged, surrounding the principal vessels throughout the length of the body. The young ova become detached from the ovaries, and attain their full development while floating about in the cœlomic fluid. Both ovaries and testes dwindle after they have given off the sexual cells, and at the non-breeding season of the year are not to be detected.

Ova and sperms, when fully ripe, are discharged, reaching the exterior probably through apertures temporarily formed by rupture of the body-wall ( $c f$. above), and impregnation takes place by contact between the two sets of elements while floating freely in the sea-water.

Nereis dumerilii is an extremely variable species. If we compare a number of specimens, we find numerous individual differences between them. The most striking of these are differences of colour and of the number of segments in the body; but a careful examination reveals many other points in which individuals differ. Thus the precise form of the lobes of the parapodia, together with the number of setæ in the two bundles, vary; so also do the relative length of the tentacles, the number of teeth on the jaws, and the number and arrangement of the denticles in the buccal cavity. Not only are such individual 
differences common, but the species occurs in two distinct forms or phases, which differ from one another so widely that they have been referred to distinct genera. One of these is the Nercis phase, which is that described in the preceding paragraphs. A Nereis dumerilii may become sexually mature in this form, or may first undergo a series of changes by which it becomes converted into the second or Heteroncreis phase (Fig. 346, B). The principal changes which take place during this metamorphosis are a great increase in the size of the eyes, and a marked modification of the parapodia in the posterior portion of the body, the lobes becoming larger and more leaf-like, and the setre of the Nereis being superseded by others which are considerably longer, more numerous, and somewhat oar-shaped. The Heteronereis, instead of creeping about on the bottom, swims about actively through the water by wriggling movements of the body combined with active paddling movements of the parapodia with their long seta. After a time the Heteronereis, like the Nereis, becomes sexually mature, developing ova and sperms, the latter of which differ remarkably in shape from those of the Nereis phase.

Development.-The egg of Nereis when first discharged is enclosed in a transparent thick gelatinous envelope, within which are two membranes-an outer very thin and delicate, and an inner (zona radiata) thicker and very distinctly striated in a radial direction. The protoplasm of the ovum contains a number of oil-drops and yolk-spherules. When fertilisation takes place the yolk-spherules move away from what is destined to become the upper pole of the egg, leaving a polar area composed of granular protoplasm. The zona radiata disappears, and the contents of the ovum undergo for a time amœboid changes of form. Then the spherical form is reassumed, two small bodiesthe polar bodics (p. 19) -are thrown off at the upper pole, and the process of segmentation begins (Fig. 35.5). Up to a fairly advanced stage this corresponds very closely with the segmentation of the Polyclad oosperm as described on page 273. The oosperm divides first into two parts, then into four. From these four cells-the megameres - there are separated off in succession three sets of micromeres, making twelve in all. One of these, belonging to the second set, somewhat larger than the others and differing from them in its subsequent history, is termed the first somatoblast (som. 1); a second somatoblast (som. 2) is soon given off from the same megamere that gave origin to the first.

The germinal layers are now all established. The micromeres constitute the ectoderm, destined to give rise to the epidermis and all its derivatives, to the cerebral ganglion and nerve-cord, to the œsophagus and rectum. The megameres eventually give origin to the cells of the endoderm, forming the internal epithelium of the alimentary canal. The second somatoblast gives rise to 
the entire mesoderm of the Annelid. As the micromeres multiply by division, they form at first a cap of small cells over the upper pole of the embryo; eventually the cap extends so as completely to cover the four megameres and the descendants of the somatoblasts except at one point, the blastopore, at the lower pole, where the investment remains for a time incomplete. When the blastopore closes, the process of epibolic gastrulation is completed. A thickening of the layer of ectoderm cells, the apical plate, in the middle of what is destined to form the head-end of the embryo, is the rudiment of the cerebral ganglion : in close relation to it are formed a pair of pigment-spots, the larval eyes. From
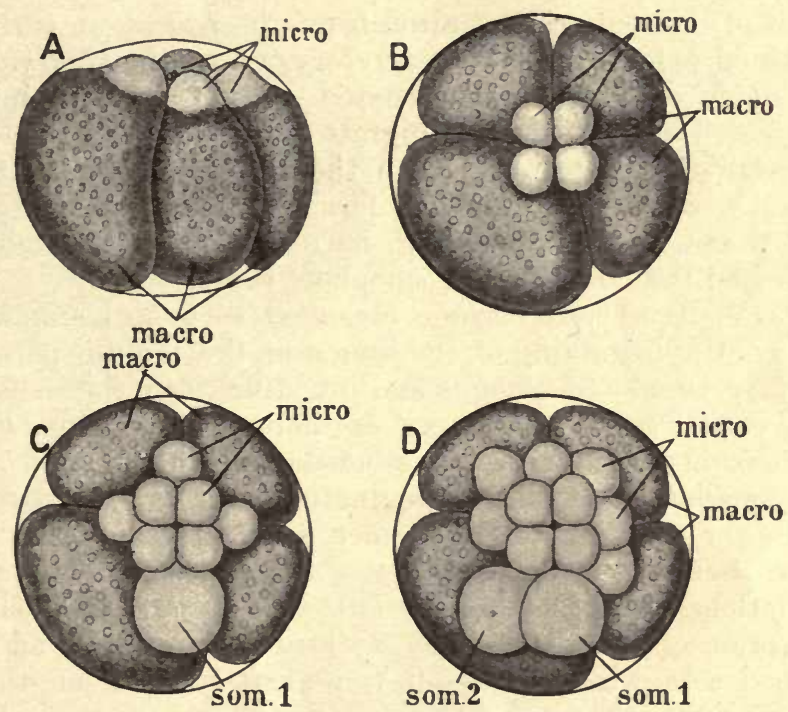

Fic. 355.- Nereis. Early stages in the development. $A$, lateral view of eight-celled stage ; $B$, the same from above ; $C$, stage of the formation of the first somatoblast; $D$, stage at which both somatoblasts are present; macro. megameres; micro. micromeres; som. 1, som. 2. first and second somatoblasts. (After Westinghausen.)

the middle of the head-end projects a tuft of cilia (Fig. 356, A, ap. cil.). Encircling the body of the larva behind this is a thickened ridge, the prototroch (prot), the cells of which develop strong cilia. Just behind the prototroch the cells of the ectoderm become pushed inwards in the middle of what will eventually become the ventral surface, so as to line a sort of depression or pouch; this is the stomodoum (st) or rudiment of the mouth and cesophagus. The anus (an) does not appear until later; the position which it will subsequently occupy is indicated at this stage by a pigmented area (pig. ur) marking the point at which the blastopore becomes closed. The first and second somatoblasts divide to form a mass of small cells which extend on the ventral surface 
behind the prototroch and mouth, constituting what is termed the rentral plate; of this plate the more superficial cells are descendants of the first somatoblast-one of the twelve original micromeres; and those situated more deeply are derived from the second somatoblast or mesomere. A superficial thickening of the ectoderm along the middle of the ventral plate is the rudiment of the ventral nerve-cord (neur. $p l$ ); the deeper cells divide and extend to form a pair of mesnderm bands or muscleplates, from which the muscles of the body-wall are developed; the muscular layers of the wall of the alimentary canal are derived from certain of the same set of cells which migrate inwards from the lower end.

A pair of micromeres separated from the rest at an early stage are destined to form the larval excretory organs, the head-kidneys or larval nephridia: at first situated at the upper end, they sink below the surface and migrate downwards till they come to lie below the prototroch; each then elongates, and a number of vacuoles which have become formed in the interior coalesce in such a way as to form a long, narrow canal. The embryo has now reached the completed trochophore stage.

The endoderm cells become arranged so as to bound a canallike space, the beginning of the lumen of the middle part of the alimentary canal (œsophagus and intestine, int.), the cells subsequently giving rise to the enteric epithelium. This canal becomes continuous in front with the stomodrum, and behind with a second smaller ectodermal invagination, the proctodoum, which arises in the position of the former pigment-area. The part of the larva behind the prototroch now elongates, and two pairs of invaginations, the setigerous sacs (set. sacs), appear at its sides: in the interior of these, to which a third pair is soon added, are developed setæ which grow out to a great relative length as the larval or provisional seto. Constrictions soon appear marking off the first three segments, and at the same time the mesoderm bands undergo a corresponding division into three pairs of mesoderm segments. The mesoderm segments of each pair grow inwards towards one another and surround the alimentary canal: in the interior of each appears a cavity which is the beginning of a segment or chamber of the colome. As the two mesoderm segments become closely applied to one another and unite around the alimentary canal, their two cavities also come into close relation, and eventually are separated from one another only by thin vertical septa, forming dorsal and ventral mesenteries which subsequently disappear. Successive mesoderm segments also come into close relationship with one another, their cavities eventually only remaining separated by thin transverse partitions, which form the intersegmental septa.

The region in front of the prototroch becomes modified to form the 

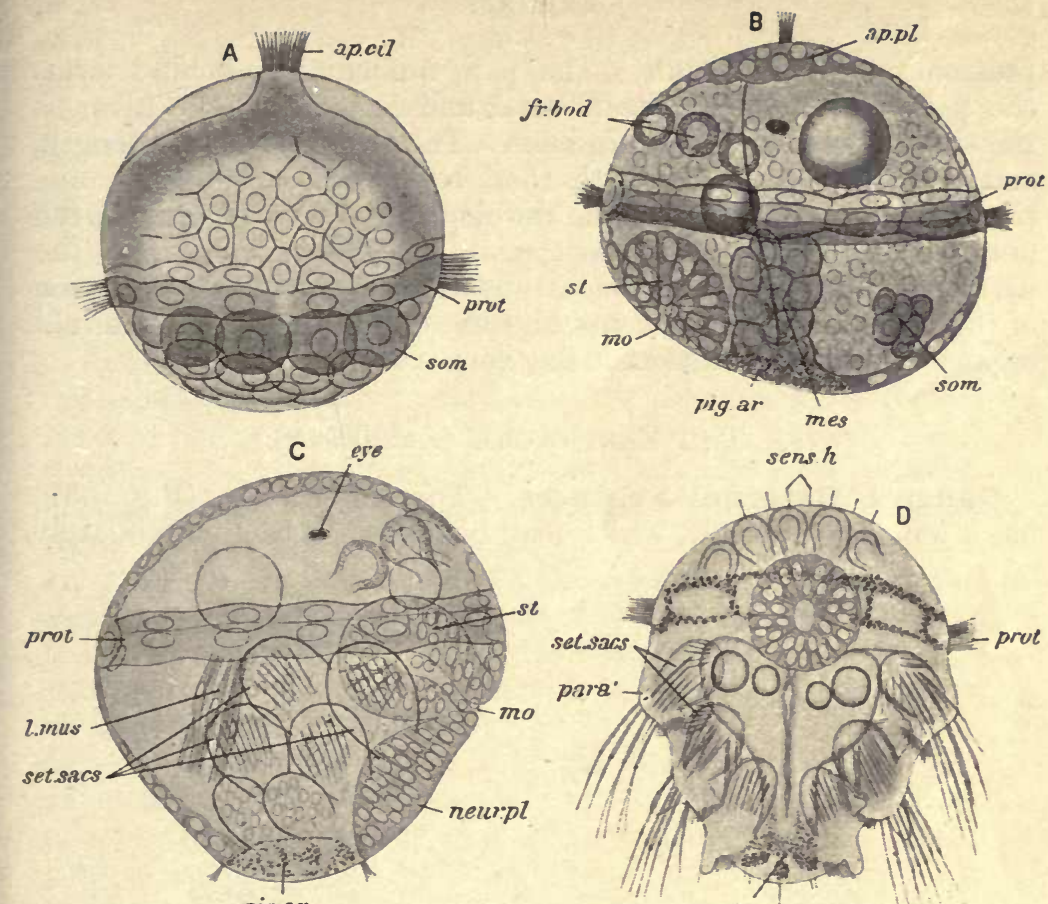

pig.ar
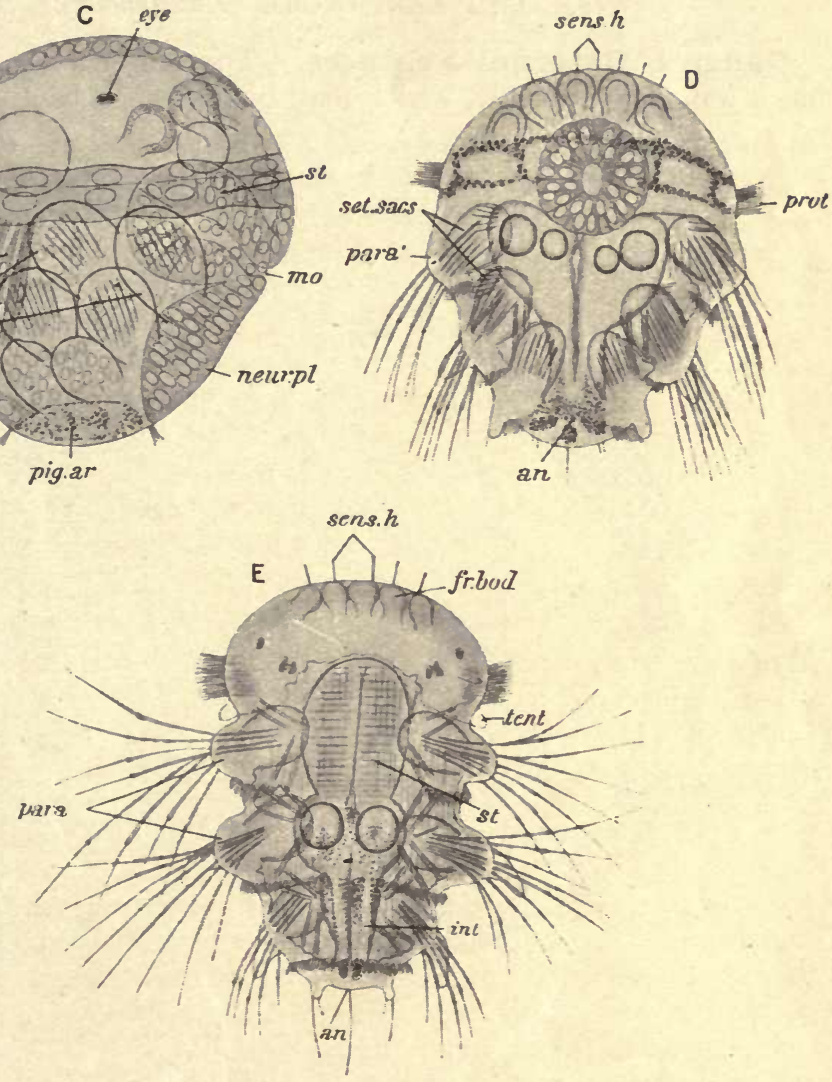

Fig. 356.- Nereis. Iater stages in the development. $A$, stage at which the prototroch and the apical tuft of cilia first become distinct. $B$, somewhat later stage, in which the stomodieal invagination is being formed, and the rudiments of the mesoderm bands are distinct; $C$, late trochophore stage in which there are rudiments of the setigerous sacs; $D$, somewhat later stage, in which the parapodia have begun to become prominent and the provisional setæe project freely ; $E$, larva with three segments. an. anus; ap. cil. apical cilia ; $a p . \quad p l$. apical plate; eye, eyc; $f$ \% bod. frontal bodies; int. intestine; $l$. mus. longitudinal muscle; mes. mesoderm; wo. mouth; neur. pl. neural plate; para. parapodia; pig. ar. pigmented area; prot. prototroch; sens. h. senscry hairs ; set. sacs. setigervus sacs: som. second somatoblast and group of cells formed from it; st. stomodrum; tent. peristomial tentacles. (After E. B. Wilson.) 
prostomium of the adult. The part immediately behind forms the peristomium, which bears setæ, and is to be looked upon as the specially modified first segment. The body increases in length, and additional segments with their setigerous sacs become distinguishable $(E)$ intil, on the development of the tentacles, the outgrowth of the parapodia (para) with their cirri and the permanent setæ (which replace those first formed), the formation of the full number of segments, and the completion of the internal organs, the adult condition of the worm is attained.

\section{b. The Earthworm (Lumbricus).}

General External Features.-The Earthworm (Fig. 357) has a long narrow body, which may be described as approximately
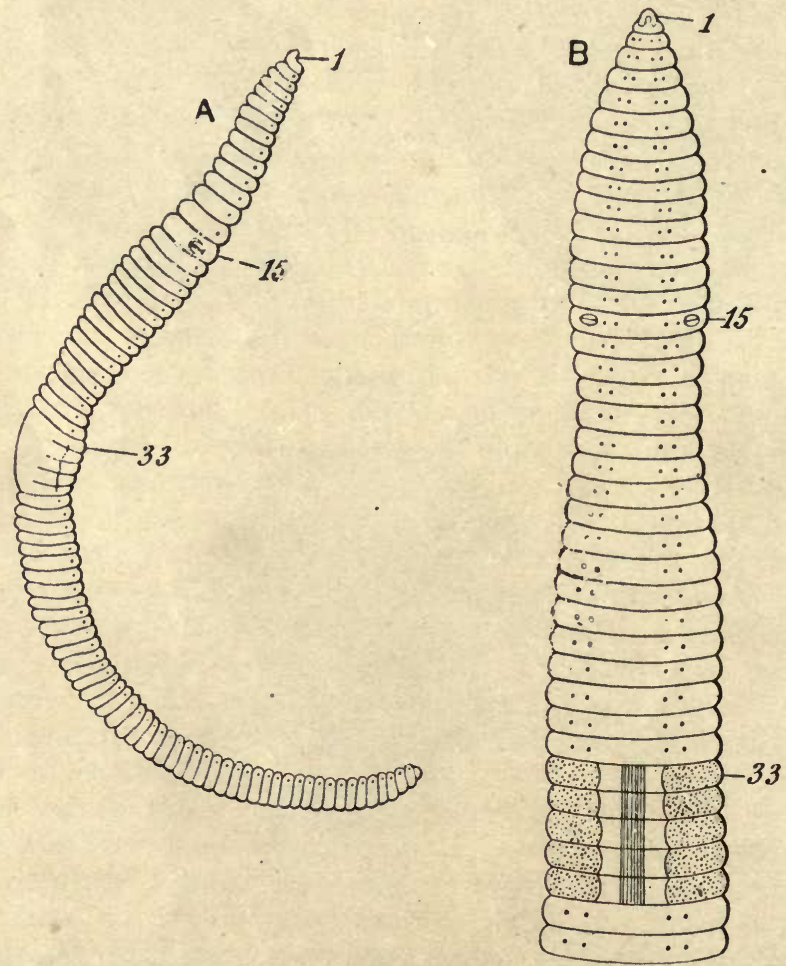

F1G. 357.-Iumbricus herculeus. $A$, entire specinen, lateral view ; $B$, ventral view of anterior portion of the body, magnifierl. 1, 15, 33, first, fifteenth, and thirty-third segments. Each of the black dots represents a pair of setæ. (After Vogt and Jumg.)

cylindrical, but slightly depressed towards the posterior end. Dorsal and ventral surfaces are readily recognisable, the latter being much paler in colour than the former, and exhibiting a 
slight flattening; the anterior end is distinguishable in the living animal as that which is directed forwards in the ordinary creeping movements of the worm. The surface, as in the case of Nereis, is very distinctly marked out into segments or metameres by a series of ring-like constrictions; the segments, which are very numerous-amounting to about 150, are somewhat longer towards the anterior end than they are further back.

At the extreme anterior end is a rounded lobe, the prostomium, immediately behind and below which is the opening of the mouth. Next to the prostomium is the most anterior segment, the peristomium, which bounds the mouth behind. The eyes and tentacles present in Nereis are not represented. On the most posterior segment, the anal segment, is a small median opening, the anal aperture. A limited region of the body in front of the middle, comprising segments from the thirty-second to the thirty-seventh, has a swollen appearance; this is termed the clitellum. There are no parapodia like those of Nereis, but running along the lower surface of the worm are to be recognised with the aid of a lens four double rows of short bristles or setæe (Fig. 358), a pair of each row occurring in each segment, which thus possesses eight altogether. The extremities of all these setie are directed backwards, and they act as fulcra for the forward movements of the worm on the surface of the ground or in the interior of its burrow. The setæ in the clitellum, and those in the neighbourhood of the genital apertures, are much slenderer than the rest. Along the middle line of the dorsal surface, from about the eleventh segment backwards,

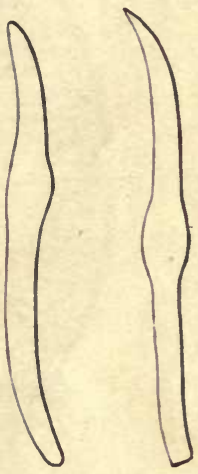

FIG. 358.- Iumbricus. Setæ, highly magnified. is a row of small apertures, one at the line of division between each contiguous pair of segments: these, which are termed the dorsal pores, perforate the body-wall and open internally into the coelome. Through these coelomic fluid is capable of being discharged, covering the surface with a thin layer which may protect the worm from desiccation or from contact with irritating substances. On the ventral surface are two rows of minute apertures-a pair on each segment-the excretcry apertures or nephridiopores. On the ventral surface of the fifteenth segment (Fig. 357, 15), is a pair of slit-like apertures with somewhat tumid lips, the male reproductive apertures; and on the segment immediately in front-the fourteenth, are two smaller rounded apertures, the female reproductive apertures. In the intervals between the ninth and tenth, and tenth and eleventh segments are two pairs of small pores, the openings of the receptcicula seminis. 
The body-wall (Fig. 359) consists of a cuticle, an epidermis or deric epithelium, a dermis, muscular layers with associated connective-tissue, and, lining the inner surface, a thin cellular membrane, the peritoneum or colomic epithelium. The cuticle (cut.) is similar to that of Nereis, and has a similar iridescent lustre; it is perforated by numerous minute apertures. The epidermis consists, except on the clitellum, of a single layer of cells elongated in the vertical direction: many of these cells have the character of unicellular glands: many others are sensory cells,

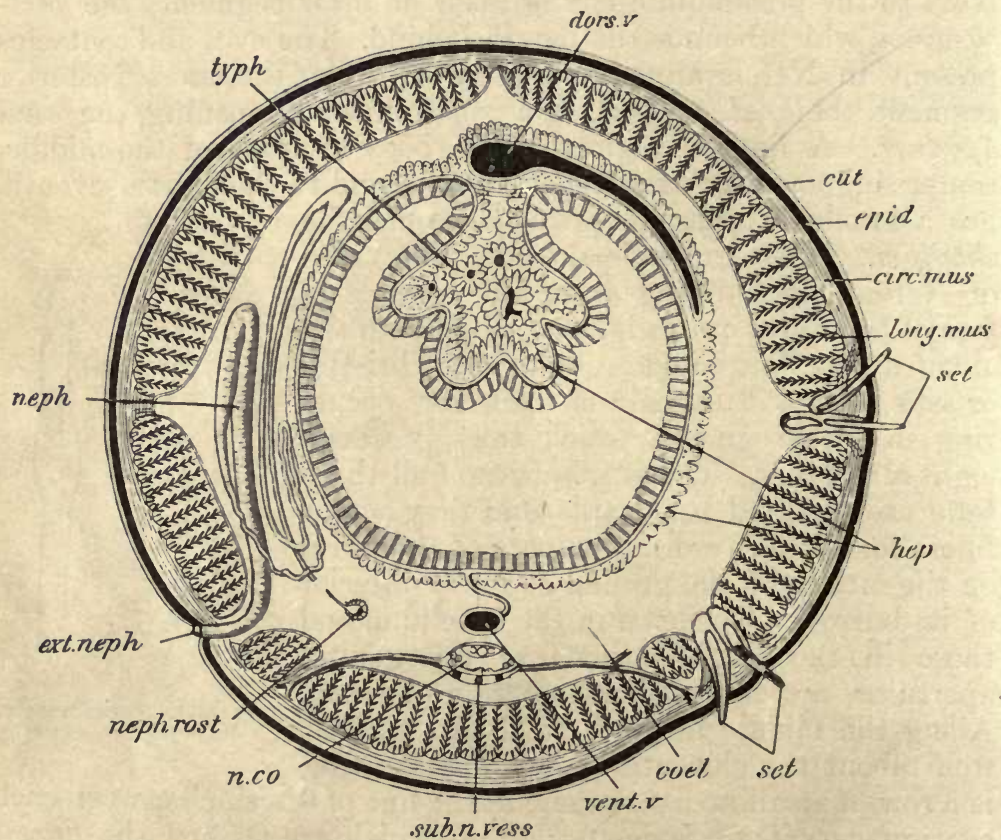

F1G, 359-Iumbricus, transverse section of the middle region of the body, cive, mus, layer of circular muscular fibres ; crel. colome ; cut. cuticle ; dois. v. dorsal vessel ; epid. epirlermis ; ext. neph. nephridiopore; hep. layer of chloragen cells; lon. . mus. longitudinal muscle; neph. nephridium; nephrost. nephrostome; n. co. nerve-eord ; eet. seta; suh. n. vess. subneural vessel; typh. typhlosole; vent. $v$. ventral vessel. (After Marshall and Hurst.)

and are connected by fine nerve-fibres with the nerve-cord. On the clitellum the epidermis is thickened, and blood-vessels extend between the cells. Below the epidermis is a layer of connectivetissue, the dermis. The muscular fibres which make up the greater part of the thickness of the body-wall are arranged in two principal sets-a layer of circularly arranged fibres (circ. mus) situated externally, immediately below the dermis and a layer of longitudinally arranged fibres (long. mus) situated internally. The circular layer is interrupted at all the intervals between the segments; the longitudinal layer is interrupted along 
a series of longitudinal lines, so as to be divided into seven bundles.

The seta (Fig. 358) are lodged in sacs, the sctigercus sacs (see Fig. 369), lined by a continuation of the epidermis. In the region of the body in which the reproductive organs are lodged some of these sacs are enlarged and glandular, and receive the special name of caps? genous glands.

The enteric canal (Fig. 360) is, as in Nereis, a tube which runs through the entire length of the body from the mouth at the anterior to the anus at the posterior end. As in the case of Nereis, it lies in a cavity, the colome, lined by a thin cellular membrane, the peritoneum or colomic epithelium, and filled with. a fluid, the colomic fluid, containing colourless corpuscles. The colome is divided into a series of chambers corresponding to the segments by a series of delicate transverse partitions, the septra or mesenteries, consisting of folds of the peritoneal membrane enclosing muscular fibres.

The mouth leads into a small buccal cavity. This is followed by a much larger, thick-

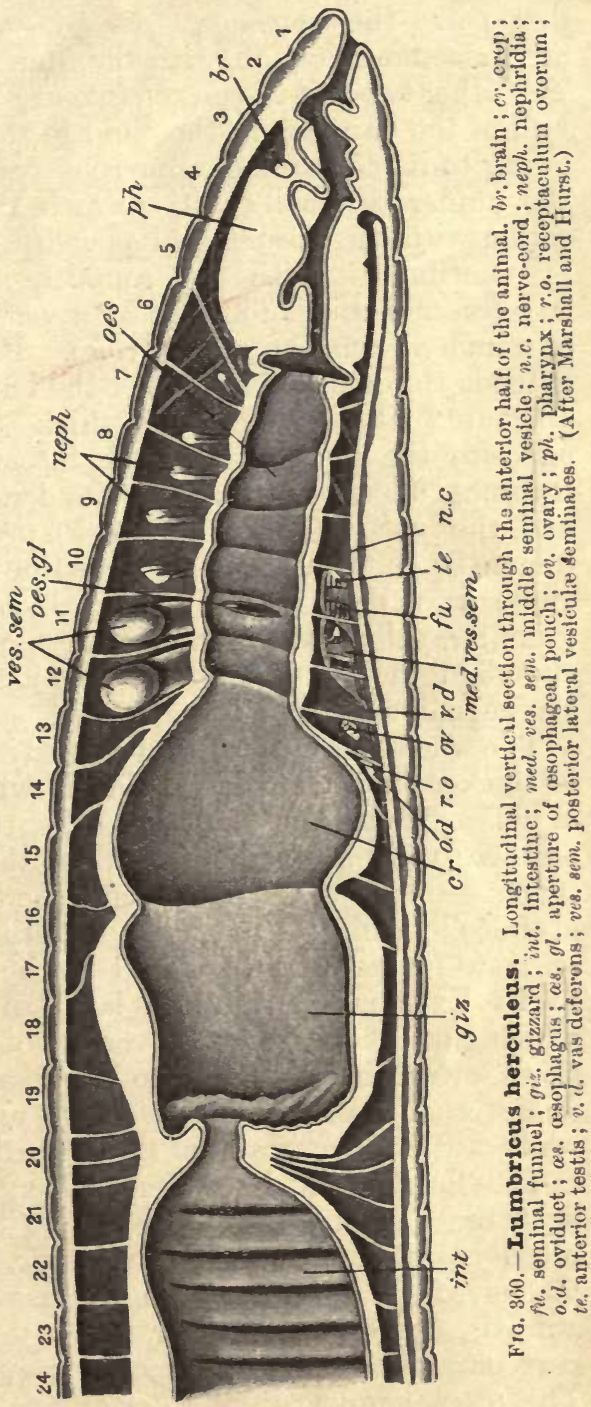
walled, rounded chamber, the pharynx (ph.). From the wall of the pharynx there run outwards to the body-wall a number of radially arranged bundles of muscular fibres which, when they contract, draw the pharynx backwards, and at the same time 
dilate it. Behind the pharynx follows a comparatively narrow tube, the oesophagus (oes), which extends through about seven segments. At the sides of the æesophagus, in each of the segments ten, eleven, and twelve, is a pair of rounded projections. The first pair-the asophageal pouches-are hollow, and their cavities are in communication with the lumen of the œsophagus (ces. $g l)$. The other two pairs - the calciferous glands - are thickenings of the wall of the œesophagus, the fluid in the interior of which is milky, owing to its containing numerous particles of carbonate of lime; the numerous small cavities which they contain are in communication with the œsophageal pouches. Posteriorly the œesophagus is continuous with a rounded thin-walled chamber, the crop $(c r)$ and this is followed by a very thick-walled chamber, also of rounded form, the gizzard (giz). From this the intestine (int) extends throughout the rest of the length of the body to the anal aperture. It is wide, with thick but soft walls, constricted opposite the septa, i.e. in the intervals between the segments. Running along the middle of its dorsal surface is a longitudinal fold, the typhlosole (Fig. 359, typh), projecting downwards into the lumen. On the wall of the intestine outside the muscular layers and surrounding the intestinal blood-vessels are a number of granular, yellow cells - the chloragen cells (hep): these are specially abundant in the typhlosole. The terminal part, situated in the last segment, is termed the rectum.

The whole alimentary canal is lined internally by a cuticle-which is thicker in the gizzard than elsewhere, and by a single layer of ciliated columnar epithelial cells, the enteric epithelium. Some of these cells, more granular than the others, grouped in certain regions-more particularly along the typhlosole, are of the nature of unicellular digestive glands, secreting a digestive fluid. Others seem to be specially concerned in the absorption of the digested food. External to this is a layer of connective-tissue, between which and the external covering of yellow cells are muscular fibres, of which there are two layers, an external longitudinal and an internal circular. These layers are greatly thickened in the walls of the pharynx and of the gizzard.

The Earthworm, like Nereis, has a well-developed vascular system, consisting of blood-vessels with well-defined walls. The blood is bright red, the colour being due to the same colouring matter, viz. homoglobin, as in the case of the blood of the higher animals, occurring, however, not in corpuscles, but in the liquid part or plasma; corpuscles are present, but they are colourless. The main trunks are the dorsal, the ventral, the sub-neural, the two lateral neural, and a series of transver'se branches. The dorsal vessel (Fig. 359, dor's. $v$ ) runs along the middle of the dorsal surface between the body-wall and the intestine; it is readily visible shining through the former in the living worm. The ventral vessel (vent.v) 
lies below the alimentary canal, the sub-neural below this again under the nerve-cord; the lateral neural lie on either side of the nerve-cord. The transverse branches correspond in number to the segments; they run round from the dorsal vessel to the ventral, giving off branches in their course. Five of them, viz. those in the seventh to the eleventh segments inclusively, are dilated and pulsate rhythmically; these have the function of driving the blood through the system of vessels, and are hence frequently termed the "hearts." The walls of the principal vessels are contractile, and assist in bringing about the movement of the blood, which is propelled in such a way as to run forwards in the dorsal vessel and backwards in the ventral, its direction of movement being regulated by a number of valves in the "hearts," the - dorsal vessel, and the chief vessels connected with it.

The nervous system (Fig. 361) - consists of a dorsal bilobed brain or cercbral ganglion and a double ventrul nerve-cord together with a pair of cesophageal connectives, by which the former is connected with the anterior end of the latter. The brain, which is of small size, is situated in the third segment, above the beginning of the alimentary canal; it is divided by a median constriction into two lateral parts of pyriform shape with their broad ends in contact. The connectives pass from this round the sides of the alimentary canal to unite in the middle below with the anterior end of the ventral nerve-cord. In this way a complete nervering or nerve-collar surrounds the anterior part of the enteric canal in the third segment. From this the ventral nerve-

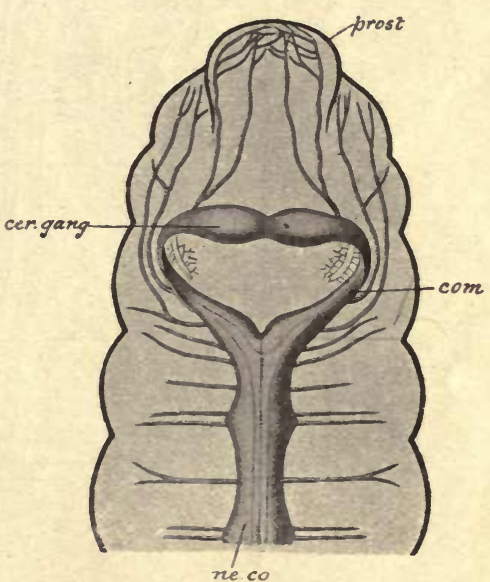

FiG. 361.-Iumbricus. Ant portion of nervous system. cer. yang. centid ganglion or brain ; com. cesophageal connectives; ne.co. ventral nerve-cord; prost. prostomium. (After Leuckart.) cord extends backwards to the posterior end of the body, and in each segment it presents a slight enlargement or ganglion, as it is usually termed, most conspicuous in the more posterior segments. The wnole cord is double, consisting of two intimately united right and left parts. From the brain, nerves are given off to the prostomium; and from the ventral cord three pairs of nerves arise in each segment. From the œsophageal connectives a series of stomatogastric nerves pass to the pharynx and neighbouring parts of the alimentary canal. 
The Earthworm is devoid of organs of sight or hearing. It exhibits sensitiveness to bright light, the sensitive elements being large cells of the epidermis devoid of pigment. The sense of

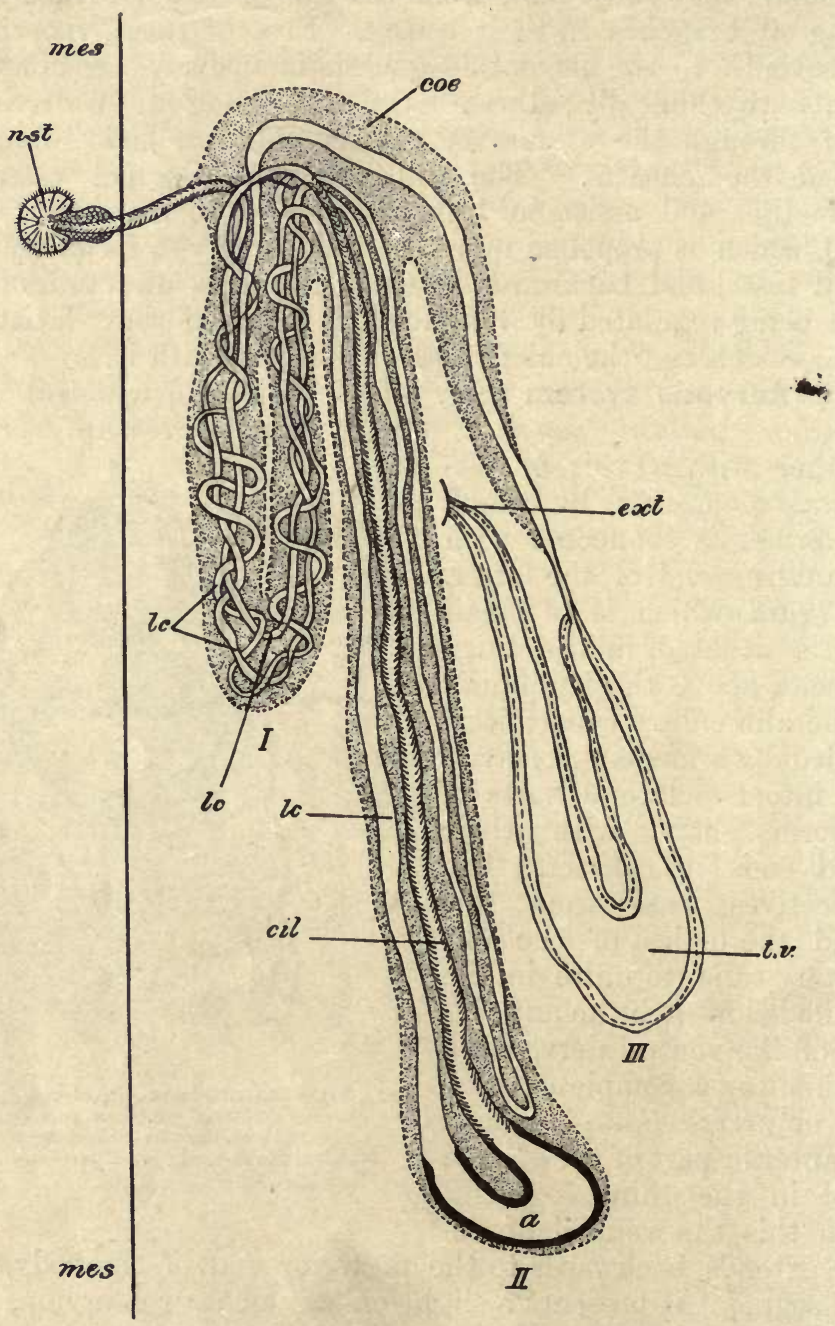

Fig. 362,-Nephridium of Tumbricus (diagrammatic). $-a$. ampulla between eiliated and nonciliated parts of the intraccllular canal; cil. ciliated part of the intracellular canal; coe. investnient derived from the colomic epithelium; ext. nephridiopore; l.c. non-ciliated part of the intracellular canal ; mes. septum ; nst. nephrostome; $t . v$. intercellular canal of the terminal vesicle. I. - III. the three principal loops. (From Meisenheimer, after Maziarski.)

hearing appears to be absent; but a faculty analogous to taste or smell, enabling the animal to distinguish between different kinds of food, is well developed. The goblet-shaped bodies, groups of 
narrow epidermal cells, most abundant on the prostomium and peristomium, have probably to do with this faculty.

Tho organs of excretion-the segmental organs or nephridia -(Fig. 362) are similar to those of Nereis, but somewhat more complicated. They are slender tubes which occur in pairs in all the segments of the body except the first three and the last. Externally each nephridium opens by one of the small nephridiopores which have already been mentioned as occurring on the ventral surface; internally it ends in a funnel-shaped ciliated extremity with a crescentic slit-like aperture, the nephrostome $(n s t)$, opening into the cavity of the segment in front of that in which the external aperture occurs. The tube is thrown into three loops attached to the posterior surface of the corresponding septum by a fold of membrane. Two parts are clearly recognisable inner narrow and an outer wide part: in the former the naw central lumen is a perforation through the axis of a string of cells, and is thus intracellular: it is lined in parts with cilia arranged in two rows; in the latter (the terminal vesicle) the passage is lined by cells, and is thus intercellular, and there is a thick muscular investment. The nephridia are abundantly supplied with blood by means of nephridial branches of the ventral vessel.

Reproductive Organs.-The Earthworm is hermaphrodite. There are two pairs of very small flattened testes (Figs. 360, 363, te, $\left.t e^{\prime}\right)$, partly divided into a number of digitate lobes, situated in the tenth and eleventh segments. A pair of comparatively large sacs, the anterior vesiculce seminales (ant. ves. sem) lie partly in the cavity of the ninth segment, but extend into the tenth, where they coalesce in the middle to form a large median sac of somewhat irregular form, the anterior sperm-reservoir (ant. sp. res). The anterior pair of testes project into this, and the cells destined to form the sperms, developed in the former, pass by dehiscence into the large median cavity. On either side is a large ciliated funnel, or rosette ( fun), leading outwards from the interior of the reservoir. A second pair of vesiculæ seminales (mid. res. scm), situated in the eleventh segment, also open into the anterior sperm-reservoir. A third pair (post. ves. sem), situated in the twelfth segment, unite in front to form the posterior sperm-reservoir (post. sp. res), which lies in the middle of the cavity of the eleventh segment. The posterior pairs of testes have the same relation to this as the anterior pair have to the anterior reservoir; and a posterior pair of ciliated funnels ( fun) lead outwards from its cavity. Each ciliated funnel passes into a narrow, somewhat convoluted duct, the vas efferens, and the two vasa efferentia of each side unite to form a vas deferens or spermiduct ( $v$. def), right or left as the case may be, which passes almost straight backwards to open by the corresponding male aperture on the fifteenth segment. 
The female reproductive organs consist of a pair of ovaries, a pair of oviducts with a pair of receptacula ovorum, and two pairs of receptacula seminis. The ovaries (ov) are minute pear-shaped bodies, which are situated in the thirteenth segment, attached to the septum between the twelfth and thirteenth. The oviducts (ov. d) are a pair of short tubes, each with a comparatively wide funnelshaped opening into the cavity of the thirteenth segment, and extending backwards and outwards in the fourteenth segment to open at the female aperture on the ventral surface of the latter. The receptacula ovorum are a pair of reniform sacs which open into

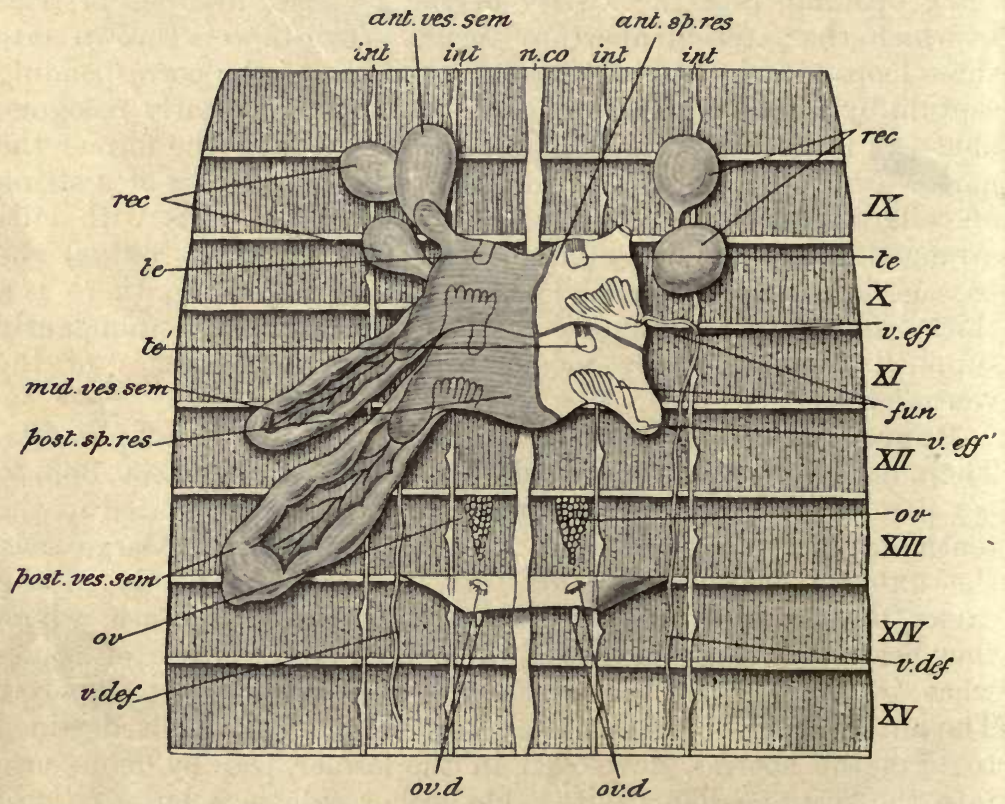

FIง. 363.-Iumbricus herculeus. Reproductive organs. ant. sp. res, anterior sperm reservoir; ant.ves. sem. anterior left vesieula seminalis; fun. funnel-like openings of vasa efferentia; int. intermuscular partitions ; mid. $v \epsilon s .8 e m$. middle vesieula seminalis ; $n$. co. nerve-cord ; ov. ovaries; $o v . d$. oviduets; post. $s p$. res. posterior spern-reservoir; post. $v e s .8 t m$. posterior vesieula seminalis; rec. receptacula seminis; te, anterior, and $t e^{\prime}$, posterior testes; $v$. eff. anterior, and $v$. eff'. posterior vas efferens; v. def. vasa deferentia. (After Vogt and Jung.)

the funnel-shaped ends of the oviducts. The recrptacula seminis (rec) are two pairs of rounded sacs which open on the exterior in the intervals between the ninth and tenth, and tenth and eleventh segments.

Though hermaphrodite, the Earthworm is not self-impregnating, but two individuals provide for mutual fertilisation by an act of copulation. The copulating individuals apply themselves together by their ventral surfaces, the heads pointing in opposite directions, and become attached in this position by the setæ of the genital region and by a viscid secretion from the clitellum and of 
the capsulogenous glands (p. 457), situated in the neighbourhood of the reproductive organs. The sperms from the male apertures of each pass along temporarily formed grooves to the receptacula seminis of the other.

When the ova are inature they are discharged from the ovary into the cavity of the thirteenth segment, whence they pass out to the exterior through the oviducts, to be enclosed in the cocoon

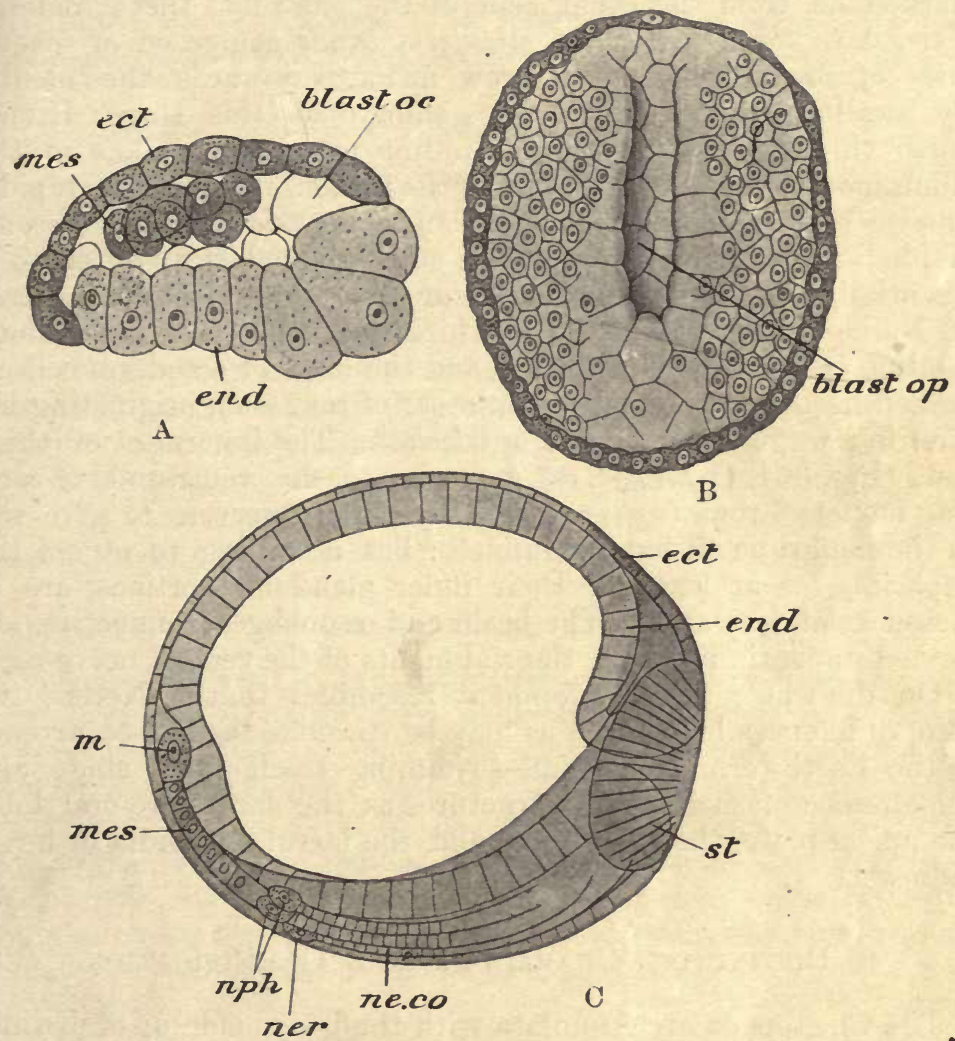

FiG. 364.-Early stages in the development of Iumbricus. A, lateral view of flattened blastula ; $B$. ventral view of gastrula with slit-like blastopore; $C$, lateral view of later stage. blastoc. blastocole ; blastop. blastopore ; ect. ectoderm ; env. eudoderm ; m. primary mesoderm cell ; mes. mesoderm bauds; ner. cell from which the primitivc nerve-cord (ne. co.) takes origin; $n p h$. cells taking part in the formation of the nephridia; $s$. stomodrum. (After Wilson.)

(vide infra), after having being detained for a time in the receptaculum ovorum.

Development. - The oosperms or fertilised ova of the Earthworm are enclosed, together with a quantity of an albuminous fluid derived from the capsulogenous glands, in a cocoon, the wall of which is formed of a viscid secretion from the glands of the clitellum, hardened and toughened by exposure to the air. The 
cocoon is deposited in the earth and the embryos develop into complete, though minute, worms before they make their escape. The segmentation is somewhat unequal. A flattened blastula (Fig. 364, A) is formed, with a large but flattened segmentationcavity. This becomes invaginated to form a cylindrical gastrula $(B)$; the blastopore narrows and subsequently gives rise to the mouth of the adult. A pair of large mesoderm cells $(m)$ are early marked off from the other cells of the gastrula; these undergo division to form a pair of mesoderm bands composed of several rows of small cells which grow forwards towards the mouth. By swallowing movements the embryo at this stage, having burst through the enclosing vitelline membrane, takes in the albuminous fluid in the interior of the cocoon, and increases rapidly in size. As the embryo elongates, the mesoderm bands become divided into segments, and the subsequent history of these is essentially similiar to what has been already described in the case of Nereis. The ectoderm is thickened on each side along the line of the mesoderm bands, and the mass of ectoderm cells so formed becomes arranged in a number of rows each originating behind in a larger rounded cell or teloblast. The innermost of these rows (Fig. 364, C, ner, ne. co) give rise to the ventral nerve-cord. The next two rows (nph) are said by some observers to give rise to the nephridia all but the funnels: but according to others the nephridia, or at least all their inner glandular portions, are of mesodermal derivation. The brain and œsophageal connectives are formed in continuity with the rudiments of the ventral nerve-cord.

On the whole the development resembles that of Nereis, the chief differences being such as may be traced to the non-occurrence in the Earthworm of any free-swimming trochophore stage, and the absence of such larval structures as the large pre-oral lobe, the apical plate, the prototroch, and the larval nephridia or headkidneys.

\section{Distinctive Characters and Classification.}

The Chætopoda are Annulata with the body made up of distinct metameres, which are usually numerous and similar throughout. The metameres are provided with chitinous setæ developed in sacs (setigerous sacs) of the epidermis, and usually elevated on muscular appendages, the parapodia. There is a large colome divided internally into chambers by transverse septa, and not in communication with the blood-vascular system, which is nearly always highly developed. The ventral nerve-cord consists of a chain of ganglia. The reproductive cells are formed by a proliferation of certain parts of the peritoneum or membrane lining the cœlome, and usually reach the exterior through cœlomoducts or through modified or unmodified nephridia. 


\section{Sub-Class I.-POLYCH ÆTA.}

Chrtopoda with the sexes distinct, and the ovaries and testes of simple character and metamerically repeated. Highly developed parapodia are present, in most instances, bearing numerous long setæ. There is usually a definite head with eyes and tentacles, and often cirri and branchiæ on the segments of the body. A clitellum is never developed. A metamorphosis takes place: the larva is a trochophore. Nearly all the Polychæta are marine.

\section{Order 1.-ARChi-Chatopoda.}

Aberrant or primitive Polychreta ${ }^{1}$ in which the nervous system is not separated from the epidermis, and the ventral cord is not segmented into ganglia. Only one genus (Succocirvus).

\section{Order 2.-PhiNerocel'HaLA.}

Polychæta with protrusible pharynx usually armed with chitinous jaws. There is a well-developed head. The segments are completeiy or nearly similar throughout the length of the body, and the parapodia are usually equally developed throughout and provided with cirri. The branchiæ, when present, are not confined to the anterior end.

\section{Order 3.-Cryptocephalat.}

Polychæta devoid of protrusible pharynx and of jaws or teeth. The head is frequently very small, and sometimes is devoid of eyes or of tentacles, the prostomium sometimes much reduced and covered over by the peristomium. The body is distinguishable, by differences in the form of the segments, parapodia, and seta?, into two or even three regions. The parapodia are little prominent in the posterior parts, and usually without cirri. The branchix, when present, are usually confined to the anterior end, and are sometimes represented by modified cephalic palpi.

\section{Sub-Class II. - OLIGOCHÆTA.}

Chrtopoda with the sexes united, the reproductive system complicated, the ovaries and testes compact and never more than two pairs of each. No definite paropodia are developed and no cirri, and only a small number of simple setæe on each segment. The head is not distinct. A clitellum is usually present. There is no metamorphosis. Mostly terrestrial or fresh-water forms.

1 The-Archi-Chretoporla are usually classed with the Polychata, but their alliances are perhaps quite as close with the Oligochata. In some respects Saccocirrus resembles Polygordius and Protodrilus (Arhci-Annelida q.e.) but is distinguished from them by the possession of setæ. 


\section{Order 1.-MICRODRILI.}

Small Oligochæta with relatively few segments, often multiplying asexually. The male genital pores are on, or in front of, the seventh segment. The vasa deferentia are short, opening on the segment immediately behind that in which the internal apertures are situated. The anterior part of the body is often distinguished from the rest by a difference in the form and arrangement of the setæ. The clitellum, which is composed of only one layer of cells is situated comparatively far forward. Eye-spots are frequently present.

\section{Order 2-MEgadriLi.}

Mostly large Oligochæta with many segments, never multiplying asexually. The male genital pores are behind the seventh segment. The vasa deferentia are elongated, passing through two or more segments. The anterior part of the body is never specialised as regards its setæ. The clitellum, which consists of two layers of cells, never begins in front of the twelfth segment. Eye-spots are not developed.

\section{Systemalic Position of the Examples.}

Nereis dumerilii is one of many species of Nereis differing from one another in certain minor details of their structure-such as the relative lengh of the palpi and tentacles, the size and form of the eyes, the shape of the parapodia, the form of the setæ, and the like. The genus Nereis differs from the other genera of the family Nereidoe, to which it belongs, in having the parapodia biramous and the cirri simple, and in the presence of a series denticles in the buccal cavity in addition to the pair of jaws. The family Nereidæ differs from all the other families of the sub-order Nereidiformia of the Phanerocephala in the union of the following characters:-The body is always elongated and made up of a considerable number of segments. The prostomium is well developed, and bears a pair of tentacles, a pair of palpi, and four eyes. The peristomium is devoid of parapodia, and has four pairs of tentacles. The parapodia are either uniramous or biramous; both dorsal and ventral cirri are present; the setæ are compound (articulated). There is a pair of anal cirri. In the pharynx there is always a pair of horny jaws, and usually a number of denticles in the buccal cavity.

The members of the sub-order Nereidiformia are all characterised by the possession of well-developed tentacles and palpi, and usually peristomial cirri. There are highly developed parapodia with acicula, jointed setæ, and dorsal and ventral cirri. The buccal 
region of the enteric canal is eversible as a proboscis, and there are usually horny jaws.

There are several species of the genus Lumbricus, differing from one another in the general form of the body, the number of the segments, the shape of the prostomial lobe, and other minor points. All of them agree in the presence of the following features, which characterise the genus and distinguish it from the many other genera of the family Lumbricide:-

The prostomium is dovetailed completely into the peristomium. The setæ are always in couples. There are longer and straighter setæ on the clitellum. The male apertures are always on the fifteenth segment. There are three pairs of vesicula seminales, in the ninth, eleventh, and twelfth segments, connected across the middle line in the tenth and eleventh by sacs enclosing the ciliated funnels. There are two pairs of receptacula seminis always situated in the ninth and tenth segments.

The family Lumbricidxe is distinguished from the other families of the sub-order Megadrili, which comprises all the Earthworms. by the combination of the following features:-

The clitellum usually begins behind the twentieth segment and occupies from six to nine segments; it is incomplete ventrally. Dorsal pores are present. The setæ on the clitellum differ from the others. The male apertures are not situated further back than the fifteenth segment. There are three or four pairs of vesiculie seminales, in the ninth to the twelfth segments. The testes and ciliated funnels are usually in the tenth and eleventh segments : the female apertures on the fourteenth.

\section{General Organisation.}

The general form of the body in the Chrtopoda is cylindrical, but in many, c.g., some members of the families Polynoidce (Fig. 365) and Amphinomida, there is a very considerable degree of dorso-ventral compression. In most the body is very long in comparison with its breadth; but this is not a universal rule, the length being in some cases not more than five or six times the breadth. The surface is marked out by a number of more or less .distinct annular constrictions or impressed lines into a corresponding series of segments or metameres, which are usually very numerous, often some hundreds in number, though in some cases there are not more than from twenty to thirty. These segments are 
usually very similar throughout the length of the body; but in the Cryptocephala (Figs. 366, 367, 373) there may be two or even more regions distinguishable from one another by the form of the segments and of their appendages. In the Oligochæta there is a thickened zone, the clitellum, comprising sometimes only one segment, sometimes a number. Each segment, with certain exceptions to be noted presently, bears either a pair of parapodia or merely a greater or smaller number of setæ. Parapodia are lateral hollow processes of the body-wall bearing a number of

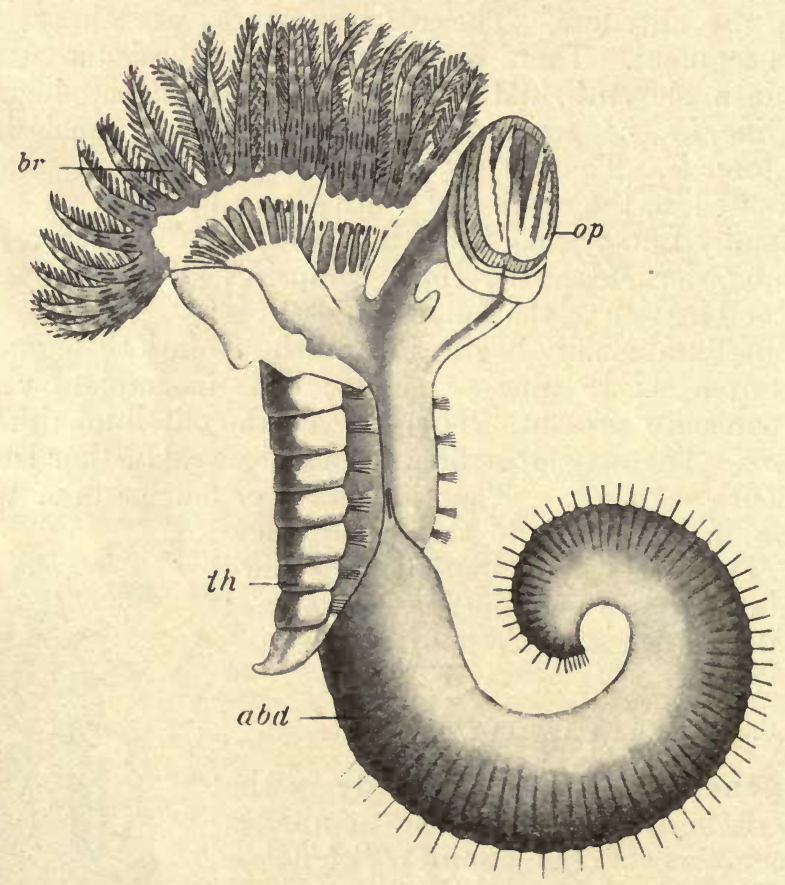

Fif. 366.--A Sexpulid (Vermilia coespitosil). Lateral view of animal removed from its tube. cbul. abdomen; $b r$. branchiæ; op. operculum; th. thorax.

bristles or setæ. Frequently the parapodium is divided horizontally into two distinct lobes or branches-a dorsal which is termed the notopodium, and a ventral which is termed the neuropodium. Even when this is not the case there may be two bundles of setæ representing the the two parts. The setæ are nearly always chitinous; in Euphrosyno they are calcified. They are always solid, except in Euphrosyno, entire, or divided into a number of joints. In shape (Fig. 368) they vary greatly in different groups ; often several very distinct forms of setæ are present in different 
parts of each parapodium of a single worm, or in parapodia of different regions of the body. Some are exceedingly delicate and hair-like, others needle-shaped, others compressed and sabre-like, others bayonet-like. Very often there is a long, straight, narrow part or handle with which is articulated a terminal blade, or bayonet, or hook. Sometimes the setæ are quite short, projecting little beyond the parapodia, and are hook-like or comb-like. Usually each bundle contains, in addition to the ordinary setæ, a stouter, straight, simple seta, which scarcely projects on the surface; this is termed the aciculum. Each seta, or each bundle of setæ, is lodged in a sac, the setigerous sac (Fig. 369), formed by an invagination of the integument, and lined by cells continuous with the epidermis. Each seta is derived from one of these cells, and is to be looked upon as a specially developed part of the cuticle of the general outer suface. The setigerous sacs are usually provided with protractor and retractor muscles, by the action of which the setæ may be thrust out or retracted.

In addition to the setæ the parapodium bears very commonly certain soft appendages of a sensory character, the cirri (Fig. 347, dors. cirr., vent. cirr.). There are usually both dorsal and ventral cirri, the latter nearly always much smaller than the former. The cirri are usually filamentous, sometimes jointed; sometimes they are laterally compressed and leaf-like. In Polynöe (Figs. 365 and 370) and its allies certain of the parapodia bear, instead of dorsal cirri, flattened scales, the elytra (el.), richly supplied

with nerves: these are sometimes looked upon as modified dorsal cirri, but in some members of the group cirri and elytra occur together on the same segment.

In Sternaspis a ventral shield formed by a thickening of the cuticle in the posterior region of the body bears a number of setæ round its edge.

In the Oligochæta (Fig. 372) the parapodia are absent as processes of the body-wall, and are merely represented by a small number of short setæ each lodged in its sac; cirri are not developed. In certain Oligochæta setæ are absent.

The first segment or prostomium, together with the second or 
peristomium, forms in many Polychæta a very distinct head; the prostomium in such a case bears eyes and tentacles and contains
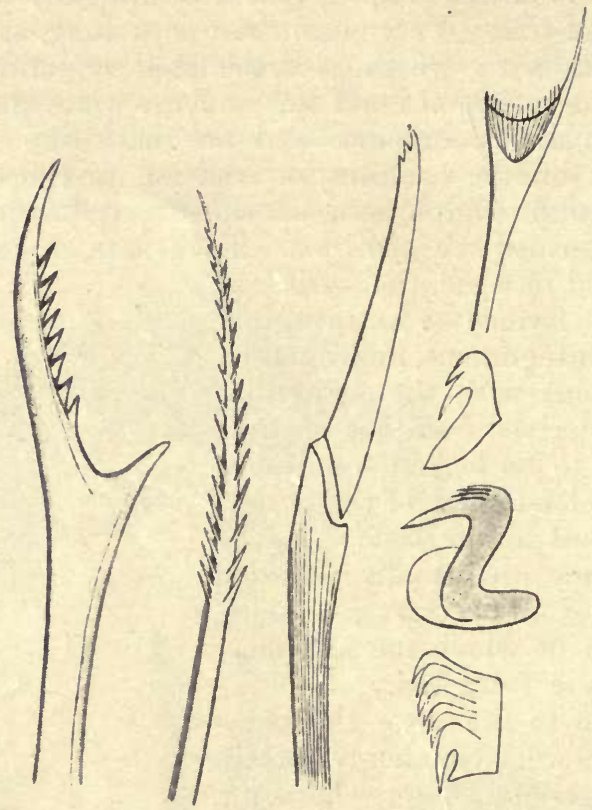

Fia. 368. - Setre of various Polychreta. (From Claparède.)

the cerebral ganglion; on the peristomium is the opening of the mouth, and from it also arise the peristomial tentacles. A

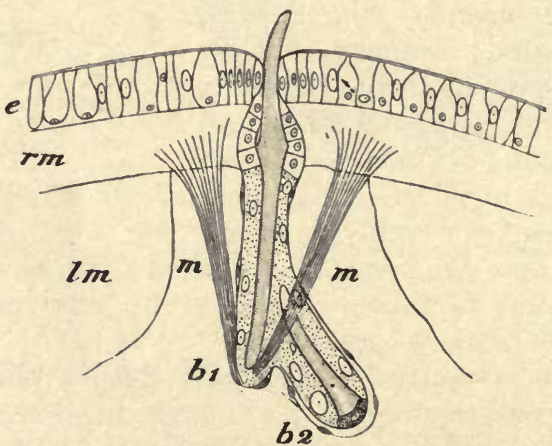

Fif 369.-Section of the setigrerous sac of an Oligochate. $b_{1}$, setigcrous sac; b., supplementary follicle with seta; e, deric epithelium (epidermis); $l m$, longitudinal muscles of body-wall ; $m$, $m$. muscles of the setigerous sac; $r . m$, circular muscular layer of body-wall. (From Hatschek, after Vejdovsky.)

ventral pair of prostomial tentacles, somewhat thicker than the rest, are sometimes to be distinguished, and are termed the palpi. 
Neither prostomium nor peristomium bears parapodia, though an aciculum is sometimes developed in the latter; the prostomium in fact, is not quite correctly termed a segment, being different from the true segments both in structure and in mode of development. In the Oligochæta there is no definite head, tentacles are entirely absent, and in the terrestrial forms the prostomium does not lodge the cerebral ganglion. In Sternaspis spinosa the prostomium is elongated and bifurcated like the proboscis of the Gephyrea armata (vide infra).

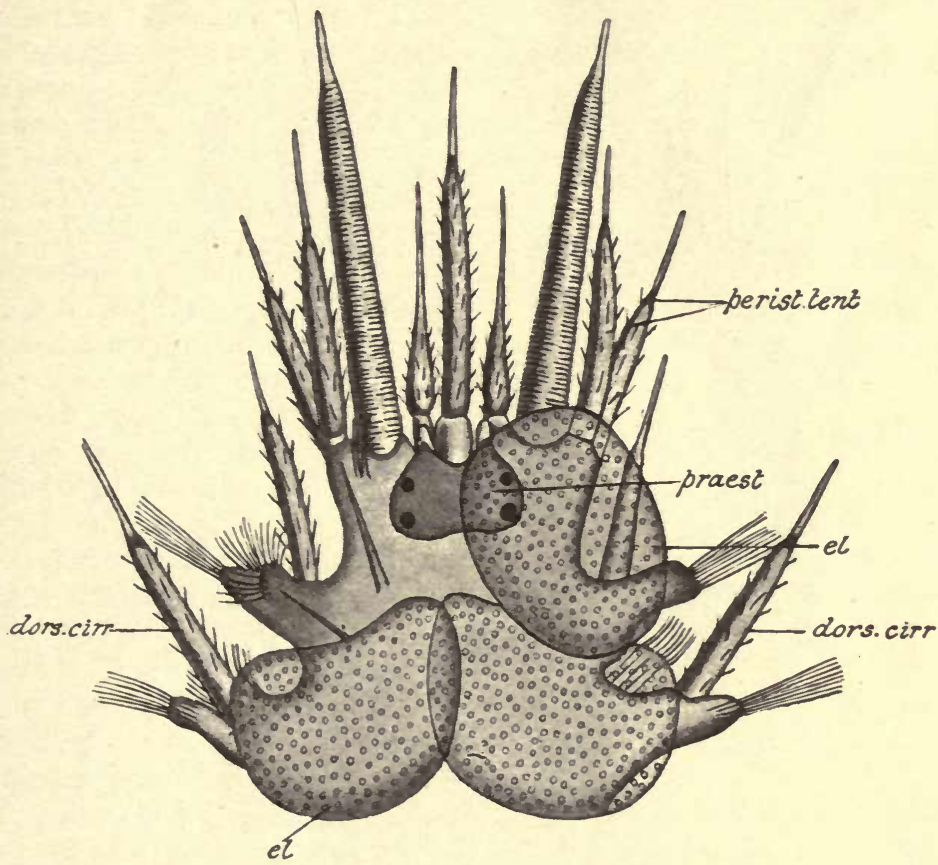

FIG. 370.- Polynoe extenuata. Dorsal view of anterior extremity. (lors. cirr. dorsal cirri ; el. elytra ; perist. tenl. peristomial tentacles; prost. prostomium. (After Claparede.)

The last segment is termed the anal segment, owing to its bearing the anal opening; it usually also differs from the preceding segments in wanting the parapodia and in having a pair of special cirri, the anal cirri.

Branchiæ are borne on the dorsal surfaces of more or fewer of the segments in many of the Polychrta. Sometimes they occur on all, or nearly all, the segments; sometimes they are confined to the middle region of the body; sometimes they are present only at the anterior end, as in the majority of the Polychæta living habitually in tubes (Figs. 366 and 373). In the 
Terebcllida (Fig. 373) the branchiæ are situated on the dorsal surfaces of some of the anterior segments. In the Serpulida (Fig. 366) they form two incomplete lateral circlets of elongated appendages situated at the anterior end of the body, apparently representing modified palpi, and sometimes supported by a cartilaginous skeleton; one of them is enlarged to form a stopper or operculum (op.), often armed with calcareous plates and spines, for the closure of the mouth of the tube in which the annelid lives. In

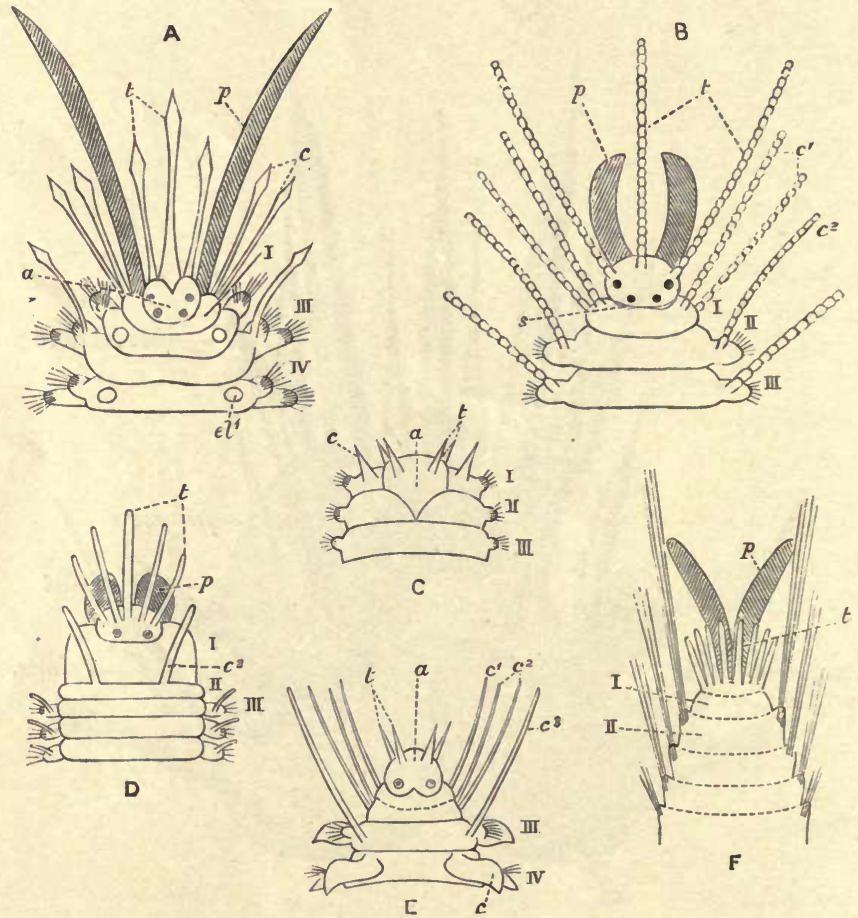

Fif. 371.-Heads of various Polychata (diagrammatic). $A$, Polynoid ; $B$, Syllid; $\&$, N $\left(p h t l_{1} y s\right.$; D, Eunice: $E$, Phyllocloce; $F$, Trophonia a, prostomium ; cirri of lody segments; $c$, peristomial cirri (tentacles); $c^{2}$, cirrus of first body-segment ; $c^{3}$, cirrus of sccond body-segment; $e l^{\prime}$, point of attachment of elytron; $p$, palp; $s$, nuchal organ ; $t$, tentacle ; $I$, peristomium: II, III, IV, segments. (From the Cambridge Natural History.)

shape the branchiæ are sometimes filiform, sometimes compressed and leaf-like, sometimes branched in a tree-like manner, sometimes pinnate. In Serpula (Figs. 366 and 383) and its allies each branchia consists of an elongated stem on which are borne two rows of short filaments. The surface of the branchiæ is usually ciliated. They are richly supplied with bloor-vessels when a blood-vascular system is developed; in Glycera, in which there are no blood-vessels, each branchia contains a diverticulum of the cœlome. 
In the Oligochrta branchire are rarely present : but in certain of the Naiidomorpha there are metamerically arranged simple or branched branchiæ, sometimes retractile, on the segments of the posterior region.

The body-wall consists of a cuticle, an epidermis, muscular layers, and a layer of peritoneum. The cuticle, composed of a chitinoid material, usually presents two systems of fine lines intersecting one another at right angles: it is perforated in many places by the ducts of the unicellular glands of the epidermis. The epidermis consists of a single row of cells, in some cases, with smaller cells of replacement intercalated between their inner ends. In shape the cells vary greatly in different families and often in different parts of the body of the same worm, being sometimes flattened, sometimes cubical or polyhedral, but more usually more or less vertically elongated. Cilia occur on the surface in certain parts in many Chrtopoda. Among the ordinary cells of the epidermis there are usually numerous unicellular glands often containing rodlike bodies. In the tubicolous forms these unicellular glands are active in secreting the material for the construction of the tube. In addition, the epidermis frequently contains sensory cells, which are in many cases contained in certain special elevations or sensory papillæ.

The muscular part of the body-wall consists of two layers, in the outer of which the fibres are disposed circularly, while in

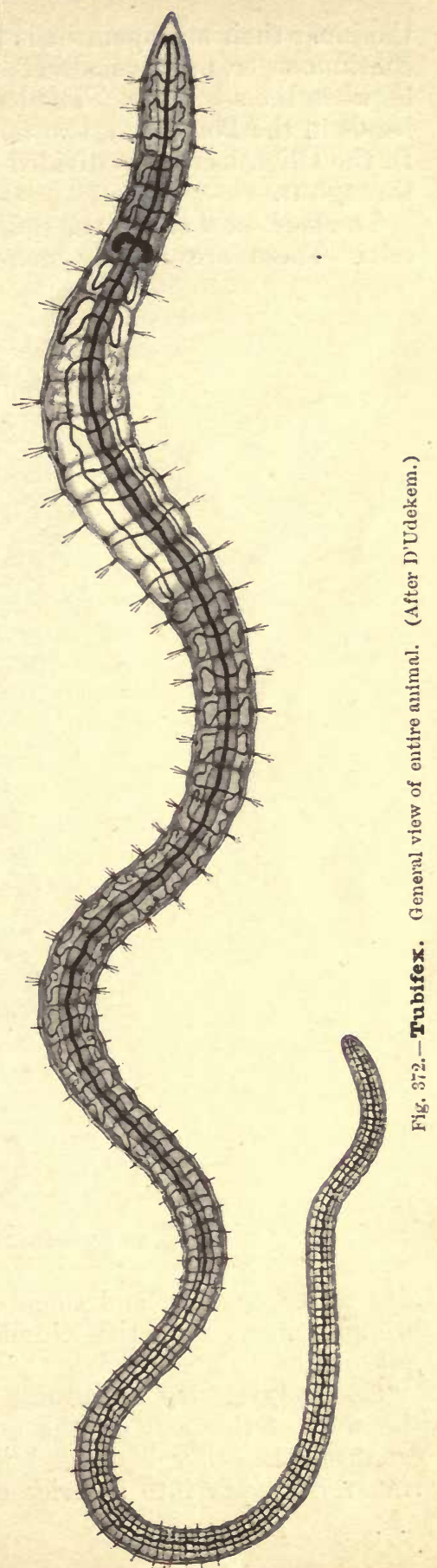


the inner their arrangement is longitudinal. The circular layer is continuous, or, more usually, is interrupted opposite the intervals between the segments. The longitudinal layer is disposed in four bands in the Polychæta, two dorso-lateral and two ventro-lateral. In the Oligochæta it is divided by the setigerous sacs which pass through it.

The peritoneal or coelomic epithelium consists of a single layer of cells. These are usually non-ciliated, but are ciliated in the

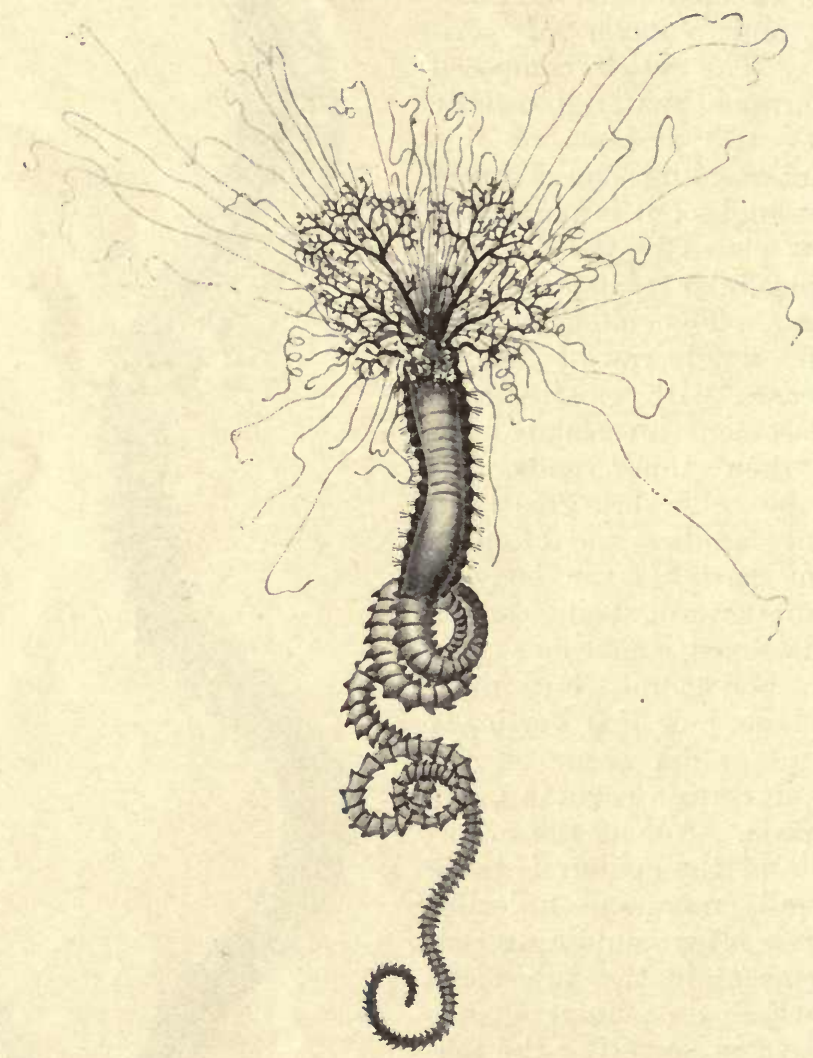

Frc. 373.-Terebella. (After Quatrefages.)

Aphroditea, Glycera, and some others, the movement of the cilia bringing about an active circulation of the colomic or perivisceral fluid in the colome.

The body-cavity or cœlome, a wide space intervening between the wall of the body on the one hand and that of the enteric canal on the other, is divided in many Chætopoda by a series of transverse septa into a series of chambers corresponding to the 
segments. The septa are not complete partitions, there being always apertures of greater or less extent by which the cavities of neighbouring segments communicate. The septa consist of double folds of the peritoneum enclosing muscular fibres.

The enteric canal is an elongated, and nearly always straight tube, running through the entire length of the body from mouth to anus. A number of different parts are usually distinguishable: but their disposition varies to a very great extent in the different groups. The buccal cavity, into which the mouth leads, is followed by a muscular pharynx; these are both formed in the embryo by invagination of the ectoderm, and therefore correspond to a stomodoeum. The muscular pharynx is absent in some of the Cryptocephala: when present it is frequently protrusible to a greater or less extent (see Figs. 349, 365); around its extremity, when it is fully protruded, are to be seen a circlet of papillæ in some forms; and in many, one or more horny teeth, situated in its interior, are brought into play. A gizzard with thick walls may follow upon this protrusible pharynx, and is sometimes preceded by an cesophagus, which may be dilated behind into a crop. The intestine is nearly always more or less deeply constricted in each segment, and in the Aphroditea, or "Sea-mice" (Fig. 374), there are in each of the segments (with the exception of one or two of the most anterior and one or two of the most posterior), a pair of cace which are to a greater or less extent branched at their extremities. In the Hesionsda and Syllida a pair of cæca which open into the anterior part of the intestine frequently contain gas,

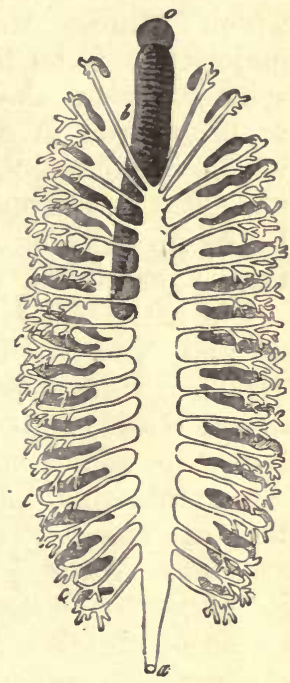

Fuc. 3i4.-Enteric canal of Aphrodite. $a$, mouth ; $b$, pharynx ; $c$, brauching caca of intestine ; $d$, anus. (From Gegenbaur's Com. parative Anatomy.) and probably have a hydrostatic function.

In some of the terrestrial Oligochæta (Earthworms) a fold of the intestinal wall, the typhlosole, projects into its lumen. The intestine is straight in most, but is somewhat coiled in the Chlorcemidce, Sternapsis, and others. The wall of the alimentary canal consists (1) of the visceral layer of peritoneum; (2) of longitudinally arranged muscular fibres; (3) of circularly arranged muscular fibres; (4) of enteric epithelium. The peritoneum on the surface of the intestine has in many Chætopoda its cells enlarged and granular to form the so-called chloragen cells, which probably have an excretory function. The enteric epithelium is very generally ciliated; it contains numerous gland- 
cells. The stomodæum and the proctodxum are lined internally by a cuticular layer, which is continuous with the cuticle of the general surface. The anus is usually terminal in position, sometimes directed towards the dorsal aspect. There is, in most instances, a longitudinal mesentery running to the alimentary canal from the dorsal body-wall; sometimes a ventral mesentery is also present bearing a corresponding relation to the ventral surface.

Some Chrtopoda are entirely devoid of blood-vessels. In one family in which this occurs (the Glycerid $x$ among the Phanerocephala), the perivisceral fluid, which assumes some of the functions of the blood, contains numerous red corpuscles, the red colour of which is due to the presence of hrmoglobin (see p. 36). In the majority of the Chrtopoda there is a highly developed vascular system. Sometimes the blood is colourless : very commonly it is bright red in colour, owing to the presence of hæmoglobin, which is not confined to the corpuscles, but is dissolved in the plasma. In Serpula and its allies the blood is bright green, owing to the presence of a green colouring matter, which has an affinity for oxygen similar to that possessed by hæmoglobin.

The chief blood-vessels are usually dorsal and ventral longitudinal trunks. These are connected together by metamerically arranged transverse branches. In some of the Cryptocephala the dorsal vessel is not present in the greater part of the length of the body, its place being taken by a circumintestinal sinus or a circumintestinal plexus of vessels lying in the wall of the alimentary canal. This gives off in front a short thick-walled dorsal vessel or "heart." 'The movement of the blood is effected in most instances by peristaltic contractions of the dorsal vessel or of a cirumintestinal sinus or plexus or of a short and wide dorsal cardiac sac given off by the latter anteriorly, which have the effect of driving the blood from behind forwards. In some instances, as in the Earthworms and some Cryptocephala, specially dilated lateral vessels are contractile, and by their pulsations bring about the circulation of the blood through the system of vessels. Plexuses of fine capillary vessels in the integument of various parts frequently aid in respiration, and are particularly well developed in certain forms in which definite organs of respiration are absent.

The nervous system consists of a cerebral ganglion or brain and a double ventral chain of ganglia. The cerebral ganglion is distinctly bilobed, and may be looked upon as composed of two intimately united ganglia. It is almost invariably situated in the prostomium, though placed a little further back in the Earthworms; it gives off branches to the eyes and tentacles. From it there run backwards and downwards the paired cesophageal connectives, which embrace the anterior part of the alimentary canal between them, and below join the anterior end of the ventral chain 
of ganglia. The latter always exhibits indications of being made up of two lateral halves in the double character of the connecting commissures and frequently of the ganglia themselves. One of these double ganglia occurs in each segment, and from it a number of nerves pass out to the various parts of the segment. In certain Cryptocephala (Serpula and others) the two halves of the chain are separated from one another by a wide space, across which transverse commissures pass between the ganglia. Connected with the cerebral ganglia, or with the oesophageal connectives, or with both, there is a system of delicate stomatogastric nerves passing to the walls of the anterior part of the alimentary canal. In the majority

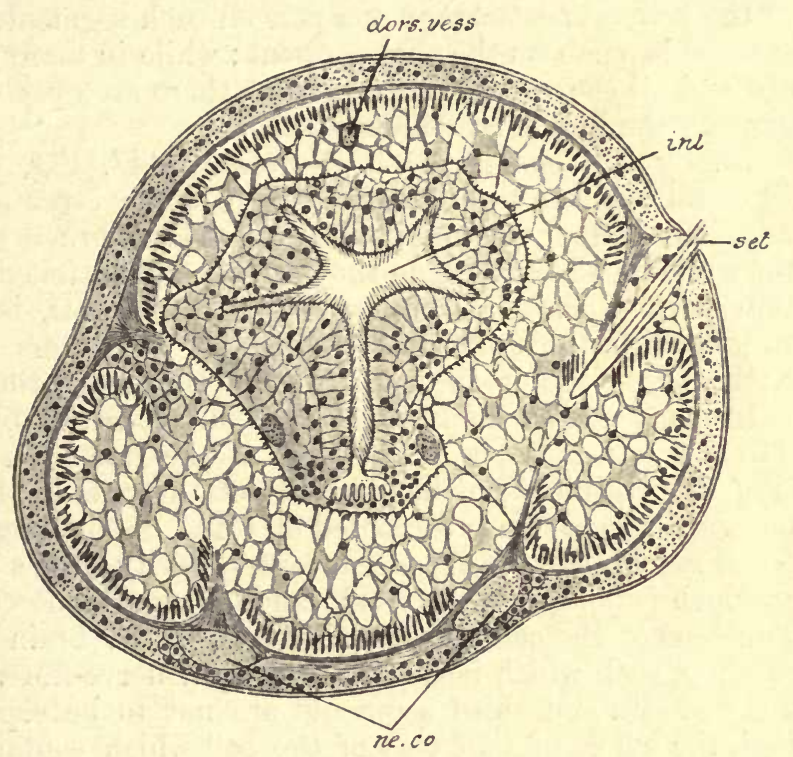

Fig. 375.-Saccocirrus, transverse section, to show the position of the nerve-cords. durs. vess. dorsal vessel; int. intestine; ne. co. nerve-cord; set. setæ. (After Fraipont.)

of the Chætopoda the cerebral ganglion and the ventral chain are separated from the epidermis by muscular layers; in some, however, the ventral chain is in contact with the epidermis, and in certain primitive or aberrant forms, the Archi-Chætopoda (Fig. 375 ) and Sternaspis, the cerebral ganglion is in close union with the epidermis; in these also the ventral cord is not segmented into ganglia. Running longitudinally through the ventral cord in many forms are certain giant fibres of very large size; though these may have rather a skeletal than a nervous function, they are simply greatly enlarged and modified nerve-fibres. Nerve-cells may be confined to the ganglia, or may be distributed over the entire sur- 
face of the ventral cord. Giant nerve-cells occur in some forms in certain regions. Small ganglia are found frequently in various peripheral parts, more especially at the bases of cirri or of sensory papillæ.

The organs of special sense are eyes, tentacles and civri, nuchal organs, and otocysts. Eyes, absent in the Oligochreta with a few exceptions and in some of the tube-forming Polychrta as well as in a few free forms of that sub-class, are very general in their occurrence. Their structure is, as a rule, very simple, but in some forms reaches quite a high grade of development. Usually they are confined to the prostomium, but Polyophthalmus, in addition to the prostomial eyes, has pairs of eye-like organs on many of the segments of the body. Leptochone has a pair on each segment, and in Fabricia there is a pair on the anal segment; while in many species of Sabella and all the species of Dasychonc there are eyes or eyelike organs on the branchial filaments.

Most usually the eye is (as in Nereis, p. 447, Fig. 353) a spherical capsule with a wall composed of a single layer of cells, which are elongated on the inner side, i.e. the side turned towards the brain, while on the outer side they are usually flattened. The outer thin part of the wall of the capsule, or cornea, is sometimes united with the epidermis; when the two layers remain distinct, the outer one is the outer cornea, the inner the inner cornea. In many cases a thickening of the surface cuticle over the cornea forms a cuticular lens. The cells of the inner portion of the wall of the capsule form the elements of the retina; they are long narrow cells, sometimes composed of three distinct segments -(1) a clear rod, directed towards the central cavity; (2) a middle segment which is densely pigmented; and (3) a segment containing the nucleus of the cell and directed towards the brain or the optic ganglion, with which it is connected by a nerve-fibre. Frequently the second and third segments are not to be separately recognised, the whole of that part of the cell which contains the nucleus being densely pigmented. A refractive mass fills the interior of the capsule, and is sometimes distinguishable into a firmer outer part, the lens, and a more fluid inner part, the vitreous body. This refractive mass is often continuous with the cuticle externally, and internally may be in continuity with the rods. In some cases the structure of the eye is very much simpler. The eyes on the branchial filaments of many tube-forming Polychæta consist each of a group of retinal cells having its own lens-like body and is quite independent of the others; the eye is thus a compound one.

Nichal organs (Fig. 371, B,s) are very general in the Polychæta. They consist of a pair of special ciliated areas or pits on the posterior part of the prostomium, eversible in certain cases.

Otocysts are only exceptionally present. They consist of capsules 
of ciliated cells, in the fluid contained in which there are one or several calcareous otoliths.

Ciliated grooves occur on the prostomium of many forms; in Aricia they are present on all the segments: they have a special nerve-supply, but their function can only be conjectured. Tactile cells of the epidermis, with or without a projecting tactile hair or stiff cilium, are very common, especially on the prostomium in the Oligochrta and on the tentacles and cirri in the Polychreta. Groups of these are often aggregated together in papilla or goblet-bodies, with special nerve-supply and often with a ganglion or a single nerve-cell at the base.

The organs of excretion of the Chrtopoda are a series of segmentally arranged tubes, the nephridia, of which a pair, as a rule, occurs in each of the segments of the body with the exception usually of a few at the anterior and a few at the posterior end. In its simplest form the nephridium is a curved tube, ectodermal in origin, ciliated internally, opening on the exterior by a laterally placed pore at the one extremity, and at the other ending in a ciliated funnel or nephrostome, which opens into the coelome either of the same segment as that on which the external aperture is situated (most Polychæta) or of the segment in front (all or most Oligochæta, some Polychæta). The nephridia thus in such cases effect a communication between the cœlome and the exterior, and serve to carry off waste-products which have passed into the colomic fluid; but in many instances the cells lining the tube are active in separating out such waste-matters, and are loaded with granules and concretions.

In many Polychæta, however, there is no ciliated colomic aperture, the tube ending blindly internally, such a blindly ending nephridium (Fig. 376) being frequently branched. On the inner extremities in such

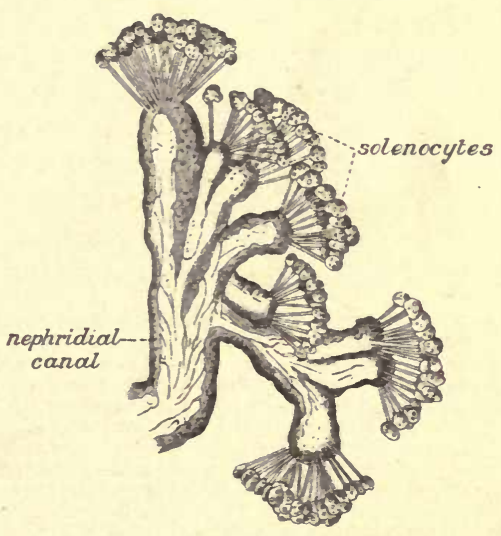

FIc. 376.- Inner branched end of nephridium of Phyllodoce paretti, showing the nephridial caual and the solenocytes. (After Goodrich.) cases, or on other parts of the tube, are situated a number of peculiarly modified cells, the solenocytes, sometimes separate, sometimes united together in groups. Each of these is a rounded cell lying in the coelome, and connected with the nephridium by a long, slender, tubular process: through the lumen of the process extends a single, extremely long, vibratile flagellum, which may 
be prolonged for some distance in the interior of the nephridium itself. The resemblance between those solenocytes and the flame-cells of Platyhelminthes will at once be recognised.

In the Polychæta another set of segmentally repeated structures are frequently intimately connected with the nephridia. These are a series of pairs of ciliated funnels, the colomoducts, opening widely into the coelome, and, in a typical case, communicating with
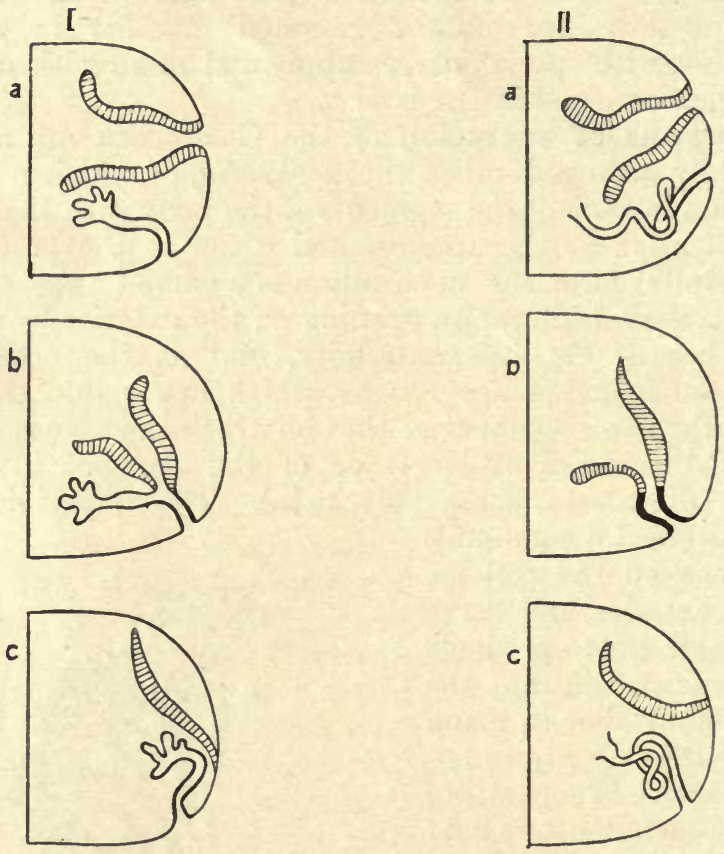

Fic. 37\%.-Diagram to illustrate the various corubinations of elosed and open nephridia and colomoducts in the Polychata.

la, Hypothetical stage with elosed neplnridia and separate celomoducts; $b$, condition in which the cuelomoducts liave becune united with the nephridia: this uccurs in Phyllodocicle and Gonianlicle; $c$, condition in which the celonoduct becomes reduced to a ciliated organ (Nephthyidet); II $u$, combination of nephridia with nephrostomes and separate coelomoducts (Dasybranchus); $b$, condition in which "segmental organs" are formed by the union of nepluridia with nephrostomes and cneloreducts (the most usual eondition); $c$, condition in which there are nephridia with nephrostemes, and the colomoducts are reduced to ciliated organs (Nereis, etc.). The nephridia are outlined with a thick line: the coelomoducts striated. (After Goodrich.)

the exterior. In Nereis they are represented by the dorsal ciliated organ, and are not known to open externally. When provided with external apertures, as is usually the case, the colomoducts act as the efferent ducts for the sexual elements. In many of the Polychrta they do not remain independent, but coalesce partially or completely with the nephridia, and the functions of excretory organs and reproductive ducts become combined in the one set of "segmental organs" (Fig. 377). In some families of Polychxta 
(Serpula and allies) there is a single pair of large nephridia in the anterior region of the body, with smaller pairs in the posterior segments, the former alone appearing to have an excretory function while the latter act exclusively as genital ducts. In Sternaspis only a single pair of nephridia are present, which, though they have small ciliated funnels, are not known to communicate with the exterior.

In the Oligochæta the nephridia are usually simple, elongated and coiled tubes, a pair or sometimes more than one pair in each segment; but in some, these are replaced or supplemented in certain of the segments, or in all, by a branching system of tubes with or without ciliated funnels. Sometimes the ordinary nephridia are not developed in the segments lodging the reproductive organs, their place being there taken by three pairs of tubes of the nature of localised colomoducts which become modified to give rise to the reproductive ducts; but ordinary nephridia may be present in these segments as well. In some Oligochæta the nephridia of the most anterior segments open into the mouth or pharynx, and have apparently taken on the function of digestive glands (peptonephridice, and all the nephridia of the posterior region of the body in one species (Allolobophora antipre), instead of opening on the exterior, communicate with a pair of longitudinal canals which posteriorly open into a median vesicle communicating with the rectum.

The permanent nephridia of the adult Chætopod are preceded in the larva by a series of provisional or embryonic nephridia of a temporary character. These have been found to occur in the head (prostomium) of many larval Oligochæta and Polychæta. They are ciliated intracellular tubes, sometimes branched, which do not open into the cavity of the prostonium. Sometimes solenocytes occur at the inner ends of the branches or of the undivided tube. Embryonic nephridia have also been shown to occur in the body in certain forms.

Phosphorescence, the production of light rendering the animal brilliantly luminous in the dark, occurs in a few cases (various Polynoids, Chatopterus, \&c.).

In the arrangement of the reproductive organs in the Chretopoda there is an essential difference between the two subclasses, the Oligochæta being hermaphrodite, and the Polychæta, with only a very few exceptions, unisexual. In the latter the gonads, ovaries or testes as the case may be, are masses of cells which are developed as the result of a proliferation of the cœlomic epithelium in certain positions (Fig. 378). Usually these organs, which are only conspicuous about the breeding season, occur in the great majority of the segments of the body; sometimes they are confined to a certain region. The exact place which they occupy in the interior of the segment varies in different cases: 
sometimes they surround one of the principal blood-vessels, sometimes they are situated laterally, in the bases of the parapodia. The sperms frequently undergo the final stages of their development after they have become detached from the testes, while floating in the colomic fluid, and the same sometimes holds good of the ova. Both sperms and ova appear to reach the exterior, in the majority of cases, through the "segmental organs," which may become modified and enlarged at the breeding season, though in some forms it is stated that the reproductive cells escape
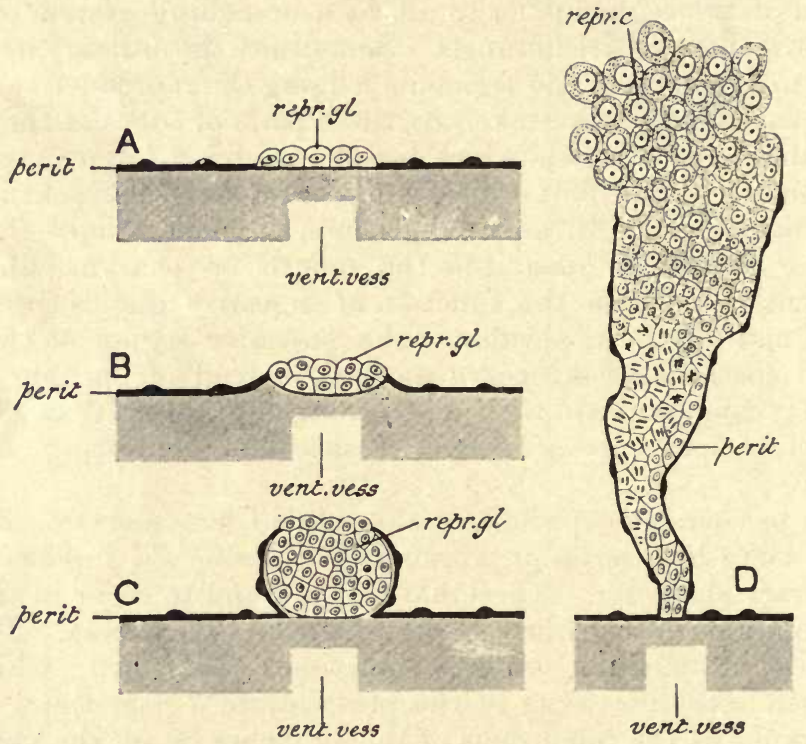

FIG. 378. - Diagram to illustrate the development of a gonad from the peritoneal (cuelomic) epithelium in one of the Polychata. perit. peritoncal membrane; repr. gl. gonad (reproduetive organ); vent. vess. ventral vessel. (After E. Meyer.)

through temporary or permanent openings in the body-wall. Impregnation takes place externally in nearly all.

In the Oligochreta the reproductive organs are confined to a certain limited region of the body. There are either, as in the Earthworms, two pairs of tcstes, or a single pair, as in the aquatic forms. The testes are small, and frequently become reduced to mere vestiges in the adult animal, having mainly become broken up into sperm-mother-cells, which in some way reach the vesiculæ seminales to undergo development into mature sperms. The vesiculce sominales are comparatively large sacs, which vary in number and arrangement in the different genera. One or two median sperm-sacs, formed by the coalescence of pairs of vesiculæ, may be present. In the same segments as the testes, and opening into the sperm-sacs when the latter are developed, are 
either two or four ciliated funnels, according to the number of the testes, leading into efferent ducts. All the four ducts, when four are present, may remain distinct, or the two ducts of each side may open into a common atrium, or they may unite to form a common elongated vas deferens, opening at the male genital aperture. In connection with the terminal part of the vas deferens in many Oligochæts is a gland known as the prostate or spermiducal gland. Near the aperture of the vas deferens in many Earthworms are special setæe, the penial setce.

There are never more than two ovaries, which, like the testes, are of very small size. The ova may become mature in the ovary, or groups of cells may be detached from the latter and one cell in each group ripen into an ovum. A receptaculum ovorum occasionally receives the ova after they leave the ovary. There are two oviducts, which open by funnel-shaped apertures into the cœlome.

Development.-The Oligochæta deposit the eggs in cocoons, either buried in the earth or attached to water-plants. The cocoon contains, in addition to a number of fertilised ova, a quantity of an albuminous fluid which serves as nourishment to the developing embryos. Segmentation is always unequal. In the forms in which food-yolk is scanty there is a process of embolic invagination (Lumbricus rubcllus); in the others (Tubifex, \&c.) the process is of the epibolic type. In the former case a blastula and an invaginate gastrula are formed in the way already described in the case of the Earthworm. In Lumbricus trapezoides the gastrula divides into two, each half subsequently giving rise to an embryo. The micromeres spread over the megameres very much as in the Polychæta. A pair of mesoderm cells early appear, and by their division forms the mesoderm bands. No free larval stage similar to the trochophore occurs in any of the Oligochæta, but the stage intervening between the completion of the gastrula and the commencement of the segmentation of the mesoderm bands corresponds to the trochophore in essential respects; and in some forms there is recognisable a feebly developed circlet of cilia comparable to the prototroch, and in some a pair of headnephridia.

Impregnation and the development of the embryo takes place externally in all the Chætopoda, with a very few exceptions in which development occurs in the cœlome or in the interior of a dilated segmental organ. In the Polychrta, in the great majority of cases, fertilisation takes place by the sperms coming in contact with the ova when both have become discharged, and the development of the embryos goes on while they are floating freely in the sea. There are a few cases in which the impregnated ova are received into a sort of brood-pouch and there pass through at least the earlier stages of their development. 
Such a brood-pouch is formed in certain Phanerocephala by the raising up of the integument on the ventral surface. In some species of Polynöe and allied genera, the fertilised ova and the

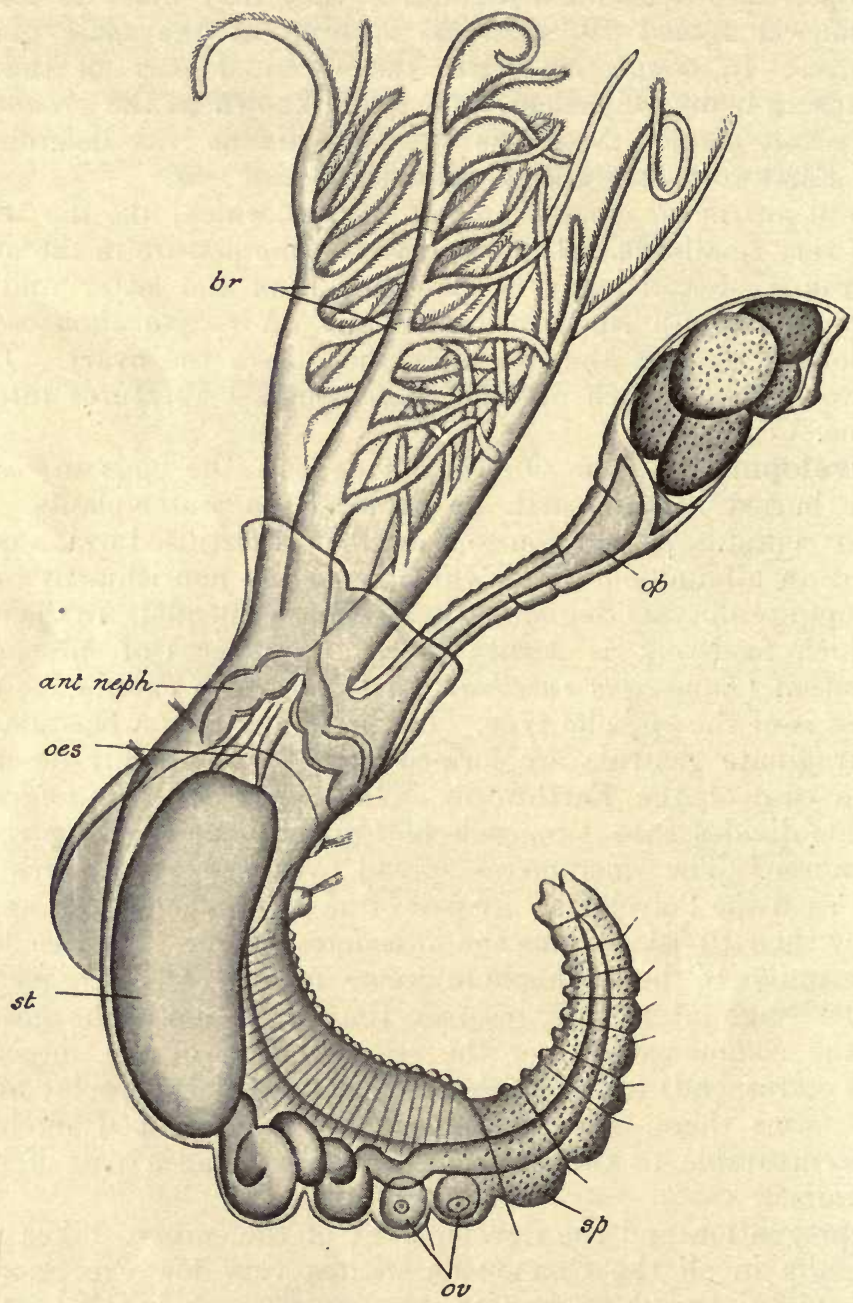

FIG. 379.-Spirorbis læevis, a hermaphrodite tubieolous Polychrt. Lateral view of entire anim ll. unt. neph. anterior nephridium; br. branchiz; $\alpha$ s. osophagus; op. operculum with developing embryos in its interior; ov. ova ; $s$ p. sperms ; st. stomach. (After Claparède.)

resulting embryos adhere in masses to the dorsal surface under the shelter of the elytra; in some other Polychæta they are stuck by means of some viscid secretion all over the dorsal surface, or they may adhere singly to the ventral cirri. In certain Crypto- 
cephala (Fig. 379) they develop in a cavity in the operculum; in others, in the interior of the tube, between the body of the worm and the inner surface of the latter, or on its outer surface. In some, again, though the ova do not remain in any way attached to the parent worm, they may be deposited in clumps or packets enclosed in gelatinous matter. Usually they have no other covering but the egg-membrane.

The segmentation of the ovum in the Polychrta is unequal. In the great majority the inequality between the megameres and micromeres is very marked. In some Serpulids, however, the difference is very slight, and the two sets of cells are at first scarcely distinguishable. In such cases the cells arrange themselves in

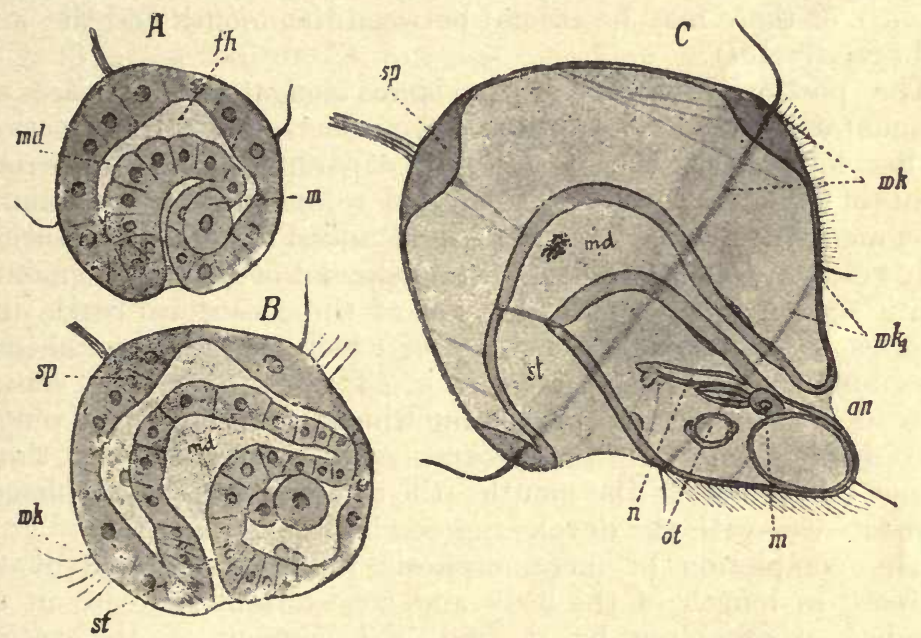

Fic. 3s0.-A, $B, C$, three stages in the development of the trochophore of Fupomatus, from the side. $a n$. anus ; $f h$. blastocole ; $m$. polar cells of the mesoderm ; md. mid.gut ; $u$. larval head-nephriảium; ot. otolith; $s p$. neural plate ; st. stonnodæum; $x k$, preoral eiliated ring; rck post-oral ciliated ring. (From Iang's Comparative Anatomy, after Hatschek.)

such a way as to form the wall of a hollow sphere, the blastula, with an internal closed cavity, the segmentation-carity. The megameres, which may or may not have been distinct from the first, lie on one side of the blastula; and soon this side becomes invaginated (Fig. $380 \mathrm{~A}$ ), the result being the formation of an embolic gastrula. In the great majority of forms however, an epibolic gastrula is formed after the manner already described in the case of Nereis; but forms of the process of gastrulation intermediate between these two extremes have been observed. The blastopore of the gastruli, however formed, does not give rise directly either to the mouth or to the anus. It becomes elongated into a slit which becomes closed up, and the anus and proctodium are formed by a fresh invagination 
in the original position of its posterior end, while another invagination of the ectoderm further forwards gives rise to the mouth and stomodrum. The embryo then passes into the trochophore stage.

The arrangement of the cilia on the surface of the trochophore varies in different Polychæta. Sometimes, though rarely, the preoral circlet is absent and the surface is covered uniformly with cilia: such larvæ are said to be atrochal. Sometimes there are two circlets close together, the one immediately in front of, and the other immediately behind, the mouth. Sometimes in addition to the pre-oral circlet, there is a peri-anal circlet round the anal end (telotrochal larvæ). In some cases, instead of a pre-oral circlet there is one further back round the middle of the body (mesoirochal), or there may be several between the mouth and the anal end (polytrochal).

The post-oral portion of the larva elongates, and traces of segmentation become visible; sometimes a series of constrictions are developed before there is any trace of parapodia, sometimes rudiments of the latter with their setæare developed first. 'The number of segments, at first very small, becomes added to from behind as the body gradually elongates. The establishment of external segmentation is accompanied by the division of the mesoderm bands into a series of segments, the history of which has been sketched in describing the development of Nereis. The ectoderm of the ventral plate develops a median thickening which gives rise to the ventral nerve-cord. Anteriorly this becames connected by a pair of thickenings at the sides of the mouth - the rudiments of the oesophageal connectives-with the developing cerebral ganglion.

The completion of metamorphosis is brought about by the increase in length of the body and concomitant increase in the number of segments, by the full development of the various systems of internal organs, and by the formation of the tentacles and other appendages. The parapodia, when first formed, very usually bear relatively long provisional seto, which are subsequently thrown off to make way for the those of the adult.

Asexual reproduction by simple fission followed by regeneration of the lost segments, or by proliferation followed by fission, occurs in certain groups of Chætopoda both among the Oligochæta and the Polychrta. Simple fission occurs in Salmacina, one of the Serpulids : a constriction becomes formed at a certain point towards the posterior end, rudiments of a new set of cephalic branchiæ bud ont on one side at this point, and this posterior part becomes a distinct zooid, which is eventually separated off and develops the full number of segments characteristic of the adult. This is not in any way a case of alternation of generations, as both parent and offspring are similar and sexual (hermaphrodite). In Nais and Chotogaster (Oligochæta) there is multiplica- 
tion by proliferation of the segments at the posterior end; then the appearance of a constriction separating off five or six of the most posterior segments followed by a fresh proliferation in front of the constriction; and then a second constriction appears five or six segments further forwards-the result being the development of a chain of zooids which remain for a time connected together. The sexual cells become fully developed only after the zooids have separated from one another.

In some of the Syllidæ there is a distinct alternation of generations. The asexual worm developed from the ovum gives rise by a process of posterior proliferation and constriction (Fig. 381) to sexual zooids, a number of which may remain for a time connected together in a string before undergoing separation. These sexual zooids become developed into mature males or females, which may be remarkably unlike the parent form in the shape of the parapodia, the character of the setæ, and other points; and in some instances the two sexes not only differ from the asexual parent form but also from one another, so that the three forms, before their relationship was known, were set down as representing three distinct genera.

Syllis ramosa (Fig 382) which occurs in the interior of certain deep-sea sponges, is exceptional among the Chætopoda in giving

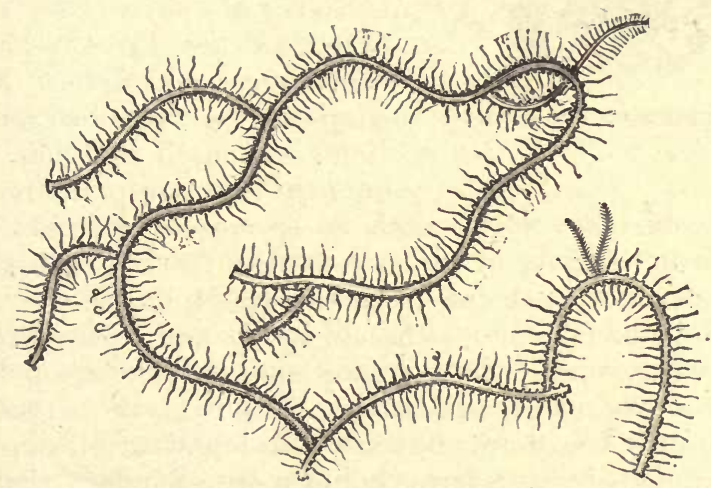

Fic. 382.-Portion of Syllis ramosa. (From the Cambridge Natural History, after MeIntosh.

rise by lateral branching to a colony from which sexual zooids afterwards become separated off. 
Modes of Life, etc.-Very few Chrtopoda are true parasites; but a considerable number are to be set down as commensals, habitually associating with another animal for the sake of food and shelter. The Earthworms burrow in soil containing decaying vegetable matter, passing the mould through their intestine and subsequently throwing it out in the shape of castings on the surface. They also feed on decaying leaves, and sometimes on animal substances. Some of the fresh-water Oligochæta (Tubificida) manufacture tubes of mud held together by a tenacious secretion from the epidermal unicellular glands. Some of the Phanerocephala form temporary tubes of a gelatinous character, or more permanent

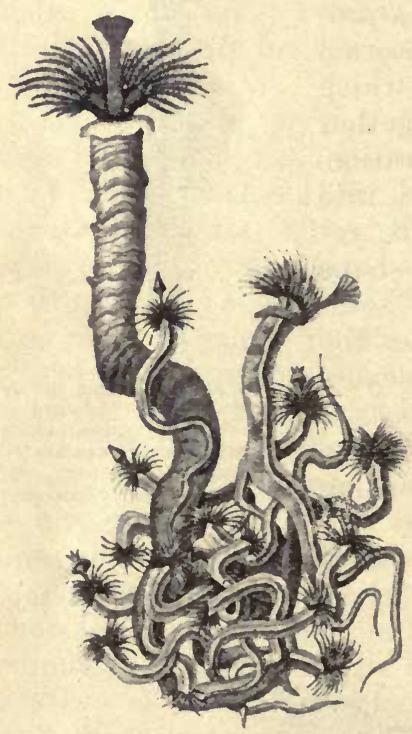

FIa. 383.-Serpulæ with their tubes. (After Quatrefages.)

parchment-like tubes sometimes strengthened by means of agglutinated sand-grains. But the majority of the Phanerocephala, which for the most part prey on other small animals, are not confined to tubes, but move about freely. Some burrow in sand; others even in harder substances, such as the shells of Mollusca, or in limestone, shale, or sandstone. Many Cryptocephala secrete tubes the substance of which is derived from the epidermal glands. These tubes are sometimes membranous or parchment-like, sometimes membranous but hardened by the leposition of grains of sand or particles of broken shells or bits of sea-weed; sometimes (Fig. 383) they are of a hard, shelly, calcareous character, sometimes composed entirely of foreign particles cemented together; very frequently they are permanently fixed to foreign objects. Some, such as species of Polydora and Stylarioides, near relatives of which construct tubes, excavate galleries in rock or coral or in the shells of various Mollusca.

A few Polychæta, such as the Alciopida and Tomopteris, as well as, in a certain phase, the Ncreidle and Syllida, are pelagic; but the majority live on the sea-bottom. They occur in the greatest abundance near the shore; but are also found at all depths in the ocean, the tube-dwelling forms being more abundant than the free forms in the deeper zones.

Owing to the soft character of most of their parts, there are comparatively few actual remains of Chætopoda in the older geological formations, though there are many burrows and tracks 
which have been ascribed to members of that class. Tubes of tubiculous Polychxta have, however, been found in formations dating from the Cambrian period onwards. Some tubes not distinguishable from those of the existing genus Spirurbis, are found as far back as the Silurian; and others, apparently closely related to the living Serpula, as far back as the Carboniferous. In addition there are a number of tubes of extinct forms ascribed to the tubicolous Polychæta. The horny jaws of various Polychæta have been detected in strata from the Cambrian period onwards ; and many tracks and burrows occurring in rocks of all ages are ascribed, some with more, some with less certainty, to this group of worms. No fossil remains of Oligochæta are known.

\section{APPENDIX TO THE CH ATOPODA.}

CIASS MYZOSTOMIDA.

The Myzostomida are a group of worms which appear to have their nearest relatives in the Chrotopoda, though possessing certain special features of their own. They are all external parasites of various Crinoids-both of the stalked and the free varieties, or internal parasites of certain Starfishes. They are disc-shaped animals (Fig. 384) devoid of any trace of external segmentation. There are patches of cilia here and there on both dorsal and ventral surfaces. At the sides there are five pairs of parapodia $(p)$, each with a chitinous hook and a supporting rod; in the intervals between these there are in Myzostoma four pairs of small "suckers"; and round the margin are a series of ten or more pairs of cirri provided terminally with motionless sensory cilia, and with a ventral groove lined by adhesive cells. The month, usually situated at the anterior extremity, leads into a muscular pharynx (Fig. 385, ph.) capable of being protruded as a proboscis; from this a narrow œesophagus leads to the stomach, which gives off a number of branched lateral diverticula ( $d a$. $)$ A short cloaca (klo.) leading from the stomach opens on the exterior, in most cases at the posterior end of the body, sometimes on the dorsal surface. There is no distinct cœlome, the space between the alimentary canal and the body-wall being filled by connective tissue (parenchyma), leaving only the eavities in which the sexual elements are lodged. Bundles of dorso-ventral nuscular fibres form imperfect transverse septa, as in some Platyhelminthes.

There is no blood-vascular system, and specialised organs of respiration are likewise wanting. There is a single pair of nephridia with funnel-shaped internal apertures and with external openings either into the cloaca or on the surface. The nervous 
system comprises a large stellate ganglion situated ventrally, probably representing a number of fused ganglia, and giving off a number of nerves; and of two nerve-rings, one round the œsophagus, the other round the pharynx, the two rings being connected together by a series of longitudinal nerves. The

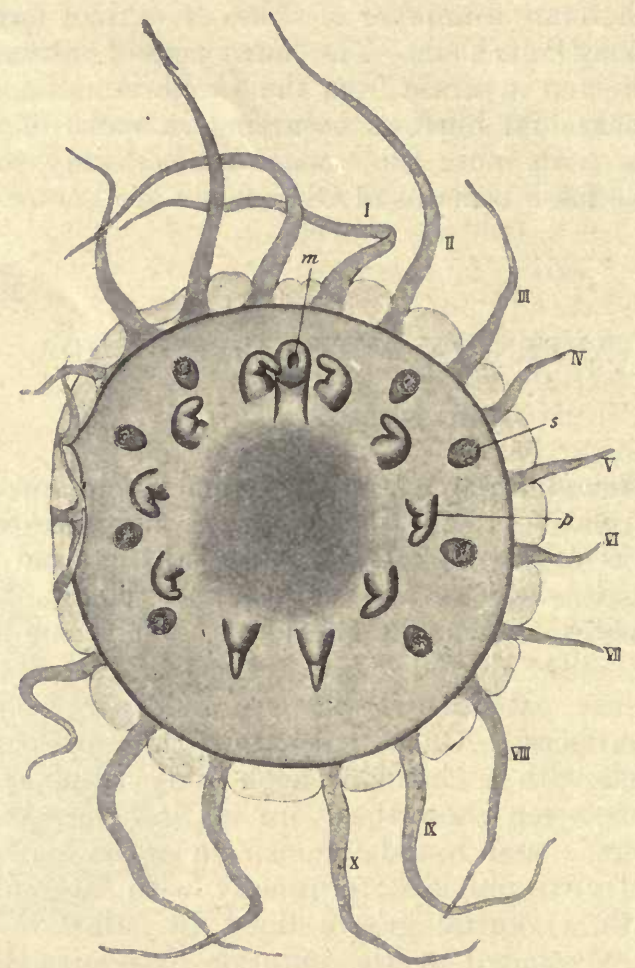

Fra. 384.-Myzostoma. I-X, cirri ; $m$, mouth ; $p$. parapodia ; s. suckers. (After von Graff.)

œsophageal ring presents a very obscure dorsal thickening, which is the only representative of a cerebral ganglion.

Most of the Myzostomida are hermaphrodite. There is a pair of ovaries formed by the proliferation of the layer of (cœlomic) epithelium covering the stomach; and in the sexually mature animal branching (cœlomic) spaces in the parenchyma, between the cæca, are found to be filled with ova $(o)$. A posterior continuation of these spaces $(u)$ opens either into the cloaca or independently of it. There are two elongated and usually branched testes (h), each of which has two vasa deferentia leading to a vesicula seminalis $(s b)$ which opens near the lateral margins. The sexes 
are united in most cases, separate in some. In the hermaphrodite forms the testes are matured before the ovaries, and may have ceased to be functional before the ova become ripe.

The development of the Myzostomida closely resembles that of the Polychæta. A trochophore larva is first formed, and this

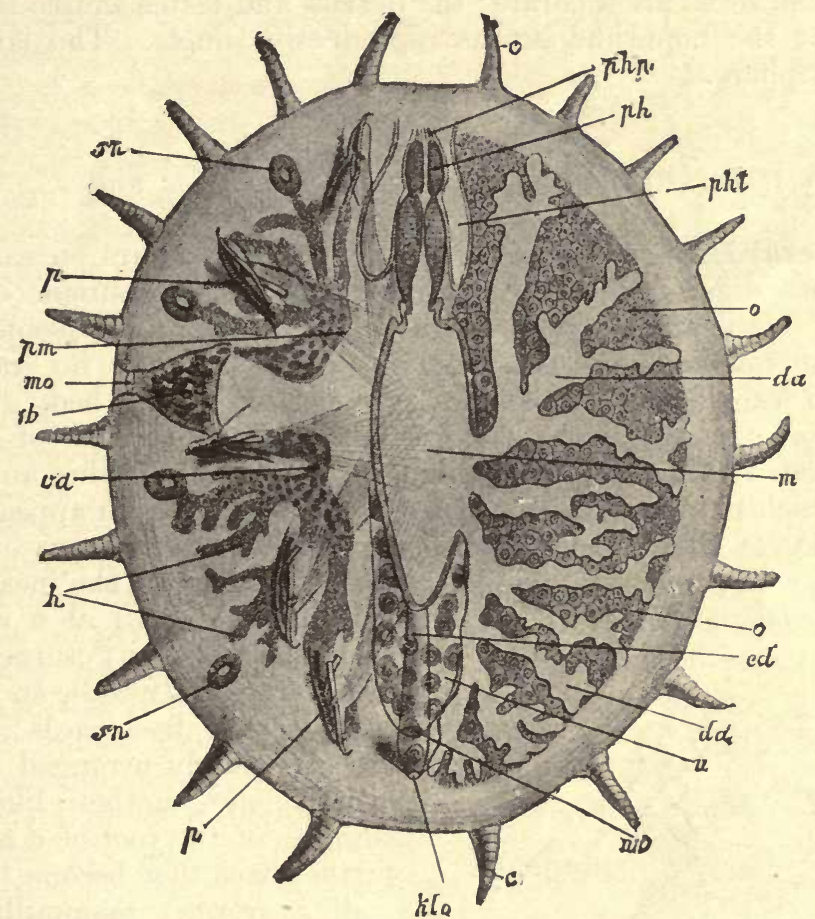

Fin. 385.-Myzostoma. Diagrammatic view of the internal organs. $c$, cirri ; da, branches of the stomach ; ed, hind-gut: $h$, testes ; $k l o$, aperture of cloaca ; $m$, stomach ; mo, male genital aperture; $o$, ovaries; $p$, parapodia, with hooks and supporting rod; $p h$, pharynx; $p h p$, pharyngeal tentacles; $p h t$, pharyngeal pouch; $s b$, vesicula seminalis; $u$, uterus; vo, female genital aperture. (From Lang's Comparative Anatomy, after von Graff.)

becomes metamorphosed into a larva with provisional setæ bearing a close resemblance to that of Nereis (p. 451).

\section{CLASS II.-GEPHYREA.}

The Gephyrea are marine Annulata devoid of any trace of segmentation in the adult condition, without parapodia, and either without setæ or with only a limited number; with either an invaginable anterior body region or introvert, at the extremity of which is the mouth surrounded by tentacles, or with a long, highly retractile proboscis representing the pre-oral lobe of the larva, and having 
the mouth situated at the base. The anus is sometimes terminal and posterior, sometimes anterior and dorsal. There is an extensive cœlome filled with a corpusculated fluid, and not divided by septa. The ventral nerve-cord is not made up of a series of ganglia. There is, as a general rule, only a single pair of nephridia. The sexes are separate; the ovaries and testes simple masses of cells; the nephridia act as reproductive ducts. The larva is a trochophore.

\section{Exampies of the Class-Sipunculus nudus.}

General External Features. - Sipunculus occurs on sand at moderate depths off the coast in most countries outside of the tropics. It is an elongated worm of a cylindrical shape, somewhat narrower towards one-the anterior-end. There is no trace of division into segments. The anterior portion of the body, to the extent of about a sixth of the total length, is capable of being involuted within the part behind. The surface of this anterior part, which is termed the introvert (Fig. 386), differs in appearance from that of the rest of the body in being covered more or less closely with chitinous papilla. The papillæ of the posterior portion of the introvert are shaped like the bowl of a spoon,

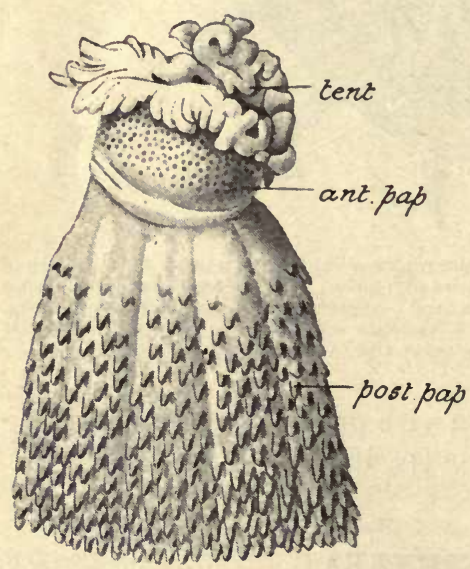

Fig. 386.-Anterior extremity of Sipunculus nudus. ant. pup. anterior papillary regiorr; post. pap. posterior papillary region ; tent. tentacular fold. (After Ward.) with the concavity turned towards the body-wall and the tip directed backwards; they are so closely arranged as to overlap one another like the shingles of the roof of a house : further back they become longer and narrower, mammilliform, and more scattered. When the introvert is fully evaginated, there appears at its extremity a horseshoe-shaped fold of the integument, the tentacular fold (tent.), which is lobed and plaited (Fig. 387) so as to assume somewhat the appearance of a circlet of tentacles. For a little space immediately behind the tentacular fold the surface of the introvert is free from papillæ. The posterior portion of the body is devoid of papillæ, but is marked out by a series of narrow impressed lines into a number of elongated foursided areas. 
Body-wall.- The surface is covered by a chitinoid cuticlc having an iridescent lustre similar to that presented by the cuticle of Nereis and Lumbricus, and due to the same cause-viz., the presence of two systems of intercrossing lines. The papillæ on the introvert are local thickenings of this cuticular layer. Beneath the cuticle is an epidermis consisting of a single layer of cells, usually sac-like, but capable of being altered as a result of contraction or compression into a spindle-like shape. Below the epidermis is a layer of connectivetissue, the dermis, in which, as well as to some extent in the epidermis itself, are a number of dermal bodics. Of these there are three kinds-bicellular glands, contained in papillæ; multicellular glands, scattered through

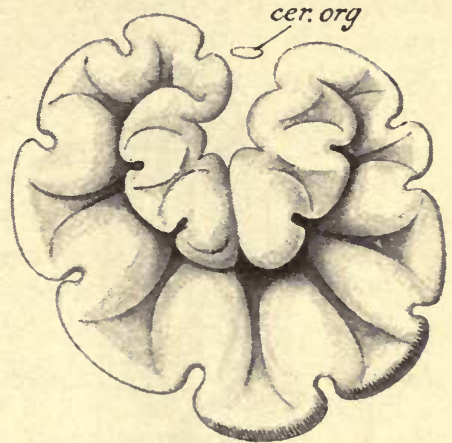

Fig. 38T.-Tentacular fold of Sipunculus nudus. cer. org. cerebral organ. (After Ward.) the integument and not contained in papillæ; and sense-papilla, small rounded thickenings of the epidermis in the anterior region of the introvert, with their summits covered with cilia. There are also numerous pigment-cells. A number of canals branch through the dermis, beneath which are three layers of muscle-(1) an outer circular layer, continuous in the introvert, but divided into annular bands in the rest of the body; $(2)$ an oblique layer, well developed only between the origins of the two retractor muscles of the introvert; (3) a longitudinal layer, which is separated by spaces into a series of parallel bands. Between the bundles of the longitudinal layer of muscle runs a series of canals which communicate with the body-cavity by transverse branches.

There is a spacious cœlome, but it is traversed in all directions by filaments and strands of connective-tissue, with which are mixed very fine muscular fibres; these mostly run from the wall of the body to the alimentary canal. Floating in the coelomic fluid are (1) colourless corpuscles; (2) reproductive elements; (3) peculiar ciliated bodies, the urrns, which are developed by proliferation from cells on the wall of the dorsal blood-vessel. These are comparable in structure and function with the ciliated funnels of the Hirudinea (q.v.).

The blood-vascular system is very feebly developed. It consists of dorsal and ventral contractile vessels, the former known as the "heart," communicating in front with a circular sinus at the base of the tentacular fold.

The alimentary canal (Fig. 388) is a cylindrical tube of uniform character throughout. It is twice the length of the body, running 
back from the mouth towards the posterior end, and then bending sharply round to run forwards to the

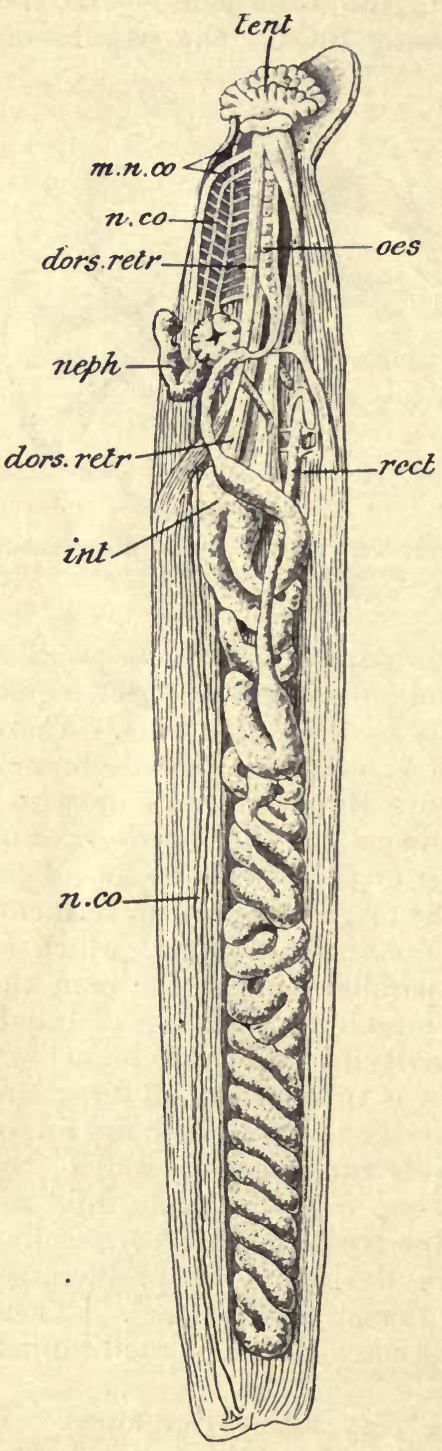

FIG. 388.-Dissection of the internal organs of Sipunculus nudus. dors. retr. dorsal retractor muscles of the introvert ; int. intestine; $m$. n. co. muscles accompanying the nerve-cord; $n$. co. nerve-cord; neph. nephridium : a.s. cesopliagus; rect. rectum; tent. tentacular fold.' (After Vogt and Jung.) anus, the two limbs being twisted spirally round one another. Running along the inner surface of the entire length of the alimentary canal, with the exception of the terminal part or rectum, is a narrow groove. Connected with the rectum is a narrow cacum of variable length, which opens into the beginning of the rectum. Two tuft-like groups of rectal glands occurclose to the anal opening.

The nervous system (Fig. 389) differs considerably from that of

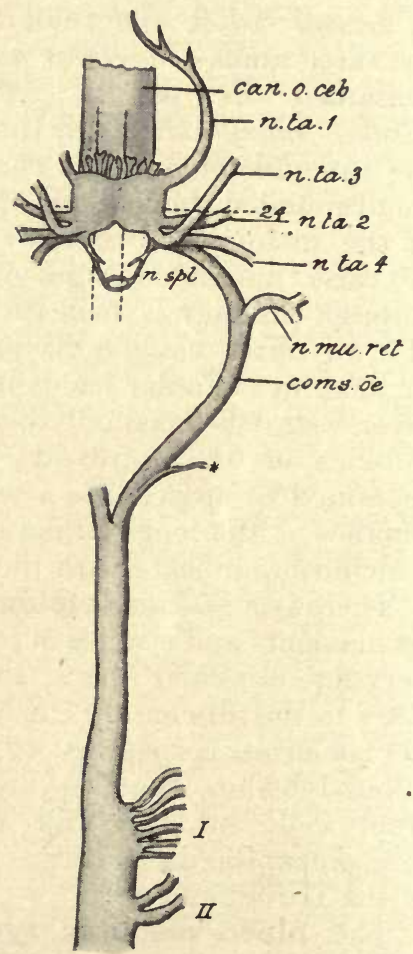

Fic. 389.-Anterior part of the nervous system of Sipunculus nudus. can. o. ceb. cerebral organ; coms. a, oesophagal comnective ; $n . m u$. ret. nerves to retractor muscles; $n . s p l$, splanchnic nerves; $n$. ta. 1-4, nerves to tentacular fold ; $I, I I$, nerves from ventral cord; 24, wain mass of brail. (After Ward.) 
the rest of the Annulata. There is a relatively small bilobed cerebral ganglion situated on the dorsal aspect just behind the tentacular circlet, to which it gives off on each side several pairs of nerves. Arising from it anteriorly and dorsally are a number of digitate processes lying in the coelome. The cesophageal connectives (coms. $\propto$ ) which it gives off behind are greatly elongated; from each arise muscular nerves $(n . m u$. ret), and also a visceral nerve $(n . s p l)$ passing to the alimentary canal. The two commissures unite behind to form a ventral cord, which extends throughout the rest of the length of the body. The ventral cord presents no appearance of ganglia: it sends off laterally a large number of pairs of nerves (I., II.); on section it appears distinctly double. Two delicate muscular bands (Fig. 388, m.n.co.), which take origin anteriorly from the body-wall, become attached to the nerve-cord, and follow it throughout its length, giving off small branch-bands to accompany the lateral nerves. A canal with folded and pigmented walls, which opens in the middle line of the dorsal surface just behind the tentacular fold (Fig. 387, cer. org.), extends backwards to the anterior ventral surface of the cerebral ganglion, where it ends blindly. It is possible that this, the cercbral organ, may be a sensory organ of some kind. Eyes are entirely absent. The digitate processes of the cerebral ganglion, which bear a number of ciliated cups along their edges, may be sensory in character.

Sipunculus has only a single pair of nephridia. These (Fig. 388, neph.) are situated tolerably far forwards, the external openings being about $2 \mathrm{~cm}$. in front of the anus. They are long, nearly straight tubes, of a brown or yellowish colour, and very mobile in the living condition. Near the external opening, which is situated at the anterior end, is the internal opening into the colome. The sexes are separate. There are no definite gonads except at a certain season of the year, when cellular elevations developed in the connective tissue covering the ventral retractor muscles of the introvert represent ovaries or testes as the case may be. These give origin to cells which become detached and develop into the fully-formed sexual elements while floating about in the coelomic fluid. The nephridia act as gonoducts.

\section{Distinctive Characters and Classification.}

The Gephyrea are Annulata with the body devoid of any appearance of segmentation in the adult condition. There is a large colome, which is not divided into chambers by mesenteries or septa. A blood-vascular system is sometimes present, sometimes absent. The ventral nerve-cord is not composed of a chain of ganglia. There is usually only one pair of nephridia. The sexes 
are separate, the gonads simple, and the nephridia act as gonoducts.

The larva is in most cases a trochophore, and may develop a metameric segmentation which disappears as development proceeds.

\section{Order 1.-Inermia (Sipunculoidea).}

Gephyrea with an introvert and usually tentacles or a tentaclar fold. The anus is dorsal. Setæ are absent. Nephridia a single pair, or absent altogether.

\section{Order 2.-Armata (Echiuroidea).}

Gephyrea with an elongated prostomial proboscis. The anus is posterior. Two or more setæ. A single nephridium, or two or three pairs of nephridia.

\section{Systematic Position of the Example.}

Sipunculus nudus is one of several species of the genus Sipunculus. Sipunculus differs from other genera of the family Sipunculide of which it is a member, mainly in having a tentacular fold around the mouth, instead of a series of distinct tentacles. The family Sipunculide is one of two families of the order Inermia; and differs from the other, the Priapulida, in the presence of either tentacles or a tentacular fold at the oral end, and the absence of filiform appendages at the aboral end.

\section{General Organisation.}

The Gephyrea are a class of worms whose position among the Annulata is determined more from a consideration of their development than of their structure in the adult condition, though the latter suggests a tolerably close affinity with the Chætopoda. The body of a Gephyrean is unsegmented, usually more or less completely cylindrical, broadest behind and narrowing towards the anterior end. The surface is covered with a chitinous cuticle developed often into papillæ, or tubercles, or hooks. In the Armata, setæ are present, but they are always very few in number and not implanted in parapodia ; in Bonellia there is only a single pair, situated about the middle of the ventral surface; in most species of Echiurus (Fig. 391), in addition to this ventral pair, there are a number arranged in one or two circlets around the posterior end. In the Inermia the anterior part of the body is capable of being invaginated within the part behind; at the extreme anterior end of this invaginable part or introvert, when it is evaginated, is the mouth, surrounded by a circlet of sometimes pinnate, sometimes simple, 
tentacles, or by a lobed and plaited tentacular fold. The prostomium is in such forms quite rudimentary. In the Armata there is no introvert, but an elongated, highly contractile, simple or bifurcated proboscis, which is the greatly produced prostomium; in Bonellia (Fig. 390 ) the proboscis, when fully extended, is many times the length of the body: in Echiurus (Fig. 391) it is much shorter: at the base of the proboscis on the rentral side is the opening of the mouth. In Priapulus (Fig. 392) there is at the posterior end an elongated simple or bifurcated caudal appendage covered with

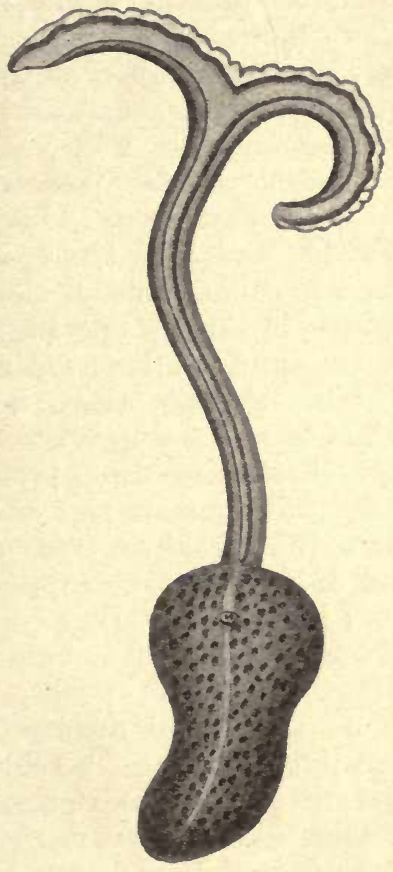

FiG. 390.-Bonellia viridis, entire animal (female) with the proboscis moderately extended. (After Greef.)

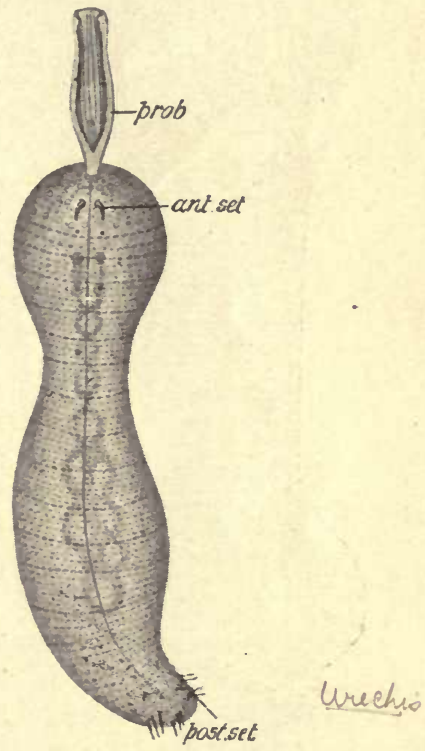

Fic. 391.-Echiurus, entire animal. ant. set. anterior seta ; post. set. posterior setæ; $p r o b$. proboscis. (After Greef.)

hollow papillæ. The anus is situated at the posterior end of the body in the Armata ; in the Inermia it lies far forwards on the dorsal surface, except in the case of Priapulus, in which it is terminal.

Body-wall.-Beneath the cuticle is an epidermis, which is composed of a single layer of cells. Among the cells are unicellular (rarely multicellular) glands, and sensory cells. Various colouring matters, such as the bright green characteristic of Bonellia, are contained in the cells of the epidermis. The muscular wall of the body consists of external circular and internal longitudinal layers,

VOL I 
sometimes with oblique and internal circular layers superadded. There is an extensive undivided cœlome, lined, as in the case of the Chretopoda, with a cuelomic epithelium, which is sometımes ciliated.

The alimentary canal in the Inermia consists of a muscular pharynx, intestine, and rectum; in the Sipunculidce (Fig. 388) the intestine is bent on itself, and spirally twisted as it runs forwards

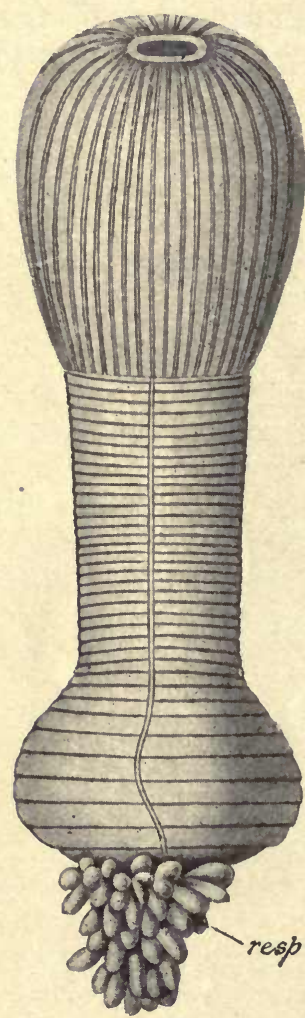

FiG. 392.-Priapulus, entire animal. resp. posterior papillie. (After Ehlers.) to the anal opening, which, as already noted, is situated far forwards on the dorsal surface: at the junction of intestine and rectum is a single simple crecum or a pair of creca; and a number of small branching glandular appendages are attached to the rectum close to the anal opening. Retractor muscles pass from the body-wall to the pharynx. In the Armata (Figs. 393 and 395) there is a thin-walled buccal cavity, and an elongated and coiled intestine, opening at the posterior extremity of the body into a dilated rectum: in most there is an elongated cocum or siphon applied to the ventral aspect of the intestine proper. Into the rectum there open a pair of remarkable cæca, the posterior nephridia (Figs. 393 and 395 , post. neph.), supposed to be excretory in function; these open into the colome by means of a number of ciliated funnels (Fig. 394).

There are no specialised organs of respiration in the Gephyrea. A bloodvascular system is sometimes present, sometimes absent. When present, as it is in most Gephyrea, it usually comprises a contractile dorsal vessel closely applied to the intestine, and a peripharyngeal ring or plexus. Cilia are present in places in the interior of the vessels.

The nervous system (Figs. 389 and 396) consists of a nervering, sometimes greatly elongated, surrounding the anterior part of the alimentary canal, with sometimes a dorsal and anterior thickening representing a cerebral ganglion; and of a nerve-cord, devoid of ganglia, running backwards from this along the middle of the ventral surface, and giving off pairs of branches at regular intervals; the branches of the same pair sometimes form complete rings (Fig. 396, ne. ri.) by uniting dorsally. Eyes of a very 
simple character, consisting of mere spots of pigment, are present in some of the Inermia.

Priapulus is devoid of nephridia. In the Armata a pair of appendages of the rectum are, as already mentioned, to be regarded as posterior nephridic. In addition there are present anterior nephridia. In Bonellia (Fig. 393, ant. neph.), and in some Inermia, there is only a single one: in the majority of cases there is one pair, while in various species of Thalassema there are from one to four pairs. They are tubes which open externally on the ventral surface, and internally communicate with the cœlome by means of ciliated apertures, the form

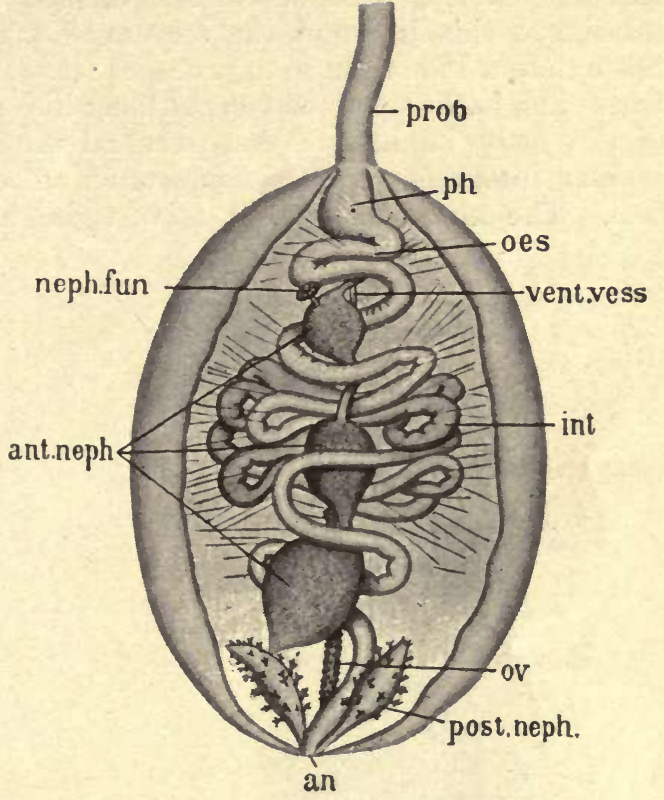

Fig. 393.-Bonellia, general view of the interual organs, an. anus; ant. neph. anterior nephridium; int. intestine; neph. Jun. nephrostome; us. oesophagus; ov. ovary; $p h$. plaryux; post. neph. posterior nephridium ; prob. proboscis ; vent. ress. ventral vessel. (After Greef.)

and position of which vary in different cases. They act as efferent ducts for the reproductive elements (gonorlucts); but

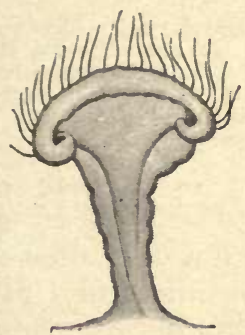

Fig. 394.-Oue of the ciliated funncls of the posterior nephridia of Echiurus. (After Greef.) their function as excretory organs has not been definitely established.

The sexes are usually distinct, and the reproductive organs are of very simple character, consisting merely of ridges or clumps of cells (gonads), sometimes enclosed in a membrane, developed at various points on the body-wall or on the wall of one of the main blood-vessels. The cells of these ovaries or testes may develop in situ into perfect ova or sperms; more usually they become detached, and undergo the later stages of their development while floating in the cœlomic fluid.

A remarkable instance of extreme sexual dimorphism occurs 
in Bonellia. The ordinary large individuals (Fig. 390), to various points in the structure of which reference has been already made, are females. The single, greatly enlarged nephridium contains a spacious cavity, which has been termed the uterus. In the interior of this is found the very small male (Fig. 397), which is not unlike a Planarian in appearance: it is compressed and covered with cilia, with a pair of ventral hook-like setæ. In the interior of the body bundles of dorso-ventral muscular fibres placed at regular intervals give an appearance of rudimentary segmentation. The alimentary canal is vestigial and completely closed,

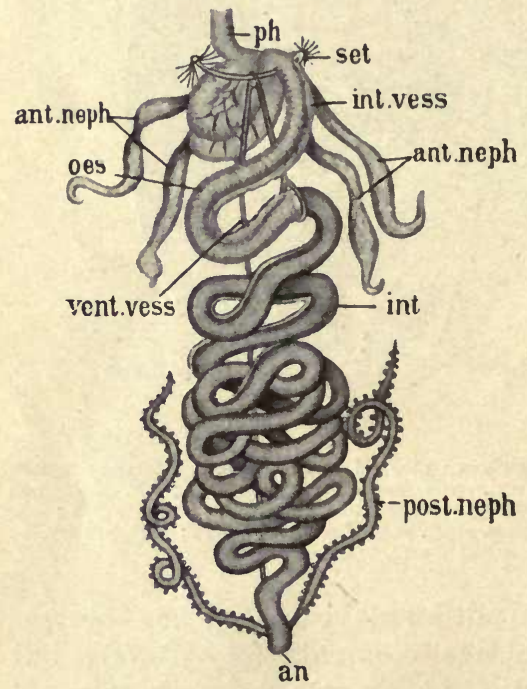

Fia. 395.-Fchiurus, internal organisation. an. anus; ant. neph. anterior nephridia ; int. intestine; int. vess. intestinal vessel; $\alpha z$. (esophagus; post. neph. posterior nephridia; vent. vess. ventral vessel. (After Greef.)

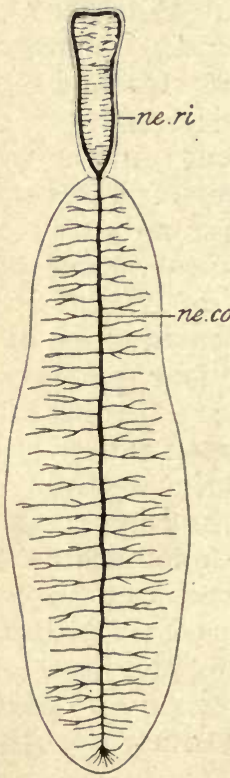

FiG. 396.-Fchiurus, general outline of the animal, witl the nervous system (diagrammatic). ne. co. nerve-eord; ne. ri. nervering. (After Greef.)

both mouth and anus being absent. There is a pair of nephridia placed posteriorly. The sperms, developed from modified colomic cells, reach the exterior through a duct, dilated externally into a vesicula seminalis, and opening internally into the coelome by a funnel-shaped aperture. In Hamingia, also, there are imperfectly developed males which are lodged in the nephridia of the female.

Development.-The larva of Echiurus (Fig. 398) has a welldeveloped pre-oral or prostomial lobe with pre-oral and postoral circlets of cilia, and in other respects closely resembles the trochophore embryo of a Chætopod. The posterior part of the 
body elongates, and the mesoderm-bands, developed as in the Chætopoda, become divided into as many as fifteen segments. A circlet of setæ is developed at the anal end, and subsequently the two ventral setæ are formed in the same manner as in the Chrtopoda. The pre-oral lobe becomes narrowed to form the cylindrical proboscis of the adult; and the rudimentary segmentation gradually disappears as development advances.

In Bonellia there is unequal segmentation, as in most Chætopoda resulting in the formation of four large megameres and eight small

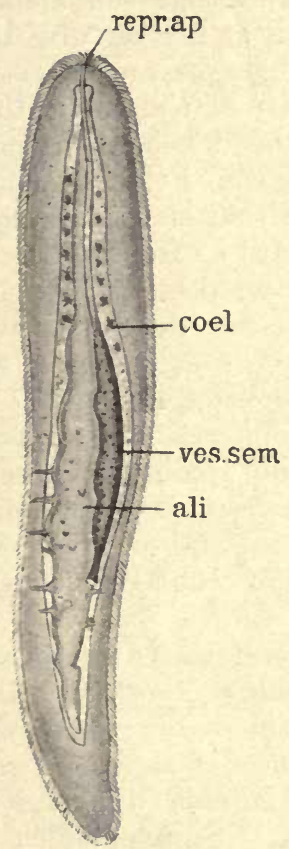

FIG. 397.-Malc of Bonellia. ali. alimentary-canal; coel. groups of crelomic cells destined to give rise to sperms; repr. ap. reproductive aperture; ves. sem. vesicula seminalis. (After Greef.) micromeres: the latter multiply rapidly, and grow over the megameres so as eventually to enclose the latter in a complete layer of ectoderm, save at one point, where there is a gap; the blastopore. Here the ectoderm bends inwards to

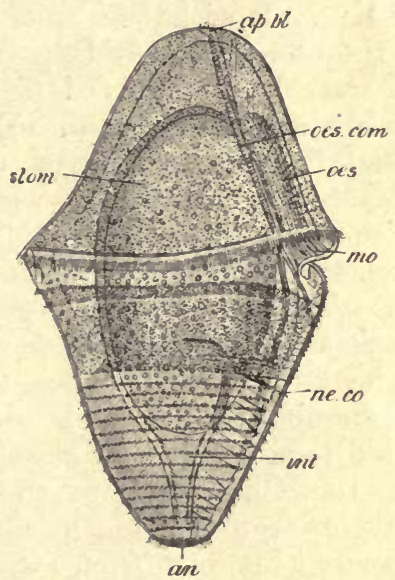

FIc. 395,-Trochophore of Echiurus. an. anus; ap. pl. apical plate; int. intestine; mo. mouth; ne. co. rudiment of nerve-cord; as. cesophing:s; as. conn. cesophageal connective; stom. stomach. (After Hatschek.)

give rise to a continuous mesoderm layer superficial to the megameres. The blastopore soon closes up. The megameres divide to form the cells of the endoderm, among which a lumen only appears comparatively late; mouth and œsophagus are developed as an outgrowth, at first solid, from the endoderm. The anus becomes formed still later by invagination at the hinder end of the body; and a pair of epidermal vesicles which appear at its sides, developed as outgrowths from the terminal part of the intestine, form the rudiments of the posterior nephridia. A rudimentary pre-oral lobe becomes established. The mesoderm remains unsegmented, 
but splits into somatic and splanchnic layers going to form the muscular system, blood-vessels, and other mesodermal organs. Before the alimentary canal is formed the larva, which had previously been spherical with two bands of cilia and a pair of eye-spots, becomes elongated and dorso-ventrally compressed, and becomes covered uniformly with cilia, so as to present the general appearance of a Planarian. It becomes converted into the adult female by a metamorphosis, including the elongation of the preoral lobe to form the proboscis and the development of the pair of setæ of the adult. The male never goes through this metamorphosis, but remains in the Planarian stage: it at first adheres to the proboscis of a female, then enters the osophagus, and afterwards, when sexually mature, passes into the cavity of the nephridium.

In the Inermia the early stages of the development closely resemble those of the embryo of one of the Polychrta, and a stage corresponding to the trochophore of that class is developed, but with the mouth situated further forward in front of the ring of cilia and with the anus in front of the posterior extremity on the dorsal side. But at no stage in the development has any trace been observed of the temporary segnientation which forms so marked a feature in the development of Echiurus.

Distribution Affinities, etc.-The Gephyrea are all marine. They are only capable of very slow creeping locomotion, and live for the most part either in natural rock-fissures, or in burrows which they excavate for themselves either in sand or mud, coral or rock. Their distribution is general; and they occur at considerable depths as well as in shallow water.

The differences between the Inermia and the Armata are so considerable that there is some doubt whether they ought to be united together in one class. The Inermia diverge most widely from the Chrtopoda in the entire absence of setæ and in the want of segmentation at any stage. Priapulus differs in such important points from the rest of the Sipunculoidea that it is sometimes regarded as constituting a distinct order.

Affinities between Phoronis (p. 355) and the unarmed Gephyrea have often been supposed to exist, and by some zoologists it has been proposed to regard Phoronis as an outlying member of that class. It seems probable, however, that the very manifest resemblances which undoubtedly exist do not indicate a near relationship, but are the result of converging modifications of originally widely different stocks. The most striking of these points of resemblance are two-(1) the approximation of the anus towards the oral aperture, and (2) the presence of the tentacular circlet. But a study of the development shows that these common features arise in totally different ways in the two cases. The forward position of the anus in the Sipunculida is brought about by a gradual displacement resulting from the growth of the aboral region of the body; and the invagination and evagination by which the corresponding 
result is attained in Phoronis do not occur. Again, while in Phoronis the tentacles of the adult may be looked upon as formed by the development of processes along the line occupied by the post-oral circlet of cilia, in the Sipunculida the tentacular lobes have nothing to do with the post-oral circlet, but are formed by the growth of a series of lobes from the margin of the mouth itself. The larva of the Sipunculida again is, as already pointed out, very nearly related to the larva of the Chrtopoda, and is a typical trochophore; while the Actinotrocha larva of Phoronis diverges somewhat widely from that type.

\section{CLASS III.-ARCHI-ANNELIDA.}

More primitive in some respects than the other Annulata are the ArchiAnnelida, comprising only the family Polygordiidae to which may perhaps be added

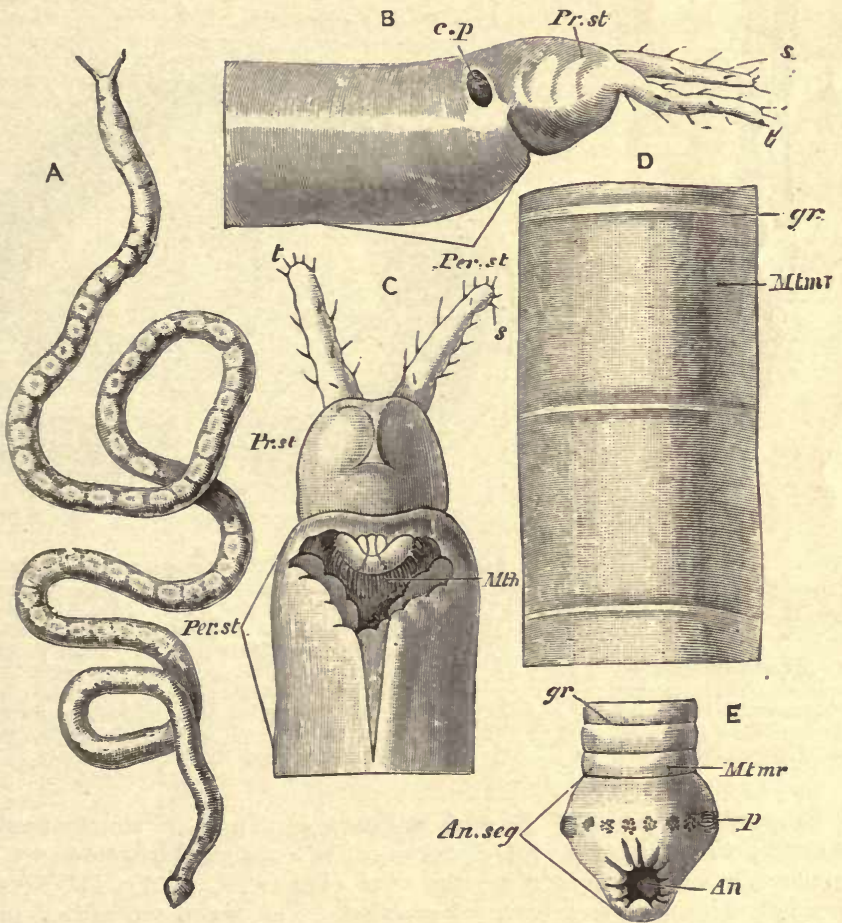

Fio. 39.-Polygordius neapolitanus. A, the living animal, dorsal aspect, about fire times natural size; $B$, anterior end, lateral view ; $C$, ventral view of the same ; $D$, portion of the hody showing the metameres; $E$, ventral view of the posterior extremity; $A n$. anus;

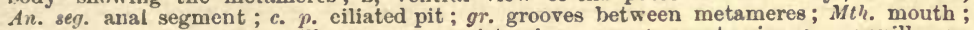
Mtmr. inetameres; $p$. papilla ; per.st. peristomium; $p r . s t$. prostomium; s. papillae on tentacles $(t)$. (From Parker's Biology, after Fraipont.)

Ctenodrilus. They are marine worms with narrow, elongated, cylindrical body. The prostomium (Fig. 399, pr. st) is small, the peristomium (per. st) large. The segments $(\boldsymbol{M t m r})$ are only faintly marked off exterually for the most part, though 
the internal division of the colome by means of septa is complete. Parapodia and setæ are absent, but the prostomium bears a pair of tentacles $(t)$. Several pairs of simple nephridia are present. The position of the nervous system (Fig. 401) is more primitive than in the Annulata in general; it is continuous with the epidermis, and not separated from it by mesodermal elements as in most of the others. A pair of ciliated grooves $(c . p$. ) are probably to be looked upon as organs of special sense.

The family Polygordiide includes two genera-Polygordius and Prolodrilus. There are a pair of prostomial tentacles, long in Protodrilus, short in Polygordius, and a pair of ciliated pits. The segmentation is only very indistinctly

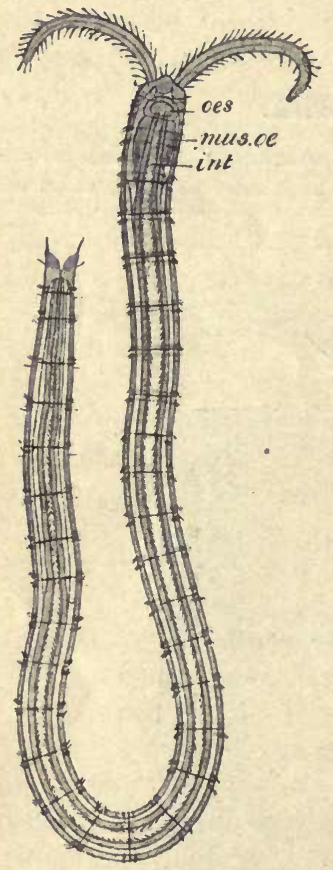

FIG. 400.-Protodrilus, entire animal. int. intestine ; mus. ${ }^{2}$. muscular append: age of oesophagus ; oes. (esophagus. (After Hatschek.) marked externally in Protodrilus by circlets of cilia; in Polygordius it is indistinct in front, but better marked behind. In Polygordius lactens a series of tooth-like processes occur round the anus, and in front a circlet of alhesive papillæ. In Protodrilus there is a ventral ciliated groove. There is a vascular system with clorsal and

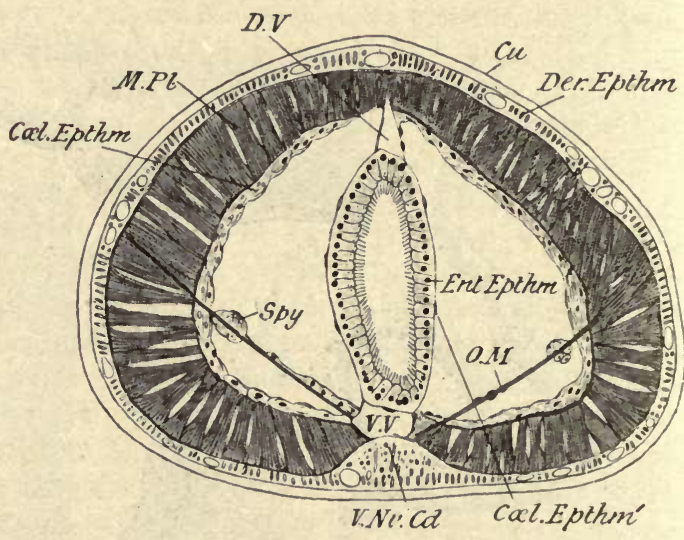

FIG. 401.-Polygordius neapolitanus, transverse section of a male specimen. Col. Epthm. parietal layer of colomic epithelium; Coel. Epthm.' visceral or splanchnic layer of the same; $C u$. cuticle; Der. Epthm. deric epithelium; D. $\boldsymbol{V}$. dorsal vessel ; Ent. Epthm. cnteric epitheliun; $M$. Pl. muscle-plates; O. M. oblique muscles; $S p u$, immature gonads; V. Nv. Cul ventral nerve cord continuous with deric epithelium ; $V$. $V$. ventral vessel. (From Parker's Biology, after Fraipont.)

ventral longitudinal vessels. In each segment is a pair of simple nephridia. In Protodrilus there are two ventral nerve-cords, connected together by trans. verse commissures: in Polygordius the cord (Fig. 401, $V . N v . C d$ ) is single; in neither genus is there any trace of ganglia. The sexes are united in most individuals of Protodrilus, ovaries occurring in all the first seven segments and testes in some of those immediately following. In Polygordius the sexes are separate; the oraries or testes (Fig. 401, Spy) are developed in the posterior segments. There are no special reproductive ducts.

The larva of Polygordius is a typical trochophore (Fig. 402), and its metamorphosis into the adult worm (Fig. 403) takes place as in the Polychrta in all essential respects.

Ctenodritus resembles Polygordius in the ectodermal position of the nervous 
system and in the presence of ciliated pits; but it has a row of comblike satæe on each segment, and it has only a single pair of nephridia, which are

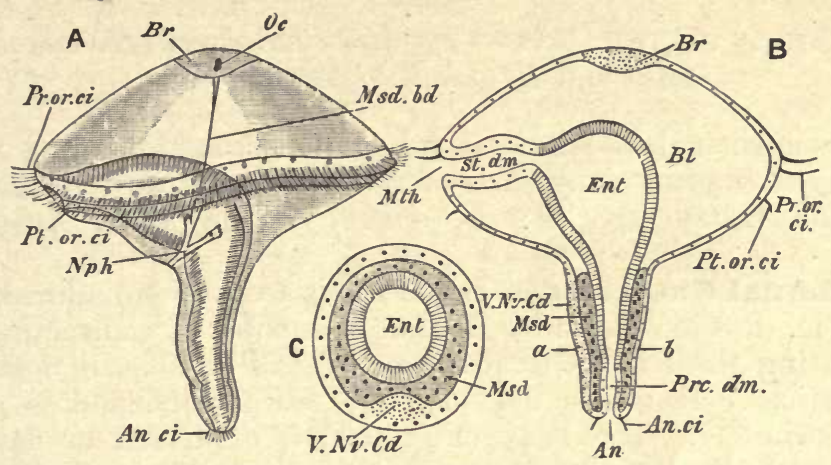

FIG. 402.-Trochophore of Polygordius neapolitanus. $A$, lateral view of entire larva ; $B$, diagrammatie vertical section; $C$, transverse section through the plane $a b$ in $B$; $A n$. anus ;

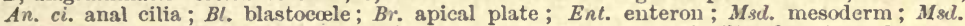
bd. mescdermal bands; $\mathrm{Nph}$. head-kidney; Oc. eye spot; $\boldsymbol{P r}$. or. ci. cilia of prototroch ; $\boldsymbol{P r c}$. dim. proctodaum; $P t$. or. ci. post-oral cilia : St. dlm. stomodæum ; $r$. Nv.Cd. ventral nerve-cord. (From Parker's Biology, partly after Fraipont.)

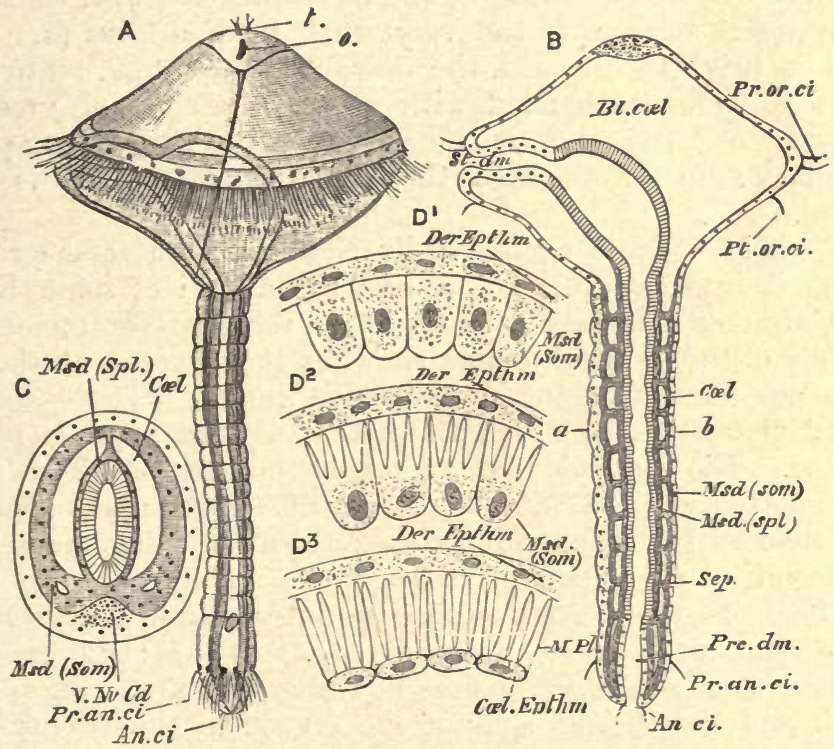

Fig. 403.-Later stage in the development of Polygordius neapolitanus, in which the rosterior part of the trochophore has become elongated and segmented; $A$, entire larva : $B$, vertical section; $C$, transverse section along the plane $a b$ in $B ; D^{\mathrm{I}}-D^{3}$, three stages in the development of the somatic mesoderm: Col. coelone : cal. Epthm. coelonic epithelium; Der. Epthm. deric epithelium; $M . p l$. muscle-plate: Msl. (som.), somatic mesoderm; $M s d .(s p l)$, splanchnic mesoderm; 0 . eye; $t$. tentacle. Other letters as in preceding figure. (From Parker's Biology, partly after Fraipont.)

situated in the peristomium. There is only a single (longitudinal) layer of muscles in the body-wall; the reproductive apparatus is not known. 


\section{CLASS IV.-HIRUDINEA.}

\section{Example of the Class-The Medieinal Leech (Hivudo medici- nalis and $H$. (Limnobdella) australis).}

The medicinal Leech is found in ponds, swamps, and slowly flowing streams in many parts of the world. H. medicinalis is the common British species: $H$. australis is an allied Australian form.

External Character. - The Leech is a vermiform animal, some $6-10 \mathrm{~cm}$. (2-3 inches) in length, but is capable of contracting and elongating itself so as to produce great alterations in form and proportions. It moves by "looping" movements, and is also a good swimmer. The body (Fig. 404) is depressed or flattened dorso-ventrally, the dorsal surface convex, the ventral flattened. The anterior end presents a ventrally directed cup-like hollow, the anterior sucker (a.s.), in the middle of which is a small aperture, the mouth (mth.). The hinder end bears a disc-like posterior sucker $(p . s$.$) , also directed downwards, and at its junction with the trunk,$ on the dorsal surface, is the very small median anus (a.). The animal is brightly coloured, the dorsal surface in $H$. medicinalis being longitudinally banded with alternate stripes of greenishgrey and rusty red, the ventral surface greenish-yellow, spotted with black: in $L$. australis the whole under-surface is rustcoloured.

The whole body is encircled by close-set transverse grooves, dividing it into annuli. These, like the annuli of some Earthworms, are more numerous than the true segments or metameres, the study of the internal organs showing that, except at the two extremities, each segment contains five annuli. There are also external characters by which the actual segmentation is plainly indicated. The rust-coloured streaks on the back of $H$. medicinctlis are spotted with black, and at every fifth annulus the spots are larger than on the intervening rings: the annuli thus marked are the second of their respective segments. Moreover, the same rings bear on the ventral surface minute paired apertures, the nephridiopores or excretory apertures $(n p .1, n p .17)$ : of these there are altogether seventeen pairs, marking the first ring of the seventh and the second ring of the eighth to the twenty-third segments.

In front of the first and behind the last pair of nephridiopores one important external mark of segmentation fails, but a further indication is furnished by the presence on the middle ring of each undoubted metamere of a number of delicate transparent elevations, the segmental papilla (s.p.), which have probably a sensory function. These structures are found along the whole length of the 
body, and as they mark the middle (third) ring of all those segments the extent of which can be checked by the nephridiopores, it is legitimate to assume their segmental value in the anterior and posterior regions, where the controlling excretory apertures are absent. By the clue thus furnished it is found that there are six segments in front of that bearing the first pair of nephridiopores, and three behind that bearing the last pair, making a total of twenty-six metameres: of these the first seven and the last three have less than the normal number of rings.

The anterior sucker bears on its dorsal surface five pairs of small black spots, the eyes (e. $1, e .5)$, the arrangement of which shows

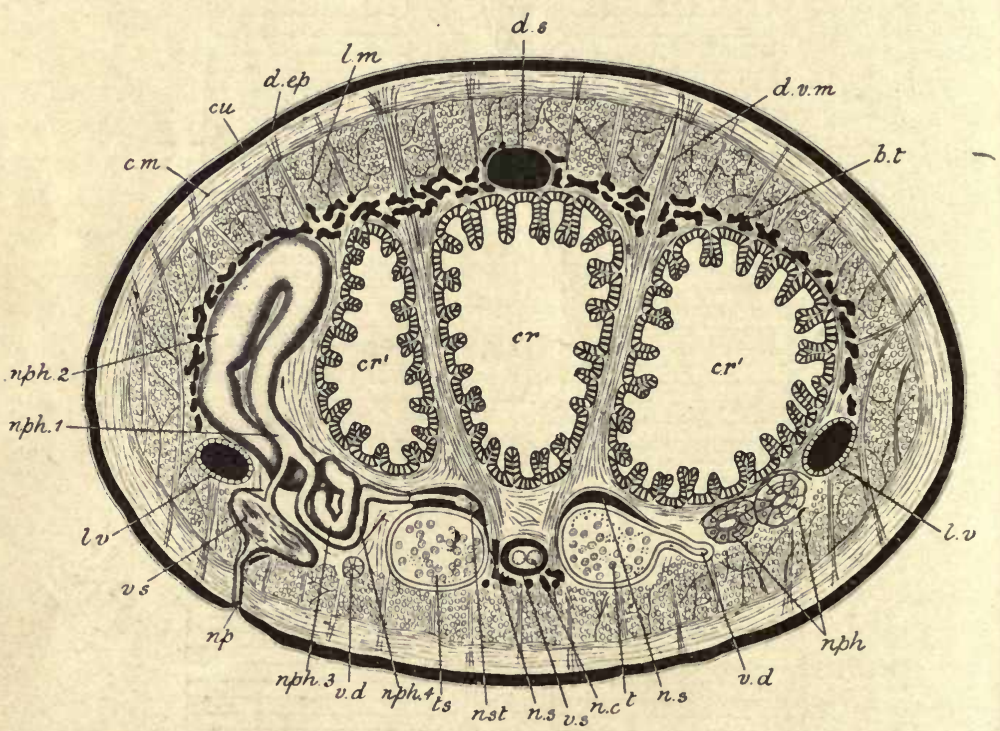

Fig. 405.-IIirudo medicinalis; transverse section. $b . t$. botryoidal tissue ; $c$. m. circular musclus; $c r$. crop ; $c \eta^{\prime}$. diverticula of crop ; $c u$. cuticle; $d . e p$. epidermis; $d$ 8. dorsal sinus; l. $v$. $m$ dolso-ventral muscles; $l$. $m$. longitudiual muscles; $l$. $v$. lateral vessel; $n$. $c$. nervecord ; nph. 1-4, nephridium; n. s. nephrostomial sinus; nst. nephrostome ; $t s$. testis; $v . d$. vas deferens; vs. vesicle of nephridium ; v. 8. ventral sinus. (After Marshall and Hurst.)

them to be special modifications of sensory papillæ, since they occupy in the first five segments the precise position occupied in the sixth and following segments by segmental papillæ.

The perfectly definite and comparatively small number of metameres in the leech offers a striking point of contrast with what we have met with in the Chrtopoda, and is to be looked upon as a mark of higher differentiation.

Body-wall. - The body is covered externally by a thin cuticle (Fig. 405, cu.), which is constantly being cast off in patches and renewed. Beneath it is an epidermis (d.ep.) consisting of hammershaped cells, separated at their inner ends by spaces in which 
blood-capillaries run. The blood is thus brought into close relation with the surrounding water, and the skin becomes a highly efficient respiratory organ. The space between the epidermis and the enteric canal is filled by a peculiar form of connective-tissue, consisting of a gelatinous matrix with interspersed cells and fibres, many of the former large and branched. More immediately surrounding the enteric canal is the peculiar and characteristic botryoidal tissue (b.t.) consisting of branched canals, the walls of which are formed of large cells loaded with black pigment. This system of canals is in communication on the one hand with the blood-vascular system and on the other with the greatly reduced colome.

Numerous unicellular glands are produced from the epidermis: the gland-cells themselves lie in the connective-tissue, and are continued into long ducts which open on the surface. Special glands in the ninth, tenth, and eleventh segments secrete the substance from which the cocoon is formed (vide infra, p. 515): the segments in question therefore constitute the clitellum.

The muscular system is well developed, and consists of an outer layer of circular $(c . m$.$) and an inner of longitudinal (l . m$. fibres. There are also dorso-ventral fibres $(d . v . m$.) passing vertically between the pouches of the crop (vide infra), and radial fibres extending from the wall of the enteric canal to the integument: these take the place of the septa of Chrtopods.

The alimentary organs are greatly modified in accordance with the bloodsucking habits of the animal. Surrounding the mouth are three jaus, one median and dorsal (Fig. 408, d. j.), the other two ventro-lateral $(v . l . j$.). Each (Fig. 406) has the form of a compressed muscular cushion, with a sharp, evenly curved, free edge covered with chitin, which is produced into numerous serrations or teeth: by means of its muscles each jaw can be moved backwards and forwards through

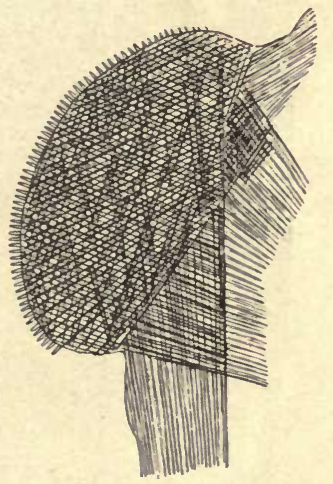

Fig. 406.-One of the jaws of Firudo medicinalis. (After Leuckart.) a certain arc, and the three, acting together, produce the characteristic triradiate bite in the skin of the animal upon which the Leech preys.

The month leads into a muscular pharynx (Figs. 407 and 408, $p h$.) situated in the fourth to the eighth segments. Radiating muscles pass from its walls to the integument, and by their contraction dilate its cavity and suck in blood from the wounds made by the jaws. Around the pharynx are numerous unicellular 


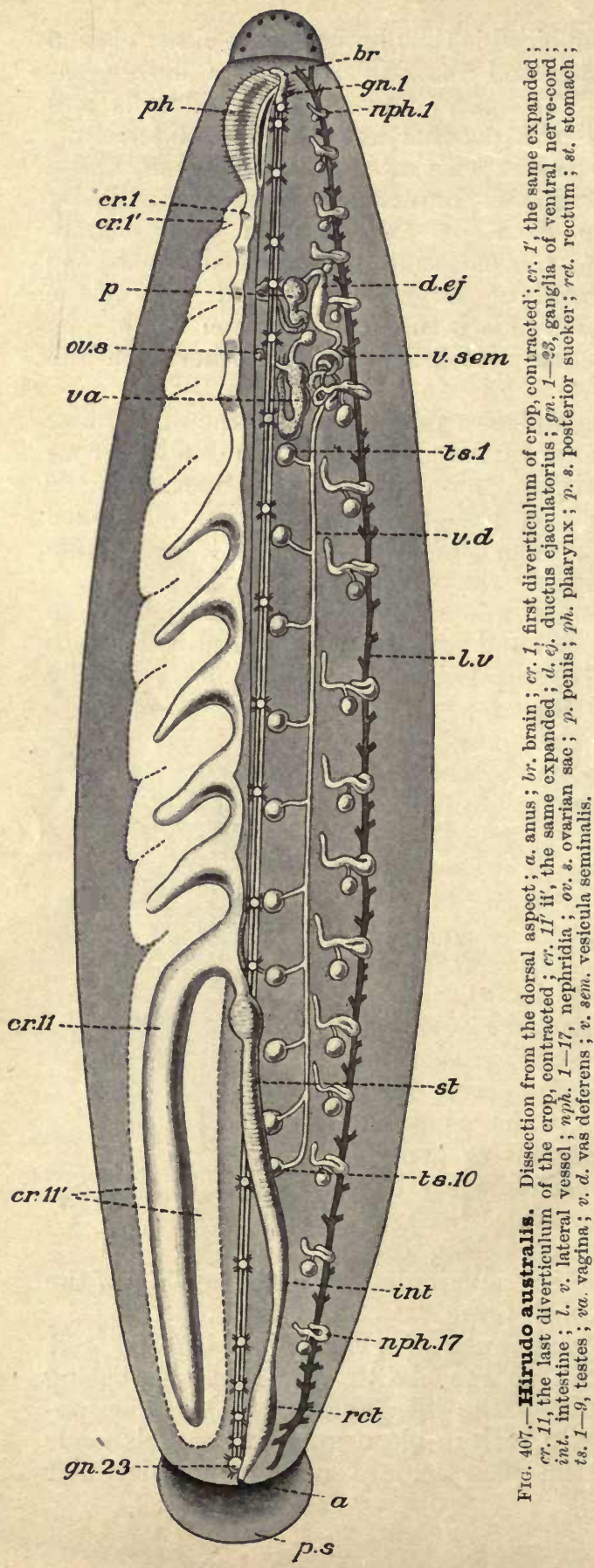

salivary glands, which open close to the mouth : their secretion has the effect of preventing the coagulation of the blood taken as food.

The pharynx communicates by a very small aperture with the second and largest division of the enteric canal, the huge crop $(c r$.$) a thin-walled$ tube extending from the eighth to the eighteenth segment, and produced into eleven pairs of lateral pouches $(c r .1, c r, 11)$, the first ten of which are directed outwards and correspond each to a segment, while the eleventh ( $c r .11)$ passes directly backwards as far as the twenty - fourth segment. The crop is capable of great dilatation, and its form varies greatly according to whether it is empty or gorged with blood. Posteriorly the crop communicates by a minute aperture with the stomach (st.), a tubular chamber with a dilated anterior end, and having its wall produced internally into a spiral fold: this is the digestive portion of the canal ; the blood is passed into it from the crop with extreme slowness, and under- 
goes an immediate change, its colour turning from red to green. The digestion of a whole cropful of blood takes many months. 'The stomach is continued into a narrow intestine (int.): this passes into a somewhat dilated rectum (rct.) which turns slightly upwards and opens by the anus (an.) in the last annulus.

The excretory system consists of seventeen pairs of nephridia (nph.1-17), situated in segments 7-23. A typical nephridium (Fig. 409) has the general form of a loop passing upwards from the ventral bodywall, produced into an offshoot which extends inwards (mesially) to the corresponding testis, and connected posteriorly with a small bladder or vesicle (vs.). The principal loop is divisible into two chief parts, the main lobe (m.l.) and the apical lobe (a.l.), connected with one another by a short rccurvent lobe $(r . l$. $)$ : the offshoot to the testis is known as the testis-lobe $\left(t_{.} l.\right)$; it is absent in $H$. australis.

All these parts are formed of a close-set mass of glandcells, traversed by a complex system of minute intra-cellular passages or ductules, which finally unite into a comparatively wide inter-cellular tube or duct: this winds through the main and apical lobes, and finally enters the vesicle, which opens posteriorly in the last annulus of the segment. The free end of the testis-lobe is swollen into a lobed mass which lies in a sinus (Fig. 405 nst) in connection with

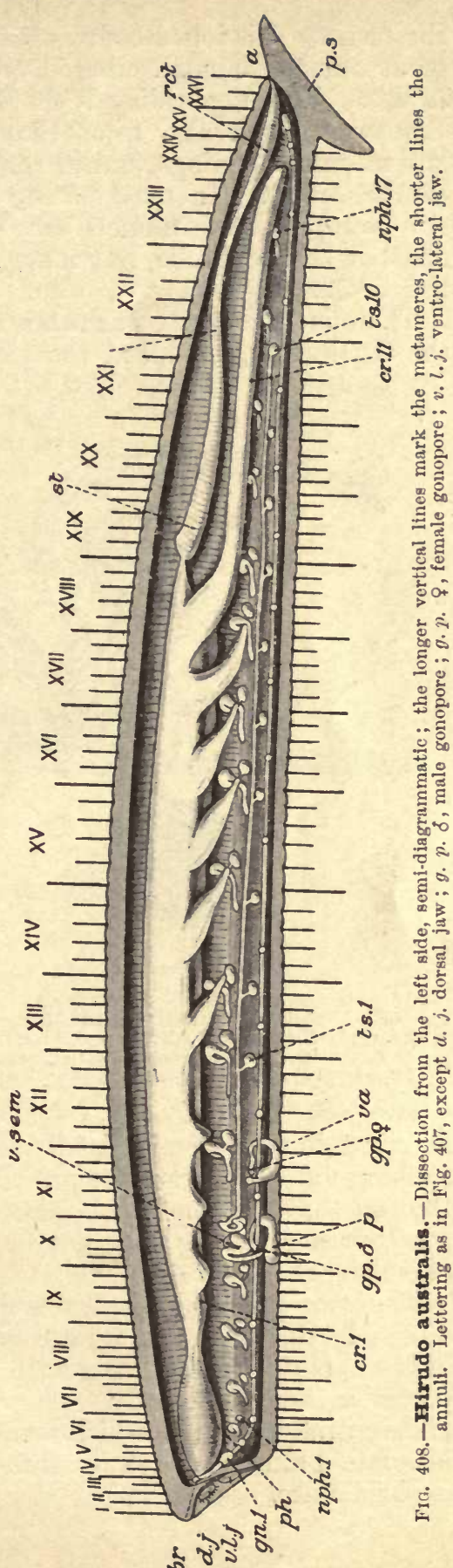


the testis. This lobed body is a modified ciliated funnel : it has a great number of small ciliated openings into the sinus in which it lies. The nephridia of the Leech differ from those of the Earthworm, and also from those of Nereis, in the absence of any internal openings, and in the absence of cilia in the interior of the canals. In most of the nephridia (all except the first six pairs) ciliated funnels are present attached to the inner ends of the nephridia, but these do not open into the canals of the latter.

There is a complex vascular system, containing, like that of the Earthworm, red blood, the plasma coloured with hæmoglobin

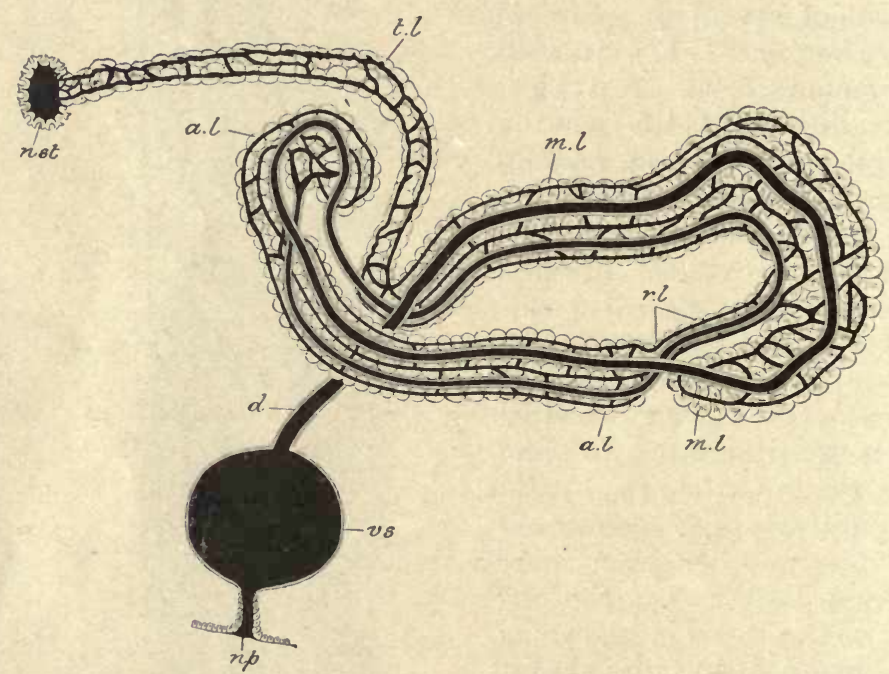

FiG. 409.-Nephridium of Irirudo medicinalis. a. $l$. apical lobe; $m . l$. middle lobe $n p$. nephridiopore; $n s t$. ciliated funnel ; r. $l$. recurrent lobe; $t$. $l$. testis-lobe; $r$. vesicle ; $\imath s$. $d$. vesiele-duct. The communication here represented as existing between the ciliated fumel and the nephridial canals does not occur. (After Bourne.)

and containing sparsely distributed colourless corpuscles. But a striking difference from the preceding annulate types is found in the fact that the blood-containing spaces are of two kinds-bloodvessels proper, having muscular walls; and blood-sinuses, the walls of which are devoid of muscle.

The two principal blood-vessels are lateral in position (Figs. 407 and $410, l$. v.), running fore and aft at the level of the middle of the nephridia and uniting with one another at the anterior and posterior ends of the body. They send off branches both dorsally and ventrally, some of which anastomose with one another. The ultimate branches break up into capillaries in the integument, nephridia, \&c. 
The two principal sinuses are respectively dorsal (Figs. 405 and $410, d . s$.$) and ventral (v. s.), the former lying just above the$ enteric canal in the middle dorsal line, the latter occupying a similar position on the ventral side, and enclosing the ventral nerve-cord. The two sinuses are in connection with one another posteriorly, and are also in communication, by means of their branches, with the capillaries of the skin. There is thus an indirect connection, by means of capillaries, between the bloodvessels and the sinuses, but no direct communication exists. The sinuses in which the ciliated funnels are lodged open into the ventral sinus. As we shall see more particularly in the general account of the class, the sinuses represent a greatly reduced cœlome.

The nervous system is of the usual annulate type. There is a small brain (Figs. 407 and 408,br.) situated above the anterior

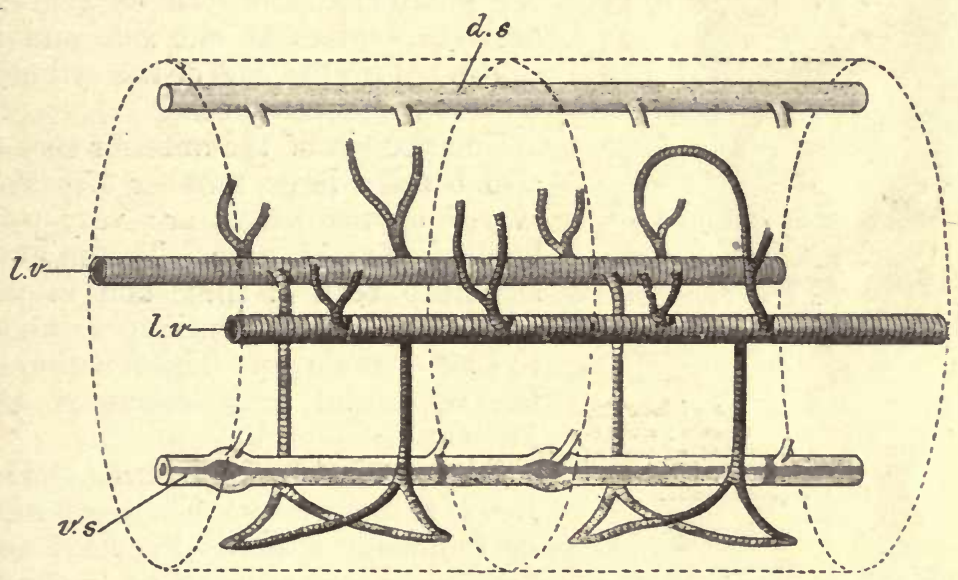

FiG. 410.-Diagram of principal blood-channels of Ieech. d. s. dorsal sinus; $l . v$. lateral vessel ; v. s. ventral sinus containing nerve-cord.

end of the pharynx immediately behind the median dorsal jaw. It is connected by a very short pair of œsophageal connectives with the ventral nerve-cord, which consists of twenty-three wellmarked rounded ganglia (gn.1-23), situated in the third or middle ring of each segment, united by delicate double connectives and a slender median strand. The ganglion-cells are regularly arranged in groups or packets. The first or sub-œsophageal ganglion is larger than the others, and is shown by development to be made up of five united pairs of embryonic ganglia: the last ganglion is also of unusual size, and results from the fusion of six pairs of ganglia distinct in the embryo. The whole ventral nerve-cord is contained in the ventral sinus. Nerves are given off from the ganglia, but not, as in the Earthworm, from the connectives, in which also, nerve-cells are wholly absent. 
The principal sense-organs are the eyes, of which there are five pairs (Figs. 404 and 411) situated round the margin of the anterior sucker, on the dorsal side, one pair in each of the first five segments. They occupy positions taken in the succeeding segments by lateral

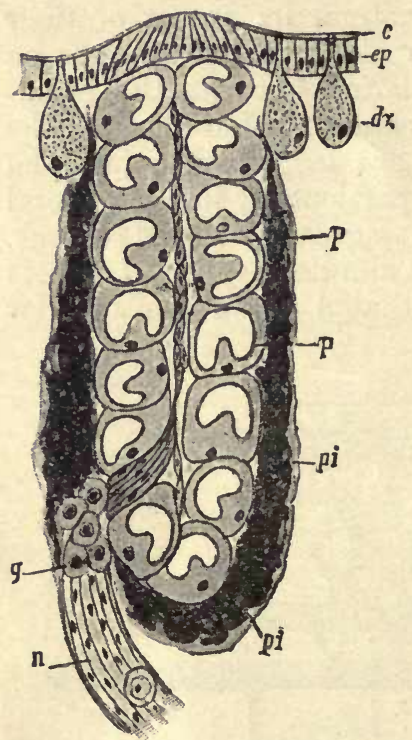

FIG. 411.-Section of cye of Ieech. $c$, cuticle ; $d 3$, gland-cells ; ep. epidermis ; $g$, nerve cells ; $n$. nerve ; $p$, refractive cells; pi. pigment. (From Lang's Comparative Anatomy.)

sense-organs, with which they are obviously homologous. The structure of the eyes is peculiar: they are cylindrical in form (Fig. 411), the long axis of the cylinder being at right angles to the surface of the body. The outer layer is formed of blaek pigmented tissue ( $\mathrm{pi}$.), surrounding a layer of large, clear, refractive cells $(p$.$) , which occupy$ the greater part of the organ. A nerve $(n$.$) enters at one side, and is$ continued up the axis of the cylinder by a row of sensory cells.

The margin of the anterior sucker also bears a large number of gobletshaped organs, which are very probably organs of taste. The minute structure both of these and of the segmental sense-organs is very similar to that of the eyes. The function of the segmental sense-organs is unknown.

Reproductive Organs. - The Leech is monœeious. There are nine or ten pairs of testes (Figs. 407 and $408, t s$.), in the form of small spherical sacs, situated in segments 12 to 20 or 21 . Each gives off from its outer surface a narrow efferent duct, which opens into a common vas deferens (v. $d$.$) . In$ the tenth segment the vas deferens increases in width and forms a complex coil, the vesicula seminalis (v. sem.), from which is continued anteriorly a somewhat dilated muscular tube, the ductus ejaculatorius $\left(d_{0} . \mathrm{ej}_{\mathrm{.}}\right)$. From each ejaculatory duct a narrow tube passes to the base of the penis (p.), a curved eversible muscular organ which opens on the ventral surface of the fourth annulus of the eleventh segment, in the middle line. The base of the penis is surrounded by a number of unicellular glands, which constitute the prostate, and secrete a substance by which the sperms are aggregated into masses called spermatophores.

The ovaries are coiled filamentous bodies, each enclosed in a small globular ovarian sac (ov. s.), situated in the eleventh segment. From each ovarian sac a short oviduct passes inwards and backwards, and unites with its fellow into a median duct, the walls of 
which are supplied with albumen-secreting gland-cells. The common oviduct opens into a curved muscular tube, the vagina (va.), which opens in the middle line on the ventral surface of the fourth annulus of the twelfth segment, i.e. one segment behind the male aperture.

It will be noticed that the ovaries of the Leech form a single pair, while the testes are multiple and segmental : also that, while the gonads and efferent ducts of both sexes are paired, the penis and the vagina are median and unpaired. In the latter respect the contrast between the Leech and the Annulata previously discussed is very striking. Further important peculiarities are the enclosure of the ovary in a sac from which a duct leads directly to the exterior, and the fact that the testes are hollow sacs discharging the sperms into a cavity from which they pass directly to the efferent ducts. In Chætopods, it will be remembered, the gonads lie freely in the coelome, their products-ova or sperms-are discharged from their external surfaces and carried off either by coelomoducts or by "segmental organs." It seems tolerably certain that in the Leech the cavities both of the ovarian sacs and of the testes represent shut-off portions of an almost obsolete cœlome, and that their ducts are cœlomoducts.

Development.-When breeding two Leeches copulate, and one impregnates the other by passing spermatophores through its penis into the vagina. Simultaneous mutual impregnation has also been described. The clitellar segments (ninth to eleventh) secrete a cocoon (Fig. 412), into which spermatophores, ova, and a quantity of albumen, secreted by the albumen-glands, are passed. The animal then withdraws its head from the cocoon, the two ends of which close up by their own elasticity, producing a closed capsule in which embryonic development takes place. Segmentation is unequal, and results in the formation of a globular

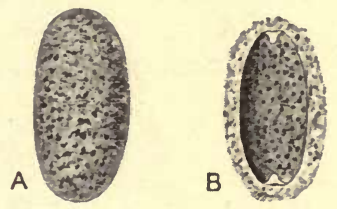

Fig. 412.-The cocoon of Irirudo. $A$, entire ; B, in section. (After Leuckart.) embryo, which, after hatching, swims about in the cocoon, actively devouring its albuminous contents, and finally escaping in a form closely resembling the adult.

\section{Distinctive Characters and Classification.}

The Hirudinea are Annulata in which the body consists of a limited and definite number of segments, and is marked externally by secondary rings or annuli, a variable number of which go to a segment. The anterior end of the body is suctorial, and several of the hindmost segments are fused to form a powerful suckingdisc, which is directed downwards and backwards. The mouth 
lies in the anterior sucker, the anus is usually dorsal and immediately in front of the posterior sucker. The cœlome is always more or less obliterated by connective-tissue, and is represented by sinuses of varying dimensions which contain blood. True blood-vessels, with muscular walls, are also present. The nervous system consists of a brain united by short osophageal connectives to a ganglionated ventral nerve-cord. The excretory organs are segmentally arranged nephridia. The sexes are united, the testes numerous and usually segmentally arranged, the ovaries a single pair. The testes have the form of sacs, and discharge their products internally: the ovaries either have a similar structure or are band-like and enclosed in ovarian sacs, into which the ova are set free. The penis and the vagina are unpaired, and open by median apertures, the male anterior to the female, on the ventral surface of the body. Development is usually direct, i.e. unaccompanied by a metamorphosis. Leeches are either freeliving, or are permanently or intermittently parasitic : they inhabit either the land, fresh-water, or the sea.

The class is divided into the following two orders :-

\section{ORDER 1.-RHYNCHOBDELLIDA.}

Hirudinea in which the anterior part of the body can be protruded and retracted so as to form a proboscis or introvert.

This order includes Clepsine (Glossiphonia), parasitic on Snails, Frogs, \&c.; Piscicola, on fresh-water Fishes; Pontobdella and Branchellion, on marine Fishes (Fig. 413).

\section{ORDER 2.-ARHYNCHOBDELLIDA.}

Hirudinea in which there is no proboscis.

\section{Sub-order 1.-Gnathobdcllida.}

Arhynchobdellida in which the mouth is provided with two, or more usually three, toothed jaws.

This sub-order includes Hirudo, the common Leech, parasitic on Vertebrata; Aulostoma, the Horse-leech, free-living and carnivorous; Homadipsa, the Land-Leech.

\section{Sub-order 2.-Herpobdellida.}

Arhynchobdellida in which the mouth is not armed with true jaws.

This group includes Herpobdella (Nephelis) Trocheta, Orobdella, etc.-all fresh-water or terrestrial forms. 


\section{Systematic Position of the Example.}

Hirudo belongs to the family Hirudinidae, of the sub-order Gnathobdellida.

The presence of jaws places it in the sub-order Gnathobdellida: the possession of ten eyes, and the presence of five rings to all the segments except a few at the anterior and posterior ends, distinguishes it as a member of the family Hirudinidæ: the genus Hirudo is distinguished by the constant presence of 26 segments and of 102 annuli.
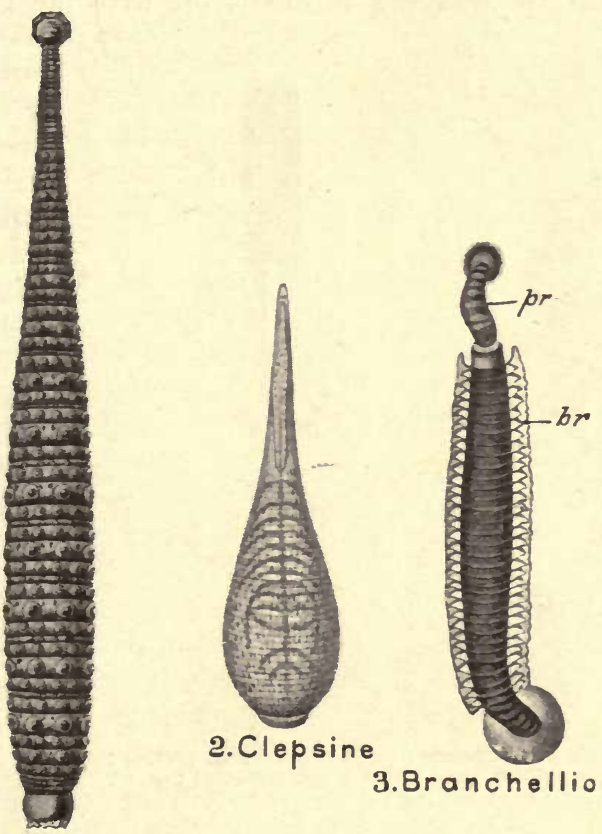

3. Branchellion

\section{Pontobdella} FIG. 413.-Three Rhynchobdellida. Ur. gills; $p r$. everted proboscis. (1, after Bourne; 2 and 3
after Cuvier.)

\section{General Organisation.}

In the essential features of their organisation the Leeches are a very uniform group: there are, however, a few interesting modifications of structure which must be referred to.

Form and Size.-Most kinds do not exceed a few centimetres in length, but the American species Macrobdella valdivania is said to attain a length of $76 \mathrm{~cm}$. ( $2 \frac{1}{2}$ feet). The number of annuli to a segment varies from three to five, but the general form of the body is remarkably uniform, the external differences between 
various species depending largely on colour and on the development of papillæ, which in some cases are large and prominent. Setæ are absent in all except one genus, Acarthobdella, which has two pairs on each side of the first five segments.

The proboscis (Fig. 414), the possession of which is distinctive of the Rhynchobdellida, is simply the retractile anterior end of the body, which, by the action of special muscles, can be drawn back into a temporary sheath. The organ is thus an introvert, like that of Gephyrea Inermia.

The chief differences in the structure of the enteric canal depend upon the varying number, or, in some cases, the total absence, of lateral pouches to the crop:

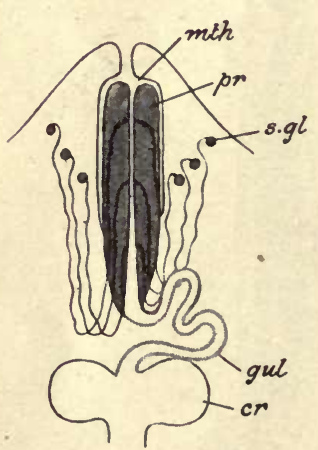

FIG. 414.-Proboscis of Clepsine. A, retracted; B, everted ; $c r$. crop; gul. gullet; $m t h$. mouth; $p r$, introvert; s. gl. salivary glancis. (After Bourne.) for instance, the horse-leech has only a single pair, corresponding to the eleventh pair in

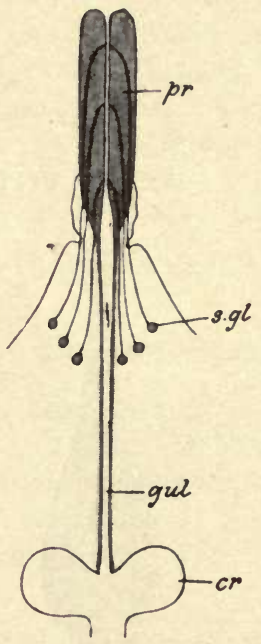

Hirudo, while $\mathrm{Ne}$ phelis has none at all. In the Rhynchobdellida there is a distinct slender gullet(Fig. 414 gul.) leading from the pharynx to the crop $(c r$.$) , and thrown$ into a coil when the proboscis is retracted. Among the Gnathobdellida the median jaw is absent in some land-leeches, and in

other species all three jaws are rudimentary or absent.

The varying development of the blood-vessels and sinuses presents many points of interest tending to explain the condition of things described above in the medicinal leech. In the latter, as we have seen, there are lateral vessels with contractile muscular walls and dorsal and ventral sinuses with non-contractile walls. In Pentobdella, one of the Rhynchobdellida, there are dorsal (Fig. 415 2, d.v.) and ventral (v.v.) as well as lateral vessels, and lateral (l.s.) as well as dorsal and ventral sinuses, and in each case the vessel is enclosed in the corresponding sinus. The ventral sinus (v.s.) also contains the nerve-cord (n.c.) and the ovaries $(o v$.), and offshoots of it surround the testes (ls.) and the nephrostomes (nst.). This arrangement clearly suggests the partial obliteration, 
by growths of connective-tissue, of an originally continuous colome. In the Rhynchobdellids in general the cœlomic spaces remain fairly extensive, and are lined by a cœlomic epithelium. Another interesting condition occurs in Nephelis (3), in which the middle region of the body contains a series of paired, metamerically arranged spaces $(c$.$) , surrounded by botryoidal tissue and containing$ the nephrostomes. Development shows that these cavities are derived from true cœlomic spaces in the embryo, formed, as in Chætopoda, by a splitting of the mesoderm in each segment. Acanthobdella, already referred to as exceptional in the possession of setæ, is also the only member of the class which has a well-developed and spacious colome, divided by mesenteries into a number of segments.

In most instances the skin, with its abundant supply of capillaries, constitutes the only respiratory organ, but in Branchellion (Fig. 413, 3) a Rhynchobdellid parasitic on the Electric Rays (Torpeclo and Hypnos) and on one of the Australasian Skates (Raja nasuta), differentiated respiratory organs or gills (br:) are present in the form of delicate lateral outgrowths of the segments.

In most members of the class the nephridia are formed on the same general type as those of Hirudo, but differ in the structure of the ciliated funnels, which may be more or less modified, as in Hirudo.

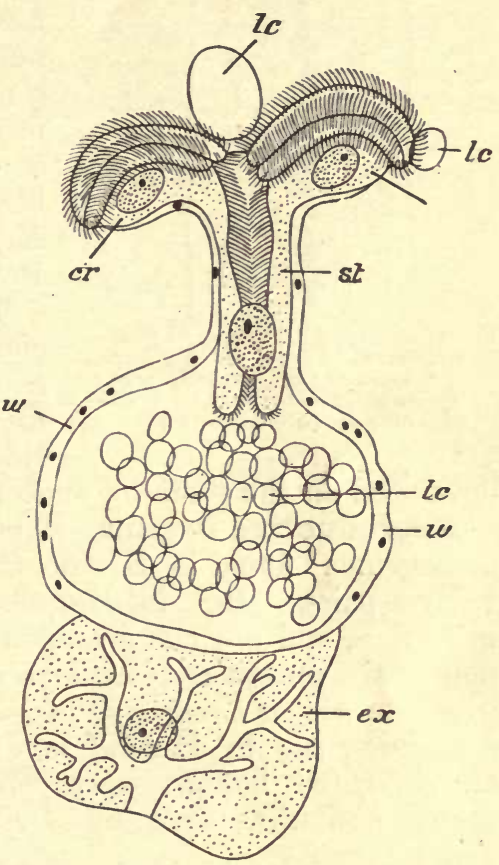

Fig. 415.-Ciliated funnel of Ferpobdella (Clepsine). $c r$. crown-cells of funnel; $e x$. terminal cell of the nephridium; $l c$. leucocytes; st. duct leading to receptacle; $w$. wall of receptacle. (From Meiscnheimer, after L. von Graff.)

The funnels, where they occur, never open into the nephridial canals. Each funnel leads by a narrow ciliated duct into a receptacle, in which leucocytes laden with waste matters are received from the cœlomic spaces and sinuses, subsequently to undergo degeneration and absorption (Fig. 415). By its outer side this receptacle is in close relation to the inner end of the nephridium, and the waste-matters from the disintegrated leucocytes are no doubt received into the nephridial canals and thus passed out to the exterior. In Hirudo and Herpobdella (Nephelis) the recep- 
tacles appear to be the organs in which new blood-corpuscles are manufactured. The ciliated funnels of the Hirudinea correspond more closely with the coelomoducts or ciliated organs of the Polychæta than with the nephrostomes; they are to be compared also with the "urns" of the Gephyrea. In the Rhynchobdellid Pontobdella a very interesting modi-

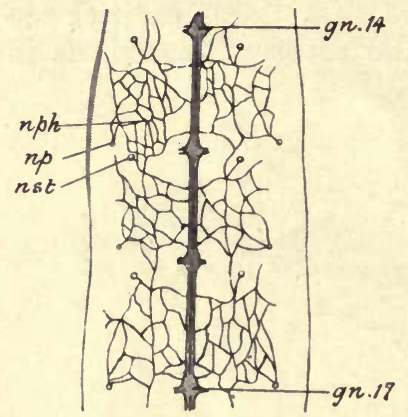

Fia. 416.-Nephridial system of Pontobdella. gn. 1/, gn. 17, ganglia of nerve-eord ; $n p$. nephridiopore ; $n p h$. nephridial network; nst. ciliated funnel. (After Bourne.)

fication of the nephridial system occurs. Instead of distinct nephridia, there is found on the ventral surface of the body a very complex network (Fig. 416, nph.), which sends off on each side of each segment a short bralch terminating in a ciliated funnel, and a similar branch which opens externally $(n p$.$) .$ A similar modification occurs in Branchellion.

The nervous system always closely resembles that of Hirudo, as also do the sense-organs. The number of eyes is subject to considerable variation: they may be developed on the posterior sucker, or may be absent altogether.

Reproductive Organs.-The testes usually have the segmental arrangement found in Hirudo, their number varying from five to twelve pairs. But in Herpobdella (Nephelis) they are very numerous, and are not arranged segmentally. In the Rhynchobdellida the muscular penis is absent, its place being taken by an eversible sac or bursa copulatris. The form of the ovary with its containing sac in Hirudo is exceptional. As a rule, there is an elongated hollow ovary, producing ova from its epithelial lining, and thus agreeing very closely in structure with the testis.

In Clepsine, a fresh-water Rhynchobdellid, copulation in the ordinary sense of the word has never been observed, but one individual has been seen to deposit one or more spermatophores on any part of the body of another-often on the back. - The spermatophore, which is nearly $3 \frac{1}{2} \mathrm{~mm}$. long, apparently exerts a solvent action on the skin, since, after a short interval, the spermatic substance streams through the skin into the cœlomic spaces, probably making its way at last to the ovaries. This extraordinary process of hypodermic impregnation probably takes place in other genera, but has been most closely fullowed in Clepsine.

It is in Clepsine that the early stages of development are best known. Segmentation is unequal, the embryo consisting, in the eight-celled stage (Fig. 417, A), of four large ventrally placed megameres $(m g$.$) and four dorsal micromeres (m i$.$) . . One of the$ 
megameres, posterior in position, divides into two cells (B): the so-called neuronephroblast and mesoblast, the latter of which at once divides into two. As shewn by their subsequent history, the neuronephroblast and the mesoblast correspond respectively to the first and second somatoblasts of Nereis. The former divides and sub-divides to form two symmetrical groups of four cells each, situated at the posterior pole. The number of micromeres increases, at first apparently by division of the megameres. The latter subsequently give off a number of small endoderm cells.

The embryo now consists of the three large megameres with a number of endoderm cells, a cap of small micromeres forming an ectodermal layer which is extending over the surface, with, at the posterior pole, two symmetrical groups of neuronephroblast cells (four in each), and, somewhat deeper, the two mesoblast cells.
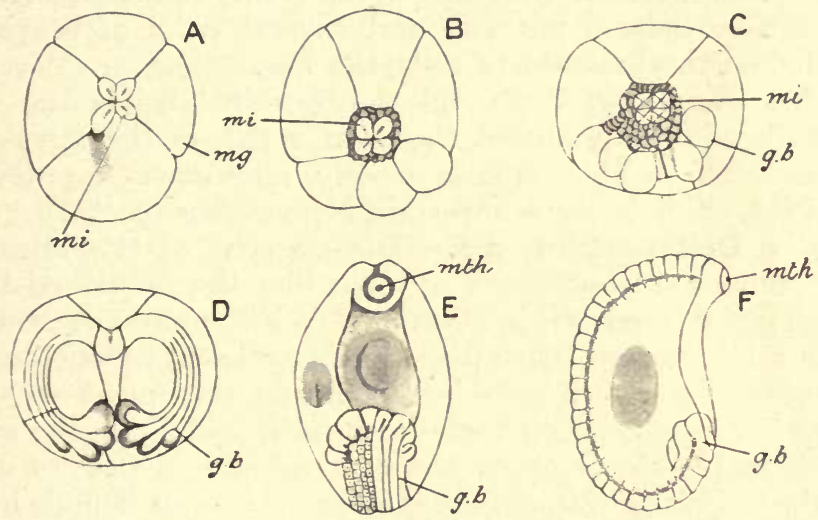

FiG. 41\%.-Six stages in the development of Clepsine. g. b. germinal bands; mg. megameres mi. micromeres; mith. mouth. (After Whitman.)

From each of the ten cells last mentioned new cells are given off in front in such a way as to form ten rows of cells, five on each side, four being derived from neuronephroblasts and one from the mesoblast cell, These two sets of rows of cells constitute the so-called germinal bands. From their subsequent fate it is clear that they correspond to the mesoderm bands of Nereis plus the neural plate. They grow forwards, the ectoderm extending with them, over the endoderm and megameres. At first they diverge widely, but their anterior ends subsequently meet towards the anterior end of the embryo. Later the intermediate parts of the bands, originally widely separated from one another owing to their divergence during growth, approach one another and meet along the middle line of the ventral surface. The germinal bands give rise to the nerve-cord, the mesodermal segments, and the nephridia. The layer of micromeres not only gives rise to the whole ectoderm 
but also forms the head-the germinal bands not extending into that region. The embryonic enteric cavity (mesenteron) becomes formed by arrangement of the endoderm cells round the three megameres, which break up to form nutrient material or yolk destined to become absorbed in nourishing the embryo. The pharynx is formed by an invagination of the ectoderm which joins the mesenteron. At this stage the embryo leaves the egg, and soon escapes from the cocoon to pass through its later stages attached to the ventral surface of the parent.

In the Gnathobdellida the young are hatched at an early stage of development, and their megameres contain but little yolk: they are nourished up to the time of leaving the cocoon on the albumen with which the latter is filled. One member of this order, Herpobdella (Nephelis) is remarkable for undergoing a metamorphosis : the anterior end of the embryo is ciliated, and it possesses a provisional pharynx and several pairs of provisional nephridia. Paired masses of cells, the head-gerns, are developed in the head, and from these and the germinal bands the whole body of the adult is produced, the greater part of the larval body being cast off. This process closely resembles the development of the pilidium larva of certain Nemerteans (p. 295).

Habits, Distribution, \&c.-The majority of the Hirudinea are inhabitants of fresh-water, and live, like the Medicinal Leech, by sucking the blood of higher animals-Vertebrates or Molluses. It is doubtless in correlation with this intermittent parasitism-the chance of finding a vertebrate host being an infrequent one-that the crop has attained such vast dimensions, holding, in the case of the medicinal leech, as much blood as takes it a year to digest. The allied species Hirudo sanguisuga has been found in the nasal passages of man, producing serious results, and being, to all intents and purposes, an internal parasite. The same is the case, with the horse-leech, Homopsis vorax, taken in, when young, by horses and cattle while drinking. It attaches itself to the pharynx and may even descend the trachea. Others are permanent ectoparasites: for instance, Branchellion occurs on the outer surface of the Skate, Electric Ray, and other Fishes, entire families of this leech, including individuals of all sizes, being sometimes found crowded together on a small area of skin, which is distinctly marked by their powerful posterior suckers. Other fish-parasites are Pontobdella, on Rays, and Piscicola on fresh-water Fish. Aulostoma, to which, as well as to Hæmopsis, the name Horseleech is applied, is carnivorous, feeding on snails and other Molluses; so also are Glossiphonia (Clepsine), Herpobdella (Nephelis) and the gigantic Macrobdella. The last-named genus and some others are of subterranean habits, living in moist earth. The Land-leeches (Homadipsa) live in the forests of many parts of 
the world, and in spite of their small size, which does not exceed $30 \mathrm{~mm}$. in length and $5 \mathrm{~mm}$. in diameter, are much dreaded for the persistent attacks they make on men and cattle.

Many genera are very widely distributed: for instance, the Land-leeches (Hamadips Indies, Japan, Australia, and South America, a distribution which seems to indicate that the group is one of great antiquity.

\section{GENERAL REMARKS ON THE ANNULATA.}

A special feature of the Annulata, as distinguished from the phyla previously dealt with, is metameric segmentation. In some of the Platyhelminthes, as we have seen, there obtains a condition to which the term pseudo-metamerism is applied. In such cases there is a serial repetition of certain of the organs-gonads, diverticula of the intestine, nerve-commissures, \&c.-in such a way as to produce a jointed appearance, though the body is not divided into definite segments. An appearance resembling segmentation is produced also in certain Rhabdocœles that multiply by budding, chains of zooids remaining connected together for a time. In the strobila of the Cestodes we recognise a condition which might be described as combining pseudo-metamerism with the formation of a chain of zooids. The condition of true metamerism, as we observe it in the Annulata, is capable of being deduced from a condition of pseudo-metamerism as it occurs in Gunda (p. 255), the pseudo-metameres becoming converted into true metameres by the development of inter-segmental constrictions and the completion of internal partitions. If we suppose that during this process serially repeated outgrowths of the enteron became separated off to form series of cœlomic sacs enclosing the gonads, a condition would be reached not far removed from that which characterises the Annulata. On the other hand, the metameric condition is deducible from the condition of a linear colony of zooids proliferating at the posterior end, the zooids, though becoming each complete in itself, not, under ordinary circumstances, becoming detached. The establishment of a closer connection between the corresponding organs of the zooids in such a colony, with the special differentiation of the anterior end, would result in a condition closely resembling the metamerism of the Annulata. It is conceivable that a condition of pseudo-metamerism was followed by that of a linear series, not of zooids, but of comparatively independent parts capable of readily reproducing the animal when detached, and that a secondary closer connection established 
between the organs of all the series of parts resulted in the metameric condition.

Metamerism is not universal in the phylum. In some (ArchiAnnelida) it may be said to be incipient or rudimentary ; in others (Gephyrea) vanishing or vestigial. The Archi-Annelida are in this, as in some other respects, the most primitive of the Annulata, and through them it seems possible to connect the higher members of the phylum with such lower forms as Dinophilus (p. 337) and the Histriobdellea (p. 338). The general occurrence of the trochophore larva may be taken as pointing to descent from an unsegmented ancestor having resemblances to the trochophore, and a
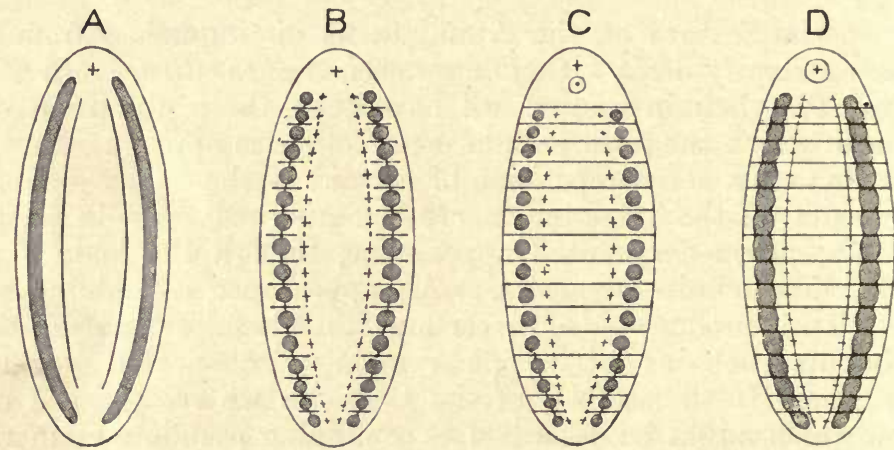

FiG. 418.-Diagram to illustrate possible relations of the unsegmented to the metamerically segmented worm. $A$, unsegmented worm with differentiated head end; $B$, pseudo-metamerism ; $C$, linear series of zooids in which the first zooid differs in character from the otliers, and in which the formation of new zooids takes place at the posterior end ; $D$, metamerically segmented worm.

form like Dinophilus would afford us an intermediate link between such a hypothetical ancestor and Polygordius or Protodrilus.

The position of the unarmed Gephyrea in the Annulata is, as already noticed, a matter of doubt; if we dissociate them from the Armata there is little to connect them positively with the other members of the phylum. But, on the whole perhaps, the evidence in favour of regarding them as allied to the Armata, and through them with the Chætopoda, is sufficiently strong.

In adult structure, particularly in the absence of parapodia and setæ and the reduction of the cœlome, the Hirudinea diverge somewhat widely from the Chætopoda; but a study of their earlier developmental stages shows unmistakably their close connection with the latter group, more particularly with the Oligochæta; and the existence of an undoubted Leech (Acanthobdella) with setæ and with a well-developed coelome traversed by mesenteries helps still further to bridge over the gap between the two classes. 
The following diagram will serve to illustrate this view of the relationships of the various groups referred to:-

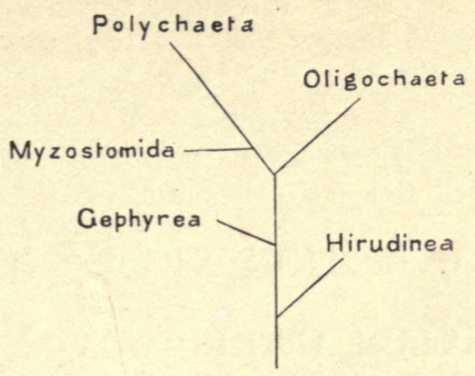

Archi-Annelida

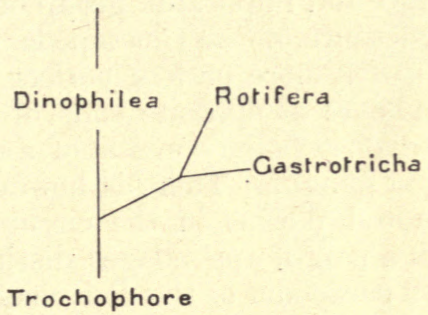

FIG. 419.-Diagram illustrating the relationships of the Annulata and the Trochelminthes.

It should be added, however, that it is not likely that the trochophore actually represents the ancestral form, since, to some extent at least, its special features, such as the special arrangement of the cilia, may be adaptations to a pelagic mode of life. 


\section{SECTION XI}

\section{PHYLUM ARTHROPODA}

In this large and important group of animals we meet with a characteristic feature of the Chætopoda, viz. metameric segmentation, and also with more or less perfect bilateral symmetry; the mouth and anus are at opposite ends of the elongated body, and the central nervous system consists of a dorsal brain and a double ventral chain of ganglia. There is, however, an important advance on the segmented Worms in the circumstance that each typical segment bears a pair of appendages, distinguished from the simple foot-stumps or parapodia of the Polychæta in being divisible into distinct limb-segments or podomeres, separated from one another by movable joints and acted upon by special muscles. Arthropods are also characterised by the almost universal absence of cilia, by their muscles being nearly always of the striped kind, and by the body-cavity largely corresponding not to a true cœlome, but to a howmocele, in free communication with the circulatory system and developed from the latter.

The following are the most important subdivisions of the phylum:-

Class 1. Crustacea, including Crayfishes, Crabs, Shrimps, Wood-lice, Barnacles, Water-fleas, \&c.

Class 2. ONychOPHORA, including only the curious caterpillarlike Peripatus, and a small number of closely related genera.

Class 3. Myriapoda, including the Centipedes and Millipedes.

Class 4. InsECTa, including the true or six-legged Insects, such as Cockroaches, Locusts, Flies, Beetles, Butterflies, and Bees.

Class 5. Arachnida, including Spiders, Scorpions, Mites, \&c.

\section{CLASS I.-CRUSTACEA.}

1. Examples of the Class.

$$
\text { a. Apus or Lepidurus. }
$$

Apus and Lepidurus are two closely allied Crustaceans found in the fresh-waters of most parts of the world, but curiously local 
in distribution and by no means common. They are so much alike that, save in minor details, the same description will apply to any species of either genus.

External Characters. - The animal (Fig. 420) is from 20 to $30 \mathrm{~mm}$. in length, and has the anterior two-thirds of the dorsal surface covered by a thin chitinous shell or carapace, beyond the posterior edge of which the hinder part of the body ( $a b d$.$) projects$ as a nearly cylindrical structure distinctly divided into segments. The last or anal segment bears a pair of long processes, the caudal styles $(a . f$.$) between$ which, in Lepidurus, is a flat scale-like postanal plate (Fig. 421). On the dorsal surface of the carapace, near its anterior border, are the paired cyes $(E)$, closely approximated in front, diverging posteriorly. Immediately in front of them is a small black median eye (e.), and between their diverging posterior ends a semi-transparent oval area, the dorisal organ (d. o.). Passing transversely across the carapace, a short distance behind the dorsal organ, is a shallow furrow, the cervical fold, immediately posterior to which a pair of coiled tubes $(s h . g l$.) are seen, one on

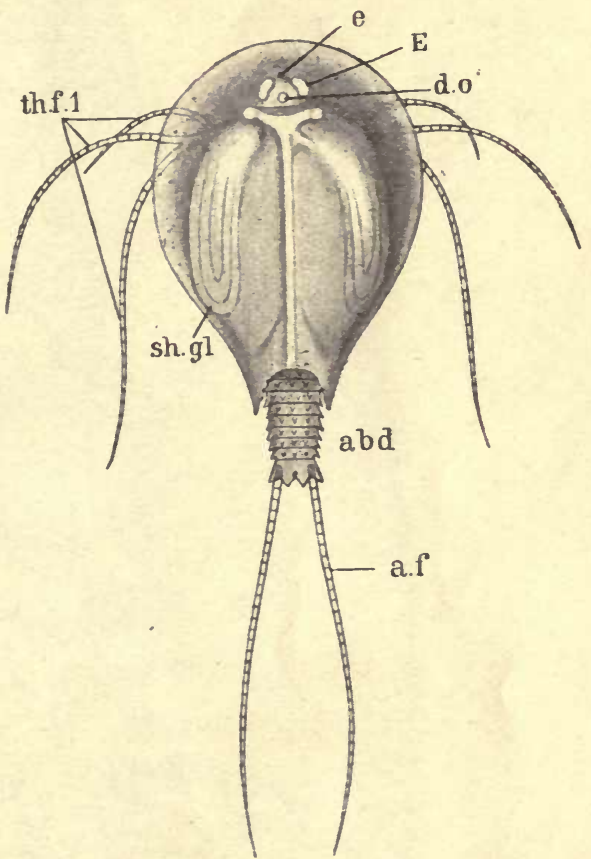

Fig. 420.-Apus cancriformis, dorsal aspect. abel. abdomen; $a$. $f$. caudal styles; $l$. o. dorsal organ ; $B$. paired eye ; e. median eye; $s h$. $g l$. shell-gland ; th.f. 1 , endites of first thoracic foot. (From Bronn's Thierreich.) each side of the carapace: these are the shell-glands or excretory organs.

The carapace is attached only as far back as the cervical fold: behind that level it is free, and, when lifted up or cut away (Fig. 421), shows the greater part of the body of the animal, divided into segments like the posterior portion. From the cervical groove backwards about twenty-eight or thirty segments can be counted: the region in front of the cervical groove shows no sign of segmentation, and is distinguished as the hcad. The segments have the form of chitinous rings, often produced into 


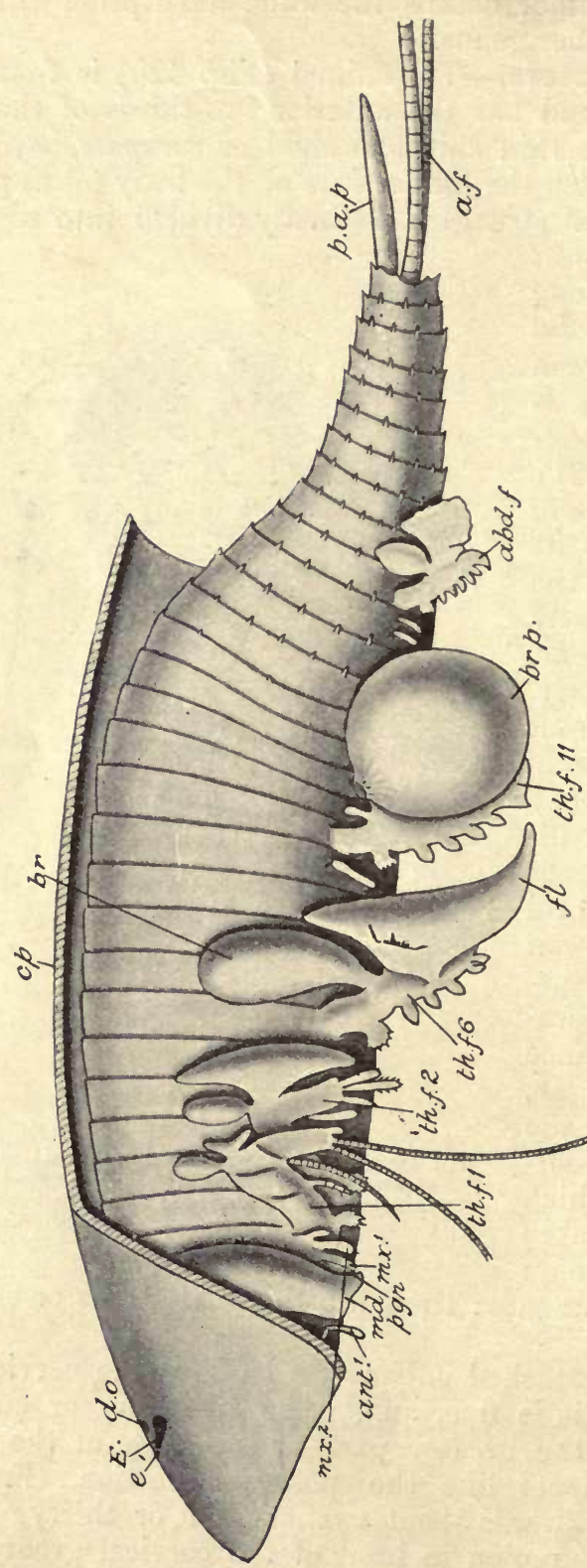

(3.

चं

...

है.

\%

的政

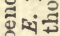
… न7

ษ

एक

흔

潒密

0

สี่

\&

\%

हึँ

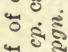

फ्...

뎐 홍ํㅀ 웅

एह है

ㅎํㅇ 군

.

룽

का

चัن

ys.

i.

类

을

드의

ज政

I-iñ

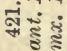


small spines: each ring slightly overlaps its successor, and is connected with it by a narrow area, the articular membrane, the chitinisation of which is less pronounced than that of the rings themselves. By this arrangement the segments are freely movable upon one another in all directions, the articular membranes acting as joints. The last or anal segment is pierced by the terminal anus (Fig. 424, an.).

The ventral surface of the head is formed by a flattened subfrontal plate (Fig. 422, s.f.pl.), continuous marginally with the carapace. The posterior edge of this plate is convex backwards, and is produced in the middle line into a shield-shaped process, the labrum or upper lip $(l b r$.$) , which over-$ hangs the mouth. From the subfrontal plate also arise, on each side, two delicate processes, the innermost called the antennule (ant. 1), the outermost the antenna (ant. 2): these are the first two pairs of appendages. The third pair consists of two strong, toothed bodies of a deep brown colour, placed one on each side of the mouth, and called the mandibles $(m d$.$) . The remaining ap-$ pendages form two rows of delicate leaf-like processes, attached to the segmented portions of the body, and overlapping one another from before backwards : their number varies from forty to nearly seventy (th.f., $a b d . f$.$) .$

\section{Appendages.-The antennule} (Fig. 423, 1) consists of a bent rod bearing delicate chitinous

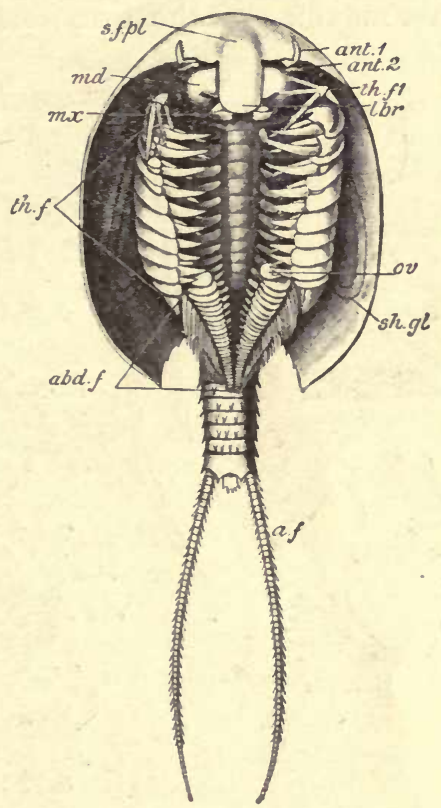

Fig. 422.-Apus glacialis, ventral aspect. abd. $f$. abdominal feet; $a n t^{1}$. antennule ; $a n t^{2}$. antenna ; lbr. labrum; $m d$. mandible; $m x$. first maxilla; ov aperture of oviduct; 8 . $f$. $p l$. sub-frontal plate ; sh. gl. shell-gland; th.f. thoracic feet; th. f. 1, first thoracic foot. (After Bernard.)

bristles or setce at its tip, and presenting, at the bend, a joint, due to the presence of an articular membrane. The appendage is thus made up of two podomeres or limb-segments, movably articulated together. Its function is probably tactile.

The antenna (2) is absent in some species both of Apus and Lepidurus : in $A$. cancriformis it is a very delicate hook-shaped unjointed structure, probably functionless. As we shall see from the study of development, it is a vestigial organ.

The mandible (3) is also an unjointed appendage. It has the form of a deeply concavo-convex plate, strongly chitinised, and pro- 
duced along its inner edge into strong teeth. The mandibles lie one on each side of the mouth, and are so articulated that, by means of muscles, their toothed edges ean be brought together in the middle line, so as to rend the food.

The fourth and fifth appendages are very small, and are probably functionless or nearly so: they follow one another just behind the mandible, and are called the first and sccond maxilla. The first maxilla (4) eonsists of two eurved chitinous plates, the second of a basal portion produced into two branches (5). Between the first maxilla and the mandibles are a pair of delicate unjointed

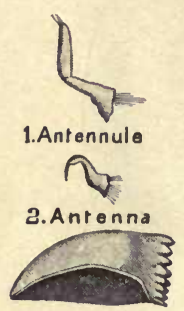

3. Mandible

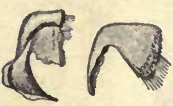

4. I' 'Maxilla

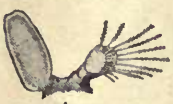

5. $2^{\text {nd }}$ Maxilla

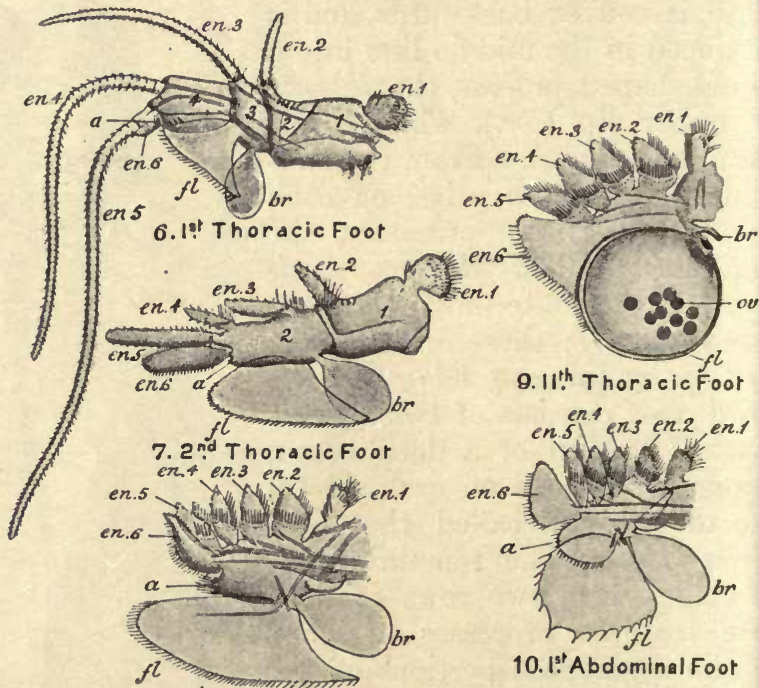

8. 7. Thoracic Foor

Fig. 423.-Typical appendages of Apus. 1-4, podomeres of axis; br. bract; en. 1, en. 7, endites ; fl. flabellum; ov. ova. (After Lankester.)

processes, the paragnatha (Fig. 421, pgn.): they form together a sort of lower lip, and are not usually reckoned as appendages.

The foregoing appendages all spring from the unsegmented anterior portion of the body or head. As we shall see, however, the sueceeding limbs arise each pair from its own segment, so that the presence of five pairs of appendages on the head may be taken provisionally as an indication that this region of the body is composed of five fused segments.

The sixth appendage $\left(6^{\circ}\right)$ springs from the ventro-lateral region of the first clearly marked segment, and is the first of the long row of appendages plainly visible in a ventral view. It consists of an axis formed of four podomeres (1-4), and bearing a number of offshoots: six of these, called endites (en. 1-en. 6), arise from 
its inner or mesial border ; two, called exites ( $b r ., f l$.), from its outer or lateral border. The proximal endite $(c n .1)$ is small, and bears strong spines; in connection with its fellow of the opposite side it is used to seize food-particles and pass them on to the mouth: it is therefore conveniently distinguished as the gnathobase. The distal endite is rudimentary $(c n .6)$ : the remaining four (en. 2-5) are long, jointed filaments. The proximal exite is nearly triangular, and is called the flabellum ( $f$. $)$; the distal exite is oval, and is known as the bract $(b r$.$) ; both probably serve a respiratory$ function.

The seventh appendage ( 7$)$ has only two podomeres in the axis, and the endites are comparatively short and flat. The next eight, i.e. those borne on the third to the sixteenth free segments, closely resemble one another: each $(S)$ has an unjointed axis and short leaf-like endites, the whole appendage having a distinctly foliaceous character. The sixteenth appendage-that of the eleventh free segment-resembles its predecessors in the male, but in the female $(9)$ is peculiarly morlified. The distal portion of the axis forms a hemispherical cup, over which the flabellum $(f$. $)$ fits like a lid: in this way a capsule or brood-ponch is produced, which serves for the reception of the eggs, and the appendage is distinguished as the oostegopod or brood-foot. The brood-feet and the adjacent genital apertures allow of a very convenient division of the body: all that region from the first free or postcephalic segment to that bearing the oostegopods, both inclusive, is called the thorax, and its appendages the thoracic feet: it consists of eleven metameres. The remaining segments, from the twelfth to the last inclusive, constitute the abdomen, and their appendages are called the abdominal feet.

The abdominal resemble the thoracic feet in general characters, having the same foliaceous form (10), with unjointed axis, small leaf-like endites, and large flabellum and bract. They gradually diminish in size from before backwards; and, from the third abdominal segment onwards, two or more pairs of appendages spring from each segment, so that while the total number of abdominal segments, in $A$. cancriformis, is twenty-two, and the five hindermost of these are without appendages, there are altogether fiftytwo pairs of abdominal feet. It seemed probable that segments bearing more than one pair of appendages represent two or more fused-or, perhaps one should rather say, imperfectly differentiated -metameres.

Body-wall. - The whole body is, as already mentioned, covered by a layer of chitin of varying thickness, which constitutes an exoskeleton or external supporting structure. Immediately underlying it is the deric epithelium or epidermis, from which the chitin is secreted layer by layer. Thus the exoskeleton of Apus is a continous cuticular structure, exhibiting segmentation in virtue of the 
fact that, while comparatively thick and strong in places where
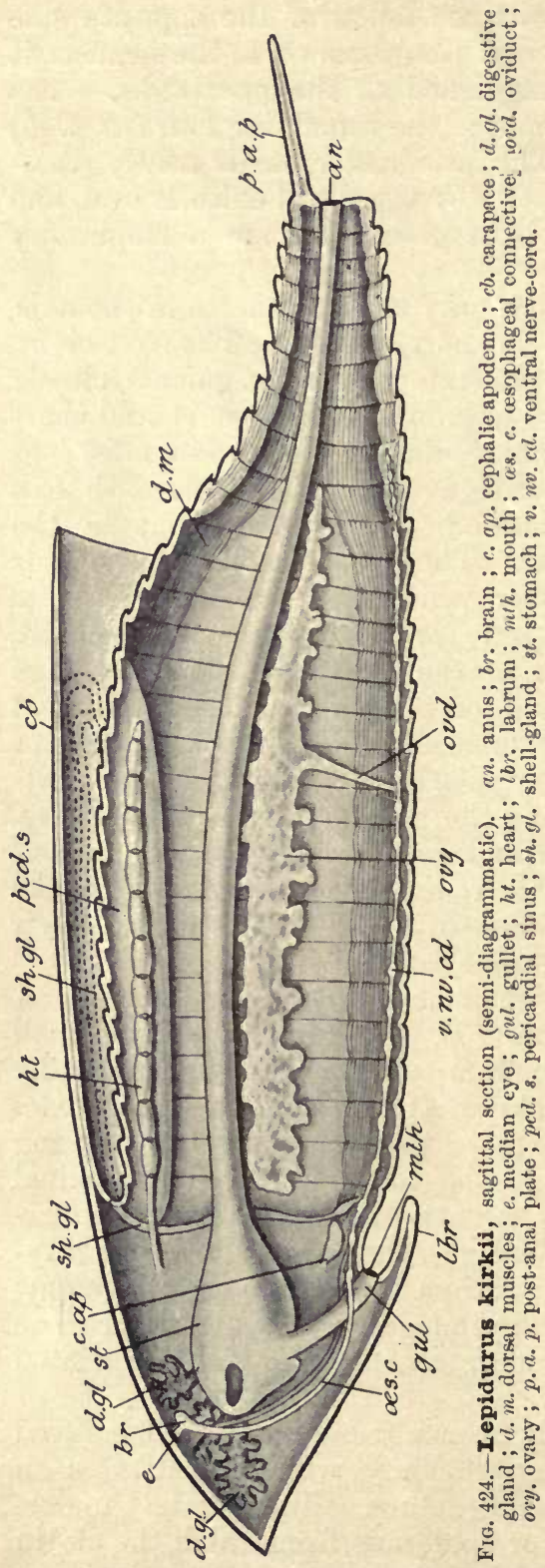
no movement is required, it is thin and flexible in the intervening spaces, and thus allows of the movement of the harder parts upon one another.

The setæ, which occur on many parts of the body, and in particular fringe the appendages, are hollow offshoots of the chitinous cuticle, containing a protoplasmic core continuous with the epidermis (Fig. 433). They thus differ fundamentally from the setæ of Chrtopods, which are solid rods sunk in muscular sacs.

The muscular system is well developed (Fig. 424). Underlying the epidermis is a layer of connective-tissue, and beneath this is found, in the posterior or limbless part of the abdomen, a layer of longitudinal muscles encircling the body and attached by connective-tissue to each segment. In this way the muscular layer is itself segmented, being divided by the connective-tissue insertions into muscle-segments or myomeres. The action of these muscles is to approximate adjacent segments : according as the fibres on the dorsal, ventral, or lateral regions contract, the abdomen will be raised, lowered, or turned sideways. In the limb-bearing portion of the abdomen and in the thorax there is no longer a continuous muscular tube, but 
paired dorsal (d.m.) and ventral bands, which pass respectively above and below the origins of the limbs: the dorsal bands arise in front from the head-region, the ventral from a strong fibrous plate, the cephalic apodeme (c.ap.), lying just behind the gullet.

Each appendage is moved as a whole by muscles passing into it from the trunk: its various parts are acted upon by delicate muscular slips running to the various podomeres of the axis and to the endites, thus rendering them separately movable. The only example we have yet met with of appendages moved by definite muscular bands is that of the curious Rotifer Pedalion (p. 330). The muscles are all striped, a character which applies to the Arthropoda generally, with the exception of the Onychophora.

Digestive Organs.-The month (Fig. 424, mth.) is situated on the ventral surface of the head, and is bounded in front by the labrum $(l b r$.$) , on each side by the mandibles, and behind by the$ paragnatha. The food appears to be pushed forwards towards the mouth by the toothed bases of the thoracic feet, and is broken up by the mandibles, which work laterally. The maxillæ are probably functionless, or nearly so.

The mouth leads into a narrow gullet (gul.), which passes upwards and forwards into the head and enters a wide stomach (st.), from which a straight intestine (int.) is continued back to the terminal anus (an). From each side of the stomach is given off a wide tube (d.gl.) which branches extensively, its ramifications finally ending in delicate creca. The larger branches of these digestive glands contain food in process of digestion : their ultimate cæca secrete a digestive juice: the walls of the stomach itself are non-glandular. The walls of the enteric canal consist of an inner layer of epithelium and an outer layer of connective-tissue and muscle. In the gullet and in the posterior end of the intestine the epithelium secretes a thin cuticle, which thus comes to form the actual lining of the cavity. It is shown by development that the portion of the canal devoid of a chitinous lining is formed from the archenteron of the embryo: the gullet is developed from the stomodæum, the posterior end of the intestine from the proctodæum.

The body-cavity is divided into several parts by membranous partitions (Fig. 425) : there is a large median cavity in which the enteric canal (i) lies, called the intestinal sinus: on each side of this are lutercl sinuses containing the muscles : and in the dorsal region is a median cavity, the pericardial sinus. All these spaces are devoid of an epithelial lining, and contain blood: as will become evident later (cf. p. 593), they do not correspond with the colome of the higher worms.

The central organ of the circulatory system is the heart (Fig. 424, $h t$, and Fig. $425, h$ ), a narrow tube contained in the pericardial sinus. It is pierced laterally by several pairs of apertures or ostia 
provided with valves opening inwards, and is continued in front into a narrow tube, the cephalic artery (c. art.), which extends into the head and gives off near its origin a pair of arteries to the shellglands (Fig. 422). When the heart contracts, the blood is driven through these arteries to the head and carapace: it then travels backwards in the intestinal sinus, passes to the limbs, and is returned to the pericardial sinus, finally re-entering the heart, during its diastole, through the ostia. The plasma of the blood is coloured red by hæmoglobin, and contains amœboid corpuscles.

As already mentioned, the function of respiration is discharged by the flabella and bracts of the feet, which are abundantly supplied with blood and the movements of which ensure a constant

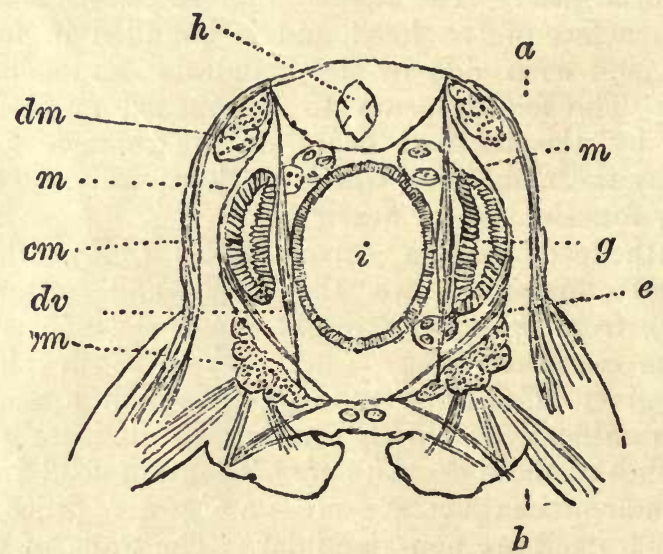

Fig. 425.-Transverse section of Apus. cm. muscles to fect; $d v$. dorso-ventral muscles; $e$. eggs ; $d m$. dorsal muscles ; $g$. ovary ; $d v$. dorso-ventral muscles; $h$. heart ; $i$. intestine ; m. partition between intestinal and lateral sinus ; vm. ventral muscles. (From Bernard.)

renewal of the water in their neighbourhood. The renal organ or shell-gland (Fig. 426) consists of a coiled urinary tube (uc.) lying between the two layers of the carapace and lined by glandcells. At one end the tube is connected with an end-sac (ts.), also lined with glandular epithelium; at the other it dilates into a small bladder $(b$.$) which opens on the second maxilla (m$.).

The nervous system (Fig. 427) is constructed on the annulate type. There is a squarish brain (bro) situated in the dorsal region of the head, beneath the eyes. From it a pair of cesophageal connectives pass backwards and downwards to join the ventrial nervecord, which consists of a double chain of ganglia (gn. 1-4) united by longitudinal connectives and transverse commissures so as to have a ladder-like appearance. The first pair of ganglia lies immediately behind the mouth, and sends off visceral nerves which join to form a ring round the gullet, swollen in front into a small visceral ganglion (v. gn.). Passing backwards the nerve-chain 
diminishes in size, and enmes to an end at about the level of the last pair of abdominal feet (Fig. 424).

The origin of the nerves given off from the central nervous system presents many points of interest. From the fourth ganglion of the ventral cord backwards each pair of appendages has its own pair of ganglia, the metameric correspondence between the limbs and the nervous system being complete. The mandibles and the first maxillæ also receive nerves, each from their own pair of ganglia, their serial homology with the more typical appendages being thus confirmed. But the second maxillæ receive their nerves $(m n .2)$ from the connectives between the third and fourth ganglia : the ganglion belonging to their segment may be assumed

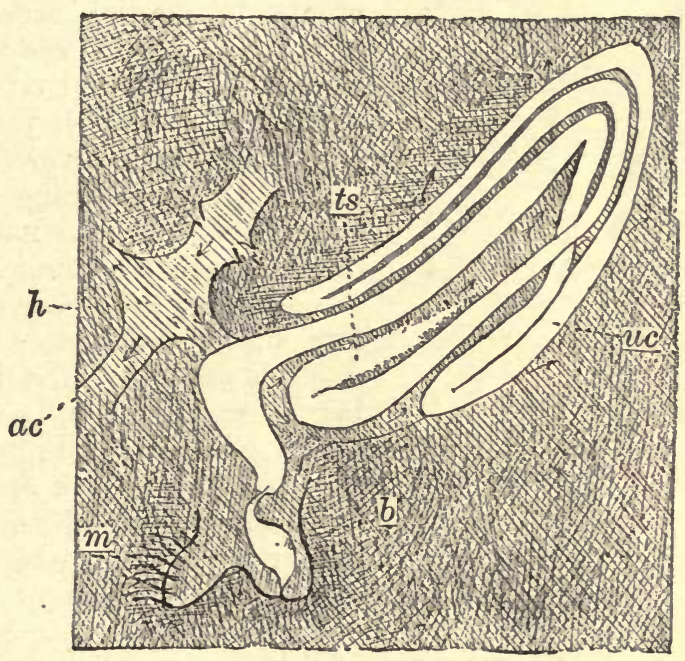

Fio. 426.-Shell-gland of Apus, diagrammatic. ac. cephalic artery; $b$. bladder; $h$. heart m. second maxilla; $t$. end-sac ; $u c$. urinary tube. (From Bernard.)

to have atrophied. The antenna is supplied by a nerve (ant. 2) which springs from the osophageal connective, but which can be traced backwards to the first ganglion of the ventral chain: this fact may be taken as an indication that the antennæe are serially homologous with the jaws and feet-that they are, in fact, metameric or post-oral appendages which have shifted forwards, one on each side of the mouth, thus becoming pre-oral. The nerve of the antennule (ant. 1) also springs from the osophageal connective, but is traceable forwards to the brain, where it is connected with a special group of nerve-cells. This has been explained by supposing that the antennule is a post-oral appendage the ganglion of which has moved forwards along the osophageal connective and fused with the brain-a process which actually takes place 
with the ganglia of the antennæ in the higher Crustacea. But it is also possible to consider the antennules as pre-oral appendages, belonging, like the prostomial tentacles of Chrtopods, to the

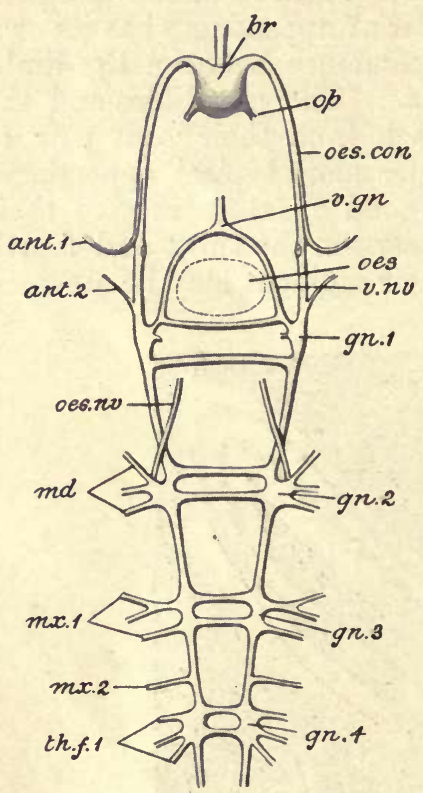

Fid. 427.-Nervous system of Apus cancriformis. ant.' nerve to antennule ; ant." to antenna ; br. brain ; gn. $1-4$, first four ganglia of ventral nerve-cord; $m d$. mandibular nerve; $m x .1$, nerve of first maxilla; $m x .2$, of second maxilla; ces. con., oesophageal connective; op., optic nerve; th. $f .1$, of first thoracic foot; $v g n$. visceral ganglion. (After Lankestcr and Pelseneer.) lens and vitreous humour. The inner portion is a group of sensory cells, constituting a retinula $(r c$. $)$, and enclosing a refractive rod, the rhabdome $(r h$.): the retinula is the actual percipient part of the ommatidium, its cells being comparable to our own rods and cones. The retinulæ of adjacent ommatidia are separated from one another by cells full of black pigment $(p$.), so that each ommatidium is in a state of optical isolation from its fellows, and the whole eye is what is called a compound eye. The optic nerve springing from the brain dilates into an optic ganglion, from which fibres pass to the retinulæ.

The median eye is an ovoid body, and consists of four groups of large sensory cells enclosing a mass of pigmented tissue: it is in immediate contact with the brain, and receives a narrow 
canal from the water-sac beneath the cuticle of the paired eyes.

Reproductive Organs.-The large majority of individuals both of Apus and Iepidurus are females; males are of comparatively rare occurrence. The ovary (Fig. 424, ovy.) is a branched tube occupying a considerable portion of the body-cavity in sexually mature individuals. The walls of the tube are lined with epithelium, and give rise to ova, which pass into the lumen of the tube and thence to a duct (ovd.) opening on the eleventh or

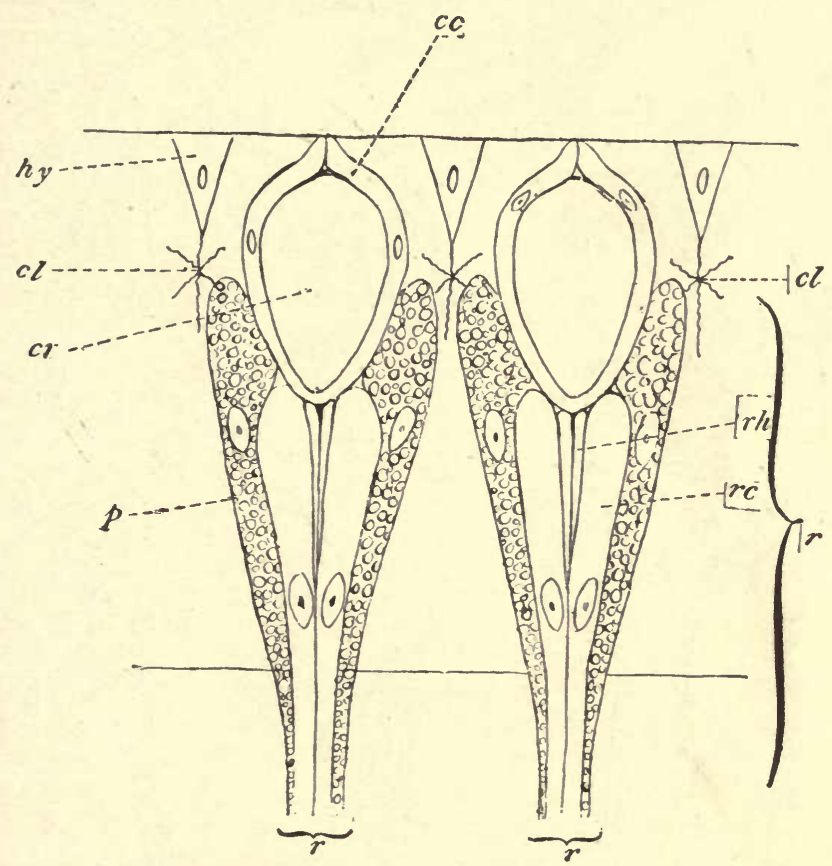

Fig. 42S.-Diagram of two ommatidia from the paired eyes of Apus. $c c$. vitreous cells ; $c r$. vitreous body $; c l$. connective-tissue fibre; $h y$. epidern cells; $p$. pigment cells ; $r$, inner parts of ommatidia ; rc. retinula ; rh. rhabdome. (From Bernard.)

last thoracic segment. As in Leeches (p. 515), there is reason for thinking that the cavity of the ovarian tube represents a shut-off portion of the colome, and the oviduct a nephridium. One species has been shown to be hermaphrodite: in others males are occasionally found, but reproduction appears to be, as a rule, parthenogenetic.

Development.-The eggs are centrolecithal, i.e., have an accumulation of yolk in the centre surrounded by a superficial layer of protoplasm. The process of segmentation and the formation of the germ-layers have not been observed. 
The embryo is hatched in the form shown in Fig. 429, A. The body is oval, and is divisible into three regions-a large anterior or head-region; an intermediate trunk-region, the hinder part of which already shows signs of segmentation $(I-V)$ and a posterior bilobed anal region. The head-region bears a single median eye, and a pair of small unjointed appendages (1), each with two large setæ at its extremity : these become the antennules of the adult. The trunk region bears two pairs of appendages, the first of which (2)
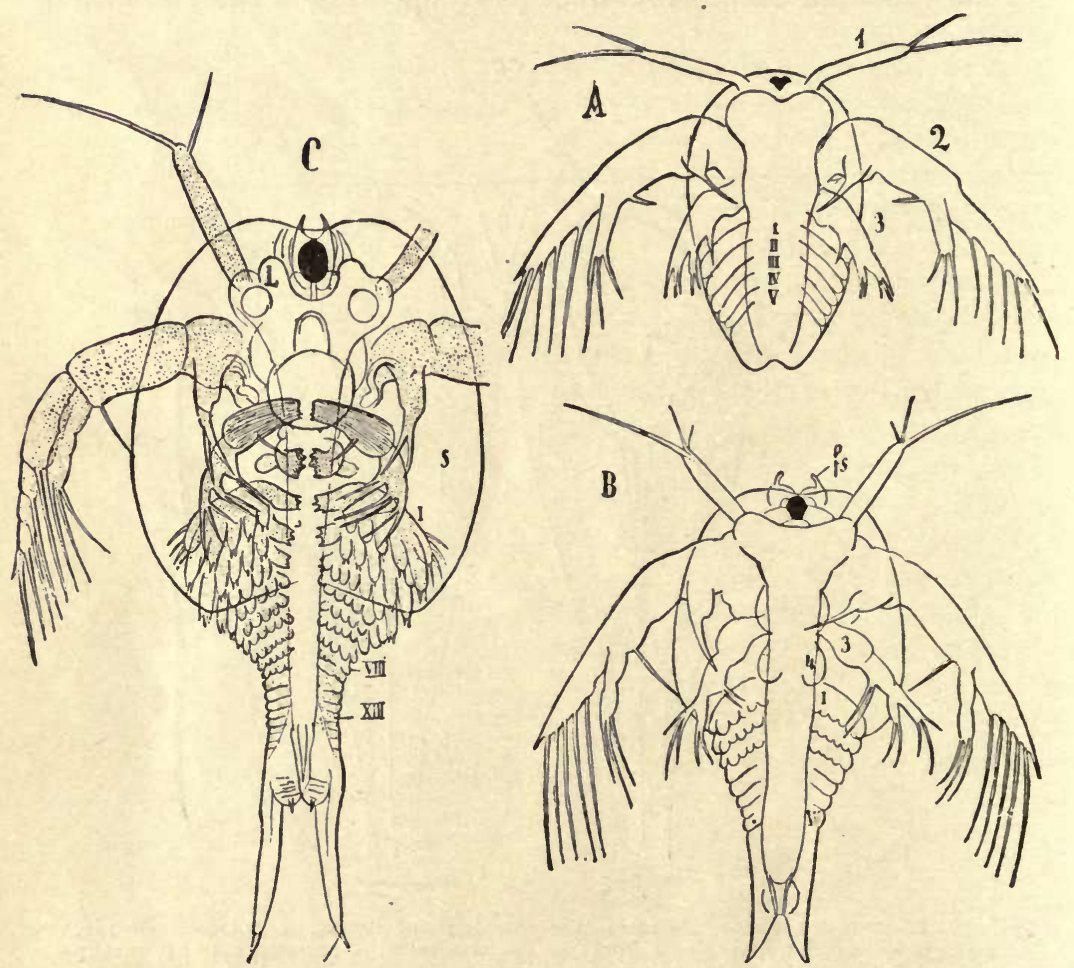

FIG. 429.-Three stages in the development of Apus. fs. frontal sensory organ; L, digestive gland; 8. carapace; $1-4$, cephalie appendages ; I-X III, body-segments and appendages. (From Lang's Comparative Anatomy.)

is very large and fringed with setæ, but is chiefly remarkable for being biramous or two-branched-being formed of a proximal portion or stem, the protopodite; a small inner branch, the endopodite; and a large outer branch, the cxopodite. This second appendage becomes the antenna of the adult, and may be called the antennary foot: it is the chief organ of locomotion of the larva. The second-trunk appendage is the mandibular foot (3), so called because it becomes converted into the mandible of the adult: it is also biramous. The only internal structure to be 
noted is the straight enteric canal with its dilated anterior end or stomach : the mouth opens between the bases of the antennary and mandibular feet, and is bounded in 'front by a large labrum : the anus is at the extremity of the anal region. This very peculiar and characteristic larval form is called a nauplius. ${ }^{1}$

The nauplius swims freely, chiefly by vigorous strokes of the great antennary feet, and after a time undergoes a series of moults or ecdyses, the cuticle being cast off and the animal emerging in the form shown in Fig. 429, B. The trunk-region has elongated, new segments having been added, as in Chrtopods, between those previously present and the anal region. The antennules have become shifted backwards, and rudiments of a fourth pair of appendages, the first maxillæ (4), have appeared. The carapace has grown out from the dorsal region of the head, and a peculiar paired sense-organ $(f s$. $)$ has appeared on the head.

After two more ecdyses the larva has assumed the form shown in Fig. 429, C. Several new segments have been added, and the anterior of these all bear leaf-like thoracic feet. The antennary feet are still very large, and the bases of the mandibular feet have become enlarged and toothed so as to form biting jaws. The carapace $(s)$ has increased greatly, and the caudal styles have attained a considerable size. Further moults occur, new segments are added with their appendages, the antennules and antennæ degenerate-the latter sometimes disappearing altogether, - the mandibles become reduced to the enlarged basal segment, and the larva passes by almost insensible gradations into the adult form.

It will be seen that the development of Apus proves clearly that the antennæ and mandibles are ordinary trunk-appendages, homologous with the thoracic and abdominal feet: a comparison of the antennary and thoracic feet of the larva supports the view that the endopodite of the former corresponds with the fifth endite of the latter, and the exopodite with the sixth endite. The antennules are from the first unbranched or uniramous, and are originally situated quite at the anterior region of the body: they do not, therefore, show a complete correspondence with the remaining appendages, and, as was inferred from their nerve supply, may perhaps be considered as prostomial and not metameric appendages.

\section{b. The Fresh-water Crayfish (Astacus fluviatilis).}

Astacus fluviatilis is common in streams and rivers in England and the continent of Europe; allied species occur in Asia and North America; and fresh-water Crayfishes belonging to other

1 More strictly metanauplius: the typical nauplius exhibits no segmentation of the trunk region. 
genera, but agreeing with Astacus in all essential features, are found in America, Australia, and New Zealand.

External Characters. - The body of the Crayfish (Fig. 430, $\mathrm{A}$ and $B$ ) is divided into two regions-an anterior, the cephalothorax

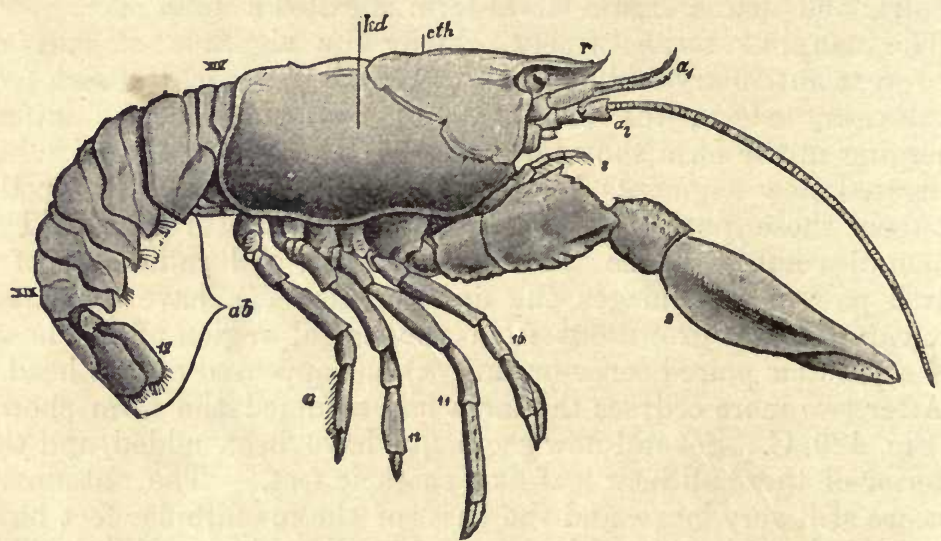

Fıg. 430A.-Astacus fluviatilus, side viow of male. $\iota^{1}$, antennule ; $\alpha^{2}$, antenna ; $a b$. abdomeu ; $c t h$. cephalothorax ; $k d$, gill-cover ; $r$. rostrum; 8 , third maxillipede; $\$$, first leg; $10-13$, remaintng legs ; 19, uropod; XIV, first abdominal segment ; XIX, sixth abdominal segment. (From Lang's Comparative Anatomy.)

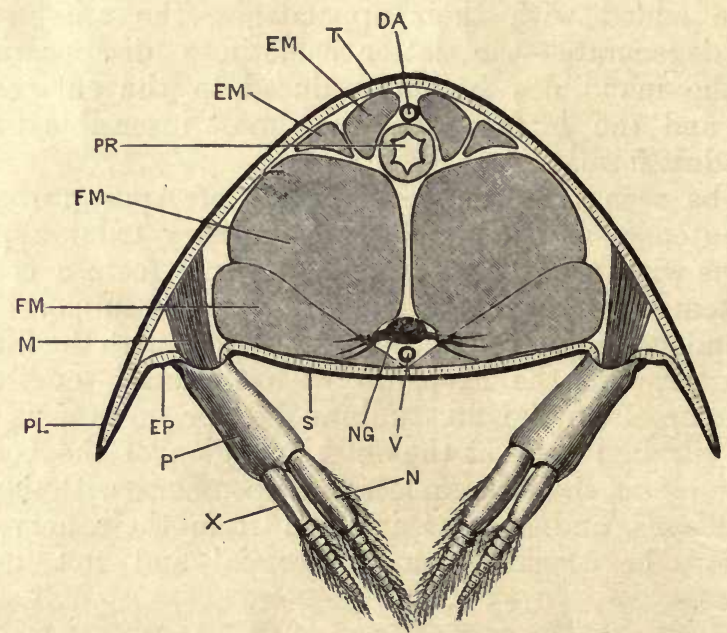

FIG. 430 B.-Transverse seetion of abdomen of Crayfish. DA, dorsal abdominal artery ; EM, dorsal museles of the abdomen; EP, space between the pleuron and the appendage; FM, ventral muscles of the abdomen; $M$, museles of the appendage ; $N$, eudopodite ; NG, nerveganglion; $\mathrm{P}$, protopodite ; PL, pleuron; $\mathrm{PR}$, hind-gut ; $\mathrm{S}$, sicruum : T, tergum ; V, ventral abdominal artery ; X, exopodite. (From Parker's Proctical Zoology, after Marshall and Hurst.

(cth.), which is unjointed, and is covered by a carapace resembling that of Apus, but of smaller proportional size; and a posterior, the abdomen $(a b)$, which is divided into distinct segments, movable upon 
one another in a vertical plane. The cephalothorax is again divided into two regions - an anterior, the head; and a posterior, the thorax - by a transverse depression, the cervical groove. The divisions of the body are thus the same as in Apus, but the abdomen alone is movably segmented, owing to the fact that the carapace, instead of being a purely cephalic structure continued backwards as a loose fold over the thorax, is developed from the dorsal and lateral regions of both head and thorax, and is free only at the sides of the thorax, where it forms a flap or gill-cover $(k d)$ on each side, separated from the actual body-wall by a narrow space in which the gills are contained (Fig. 436). The carapace is made of chitin, strongly impregnated with carbonate of lime so as to be hard and but slightly elastic.

The abdomen is made up of six segments and a tail-piece or telson: the six segments (XIV-XIX) have a ring-like form, presenting a broad dorsal region or tergum, a narrow ventral region or sternum, and downwardly directed lateral processes, the pleura - the latter quite unrepresented in Apus. The telson is flattened horizontally, and divided by a transverse groove into anterior and posterior portions. All the segments and the telson are calcified, and are united to one another by chitinous articular membranes: the first segment is similarly joined to the thorax. Thus the exoskeleton of Astacus resembles that of Apus in being a continuous cuticular structure, but differs from it in being discontinuously calcified, so as to have the character of a hard jointed armour.

It has been stated that the abdominal segments are movable upon one another in a vertical plane-i.e. the whole abdomen can be extended or straightened, and flexed or bent under the cephalothorax: the segments are incapable of movement from side to side. This is due to the fact that, while adjacent segments are connected dorsally and ventrally by flexible articular membranes, they present at each side a hinge (Fig. 434, h), placed at the junction of the tergum and pleuron, and formed by a little peglike process of one segment fitting into a depression or socket in the other. A line drawn between the right and left hinges constitutes the axis of articulation, and the only possible movement is in a plane at right angles to this axis.

Owing to the presence of the carapace, the thoracic region is immovable, and shows no distinction into segments either on its dorsal (tergal) or lateral (pleural) aspect. But on the ventral surface the sterna of the thoracic segments are clearly marked off by transverse grooves, and the hindmost of them is slightly movable. Altogether eight thoracic segments can be counted.

The ventral and lateral regions of the thoracic exoskeleton are produced into the interior of the body in the form of a segmental series of calcified plates, so arranged as to form a row of lateral chambers in which the muscles of the limbs lie, and a median 
tunnel-like passage or siernal canal, containing the thoracic portion of the nervous system. 'The entire endophragmal system, as it is called, constitutes a kind of internal skeleton: its anterior end is formed by a plate, the cephalic upodeme, having the same anatomical relations as the similarly named structure in Apus.

The head exhibits no segmentation: its sternal region is formed largely by a shield-shaped plate, the epistoma, nearly vertical in position. The ventral surface of the head is, in fact, bent so as to face forwards instead of downwards. The epistoma is bounded laterally by the free edge of the carapace instead of passing insensibly into it like the sub-frontal area of Apus, with which, however, it agrees in having the labmum attached to the middle of its posterior border. The cephalic region of the earapace is produced in front into a large median spine, the rostrum (Fig. $430, r$ ): immediately below it is a plate from which spring two movably articulated cylindrical bodies, the eye-stalks, bearing the eyes at their ends.

The appendages are seen at a glance to differ from those of Apus in their vastly greater degree of differentiation: obvious at a glance are the long feelers (Fig. $430, \alpha .1, \alpha$. 2) attached to the head, the five pairs of legs $\left(9-13^{3}\right)$ springing from the thorax, and the little fin-like bodies arising from the sterna of the abdomen. It will be convenient to begin with the last-named region.

The third, fourth, and fifth segments of the abdomen bear each a pair of small appendages, the swimming feet or pleopords (Fig. 431, 10), the resemblance of which to the biramous limbs of the larval Apus is obvious. There is an axis or protopodite consisting of a very short proximal $(p r .1)$ and a long distal $(p r .2)$ podomere, and bearing at its free end two jointed plates, fringed with setæ, the endopodite $(e n)$ and exopodite $(e x)$. These appendages act as fins, moving backwards and forwards with a regular swing, and probably aiding in the animal's forward movements.

In the female a similar appendage is borne on the second segment, while that of the first is more or less vestigial. In the male the first and second pleopods (9) are modified into incomplete tubes which act as copulatory organs, serving to transfer the spermatophores to the body of the female. The sixth pair of abdominal limbs (11) are alike in the two sexes: they are very large, both endopodite and exopodite having the form of broad tlat plates: in the natural position of the parts they lie one on each side of the telson, forming with it a large five-lobed tail-fin capable of being spread out after the manner of a fan; they are therefore conveniently called uropods or tail-feet. The telson itself bears no appendages.

The thoracic appendages are very different. The four posterior segments bear long slender, jointed legs $(S)$, upon which the animal 
walks : in front of these is a pair of very large legs terminating in huge claws or chelee, and hence called chelipeds (Fig. 430,9). The three anterior segments bear much smaller appendages more or less leg-like in form, but having their bases toothed to serve as jaws: they are distinguished as maxillipeds or foot-jaws (Fig. 431, 5-7).

The structure of these appendages is best understood by a consideration of the third maxilliped ( $y)$. The main portion of the
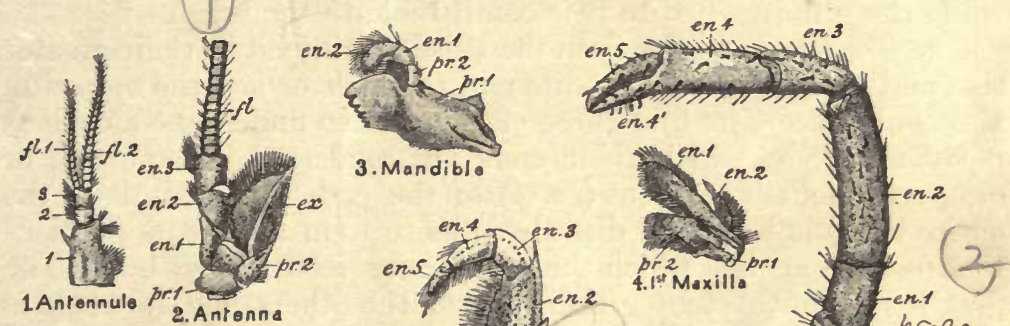

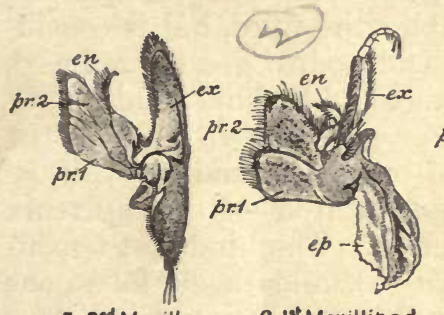

5. 2nd Maxilla

6. Is Maxilliped

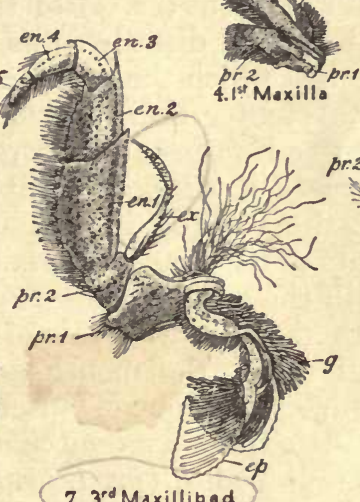

7. 3 ?d Maxilliped

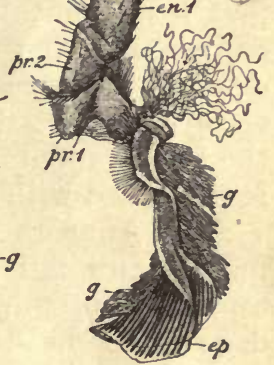

8. $30^{d} \mathrm{~L} \in 8$

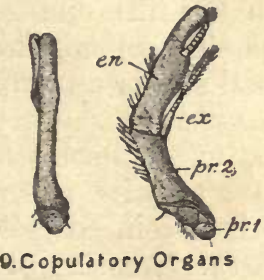

9. Copulatory Organs

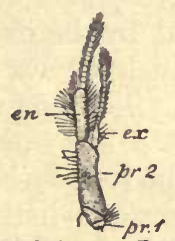

10.Swimming Foor

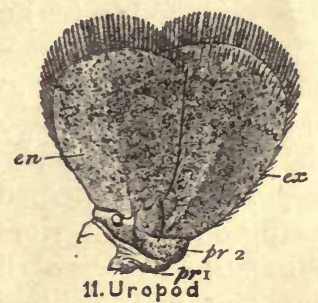

Fit. 431.-Typical appendages of Astacus. en. 1-5, podomeres of endopodite ; ep. epipodite ; ex. exopodite; fl. flagella ; $g$. gill ; $p r .1, p r .2$, podomeres of protopodite ; $1-3$, podomeres of axis of antennule. (After Huxley.)

limb is formed of seven podomeres arranged in a single series, strongly calcified, and-with the exception of the second and third, which are fused-movably articulated with one another. The second podomere, counting from the proximal end, bears a many-jointed feeler-like organ $(e x)$, and from the first springs a thin folded plate $(e p)$ having a plume-like gill $(g)$ attached to it. Obviously such an appendage is biramous, but with one of its branches 
greatly in excess of the other: the first two segments of the axis (pr. 1, $p$. 2) form the protopodite, its remaining five segments (en. 1-5) the endopodite, and the feeler, which is directed outwards, or away from the median plane, the exopodite $(e x)$. The folded plate $(e p)$ is called the epipodite: in the natural position of the parts it is directed upwards, and lies in the gill-cavity between the proper wall of the thorax and the gill-cover (Fig. 438). Its position is thus very similar to that of the flabellum of Apus, while the gill attached to it is comparable to the bract.

The five legs $(8)$ differ from the third maxilliped in their greater size, and in having no exopodite : in the fifth or last the epipodite also is absent. The first three of them have undergone a curious modification, by which their ends are converted into pincers or chela : the fourth segment (en. 4) of the endopodite (sixth of the entire limb) is produced distally so as to form a claw-like projection $\left(e n .4^{1}\right)$, against which the terminal segment (en. 5) bites. The first leg is much stouter than any of the others, and its chela is of immense size and forms an important weapon of offence and defence. The second maxilliped resembles the third, but is considerably smaller: the first (6) has its endopodite greatly reduced, the two segments of its protopodite large and leaf-like, and no gill is connected with the epipodite.

As in Apus, the head bears a pair of mandibles and two pairs of maxillæ in relation with the mouth, and in front of that aperture a pair of antennules and one of antennæ. The hindmost appendage of the head is the second maxilla (5), a markedly foliaceous appendage: its protopodite $(p r .1, p r .2)$ is cut up into lobes comparable with the four proximal endites in the thoracic feet of Apus : its endopodite (en) corresponds with the fifth endite, while the sixth endite is represented by the exopodite $(e x)$, modified into a boomerang-shaped plate, which, as we shall see, is an important accessory organ of respiration. The first maxilla (4) is a very small organ, having neither exopodite nor epipodite. The mandible (3) is a large strongly calcified body, toothed along its inner edge, and bearing on its anterior border a little three-jointed feeler-like body, the palp, the two distal segments (en. 1, en. 2) of which represents the endopodite, its proximal segment ( $p v^{\circ}$. 2) together with the mandible proper $(p r .1)$, the protopodite.

The antenna (2) is of great size, being nearly as long as the whole body. It consists of an axis of five podomeres, the fifth or last of which bears a long, flexible, many-jointed structure, or flagellum $(f)$, while from the second segment springs a scale-like body or squame (ex). It is fairly obvious that the two proximal segments represent the protopodite, the remaining three, with the flagellum, the endopodite, and the squame the exopodite.

The antennule (1) has an axis of three podomeres $(1-3)$ ending in two many-jointed flagella $(f .1$. and 2$)$, which are sometimes 
considered as endopodite and exopodite. But in all the other limbs, as we have seen, the exopodite springs from the second segment of the axis, and the probabilities are that there is no exact correspondence between the parts of the antennule and those of the remaining appendages.

The eye-stalks, already noticed, arise just above the antennules and are formed each of a small proximal and a large distal segment. They are sometimes counted as appendages serially homologous with the antennæ, legs, \&c. But, as we have seen in the case of Apus, the appendages of Crustacea are always formed in regular order from before backwards; the eye-stalks, on the other hand, always appear later, both in individual development and in the Crustacean series, than the normal anterior appendages. They are therefore more properly to be looked upon as articulated processes of the prostomium, developed in connection with the need for an increased range of vision. Assuming this to be the case, it will be seen that the body of the Crayfish consists of a prostomium, nineteen metameres, and a telson. The prostomium bears eye-stalks: the first five metameres are fused with the prostomium to form the head, and bear the antennules, antennæ, mandibles, first maxillæ, and second maxillæ: the next eight metameres (5th-12th) constitute the thorax, and bear the three pairs of maxillipeds and the five pairs of legs : the remaining six metameres (13th-18th), together with the telson, constitute the abdomen, and bears five pairs of pleopods and one of uropods.

The articulation of the various podomeres of the appendages is on the same plan as that of the abdominal segments (p. 541). The podomeres are, it must be remembered, rigid tubes: they are connected with one another by flexible articular membranes 
(Fig. 432, art. m.), but at two points the adjacent ends of the tubes come into contact with one another and are articulated by peg-and-socket joints $(h$.$) , the two joints being at opposite ends of$ a diameter which forms the axis of articulation. 'The two podomeres can, therefore, be moved upon one another in a plane at right angles to the axis of articulation and in no other direction, the joints being pure hinge-joints. As a rule, the range of movement is from the perpendicular to a tolerably extensive flexion on one side-the articulations are single-jointed, like our own elbows and knees. The whole limb is, however, capable of universal movement, owing to the fact that the axes of articulation vary in direction in successive joints : the first joint of a limb bending, for instance, up and down, the next backwards and forwards, the next obliquely, and so on. In some cases, e.g. in the pleopods, peg-and-socket joints are absent, the articulation being formed merely by an annular articular membrane and movement being therefore possible in any plane.

Body-wall. - The exoskeleton is produced into spines of varying form and size, and many parts of it bear tufts or fringes of

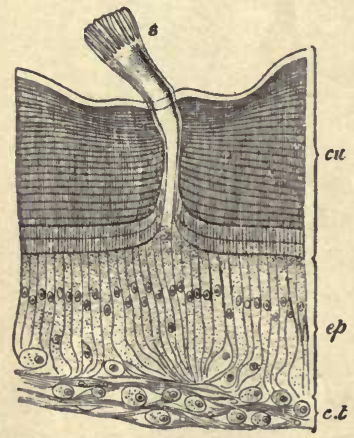

FrG. 433. - Vertical section of skin and exoskeleton of Iobster. c.t. connective-tissue; $c u$. cuticle; ep. epidermis; 8. seta. (After Gerstaecker.) setæ, which also exhibit a wide variation in size and form. It is composed of a thick laminated chitinous membrane (Fig. 433, cu.), more or less impregnated with lime-salts, and is shed periodically - once a year during adult life. Beneath it is the epidermis (ep.) composed of a single layer of cells from which the chitin is secreted, and underlaid by a layer of connective-tissue (c.t.) to which the muscles are attached.

The muscular system, like the exoskeleton, shows a great advance in complexity over that of Apus. In the abdomen (Fig. 434) the muscles are of great size, and are divisible into a smaller dorsal and a larger ventral set.

The dorsal muscles $(d . m$.) are paired longitudinal bands, divided into myomeres, and inserted by connective-tissue into the anterior border of each segment: anteriorly they are traceable into the thorax, where they arise from the side-walls of that region. When these muscles contract, they draw the anterior edge of each tergum under the posterior edge of its predecessor, and thus extend or straighten the abdomen.

The ventral muscles are extraordinarily complex. Omitting details, there is on each side a wavy longitudinal band of muscle $(c . m$. $)$, nearly circular in section, which sends off a slip $(e x$.) to be inserted into each segment above the hinge $(h$.$) : the contraction of this$ 
muscle must obviously tend to approximate the terga, and so aid the dorsal muscles in extending the abdomen. Around this central muscle is wrapped, in each segment, a band of muscle (env. m.) in the form of a loop, the outer limb of which turns forwards and is inserted into a sternım, while the inner limb turns backwards and is inserted into another and more posterior sternum. The contraction of this enveloping muscle produces an approximation of the sterna, and thus flexes the abdomen, the central muscle always
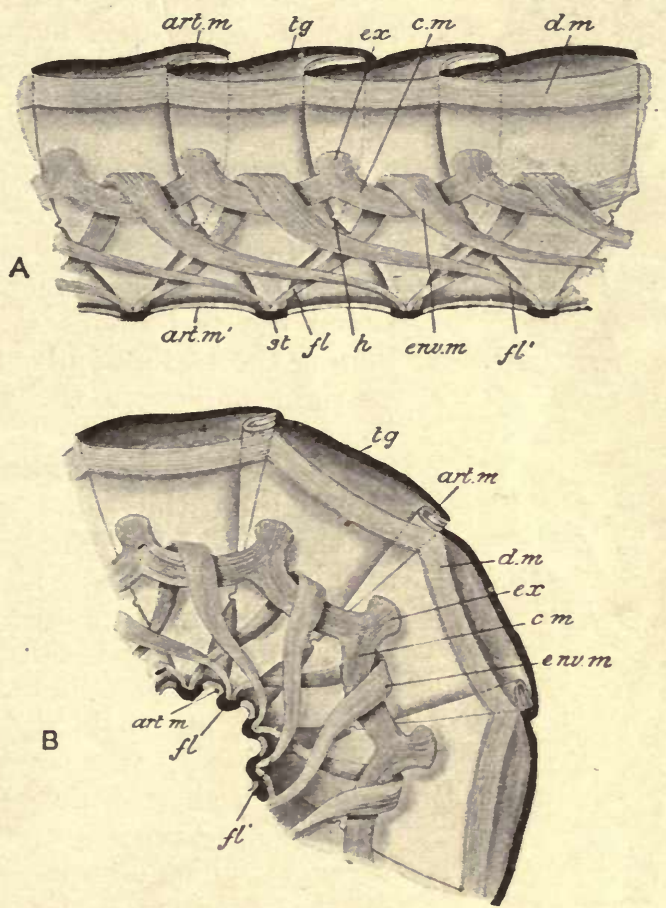

Fig. 434.-Fourisegments of abdomen of Crayfish in sagittal section, with muscles (diagrammatic). A, extension; B, flexion; art. m., avt. $m^{\prime}$. , articular membranes; $c$. m. central muscles; d. $m$. dorsal muscle; $e x$. extensor slip of central muscle; $e n v$. m. enveloping muscle; $\lambda_{\text {., }} f . .^{1}$, flexor slips; $h$. hinge; st. sternum ; $t g$. tergum.

keeping the middle of the loop in place. The ventral muscles are, like the dorsal, traceable into the thorax, where they arise from the endophragmal system (p. 542): their various parts are connected by a complex system of fibres extending between the central and enveloping muscles, and connecting both with their fellows of the opposite side. The flexor muscles are immensely powerful, and produce, when acting together, a sudden and violent bending of the abdomen upon the cephalothorax, causing the Crayfish to dart backwards with great rapidity. 


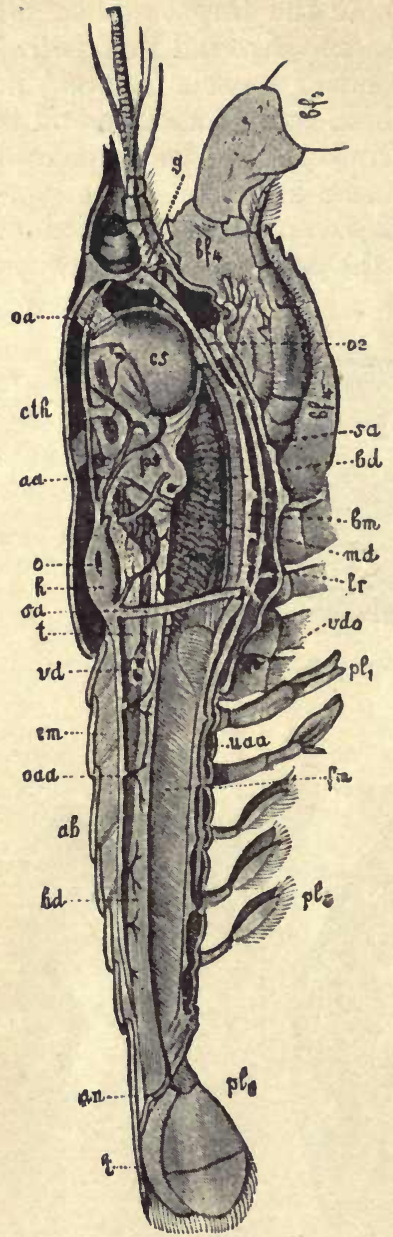

FIG. 435.-Astacus fluviatilis, dissection from the right side. $a a$. antennary artery; $a b$. abdomen; $a n$. anus; $b$. $d$. aperture of duet of right digestive gland; bf. 4 , cheliped; bm, ventral nerve. cord ; cs. anterior division of gizzard; cth. cephalothorax; em, dorsal muscles; fin, ventral muscles; $g$, brain; $h$. heart; $h d$, posterior part of intestine; $l v$. left digestive gland; $m d$, mid-gut ; $o$ ostium of heart; on. right lateral artery ; oan, dorsal abdominal artery; $\alpha$, gullet; $p l .1-5$, pleopods; $p l$. 6, uropod; p8, posterior division of gizzard; sa. sternal artery; $t$. testis and telson; uaa, ventral abdominal artery; $v \imath l$. vas deferens; vdlo, male genital aperture. (From Lang, after Huxley.)
It will be seen that the bodymuscles of the Crayfish cannot be said to form a layer of the body-wall, as in Chrtopods, the abdomen of Apus, \&c., but constitute an immense fleshy mass, filling up the greater part of the body-cavity, and leaving a very small space around the enteric canal.

In the limbs (Fig. 432) each podo. mere is acted upon by two muscles situated in the next proximal podomere. These muscles are inserted, by chitinous and often calcified tendons, into the proximal edge of the segment to be moved, the smaller on the extensor (ext.), the larger on the flexor $(f$.$) side, in each case half-way be-$ tween the two hinges, so that a line joining the two muscular insertions is at right angles to the axis of articulation.

The digestive organs are constructed on the same general plan as those of Apus, but present many striking differences (Fig. 435). The mouth lies in the middle ventral line of the head, and is bounded in front by the labrum, at the sides by the mandibles, and behind by a pair of delicate lobes, the paragnatha. It leads by a short wide gullet (oc) into a capacious gizzard (sometimes termed stomach), which occupies a great part of the interior of the head, and is divided into a large anterior division (c.s), and a small posterior division $(p s)$ : the latter passes into a narrow and very short portion of the intestine, the mid-gut ( $m d$ ), from which the rest of the intestine (hind-gut, $h d$ ) extends to the anus (an), situated on the ventral surface of the telson.

The outer layer of the enteric canal consists of connective-tissue containing striped muscular fibres: within this is a single layer of columnar epithelial cells. In the gullet and gizzard, 
and in the hind-gut, the epithelium secretes a layer of chitin, which thus constitutes the innermost lining of those cavities. It is proved by development that the mid-gut, which has no chitinous lining, is the only part of the enteric canal developed from the mesenteron: the gullet and gizzard arise from the stomodrum, the hind-gut from the proctodæum. 'Thus a very small portion of the enteric epithelium is endodermal.

In the anterior division of the gizzard the chitinous lining is thickened and calcified in certain parts, so as to form a complex articulated framework, the "gastric mill," on which are borne a median and two lateral teeth, strongly calcified and projecting into the cavity of the gizzard. Two pairs of strong muscles arise from the carapace, and are inserted into the gizzard: when they contract they move the mill in such a way that the three teeth meet in the middle and complete the comminution of the food begun by the jaws. The separation of the teeth is effected partly by the elasticity of the mill, partly by delicate muscles in the walls of the gizzard. The posterior division of the gizzard forms a strainer: its walls are thickened and produced into numerous setie, which extend quite across the narrow lumen and prevent the passage of any but finely divided particles into the intestine. Thus the gizzard has no digestive function, but is merely a masticating and straining apparatus. On each side of the anterior division is found at certain seasons of the year a plano-convex mass of calcareous matter, the gastrolith.

The digestion of the food and to some extent the absorption of the digested products are performed by a pair of large glands $(l r$.$) ,$ lying one on each side of the gizzard and anterior end of the intestine. They are formed of finger-like sacs or cсесa, which discharge into wide ducts opening into the mid-gut, and are lined with glandular epithelium derived from the endoderm of the embryo. The glands are often called livers, but as the yellow fluid they secrete digests proteids as well as fat, the name hepato-pancreas is often applied to them, or they may be called simply digestive glands. The Crayfish is carnivorous, its food consisting largely of decaying animal matter. Microscopic glands occur in the wall of the gullet.

The digestive organs and other viscera are surrounded by a body-cavity, which is in free communication with the bloodvessels and itself contains blood. As will be pointed out more particularly hereafter, this cavity is to be looked upon as an immense blood-sinus, and not as a true coelome.

There are well-developed respiratory organs, in the form of gills, contained in a narrow branchial chamber, bounded internally by the proper wall of the thorax (Fig. $438, c p$ ), externally by the gill-cover or pleural region of the carapace $(k l)$. Each gill consists of a stem giving off numerous branchial filaments, so that 
the whole organ is plume-like. The filaments are hollow, and communicate with two parallel canals in the stem-an external, the afferent branchial vein, and an internal, the efferent branchial vein. The gill is to be considered as an out-pushing of the body-wall, and contains the same layers-a thin layer of chitin externally, then a single layer of epithelial cells, and
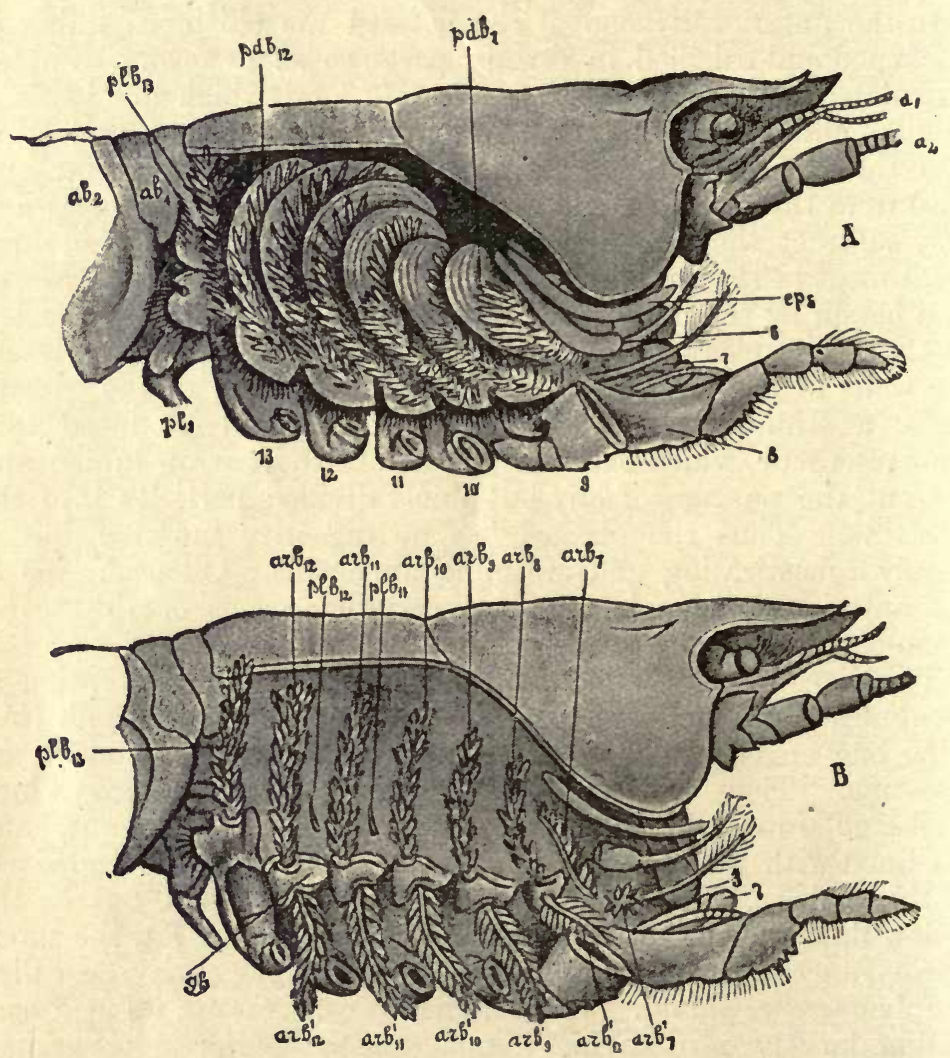

F1G. 436.--Respiratory organs of Astacus fluviatilis. In A the gill-cover is removed and the gills undisturbed; in $\mathbf{B}$ the podobranchiæe are removed and the outer arthrobranchiat turned down. $a_{1}$, antennule; $a_{2}$, antenna; $a b_{1}$, first; $a b_{2}$, second abdominal segment ; arb. 7-12, iuner arthrobranchia; $a r b^{\prime} .7-12$, outer arthobranchix ; ep. 5 , scaphognathite; plb. $11-13$, pleurobranchiæ; $p d b$. $7-13$, podobranchs ; $p l .1$, first pleopod ; $6-13$, thoracic appendages. (From Lang's Comparative Anatomy, after Huxley.)

beneath this connective-tissue, hollowed out for the blood channels and containing gland-cells, which will be referred to presently (p. 551).

According to their point of origin, the gills are divisible into three sets-first, podobranchice or foot-gills, springing from the epipodites of the thoracic appendages, from which they are only 
partially separable; secondly, arthrobranchia or joint-gills, springing from the articular membranes connecting the thoracic appendages with the trunk; and thirdly, pleurobranchice, or wallgills, springing from the lateral walls of the thorax, above the attachment of the appendages. It is inferred from the study of other Crayfishes, that a typical thoracic segment bears four gills, one podo-, two arthro-, and one pleurobranchia. But in Astacus one or more of the gills in every segment are absent or vestigial, and the following table, or "branchial formula," shows the actual number and arrangement of these organs, ep standing for epipodite, and $r$ for the vestige of a gill.

\begin{tabular}{|c|c|c|c|c|c|c|c|c||c|}
$\begin{array}{c}\text { Tnokacic } \\
\text { SEgments. }\end{array}$ & I. & II. & III. & IV. & V. & VI. & VII. & VIII. & TotaL. \\
\hline Podobranchiæ... & $0+e p$ & $1+e p$ & $1+c p$ & $1+e p$ & $1+e p$ & $1+e p$ & $1+e p$ & 0 & $6+7 e p$ \\
\hline Arthrobranchix & 0 & 1 & 2 & 2 & 2 & 2 & 2 & 0 & 11 \\
Pleurobranchiæ & 0 & 0 & 0 & 0 & 0 & $r$ & $r$ & 1 & $1+2 r$ \\
\hline Total ... & $0+e p$ & $2+c p$ & $3+e p$ & $3+e p$ & $3+e p$ & $3+r+e p$ & $3+r+e p$ & 1 & $18+2 r+7 e p$ \\
\hline
\end{tabular}

By adding up the columns vertically we get the number of gills in each segment; by adding them horizontally, the number of each kind of gill; and by adding together the results obtained by both methods, the total number of gills, viz., eighteen complete gills with two vestiges and seven epipodites.

The excretory organs differ both in position and in form from those of Apus. There are no shell-glands, but at the base of each antenna is an organ of a greenish colour, the antennary or grecn gland, by which the function of renal excretion is performed. The gland (Fig. 437) is cushion-shaped, and consists of three parts-(1) a central saccule (s.) of a yellowish colour, occupying the mid-dorsal region, and consisting of a sac divided into numerous compartments by partitions, and communicating with (2) the outer or cortical portion (c.p.), of a green colour, consisting of a glandular network formed of anastomosing canals, and communicating in its turn with (3) a white portion (w. p.), formed of a single tube partly converted into a sponge-work by ingrowths of its walls. The whole organ is lined by glandular epithelium, and the white portion discharges into a thin-walled sac or urinary bladder (bl.) which opens by a duct (d.) on the proximal segment of the antenna. The glands already referred to as occurring in the gills are also supposed to have an excretory function.

The circulatory organs are in a high state of development. The heart (Figs. $435,438, h$.) is situated in the dorsal region of the 
thorax, and is a roughly polygonal muscular organ pierced by three pairs of apertures or ostia (o.), guarded by valves which open inwards. It is enclosed in a spacious pericaidial sinus (Fig. 438, pc.), which contains blood. From the heart spring a number of narrow tubes, called arteries, which serve to convey the blood to various parts of the body. At the origin of each artery from the heart are valves which allow of the flow of blood in one direction.

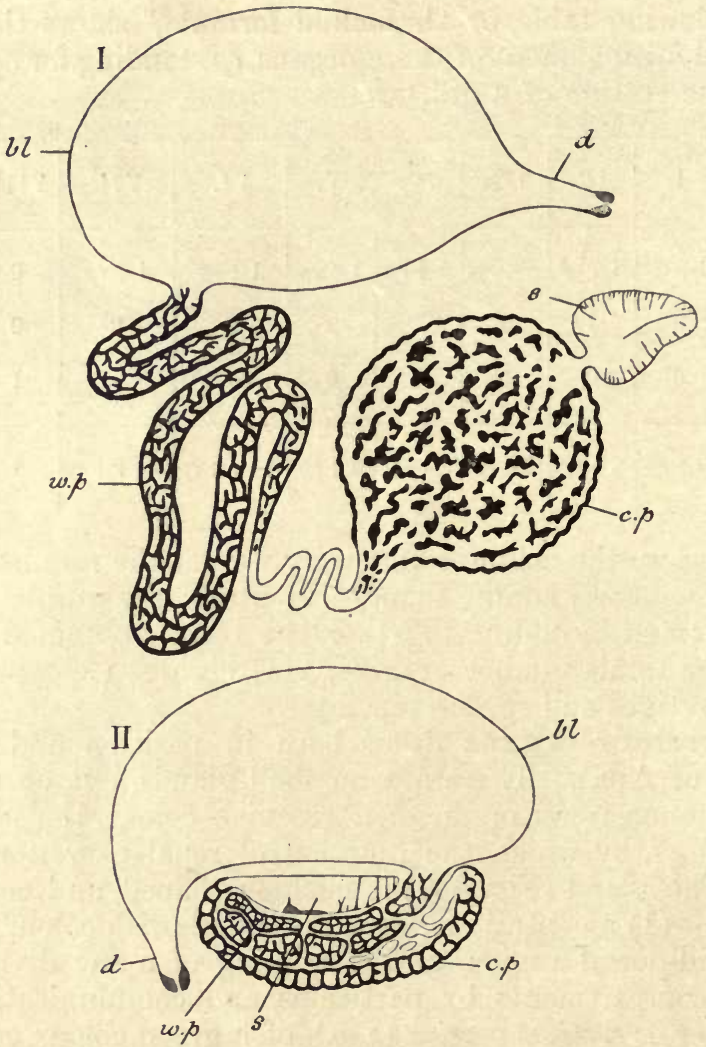

FiG. 437.-Diagram of kidney of Astacus fluviatilis. I, unravelled; II, the parts in their natural relations. $b l$. bladder; $c . p$. cortical portion; $d$. duct; s. saccule ; $w . p$. white portion. (After Marchal.)

only, viz., from the heart to the artery. From the anterior end of the heart arise five vessels-the median ophthalmic artery (Fig. 435, oa.), which passes forwards to the eyes; paired antennary arteries (aa.), going to the antennules, antennæ, green glands, \&c., and sending off branches to the gizzard; and paired hepatic arteries, going to the digestive glands. The posterior end of the heart gives off two unpaired arteries practically united 
at their origin, the dorsal abdominal artery (oaa.), which passes backwards above the intestine, sending branches to it and to the dorsal muscles; and the large sternal artery (sa.), which extends directly downwards, indifferently to right or left of the intestine, passing between the connectives uniting the third and fourth thoracic ganglia, and then turns forwards and runs in the sternal canal, immediately beneath the nerve-cord, sending off branches to the legs, jaws, \&c. At the point where the sternal artery turns forwards it gives off the median ventral abdominal artery (uaa.), which passes backwards beneath the nervecord, and supplies the ventral muscles, pleopods, \&c.

All these arteries branch extensively in the various organs they supply, becoming divided into smaller and smaller offshoots, which finally end in microscopic vessels called capillaries. These latter end by open mouths which communicate with the bloodsin?ses (Fig. 439, s.), spacious cavities lying among the muscles and viscera, and all communicating, mediately or immediately, with the sternal sinus (st.s.), a great median canal running longitudinally along the thorax and abdomen, and containing the ventral nerve-cord and the sternal and ventral abdominal arteries. In the thorax the sternal sinus sends an offshoot to each gill in the form of a well-defined vessel, which passes up the outer side of the gill and is called the afferent branchial vein (af.br:v.; see also Fig. 438). Spaces in the gill-filaments place the afferent in communication with the efferent branchial vein (ef.br.v.), which occupies the inner side of the gill-stem. The eighteen efferent branchial veins open into six branchiocardiac veins (br.c.v.), which pass dorsally in close contact with the lateral wall of the thorax and open into the pericardial sinus ( $p c d . s$.$) .$

The whole of this system of cavities is full of blood, and the heart is rhythmically contractile. When it contracts, the blood 
contained in it is prevented from entering the pericardial sinus by the closure of the valves of the ostia, and therefore takes the only other course open to it, viz., into the arteries. When the heart relaxes, the blood in the arteries is prevented from regurgitating by the valves at their origins, and the pressure of blood in the pericardial sinus forces open the valves of the ostia and so fills the heart. Thus in virtue of the successive contractions of the heart and of the disposition of the valves, the blood is kept constantly moving in one direction-viz., from the heart by the arteries to the various organs of the body, where it receives carbonic acid and other waste matters; thence by sinuses into the great sternal sinus; from the sternal sinus by afferent branchial veins to the gills, where it exchanges carbonic acid for oxygen; from the gills by efferent branchial veins to the branchiocardiac

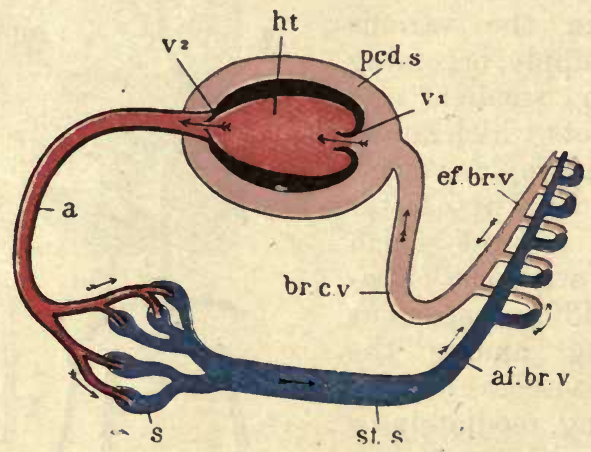

FrG. 439.-Diagram of the circulation in the Crayfish; heart and arteries scarlet, veins and sinuses containing non-aërated blood, blue; those containing aërated blood, vink. $a$. artery ; $a j: b r \cdot v$. afferent brunchial vein; $b r . c . v$. branchio-cardiac vein; ef.br.v. efferent branchial vein; ht. heart; pcd.s. pericardial sinus; s. sinus; st.s. sternal sinus; $v 1$. ostium with valves; $v^{2}$. arterial valves. The arrows sliow the direction of the current.

veins, thence into the pericardial sinus, and so to the heart once more.

It will be seen that the circulatory system of the Crayfish consists of three sections-(1) the heart or organ of propulsion; (2) a system of out-going channels, the arteries, which carry the blood from the heart to the body generally; and (3) a system of returning channels, some of them, the sinuses, mere irregular cavities; others, the veins, with definite walls, which return it from the various organs back to the heart. The respiratory organs, it should be observed, are interposed in the returning current, so that blood is taken both to and from the gills by veins.

Comparing the blood-vessels of Astacus with those of a Chætopod, it would seem that the ophthalmic artery, heart, and - dorsal abdominal artery together answer to a dorsal vessel, part of which has become enlarged and muscular and discharges the 
whole function of propelling the blood. The horizontal portion of the sternal artery, together with the ventral abdominal, represent a ventral vessel; while the vertical portion of the sternal artery is a commissure, developed sometimes on the right, sometimes on the left side, its fellow being suppressed.

The blood when first drawn is colourless, but after exposure to the air takes on a bluish-grey tint. This is owing to the presence of a colouring matter called hamocyanin, which becomes blue when combined with oxygen; it is a respiratory pigment, and serves, like hæmoglobin, as a carrier of oxygen from the external medium to the tissues. The hæmocyanin is contained in the plasma of the blood: the corpuscles are all colourless leucocytes.

The nervous system (Fig. 440) consists, like that of Apus, of a brain $(g)$ and a ventral nerve-cord, united by œsophageal connectives (sc). But the right and left halves of the ventral cord have undergone partial fusion, so that the ganglia, and in the abdomen the connectives also, appear single instead of double. Moreover, the brain supplies not only the eyes and antennules, but the antennæ as well, and it is found by development that the two pairs of ganglia belonging to the antennulary and antennary segments have fused with the brain proper. Hence we have to distinguish between a primary brain or archicerebrum, the ganglion of the prostomium, and a secondary brain or syn-cerebrum formed by the union of one or more pairs of ganglia of the ventral cord with the archi-cerebrum. A further case of concrescence of ganglia is seen in the ventral nerve-cord, where the ganglia of the last three cephalic and first three thoracic segments have united to form a large compound sub-cesophageal ganglion $(b g)$. All the

\section{B}

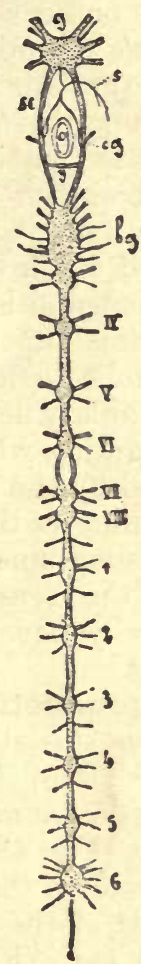

Fig. 440.-Nervous system of Astacus fluviatilis. bg. sub-oesophageal ganglion ; $c g$. commissural ganglion; $g$, brain ; $s$, visceral nerve; $s c$, œesophageal connective; $y$, postcesophageal commissure ; IV-VIII, thoracic gang: lia ; $1-6$, abdominal gang. lia. (From Lang's Com. parative Anatomy, after Vogt and Yung.) remaining segments have their own ganglia, with the exception of the telson, which is supplied from the ganglion of the preceding segment. There is a visceral system of nerves (s) supplying the gizzard, originating in part from the brain and in part from the œesophageal connectives. 
Sensory Organs. - The eycs have the same essential structure as the compound eye of Apus. The chitinous cuticle covering the distal end of the eye-stalk is transparent, is divided by delicate lines into square areas or facets, and constitutes the cornea. Beneath each facet of the cornea is an ommatidium, optically separated from its neighbours by black pigment, and consisting of an outer segment or vitreous body, and an inner segment or retinula, formed of sensory cells enclosing a rhabdome.

The antennules contain two sensory organs, to which are assigned the functions of smell and hearing respectively. The olfactory organ is constituted by a number of extremely delicate olfactory setce, borne on the external flagellum and supplied by branches of the antennulary nerve. The so-called auditory organ is a sac forined by invagination of the dorsal surface of the proximal segment, and is in free communication with the surrounding water by a small aperture. The chitinous lining of the sac is produced into delicate feathered auditory setce, supplied by branches of the antennulary nerve; and in the water which fills the sac are minute sand-grains, which take the place of otoliths, but, instead of being formed by the animal itself, are taken in after each ecdysis, when the lining of the sac is shed. Probably the main function of these organs is connected with the equilibration of the body rather than with the sense of hearing. Many of the setæ on the body generally have a definite nerve-supply, and are probably tactile organs.

Reproduction.-The Crayfish is diœeious, and presents a very obvious sexual dimorphism. The abdomen of the female is much broader than that of the male: the first and second pleopods of the male are modified into tubular or rather spout-like copulatory organs (Fig. 431, 9); and the reproductive aperture is situated in the male on the proximal podomere of the fifth leg, in the female on that of the third.

The testis (Fig. 441, B, $t, u$ ) lies in the thorax, just beneath the floor of the pericardial sinus, and consists of paired anterior lobes $(t)$ and an upaired posterior lobe $(u)$. From each side goes off a convoluted vas deferens $(v d)$, which opens on the proximal segment of the last leg. The sperms are curious amœboid bodies produced into a number of stiff processes or pseudopodia (Fig. 23, $f$ ): they are aggregated into vermicelli-like spermatophores by a secretion of the vas deferens.

The ovary $(\mathrm{A}, o v, u)$ is also a three-lobed body, and is similarly situated to the testis: from each side proceeds a thin-walled oviduct $(o d)$, which passes downwards, without convolutions, to open on the proximal segment of the third or antepenultimate leg. The eggs are of considerable size and are centrolecithal.

As in Apus, both ovary and testis are hollow organs, discharging their products internally. The ova, when laid, are fastened to the 
setæe on the pleopods of the female by the sticky secretion of glands occurring both on those appendages and on the segments themselves: they are fertilised immediately after laying, the male
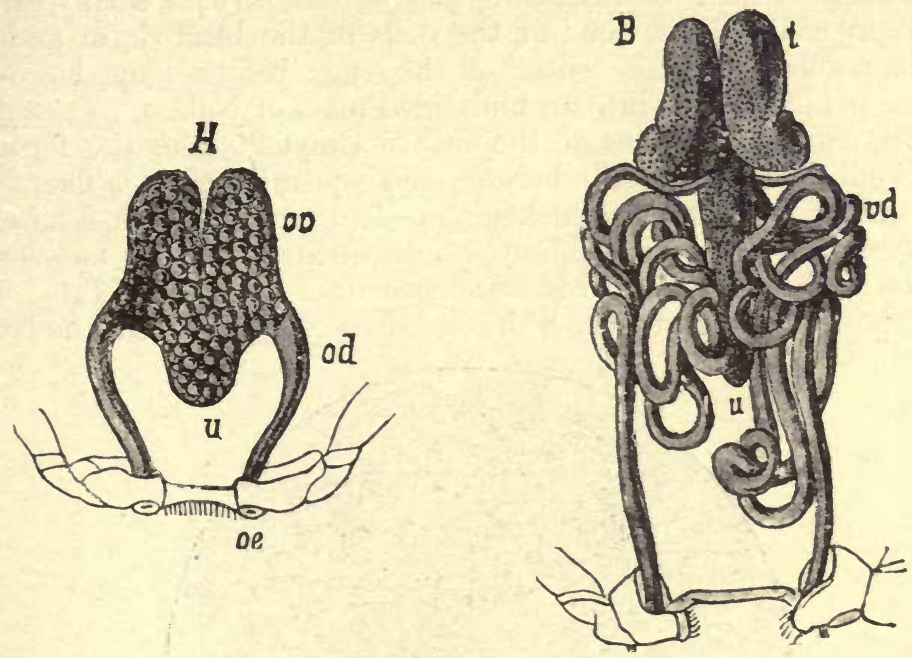

Fig. 441. -Reproductive organs of Astacus fluviatilis. A, female; B, male. od. oviduct; $o$, its external opening ; ov. ovary; $t$. testis ; $u$, unpaired posterior portion of gonad ; vd. vas deferens. (From Lang's Compurative Anatomy, after Huxley.)

depositing spermatophores on the ventral surface of the female's body just before oviposition.

Development. - The process of segmentation of the oosperm presents certain striking peculiarities. The nucleus (Fig. 442, A, nu) divides repeatedly, but no corresponding division of the protoplasm takes place, with the result that the morula-stage, instead of being a heap of cells, is multinucleate but non-cellular. Soon the nuclei
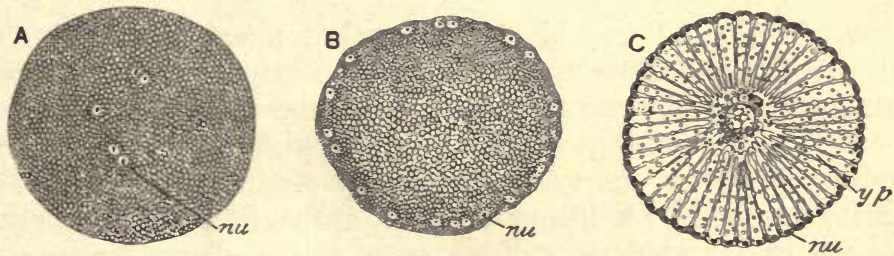

FiG. 442.-Three stages in the formation of the blastodern of Astacus fluviatilis. nu. nuclei ; yp. yolk-pyramids. (From Korschelt and Heider, after Morin and Reichenbach.)

thus formed retreat from the centre of the embryo, and arrange themselves in a single layer close to the surface $(\mathbf{B})$ : around each of these protoplasm accumulates, the central part of the embryo consisting entirely of yolk-material. We thus get a superficial 
segmentation, characterised by a central mass of yolk and a superficial layer of cells collectively known as the blastoderm (C). Subsequently the yolk itself undergoes a process of segmentation, becoming divided into radiating yolk pyramids (y.p.), each with its base in contact with one of the cells of the blastoderm and its apex pointing to the centre of the egg: before long, however, these pyramids fuse into an undivided mass of yolk.

The first indications of the future Crayfish take the form of thickenings on what will become the ventral surface. There are at first five of these thickenings-two anterior, the head-lobes (Fig. $443, K$ ), on which the eyes subsequently appear; two somewhat further back, the thoracico-abdominal rudiments $(T A)$; and one, posterior and unpaired, the endoderm-disc (ES). On the latter

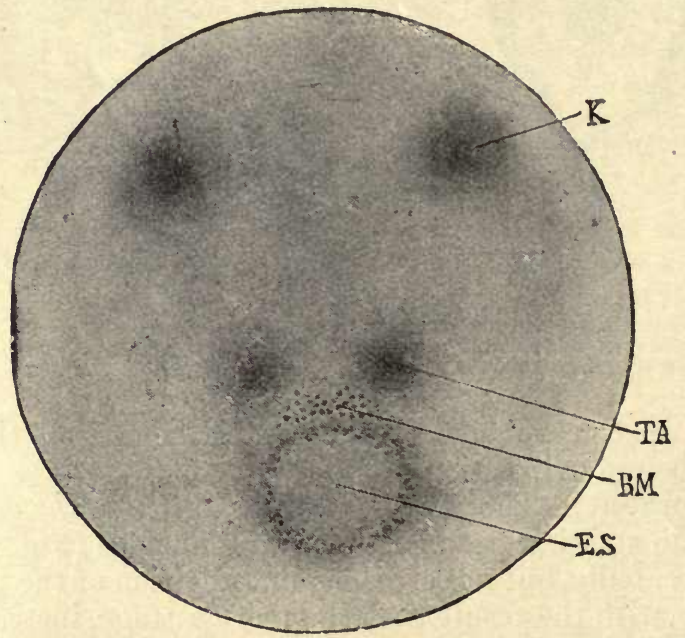

FIG. 443.-Early embryo of Astacus. $B M$, mesoderm ; $E S$, endoderm disc ; $K^{\prime}$, head-lubes ; $T A$, thoracico-abdominal rudiments. (From Lang's Comparative Anatomy, after Reichenbach.)

an invagination of the blastoderm takes place, giving rise to a small sac, the archenteron, which communicates with the exterior by an aperture, the blastopore. By this process the embryo passes into the gastrula-stage, which, however, differs from the corresponding stage in the types previously studied in the immense quantity of food-yolk filling up the space (blastocœle) between ectoderm and endoderm. Very soon the embryo become triploblastic, or three-layered, by the budding off of cells from the endoderm in the neighbourhood of the blastopore : these accumulate between the ectoderm and endoderm, and constitute the mesoderm.

Before long the blastopore closes, converting the archenteron into a shut sac (Fig. $445, A)$ : the thoracico-abdominal rudiments unite with one another, forming a well-marked oval elevation 
(Fig. 444, TA), and three pairs of elevations appear between it and the head-lobes. These are the rudiments of the first three pairs of appendages, the antennules $\left(a_{1}\right.$.), antennæ $\left(a_{2}\right)$, and mandibles $(m$.$) : by their appearance the embryo passes into the$ nauplius-stage, which in this case is passed through in the egg, instead of being active and free-swimming as in Apus.

Between the bases of the antennules and antenna a pit appears, which soon deepens and widens: it is the stomodceum (Fig. 455, stdm.), and its aperture the mouth. A similar but narrower and more cylindrical pit appears on the thoracico-abdominal rudiment : it is the proctodaum $(\operatorname{pcdm}$.$) , and its aperture the anus. For a$

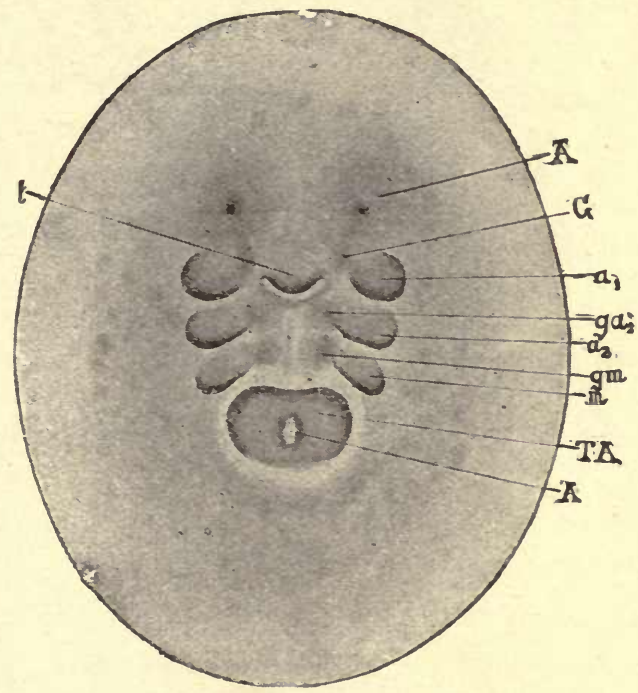

FIG. 444.-Nauplius-stage of Astacus. $A$, (above) eye; $A$, (below) anus; $a_{1}$. antennule ; a. antenna; $G$, cerebral ganglion; gu, antennary ganglion; gm, mandibular ganglion ; $l$. labrum ; $m$. mandible; $T d$, thoracico-abdominal rudiment. (From Lang's Comparative Anatomy, after Reichenbach.)

considerable time both stomodxum and proctodæum remain in the condition of blind sacs, but after a time they open into the archenteron, a complete enteric canal being thus constituted. In the meantime the endoderm cells lining the archenteron grow outwards in a radial direction, ingesting the yolk as they do so, until they take the form of long columns, in contact by their outer ends with the ectoderm (Fig. 446, B).

The thoracico-abdominal rudiment soon begins to increase rapidly in length, but, being enclosed in the egg-membranes, it grows not backwards but forwards, being in fact folded upon the anterior part of the body in much the same way as the abdomen of the adult during extreme flexion. Thus in Fig. 447 the ventral surface of the head and anterior thoracic region faces the observer, 
but the dorsal surface of the posterior thoracic and abdominal regions : in order to bring the parts into their adult position, the abdomen must be supposed to be lifted up and turned backwards.

In the meantime the post-mandibular appendages are formed in regular order from before backwards: the eye-stalks appear
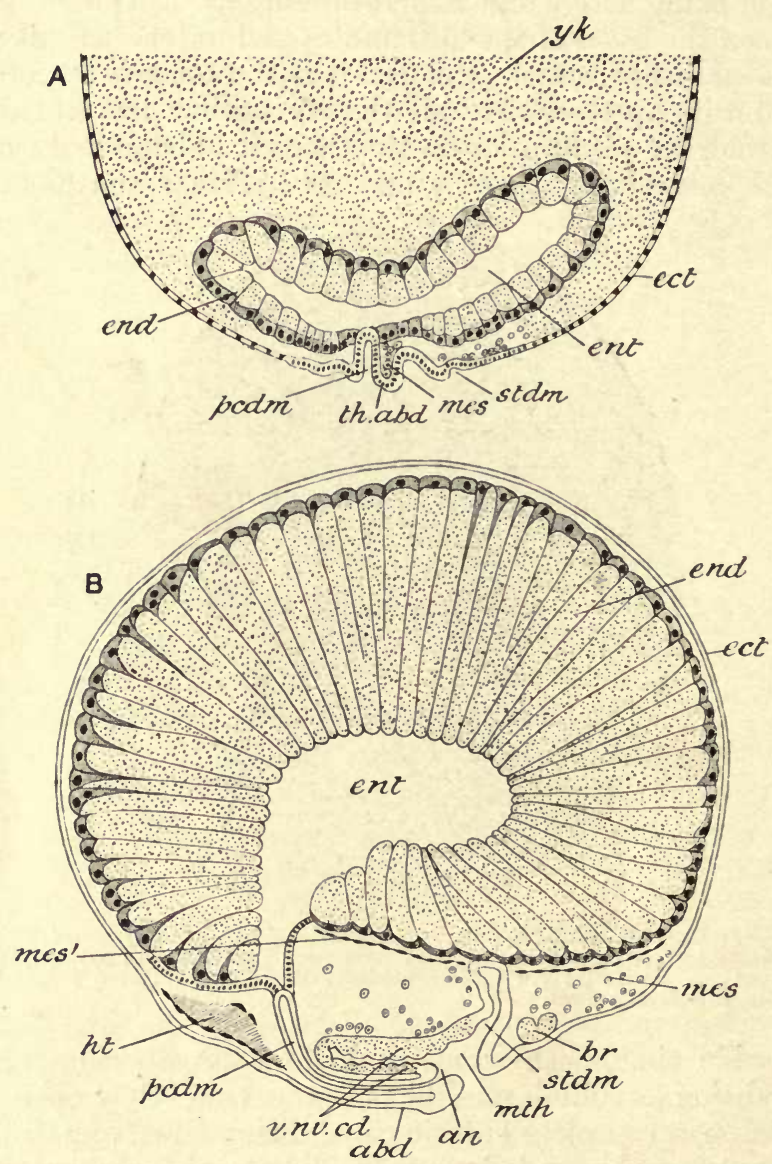

Figs. 445 and 446.-Sections of embryus of Astacus. A, Nauplius-stagc (cj. Fig. 444; B, after development of thoracic appendages ( $f f$. Fig. 447$)$. abd. abdomen; an. anus; br. brain; ect. ectoderm ; end. endoderm ; ent. enteron ; ht. heart ; mes. mesoderm; mes.' splanelinic layer of mesoderm; inth. mouth ; $p c d m$. proctodæum; stdm. stomodæun ; th. abd. thoracicoabdominal rudiment; $v$. nv. $c d$. ventral nerve-cord. (From Korschelt and Heider, after Reichenbach.)

(Fig. $447, A$ ), as well as the labrum (l.), and a fold on each side of the thorax, which is the rudiment of the carapace $(t s)$, and this gradually extends dorsally until it meets with its fellow of the opposite side and covers in the cephalothorax. The embyro now consists of a nearly globular cephalothorax with a small abdomen 
and a nearly complete set of appendages, all tucked in under the cephalothorax and closely packed together within the egg-membranes. In this condition the embryo is hatched, and for some time clings to the pleopods of the mother by means of the peculiarly hooked chelæ of its first pair of legs.

The development of the principal internal organs must be referred to very briefly. From the ectoderm arise, not only the epidermis of the adult, but the epithelium of the gullet and gizzard and of the hind-gut, the epithelium of the gills, the nervous system, the vitreous cells and retinula of the eyes, and

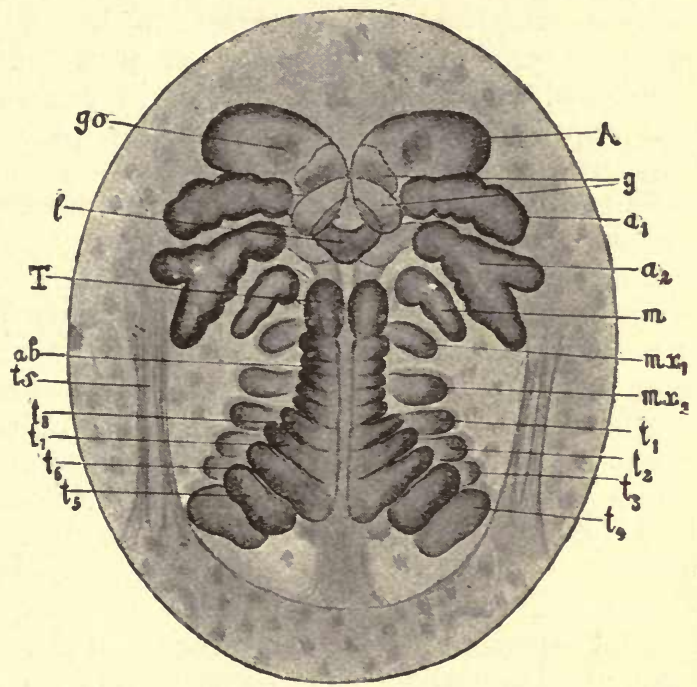

Fig. 447.-Embryo of Astacus after derelopment of thoracic appendages. $A$, eyes; $a_{1}$. antennule; $a_{2}$ antenna; $a b$. abdomen; $g$, archicerebrum and ganglion of anteniule; $g o$, optic ganglion; $l$. labrum; $m$. mandible; $m x_{1}$. $m x_{2}$. maxilla; $t .1-s$, thoracic appendages; t. telson; ts, carapace. (From Lang's Comparative Anatomy.)

the epithelium of the auditory sac. From the endoderm arises the epithelium of the mid-gut and of the digestive glands, the latter being formed as tubular branching outgrowths of the archenteron. The connective-tissues, the muscles, the vascular system, the gonads, and perhaps the kidneys, are all of mesodermal origin.

\section{Distinctive Characters and Classification.}

The Crustacea are Arthropods in which the five anterior segments are fused with the prostomium to form the head, while the rest are usually divisible into two regions, the thorax and the abdomen. More or fewer of the thoracic segments may be fused with the head to form a cephalothorax. The head may bear a 
median eye, which frequently disappears in the adult, and a pair of compound eyes, both belonging to the prostomial region: the latter frequently become elevated on jointed eyestalks. The appendages of the head are (1) the antennules, which are usually considered as belonging to the first metamere; (2) the antennæ, which are certainly post-oral or metameric appendages shifted forwards to a pre-oral position ; (3) the mandibles or crushing jaws ; (4) the first maxillæ; and (5) the second maxillæ. The thoracic and abdominal appendages are variously modified as jaws, legs, fins, or accessory reproductive organs. With the exception of the antennules, the appendages are typically biramous, consisting of a stem or protopodite bearing two branches, the endopodite and exopodite.

The body is covered externally by a chitinous cuticle, which becomes thickened and sometimes calcified in regions where no movement is required, forming a series of hard parts or sclerites, separated by flexible chitin: the whole chitinous cuticle thus constitutes an exoskeleton. Typically there is one sclerite to each metamere behind the head, and to each podomere in the appendages, but concrescence of sclerites frequently takes place. The exoskeleton is produced into setæ, which are hollow processes of the cuticle containing prolongations of the underlying epidermis.

Respiration takes place either by the general surface of the body or by gills, which are hollow offshoots of the thoracic wall or of the thoracic or abdominal limbs. The stomodæum and proctodxum form a considerable portion of the enteric canal, and are lined with chitin: the mesenteron gives rise to digestive glands. The body-cavity is divided into comparments, most of which contain blood and are portions of the vascular system: the true cœlome may be represented by compartments of the body-cavity not containing blood and by the cavities of the reproductive organs. There is a vascular system which nearly always includes a contractile heart, formed as a muscular dilatation of a dorsal vessel, and communicating by valvular ostia with an enclosing pericardial sinus. The blood is taken from the heart to the various organs by arteries, and is returned to the pericardial sinus by sinuses and veins: the respiratory organs are interposed in the returning current. The renal organs are peculiarly modified nephridia, which may take the form either of shell-glands opening on the second maxillæ, or of antennary (green) glands opening on the antennæ.

The nervous system consists of a brain united by œsophageal connectives with a ventral nerve-cord, formed of a double chain of ganglia joined together by commissures and connectives. The first three pairs of embryonic ganglia commonly unite to form the brain, which is therefore a syn-cerebrum. The sexes are separate or united: sexual dimorphism is common: parthenogenesis frequently occurs. The sperms are either amœboid with 
radiating pseudopodia, or flagellate: the eggs are usually centrolecithal but may be telolecithal, or almost alecithal. The muscles are striped, and there are no cilia.

Segmentation of the oosperm is usually superficial, but may be complete or discoid. The embryo very usually has a distinct nauplius-stage, which may be a free-swimming larva or may be passed through before hatching, and is characterised by the presence of three pairs of appendages which become the antennules, antennæ, and mandibles of the adult.

The Crustacea are classified as follows :-

\section{Sub-class I.-Branchiopoda.}

Crustacea with a varying number of body-segments, provided with appendages of a uniform character, usually foliaceous, rarely leg-like, the posterior region (abdomen) devoid of appendages and provided with a pair of many-jointed or unjointed caudal styles. A cephalic carapace is sometimes absent: when present it may be either shield-like or bivalve. Paired eyes are usually present. The antennules and the maxillix are reduced or absent: the mandibles devoid of, or with a vestigial, palp. The larva is a nauplius or metanauplius.

\section{Order. 1.-ANostraca.}

Branchiopoda in which a carapace is not developed. The eyes are stalked: the antennæe are prehensile in the male, reduced in the female. The appendages of the body-segments number 11 or 19 pairs. The caudal styles are not jointed.

This order includes Branchipus and Artemia.

\section{ORDER 2.-NOTOSTRACA.}

- Branchiopoda in which there is a large dorsal shield-shaped carapace. The eyes are sessile. The antennæ are reduced. There are 40 to 63 pairs of trunk appendages. The caudal styles are many-jointed.

Including Apus and Lepidurus.

\section{Order 3.-Conchostraca.}

Branchiopoda with a carapace divided into two lateral portions or valves like the shell of a bivalve mollusc, and enclosing the entire animal. The antennæe are biramous and are used as swimming appendages. The eyes are sessile, coalescent. The 
appendages of the body-segments number 10 to 27 pairs. The caudal styles are in the form of unjointed, curved claws.

In this order are included Estheria, Limnetis (Fig. 448) and one or two other genera.

\section{Order 4.-Cladocera.}

Branchiopoda of small size with a bivalved carapace which encloses the trunk but not the head. The eyes are sessile and united together. The antennæe are biramous and used as swimming appendages. Only 4 to 6 trunk appendages; caudal styles unjointed, claw-like.

To this order belong Daphnia, Polyphemus, Leptodora (Fig. 449 ), etc.

\section{Sub-class II.-0stracoda.}

Crustacea with unsegmented, or indistinctly segmented, body, bearing not more than four pairs of appendages on the trunk, the limbless posterior part provided with a pair of caudal styles. There is a well-developed bivalved carapace. Paired eyes may be present or absent. Both antennules and antennæ are used in swimming; the latter are generally biramous. The mandibles have a palp. The young escapes from the egg as a nauplius.

In this sub-class are comprised Cypris, Cythere, etc. (Fig. 450).

\section{Sub-class III.-Copepoda.}

Crustacea with elongated, distinctly segmented body, bearing usually five pairs of limbs, the last four having the character of biramous swimming appendages, sometimes with a sixth pair which may be vestigial : the posterior region (abdomen) without appendages, provided with a pair of caudal styles. The cephalic dorsal shield is not extended backwards, but usually coalesces with the exoskeleton of the first (and sometimes also the second) bodysegment. Paired eyes are absent except in the Branchiura. Both antennules and antennæ are usually well developed, and the latter are sometimes biramous: they may both be used as swimming organs or for prehension. The mandibles may be provided with a palp. The young is a nauplius. In the parasitic forms more or fewer of these general characteristics may become lost in the adult.

\section{ORder 1.-EuCOPEPODA.}

Free or parasitic Copepoda without paired compound eyes. The appendages of the body-segments are devoid of a flagellum. The genital apertures are situated on the seventh body-segment. In this group are included $(a)$ free-swimming forms, such as Cyclops (Water-flea) (Fig. 451) and (b) parasitic forms or Fishlice-c.g, Ergasilus, Chondracanthus, Lernsea (Fig. 452). 


\section{Order 2.-BranchiURA.}

Parasitic Copepoda with compound eyes and a suctorial mouth. Some of the appendages of the body-segments are usually provided with peculiar appendages-the flagella. The genital apertures are situated on the fifth body-segment. This order includes the Carp-lice, Argulus (Fig. 453) and two other genera.

\section{Sub-class IV.-Cirripedia.}

Imperfectly segmented Crustacea, which are always fixed in the adult condition, and may be parasitic. There are usually six pairs of biramous cirriform appendages of the body region. The limbless posterior region (abdomen) is rudimentary, and is usually provided with a pair of caudal styles. The carapace forms a pair of folds, the mantle, completely enclosing the animal, and usually supported by a system of calcareous plates giving rise to a hard shell. Paired eyes are absent in the adult. The antennules of the larva give rise to organs of attachment and become vestigial in the adult: the antennæ usually disappear. The mandibles have no palp. The sexes are united in the great majority. The young animal is hatched in the nauplius form and passes later through a stage-the cypris stage-in which it is provided with a bivalved shell.

\section{Order 1.-EUCIRRIPEDIA.}

Cirripedia some of which are parasitic, while the rest are nonparasitic but are permanently fixed in the adult condition. There are usually six pairs of biramous trunk appendages.

In this order are included (a) fixed forms such as Lepas (Barnacle) (Fig. 454) and Balcunus (Acorn-shell) (Fig. 455) and (b) parasites-e.g., Petrarca, Alcippe, Proteolepas.

\section{ORDER 2.-RHIZOCEPHALA.}

Parasitic Cirripedia in which the body has undergone extreme degeneration, and has lost all trace of appendages and of alimentary canal in the adult condition.

Including Sacculina (Fig. 456) and Peltogastcr.

\section{Sub-class V.-Malacostraca.}

Crustacea in which the body is always distinctly segmented and is made up in all cases except the Leptostraca of an anterior region (thorax) of eight segments, and a posterior (abdomen) of six, with a terminal tail-piece or telson-the total number of segments, leaving the prostomium and the telson out of account, being always nineteen. The appendages of the thorax and abdomen are sharply 
marked off from one another. The abdomen is devoid of caudal styles. The exoskeleton of the head unites with that of more or fewer of the thoracic segments to form a cephalothoracic carapace. Paired eyes are usually present and may be sessile or stalked. The antennules are biramous in most cases. The mandibles are provided with a palp. There is usually a metamorphosis, but a nauplius-stage rarely occurs.

\section{Series I.-Leptostraca (Phyllocarida).}

Malacostraca in which the abdomen contains seven segments and a telson-the last segment devoid of appendages, the telson bearing a pair of caudal styles. There is a large bivalved carapace with an adductor muscle, enclosing the greater part of the body. The thoracic appendages are foliaceous, the abdominal biramous.

Includes only one order, the Nebaliacea, with Nebalia (Fig. 457) and three allied genera.

\section{Series II.-Eumalacostraca.}

Malacostraca with six segments and a telson in the abdomen, the latter never provided with caudal styles. Carapace never bivalve. Thoracic appendages nearly always leg-like, but seldom all uniform: their protopodite always made up of two podomeres except in the Stomatopoda.

\section{Division 1.-Syncarida.}

Eumalacostraca devoid of carapace, with the first thoracic segment united with the head or marked off from it by a groove. Heart elongated, tubular.

\section{ORDER-ANASPIDACEA.}

Syncarida in which the thoracic appendages are provided (except the last or the last two) with exopodites, and (except the last) with a double series of lamellar epipodites (gills). The abdominal appendages, except the first two in the male and the last in both sexes, have the endopodite reduced or absent. The last pair of abdominal appendages (uropods) expanded, and forming with the telson a fan-like tail-fin. This, the only order of the Syncarida, comprises the genera Anaspides, Koonunga, and Paranaspides (Fig. 458).

\section{Division 2.-Peracarida.}

Eumalacostraca in which the carapace, when present, leaves at least four of the thoracic segments free. Heart elongated, tubular. 


\section{ORDER 1.-MYSIDACEA.}

Peracarida in which, though the carapace extends over the greater part of the thorax, it does not coalesce dorsally with more than the first three segments. The eyes, when present, are supported on movable stalks; the antennules are biramous, and the antennæ have a scale-like exopodite or squame. The first pair of thoracic appendages are specialised as maxillipedes. The thoracic appendages (except sometimes the first and second pairs) are biramous. The uropods with the telson form a broad fan-like tail-fin.

This order includes Mysis (Fig. 459), Lophogaster, and other genera.

\section{Order 2.-Cumacea.}

Peracarida in which the carapace coalesces with the first three or four segments of the thorax, is produced on each side to enclose a branchial cavity, and in front is drawn out into a rostrum. The eyes usually coalesce into one, which is not borne on a movable stalk. The antennules are sometimes biramous; the antennæ have no exopodites. Some of the thoracic appendages are biramous. The telson may coalesce with the last segment of the abdomen. The uropods are styliform, and there is no fanlike tail-fin.

Includes Cuma (Bodotria), Diastylis, etc. (Fig. 460).

\section{Order 3.-TANAIDACEa.}

Paracarida in which the carapace coalesces with the first two thoracic segments and is produced on each side to enclose a branchial cavity. The eyes, when present, are usually supported on short stalks which are not movable. The antennules are sometimes biramous: the antennæ may possess small exopodites. The first pair of thoracic limbs are modified as maxillipedes. The second and third thoracic limbs sometimes have vestigial exopodites. The uropods are usually narrow.

This order includes Apseudes, Tanais, Leptochelia, etc.

\section{ORDER 4.--IsOpoda.}

Peracarida in which the dorsal exoskeleton of the head is not produced into a carapace, but in which the first and sometimes also the second segment of the thorax coalesce with the head. The eyes are sessile or borne on short processes which are not movable. The antennules are nearly always uniramous: the antennæ sometimes bear a minute exopodite. The thoracic limbs have no exopodites: the first pair are modified as maxillipedes; 
the rest are usually alike in character. The abdominal appendages are usually biramous; the rami function as branchix. The body is nearly always dorso-ventrally compressed. This is a large order including many families, e.g.-Asellus, Phreatoicus, Anthura Sphacroma, Idotea, Oniscus, Bopyrus (Figs. 462, 464).

\section{ORDER 5.-AMPHIPODA.}

Peracarida with the characters of the preceding order, except that (1) the body is nearly always laterally compressed; (2) the second and third pairs of thoracic appendages are nearly always modified as prehensile organs (gnathopods); (3) there are vesicular or lamellar branchix attached to the bases of more or fewer of the thoracic limbs; (4) the abdominal appendages are distinguishable into two sets, the three anterior pairs with many-jointed rami, the three posterior (including the uropods) with unjointed styliform rami.

Includes Orchestia, Cammarus (Fig. 461), Hyperia, Caprella, Cyamus (Fig. 463), and many other genera.

\section{Division 3.-Eucarida.}

Malacostraca in which the carapace coalesces with all the thoracic segments, forming a cephalothorax. The eyes are borne on movable stalks. The heart is short, sac-like, and situated in the thorax.

\section{Order 1.-Euphausiacea.}

Eucarida in which none of the thoracic limbs take the form of maxillipedes; with a single series of branchiæ (podobranchs) attached to the bases of the thoracic limbs. The larva is a nauplius.

This is a comparatively small order of pelagic Malacostraca, including Euphausia (Fig. 472), Thysanopoda, Nyctiphanes, and a few other genera.

\section{ORDER 2.-DECAPODA.}

Eucarida in which the first three pairs of thoracic appendages are modified as maxillipedes, with the branchiæ usually in several series-podobranchs, arthrobranchs, and pleurobranchs.

\section{Sub-order 1.-Macrura.}

Decapoda with well-developed, elongated abdomen, which is usually held in the extended position, and terminates in an expanded fan-like tail-fin composed of the telson and the uropods. The eyes are not enclosed in orbits. The antennules and antennæ 
are both large; the former are not sunk in pits, and the antennæe usually have a scale-like exopodite (squame).

Included among the Macrura are $(a)$ swimming forms-Penceus and Palcemon (Prawns), Crangon (Shrimps), Lucifer, etc.; and (b) creeping forms-Homarus (Lobster), Astacus, Astacopsis, Puranephrops, Cambarus (Fresh-water Crayfishes), Palinurus (Rocklobsters), Scyllarus, etc. (Figs. 465, 466).

\section{Sub-order 2.-Anomura.}

Decapoda with the abdomen more or less reduced, usually held in a flexed position, and not provided with such a well-developed tail-fin as in the Macrura.

In most respects the Anomura are intermediate between the Macrura and the Brachyura. Examples are the Hermit-crabsPagurus (Fig. 467), and other geners, the Cocoa-nut crab-Birgus -Galathca, Hippa, Porcellana, etc.

\section{- Sul-order 3.-Brachyura.}

Decapoda in which the abdomen is greatly reduced, shorter than the cephalothorax, and permanently flexed beneath it. The antennules and the eyes are both capable of being retracted into cavities. There is a metamorphosis comprising zccec ard megalopa stages.

Including the true Crabs such as Cancer, Maia, Grapsus, etc. (Figs. 468, 469).

\section{Division 4.--Hoplocarida.}

Malacostraca in which the carapace does not coalesce with at least the last four thoracic segments, so that the cephalothorax is relatively short. In front of the head proper are two movable segments, one bearing the stalked eyes, the other the antennules. The branchix are borne on the abdominal appendages. The heart is elongated. There is a metamorphosis, but a nauplius stage is not known to occur.

\section{ORder Stomatopoda.}

This, the only order of Hoplocarida, includes Squilla (Fig. 470), Gonodactylus, and other genera.

\section{Syteriatic Position of the Examples.}

The genera Apus and Lepidurus belong to the family Apcdide of the order Notostrace of the sub-class Branchiopoda.

The foliaceous character of the swimming-feet is alone sufficient to assign them to the Branchiopoda, and the large number of seg- 
ments (considerably more than ten) and swimming-feet, together with the presence of the large shield-like carapace, decides their position among the Notostraca.

They are placed in the family Apodidæ in virtue of the elongated body with 40-60 pairs of swimming-feet, diminishing in size from before backwards and showing considerable differentiation ; and of the elongated heart reaching to the twelfth post-cephalic segment.

Apus is distinguished by the absence of a post-anal plate, and by elongated flagella (endites) to the first pair of thoracic feet: in Lepidurus the post-anal plate is present, and the flagella of the first thoracic feet are short.

Astacus fluviatilis is one of several species of the genus Astacus, belonging to the family Potamobiidce, tribe Astacoidca, sub-order Macrura, order Decapoda, and sub-class Malacostraca.

The possession of a thorax made up of eight segments and an abdomen of six, places it among the Malacostraca : the presence of a cephalothorax formed by coalescence of head and thorax, together with that of movable eye-stalks, determines its position in the division Eucarida: the modification of the first three pairs of thoracic appendages as maxillipedes, and the arrangement of the branchiæ in three sets, place it in the Decapoda.

The possession of a squame to the antenna, and of legs having all seven podomeres distinct-the first three pairs chelate, and the first pair greatly enlarged-determine its position in the tribe Astacoidea, which includes all the fresh-water Crayfishes and the true Lobsters. The family Potamobiidæ is distinguished by having the podobranchiæ partly united to the epipodites, and by possessing appendages on the first abdominal segment of the male and usually on that of the female.

\section{General Organisation.}

There is no class in the animal kingdom which presents so wide a range of organisation as the Crustacea, or in which the deviations in structure from the "type-form" are so striking and so interesting from their obvious adaptation to the mode of life.

The most interesting modifications are those connected with the external characters and the structure of the appendages. As we have seen, the body consists of a prostomium, a variable number of metameres, and an anal segment. The first five metameres fuse with the prostomium to form a head, which, as well as the anal segment, is homologous throughout the class. On the other hand, there is no strict homology between the various post-cephalic metameres in different forms until we come to the Malacostraca, in which their number is constant.

There is considerable diversity of form among the Branchiopoda. Apus has already been described. Branchipus (Fig. 448, 1) and 
Artemia (the Brine-shrimp) (Anostvaca) are small shrimp-like forms, the former living in fresh-water lakes, the latter in brinepools; they have no carapace, and the eyes are raised on unjointed stalks. In Limnetis (2), on the other hand, and in Estheria (3) the carapace takes the form of a shell, formed of two parts or valves, united in Estheria by a hinge, and resembling the shell of a cockle or other bivalved mollusc. The limbs have the same general structure as those of Apus, but the antennæ are
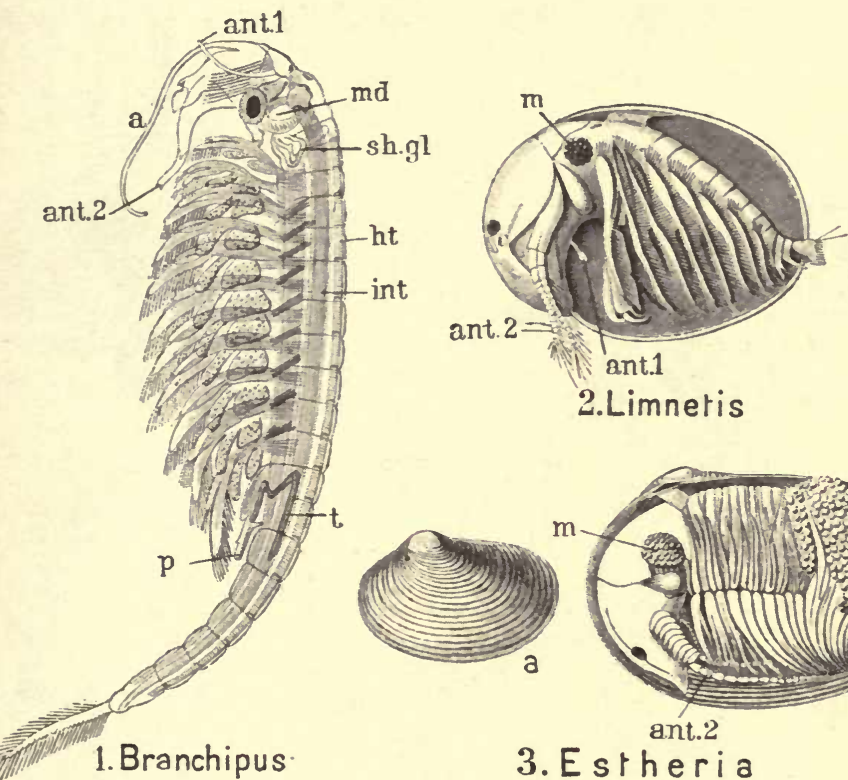

Fig. 44S.-Three Branchiopoda. In $3, a$ is the shell $; b$ the animal with one valve of the shell removed. "n $t^{1}$. antenuule; ant ${ }^{2}$. antenua; $h t$. heart; $m$. adductor musele; md. mandible; ov. ovary; $a$. unpaired process from head; $p$. copulatory appendages; sh.gl. shell-gland; $t$. testis. (After Gerstaecker.)

often of considerable size, and are sometimes modified into prehensile organs.

In the Cladocerc, of which the common fresh-water Daphnia (Fig. 449,1 ) is a good example, there is a great reduction in size (1-2 mm.). and a corresponding shortening of the body by a reduction in the number of metameres. Segmentation is very imperfect, and the whole body, but not the head, is covered by a large folded carapace. The abdomen is turned downwards and is in constant movement, sweeping out any foreign particles which may have made their way among the feet. Between the abdomen of the female and the posterior part of the carapace is a large brood-pouch (br.p), in which the eggs are stored. The 
paired eyes ( $E$ ) have fused into a single organ, which exhibits a contstant trembling movement. The antennules (ant. 1) are small, the antennæ (ant. 2) very large, biramous, and constitute the chief organs of locomotion. The mandibles are large, the second maxillæ absent in the adult, and there are usually five pairs of leaf-like swimming-feet $(f)$ on the thorax. The abdomen is devoid of appendages. Many of the Cladocera have an extraordinarily grotesque form $(2,3)$, owing to the peculiar

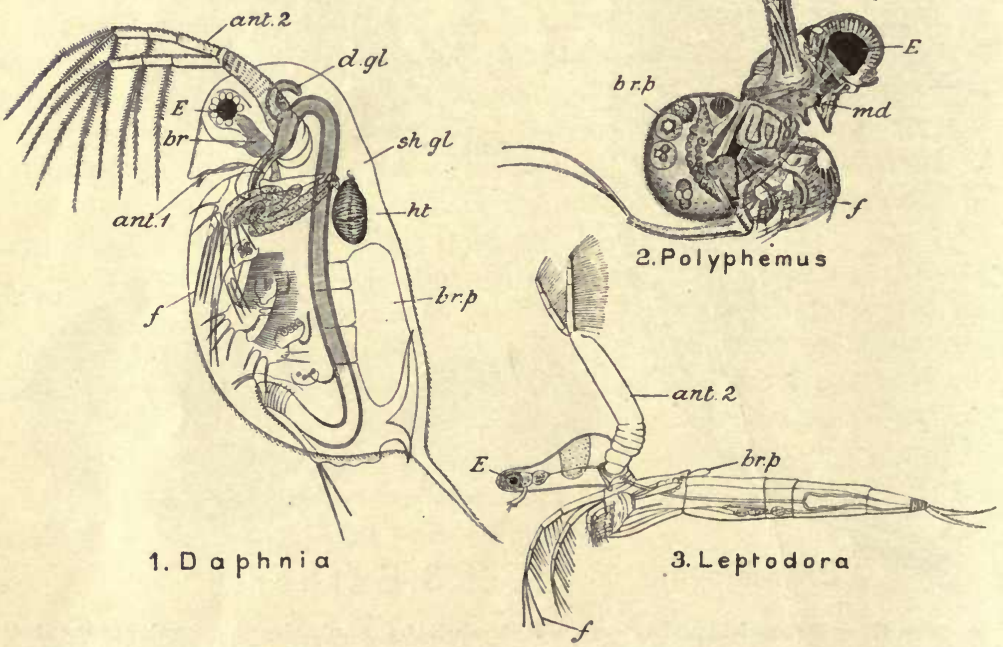

G. 449. - Three Cladocera. ant. 1 , antemule ; ant. 2 , antenna ; $u n$ brain ; $m \cdot p$. brood-poneh ; $E$. eye; $d . g l$. digestive gland; f. swimming-feet; $h t$. heart; md. mandible; sh.gl. shell. gland. (1 after Claus, 2 and 3 after Gerstaecker.)

shape of the head, the immense antennæ, and the great hump-like brood-pouch.

The Ostracoda are usually not more than 1-2 $\mathrm{mm}$. in length, and are found both in fresh and sea-water. One of the commonest genera is Cypris, which occurs in immense numbers in stagnant pools. Cythere is a common marine form.

The body (Fig. 450) is unsegmented, and is completely enclosed in a carapace (A), the right and left halves of which are articulated together along the dorsal edge so as to form a bivalved shell (C), which may be variously ornamented or sculptured. The valves are opened by the elasticity of a ligament, which passes 
from one to another at the hinge, and are closed by a large adductor muscle $(m$.), which extends transversely from valve to valve, its insertions giving rise to markings on the shell $(\mathbf{A}, m$.), often of systematic value.

At the anterior end is a median eye (e), and in some forms compound eyes are present as well. There are only seven pairs of appendages. The antennules (ant.1) and antennæ (ant.2) are large and uniramous. The mandible $(m \dot{d}$.$) has a large leg-like palp$ and a flabellum-like offshoot. The first maxilla ( $m x .1)$ also bears a large plate resembling a flabellum of Apus. The last cephalic
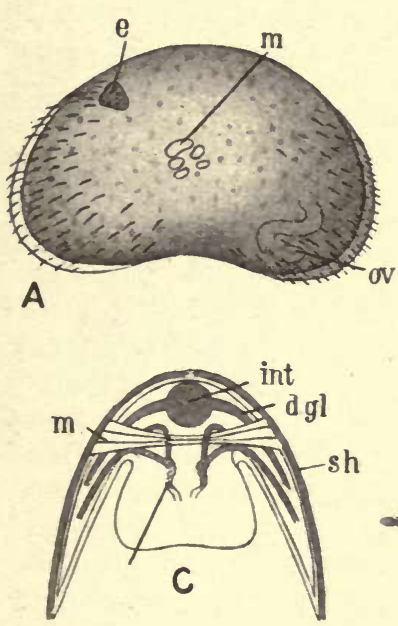

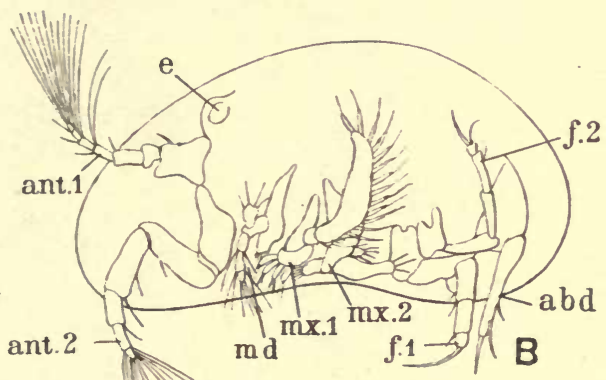

FiG. 450.-A, external view of Cypris ; B, the same with the appendages exposed by the removal of the left valve of the shell; C, transverse section; D, a single sperm. abl. abdomen ; ant.1, antennule; ant.2, antenna; $\boldsymbol{c l . g l}$. digestive gland; $e$. median eye; f.1, f.., thoracic feet; int. intestine; $m$. adductor muscle; mel. mandible; mx. $1, m x .2$, maxilla; or. ovary; $s h$. shell; $t$. testis. (After Gerstaecker.)

appendage (second maxilla, $m s .2$ ) is jaw-like in some forms (Cypris), leg-like in others (Cythere). The only thoracic appendages are two pairs of slender legs $(f .1, f . P)$. The abdomen $(a b d$.$) is$ devoid of appendages, and is terminated by a pair of small caudal styles.

The diversity of form among the Copepoda is so great that it will be advisable to consider separately the free-surimming Eucopepoda, the parasitic Eucopepoda, and the Branchiura.

The free-swimming Eucopepoda are well represented by the common water-flea (Cyclops), found everywhere in fresh and brackish water, and easily recognisable, in spite of its minute size, by its elongated form, its rapid, jerky movements, and by the egg-sacs of the female. 
SECT ,

Cyclops (Fig. 451, 1) has been compared in form to a split pear, the broad end being anterior, and the convex surface dorsal. The first thoracic segment is fused with the head, and the

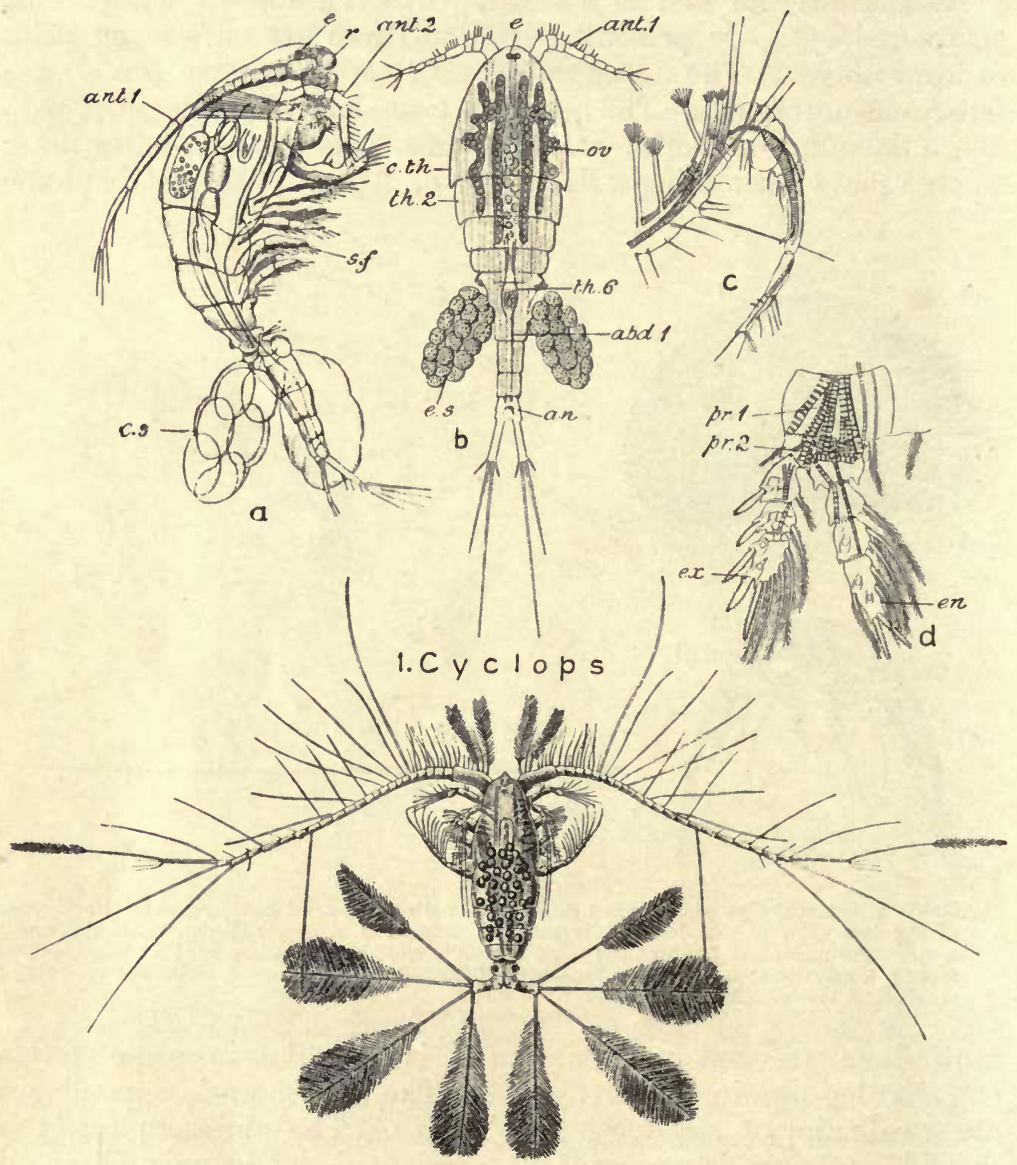

2. Calocalanus

FiG. 451.-Free-swimming Eucopepoda. Ia, female Cyclops, from the right side; $b$, dorsal view; $\mathrm{C}$, antenna of male; $\mathrm{D}$, swimming-foot. abd.1, first abdominal segment; ant.1, antennule; ant.2, antenna; $c$. th. eephalothorax; e. median eye ; en. endopodite; e.s. eggsac; $e x$. exopudite; ov. ovary; $p r .1, p r .2$, protopodite; $r$. rostrum; s.f. swimming-feet; th.2, th.6, thoracic segments. (After Huxley, Gerstaecker, Hartog, and Giesbrecht.)

cephalothorax (c. th.) thus formed is covered with a carapace produced in front into a short spine or rostrum $(r)$, near the base of which, on the dorsal surface, is the median eye $(e)$. There are five free thoracic segments: the last $(t h .6)$ bears the genital 
aperture, and is fused in the female with the first abdominal (abd. 1). There are four abdominal segments: the last bears the dorsal anus $(a n)$, and a pair of caudal styles produced into plumed setæ.

The antennules (ant. 1) are very large, and are the principal organs of locomotion. In the male they are modified (C.) - by a peculiar form of joint and long setæ-as clasping organs, used for holding the female during copulation. The antennæ (ant. 2) are comparatively short and uniramous. Mandibles and maxillæe are present, and the first four thoracic segments bear biramous swimming-feet ( $1 a$ s. $s$. .), those of the right and left sides being connected by transverse plates or couplers. The fifth thoracic segment bears a pair of vestigial limbs: the abdominal segments are limbless.

Some of the pelagic marine Eucopepoda (Fig. 451, 2) are remarkable for their brilliant colours and for the extraordinary development of their setæ, especially those of the caudal styles.

The parasitic Eucopepoda, or Fish-lice, present a very interesting series of modifications, illustrating the degeneration of structure which so often accompanies parasitism. Ergasilus (Fig. 452, 1) is found on the gills of the Bass (Morone labrax); it is readily recognisable as a Copepod, but the appendages are greatly reduced, the antennæ modified into hooks for holding on to the host, and the eyes absent. Anthosoma (2), found in the mouth of the Porbeagle Shark (Lamna cornubica), has recognisable appendages, but the form of the body is much modified by the development of curious overlapping lobes. Nicothöe (3), found on the gills of the Lobster, has antennæ and mouth-parts modified for suction: the abdomen is normal, but the thorax is produced into huge lobes, which give it a curiously deformed appearance. In Chondracanthus (4), the various species of which are parasites on the gills of Bony Fishes, there is, at the first glance, nothing to suggest that the animal is a Crustacean, except the characteristic copepod egg-sacs: the body is depressed, unsegmented, and produced into crinkled lobes, and it requires careful examination to discover that antennules, hooked antennæ (ant.2)-used for attachment-mandibles, maxillæ, and two pairs of legs $(f .1, f .2)$ are present. The male $(b)$ is of higher organisation than the female, but of minute size-about $\frac{1}{12}$ the length of its mate-and is permanently attached to her body, close to the genital aperture $(a, M)$. In Lerncea ( 7$)$ and its allies the body is vermiform with a curiously lobed anterior end: the maxillæ are adapted for piercing the skin of the host and sucking its juices, and there are minute vestiges of feet. In Lesteira (5) the degradation is even more marked: the female reaches a large size- $70 \mathrm{~mm}$. in length, excluding the egg-sacs-and is found with the swollen head between the skin and flesh of a fish 
(Genypterus blacodes), and the rest of the body hanging freely into the water. Lastly, in Tracheliastes $(G)$ the second maxillæ

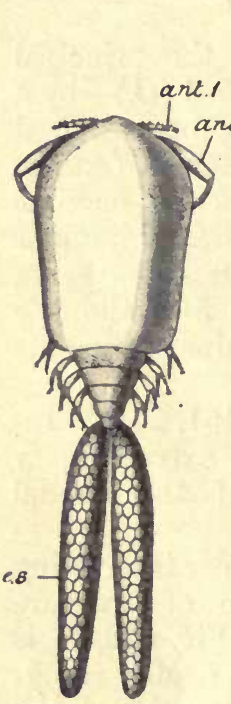

1. Ergasilus

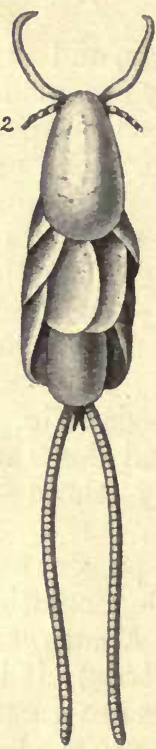

2.Anthosoma

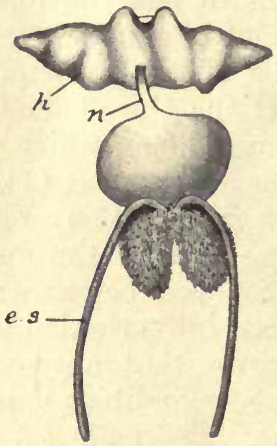

5. Lesteira

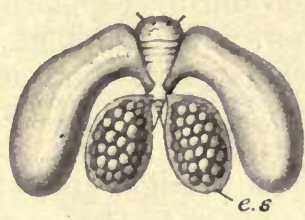

3.Nic othoe
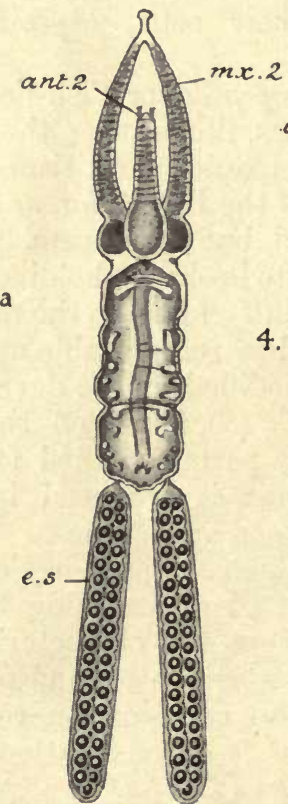

6.Tracheliastes

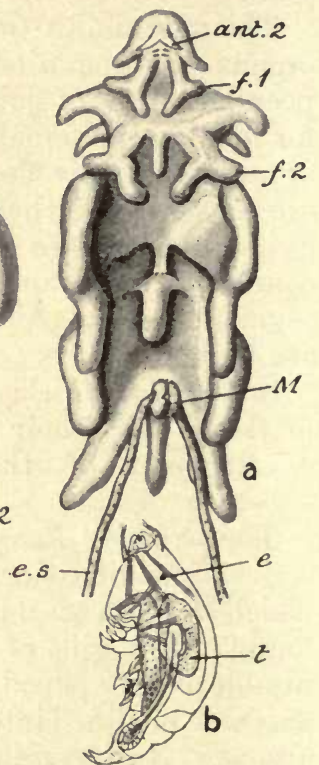

4. Chondracanthus

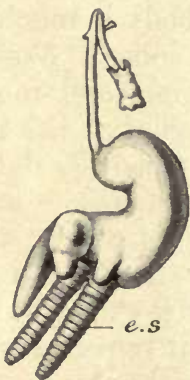

7. Lernaea

Fif. 452.-Various forms of parasitic Eucopepoda. 4a, female; 4 b, malc. ant.1. antennule ; ant.2, antenna ; $e$. median eye ; e.s. egg-sac; $f .1, f .2$, thoracic feet; $M$. male ; inx. 2 , second maxillæ. (After Gerstaccker, Claus, Cuvier, and G. M. Thempson.)

(mx.2) are greatly enlarged, and form a characteristic organ of attachment.

Argulus (Fig. 453) is the most familiar example of the Branchiura, or Carplice. It is an external parasite on fresh-water Fishes (Carp, Stickleback, \&c.), not 
permanently attached like the degenerate forms just described, but crawling freely over the surface of the host. The body consists of an oval flattened cephalo-thorax, and a small bilobed abdomen $(a b$.$) . The mandibles and maxillæ$ are piercing organs enclosed in a sucking-tube or proboscis $(r$.$) , in front of which$ is a median tube ending in a spine (st.). The second maxilla are divided into two portions, the anterior of which $(k f .1)$ are modified into sucking-dises by

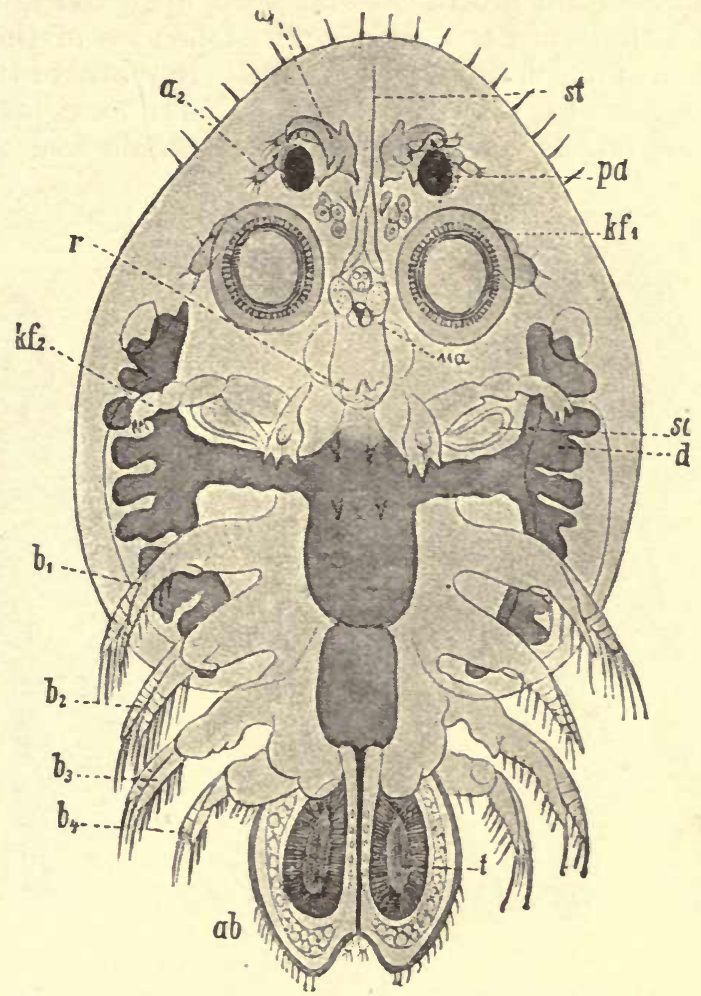

Fig. 453.-Argulus foliaceus, young male. $a_{7}$, antennulc; $a_{2}$, antenna; $a b$. abdomen; $b_{1}-b_{4}$, thoracic fect; $d$. digestive glands connected with intestine; $l f f$, anterior or suctorial feet; $k \cdot f$, posterior or leg-like portion of second maxillae ; pa." paired eye ; $r$. rostrum : sd. shellgland; st. stylet; ts. testis ; u, median eye. (From Lang's Comparative Anatomy.)

which the parasite clings to the surface of its host, and there are four pairs of swimming-feet $(b 1-b / 4)$. Alone among the Copepoda the Branchiura have no egg-sacs, and they are exceptional also in the possession of compound eyes (pa.).

The most familiar examples of the Eucirripedia are the Barnacles found on ships' bottoms, piles, \&c., and the Acorn-shells or Sessile Barnacles, which occurin immense numbers on rocks between tidemarks in all parts of the world.

The common Barnacle (Lepas anatifera) is attached by a long stalk or peduncle (Fig. 454, A, p), covered with a wrinkled skin, and bearing at its distal end the body proper enclosed in a sort of 
bivalved carapace, formed by a fold of the skin, and strengthened by five calcareous plates. Of these one is median and dorsal, and is called the carina $(c)$; two are lateral and proximal, the scuta $(s)$; and two lateral and distal, the terga $(t)$. During life the carapace is partly open, and from the ventrally placed aperture delicate setose filaments are protruded and keep up a constant grasping movement: these are the endo- and exopodites of the biramous thoracic feet, of which there are six pairs. Removal of the carapace shows the feet to arise from a vermiform unsegmented body (B), attached on the ventral aspect to the stalk and carapace by
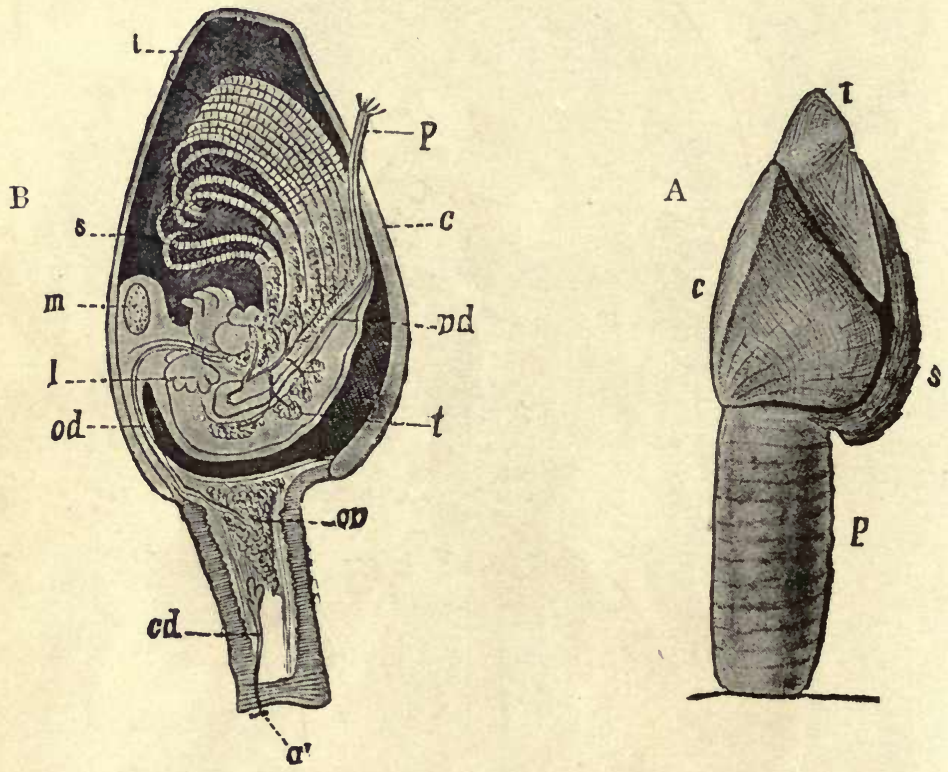

FIG. 454.-Iepas anatifera. A, the entire animal; B, anatomy. $\iota_{1}$, antennule ; c. carina ; $c l$. eement-gland; $l$. digestive gland; $m$. adduetor muscle; od. oviduet; ov. ovary ; $p$. (in B) penis and (in A) peduncle ; s. scutum ; $t$. tergum and testis; vu. vas deferens. (From Lang's Comparutive Anatomy, after Darwin and Claus.)

its anterior end, while its posterior end is free and terminates in a long filament, the penis $(p)$, immediately dorsal to which is the anus. The mouth is ventral and anterior, and is provided with a pair of mandibles and two pairs of maxillæ. There are no antennæ: at first sight the antennules appear to be absent, but a careful examination shows the presence of a pair of minute structures $\left(a^{\prime}\right)$ on the proximal or attached surface of the stalk, and embedded in the cement by which the animal is fixed to its support; these are the antennules, and their position relatively to the mandibles shows that the stalk is formed by an elongation of the anterior region of the head. 
The Sessile Barnacles or Acorn-shells (Balanus) have no stalk (Fig. 455), the head-region being short and broad. The scuta $(s)$ and terga $(t)$ support a valvular carapace, through the opening of which the feet are protruded, and the whole animal is surrounded by a sort of parapet (sk) formed of six calcareous pieces. One of these, dorsal in position, is the carina, the others appear to be represented by small ossicles developed on the peduncle of certain stalked forms such as Pollicipes.

Many of the Eucirripedia are parasitic. Some of these (Petrarca, \&c.), parasitic in Actinozoa, resemble the attached forms in essential respects; others (Alcippe), parasitic in the shells of Molluses and Cirripedes, have abdominal but no thoracic feet.

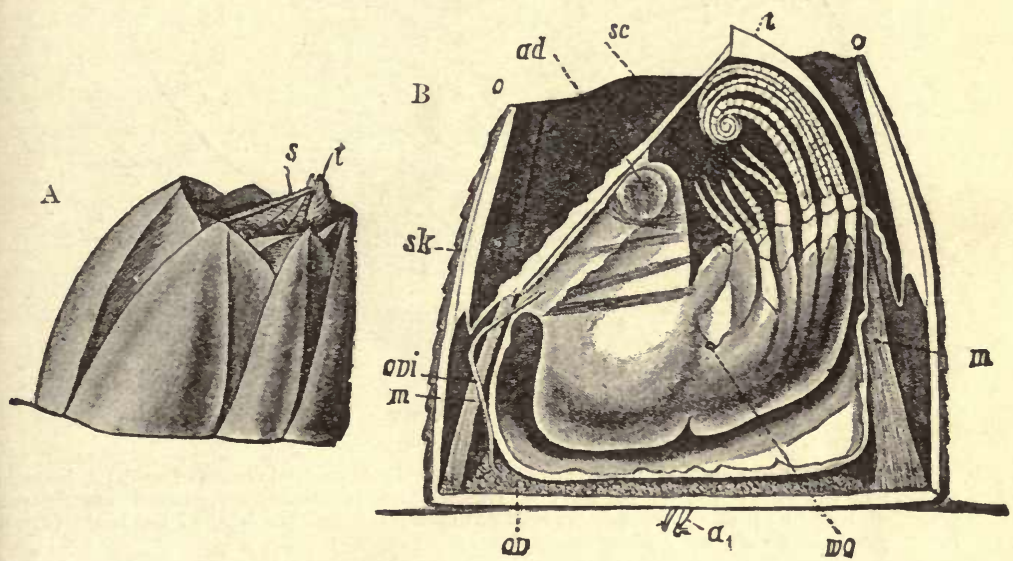

Fig. 455.-Balanus. A. external view; B, anatomy. $a_{1}$. antennules; ad. adductor muscle ; $m$. muscles of scuta and terga; $o$, edge of parapet; ov. ovary; ovi. oviduct; 8 . scutum; $s k$. parapet; $t$. tergum; 200. female aperture. (From Lang's Comparative Anatomy, after Darwin.)

Proteolepas, also parasitic on other Cirripedes, has a maggot-like, segmented, limbless body, and a suctorial mouth.

The Rhizocephala are represented by Sacculina (Fig. 456), parasitic on Crabs, and Peltogaster on Hermit-Crabs. Both genera have the appearance of an immense tumour $(k s)$ on the abdomen of the host, showing no sign of segmentation, no appendages, no mouth or anus. From the attached end go off a number of delicate rootlike filaments, which extend through the body of the host and absorb nutriment. Obviously degeneration is here as complete as it can well be, and nothing but the developmental history of the parasite (p. 599) would justify its inclusion among the Crustacea.

The most striking general character in the external features of the Malacostraca is the limitation in the number of segments. The 
head has the same composition as in the Entomostraca, but the thorax is invariably formed of eight segments, and, except in the Phyllocarida, the abdomen of six segments and a telson.

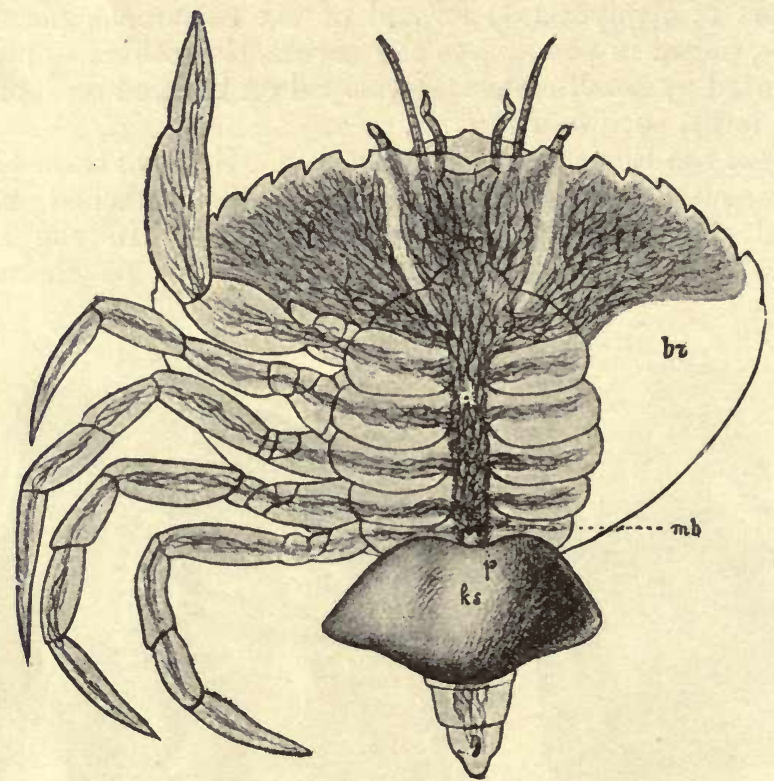

FiG. 456. - Sacculina carcini, on abdomen of crab. $l r$. branchial region of crab; $l$, hepatic region; $d$, intestinal region; $k s$, body of parasite; $p$. peduncle; $m b$, basilar membrane, giving off root-like processes which are seen extending through the body of the host. (From Iang's Comparative Anutomy, after Delage.)

The limbs are strikingly modified for the performance of various functions.

The Phyllocarida are interesting from the fact that they are annectent or linking forms between the Branchiopoda and the Copepoda on the one hand, and the higher Crustacea, particularly the Schizopoda and Decapoda, on the other. The order contains only three genera, the commonest of which, Nebalia (Fig. 457), is a little shrimp-like marine Crustacean about 6-8 $\mathrm{mm}$. in length. The body is divisible into head, thorax, and abdomen, all having the normal malacostracan number of segments except the abdomen, which is formed of eight segments, the last bearing caudal styles-structures not found elsewhere in the sub-class. There is a bivalved cephalic carapace $(s)$, closed by an adductor muscle $(\mathrm{sm})$ and extending backwards to the fourth abdominal segment: it is terminated in front by a movable rostrum $(r)$. 
The eyes $(a)$ are large, compound, and raised on movably articulated stalks. The antennules $\left(a_{1}\right)$ and antennæ $\left(\alpha_{2}\right)$ are large, the mandibles (md.) have palps $(m t)$, and the exopodite of the second maxilla (mxt) has the form of a slender filament which acts as a "cleaningfoot" to keep the cavity of the carapace free from foreign bodies. There are eight thoracic appendages $(b \cdot f)$, all of them leaf-like, and recalling those of Apus. The first four abdominal appendages $(p 1-p 4)$ are large biramous swimming-feet, like those of Copepods; the fifth and sixth $(p .5, p 6)$ are small and uniramous.

The Syncarida (Anaspidacca) (Fig. 4.58) are small, shrimp-like, fresh-water Crustaceans, which, though resembling the rest of the Malacostraca (Eumalacostraca) in the presence of only six segments in the abdomen and the absence of caudal styles, differ from them in the possession of a combination of features which connect them more closely with certain fossil forms of Carboniferous age. Thus there is no carapace, the thoracic appendages are provided with slender respiratory exopodites, and bear a double series of epipodites or branchiæ; there are stalked eyes and a fan-like tail-fin formed of the telson and the expanded uropods.

The Mysidacca (Fig. 459)

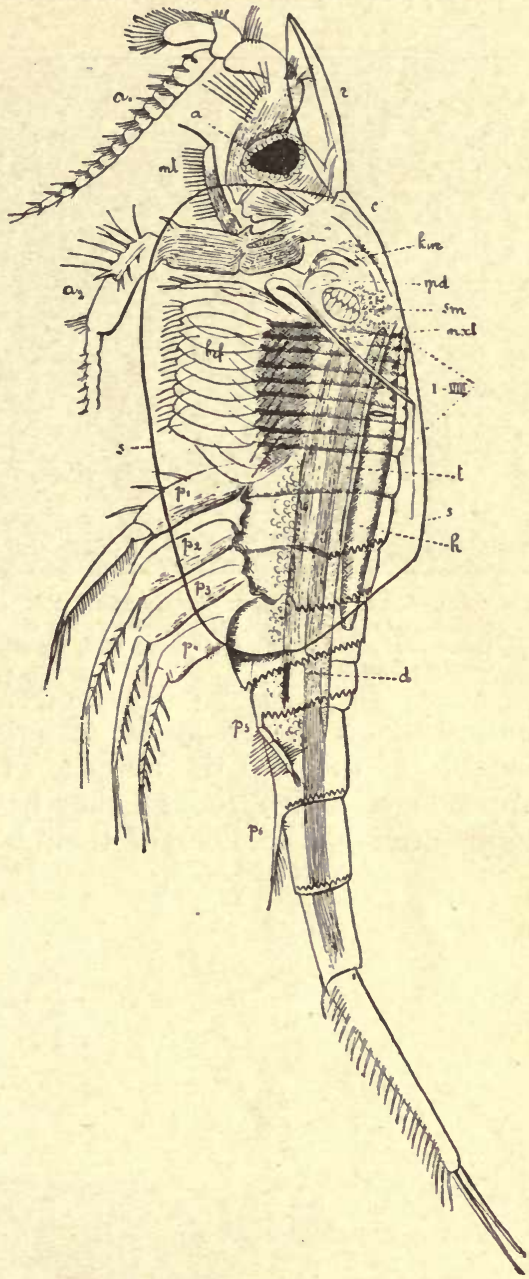

FIG. 457.-Nebalia geoffroyi, male, a. eye; $a_{1}$, anteunule; $a_{2}$, antenna ; $c$, head ; brf. thoracic feet; $d$, intestine; $h$. heart; $l m$, gizzard ; $m l$. mandible; $m t$, mandibular palp; mnt. exopodite of secund maxilla; $p_{1}-p_{4}$, pleopods ; $v$. rostrum ; 8 , carapace ; $8 m$, adductor muscle ; $t$. testis; I-VIII, thoracic segments. (From Lang's Comparative Anatomy, after Claus.)

are small, transparent, shrimp-like forms, mostly from $2-6 \mathrm{~mm}$. in length. They agree with the Crayfish in the general form of the body, in the union of the head and thorax, in the 
presence of a carapace-which leaves some of the posterior thoracic segments free-and in the number both of segments and appendages, but present several interesting characters

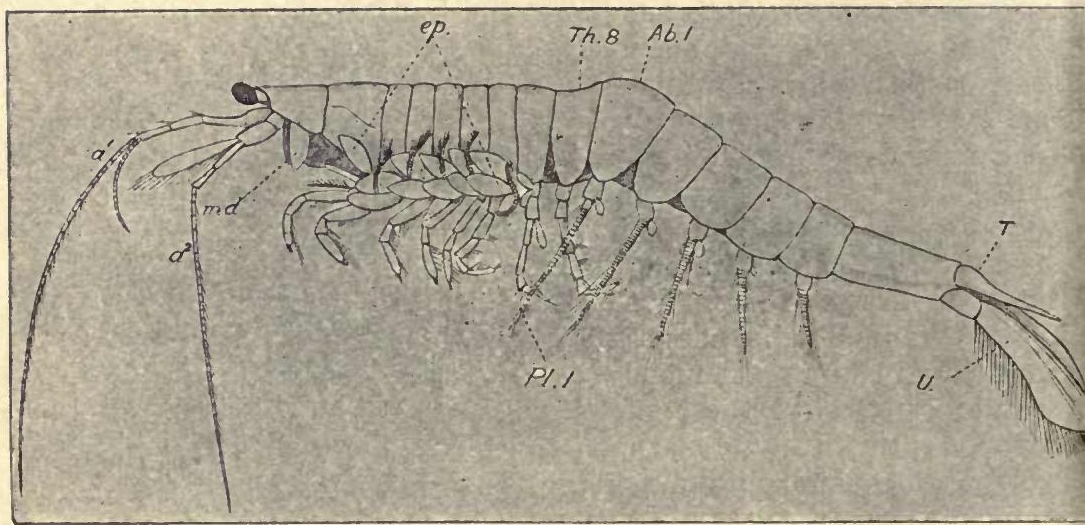

Fig. 458.-Taranaspides lacustris, $\times 4$. $a^{1}$, antennules; $a^{2}$, antennæ; $A b .1$, first abdominal segment : ep, epipodites or gills on the thoracic legs; ned, mandible; Pl.1, first abdominal appendage; $T$, telsun; $T h .8$, eighth free thoracic segment; $U$, uropod. (After Geoffrey Smith.)

indicating a lower grade of organisation. One of the most notable of these is the absence of differentiation in the thoracic appendages, which, though they have a leg-like and not a leaf-like form, are all alike, none of them being modified into maxillipedes,

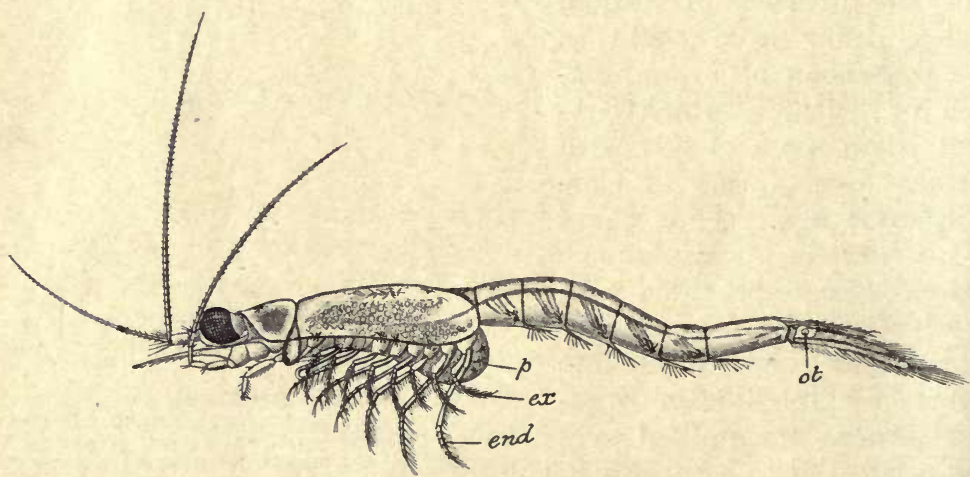

Fig. 459.- Mysis oculata. end. endopodite; ex. exopodite ; ot. otocyst. (After Gerstaeeker.)

except to a very slight degree in some forms. Moreover, the legs all possess exopodites $(e x)$, thus retaining the primitive biramous or "split-footed" form which is lost in the Decapoda. The first five pleopods are large in the male, small in the female: the sixth 
is a uropod, i.e., assists the telson in the formation of the characteristic malacostracan tailfin: there is no trace of the entomostracan caudal styles

The Cumacea are also a very small group: Diastylis (Fig. 460) is a good example. They are little shrimp-like animals, differing from all the Malacostraca previously considered in having poorly developed sessile eyes, sometimes fused together, and in some genera altogether absent. The carapace $(c t h)$ is so small as to leave the five posterior segments ( $t h I V-$ $V I I I)$ uncovered. The first two pairs of thoracic limbs are maxillipedes, the last six, legs: of these two or three pairs have exopodites (ex).

The Tanaidacea, the Isopoda and the Amphipoda are often grouped together under the heading of Arthrostraca. These orders, particularly the two last, comprise a great number of genera and species, many of them strangely modified in correspondence with special habits of life. The best known examples of the Amphiporla are the little Fresh-water Shrimp (Gammarus, Fig. 461) and the Sandhoppers (Talitrus, Orchestic) so common on the sea-shore. Of the Isopoda very convenient examples are Asellus (Fig. 462), common in fresh-water, and the well-known Wood-lice or Slaters (Oniscus, Fig. 464, 1), found under almost any piece of wood, stone, \&c., which has lain undisturbed on the ground for a few weeks.

The body is usually compressed or flattened from side to side in Amphipods (Fig. 461), depressed or flattened from above down-

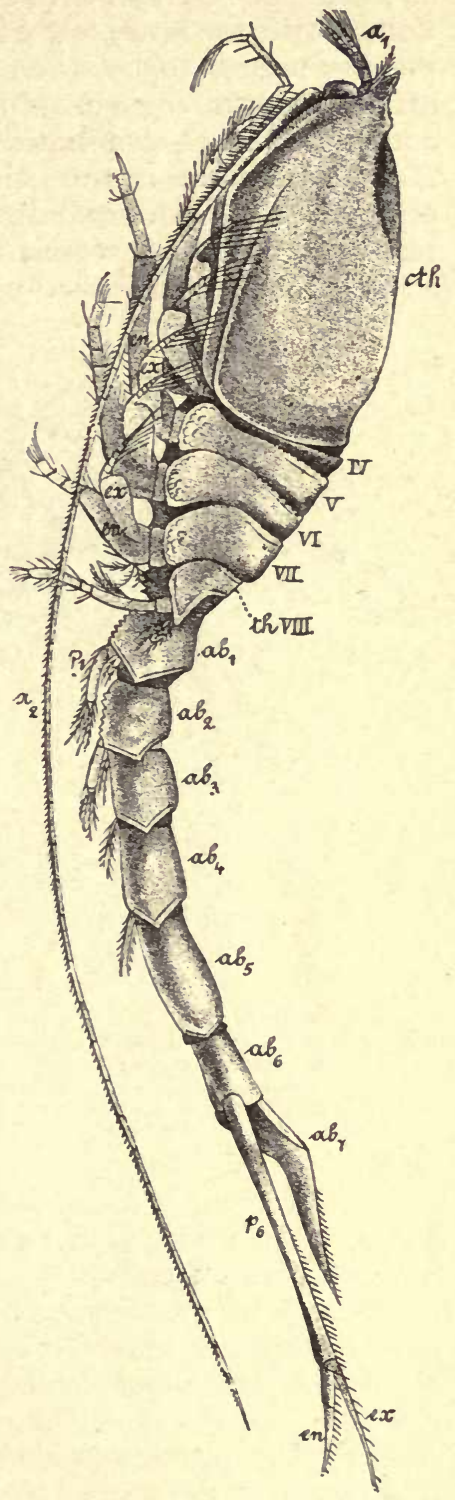

FIf. 460.-Diastylis stygia. $a 1$, antennule; $a^{2}$, antenna; ab.1-ab.7, abdominal segments; eth. cephalothorax ; en, endopodite; ex, exopodite; p.1,p.6, pleopods ; IV-VII, th $V I I I$, free thoracic segnients. (From Lang's Comparative Anatomy, after Sars.) 
wards in Isopods (Fig. 462). The normal malacostracan number of segments is present, but the first thoracic segment is always united with the head, so that the apparent head is really an incomplete or partial cephalothorax (c.th). In the Tanaidacea (Tancis, \&c.) the second segment of the thorax also unites with the head, and such forms-sometimes included under a distinct sub-order, Anisopoda-form a transition to the other Malacostraca, and especially the Cumacea. In the Amphipoda and Isopora, the posterior seven tharacic segments $\left(t h .2-t h \delta^{\prime}\right)$ are free, and those of the short abdomen are usually free in Amphipods (Fig. 461, abd.

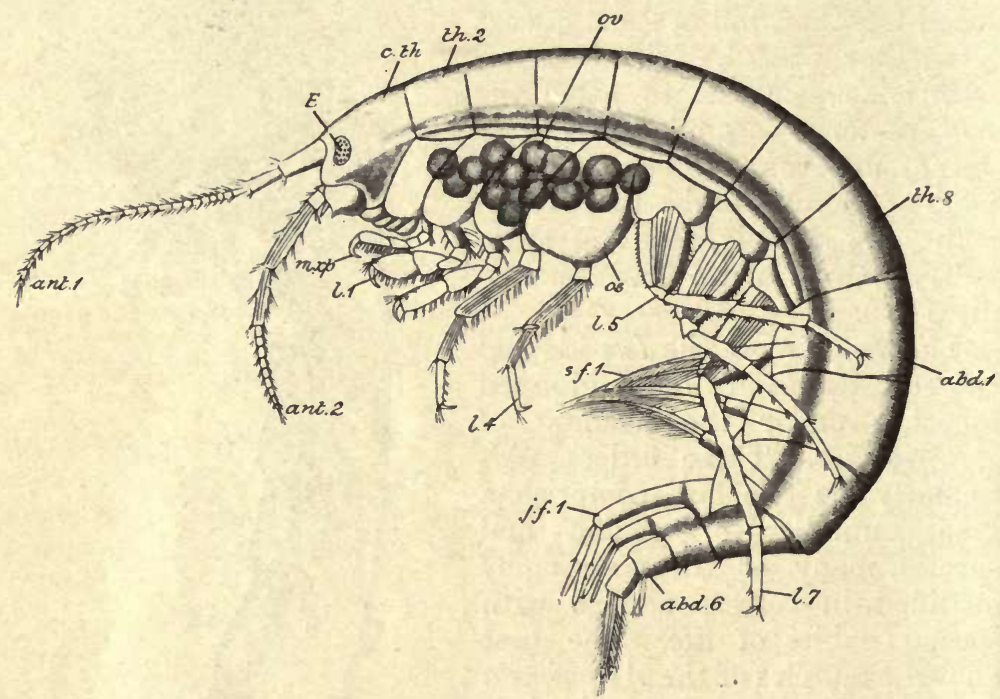

Fig. 461.-Gammarus neglectus. alul.1-ubd.6, abdominal segments; ant.1, antennule; ant. 2, antenna; cth. cephalothorax ; $E$. eye ; $j$. $f$. 1 , first jumping foot ; $l$. $1-l$. 7 , legs; mxp. maxillipsde; os. onstegite; ov. ova; $8 . f .1$, first swimming foot; th.s-th. , free thoracic segments. (After Gerstaecker.)

1-6), often more or less fused in Isopods (Fig. 462, $a b d$ ). In some Isopoda the thoracic segments are produced laterally into large and prominent pleura.

The eyes $(E)$ are compound and usually sessile : they are, however, stalked in some of the less specialised members of the order, a circumstance which lends support to the view that the sessile eyes have, in this particular group, arisen by the atrophy of eyestalks. The antennæ (ant.2) as well as the antennules (ant.1) are uniramous, or the former bear a minute exopodite. The first pair of thoracic appendages $(m x p)$ are modified to form maxillipedes, which are sometimes united together in the middle line so as to form a sort of lower lip. The remaining seven thoracic appendages take the form of legs $(l .1-l .7)$ which are usually arranged in 
two groups, four of them directed forwards and three backwards, or vice versâ. The-legs end either in simple claws or in large subchelæ: vestigial exopodites are present in some Tanaidacea. In the female, certain of the legs bear flat plates, the oostegites (Fig. 461, os), probably modified epipodites, which enclose a broodpouch for the reception of the eggs. In Amphipods the gills are also borne on the legs.

The abdominal appendages are very different in the two orders. In Amphipoda the first three are biramous swimming-feet (Fig.
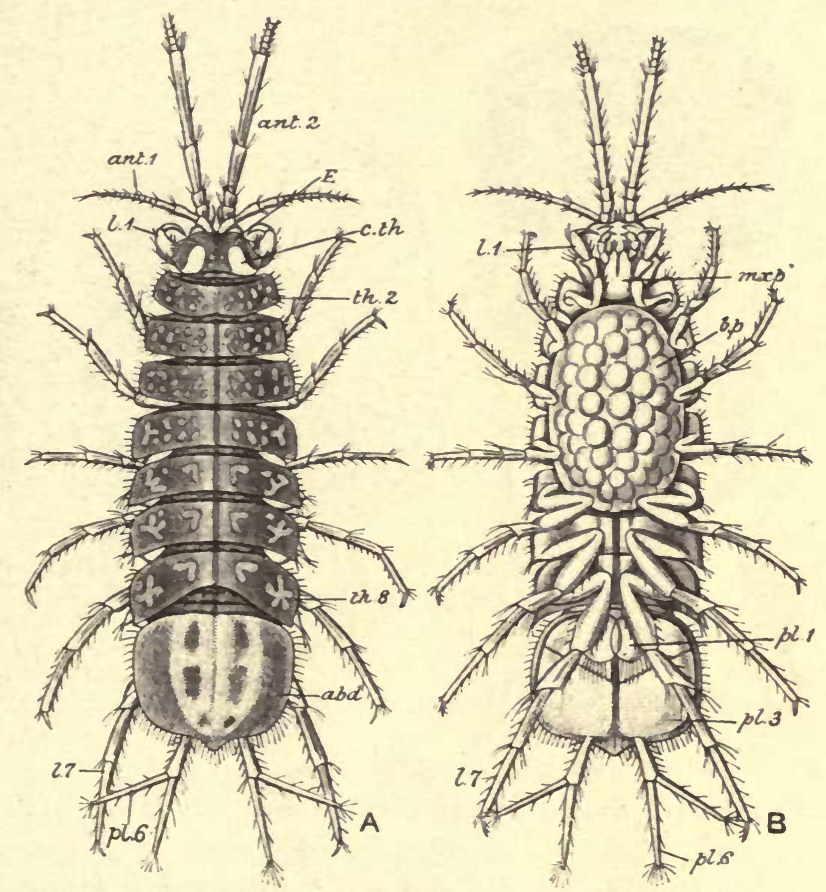

FIG. 462.-Asellus aquaticus. A, dorsal ; B, ventral view. abl, abdomen; ant.1, antennule ; int.2, antenna; $b p$. brood-pouch; $c . t h$, cephalothorax: $E$, eye; $l .1-l .7$, legs; $p l .1-p l .7$, pleopods ; th.2-th.8, free thoracic segments. (After Gerstaecker.)

$461, s f$.), the last three peculiar stiff processes used for jumping $(j f)$. In Isopods more or fewer of the pleopods have broad platelike endo- and exopodites (Fig. $462, p l .3$ ), the former thin and vascular and acting as gills: the sixth pair $(p l .6)$ are either leglike or aid in the formation of a tail-fin.

Interesting modifications occur in both sub-orders. Among the Amphipoda, Phronima (Fig. 463,1) is a marine form of glassy transparency, the female of which inhabits a transprarent barrellike structure-the test of a pelagic Tunicate-in which she 
brings up her young. Caprella (3) is a singular creature in which the abdomen is quite vestigial, and the rest of the body, as well as the appendages, extremely slender. It creeps about on colonies of Hydrozoa and Polyzoa, to the branches of which its own form and colour are so closely assimilated as to render it difficult of detection. The allied Cyamus (Whale-louse, 2) is parasitic on the skin of whales: it also has a vestigial abdomen, but the body -exceptionally among Amphipods-is broad and depressed, and the legs are curiously swollen.

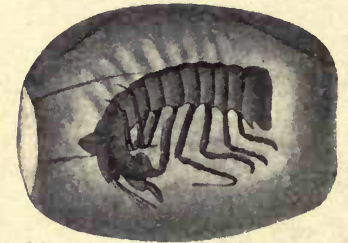

1.Phronima

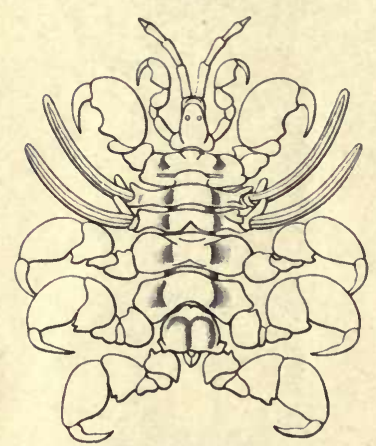

2. Cy a mus

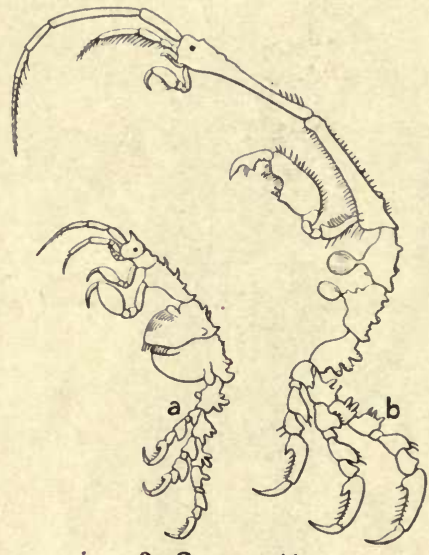

3. Caprella

Fig. 463.-Amphipoda. 3, $a$, male; $b$, female. (After Gerstaecker, and Bate and Westwood.

Among the Isopoda, one of the most interesting forms is the common Wood-louse (Fig. 464, 1), which is almost unique among Crustacea for its perfect adaptation to terrestrial life. The allied "Pill-bugs" (Armadillidium, 2) have the habit of rolling themselves up into a ball when disturbed. Cymothoa and its allies are large species $(6-8 \mathrm{~cm}$. in length) parasitic in the mouths of Fishes, where theyhold on to the mucous membrane with their short, clawed legs: their mouth-parts are often modified for sucking. In the Bopyrini, found in the gill-cavities of various Crustacea, parasitism is accompanied by great degeneration and asymmetry (3) as well as by a notable degree of sexual dimorphism, the males $(3, \mathrm{~b}, \mathrm{~m})$ being very small and permanently attached to the bodies of the females. Lastly, in Cryptoniscus, parasitic on Crabs, the adult female $(4, \mathrm{~b})$ has 
no trace of crustacean organisation, and it is only by the study of development that its true systematic position can be guessed.

In the division Eucarida, the Euphausiacea (Fig. 472) are pelagic forms in which none of the thoracic appendages are modified so as to take the form of maxillipedes, and in which there is only a single series of branchiæ (podobranchs).

Amongst the Decapoda are included nearly all the largest and most familiar Crustacea-the Prawns and Shrimps, Lobsters, Crayfishes, and Crabs. The cephalothorax is always completely covered by the carapace. The three anterior pairs of thoracic appendages are modified into maxillipedes, which retain the original biramous character, but the five posterior pairs are enlarged, and form legs,

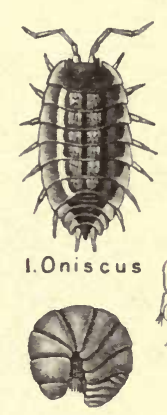

2. Armadillidium.

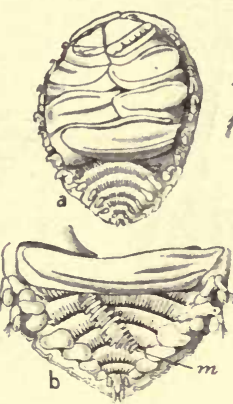

3. Gyge.
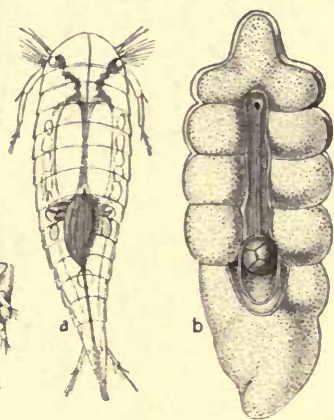

4. Cryptoniscus.

FIG. 464.-Isopoda. $3, a$. entire animal ; $b$, posterior end with attached male $(m)$; $4, a$, larva ; $b$, adult female. (After Cuvier, Claus, and Gerstaecker.)

which are always-except as an individual variation-devoid of exopodites in the adult.

In the Shrimps and Prawns (Fig. 465) the body is compressed, and the exoskeleton is not calcified. The abdomen is very large in proportion to the cephalothorax, and has a peculiar bend close to its junction with the thorax. The legs are very slender, are used for swimming, not walking, and sometimes one pair, sometimes another, is enlarged to form the chelipeds. The rostrum is large - sometimes longer than the rest of the carapace-and the eyestalks, antennæ, and legs may attain extraordinary dimensions.

The Lobsters and fresh-water Crayfishes agree with Astacus in all essential details, but the sea-Crayfishes (Palinurus) present some striking modifications. There are no chelæ, the legs all ending in simple claws : the antennæe are of immense size, and their proximal segments are fused with one another and with the carapace, quite crowding out the epistoma: the rostrum is reduced, or even vestigial, and the pleopods are very broad and fin-like. In Scyllarus (Fig. 466) and its allies the body is broad and depressed, the bases 
of the legs widely senarated from one another by the broad sterna, the antennæ (ant. 2) short and plate-like, and the eye-stalks $(E)$ enclosed in socket-like grooves of the carapace. Most of

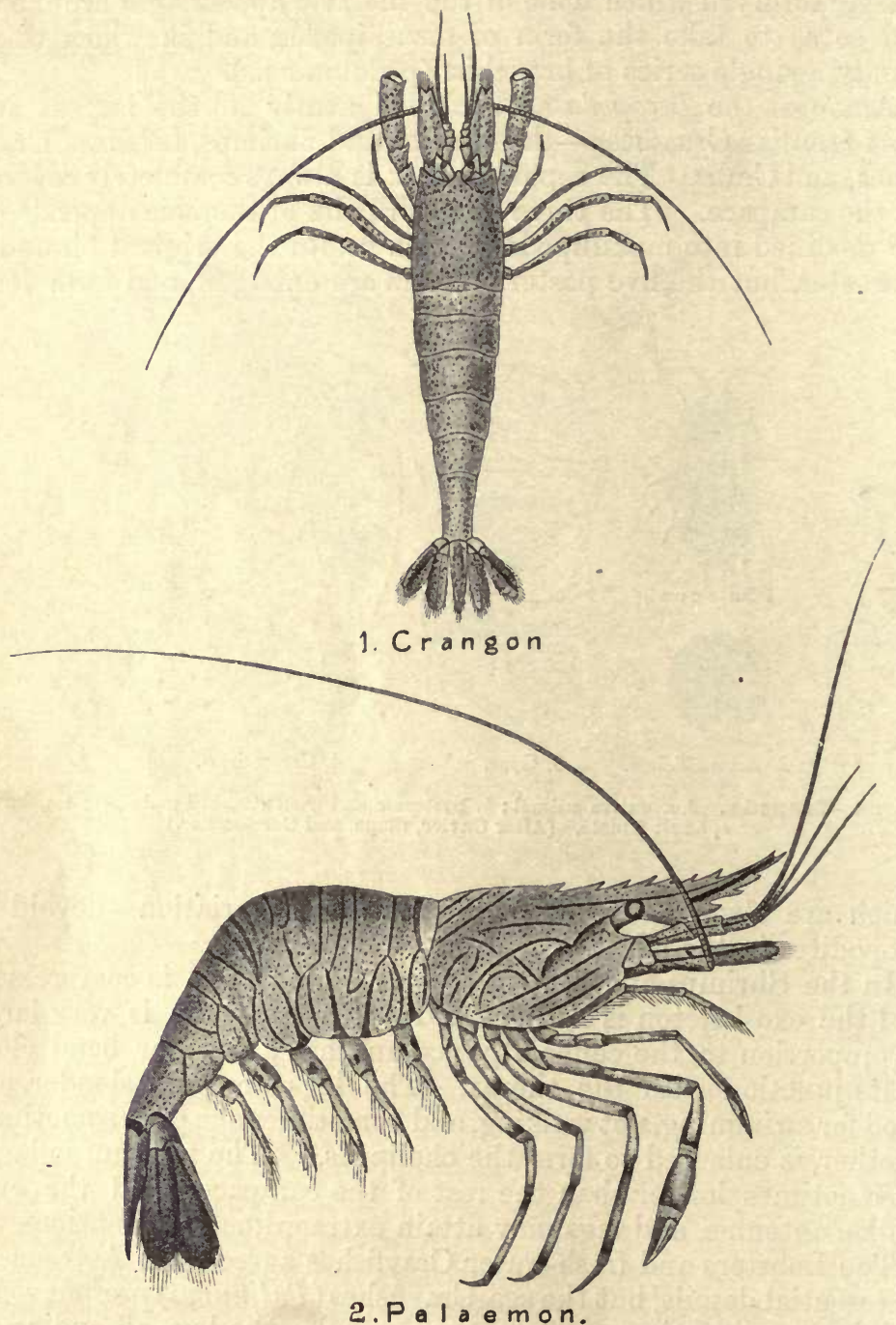

I'IG. 465.-Shrimp (dorsal view) and Prawn (side view). (After Cuvier.)

these characters show an approximation to what is found in the Crabs.

Of the Anomura, the Hermit-crabs (Pagurus, \&c., Fig. 467) are very strangely modified in relation with their peculiar mode 
of life. They are always found inhabiting the empty shells of Gastropods (Whelks, Periwinkles, \&c.), the abdomen, which has become spirally twisted, completely enclosed within the shell and only the cephalothorax protruding. In correspondence with this mode of protection, the abdomen is soft, having only vestiges of terga $(t)$ on the dorsal side, and its appendages are more or less atrophied except the sixth pair (up), which take the form of a pair of hooks, and are used to hold on to the columella of the shell. The fifth pair of legs (l.5) are much reduced, and in

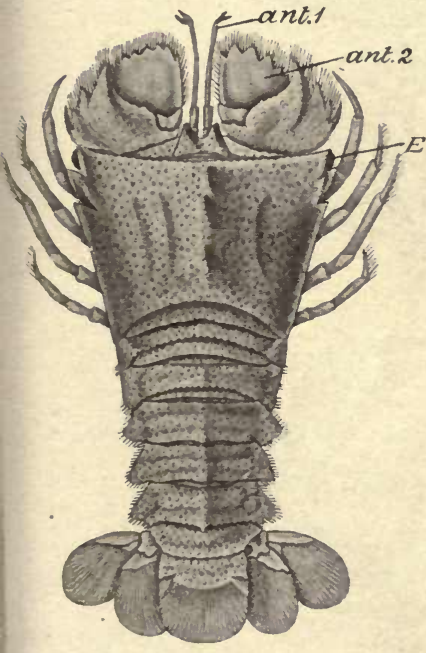

Fig. 466.-Scyllarus arctus. ant.1, antennule; ant.2, antenna; $B$, eye. (After Cuvier.)

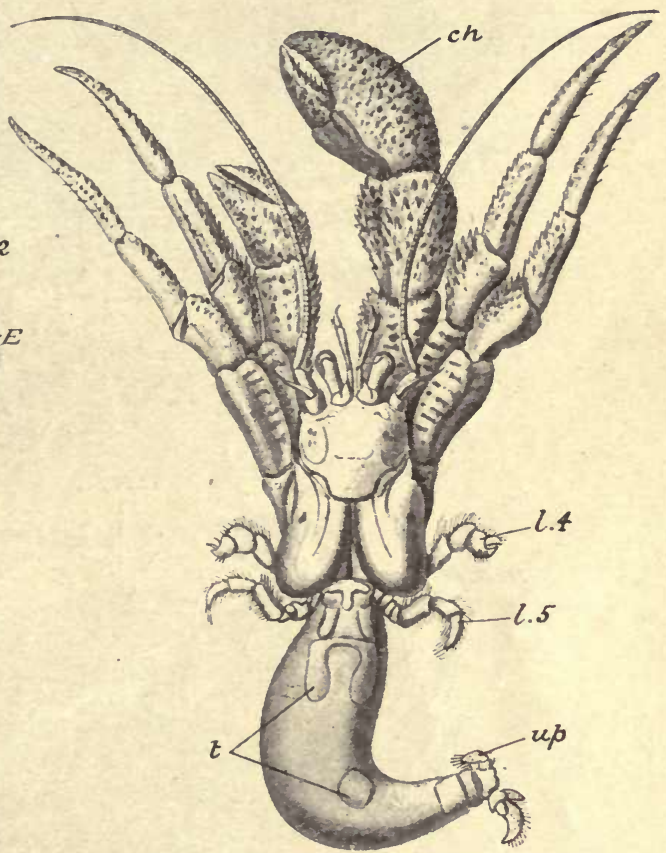

Fig. 467:- Pagurus bernhardus. ch. chela of first right leg; $l .4, l .5$, fourth and fifth legs; $t$, abdominal terga; $\boldsymbol{w}$. urupods. (After Bell.)

some species one of the chelipeds is greatly enlarged and its chela (ch) acts as an operculum, completely closing the mouth of the shell when the animal is retracted. As the Hermit-Crab grows it takes up its abode in larger and larger shells, sometimes killing and removing piecemeal the original inhabitant.

Other Anomura, such as the Cocoa-nut Crab (Birgus), Hippa, \&c., approach the Brachyura in the short, more or less permanently flexed abdomen, but are clearly separated from them by the structure of the head and its appendages.

In the Brachyura, or true Crabs, we reach the highest degree of 
specialisation known among the Crustacea. The cephalothorax (Fig. 468) is always of great proportional breadth, and is frequently much broader than long. The abdomen, on the other, hand is greatly reduced, its sternal region is uncalcified, and it lies permanently
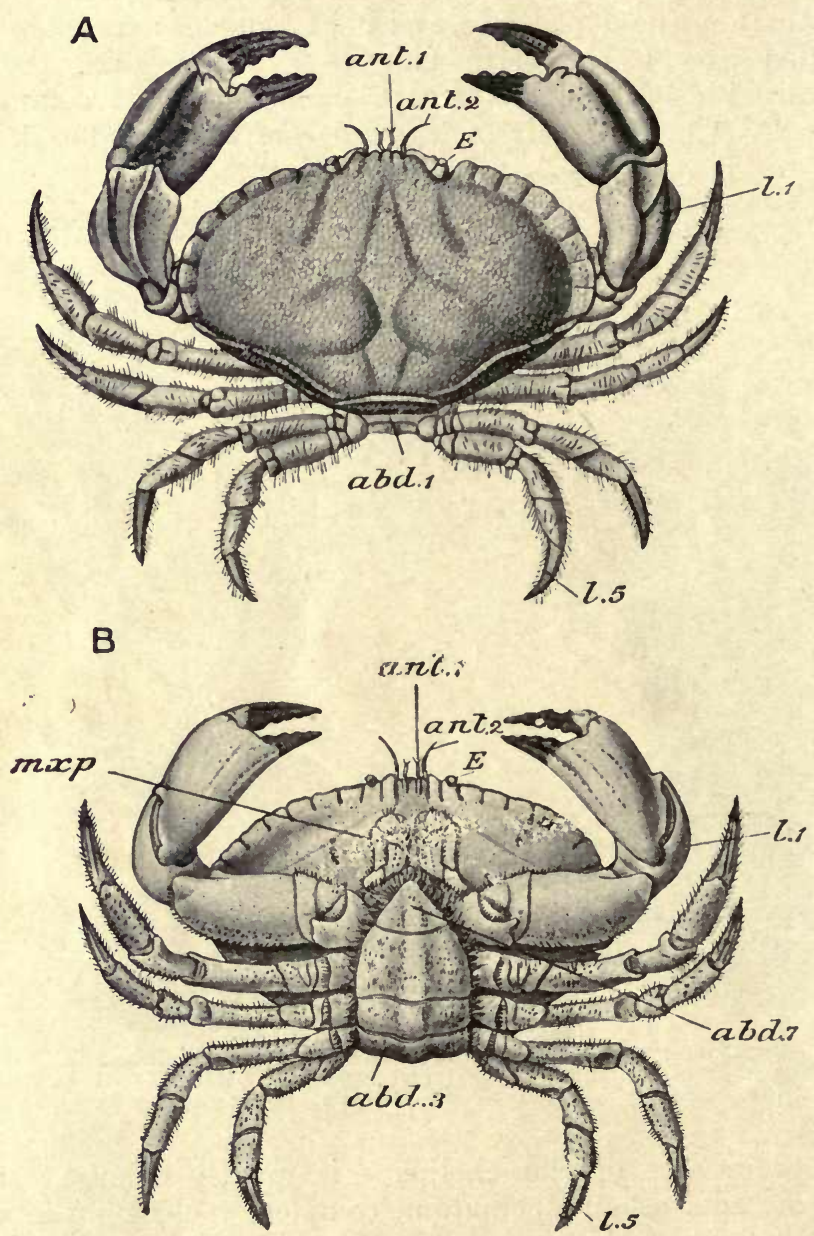

FiG. 46S.-Cancer pagurus. A, dorsal, B, ventral aspect. ant.1, antennule ; ant.2, antenna ; $a b d .1, a b d .3, a b d .7$, abdominal segments ; $B$, eye-stalk; $l .1, l .5$, legs ; mxp.s, third maxillipedes. (A, after Bell.)

flexed in a groove on the very broad thoracic sterna, so as to be often quite hidden in a view from above. In correspondence with this the pleopods are much reduced, the male retaining only two pairs as copulatory organs, the female four pairs for the attachment of the eggs. The uropods are absent, so that there is no tail-fin. 
The eye-stalks $(E)$ are contained in orbits or sockets of the carapace, which are so prolonged that the eyes appear to arise behind the antennules and antennæ. Both pairs of feelers are small, and the
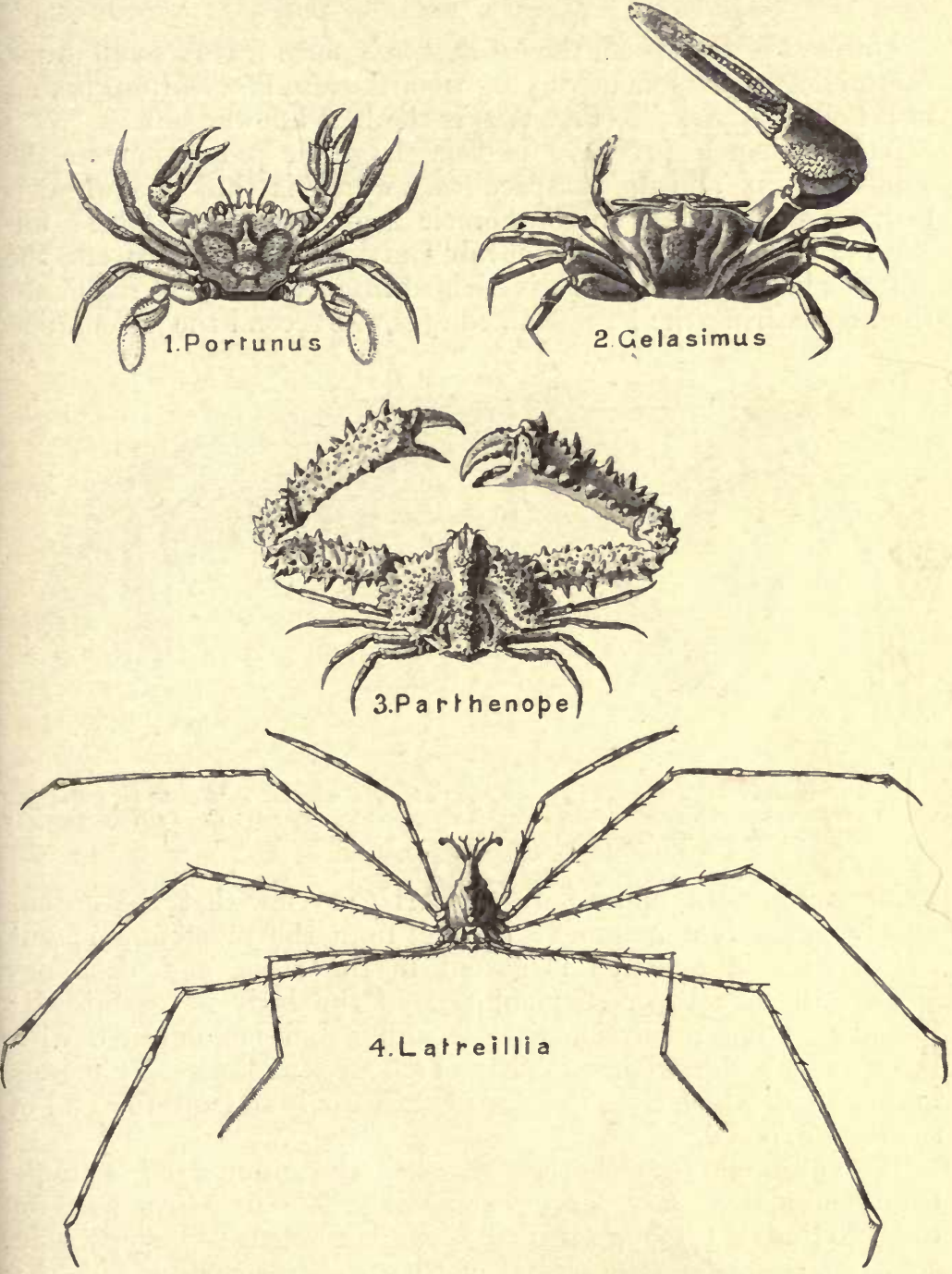

Fig. 469.-Typical Brachyura, (After Bell_and de:Haan.)

bases of the antennules are contained in sockets or fossettes. The third maxillipedes (mxp.) are broad, flat, and valve-like, not leglike as in the Macrura. The first legs (l.1) form chelipeds often of great size : the remaining legs generally end in simple claws, 
SECT.

but in the Swimming-crabs (Fig. 469, 1) the distal segment in the fifth pair is flattened and forms a tin. The range of variation in form, proportions, colour, markings, \&c., among Crabs is very great (Fig. 469).

Unlike the Decapoda, the Stomatopoda form a very small order, comprising a few genera varying from the size of a Shrimp to that of a Lobster. Squilla (Fig. 470) is the best known genus.

The abdomen $\left(a 1-a^{y}\right)$ is very large in proportion to the cephalothorax, and the carapace (cth), which is thin and uncalcified, leaves the last three thoracic segments (VI-VIII) uncovered. The rostrum is movably articulated, and covers the anterior head-region, which is divided into two distinct segments, the first bearing the large stalked eyes, the second the antennules.

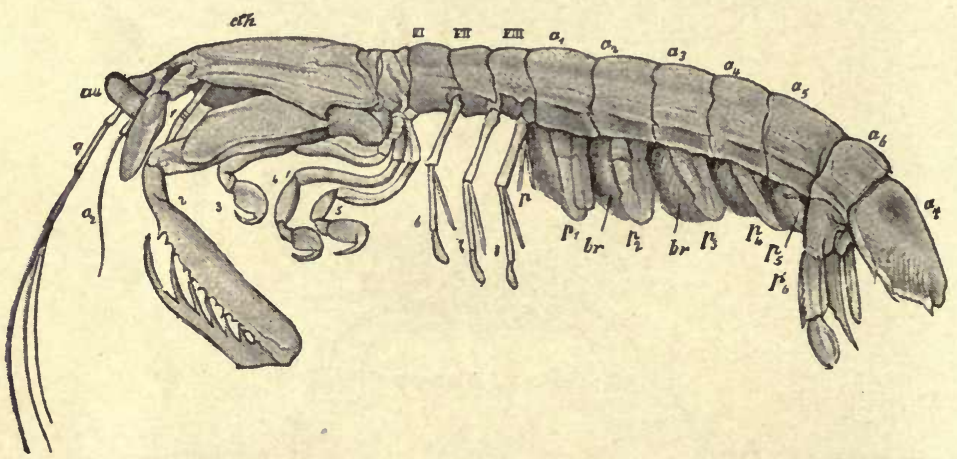

Fia. 470.-Squilla. $a 1$, antennule; $a 2$, antenna ; $a 1-a 7$, abdominal segments; $b r$, gills ; $c t h$, cephalothorax ; $p$, copulatory organ ; $p 1-p 5$, pleopods : $p 6$, uropods ; $V I-V I I I$, free thoracic segments; $1-8$, thoracic appendages. (From Lang's Comparative Anatomy.)

This arrangement appears to support the view that the antennulary region is a metamere distinct from the prostornium; but the division in question is absent in the larva, and does not appear till the proper segmentation of the body is established: probably it has a physiological meaning, and is connected with the necessity of extreme mobility of the eyes and olfactory organs in an animal which lives in a burrow with only the anterior end of the head exposed.

The antennule ( $\alpha 1)$ has three flagella; the antenna (a2) a single flagellum and a very large exopodite. The first five pairs of thoracic limbs $(1-5)$ are turned forwards towards the mouth, and act as maxillipedes; the second of these-corresponding with the second maxillipede of Astacus - is very large (2), and its distal segment is turned back and articulated to the penultimate segment like the blade of a pocket-knife to the handle. In this way a very efficient weapon called a sub-chela is produced, both of the segments of which are produced into strong spines. The re- 
maining three thoracic appendages $(6-\delta)$ are slender legs provided with exopodites: the last of them has a styliform copulatory organ $(p)$ developed from its proximal segment. The pleopods are large and biramous : the first five $(p l, p .5)$ have gillfilaments $(b r)$ attached to their plate-like exopodites: the sixth ( $p 6$ ) form large uropods or lateral tail-lobes, as in Astacus.

With regard to the texture of the exoskeleton, there is every graduation from the delicate polished cuticle of most Branchiopoda, Ostracoda, Copepoda, \&c., through the calcified but still flexible cuticle of Astacus, to the thick, tuberculated, stony armour of many Crabs (Fig. 469,3), or the shelly pieces of Cirripedes. The exoskeleton is secreted from a single-layered ectoderm, and undergoes periodical moults or ecdyses. There is no transverse layer of muscle, and the longitudinal layer is broken up into paired dorsal and ventral bands. As a rule, each limbsegment is acted upon by two muscles: the joints are nearly always hinge-joints.

The body-cavity consists of several chambers separated from one another by partitions. In Palamonetes, one of the Prawns, there is a median dorsal chamber enclosing the ophthalmic artery, and not containing blood: it is probably a portion of the coelome in the strict sense of the word. The cavities of the gonads are also coelomic, and the ducts by which they communicate with the exterior are probably modified cœlomoducts. In addition to these cavities there is a large central space, in which the enteric canal, digestive glands, gonads, \&c., lie ; paired lateral spaces containing portions of the shell-gland; spaces in the limbs; and the pericardial sinus, in which the heart lies. All these cavities contain blood, and constitute a kind of secondary body-cavity, formed by the enlargement of blood-vessels, which have largely replaced the true colome. Such a secondary or blood-containing body-cavity is called a homocoele.

The enteric canal consists of a vertical gullet, an expanded "stomach," and a nearly straight horizontal intestine. In some of the Cladocera the intestine is coiled, but this is quite exceptional. In the lower Crustacea, part or the whole of the "stomach" is formed from the mesenteron, but in Malacostraca both gullet and "stomach" (gizzard) are developed from the stomodæum. A "gastric mill" is present in Malacostraca, and a rudiment of such an apparatus occurs in Ostracoda. The digestive glands are usually branched cæea formed as offshoots of the mesenteron: in the Isopoda and Amphipoda (Fig. 471, l) they are unbranched crca extending into the abdomen : in Stomatopoda they consist of ten metamerically arranged organs opening into the intestine. In Amphipods there are intestinal cæca ( $\iota d)$ which may have an excretory function. So-called salivary glands, opening on the labrum, have been found in several genera. 
In most of the the Branchiopoda, Ostracoda, Copepoda, and Cirripedia, respiration takes place by the general surface of the body, and the only respiratory organs are specially modified parts of the appendages. In the stalked Barnacles, however, there are delicate

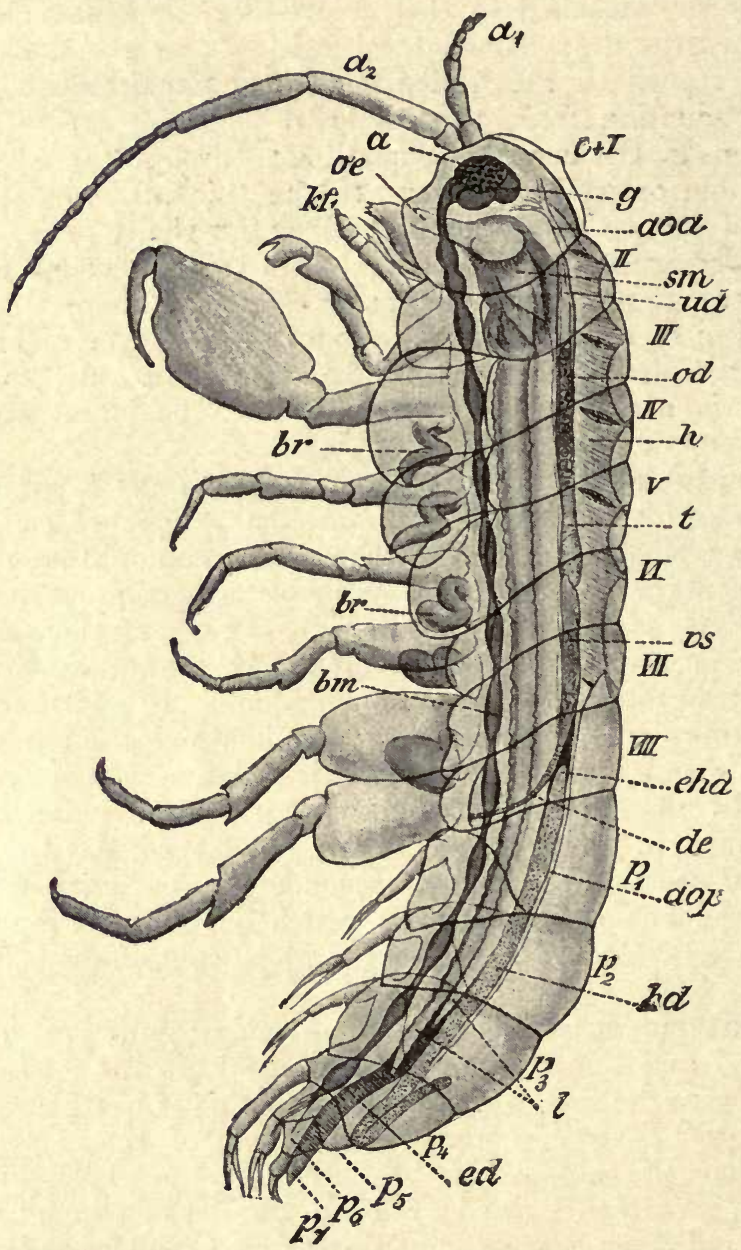

Fig. 471.-Orchestia cavimana, male. $a$, eye ; $a_{1}$, antennule ; $a_{2}$, antenna ; $a \circ a$, anterior aorta ; $a o p$, posterior aorta ; $b m$, ventral nerve-cord; $b r$, gills ; $C+T$, eephalothorax; $d e$, vas deferens ; ed, rectum; $g$, brain; $h$, heart ; $h l$, intestine ; $k f$, maxillipede ; $l$, digestive glands ; $a$, gullet; $p$ 1-p 7, abdominal segments ; sm, "stomach"; ud, intestinal execum ; vs. vesicula seminalis; $t$, testis; $I I-V I I I$, free thoracic segments. (From Lang's Comparative Anatomy, after Nebesky.)

processes attached to the feet, which are supposed to be rudimentary gills. Amongst the Malacostraca also, the Phyllocarida, many Mysidacea, and the Cumacea have no specialised respiratory organs, but the Euphausiacea possess tufted podobranchiæ (Fig. 472) quite 
uncovered by the carapace. In the Decapoda the gills may be either plume-like, as in Astacus and its allies, or the delicate cylindrical gill-filaments may be replaced by flat plates, as in Crabs and many Prawns. It is in this order only that we find the three types of gill described in Astacus, and the examination

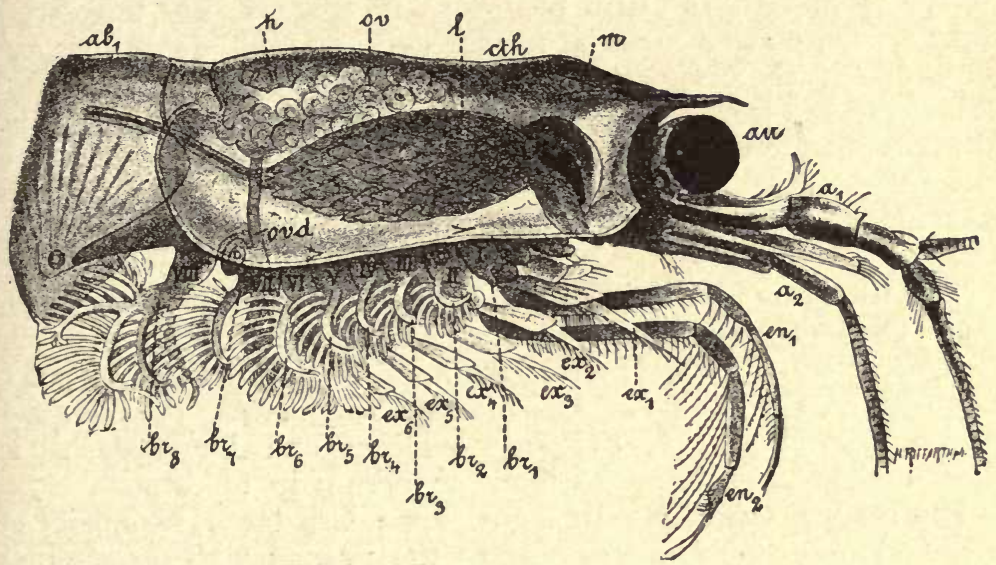

Fig. 472.-Anterior portion of Euphausia pelucida. $a_{1}$, antennule; $a n t .^{2}$, antenna; $a b .1$, first abdominal segment; $a u$, eye; $b r .1-8$, podobranchia ; cth. cephalothorax; en.1, en.2, endopodites of first two thoracie limbs; $e x .1-e x .6$, exopodites of first six thoracic limbs ; $h$. heart ; $l$, digestive gland ; $m$, "stomach" ; ov. ovary ; ovd. oviduct ; $I-V I I I$, protopodites of thoracic limbs. (From Lang's Comparative Anatomy.)

of numerous forms leads to the conclusion that the typical or theoretical branchial formula for the group is as follows :-

\begin{tabular}{|r|c|c|c|c|c|c|c|c|c|}
\hline $\begin{array}{c}\text { Thoracic } \\
\text { SEgmants. }\end{array}$ & I. & II. & III. & IV. & V. & VI. & VII. & VIII. & Total. \\
\hline Podobranchia & $1+e p$ & $1+e p$ & $1+e p$ & $1+e p$ & $1+e p$ & $1+e p$ & $1+e p$ & $1+e p$ & $8+8 e p$ \\
Arthrobranchiæ & 2 & 2 & 2 & 2 & 2 & 2 & 2 & 2 & 16 \\
Pleurobranchire & 1 & 1 & 1 & 1 & 1 & 1 & 1 & 1 & 8 \\
\hline Total ... & $4+e p$ & $4+e p$ & $4+e p$ & $4+e p$ & $4+e p$ & $4+e p$ & $4+e p$ & $4+e p$ & $32+8 e p$ \\
\hline
\end{tabular}

Actually, however, this formula never occurs, as there is always more or less reduction in the number of gills. Palinurus has the highest number known, viz., twenty-one, and in the Common Crab the total number is only nine.

Many Crabs live on land, and their gills are enabled to discharge their function in virtue of the moisture retained in the nearly closed gill-chamber. In the Cocoa-nut Crab (Birgus) the upper part of the gill-chamber is separated from the rest and forms an almost closed cavity into which vascular tufts project: it thus 
functions as a true lung. Probably the inner surfase of the gillcover or branchiostegite performs a respiratory function in the Crayfishes.

In Amphipoda, also, the gills (Fig. 471, br) are outgrowths of the thoracic limbs: in Isopods they are the modified endopodites of the second to the fifth pleopods; in some of the terrestrial forms, in adaptation to äerial respiration, a system of air-tubes are developed in the exopodites; in Stomatopoda, gill-filaments (Fig. $470, b r)$ spring from the exopodites of the first to the fifth pleopods. Moreover many Crustacea perform rhythmical contractions of the intestine, taking in and expelling water: such anal respiraticn is common among the lower groups, and is especially noticeable in Cyclops.

The heart is absent in many Copepods (including Cyclops), in some Ostracoda (including Cypris), and in Cirripedia: it is an elongated tube with several pairs of ostia in Euphyllopoda, Leptostraca, Stomatopoda, Anaspidacea, Tanaidacea, Isopoda, and Amphipoda (Fig. 471, h); in Cladocera and Decapoda it is shortened to an ovoid sac with one or more pairs of ostia.

Excretory Organs.-In many larval Crustacea two pairs of modified mesonephridia are present-the antennary glands opening on the bases of the antennix, and the maxil-

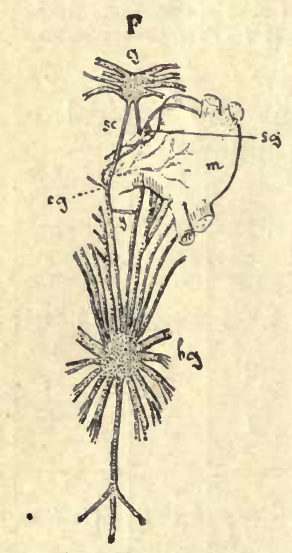

FIc. 473.-Nervous system of a Crab (Maja squinado). $b g$, thoracic ganglion; $c g$. commissural ganglion; $g$, brain ; $m$, "stomach"; $s c$, cesophageal connective; $8 g$, visceral nerves ; $y$, post-oesophageal connective. (From Lang's Comparative Anatomy, after Milne-Edwards.) lary or shell-glands opening on the bases of the second maxillæ. But as development proceeds one pair nearly always atrophies, the maxillary gland alone being usually retained in the Branchiopoda, Ostracoda, Copepoda and Cirripedia, the antennary gland in the Malacostraca. In the Stomatopoda, however, there is no antennary gland, and the function of renal excretion may be discharged by a pair of glandular tubes opening into the rectum; and in Amphipoda a similar function is assigned to cæca opening into the posterior end of the mesenteron. In some of the Cirripedia the maxillary gland is described as opening into one of the compartments of the body-cavity like a typical nephridium.

The nervous system is always formed on the ordinary arthropod type, as described in Apus and Astacus, and the chief variations it presents are connected with the greater or less amount of concrescence of ganglia. In the sessile Barnacles and in the Crabs (Fig. 473) this process reaches its limit, the whole ventral nerve-cord being represented by a single immense thoracic ganglion $(b g)$. 
The sense-organs are mostly of the same character as those of the two examples. The median or nauplius-eye always occurs in the larva, and can frequently be shown to exist in the adult of even the higher groups (Decapoda). The Cirripedia and many parasitic Copepods are eyeless in the adult, as also are certain subterranean Malacostraca. - Olfactory setæ occur, as a rule, on the antennules, and the auditory organs (or statocysts) of Decapoda are open sacs in the basal segment of the same appendages, but in Mysidacea they occur as closed cysts (Fig. 459, ot) in the endopodites of the uropods.

Reproduction.-In most Crustacea the sexes are separate, but hermaphroditism occurs in some Branchiopods, in nearly all Cirripedes, and in certain parasitic Isopods (Cymothoa). In the latter case the animals are protandrous, male organs being developed first, and female organs at a later stage. In many Cirripedia minute complemental males are found attached, like parasites, to the body of the ordinary or hermaphrodite individual, the male organs of which appear to be inadequate for the full discharge of the fertilising function. Sexual dimorphism is almost universal, and reaches its maximum in the parasitic Copepods and Isopods already referred to.

The gonads are always a single pair of hollow organs discharging their products into a central cavity or lumen, whence they pass directly into the gonoducts and so to the exterior. The gonads may be single or branched, and frequently there is more or less concrescence between those of the right and left sides, as in Astacus and Cyclops. The sperms vary greatly in form, and are usually motionless: in Cirripedia, however, they are motile, and in Ostracoda they perform movements after reaching the female ducts. In some Ostracoda they are about three times as long as the animal itself (Fig. 450, D). In many Branchiopoda and Ostracoda reproduction is parthenogenetic. In Daphnia, for instance, the animal reproduces throughout the summer by parthenogenetic summer eggs, which develop rapidly in the broodpouch (Fig. 449, 1, br.p.). In the autumn winter eggs are produced, which are fertilised by the males: they pass into the brood-pouch, a portion of which becomes specially modified and forms the ephippium or saddle. At the next moult the ephippium is detached and forms a sort of bivalved capsule in which the eggs remain in an inactive state during the winter, developing in the following spring.

Development.-In some Crustacea segmentation is complete, and a hollow blastula is formed: in others segmentation is followed by an accumulation of yolk in the interior, resulting in the formation of a superficial blastoderm, as in Astacus: in others, again, the egg is telolecithal, and the protoplasm, accumulated at one pole, divides so as to form a disc of cells 
which afterwards spreads over the whole yolk. But in most cases the egg is centrolecithal and segmentation superficial, as in Astacus.

Development is always accompanied by more or less metamorphosis. In most Branchiopoda the young is hatched in the form of a nauplius (Fig. 429, A), and further changes are of the same character as in Apus. In Cladocera development is direct, the naupliusstage being passed through in the egg, and the young hatched in a form closely resembling the adult. In one of the Cladocera, however, Leptodor a (Fig. 449,3), while development of the summer eggs is indirect, the winter eggs give rise to free nauplii. In the Ostracoda the nauplius is peculiar in having a bivalved shell and all three pairs of appendages uniramous. In all the Copepoda there is a free nauplius, which, in the parasitic forms, leads a free existence for a time, and then attaches itself to its particular host and undergoes retrograde metamorphosis.

In the Cirripedia, also, there is a free nauplius, the body of which is often produced into long spines. After several moults, the

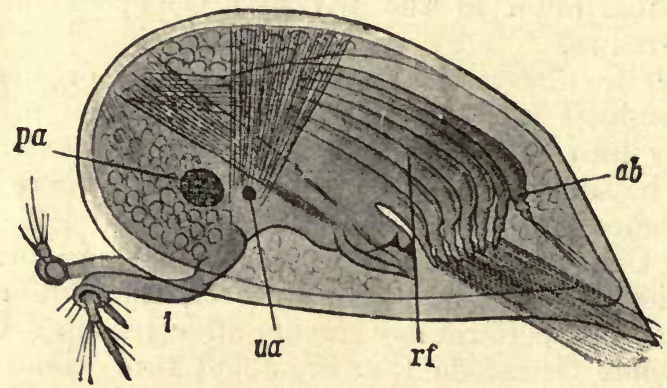

FIG. 474.-Cypris-stage of Iepas fascicularis. al, abdomen ; pa. paired eye ; $r /$, thoracic feet; ua, unpaired eye ; 1 , antennule. (From Lang's Comparative Anatomy, after Claus.)

nauplius passes into a form called the Cypris-stage (Fig. 474), characterised by the presence of a bivalved shell, like that of an Ostracod: the antennules (1) also have become modified into organs of adhesion by the development of the penultimate segment into a disc, the antennæ have disappeared, and six pairs of swimming-feet like those of a Copepod have made their appearance: there are paired compound eyes, and the shell is closed by an adductor muscle. After leading a free existence for a time, the Cyprislarva attaches itself by its antennules, aided by the secretion of cement-glands, and becomes a pupa: the carina, terga, and scuta appear beneath the shell, and within the skin of the mouthparts and legs of the pupa appear the corresponding appendages 
of the adult. In Lepas the anterior region of the head grows out into a peduncle. The pupal integument is then thrown off, the paired eyes disappear, and the adult form is assumed.

In Sacculina a still more extraordinary metamorphosis takes place. The young is hatched as a nauplius, and passes into a Cypris-stage. In this condition, after a brief free existence, it attaches itself to the body of a young Crab, near the base of a seta. The thorax with its appendages is thrown off, and the rest of the body is converted into a rounded mass, from the anterior end of which an arrow-like process is developed. This perforates the cuticle of the host, and, through the communication thus formed, the whole body of the parasite passes into the interior of the Crab, and becomes surrounded by a new cuticle, the old cuticle being left empty on the outside of the Crab's body. The Sacculina now sends out root-like processes, grows immensely, and, pressing upon the body-wall of the Crab, causes atrophy of the tissues: this allows the now greatly-swollen parasite to project on the exterior as the tumour-like adult described above (p. 579).

The embryo of Euphausia leaves the egg as a typical free-swimming nauplius; this passes into what is called the protczocea-stage, distinguished by the possession of an elongated, unsegmented abdomen without appendages. After successive moults, the rest of the appendages appear, and the adult form is assumed. In Mysis (Fig. 459) the nauplius is maggot-like, and undergoes development in the brood-pouch, emerging in a condition closely resembling the adult.

The development of the Decapoda presents a very interesting series of' modifications. In two genera of prawns (Penceus and Lucifer) the embryo leaves the egg as a nauplius, and passes by successive moults through a protozoæa stage, a zocea-stage, with segmented but limbless abdomen, and a mysis or schizopod-stage in which it resembles an adult Mysis, having exopodites to all the thoracic limbs.

In the Crabs the nauplius stage is passed through in the egg, and the young is hatched in the form of a peculiarly modified zorea (Fig. $475, \mathrm{~A}$ ), with an immense cephalothorax produced into spines, large stalked eyes, and a slender abdomen. This passes by successive moults into the megalopa-stage (B), which resembles an adult Macruran, having an extended abdomen with welldeveloped pleopods. The megalopa passes by successive moults into the adult form.

In the Lobster (Homarus) both nauplius and zoæa-stages are passed through in the egg, and the embryo is hatched in the mysis-stage with exopodites to all the thoracic limbs. In the Rock-lobster (Palinurus) and its allies, the newly hatched young is a strangely modified Mysis-form called a Glass-Crab or Phyllo- 
soma: it has broad, depressed cephalic and thoracic shields of glassy trausparency: the abdomen is very small and the legs extremely long and biramous. Lastly, in the Fresh-water Crayfish the young resemble the adult in all but proportions and certain unimportant details of structure. Thus in the series of Decapoda we get a gradual abbreviation in development, stages which are free larval forms in the lower types being hurried through before hatching in the higher.

The larva of Stomatopoda are grotesque little creatures with a very large spiny carapace. In Amphipoda there is no free larval

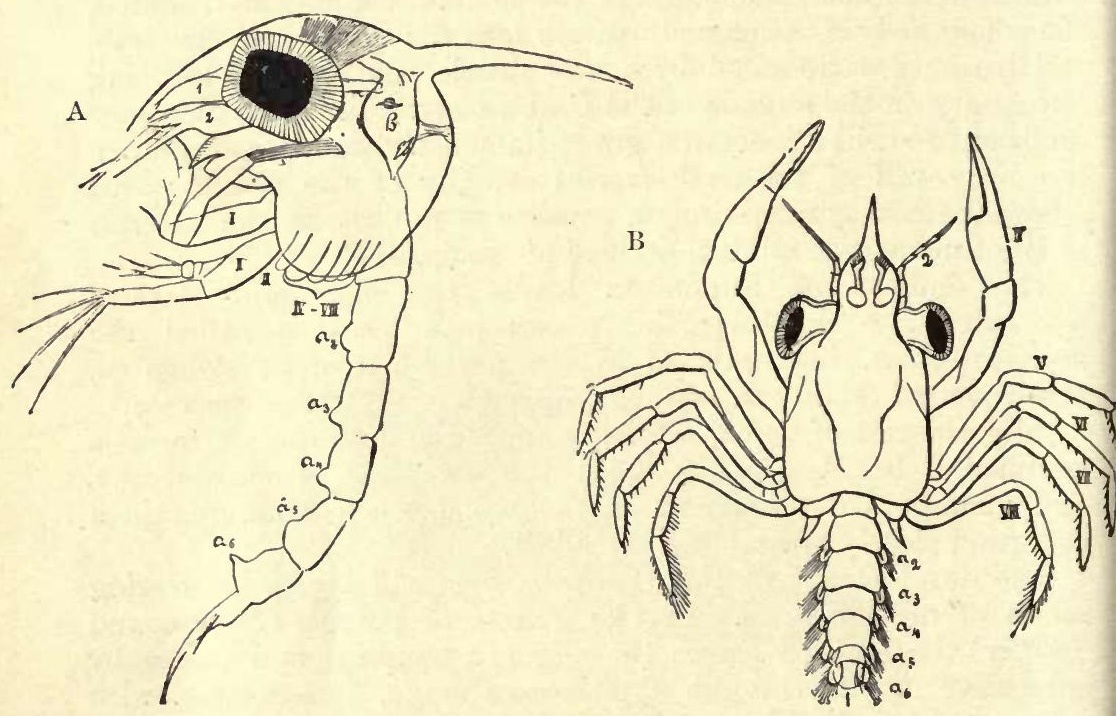

Fig. 475.-Larves of Crabs. A, Zouta-stage of Maja; B, Megalopa-stage of Portunus. $h$, heart; $a_{2}-a_{6}$, abdominal segments; 1 , antennule ; 2 , antenna ; $1-V I I 1$, thuracie appendages. (From Lang's Comparatıve Anatomy, after Claus.)

form, but in Isopoda the young leave the egg in the form of a curious maggot-like modification of the nauplius, which remains in the brood-pouch until it has attained the adult form.

Ethology.-The Crustacea are remarkable for their very perfect adaptation to the most various conditions of life: they occur in fresh-water, in the sea, in brine-pools, in subterranean caves, and on land: of the marine forms some are littoral, some pelagic, some abyssal, descending to over 3,000 fathoms. One species of Copepod, Puntellina mediterranea, may almost be considered as aërial: it is described as taking long flying leaps out of the water, after the manner of a Flying-fish. Some, like Lobsters, Crayfishes, \&c., are 
solitary ; others, like Shrimps, are gregarious, occurring in immense shoals. Most of them either prey on living animals or devour carrion, but, as we have seen, the barnacles are fixed, and feed on minute particles after the fashion of many of the lower animals, and the members of more than one order are parasites remarkable for their deviation from the typical structure of the class and their adaptation to their peculiar mode of life. In size they present almost every gradation from microscopic Water-fleas to Crabs two feet across the carapace, or four feet from tip to tip of legs.

As to geographical distribution, all the chief groups are cosmopolitan, and it is only among the families, genera, and species that matters of interest from this point of view are met with. Fossil remains are known from very ancient periods. The oldest forms are usually referred to the Phyllocarida, and occur from the Cambrian to the Trias. The shells of Ostracoda are also known from the Cambrian upwards, and those of Cirripedia from the Silurian. Peracarida are known from Palæozoic times, but are rare as fossils: the earliest Macruran is a shrimp-like form from the Devonian, while the highly differentiated Brachyura are not known with absolute certainty until the Cretaceous period.

It was in the Crustacea that the recapitulation theory so often alluded to was first worked out in detail. Embryology shows that all Crustacea may be traced back in individual development to the nauplius, upon which follows some kind of zoæa-stage, many of the lower forms progressing no further. But in Malacostraca the zoæa is followed by the mysis-stage, which is permanent in Schizopods, transient in Decapods. It was certainly a tempting hypothesis that this series of forms represented as many ancestral stages in the evolution of the class. But we have to remember that all such free larvæ are subject to the action of the struggle for existence, and have no doubt been modified in accordance with their own special needs and without exclusive reference to their ancestors or to the adult species into which they finally change.

Many Crustacea present instances of protcctive and aggressive characters, i.e., modifications in form, colour, \&c., which serve to conceal them from their enemies or from their prey. Probably the most striking example is that of certain crabs (Paramithrax), which deliberately plant Sea-weeds, Sponges, Alcyonarians, Zoophytes, \&c., all over the carapace, and are thus perfectly concealed except when in motion. Another Crab, a species of Dromia, carries a relatively immense Ascidian or Sea-squirt on its back, and in another member of the same family the hinder legs are used to hold umbrella-wise over the back a single valve of a bivalve shell. 
Several instances of commensalism occur in the class. The association of Hermit-crabs with sea-anemones, has already been referred to (p. 208): another interesting example is the occurrence of the little Pea-crab (Pinnotheres) in the mantle-cavity of Mussels. Other Decapods are found in the intestines of Sea-urchins and Holothurians, and one genus of Crab lives in a cavity in a Coral, the aperture being only just sufficient to allow of a due supply of food and water.

It is in Crustacea that we find the first indication of characters the purpose of which appears to be their attractiveness to the opposite sex. The immensely enlarged and highly coloured chelx of some male crabs (Gelasimus, Fig. 469, 2) are said to be used for attracting the female as well as for fighting. The sound-producing organs of some Decapoda have probably also a sexual significance. The Rock-lobster (Palinurus vulgaris) has a soft chitinous pad on the antenna, which it rubs against a projecting keel on the sternal region of the head, producing a peculiar creaking sound; and Alpheus, another Macruran, makes noises by clapping together the fixed and movable fingers of its large chelæ. The fact that these sounds can be produced at the will of the animals seems to show that the latter undoubtedly possess a sense of hearing, and that the auditory sac is not merely an organ of the sense of direction.

Affinities and Mutual Relationships.-That the Crustacea belong to the same general type of organisation as the articulated worms is clear enough. The advance in structure is shown in the reduction in number and in the differentiation of the segments, and in the concrescence of those at the anterior end to form a head; in the hardening of the cuticle into sclerites so as to form a jointed armour; in the jointing and mobility of the limbs; and in the differentiation of the dorsal vessel into a heart by which the propulsion of the blood is alone performed. The resemblance of the foliaceous limbs of Phyllopods to the parapodia of the higher worms is so striking that one can hardly believe it to be without significance. On the other hand, the absence of transverse muscles and of cilia, and the replacemert of the cœlome by blood-spaces, are fundamental points of difference from any known Chretopod.

As to the mutual relations of the various orders, the Branchiopoda, with their very generalised structure and parapod-like limbs, may be taken as the base of the series. The Ostracoda, Copepoda, and Cirripedia are best conceived as derivatives, along 
separate lines, of an ancestral form common to them and the Branchiopoda. By a differentiation of the post-cephalic limbs, and a reduction in the number of segments, the branchiopodtype easily passes into that of the Phyllocarida, which, though they nearly conform to the malacostracan type of segmentation, have still marked traces of relationship with lower groups in the presence of caudal styles and in their bivalved carapace and foliaceous thoracic appendages. Next to these in ascending order would come the Cumacea with their cephalic carapace coalescent with the first three or four thoracic segments and bounding branchial cavities at the sides of the thorax, but with - as more primitive features-a biramous character in some of the thoracic appendages and the absence of the fanlike tail-fin. Then a little higher, the Arthrostraca ('Ianaidacea, Isopoda and Amphipoda) and the Anaspidacea may be supposed to have branched off from the main trunk at about the same level, and may be regarded, on account of a number of resemblances, as having had a common origin from it. Probably the Anaspidacea are to be looked upon as more primitive than the other two groups in view of their less advanced coalescence of the first thoracic segment with the head, the absence of specialised maxillipedes, and the biramous character of the thoracic limbs; but, on the other hand, they show a higher development in the possession of the fan-like tail-fin and the stalked movable eyes such as characterise the Decapoda.

A stage nearer the latter group are the Mysidacea, with their single pair of maxillipedes, their stalked eyes, their rudimentary podobranchiæ and their fan-like tail-fin; but these still show some primitive features, more especially in their incomplete cephalothorax and their biramous thoracic appendages. But without doubt it is in the Euphausiacea that we find the nearest connections with the Decapoda. This is shown, in spite of the absence of maxillipedes, in their completed cephalothorax, their series of podobranchiæ, and sac-like heart, in addition to their stalked eyes and fan-like tail-fin.

From the Euphausiacea the Macrura are derivable by the differentiation of three pairs of foot-jaws and the disappearance of the exopodites of the legs. In the series of the Macrura we find, on passing from the Prawns through such forms as Astacus, Palinurus, and Scyllarus, a gradual shortening of the abdomen, accompanied by a broadening and flattening of the whole body. In Birgus, Hippa, \&c., this process goes a step further, and the abdomen becomes permanently flexed under the cephalothorax, thus leading to the high degree of specialisation found in the Crabs. 
These relationships are expressed in the following diagam :-

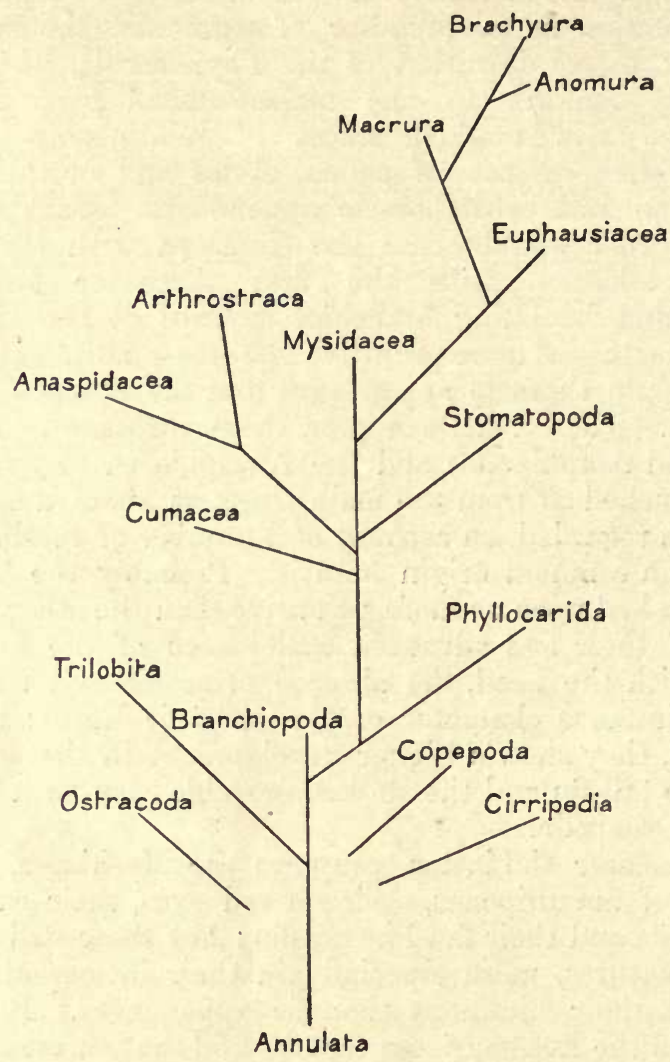

FiG. 476.-Diagram illustrating the mutual relationships of the orders of Crustacea.

\section{APPENDIX TO CRUSTACEA.}

\section{Class Trilobita.}

The Trilobita are extinct Arthropods peculiar to, and characteristic of, the Palæozoic rocks : they are specially abundant from the upper Cambrian to the Carboniferous. They are often found in a wonderfully good state of preservation, owing to the hard exoskeleton covering the dorsal surface : the greater part of the ventral region and the appendages were, however, very delicate, and are preserved only in exceptionally favourable cases.

The body is depressed, more or less oval in outline, and divided into three regions, the head (Fig. 477, c.sh), the thorax (th), and the abdomen $(p)$, all of which usually present an elevated median ridge and depressed lateral portions, whence the trilobation generally characteristic of the group. The head is 
covered by a carapace or cephalic shield $(c . s h)$, the elevated median region of which, known as the glabella $(g l)$, usually presents three or four transverse grooves, probably indicating the presence of four or five segments. The lateral regions of the carapace are divided by an oblique line of separation, the frontal or facial suture $(f . s)$, into an inner or mesial portion, the fixed cheek $(f . c)$, continues with the glabella, and an outer free portion, the movable cheek (m.c); the latter bears the large paired compound eye $(e)$. In some cases there is an indication of a dorsal organ, like that of Apus, on the last cephalic segment. Ventrally the carapace is continued, as in Apus, into a sub-frontal plate $(\mathrm{B}, s \cdot f \cdot p)$, to the posterior edge of which is attached a large labrum or hypostome $(l b v)$. In many Trilobites the hypostome bears a pair of small compound eyes. The posterior angles of the carapace are often produced into spines.

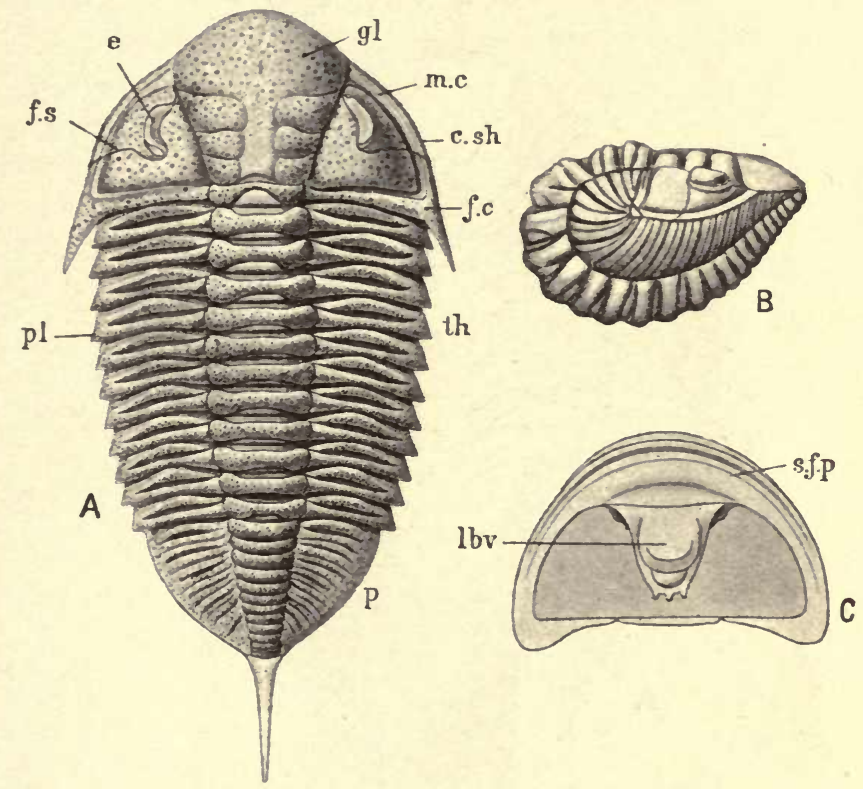

F1G. 477.-Dalmanites socialis, dorsal aspect; $B$, the same rolled up; $C$, under-side of head of Phacops fecundus. c.sh. cephalic shield; $e$. eye; f.c. fixed cheek; f.s. frontal suture; $g l$. glabella; $l b x$. labrum : m.c. movable cheek; $p$. pygidium; $p l$. pleura ; \&.f.p. sub-froutal plate; th. thorax. (After Gerstaecker.)

The thorax $(t h)$ is composed of a variable number (2-29) of movably articulated segments, which are commonly trilobed, consisting of a median region or axis, and of lateral pleura $(p l)$ often produced backwards and downwards into spines. The abdomen is covered by a caudal shield or pygidium $(p)$, formed of a variable number of fused segments. Owing to the mobility of the thorax, the Trilobites were able in many cases to roll themselves up like Wood-lice (B). Each of the segments, with the sole exception of the last or anal, bore a pair of appendages.

The appendages are known only in a few cases. Quite recently a single pair of antennæ (Fig. 478) has been shown to exist in one species, probably attached to the sub-frontal plate. Four pairs of biramous leg-like cephalic appendages have been demonstrated, and the thorax bears slender biramous legs with endo- and exo-podites, and bearing spiral gills. Similar limbs are present on the abdomen. 
The larvæ of several species of Trilobites have been found in the fossil state. In some of these the body consists only of carapace and pygidium in the youngest stages, and the thoracic segments are subsequently intercalated in regular order. In other species the earliest stage has the form of a rounded plate, the posterior portion of which elongates and segments to form the thorax and abdomen.

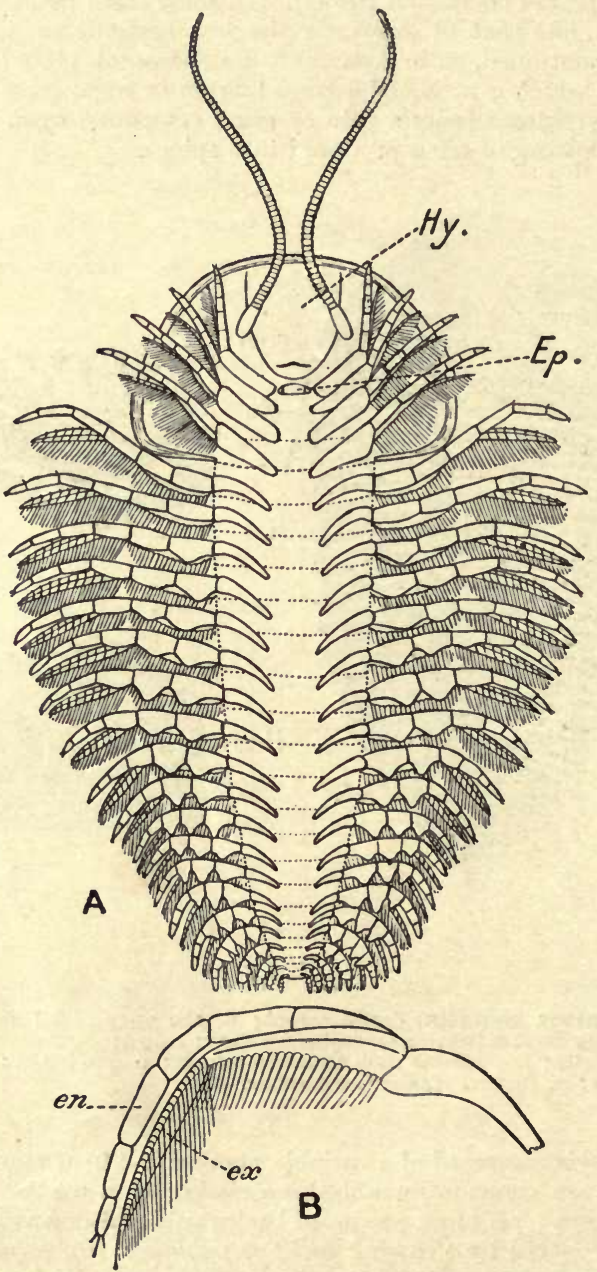

FIG. 478. - Triarthrus becki, $\times 2 \frac{1}{3}$. A, ventral surface with appendages; $E p$, metastome; $H y$, hypostome. B, second thoracic appendage. en. cndopodite; ex. exopodite $\times 12$. (From the Cambridge Natural History, after Beecher.)

Nothing is known of the larval appendages, and none of the stages hitherto discovered can be considered as nauplii.

The precise systematic position of the Trilobites is uncertain, but their nearest affinities seem to be, on the whole, with such Branchiopoda as Apus: but the relationship is by no means a close one. 


\section{CLASS II.-ONYCHOPHORA.}

The class Onychophora comprises only the aberrant arthropod genus Peripatus, with several sub-genera, which differs very widely in certain important features of its organisation from all the rest of the Arthropoda, and in some respects enables us to bridge over

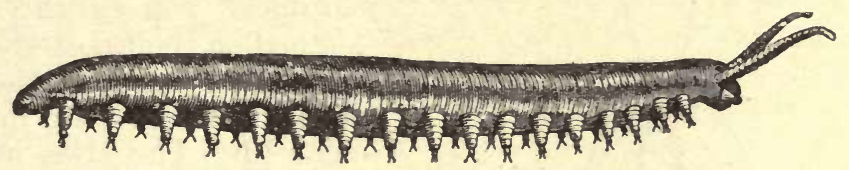

Fig. 479.-Peripatus capensis, lateral view. (From Balfour.)

the interval between the latter and some of the lower phyla, more particularly the Annulata.

General external features.-Peripatus (Fig. 479) is a caterpillar-like animal of approximately cylindrical form, and not divided into segments: it has a fairly well-marked head, and a series (1442 , according to the species) of pairs of short stumpy appendages.

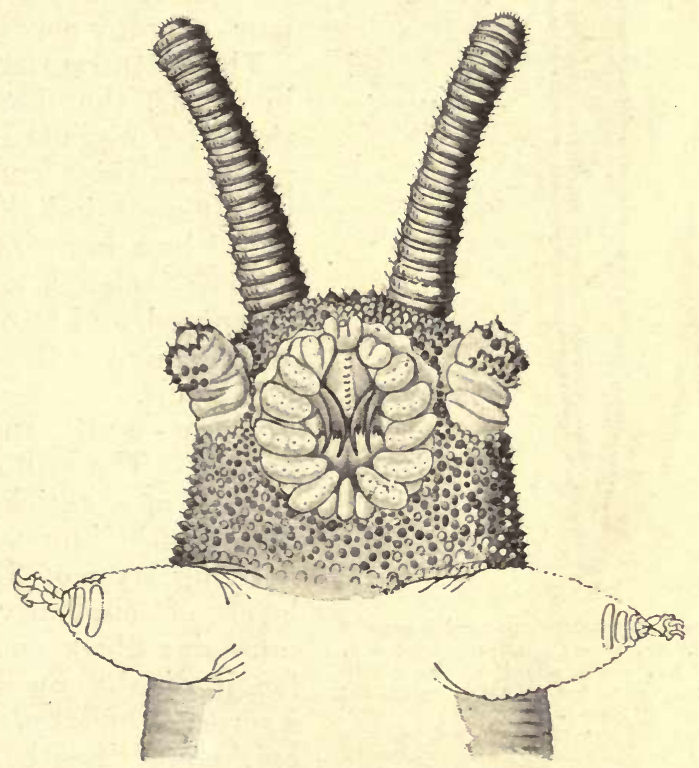

Fı'. 4S0.-Ventral view of head of Peripatus capensis, with antennæ, jaws, oral papillæ, and first pair of legs. (After Balfour.)

The integument is thrown into a number of fine, transverse wrinkles. and is beset with numerous conical papillæ, each capped with a little chitinous spine. The head (Fig. 480) bears a pair of antennæ, a pair of eyes, a pair of jaws, and a pair of short processes known as the oral papilla. The antennæ are made 
up of a number of short rings bearing minute spines. The eyes are constructed somewhat after the model of the chætopod eye as described on p. 478. On the surface of the oral papillæ are situated the apertures of a pair of glands-the slime glands.

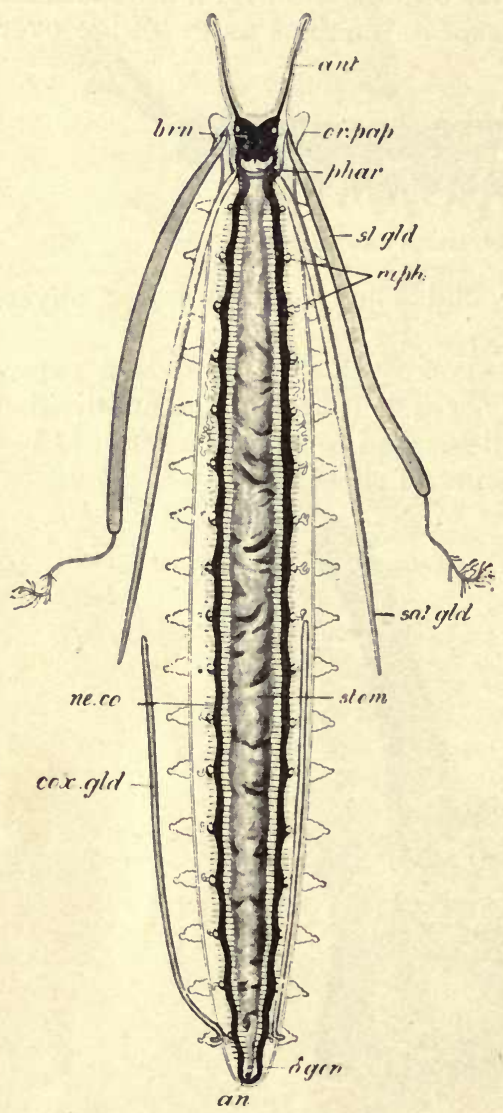

Fir. 481.-Dorsal view of the internal organs of Peripatus, an. anus; ant. antennæ; $b$ n. brain; rox. gld. coxal gland of the seventeenth leg; \& gen. male genital aperture; ne. co. nerve-cord; neph. nephridia; or. pap. oral papillæ ; phar. pharynx ; sal. glel. salivary gland; sl. gll. slime-gland; stom. stomach. (Combined from Balfour.)

Each jaw is composed of two curved, falciform, pointed, chitinous plates, the inner toothed on their posterior concave edge ; they lie at the sides of the mouth enclosed by a circular lip. The jaws, as well as the oral papillæ, are developed as modified limbs.

The legs are not jointed, but rows of papillæ give thern a ringed appearance; each consists of a conical proximal part and a small distal part or foot, the latter terminating in a pair of horny claws.

The ventral surface is reddish in colour, the dorsal darker: the latter presents an elaborate pattern-which varies greatly in different individuals-produced by minute mottlings of varions colours and tintsgreen, red, and brown, and the arrangement of these in stripes and bands.

Body-wall and body cavity.-The wall of the body consists of a cuticle, a layer of deric epithelium with an underlying layer of fine fibres, a layer of circularly arranged muscular fibres, and a layer of longitudinal fibres divided into a series of bundles. A layer of epithelium lines the wall of the body-cavity and invests the contained organs. Incomplete muscular partitions divide the cavity into a median and two lateral compartments, in addition to the pericardium, or space in which the heart is lodged; the lateral compartments send prolongations into the legs. As in the Arthropoda in general, the body-cavity is a hæmocoele, and is filled with blood. The enteric canal (Fig. 481) begins with a small buccal cavity, 
enclosed by the circular lip raised up into a number of papillæ bearing a few spines, and having on its roof a slight prominence, the tongue, with a row of small spines or teeth. This is followed by a thick-walled pharynx (phar.) leading to a narrow osophagus. The part which follows, the mesenteron or stomach-intestine, a wide somewhat thin-walled tube, extends nearly to the posterior end of the body. The narrower rectum leads to an anal aperture situated on the last segment of the body. A diverticulum leading backwards from the buccal cavity receives the secretion of two long narrow tubular salivary glands (sal. gld.).

Circulatory system.-The heart is an elongated tube running through nearly the entire length of the body. It presents a number of pairs of ostia arranged segmentallyi.e., one opposite each pair of legs. It is enclosed in a pericardial sinus imperfectly cut off from the general body-cavity by a longitudinal partition. The only other vessel is a median ventral vessel.

The organs of respiration (Fig. 482) are delicate, unbranched or rarely branched tracheal tubes, lined with a thin chitinous layer exhibiting fine transverse striations. Groups of these open in

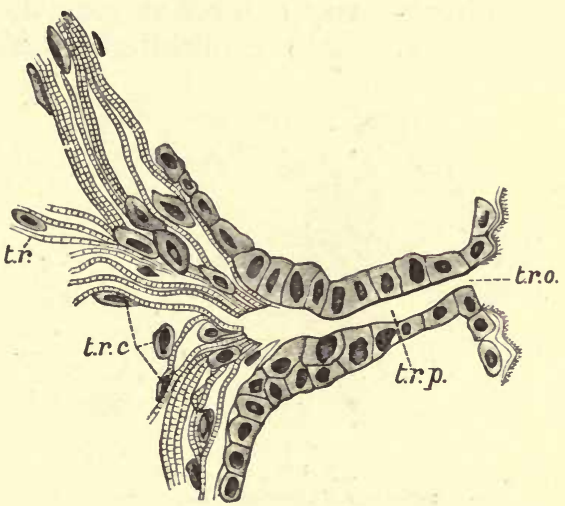

FiG. 4S2,- Section through a tracheal pit and diverging bundles of tracheal tubes of Peripatus. $t r$. trachea; $t r$. $c$. cells in walls of trachea; $t r . v$. tracheal stigma; $t i \cdot p$. tracheal pit. (From Cumb. Nat. Hist., after Balfour.)

little depressions of the integument, the tracheal pits (tr.p.), the external openings of which are known as the stigmata (tr.o.). The stigmata in some of the species are distributed irregularly over the surface; in others are arranged in longitudinal rows. By means of these tubes air is conveyed to all parts of the body.

A series of pairs of glands, the coxal glands (Fig. 481, cox. gld.), lie in the lateral compartments of the body-cavity, and their ducts open on the lower surfaces of the legs just outside the nephridial apertures. Their distribution varies in the two sexes and in the different species: in one species-P.edvardsii-they are only developed in the male. A pair of larger glands-the slime glands $(s l . g l d$.$) - opening at the extremities of the oral$ papillæ, may be inodified coxal glands: the secretion of these is discharged in the form of a number of fine viscid threads when the animal is irritated, and appears to serve a defensive purpose.

The nervous system consists of a brain (brn.) situated in the head, and of two longitudinal nerve cords (ne.co.) which run parallel 
with one another throughout the body to the posterior end, where they join together behind the anal aperture. A number of very fine transverse commissures, more numerous than the segments, (i.c., than the pairs of limbs) connect the two cords together to form a ladder-like nervous system comparable to that of some of the Flat Worms. The cords are very slightly swollen opposite each pair of limbs: nerve-cells cover them uniformly throughout their entire length. The brain gives off nerves to the antennæ. The nerves to the jaws are just where the brain passes into the longitudinal nerve cords.

The excretory organs are nephridia (Fig. 483) of the type of those of the Annulata, situated in pairs in the lateral compartments of the body-cavity, and opening on the lower surfaces of the legs at their bases. Each nephridium consists of a thin-walled closed

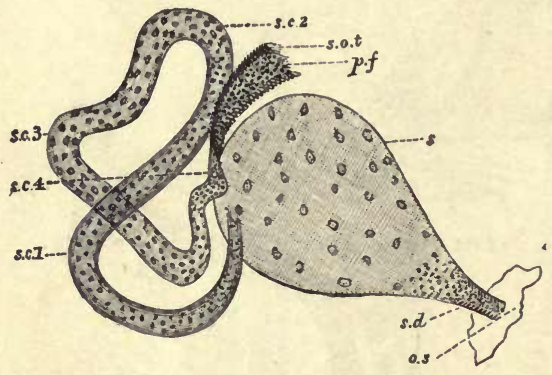

Fig. 483.-Peripatus capensis, uephridium from the ninth pair of legs. o.s, exterual opening; $p . f$, internal opening into the lateral compartment of the body-cavity; $s$, vesicle of nephridium; s.c. 1 , s.c. 2 , s.c. 3 , s.c. 4 , successive regions of coiled portion; s.o.t., third portion of nephridiurn broken off at $p . f^{\prime}$ from the internal vesicle, which is not shown. (From the Camb. Nat. Hist., after Balfour.) internal vesicle, a looped tube (s.c.), and a dilated terminal vesicle (s.), situated close to the external opening. The salivary glands and the reproductive ducts are, as shown by the study of their development, specially modified nephridia, as apparently also are a pair of glands-the anal glands -opening close to the anus.

\section{Reproductive organs.} -Peripatus has the sexes distinct. In the female there are two tubular ovaries and two uteri, the latter in the form of long curved tubes which unite behind in a median vagina opening on the exterior on the ventral surface just behind the anus, between the legs of the last pair or behind them. In the oviparous forms the opening is situated at the end of a long cylindrical processthe ovipositor. In some species, connected with each uterus where it leaves the ovary, are two diverticula-the receptaculum seminis and receptaculum ovorum. In certain species one or other of these may be absent.

In the male there are two tubular testes, each with a narrow vas efferens opening by a funnel-like aperture into a vesicula seminalis; this is followed by a long, narrow, coiled ras deferens. The two vasa deferentia unite together to form a median tube-the ductus ejaculatorius-opening on the exterior, in the same position as the vagina of the female. The wall of the proximal part of the ejaculatory duct is glandular, and secretes a substance forming 
complicated cases which enclose masses of sperms to form spermatophores.

Development. - The differences between the species of Peripatus as regards the segmentation of the egg and the formation of the germinal layers as described by various observers are very considerable. Nearly all the species are viviparous, but in some the egg, before the completion of embryonic development, is enclosed in a well-formed shell, and in certain species the eggs pass out to the exterior before the emergence of the embryo. In some species the egg encloses a considerable amount of food-yolk, in others the quantity of food-yolk is small, and nutriment is obtained from the parent.

In $P$. nove-zealandice there is a superficial segmentation. The first segmentation-nucleus is itself superficial, and segmentation
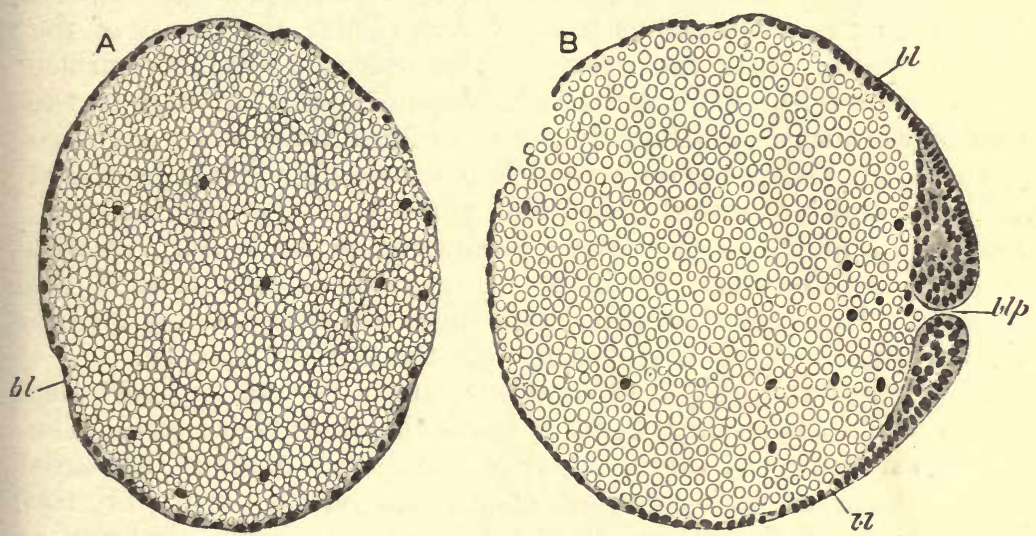

FIG. 4S4.-Two early stages in the development of Peripatus novær-zealandix. A, transverse section of an ovum in which the yolk is nearly covered by the blastoderm $(b l) ; \mathrm{B}$, transverse section of an ovum in which the blastopore (blp.) is formed. (After Sheldon.)

results in the development of a number of nuclei, each with its island of protoplasm, which arrange themselves on what is destined to become the dorsal side (Fig. 484 $A$ ), opposite the site of the future blastopore, while some pass inwards to the central part of the ovum. The peripheral nuclei multiply rapidly and grow round the yolk so as completely to enclose it except on a small space (blastopore) in the middle of the ventral side $(B)$. There a thickening takes place, and an involution of the lips of the blastopore results in a sort of invagination, the floor of the invagination-cavity being formed of yolk with scattered nuclei.

In another species- $P$. capensis-the segmentation has the appearance of being total; but the cells, though separated by fissures externally, are fused internally. A peripheral ectodermal layer becomes formed, enclosing a central, nucleated, vacuolated 
mass, except at one point where a small area, the blastopore, is uncovered. The central mass is the endoderm; the lumen of the enteron is formed by coalescence of the vacuoles.

In accordance with the smaller size of the ova and the relationship of the embryo with the wall of the uterus, the American species show a totally different mode of development. The eggs, which are almost entirely devoid of yolk, undergo a total and tolerably equal process of segmentation. Even at this stage the embryo, which increases considerably in size, appears to receive nutrient lymph from the uterine wall. When it has reached the 32 -cell stage the embryo, according to one observer, consists of a solid mass closely invested by the epithelium of the wall of the uterus. It then becomes reduced in size, and owing to exosmosis, assumes the form of a disk placed in close apposition to one side of the wall of the uterus. The embryo subsequently loses its flattened form and becomes somewhat vesicular, the cavity of the vesicle opening into the cavity of the uterus. From its surface are given off isolated cells which become applied in part to the wall of the uterus, and finally unite to form a complete envelope (amnion) enclosing the embryo. The vesicle then becomes closed and the embryo raised from the surface of the uterine wall, the part applied to the latter narrowing so as to form a sort of stalk, at the base of which is a growth of cells termed the placenta. Into close relation with this placenta comes a ringshaped thickening of the uterine wall, the uterine placenta.

In $P$. capensis (Fig. 485) proliferation of cells gives rise to an oval thickening behind the elongated blastopore. The mesoderm takes its origin at this point and extends forwards in the form of two germinal bands, one on the right of the blastopore and the other on the left. These bands undergo a division into rudiments of segments-the division beginning in front. The lips of the blastopore meanwhile become approximated, and fuse throughout the greater part of their length, leaving only an anterior and a posterior opening; these go to form the mouth and the anus respectively. The division into segments soon becomes well marked. The cavities of the segments give rise only to the nephridia and the generative ducts, which thus alone represent the colome. At the anterior end the head lobes become distinguishable. The body elongates, and the head and trunk become differentiated. The limbs now arise as ventrolateral outgrowths which are developed from before backwards.

Distribution.-The various species of Peripatus are all terrestrial, and are found in damp localities, under bark, or dead timber, or stones. Some twenty-nine species occur in the Neotropical region; one in South America; eight in Africa; four in Malaya; one in New Britain, and eight in Australasia.

Relationships.-Peripatus is the most primitive of existing 
Arthropods, and presents some striking points of resemblance to the Chrtopoda. The development is in the main arthropodan, especially as regards the mode of segmentation (at least in the forms with much food-yolk, which are probably the more
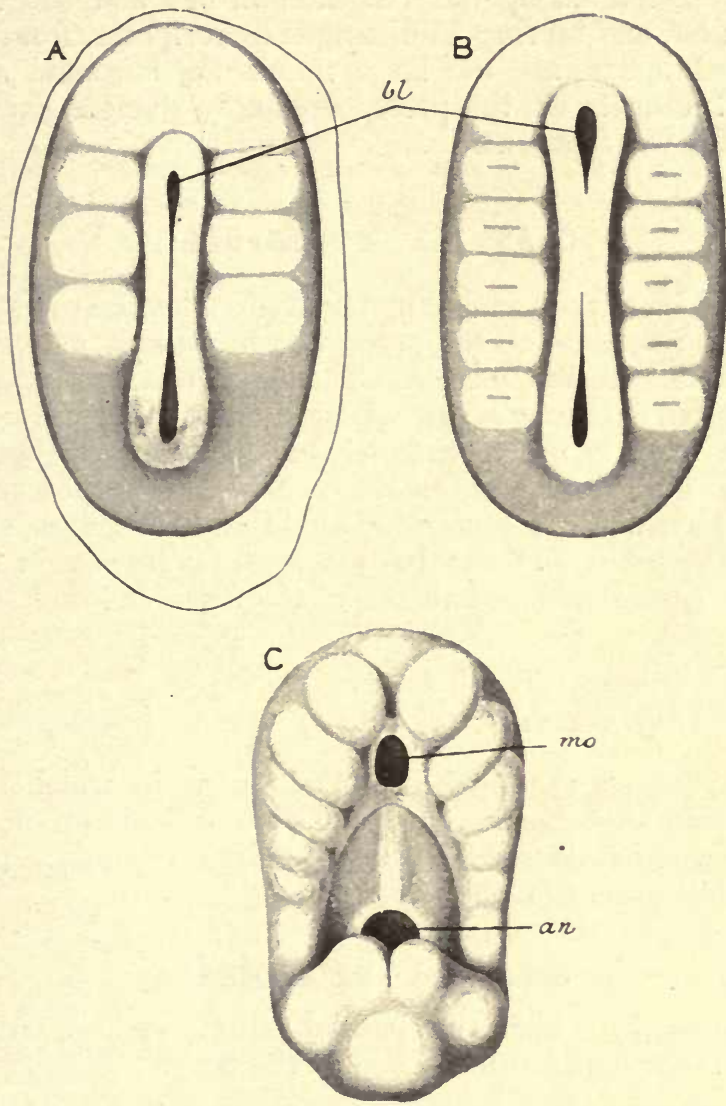

FIG. 4S5. - Three somewhat later stages in the development of Peripatus capensis, showing the mode of closure of the blastopore and the appearance of the prinitive segments. A, stage in which the blastopore $(b l$.) has the form of an elongated slit; B, stage in which the blastopore is closing up in its midule part; $\mathrm{C}$, stage in which the blastopore has become closed up except the anterior part which has gone to form the mouth (mo.), and the posterior part which has formed the anus (an.): the whole embryo has now become strongly curved towards the dorsal side. (After Balfour.)

primitive), the mode of closure of the blastopore, and of the development of the mesodermal strands. Arthropodan also are the relatively large size of the brain and the presence of tracheæ, the character of the heart with its pairs of ostia, together with the clawed appendages, and the jaws in the form of modified 
limbs. The nephridia on the other hand, and their modification in certain segments to form the gonoducts, which are ciliated internally, are annulate in character; and in all probability the slime-glands and coxal glands correspond to the setigerous glands of the Chrtopoda. The nervous system is peculiar, and is most nearly paralleled among the Platyhelminthes and the Mollusca. Also peculiar, and serving to distinguish Peripatus from the rest of the Arthropoda, are the large number of stigmata and their irregular arrangement, the presence of only a single pair of jaws, and the nature of the cuticle.

\section{CLASS III.-MYRIAPODA. ${ }^{1}$}

The class Myriapoda, including the Centipedes and the Millipedes, consists of tracheate Arthropoda, which present many features of resemblance to the Insects. There is a distinct head, bearing many-jointed antennæ, a pair of eyes, and two or three pairs of jaws; the body is not distinguishable into regions, but consists of a number of similar segments, each bearing either one pair of legs or two pairs. A system of air-tubes or tracheæ, similar to those of Peripatus and the Insects, open by a series of stigmata, usually in considerable numbers, on the sides or lower surfaces of the segments.

\section{A.-Distinctive Characters and Classification.}

The Myriapoda are tracheate Arthropoda in which there is a head, bearing antennæ and jaws, and a trunk made up of a number of similar segments, provided with leg-like appendages. Groups of ocelli are present on the head.

\section{Sub-Class I.-PROGONEATA.}

Myriapoda in which the genital apertures are situated far forwards towards the anterior end of the body.

\section{Order 1.-Pauropoda.}

Progoneata with ten trunk-segments and nine pairs of legs, one pair to each segment except the first. Antennæ with several Hlagella. Tracheæ not known. The order includes only the single genus Pauropus (Fig. 489).

1 As will appear subsequently, the class Myriapoda, as formerly understood, comprises two groups which are separated from one another by such important differences that they might very well be looked upon as constituting two distinct and independent classes. The old class Myriapoda is retained here as a matter of convenience, and the two constituent groups are ranked as sub-classes. 


\section{Order 2.-Diplopoda (Chilognatha).}

Progoneata with a body composed of a considerable number of apparent segments, each of which, with the exception of the first three, bears two pairs of legs. There are no maxillipedes.

This order includes the Millipedes.

\section{ORDER 3.--SYMPHYLA.}

Progoneata in which there are not more than twelve leg-bearing segments, and in which there is only a single pair of branching tracheæ, the external apertures of which are situated in the head. Not more than three pairs of jaws. Feet with two claws.

This order includes only the two genera Scolopendiella (Fig. 486), and Scutigerella.

\section{Sub-Class II.-OPISTHOGONEATA.}

Myriapoda in which the genital apertures are situated at the posterior extremity of the body.

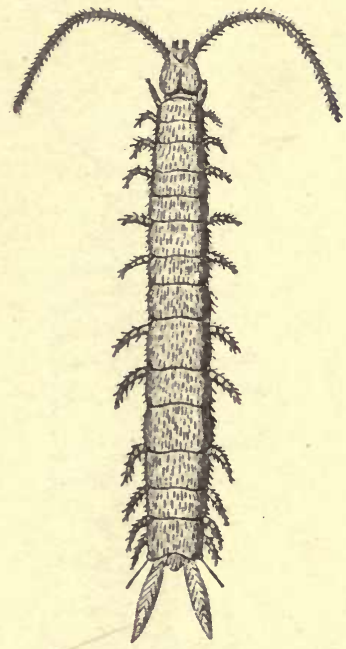

FIG. 4S6. - Scolopendrella immaculata. (From Léuckart, after Latzel.)

\section{Order 1.-Chilopoda (Synganatha).}

Opisthogoneata with numerous (15-173) trunk-segments, each bearing a single pair of legs. Numerous tracheæ opening in pairs of stigmata on the sides of a number of the segments. Four pairs of jaws, including a pair of poison-jaws. Feet with a single claw.

This order includes the Centipedes (Fig. 487) and Scutigerc.

\section{General Organisation.}

External features.-The head in the Myriapoda is as well marked off as in an Insect; it appears to be composed of about four fused segments. The antennce consist sometimes of many, sometimes of comparatively few segments; in Pauropus they are branched. A pair of eyes, situated on the dorsal surface of the head, consist of aggregations of ocelli except in Scutigera, in which there are compound eyes, differing, however, in their structure from those of Insects. There is a movable labrum, a pair of mandibles, and two pairs of maxilloe. The mandibles have no 
palps; one or both pairs of Inaxillæ usually possess palps; the second pair of maxillæ are in some groups more or less united together. In the Chilopoda the first pair of legs of the trunk are specially modified to act as poison-jaws (maxillipedes), by means of which the Centipede inflicts its poisonous bite.

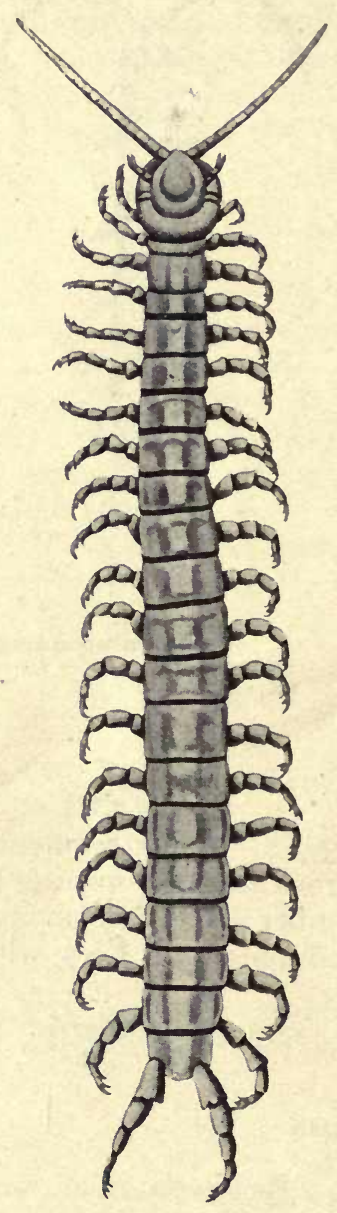

Fic. 487.-Scolopendra. (From Cuvier's Animal Kingdom.)

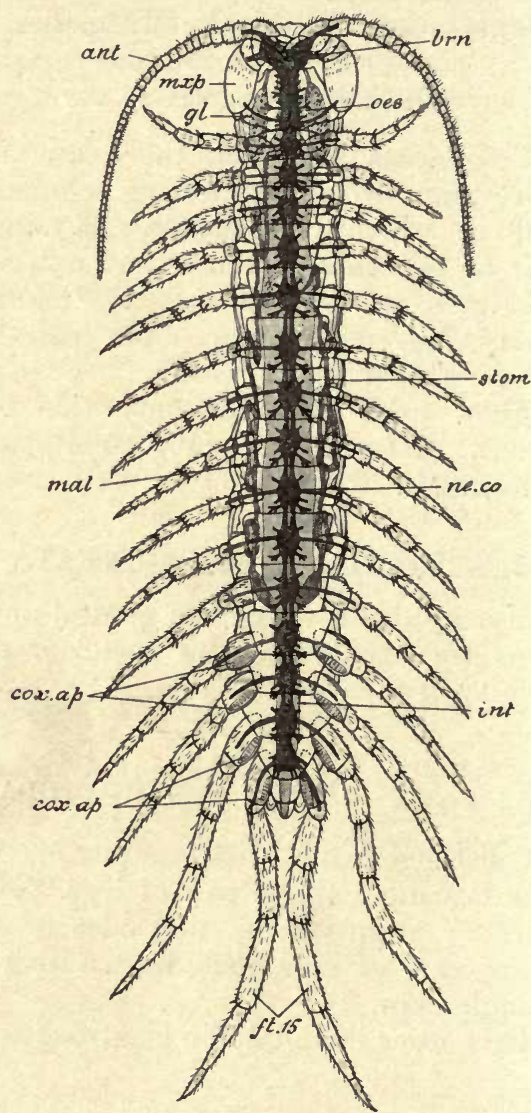

FIG. 4SS.-Iithobius forficatus scen from the ventral side. ant. antenna; brn. brain : cox. ap. coxæ of aprendages; $j t .15$, fifteenth pair of legs; int. intestine; mal. Malpighiı tubes; mxp. maxillipedes; ne. co. nerve cord; $\alpha s$. cesophagus; stom. stomach. (From Leuckart.)

The number of segments in the body varies from 10 to 173 . In the Millipedes the dorsal walls of the segments are very strongly arched; in the Centipedes the segments are all dorso-ventrally compressed, with distinct tergal and sternal shields separated laterally by intervals of comparatively soft skin on which the 
stigmata open. In the Chilopoda each segment bears a pair of jointed legs; of these the most anterior pair is extended forwards, as already stated, to form a pair of poison-jaws (maxillipedes), at the extremity of the pointed terminal joint of which opens the duct of a poison-gland. In the Diplopoda each segment behind the fourth or fifth bears two pairs of legs, the four or five most anterior having only one pair each. In most of the Diplopoda the appendages of the seventh segment are modified in the male to form copulatory organs.

The integument and body-wall do not differ widely from those of Insects (see p. 636). The exoskeleton is a thickened chitinous cuticle which is calcified in Diplopoda. Odoriferous glands are present in most Diplopoda on some of the bodysegments, and open on the dorsal surface. Scolopendrella possesses spinning glands.

The alimentary canal is straight, and is much simpler in character than that of the Insecta. There are a pair of salivary glands; and one or two pairs of Malpighican tubcs, having a renal function, open into the beginning of the hind-gut.

The heart is a greatly elongated tube, divided into a number of chambers.

The respiratory system resembles that of Insects, which will be fully dealt with later (p. 626, Fig. 497), consisting of air-tubes or trachea. There is one pair of stigmata in each segment in the Diplopoda, and the branching tracheæ do not anastomose. In the Chilopoda the number of stigmata is in most cases less than the number of segments, and the tracheæ anastomose, often forming longitudinal trunks which may extend throughout the body. In Scutigera, the stigmata are unpaired and dorsal, and lead, not into tracheæ, but into airsacs or lunys. In the Symphyla there are only two stigmata, and these are situated on the head.

The nervous system is, in accordance with the form of the body, much less concentrated than in the Insecta (see below, p. 643). There is a brain, a pair of esophageal connectives, and a ventral nerve-cord consisting of a series of double nerve-ganglia, one in each segment, with double connectives between them. The double eharacter of the ventral cord is much

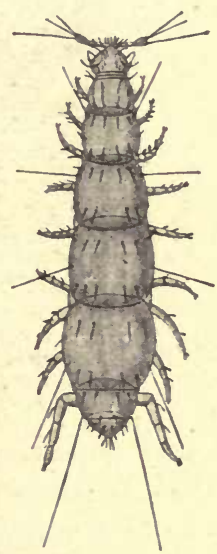

Fin. 4S9.-Pauropus huxleyi. (From Leuckart, after Latzel.) more distinctly marked in the Chilopoda than in the Diplopoda, the ganglia are more distinct, and the first three are intimately united together into an infra-œsophageal mass. A sympathetic or visceral nervous system is present, at least in the Diplopoda. 
The sexes are always separate. There is usually an unpaired gonad with paired ducts. In the Chilopoda the single genital aperture is situated at the posterior end of the body: in the Diplopoda and Pauropoda the two apertures are placed far forwards towards the anterior end.

The ovum, as in most Arthropods, contains a large quantity of food-yolk. The centrally-placed segmentation-nucleus divides so as to give rise to a number of nuclei, this division being accompanied by a division of the yolk into a number of masses, which, however, are more numerous than the nuclei. The nuclei then, for the most part, migrate to the surface, some being left behind in the yolk. Those that reach the surface, surrounded each by its little clump of protoplasm, become arranged into a continuous superficial layer of cells-the blastoderm. On the surface of this

A

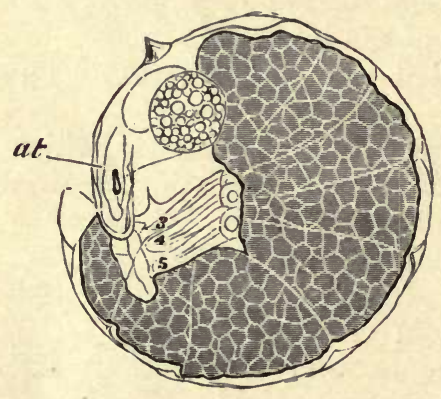

$\mathbf{B}$

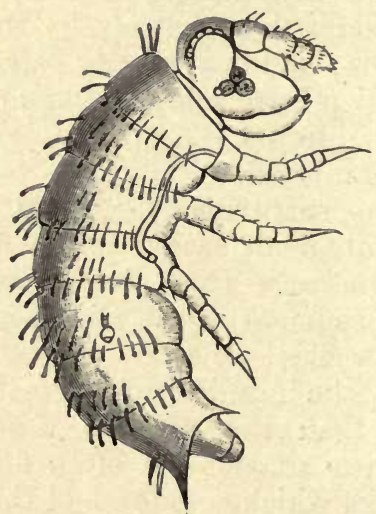

F1G. 490.-Two stages in the development of Strongylostoma, one of the Diplopoda. A, early stage in the formation of the larva, which already exhibits distinct segments. B, larva immediately after hatching. (From Balfour, after Metschnikoff.)

appears a thickening, and along the thickening is formed a groove which may perhaps represent the blastopore, though the endoderm is formed by direct modification of the cells in the interior of the yolk. Stomodæum and proctodæum are developed as invaginations of the surface layer. The thickening of the blastoderm gives rise to a germinal band in which rudiments of the segments soon become recognisable. Larval membranes do not occur.

In some of the Diplopoda there is a metamorphosis, such as will shortly be described in the embryo Insect, and the larva (Fig. $490, \mathrm{~B}$ ) has a singular superficial resemblance to an Insect, owing to the presence at first of only three pairs of appendages on the anterior trunk region.

Fossil remains of Myriapoda have been found in strata as far back as the Devonian. The more ancient fossil forms are not 
capable of being grouped in the same orders as the living representatives of the class, and are looked upon as constituting at least two orders, the members of which are all extinct. While the Progoneata, and, more especially, the Symphyla, show marked resemblances to the Insecta-more particularly to some of the members of the order Aptera, the Opisthogoneata have features connecting them through the Onychophora with the Annulata.

\section{CLASS IV.-INSECTA.}

The class of Insects (comprising the Cockroaches, Grass-hoppers, Dragon-flies, House-flies, Butterflies, Beetles and Bees, with their many allies) though it is a very extensive one-including as it does a larger number of species than any of the other classes of the Arthropoda - is yet characterised by a remarkable degree of uniformity, no such extremes of modification occurring as are observable within the class Crustacea.

Characteristic of all the members of the class is the presence of three clearly-defined regions-the head, thorax, and abdomen. There are present on the head, antennæ, mandibles, and tivo pairs of maxillæ, the jaws being variously modified in the different orders. All Insects have three pairs of thoracic legs, and most have either one or two pairs of wings likewise borne on the thorax ; the abdomen is not provided with paired appendages.

The organs of respiration are tracheæ similar to those of the Myriapoda.

The various systems of internal organs attain a very high grade of structure in all the higher groups of Insects. In most the development is complicated by the occurrence of a stronglymarked metamorphosis. Insects are terrestrial or aerial, only a few groups living on the surface of fresh or salt water; but many are aquatic throughout their larval condition.

Many groups of Insects are remarkable for the high grade of their intelligence as compared with the members of other classes of the animal kingdom. This manifests itself mainly in a number of instincts, often of a remarkable character, having to do with the protection and rearing of the young; and in some cases leading to the formation of communities consisting of individuals of various different kinds (workers, soldiers, sexual individuals) for mutual support and protection.

\section{Example of the Class-The Cockroach (Periplaneta orientalis or $P$. americana).}

The Cockroach, familiarly known by the misleading title of "Black Beetle," is a common pest of kitchens, bakeries, and storerooms. It is nocturnal in its habits, rarely coming out of its 
lurking-places in the day-time, and is almost omnivorous in its diet. It is a good example of the Insecta, not only on account of its large size, which renders it convenient for dissection, but also because of its generalised structure, which makes it a fairly central member of the class, devoid of any extreme modifications.

Three regions are very distinctly recognisable in the body of the Cockroach (Fig. 491). In front is the head, elongated vertically, bearing the very long slender feclers or antenne and the
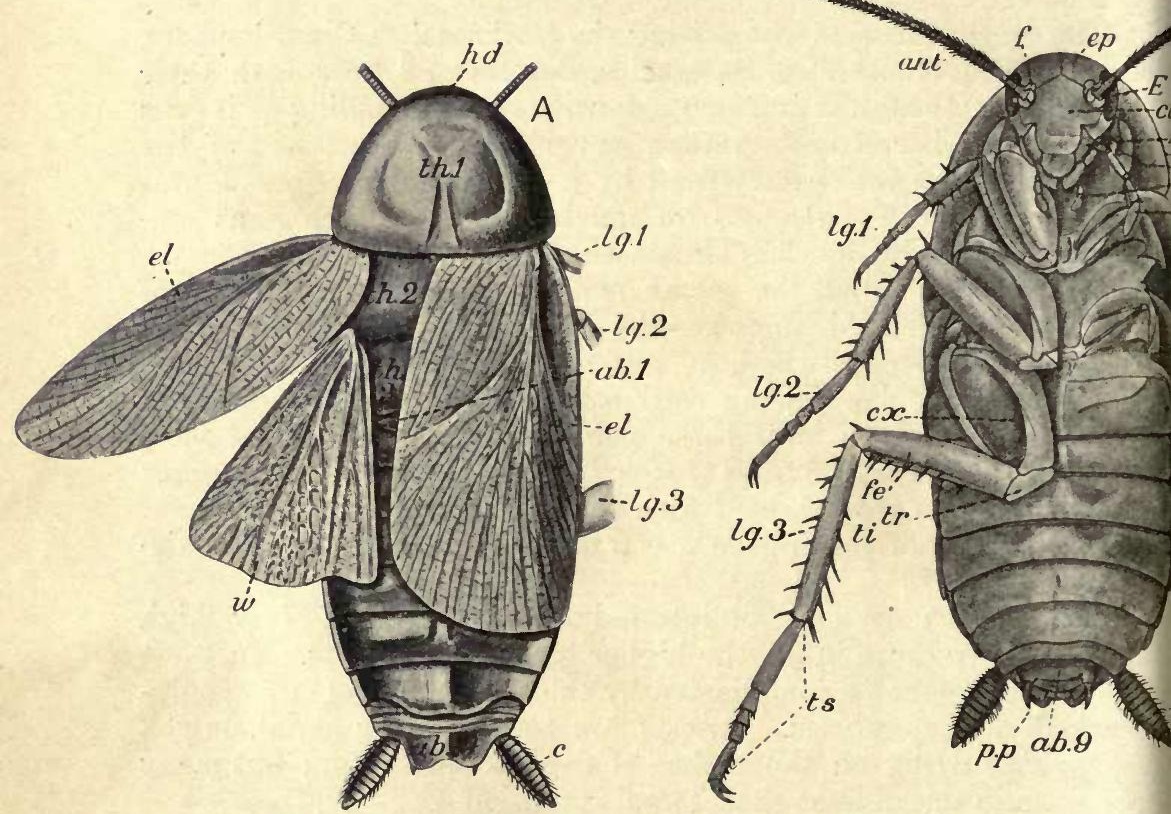

FiG. 491.- Periplaneta orientalis, male. A, dorsal view. B, ventral view $\times 2 \frac{1}{2} . a b^{1} . a b^{2}$ $a b 9 . a b 10$. first, second, ninth, and tenth segments of abdomen; ant. antennæ; $c$. cerci; $c l$. clypeus ; $e x$. coxa of third leg; $E$. eye; el. elytra ; ep. epicranium; $f$. fcnestra; fe. femur of

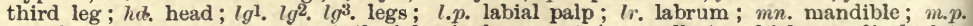
maxillary palp; p.p. style on ninth abdominal segment, internally to which a podical plate is seen; th1. (th. in B) $t h^{2}$. $t h^{3}$. segments of thorax; $t i$. tibia; $t r$. trochanter; ts. tarsus; w. posterior wing.

large eyes, and contracted behind to form a narrow neck. In the middle is the thorax, consisting of three segments, bearing the three pairs of legs and the two pairs of wings. Behind is the abdomen, consisting of ten segments covered over above by the wings in the male. The entire surface is invested by a chitinous cuticle, which is especially thickened on the head, on certain parts of the thorax, and on the anterior pair of wings.

The head consists of four parts-the epicranium behind, comprising the region between and behind the eyes; the clypers, or portion extending vertically downwards; and two lateral parts, 
the gence, in front. The eyes are a pair of reniform black patches on the sides of the head; each is seen when examined with a lens to be divided into a number of minute hexagonal areas or facets, like those in the eye of the Crayfish. Borne in sockets just below the eyes are the long, slender, highly mobile feelers or antennce, each made up of a large number of small segments, the first three being larger than the others. Internal to the base of each antenna is a rounded white space - the fenestra - the nature of which is not known, but which may be an abortive representative of the simple eyes or ocelli found in most Insects.

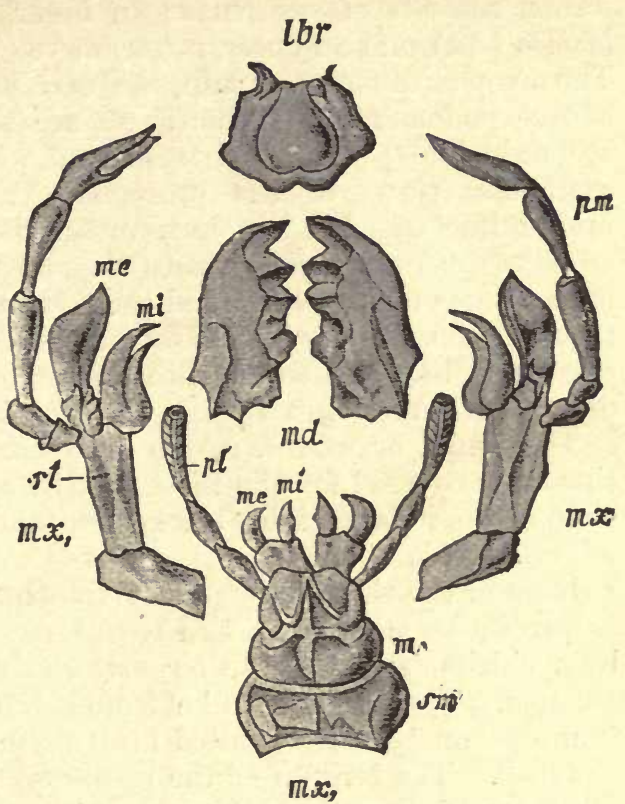

Fig. 492.-Mouth parts of the Cockroach. lbr. labrum ; $m$. mentum ; mul. mandible; $m x^{1}$. anterior pair of maxilla ; $m e$ and $m i$. outer and inver divisions of the first and second pair of maxilla; $m x_{2}$. second maxillæ; $p$. labial palp ; pm. maxillary palp; st. stipes; sm. submentum. (From Lang's Comparative Anatomy.)

Movably articulated with the lower or ventral end of the clypeus is a broad plate, the labrum or upper lip (Fig. 492, lbr.) overhanging the aperture of the

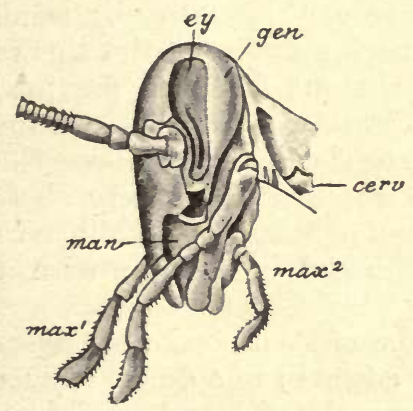

Fro. 493.- Periplaneta americana. Lateral view of the head and its appendages. cerv. one of the cervical sclerites; ey. cye; gen. gena ; man. mandible; $m_{x} x^{1}$., first pair of maxilla; max². second pair of maxillæ (labium). mouth. Below the genæ and articulating with the sides both of the epicranium and of the clypeus are a pair of stout mandibles (Fig. 492, $m d$., and 493 , man.) which work horizontally like those of the Crayfish; their inner edges are divided into a number of teeth. Behind the mandibles are a more flexible pair of jaws - the first pair of maxilla $\left(m x .{ }^{1}, \max { }^{1}\right)$. Each maxilla exhibits a structure comparable to the fundamental type of the appendages of the Crayfish :- a basal part or protopodite, consisting of two segments (podomeres), supporting an internal 
ramus or endopodile, and an external ramus or exopodite. The former consists of two parts : an inner, pointed, hard blade-the lacinia (mi.), and an outer, softer, more elongated-the galea (me.). The exopodite forms a palp, the maxillary palp ( $\mathrm{nm}$.), consisting of five podomeres. Behind these are the second maxilla, which are reducible to the same type, but which have their two basal segments (those of the protopodites) united together in the middle line to form two median sclerites, known respectively as mentum $(m$.) and submentum $(\mathrm{sm}$.$) , so that the two appendages$ form a sort of lower lip called the labium. The endopodites taken together constitute what is termed the ligula; each is divided into two parts like the endopodite of the first maxillæ. The exopodites form three-jointed palps, the labial palps $(p l$.).

The neck, or narrow region between the head proper and the thorax, is covered for the most part by a thin flexible cuticle, but supporting it are eight thickened and hardened patches-the cervical sclerites (cerv.).

Each of the three segments of the thorax-known respectively as prothorax, mesothorax, and metathorax - is covered over dorsally by a chitinous plate-the tergum, and ventrally by another-the sternum. The tergum and sternum of each segment are distinct from one another, not united into a continuous sclerite as in the Crayfish. The tergum of the prothorax is larger than that of the other two segments, and overlaps the neck above. Attached to the anterior border of the tergum of the mesothorax in the male are the anterior wings or elytra-a pair of thick opaque plates, which, in their ordinary position, extend backwards over the abdomen to some little distance beyond its extremity. Articulating with the tergum of the metathorax are the posterior wings - a pair of extremely delicate membranous expansions, which, when at rest, are folded up longitudinally, like a fan, under the elytra. In the female of $P$. orientalis the wings are only represented by small vestiges. Attached to the sternum of each segment of the thorax is a pair of legs. Each leg consists of a stout flattened proximal podomere or coxa ; a small second, or trochanter; a third, the femur, similar to the coxa but narrower; a fourth slender and spinose, the tibia; and finally the tarsus or foot, composed of six very short segments provided ventrally with patches of setæ to give adhesive power; the last segment (pulvillus) is armed in addition with a pair of claws (Fig. 491).

Of the segments of the abdomen the most posterior are overlapped by those just in front. Each is enclosed in a dorsal tergum and a ventral sternum, both of which are thinnish and flexiblethe terga and sterna of succesive segments overlapping one another from before backwards. The eighth and ninth terga are hidden from view by being overlapped by the seventh. The tenth is produced backwards into a thin flexible plate, the posterior border 
of which presents a deep notch; below this is the opening of the anus, at the sides of which are a pair of small hard plates-the podical plates; at the sides of the tergum are a pair of manyjointed palp-like bodies-the cerci. The sternum of the first abdominal segment is rudimentary. In the male that of the ninth bears a pair of short styles. In the female the sternum of the seventh is very much more prominent than in the male. The genital aperture is situated on the ventral aspect of the posterior extremity of the abdomen beneath the anal opening.

When compared with the Crayfish, as regards the external anatomy, the Cockroach is found to differ (1) in the arrangement of the segments into regions; $(2)$ in the form and position of the appendages. The head and thorax together correspond to the cephalothorax of the Crayfish, but comprise fewer segments ; the abdomen contains a larger number of segments. The single pair of antennæ probably correspond to the antennules of the Crayfish -the antennæ of the latter not being represented. On this view the homologies of the anterior appendages in the two animals may be expressed in the following table:-

\section{Crayfish.}

Antennules.

Antennæ.

Mandibles.

First maxillæe.

Second maxillæ.

First maxillipedes.

Second maxillipedes.

Third maxillipedes.

\section{Cockroach.}

Antennæ.

Absent.

Mandibles.

First maxillæ.

Second maxillæ (labium).

First legs.

Second legs.

Third legs.

Representatives of the five pairs of thoracic legs of the Crayfish would thus appear to be absent in the Cockroach, and evanescent rudiments, no traces of which remain in the adult, alone represent in the latter the well-developed abdominal appendages of the former.

In the living Cockroach respiratory movements are to be observed, in which the abdomen becomes alternately expanded and contracted; these movements bring about the alternate inhalation and exhalation of air through certain apertures-the stigmata - at the sides of the body. Two of these are situated on each side of the thorax, one between the prothorax and mesothorax, and the other between the mesothorax and the metathorax. Eight occur on each side in the abdomen between the terga and sterna of the segments. Just internal to each spiracle the main air-tube or trachea into which it leads presents an elastic ring or spiral, acting as a valve for closing the passage. 
The principal sets of muscles of the trunk of the Cockroach are (1) the longitudinal sternal muscles (Fig. 494, long. stern.), which form a transversely segmented sheet, extending between adjoining sterna of the thorax and abdomen: (2) oblique sternal muscles (obl. stern.), confined to the abdomen; and (3) longitudinal tergal muscles, best developed in the abdomen. The various segments of the limbs are capable of being flexed or extended on one another, as in the Crayfish, by the contractions of special muscles. The wings are little used, the female Cockroach being incapable of flight, and the male not a strong flier: accordingly

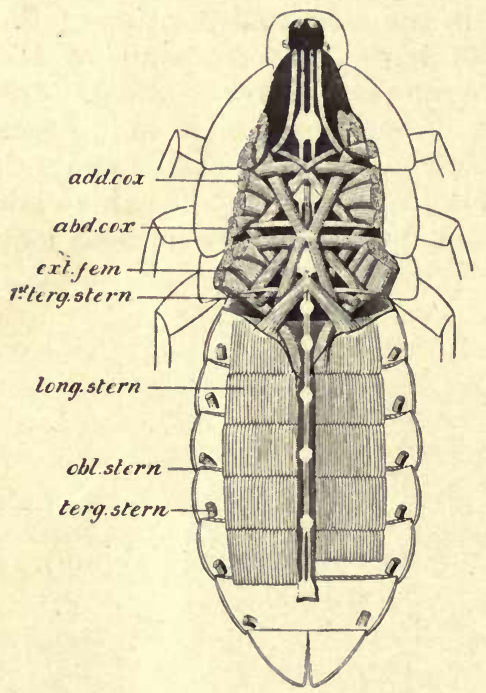

FIo. 494.-Ventral portion of the muscular system of the Cockroach. $a d d$. cox. adductor of coxa; abd. cox. abductor of coxa; ext. fem. extensor of fcmur; $18 t$ terg. stern. first tergo-sternal ; long. stern. longitudinal sternal; obl. stern. oblique stemal. (After Miall and Denny.) the wing muscles are not very strongly developed.

Between the body-wall and the alimentary canal is a cavity taking the place of the coelome, but in reality forming a specially developed part of the blood-vascular system (homocole). This is bounded externally by an irregular wall, formed of a mass of polygonal cells constituting the fat-hody.

Digestive system. - The mouth opens into a buccal cavity, which receives the ducts of the salivary glands (Fig. 495, sal.gld.). Each gland is divided into two lobes, each made up of numerous ramifications. In close relation to each gland is an elongated thin-walled sacthe salivary receptacle (sal. rcc.). The duct given off from the salivary receptacle joins that of the opposite side, and the median duct thus formed is joined by a single duct (sal. du.), formed by the union of the two ducts of the salivary glands; the common duct thus formed opens into the buccal cavity (Fig 496). A chitinous fold of the floor of the mouth forms the lingua or tongue.

From the buccal cavity there proceeds backwards a narrow oesophagus (ces.), which leads to an elongated saccular dilatable sac - the crop (cr.). On this there follows the proventriculus or gizzard (gizz.) - a pear-shaped chamber with the broad end directed forwards, its chitinous internal lining raised up into a number of teeth. A narrow passage leads from this to the stomacha wide tube, with glandular walls; from its anterior end are 
given off eight tubular hepatic coeca (hep. cce.)-blind tubes somewhat narrower than the stomach. The point of junction of the stomach with the intestine is marked by the presence of

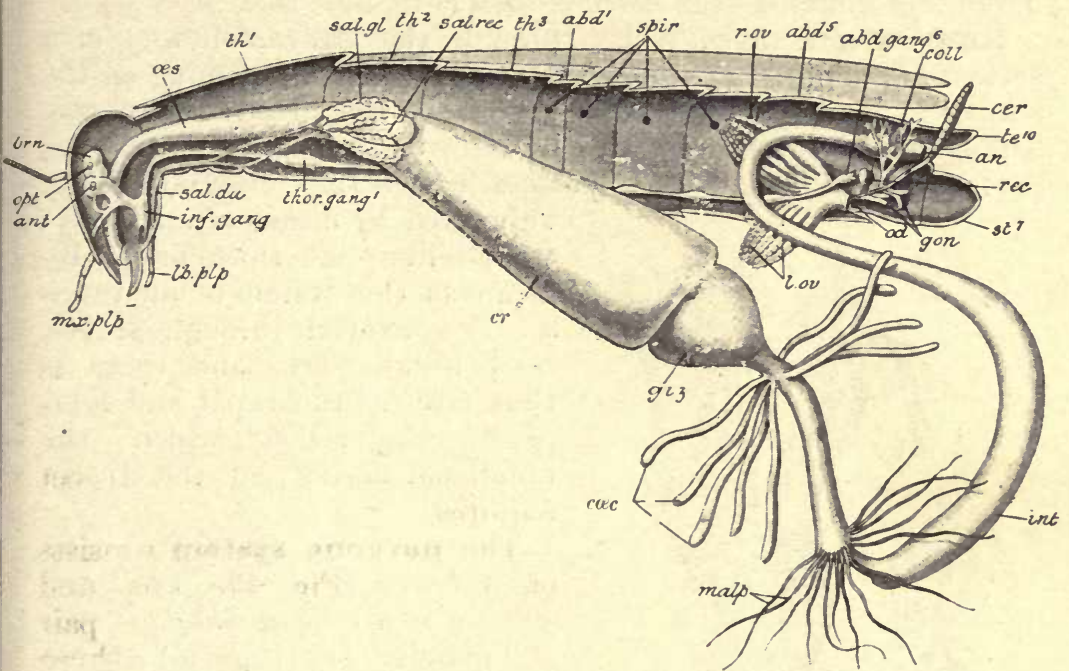

Fig. 495.-Semi-diagrammatic view of the internal organs of female Cockroach, dissected from the left side. The heart is $110 t$ represented. $a b l_{.1}, a b l^{5}$, , first and fifth abdominal segments; $a b d$. yang. ${ }^{6}$ sixth abdominal ganglion; an. anus; ant. antennary nerve; brn. brain ; cer. cercus; cac. cæca; coll. colleterial glands; cr. crop; gizz. gizzard; gon. gunapophyses; inf. gang. sub-œsophageal ganglion; int. intestine; $l b . p l p$. labial palp; $l . o v$. left ovary; malp. Malpighian tubes; $m x . p l p$. maxillary palp.; od, points to the external opening of the median oviduct (vagina); as. osophagus; out. optic nerve; rec. rectum; r.ov. right ovary ; sal. gld. salivary glands; sal. rec. salivary receptacle (left); sal. du. salivary ducts, indicating the point at which the median duct of the salivary glands unites with the median duct of the salivary receptacles; spir. stigmata; st. 7, sternum of the sevcnth segment; te. 10, tergium of the tenth segment; $t h^{1}, t h^{2}, t h^{3}$, first, second, and third segunents of the thorax; thor. gangl, first thoracic ganglion.

very numerous thread-like yellow appendages-the Malpighian tubes (malp.) - which are the renal organs of the animal. The intestine (int.) terminates in a dilated portion-the rectum (ret.) - the walls of which are longitudinally folded. Of the entire alimentary canal only a small part - the stomach - with the appended hepatic cæca, is of the nature of a mesenteron, the region in front being a stomodæum, and

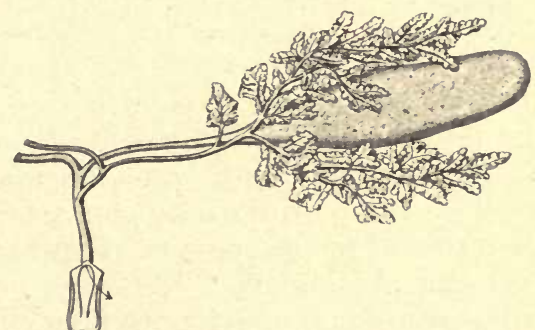

F16. 496.-Right salivary glauds and salivary receptacle of Cockroach. (After Miall and Denny.) that behind a proctodæum.

The heart is an elongated tube, closed behind, open in front, running along the middle line of the abdomen and thorax, immediately beneath the terga. Internally the tube is divided into a 
number of chambers; its walls are perforated by a series of pairs of valvular apertures or ostia. Running from the wall of the heart to the terga are a series of segmentally-arranged fan-shaped bundles of muscles - the alary muscles (Fig. 522, m.).

Respiration takes place through the instrumentality of a system of air-tubes or trachece (Figs. 497 and 498), opening on the surface at the stigmata, to which reference has already been made. These tracheæ form a richly ramifying system extending to all parts of the body. They possess a chitinous internal lining,

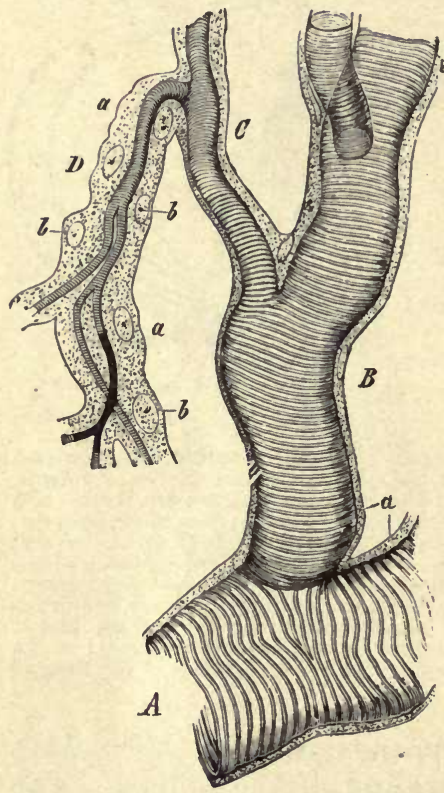

Fia. 497.-Portion of a trachea of a Caterpillar. $B, C, D$, branches; $a$, cellular layer; $b$, nuclei. (From Gegenbaur.) supported by means of a spirallywound, fibre-like thickening. By means of this system of air-tubes air is conveyed throughout the body to all parts, and there is thus ensured the rapid and complete oxygenation which the functional activity of the Insect requires.

The nervous system consists of a brain (Fig. 495, lrn., and 499, br.), a sub-cesophageal pair of ganglia (infr. gang.), three thoracic (Fig. 499, thor. 1, 2, and 3), and six abdominal pairs of ganglia (the members of each pair being united), a system of connectivcs uniting the ganglia together, and a series of nerves given off to the various parts of the body. The brain consists of a bilobed mass of nerve-matter situated in the head, and divisible into two parts, anterior and posterior. From the anterior part is given off on each side the optic nerve passing to the eye to become expanded into an optic ganglion, and from the posterior part the nerves to the antennæ. It is supported by a chitinous framework - the tentorium. From the brain there run backwards a pair of cesophageal connectives (conn.), passing, one on each side of the oesophagus, downwards and backwards to the subœesophageal ganglia. The latter, which are situated between the submentum and osophagus, give off a pair of conrectives, passing backwards to the first thoracic ganglia. From the sub-œesophageal ganglia are given off the nerves to the labrum, the mandibles and both pairs of maxillæ. The three pairs of thoracic and six of abdominal ganglia are connected together into a ehain by a series of double connectives; the last pair of abdominal ganglia, situated 
in the sixth segment of the abdomen $\left(a b d^{6}\right)$, are larger than the others, and supply the segments behind. A visceral nervous system, ramifying on the anterior part of the alimentary canal, is connected with the two œesophageal connectives by two nerves, which join above the osophagus to form a median frontal ganglion.

The organs of special sense are the eyes, the antennæ, and the palpi. The eyes are compound-each being made up of a large number of simple elements similar to those that go to make up the eye of Apus (see p. 537). The antennæ and palpi,

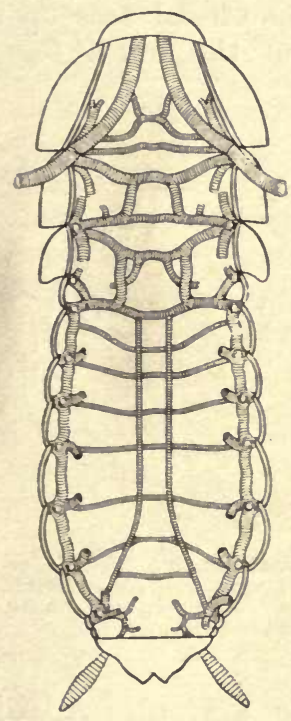

Fig. 498.-Cockroach. View of the arrangement of the principal trunks of the tracheal system. (After Miall and Denny.)

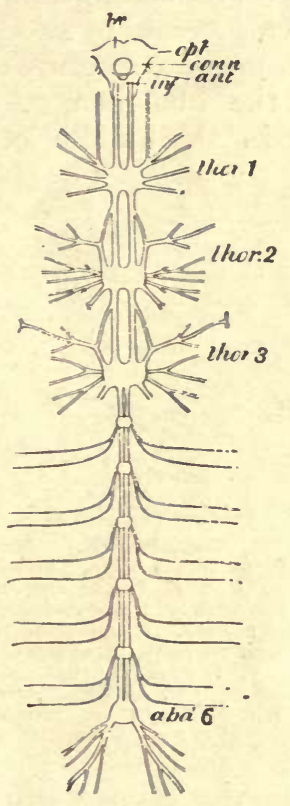

Fig. 499.-Cockroach. General view of the nervous system. abd ${ }^{\text {s }}$, sixth abdominal gang. liou; ant. antennary nerve; br. brain; conn. cesophageal connective; inf. sub-cesophageal gangliun; opt. optic nerve ; thor, ${ }^{1}$ thor, 2 thor, ${ }^{3}$ first, second, and third thoracic ganglia. (After Miall and Denny.)

together with the anal cerci, act as organs of touch. In addition, certain setæ on the antennæ appear to have an olfactory function.

Reproductive organs.-In the male the testes (Fig. 500, test.), are a pair of small bodies which lie in the fourth and fifth segments of the abdomen immediately below the terga. From these a pair of delicate tubes, the vasa deferentia, lead to the vesiculce seminales, two tufts of whitish cæca, which together constitute what is known as the "mushroom-shaped gland"; these open into the anterior end of the ejaculatory duct (duct. $e j$.), an un- 
paired tube with muscular walls opening on the exterior immediatcly below the anus. Around the genital aperture are a series of chitinous processes, the gonapophyses, which subserve copulation.

In the female there are two groups of ovarian tubes or ovarioles, each group or ovary (Fig 501, or.) consisting of eight. The ovarioles of each group are united together anteriorly, where they are connected by a ligament to the dorsal body-wall. Posteriorly each group is connected with a lateral oviduct (od.). Each ovarian tube has a beaded appearance, owing to its containing a row of ova, which increase in size posteriorly. The two oviducts unite to open by a median aperture on the sternal surface of the eighth segment of the abdomen, A pair of unsymmetrical sacs opening together in the middle of the sternum of the ninth segment

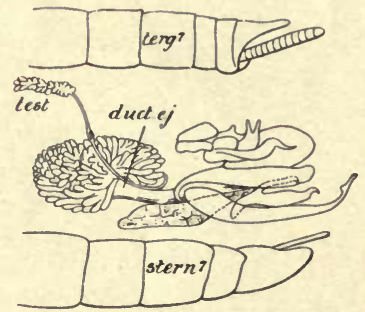

FiG. 500.-Cockroach. Male reproductive organs, lateral view. duct. ej. ductus ejaculatorius with mushroom-shaped gland; stern. 7 , sternum of seventh segment of abdomen; terg. 7, tergum of the same segment; test. testis. (After Miall and Denny.)

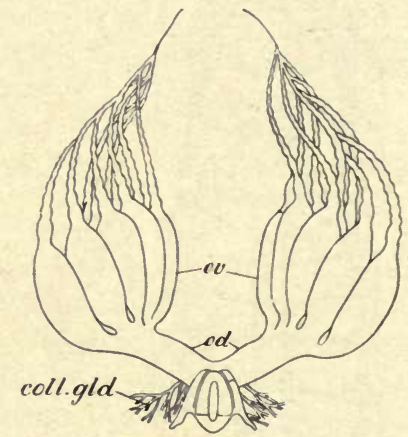

FIG. 501.-Cockroach. Fenale reproductive organs. coll. gld. colleterial glands; oul. oviducts; ov. ovaries. (After Miall and Denny.)

constitute the spermatheca or receptaculum seminis. A pair of ramifying glandular tubules, the colleterial glands (coll. gld.), open behind the spermatheca. A series of chitinous gonapophyses, which aid in carrying the eggs, are situated between the female genital aperture and the anus.

Development. - The eggs are enclosed about sixteen together in chitinous capsules, the substance of which is secreted by the colleterial glands. They are laterally compressed, concave on one side (the future ventral side), convex on the other (the future dorsal side).

The mature egg in the lower part of the ovary is enclosed in a follicle composed of a single layer of cells, within which is the thin chitinoid egg-shell or chorion perforated by a number of micropylar apertures. After the processes of maturation and fertilisation, the segmentation-nucleus undergoes division, the result being the formation of a number of irregular amœboid cells, which are 
distributed through a considerable portion of the yolk. These (Fig. 502) all migrate to the surface, where they multiply rapidly and form a layer, the blastoderm, which becomes thickened along the ventral surface by the cells being elongated in a vertical direction. From the blastoderm a number of cells pass inwards into the substance of the yolk, where their function is to convert the yolk-material into various soluble substances for the nourishment of the blastoderm. The ventral thickening of the latter is the ventral plate: its cells proliferate, and the plate comes to be several cells thick: in front it becomes broader-an indication of the position of the future head-lobes. At the opposite end there is a specially thickened area of the ventral plate with a slight depression on its surface; the depression perhaps represents the blastopore, since it is from this point forwards that the formation of the mesoderm proceeds. The latter is formed as a longitudinal band which bifurcates in front in the position of the head-lobes.

The mode of origin of the endoderm in the Cockroach is not known with certainty. It appears beneath the mesoderm, in two separate portions, as a thin layer of cells-one portion, the anterior, coming into relation with the beginnings of the stonıodæum, which arises as an invagina-
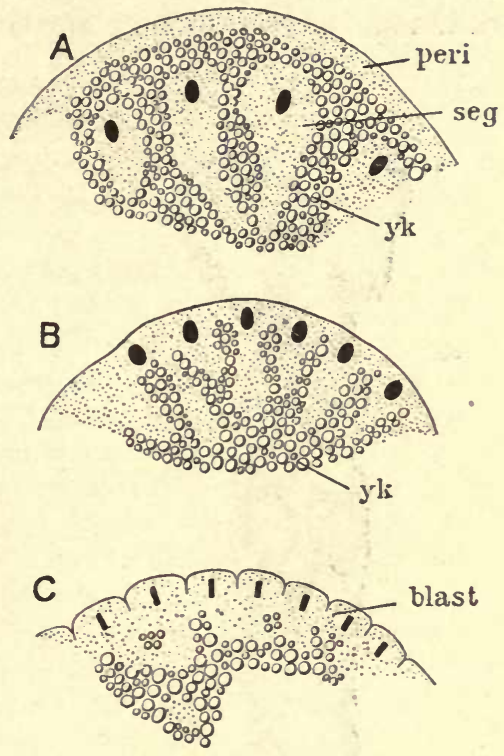
tion from the surface in the region of the head-lobes-and the other, the posterior, uniting with the proctodæum, a similar ectodermal invagination at the posterior end of the ventral plate. These two rudiments of the endoderm grow towards one another, and eventually meet to form a continuous layer destined to form the wall of the mesenteron. The ventral plate early becomes divided by a 
number of narrow transverse lines which indicate the boundaries of the future segments.

Rudiments of appendages (Figs. 503, 504) appear on the head and thorax, and a series also appears on the abdomen; all of the latter, however, subsequently disappear with the exception of the last pair, which give rise to the cerci. The segment on which the rudiments of the antennæ appear is at first post-oral in position,

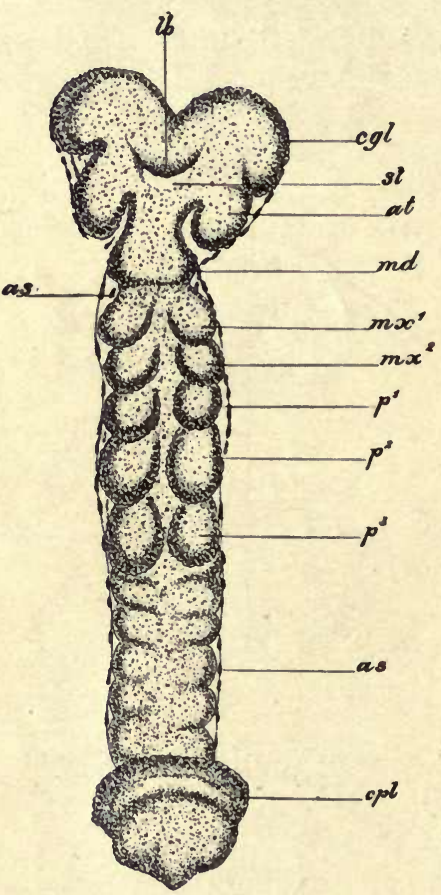

Fig. 503.-Ventral plate of embryo Cockroach (Blatta germanica), isolated from the yolk. as. amnion and serosa ; at. antennary lobe; $c g l$. brain; $c p l$. caudal plate; $l b$. labrum ; md. mandible; $m x^{1}, m x^{2}$, first and second maxillæ; $p^{1}, p^{2}, p^{3}$, legs. (After Wheeler.)

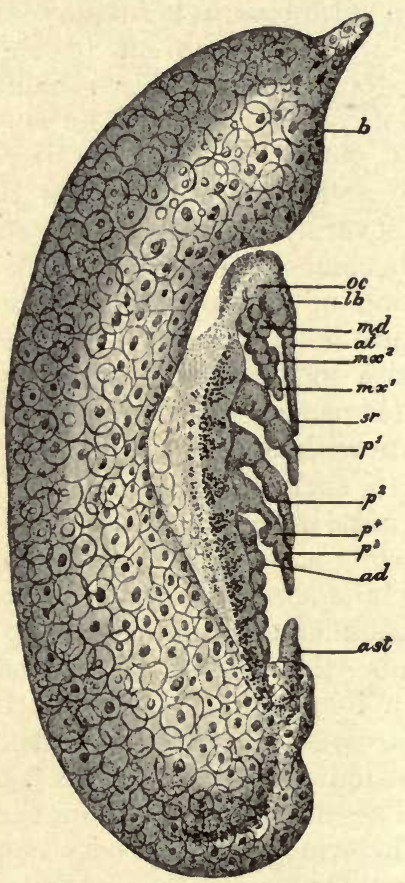

Fig. 504.-Embryo Cockroach just after the rupture of the amnion and serosa, lateral view of entire egg. Letters as in preceding figure. In addition, at, fatty body; ast. caudal styles; $b$. cephalic end of yolk; oc. eye. (After Wheeler.)

but subsequently becomes fused with a pre-oral segment (prostomium), so that the antennæ acquire their permanent pre-oral position only secondarily. The prostomial segment, the antennary segment, a segment devoid of appendages, the segment bearing the rudimentary mandibles, and those bearing the two pairs of maxillæ, all unite to form the head of the adult.

Then follows the appearance of the larval membrancs. On either side arises a fold of the blastoderm; and the two folds grow inwards and eventually unite over the body of the embryo, 
forming a complete two-layered covering for it. The outer layer is termed serosa, the inner amnion. ${ }^{1}$

Each of the two mesoderm bands undergoes transverse division into a series of segments, which become hollow and are then closely applied to one another, eventually coalescing, so that the cavities of all of them unite to form the colome, the outer walls becoming applied to the ectoderm to form a somatopleure, or lamina consisting of somatic layer of mesoderm and of ectoderm; the inner being applied to the endoderm to form a splanchnopleure, or lamina consisting of splanchnic layer of mesoderm and endoderm. The body-cavity of the adult (hcemocoele) is not derived from the cœlome of the embryo.

The ventral plate gradually grows upwards at the sides, and eventually its borders meet and unite along the dorsal middle line, the entire yolk thus becoming enclosed by it.

The ventral nerve-chain is developed from a groove of the ertoderm, bounded by thickenings which become detached from the surface-ectoderm and form the chain of ganglia. The brain is developed from a pair of ectodermal thickenings. That part which is developed in the prostomial region-the archicerebrumbecomes united with that developed in the following two segments to form the completed brain or syncerebrum.

It can hardly be said that the Cockroach undergoes a netamorphosis, the young Insect when it escapes from the egg differing from the adult only in its smaller size and in the absence of wings, which grow out subsequently from the terga of the mesoand metathorax. Between its hatching and its complete development the young Cockroach undergoes no fewer than seven "moults" or ecdyses, in which all the chitinous parts become thrown off and renewed.

\section{Distinctive Characters and Classification.}

The Insecta are air-breathing Arthropoda in which the body consists of three well-marked regions-head, thorax, and abdomen; the head devoid of external segmentation, nearly always bearing compound eyes, a pair of antennæ situated on the prostomium, mandibles, and two pairs of maxillæ; the thorax of three segments each bearing a pair of legs, and the second and third usually bearing wings; the abdomen composed of a varying number of segments $(7-11)$, which are devoid of appendages in the adult condition. A liver is absent, but salivary glands are always present. There is an elongated tubular heart,

1 This term is derived from one used in the Vertebrata, in which there is an analogous membrane, occupying, however, a dorsal instead of a ventral position as regards the body of the embryo. 
divided into eight chambers, situated in the abdomen; the vessels themselves are not highly developed. The Insecta are, almost without exception, air-breathers, and the organs of respiration take the form of branching

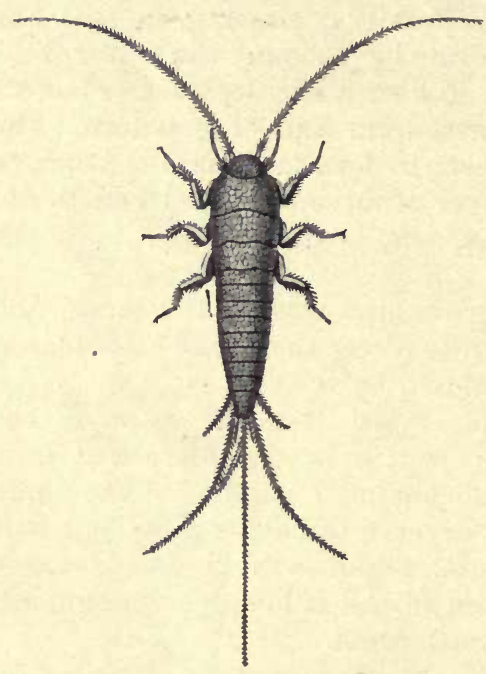

F1G. 505.-Iepisma. (After Guèrin and Percheron.) tubes, the tracheæ, by means of which air is conveved to all parts of the body. The nervous system and sense-organs are highly developed. The excretory organs are a number of blind tubes, the Malpighian tubes, appended to the intestine. The sexes are separate; development is sometimes direct, more usually complicated by a metamorphosis.

\section{ORder 1.-ApterA (Collem-} bola and Thysanura).

Insecta in which the wings are absent, and the surface is covered either with scales or hairs. Eyes are sometimes

absent; sometimes there are groups of ocelli; sometimes compound eyes. The segments of the thorax are not fused. Some progress by running, others by springing movements effected by a special springing apparatus on the abdomen. Some have elongated, many-jointed filaments or cerci at the extremity of the abdomen. Development is direct.

This order includes the Spring-tails (Podura, Fig. 506), and "Silver-fish" (Lepisma, Fig. 505).

\section{ORDER 2.-ORTHOPTERA.}

Insects in which there are two pairs of wings, of which, in most cases, the anterior pair are hard and tough, and the posterior pair delicate and transparent. The parts of the mouth are masticatory. The prothorax is not united with the other segments of

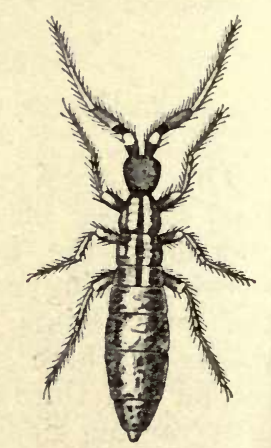

Fig. 506.-Podura. (After Guèrin and Percheron.) the thorax. Development is direct, or there is a gradual and incomplete metamorphosis. 
This order includes Earwigs, Cockroaches, Stick- and Leafinsects, Grasshoppers, and Locusts (Fig. 507).

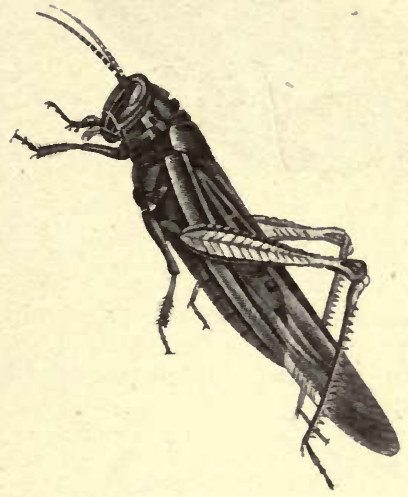

Fin 50\%.-Locusta. (From Cuvier's Animal Kingdom.)
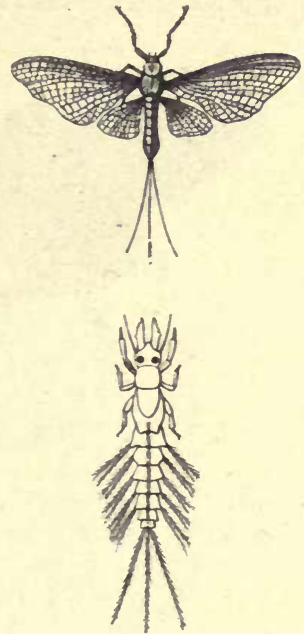

Fig.508.-Ephemera (May-fly) and larva. (After Guèrin and Percheron.)

ORder 3.-NeNroptera.

Insects with two pairs of netted membranous wings. The parts of the mouth are adapted for biting. The prothorax is free from the other segments of the thorax. The metamorphosis is sometimes complete, sometimes incomplete.

This order includes Termites ("White Ants"), May-fiies (Fig. 508), Dragon-flies, Ant-lions, and Caddis-flies.

\section{Order 4.-Hemiptera.}

Insects in which wings are usually present, sometimes similar, sometimes dissimilar, and in which there is a jointed suctorial
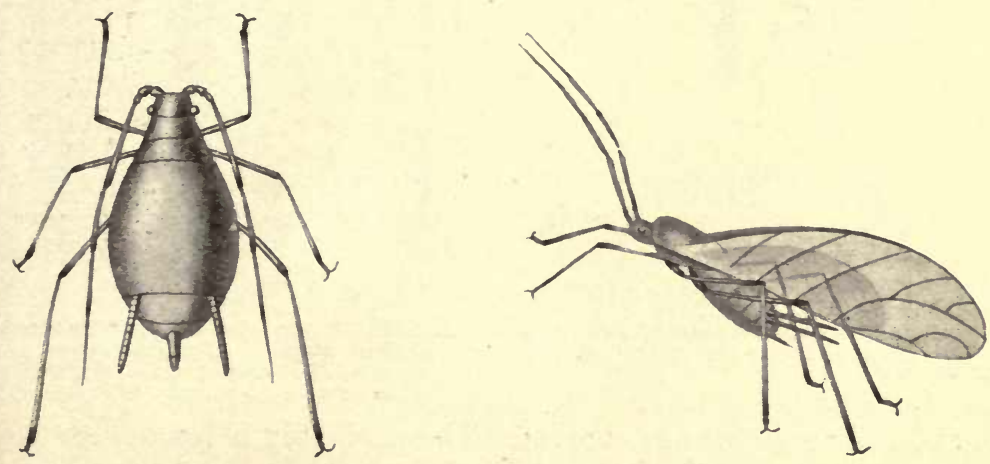

FiG. 509.-Aphis rosæ and larva. (From Cuvier's Animal Kingdom.) 
rostrum formed from the labium, enclosing the jaws in the form of piercing organs. The prothorax is free from the other segments of the thorax. The metamorphosis is incomplete.

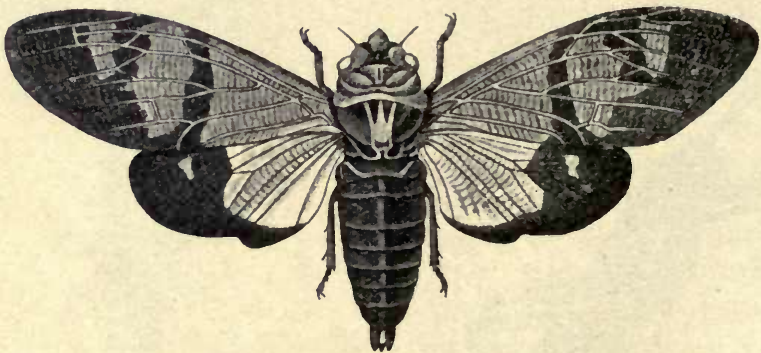

Fir. 510.-Cicada. (After Guèrin and Percheron.)

This order includes Bugs, Water-bugs, Lice, Scale-insects, Plantlice (Fig. 509), and Cicadas (Fig. 510).

\section{ORder 5.--Diptera.}

Insects provided (except in the Fleas) with a single pair of transparent membranous wings, representing the anterior pair of other orders. The mouth parts are

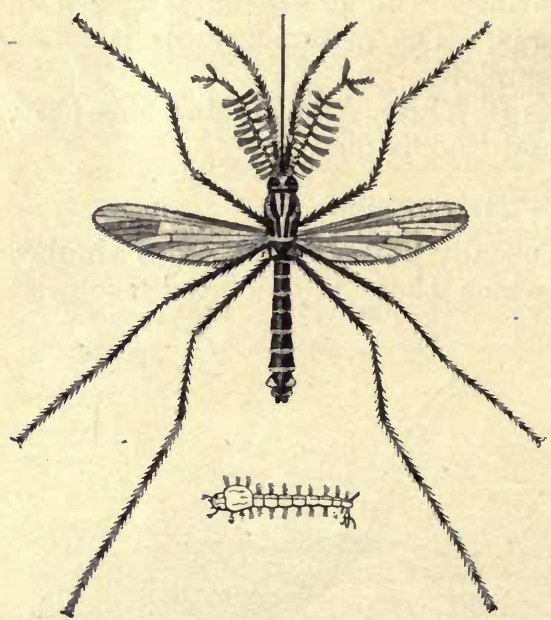

Fra. 511.-Culex (mosquito) and larva. (After Guèrin and Percheron).
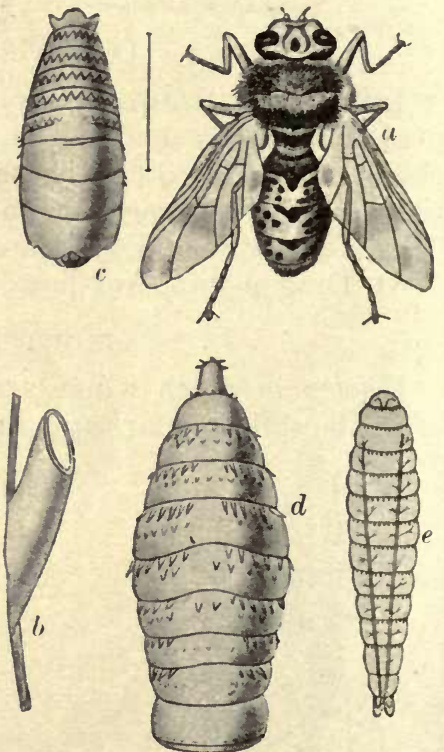

F1G. 512.-Bot-fly of $\mathrm{j}$ the philus equi). $a,[$-mature insect; $b$, egg attached to a hair; $c, d$, and $e$, stages in the larval development. (After Brehm.)

adapted for piercing and sucking. 'The prothorax is fused with the other segments of the thorax. There is a complete metamorphosis. 
This order includes Fleas, Gnats, and Mosquitoes (Fig. 511), House-flies and Blow-flies, Bot-flies (Fig. 512), Crane-flies, and "Daddy-long-legs."
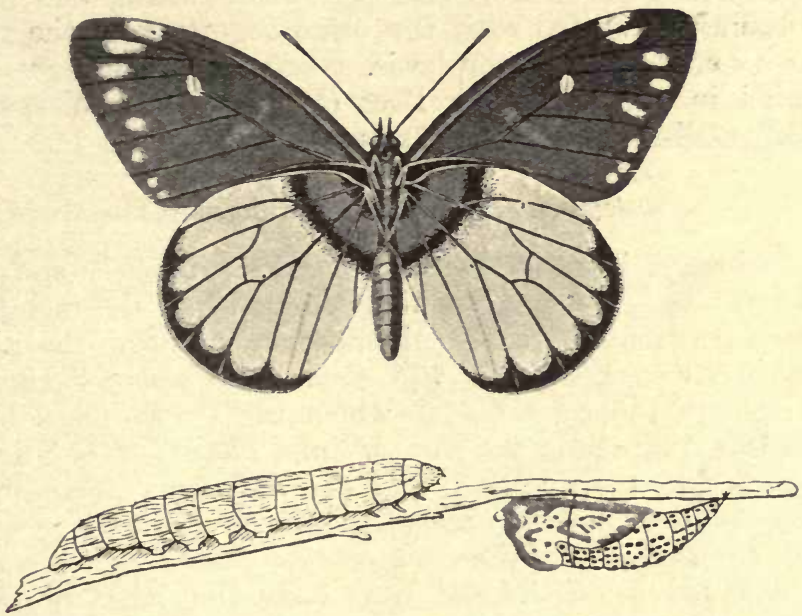

Fig. 513.-Butterfly (Pieris), with caterpillar and chrysalis stages: (After Guèrin and Percheron.)

\section{ORJER 6.-LEPIDOPTERA.}

Insects with both pairs of wings well developed and covered with scales (modified hairs). The maxillæ are modified to form an elongated sucking tube, which can be rolled up spirally; the other parts of the mouth are rudimentary, with the exception of the labial palpi. The prothorax is fused with the mesothorax. The metamorphosis is complete.

This order includes Butterflies (Fig. 513) and Moths.

\section{Order 7.-COLEOPTERA.}

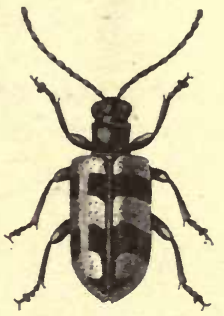

Insects in which the anterior pair of wings take the form of hard horny wing-cases, or elytra, which, when at rest, are folded up along the back and cover over the folded-up membranous posterior wings. The prothorax is movable on the other segments. The jaws are fully developed, and adapted for biting and chewing. The metamorphosis is complete.

This order includes the true Beetles (Fig. 514). 


\section{ORDER 8.-HYMENOPTERA.}

Insects in which both pairs of wings are present and membranous. The mouth parts are adapted both for biting and licking. The prothorax is united with the other segments of the thorax. There is a complete metamorphosis.

Included in this order are Bees (Fig. 531) and Wasps, Ants (Fig. 532), Gall-flies, and Ichneumons.

\section{Systematic Position of the Example.}

The Cockroach is a member of the order Orthoptera and of the sub-order Orthoptera genuina, which comprises all the members of the order with the exception of the aberrant group of the Earwigs (sub-order Dermaptera). Of the Orthoptera genuina there are three divisions, the Cursoria, to which the Cockroaches belong; the Gressoria, comprising the Mantidoe and Phasmido, or Stick-and Leaf-insects and their allies; and the Saltatoria, including the Grasshoppers, Locusts, and Crickets. The division Cur'soria comprises the single family of the Cockroaches (Blattida), characterised by the deflexed head, the flat oval body, the large prothoracic tergum, the long antennæ, the three pairs of legs similar, with large coxæ entirely covering the sternal surface of the thorax, the five-jointed tarsi, and the presence of anal cerci. Periplaneta belongs to a section of the family distinguished from the rest by the femora being spiny underneath, and by the valvular character of the last sternum in the female.

\section{General Organisation.}

The exoskeleton of the Insecta (Fig. 515) consists of a chitinous cuticle (cut.), which varies in hardness and thickness in different

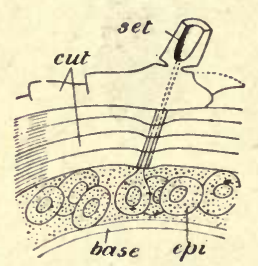

Fig. 515.-Section through the integument of an Insect. base. basement membrane; cut. layers of the cuticle; epi. epidermis; set. seta. (After Miall and Denny.) Insects and in different parts of the body of the same Insect, but is very rarely calcified. Frequently it presents hexagonal markings; sometimes it is perforated by numerous pores; sometimes it is covered with thin scales; in many cases it is developed into tactile hair's or seta, which may be scattered over the body, or may be located only on certain of the appendages - the antennæ, the maxillary and labial palpi, and the tarsi of the legs. In some, glands are present in the integument-odoriferous, honeysecreting, or wax-forming glands; poison glands are present in connection with an abdominal sting in certain Insects; spinning glands, forming a silky material, are confined to the larvæ. 
The head presents no trace of segmentation, but the history of its development indicates that it may be looked upon as composed of a prostomium and about five seginents, intimately united together. It varies a good deal in shape, but always presents the regions that have already been described in the case of the Cockroach. Of these the epicranium is the most extensive; the clypers, situated in front of it, supports the labrum; the gence are situated laterally, and a median piece, the gula, occupies the middle of the ventral surface. Sometimes the head is sunk within the anterior part of the thorax; sometimes it is free from the latter; and there may be, as in the Cockroach, a short narrow region or neck, covered with soft skin. supported only by isolated cervical sclerites, on the ventral aspect.

The three segments of the thorax-pro-, meso-, and meta-thorax - are usually firmly united together; but in some Insects the prothorax is movable upon the other segments: it is usually the smallest of the three segments. In each the exoskeleton consists of dorsal or tergal and ventral or stcrnal elements, sometimes separate from one another laterally, sometimes united together in such a way as to form complete rings round the segments.

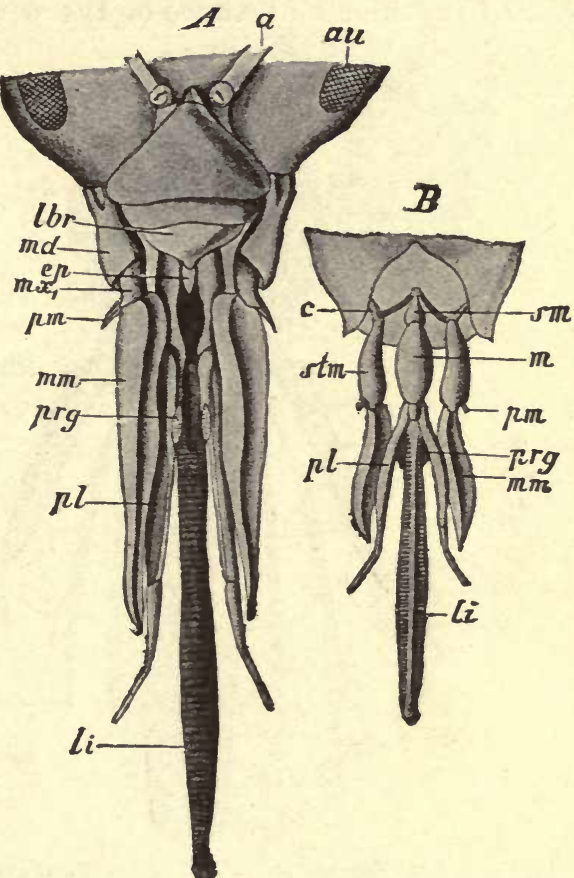

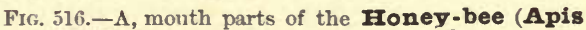
mellifica); $B$, the two pairs of maxilla. au. eye; c. antenua; $c$. cardo; ep. epipharynx; lbr. labrum; li. ligula ; m. mentum; mm, $m x^{1}$, first pair of maxillæ; mel. mandible; $p l$. labial palpi ; pm. palp of the first pair of maxille; prg. paraglossa ; sm. sulbmentum ; stm. stipes of the first maxilla. (From Lang.)

Laterally projecting processes or pleura are sometimes developed.

The abdomen contains from seven to eleven segments, enclosed in tergal and sternal shields. In some Insects the first abdominal segment is united with the thorax so as to appear to belong to the latter region.

The appendages of the head are four pairs, as in the Cockroach; but a considerable variation is observable in the different orders, especially as regards the jaws. In certain of the Aptera 
an additional pair-the so-called maxillulce-occur between the mandibles and first maxillæ. In a few eyes are absent. Most have large compound or faceted eyes, and many have simple eyes or ocelli as well; in a few groups the latter are alone present. The antennæ vary in shape in different groups and sometimes even in the sexes of the same species. They may be tapering, moniliform, club-shaped, pectinate, or plume-like. In addition to functioning as tactile appendages they bear the olfactory setæ, and there seems reason to believe that they act also as organs concerned in the maintenance of the equilibrium of the body. The

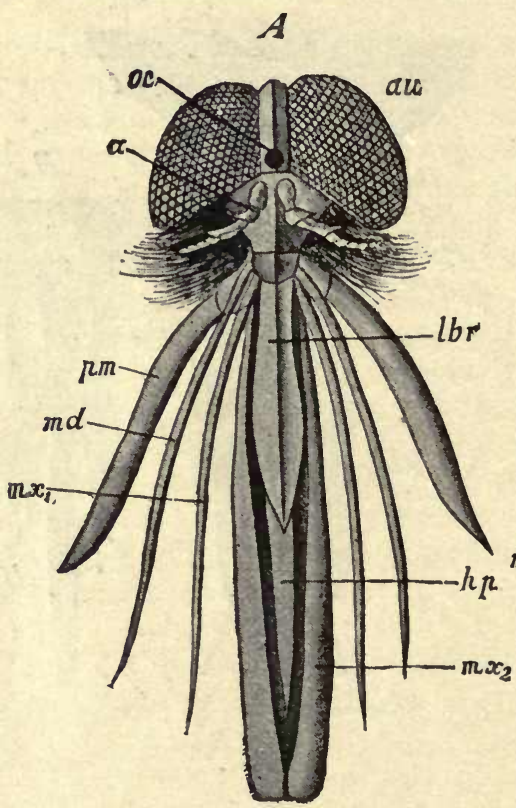

B

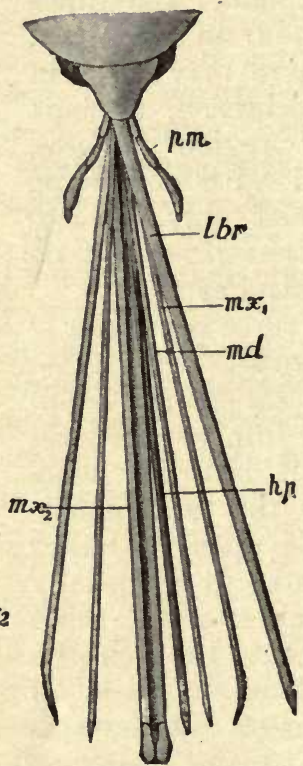

Fia. 517.-Mouth parts of the Diptera. A, of Tabanus; B, of Culex. Lettering as in preceding figure: $p p$. hypopharynx; oc. ocellus. (From Lang.)

mandibles are always one-jointed, and differ from those of the Crustacea in never being provided with a palp. An arrangement of the mouth-parts adapted for biting or chewing has already been described in the case of the Cockroach : this type is characteristic of the order Orthoptera, to which the Cockroach belongs, and a very similar type characterises the Coleoptera. In the Hymenoptera(Fig. 516) the mouth-parts are adapted both for biting and for licking and sucking; the mandibles (md.) and maxilli $\left(m x^{1}\right.$.) are sharp and lancet-like, the middle part of the labium is produced into a long median tongue (ligula, li.) at the sides of which are a pair of accessory tongues or paraglossa (prg.). In the Hemiptera there is a proboscis formed from the labium and enclosing the stylet-like 
mandibles and maxillæ. In the Diptera (Fig. 517) the mandibles (md.), usually not developed in the males, are biting or piercing organs, while the basal parts of the labium form a proboscis $\left(m x^{2}\right.$.) enclosing a spine or seta ( $h p$.) - which is a process from the hypopharynx, a chitinous process on the floor of the mouth-and sometimes stylet-like maxillæ $\left(m x^{1}\right.$.). In the Lepidoptera (Fig. 518) the mandibles are aborted in the adult, and the maxillæ are developed into elongated half-tubes, which when applied together form a complete tube (sr.) capable of being coiled up in a spiral manner under the head, the extremity provided with hooks or spines for rupturing the nectaries of flowers.

Appendages of the thorax. - Each of the segments of the thorax bears a pair of five-jointed legs; the terminal section or tarsus being made up of a number of short segments and ending in a pair of claws, often with an adhesive pad or sucking disc between them. In accordance with variations in the uses to which they are put, considerable differences are observable in the form of the legs in different groups of Insects. In most they are adapted for walking, and are long and slender; in some they are expanded to enable them to act as swimming paddles; in some the first

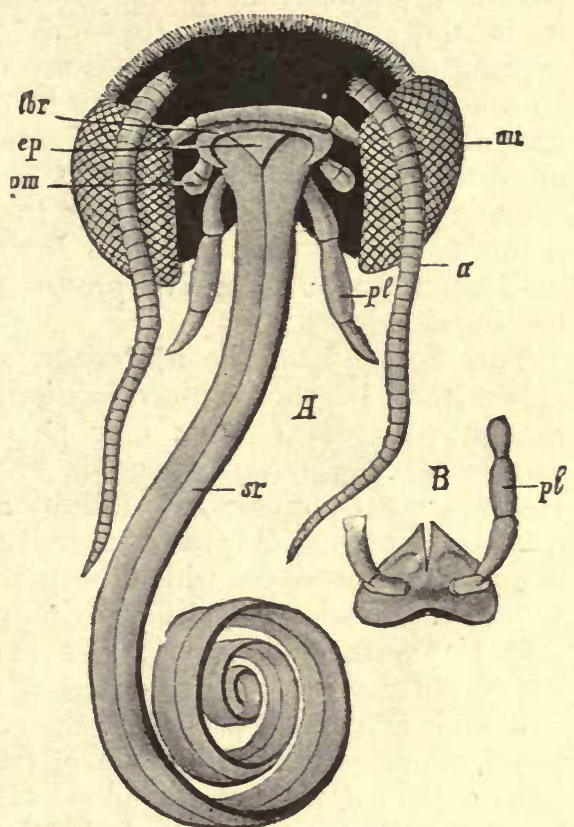

Fia. 518.-Mouth parts of the Iepidoptera. B, the second maxillw. Lettering as in preceding figures: $p l$. labial palp; $\mathrm{mn}$. palp of the anterior maxilla; sr. sucking tube. (From Lang.) pair are prehensile, and develop a sub-chelate extremity; in others, again, the legs, or the first pair of them, are stout and adapted for burrowing. In addition to the legs the meso- and meta-thorax may each bear a pair of wings. The wings are thin transparent expansions of the integument of the body, supported by a system of branching ribs or nervures consisting of chitinous material with branches of the tracheæ, nerves, and tubular diverticula of the body-cavity. In most Lepidoptera the wings are opaque, owing to their being covered with numerous overlapping microscopic scales, to which the various colours of the wing are 
due. In some insects-e.g., Beetles and Orthoptera-the posterior wings alone are delicate and membranous, the anterior pair being converted into hard or tough cases-the elytra-which when folded up cover over and protect the delicate posterior wings. In some Beetles the elytra are permanently united together along the back of the Insect. In some Insects (Bugs) the anterior wings are chitinous at the bases only. In the Diptera the anterior wings alone are developed, the posterior being represented by vestiges - the halteres or balancers. In the Strepsiptera, or Beeparasites, an aberrant group of Neuroptera, on the other hand, it is the anterior pair that are vestigial. In some Insects (Spring-tails, Lice, Fleas) wings are entirely absent in all stages. In others again they are present in one sex-usually the maleand absent or vestigial in the other. In the Aptera there is no vestige whatever of wings at any stage, and this, taken in connection with the simplicity of the structure in other respects, seems to indicate that in these Insects we have to do with the descendants of a primitive group in which wings had not yet become developed.

The segments of the abdomen are entirely devoid of paired appendages in the adult condition (except in the Thysanura), though vestiges of them may be present in the young at an early stage. Each segment is enclosed in dorsal tergal and ventral stcrnal plates, which usually remain separate laterally, but may be united. At the extremity of the abdomen there are frequently appendages which are perhaps of the nature of limbs, having the function of stings, ovipositors, and genital processes.

Hæmocœle. - The cavity intervening in an Insect between the body-wall and the various internal organs does not correspond, as already explained (p. 631), to the cœlome of other groups; but is found, when we study its mode of development, to be a homoccele -an extended part of the blood-vascular system. The colome is apparently represented only by the lumen of the reproductive organs.

A fat-body is always present, either in the larval condition or throughout life. It consists of a mass of polygonal cells bounding the hæmocœle externally. When young the cells are nucleated and possess a protoplasmic body. At a later stage a fluid loaded with minute granules takes the place of the protoplasm, and crystals containing uric acid are formed. These crystals afterwards become absorbed; their appearance and subsequent absorption would seem to point to the probability that the fat-body is concerned in separating out nitrogenous waste matters, which subsequently reach the exterior through the Malpighian tubes. Its chief function is to serve as a reserve-store of nutrient material.

Digestive system.- Some Insects do not feed in the adult condition, and when this is the case the mouth may be absent, 
as, for example, is the case in the Day-flies (Ephemerido). When it mouth is developed, as it is in the vast majority of Insects, it is situated on the lower aspect of the head, bounded in front by the labrum, and behind by the labium. It leads into the buccal cavity, into which open the ducts of a pair of salivary glands, each of which often has associated with it a thin-walled sac or salivary receptacle. Also in the neighbourhood of the mouth in such larval Insects as spin a cocoon, the ducts of a pair of spinning glands open. A projection of the roof of the mouth-cavity (epipharynx) is present in some Insects; in others it is replaced by a projection from the floor, the hypopharynx or lingua.

The alimentary canal is nearly always considerably longer than the body; it is longer in vegetable-feeding than in carnivorous forms. The mouth leads into a long, narrow passage-the osophagus (Figs. 519 and 520 œ.) which dilates behind into a crop (in) for the storage of food. The place of this in sucking Insects is taken by a stalked sac, usually termed the sucking stomach. The essential processes of digestion are carried on in an elongated chamber with glandular walls-the stomach $(c d)$-which may be divided into several parts. Sometimes between the crop and stomach is intercalated a muscular-walled chamber, frequently containing chitinous teeth, the proventriculus or gizzard ( $p v)$. Appended to the stomach at its anterior end are, in many Insects, a varying number of tubular blind pouches, the hepatic caca. At its junction with the small intestine, or further back, there open a number (from 2 to over 100) of narrow

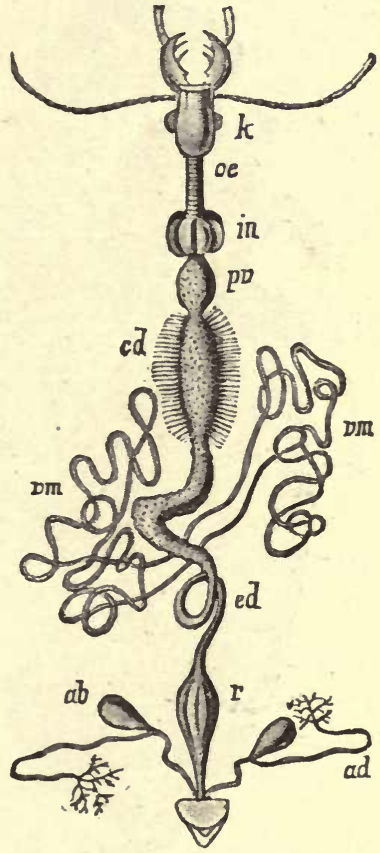

FIG. 519.-Digestive apparatus of a Beetle (Carabus auratus). $a d$, anal glands : $a b$, their muscular appendages; $c d$, stomach ; $e d$, hind gut ; in, crop; $k$, head with mouth parts; $e^{\text {. oesopha. }}$ gus ; $p v$. proventriculus; $v m$. Malpighian tubes. (From Lang, after Dufour.) tubular appendages, the Malpighian tubes $(v m)$, which are the organs of renal excretion. In the cases in which the development of the alimentary canal has been traced, it has been found that the Malpighian tubes mark the point where the mesenteron passes into the proctodæum, and it is assumed that this holds good generally. The lumen of the tubes is sometimes filled up with cells. In some insects, the Malpighian tubes open into a paired or unpaired sac-the urinary bladder. The intestine is usually 
elongated, and its posterior portion (ed.) is dilated to form a wide rectum ( $r$.), which opens on the exterior by an anal aperture situated on the ventral side of the last segment of the abdomen. Anal glands (ad.), producing an odoriferous secretion, often open into the rectum.

The tracheal system (Fig. 520) communicates with the exterior through a number of apertures - the stigmata (st)-which

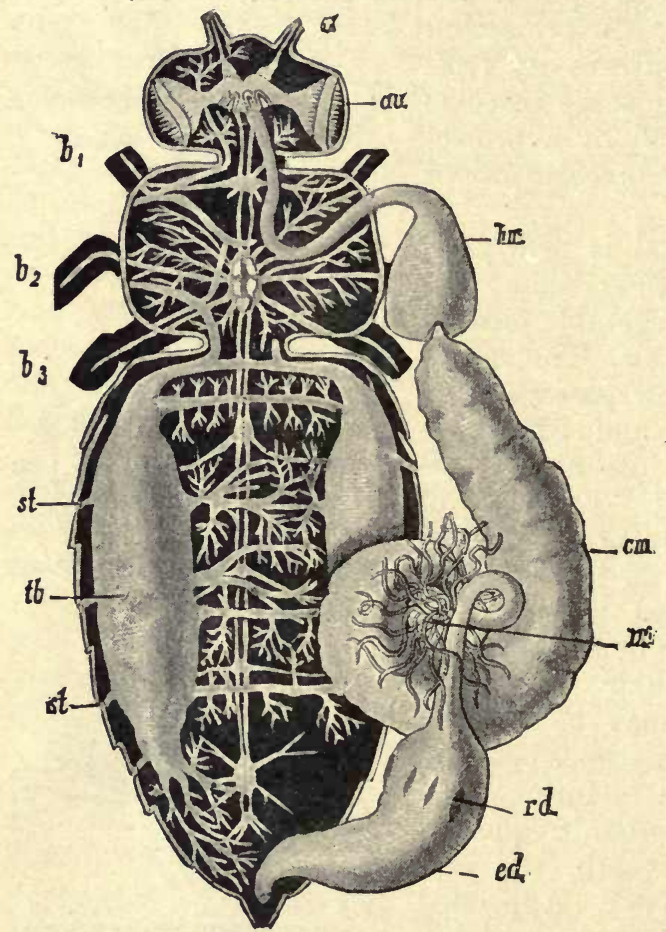

Fic. 520.-Nervous, tracheal, and digestive systems of the Froney-bee. $a$. antenna; $a u$, compound eye; $b_{1}, b_{2}, b_{3}$, the three pairs of legs; $\mathrm{cm}$, stomach; $\mathrm{ed}$, hind-gut; $\mathrm{hm}$, honey stomach (crop); rd, rectal glands ; st, stigmata; th, vesicle of tracheal system; vm, Malpighian vessels. (From Lang's Comparative Anatomy.) vary in the details of their arrangement in the different orders. They are always protected against the entry of foreign particles by some means - either by being surrounded by special bundles of hairs, or by being provided with a special sievelike membrane. In most cases they are capable of being closed by muscular action. In some Insects, mainly those adapted for active flight, such as the Hymenoptera, the tracheal system is dilated in certain parts of the body to form comparatively large air-sacs or airreservoirs $(t b)$. In the aquatic larvæ of some Insects there is a series of soft external, simple or divided, processes - the tracheal gills (Fig. 521) - attached to the abdominal segments and richly supplied with tracheæ, which have no communication with the exterior.

The blood-vascular system is, in comparison with the other systems of organs, not very highly developed, the need of an elaborate system of vessels being greatly diminished by the way in which all the tissues and organs are supplied with oxygen through the system of tracheæ. The blood is colourless or faintly yellowish or greenish, and contains colourless corpuscles. A contractile 
dorsal vessel or heart (Fig. 522) extends through the abdomen-and sometimes thorax-immediately below the terga. Its cavity is divided internally into a series usually of eight chambers by a system of valves. In its walls are a series of slits or ostia, by which a communication is effected between the internal cavity and a surrounding pericardial sinus. In front the heart gives origin to a main vessel, or aorta (a), by means of which the blood is conveyed throughout the body to enter a system of sinuses in free communication with the general body-cavity, from the various parts of which it finds its way back to the pericardial sinus.

The nervous system (Figs. 520 and 523) is on the same general plan as in the Crustacea. There is a double supra-cesophageal

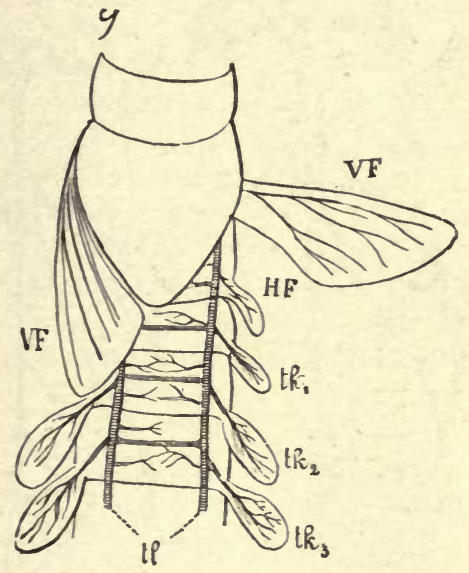

FIG. 521.-Thorax and anterior abduninal segments of a larval Ephemerid with tracheal gills. $H F$, hind wings; $t r^{1}, t r^{2}, t r^{3}$, tracheal gills; $t l$, longitudinal tracheal trunks; $V F$, fore wings. (From Lang's Comparatice Anatomy.)

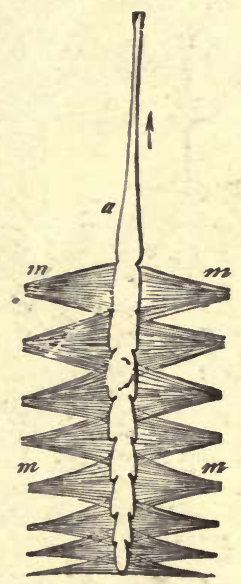

Fis. 5\%:- - Heart of Cuckchafer (Melolontha). $a$ aorta; $m$, m, alary muscles. (From Gegenbaur.)

ganglion or brain, a sub-cesophageul ganglion, also double, and a series of thoracic and abdominal pairs of ganglia, which are closely united together in the middle line. The brain is relatively large in the higher Insects, and is divided into several lobes. It gives off nerves to the antennæ, the ocelli and the labrum, and on each side arises a large lobe-the optic ganglion-on which the compound eye rests. A pair of oesophageal connectives pass backwards on either side of the mouth from the brain to the sub-osophageal ganglia. These connectives are very short, and, as a consequence, the brain and sub-nesophageal ganglia are closely approximated. From the latter there originate nerves to the appendages of the mouth-the mandibles and the two pairs of maxillæ. There are sometimes three pairs of thoracic, and as many as eight of abdominal ganglia in the adult insect; but in many cases there is a greater or less 
degree of concentration of the ventral ganglionic chain (Fig. 523), and in some of the Diptera this reaches such an extreme that all the ventral ganglia, with the exception of the sub-œsophageal, are united into one continuous elongated mass. The Insects, like the higher Crustacea, possess a visceral or sympathetic nervous system, connected with the œsophageal connectives, and passing backwards on the œsophagus and crop.

The most highly-developed organs of special sense are the large compound eyes. The surface of the compound eye is marked out, as in the case of the Crayfish, into a great number of minute hexagonal facets, each of which represents one of the
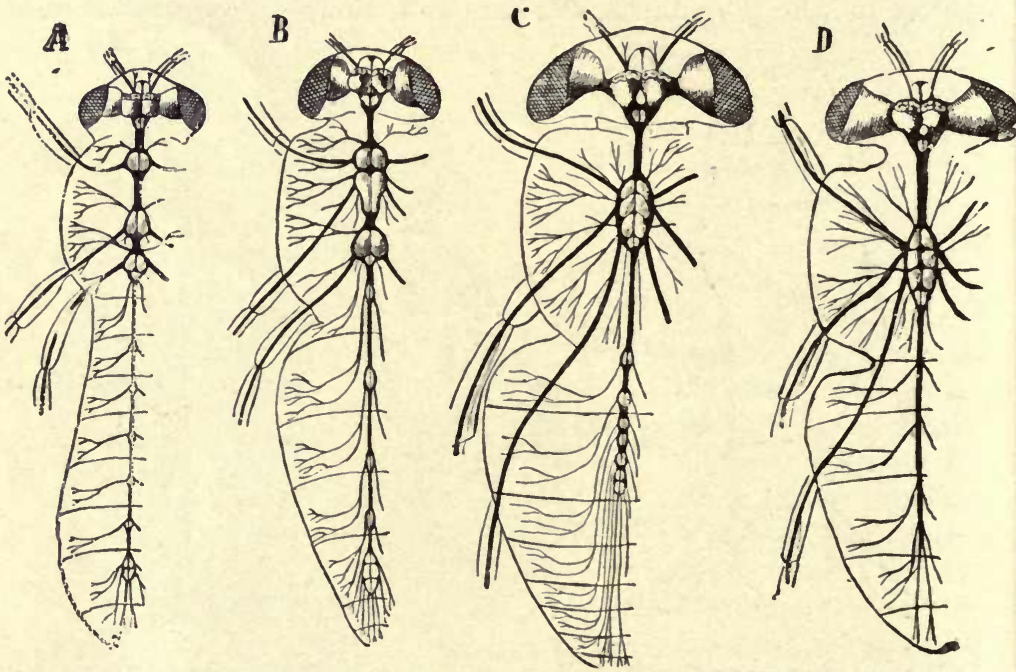

FIG. 523 -Nervous systems of four speeies of Diptera to illustrate various degrees of concentration. A, non-concentrated nervous systems of Chironomus plumosus with three thoracic, and six abdominal ganglia; B, nervous system of Empis stercorea with two thoracic and five abduminal ganglia ; C, nervous system of Tabanus bovinus, with one thoracie ganglion and with the abdominal ganglia elosely approximated; D, nervous system of Sarcophaga carnaria, with all the ganglia of the ventral chain united together with the exception of the sub-oesophageal. (From Lang's Comparative Anatomy.)

elements (ommatidia) of the eye. Of these there may be as many as 28,000 (Dragon-fly). When the eye is examined in section, each ommatidium is found to consist of a cornea-lens-the outer surface of which forms the facet, a crystalline cone, and a rhabdome. The crystalline cone is not always developed, its place being taken in the eyes of some Insects by four crystal cells. The rhabdome is an elongated rod. Beneath the rhabdomes is a fenestrated membrane, beneath which, again, is a dense plexus of nerve-fibres. Nerve-fibres pass through the fenestrated membrane and terminate in a delicate sheath which incloses each rhabdome, the sheath, together with the nerves that end in it, constituting the retinula. Pigment surrounds the crystalline cones and retinulæ. 
The ocelli, or simple eyes (Fig. 524), consist of a bi-convex transparent thickening of the cuticle-the lens-and beneath it of a group of specially modified epidermal cells. Some of these, situated beneath the lens, form a transparent mass, the vitreous body, another set of elongated cells being arranged to form the retina.

The antennæ and palpi are the organs of touch, and these. appendages seem to be also the seat of the olfactory sense. A number of minute processes sometimes - sunk in pits, and each having a special nerve-plate con-

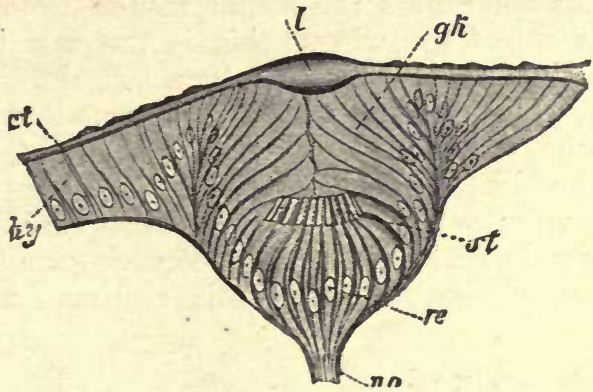

FIG. 524.-Section through the ocellus of a young Dytiscus larva. ct. cuticle : gk, cells of the vitreous body; $h y$, epidermis; $l$, cuticular lens ; no, optic nerve: re, retinal cells; st, rods. (From Lang, after Grenacher.)

nected with it, are regarded as being specially concerned with this sense; and similar processes or pits on the maxillæ and the epipharynx are perhaps connected with the sense of taste. The results of experiments on the action of the antennæ seem. to lead to the conclusion that one of their main functions is to

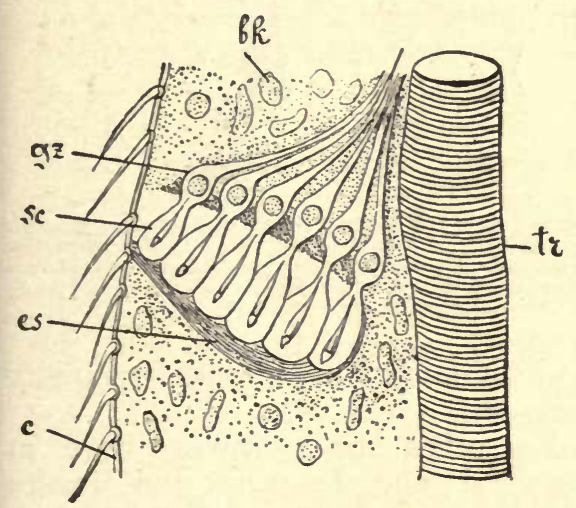

FIG. 525.-Chordotonal (auditory) organ in the tibia of Isopteryx apicalis. bl, blood-corpuscles; c. integument; es terminal fibrous strands attached to the integument; $g z$, nerve-cells: $s c$. terminal rods; $t r$. trachea. (From Lang, after v. Graber.) act as organs for regulating the equilibrium of the body.

Pcenliar nerve-endings, supposed to be auditory, have been found in various parts of the body. Each consists of a ganglion-cell (Fig. 525, gz.) giving off a process which is enclosed in an elongated tube, and which ends externally in a slender rod (sc.). Groups of these are associated together to form the auditory organ.

In certain Insects-the Fireflies and Glowworms, belonging to the order Coleoptera, occur luminous organs for the production of light.

Sounds are emitted by many Insects, and are produced by a variety of different means. Often the sound is the result of the rubbing together of opposed rough surfaces of the integument. The chirp of the Grasshopper, for example, is produced by the 
rubbing of the femur of the last pair of legs over a series of ridges on the anterior wing, and that of the Locust by the rubbing against one another of the roughened basal parts of the first pair of wings. In other cases the sound results from the rapid vibratory movement of the wings; this is the case with the buzzing of many Diptera and Hymenoptera. Again, the humming sounds characteristic of many of the last named order are produced partly by the vibrations of the wings in flight, partly by the vibration of leaf-like appendages in the tracheæ set in mation by strong expiratory currents of air. The loud shrill note of the Cicada is produced by the rapidly recurring contractions of the fibres of a muscle inserted into a stiff chitinous membrane, the result being
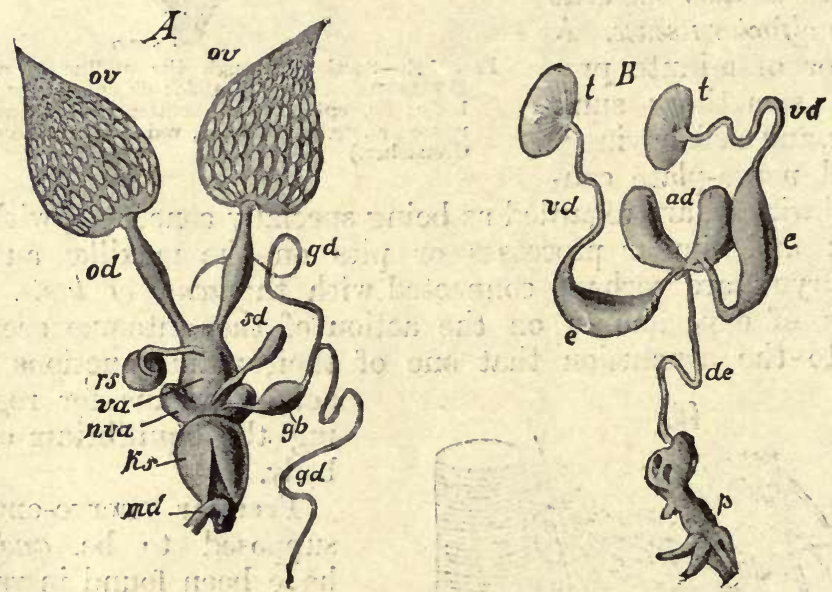

Fic. 526. $-A$, femalc and $B$, male sexual apparatus of the Foney-bee; $a d$, accessory glands ; de, c mímon ejaculatory duct; $g d$, poison-glands; $g b$, poison-vesicle; $l \cdot s$, bulb of the stinging apparatus; med, rectum, twisted back and cut off; nva, accessory sac of the vagina (bursa copu'atrix); od, oviduct ; ov, ovary; $p$, penis ; $r$, receptaculum seminis ; sd, colleterial gland ; $t$, testes ; va. vagina ; $r d$, sperm-ducts. (From Lang's Comparative Anatomy.)

a series of crackling sounds, which follow one another so rapidly as to give rise to a continuous note.

Reproductive organs.-The sexes are always separate in Insects, as in Arthropoda in general; and the males and females are very commonly distinguishable from one another by various inodifications of form and of coloration. There are two ovaries eách of which consists of a greater or smaller number of narrow tubes or ovarioles; in each of these the ova are arranged in a single row-the early stages in their formation being situated at the anterior end, the more mature ova towards the posterior extremity. Each group of ovarian tubes opens into a lateral oviduct, and the two lateral oviducts, right and left (Fig. 526, $A$, od.), in most cases unite behind to form a median oviduct or vagina ( $v a$.), which opens towards the posterior end of the abdomen. Connected with 
this median oviduct, or opening close to it, are receptacula seminis ( $r s$.$) and colleterial or cement-glands (sd.). Sometimes there is a$ copulatory sac, or bursa copulatrix (nva.). In the male the paired testes $(B, t$.) vary greatly in form: sometimes each is a long narrow tube; sometimes several such tubes combine to form the testis; or it may be of more compact rounded form and entire or lobed. Each testis has a slender duct or vas deferens $(\mathrm{B}, v d)$, the two vasa deferentia uniting to form a median ejaculatory duct. A vesicula scminalis is appended to each vas deferens or to the ejaculatory duct. Accessory glands, opening into the vas deferens or the ejaculatory duct, secrete cementing material for uniting the sperms into masses, the spermatophores. In most instances the eggs are laid shortly after their fertilisation, only a comparatively few forms, such as the Aphides or Plant-lice, many Diptera, and some Coleoptera, being viviparous. Some Insects, such as the Aphides and the Rees and Wasps, as well as some Lepidoptera and Neuroptera, present us with the unusual phenomenon of parthenogenesis; i.e., ova are formed, as in ordinary female insects, in organs corresponding to the-ovaries of the latter, and are developed without fertilisation. In the case of the Aphides, an autumn generation of completely-developed males and females is followed by a spring generation consisting entirely of females; these are both parthenogenetic and viviparous. In the Bees, the workers (imperfectly developed females) occasionally produce ova which, without fertilisation, develop into drones (males). In one or two groups, including the Scale-Insects (Coccida) and Gall-Insects (Cynipida), males are never developed, so that reproduction is exclusively parthenogenetic. Pcedogenesis accompanies parthenogenesis in certain Diptera ; i.e., the larvoc produce ova and embryos without impregnation.

The eggs when laid are protected from injury by a number of methods; they may be firmly fixed to the substratum, buried in the earth, or laid in the interior of certain plants or even of animals. The deposition of the eggs, by means of ovipositors, in the leaves or other parts of plants gives rise to swellings-the so-called galls, in the interior of which the young Insects live. In the case of many Insects the eggs are enclosed in a cocoon; in others they are surrounded by gelatinous or waxy material. The eggs are, for the most part, of relatively considerable size. In form they vary, but the long oval prevails in most instances. The ripe egg is enclosed in two egg-membranes-an inner, the vitelline membrane, produced by the egg itself, and an outer, the chorion, formed from the follicle-cells. The chorion, which usually exhibits a more or less elaborate pattern, has one or more apertures or micropyles for the entry of the sperm. The contents are distinguishable into two layers-a superficial, consisting of protoplasm, and a central, of nutrient yolk. 
Development.-The segmentation is usually of a type already referred to (p. 597) as very common among the Crustacea, viz., supcrficial segmentation. The actual segmentation (Fig. 527) has chiefly been observed in the case of certain Insects with very little yolk; but there can be very little doubt that in ordinary

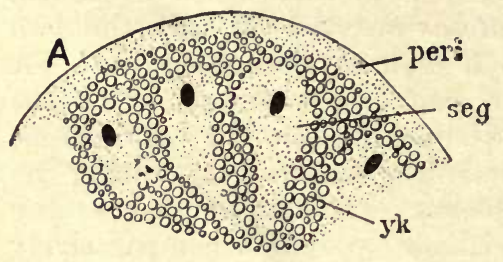

forms with abundant yolk the process is in essence the same. The segmentation-nucleus, originally situated near the middle of the ovum, divides into a number of nuclei, and most or all of these migrate towards the surface, and arrange themselves in the form of a sphere
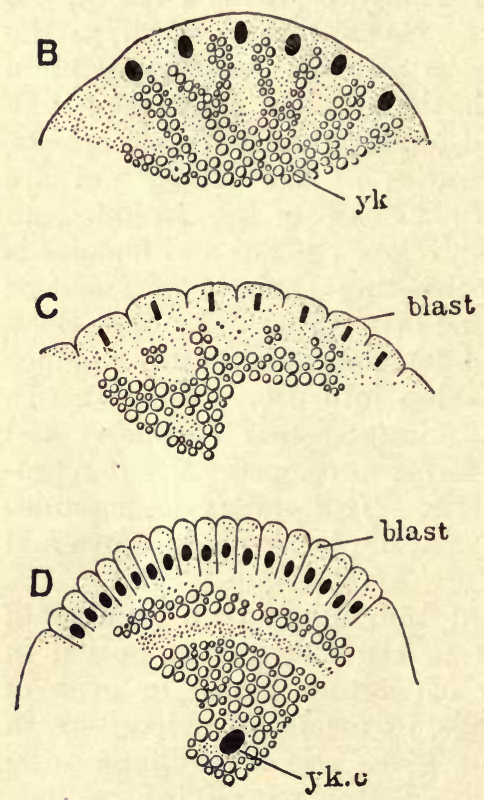

F1,. 527.-A-D, successive stages in the segmentation of the ovum of an Insect b:ast. blastoderm; peri. peripheral protoplasm; seg. segmentation-cclls; $y k$. yolk; $y k c$. yolkcells. (From Korschelt and Heider, after Blochmann.) almost parallel with the latter; eventually they reach the surface and coalesce with the peripheral protoplasm, which then becomes divided into cell-areas corresponding with the nuclei. The layer of cells thus formed constitutes the blastoderm. This thickens along one side to form the ventral plate, as already described in the case of the Cockroach (p. 629), and the changes which this structure undergoes, together with the mcde of formation of the appendages, are similar in most members of the class, except that in most Insects the formation of the lower layers is associated with a more or less distinct invagination (Fig. 528). The same holds good of the formation of the amnion and the development of the mesoderm and endoderm. In some cases there is developed between the serosa and the true

amnion a space filled with yolk, and the ventral plate appears sunk within the yolk. The nervous system is developed from the ectoderm in the manner indicated in the account of the Cockroach (p. 631). The tracheal system is derived from a series of pairs of segmentally arranged ectodermal involutions (Fig. 530, st). 
Metamorphosis.-In some instances the young Insect, when it escapes from the egg-membranes, has exactly the form of the parent, except that, as a rule, the wings have not yet grown. But in most cases there is a metamorphosis. In some this is comparatively slight and gradual, the adult Insect differing from the larva only in comparatively unimportant points, and the segments and appendages of the latter becoming directly converted into those of the former. Such a metamorphosis, in which there is no quiescent stage, is said to be incomplete. The term complete is applied to the metamorphosis of the majority of Insects, in which the larva differs so completely from the imago, or perfect Insect, in external form, the nature of the appendages, and the internal organisation, that there is need of a quiescent or pupa stage, during which the whole animal, or a considerable part of it, undergoes an entire transformation. The metamorphosis is complete in the Diptera, Lepidoptera, Coleoptera, and Hymenoptera, absent or incomplete in the other orders. In the most lowly organised larvæ (many Diptera) the body of the larva or "maggot" is completely worm-like, without any appendages, and without any distinct head. In other cases (Lepidoptera, \&c.), there is a distinct head; the three thoracic segments have three pairs
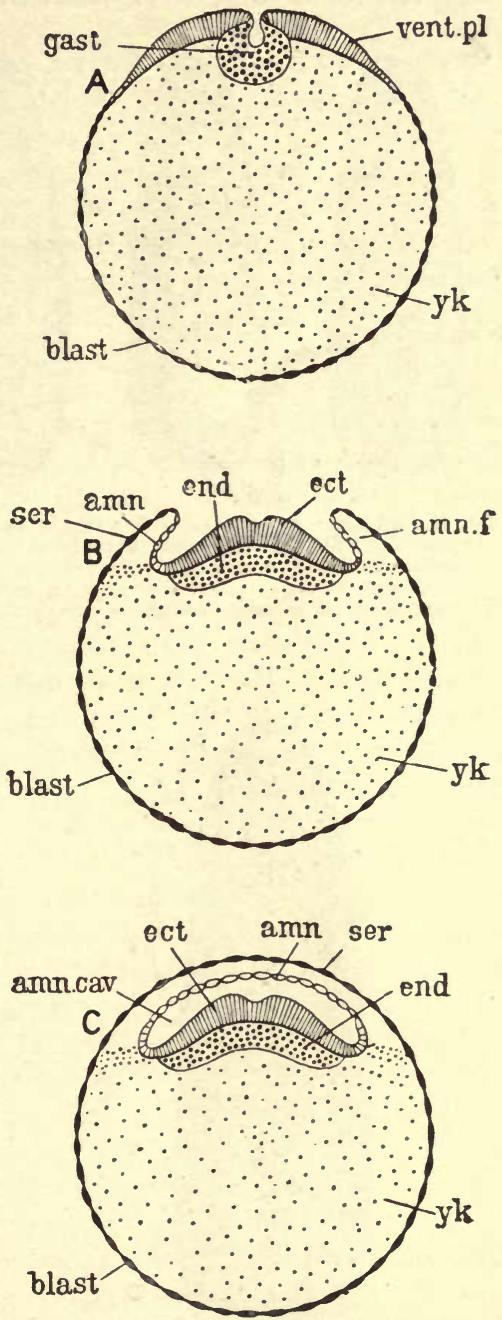

Fig. 528. - A-C, transverse sections through the developing ovum of an Insect at successive stages to show the mode of development of the germinal layers and of the amnion. amn. amnion; amn. $f$. fold of the amnion; amn. cav. cavity of the amnion ; blust. blastoderm covering the yolk; ect. cetoderm; end. endoderm; gast. invagination of ventral plate; ser. serosa; vent. pl. ventral plate; $y k$. yolk. (After Korschelt and Heider.) of jointed legs, and the abdominal segments short unjointed prolegs (Fig. 513). In most instances the larvæ differ widely from the 
adults in their food and mode of life; very generally the jaws are adapted for biting, even when the mouth of the adult is suctorial.
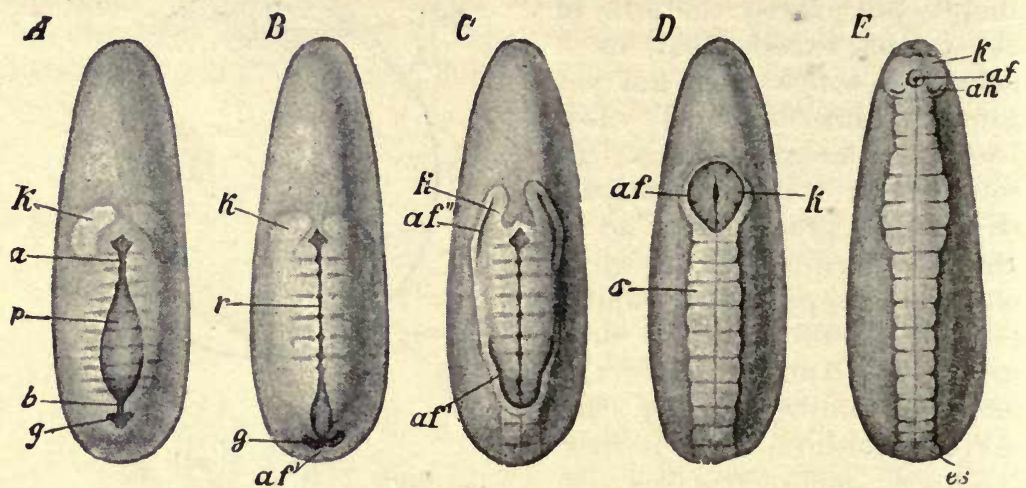

FIG. 529.-A-E, ventral view of five stages in the development of Fy ydrophilus. $a$ and $b$, points at which the blastopore first closes; $a f$. edge of the amnion fold ; $a f^{\prime}$, caudal fold ; $a f^{\prime \prime}$, paired head-fold; an. antenna; es, terminal segment; $g$, pit-like invagination to form the rudiment of the amnion cavity; $k$, procephalic lobes; $r$, groove-like medio-ventral inragination; 8 , germinal bands covered by the amnion. (From Lang, after Heider.)
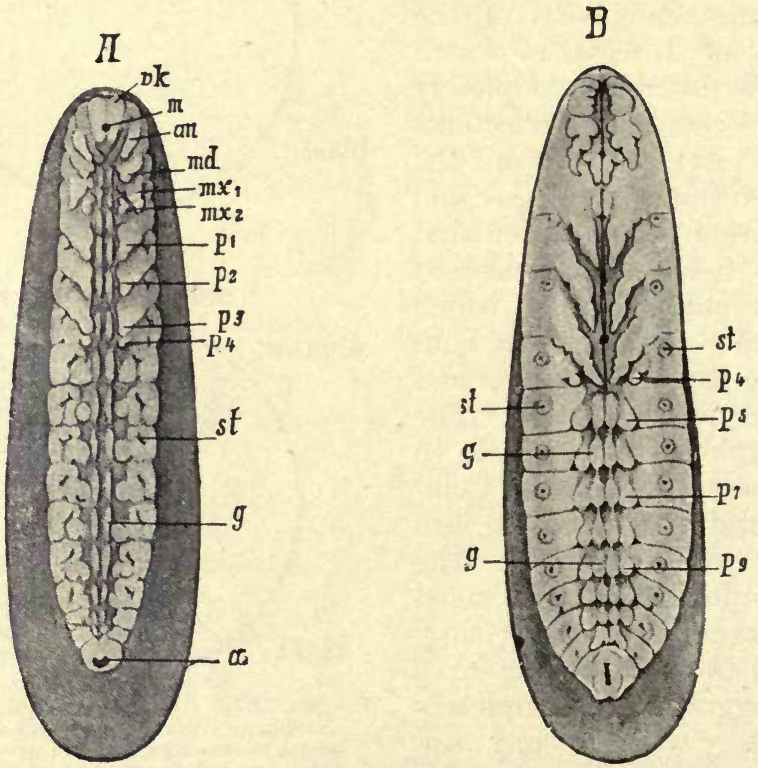

FiG. 530.-A and $B$, later stages of the embryo of Fydrophilus with the rudiments of the extremities ; in $B$ the abdominal appendages are visible. $a$. anus; $a n$. antenna ; $g$, rudiment of the ventral nerve-chain; $m$. mouth; md. mandible; $m x^{1}$, first maxilla; $m x^{2}$, sccond maxilla; $p_{1}, p_{2}, p_{3}$, thoracic legs; $p_{4}, p_{5}, p_{7}, p_{9}$, rudiments of the appendages of the first, second, fourth, and sixth abdominal appendages; st. stigmata; $v k$, prostomium. (From Lang, after Heider.)

After a longer or shorter period passed in this larval condition, in which it is usually active and very voracious, the young Insect 
passes into a quiescent or pripa stage, during which it remains passive, enclosed in a tough integument, while a more or less complete reconstruction of the organs goes on, resulting in the development of all the parts of the perfect Insect. The development of the new parts takes place from certain patches of cells, the imaginal discs, present in the larva.

In the Diptera the larva or maggot is sometimes completely devoid of jaws. In some Diptera, however, the jaws are well developed, and there is a distinct head. After frequent moultings the maggot passes either into a quiescent or pupa stage enclosed in a hard skin, or into the stage of an active aquatic pupa, which swims about actively in water and may possess tracheal gills.

In the Lepidoptera the larvæ ("caterpillars") are worm-like, but with well-developed jaws, three pairs of jointed thoracic legs, and a number of unjointed stumpy abdominal legs (pro-legs). Lepidopterous larvæ are often brilliantly coloured, are very active, and feed with voracity, chiefly on leaves and other succulent parts of plants. Eventually they spin a cocoon of a silky substance, enclosed within which, and covered with a tough skin, they pass through a quiescent or pupa condition-the condition of the chrysalis (Fig. 513). From the interior of this the imago subsequently emerges with all the parts of the adult Insect fully formed.

In mode of life there is a very considerable difference between different orders and families of Insects. Some are parasites in the strict sense throughout life. This is the case, for instance, in the Strepsiptera (Bee-parasites), the females of which live permanently lodged between the joints of the abdomen of their hosts. The Lice and Bird-lice are external parasites throughout life ; Bugs and Fleas, though not adhering to their hosts, are parasites as regards their diet. Many Insects are parasites in the larval condition, though free in the adult state. This holds good, for example, of the larvæ of the Ichneumons, which develop in the interior of the bodies of other insect-larvæ; also of the larvæ of the Bot-flies (Fig. 512), which inhabit the alimentary canal of mammalian hosts (Horses, Oxen, Sheep, Rhinoceroses, Tapirs). The blood-sucking Insects act in certain cases as the carriers or intermediate hosts of the protozoan or bacterial parasites that are the causes of various diseases in man. Thus, as was stated in the account of the malaria-parasite (Section II, p. 86), mosquitoes are the means of conveying that disease from one person to another.

In accordance with the high grade of the structure of their various system of organs, Insects exhibit a correspondingly high degree of functional activity. The quantity of food consumed and assimilated is great in comparison with the bulk of the body, and the energy expended in muscular contractions is of very considerable amount. It is estimated that while the muscular force exerted by a Horse bears a ratio of about 0.7 to its own weight. 
(reckoned as 1) the muscular force of an Insect bears a ratio to its weight of from about 14 to about 23 . Insects are also distinguished among the Invertebrata by the keenness of their senses. The sense of sight is, as we should expect from the elaborate character of the optic organs, the most highly developed, many Insects having been shown by experiment to have a keen sense of colour; but a sense of smell, the seat of which is in the antennæ and palpi, can be shown to exist in a high degree, and

$a$

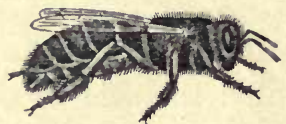

$b$

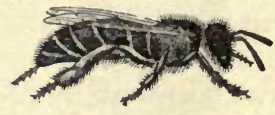

$c$

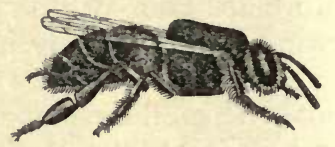

FIG. 531.-Honey-bee (Apis mellifica). $a$, queen (perfect fernale); $b$, worker (imperfeet female), and $c$, drone (male). (After Brehm.)

the parts about the mouth bear nerve-endings concerned in a welldeveloped sense of taste. A sense of hearing does not appear to be universally present, but is well marked in such forms as produce sounds. At the same time Insects are remarkable for the instincts, often leading to results of an elaborate character, which guide them in the pursuit of food and the protection and rearing of their young. Among the insects which are the most highly endowed in this respect are some-the $A^{+} s$, Bees, Wasps, and Termites-which live together in organised associations or communities, the various individuals composing which are distinguishable into sexual individuals, neuter vorkers, and soldiers (Figs. 531
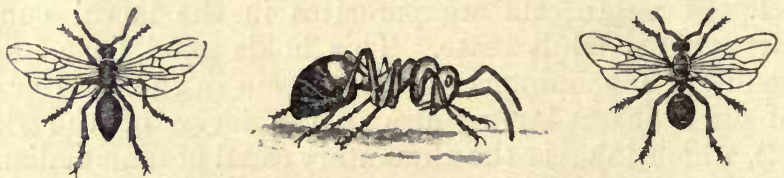

Fia. 532.-Red Ant (Formica rufa); male, worker, and female. (After Brehm.)

and 532), each specially organised for the part which it has to play in the economy of the conmunity.

Distribution in time.- The earliest known fossil remains of Insects have been found in rocks of Silurian age. A good many fossil Insects have been found in the Devonian; but they only become abundant in the Carboniferous. All the Palæozoic Insects belong to a group which has been regarded as a distinct order, and has been named the Palocodictyopterc. The members of this group are characterised rather by the absence of the special characteristics of any of the existing orders than by any positive 
features of their own; but different families of the order approximate to a certain extent towards the groups of living Insects. Amongst them, for example, are forms representing the Cockroaches and the Phasmidæ among the Orthoptera; others representing the modern Day-flies among the Neuroptera; others the Coleoptera.

Of the existing orders, the Neuroptera, Orthoptera, and Coleoptera are first found in the Trias ; the Hemiptera, Diptera, Hymenoptera, and Lepidoptera in the Jurassic.

\section{$A R$ UI DLASS V.-ARACHNIDA.}

The class Arachnida, comprising the Scorpions and Spiders, the Mites and Ticks, the King-crabs, and a number of other families, is a much less homogeneous group than the Insecta, approaching the Crustacea in the variety which it presents in the arrangement of the segments and their appendages. In most members of the class, however, there is an anterior region of the body-the cephalothorax-representing both head and thorax, and a posterior part, or abdomen, which is typically composed of a number of distinct segments ; in some cases cephalothorax and abdomen are amalgamated. There are no antennæ in the adult Arachnid, though rudiments of them have been found in the larvæ of some species. The first pair of appendages of the cephalothorax (probably representing the antennæ of the Crayfish) are the cheliceros; the second are the pedipalpi, the representative of the Crayfish's and Cockroach's mandibles. Behind these are four pairs of legs. The organs of respiration are sometimes trachex, similar to those of the Insects, sometimes book-lungs, or sacs containing numerous book-leaf-like plates: sometimes leaf-like external appendages or gills.

\section{Example of the Class.-The Scorpion (Euscorpio or Buthus).}

Scorpions are inhabitants of warm countries-the largest kinds being found in tropical Africa and America. They are nocturnal animals, remaining in holes and crevices during the day, and issuing forth at night to hunt for their prey, which consists of Spiders and Insects. These they seize with their pincer-claws and sting to death with their caudal spine, afterwards sucking their juices.

There are a number of different species of Scorpions, divided into several genera, which differ from one another in comparatively unimportant points, so that the following general description will apply almost equally well to any of them. 
External features.-A Scorpion (Fig. 533) has a long narrow body, in superficial appearance not unlike that of a Crayfish. There is a small cephalothoracic shield or carapace, covering over dorsally a short anterior region, cephalothorax or prosoma. This is followed by a long posterior region or abdomen, the terminal part of which in the living animal is habitually carried over the back (536), constituting the "tail," at the end of which the sting is placed. The carapace bears a pair of large eyes about its middle, and several pairs of smaller eyes on the antero-lateral

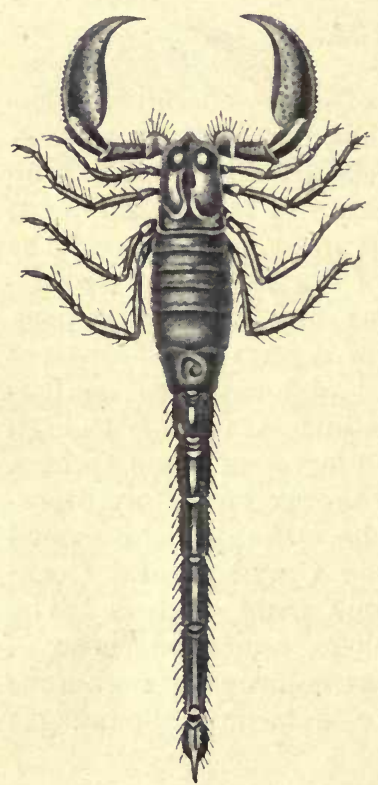

FIG. 533.-Fuscorpio. (From Cuvier's Animal Kingdom.)

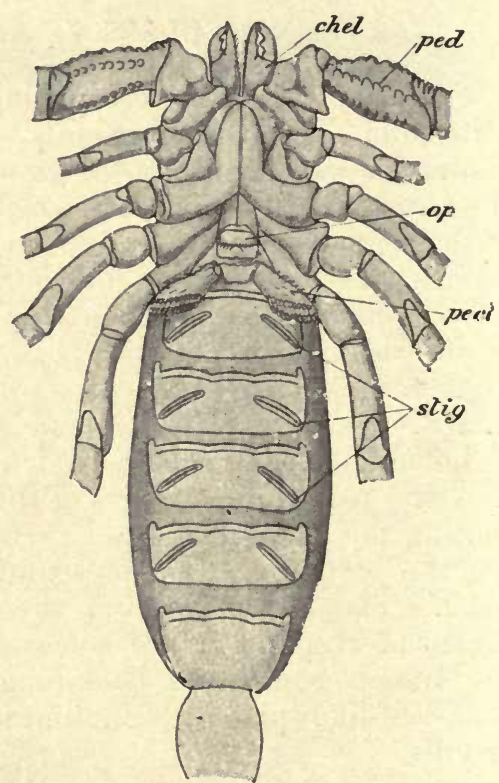

FIG. 534.-Scorpion. Ventral surface of the cephalothorax and pre-abdomen. chel. cheliceræ; op. operculum; pect. pectines; ped. pedipalpi ; stig. stigmata. (From Leuckart and Nitsche's Diagrams.)

margin. The anterior, broader part of the abdomen, which is termed the pre-abdomen or mesosoma, consists of seven segments, each of which is enclosed in firm, chitinous, dorsal and ventral plates, or terga and sterna. The tergum and sternum of each segment are separated from one another laterally by intervals of soft skin, except in the seventh, where they are united laterally for a longer or shorter distance. The posterior, narrower part of the abdomen, known as the post-abdomen or metasoma, consists of five segments, each enclosed in a complete investing ring of hard chitinous matter. Articulating with the last segment of the 
post-abdomen is a terminal appendage, the caudal spine or sting, swollen at the base and acutely pointed at the apex, where open the ducts of two poison-glands. The anal opening is situated on the ventral surface of the last segment of the post-abdomen, immediately in front of the sting.

The aperture of the mouth, which is very small, is at the anterior end of the cephalothorax on its ventral aspect; a lobe which overhangs it in front is the labrum. On each side of the mouth is a three-jointed appendage-the chelicera (Fig. 534, chel.) - which is terminated by a chela. Behind these are the very large pincerclaws or pedipalpi (ped)., each composed of six podomeres and terminating in a powerful chela. The basal joint of each pedipalp has a process which bites against the corresponding process of the other pedipalp, these processes thus performing the function of jaws. Following upon the pedipalpi are four pairs of walking legs, each composed of seven podomeres, the last of which is provided with curved and pointed horny claws. The basal segments of the first two pairs of walking legs are modified so as to perform to some extent the function of jaws.

All the six pairs of appendages hitherto described - the cheliceræ, the pedipalpi, and the four pairs of walking legs-belong to the cephalothorax. The first segment of the pre-abdomen (Fig. 534) has a narrow sternum, on which there is a soft rounded median lobe divided by a cleft; this is termed the genital operculum (op.); at its base is the opening of the genital duct. To the sternum of the second segment of the pre-abdomen are attached a pair of remarkable appendages of a comb-like shape-the pectines (pect.)-each consisting of a stem, along the posterior margin of which is a row of narrow processes, somewhat like the teeth of a comb; the function of these appendages is doubtful, but is probably sensory. The remainder of the segments of the pre-abdomen, and all those of the post-abdomen, are devoid of appendages. The sterna of the third, fourth, fifth, and sixth segments of the pre-abdomen, which are very broad, bear each a pair of oblique slits-the stigmata (stig.)-leading into the pulmonary sacs.

In the interior of the cephalothorax, over the nervous system, is a cartilaginous platethe endosternite (Fig. 535)-which serves to give attachment to muscles, and is comparable to the cephalic apodeme of Apus (p. 533).

All the appendages of the Scorpion are postoral in position, and the most anterior - the

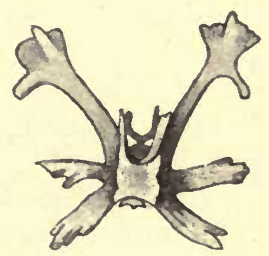

FIG. 535.-Endosternite of Scorpion. (After Lankester.) cheliceræ-are probably best regarded as corresponding to the antennæ of the Crayfish, the equivalent of the Crayfish's antennules and of the antennæ of the Cockroach 
not being present. The pedipalpi would then be the homologues of the mandibles of the Insect and the Crustacean.

\section{Crayfish.}

Antennules.

Antennæ.

Mandibles.

First maxillæ.

Second maxillæ.

First maxillipedes.

Second maxillipedes.

Third maxillipedes.

\section{COCKROACH.}

Antennæ.

Absent.

Mandibles.

First maxillæ.

Second maxillæ.

First legs.

Second legs.

Third legs.

\section{SCORPION.}

Absent.

Cheliceræ.

Pedipalpi.

First legs.

Second legs.

Third legs.

Fourth legs.

Digestive system.-The narrow mouth leads into a large chamber with elastic walls, the pharynx; this is capable of being greatly dilated by the action of a number of radiating bundles of muscular fibres which run outwards from it to the walls of the cephalothorax, the result of this being to cause suction through the mouth, by which means the juices of the Scorpion's prey are drawn in. A second dilatation, to which a narrow oesophagus leads, receives the ducts of a pair of salivary glands (Fig. 537, sal. gld.). Upon this follows the mesenteron (mesent.), which is an elongated, wide, straight tube, with glandular walls corresponding to the stomach of the Insect. Opening into the mesenteron are five pairs of narrow tubes (Figs. 536 and 537, hcp. du.) leading into the substance of a large glandular body, usually termed the liver (hep.), though its hepatic functions are doubtful. Into the long narrow intestine, in the first segment of the post-abdomen, open one or two pairs of delicate tubesthe Malpighian tubes (mal.)--which act as the organs of renal excretion.

Circulatory organs.-An elongated tubular heart (Fig. 536, $h r t$.) lies in the pre-abdomen enclosed in a pericardial sinus; it is divided internally into a series of eight chambers by transverse partitions ; into each of these chambers the blood passes by a pair of valvular apertures or ostia. The heart ends both in front and behind in main arteries, the anterior and posterior aorto (ant. art., post. art.); and a series of pairs of lateral arteries are given off from the various chambers. The anterior aorta soon bifurcates to form a pair of vessels which embrace between them the esophagus, and meet below in a median ventral trunk which runs backwards above the nerve-cord. The blood carried to the various parts of the body by the arteries is gathered up into a large ventral sinus from which it passes to the book-lungs. From these it is carried by a series of veins to the pericardial sinus to enter the heart through the ostia. 
The organs of respiration in the Scorpions are in the form of pulmonary sacs or book-lungs (pul.), the stigmata or external openings of which have already been referred to. Each pulmonary sac is

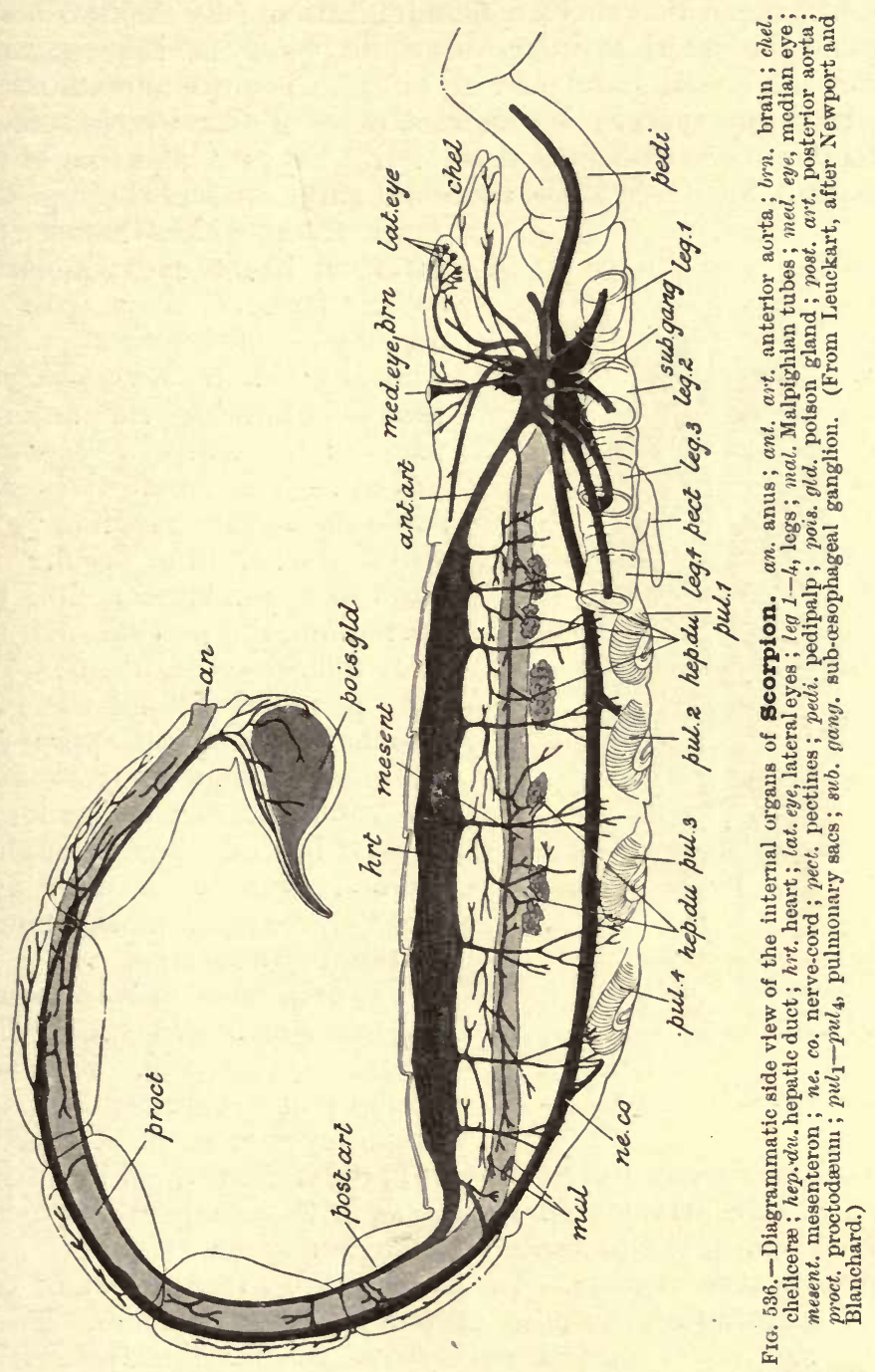

a compressed chamber lined with a thin cuticle. The lining membrane is raised up into numerous delicate laminæ lying parallel with one another like the leaves of a book. Into the numerous narrow spaces between the laminæ the air penetrates, 
and oxygenates the blood which enters the interior of the laminæ from the ventral sinus.

A pair of coxal glanils, situated near the base of the fifth pair of appendages, are, in the embryo Scorpion, represented by tubes which, like nephridia, effect a communication between the bodycavity and the exterior; in the adult Scorpion the tube assumes the form of a closed gland, and its function is quite uncertain.

The nervous system is constructed on a plan which bears a considerable resemblance to that of the Crayfish and that of the Cockroach. There is a bilobed cerebral ganglion or brain (Fig. 536,

trn.) from which nerves are

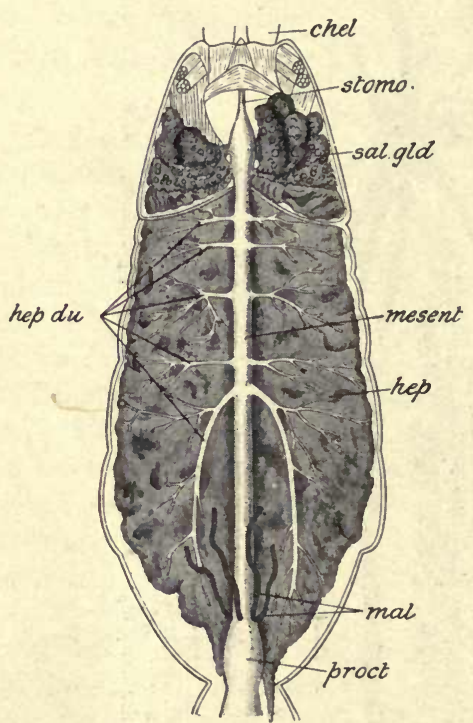

Fig. 537.-Dorsal view of the internal organs of Scorpion. chel. cheliceræ; hep. liver; hep. du. hepatic duets; mal. Malpighian tubes; mesent. mesenteron; proct. intestine; sal. gld. salivary glands; stomo. stomodæum. (From Leuckart, after Blanchard.) given off to the eyes; a nervecollar formed of a pair of cesophageal connectives unites ventrally in a sub-cesophageal ganglion, forming the anterior part of a ventral nerve-cord (ne.co.). The connectives and sub-œsophageal ganglion give rise to the nerves of the first six pairs of appendages and of the operculum, the pectines, and the two following segments. The first ganglion behind the subœesophageal ganglion appears in the eleventh segment (reckoning the cephalothorax as made up of six); behind which a ganglion occurs regularly in each segment as far back as the fourth of the post-abdomen.

The organs of special sense are the eyes and pectines. The lateral eyes (Fig. 556) are similar in character to the simple eyes or ocelli of Insects.

The two larger central eyes (Fig. 557) differ from them in having the retinal cells arranged in groups as in the compound eye, but resemble them in the presence of a single cuticular lens.

Reproductive organs. - In the male the testes consist of two pairs of longitudinal tubules united by cross branches. These are connected with a median vas deferens, the terminal portion of which, provided with accessory glands, is modified to form a double penis; its external opening is just behind the operculum, as already noticed. There is an unpaired ovary, which is made up of three longitudinal tubules with transverse connecting branches; the oviducts open on the operculum. 
Scorpions are viviparous. The eggs, which are spherical or oval, and in most species contain a large amount of food-yolk, lie in a follicle formed of a diverticulum of the oviduct. Fertilisation either occurs in the follicle or after the egg has escaped into the oviduct. The further development takes place in the oviducts ; and, when born, the young Scorpion differs from the parent very little save in size.

Development.-The segmentation is of the type to which the term discoidal is applied. On one side are formed a number of cells in the form of a one-layered disc or cap, which gradually spreads over the yolk. On this appears a thickening - the ventral plate (Fig. 538) corresponding to that of the Insect. A longitudinal groove which
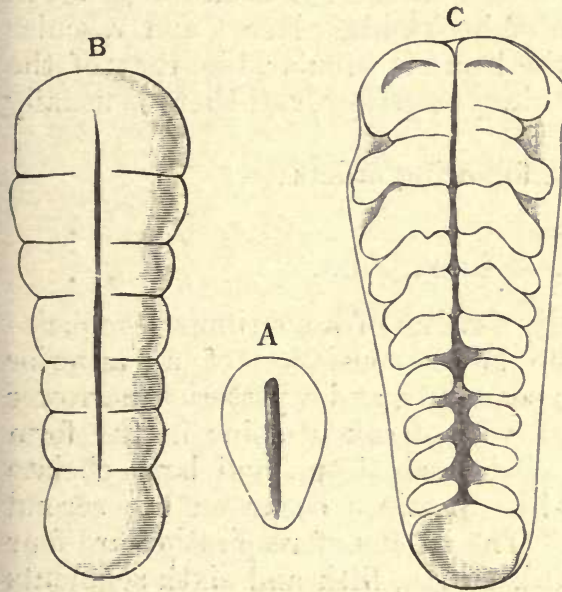

Fig. 538.-Three surface views of the ventral plate of a developing Scorpion. A, before the appearance of segments; B, after five segments have become formed; C, after the appendages have begun to be formed. (From Balfour, after Metschnikoff.)

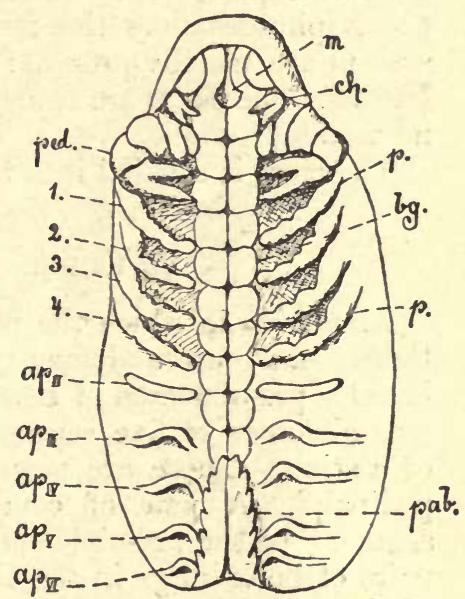

Fig. 539.-Embryo of Scorpion (2uscor: pius italicus), later stage. $a p$. II.-VI., abdominal appendages ; $c h$. cheliceræ ; $p .1-4$, legs; $m$. mouth ; ped. pedipalpi ; pab. post-abdomen, (From Korschelt and Heider, after Metschnikoff.)

appears on the surface of this may be regarded as representing an elongated blastopore (Fig. 538, A). The cells of the blastoderm of the ventral plate become divisible into three layers-ectoderm, endoderm, and mesoderm. The mesoderm undergoes division into a series of masses which are hollowed out to form the primitive segments (B) and their cavities. Embryonic membranes-serosa and amnion-are formed as in the Insects. When about ten segments have become distinguishable, the rudiments of appendages (Fig. $538 \mathrm{C}$, and Fig. 539) appear in the form of hollow processes of the segments on either side of the middle line. Behind the rudiments of the thoracic limbs appear a series of six pairs of abdominal appendages ( $a$, J.-VI.); the place of the first of these 
is afterwards taken by the operculum; the second develops into the pectines. The four posterior pairs become aborted, though they apparently have some relation to the development of the book-lungs.

\section{Distinctive Characters and Classification.}

The Arachnida are air-breathing Arthropoda in which the body is usually distinguishable into two regions-cephalothorax and abdomen. The cephalothorax bears sessile, usually simple, eyes, two pairs of jointed appendages - the cheliceræe and pedipalpi - and four pairs of legs. There are no antennæ. The organs of respiration, when present, are usually either tracheæ or book-lungs, but in the Xiphosura take the form of book-gills. Heart and vascular system are usually present; the heart is tubular, like that of the Insects. The sexes are nearly always separate, and there is usually no metamorphosis.

The class is divided into the following orders :-

\section{ORDER 1.-SCORPIONIDA.}

Arachnida in which the body consists of a continuous cephalothorax and an abdomen, the latter consisting of an anterior broader pre-abdomen of seven segments, and a posterior, narrower post-abdomen of five segments, with a caudal spine in the form of a sting. There are small chelate cheliceræ and large chelate pedipalpi. A pair of comb-like pectines occur on the second segment of the pre-abdomen. The organs of respiration are four pairs of book-lungs in the third, fourth, fifth and sixth segments of the pre-abdomen.

This order includes the Scorpions.

\section{Order 2.-PSEudoscorpioNida.}

Arachnida in which there is a continuous cephalothorax, sometimes marked dorsally with two transverse grooves, and a broad abdomen, not divided into pre- and post-abdomen, and not provided with a sting. The cheliceræ are very small, the pedipalpi similar to those of the Scorpions. The organs of respiration are a system of tracheæ. A pair of spinning-glands are present.

This order includes the Book-scorpions (Fig. 540).

\section{Order 3.--PeDipalpida.}

Arachnida in which the body consists of unsegmented cephalothorax and flattened abdomen of eleven to twelve segments. The chelicerse are simple, the pedipalpi simple or chelate, and the 
first pair of legs terminate in a many-jointed flagellum. The organs of respiration are two pairs of book-lungs on the second and third segments of the abdomen.

This order includes the Scorpion-spiders (Fig. 541).

\section{ORDER 4.-SOLPUGIDA.}

Arachnida with three regions-head, thorax (of three segments) and abdomen (of ten segments). The cheliceræ are chelate; the pedipalpi elongated and leg-like. The organs of respiration are tracheæ.

This order includes Galeodes (Fig. 542).

\section{Order 5.-Phalangida.}

Arachnida with an unsegmented cephalothorax, and an abdomen of six segments. The cheliceræ are chelate, the pedipalpi leg-like. The organs of respiration are tracheæ. No spinning glands are developed.

This order includes the Harvest-men.

\section{ORDER 6.-ARANEIDA.}

Arachnida in which the body is composed of an undivided cephalothorax and an unsegmented abdomen, which is usually soft and rounded, and attached to the cephalothorax by a narrow neck. The cheliceræ are sub-chelate, with poison-glands; the pedipalpi simple. The organs of respiration are book-lungs alone, or booklungs combined with trachex.

This order comprises all the true Spiders (Fig. 543).

\section{ORDER 7.-ACARIDA.}

Arachnida in which the body exhibits no division into regions. The mouth-parts are adapted either for biting or piercing and sucking. The organs of respiration, when present, are in the form of tracheæ.

This order includes the Mites and Ticks (Figs. 546 and 547).

\section{ORDER 8.-XIPHOSURA.}

Arachnida in which the body consists of a cephalothorax covered over by a broad carapace, and an abdomen of seven firmly united segments, with a long narrow tail-piece or telson. The cephalothorax bears a pair of short chelate appendages and five pairs of legs. The abdomen bears in front a pair of united plate-like appendages, forming the operculum, followed by five pairs of flat appendages overlapped by the operculum.

VOL. I 
The organs of respiration are lamelliform gills attached to the abdominal appendages.

This order includes the King-crabs (Limulus, Figs. 548 and $549)$.

\section{ORDER 9.-EURYPTERIDA.}

Arachnida with a. relatively small cephalothorax, followed by twelve free segments and a terminal, elongated, narrow telson. There are a pair of pre-oral leg-like or chelate appendages and four more leg-like appendages on the cephalothorax, the last expanded to form swimming paddles. A broad operculum is situated immediately behind the cephalothorax. There are pairs of lamellate appendages on certain of the anterior free segments. The exoskeleton is characteristically sculptured.

This order includes only a number of extinct (Palæozoic) forms of large size (Fig. 550).

\section{General Organisation.}

The external form in the Scorpionida has already been sufficiently described. Most nearly related to that order in this respect are the Pseudoscorpionida or Book-scorpions and their allies. In these (Fig. 540) there is an unsegmented cephalo-

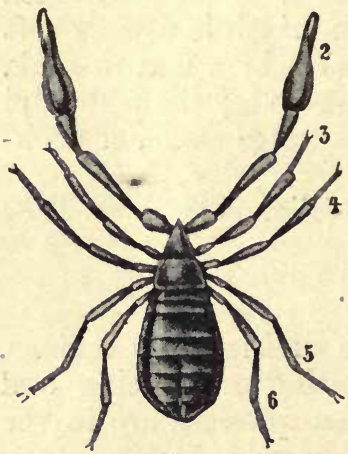

FIG. 540.-Chelifer bravaisii. $2-6$, second to sixth pairs of appendages. (From Lang's Comparative Anatomy.) thorax, or the carapace is crossed by two transverse grooves which may indicate segmental divisions. There is a broad abdomen consisting of eleven, or more rarely ten, segments; the postabdomen is not represented, nor the caudal sting. 'The cheliceræ are small; the pedipalpi are large, and resemble those of the Scorpions in their chelate form. Spinning glands are present.

The Pedipalpi, or Scorpion-spiders (Fig. 541), are intermediate in some of their external features between the Scorpions and the Spiders. The abdomen is broad and marked out into a series of eleven or twelve distinct segments; in one of the genera of the order there is a short post-abdomen formed of the last three segments, with an elongated, many-jointed anal filament. The cheliceræ end in simple claws; they are probably provided with poison-glands; the pedipalps are very long, either claw-like or chelate; the first pair of legs are very long and slender, their terminal part made up like antennæ of numerous short joints. There are eight eyes on the carapace, two larger central, and six smaller marginal. The Solpugida. (Fig. 542) have, at least superficially, the 


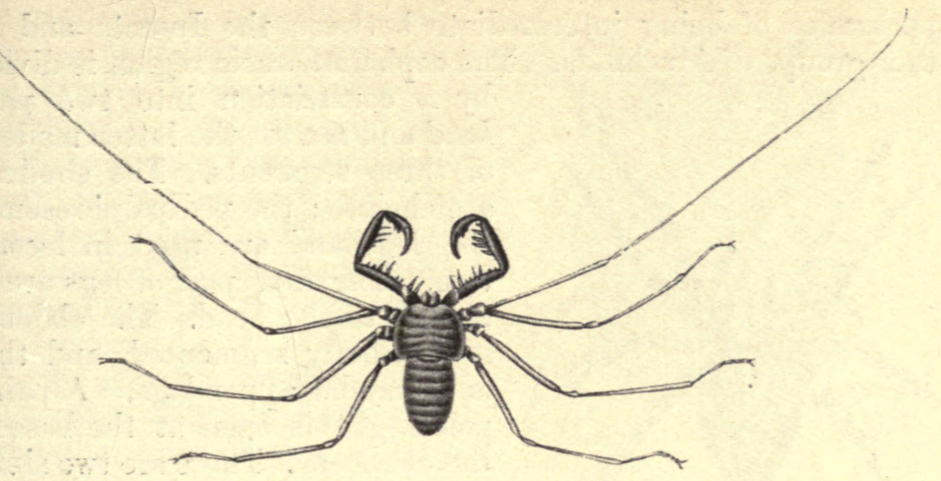

Fig. 541.-Phrynus. (From Cuvier's Animal Kingdom.)
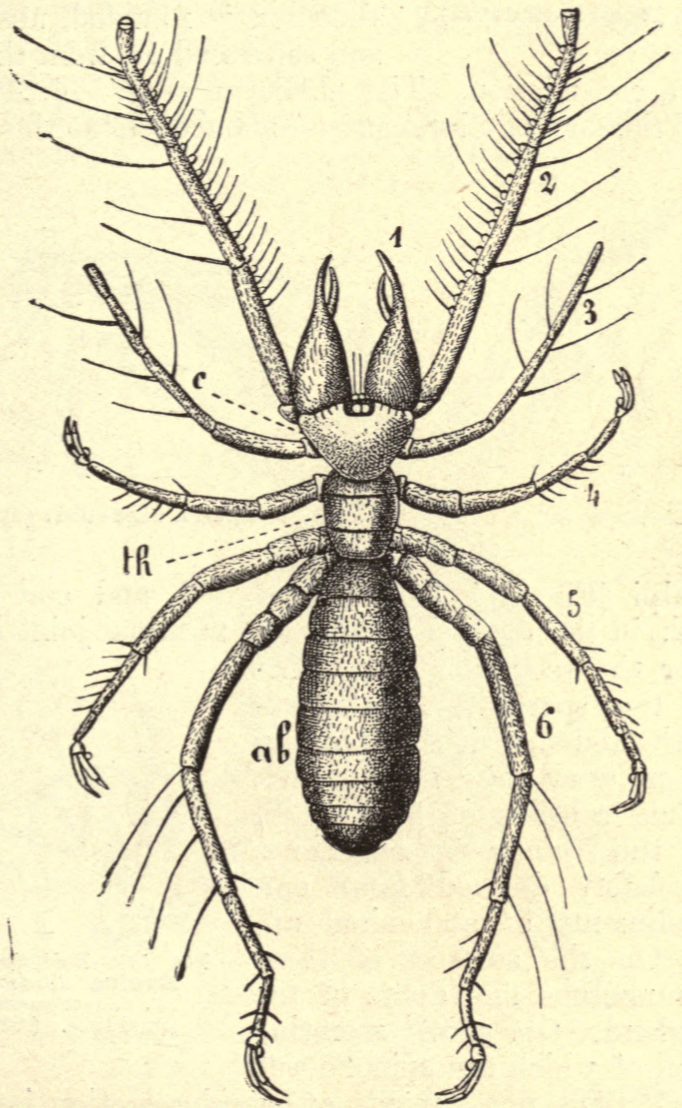

EIG. 542.- Galeodes dastuguei $q$, natural size. $1-6$, the six pairs of appendages; 1, cheliceræ; 2, pedipalpi ; $c$, head ; th. thorax ; $a b$. abdomen. (From Lang, after Dufour.) 
appearance of being intermediate between the Insecta and the other groups of Arachnida. The cephalothoracic region is divided

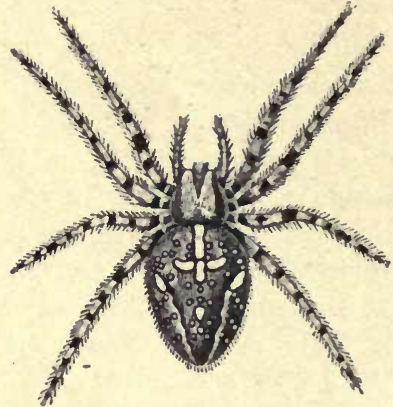

FIG. 543.-Spider (Epeira diadema). by a constriction into two parts, head and thorax, the latter made up of three segments. The cheliceræ are chelate; the pedipalpi resemble the legs, and are used in locomotion. The first pair of legs are attached to the head. The abdomen is distinctly segmented, and there is no caudal appendage. A pair of poison-glands open at the bases of the cheliceræ. 'There are two simple eyes on the head.

In the true Spiders (Fig. 543) the abdomen is rounded, unsegmented, and separated off from the cephalothorax by a constriction. The cheliceræ (Fig. 544, $A$ ) are subchelate, and the duct of a large poison-gland opens at the extremity.
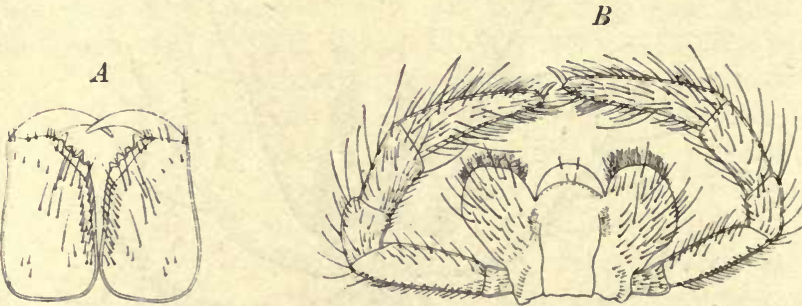

FiG. 544. $-A$, Cheliceræ, and $B$, podipalpi of female of Epeira diadema. (After Leuckart.)

The pedipalpi (Fig. 544, B) are elongated, and end in simple extremities; in the male (Fig. 545) the terminal joint is modified to serve for the reception and transference of the sperms. At the extremity of the abdomen is the spinning apparatus or arachnidium (Fig. 551, arach.). This consists of four or six elevations, the spinnerets, sometimes jointed, probably derived from embryonic rudiments of abdominal appendages. On the surfaces of these open the numerous fine ducts of the spinning glands (sp. glds.), secreting

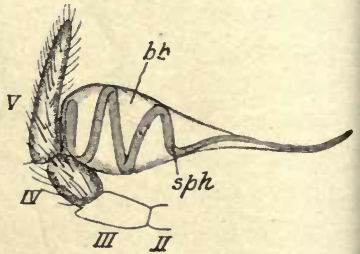

Fig. 545.-Pedipalpi of male of Epeira diadema. 11 . $11 I$. $I V . V$. podomeres: $b b$, sac; $s p h$. spiral tube. (After Leuckart.) the material of which the spider's web is composed. The fine threads of viscid secretion issuing from the ducts harden on, exposure to the air, and are worked up 
into the web by means of the posterior legs. There are six or eight eyes on the carapace.

In the spider-like Phalangida, or "Harvest-men," the cephalothorax is not constricted off from the abdomen. The cheliceræ are chelate, the pedipalpi short and leg-like, the legs long and slender.

In the Acarida, or Mites and Ticks (Figs. 546 and 547), the distinction into regions is no longer recognisable. The form of the mouth parts varies somewhat in the different families. Sometimes the basal portions of the pedipalpi form a sucking

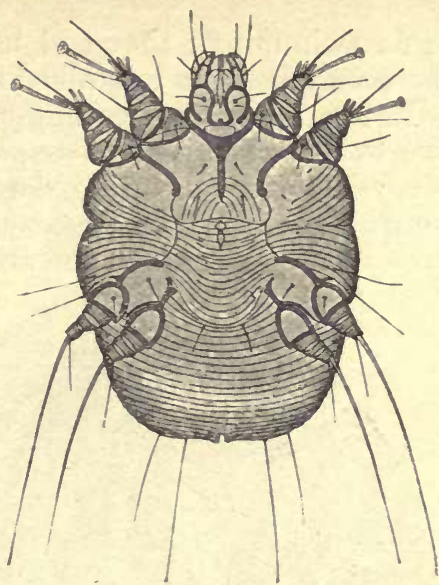

FIG. 546.-Itch mite (Sarcoptes scabiæi). (After Leuckart.) proboscis enclosing the stylet-like cheliceræ, modified to form piercing organs; sometimes these appendages are claw-like or

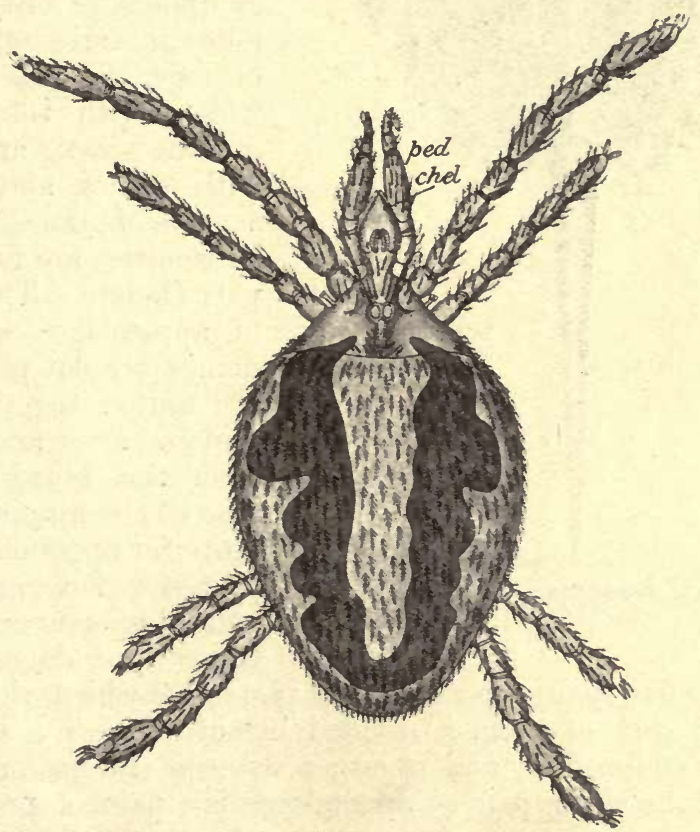

FiG. 547.-Water mite (Trombidium fuliginosum), female chel. chelicera ; ped. pedinalpi. (After Lenckart.)

chelate. The legs vary somewhat in shape in the different groups, according as they are used for prehension, for creeping, for running, 
or for swimming; they end usually in two claws, between which there may be dises or stalked suckers.

In the Xiphosura or King-crabs (Fig. 548), the body consists of two well-marked regions-cephalothorax and abdomen. The former is covered over by a wide, dorsally convex, sub-crescentic shield or carapace, bearing two large compound eyes, and two smaller simple eyes. The segments of the abdomen (seven in number) are united together, being covered dor-

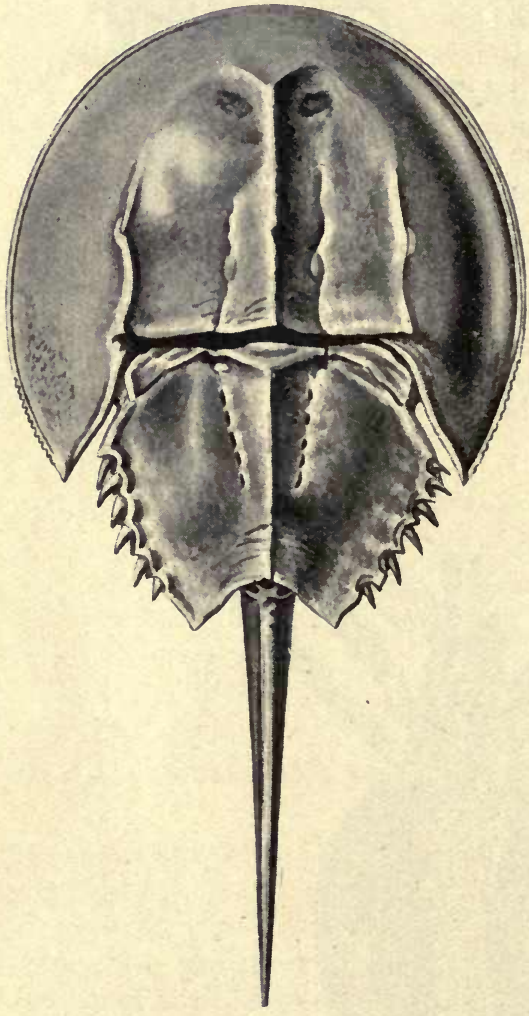

Fif. 548.- Limulus. Dorsal aspect. (After Leuckart.) sally by a continuous abdominal carapace. At the posterior end is attached a very long, narrow, caudal spine or telson. The anterior appendages (Fig. 549) resemble those of the Scorpion. In front of the mouth is a pair of short, threejointed, chelate appendages, the chelicerce (1), at the sides of a labrum (rostrum) or upper lip. Behind these follow a series of five pairs of legs, the bases of all of which, with the exception of the last, are covered with spines, and have the action of jaws, while the extremities are for the most part chelate. The first pair of appendages of the abdomen are flat plates, which are united together in the middle line and together form the broad operculum (operc.), overlapping all the posterior appendages ; on its posterior face are the two genital apertures. The posterior appendages, of which there are five pairs, are thin flat plates to which the gills are attached; each of them is divided by sutures into a small inner ramus or endopodite, and a larger external ramus or exopodite. Between the sixth pair of appendages is a pair of processes, the chilaria.

In the Eurypterida (Fig. 550) there is a small cephalothorax bearing a pair of large eyes and a pair of ocelli, and an elongated segmented region containing twelve segments, followed by a 
narrow pointed telson. There are usually five pair of limbs surrounding the mouth and, with the exception of the first, toothed at the bases in order to perform the functions of jaws; the last pair are stouter than the others and are expanded so as, apparently, to assume the character of swimming paddles. Certain of the more anterior of the free segments bear paired lamelliform appendages which probably carried the branchiæ, as in the Xiphosura. The exoskeleton is in many cases elaborately sculptured.

A cartilaginous internal endosternite of the same nature as that which has been described as occurring in the Scorpions is found in Limulus and in certain: Spiders, but not in the other groups.

\section{Coxal glands,} similar to those that have been described in the Scorpion, occur also in most Spiders, in the Solpugida and Phalangida, in some Acarida, and in the Xiphosura. In the Solpugida and Phalangida they occur on the bases of the last pair of legs: in the Araneida and Xiphosura, as in the Scorpion, they are found on the bases of the fifth pair of appendages.

Alimentary system.-The œsophagus (Fig. 551, os.) of the Spiders is expanded behind into a special sucking stomach (suck. st.). The mesenteron (mesent.) gives off in the cephalothorax a pair of large diverticula from each of which arise five narrow diverticula (cacc.) which enter the bases of the pedipalps and legs ; in the abdomen it is surrounded by a mass of cells commonly 
termed "liver" (hep.), the ducts of which open into it. The rectum or proctodoeum (rect.) is dilated; the dilated portion (rect. cace.) gives off two pairs of Malpighian tubes (mal.).

In the Pseudoscorpionida the mesenteron, which is bent into a loop, gives off three diverticula; the proctodæum has also a diverticulum. In the Solpugida the mesenteron also gives off diverticula; the occurrence of Malpighian tubes is doubtful. In the Acarida there are always diverticula, the number and arrange-

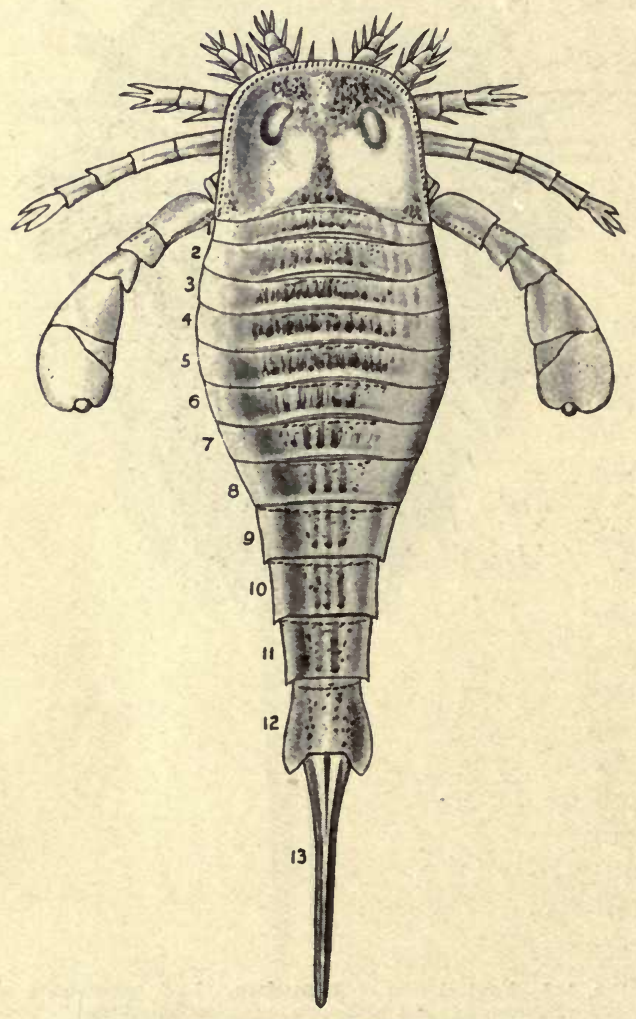

Fid. 550.-Furypterus fischeri (Silurian).

(From Nicholsun and Lydekker.) ment of which vary, connected with the mesenteron. There are usually two long coiled Malpighian tubes.

In the Xiphosura, the mouth (Fig. 552, mo.), which is situated some distance behind the anterior extremity of the body, leads into a suctorial pharynx, followed by a stomach, which opens into the elongated mesenteron; the proctodæum, a short tube with folded walls, opens on the exterior at the posterior extremity of the abdomen. Into the mesenteron, as in the Scorpion, open the ducts of a large gland, usually termed the "liver" (l. liv.).

A heart is absent in all the Mites with the exception of one family. In the other Arachnida a heart is present and has the same general form as in the Scorpions, though always more concentrated.

In the various orders the organs of respiration differ a good deal in their character. In the Pseudoscorpionida they take the form of branching trachea similar to those of Insects. In the Pedipalpi there are two pulmonary sacs or book-lungs similar to those of the Scorpions. In the Solpugida there is a system of 
trachex. In the Spiders there are either four pulmonary sacs (Fig. 553), or two pulmonary sacs and a system of tracheæ (Fig. 554).
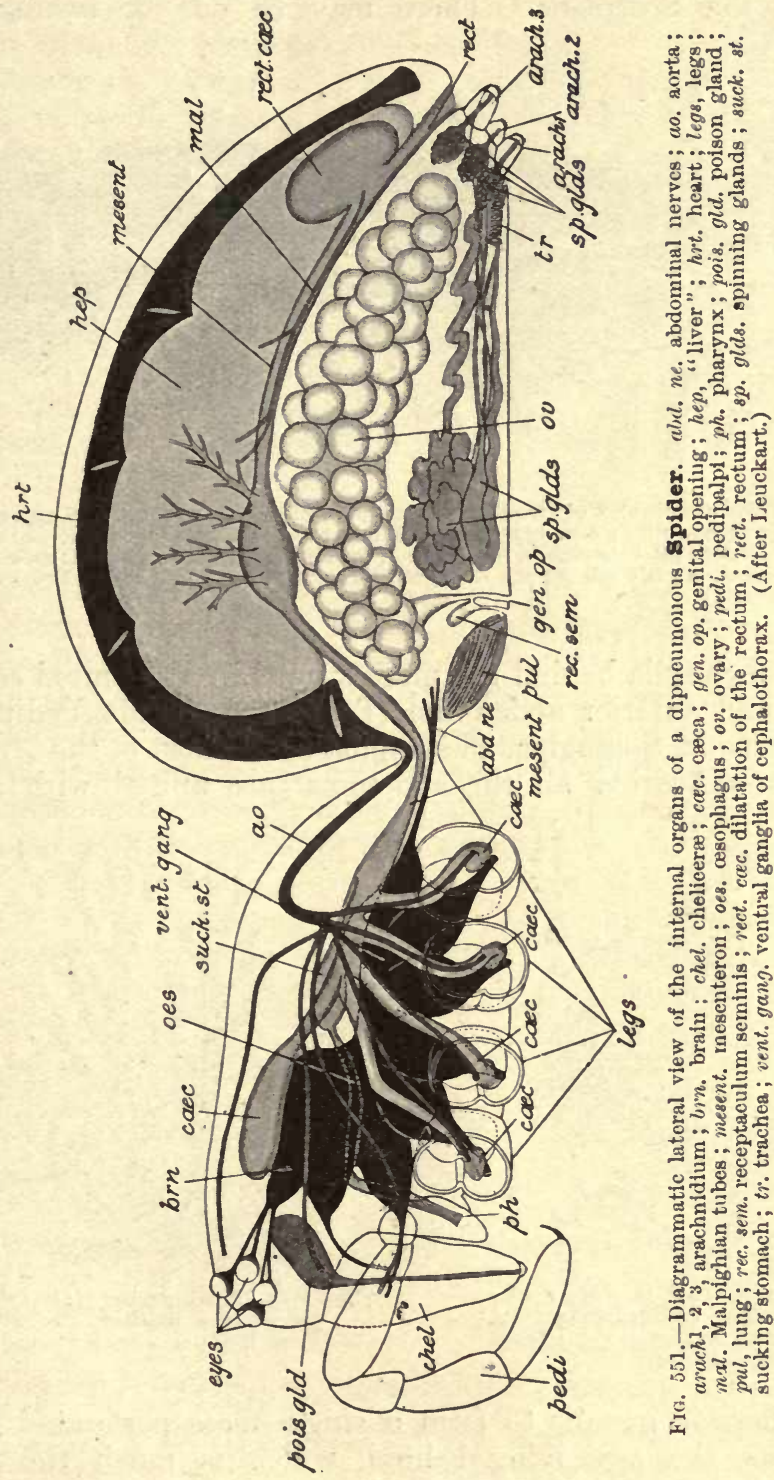

Trachex are present in the Phalangida and also in the majority of the Acarida. In the Xiphosura the organs of respiration are 
external appendages or gills (book-gills), in the shape of delicate laminæ attached to the abdominal appendages (Fig. 555).

The nervous system is, in most instances, more concentrated than in the Scorpions. There may be one or two separate

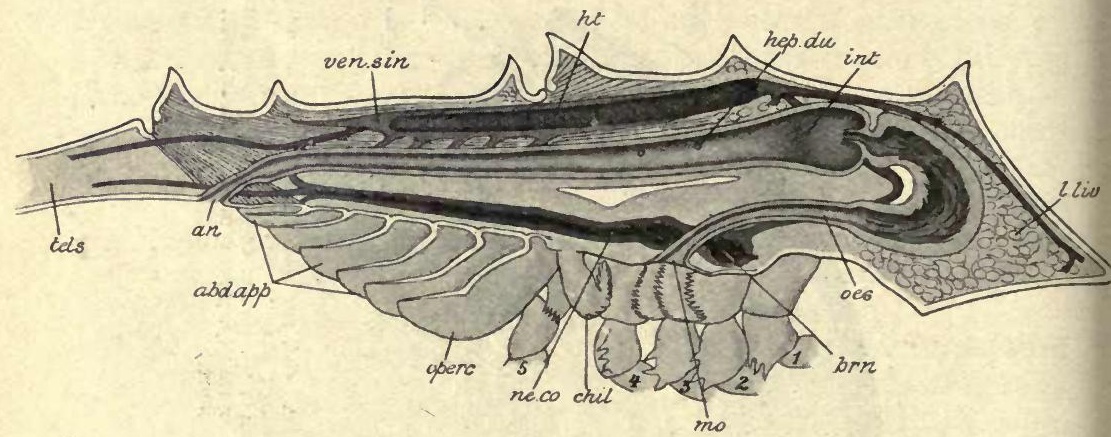

Frc. 552.-Diagrammatic view of a median longitudinal section of Limulus. ald. app. abdominal appendages; an. anus; brn. brain: chil. chilaria; hep. du. opening of one of the hepatic ducts; $h t$. heart ; int. intestine; l. liv." liver" ; mo. mouth ; ne. co. nerve-cord ; cs. nesophagus ; operc. operculum ; tels. telson; ien. sinus, venous sinus; $1-5$, legs. (From Leuckart, partly
after Packard.)

abdominal ganglia behind the mass formed by the united cephalothoracic and anterior abdominal (Pseudoscorpionida, Pedipalpida, some Araneida, Solpugida, Phalangida). In most of the Araneida and in the Acarida all the abdominal are united with all the

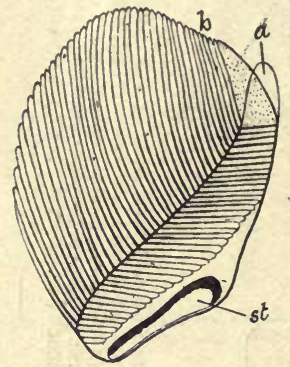

FIa. 553,-Book-lung of a Spider (Zilla callophylla). $a$, axis; $b$, laminæ; st. stigma. (From Hertwig.)

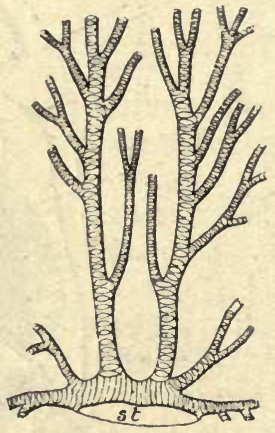

FIG. 554.-Main branches of the tracheal system of a Spider. st. stigma. (From Hertwig, after Bertkau.)

cephalothoracic ganglia to form a single mass perforated by the œsophagus, the part lying behind, which is much the larger, representing the ventral nerve-cord.

Sense-organs.-Eyes are present in all except in some of the Acarida. Their number and arrangement have been given with 
the external characters of the groups. They are all (Fig. 556) of the type of the ocelli or simple eyes of Insects, except the central eyes of the Scorpions (Fig. 557) and the compound eyes of Limulus. The former are intermediate in character between ocelli and faceted eyes, possessing the single cuticular lens (lens) of the ocellus, and resembling the faceted eye in having the retinal cells arranged in groups corresponding to ommatidia. Each retinula, composed of five cells, contains a thick axial rod or rhabdome ( $r h a b d$.).

In Limulus the com-

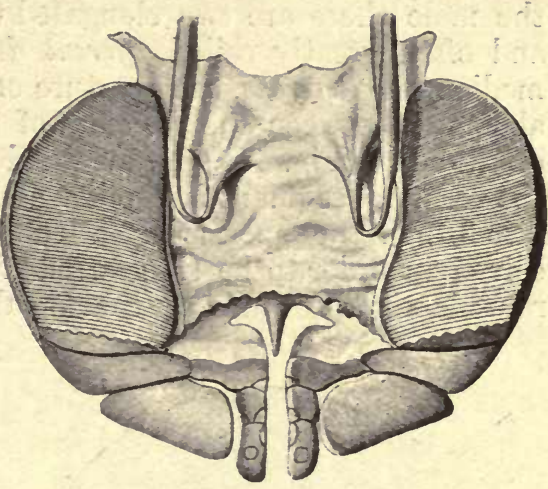

Fig. 555.-One of the book-gills of Iimulus, with the appendage to which it is attached. (After Lankester.) pound eye has a continuous chitinous cornea-lens of the nature of a thickening of the cuticle. This, though non-faceted, differs from the corresponding part in the compound eye of the Scorpion in being produced internally into a number of conical papillæ, each of which lies over one of the ommatidia and may be looked upon as its lens.

A considerable variety is observable in the exact arrangement of the parts of the reproductive apparatus in different groups

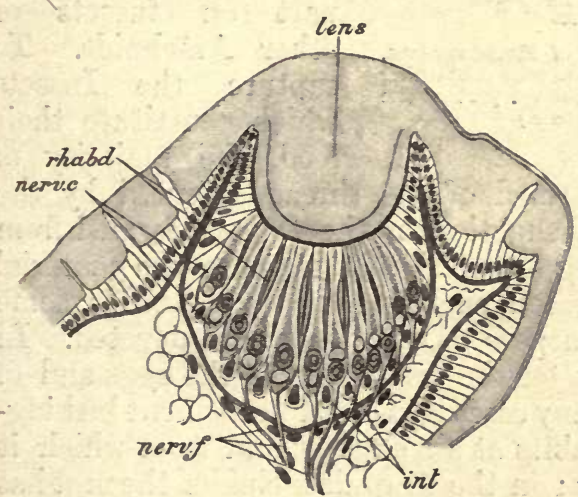

F1G. 556.-Section of the lateral eye of ruscorpius italicus: int. intermediate cells;:lens, cuticular lens; nere.c. - terminal nerve-cells; nerv. $f$. nervefibres of optic nerve; rhabd. rhabdomes. (After Lankester and Bourne.) of the Arachnida. In general, testes or ovaries are either paired or (more rarely) unpaired tubes, with paired vasa deferentia or oviducts, which unite in a median duct opening on the exterior by an unpaired genital opening. Viviparity is exceptional. In the Spiders the ovaries (Fig. 551, ov.) are two wide tubes, on the surface of which follicles project prominently; sometimes they unite into a single circular ovary. There are two short oviducts even when the ovary is single; these unite in a median vagina, which opens on the 
exterior by a median genital aperture at the base of the abdomen. One, two; or three receptacula seminis (rec. sem.) are present, and either open into the vagina or independently on the surface. In the male there are two elongated tubular testes with two narrow and often greatly coiled efferent ducts, which unite in a short median vas deferens, the aperture of which is on the base of the abdomen between the stigmata of the first pair. The pedipalpi of the male (Fig. 545) are modified to act as intromittent organs : the terminal segment is swollen, and contains a twisted tube (sph.) into which the sperms from the reproductive aperture are received in order to be transferred in the act of copulation to the reproductive aperture of the female. The eggs of spiders are

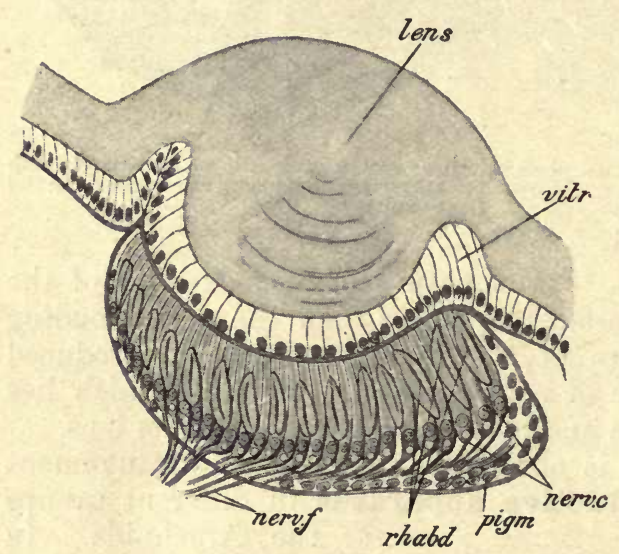

Fuc. 557.-Section of the central eye of Fuscorpius. Letters as in preceding figure. pigm. cells containing pigment; $v t r$. vitreous body (a specialised part of the ectoderm). (After Lankester aud Bourne.)

laid in nests or cocoons, and are usually guarded by the mother, sometimes carried about by her.

In their mode of life the Arachnida present almost as great a diversity as the Insecta. Some Acarida are parasites throughout life. Most of the other groups of Arachnida are predaceous - preying for the most part on Insects or other Arachnids. To capture the Insects which constitute their food, the majority of Spiders construct a web formed of the threads secreted by the arachnidium. The primary function of the threads formed from the secretion of the spinning organ is to constitute the materia! for the manufacture of a cocoon enclosing the eggs, and in some Arachnids this is the sole purpose to which they are devoted. In others there is added a nest for the protection of the eggs and of the parent itself ; this in many cases becomes a permanent lurkingplace which the Spider inhabits at all seasons, and from which it darts out to capture its prey; in the Trap-door Spider the nest has a closely fitting hinged lid. In very many Spiders the secretion is used mainly to form the web by means of which the prey is snared, and frequently also a nest in which the Spider. lies in wait. A subsidiary function of the threads is to aid in locomotion, the Spider being enabled by means of them to let itself down safely from considerable heights, and even to float in the air. 
Some of the Mites, as already mentioned, are parasitic; others feed on various kinds of fresh or decaying animal or vegetable substances. Most free Acarida are terrestrial; some are aquatic.

The Xiphosura are marine, living at a depth of a few fathoms in warm seas, burrowing in sand; their food consists of various kinds of marine Annelids.

Geological History.-The most ancient of the living groups of the Arachnida are the Scorpions, which are represented in Silurian rocks by various fossil forms not differing very widely from those existing at the present day. The earliest known fossil Spiders have been found in deposits of Carboniferous age; and remains of Pedipalpida occur in the same formation. In Tertiary deposits there have been found representatives of all the principal groups of living Arachnida.

The earliest fossil-remains of Xiphosura that have been found occur in strata of the Triassic period. Other fossil species occur in later formations. These are all nearly related to the living species of Limulus. The Eurypterida, as already noted, are entirely Palæozoic, ranging from the Lower Silurian rocks to the Devonian.

\title{
APPENDIX TO THE ARACHNIDA.
}

\author{
THE PYCNOGONIDA, LINGUATULIDA, AND TARDIGRADA.
}

These three groups, though not in any way related to one another, and of doubtful relationships to the Arachnida, are, as a matter of convenience, mentioned together here.

\section{The Pycrogonida.}

These are marine Spider-like Arthropods (Fig. 558) in which the body cousists of a cepthalothorax composed of an anterior proboscis $(s)$, three head-segments, and one thoracic segment, followed by three free thoracic segments and a rudimentary abdomen $(a b$.$) . The cephalothorax bears usually four simple eyes and$ four pairs of appendages, one or both of the first two of which may be chelate. To these succeed a pair of usually ten-jointed ovigerous legs (3), and the first pair of thoracic legs (4). The free thoracic segments bear lateral processes for the articulation of the remaining three pairs of legs. The rudimentary abdomen $(a b$.$) is devoid of appendages.$

Diverticula from the mesenteron penetrate for a considerable distance into the limbs. Malpighian vessels are absent. There is a tubular heart with two or three pairs of ostia. Organs of respiration are absent. The nervous system consists of brain, sub-œsophageal ganglia and three other ganglia in the cephalothorax, and one or two small pairs in the abdomen. The testes in the male are partly, and the ovaries in the female either partly or completely contained in the bases of the thoracic appendages on which they open. In the male 4-7 cement-glands are situated in the fourth joints of certain of the appendages; their secretion cements the eggs together into masses which are carried on the ovigerous legs of the male, and in one species on those of the female also.

A metamorphosis occurs in most cases. The larva usually has three pairs of appendages, so that it bears a superficial resemblance to a nauplius; but the 
appendages are simple, and in other respects the larva has no essential likeness to the nauplius form. Additional segments with their appendages are formed

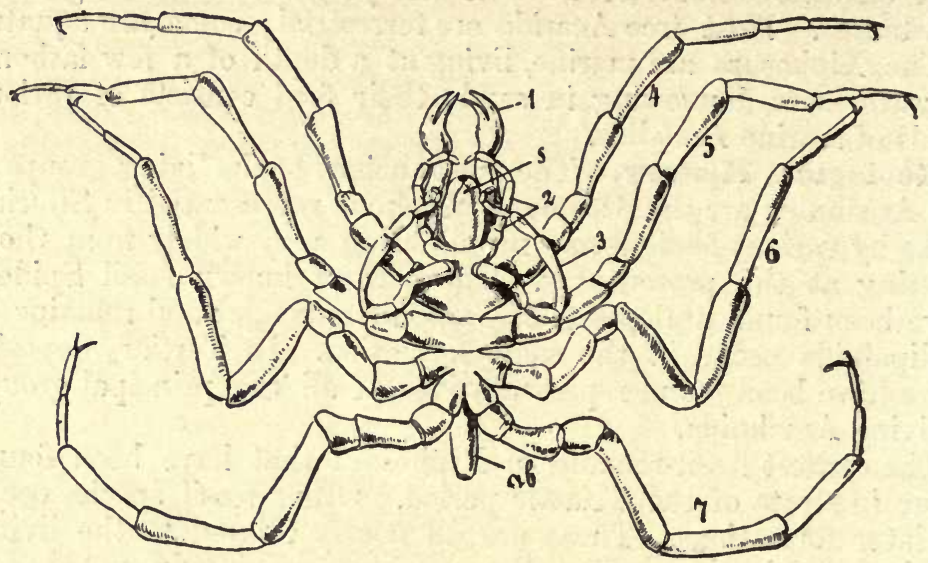

FIG. 558.-Nymphon hispidum. 1-7, appendages ; $a$. abdomen ; s. proboscis. (Frum Laug, after Hoek.)

behind the original three until the form of the adult is completed. Different kinds of Pycnogonids occur at various depths from between tidal limits to considerable depths in the ocean. The larvæ of the species of one genus are

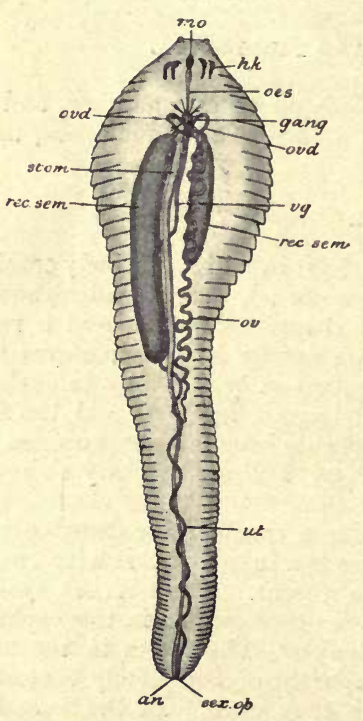

Fua. 559.-Pentastomum tanioides, young female. an. anus; gang. ganglion; $h k$. hooks; mo. mouth; ars. aesophag us ; ov. ovary; ovd. oviduct; rec. sem. receptaculum seminis; sex. ap. sexual aperture; stom. stomach; $u t$. uterus. -(After Leuckart.) internal parasites in certain hydroid Zoophytes.

\section{The Linguatulida or Pentastomida.}

The Linguatulida (Fig. 559) are parasitic aninials, which, when superficially examined, present little appearance of affinity with the Arthropoda. The body is completely worm-like, not divided into regions, and presenting only a superficial annulation, which in no way corresponds with division of the body into segments. The sole representatives of limbs are four hooks $(h k$.) at the sides of the mouth. The muscular fibres are striated. The alimentary canal is simple and straight, and Malpighian tubes are absent. Heart and organs of respiration are wanting. The nervous system is greatly reduced. A narrow nerve-collar surrounds the œsophagus, presenting no brain-enlargement, and connected behind with a single ventral nerve-mass. Organs of special sense are absent.

Some species of Pentastomum are in the adult condition parasites in the lungs of snakes. One species (Pentastomum tanioides) inhabits certain cavities - the frontal sinuses and maxillary antra connected with the nasal chambers-in the 
Dog and Wolf. Its embryos, escaping and falling on grass and other herbage, which form the food of Hares and Rabbits, are taken up by these animals, and perforating the wall of the alimentary canal, by means of a boring apparatus composed of several chitinous pieces, lodge themselves in the liver, where they become encysted and undergo a metamorphosis. Afterwards they leave the eysts and move about. If the young Pentastomum should be received into the mouth of a Dog (still contained probably in most cases in the tissues of the Hare or Rabbit) it may find its way to the frontal sinuses or maxillary antra, there to undergo its final transformation into the adult form. The larva possesses two pairs of short legs.

\section{The Tardigrada.}

The Tardigrada (" Bear-animalcules") are soft-skinned animals (Fig. 560) of minute size, not exceeding a millimetre in length. The body is unsegmented and not distinguishable into regions, except that in some a slight constriction separates off an anterior part or head from the rest. The mouth is provided with a sucking proboscis. There are four pairs of short unjointed legs (I.-IV.), the last of which is terminal, and each is provided with two or four claws. The mouth is surrounded by papillæ; the buccal cavity contains a pair of horny, sometimes partly calcified, teeth (styl.). The ducts of a pair of salivary (?) glands (sali) open into the cavity of the mouth; there is a muscular pharynx (ph.), a narrow œesophagus, and a wide mesenteron (stom.); the anus is sub-terminal, situated in front of the last pair of limbs. A pair of tubes (mal.) which open into the terminal part of the intestine are perhaps representatives of Malpighian tubes. The muscles are all non-striated. There are no organs of respiration, and heart and blood-vessels are likewise absent. There is a brain and a ventral nerve-cord with four ganglia. Two eye-spots situated at the anterior end are the only representatives of organs of special sense. The gonads in both sexes are saccular, and open into the terminal part of the intestine. Segmentation is complete and regular. The young animal at one stage has only two pairs of rudimentary legs, but develops the

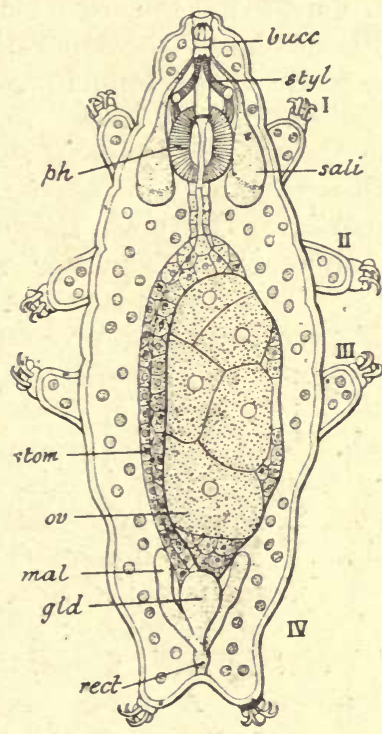

FiG. 560.-Macrobiotus hufelandi. I-IV, appendages; bucc. buccal cavity; gll. accessory glaud; mal. Malpighian tube; ov. ovary; rect. rectum; suli. salivary glands; stom. stomach; styl. teeth. (From Hertwig's Lehrbuch, after Greef and Plate.)

full number before being hatched. The larva possesses a head and four distinct segments.

Some of the Tardigrada live among damp moss; others in fresh or in salt water. 


\section{Relationships of the AiR-BReathing ARthropoda. ${ }^{1}$}

Notwithstanding the existence of some striking superficial resemblances between the Arachnida and the Insecta, the evidence afforded by anatomy and embryology points to the conclusion that there is no direct genetic relationship between the two groups. The occurrence in both of a peculiar form of respiratory organs, the tracheæ, seems at first sight to indicate such a relationship; but the evidence of an independent origin is so strong that it must be supposed that the tracheæ have been independently developed in the two classes. The most important points of difference are-the separation of head and thorax in the Insecta, the mode of development of the eyes, the presence in the Arachnida of an extensive "liver" and (perhaps) the endodermal origin of the Malpighian tubes in the latter class.

Resemblances between Limulus and the Scorpions are readily apparent. In both there is a cephalothorax bearing six pairs of appendages, together with two median and several lateral eyes. The appendages in both are all originally post-oral, the first pair becoming pre-oral in course of growth, and the ganglia belonging to it coalescing with the brain. The upper lip between the bases of these appendages is similarly developed in both. The pair of processes situated behind the sixth pair of appendages, which in Limulus form the chilaria, are represented in the Scorpions by a small pentagonal plate in front of the operculum. The abdomen of Limulus corresponds to the pre- and post-abdomen of the Scorpion; it contains only eight segments, inclusive of the telson; but there is evidence, from a comparison with certain fossil forms, that the telson represents several united metameres. A certain amount of correspondence is also traceable in the appendages of the abdomen. In both the first pair form the operculum; in the Scorpion the second pair form the pectines, while the rest disappear; in Limulus all persist as the lamelliform appendages to which the buok-gills are attached. In structure there is considerable similarity between the book-gills of Limulus and the book-lungs of the Scorpion, but how far they are equivalent to one another remains doubtful in view of the difference in their position, the book-gills being attached to the dorsal surface of the abdominal appendages and the book-lungs sunk within the segments.

The presence in both of the large "liver," of a circum-œsophageal artery, of a cartilaginous endosternite, and of a pair of coxal

1 The Xiphosura, and also the Pentastomida, though not air-breathing, are discussed here. 
glands on the basal joints of the fifth pair of appendages, are some of the points of correspondence in the internal anatomy.

While Limulus is thus closely related to the Scorpions on the one hand, it exhibits, on the other, indications of affinities with the Trilobites, a group of extinct Arthropods probably finding their nearest existing allies in the Branchiopod Crustacea (p. 563). This resemblance to the Trilobites is most marked in the stage-the trilobite-stage-in which the young King-crab escapes from the egg. Certain fossil representatives of the Xiphosura come still nearer to the Trilobites than the adult Limulus, and thus increase the probability that there is a genetic connection between the two groups.

It seems probable that the air-breathing Arachnida were derived through Limulus-like ancestors from primitive Crustacea, and that the tracheæ were developed without genetic relationship with those of the other air-breathing groups-perhaps as modifications of the pulmonary sacs, the latter having been originally derived from gills like those of Limulus. That air-tubes can be developed in air-breathing members of what are, fundamentally, aquatic groups, is shown by the case of certain terrestrial Isopoda among the C'rustacea (p. 596).

There is a very evident close relationship between the Myriapoda i.e., the Progoneata, and the Insecta. The Insects are more highly specialised, and have their structure modified in adaptation to a special mode of locomotion, but the resemblances in many respects are very strong. One of the most striking points of difference is the indefiniteness in the number of the segments in the Myriapoda, and their constant and definite arrangement in the Insecta. The well-defined thorax of the Insects is wanting in the Myriapods in general, but certain of the segments following the head differ from the rest in various respects, and might be looked upon as constituting a thoracic region. The presence in both groups of a sharply marked-off head bearing antennæ and jaws is an important point of resemblance; so is the absence in both of the voluminous "liver" of the Crustacea and Arachnida. The gap between the two classes is narrowed by two converging groups - the Symphyla among the Myriapoda on the one hand, and the wingless-and in other respects primitive-Aptera among the Insecta on the other.

While the Insecta thus appear to be nearly related to the Progoneata, there are indications of relationship between the Opisthogoneata and the Onychophora, and, through these, the Chætopoda. The elongated, homonomously segmented body, the well-defined head with its antennæ, the occurrence of similar appendages on all the body-segments, all point in this direction. Accordingly, instead of placing the branchiate Arthropoda in one group and all the air-breathing forms in another, and 
deriving the latter from the former, we should probably express more correctly the affinities of the various groups of Arthropods by some such scheme as that expressed in the diagram (Fig. 561).

Here an intermediate link between Annelida and the existing Arthropoda is supposed to have been constituted by hypothetical primitive forms from which Peripatus, the Insecta, and the Myriapoda are supposed to have been evolved in the one direction,

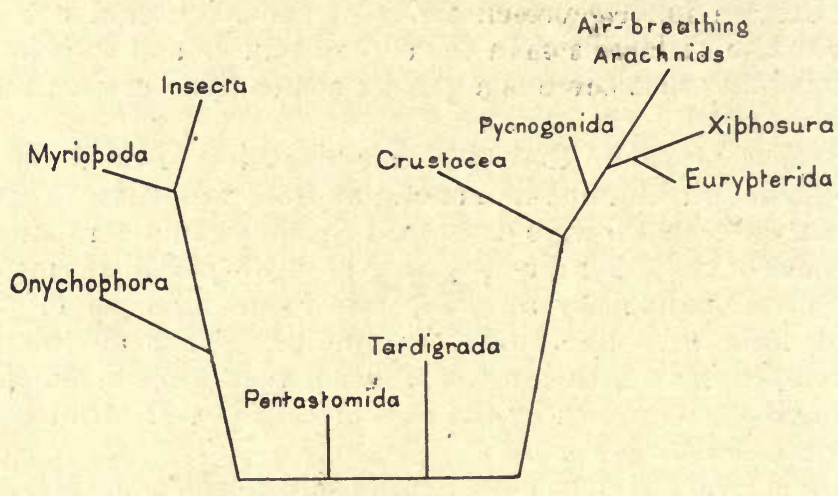

Primitive Arthropods

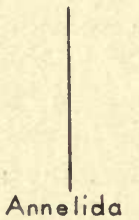

FIG. 561

and the Crustacea, Eurypterida, Xiphosura, and air-breathing Arachnida in the other.

On account mainly of general resemblances to the Spiders, the Pycnogonida have frequently been grouped with the Arachnida, and attempts have been made to homologise their appendages with those of the Spiders and Scorpions. There is one pair more in the Pycnogonida; and either the last pair would have to be set down as corresponding to the vestigial first abdominal pair of the ordinary Arachnida, or the ovigerous legs would have to be reckoned, not as independent appendages, but as parts of the second pair, a view for which there is some ground. A close relationship with the Arachnida, however, cannot be traced, and their affinities are perhaps best expressed, as in the diagram, by connecting them with the Arachnid branch of the Arthropod tree at a point below that at which the air-breathing forms had become developed from forms allied to the Xiphosura. 
The position of the Pentastomida is a matter of uncertainty. In the absence of organs of respiration and excretion, the only feature in the adult which distinctly points to arthropod affinities is the striated character of the muscular tissue. The presence of two pairs of legs in the larva, however, is sufficient to confirm the view that they are aberrant and probably degenerate Arthropods, while leaving it uncertain in what class they find their nearest allies. The Tardigrada are still more aberrant in some respects. They differ from Arthropods in general in the absence of external segmentation in the adult state, in the simple unjointed character of the appendages, in the absence of striation in the muscular fibres, and in the absence of organs of respiration and circulation. It is impossible to place them in any of the great classes, and they are perhaps best looked upon as a special offset of the Arthropod tree given off near the base. 


\section{SECTION XII}

\section{PHYLUM MOLLUSCA}

The Mollusca, like the Arthropoda, form one of the chief divisions of the animal kingdom, both as regards diversity of organisation and number of genera and species. They are sharply distinguished from Arthropods by the absence of segmentation, and by having, as a rule, an exoskeleton in the form of a shell, usually external, sometimes internal. An enumeration of the Classes of the Phylum will serve to give some notion of its extent.

Class 1.-PELecypoda, including the bivalved shell-fish, such as Mussels, Cockles, Oysters, \&c.

Class 2.-Amphineura, including the Chitons and their allies.

Class 3.-GaSTROPODA, including the univalved Shell-fish, such as Periwinkles, Whelks, Snails, Slugs, \&c.

Class 4.-SCAPHOPODA, including the Tooth-shells.

Class 5.-CephalopodA, including the Cuttle-fishes, Squids, Octopods, and Nautili.

\section{CLASS I.-PELECYPODA.}

\section{Example of the Class-The Fresh-water Mussels (Anodonta and Unio).}

Fresh-water Mussels are found in rivers and lakes in most parts of the world. Anodonta cygnea, the Swan-mussel, is the commonest species in England; but the Pearl-mussel, Unio margaritifer, is found in mountain streams, and other species of the same genus are universally distributed.

The Mussel (Fig. 562) is enclosed in a brown shell formed of two separate halves or valves hinged together along one edge. It lies on the bottom, partly buried in the mud or sand, with the valves slightly gaping, and in the narrow cleft thus formed a 
delicate, semi-transparent substance $(m$.$) is seen-the edge of the$ mantle or pallium. The mantle really consists of separate halves or lobes corresponding with the valves of the shell, but in the position of rest the two lobes are so closely approximated as to appear simply like a membrane uniting the valves. At one end, however, the mantle projects between the valves in the form of two short tubes, one (ex. sph.) smooth-walled, the other (in. sph.) beset with delicate processes or fimbrio. By diffusing particles of carmine or indigo in the water it can be seen that a current is always passing in at the fimbriated tube, hence called the inhalant siphon, and out at the smooth or exhalant siphon. Frequently a semi-transparent, tongue-like body $(f t$.) is protruded between the valves at the opposite side from the hinge and at the end furthest from the siphons: this is the foot, by its means the animal is able slowly to plough its way through the sand or mud. When the

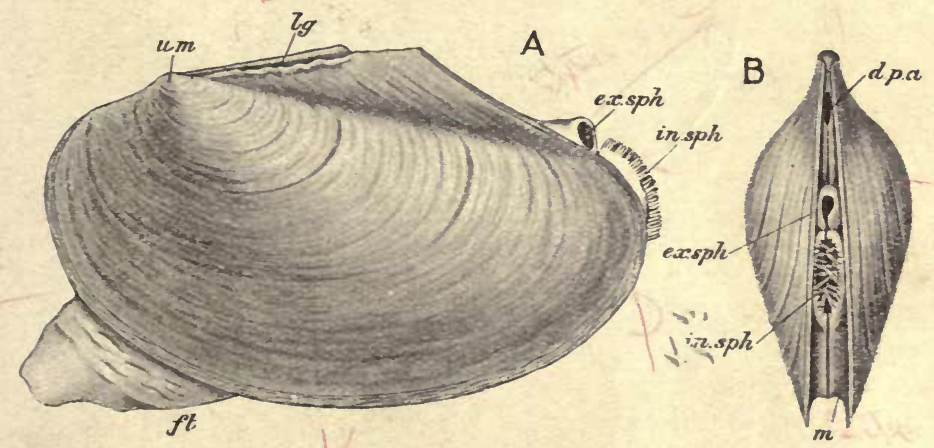

Fig. 562.-Anodonta cygnea. The entire animal. A, from the left side; $B$, from the posterior end; $d . p_{0}$. dorsal pallial aperture; $e x .8 p h$. exhalant siphon; $f t$. foot; $i n .8 p h$. inhalant siphon; $\mathrm{lg}$. ligament ; $m$. mantle ; um. umbo. (After Howes.)

Mussel is irritated the foot and siphons are withdrawn and the valves tightly closed. In a dead animal, on the other hand, the shell always gapes, and it can then be seen that each valve is lined by the corresponding lobe of the mantle, and that the exhalant siphon is formed by the union of the lobes above and below it and is thus an actual tube; but that the boundary of the inhalant siphon facing the gape of the shell is simply formed by the approximation of the mantle-lobes, so that this tube is a temporary one.

The hinge of the shell is dorsal, the gape ventral, the end bearing the siphons posterior, the end from which the foot is protruded anterior: hence the valves and mantle-lobes are respectively right and left.

In a dead and gaping Mussel the general disposition of the parts of the animal is readily seen. The main part of the borly 
lies between the dorsal ends of the valves: it is produced in the middle ventral line into the keel-like foot, and on each side, between the foot and the corresponding mantle-lobe, are two delicate, striated plates, the gills (Figs. 566-568). Thus the whole animal has been compared to a book, the back being represented by the hinge, the covers by the valves, the fly-leaves by the mantlelobes, the two first and the two last pages by the gills, and the remainder of the leaves by the foot.

The Shell.-When the body of the mussel is removed from the shell the two valves are seen to be united, along a straight

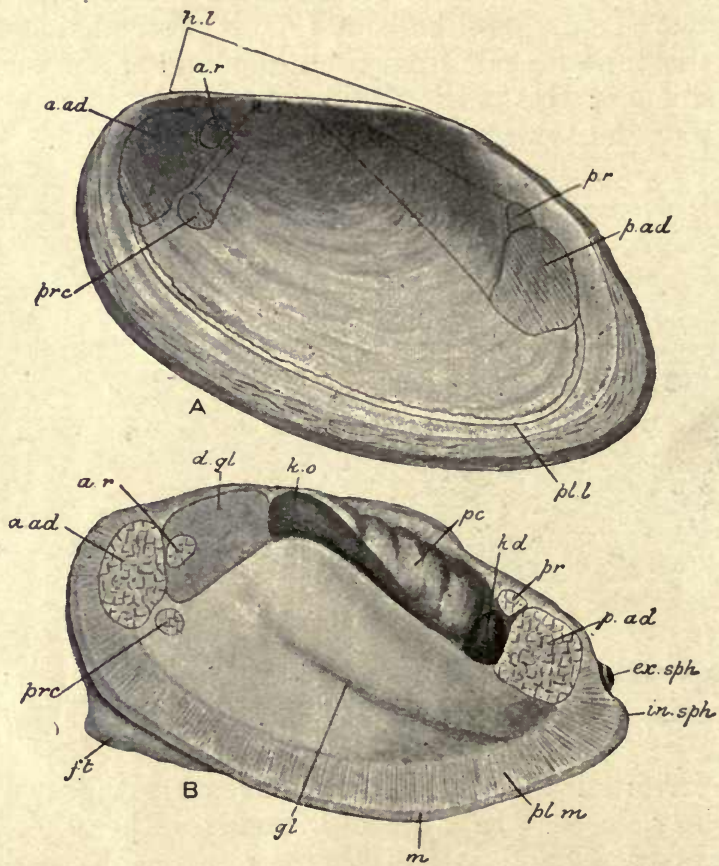

Fic. 563.-Anodonta cygnea. A, interior of right valve ; B, the animal removed from th shell. a. ad. anterior adductor or its impression; $a . r$. anterior retractor or its impression; d. $g l$. digestive gland, seen through mantle; $e x . s p h$. exhalant siphon ; $f t$. foot; $g l$. gills, seen through mantle; $h . l$. hinge-line ; in. sph. inhalant siphon; $k d$. kidney, seen through nantle; $k$. o. Keber's organ, seen through mantle; $m$. mantle; $p$. ad. posterior adductor or its impression ; $p c$. pericardiun, seen through mantle ; $p l . l$. pallial line; $p l . m$. pallial muscles n. $r$. posterior retractor or its impression; $p v c$, protractor or its impression.

hinge-line (Fig. 563, A, h.l.), by a tough, elastic substance, the hinge-liganient (Figs. 562 and 568,lg.) passing transversely from valve to valve. It is by the elasticity of this ligament that the shell is opened: it is closed, as we shall see, by muscular action: hence the mere relaxation of the muscles opens the shell. In Anodonta the only junction between the two valves is afforded by the ligament, but in Unio each is produced into strong projections and 
ridges, the hinge-teeth, separated by grooves or sockets, and so arranged that the teeth of one valve fit into the sockets of the other.

The valves are marked externally by a series of concentric lines (Fig. 562) parallel with the free edge or gape, and starting from a swollen knob or elevation, the $u m b o(u m$.), situated towards the anterior edge of the hinge-line. These lines are lines of growth. The shell is thickest at the umbo, which represents the part first formed in the young animal, and new layers are deposited under this original portion, as secretions from the mantle. As the animal grows each layer projects beyond its predecessor, and in this way successive outcrops are produced giving rise to the markings in question. In the region of the umbo the shell is usually more or less eroded by the action of the carbonic acid in the water.

The inner surface of the shell also presents characteristic markings (Fig. 563, A). Parallel with the gape and at a short distance from it is a delicate streak $(p l . l$.$) cansed$ by the insertion into the shell of muscular fibres from the edge of the mantle: the streak is hence called the pallial line. Beneath the anterior end of the hinge the pallial line ends in an

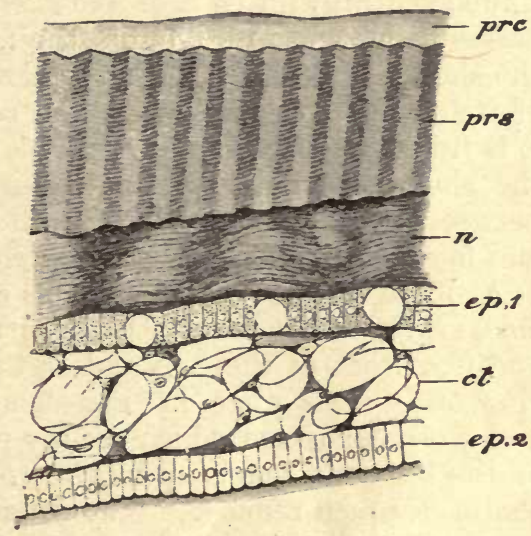

Fig. 564.-Vertical section of shell and mantle of Anodonta. c. $t$. connective-tissue layer of mantle; $e p$. 1, its outer epithelium; ep. 2, its inner epithelium; $n$. nacreous layer of shell; prc. periostracum; prs. prismatic layer. (After Claus.) oval mark, the anterior adductor impression (a. ad.), into which is inserted one of the muscles which close the shell. A similar but larger posterior adductor impression ( $p$. ad.) lies beneath the posterior end of the hinge. Two smaller markings in close relation with the anterior adductor impression mark the origin of the anterior retractor $(a . r$.) and of the protractor (prc.) of the foot: one connected with the posterior adductor impression is that of the posterior retractor ( $p . r$. of the foot. From all these impressions faint converging lines can be traced to the umbo: they mark the gradual shifting of the muscles during the growth of the animal.

The shell consists of three layers. Outside is a brown horn-like layer, the periostracum (Fig. 564, prc.), composed of conchiolin, a substance allied in composition to chitin. Beneath this is a 
prismatic layer (prs.) formed of minute prisms of calcium carbonate separated by thin layers of conchiolin ; and, lastly, forming the internal part of the shell is the nacre $(n$.$) , or "mother-of-pearl,"$ formed of alternate layers of carbonate of lime and conchiolin arranged parallel to the surface. The periostracum and the prismatic layer are secreted from the edge of the mantle only, the pearly layer from the whole of its outer surface. The hingeligament is continuous with the periostracum, and is to be looked upon simply as a median uncalcified portion of the shell, which is therefore, in strictness, a single continuous structure.

By the removal of the shell the body of the animal (Fig. 563, B) is seen to be elongated from before backwards, narrow from side to side, produced on each side into a mantle-lobe $(m$.$) and con-$ tinued ventrally into a keel-like visceral mass (Fig. 565, v.m.), which passes below and in front into the foot $(f t$.). Thus each valve of the shell is in contact with the dorso-lateral region of the body of its own side together with the corresponding mantle-lobe, and it is from the epithelium (Fig. 564, ep..$^{1}$ ) covering these parts that the shell is formed as a cuticular secretion. The whole space between the two mantle-lobes, containing the gills, visceral mass, and foot, is called the mantle-cavity.

A single layer of epithelial cells covers the whole external súrface, i.e. the body proper, both surfaces of the mantle, the gills, and foot; that of the gills and of the inner surface of the mantle (Fig. 564,ep..$^{2}$ ) is ciliated. Beneath the epidermis come connective and muscular tissue, which occupy nearly the whole of the interior of the body not taken up by the viscera, the colome being, as we shall see, much reduced. The muscles are all unstriped, and are arranged in distinct bands or sheets, many of them very large and conspicuous. The largest are the anterior and posterior adductors (Figs. 563 and 565, a. ad., p. ad.), great cylindrical muscles, passing transversely across the body and inserted at either end into the valves of the shell, which are approximated by their contraction. Two muscles of much smaller size pass from the shell to the foot, which they serve to draw back; they are the anterior $(a . r$.$) and posterior (p.r.) retractors of the foot. A third muscle$ $\left(p^{\circ} c\right.$.) arises from the shell, close to the anterior adductor, and has its fibres spread fan-wise over the visceral mass, which it serves to compress, thus forcing out the foot and acting as a protractor of that organ. The substance of the foot itself consists of a complex mass of fibres, the intrinsic muscles of the foot, many of which also act as protractors. Lastly, all along the border of the mantle is a row of delicate pallial muscles (Fig. 563, pl. m.), which, by their insertion into the shell, give rise to the pallial line already seen.

The cœlome is reduced to a single ovoidal chamber, the pericardium (Fig. 565, pc.), lying in the dorsal region of the body and containing the heart and part of the intestine; it is lined by 
colomic epithelium. In the remainder of the body the space between the ectoderm and the viscera is filled by the muscles and connective-tissue.

Digestive organs.-The mouth (Fig. 565, mth.) lies in the middle line, just below the anterior adductor. On each side of it are two triangular flaps, the internal and external labial palps (l. int.plp., l. ext.plp.); the external palps unite with one another in front of the mouth, forming an upper lip; the internal palps are similarly united behind the mouth, forming a lower lip; both are ciliated externally. The mouth leads by a short gullet (Fig: 566, gul.) into a large stomach (st.), which receives the ducts (d.d.) of a pair of irregular, dark-brown digestive glands (d.gl.). The intestine

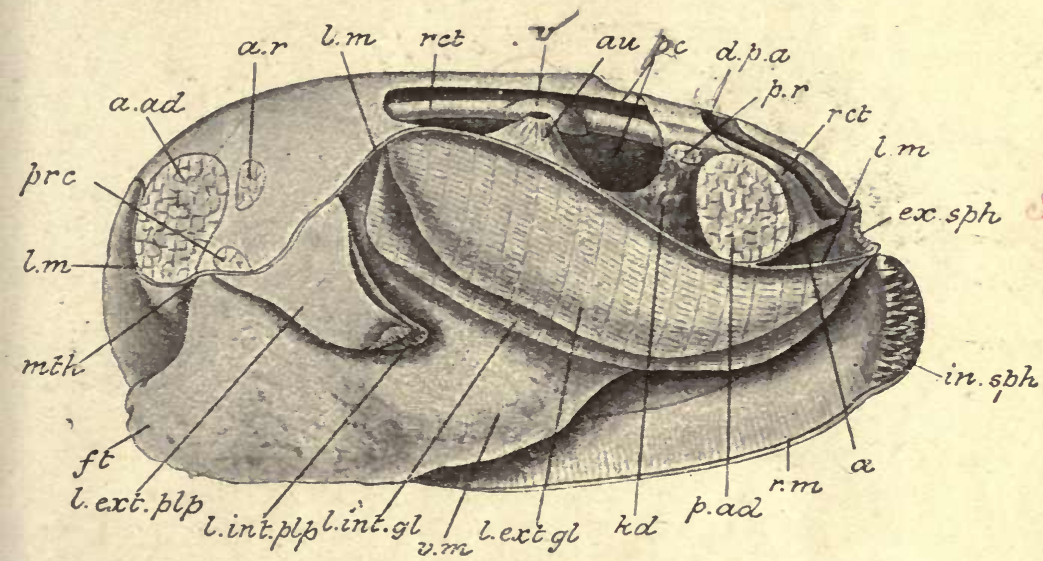

FIG, 565.-Anodonta cygnea. The animal with most of the left mantle-lobe removed. a. anus; $a_{\text {. }}$ ad. anterior adductor; $a$. $r$. anterior retractor; $a u$. auricle; $d . p$. $a$. dorsal pallial aperture; $e x .8 p h$. exhalant siphon; $f$. foot; in. sph. inhalant siphon ; kd. kidney; $l$. ext. $g l$. left external gill-lamina ; $l$. ext. plp. left external labial palp; $l$. int. gl. left internal gill-lamina; $l$. int. $\rho l p$. left internal labial palp; $l . m$. cut edge of left mantle-lobe; mth. mouth; $p$. ad. posterior adductor ; $p c$. pericardium; $p . r$. posterior retractor ; pre. protractor; $r c t$. rectum ; $r$. $m$. right mantle-lobe; $v$. ventricle; $v$. $m$. visceral mass.

(int.) is given off from the posterior end of the stomach, descends into the visceral mass, where it is coiled upon itself, then ascends parallel to its first portion, turns sharply backwards, and proceeds, as the rectum (rct.), through the pericardium - where it traverses the ventricle of the heart-and above the posterior adductor, finally discharging by the anus (a.) into the exhalant siphon, or cloaca. The wall of the rectum is produced into a longitudinal ridge, or typhlosole (ty.), like that of the Earthworm, and two similar ridges begin in the stomach and are continued into the first portion of the intestine. The stomach contains, under certain conditions, a gelatinous rod, the crystalline style.

On each side is a single gill or ctenidium composed of two plates or lamino, an inner and an outer. We have thus right outer 
and inner gill-laminæ, and left outer and inner gill-laminæ (Fig. 565 , l.ext. gl., l.int. gl.). Seen from the surface, each lamina presents a delicate double striation, being marked by faint lines running parallel with, and by more pronounced lines running at right angles to, the long axis of the organ. Moreover, each lamina is double, being formed of two similar plates, the inner and outer lamellc, united with one another along the anterior, ventral, and posterior edges of the lamina, but free dorsally. The lamina has thus the form of a long and extremely narrow bag open above (Figs. 566, 567 and 568): its cavity is subdivided by vertical bars of tissue, the inter-lamellar junctions $(i . l . j$.$) , which extend between the$

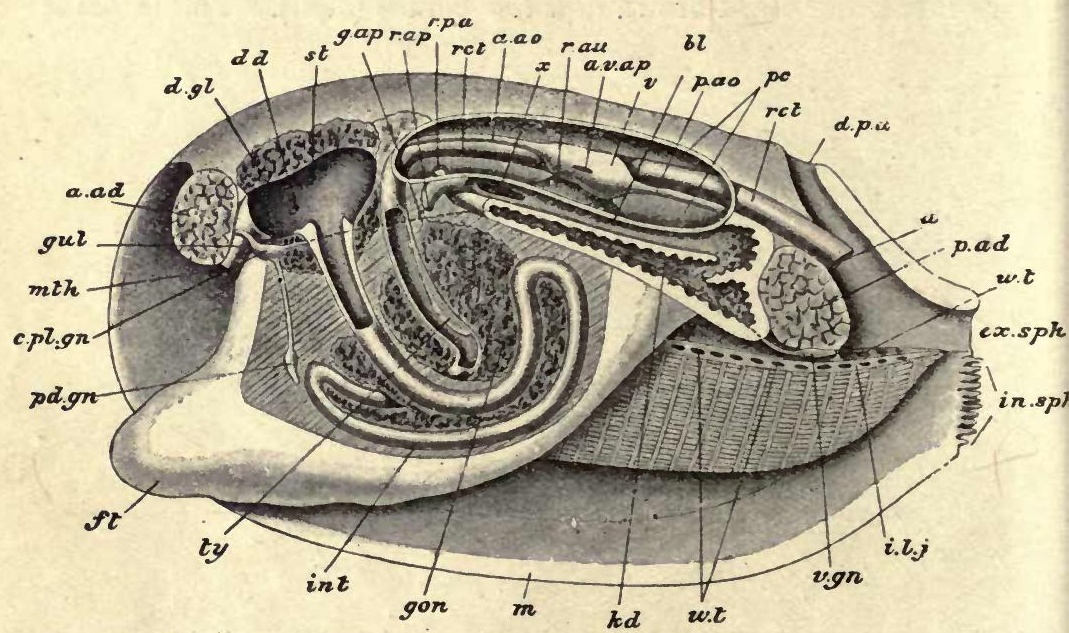

Fic. 566. - Anodonta cygnea. Dissection from the left side. $a$. anus; $a$. ad. anterior adductor; $a . a o$. anterior aorta ; a. $v . a p$. auriculo-ventricular aperture ; $b l$. urinary bladder; $c . p l$. gn. cerebro-pleural ganglion; $d . d$. duct of digestive gland; $d$. gl. digestive gland; d. $p$. a. dorsal pallial aperture; $e x$. sph. exhalant siphon; $f t$. foot; $g$. ap. genital aperture; gon. gonad; gul. gullet; i. $l$. j. inter-lamellar junction : in. sph. inhalant siphon ; int. intestine ; kd. kidney ; m. mantle ; $m$ th. mouth $; p$. ao. posterior aorta $; p$. ad. posterior adductor; $p c$. pericardium; $p d . g n$. pedal ganglion; $r$. $a p$. renal aperture; $r$. au. right auricle ; rct. rectum; r. p. a. reno-pericardial aperture; st. stomach; ty. typhlosole ; $v$. ventricle ; $v$. gn. visceral ganglion; $v$. $t$. water tubes.

two lamellæ, and divide the intervening space into distinct compartments or water-tubes (w.t.), closed ventrally, but freely open along the dorsal edge of the gill. The vertical striation of the laminæ is due to the fact that each lamella is made up of a number of closeset gill-filaments $(f$.$) : the longitudinal striation to the circumstance$ that these filaments are connected by horizontal bars, the interfilamentar junctions $i . f_{. j}$. .). At the thin free or ventral edge of the lamina the filaments of the two lamellæ are continuous with one another, so that each lamina has actually a single set of V-shaped filaments, the outer limbs of which go to form the outer lamella. their inner limbs the inner lamella. Between the filaments, and bounded above and below by the inter-filamentar junctions, are 
minute apertures, or ostia (os.), which lead from the mantle-cavity through a more or less irregular series of cavities into the interior of the water-tubes. The filaments themselves are supported by chitinous rods $(r$.$) , and are corered with ciliated epithelium, the$ large cilia (Fig. 567, D) of which produce a current running from the exterior through the ostia into the water-tubes, and finally

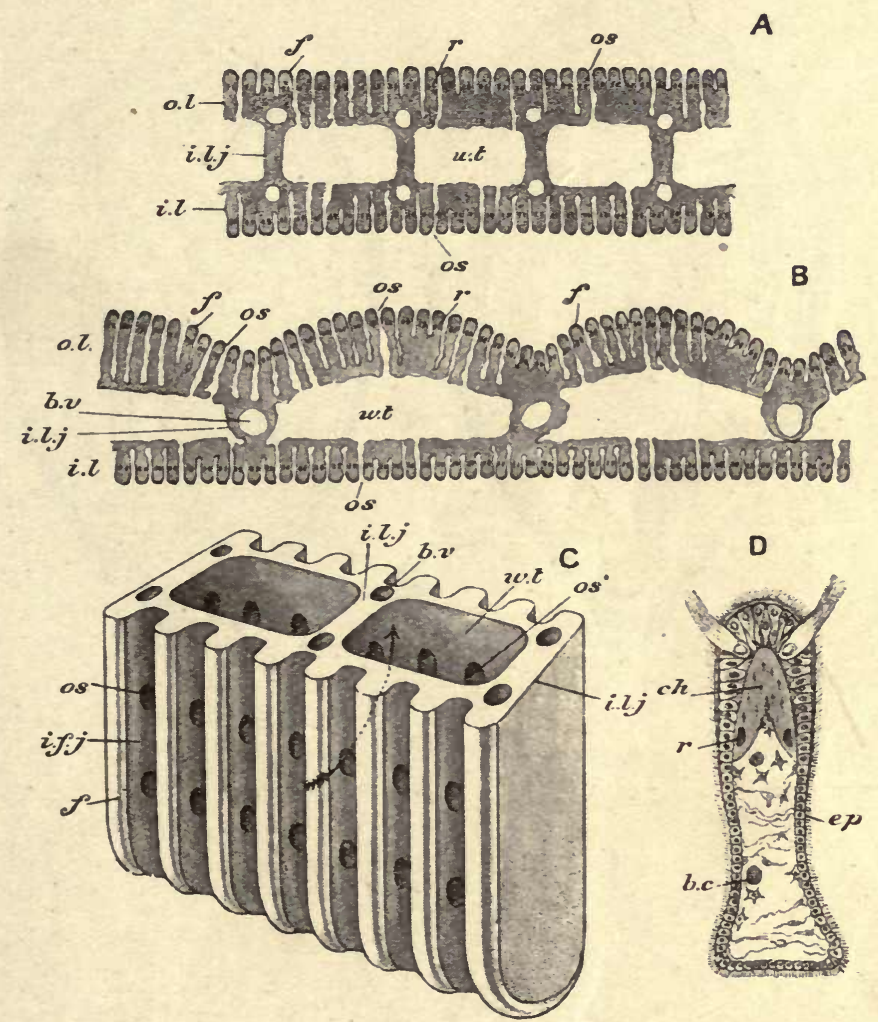

FIG. 567.-Anodonta cygnea. A, transverse section of outer, and B, of inner gill-lamina ; $\mathrm{C}$, diagram of gill-structure; $\mathbf{D}$, transverse section of gill filament. $b$. $c$. blood-corpuscle ; b. $v$. blood-vessels; $c h$. chitin; $f$. branchial filaments; $e p$. epithelium; $i . ~ f . j$. interfilamentar junction; $i . l$. inner lamella ; $i . l . j$. inter-lamellar junction; $0 . l$. outer lamella; os. external ostium; of' internal ostium; $r$, chitinous rods; $v$. $t$. water tubes. (A, B, and D, after Peck.)

escaping by the wide dorsal apertures of the latter. The whole organ is traversed by blood-vessels $(b . v$.).

The mode of attachment of the gills presents certain features of importance. The outer lamella of the outer lamina is attached along its whole length to the mantle (Fig. 568): the inner lamella of the outer and the outer lamella of the inner lamina are attached together to the sides of the visceral mass a little below the origin 
of the mantle: the inner lamella of the inner lamina is also attached to the visceral mass in front (A), but is free further back (B). The gills are longer than the visceral mass, and project behind it, below the posterior adductor (C), as far as the posterior edge of the

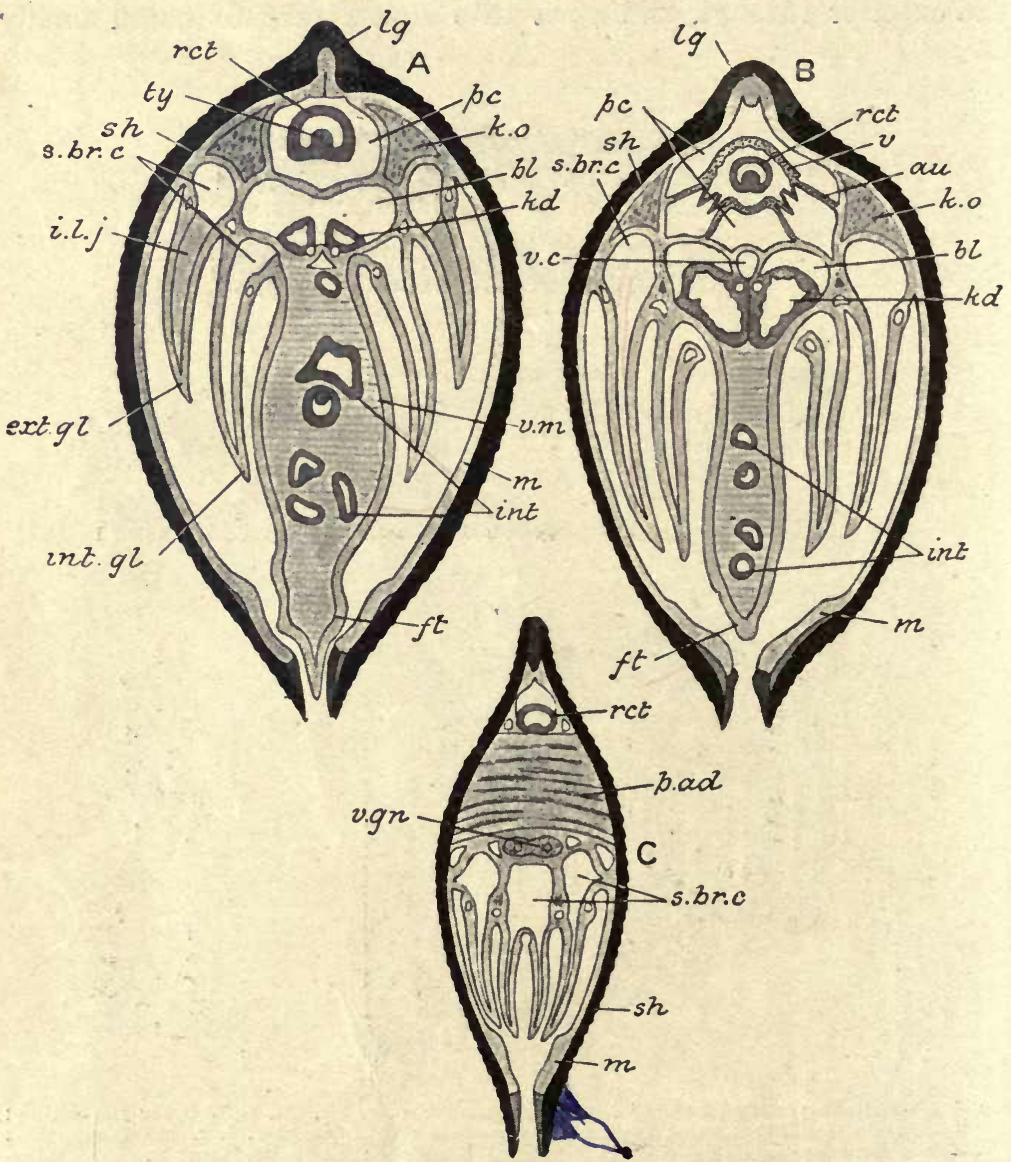

FIG. 568.-Anodonta cygnea. Three transverse sections, A. B. C. au. auricle; bl. urinary bladder ; ext. gl. external gill-lamina ; ft. foot; $i . l . j$. inter-lamellar junction ; int. intestine ; int. gl. internal gill-lamina ; $k d$. kidney; $k . o$. Keber's organ ; lg. ligament; $m$. mautle ; $\rho . a d$. posterior adductor; $p c$. pericardium ; ret. rectum ; $8 . b r c$. supra-branchial chamber; sh. shell ; ty. typhlosole; $v$. ventricle ; vc. vena cava; v. gn. visceral ganglion. (After Howes, slightly altered.

mantle: in this region the inner lamellæ of the right and left inner laminæ are united with one another, and th dorsal edges of all four laminæ constitute a horizontal partition between the paliial cavity below and the exhalant chamber or cloaca above. Owing to this arrangement it will be seen that the water-tubes all 
open dorsally into a supra-branchial chamber (s. br. c.) continuous posteriorly with the cloaca and thus opening on the exterior by the exhalant siphon.

The physiological importance of the gills will now be obvious. By the action of their cilia a current is produced which sets in through the inhalant siphon into the pallial cavity, through the ostia into the water tubes, into the supra-branchial chamber, and out at the exhalant siphon. The in-going current carries with it not only oxygen for the aëration of the blood, but also Diatoms, Infusoria and other microscopic organisms, which are swept into the mouth by the cilia covering the labial palps. The out-going current carries with-it the various products of excretion and the fæces passed into the cloaca. The action of the gills in producing the food-current is of more importance than their respiratory function, which they share with the mantle.

The excretory organs are a single pair of curiously-modified nephridia (portions of the true cœlome), situated one on each side of the body just below the pericardium. Each nephridium consists of two parts, a brown spongy glandular portion or kidney (Fig. 566, $k d$.), and a thin-walled non-glandular part or bladder (ól.), which communicates with its fellow anteriorly by a large oval aperture. The two parts lie parallel to one another, the bladder being placed dorsally and immediately below the floor of the-pericardium : they communicate with one another posteriorly, while in front each glandular part opens into the pericardium ( $r . p . a p$.$) , and the$ bladder on to the exterior by a minute aperture ( $r . a p$.), situated between the inner lamina of the gill and the visceral mass. Thus the whole organ, often called after its discoverer, the organ of Bojanus, is simply a tube bent upon itself, opening at one end into the colome, and at the other on the external surface of the body: it has thus the normal relations of a nephridium. The epithelium of the bladder is ciliated, and produces an outward current.

An excretory function is also discharged by a large glandular mass of reddish-brown colour, called the pericardial gland or Keber's $\operatorname{organ}$ (Fig. 568, B, k.o.). It lies in the anterior region of the body just in front of the pericardium, into which it discharges.

The circulatory system is well developed. The heart lies in the pericardium and consists of a single ventricle (Figs. 566, 568, and $569, v$.) and of right and left auricles (au.). The ventricle is a muscular chamber which has the peculiarity of surrounding the rectum (Figs. 566 and 568, B): the auricles are thin-walled chambers communicating with the ventricle by valvular apertures opening towards the latter. From each end of the ventricle an artery is given off, the anterior aorta (Fig. 566, a. ao.) passing above, the posterior aorta ( $p$. ao.) below the rectum. From the aortæ the blood passes into arteries (Fig. 569, art. ${ }^{1}$ art..$^{2}$ ) which ramify all over the body, finally forming an extensive network of 
vessels, many of which are devoid of proper walls and have therefore the nature of sinuses. The returning blood passes into a large longitudinal vein, the vena cava (v.c.) placed between the nephridia, whence it is taken to the kidneys themselves ( $n p h . v$.), thence by afferent branchial veins ( $a f . b r . v$.) to the gills, and is finally returned by efferent branchial veins (ef. br. v.) to the auricles. The mantle has a very extensive blood supply, and, as mentioned above, probably acts as the chief respiratory organ: its blood (art. ${ }^{1}$ ) is returned directly to the auricles without passing through either

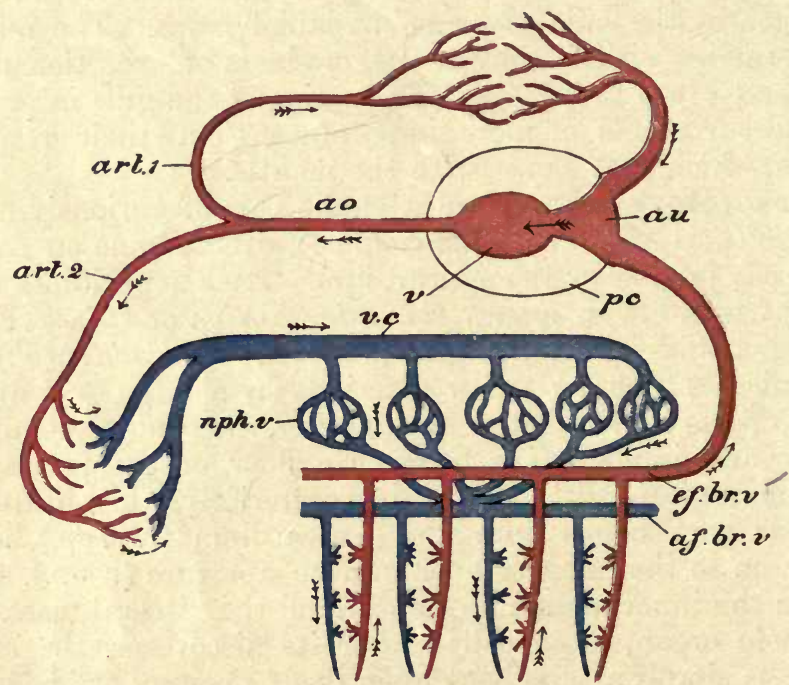

Fic. 569.-Diagram of the circulatory system of Anodonta. Vessels containing aërated blood red, non-aërated blue. af. br. $v$. afferent branchial veins; ao. aorta ; art. 1 , artery to mantle ; art. 2 , artery to body generally; $a u$. auricle; $e f . b r . v$. efferent branchial veins; $n p h . v$. nephridial veins; $p c$. pericardium; $v$. ventricle; $v$. $c$. vena cava. The arrows show the direction of the current.

the kidneys or the gills. The blood is colourless and contains leucocytes.

The nervous system is formed on a type quite different from anything we have yet met with. On each side of the gullet is a small cerebro-pleural ganglion (Fig. 566, c. pl. gn.) united with its fellow of the opposite side by a nerve-cord, the cerebral commissure, passing above the gullet. Each cerebro-pleural ganglion also gives off a cord, the cerebro-pedal connective, which passes downwards and backwards to a pedal ganglion ( $p d$. gn.) situated at the junction of the visceral mass with the foot: the two pedal ganglia are so closely united as to form a single bilobed mass. From each cerebro-pleural ganglion there further proceeds a long cerebrovisceral connective which passes directly backwards, through the kidney, and ends in a visceral ganglion $(v . g n$.) placed on the ventral 
side of the posterior adductor muscle. The visceral, like the -pedal ganglia, are fused together. The cerebro-pleural ganglia supply the labial palps and the anterior part of the mantle; the pedal the foot and its muscles; the visceral the enteric canal, heart, gills, and posterior portion of the mantie.

It will be seen that the cerebral commissures and cerebro-pedal connectives together with the cerebro-pleural and pedal ganglia, form a nerve-ring which surrounds the gullet: the cerebro-pleural ganglia may be looked upon as a supra-œesophageal nerve mass corresponding in part with the brain of Annelids and Arthropods, and the pedal ganglia as an infra-œsophageal mass representing the ventral nerve-cord.

Sensory organs are poorly developed, as might be expected in an animal of such sedentary habits. In connection with each visceral ganglion is a patch of sensory epithelium forming the so-called olfactory organ or, better, osphradium, the function of which is apparently to test the purity of the water entering by the respiratory current. Close to the pedal ganglion a minute statocyst ("otocyst") (Fig. 570) is sometimes found, the nerve of which is said to spring from the cerebro-pedal connective, being probably derived from the cerebral ganglion. Sensory cells - probably tactile-also occur

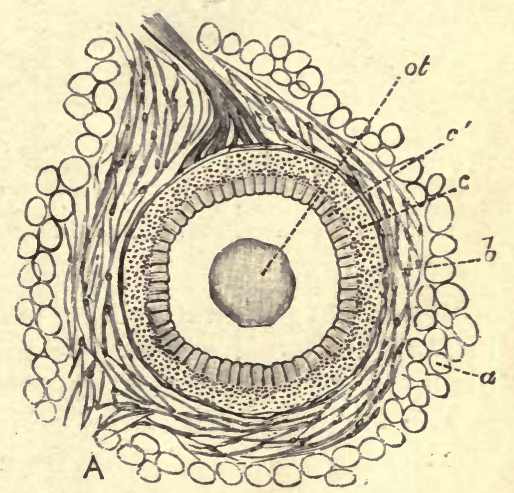

FIG. 570.-Statocyst of Anodonta. $a, b, c, c^{\prime}$, cellular layers surrounding the statocyst; ot. statolith. (From the Cambridge Natural History.) round the edge of the mantle, and especially on the fimbriæ of the inhalant siphon.

Reproductive organs.-The sexes are separate. The gonads (Fig. 566, gon.) are large, paired, racemose glands, occupying a considerable portion of the visceral mass amongst the coils of the intestine: the testis is white, the ovary reddish. The gonad of each side has a short duct which opens ( $g . a p$.) on the surface of the visceral mass just in front of the renal aperture.

In the breeding season the eggs, extruded from the genital aperture, pass into the suprabranchial chamber and so to the cloaca. There, in all probability, they are impregnated by sperms introduced with the respiratory current. The oosperms are then passed into the cavities of the outer gill-laminæ, which they distend enormously. Thus the outer gill-laminæ act as broodpouches, and in them the embryo develops into the peculiar larval form presently to be described. 
Development.-Segmentation of the oosperm is complete, but unequal. A gastrula is formed by the invagination of the mega-

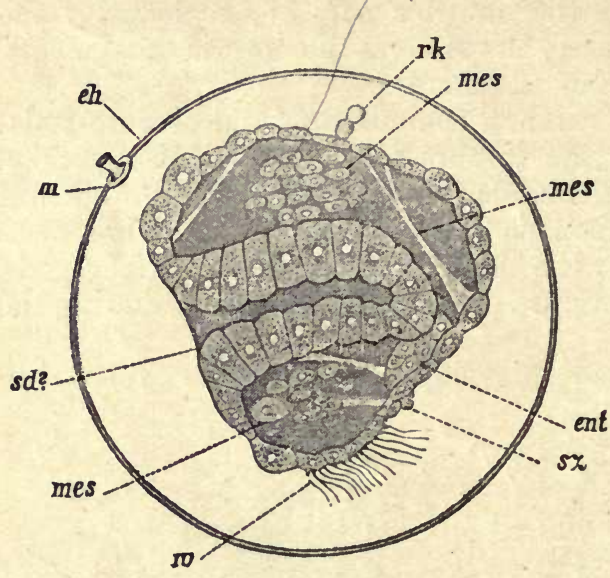

FIc. 571.-Early embryo of Anodonta. eh, vitelline membrane ; ent. archenteron ; $m$. micropyle ; mes. mesoderm ; $r k$, polar cells ; scl, shell-gland ; $s k$, lateral cells ; ve, cilla. (From Korschelt and Heider's Eirbryology.) meres into the micromeres, but the archenteron (Fig. 571, ent.) thus formed is quite small and insignificant, and has no physiological importance until a late period of larval.life. Certain of the cells of the gastrula are budded off into the blastocole, where they accumulate and form the mesoderm (mes.). At about the same time a deep invagination $(s t$.) is formed, which might easily be mistaken for the archenteron, but is really a very characteristic molluscan organ, the shell-gland: it marks the dorsal surface of the embryo. The posterior end is distinguished by a tuft of long cilia.

The shell-gland becomes converted into a plate of long, cylindrical cells (Fig. 572, sd.), from which an unpaired shell (s.) is

H.

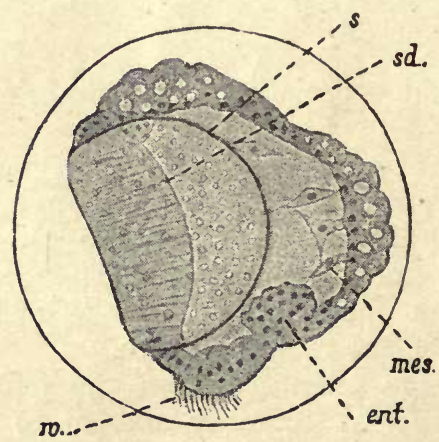

$B$.

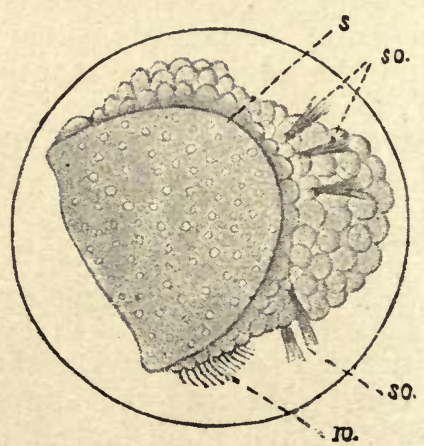

Fic. 572.-Two later stages in the developinent of Anodonta. ent. arehenteron; mes. mesoderm ; 8. shell; $s d$, shell-gland ; so. sense-organs; 20 , cilia. (From Korsehelt and Heider's Embryology.)

secreted. This is replaced before long by a bivaived shell of triangular form, its ventral angles produced into incurved hooks 
beset with spines (Fig. 573, sh). At the same time the body of the larva, which has hitherto been an undivided mass projecting between the two valves of the shell, becomes cleft from below upwards, and thus divided into a single dorsally-placed body proper, and paired-right and left-mantle-lobes. Upon the latter peculiar brush-like sense-organs make their appearance, and on the ventral surface of the body is formed a glandular pouch, which secretes a long thread, the provisional byssus $(f)$. The mesoderm cells give rise to a single immense adductor muscle $(\mathrm{sm})$, the fibres of which extend from valve to valve.

The larva is now called a glochidium: it remains in the broodpouch, nourished by a secretion from the walls of the latter, and entangled with its fellows by means of the byssus. At this stage the outer gill-lamina appears as if stuffed full of closely aggregated sand-grains. Before long the larvæ are ejected
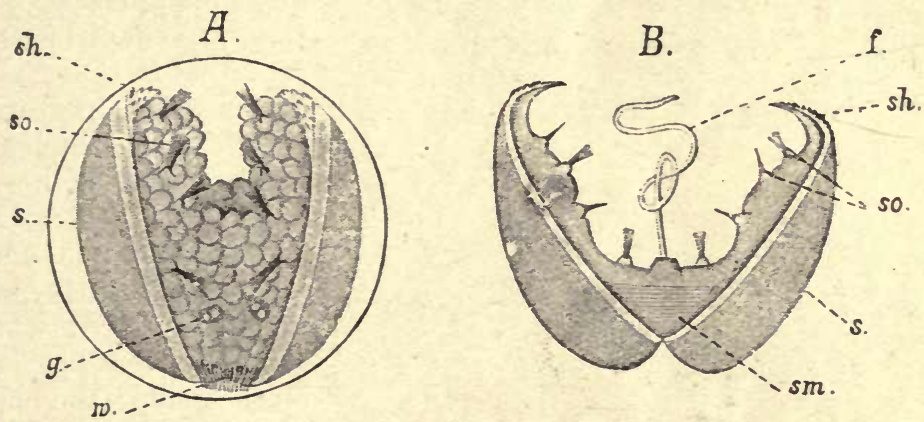

F1G. 573.-A, advanced embryo of Anodonta. B, free glochidium; $f$, provisional byssus 8. shell; $s h$, hooks; sm, adductor muscle; 80 . sense-organs; 20 , cilia. (From Korschelt and Heider's Embryology.)

through the exhalant siphon, and if they happen to come in contact with a passing Stickleback or other fresh-water Fish, fix themselves on some part of its body by means of the hooked valves. The glochidia of Unio usually become attached to the gills, those of Anodonta to the skin or the fins. In this position they become encysted by an overgrowth of the skin or mucous membrane of the host, and are nourished by its juices absorbed through processes of the mantle. They thus lead a truly ectoparasitic existence for about ten weeks.

While in this condition a metamorphosis takes place. The provisional byssus and sense-organs disappear (Fig. 574), and immediately posterior to the former an invagination, the stomodceum $(m)$, is formed, and soon communicates with the archenteron. The posterior end of this cavity is in close contact with the ectoderm, so that the anus is formed by a simple process of rupture, and without the development of a proctodæum. The foot $(f u)$ arises as a median ventral elevation behind the mouth, and on each side of 
it two papillæ (k) appear, the rudiments of the gills. The larva is now fitted for free existence; it drops from its host, and gradually assumes the adult form and mode of life.

\section{Distinctive Characters and Classification.}

The Pelecypoda are bilaterally symmetrical, compressed Molluscs, in which the mantle consists of paired right and left lobes, secret-

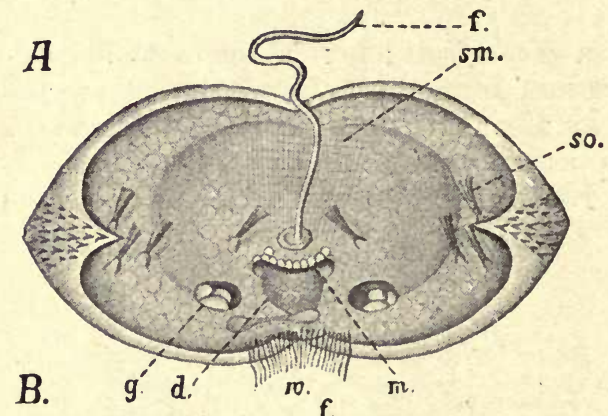
ing a bivalved calcareous shell. There is no distinct head. The ventral region of the body is differentiated into a muscular foot, which is usually ploughshare- or tongue - shaped: in some cases there is a byssus-gland posterior to the foot, which secretes a mass of horny fibres, the byssus, by which the animal may be permanently attached. There are two gills or ctenidia, one on each side: the chief function of the gills is the production of a respiratory and food-

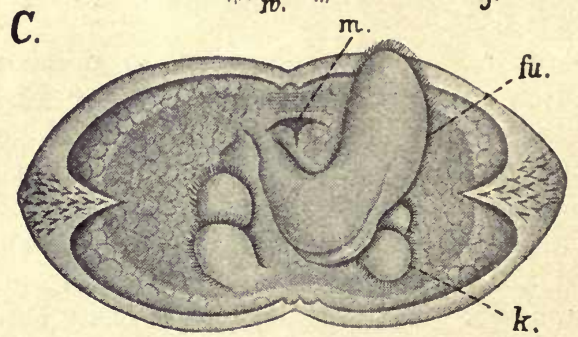

Fig. 574.-Three stages in the metamorphosis of Anodonta. $d$, enteric canal; $f$. provisional byssus; $f u$, foot ; $g$, lateral pits ; $k$, rudiments of gills ; m. mouth; $s h$. shell; sm, adductor muscle; so. sense-organs; 20 , cilia. (From Korschclt and Heider's Bmbryology.) carrying current of water. The body is covered by a onelayered epidermis, which is ciliated on the gills and on the inner surface of the mantle. The muscular system is well-developed, the largest muscles being either one or two adductors, which close the shell, and several bands connected with the foot and byssus; the muscles are usually unstriped. The cœlome is reduced to a dorsally-placed pericardium. The mouth is bounded by two pairs of flat, triangular tentacles or labial palps, the cilia of which 
serve to carry food-particles to the mouth: the enteric canal is coiled, and is formed mainly from the mesenteron: there are large paired digestive glands : the rectum passes through the pericardium, usually perforates the ventricle, and ends above the posterior adductor. The heart is contained within the pericardium, and consists of a median ventricle and of right and left auricles : the blood, which is usually colourless, is taken from the ventricle to the body by one or two aortæ, and is returned partly directly, partly by way of the renal organs and gills, to the auricles. The renal organs are a single pair of cœlomic nephridia, which usually open at one end into the pericardium, at the other on the exterior. The nervous system consists typically of four pairs of ganglia called respectively cerebral, pleural, pedal, and visceral : the cerebral and pleural of each side are usually fused into a single cerebro-pleural ganglion. The chief sense-organs are statocysts and osphradia or water-testing organs. The sexes are separate or united: there are no accessory organs of reproduction. Development is accompanied by a metamorphosis, which usually includes a trochophore stage.

The classification of the Pelecypoda is as follows :-

\section{ORdER 1.-PROTOBRANCHIA.}

Pelecypoda in which the gills take the form of a single pair of plume-like organs or ctenidia, each with two rows of flattened gill-filaments. The foot is not compressed, but has a flattened ventral surface or sole upon which the animal creeps. There are two adductor muscles.

This group includes only four genera-Nucula (Fig. 586), Yoldice, Leda, and Solenomya.

\section{Order 2.-Filibranchia.}

Pelecypoda in which there is a pair of plate-like gills formed of distinct $\mathrm{V}$-shaped filaments : interfilamentar junctions are either absent or formed by groups of interlocking cilia: interlamellar junctions are either absent or non-vascular. As a rule there are two adductor muscles, but the anterior may be greatly reduced or absent.

Including the Noah's ark shell (Arca), Sea-mussel (Mytilus, Fig. 585), Anomia, Trigonia, \&c.

\section{ORder 3.-PSEUdO-LAMELLIBRANCHIA.}

Pelecypoda in which the gills are plaited so as to present vertical folds: the interfilamentar junctions may be ciliary or vascular: the interlamellar junctions vascular or non-vascular. 
There is a single large (posterior) adductor muscle. The shell is frequently inequivalve.

Including the Scallop (Pecten, Fig. 575), Oyster (Ostrea), Pearl Oyster (Meleagrina), Lima, Pinna, \&c.

\section{Order 4.-EUlamellibranchia.}

Pelecypoda in which the gill-filaments are united by vascular interfilamentar and interlamellar junctions, firm, basket-like gills being the result: the gills may be smooth or plaited. There are two equal-sized adductor muscles.

\section{Sub-order a.-Integripalliata.}

Eulamellibranchia in which the siphons are small or absent, and the pallial line on the shell is entire.

Including the Fresh-water mussels (Anodonta and Unio).

\section{Sub-order b.-Sinupalliata.}

Eulamellibranchia in which the siphons are of considerable size, and the pallial line is inflected to form a sinus.

Including the Cockle (Cardium), Mya, Pholas, Teredo (Shipworm), Aspergillum, \&c. (Figs. 577-580).

\section{ORDER 5.-SEPTIBRANCHIA.}

Pelecypoda in which the gills are reduced to a horizontal muscular partition. There are two adductor muscles.

Including Poromya, Cuspidaria, \&c.

\section{Systematic Position of the Examples.}

Anodonta and Unio are two genera belonging to the family Unionida, sub-order Integripalliata, order Eulcmellibranchia.

Their complex basket-like gills are alone sufficient to place them among the Eulamellibranchia. The incomplete ventral siphon and the correlated entire pallial line (see p. 683) indicate their position among the Integripalliata. The regular shell, with thick brown periostracum and large external ligament, the elongated branchial or inhalant aperture, the long, compressed foot, and the absence of a byssus, place them among the Unionidæ. Anodonta is distinguished from Unio by the absence of hingeteeth.

\section{General Organisation.}

The most important variations in structure in the present class are connected with modifications of the gills, the foot, the muscular system, and the siphons. With the structure of the muscles and of the siphons are correlated important variations in the shell 
which are of great systematic value, especially in cases where, as with fossils, the shell is the only part available for examination.

In all the Protobranchia, some of the Filibranchia, such as Arca, and all the Eulamellibranchia and Septibranchia, there are two almost equal-sized adductor muscles, as in Anodonta. In many Filibranchs, such as the common sea-mussel (Mytilus edulis), the anterior adductor becomes greatly reduced and the posterior correspondingly enlarged; and in another species of the same genus (M. latus) the anterior adductor has completely atrophied, the function of closing the shell being performed by the

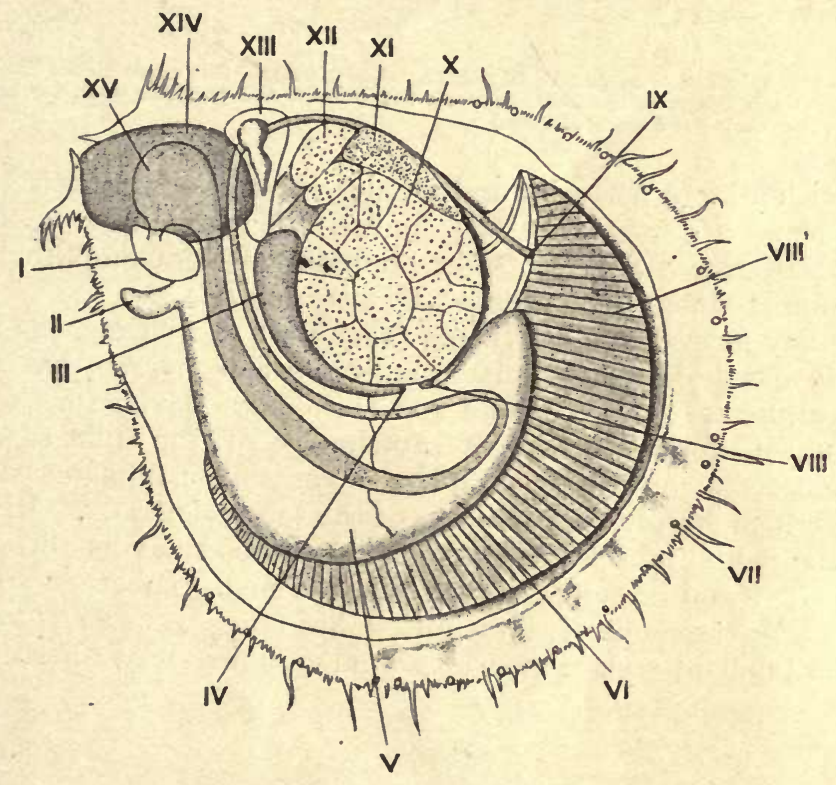

Fig. 575.-Anatomy of Pecten. I, palpi ; II, foot; III, aperture of gonad into kidney ; IV, external renal aperture; V, male, and VI, fernale portion of gonad; VII, pallial eye ; VIII, visceral ganglion ; VIII', gill ; IX, anus; $\mathrm{X}$, striated portion of adductor ; XI, smooth portion; XII, retractor of foot ; XIII, heart; XIV, liver; XV, stomach. (From Pelseneer's Mollusques.)

great posterior adductor alone. In Anomia and in the Pseudolamellibranchs there is a single immense adductor (Fig. 575, X, XI) placed nearly in the middle of the greatly shortened body, and known to represent the posterior adductor-both from the fact that the rectum passes over it, and from the circumstance that, in the embryo Oyster, two adductors are present, the anterior of which atrophies, while the posterior enlarges to form the single muscle of the adult.

These peculiarities in the muscular system bear their mark upon the shell, in which impressions corresponding to the 
adductors are clearly marked on the inner surface (Fig. 576). The whole class is, in fact, frequently classified on this basis, species with equal-sized adductors (Protobranchs, some Filibranchs, and all Eulamellibranchs and Septibranchs) being called Isomyaria (A),

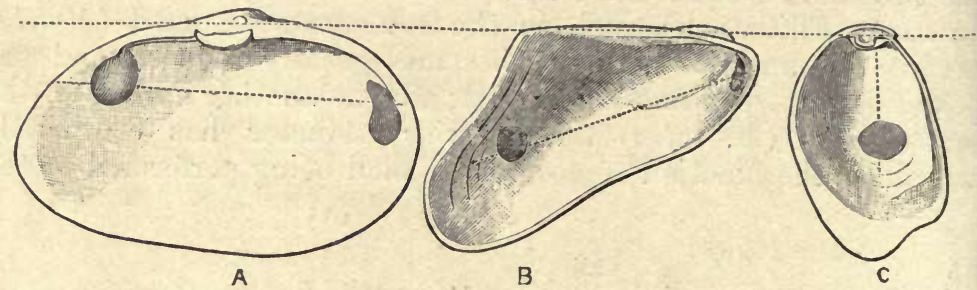

FiG. 576.-Left valves of A, Mya; B, Modiola; C, Vulsella. The upper dotted line passes through the hinge-lines, the lower connects the anterior and posterior adductor muscles. (From the Cambridge Natural History.)

those with a large posterior and a reduced anterior adductor (most Filibranchs) Heteromyaria (B), and those with large centrally placed posterior and no anterior adductor (Pseudolamellibranchs and Anomia among Filibranchs) Monomyaria (C).

In many forms, such as Nucula (Fig. 586), Ostrea, \&c., the right and left mantle-lobes are quite free from each other, so that there are no siphons. In Anodonta and Unio, as we have seen, the two lobes unite along the line of attachment of the gills so as to enclose a dorsal or exhalant siphon, a ventral or inhalant siphon being formed simply by apposition of the lobes ventrally. In such cases the pallial muscles in their neighbourhood act as retractors of the short and imperfect tubes thus formed. In other species a second concrescence of the mantle-lobes takes place so as to convert the inhalant siphon into an actual circumscribed aperture or

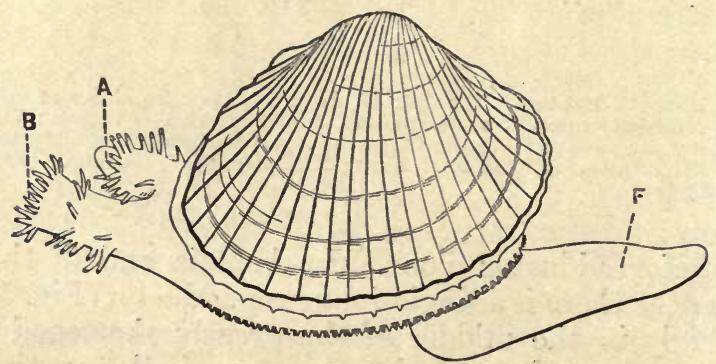

FIG. 577.-Cardium edule. A, exhalant siphon; B, inhalant siphon; F, foot. (From the Cambridge Natural History.)

short tube. In the Sinupalliata the two siphons are prolonged into distinct muscular tubes (Fig. $577, \mathrm{~A}, \mathrm{~B}$ ) which, in the position of extension, project beyond the posterior margin of the shell and may even be considerably longer than the body. Under 
these circumstances the posterior pallial muscles become enlarged to form retractors of the siphons, and the portion of the pallial line from which they arise becomes, as it were, pushed forwards so as to form a bay or pallial sinus (Fig. 578, p.s). Thus the shells of species with welldeveloped siphons are sinupalliate, or have an indented pallial line, while those with small or no siphons are integripalliate, or have an entire pallial line. The larger the siphons the stronger are their muscles and the deeper is the pallial sinus: when very large they cannot be completely retracted, and the posterior border of the shell then gapes permanently. The siphons may be separate (Fig. 579) or united (Fig. 580). They are specially adapted for species of burrowing habits, which are able to remain buried in the

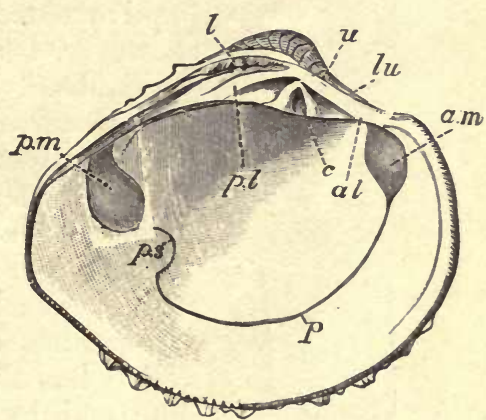

FIG. 578.- Venus gnidia, inner surface of left valve. al, anterior lateral tooth; $a m$, anterior adductor impression; $c$, cardinal teeth; $l$. ligament; $l u$. lunule; $p$, pallial line; $p . l$, posterior lateral tooth ; $p . m$, posterior adductor impression; $p$. 8 . pallial sinus; $u$. umbo. (From the Cambridge Natural History.) mud or sand, only the ends of the siphons being exposed for the supply of aërated water and food, and even these can be instantly withdrawn in the event of danger.

In addition to their union posteriorly to form the siphons, the mantle-lobes may concresce to a greater or less extent along their ventral border (Fig. 581), forming a more or less tubular investment for the body, and leaving an anterior pedal aperture for the

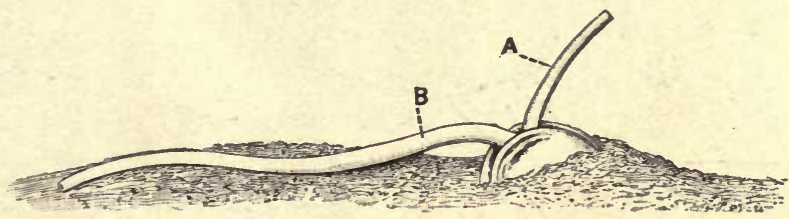

Fig. 579.-Scrobicnlaria piperata, in its natıral position, partly buried in sand. A, exhalant siphon; B, iuhalant siphon. (From the Canbridge Natural History.)

protrusion of the foot. Their anterior portions may also be united to form a sort of hood.

To return to the shell, the muscular impressions and the pallial line on which have already been referred to. As a general rule the right and left valves are alike, or nearly so, the shell being therefore equivalve. Each valve is inequilateral, being divided into 


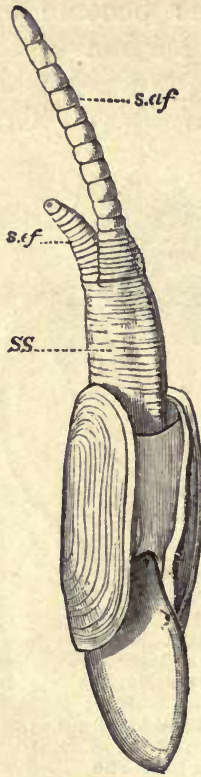

Fra. 5s0.-Solecurtus strigillatus. $8 . a f$, inhalant siphon, 8. ef, exhalant siphow, the two united at SS. (From the Cambridge Natural History.)

unequal portions by a line drawn from the umbo to the gape. It will be remembered that in the Brachiopoda, the only other class of bivalved animals, the precise opposite is the case, the shell being equilateral and inequivalved. Some Psendolamellibranchs are, however, nearly equilateral and markedly inequivalved, such as the scallop (Pecten), and the inequivalve character is still more marked in the oyster, in which the right valve is deeply concavo-convex and permanently attached to a rock, while the left is flat and forms a sort of lid. This condition of things reaches its maximum in the extinct Hippurites (Fig. 582, B), in which the left valve has the form of a long tube closed at one end by the flat lid-like right valve. In the extinct Requienia (A) the left valve is spirally coiled, so that it resembles a snailshell, and its aperture is closed by the flat lid-like right valve: in Diceras, also extinct, both valves are coiled.

The hinge-teeth (Fig. 578) vary greatly in form and size or may be absent altogether: the hinge-ligament is usually band-like, but in Pecten takes the form of a cylindrical cord. The variations in form, ornamentation, colour, \&c.; among the many thousand known species of shell are too numerous to mention; but reference must be made to peculiar modifications found in certain burrowing forms. In Pholas, a
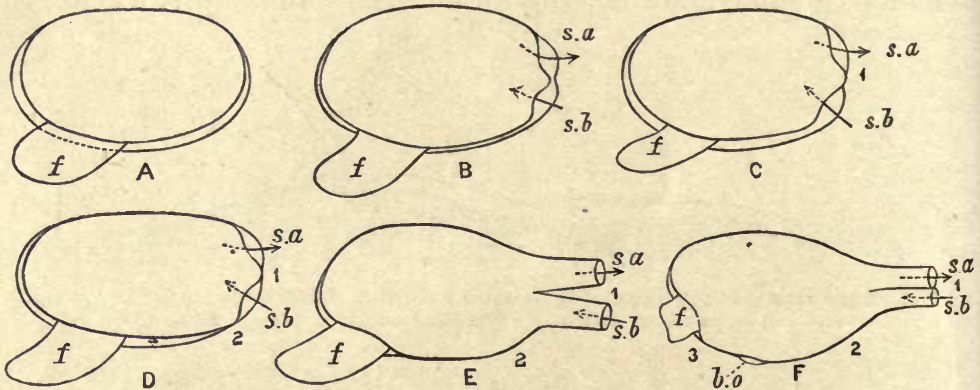

FIG. 581.-Diagram illustrating the various degrees of union of the mantle-lubes. b.o, byssal aperture; $f$. foot; $s, a$, exhalant siphon; $s . b$, inhalant siphon; 1 , first point of union between siphons; 2 , second, between inhalaut siphon and foot; 3 , third, between byssal aperture and foot. (From the Cambridge Natural History.)

siphonate genus which burrows in stone, the shell is weak and brittle, and additional calcareous pieces are developed between 
the two valves. In Teredo (Fig. 583), the so-called Ship-worm, which causes great destruction by boring into piles, ships'-timbers, \&c., the valves $(v$.) remain very small and weak but movable, and the general surface of the mantle secretes a continuous shelly tube which lines the burrow. In Aspergillum (Fig. 584), which lives buried in sand, there is a similar, but wider calcareous tube, with which the valves are completely fused, and the anterior end of the tube which appears above the surface of the sand is closed by a plate perforated with numerous holes like the rose of a watering-pot. The larval shell is
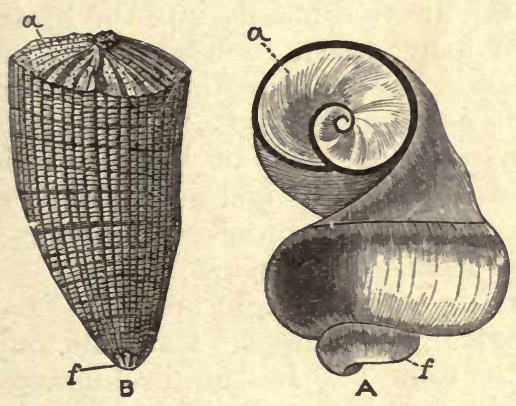

Fig. 582.-Requienia ammonea; B, IIppurites cornu-vaccinum. $a$, right valve: $f$, point of fixation. (From the Cam. bridge Natural History.)

sometimes, though not always, distinguishable at the apex of each valve in the Pelecypoda in general.

In Nucula, Arca, \&c., the foot (Fig. 586, ft.) presents what may be considered as its most primitive form, having a flat ventral surface or sole upon which the animal creeps. Far more common is the ploughshare-like form we are already familiar with in Anodonta and Unio, adapted for slowly making its way through sand or mud. In a few forms, c.g. Trigonia and

Cardium (Fig. 577), it is bent upon itself and is capable of being suddenly straightened so as to act as a leaping organ : in Mytilus it is cylindrical (Fig. 585, F): in the Oyster it is absent. In addition to the anterior and posterior retractors and a pair of protractors, 
the foot is sometimes provided with a levator muscle, particularly well developed in Nucula and its ailies.

Immediately posterior to the foot a byssus-gland is frequently found: it secretes a silky substance in the form of

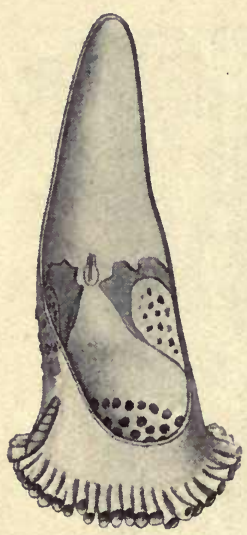

FIG. 584.-Aspergillum. (After sowerby.) threads which serve to anchor the animal permanently or temporarily. It is by means of the byssus that the Sea-mussel (Mytilus) is attached to the rocks (Fig. 585, By): in Pinna the threads are fine enough to be woven in a fabric. In Lima the threads of the byssus are spun into a kind of nest in which the animals lie protected, and in species of Mocliola similar modifications of the byssus occur. In such forms as Mytilus the muscles which ordinarily serve to retract the foot are inserted mainly into the byssus : the latter being fixed, they serve to rotate the animal in various directions or, in other words, act as adjustors and also as retractors of the byssus. It must be borne in mind that the definite byssus just described is not homologous with the provisional byssus of Anodonta (p. 693) which lies in front of the mouth.

The gills or ctenida are two in number, right and left. Each consists of a horizontal axis bearing two rows of filaments, outer and inner, which are outgrowths from it. In the Protobranchia (e.g. Nucula) the filaments are short, compressed, and free from one another (Figs. 586, $g$, and 587, A). In Amusium (B) the gill-filaments are much elongated and thread-like instead of triangular.

In the cornmon Arkshell $(A r c a, C)$ a great change is seen. The gill-filaments are delicate and somewhat flattened threads, each bent upon itself into the form of an elongated $\mathrm{U}$, and therefore con-

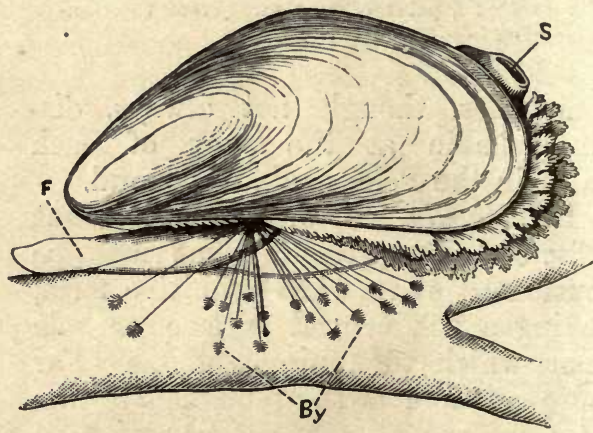

Fra. 585.-Mytilus edulis, attached by byssus (E!). to a piece of wood. F. foot: $S$, exhalant siphun. (From the Cambridge Natural History.) sisting of a proximal or fixed limb and a distal or free limb. The flexure takes place in such a way that the free limb is external in the outer row of filaments, internal in the inner row. Adjacent filaments are loosely united by groups of large interlocking cilia (see Fig. 588), 
placed at regular intervals, and in this way the outer and inner limbs of the filaments are respectively joined together so as to convert each longitudinal row of U-shaped filaments into a double plate, fairly coherent unless the ciliary junctions are forcibly separated. In this way the single ctenidium of Nucula has given place to two plate-like simple laminæ, each formed of an outer and an inner lamella : the inner lamella of the outer and the outer lamella of the inner laminæ are united along their dorsal edges, the line of junction representing the axis of the

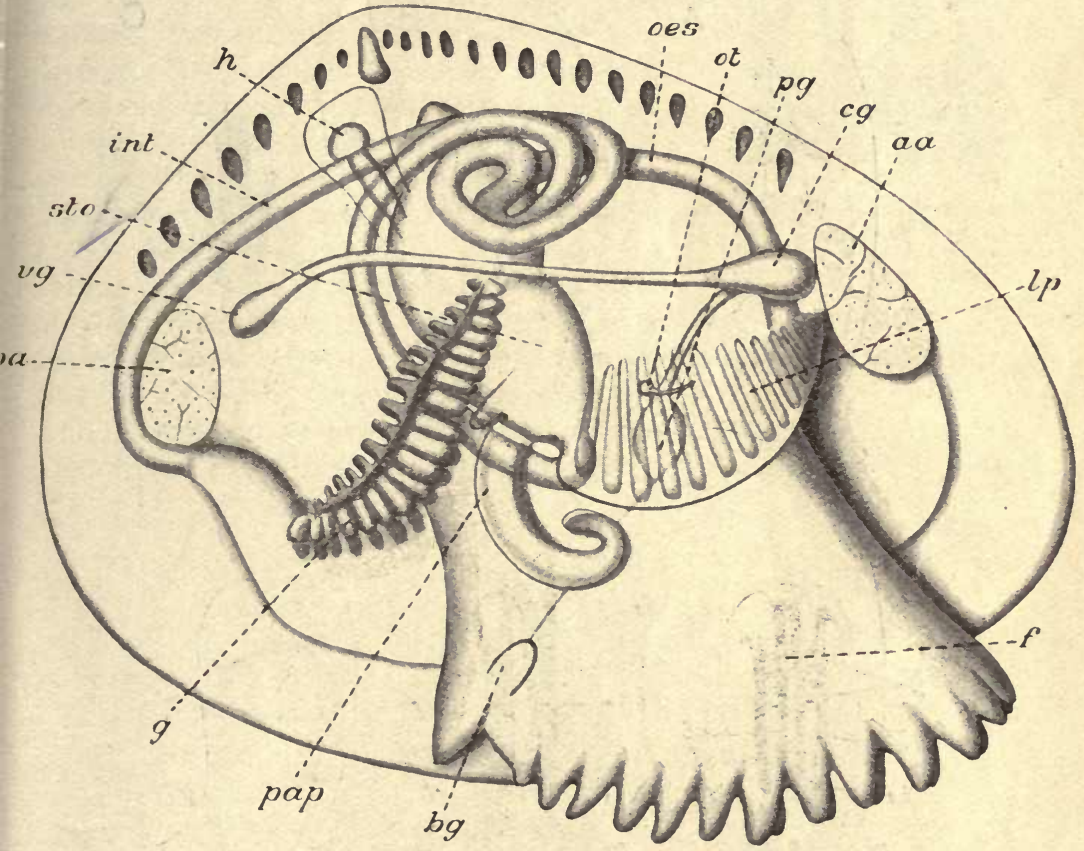

Fig. 586.- Adult specimen of Nucula delphinodonta, represented as seen from the right side. Reconstructed to show internal organs. Fully grown specimens are $4 \mathrm{~mm}$. long. $a a$. anterior adductor muscle; $b g$. byssal gland; $c g$. cerebral ganglion ; $f$. foot; $g$. gill : $h$. heart ; int. intestine ; $I p$. labial palp ; $\propto 8$. œesophagus ; ot. statocyst ; $p a$. posterior adductor muscle; pap. palp-appendage; $p g$. pedal ganglion; sto. stomach; vg. visceral ganglion. (After Drew.)

ctenidium: the outer lamella of the outer and the inner lamella of the inner laminse are free dorsally.

In Mytilus (Fig. 587, D) the gill is strengthened by the development of delicate non-vascular bars or interlamellar junctions between the two limbs of each filament. In Lucina these junctions are large and provided with blood-vessels; and vascular bars of tissue, the interfilamentar junctions, replace the ciliary junctions of the lower forms. Thus by a regular series of gradations the ctenidium is replaced by the complex double gill we are 
already familiar with in Anodonta. In all the higher forms the outer lamella of the outer lamina unites with the mantle and the inner lamella of the inner lamina with the visceral mass, while, posterior to the latter, the inner lamellæ of the right and left inner laminæ unite with one another. The blood-vessels, which are confined to the filaments in the simpler types, occur also in the interfilamentar and interlamellar junctions in the
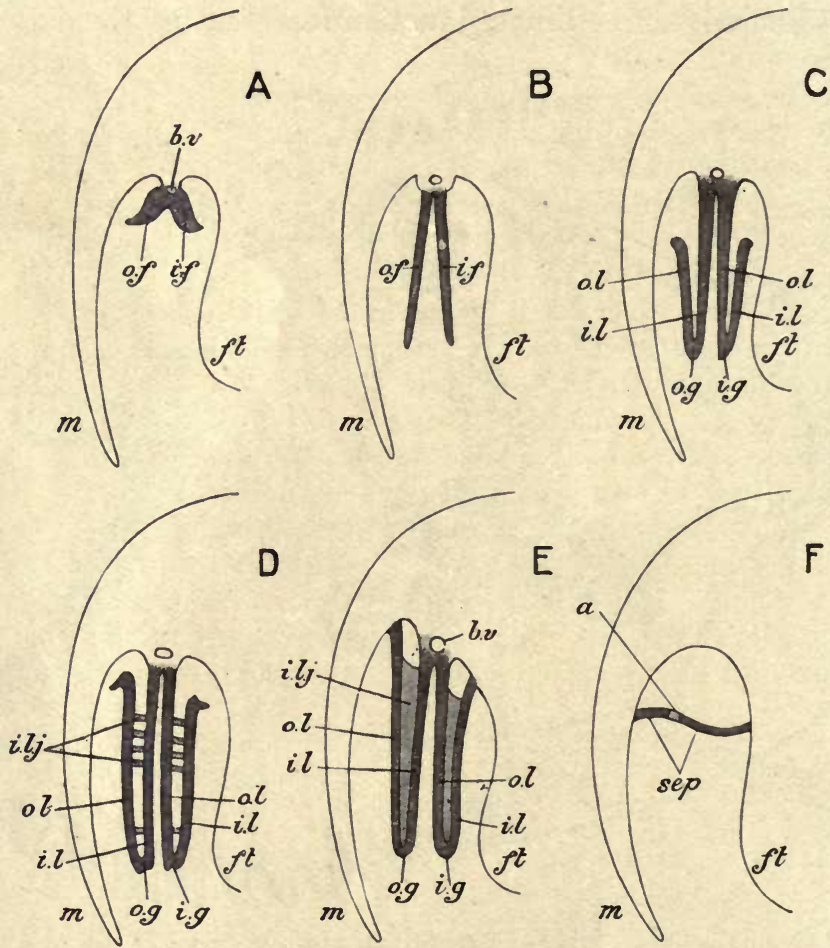

Fic. 587.-Half transverse sections of various Pelecypoda to show the chief kinds of gill. A, Nucula; B, Amusium; C, Arca ; D, Mytilus; E, Anodonta; F, Poromya. $a$. aperture in branchial septum; $b . v$. blood-vessel ; $f t$. foot; $i$. $f$. inner row of filaments; $i . g$. inner lamina ; $i . l$. immer lamella; $i . l . j$. interlamellar junctions; $m$. mantle; $o$. $f$. outer row of flaments; o. g. outer lamina; $o . l$. outer lamella; sep. branchial septum. (Modified from Korschelt and Heider, and Lang.)

inore complex forms of gills. In the Septibranchia the gills are degenerate, being represented by a horizontal muscular partition or septum (Fig. 587, F, and Fig. 589 IX), which divides the inhalant and exhalant chambers from one another. Respiration in this case is performed entirely by the internal face of the mantle.

Digestive Organs. - The mouth is anterior; in forms with two adductor muscles it is always placed immediately behind the 
anterior adductor. It is usually bounded by two pairs of labial palps which sometimes attain an immense size (Fig. 586); there is never any trace of jaws or other masticatory apparatus. The convolutions of the intestine are sometimes very complex. The crystalline style either lies freely in the stomach and anterior part of the intestine, or is contained in a creal pouch of the stomach (Fig. 590), which may be prolonged into one of the lobes of the mantle. The anterior end of the style, which projects into the stomach, appears to be slowly dissolved by the digestive juice, forming a sort of cement to enclose the hard particles of the food and prevent any harmful effect on the mucous membrane. It is possible also that the dissolved substance of the style may play the part

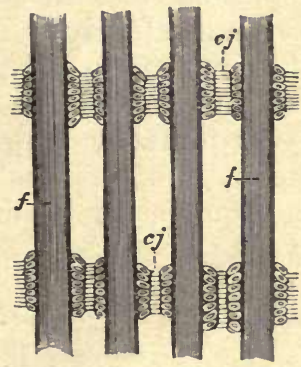

Fig. 58s.-Four gill-filaments of Mytilus. e. $j$. ciliary junetion ; $f$. filaments. (From the Cambridge Natural History.) of a digestive secretion, since it appears to contain a substance of the nature of a digestive ferment capable of acting upon starchy matters.

The excretory organs occur in their simplest form in the Protobranchia, in which they have the form of cylindrical curved

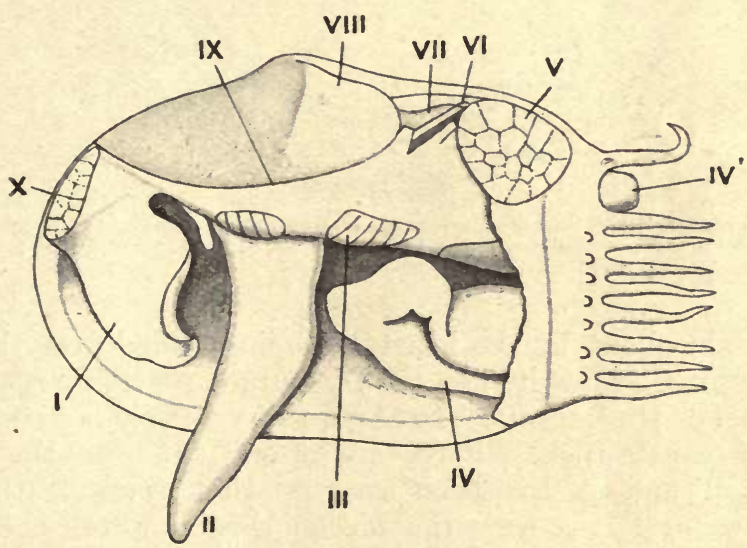

FIG. 589.-Dissection of Poromya. I, anterior palp; II, foot ; III, lamella on brunchial septum ; $I V$, valve of branchial aperture ; $I V^{\prime}$, anal siphon; $V$, posterior adductor; VI, posterior retractor of foot ; VII, heart ; VIII, ovary ; IX, branchial septum ; X, anterior adduetor. (From Pelseneer.)

tubes or nephridia, opening at one end into the pericardium and at the other on to the exterior; the whole nephridium is lined with glandular epithelium, and has no communication with its fellow of the opposite side. In the higher forms the organ 
becomes differentiated into a secreting portion or kidney-which is very spongy in texture, and opens into the pericardium, and a non-secretory portion or bladder, which opens externally. Frequently there is a communication between the right and left nephridia, and in some genera, such as the Oyster, the organs become extensively branched. Also taking a share in the process of excretion are the pericardial glands, or Keber's organs, glandular developments of the wall of the pericardium.

Circulatory Organs.-The heart is usually perforated by the rectum, but lies altogether above it in Nucula (Fig. 586, h) and some other genera; the ordinary arrangement seems to have

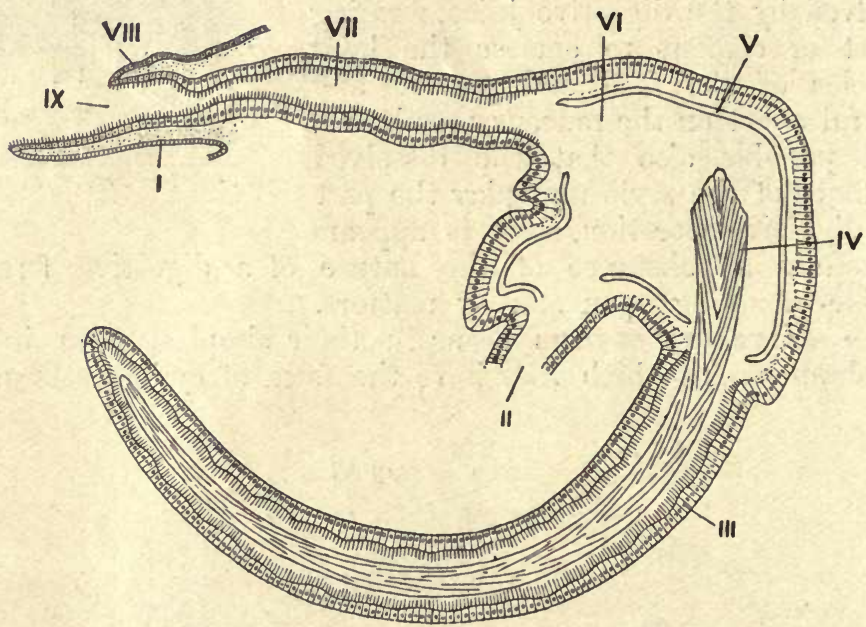

Frg. 590.-Sagittal section of part of enteric canal of Donax. I, lower lip; II, intestine ; III, pyloric cecum; IV, crystalline style; V, cuticle; VI, stomach; VII, gullet; VIII, upper lip; IX, mouth. (From Pelseneer.)

been brought about by the heart becoming folded over the intestine and united below it. In the Oyster and some other forms the heart is below the rectum. In Arca the ventricle is divided into two by a constriction. Pores are often found on the surface of the foot, and it has been asserted that through them the external water mixes with the blood; this, however, is certainly not the case: the blood-system is everywhere closed. The blood is red in some forms (e.g., Arca) owing to hæmoglobin in the corpuscles; in some cases it is of a bluish tint owing to the presence of hæmocyanin.

The nervous system is found in its most primitive condition in Nucula (Fig. 591). Instead of a single cerebro-pleural ganglion there are, on each side, distinct cerebral (XVI) and pleural (I) ganglia, each united by a connective with the pedal, 
The most characteristic sense-organs are the statocysts ("otocysts") and the osphradia. The statocyst_- auditory" or directive organ-is always placed in the foot, in close relation to the pedal ganglion, sometimes embedded in the latter. The statocysts are developed as involutions of the ectoderm and retain their connection with the exterior in Nucula (Fig. 586 ot) and some others. In most cases they become closed sacs. The cavity is usually ciliated, but the cilia may be wanting. Each statocyst may contain a number of minute statocones or, more usually, a single, larger statolith. The nerves supplying the statocysts are given off not from the pedal ganglia, but from the cerebropleural connectives, and their fibres are derived from the cerebral ganglia.

The osphradia- "olfactory" or watertesting organs-are patches of sensory epithelium with an accessory or osphradial ganglion situated in the immediate relation with the visceral ganglia (Fig. 591 , viii), but connected by nerve-fibres with the cerebral ganglion. Patches of sensory epithelium, very similar to the osphradia, and called the ahdominal senseorgans, occur one on each side of the anus in Arca and other forms devoid of siphons, and a similar organ has been described beside the retractor muscles of the siphons in several Sinupalliata.

In a few instances eyes are present, but never in what we are accustomed to consider as the normal position for such organs, at the anterior or head-end of the body. They occur, in fact, in the only situation where they can be of any use, namely, along the edge of the mantle. The best-known form in which they occur is the common Scallop (Pecten), which has a single row (Fig. 575, VII) all round the

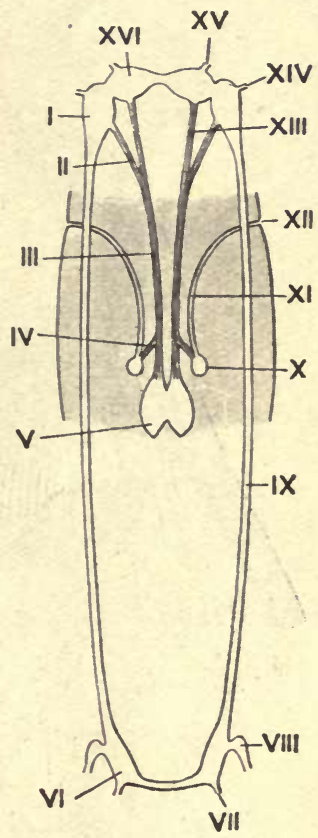

FiG. 591.-Nervous system and "auditory" organs of $\mathbf{N u}$ cula. I, pleural ganglion ; II, pleuropedal-connective ; III, common connective from cerebral and pleural to pedal gauglia ; IV, "auditory" nerve; V, pedal ganglion; VI, visceral ganglion; VII, posterior pallial nerve ; VIII, osphradial ganglion ; IX, visceral connective ; $\mathbf{X}$, statocyst; XI, canal of statocyst; XII, its external aperture; XIII, cerebro-pedal counective; XIV, anterior pallial nerve ; XV, nerve to palps ; XVI, cerebral ganglion. (From Pelseneer.) mantle-border. Each has a cornea (Fig. 592 1), a cellular (not cuticular) lens (2), a retina (5) - formed of cells, the inner ends of which are modified into visual rods, and an optic nerve ( $/ 7)$, one branch of which spreads over the front of the retina and sends branches backwards to the visual rods. In this peculiarity, as well as in the cellular lens, the eye of Pecten is singularly 
like that of Vertebrates. The pallial eyes of Pelecypoda are probably to be looked upon as modified tentacles. The only cephalic eyes that occur in this class are a pair of small but welldereloped organs which occur in the bases of the most anterior

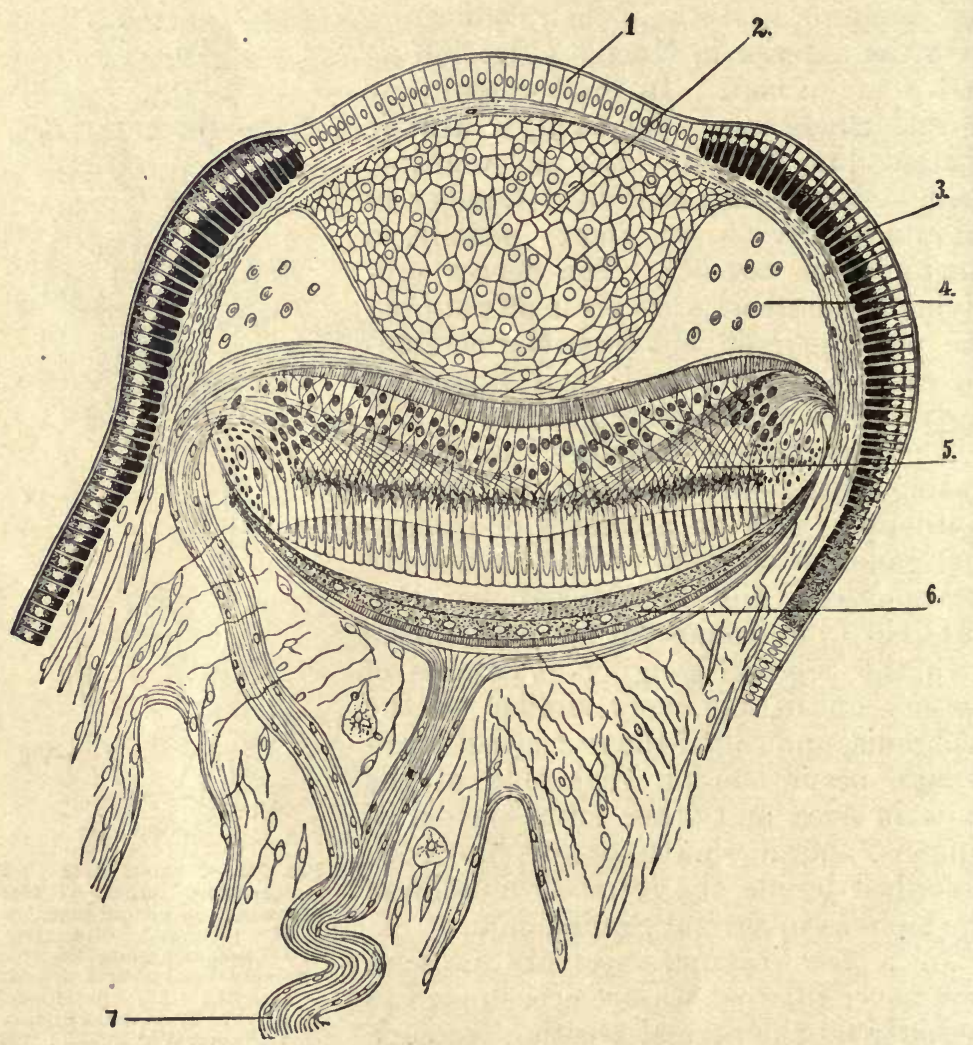

Fic. 592.-Vertical section of eye of Pecten. 1, cornea ; 2, lens; 3, external epithelium ; 4 , blood-sinus; 5 , retina; 6 , pigmentary layer; 7 , optic nerve. (From Korschelt and Heider.)

filaments of the inner lamina of the gill in Mytilus and some other genera.

Reproduction and Development.-Most Pelecypoda are diœcious, but several hermaphrodite forms are known. Some of these, such as the Oyster, are protandrous, the gonad producing first sperms and afterwards ova : in others part of the gonad serves as an ovary, part as a testis, the two opening into a common duct: in others again there is a distinct ovary and testis on each side opening by separate ducts. There are never any accessory organs of reproduction, such as spermatheca, penis, \&c. Fertilisation 
frequently takes place in the water after the eggs are laid. Segmentation is total but unequal, and the gastrula is formed either

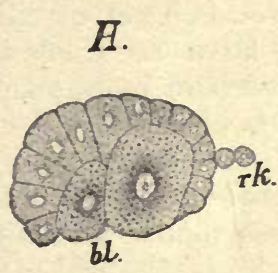

B.

C.
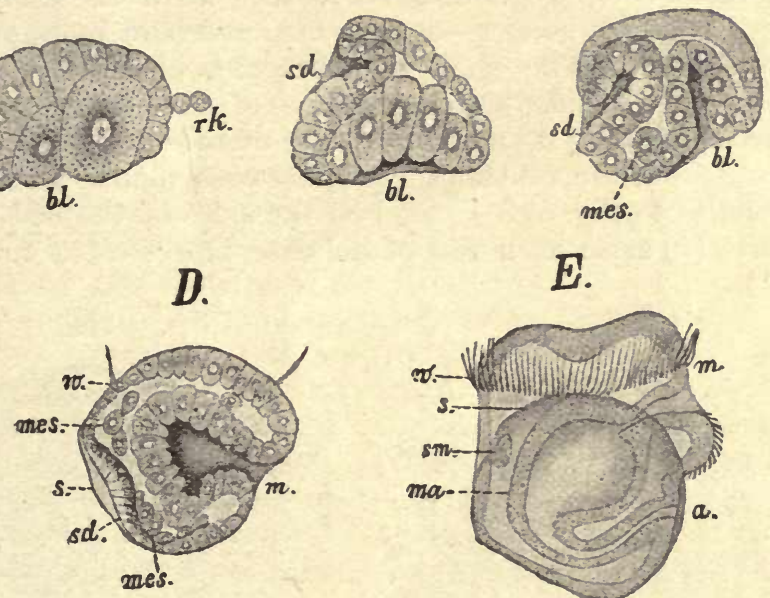

E.

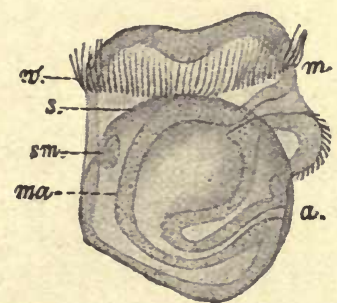

Fig. 593.-Five stages in the development of Ostrea. a. anus; $b l$. blastopore; m. mouth ; ma, stomach ; mes. mesoderm : rl, polar bodies ; s. shell ; sd, shell-gland ; sm. anterior adductor; w, pre-oral circlet of cilia. (From Korschelt and Heider.)

by invagination or by epiboly. A shell-gland (Fig. 593, sil.) is formed as an invagination of the dorsal surface, a stomodæum $(\mathrm{m})$ as an invagination of the ventral surface, and the larva of most forms, unlike that of Anodonta or Unio, passes into a stage in which it closely resembles the trochophore of Chætopods (Fig. 593), having a pre-oral and a post-oral circlet of cilia, a tuft of cilia round the anus, and an apical tuft in the middle of the prostomium. There is also an ectodermal thickening on the prostomium which becomes the cerebral ganglion, and a similar ventral thickening which gives

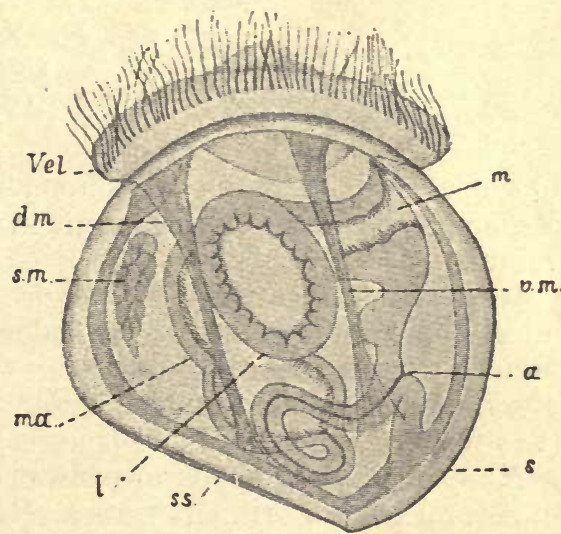

Fio. 594. - Veliger larva of Ostrea. a. anus; $d m$. dorsal longitudinal muscle ; $l$. "liver" ; m. mouth ; ma, stomach; s. shell; sm. adductor muscle; ss. hinge of shell; Vel. velum; vm. ventral longitudinal muscle. (From Korschelt and Heider.) rise to the pedal ganglion and corresponds with the rudiment of the ventral nerve-cord in Polychæta. The pelecypod 
trochophore is, however, distinguished from the corresponding stage in Worms by the presence of the shell-gland, which soon secretes a delicate unpaired shell. The prostomial region grows out into a thickened retractile rim bearing the pre-oral circlet of cilia, and called the velum (Fig. 594 vel.): the larva at this stage is distinguished as a veliger-a very characteristic molluscan phase of development. The shell soon becomes bivalved and extends ventrally on each side, paired processes of the dorsal region of the body accompanying it and forming the mantle-lobes. A projection grows out from the ventral surface, between mouth and anus, and forms the foot (Fig. 595, $f$ ), and on the sides of the body the gillfilaments $(k)$ arise as a row of delicate processes, at first simple
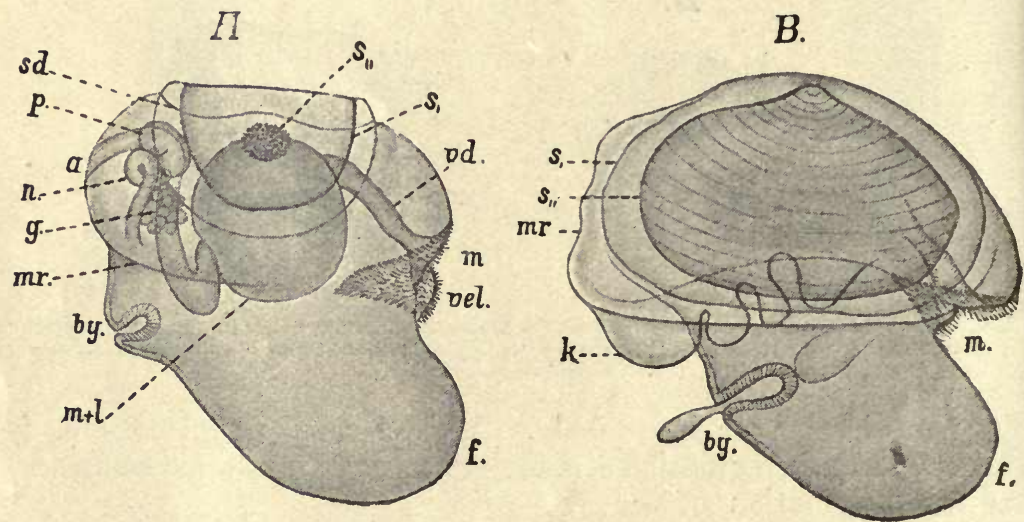

Fia. 595. - Two embryos of Cyclas. $a$. anus; $b y$. byssus-gland; $J$. foot; $g$. gonad; $k$, gill ; $m$. mouth; $m+l$. stomach and "liver" ; $m$ ". edge of mantle; $n$. kidney ; $p$. pericardium ; $g^{\prime}$. umpaired shell; $8^{\prime \prime}$. rudiment of paired shell; $8 d$. shell-gland; vd. gullet; vel. velar area. (From Korschelt and Heider.)

but afterwards becoming bent upon themselves so as to assume a $\mathrm{V}$-shape. Eyes are often present in the larva at the base of the velum.

General Remarks.-Although none of the Pelecypoda are microscopic, they present a considerable range in size, from the minute Nucula, about $4 \mathrm{~mm}$. long, to the Giant Clam (Tridacna gigas) of the Indian and Pacific islands, which is sometimes $60 \mathrm{~cm}$ : (two feet) in length and 500 pounds in weight.

Many pelecypod shells are white or dull brown in colour, but in several genera brilliant tints are the rule, the various species of Scallop (Pecten) being specially remarkable in this respect. The inner surface of the shell often exhibits beautiful iridescent tints, noticeably in the so-called Pearl-oyster (Meleagrina) and the Australian Trigonia. As far as is known, the colours are all what are called " non-significant," i.e. are of no physiological or ethological importance. In this connection the formation of pearls by some 
species must be mentioned: they are deposits of nacre formed usually round encysted parasitic worms, either between the mantle and shell or in the soft parts. They are produced, amongst other species, by the "Pearl-oyster" (Meleagrina inargaritifera) and by the Pearl-mussel (Unio margaritifera). Some species, such as the common boring Pholas, are phosphorescent.

Most Pelecypoda are sluggish in habit, progressing only by slow contractions of the foot, and some are permanently fixed during adult life by the byssus, or are only able to change their position after throwing off the byssus, which becomes replaced by a new one. The Scallops, however, swim freely by clapping the valves together. The Cockles (Cardium), Trigonia, \&c., jump by sudden movements of the foot, and the Razor-fish (Solen) jerks itself forward by suddenly withdrawing its foot and thus ejecting water through the siphons. The only parasitic genus is Entovalva, found in the gullet of a Holothurian.

Pelecypoda are abundant both in fresh water and the sea; the marine forms are mainly littoral. None are pelagic or terrestrial. They are very abundant in the fossil condition, occurring in all formations from the. Upper Cambrian upwards, and, owing to their gregarious habits, frequently forming extensive deposits or shell-beds. The oldest forms are all iso- or hetero-myarian; the monomyarian types (Pseudolamellibranchia) appear first in the Carboniferous, and the Siphoniata not until the Triassic period. The modern genus Arca dates from the Upper Cambrian, and thus furnishes as striking an example of a "persistent type" as some of the Brachiopods.

There seems to be little doubt that the Protobranchia, and especially Nucula, exhibit the most primitive type of pelecypod organisation, as indicated by the plume-like gills with separate filaments, the simple nephridia, and the distinct cerebral and pleural ganglia; absence of concrescence is always a mark of low or generalised organisation. The Filibranchia with imperfectly united gill-filaments come next, and are divisible into two groups - isomyarian with equal-sized adductors, and heteromyarian with more or less atrophied anterior and proportionally enlarged posterior adductor; the latter group is to be looked upon as the more specialised, and leads to the Pseudolamellibranchia (monomyarian type) in which the anterior adductor disappears completely in the adult, while the posterior is immensely enlarged and assumes a central position. Similarly, the isomyarian Filibranchia lead to the Eulamellibranchia, which are equal-muscled, but have the gillfilaments united into a complete basket-work. In the Eulamellibranchia, lastly, there is a gradual series of stages from comparatively generalised forms with free mantle-lobes up to the highly specialised species with large siphons. That the Pseudolamellibranchia and the siphoniate Eulamellibranchia are to be looked 
upon as the highest members of the class is indicated, not only by morphological evidence but by their comparatively late appearance in time.
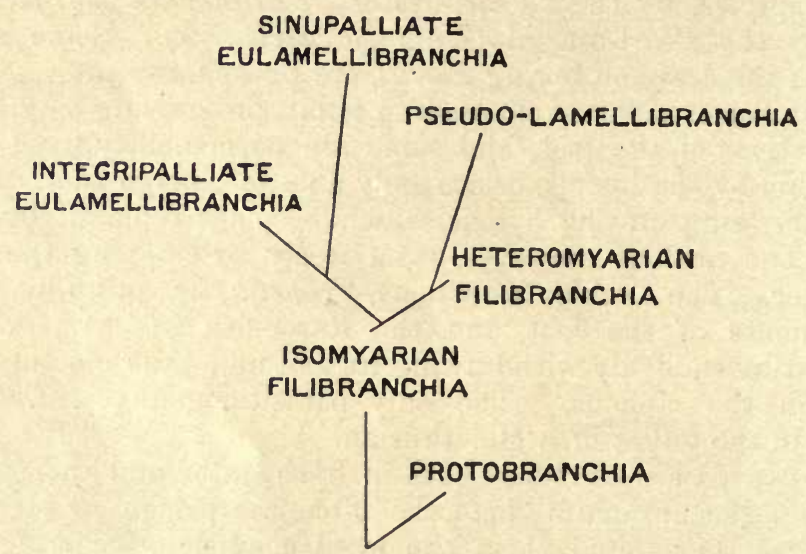

Fig. 596.-Diagram illustrating the mutual relationships of the Pelecypoda.

\section{CLASS II.-AMPHINEURA.}

The Amphineura are a class of marine Mollusca formerly grouped with the Gastropoda, but now recognised as sufficiently far removed from the latter to require separation as a distinct class. The commonest, as well as the most highly organised, of the Amphineura are the Chitons, a group of remarkably sluggish Limpet-like Molluscs with a shell composed of eight pieces. The other members of the class are lowly organised, comprising the most primitive forms of the entire phylum, all of which are devoid of a shell.

\section{Distinctive Characters and Classification.}

The Amphineura may be defined as bilaterally symmetrical, more or less elongated Mollusca, with terminal mouth and anus, either devoid of a shell, or possessing one which consists of eight median valves. The mantle contains numerous spicules of carbonate of lime, and is not divided into paired lateral lobes. The ctenidia are either absent, or there is a single pair, or they occur as a circlet round the anus, or as two lateral rows situated between the edge of the mantle. and the side of the foot. A radula (vide infra) is sometimes present, sometimes absent. The nervous system consists of two pairs of nerve-cords, pedal and pallial, connected in front with a nerve-ring.

The class is divisible into two orders : 


\section{Order 1.-Placophora.}

Amphineura with a broad foot, and with a shell which consists of eight transverse valves. There is a row of ctenidia on either side. This order includes the Chitons.

\section{Order 2.-Aplacophora [Solenogastres].}

Amphineura with an elongated body covered completely by the mantle, without shell, but with calcareous spicules. There is no foot, but generally a ventral longitudinal groove along which usually runs a low ciliated ridge. In some there is a posterior cavity (cloaca or mantle-cavity), containing a pair or a circlet of ctenidia.

This order includes Neomenic, Proneomenia, Chetoderma, and a number of other genera.

\section{General Organisation.}

External Features.-The Aplacophora are distinguished by their worm-like body, sometimes elongated and narrow and capable of being coiled into a spiral, sometimes comparatively short and thick. In most instances there is little difference in external appearance between the anterior and posterior ends. In Chcetoderma (Fig. 597) alone is there a distinct head, separated off from the body by a constriction, as well as a posterior cloacal region which is similarly marked off. A shell is completely absent. The mantle covering the surface possesses a cuticle, in the substance or on the surface of which are spicules of calcified material. Along the middle of the ventral surface runs, in most instances, a groove, in some cases merely represented by a narrow strip from which the cuticle and spicules are absent. The ventral groove, when present, usually contains a slight longitudinal ridge, and this is all that in these simple forms represents the foot, an organ so highly developed in other Molluses. In Chætoderma it is entirely absent. With the ventral groove is connected in front an anterior ciliated groove, while behind it is in direct communication with the cavity of the cloaca.

In Proneomenia ctenidia are absent. In the remaining genera there is either a pair or a circlet of gills situated in the cloaca 
- a cavity at the posterior end of the body into which the anus opens (Fig. 602).

In Chiton (Figs. 599 and 600) the body is dorso-ventrally compressed, convex above, and presents below a broad flat foot

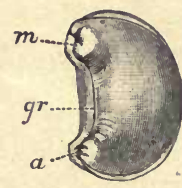

FIG. 598.- Neomenia carinata. a. anus gr. ventral groove; m. mouth. (From the Cambridge Natural History.)

\section{valves, arranged in} (narrow in Chitonellus) which acts not only as an organ for effecting creeping movements, but also as a sucker for enabling the animal when at rest to adhere firmly, like a Limpet, to the surface of a rock. The head region is not distinctly separated off, and is not provided with eyes or tentacles. The most remarkable external feature of Chiton is the presence on the dorsal surface of a calcareous shell (Figs. 599 and 601) made up of no fewer than eight transversely elongated pieces or valves, arranged in a longitudinal row, articulating together and partly overlapping one another. They are sometimes partly, sometimes completely, covered over by the mantle. Each valve consists of two very distinct layers, a more superficial and a deeper, the latter formed of compact calcareous substance, the former perforated by

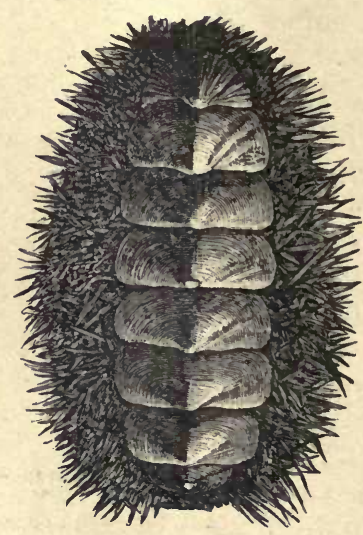

FIG. 599.-Chiton spinosus, dorsal view. (From the Cambridge Natural History.)

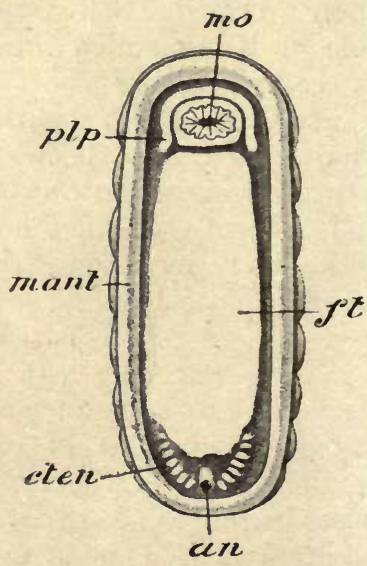

FIG. 600.-Chiton, ventral view. an. anus ; cten. ctenidia ; $f t$. foot; mant. mantle edge ; mo. mouth; $p l_{p}$. palp. (After Pelseneer.)

numerous vertical canals for the lodgment of the sense-organs to be presently referred to; the former alone represents the shell of other Molluses. External to the valves the dorsal integument (mantle) of Chiton and its allies is usually beset with a number of horny or calcified tubercles and spicules. The mantle develops only very slight lateral flaps, and under cover of these are a series of small etenidia (Figs. 600 and 606 , cten.) to the number of 
from fourteen to eighty. The mouth and anus are both median, situated at the anterior and posterior extremities respectively.

Alimentary System.-In the Aplacophora the mouth is usually a longitudinal, rarely (Chotoderma) a transverse, slit, situated ventrally near the anterior extremity. There is a buccal cavity, with a radula ${ }^{1}$ in some cases (Fig. $602 . \mathrm{rad}$ ), and in others a single chitinous tooth supporting smaller denticles: sometimes teeth are entirely absent. There are both salivary and buccal glands. Very characteristic of the group as compared with other Molluses is the presence of a straight intestine devoid of coils, and having connected with it either a single cæcum or a double row of cxea. In the Placophora the buccal cavity always contains a welldeveloped odontophore and radula. The intestine is elongated and coiled. There are salivary glands and a large paired liver (Fig. 603, liv.).

Body-Cavity. - In the Aplacophora the interstices between the organs and the body-wall are filled with a form of connective-tissue with muscular fibres; a vertical diaphragm (Fig.602, dia.) separates the posterior part of the body, containing the pericardium (peri), from the rest. In the Placophora the colome (Fig. 603) is an extensive cavity, lined with a coelomic epithelium, and divided into three completely separated partsthe pericardium, the genital cavity, and the general body-cavity.

Vascular system.-The vascular system of the Aplacophora is very rudimentary. There is a heart enclosed in a pericardium, and composed, when best

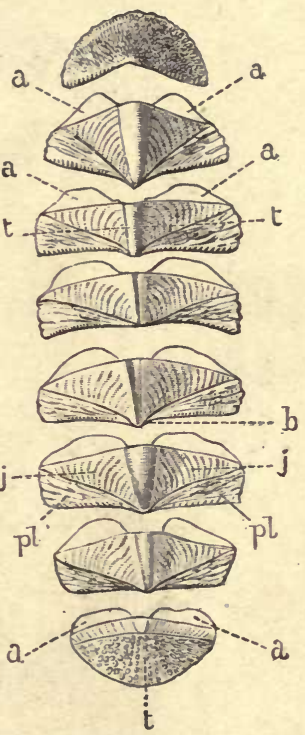

Fig. 601.-Chiton, valves of shell. (From the Cambridge Nutural History.) developed, of an auricle and a ventricle (Figs. 602 and 605, peri).

In Chiton there is a well-developed heart (Fig. 603, $h l$.) consisting of a median ventricle and two lateral auricles. The pericardial cavity in which it lies is a space of considerable extent in the posterior region of the body, below the two last valves of the shell.

The Nervous System consists in the Aplacophora (Fig. 604, $\left.A, B, C^{\prime}\right)$ of four longitudinal nerve-cords-two pedal and two pallial. These are connected together by an œsophageal ring, thickened dorsally into a single or double cerebral ganglion; and in front of this is a second, more slender stomatogastric nerve-ring with small

1 For a description of the structure of this characteristic organ, see the account of Triton (p. 726). 
ganglia. The pedal cords (v.v) may present in front a pair of ganglionic thickenings connected by a commissure, and further back there may be a series of enlargements united by commissures, The pallial cords $(l, l)$ are connected behind, above the rectum, by a commissure $(p, c)$ which usually bears a median enlargement. Sometimes a union takes place posteriorly between the cords of the two pairs. There are no eyes, or statocysts, or tentacles, Some have a sensory frontal lobe and a sensory pit or elevation in the middle line of the dorsal surface near the posterior end.

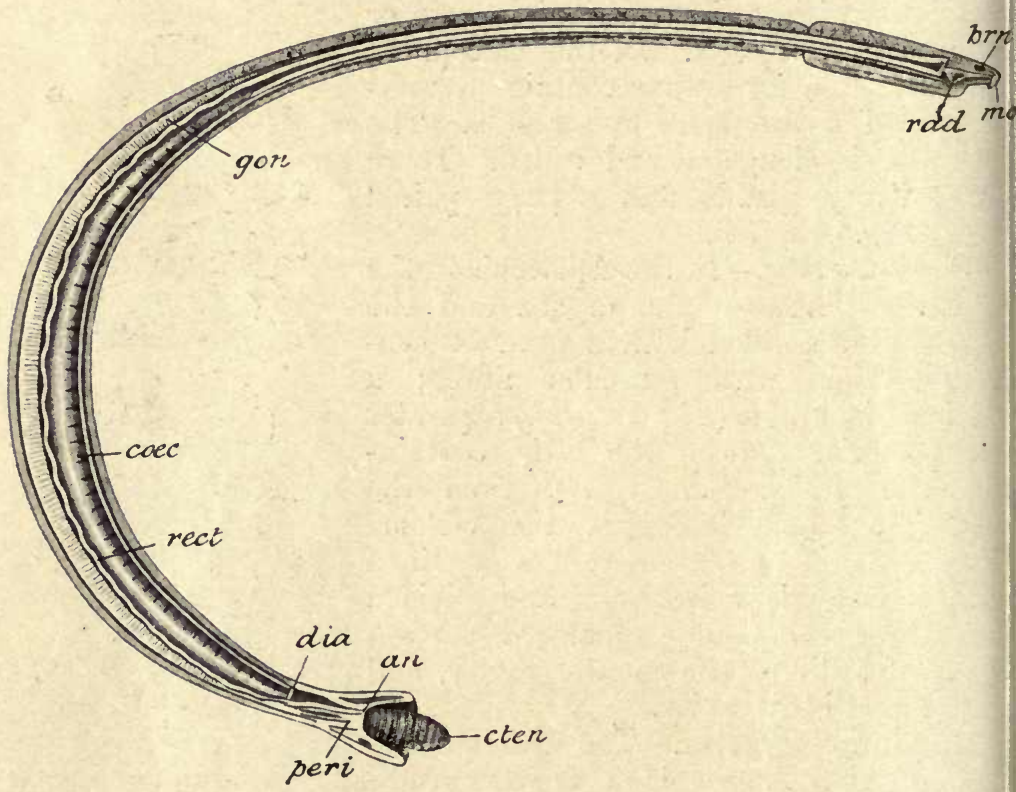

FIG. 602.-Chætoderma nitidulum, longitudinal section. an. anus; brn. brain ; cac. gland ular caca of mesenteron; cten. ctenidium; dia. diaphrigm separating off the posterior portion of the body; mo. mouth; peri. pericardium and heart; rad. radula; rect. rectum. (Afte Simroth.)

In the Placophora (Fig. 604, D) there is an œsophageal nerve ring consisting of a thicker dorsal cerebral portion not differentiated into ganglia, and a thinner ventral buccal commissure. The cerebra part sends off nerves to the labial palps, the lips, and the buccal apparatus. 'Two pairs of longitudinal nerve-cords, pedal and pallial are given off posteriorly: the former, from which arise nerves to the foot, are joined by numerous commissures passing beneath the enteric canal; the latter, which send off nerves chiefly to the mantle and the ctenidia, are united together by a suprarectal commissure at the posterior end of the body. Just behin its origin each pallial cord gives off a slender visccral commissum 
which unites with its fellow of the opposite side: two small ganglia lie in this visceral commissure near the middle line. The large cords contain nerve-cells throughout their length.

The conspicuous organs of special sense, present on the head of Gastropods (vide infra), are absent in the Placophora, as in the

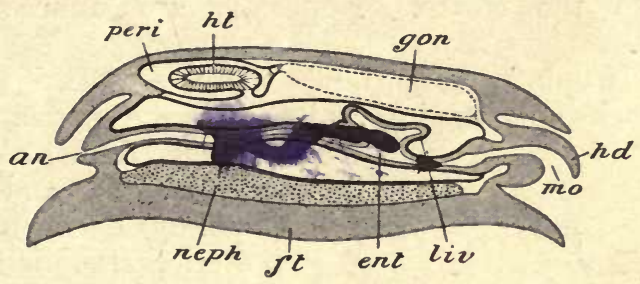

FIc. 603.-Diagrammatic longitudinal section of Chiton, specially intended to show the relations of the parts of the colome, whlch, except the genital parts, are bordered with a thick line. an. anus; ent. enteric cavity; ft. foot; gon. gonad; hl. head-lobes; ht. heart; liv. liver; mo. mouth; neph. nephridium; peri. pericardial cavity. (From Simroth, after Haller.)

Aplacophora. A pair of processes situated in front, at the sides of the mouth, have the character of labial palps. In the buccal cavity there are cup-shaped gustatory organs supplied with nerves from the cerebral commissures, and in front of the odontophore is a thickening of the epithelium-the subradulur organ-containing nerve-endings. Remarkable sensory organs, the microsthetes and the megalcesthetes, lie in the canals already
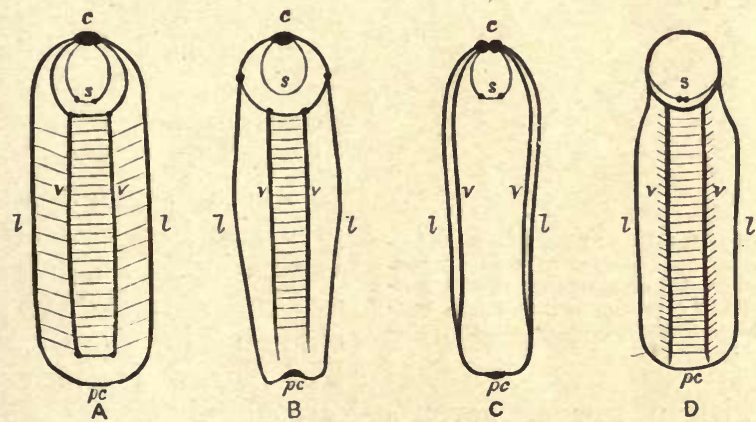

Fir. 604.-Nervous system of Amphineura. A, Proneomenia; B, Ntomenia; C, Chatodemna : $\nu$, Chilon. $c$, cerebral ganglia ; $l, l$, pallial cords ; $p c$. posterior commissure ; $s$, stomatogastric commissure or ring, with ganglia ; $v, v$, pedal cords. (From the Cambridge Natural History, after Hubrecht.)

mentioned as occurring in the superficial layer of the shell-valves. The megalæsthetes may take the form of eyes, with cornea, lens, pigment-layer, jris, and retina; in some cases the lens is absent. 
Reproductive and Renal Organs.-In the Placophore the sexes are distinct: in the Aplacophora, with the exception of Chætoderma, they are united. In the Aplacophora (Fig. 60.5) with the exception of Chætoderma, the gonads are paired. The sexual products pass into the pericardial cavity and thence are carried to the exterior by a pair of ducts (colomoducts) opening into the cloaca. Nephridia are unknown in the Aplacophora.

In the Placophora (Fig. 606) there are two symmetrical nephridia, each opening internally into the pericardium by a ciliated funnel-like opening

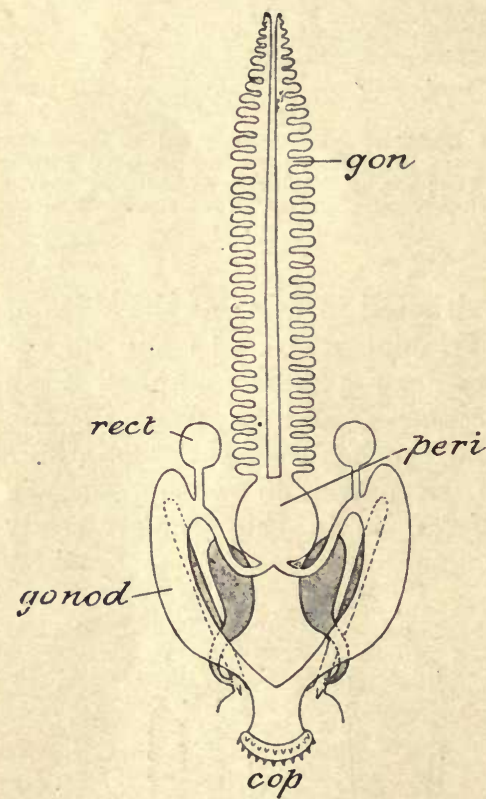

FI\%. 605.-Neomenia carinata, reproductive organs. cop. copulatory organs; gon. gonads enclosed in extensions of the pericardial cavity ; gonod. gonoducts; peri. pericardium ; rect. receptaculum seminis. (From Simroth, after Wirèn.) The larva is provided for a time with a row of seven calcareous plates on the dorsal surface.

The eggs of Chiton are fertilised in the mantle-cavity, where in one species they are retained until the embryos are fully developed. At first the segmentation is tolerably equal-the ovum becoming divided into four approximately equal blastomeres; but at the stage of eight cells, four on one side are to be distinguished as larger than the other four. These two sets undergo further divisions and arrange themselves in such a way as to form

(n. peri. $a p$ ), and externally (neph. ap) between two of the posterior ctenidia some little distance in front of the anus. Each consists of a looped main tube, into which open numerous minute tubules which ramify among the viscera. The testis and ovary (gon) are similar in appearance, differing only in colour when the products are mature. Each is an unpaired sac marked by a series of slight lateral constrictions. There are two gonoducts, each opening immediately in front of the corresponding nephridial duct.

Little is known of the development of the Aplacophora. The eggs undergo complete segmentation, and give rise to a gastrula by invagination. This develops into a form of trochophore with a ciliated ring, the prototroch. 
a somewhat flattened blastula, one side of which (vegetal pole) is composed of a comparatively small number of large cells. Then follows the invagination of the cells of the vegetal side and the resulting formation of a gastrula: this soon becomes elongated in the direction of the future long axis. Two endoderm cells of specially large size in the neighbourhood of the blastopore, with several others in their proximity, constitute the rudiments of the mesoderm (Fig. 607, B, mes.); these pass

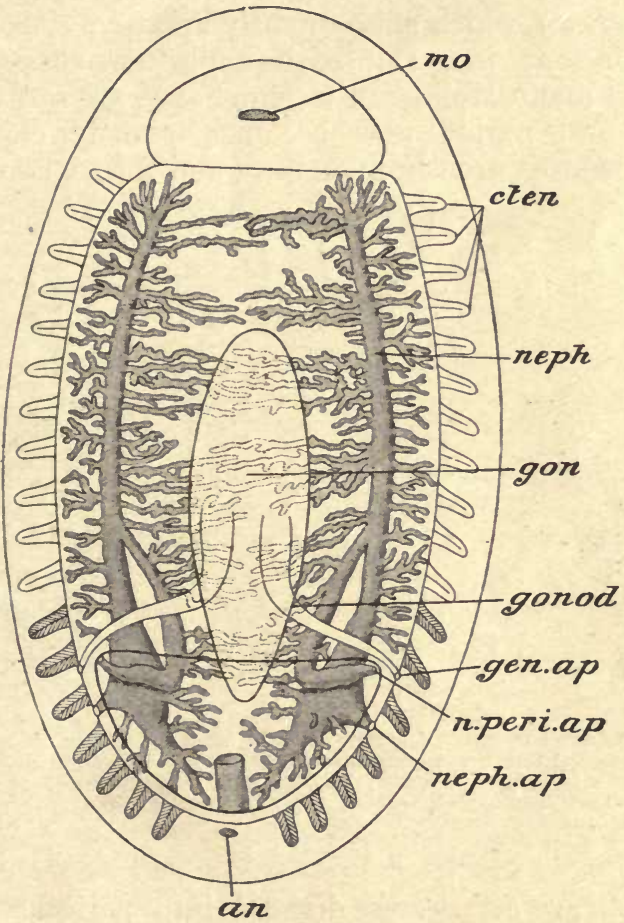

606.-Chiton, nephridial and genital systems. an. anus; cten. ctenidia; gen. ap genital aperture; yon. gonad; gonod.gonoduct; mo. mouth; neph.ap. nephridial aperturc; n. por ap. aperture from nephridia to perieardium. (From Simroth, after Haller and Lang.

into the segmentation-cavity and speedily assume a bilateral arrangement.

Two rings of cells surrounding the embryo develop cilia (cil.), and owing to the double circlet thus formed an anterior and a posterior region are distinguishable in the larva. The blastopore becomes shifted from its original posterior position forwards on the ventral surface until it comes to be situated just behind the circlet of cilia ; it undergoes elongation, and an invagination of ectoderm round its anterior end forms the mouth $\left(m_{0}.\right)$ and stomodæum. A 
ventral diverticulum of this forms the rudiment of the radular sac $(r d$.$) . By greater relative growth of the post-oral part the embryo$ assumes the form of a pear; and in this trochophore stage, with a pre-oral circlet and a bunch of cilia in the middle of the apical area, it becomes free in the case of certain of the species, while in others it remains enclosed in the egg up to a later stage of development. As yet there is no anus, that aperture, with the proctodæum, being formed later by invagination. An apical plate is not present in the early larva; but the rudiments of the cerebral ganglia (C, cer. g.), which subsequently appear at the apical pole, probably represent it. Primitive nephridia, such as occur in Annulate and many Molluscan trochophores, are not present.

The post-oral region now becomes greatly elongated; the mesoderm increases greatly in extent, and forms two well-defined

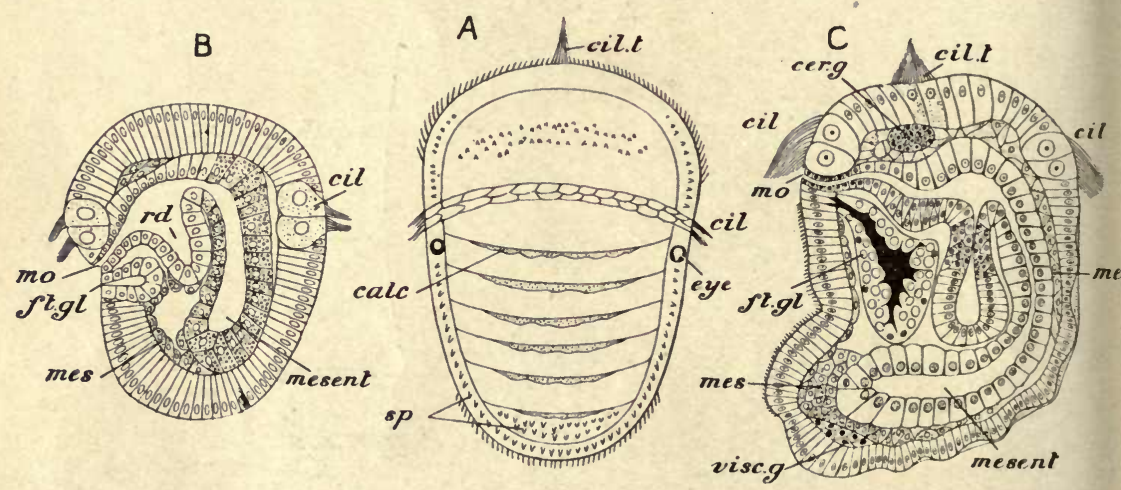

Fig. 607.-Chiton, development. $A$, general view of larva ; $B$, section of early, and $C$, of later trochophore. calc. calcifications (rudiments of shell); cer.g. cerebral ganglion ; cil. ciliary ring; cil. $t$. ciliary tuft at apical pole ; cye, eye; ft.gl. foot-gland; mes. mesoderm ; mesent. mesenteron; mo. mouth; rl. radular sac; $8 p$. spines; visc. g. visceral ganglion. (Frum Korschelt and Heider, after Kowalewsky.)

streaks, which are afterwards divided into parietal and visceral layers with a colomic space between them. The post-oral part of the embryo now presents an appearance resembling rudimentary segmentation. This is due to the development of a series of rudiments of the eight pieces of the shell $(B$, calc. $)$, each of which is formed independently after the fashion of the entire shell of other Mollusca.

Ethology, Distribution, \&c.-All the Amphineura are marine. The Placophora occur at all depths, though most abundant on the shore between tidal limits. The Aplacophora, on the other hand, are rare in very shallow water, and absent altogether from the littoral zone: some have been found at considerable depths (down to 1,250 fathoms). Some of them burrow 
in mud, others live in association with various colonial Coelenterates. The Placophora are all vegetable feeders, their food consisting of minute algæ and diatoms. The Placophora when at rest adhere firmly to the surface of a rock or a block of coral by means of the sucker-like foot. When forcibly detached the animal curls itself up into a ball, and will only after a considerable time slowly extend itself again: all its movements are extremely sluggish.

The Aplacophora have no hard parts that would be recognisable in the fossil condition; but numerous fossil Placophora are known from the Silurian onwards. The valves of the Silurian genera differ from those of recent forms in the absence of the articulations.

\section{CLASS III.-GASTROPODA.}

The Gastropoda, including the Snails and Slugs, Limpets, Whelks, Periwinkles, Sea-hares, and the like, are Mollusca in which there is, as a rule, a shell composed of a single piece, and in which the mantle is not divided into two lateral folds as in the Pelecypoda. The body is inequilateral, owing to the one-sided development of the visceral mass. There is a welldeveloped ventral foot, usually with a broad, flat surface on which the animal creeps. A head-region bearing eyes and tentacles is distinguishable in front of the foot. The alimentary canal is characterised by the presence in the buccal region of a peculiar organ, the odontophore, present also in some of the Amphineura, bearing rows of minute chitinous teeth. Plume-like ctenidia are usually present. A metamorphosis occurs in the development, during which the young Gastropod passes successively through trochophore and veliger stages. The majority of the families of Gastropoda are marine, a few of these being pelagic; but some inhabit fresh water, and others are terrestrial.

\section{Example of the Class.-The Triton (Triton nodiferus).}

Triton is a marine Gastropod living in shallow water, usually close inshore. The species to which the following description specially applies has a very wide range, from the English Channel to the South Pacific, and occurs as a fossil as far back as the Miocene. In most respects the English Whelk (Buccinum undatum) will be found to conform to the description.

The shell (Fig. 608) is a very hard and dense calcareous structure, presenting no trace of division into valves such as compose the shell of the fresh-water Mussel, and lacking also its bilateral symmetry. It is in the form of an elongated hollow cone closely wound round a central axis. The apex of the cone 
is the organic apex of the shell, corresponding to the umbo of the fresh-water Mussel, and is the point from which the growth of the shell has proceeded: the base is represented by the wide oblique opening - the mouth or peristome of the shell. Starting from the apex along the internal cavity of the spirally-wound cone, in order to reach the mouth in an adult shell, we pass completely round the central axis five times-i.e. the spiral consists of five turns. In following the turns, the direction taken is to

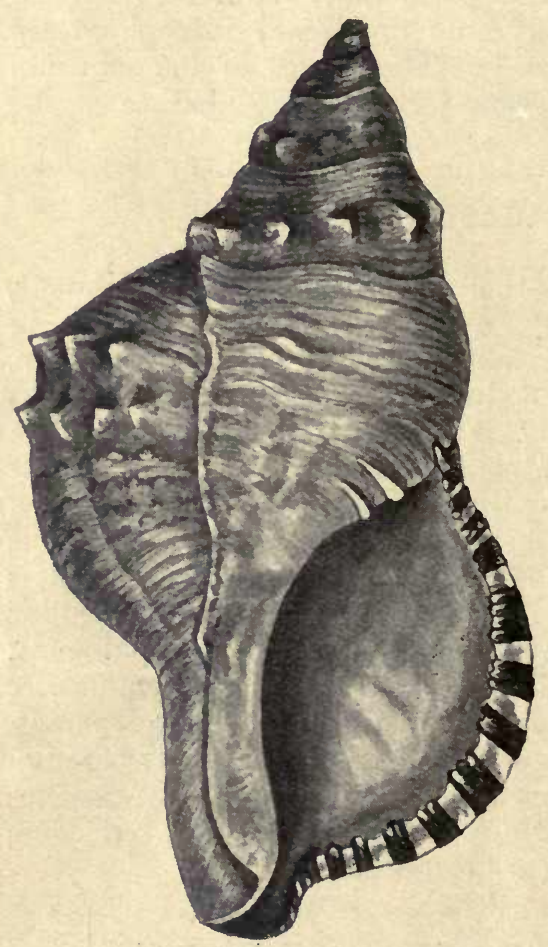

FIG, 608,-Shell of Triton nodiferus. Natural size. the right, that is to say, the spiral of the shell is a righthanded or dextral one. The axis (Fig. 609) is in the shape of a twisted shelly rod - the columella - containing a narrow lumen; it is formed by the close union of the axial portions of the wall of the spiral. The windings of the spiral are marked on the outer surface of the shell by a narrow impressed spiral line or suture, parallel with which are numerous fine ridges and depressions-the lines of growth; the increase in size of the shell takes place in the direction of these lines, not at right angles to them as in the shell of the freshwater Mussel, and the lines that more strictly correspond to the lines of growth of the latter are excessively fine striæ which run transversely to the stronger lines. At certain points, usually three in a full-grown shell, the spiral is interrupted by a transversely directed edge which appears to overlap the succeeding portion; this edge marks the position which the mouth of the shell has occupied during regularly recurring periods of arrest of growth, probably annual.

The mouth of the shell is bordered on the side turned away from the columella by a prominent rim or outer lip of the peristome ; this is produced at the extremity farthest from the apex of the shell into a spout-like process-the siphonal process. The prominent edge of the peristome is in relation to the dorsal 
surface of the body of the animal; the opposite side has no prominent edge, but is rounded off to form a smooth inner lip ; a couple of ridges on this inner lip towards the apical end aid the animal in drawing itself out after it has become retracted into the interior of the shell. The outer lip is in relation to the dorsal surface of the body of the animal, the inner lip in relation to the ventral surface; the siphonal process is for the lodgment of a spout-like process of the edge of the mantlethe siphon.

When removed from the water, or disturbed in any other way, the animal becomes completely withdrawn into the interior of the shell, when the latter is observed to become closed by a plate-the operculum (Fig. 610) - which fits accurately across the passage some distance internal to the peristome. The operculum is an oval plate of chitinoid material hardened by calcareous deposits; like the shell itself, it exhibits lines of growth marking what has been its edge at successive stages in the development of the shell.

The minute structure of the shell is in the main similar to that of the freshwater Mussel (p. 683). Its outer surface is covered with a thin layer of uncal-

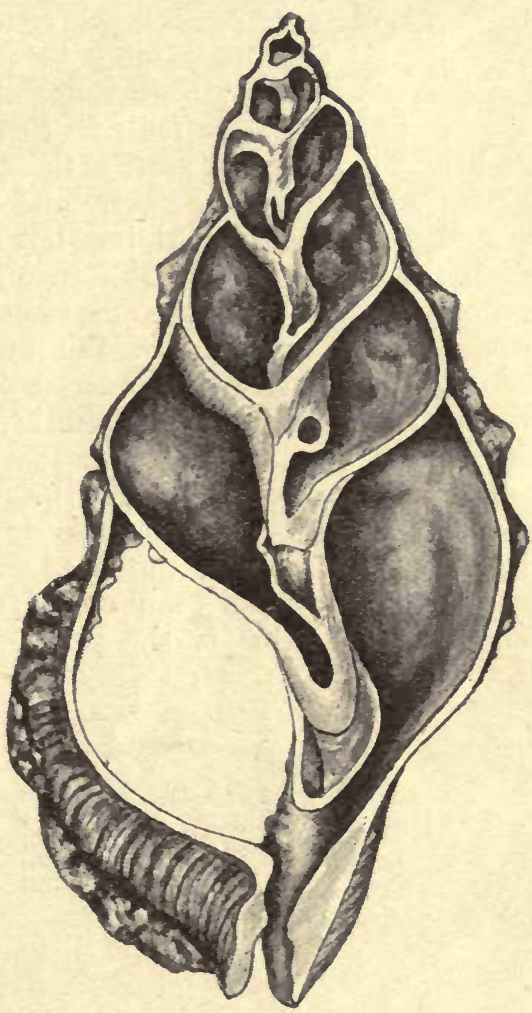

FIG. 609.-Longitudinal median section of the shell of Triton nodiferus. cified chitinoid material, the periostracum, beneath which is a thick prismatic layer, and, lining the inner surface, a layer of nacre.

External Features of Soft Parts.-The Triton is able to extend itself to a considerable degree beyond the mouth of the shell; but a portion of the body always remains concealed in the interior, even when the animal has extended itself to its utmost, the body being, like that of the fresh-water Mussel (and of nearly all the Mollusca) organically connected with the shell. In Triton the connection is by means of a strong muscle-the columellar 
muscle (Fig. 611, col. m.)-which extends from the concave right side of the animal to the columella, into which it is inserted; it is by means of this muscle that the anterior portion of the body, capable of being thrust out through the

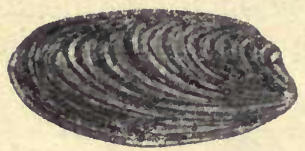

Fig. 610.-Operculum of Triton nodiferus. mouth of the shell, may again be withdrawn.

If the Triton be examined in the extended condition (Fig. 611) it will be found to present a distinct head, which bears dorsally a pair of appendages - the tentacles (tent) - of a sub-cylindrical shape, slightly compressed towards their bases, and narrowing somewhat towards their free extremities; these are capable of being extended and contracted, but not of being completely retracted. Each bears on its outer side, some little distance from the base a prominent eye. At the anterior end of the head on its ventral aspect is the opening of the mouth. When the animal is feeding, an elongated cylindrical introvert (Fig. 612), comparable to that of Sipunculus (p. 492), is extended forwards, bearing the mouth at its anterior end; at other times the introvert is completely involuted within the head and anterior portion of the body. In the male, on the right-hand side of the body some little distance behind the head, is a long, narrow fleshy process, broader at the base than at the free end, and deeply grooved longitudinally; this is the penis. Running back from its base

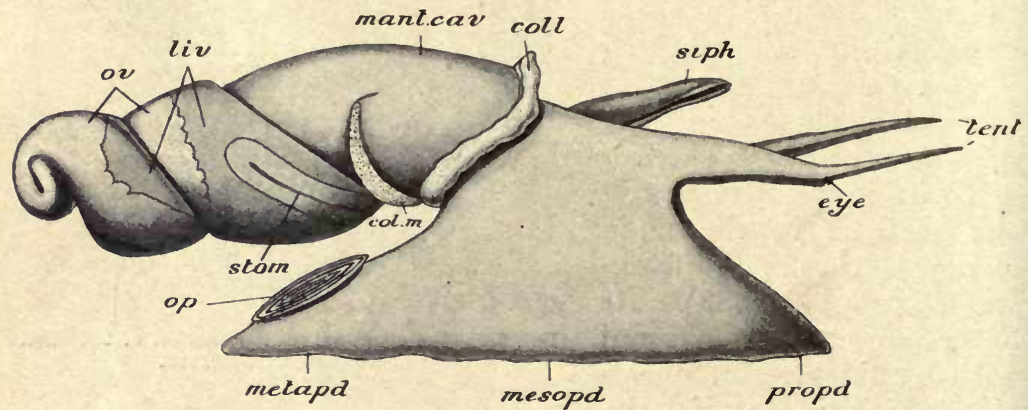

Fir. 611.-Lateral view of the body of a female specimen of Triton nodiferus, removed from the shell, moderately extended. col. m. columellar muscle; coll. collar of mantle; eye, eye ; liv. liver; mant. cav. mantle-cavity; meso. mesopodium; neph. nephridium ; op. operculum ; ov. ovary; prop. propodium ; stom. stomach ; tent. tentacles

is a narrow grove with prominent lips-the sperm-groove, continuous with that of the penis; in the female these parts are not represented.

Foot.-On the side of the body (ventral) which the animal applies to the surface of the ground when it extends from the shell is a flat surface elongated in the antero-posterior direction. 
The wall of the body in this region is composed of a dense mass of muscular fibres: this is the principal part of the foot (propodium and mesopodium combined); the posterior portion (metapodium) is a thick process projecting behind this and bearing the operculum on its surface. The foot is highly contractile, and it is by means of contractions passing over it in a succession of undulations that the animal creeps along, dragging after it the rest of the body enclosed in the shell. In the middle line of its flat surface, nearer the anterior than the posterior end, is a slitlike aperture leading into a cavity lined with unicellular glandsthe pedal gland.

When the remainder of the body has been removed from the shell, it is found to be twisted up into a coil-the visceral spiral, corresponding to the spire of the shell within which it was lodged. This is unsymmetrical, the axis of the spiral being directed not straight backwards, but backwards, upwards, and to the right. The external asymmetry of the body is not strongly marked in the part which is capable of being protruded from the shell, but is still recognisable; and an examination of the internal organs shows a marked excess of development on the left-hand side, i.e. the side which corresponds with the longer outer side of the spiral of the shell. The surface of the part of the animal which is capable of being pushed out from the shell is covered with a thick integument, which is darkly pigmented except on the lower surface of the foot. Over the visceral spiral the mantle forms a thin, delicate, colourless layer. Anteriorly the mantle becomes thickened and pigmented, and at the posterior limit of the protrusible part gives rise to a thickened ridge, the collar (Fig. 611, coll.), forming a semicircle over the dorsal and lateral regions. In the middle the collar is not in close contact with the body, but leaves a large cleft leading into a very wide space extending backwards for a considerable distance. This space, which is formed by an infolding of the mantle, is termed the mantle- or pallial cavity. In it are to be found the ctenidium, the osphradium, and the anal, excretory, and reproductive apertures. The wall of the cavity is much folded and plaited, and contains a quantity of glandular tissue, the plaits being most numerous on the right-hand side in front of the anus.

The ctenidium or gill (Fig. 613, cten.) is closely applied to the wall of the mantle-cavity to the left of the middle. It consists of 
a main stem, with which are connected a row of delicate flexible laminæ set at right angles to it: these are broadest in the middle, becoming smaller towards the ends.

The osphradium (osph.) lies close to the ctenidium on its righthand side, i.c. nearer the middle of the body. It presents a central axis, connected at right angles with which are two rows of close-set delicate lamellæ. Like the ctenidium, it is closely applied to the wall of the mantle-cavity throughout its length. The laminæ of the osphradium are supplied with nerves which ramify over them, and its function seems to be to ascertain the condition of the water that enters the mantle-cavity.

Digestive System.-The mouth, situated at the anterior end of the introvert, leads into a large chamber with muscular walls, the buccal cavity (buc.). At the sides of the entrance to this cavity the investing cuticle is thickened to form two distinct horny plates - the jaws (Figs. 613, 614, and 615, jaw). The jaws are Hexible, and on examination under the microscope are found to be composed of numerous rows of minute bodies, the denticles; the anterior edge is minutely denticulated. From the floor of the cavity rises an elevation, the odontophore (Fig. 613, od., Fig. 614, odont.), which is somewhat elongated in the direction of the long axis of the body and compressed laterally. Over the summit of the odontophore runs longitudinally a narrow strap-like body, the radula or lingual ribbon (Figs. 614, and 615, rad.), beset with numerous minute horny teeth arranged in transverse rows. Posteriorly this toothed ribbon passes into a narrow curved pouch -the radular sac (Fig. 613, rad. s, Fig. 615, rad sac.)-extending backwards from the posterior and lower aspect of the buccal cavity. Anteriorly it does not extend beyond the odontophoreprominence. The latter contains cartilages (Fig. 615, cart.) serving for the support of the whole apparatus, and is capable of being extended, with the radula which it bears, through the opening of the mouth, by the contraction of sets of protractor muscular fibres. Muscles inserted into the odontophore also effect the movements of the radula by means of which it produces a rasping effect on the food, and probably the radula itself is capable of a certain degree of to-and-fro movement on the membrane (sub-radular membrane) which lies beneath it. The entire buccal cavity is capable of being drawn forwards towards the mouth opening, or backwards into the introvert, by the contraction of strands of muscular fibres passing from its wall to the wall of the body.

From the buccal cavity runs backwards a long narrow tube with sacculated walls - the cesophagus (Figs. 613 and 615 , oes.). Posteriorly this opens into a large ovoid sac-the crop (Fig. 613, crop). The outer surface of the crop appears marked with numerous close-set fine lines, transverse or oblique in 


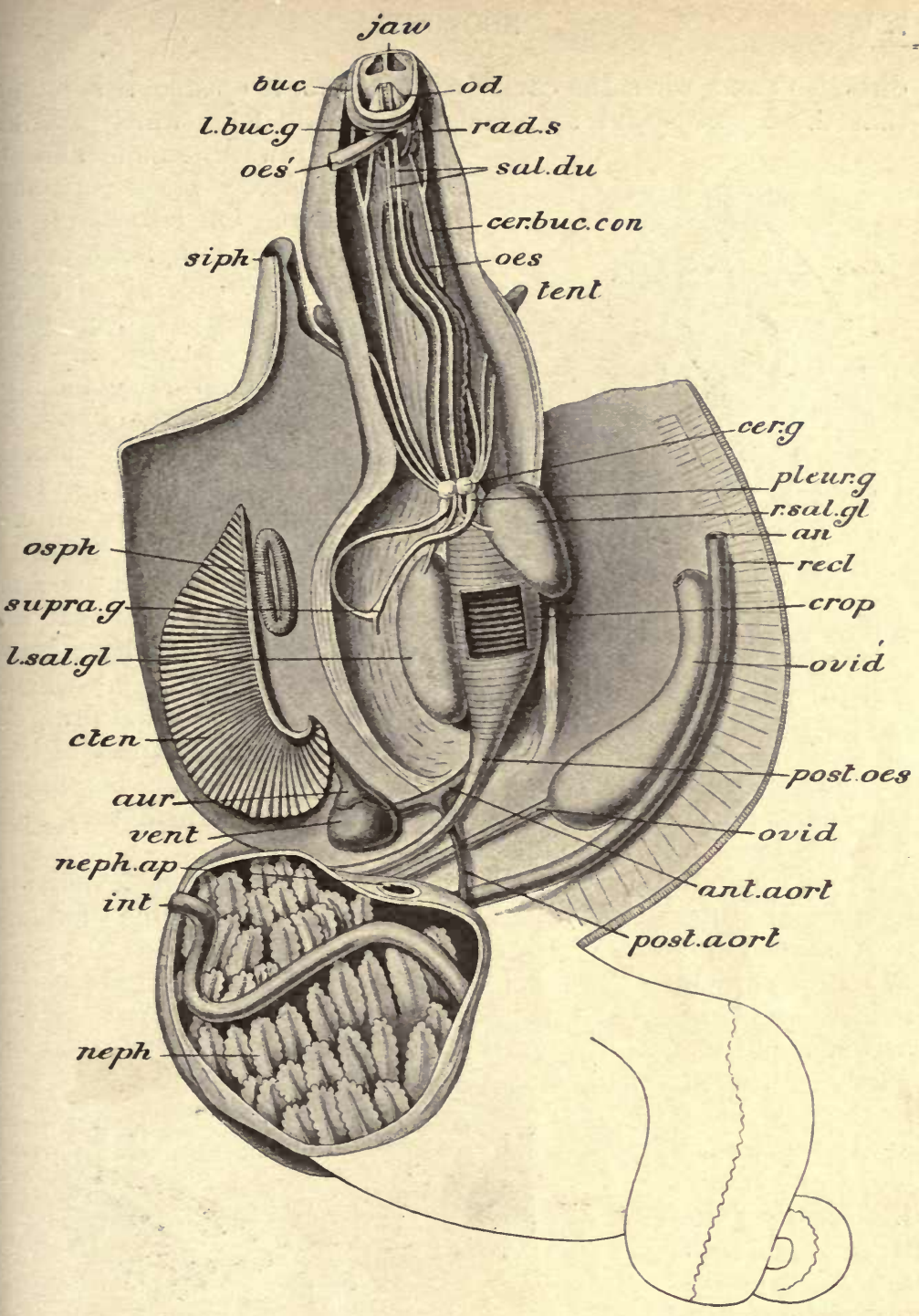

FiG, 613.-Triton nodiferus. Dissection of the internal organs of a female, viewed from the dorsal side. The roof of the mantle-cavity has been divided by a longitudinal incision and the flaps laid out, that on the left bearing the ctenidium and osphradium, and that on the right the rectum and terminal part of the oviduct. The muscular dorsal wall of the body and the introvert bave been divided 80 as to bring into view the anterior part of the alimentary canal and a portion of the nervous system. The buccal cavity has been tilted up and opened so as to show the odontophore, and the cesophagus has been cut through near the anterior end. A portion of the ventral wall of the crop has been removed so as to bring the internal folds into view, and the interior of the nephridium with the contained portion of the intestine bas been exposed. Note. The complete course of the intestine through the nephridium is not shown; the stornach is not seen, being hidden by the nephridium, and the ovary is not represented. an. anus ; ant. aort. anterior aorta ; eur. auricle ; buc. buccal cavity; cer. luc. con. cerebro-buccal connective; cer. g. cerebral ganglia; crop, crop ; cten. ctenidium ; int. intestine; jaz, jaw; $l$. buc. g. left buccal ganglia; $l$. $8 a l$. gl. ieft salivary gland; neph. nephridium; neph. ep. nephridial aperture; od. odontophore; $u$ s. ossophagus; ' $\varepsilon^{\prime}$. anterior end of same, cut and turned aside; osph. osphradium; ovid. oviduct; ovid'. terminal thick-walled portion of oviduct; pleur. $g$. pleural ganglion; post. aort. posteriur aorta ; post. $a$. . posteric $\mathbf{r}$ osophagus; roul. $s$. radula sac; rect. rectum ; $r$. sal. gl. right salivary gland; \&al. du. salivary duct; siph. siphon; supra. g. supra-œesophageal visceral ganglion; tent, tentacle; tent. $x$. teutacular nerve; rent, ventricle. 
direction; and, when the cavity of the organ is opened, it is found that these correspond to numerous delicate folds which extend far inwards, and almost

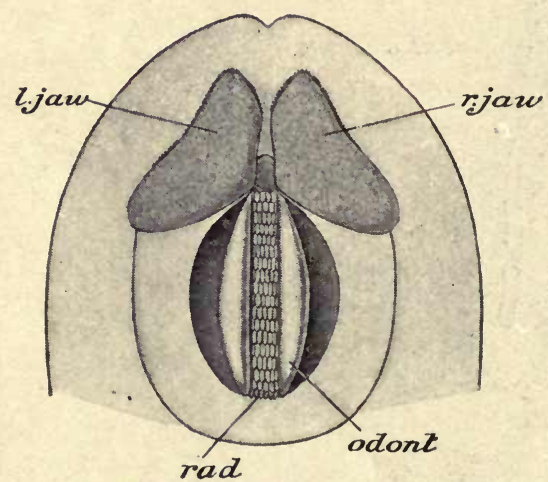

Fig. 614.-Triton nodiferus. Interior of the buccal mass, from above, magnified. $l$. javo, left jaw : oclont. lateral surface of odontophore; rad. radula; $r$ jaw, right jaw. completely block up the lumen. On either side of the crop is a large gland - the salivary gland (Fig. 613, l. sal. gl., Fig. 617, r.sal.gl.) - partly composed of a compact glandular substance, partly of spongy tissue in which the secretion collects. The two salivary glands are unlike in size and shape, that on the left-hand side being much longer than that on the right. Each has a narrow duct (sal. du.) which runs forwards and inwards to the dorsal aspect of the œsophagus, where the two come into close apposition, becoming embedded in the wall of the oesophagus, along which they run forwards to open into the buccal cavity.

From the crop leads backwards and to the left a narrow cylindrical tube-the posterior cesophagus. On this follows a stomach (Fig. 611 , stom.) which is in the form of a U-shaped tube partly embedded in the substance of the digestive gland or "liver," the hepatic ducts from which open into it. The tubular stomach is followed by a somewhat narrower tube-the intestine (Fig. 613 , int.). This

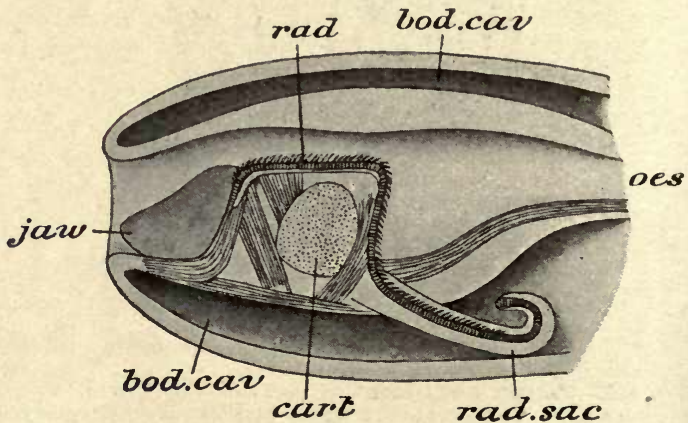

FIG. 615.-Triton nodiferus. Diagrammatic longitudinal vertical section of buccal cavity. bod. cav. body-cavity; curt. cartilage of odontophore; javo, right jaw; as. osophagus; ral. radula; rad. sac. radula sac. enters the cavity of the nephridium, round the interior of which it bends; and, leaving it at its right-hand side, runs forwards in a straight course as the rectum (rect.), embedded in the glandular wall of the 
mantle-cavity, to near the anterior end, where it terminates in a short, freely projecting, spout-like portion, with the anus (an.) at its extremity.

The digestive gland or "liver" forms a mass of reddish-brown glandular follicules which compose the greater part of the bulk of the visceral coil.

Vascular System.-Close to the base of the ctenidium, behind it and a little to the right, is the heart, lodged, like that of the fresh-water Mussel, in a cavity, the pericardium, lined by a transparent membrane-the pericardial membrane. The heart consists of two chambers, an auricle (Fig. 613, aur.) and a ventricle. The auricle, which is the smaller of the two, is situated somewhat in front of the ventricle, close to the ctenidium, from the main central vessel of which it receives the blood. The ventricle (vent.) is of somewhat pyramidal shape, but with the edges rounded off. Its wall is extremely thick and muscular. Passing out from the ventricle towards the right is a thick artery, which soon divides into two, one running forwards, the other backwards-the anterior (ant. aort.) and posterior (post. aort.) aortce. The former is a very large trunk which runs forwards below the posterior oesophagus, crop, and anterior œesophagus, giving off branches to the region of the head as it goes. The posterior aorta, narrower than the anterior, passes into the visceral spiral, where it breaks up into branches for the supply of the various parts. The bloodsystem consists in large measure of sinuses, as in the fresh-water Mussel, and the general course of the circulation is similar to what has already been described in that Mollusc (p. 690).

Excretory System.-There is only one nephridium (neph.), a large organ situated dorsally, behind the pericardium. It is a sac with thick, glandular, and highly vascular walls, the inner surface of which is thrown into numerous complex folds. In front it communicates directly by a large aperture (neph. ap.) with the mantle-cavity, and by a narrower passage with the pericardium.

The nervous system (Figs. 616 and 617) is more highly elaborated than in the fresh-water Mussel. Two pairs of nerveganglia-the cerebral (cer. g.) and the pleural (pl. g.)-lie close together over the posterior part of the œesophagus, just where it passes into the crop. The right and left cerebral ganglia are fused together in the middle line, though separated by a constriction, and the ganglia of the two pairs are placed very close together, though quite distinct. From each cerebral ganglion there passes forwards a stout cerebro-buccal connective (cer. buc. con.) to a buccal ganglion $(r$. buc. $g$.) situated on the posterior surface of the buccal chamber. Also given off anteriorly from the cerebral ganglia are optic nerves (opt. n.) to the eye and tentacular nerves (tent.n.) to the tentacles. From each cerebral ganglion passes 


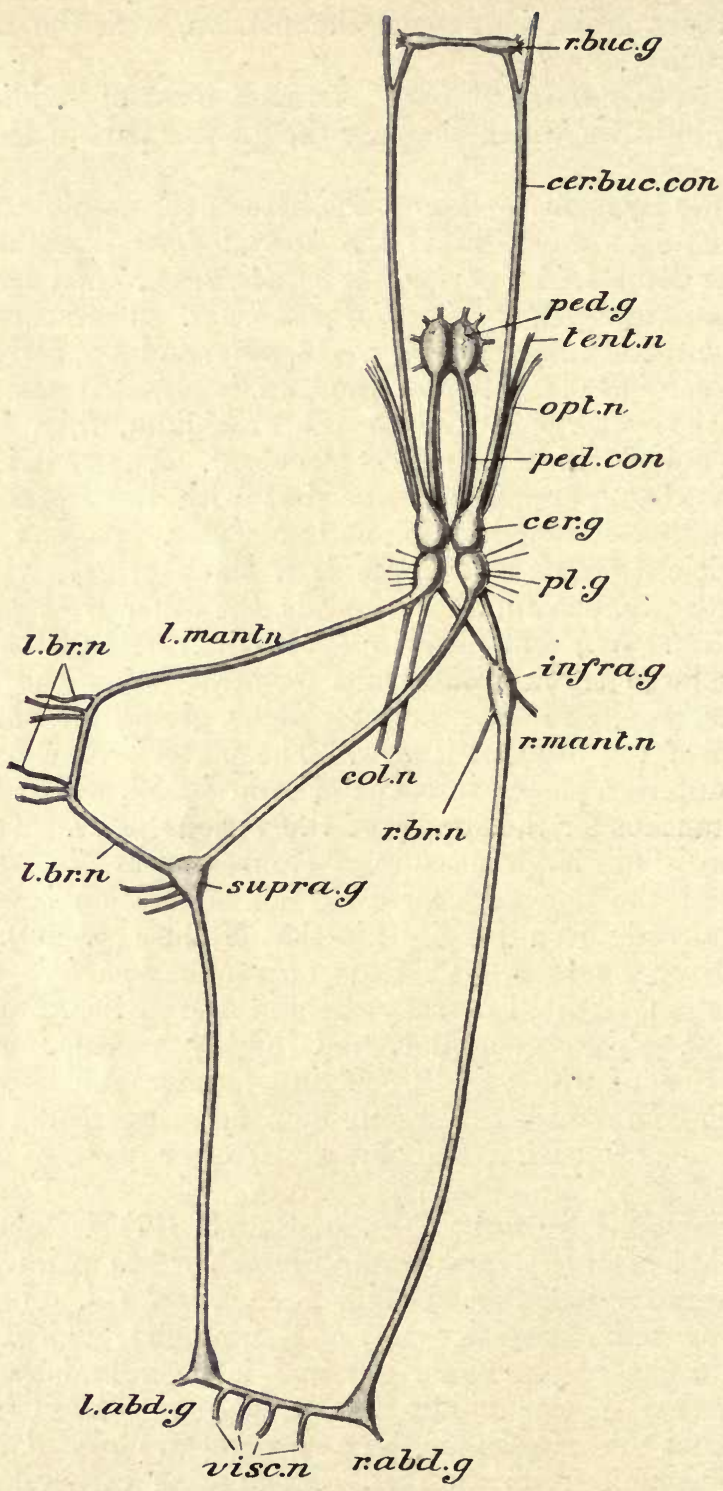

Frg. 616.-Triton nodiferus. Nervous system, from the dorsal side. cer. buc. con. cerebrobuccal connective; cer. g. cerebral ganglion; col. $n$. nerves to the columellar muscle; infra. $g$. infra-cesuplageal visceral ganglion; $l$. abd. $g$. left abdominal ganglion; $l$. $b r . n$. Ieft branchial nerve; $l . b r . n^{\prime}$. nerves to brauchia and osphradium; $l$. mant. $n$. left inantle-nerve; opt. $n$. optic nerve; ped. con. cerebro-pedal and pleuro-pedal connectives; ped. $g$. pedal ganglia; pl. g. pleural ganglion; $r . a b d . g$. right abdominal ganglion; $r . b r . n$. right branchial nerve; $r . b u c$. gang. rigint buccal ganglion; $r$ mant. $n$. right mantle-nerve; supr $x_{\text {. }} g$. supra-intestinal visceral ganglion; tent. n. nerve to tentacle; visc. $n$. visceral nerve-uranches. 
downwards and forwards a stout cerebro-pedal connective, and from each pleural ganglion a pleuro-pedal connective, to a large pair of closely-united pedal ganglia (Figs. 616 and 617, ped.g.) embedded in the upper layers of the muscles of the foot, to which they give off numerous nerves. The right pleural ganglion gives off behind a supra-intestinal visceral connective, which bends across to the left, over the osopbagus, and, some distance to the left of the alimentary canal, expands into a triangular supra-intestinal viscercal ganglion (supra.g.), situated below the superficial layer of muscular tibres. The left pleural ganglion gives off an infra-intestinal visceral connective, which passes obliquely backwards and to the right, below the alimentary canal, to a ganglion situated a little to the right of the middle line-the infira-intestinal visceral ganglion (infra. g.). The supra-intestinal ganglion gives off a nerve which

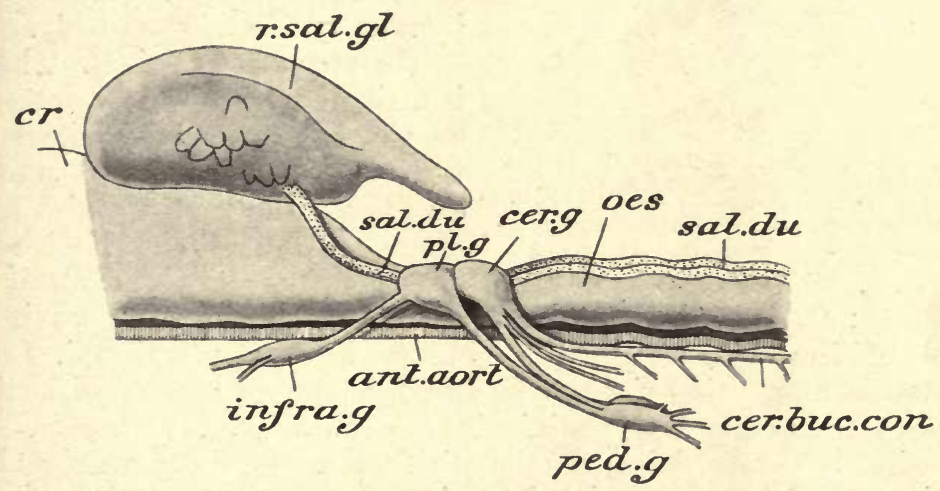

FiG. 617.- Triton nodiferus. Lateral view of nerve-ganglia and related parts. Letters as in Fig. 616 ; in addition-ant. aort. anterior aorta ; $\mathrm{cr}$. crop ; es. oesophagus ; sal. lu. salivary duct ; $r$. sal. $g l$. right salivary gland.

runs towards the osphradium and ctenidium, which it supplies with branch nerves, and unites with a stout mantle-nerve (l. mant. n.), which is given off from the left pleural ganglion. The right pleural also gives off a stout connecting nerve to the infra-intestinal ganglion. From the supra- and infra-intestinal ganglia the left and right visceral connectives are continued backwards and unite behind in the neighbourhood of the stomach; each ends in a triangular abdominul ganglion (l.abd.g.; r.abd.g.), and these are joined by a transverse commissure, from which a number of visceral nerves (visc. $n$.) are given off. A remarkable torsion of the nerve connectives is here to be observed, the two visceral connectives becoming twisted into the form of the figure 8 .

The organs of special sense of 'Triton, in addition to the tentacles and the osphradium, which have been already referred to, are the eyes and the statocysts. The eye (Fig. 618) is a rounded invagination of the epidermis with an inner wall or retina (ret.) 
composed of pigmented and sensory cells. The latter (retinophores) are elongated cells narrowed at their central free ends, and produced at the opposite extremity to become continuous with nervefibres of the optic nerve. The former (retinulce) have their free

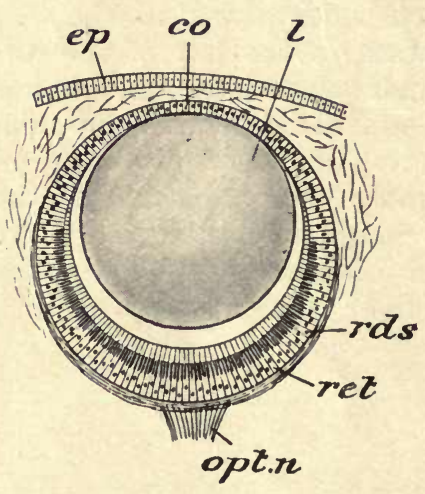

FIG. 618.-Triton. Section of the eye. co. cornea; ep. epidermis ; $l$. lens ; opt. optic nerve; rds. layer of rods (the line is not continued far enough inwards); ret. retina.

extremities much enlarged, and surround the slender ends of the retinophores. A layer of short rods $(r d s$.$) lies within the retina$ proper. The outer wall is thin, and, with the overlying epidermis, formis a transparent cornea. In the interior of the eye is a clear rounded lens $(l$.) of dense cuticular matter secreted by the cells of the retina; this is surrounded by a less dense vitreous body.

The sexes are distinct. There is a single gonad-ovary or testis as the case may be-lodged in the visceral spiral. The spermduct is a white tube, thickish and much convoluted where it leaves the testis, narrower and straight

distally; it opens in front in the mantle-cavity into the proximal end of the sperm-groove, which, as already mentioned, runs forwards along the right side and becomes continous with the groove traversing the penis. The oviduct (Fig. 613, ovid.) is proximally a very delicate tube with colourless, transparent walls. This runs forwards to the right side of the mantle-cavity, where it assumes the character of a stout tube (ovid.') with thickened glandular walls, which passes forwards close to and parallel with the rectum, and opens on the exterior near the anus.

\section{Distinctive Characters and Classilication.}

The Gastropoda are unsymmetrical Mollusca, with a mantle which is not divided into two lateral portions and usually a shell, which does not consist of two lateral valves, but of a single, unsymmetrical, usually spirally coiled valve, enclosing a visceral mass of corresponding form. There are, typically, two plume-like ctenidia enclosed in a mantle-cavity, but there may be only one; and in air-breathing forms ctenidia are not developed, respiration taking place through the wall of the mantle-cavity itself. A disdinct head bearing eyes and tentacles is present in the majority. The foot is situated behind the head, and usually has an extensive flattened ventral surface. The buccal cavity contains an odontophore. The nephridium is usually single. The nervous system 
contains distinct cerebral and pleural, besides pedal, visceral, abdominal, and buccal ganglia. The sexes are sometimes separate, sometimes united. The larva passes through trochophore and veliger stages.

\section{Sub.Class I.-Streptoneura.}

Gastropoda in which the visceral connectives are in most cases twisted into a figure of 8 , and in which the sexes are distinct.

\section{ORDER 1.-ASPIDOBRANCHIA.}

Streptoneura with the nervous system but little concentrated : the pedal ganglia are produced into long cords with the anterior ends of which the pleural ganglia are fused; the cerebral ganglia wide apart; the osphradium little developed. There is nearly always a single ctenidium or a pair, plume-like and free distally. The auricles and the nephridia are usually paired.

\section{Sub-Order 1.-Docoglossa.}

Aspidobranchia in which the pleural ganglia are not connected with the opposite visceral connective. The eye is in the form of an open pit, without lens. There are two osphradia, a single jaw, and no operculum. The visceral mass is conical.

This section includes the Limpets (Patellida).

\section{Sub-Order 2.-Rhipidoglossa.}

Aspidobranchia in which each pleural ganglion is connected with the opposite visceral connective. The eye is a closed sac and contains a lens. There are nearly always a single osphradium, a pair of jaws, and two auricles.

This sub-order includes the Ear-shells (Haliotida), Trochus, Turbo, and others.

\section{Order 2.-PECTINIBRANCHiA.}

Streptoneura with a somewhat concentrated nervous system. There is a single osphradium which is often pectinate. The primarily right ctenidium is alone developed. The heart has a single auricle. The ctenidium consists of a stem with a single row of lamellæ, attached throughout its length to the wall of the mantle-cavity.

\section{Sub-Orcler 1.-Platypcda.}

Pectinibranchia with the foot flattened ventrally, at least in front. Jaws are nearly always present.

This sub-order includes the Cowries, the Vermetes, the Tritons, the Whelks, the Cones, and a number of other groups. 


\section{Sub-Order 2.-Heteropoda.}

Pelagic Pectinibranchia with the foot laterally compressed and bearing, at least in the male, a ventral sucker. The visceral sac and mantle form only a small part of the mass of the body. Jaws are absent.

\section{Sub-Class II.-Euthyneura.}

Gastropoda in which the visceral connectives are not twisted into a figure of 8 , and in which the sexes are united.

\section{ORDER 1.-OPISTHOBRANCHIA.}

Marine Euthyneura with aquatic respiration, the auricle of the heart usually posterior to the ventricle. The mantle-cavity, when present, opens by a wide aperture.

\section{Sub-Order 1.-Tectibranchia.}

Opisthobranchs provided in nearly all cases with a mantle and a shell, nearly always with a true ctenidium, and an osphradium.

This section comprises the Aplysiida, or Sea-hares, and several other families, including certain pelagic Gastropoda, some shellbearing, some shell-less, formerly regarded as constituting a distinct class-the Pteropoda.

\section{Sub-Order 2.-Nudibranchia.}

Opisthobranchs which are devoid of a shell in the adult condition, and have no true ctenidia or osphradia, respiration being carried on by means of secondary branchiæ usually arranged in a circlet around the anus, or in rows on the dorsal surface, or laterally under the edge of the mantle.

This sub-order includes Doris, Eolis, Tethys, and other shell-less forms.

\section{ORDER 2.-PUlMONATA.}

Euthyneura devoid of ctenidia, respiration being carried on through the walls of the mantle-cavity, which has a narrow contractile aperture.

This sub-order includes the Land-Snails and Slugs.

\section{Systematic Position of the Example.}

Triton nodiferus is one of several species of the genus Triton, which is the only member of the family Tritonida, belonging to the sub-order Platypoda. The family Tritonidæ differs from the 
other families of the sub-order in the possession of a proboscis, of a well-developed, but not greatly elongated, siphon, and of a short foot.

\section{General Organisation.}

External Features, Symmetry, \&c.-Few Gastropods make an approach towards even superficial symmetry, and in cases in which there is a near approximation towards such a state of things, it seems clear from the results of the study of development and of a comparison with allied forms that the symmetry presented is not primitive, but has been secondarily acquiredsuch symmetrical forms having been derived from unsymmetrical ancestors.

The departure from symmetry is most marked in the majority of the Streptoneura. It may be said to be due to the development of a protective shell composed of one piece and extensive enough to be capable of enclosing all the soft parts; and to the extension of the foot on the ventral side as an elongated muscular creeping organ. The development of the shell rendered necessary an arrangement of the parts whereby the mantle-cavity with the anus, the ctenidia and the excretory apertures, should come to be situated in the neighbourhood of the opening of the shell, i.e., towards the head-end of the animal. The mantlecavity and associated parts (pallial complex, as the whole is termed) had, therefore, to be shifted forward from its primitive posterior position, and this was probably effected by arrest or retardation of growth on one side and active extension on the other. In the majority of cases it is the right side the growth of which becomes retarded, and, in consequence, it is on the right side that the pallial complex comes to travel forwards. The effect is as if, the head retaining its symmetry, the parts between it and the anus had undergone a process of rotation or torsion through about $180^{\circ}$ around a vertical axis passing in a dorso-ventral directionthe direction of torsion being opposite that of the movement of the hands of a watch (Fig. 619).

With regard to the spiral form assumed by the shell in all highly developed Gastropods, it can only be pointed out here that, given the necessity for complete protection, compactness, and a provision for continuous growth, the spirally-coiled cone is the form of shell best adapted to all the conditions. A straight cone, however directed, would be a great impediment to active progression, and the coiling in a compact spiral would seem to be the line of development best adapted to secure concentration and strength.

The rotation around a dorso-ventral axis is not the only form of torsion leading to the markedly unsymmetrical disposition of parts observable in most Gastropoda. There is also a process of torsion 
around a horizontal axis which, the head and foot being regarded as fixed, results in the visceral mass enclosed in the spiral shell coming to occupy a more or less dorsal position with the apex directed backwards and to the right.

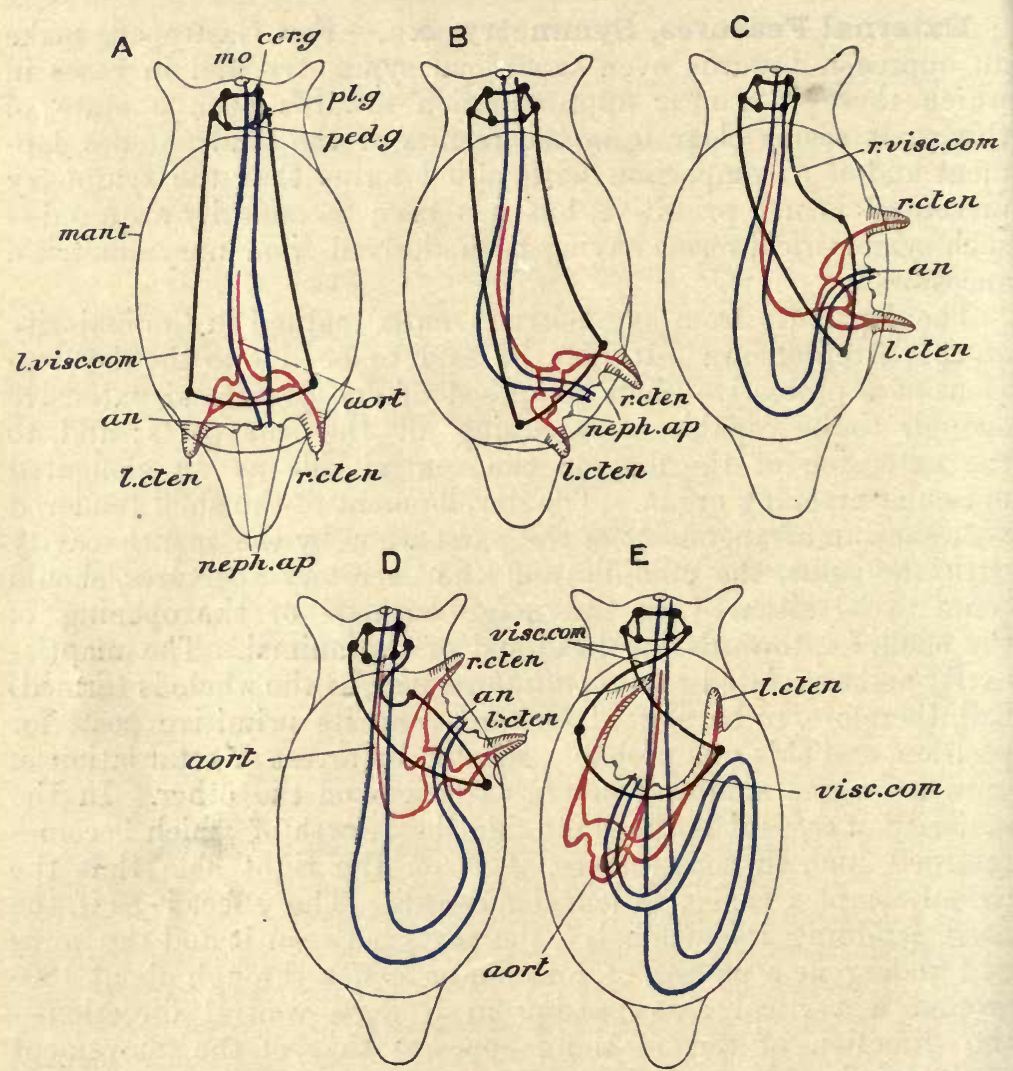

FiG. 619.-Diagrammatic representation of the displacement of the mantle-cavity and associated parts in the Gastropoda. Enteric canal blue, blood-system red. A, represents a ncarly symmetrical arrangement ; in B, C, D, are represented successive stages of displacement of the mantle-carity to the right and forwards; in $\mathrm{E}$, the anus and (primarily) right ctenidium have passed the middle line. an. auus : aort. aorta; cer. g. cerebral ganglion; $l$. cten. left ctenidium ; $l$. visc. corn. left visceral connective; mant. mantle; mo. mouth; neph. a $\rho$. nephri. dial apertures; $p e d . g$. pedal ganglion; $p l . g$. pleural ganglion; $r$. cten. right ctenidium ; $r$. visc. com. right visceral connective; visc. com. visceral connectives. (After Korschelt and Heider.)

The shifting of the pallial complex in many Streptoneura proceeds so far that the complex completely or partially passes across the middle line, and the anus comes to be situated to the left of the mouth. The displacement of the pallial complex involves two important series of changes in the internal organs, in addition to the suppression of certain structures to be referred to 
presently. In the first placed there is necessarily involved a throwing of the enteric canal into a loop (Fig. 619), and in the second place the long pleuro-visceral connectives (visc. com.) become twisted in such a way as to assume the form of the figure 8 -the right connective becoming supra-intestinal and the left infra-intestinal.

Universally accompanying the process of forward displacement of the pallial complex, except in Haliotis and Fissurella and allied Rhipidoglossa, occurs the reduction of its paired parts. Thus in all the more highly developed Streptoneura the primitively right (topographically left) ctenidium alone persists, and one of the two nephridia is alone fully developed and functional.

In the Euthyneura there are distinguishable various stages in a process of detorsion by which the torsion tends to be reversed and the pallial complex carried back towards the posterior end along the right side. The pleuro-visceral connectives lose their twisted arrangement in nearly. all such cases; but there is the same reduction of the paired parts of the pallial complex.

The shell in the adult limpets (Patella and allied genera) is in the form of a short cone. In most of the Gastropoda it is in the shape of a spiral with the turns usually in close contact with one another, the inner walls of the turns coalescing to form an axial, hollow or solid column-the columella. The portion of the shell projecting inwards between the turns of the spiral sometimes becomes absorbed. In certain cases, on the other hand, the cavity of the apical portion of the spiral may be cut off from the cavity of the rest of the shell by the formation of a transverse partition, the animal then becoming restricted to the basal portion; or several such partitions may be formed. By far the greater number of such spiral shells are dextral, i.e. if we begin at the apex of the spiral, to reach the opening of the shell we have to pass from left to right, with the columella always on our righthand side: in a few cases, however, the spiral is sinistral, taking the opposite direction from that of the ordinary dextral shell. The form of the shell varies with the degree of obliquity with which the whorls are set on the axis. When the obliquity is very slight (Fig. 620) the spiral is nearly flat; when the obliquity is great, an elongated tapering shell such as that represented in Fig. 621 is the result. Sometimes the later whorls completely cover over the earlier ones, so that the spiral form of the shell 
is concealed. Sometimes only the apical portion of the shell is spiral, the remainder being a straight or sinuous cylinder. The spiral form of the shell and the parts enclosed in it, as well as the direction of the spiral - whether dextral or sinistral - are, it may be here pointed out, very fundamental features of the organisation of the Gastropod, and are foreshadowed at an early stage in the segmentation of the ovum. The mouth of the shell has usually a prominent margin or peristome, which is

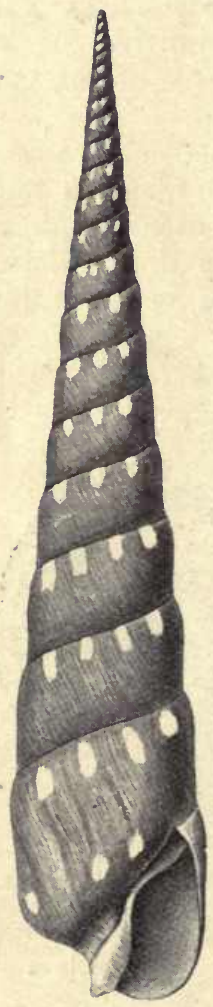

FIG. 621.-Shell of Terebra oculata. sometimes entire and continuous, sometimes broken by a deep notch or a spout-like process or canal, formed in connection with the development of a spout-like prolongation of the mantle, the siphon, which lies in it. The mouth of the shell in many Gastropoda is capable of being closed by means. of an operculum borne on the foot. In some terrestrial forms in which an operculum is absent, the opening may be closed up during winter by a layer of hardened mucous matter to which the name of epiphragm is applied. The margin of the mantle in some cases bears a series of tentacles. Lateral folds of the mantle are in some of the Gastropoda (Fig. 622) reflected over the shell and may completely cover it. In some cases these folds unite by their edge, so that the shell comes to be enclosed in a complete sac of the mantle; such enclosed shells are always imperfectly developed and incapable of covering the body. Thus in Aplysia and some other Opisthobranchs the shell is greatly reduced, thin and horn-like, and concealed within the mantle, while in the nudibranch members of the same sub-order it is entirely absent (Fig. 623). The shell is also completely absent in some of the pelagic forms (Ileteropoda and Pteropoda); in others, though present and external, it is too small to enclose the animal (Fig. 624). In the slugs, among the Pulmonata, the shell is vestigial and in most cases is concealed by the mantle (Fig. 625).

The foot varies in the extent of its development in the different families of the class. It usually presents an elongated flat ventral surface on which the animal creeps by wave-like contractions of the muscular tissue. An exceptional case is that of Cæcum, in which the creeping movement is entirely due to the action of cilia covering the ventral surface. In the typical Gastropods the foot is usually distinguishable into three parts, a middle part 
or mesopodium, which is the most important, with a smaller anterior propodium and posterior metapodium. In many burrowing forms (Fig. 626) the propodium is well developed and sharply marked off to act as a burrowing organ. In a few cases a pair of tentaclesthe pedal tentacles-

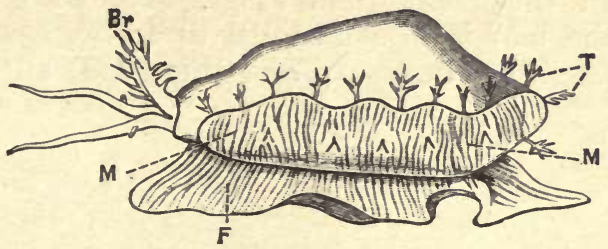

Fig. 622.-Cypræa moneta (Cowrie). Showing the mantle, provided with marginal tentacles, partly enveloping the shell. $B r$. siphon; $M$. M. mantle; $F$. foot $;$. tentacles at the edge of the mantle. (From Cooke, after Quoy and Gaimard.)

are situated at the anterior end of the foot; still rarer is a pair of similar appendages at the posterior end. The whole foot

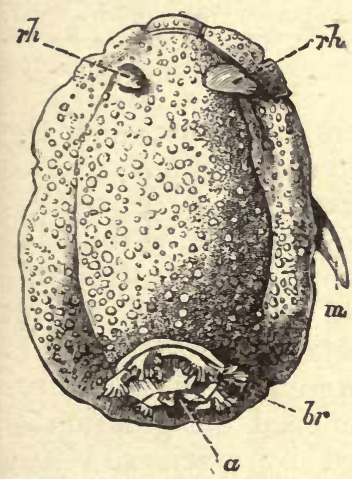

Fig. 623.-Doris (Archidoris) tuberculata. $a$. anus; $b r$. branchia; $m$, penis ; $r h$, $r h$, tentacles. (From the Cambridge Natural History.) becomes reduced in the few Gastropods that remain fixed. The metapodium very usually in the Streptoneura bears

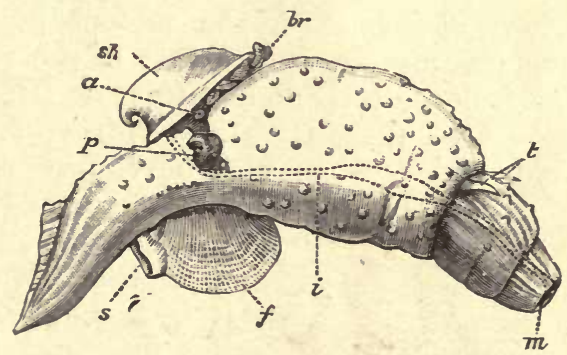

Fig. 624.-Carinaria mediterranea. a. anus; $b r$. branchia; $f$. foot; $i$. intestine; m. mouth; $p$. penis ; 8 . sucker; $s h$. shell; $t$. tentacles. (From the Cambrielge Natural History.)

a disc or stopper-the opcrculum already referred to-usually horn-like, rarely completely calcified, more commonly horn-like with

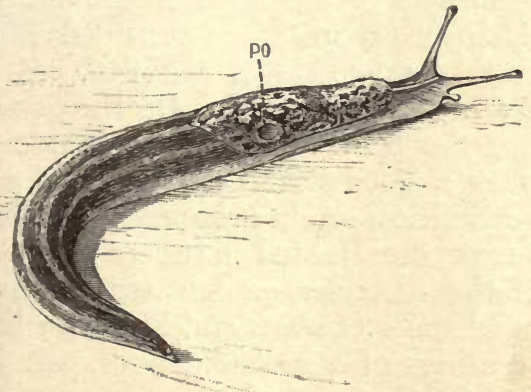

F1G. 625.-A Slug (Limax). PO, pulmonary aperture. (From the Cambridge Natural History.) a thin calcareous investment-by means of which the aperture of the shell is closed when the animal is retracted.

In some forms, such as the Sea-hares (Aplysia, Fig. 627 ), the foot develops a pair of lateral lobes-the parapodia-which act as fins; and in the Pteropods (Fig. 628) which are specially modified for a 
pelagic existence, these constitute the largest part of the foot. In the Heteropoda (Figs. 629, 630) which are also pelagic, the foot is also modified to act as a swimming organ. In one family of this sub-order (Fig. 629) all three parts of the foot

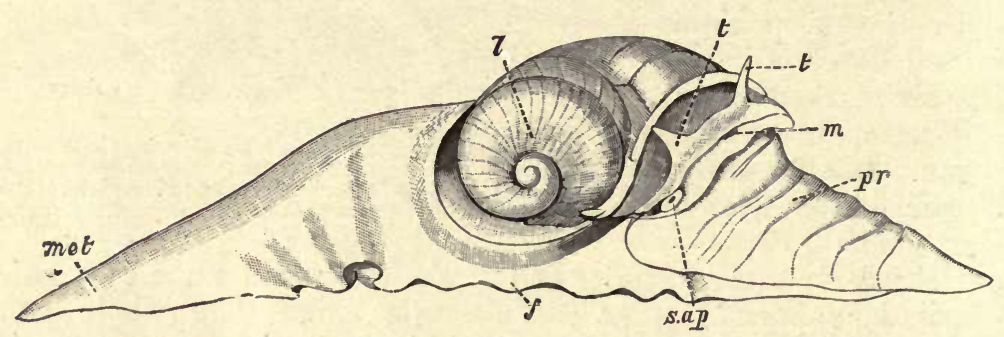

FIf. 626.-Sigaretus Iævigatus, exemplifying great development of propodium $(p r$.) and metapodium (met.), in a burrowing Gastropod. The shell has been removed. $f$. mesopodium; $l$. "liver"; s. ap. aperture of siplion; $t . t$. tentacles. (From the Cambridge Natural History, after Quoy and Gaimard.)

are well-developed, the mesopodium bears a sucker, and the metapodium an operculum; in the rest the mesopodium is alone well developed and forms a laterally-compressed, verticallyelongated fin. The term epipodium is applied to a ridge or fold, which, when best developed, runs around the entire edge

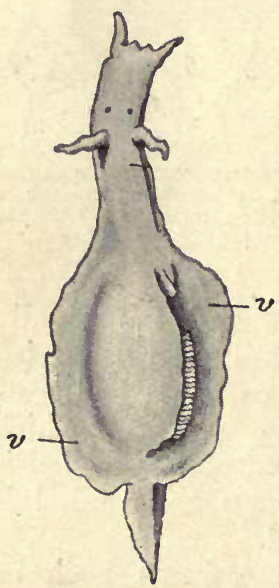

Fia. 627.-Aplysia, dorsal view. $v$, parapodia. (After Keferstein.) of the creeping sole of the foot, and is beset with papillæ or tentacle-like processes.

A pedal gland is present in the majority: it is a simple or branched invagination of the integument, lined by mucus-secreting cells. Very commonly, as in Triton, it opens on the exterior in the middle line of the ventral surface of the foot.

The Gastropoda have a well-marked head, separated from the body by a constriction or neck. The mouth, situated at the anterior end of the head on its ventral aspect, is in many instances provided with a protrusible proboscis or introvert, sometimes of considerable length. On the dorsal surface of the head are a pair of tentacles which vary a good deal in shape, but are usually cylindrical or club-shaped. In most cases the eyes are situated on tubercles at the bases of the tentacles, or elevated towards the middle; but in the snails and slugs (Pulmonata, Fig. 631) the eyes are elevated on the extremities of a second, longer, pair of tentacles (oc. tent) placed behind the first.

The mantle is usually developed into a fold-the mantle flap- 
originally posterior, but subsequently becoming shifted round, in the course of the displacement already referred to, to the righthand side. This covers over a cavity-the mantle-cavitysituated anteriorly, in which are situated the anal and nephridial
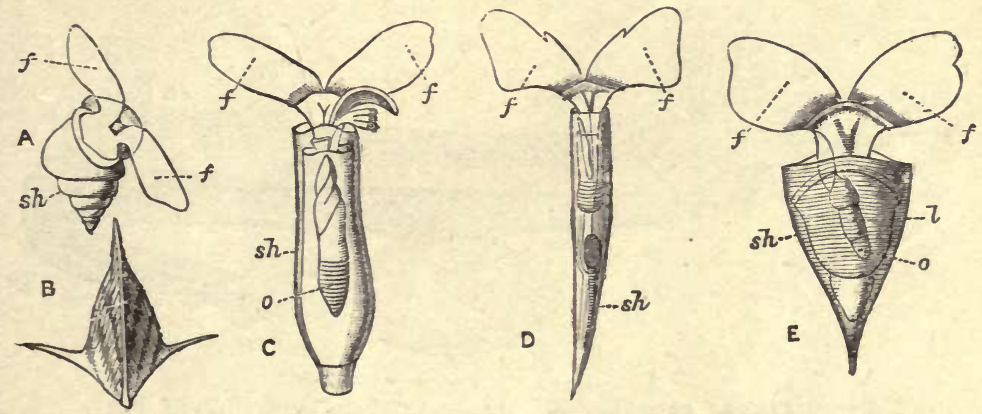

FIG. 628.-Shell-bearing Pteropoda. f. $f$. fins; $l$. liver; 0. ovary; sh. shell. (From Cooke after Souleyet.)

apertures and the ctenidia. The edges of the mantle-flap may become united together in such a way as to form a chamber opening on the exterior by a comparatively narrow opening. In many of the Prosobranchia the edges of this aperture are drawn out into a spout-like prolongation open ventrally-the

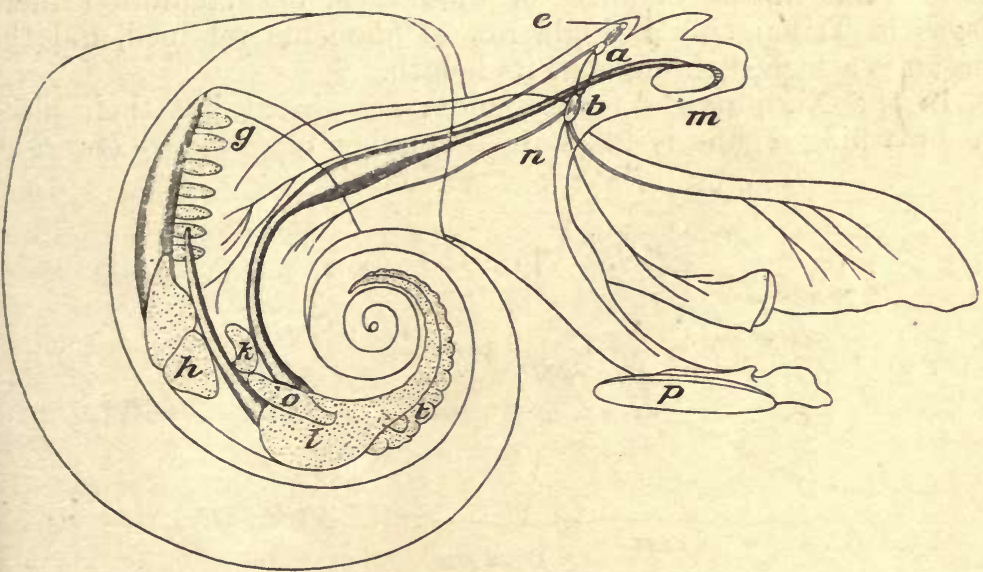

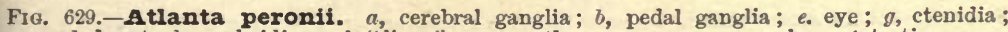
$h$. heart; $l$, nephridium; $l$. "liver"; $m$. mouth; $o$. ovary; $p$, operculum; $t$. testis

siphon-which lies in the corresponding prolongation of the peristome of the shell and serves as a channel for the ingress and egress of water. In some Gastropods, however, there is no definite mantle-cavity, the anus, nephridial apertures, and ctenidia merely lying under cover of a comparatively slightly-developed 
lateral mantle-flap. Usually there is on the inner surface of the mantle a glandular area-the pallial mucus-gland.

Respiratory Organs. - There are typically two ctenidia, one on the right side and the other on the left, contained in the mantle-

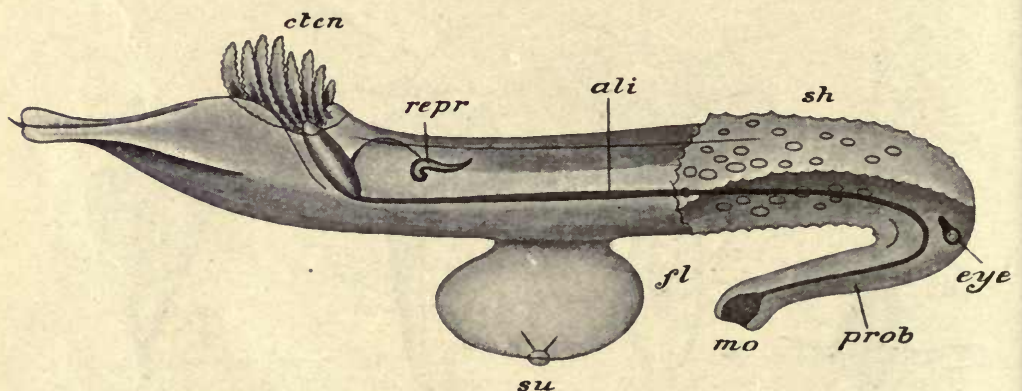

FIG. 630.-Pterotrachea scutata. ali. alimentary canal; cten. gills; eye, eye; fl. float; mo. mouth; prob. proboscis ; repr. gonad; $s h$. shield covering a portion of the dorsal surface; siı. sucker.

cavity ; but in the great majority of the Streptoneura and branchiate Euthyneura the primitively right (actually left) ctenidium alone is retained. In those Gastropoda that possess two ctenidia, and in many forms with only one, the axis of the ctenidium bears two rows of compressed filaments, and is attached only towards its base. But in the majority of those with one ctenidium there is, as in Triton, only a single row of filaments retained, and the organ is attached throughout its length.

In the Nudibranchs true ctenidia are absent, but their place as breathing organs is taken by a number of secondary branchice,

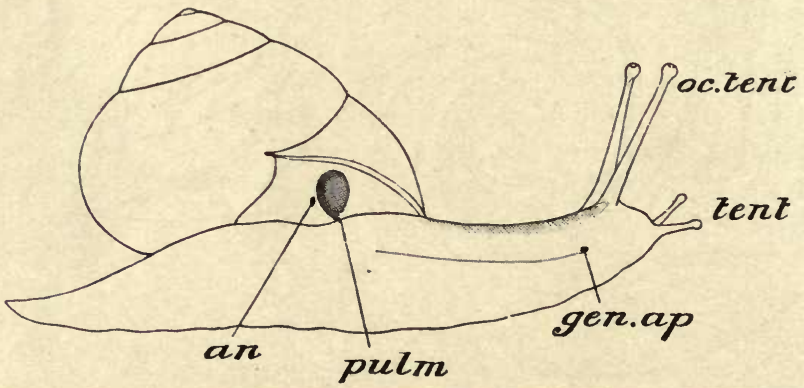

FiG. 631. - Felix nemoralis. an. anus ; gen. ap. genital aperture ; oc. tent. posterior eye-bearing tentacles; pulm. opening of pulmonary sac; tent. anterior tentaclcs. (After Pelseneer.)

sometimes simple, sometimes branched or pinnate processes, which are distributed over the dorsal surface, as in Eolis; or, as in Doris (Fig. 623), form a circlet surrounding the anus; or, as in Pleurophyllidia (Fig. 632), a row on each side beneath the mantle-flap. 
In the limpets (Patella and its allies, Fig. 633) the true ctenidia are represented only by a pair of vestiges, and respiration

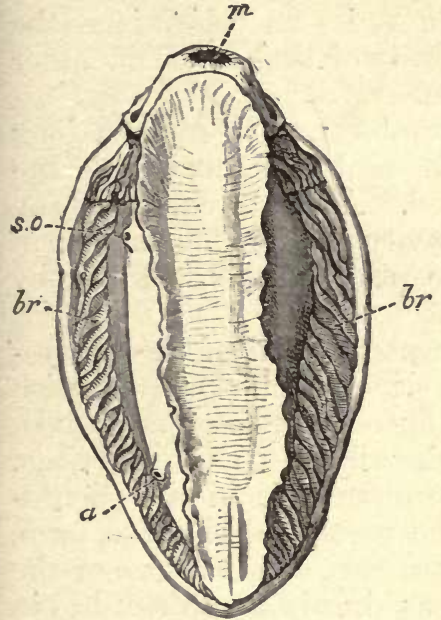

FIG. 632.-Pleurophyllidialineata, from the ventral surface. $a$. anus: $m$. secondary branchiæ: $m$. mouth; 8. o. sexual opening. (From the Cumbridge Natural History.)

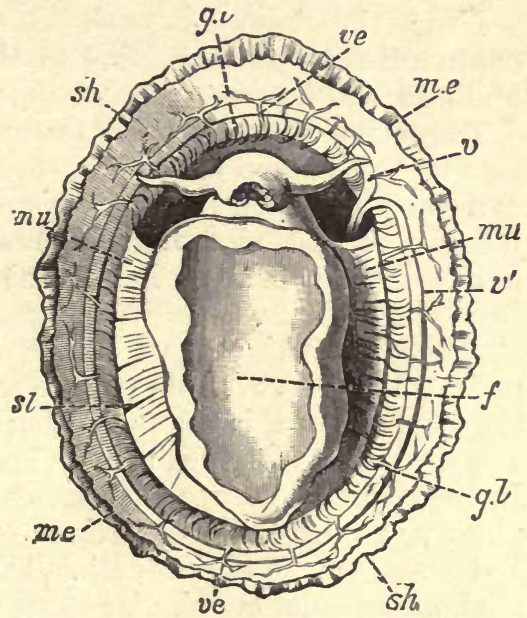

Fig. 633.- Patella vulgata, iseen'from the ven tral side. $f$. foot; $g$. l. circlet of gill-lamella; m. e. edge of the mantle; mu. attachment-muscle; sl. slits in the attachment-muscle; $s h$. shell $v$. efferent branchial vessel $; v^{\prime}$. aorta ; $v$. smaller vessels. (From the Cambridge Natural History.)

is carried on by a number of secondary branchiæ $(g . l$. $)$ in the form of lamellæ situated between the short lateral fold of the mantle and the foot. In the Pulmonata, and in some members of other groups, ctenidia are absent, and the mantlecavity, completely enclosed except for a small rounded opening, has the function of a pulmonary sac or lung (Fig. 634), its roof being richly supplied with blood-vessels: in the aquatic forms its function is apparently as much hydrostatic as respiratory. In one family of Pulmonata,

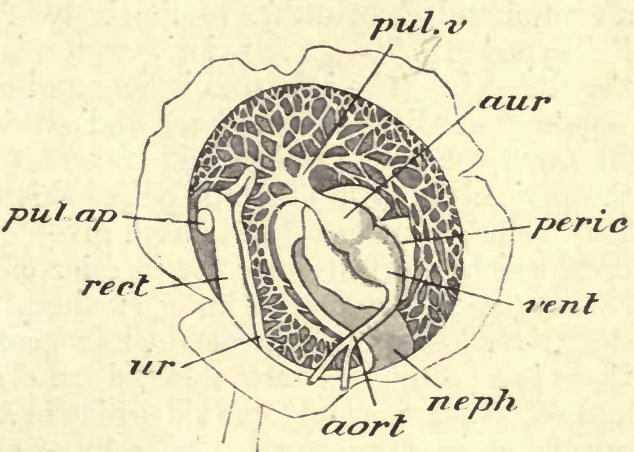

FIG. 634.-Pulmonary cavity and related parts in a slug (Limax). aort. aorta; aur, auricle; neph. nephridium; peric. pericardium, laid open; $p u l$. ap. pulmonary aperture; $p u l . v$. pulmonary vein with its ramifications; rect. rectum; $u r$. ureter; vent. ventricle. (After Pelsenecr.)

the pulmonary chamber gives off a number of branching air-tubes or trachece. In some of the Pulmonata there is a return to 
a completely aquatic mode of respiration accompanied by the development of secondary gills-vascular processes of the wall of the mantle-cavity.

Near the base of each ctenidium is an elevation-the osphradium-corresponding to the body of that name in other Mollusca and having a similar function.

Digestive Organs. - In many Streptoneura there is a long introvert, capable of being everted and retracted, at the extremity of which the mouth is placed. A single curved horny jaw lies on the roof of the buccal cavity in the Pulmonata; in most Streptoneura (as in Triton) the place of this is taken by two lateral pieces.

A characteristic feature of the alimentary canal of the Gastropoda, which, however, they share with some Amphineura and with the Cephalopoda, is the possession of an odontophore and radula, a typical example of which has been described in that of Triton. In the different groups differences are observable in the odontophore as regards the proportions of the parts, and the size, form, and arrangement of the teeth. The structure and relations of the alimentary canal are similar to what has already been described in Triton, and salivary glands and "liver" (hepato-pancreas) are always present. The former may be tubular, but are usually botryoidal : the latter varies in relative extent and in the arrangement of its lobes in different forms.

In some Opisthobranchia the stomach contains a series of teeth which are sometimes sharp and chitinous, sometimes plate-like and calcified. Frequently a special development of the cuticular lining of the stomach forms a hard rod-the crystalline style, lodged in a cæcum and comparable to the body of the same name in the Pelecypoda (p. 705). A pyloric cæcum is frequently appended to the stomach. The intestine is long and thrown into folds in the vegetable-feeding forms, short and straight in the carnivorous. In some cases, e.g. Haliotis, it traverses the ventricle, in others the pericardium ; in others it passes through the nephridium. In Eolis (Nudibranchia) the stomach gives off a number of glandular cæca which penetrate into the interior of the secondary branchix or cerata on the dorsal surface; these cæca take the place of the "liver" of other Gastropoda. In some of the Pectinibranchia there is a peculiar adrectal gland, situated at the side of the rectum and secreting a colourless fluid, which in Murex and Purpura turns purple on exposure to the air, and was anciently used as a dyethe "Tyrian purple."

The heart is, as in other Molluscs, enclosed in a special cavitythe pericardium - a specialised part of the colome, communicating with the cavity of the nephridia. It consists usually, as in Triton, of two chambers-auricle and ventricle; but in some, e.g., Haliotis, there are two auricles and a ventricle. In the 
Opisthobranchia, as already mentioned, it lies in front of the ctenidia; in the Streptoneura at the side or behind. Given off from the apex of the ventricle is. a large vessel which soon bifurcates to form anterior and posterior aortæ. These are the main trunks of the arterial system, which is more highly developed than in the Pelecypoda; the finest branches terminate in sinuses, as in the latter class.

The nervous system varies considerably in the different groups in regard to the arrangement of the ganglia and their commissures and connectives.

In the majority the arrangement is nearly that which has been described as occurring in Triton. There is a pair of cerebrai ganglia usually closely united, but in Patella (Fig. 635) widely separated, situated over the gullet, and giving off behind a pair of visceral nerve-cords, in the course of which there is placed laterally a pair of pleural ganglia, which are united together behind in a median abdominal ganglion (or a paired ganglion, as in Triton). In the course of these visceral cords there is a pair of visceral ganglia. A pair of pedal ganglia united together by a transverse commissure and joined to the cerebral ganglia by connectives, give off behind one or two pairs of pedal nerves, as already mentioned.

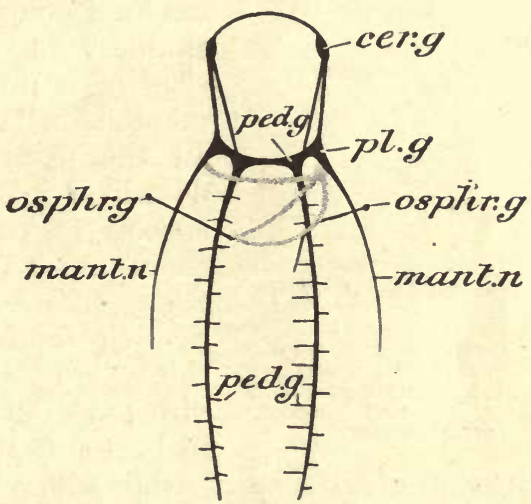

Fig. 635.-Nervous system of Patella. cer. g. cerebral ganglia; mant. n., mantle-nerves ; osph. g. osphradial ganglia; ped. $g$. pedal ganglia and pedal nerve-cords; $p l . g$. pleural ganglion. (After spengel.) A pair of buccal ganglia are connected by slender nerves with the cerebral. At the base of each osphradium is usually a small osphradial ganglion connected by a slender nerve with the visceral. In most Streptoneura (Fig. 619), in accordance with the displacement of the anus, the visceral cords are twisted, as already described in the case of Triton, into a figure of 8.

In Patella (Fig. 635) the pedal ganglia (ped. g.) give origin to a pair of elongated pedal nerve-cords. In Haliotis and Fissurella there is a similar pair of pedal cords which are connected together by transverse commissures, and, in the latter genus, join one another posteriorly.

In the Euthyneura (Fig. 636) except in Actoon and Chilina, the visceral cords are not caught $u$ in the twist of the visceral mass, and do not cross one another. 
In the Snails and other Pulmonata (Fig. 637) the ganglia of the nervous system are more closely aggregated together. A pair

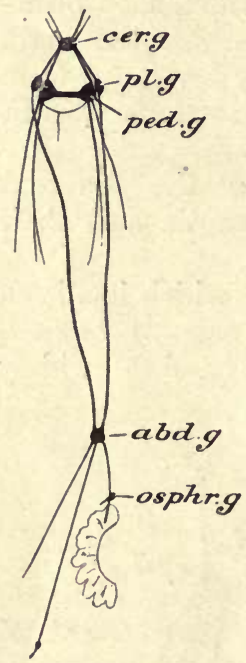

FiG. 636.-Nervous system of Aplysia (Opisthobranchia). abd. abdo. minal ganglion; cer. $y$. cerebral gangliou; osphr. g. osphradial ganglion ; ped. g. pedal ganglion; pl. g. pleural ganglion. (After Spengel.)

instead of towards it of cerebral ganglia overlie the esophagus, and below it is a mass of ganglia in which are to be made out a pair of pedal ganglia and at least two pairs of ganglia representing the visceral and pleural. A pair of small buccal ganglia are connected with the cerebral by means of slender connectives.

The organs of special sense are the eyes, the statocysts, and the osphradia. In nearly all cases there are two cephalic eyes (Fig. 638), the position of which has already been referred to in the account given of the external characters. In structure they are simplest in Patella $(A)$, where each consists of a pit-like depression, lined by pigmented cells connected with nerve-fibres. In the majority they have the structure described in the case of Triton. In certain species of Oncidium, a littoral Pulmonate, there are numerous eyes of a simple type scattered over the dorsal surface. In this case the optic nerve pierces the retina and the cells of the latter have their free ends directed away from the centre of the eye, as in Pecten (see p. 707) and in the Vertebrata, of the eye is occupis in other Mollusca. The internal cavity of the eye is occupied by a
large transparent cells. The statocysts are usually placed in close relation to the pedal ganglia, but are always innervated from the cerebral. An olfactory organ is present in the shape of groups of cells on the tentacles, in which the fibres of an olfactory nerve terminate.

The osphradia are prominences, usually of simple form, situated close to the base of the ctenidium. In many of the branchiate Streptoneura (Fig. 639), as already mentioned in the case of Triton (see p. 726, Fig. 613), the

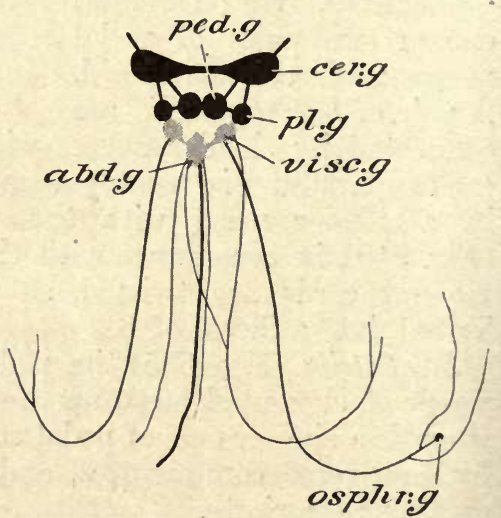

FIG. $637 .-$ Nervous system of Iimnæus (Pulmonata). abd. $g$. abdominal ganglion; cer. $y$. cerebral ganglion; osphr. g. osphradial gang: lion; ped. g. pedal ganglion; $p l$. g. pleural ganglion. (After Spengel.) 
primitively right osphradium, which is alone developed, assumes the form of a pectinate body with a central ridge, on either side
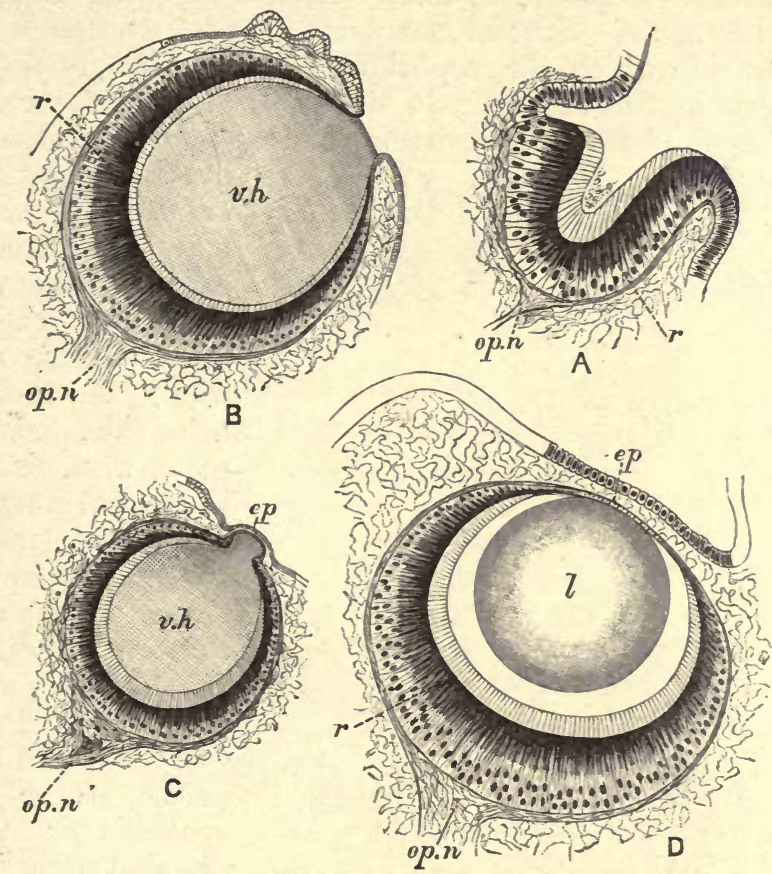

Fig. 638.-Eyes of Gastropoda. A, Patella; B, Trochus; C, Turbo; D, IMurex. ep. epidermis; $l$. lens; op. $n$. optic nerve; $r$. retina; $v . h$. vitreous humour. (From the Cambridge Natural History, after Helger.)

of which is a row of close-set lateral laminæ, and is commonly termed the parabranchia from its resemblance in appearance to a gill. In some cases it is of even more complicated shape than in Triton, owing to the branching of the lateral ridges.

The nephridia of the Gastropoda are dorsally placed glandular tubes or chambers, which communicate internally with the pericardium, and open on the exterior, either directly or through a duct-the ureter. Both right and left neph-

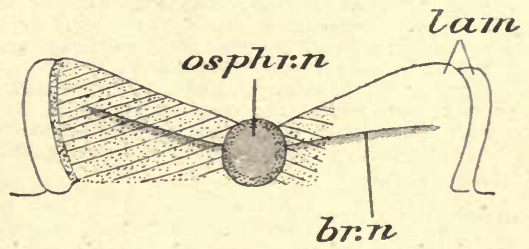

FIG. 639.-Transverse section of osphradium of Murex. $b r$. $n$. branch nerve passing to lamina ; lam. laminæ; osphr. n. main osphradial nerve. (After Spengel.)

ridia may be present, though unequal in size, the one situated to the right of the anus being larger than that situated to the left; or the former may alone be developed (Euthyneura). 
In a very limited number of Gastropoda the gonad opens into the nephridium.

The sexes are separate in nearly all the Streptoneura, united in the Euthyneura. Special gonoducts are present, except in one or two forms in which the nephridia perform that function. In the unisexual forms the reproductive apparatus is of a comparatively simple character, consisting merely of a racemose repro-

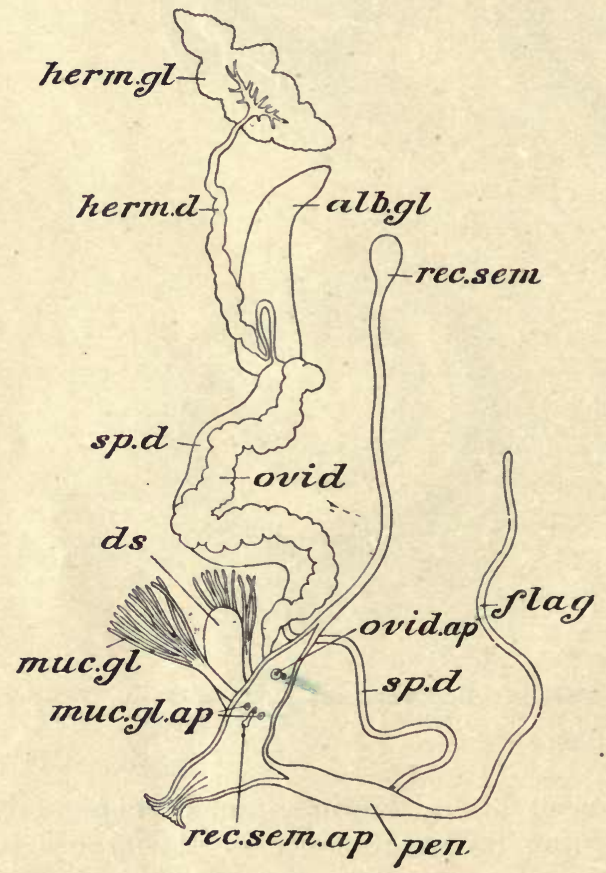

FıG. 640.-Reproductive organs of Felix. alb. gl. albumengland; d. s. dart-sac; flag. flagellum of the penis ; herin.gl. hermaphrodite gland or ovotestis ; herm. $l$. duct of ovotestis; muc. gl. mucous gland; mvc. gl. ap. apertures of mucous glands into vestibule; ovid. oviducal part of the eommon duct; ovid. ap. aperture of oviduct into vestibule; pen. penis; rec. sem. receptaculum seminis; rec. sem. ap. aperture of receptaculum seminis ; $8 p . d$. sperm duct; $s p . d^{\prime}$. spermiducal part of common duct. (After Pelseneer.)

ductive organ, ovary or testis as the case may be, situated dorsally in the visceral spiral, with the gonoduct opening far forwards on the righthand side, and, in the male, a penis, which is grooved longitudinally and non-retractile. In the hermaphrodite forms, such as the Pulmonata (Fig. 640), on the other hand, a considerable degree of complexity is observable. There is an ovotestis or "hermaphrodite gland" (herm. gl., Fig. 641, A)-some of the follicles of which produce ova, while others produce sperms, a convoluted "hermaphrodite duct" (herm. d.), an albumen-gland, in which the albumen of the relatively large eggs is formed, and sometimes a separate oviduct and sperm duct leading to a common genital opening; sometimes there is a single duct undivided throughout. A receptaculum seminis (rec. sem.) is connected with the oviduct, and also a number of narrow accessory oviducal glands (muc. gl.); frequently a gland termed prostate is connected with the sperm duct, and there is an eversible sac-the sac of the dart $(d . s$.$) containing a$ crystalline stylet, and a penis (pen.), which is perforated by a canal and is capable of being retracted by a special muscle. The duct 
may be simple or may be incompletely divided longitudinally into two parts. In the Pulmonata the first part (" hermaphrodite duct" proper) is simple, and serves for the passage both of ova and sperms: the middle part is incompletely divided internally into two passages, one serving as oviduct, the other as sperm-duct. In the distal part oviduct and spermduct are completely separate. Where the sperm-duct enters the penis, there is given
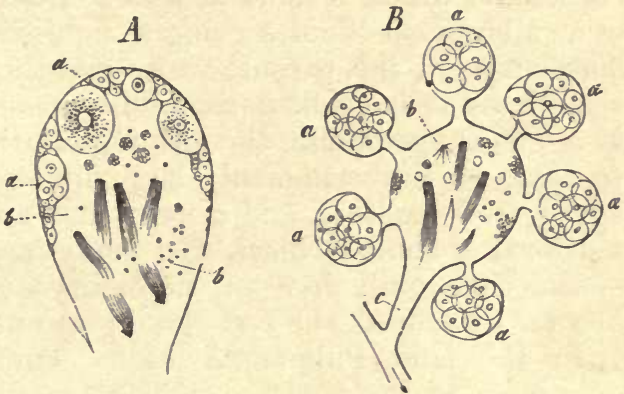

FiG. 641.-Follicles of the ovotestis of the Gastropodo. A of Felix hortensis (Pulmonata): $B$, of the rolida. $a$. $a$, ova ; $b$, masses of sperms; $c$. common efferent duct. (From Gegenbaur.) off a long, slender, tapering diverticulum, the flagellum (flag.), in which the sperms are made up into elongated masses or

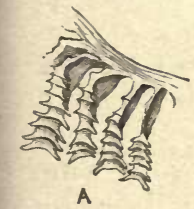

A

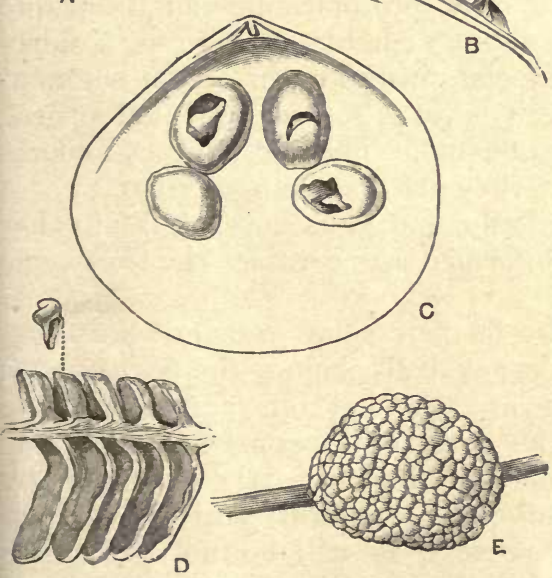

Fig. 642.-Forms of egg-cases in Gastropoda. $A$ and $D$, Pyrula or Busycon; $B$, Conus; $C$, voluta musica; $B$, Ampullaria. (From the Cambridge Natural History.) spermatophores.

Development. - The limpets (Patella) are exceptional in laying the eggs one by one and unfertilised-impregnation taking place in the water after they have been discharged. In almost all the Gastropoda fertilisation is internal, and the eggs are laid in great masses, embedded in jelly, each egg having its own hyaline envelope. Very often the mass of spawn consisting of the jelly-like substance, with the eggs embedded in it, attains a relatively considerable size. In form it varies greatly: frequently it is in the shape of long strings which are cylindrical or band-like: sometimes several such strings are twisted together into a cord. Sometimes the spawn is fixed to sea-weed or other objects; sometimes it is unattached, and may 
float about freely. In the Streptoneura (Fig. 642), instead of a jelly-like mass, the eggs are enclosed in a firm parchment-like capsule, in which is contained, in addition to the eggs, a quantity of an albuminous fluid, serving to nourish the developing embryos. The shape of the capsule varies greatly in the different genera: sometimes it is stalked, sometimes sessile; in some cases there is a lid or operculum, the opening of which permits the embryos to escape. Very commonly large numbers of these capsules are aggregated together, and usually they are attached to a rock or a sea-weed or similar object. In many cases only a limited number - sometimes only one-of the embryos contained in the capsule become developed, the rest serving as nutriment for the survivors.

In the land Pulmonata each ovum may be embedded in gelatinous matter enclosed in a firmer envelope, and a number of them are arranged in a string; sometimes a large number are embedded in a rounded gelatinous mass. Usually, as in some species of Helix and other genera, the outer layers of the albumen-like substance enclosing the egg become toughened and impregnated with salts of lime, so as to assume the character of a calcareous shell; a number of such eggs, which are of relatively considerable size, are laid in holes excavated in the earth.

In a few marine and fresh-water Gastropoda the ova undergo their development in the body of the parent, enclosed in an enlargement of the oviduct which serves as a uterus.

The egg contains a considerable quantity of food-yolk, which may be evenly distributed, or a clear protoplasmic and an opaque yolk-laden segment may be distinguishable. There is a fairly close agreement throughout the class in the nature of the segmentation (Fig. 643). In all cases it is total, sometimes equal at first, but soon afterwards becoming unequal. The first four blastomeres are usually equal or nearly so; they are so arranged that two of them are in contact in the middle, and thus separate the other two: the line of contact of the former pair becomes the transverse axis of the embryo.

From the four first-formed cells four small cells or micrcmeres become constricted off, the larger cells being the mcgameres; then four more micromeres are divided off, and again the same process is repeated. The embryo now consists of the four megameres and twelve micromeres. The latter then increase by division and form a cap of small cells (ectoderm) on the surface of the megameres. The whole process, as will be noticed, has a remarkably close resemblance to the process of segmentation of the ovum of a Polyclad as described on p. 273.

The megameres then give off internally four small endoderm cells, and from one of these (endo-mesoderm cell) are formed two primitive mesoderm cells, from which the cells of the mesoderm are developed. In some cases (Paludina) the mesoderm is formed 
entirely from cells that migrate inwards from the ectoderm and come to fill the segmentation-cavity. The megameres themselves eventually become converted into endoderm cells. A segmentationcavity is developed between the micromeres and the megameres, and the result is the formation of the blastula, one side of which (vegetal pole) is greatly thickened owing to its consisting of the large megameres, the opposite side (animal pole) being made up of micromeres. This may become a gastrula by epiboly or over-growth
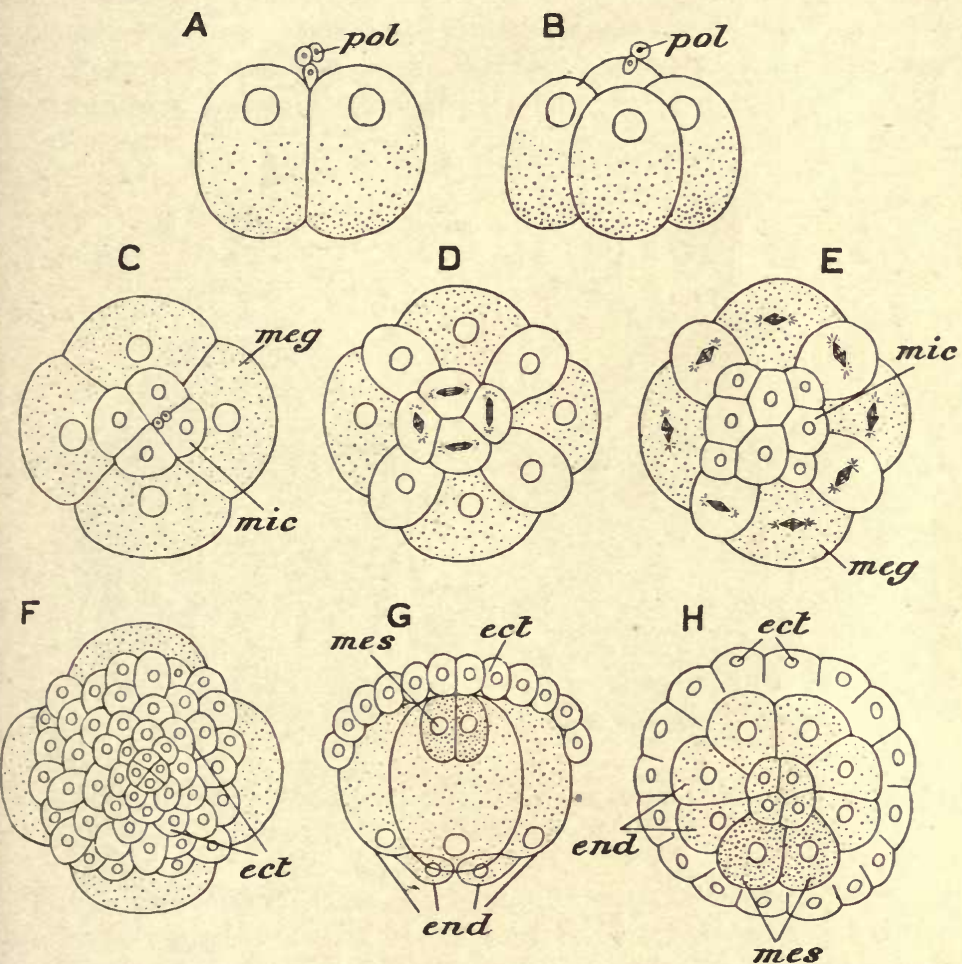

FIg. 643 -Diagram of the scgmentation and formation of the germinal layers of the Gastropoda $A$ and $B$, lateral view ; $C-F$, viewed from the auimal (upper) pole; $H$, from the vegetal (lower) pole ; $G$, in optical section ; ect. ectoderm ; $₫$ ind. enduderm ; mic. nicromeres ; meg. megameres ; mes. mesodern ; pol. polar bodies. (After Korschelt and Heider.)

of the ectoderm over the megameres; or, if the segmentationcavity is of considerable size, an invagination takes place.

The two larval stages, the trochophore and the veliger, are characteristic of the development of the Gastropoda. The former is most typically developed in Patella; in other Gastropods it undergoes more or less modification. In Patella (Fig. 644) there is a ciliated blastula $(A)$ which has on one side the large megameres The latter become enclosed by the micromeres, and the foundation 
of the mesoderm is laid in the manner already described. The blastopore is situated at the vegetal pole, destined to become the hinder end of the larva, but it soon changes its position and extends forwards on the ventral side, and a ciliated ring - the prototroch or future vcluin-is formed. Subsequently
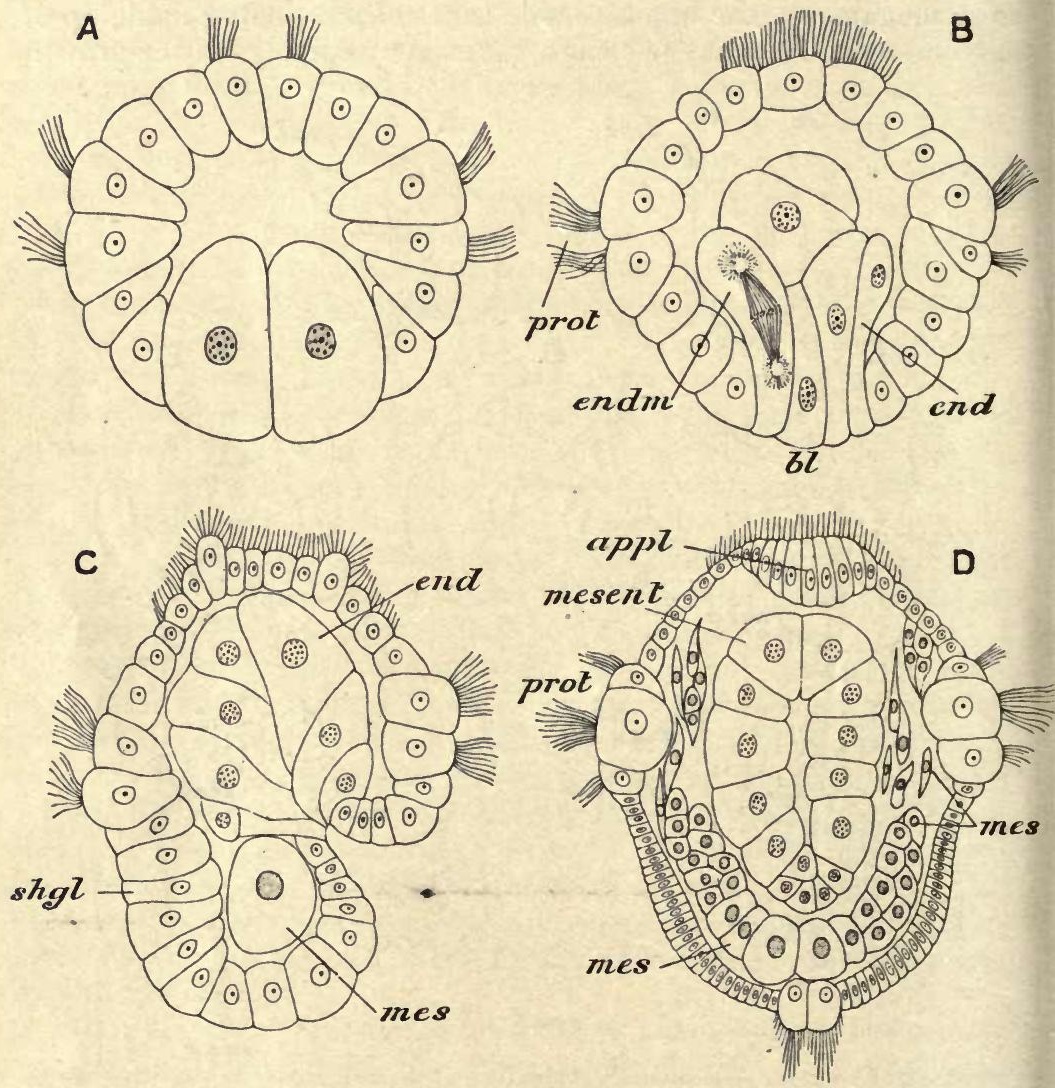

FIG. 644.-Earlier stages in the development of Tatella. $A$, blastula ; $B$, beginning of endodermal invagination ; $C$, completion of gastrula; $D$, frontal section of somewhat later stage. ap. $p l$. apical plate; $b l$. blastopore ; endm. endo-mesoderm cell ; encl. endoderm ; mes. mesoderm ; mesent. mesenteron; proto. prototroch; sh. gl. shell-gland. (From Korschelt and Heider, after Patten.)

the position of the blastopore becomes still further shifted and its form U-shaped and then slit-like. It undergoes elongation (Fig. $645, A$ ) and eventually becomes partly closed up, the closure taking place from behind forwards; the most anterior part remains open to form the mouth-or, perhaps more correctly, there is in the position of the anterior part a sinking-in of the 
ectoderm which pushes the blastopore inwards and forms the rudiment of the stomodæum. The originally solid mass of endoderm develops a lumen, and its cells become arranged to form the enteric epithelium. From the posterior end, where the mesoderm cells are situated, proceed two very regularly formed mesoderm-bands (Fig. 645, B). On the dorsal surface the shell-gland has already appeared as a pit lined by elongated ectoderm cells; on the surface of this appears the embryonic shell. The rudiment of the foot (Fig. 645, $A$ ) arises at a remarkably early stage as two protuberances lying on the ventral side of the posterior end of the larva at the sides of the blastopore; these coalesce to form the median foot.
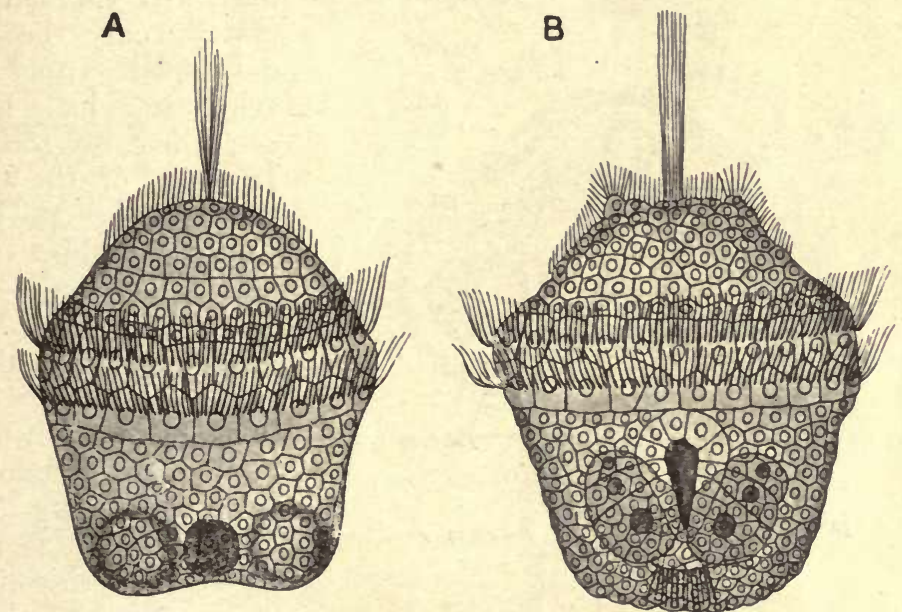

Fig. 645. $-A$ and $B$, Trochophores of Patella at different stages. In $A$ are to be seen the circular blastopore and the two foot-elevations; in $B$ the blastopore is drawn out, at the sides of it are the two mesoderm bauds. (From Korschelt and Heider, after Patten.)

The larva (Fig. 646) has now assumed the trochophore form. The pre-oral part is large and convex, with an apical plate on which is borne a bunch of long cilia, and near it two small ciliated elevations, each consisting of a single cell. The pre-oral part of the larva then becomes much flattened, and the apical plate $(a p . p l)$ increases in size and importance. At the posterior end is a bunch of cilia which are borne on two special large cells, the anal cells (an.c). The embryonic shell becomes saucer-shaped. A slight ridge in the neighbourhood of the shell represents the border of the mantle. The mid-gut (mesent) has become considerably widened: a diverticulum from it is recognisable, and this afterwards opens on the exterior to form the anus. A diverticulum of the foregut $(\mathrm{rad})$ at the same time forms the rudiment of the radular sac. The statolith-sacs appear as depressions of the ectoderm at 
the sides of the mouth : these grow inwards and become sac-like, subsequently lying at the sides of the foot, which has meantime attained a considerable size.

The trochophore-stage, which is so well marked in the case of Patella, occurs in other Gastropods, though, as a rule, presenting modifications perhaps traceable to the enclosure of the embryo in an egg-shell and to the presence of much food-yolk. The history of the blastopore is not the same in all cases; in Paludina it becomes converted into the anus; in some the mouth is developed from its anterior portion ; in others the stomodæal in-

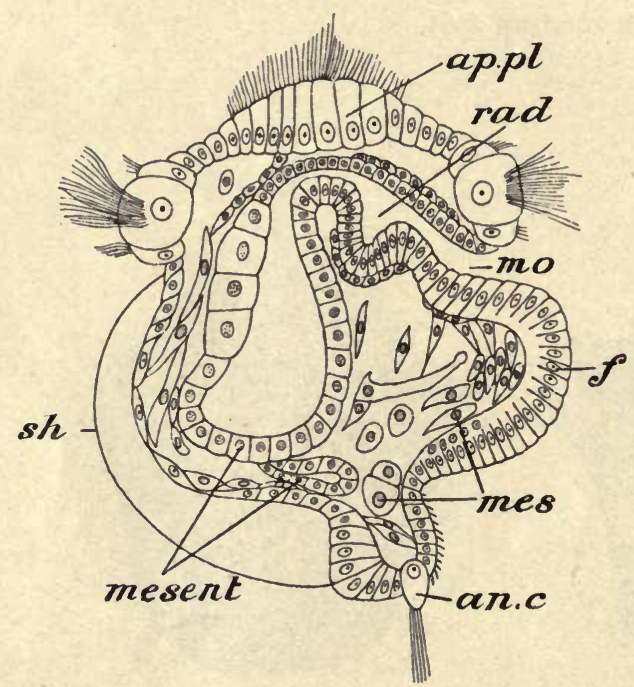

FIa. 646.-Later truchophore of larva of Patella in longitudinal section. $a n$. $c$. anal cells with cilia ; $a p . p l$. apical plate; $f$. foot; mes. mesoderm cells ; mesent. mesenteron; mo. mouth; rad. rudiment of radula-sac; sh. shell. (From Korschelt and Heider, after Patten.)

vagination arises after its complete closure, or may, with the mantlecavity, only become developed after the symmetry has been disturbed by torsion. In most of the Gastropoda the pre-oral circlet or velum (Fig. 647, vel.) undergoes a development not observable in the Pelecypod embryo, and becomes greatly extended as a bilobed flap, the strong cilia with which it is bordered rendering it a very efficient organ of locomotion for the larva. With the full development of the velum the larva passes

into the veliger stage (Fig. 647). In this stage the shell (sh.) increases in size, loses its simple form, and begins to develop a spiral. A cleft-like depression in the border of the mantle on the right-hand side forms the rudiment of the mantle-cavity in which, later, the gills are developed. The anus when it first appears may be symmetrically placed, but later becomes shifted to the right side and forwards as well as dorsally. A pair of larval nephridia are developed, having a remarkable resemblance to the excretory canals of the Flat-worms. Each consists of a longer or shorter tube sometimes intra-cellular, sometimes inter-cellular, opening on the exterior at one end, and at the other terminating in one or several flame-cells. The foot $(f$.) may attain a considerable development during the veliger stage, and on its posterior and dorsal part appears 
the operculum. Two little processes on the velar area develop into the tentacles (tent.), and the eyes (ey.) appear at their bases. As the foot and other organs advance in development the velum decreases in size and gradually aborts, but in some cases a portion of it persists as the subtentacular lobes or labial tentacles in the neighbourhood of the mouth.

In the Pulmonata the velum is not well developed, except in Oncidium, though the trochophore stage is well marked.

The young Gastropod is at first bilaterally symmetrical; the prevailing asymmetry is the result of unequal growth of the two sides of the body. In the majority of cases it is the left side that grows more actively than the right, a result of which is that the
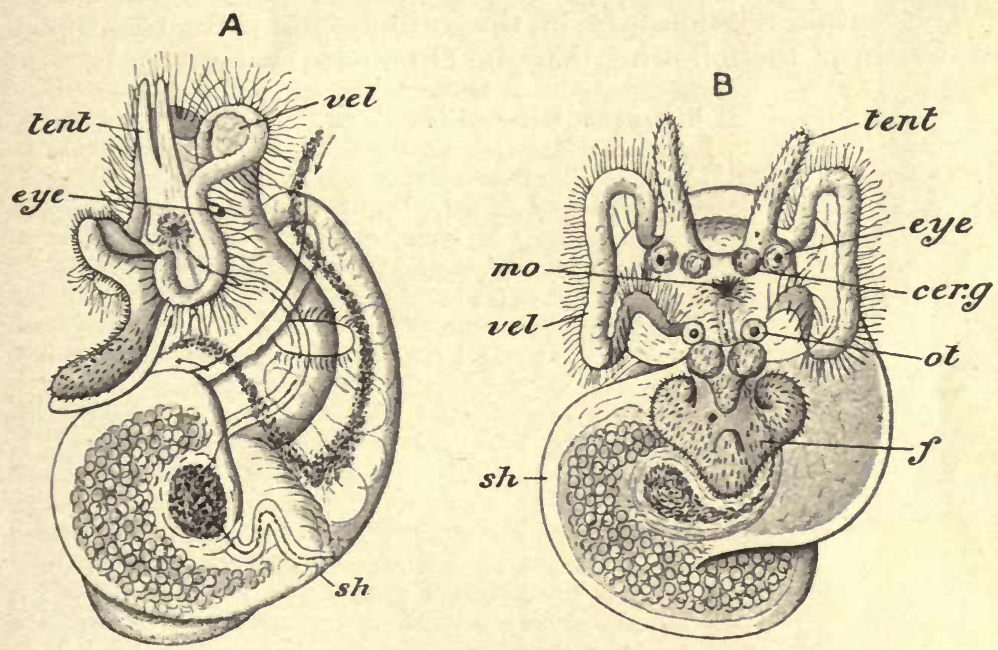

Fic. 647.-Veliger stage of Vermetus. cer. $g$. cerebral ganglia; $t y$. eye; $f$. foot; mo. mouth; ot. statocyst; sh. shell; tent. tentacle; vel. velum. (After Lacaze-Duthiers.)

posterior parts-the anus and the region surrounding it-are displaced forwards towards the right, the space between the anus and the mouth on that side undergoing little or no increase in length. In the Opisthobranchia and the Pulmonata the anus with the mantle-cavity and its contents become displaced forwards towards the anterior end; in most of the Streptoneura the anus, \&c., in their displacement forward pass beyond the middle line, one of the most striking effects of which is the crossing of the pleuro-visceral connectives already referred to (p. 737).

Ethology and Distribution.-Only a few aberrant families of Gastropoda are parasites. Most are aquatic, all the most primitive forms being inhabitants of the sea. Of the marine families the majority move by creeping over the sea-bottom, some burrowing in mud or sand, some in solid rock; some are able to float in 
a reversed position, adhering to frothy mucus secreted by the glands of the foot; certain exceptional forms such as Vermetus are fixed in the adult condition by the substance of the shell. A few families - the Heteropoda and the Pteropoda-are specially modified for a pelagic mode of existence, and swim through the water by flapping movements of the lobes of the foot, which act as fins. Gastropods are found in the ocean at considerable depths-up to nearly 3,000 fathoms. Many forms, however, are inhabitants of fresh water, while many Pulmonata are terrestrial, and occur even towards the summits of the highest mountains.

Fossil Gastropoda are known from almost the earliest fossilbearing rocks, and all the major divisions of the class are represented in formations of Palæozoic age.

The mutual relationships of the various groups of Gastropoda are shown in the following diagram (Fig. 648):-

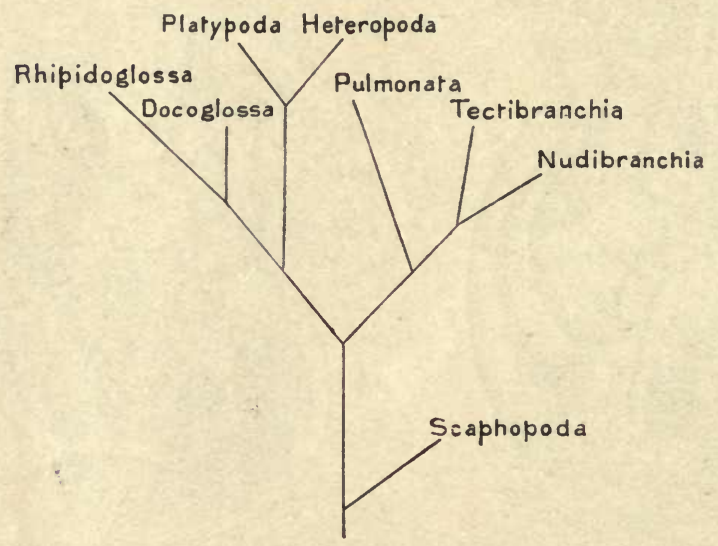

FIส. 648.-Diagram to illustrate the relationships of the Gastropoda.

\section{APPENDIX TO THE GASTROPODA.}

\section{A. CLASS IV. -SCAPHODA.}

The Scaphopoda or Elephant's tusk-shells are aberrant marine. Molluses comprising only three genera-Dentalium, Siphonodentalium, and Pulsellum. The body is elongated so as to be almost worm-like, with complete bilateral symmetry. The mantlefolds are almost completely united to form a cylindrical tube enclosed by the shell (Fig. 649), which is in the form of a delicate, curved tube, open at both ends

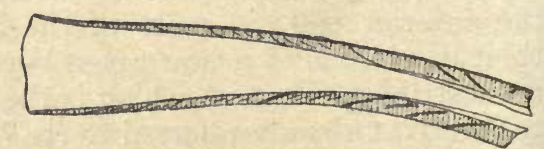

FIG. 649.-Dentalium, longitudinal section of shell. (After Keferstein.) and wider at the anterior or oral end than at the other. The foot (Fig. 650, $f$ ) is narrow, trilobed at the extremity or provided with a terminal disc, capable of being protruded through the oral opening of the shell, and used for burrowing in sand. The mouth is 
situated on a short oral proboscis, and is sometimes surrounded by lobed processes or pinnate palpi. Further back are a pair of tentaculiferous lobes, each bearing a large number of filiform tentacles, which are probably respiratory in function. The mouth leads into a buccal cavity containing an odontophore. Connected with the mesenteron is a large bilobed digestive gland $(l$.$) .$ The anus is situated ventrally behind the base of the foot. 'The vascular system is extremely simple, consisting of sinuses without definite walls, and there is no distinct heart, though in the neighbourhood of the rectum there is a specially contractile part of the principal sints. Two nephridia open near the anus, the right one acting as a gonoduct, the left $(k)$ entirely renal in function. The sexes are distinct. There is an elongated unpaired gonad $(g$.$) , divided by lateral in-$ cisions into a number of lobes, occupying all the posterior and dorsal parts of the body. Anteriorly it narrows to form a duct opening into the right nephridium.

The nervous system consists of paired cerebral, pleural, pedal, and visceral ganglia; the cerebral ganglia are situated close together. There are no eyes or statocysts.

In the gastrula stage the embryo, which is provided with cilia, becomes free. The ciliated cells are arranged in a characteristic manner in three rows which, at first situated close together about the middle of the body, become shifted at a later stage near the apical pole, and amalgamated into a broad band representing the preoral circlet of other molluscan larvæ; at the same time a bunch of cilia previously developed at the apical pole becomes more conspicuous and a considerable part of the general surface covered with more delicate cilia. The blastopore, at first terminal, is shifted forwards on the ventral surface until it comes to be immediately behind the ciliated circlet. At its anterior end an in-

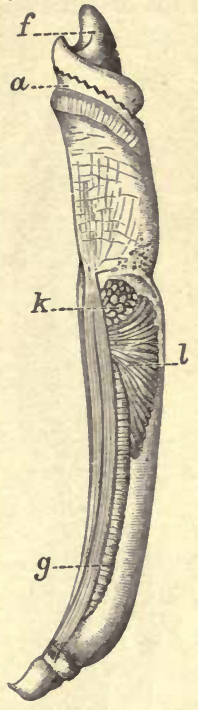

Fic. 650.-Dentalium, anatomy. $a$. anterior aperture of mantle; $f$. foot; $g$. gonad ; $k$, nephridium; $l$. digestive gland. (From the Cambridge Natural History, after Lacaze-Duthiers.) vagination gives rise to the mouth and stomodæum.

The larva (Fig. 651) has now attained the stage of a trochophore, in which, however, both apical plate and primitive nephridia are wanting. A shell-gland

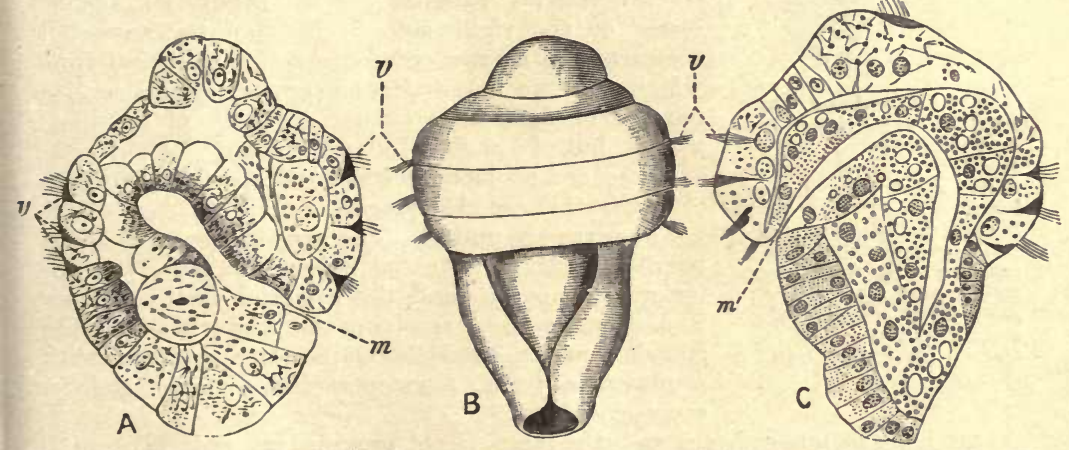

FIG. 651.-Veliger of Dentalium. A, longitudinal section of a larva 14 hours old; B, larva of 37 hours; C, longitudinal sectiun of larva of 34 hours. m. mouth; $v, v$. velum. (From Cooke, after Kowalewsky.)

is developed, and soon the rudiment of the shell. The post-oral region, at first inconsiderable in size, soon undergoes an increase, until it forms eventually by 
far the longest part of the body, while the pre-oral region almost completely aborts. When the post-oral region has attained a certain size, there are developed on it two lateral folds, the rudiments of the mantle (B), which grow inwards towards the middle ventral line, and later on unite by their free margins. The pre-oral circlet or velum changes its form-at first it is conical, later it becomes plate-like, and is then gradually reduced, the larva sinking to the bottom; and though still occasionally swimming with the aid of the velum, coming to use the foot as a creeping organ. The shell now increases in size step by step with the growth of the mantle, and bends round the body of the larva until its edges meet and coalesce in the ventral median line. Later it assumes the elongated conical form, curved towards the dorsal side, characteristic of the adult. The foot at the same time elongates and takes on the characteristic three-lobed shape.

\section{B. RHODOPE.}

Rhodope (Fig. 652) is a minute, elongated, fusiform animal, ciliated externally, with complete (external) bilateral symmetry. There is no shell, but within the body-wall, in the parenchyma between it and the enteric canal, are numerous irregularly shaped calcareous spicules. There are neither jaws nor odontophore. The enteric canal - which is a narrow tube, consisting of buccal cavity, with salivary glands, œsophagus, mid-gut with a cæcum, and rectum-opens in an anal aperture situated to the right of the posterior extremity of the body. A digestive gland is absent. The central part of the nervous system consists of a supra-œsophageal mass made of three pairs of ganglia-cerebro-visceral, pedal and buccaland a single ventral ganglion. An eye and a statocyst are situated on each side in close relation to the ('erebro-visceral ganglion. The nephridial system opens on the right side in front of the anus : it consists of a narrow ciliated canal, running out from which are two longitudinal excretory canals with a number of flame-cells similar to those of the Flatworms, but of a multicellular structure.

There are no blood-vessels, and specialised organs of respiration are also absent.

The sexes are united. The gonads consist of about twenty ventrally situated masses of cells, the anterior being ovaries and the posterior testes. There is a common duct receiving the products of all the gonads; and a single hermaphrodite aperture, with a muscular penis, a receptaculum seminis, and an accessory gland.

There is no metamorphosis, and the larva is not provided at any stage with any representatives of either shell-gland or foot.

Though the occurrence of flame-cells is unique, there can be little doubt that Rhodope is best regarded as a degenerate member of the Mollusca, and it probably finds its nearest relatives among the Gastropoda. 


\section{Class V.-CEPHALOPODA.}

The Cephalopoda, including the Cuttle-fishes, Squids, Octopods, and Nautili, are marine Mollusca of a high grade of organisation. There is a very definitely-formed head, bearing a pair of highlydeveloped eyes, and surrounded by the anterior portion of the foot, modified into arms or tentacles. The body is bilaterally symmetrical. The posterior part of the foot is modified to form a funnel leading out from the large mantle-cavity. A shell is sometimes present, sometimes absent. When present it is usually internal, but sometimes external, and in the Nautili is capable of containing the body of the animal.

\section{Examples of the Class.}

\section{i. The Cuttle-Fish (Sepia ${ }^{1}$ ).}

Cuttle-fishes are marine Molluscs, which live usually at a depth of a few fathoms, but often come into shallower water, and are frequently caught in the trawl or the seine. The animal arrests attention when compared with Unio or Triton by the strength, and more particularly the rapidity, of its movements; by the possession of a pair of eyes resembling in size and complexity those of a Fish; and by various other features, all pointing to a higher grade of organisation than is attained by the members of the classes of Mollusca dealt with in the preceding pages.

External Features. - The Cuttle-fish (Fig. 653) has a distinct head, bearing ten long arms, and a pair of large, highly-developed eyes. The head is connected with the body by a constricted region or neck. The trunk is elongated and shield-shaped, the base of the shield being towards the head. The long axes of head and trunk are in line with one another. Not only the head, but also the trunk, are completely equilateral, in which respect there is a marked contrast to Triton; and this symmetry extends to most of the systems of internal organs. 'The free extremity of the head bears the mouth, and is accordingly termed the oral extremity, the opposite extremity, the apex of the shield-shaped body, is the aboral end. The surfaces of the shield are anterior or antero-dorsal and posterior or postero-ventral, its borders right and left. The anterior surface is to be distinguished by its darker colour, and by the firmness of the body-wall, due to the presence in this position of a hard internal shell.

1 Most of the figures have reference to a common Australian species-S. cultrata - but the differences between the various species of the genus are slight and unimportant, and the description given will apply fairly well to any other species. 
The aperture of the mouth is surrounded by the bases of the ten arms. These are in pairs, situated to the right and left of the median plane. All of them, with the exception of the fourth pair (the most anteriorly situated pair being reckoned as the first), are stout at the base and taper towards the extremity. When extended

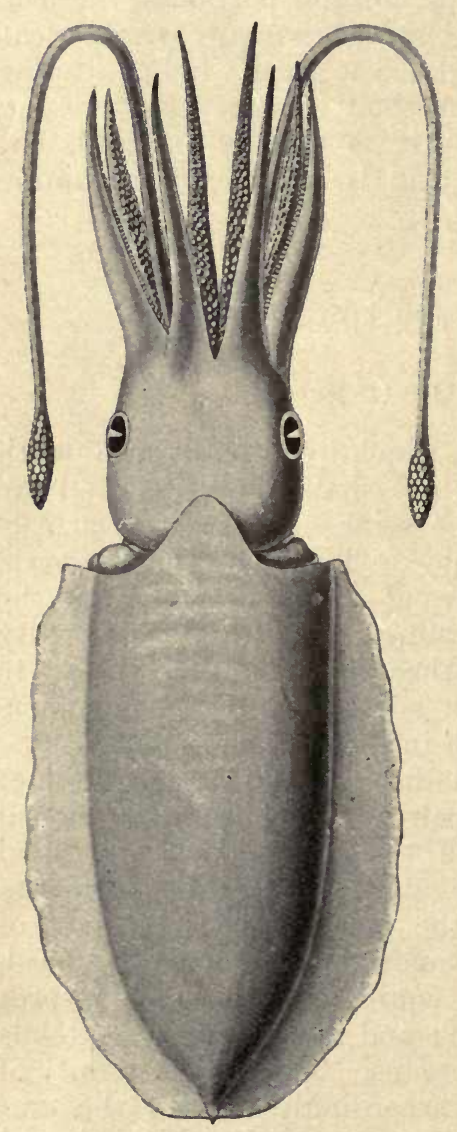

Fis. 653.-- Sepia cultrata. Fntire animal viewed from the antero-dorsal aspect.

they are about two-thirds of the length of the body. The outer surface of each (i.e. that turned away from the mouth) is strongly convex, the inner flat, and beset throughout its length with a number of suckers, which are arranged in four longitudinal rows. Each sucker is in the form of a shallow cup, supported on a short, thick stalk (st.); the lip of the cup is membranous, and immediately within it is a narrow, horny rim (dent.). Into the floor and walls of the cup are inserted numerous muscular fibres. When the sucker is being brought into use it is firmly applied to the surface of the object; by the contraction of the muscular fibres the cavity of the cup is then enlarged, and a partial vacuum is formed, the result being firm adhesion, owing to the pressure of the surrounding water. The fourth pair of arms, usually known as the tentacles, are comparatively long and narrow, and provided with suckers oniy towards their free ends, which are somewhat thickened and club-like. In the male the fifth arm on the left side presents a slight modification, some of the suckers being absent. This is an indication of a change termed hectocotylisation, which, as will be pointed out in the general account of the class, assumes in some cases a very remarkable character. As the nerves which supply them are derived from the pedal ganglia, there is no doubt that the arms of Sepia represent a portion of the foot of other Molluses; but there is some doubt as to whether they correspond to the fore-foot or to the epipodia of the Gastropoda. 
The head-region, comprising as it does the arms (which are the chief part of the foot) and the head proper, is termed the cephalnpodium.

The trunk is covered over by the thick integument of the mantle, which terminates toward the oral end in a ridge round the neck. Anteriorly this ridge projects as a prominent rounded lobe under cover of which the head can be partially retracted. Posteriorly it forms the posterior lip of the opening of a large cavity bounded by the mantle-the mantle-cavity-which extends along the entire posterior face of the body almost to the apex. The wide cleft between the oral edge of the mantle and the posterior surface of the body is not the only aperture leading into the mantle-cavity. On the oral side of this cleft is a large tube-the funnel (Fig. 658, inf.)-opening on the exterior behind the neck, and internally communicating by a wide aperture with the mantle-cavity. The cleft is capable of being almost completely closed by the apposition of a pair of oval projections (mant. cart.) of the inner surface of the posterior mantle-wall near its oral border, and a pair of concave depressions (inf. cart.) on the opposite (posterior) face of the funnel. The funnel is thus, under ordinary circumstances, the main outlet of the mantle-cavity. As such it not only carries to the exterior the effete water of respiration, the freal matters from the intestine, and the products of the excretory and reproductive organs, but also takes an important part in locomotion, the most important movements of the Cuttle-fish-by which it darts rapidly through the water in the direction of the aboral pointed end of the bodybeing effected by rhythmical contractions of the muscular walls of the mantle-cavity causing jets of water to be forced in the oral direction through the funnel. The free passage of water inwards through the funnel is prevented by the presence in its interior of a flap-like valve opening outwards. The water required for respiration and in locomotion is thus drawn in, not through the funnel, but through the partially-closed slit-like pallial aperture previously referred to. The funnel seems, from the source of the nerves which supply it, to be, like the arms, a specially modified part of the foot.

Fringing each lateral margin of the body is a thin muscular fold-the fin-which is used as a swimming organ.

The anterior wall of the body exhibits, as already mentioned, a hard and resistant character owing to the presence of the internal shell (Fig. 654). This is completely enclosed in a sac of the mantle. Like the body itself, it is bilaterally symmetrical. In shape it may be described as leaf-like, with a rounded and comparatively broad oral end, and a narrower aboral end, provided with a sharp, anteriorly-projecting spine. The posterior surface is convex; the anterior convex towards its oral end, but deeply 
concave aborally, and bounded laterally by thin prominent winglike ridges which converge to meet at the aboral extremity. The

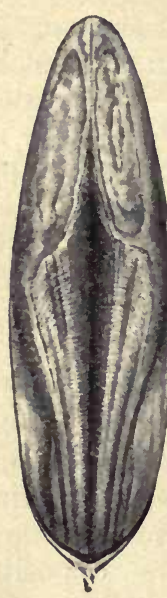

FIc. 654.- -Shell of Sepia cultrata, posterior view. Re. duced. main mass of the shell consists of numerous, closely-arranged, thin laminx of calcareous composition, between which are interspaces containing gas. On the surface is a thin layer of chitinoid material, and slightly thicker strips of similar composition run along the margins.

The living Cuttle-fish will be observed to undergo frequent changes of colour, and blushes of different hues are to be observed passing over the surface. These are due to the presence of numerous contractile pigment-containing cells or chromatophores (Fig. 655) situated in the deeper layers of the integument over the entire surface. The chromatophores are flattened sacs with elastic walls, the contracting tendency of which is capable of being counteracted by the action of bundles of muscular fibres radiating outwards from the edge of the sac into the surrounding tissues. When these radiating fibres are in action the edge of the chromatophore is drawn outwards in different directions, and as a result the flattened sac becomes more expanded and thinner, the pigment being spread out into a thinner layer. When the fibres are relaxed the elasticity of the wall comes into play, and the chromatophore contracts, the contained pigment resuming its former arrangement. A peculiar iridescence which, in addition to the play of colours, is recognisable in the integument of Sepia, is due to the presence of a number of cells, the iridocysts.

When the mantle-cavity is laid open (Fig. 658) there is seen on each side of it one of the two plume-shaped ctenidia(cten.). In the middle line of the posterior surface, close to the internal opening of the funnel, is the anal aperture (un.) situated at the oral extremity of a longitudinal tube-the rectum. On either side of the rectum is a much narrower projecting tube with a terminal opening-the 
nephridial aperture (neph.). On the left-hand side is the opening of the sperm-duct or oviduct (ovid.) as the case may be.

In addition to the shell, which is an important protective structure, and gives support to the muscles of the fins, Sepia also has a remarkably well developed internal skeleton composed of cartilage. An important part of this-the cranial cartilage (Fig. 656 ) - protects the principal nervecentres, encloses the statocysts, and gives support to the eyes. Other cartilages support the bases of the arms. A thin shieldshaped plate-the nuchal cartilage (Fig. 657)-lies on the posterior surface of the neck. The pair of elevations on the posterior wall of the funnel and the corresponding depressions on the anterior surface of the body are borne each on a thin plate of cartilage, and other thin cartilages support the bases of the fins.

\section{Alimentary System. - The}

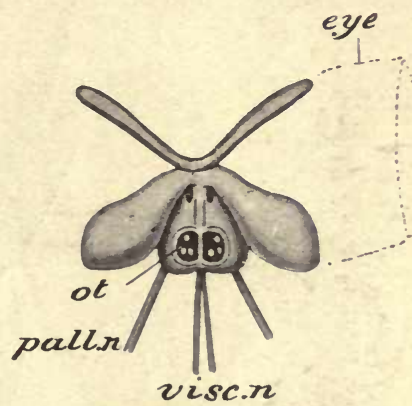

FIG. 656.-Eepia cultrata, cranial cartilage seen from the posterior aspect, with the cavities of the statocysts exposed. eye, position of eye indicated by dotted line; ot. statocyst; pall. $n$. pallial nerve; visc. $n$. visceral nerves. mouth is surrounded by a thin peristomial membrane, within which is a circular lip beset with numerous minute elevations. Lodged within the circular lip is a pair of powerful horny jaws (Fig. 659, Fig. 660, jaw ${ }^{1}$, jaw ${ }^{2}$; Fig. $661, j$.; Fig. 663, juw). These have somewhat the appearance of the beak of a parrot, the posterior jaw being larger and more strongly bent than the other, which it partly encloses. The mouth leads into a thick-walled buccal cavity, which

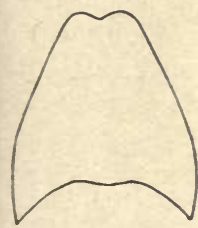

FIf. $65 \%$. - Sepia culirata, nuchal cartilage. contains an odontophore bearing numerous minute horny teeth. The oesophagus (Figs. 660 and 661, $\propto$; Fig. 663, oes), following on the buccal cavity, is a narrow straight tube, which runs between the halves of the "liver" towards the aboral end of the body. It opens into a rounded thick-walled stomach (st.), and, close to the pyloric aperture leading from the latter into the intestine, opens a wide cacum (c.). The alimentary canal at this point bends sharply round upon itself, and the intestine runs nearly parallel with the œesophagus to open into the mantle-cavity as already described.

A pair of glands (Fig. 661, s.g.; Fig. 663, sal.), which are commonly termed salivary, though their functional correspondence with salivary glands has not been proved, are situated in the head behind the cranial cartilage. The ducts of these two glands run inwards and unite to form a median duct, which opens into 
the buccal cavity. The name of "liver" (Fig. 660,l.l.; Fig. 662, liv.) or digestive gland is given to a large brown glandular mass which extends from the neighbourhood of the salivary glands nearly to the aboral end of the body. It consists of two partly united right

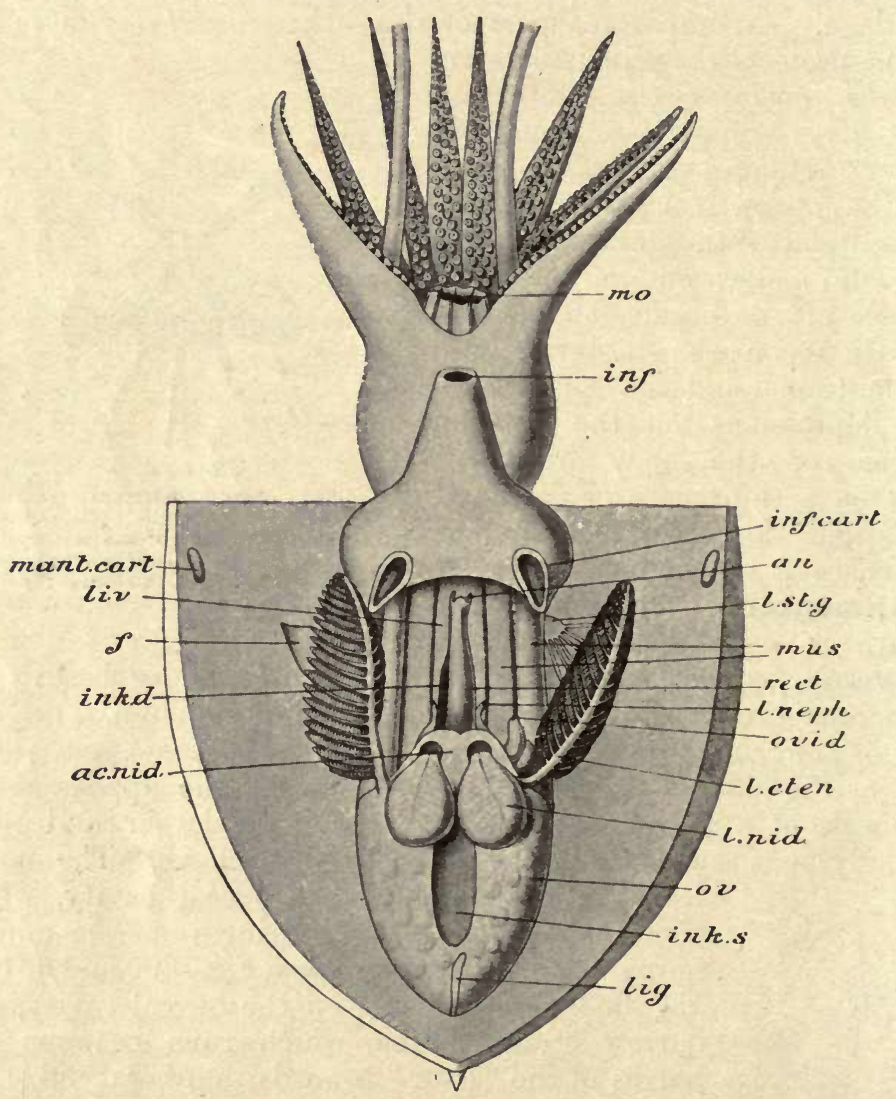

FiG. 658.- Sepia cultrata, female seen from the posterio-ventral aspect, the wall of the mantle cavity divided along the middle line and the twu flaps thus formed spread out so as to expuse the contents. ac. nid. accessory nidamental glands; $a n$. anal aperture with its lateral appendages; $f$. inembranous fold attaching the ctenidium to the wall of the mantle-carity; inf: external opcning of funnel; inf. cart. infundibular cartilagc ; ink. $d$. iuk-duct; ink. 8 . ink-sac ; lig. ligamentous band which extends from the anterior wall of the mantle-eavity to the ovary, cut across ; liv. "liver" ; $l$. cten. left ctenidium; $l$. neph. left nephridial aperture; $l$. ninl. left nidameutal gland; $l$. st. g. left stellate ganglion; mant. curt. mantle-cartilage; mo. mouth; mus. neck-nuscles; ov. ovary ; ovil. oviduct ; rect. rectum.

and left portions, each of which has a duct opening into the cavity of the alimentary canal opposite the point where stomach, cæcum, and intestine meet. Surrounding the ducts and opening into them are masses of minute vesicles (Fig. 661, b, d.); the secretion of these has the property of converting starchy matters 
into sugar; they sometimes, though without sufficient reason, receive the name of pancreas.

Immediately below the thin integument of the anterior wall of the mantle-cavity lies a characteristic organ-the ink-sac (Fig. 658, ink. s. ; Fig. 661, i). This is a pear-shaped body, a portion of the interior of which is glandular and secretes a black substancethe ink or sepia - which collects in the main cavity of the sac and is discharged by a cylindrical duct opening into the rectum close to the anal aperture. When the Cuttle-fish is startled it discharges the ink, which, mixing with the water in the mantle-cavity, is ejected through the funnel as a black cloud, under cover of which the animal may escape from a threatened attack.

Vascular System.-The heart (Figs. 662, 663, and 665) of the Cuttle-fish consists of a ventricle and two auricles. The ventricle (vent.) which is divided into two lobes by a constriction, is somewhat obliquely placed, but the rest of the vascular system is almost completely equilateral. At its oral end the ventricle gives off a large vessel-the oral aorta (aort.); aborally it gives origin to be a much smaller aboral aorta (aort'), which bends over the ink-sac and supplies the aboral portions of the body. The arteries which lead off from the aortæ communicate by their ultimate branches with a system of capillaries, and these with a

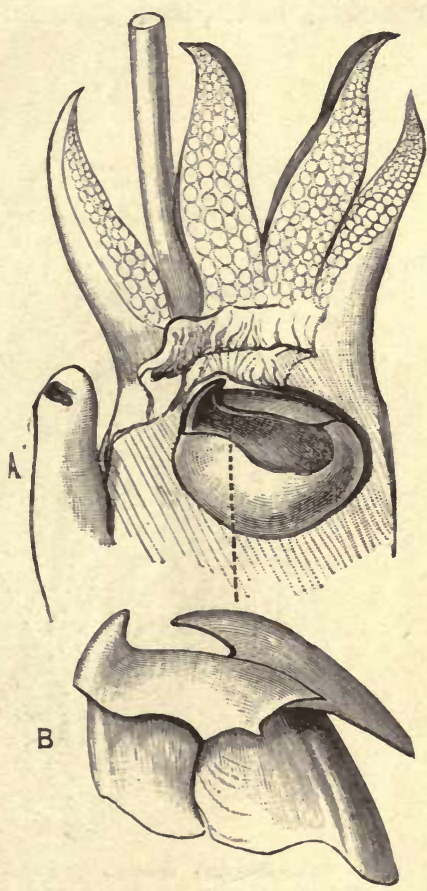

FIG. 659.-Sepia officinalis, jaws. A, in situ; $\mathrm{B}$, removed and slightly enlarged. (From the Canbridge Nutural History.)

system of veins. A large median vein, the rena cava (c. cav.), runs from the head to the neighbourhood of the rectum, in front of which it bifurcates to form the left and right afferent branchial veins $(l . a f f . b r . v ., r . a f f . b r . v$.), each running through the cavity of the corresponding renal organ to the base of the gill, where it is joined by veins from the aboral region. At the base of the gill the afferent branchial vein becomes dilated to form a contractile sac-the branchial heart $(r . b r . h t$.) -appended to which is a rounded body of a glandular character-the appendage of the branchial heart, representing the pericardial glands of the 
Pelecypoda. The afferent branchial vein runs through the axis of the branchia, giving off branches as it goes. The blood is carried back to the ventricle on either side by a dilated contractile vessel, the auvicle or efferent branchial vein (l. aur., $r$. aur.).

The cœlome (Fig. 671) is a pouch of considerable size, divided by a constriction into oral and aboral parts. The former is the pericardium, or cavity in which the heart is lodged; it gives off a pair of diverticula, right and left, each lodging the corresponding branchial heart, and communicates by a pair of apertures with

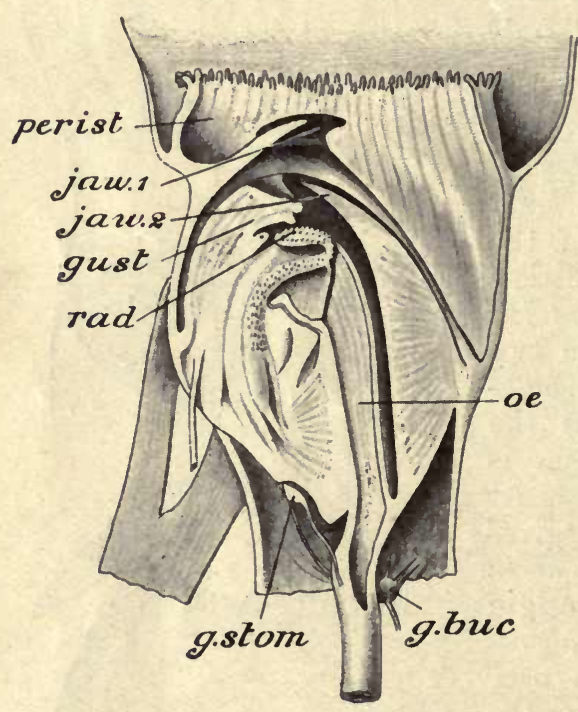

FIG. 660.-Sepia, median section through the buccal mass. g. buc. buccal ganglia; g. stom. stomatogastric ganglia; gust. supposed gustatory organ ; jawl, posterior jaw ; javo2, anterior jaw; $\alpha$. oesophagus ; perist. peristomial membrane ; rad. radula. (After Keferstein.)

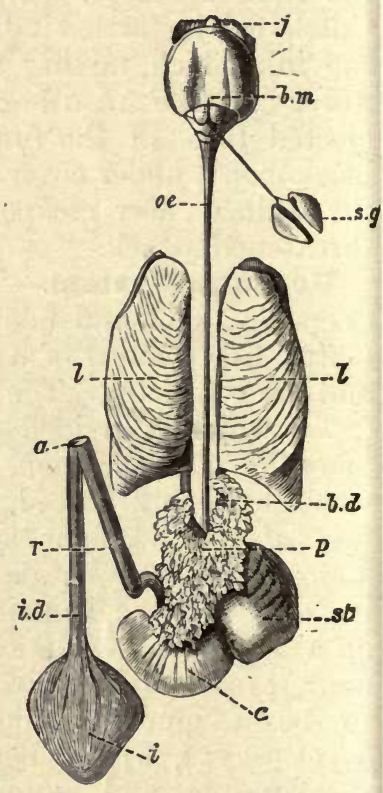

F1G. 661.-Sepia officinalis, enteric canal. $a$. anus; $b . d$. duct of one of the portions of the digestive gland; $b$. $m$. buccal mass ; c. cæcum ; $i$. inksac; $i . d$. ink-duct; $j$. jaws; $l . l$. digestive gland; $\alpha$. cesophagus; $p$. pancreatic appendages; $r$. recturn; s. g. salivary glands; st. stom:ch. (From the Cambridge Natural History.)

the cavities of the nephridia or renal sacs. The aboral part of the colome forms the capsule (gonocole) which encloses the ovary or testis.

The paired, plume-shaped ctenidium lies parallel with the long axis of the body. It is attached throughout the greater part of its length to the wall of the mantle-cavity by a thin muscular fold, and consists of numerous pairs of delicate lamellæ, the surface of which is increased by the presence of a complex system of foldings. Internally the lamellæ are not completely in contact, an 
axial canal being left through which the water penetrates freely to all parts of the gill. The blood carried to the gill by the afferent branchial vessel passes in a system of minute branches through the lamellæ, and is gathered up again into vessels which open into the main efferent vessel leading to the auricle.

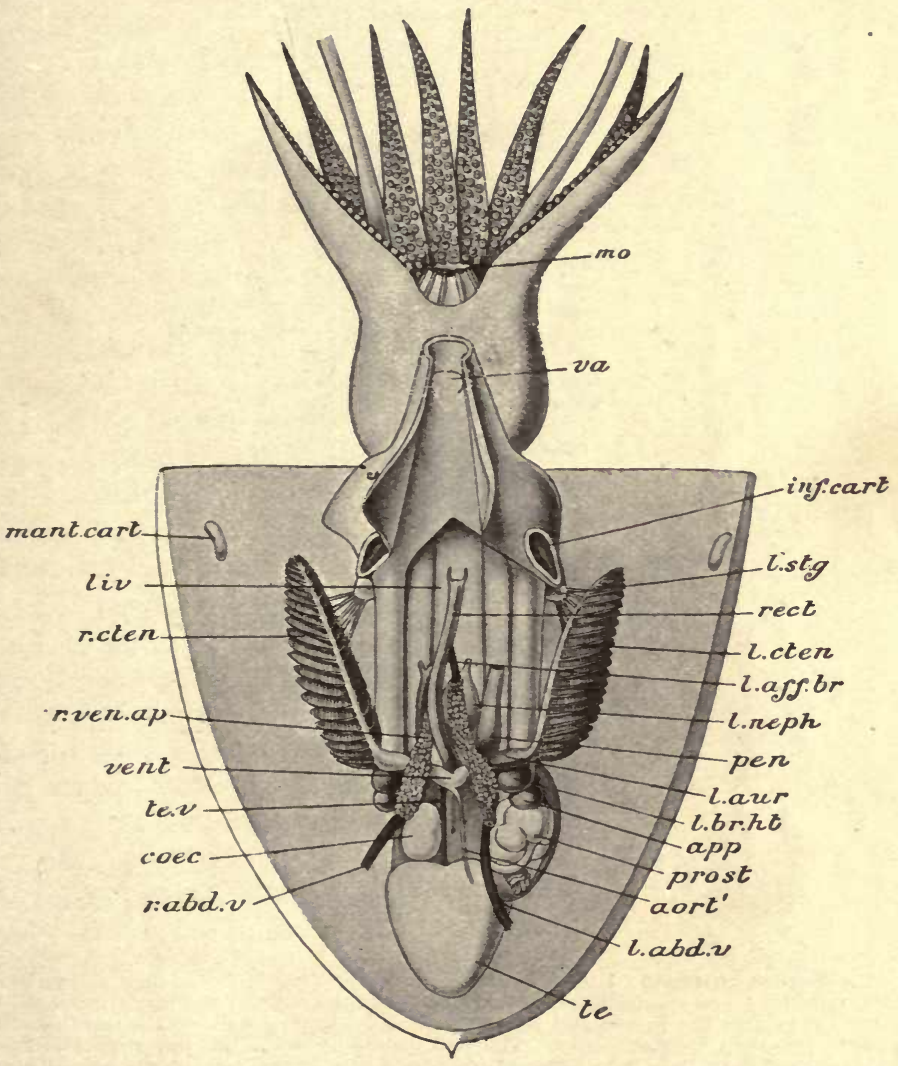

FiG. 662.-Sepia cultrata, male specimen seen from the postero-ventral aspect, the mantlecavity opened as in Fig. 65s, the posterior body-wall partly dissected off, so as to expose the organs in the visceral sac, the ink-sac and duct renoved. aort. main aorta ; aort.' aboral aorta; $a p p$. appendage of left branchial heart; cce. cacum; inf. cart. funnel cartilages; lic. digestive gland ; $l$. alvl. $x$. left abdominal vein; $l$. aff. $b r$. left afferent branchial vessel ; $l$. aur. left auricle; $l$. $b r$. ht. left branchial heart; $l$. cten. left ctenidium; $l$. st. $g$. left stellate ganglion; mant. cart. mantle-cartilage; mo. mouth; pen. penis; prost. prostate; $r$. abd. $v$. right abdominal vein; $r$. cten. right ctenidiun ; rect. rectum; r. ren. app. appendages of right afferent branchial ressel; te. testis; $t e . v$. vein to testis; ra. valve of funnel; vent. ventricle.

Nervous System.-Though parts homologous with those of Triton are recognisable in the nervous system of Sepia, their proportions and arrangement indicate a higher grade of organisation. The cerebral, pedal, and pleuro-visceral ganglia (Fig. 666), all of relatively large size, are closely aggregated together around the 
œsophagus, supported and protected by the cranial cartilage. The cerebral ganglia (cer.g.) are fused together into a rounded mass, lodged in a hollow of the cranial cartilage, and covered over anteriorly by a strong fibrous membrane. Laterally are given off

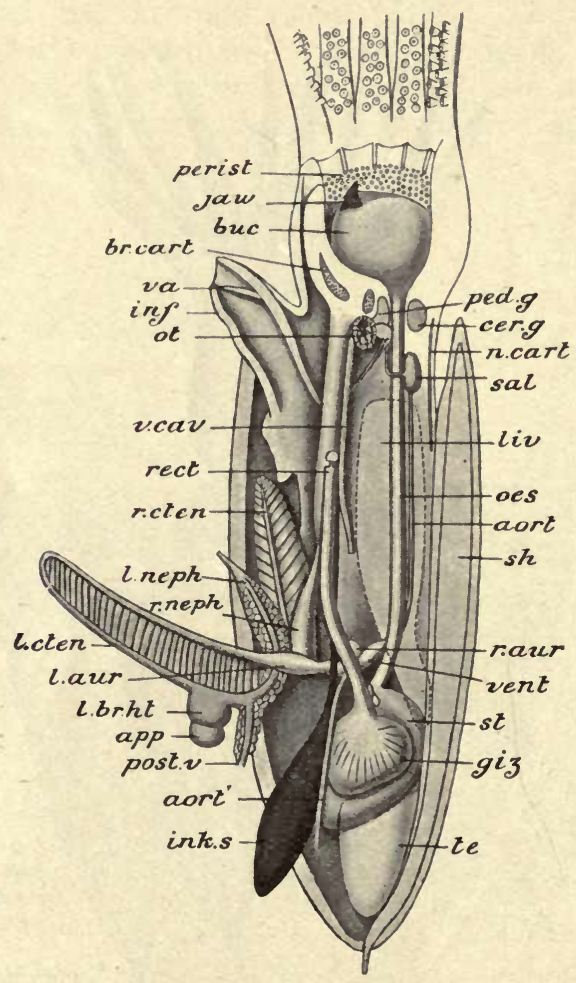

Fig. 663.-Sepia cultrata, lateral dissection of male. The left-hand half of the head has been removed by an approximately median longitudinal section, the buccal mass, huwever, being left intact; the funnel and the anterior and posterior walls of the mantle-cavity are likewise bisected longitudinally. The left etenidium with the left nephridial sac and left branchial heart have been romoved from their natural position and displaced backwards so as to expose the other organs. The digestive gland with its ducts and the pancreatic appendages have been removed, but the position of the former is indicated hy a dotted line. app. appendage of left branchial heart ; aort. arrta ; wort'. aboral aorta ; buc. buccal mass; br. cart. section of cartilage supporting the arms; cer. g. cerebral ganglia; giz. gizzard; ink: s. ink-sac ; inf, funnel ; jaw, jaw; $l$. aur. left auricle; $l$. br. ht. left branchial heart; $l$. cten. left ctenidium; liv. position of digestive gland; $l$. neph. left nephridial sac; $n$. cart. nuchal cartilage ; oss. aesophagus; ot. cavity of statocyst laid open; ped. $g$. seetion of pedal ganglion; perist. peristomial membrane; post. $v$. abdominal vein ; $r$. aur. right auricle; $r$. cten. right ctevidium; rect. rectum; sal. salivary gland; sh. shell; st. stomach; te. testis ; $r a$. valve of fumel; $v$. cav. vena cava; vent. ventricle.

a pair of short thick processes-the optic nerves or optic stalks (opt. st.) - which expand almost immediately into large massesthe optic ganglia (opt. g.) -in immediate contact with the eyes. At the sides and posteriorly a pair of very thick commissural bånds of nerve-matter pass round the œsophagus to unite with the 
pedal and pleuro-visceral ganglia, which lie behind. The pedal ganglia (Fig. 667) are, like the cerebral, united into a single mass; orally this is prolonged and expanded into a broad mass from which the ten brachial nerves $(b r . n$.) are given off to the arms. The pleuro-visceral ganglia, also united into one, are in immediate contact with the pedal behind the osophagus.

Besides the optic nerves the cerebral ganglia also give off a pair of slender nerves which join a smaller pair of closely united buccal ganglia (Fig. 666, buc.), situated close to the buccal mass on the anterior aspect of the cesophagus. The buccal ganglia again (which are sometimes looked upon as separated portions of the cerebral) are connected by slender connectives with a pair of stomatogastric ganglia (Fig. 660, g. stom.), also closely united, situated on the posterior aspect of the œsophagus. Besides the ten brachial nerves, each of which, expanding at the base of the arm into a brachial ganglion, runs along the axis of the arm to its extremity, the pedal ganglia also give off nerves to the funnel, and also a pair to the statocysts; but the latter are found, when their fibres are traced to their origin, to be derived from the cerebral ganglia. The pleuro-visceral ganglia give off two visceral nerves (Fig. 667, visc. n.) supplying the various internal organs, one pair of branches, the branchials, having each a branchial ganglion at the base of the ctenidium, and running along its axis to its extremity. Two other ganglia of considerable size-the visceral and the gastric-occur in the course of this system. The pleuro-visceral ganglia also give off two very stout pallial nerves (pall: n.) which run through the neck to the inner surface of the mantle-cavity,

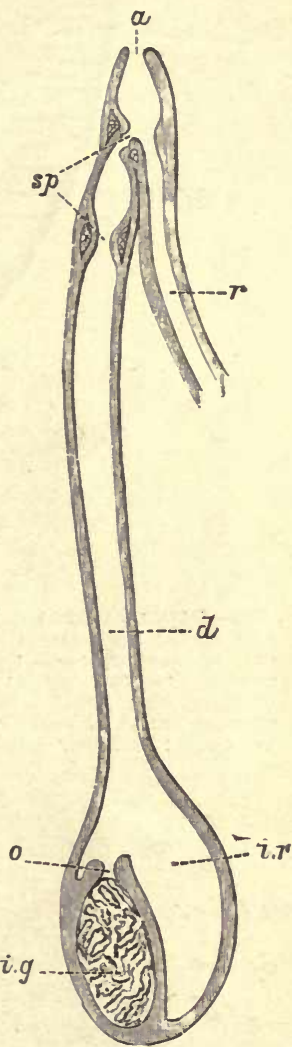

FIG. 664.-Sepia officinalis, longitudinal section of inksac. $a_{\text {. }}$ anus ; $d_{\text {. ink-duct ; }}$ $i$. g. ink-gland; $i$. $r$. cavity of ink-sac; $o$. orifice of inkgland; $r$. rectum; sp. sphincter muscles. (From the Cambridge Natural History, after Girod.) where each expands into a large, flat, pallial or stellate ganglion (Fig. $658, l$. st. g.), which is visible in front of the ctenidium when the mantle-cavity is opened. From the outer edge of this arise a number of nerves supplying the various parts of the mantle.

The organs of special sense of the Cuttle-fish are much more highly developed than those of Triton. The eyes (Fig. 668) are 
supported by curved plates of cartillage forming a sort of orbit, connected with the cranial cartilage. The significance of the

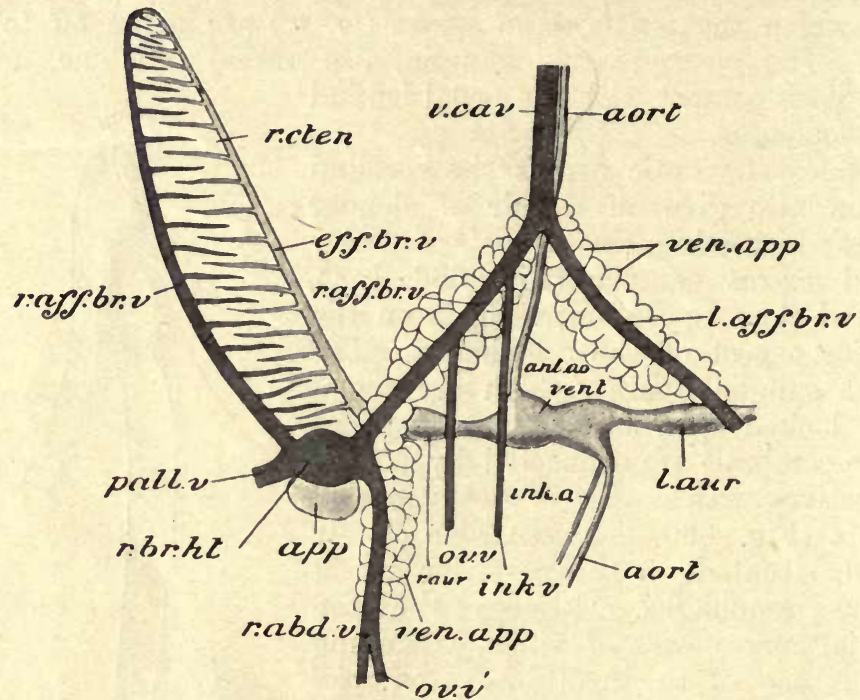

FiG. 665.-Sepia cultrata, heart and main blood-vessels from the posterior aspect. ant. ao, aort. aorta ; aort'. aboral aorta; $a p p$. appendage of right branchial heart; $e f f^{\circ} . v^{\circ} . v$. right efferent branchia] vessel ; ink. $a$. artery to ink-sac; ink. $v$. vein from ink-sac; $l . a f b^{\circ} b r \cdot v$. left afferent branchial vessel; $l$. aur. left auricle; $o v$. $v$. deep ovarian vein; $o v$. $v^{\prime}$. superficial ovarian vein; pall. $v$. pallial rein; $r$. abd. $v$. right abdominal vein; $r$. aff. $b r$. $v$. right afferent branchial vein; $r$. cten. right ctenidium; $r . b r$. ht. right branchial heart; $v$. cat. vena cava: ven. app. venous appendages; vent. ventricle.

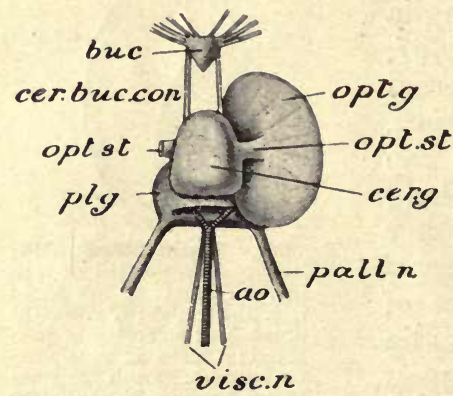

FIG. 666.-Sepia cultrata, cephalic gang. lia from the anterior aspect. wo. aorta; lnec. buccal ganglion; cer. buc. con. cere. bro-buccal connective; cer. g. cerebral ganglion ; opt. $g$. optic ganglion (removed on the left side); opt. st. optic stalk; pall. n. pallial nerve; $p l .$, . pleural ganglion; visc. $n$. visceral nerves.

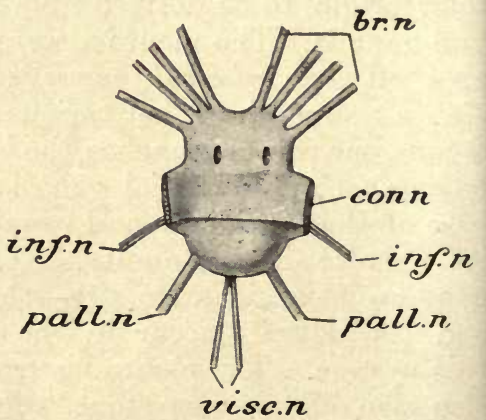

Fig. 667.-Sepia cultrata, anterior view of pedal and pleuro-visceral ganglia after removal of the cerebral and optic. $b r . n$. brachial nerves; conn. connectives be. tween the cerebral and the pedal and pleuro-visceral ganglia (cut across); int. $n$. nerve to funnel; pall. $n$. pallial nerves; visc. $n$. visceral nerves.

various parts of the eye will not be fully understood till the structure of that of the Vertebrata has been studied. A transparent 
portion of the integument covering the exposed face of the eye is termed the false cornea (corn). The eye-ball has a firm wall, or sclerotic, strengthened by plates of cartillage (scl.cart). Externally, i.e., on the side turned towards the surface of the head, this presents a large opening - the pupil. The part of the sclerotic which imme-

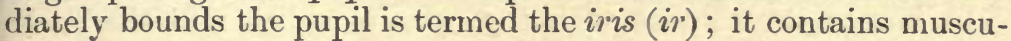
lar fibres by whose action the size of the pupil can, to a limited extent, be increased or diminished. Just internal to the iris and projecting slightly through the pupil is the lens-a dense glassylooking body of a spherical shape. The lens consists of two planoconvex lenses in close apposition; it is supported by an annular

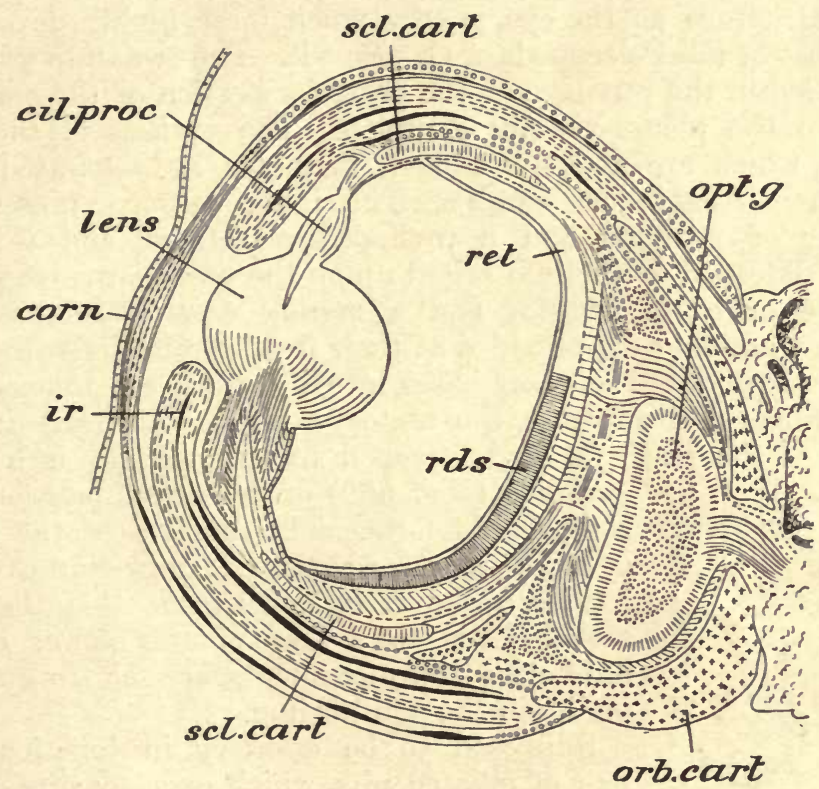

FIG. 66S.-Sepia, section of eye. cil. proc. ciliary processes ; corn. false cornea ; ir. iris ; lens, lens; opt. g. optic ganglion; orb. cart. orbital cartilage; rels. rods; ret. retina ; scl. cart. sclerotic cartilage. (From Yogt and Jung, after Hensen.)

process - the ciliary process (cil. proc.) -projecting inwards from the sclerotic. Between the two parts of the lens lies a thin layer of cells-the cornea. The lens with the ciliary process divides the cavity of the eye into two portions, a smaller outer-the cavity of the aqueous humour,-containing water, and a larger inner, containing a gelatinous substance-the vitreous humour. Over the wall of this inner chamber extends the retina (ret), the sensitive part of the eye, in which the optic nerve-fibres derived from the optic ganglion terminate. The retina is of somewhat complicated structure, consisting of a number of layers: of these that which 
immediately bounds the internal cavity of the eye is a layer of short narrow prismatic bodies-the layer of rods $(r d)$, while the outermost is a layer of optic nerve-fibres connected with the nervecells of the optic ganglion on the one hand, and with the other elements of the retina on the other.

In immediate contact with the eye, in addition to the optic ganglion, is a large soft body of unknown function, the so-called optic gland or white body, Bundles of muscular fibres bring about limited movements of the eyeball in various directions. A pair of integumentary folds of the character of eyelids are capable of being drawn over the cornea.

The statocyst ("otocyst") (Fig. 656), though not of such complicated structure as the eye, is very much more highly developed than that of the Pelecypoda or Gastropoda. The two statocysts are embedded in the cartilage of the posterior portion of the cranium close to the pleuro-visceral ganglion. The cavities of the two organs, which are about $3 \mathrm{~mm}$. in diameter, are separated by a median cartilaginous septum. The inner surface presents a number of rounded and pear-shaped elevations, and is lined with a flattened epithelium raised up on the posterior surface into a ridge or crista acustica and a macula acustica composed of large cylindrical cells provided at their free extremities with short cilia, and produced at their bases into processes continuous with nerve-fibres derived from the statocyst-nerve. Enclosed in the cavity of the statocyst and attached to the macula is a large statolith (Fig. 669) of dense composition and

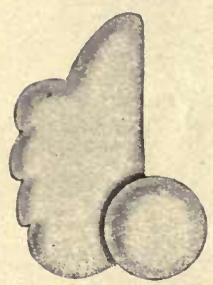

Fra. 669.- Sepia cultrata. Statolith, highly magnified. complicated form. The function of the statocysts as organs of hearing is quite unproved; it has been shown by experiment that their removal leads to a loss of the power of coordinating the movements in such a way as to maintain the equilibrium.

Supposed to be olfactory in function is a pair of eiliated pits, which open by slits on the surface behind each eye; among the ciliated cells lining the pit are numerous narrow sensory cells connected at their bases with the fibres of a nerve derived from a small ganglion situated close to the optic ganglion. A small elevation (Fig. 660, gust), covered with papillæ, on the floor of the buccal cavity just in front of the odontophore, is perhaps an organ of taste.

The excretory organs or nephridia of Sepia (Figs. 670 and 671 ) are a pair of thin-walled sacs, which open into the mantlecavity by the conspicuous excretory apertures already described. On either side is an aperture $\left(a p .^{1}\right)$ placing the cavity of the sac in communication with the pericardium, and the right and left sacs communicate with one another anteriorly and posteriorly. From 
their posterior junction is given off a median diverticulum (Fig. 671, med.s), into which the pancreatic follicles (panc.) project. Through each excretory sac runs the corresponding afferent branchial vein, formed by the bifurcation of the vena cava, and surrounding it are masses of glandular tissue (Fig. 670, ven. app), by whose agency the process of renal excretion (the products of which, in the shape of a nitrogenous excretory

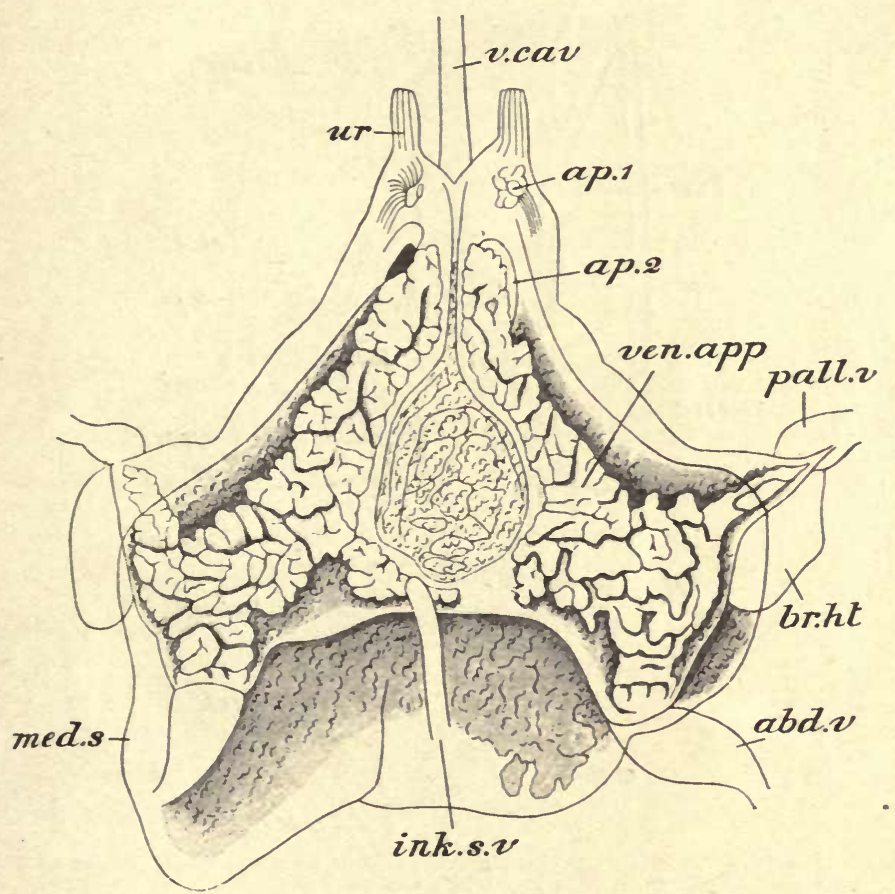

FIf. 6\%0.-Sepia officinalis, excretory organs. abl. 2 abdominal vein ; $a p l$, funnel-like opening from the pericardium; $a p^{2}$, aperture of communication between the left and the median nephridial sac; ink. $s . v$. ink-sac vein; med. s. median sac; pall. $v$. pallial vein; ur. ureter: $v$. car. vena cava; ren. app. venous appendages of the afferent branchial veins. (From Vogt and Jung, after Grobben.)

substance called guanin, are to be detected in the internal cavity) is carried on.

Reproductive system.-In the male the testis (Fig. 672, te.) forms a compact mass of minute tubules situated in the aboral region of the body and enclosed in a capsule. The single spermiduct $(v$. def) is a greatly convoluted tube which leads from the cavity of the capsule towards the right; it opens into an elongated vesicula seminalis (ves.), to which is appended a glandular body, the prostate $(p r$.$) . In the interior of the vesicula seminalis$ 
the sperms are rolled up by the action of a system of grooves and ridges into long narrow bundles of about $2 \mathrm{~cm}$. in length, each of which becomes enclosed by a chitinoid capsule of a narrow cylindrical shape, forming a spermatophore (Fig. $673, B$ ); at one end of the spermatophore is a complicated apparatus of the nature of a spring for causing the rupture of the wall and the discharge of the sperms. The vesicula seminalis expands into a wide sac-

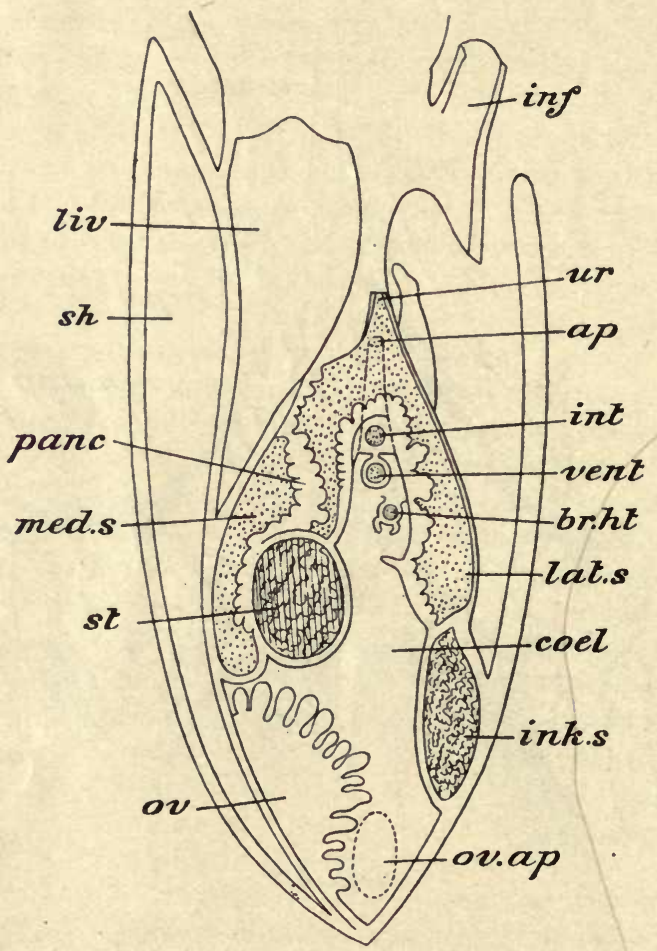

FIG. 671.-Sepia officinalis, diagram of a median vertical section of a female specimen, to show the relations of the cavities, ap. aperture between the secondary body-cavity (pericardium) and the lateral nephridial sac; $b r . h t$. branchial heart ; inf. funnel ; ink. s. in k-sac ; int. intestine; lat. 8. lateral nephridial sac; liv. liver; med. 8. median nephridial sac; or: ovary; ov. ap. aperture leading from oviduct to secondary body-cavity; panc. pancreatic appendages; $s h$. shell ; st. stomach ; ur. ureter; vent. ventricle. (From Vogt and Jung, after Grobben.)

the spermatophoral sac or Needham's sac (Fig. 672,sp. s.) -in the interior of which the spermatophores are stored. This opens into the mantle-cavity by the aperture already described at the extremity of the penis to the left of the middle line.

In the female the ovary (Fig. 658,ov.) occupies a position exactly corresponding to that of the testis in the male, and is enclosed in a similar capsule, with the cavity of which the lumen of the oviduct is continuous. An axial swelling bears numerous follicles, 
each containing a single ovum at various stages of development, and supported on a long slender stalk. At the breeding season the ovary becomes a compact mass of ova, which assume a polygonal shape owing to mutual pressure. The oviduct (ovid.) is a wide tube, opening, as already described, into the mantlecavity to the left of the rectum. Occupying a conspicuous position on the anterior wall of the mantle-cavity of the female is a pair of large flattened glands of somewhat oval outline, the

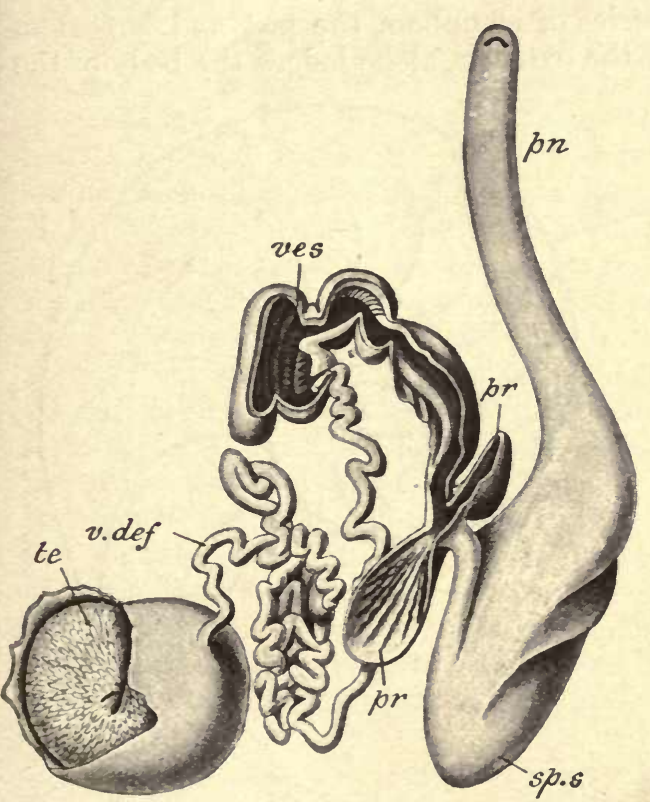

FiG. 6r2.-Sepia, reproductive organs of male. $p n$. penis pi. prostate; $s p$. s. sperm-sac; te. testis; $v . d e f$. vas deferens; ves. vesicula seminalis. (After Keferstein.)

nidamental glands (nid), situated to the right and left of the ink-duct. In the long axis of each is a median canal, on either side of which is a range of closely-set delicate lamellæ; the median canal opens into the mantle-cavity by a slit bounded by a number of plaits situated at the narrower oral end. The nidamental glands secrete the viscid material by means of which the eggs when deposited adhere together in masses. A glandular mass of unknown function, known as the accessory nidamental glands (ac. nid.), lies at the sides and around the oral ends of the nidamental glands proper. 


\section{ii. The Pearly Nautilus (Nautilus pompilius).}

The three living species of Nantilus, of which $N$. pompilius is the best known, are inhabitants of moderately shallow water about the shores and coral-reefs of the South Pacific, usually swimming near the bottom, and probably rarely, if ever, coming voluntarily to the surface. The body is enclosed in a calcareous, spirallycoiled shell (Fig. 674), into which the entire animal can be withdrawn for protection. The cavity of the shell is divided by a system of septa into a series of chambers, the last and largest of which, opening widely on the exterior, alone lodges the body of the

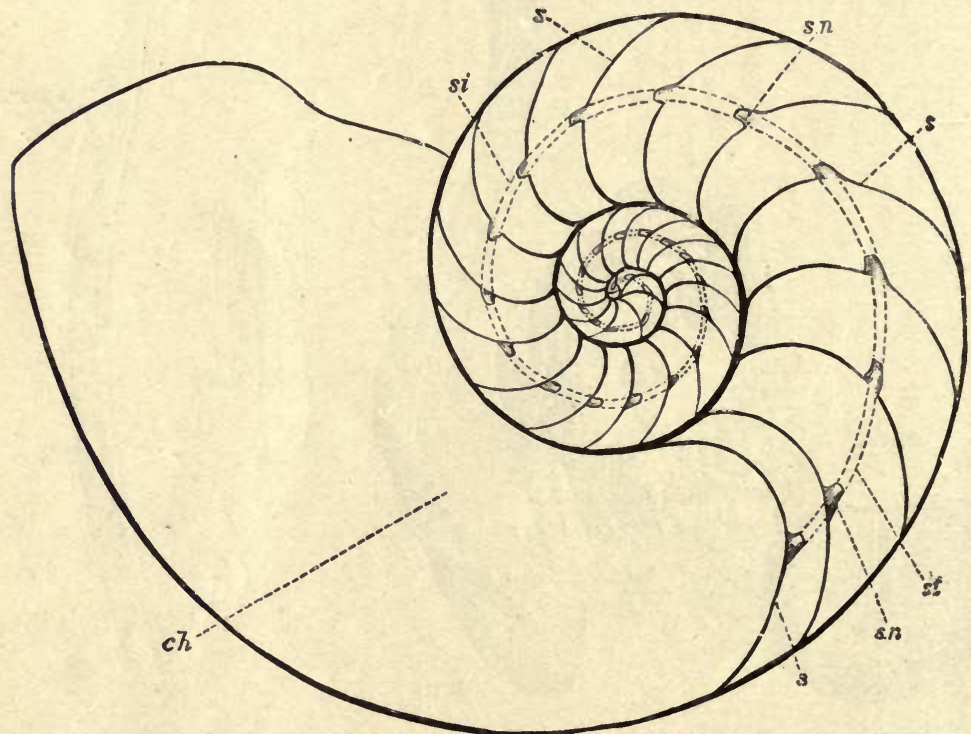

Frf. 674 .- Section of the shell of Nautilus pompilius, showing the septa $(8,8)$, the septal necks (s. n., s. n.), the siphuncle, si. (represented by dotted lines), and the large body-chamber (ch). (From the Cambrilge Natural History.)

animal. Between the animal and its shell there is a direct organic connection through the intermediation of a narrow, tubular, vascular prolongation of the visceral region, which perforates the entire series of the septa to the apex of the spiral. This tube, which is termed the siphuncle (si.), has its wall supported by scattered spicules of carbonate of lime; but, in addition, as it passes through each septum, there is produced over it for some distance a shelly tube-the septal neck (s.n.)-continuous with the substance of the septum. The apical or initial chamber presents a small scar, the cicatrix, which may indicate the original presence of the larval shell, or protoconch, which has fallen off in the course of development. 
When the shell of the Nautilus is compared with that of Triton some points of resemblance, together with important points of difference, will be at once recognised. In both the growth of the shell has taken place in such a way as to produce a gradual and regular increase in the width of the internal cavity, from the apex to the mouth, the result being a form of shell which, if it were straightened out, would be a long cone. In both the growth has not taken place in a straight line, but in a spiral, and a spiral of so close a character that successive turns are in immediate contact and their walls fused together. But in Nautilus all the turns of the spiral are in the same plane; the spiral in other words, is a flat one, as has already been found to be the case in certain of the Gastropoda (p. 737), whereas in Triton the spiral is an elongated helix: in other words, the spiral of Nautilus is that of a watchspring, that of Triton that of a corkscrew. The possession by Nautilus of the series of septa marking the position which the animal has occupied at successive stages in its growth is another striking difference. Moreover the relations of the soft parts of the shell are radically different in the two cases. In Triton the body is attached to the shell by the columellar muscle; in Nautilus the main organic connection is by means of the siphuncle; for, though it is chiefly through the pressure exerted by two great lateral masses of muscle (Fig. 675, onus.) that the Nautilus retains its hold of the shell, the muscular fibres are not attached to the latter in the same intimate way as those of the columellar muscle of Triton, but are inserted into a horny cuticular membrane intervening between the muscle and the shell. Again, while the curvature of the body of Triton with the enclosing shell is towards the ventral side (endogastric), in Nautilus it is towards the dorsal side (exogastric).

When the animal is removed from the shell it is found to possess two regions, a distinct and relatively large, obtusely conical head bearing eyes and a system of tentacles, and a rounded sac-like trunk. Both head (or cephalopodium) and trunk are very slightly compressed, the direction of the compression being, as in Sepia, from the antero-dorsal towards the postero-ventral side, and they are almost complete bilaterally symmetrical, only a very slight disturbance of the symmetry being discernible. The mouth, situated at the free extremity, is provided with a pair of relatively enormous, partly calcified jaws (Fig 675). Surrounding the mouth is a series of bilaterally arranged lobes which represent the forefoot or the epipodia of other Molluses. These are beset with numerous slender, three-sided tentacles, each provided with an elongated tubular sheath, in the interior of which the greater part of the tentacle in the retracted condition lies enclosed, only a small portion protruding. Minute ring-like markings on the tentacle are due to the presence of a number of annular constric- 
tions, which give the tentacle a transversely ridged character. There are no suckers: but the ridged surfaces enable the tentacle to adhere firmly to rough objects. The tentacles are arranged in two series, an outer and an inner. The outer, which are borne on an annular muscular ridge of the foot, are nineteen on each side in both sexes. Anteriorly this muscular ridge is thickened to form a massive lobe-the hood (Figs. 675, 676, hd.)-in which there is a concavity for the reception of the coil of the shell. The

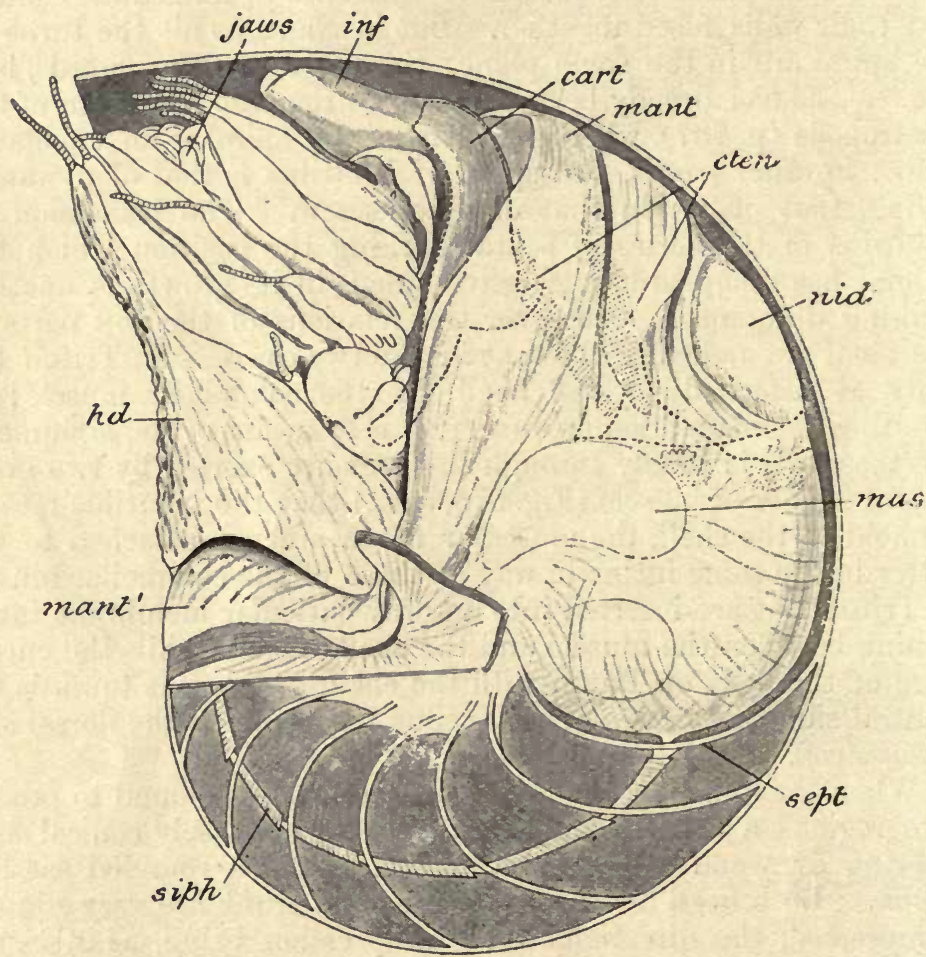

Fig. 675.-Wrautilus pompilius, diagrammatic lateral view of a female specimen enclosed in its shell. cart. cartilage ; cten. etenidia ; hd. hood ; inf. funnel ; jaros, jaws ; mant. mantle ; mant'. dorsal mantle fold overlapping the coil of the shell ; mus. position of lateral mass of muscle : nid. nidamental glands ; sept. first septum ; siph. siphuncle. (After Keferstein.)

hood bears two tentacles, and has the appearance of being composed of the immensely developed sheaths of these, completely fused together in the middle line: on each side the enlarged sheaths of a second pair of tentacles are closely applied to, though not completely coalescent with, the hood, being separated from the latter by a narrow groove. The hood, with these two enlarged sheaths, is covered with a thickened tuberculated skin, and acts after the manner of an operculum for protecting the tentacles 
and other soft parts about the head. Altogether there are fortytwo tentacles of the outer series, including four ophthalnic tentacles, one situated on the oral and another on the aboral side of each eye. The latter (ophthalmic) differ from the rest in being highly

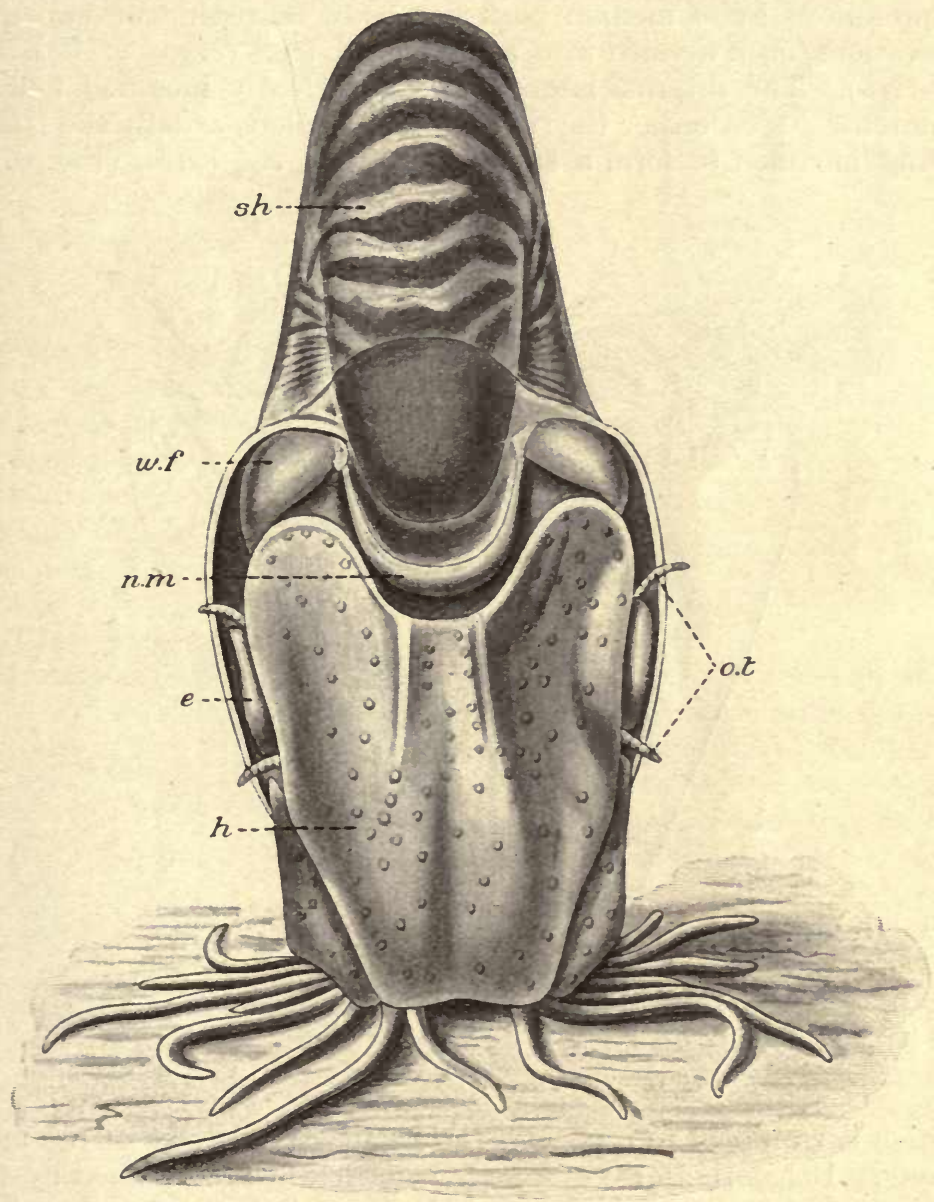

Fig. 676.-Nautilus macromphalus, adhering to the substratum in a vertical position by

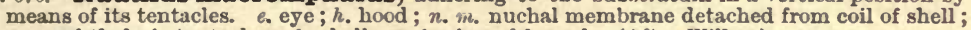
o. $t$ ophthalmic tentacles; sh. shell; $20 . f$. wing of funnel. (After Willey.)

sensitive, ciliated, and with the ridges on the inner side produced into lamellæ. The tentacles of the inner series differ strikingly in number and arrangement in the two sexes. In the female there are two inner lateral lobes, right and left, quite symmetrically developed, each bearing twelve tentacles, and an inner posterior 
lobe (Fig. 677) divided by a deep median notch into two, each half bearing twelve to fourteen tentacles. On the middle of the oral surface of the latter, close to the median notch, is an oval patch raised up into numerous closely set ridges (organ of Owen).

In the male the inner posterior lobe with its ridged organ is only represented by a median posterior body consisting of two oval elevations, each divided into a number of folds (organ of $V$ an der Hoeven). The internal lateral lobes are greatly modified, four of the tentacles on either the right side or the left, usually the latter, being modified to form a structure termed the spadix (Fig. 678),

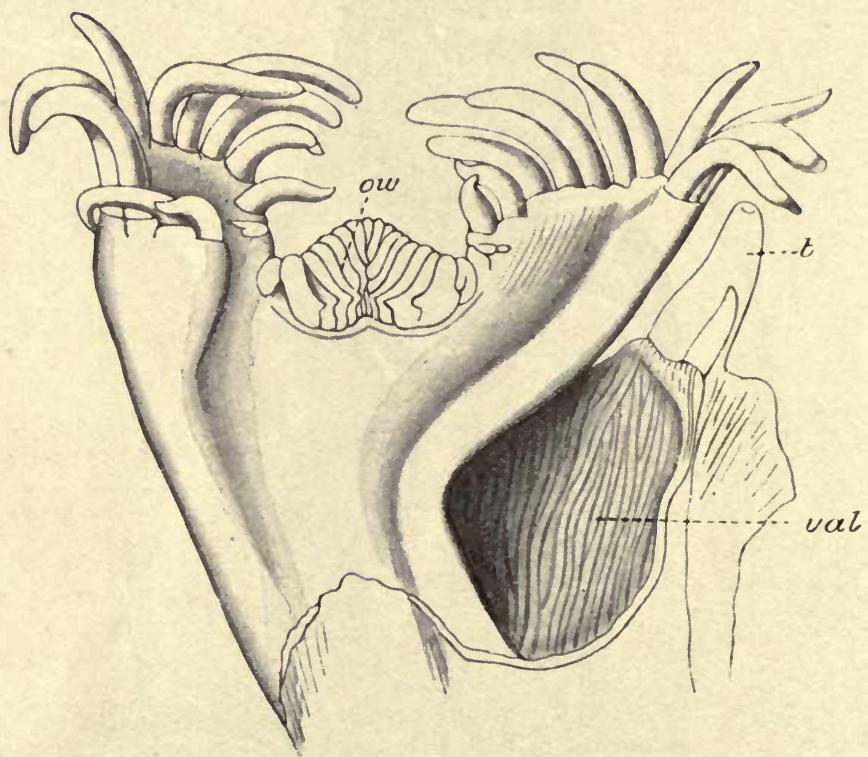

FiG. 677.-Inner posterior lobe of foot of female of Nautilus pompilius, with neighbouring parts of cephalopodium. ov. organ of Owen; $t$. one of the tentacles of the outer wing; val. organ of Valenciennes. (After Willey.)

which is supposed to represent the hectocotylised arm of the male Sepia. It has the form of a large compressed cone formed by the union of the enlarged sheaths of three of the tentacles. The corresponding tentacles themselves are in the adult male enormously thickened, and the outer surface of the most posterior (3) is covered with regularly arranged rows of minute pits. A fourth tentacle, much smaller than the others, is closely applied to the outer surface of the organ. In the internal lateral lobe, right or left as the case may be, opposite that bearing the spadix, the latter is represented by a group of four tentacles forming what is termed the anti-spadix. 
A further difference between the male and the female with regard to the foot is the presence in the latter, but not in the former, on the inner surface of the outer ring, close to the inner posterior lobe on either side, of an area thickly beset with delicate membranous ridges (organ of Valenciennes, Fig. 677, val.).

On the posterior side of the head is a funnel corresponding with that of Sepia, but extending further forwards ; this, however, does not form a completely closed tube, the edges of its right and left moieties being simply in apposition posteriorly without being united together. Near the oral end is a large, somewhat triangular valve arranged like that of Sepia.

There is an internal skeleton of cartilage (Fig. 679), as in Sepia, but its relationships with the nerve-ganglia are much less intimate in the case of Nautilus than in that of Sepia.

Mantle and Mantle-Cavity.-The mantle is produced around the head into a free flap, longer and looser than the mantle-flap of

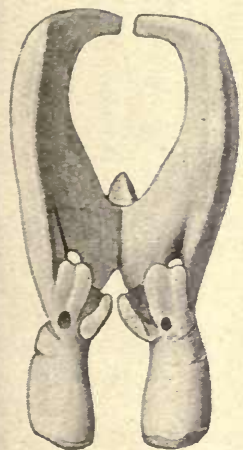

FIG. 679.- Nautilus pompilius, cartilaginous internal skeleton. (After Keferstein.)

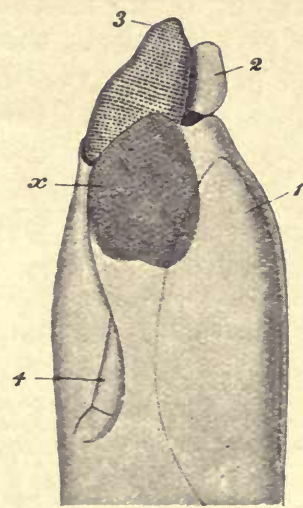

FIG. 675.-Nautilus pompilius, spadix of full-grown male, seen from the outer side. $1,2,3,4$, modified tentacles; 1 , withdrawn into its sheath, its position and shape indicated by the dotted line: 3 , the flattened tentacle with the rows of minute cavities ; $x$, patch of modified integument. Two-thirds of the natural size. (After Haswell.) Sepia. Dorsally this splits into two layers, reflected over the convexity of the shell, which fits into a hollow behind the hood. Ventrally and posteriorly the mantle encloses a large mantle-cavity (Fig. 680), corresponding to that of Sepia. In this are lodged two pairs of ctenidia (cten.), having the same general structure as the single pair present in Sepia. Between the bases of the ctenidia of each side is a small knoblike elevation, the oral osphradium (ant.os.), ${ }^{1}$ and behind the bases of the more aborally situated pair are two compressed, bilobed projections, more or less completely united in the middle so as to form a transverse ridge; these are the aboral osphradia (p.os.). In the middle line of the mantle-cavity is the anus (an.), a large

1 As in Sepia, it is convenient to use the term oral for parts towards the mouth end, and aboral for those situated towards the opposite extremity, the same terms being also used to indicate relative position of different parts. The relative position of the parts is, however, for the sake of simplicity given here as they lie when the mantle-cavity is opened by turuing back its thin posteroventral wall. 
aperture with minutely lobed margin, situated on a slight elevation, but by no means so prominent as in Sepia. On each side are two apertures, the oral and aboral nephridial apertures (Fig. 680 , a. l. neph., pt. neph.), corresponding to the single pair of Sepia, but not elevated on papillæ. Close to each posterior

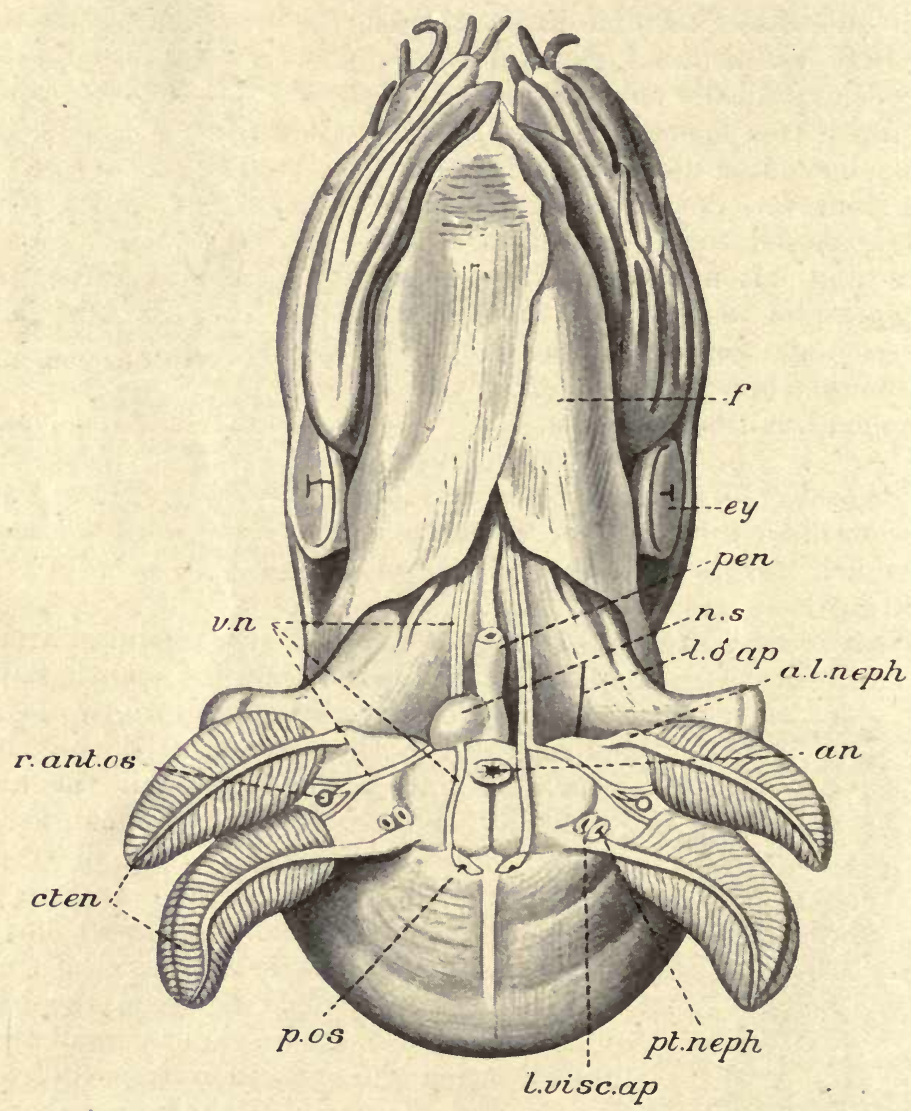

FiG. 680.- Tautilus pompilius, interior of mantle-cavity of a male speeimen with the postero-ventral wall reflected. $a . l$. neph. oral left nephridial aperture; an. anus; cten. ctenidia ; ey. eye ; $f$. fumnel ; $l$. $\delta$ ap. left reproductive aperture indicated by a bristle passed through it; $l$. vise. ap. left viscero-perieardial aperture; $n$. 8 . Needham's sae; pen. penis ; $p t$. neph. aboral left nephridial aperture; $p$. os. aboral osphradia; $r$. ant. os. right oral osphradium ; v. n. visceral nerves. (After Willey.)

nephridial aperture is an opening-the viscero-pericardial (1.visc.ap., r. visc. ap.)-leading into the pericardial section of the cœlome; these are not represented in Sepia. In both sexes there are two reproductive ducts, right and left; but in both the right alone appears to be functional, and the left is much the smaller. The 
opening of the right sperm-duct of the male is situated on a cylindrical prominence-the penis (pen.) - placed close to the middle line. In the female the nidamental glands are, as in Sepia, conspicuous objects when the mantle-cavity is exposed; but they are mainly situated on its posterior instead of its anterior wall.

Enteric Canal. - The mouth is surrounded by a peristomial - membrane beset with numerous papillæ. There is a pair of jaws (Fig. 681, jaw') of similar shape to those of Sepia, but much more powerful, and calcified towards the tips. The buccal mass is a large rounded body with thick muscular walls. On the floor of the contained cavity is a large and prominent odontophore (odont.), with long and pointed, curved teeth. In front of the odontophore is a large bilobed soft prominence, the tongue (tong.). Behind the odontophore, between it and the opening of the œsophagus, are one large median and two lateral tongue-like prominences beset with papillæ; on the inner surface of the latter are the apertures of a pair of salivary glands.

The œesophagus (œs.) becomes dilated aborally into a very spacious crop (cr.) for the storage of the food, which consists of small prawn-like Crustaceans and small Fishes broken up by the jaws and radula. This opens into a rounded stomach (stom) having very much the appearance of the gizzard-like cæcum of Sepia. The intestine (int.), shortly after it leaves the stomach, develops a rounded cæeum (cœe.) with complexly folded walls, into which the ducts of the digestive gland or "liver" open. The intestine does not pass straight to the anus as in Sepia, but first bends round in a short coil. The ink-sac and duct of Sepia are not represented. There is a very large digestive gland divided into four main portions or lobes, each of which is made up of a number of lobules. The ducts ("bile-ducts," $b . d u_{\text {. }}$ ), opening as above mentioned into the cæcum, have a series of small diverticula which may represent the pancreatic appendages of Sepia.

The cœlome consists of the pericardium and the gonocoele-the cavity in which the gonad is enclosed: these communicate with one another by three apertures. The pericardium contains the ventricle, the four auricles, and parts of the renal glandular appendages. It communicates with the exterior by the visceropericardial apertures.

Heart and Vascular System.-The vascular system consists of the heart, the arteries and veins, and certain large spaces constituting the hæmocœle. The latter consists of three chief parts-the peristomial, peri-œsophageal and peri-hepatic hæmocœles, the first surrounding the buccal mass, the second the osophagus, and the third the liver.

The ventricle (Figs. 681 and 683 , vent.) is a bilobed, transversely placed, muscular sac, very similar to that of Sepia. On either side 


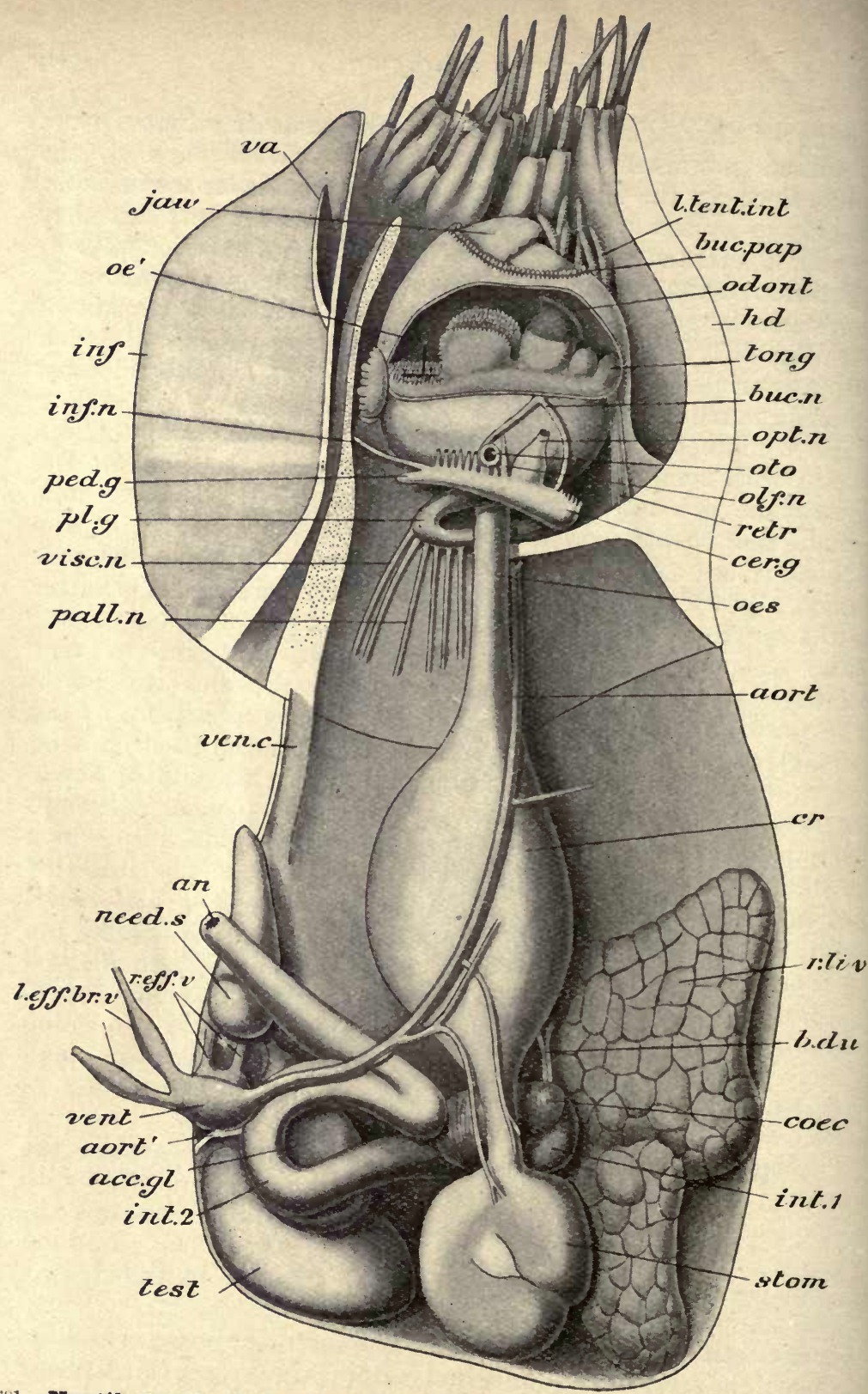

Fig. 681.- Nautilus pompilius, dissection of the internal organs of a male, from the left side. T buccal cavity has been removed to show the longitudinal median section. A portion of the wall of th an. anus; aort. oral aorta; wort'. posterior padontophore and the tongue. acc. gl. vesicula seminalis pap. papillæ of peristomial membrane; pallial artery ; $b . d u$. bile-ducts; buc. n. buccal nerves; buc funnel; inf. $n$. infundibular nerve ; intestine following cæcum; jaze, larger in part of intestine between stomach and crecum ; int.2 part int. left internal tentacular lobe ; neel. 8 . Needham jaw ; $l$. eff. $b l . v$. left efferent branchial vessels; $l$. tent cavity into the opening of the ; need. 8 . Needham's sac ; ollont. odontophore; $\alpha^{\prime}$. style passed from buce oto. statocyst; pall. n. pallial nerves;us; ws. cesophagus; olf. $n$. olfactory nerve; opt. n. optic nerve

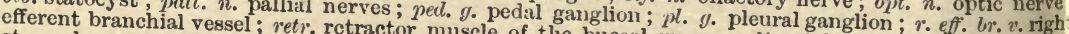
stomach; test. testis ; tong. tongue-shaped ven. c. vena eava; vent. ventricle. 
there open into it two auricles or efferent branchial vessels (a.or.), one from each of the four ctenidia. The ventricle gives off a large main aorta (cort.), which passes to the head after giving off arteries to the stomach, the crop, the digestive gland, and the mantle. From the aboral surface of the ventricle arises a smaller artery, the lesser aorta, which immediately bifurcates. One of its branchesthe posterior pallial artery (Fig. 682 , post. pall. a.)-passes to the area of the mantle applied to the septum, bifurcates to supply this area, and gives off a branch to the siphuncle. The other-anterior

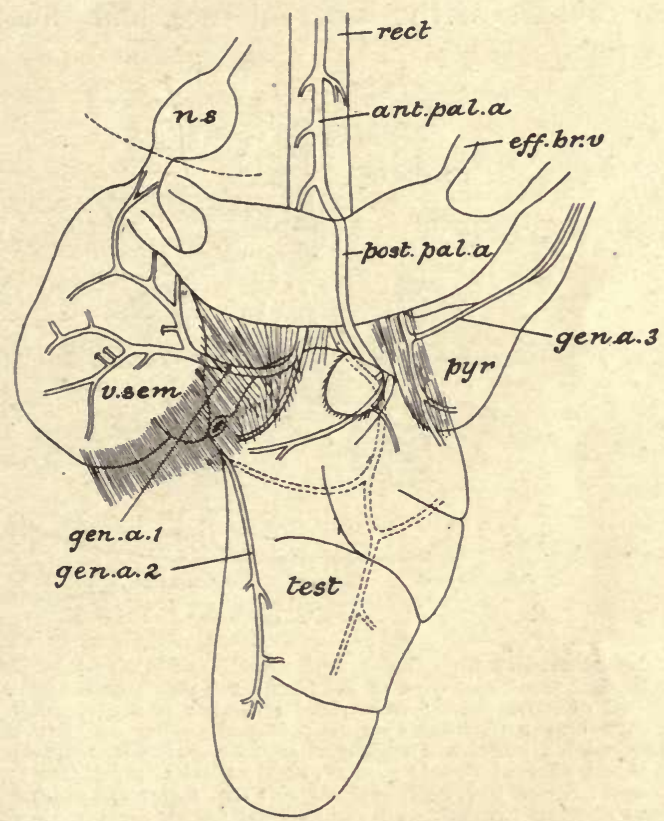

FIG. 6S2. - Nautilus pompilius (male), origin of pallial and genital 2rteries. ant. pal. a. anterior pallial artery; $e f f . b{ }^{\circ}$. $v$. efferent branchial veins; gen. $a .1$, artery to vesicula seminalis $(v .8 \mathrm{em}$.$) ; gen. a. 2, testicular artery and its branches; gen. a .3$, artery to pyriform sac; n. s. spermatophore-sac; post. pall. a. posterior pallial artery; pyr. pyriform sac; rect. rectum; test. testis. (After Willey.)

pallial (ant. pall. a.)-after giving off arteries to the intestine and rectum, and to the branchiæe and osphradia, passes to the nuscular edge of the mantle, bifurcating anteriorly. Three genital arteries (gen. a. 1, 2, 3), supplying the various parts of the reproductive apparatus, are likewise given off directly from the ventricle.

A large vena cava (Figs. 681 and 683, ven. c.) occupies a position corresponding closely with that which it occupies in Sepia. It presents the remarkable peculiarity of being in free communication by numerous (valvular) apertures with the general cavity of the 
hæmocœle. At its aboral end it presents a dilatation from which four afferent branchial veins (Fig. 683 a.l.aff, p.l.aff, p.r.aff, r.ant. aff.) - two right and two left-proceed to the corresponding ctenidia, at the bases of which veins from the aboral region join them. There are no branchial hearts.

The renal organs (Fig. 683) are, like the ctenidia and the afferent and efferent vessels, four in number, instead of two as in Sepia. Each renal sac (l.neph. s., r. neph. s., l. post. neph. s., r. post. neph. s.) opens into the mantle-cavity, as already stated, by an orifice which is not drawn out into a tube. There is no communication between the cavities of the different sacs, and thus no median

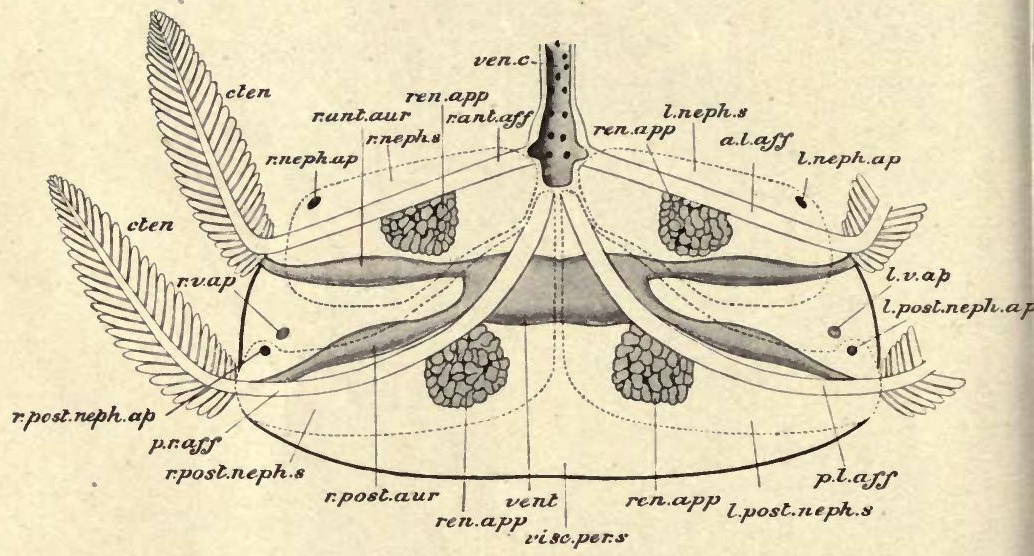

FIG. 683.- Nautilus pompilius, renal sacs, with ctenidia and other related parts, as seen from the posterior aspect; the boundaries of the four renal sacs represented by dotted lines. a. $l$. aff. left oral afferent vessel; cten. right ctcuidia; $l$. neph. s. left nephridial sac; l. neph. ap. left oral nephridial aperture; $l$. post. neph. ap. left aboral nephridial aperture; l. post. neph. 8. left aboral nephridial sac; l.v.ap, left viscero-pericardial aperture ; p. $l$. aff. left aboril afferent vessel; $p, r$. aff. right aboral afferent vessel; $r$. ant. aff. right oral afferent vessel; $r$. ant. aur. right oral auricle; ren. app. renal appendages; $r . n e p h . a p$. right nephridial aperture; $r$. post. aur. right aboral auricle; $r$ post. neph. 8 . right aboral nephridial sac; $v$. v. ap. right viscero-pericardial aperture; ven. $c$. vena cava; vent. ventricle; visc. per. 8. viscero-pericardial sac.

chamber as in Sepia, and there is also no communication with the pericardium. The cavities are found to contain phosphate of lime. Into each projects, from the corresponding afferent branchial vein, a compact rounded group of venous appendages (ren. app.), consisting of two symmetrical portions. Internal to these, each afferent vein has connected with it a second group of glandular appendages, which are cylindrical or club-like in form; they project, not into the nephridial sac, but into the pericardial compartment of the colome. They have been compared with the appendages of the branchial heart of Sepia, but differ in their relations to the renal appendages. 
Nervous System.-Nautilus differs strikingly from Sepia, and somewhat resembles Chiton (p. 716, Fig. 604) in the form assumed by the central parts of the nervous system (Fig. 681, cer. g.), distinct ganglia being absent. A very thick nerve-collar, the posterior portion of which is double, surrounds the œesophagus just behind the buccal mass. The anterior part of the collar (Fig. 681, cer.g.) represents the cerebral ganglia, the oral portion of the posterior part the pedal (ped.g.), the aboral portion the pleuro-visceral $(p l . g$.$) ; while the lateral parts, not$ distinctly marked off from the rest, represent the cerebro-pedal and cerebro-pleural connectives. From the cerebral ganglia pass nerves to the buccal mass, to the olfactory organs (olf. n.) and the statocysts, and a pair of very thick optic nerves supply the eyes $(o p t . n$.). The pedal ganglion gives off numerous nerves to the tentacles and the funnel, and from the pleuro-visceral arise pallial and visceral nerves.

Sense Organs.-The statocysts (otocysts) are a pair of sacs embedded in recesses close to the cerebral ganglia, but not enclosed in the cartilage of the endoskeleton; each contains a number of microscropic statocones. An olfactory function is ascribed to a. process (the rhinophore) with a ciliated pit at its base, situated on the aboral side of the eye. The ophthalmic tentacles (Fig. 676, o.t.) are supposed to act as accessory olfactory organs. The osphradic (p. 781) contain ganglion-cells, are beset with sensory cilia, and are undoubtedly organs of special sense.

The enes, situated at the sides of the head, are very large but extremely simple in structure, presenting a marked contrast to those of Sepia, and scarcely comparable to those of any other animal with the exception perhaps of Patella (p. 746). Each is of the shape of a saucer, attached to the head by its convex side by means of a short thick stalk, the mouth being closed in by a slightly convex disc, with a circular aperture at about its centre. A slightly raised rim runs round close to the margin on the posterior half, and a narrow groove extends inwards from this to the central aperture. In the interior of the cup is neither lens, vitreous humour, nor iris. The sea-water, passing in through the central aperture, directly bathes the retina, which is spread over the interior in a thick layer.

Reproductive Organs.-The gonad (testis, Fig. 684, test., or ovary, Fig. 685, ov.), like that of Sepia, is single and median, enclosed in a special sac towards the aboral end of the body. The duct is paired in both sexes, but in both the right alone appears to be functional. In the male a large glandular vesicula seminalis, in which the spermatophores are formed (acc.) is connected with the right duct, and this appears to be represented on the left-hand side by a vestige-the so-called pyriform sac (pyr.), situated close to the ventricle. The distal part of the right duct dilates to form 
a receptacle, the spermatophoral sac or Needham's sac (sp. s.), and opens, nearly in the middle line at the end of a prominence-the penis (Fig. 680, pen.). In the female the right oviduct has a

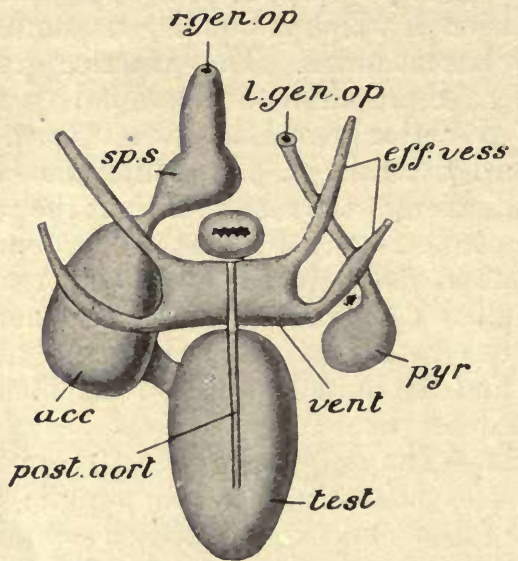

FrG. 684.-Nautilus pompilius, male reproductive organs. acc. vesicula seminalis; eff. vess. efferent branchial vessels; $l$. gen. op. left genital opening; post. ao. posterior aorta ; pyr. pyriform appendage; $r$. gen. op. right genital opening; $8 p$. s. spermatophore-sac; test. testis; vent. ventricle.

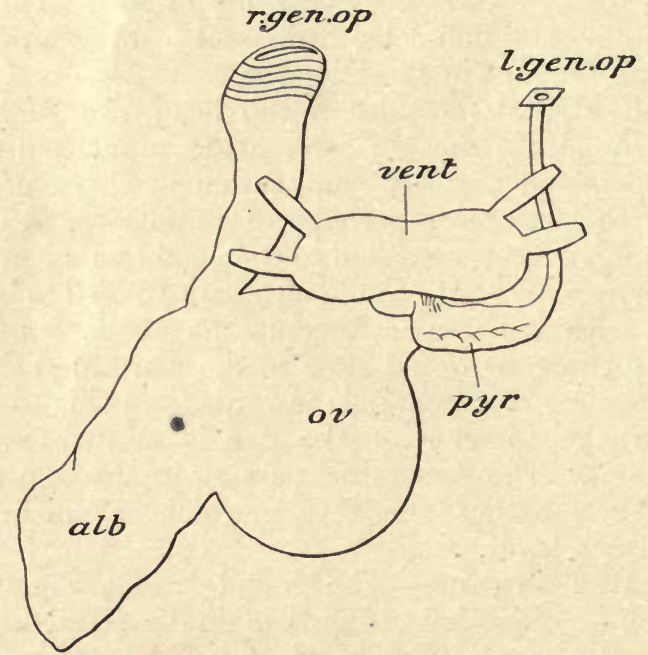

FIG. 685.-Nautilus pompilius, female reproductive organs. alb. albumen-gland; $l$. gen.op. left genital opening ; ov. ovary; pyr. pyriform appendage ; $r$. gen. op. right genital opening ; vent. ventricle. (After Lankester and Bourne.)

glandular dilatation, which is supposed to be an albumen gland. The ova are of large size, greatly exceeding those of Sepia in dimensions, containing a large proportion of food-yolk. Nidamental 
glands are present, but are mainly situated, as already pointed out, on the posterior instead of the anterior wall of the mantlecavity. Each egg becomes enclosed in an elaborate capsule (Fig. 686), probably moulded by the agency of the organ of Owen, on the inner posterior lobe of the foot of the female (Fig. 677). The development is not known.

\section{Distinctive Characters AND Classification.}

The Cephalopoda are bilaterally symmetrical Mollusca, which have the main part of the foot displaced forwards to the neighbourhood of the mouth and divided into a series of arms bearing suckers, or of lobes bearing tentacles, while the remainder of the foot forms a funnel for the

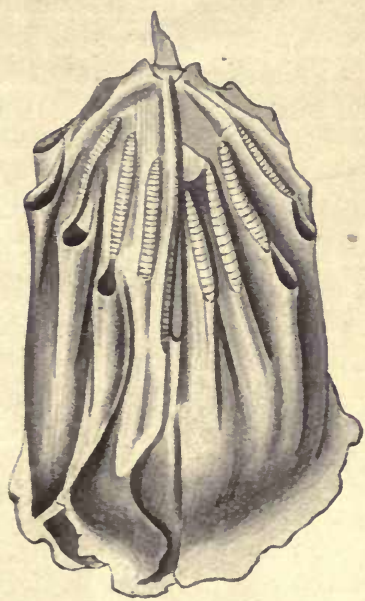

FIt. 686.-Egg of Nautilus macromphalus, enclosed in its capsule. (After Willey.) egress of water from the mantle-cavity.

The visceral mass is symmetrical and not coiled. The mantle encloses posteriorly and ventrally a large mantle-cavity, in which are situated the ctenidia and the nephridial, reproductive, and anal apertures. The shell may be absent or rudimentary; when present and well developed,it may be internal or external, undivided, or divided internally by septa into a series of chambers. There is an internal cartilaginous skeleton, supporting and protecting the nerve-centres and giving attachment to muscles. The mouth is provided with a pair of horny jaws, and an odontophore is present. In the majority there is an ink-gland with a duct opening into the rectum. The ctenidia and nephridia are either two or four in number. The nervous system is highly developed; and the principal nerve-ganglia are aggregated together around the œsophagus. The sexes are separate; the segmentation of the ovum is meroblastic, and there is no metamorphosis.

\section{Sub-Class I.-Dibranchiata.}

Cephalopoda in which the main part of the foot assumes the character of a circlet of either eight or ten arms, bearing suckers, and surrounding the mouth. The funnel forms a complete tube. The shell is usually internal ; when external its cavity is not divided by septa. There are two ctenidia, two nephridia, and two branchiocardiac vessels or auricles. An ink-gland and duct are present. 


\section{ORDER 1.-DECAPODA.}

Dibranchiata possessing ten arms, with stalked suckers provided with horny rims, and with a well-developed internal shell.

This order includes the Cuttle-fishes, Squids, Spirula, and others, as well as the extinct Belemnites.

\section{ORDER 2.-OCTOPODA.}

Dibranchiata provided with eight arms, the suckers on which are sessile and devoid of horny rims: with or without slight vestiges of an internal shell. An external shell, secreted by a speciallymodified pair of arms, is present in the female Argonaut only.

This order includes the Octopods and the Argonauts.

\section{Sub-Class II.-Tetrabranchiata.}

Cephalopoda in which the main part of the foot has the character of lobes bearing numerous tentacles. The funnel does not form a complete tube. There is an external, spiral, chambered shell. There are four ctenidia, four nephridia, and four auricles. An ink-gland is absent.

This sub-class includes only one living genus, Nautilus, but the Ammonites and other extinct forms are usually referred to it.

\section{Systematic Position of the Examples.}

The genus Sepia is a member of the family Sepiidoe of the order Decapoda, which is distinguished from the seven other families of the order by the combination of the following features:-The body is compressed and comparatively broad; the fins are narrow and elongated; the internal shell consists almost entirely of calcareous material.

Nautilus is the sole living representative of the sub-class Tetrabranchiata.

\section{General Organisatiun.}

The uniformity of structure among the Dibranchiate Cephalopoda is very great, and, as already stated, Nautilus is the only living member of the Tetrabranchiata, so that comparatively little has to be said to supplement the descriptions of the two examples.

External Features. - The general external shape differs very little in the different members of the Dibranchiata: the body in some is more elongated, in others, less; the degree of compression likewise varies. Fins may be absent, and the animal may pro- 
gress entir!'s by creeping with the aid of the long arms, or by swimming by the movements of the arms, or under the propulsion of a current of water forcibly ejected through the funnel by the contraction of the muscular mantle (Fig. 687). When fins are present they may take the form of a continuous lateral flap as in Sepia, but, more usually, are of the nature of flattened lobes situated towards the aboral extremity of the body (Fig. 688); in Ctenopteryx they have the character of fringes of filaments. The arms vary in length and proportions and in the form and arrangement of the suckers. Eight arms are present in the Octopoda and ten in the Decapoda. In the former group the

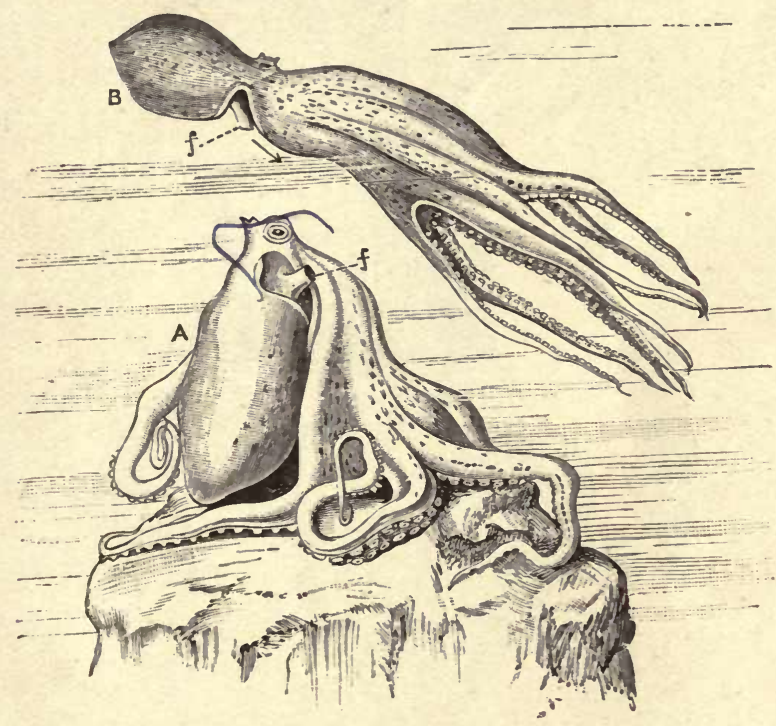

Fic. 687.-Octopus vulgaris. $A$, at rest; $B$, in motion; $f$. funnel, the arrow showing the direction of the propelling current through the water. (From Cooke, after Merculiano.)

Argonauts (Fig. 689) have, in the female, one pair of arms (wa.) flattened and expanded at the extremities for the secretion and support of the shell $(s h$.). In the Decapoda one pair of arms, the fourth, is always specially modified, as in Sepia, to act as prehensile appendages or tentacles capable of being partly or entirely retracted within certain sacs situated at their bases. In nearly all one of the arms is specially modified (or hectocotylised) to act as an intromittent organ. This modification is only very slight in Sepia and confined to the base, and is most marked in certain of the Octopoda (Fig. 690), including the Argonauts. In the latter, before the breeding season, the third arm in the male is found to be represented by a rounded sac, which subsequently 
bursts and sets free the elongated hectocotylised arm. Spermatophores are taken by the arm from the genital opening, and in the act of copulation the entire arm is detached and left in the mantle-cavity of the female. In other cases the arm is not detached. The suckers are sometimes stalked, sometimes sessile, sometimes armed with hooks, sometimes replaced by hooks. In many cases the arms are united by a web-like fold, the inter-

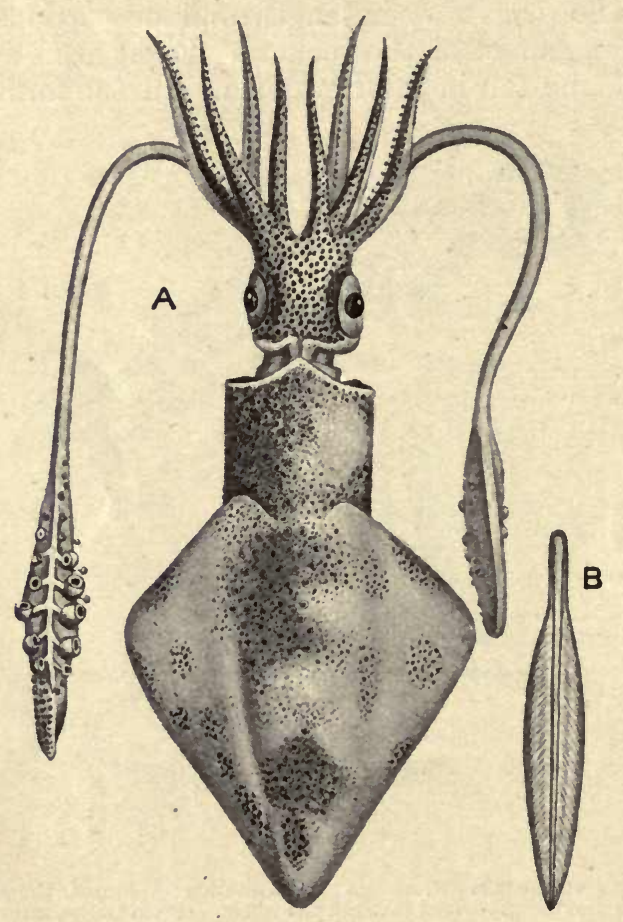

Fic. 688.-Ioligo vulgaris. A, entire animal, dorsal view; B, horny internal shell or pen. (From Keferstein.)

brachial membrane (Fig. 691), which may reach nearly to their extremities.

In the Tetrabranchiata the series of groups of slender, ringed, sheathed tentacles, situated on lobes of the foot surrounding the mouth, take the place of the arms, and suckers are not present. In the males the spadix probably represents, functionally at least, the hectocotylised arm of the Dibranchiata.

In all the Dibranchiata the funnel is a complete tube. In the Nautilus, on the other hand, as we have seen, the folds which form the funnel have their edges merely in apposition, and not united. 
A valve, such as has been described in Sepia, occurs in most Decapoda and in Nautilus, but is absent in the Octopoda.

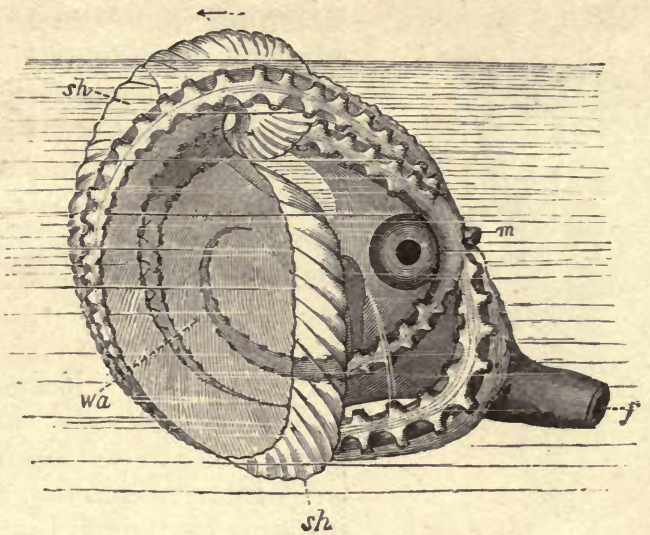

Fig. 659.-Argonauta argo, female, showing the relations of the animal to the shell in the living state, the arrow showing the direction of movement. $f$. funnel ; $m$. mouth, with jaws projecting; $8 h$. shell, with arms as seen through it; $v a$, webbed arm clasping the shell. (From Cooke, after Lacaze-Duthiers.)

Chromatophorcs, similar to those of Sepia, are universal in the Dibranchiata but absent in Nautilus.

Shell.-The shell of Nautilus is the most complete and yet in a certain sense the most primitive. As already stated, it is an external shell of a spiral character, divided internally by septa into a series of chambers. The last of the chambers is occupied

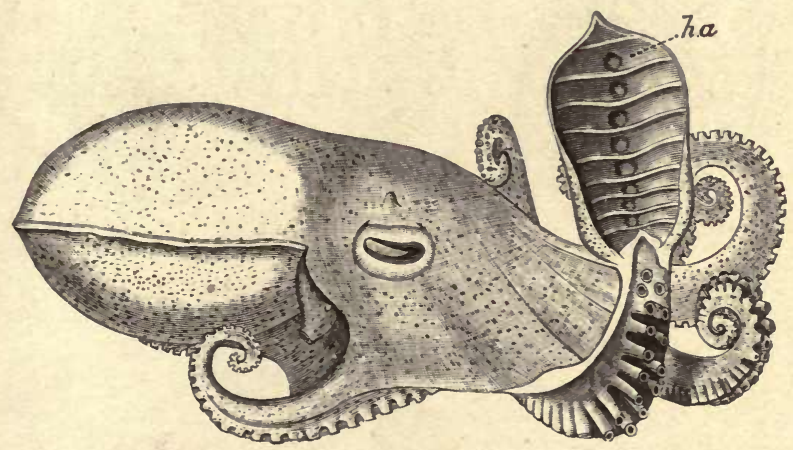

FIG. 690.-Octopus lentus, male specimen, showing the structure of the hectocotylised arm (h. a). (Frmm Ccoke, after Verrill.)

by the body of the animal; the rest are filled with gas. Perforating the middle of all the septa in succession is a spiral tube-the siphuncle-continuous with the centro-dorsal region of the visceral 
prominence. In the course of its growth the body of the Nautilus shifts forwards at intervals into a newly formed chamber, and a

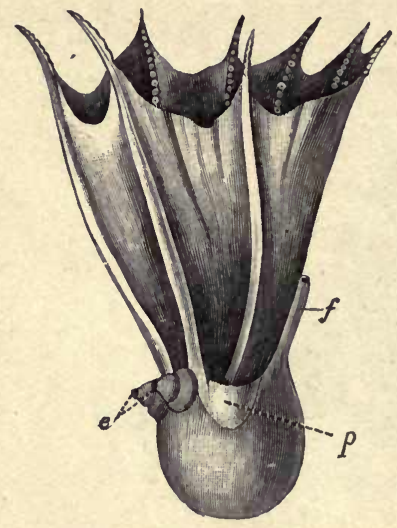

FiG. 691.-Amphitretus pelagicus, an Octopod with the arms united by a web. $e$. eyes; $f$. funnel; $p$. poueh in the mantle: (From Cooke, after Hoylc.) new septum is formed closing the latter off from the cavity last occupied. It is only after the last septum has been formed that the animal attains sexual maturity.

Of existing Dibranchiata, Spirula alone has a shell (Fig. 692) comparable to that of Nautilus. The shell of Spirula is of spiral form, the turns of the spiral, however, not being in close contact. Internally it is divided into chambers by a series of septa, and these are perforated by a siphuncle. But the initial chamber (protoconch) instead of being, like the initial chamber in Nautilus, similar to the others though smaller, is dilated into a spherical shape, constricted off from the succeeding chamber, and has passing through it a tube-the prosiphon-not continuous with the siphuncle. Again, as will be seen by comparing Figs. 675 and 693 , the relation of
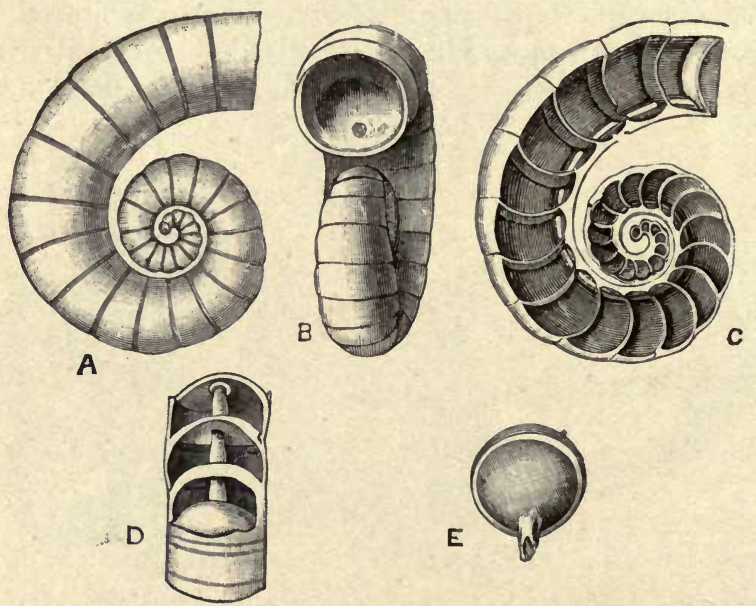

Frg. 692.-Shcll of Spirula. A, outside view; B, showing last chamber and position of siphuncle; C, in section, showing the septa and the course of the siphuncle; $\mathbf{D}$, shell broken to show the convexity of the inner side of the septa; $\mathbf{E}$, portion of a septal neck. (After
Cookc.)

the soft parts to the shell is the reverse of what obtains in Nautilus, the shell of Spirula curving backwards (endogastric 
curvature), that of Nautilus forwards (exogastric curvature). Moreover the shell of Spirula is an internal structure, being almost completely covered by the mantle.

The shell of the extinct Ammonites (Fig. 694), which are usually referred to the Tetrabranchiata, resembles that of the Nautilus in many respects, being a chambered spiral shell with a large terminal chamber, and with a siphuncle. The chief external difference is in the form of the sutures, or lines of union of the edges of the septa with the side wall of the shell; these are more or less complexly lobed, instead of being entire as in Nautilus. But in one important respect the shell of an Ammonite differs from that of Nautilus and approaches that of the dibranchiate Spirula. At the apex of the spiral is an initial chamber or protoconch, which is dilated and separated from the first of the ordinary chambers by a constriction, and has passing into it a prosiphon not continuous with the siphuncle. The Ammonite was also characterised by the possession of a paired or unpaired structure, sometimes horny, sometimes calcareous, called the aptychus, not represented

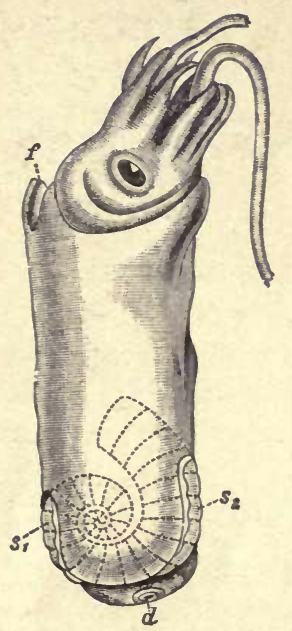

FiG. 693.-Spirula peronii, lateral view. $d$, terminal sucker; $f$. funnel; $8, s^{1}, 8^{2}$. projecting portions of the shell, the internal part of which is indicated by dotted lines. (From Cooke.) in any existing form. The aptychus, which was composed of two parts, may have been of the nature of an operculum for closing the mouth of the shell, but was more probably endoskeletal. Young Ammonites, each with its aptychus, have been found within the

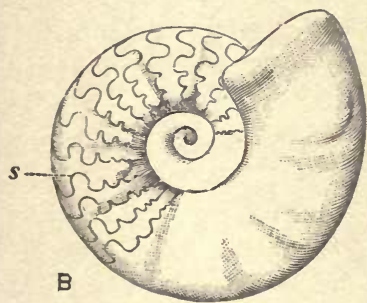

Fig. 694.-An Ammonite (Ceratites nodosus). shell of the parent, in which they must have remained protected during their development.

In the ordinary decapod Dibranchiata the shell may consist of three parts-a horny pen or pro-ostracum, a calcareous guard, and a part termed the phragmacone. The last, which alone represents the shell of Spirula, has the form of a cone divided internally by a series of septa perforated by a siphuncle. These parts are most completely developed in the extinct genus Belemnites, in which the shell (Fig. 695) consists of a straight, conical, chambered phragmacone (phr.), with a siphuncle, enclosed in a calcareous 
sheath, the guard, produced into a horny or calcareous plate, the pro-ostracum (pen.). In Sepia the spine-like projecting point represents the guard, and the main sub-

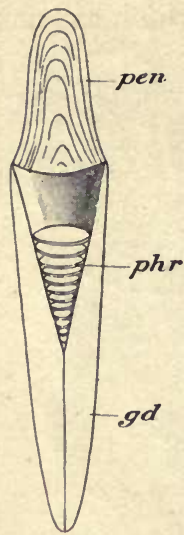

FiG. 695.-Shell of a Belem nite. gd. guard; pen, pro-ostracum; phr: phrag. mocone. (From Nicholson and Lydekker's Pulceonto. logy.) stance of the shell is to be looked upon as the pro-ostracum and phragmacone, the septa of the latter being represented by the calcareous lamellæ. In the Squids (e.g., Loligo) the shell (Fig. 688, B) is long, narrow, and completely horny; it corresponds to the pro-ostracum, the phragmacone being entirely absent.

In Octopus the shell is represented only by a pair of vestiges with which muscles are connected. In Argonauta there is no shell in the male, but the female has an external shell (Fig. 696) of a rernarkable character. This is a delicate spiral structure the internal cavity of which is not divided into chambers. It is not secreted by the mantle like the shells of other Mollusca, but by the surfaces of a pair of the arms ending in expanded disc-like extremities, which become applied to its outer surface (Fig. 689); its chief function is to carry the eggs. An internal cartilaginous skeleton is present not only in Sepia and Nautilus, as already described, but in all the Cephalopoda. Such an internal skeleton occurs in other groups-some

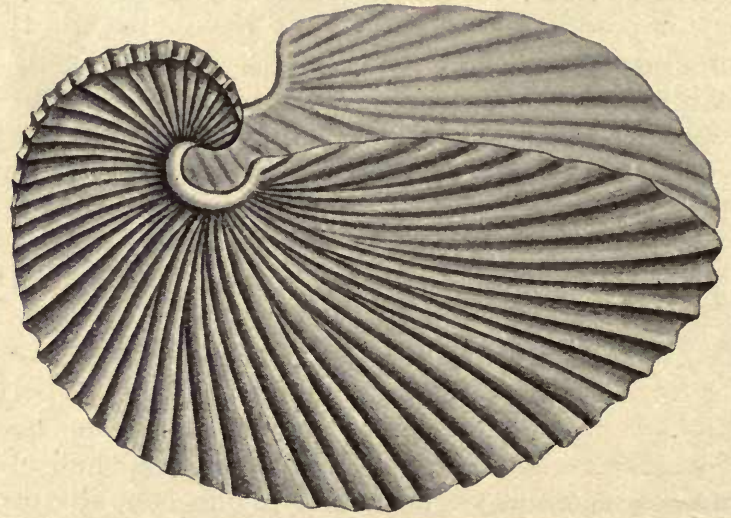

FIG. 696.- Shell of Argonauto argo.

Chretopoda (p. 472), Crustacea, and Arachnida (p. 667)-but attains a much more elaborate character in the present group than in any other Invertebrates. 
The plume-shaped gills, lodged in the mantle-cavity, are two in number in all the Dibranchiata, as in Sepia. In the Tetrabranchiata there are four gills, similar in general character to those of the Dibranchiata.

The cœlome in the Dibranchiata has the the extent already indicated (p. 766) in the case of Sepia, except that in the Octopoda the oral part does not exist. In Nautilus it encloses, besides the heart and gonad, a part of the glandular appendages of the afferent branchial vessels. In the Dibranchiata the pericardial portion communicates with the nephridia; in Nautilus this communication is absent, but the colome opens on the exterior by two symmetrical viscero-pericardial orifices placed at the side of the openings of the aboral nephridia.

Alimentary Organs.-Jaws similar to those of Sepia are present in all the members of the class; in Nautilus, instead of being completely horny, they are partly calcified. Buccal mass, œsophagus, stomach, intestine, salivary glands, and digestive gland are all of the same general character throughout all the members of the class. In some of the Dibranchiata, such as Octopus, there are two pairs of salivary glands. In Nautilus the salivary glands are absent, so far as known, the cesophagus is dilated to form a sort of crop, and the stomach is gizzard-like. In that genus also the ink-gland, general in the Dibranchiata, is absent, and there is a cæcal appendage to the intestine; the digestive gland is four-lobed, each lobe having its duct. The so-called pancreas, described in Sepia, is similarly developed in all the Dibranchiata, and is present also, though only feebly developed, in the Tetrabranchiata.

Heart and vascular system are well developed in the Cephalopoda, and their structure and arrangement closely correspond with what has been described in Sepia, except that in Tetrabranchiata there are, as already stated, in accordance with the double number of gills, four auricles instead of two, and branchial hearts are absent.

Nervous system and sense-organs. - The ganglia of the central nervous system are in all closely aggregated together round the œsophagus, as already stated to be the case in Sepia; and the general disposition is the same as that described. In Octopus the ganglia are much less sharply marked off. In Nautilus, as already mentioned, there is less concentration, and distinct ganglia are not recognisable. All the Dibranchiata possess highly developed eyes similar to those of Sepia; but in Nautilus the eyes are of a much simpler character, each consisting of a sac opening on the exterior by a small rounded aperture, lined internally by a two-layered retina similar to that of Sepia, but without lens, vitreous humour, or cornea. In the embryo of the Dibranchiata, the eye passes through a stage in which it is in the condition of an open cup similar to the adult eye of Nautilus. Osphradia are present, as 
already mentioned, only in the Tetrabranchiata; but in both the Dibranchiata and the Tetrabranchiata certain sensory processess or depressions conjectured to possess an olfactory function are developed on the head. Statocysts are universally present.

All the Dibranchiata have two nephridia similar in character to those of Sepia, and communicating with one another; in Octopus they are completely united. In the Tetrabranchiata there are four nephridia, each opening on the exterior.

The sexes are distinct in all the Cephalopoda, and in addition to the hectocotylised arm, there are frequently other external differences between male and female. In all the Dibranchiata the arrangement of the gonads and gonoducts is, as regards general features, similar to what we find in Sepia. In Octopus, however, there are two oviducts instead of one, and in one other member of the Octopoda (Eledone moschata) the same holds good of the spermiducts.

Development.-The development of the Dibranchiata alone is known. The eggs are very large, containing a relatively large amount of food-yolk. They are usually laid in masses or strings embedded in a soft gelatinous, or a tougher, more leathery substance, usually attached to some foreign body; in some cases each egg, enclosed in a gelatinous sheath, has a longer or shorter stalk. A chorion or delicate transparent egg-membrane, in which there is an aperture-the micropyle-immediately invests the egg itself. In shape the egg is oval or spherical. The greater part of the comparatively small quantity of protoplasm lies as

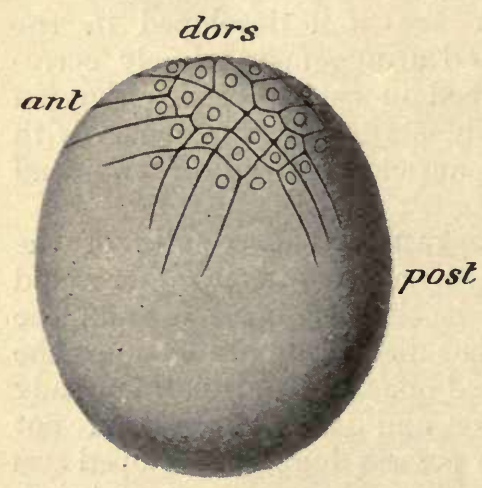

vent

FiG. 697.-Segmenting ovum of Ioligo. (From Korschelt and Heider, after Watasé.) a disc-like elevation on the surface of the yolk on the side of the egg at which the micropyle is situated. Continuous with this germinal disc is a thin layer of peripheral protoplasm investing the entire ovum.

Segmentation (Figs. 697 and 698 ) is incomplete, being confined to the germinal disc. At an early stage in the process of division, the blastoderm exhibits a distinct bilateral symmetry. This meroblastic segmentation results in the formation of a nearly circular blastoderm, the outer cells of which tend to separate off. At first the blasto lerm consists of only a single layer of cells-the ectoderm, which gradually extends. At a later stage a second layer (Fig. $699, B, C$ ) appears below the margin of the blastoderm, and 
extends inwards until it comes to underlie the whole of the embryonic part of the blastoderm : separating this trom the yolk

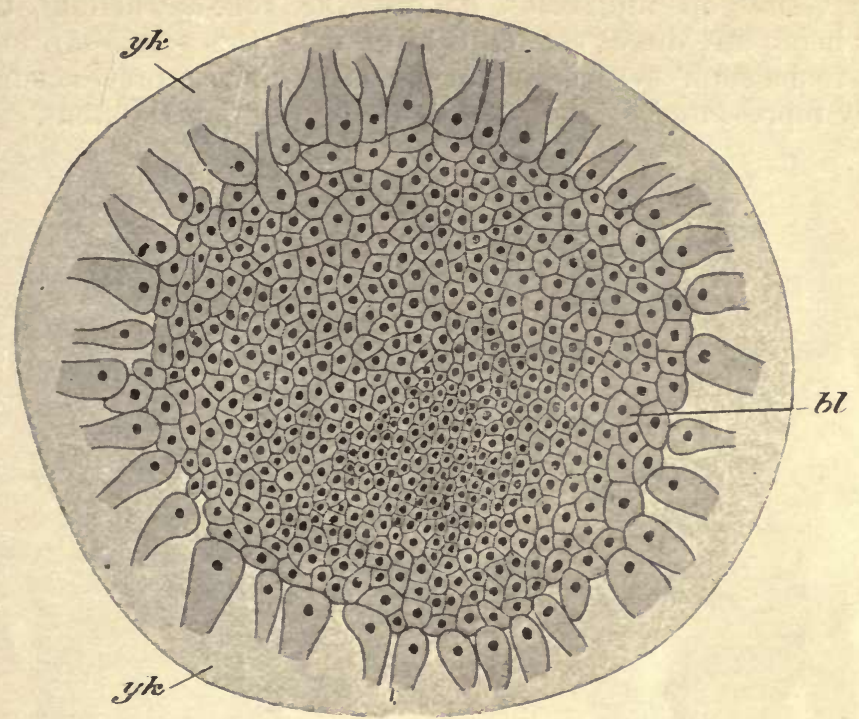

FIG. 698.-Sepia, blastoderm at a late stage of segmeutation. bl. blastoderm; yl. yolk. (From Korschelt and Heider, after Vialleton.)

is a thin layer of uncertain derivation-the yolk-epithelium (Fig. $699, y k$. ep.). There is some doubt as to the nature of the second

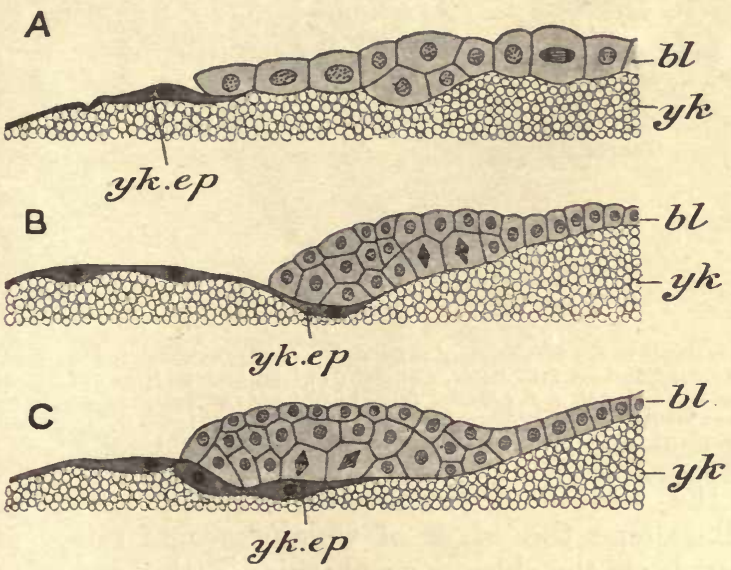

FiG. 699.-Sections through the edge of the blastoderm of Sepia at three successive stages; bl. blastoderm; $y k$. Jolk; $y k$. ep. yolk-epithelium. (From Korschelt and Heider, after Vialleton.)

layer; it certainly gives rise to the mesodermal structures, and by some observers it is also said to form the epithelium of the 
mesenteron. From whatever source it may be derived, the latter becomes distinguishable as a cell-plate which is converted into a vesicle opening below against the yolk-epithelium, there never being any direct communication with the yolk. An extensive stomodæum eventually opens into this; a proctodæum is merely represented by the ectodermal pit forming the anus.

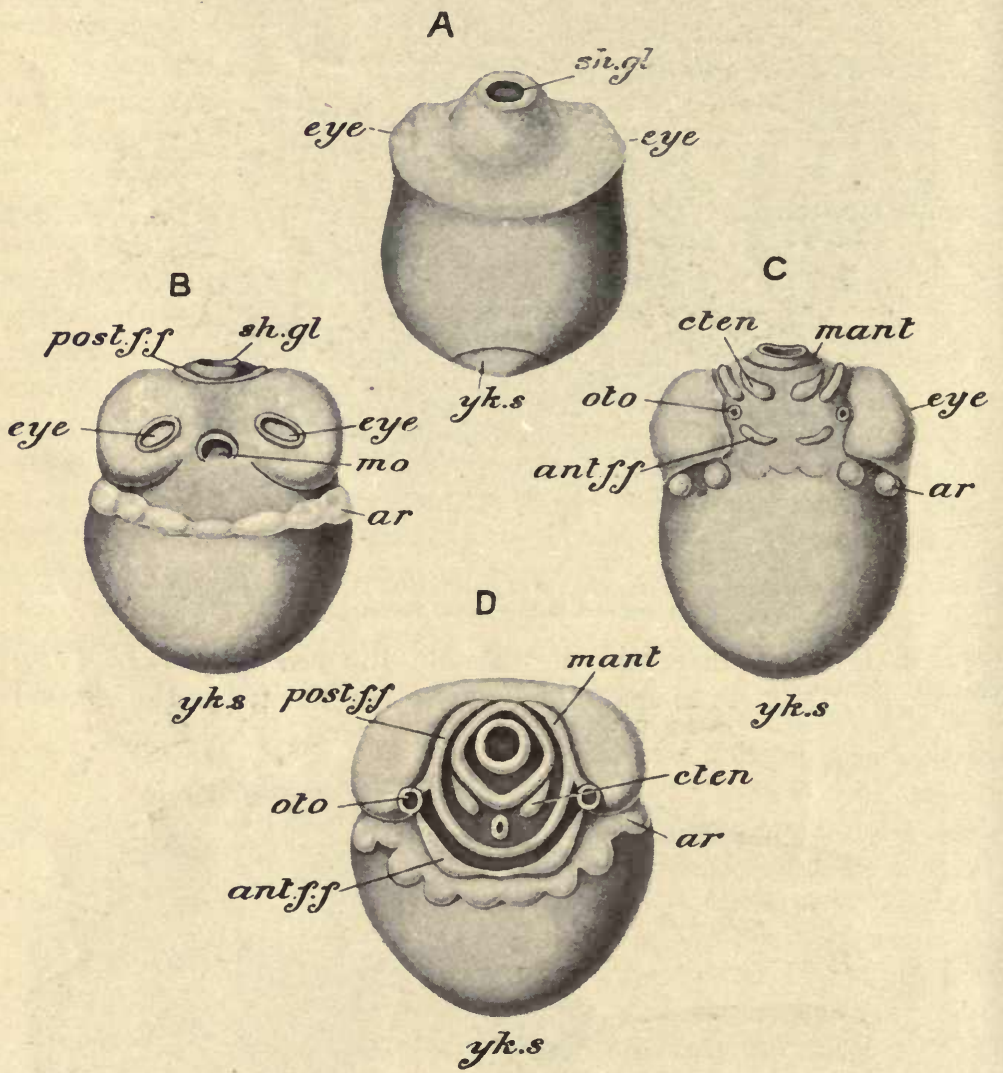

Fig. 700.-Early stages in the development of Ioligo. A, stage at which the rudiments of the eyes and of the shell-gland are first distinguishable ; $B$, later embryo from the oral side; $C$ and $D$, from the anal side. ant. $f_{.} f_{0}$ anterior funnel-fold; $a r$. rudiments of arms; cten. ctenidia; eye, eye ; mo. mouth ; mant. rudiment of mantle ; ot. statocyst; post. $f . f$. posterior funnel-fold ; sh. gl. shell-gland; $y k$. s. yolk-sac. (After Korschelt and Heider.)

About the middle of the blastoderm appears a thickening of a cap-like shape, the edges of which become raised above the general level of the blastoderm; this is the rudiment of the mantle. On the surface of this is developed a depression which subsequently forms a closed sac-the shell-gland (Fig. 700, sh.gl.). Below the mantle-i.e. nearer the vegetal pole-appear two elevations each with a pit-like depression, which are the rudiments of 
the eyes; and still nearer the vegetal pole a series of paired elevations, the rudiments of the arms.

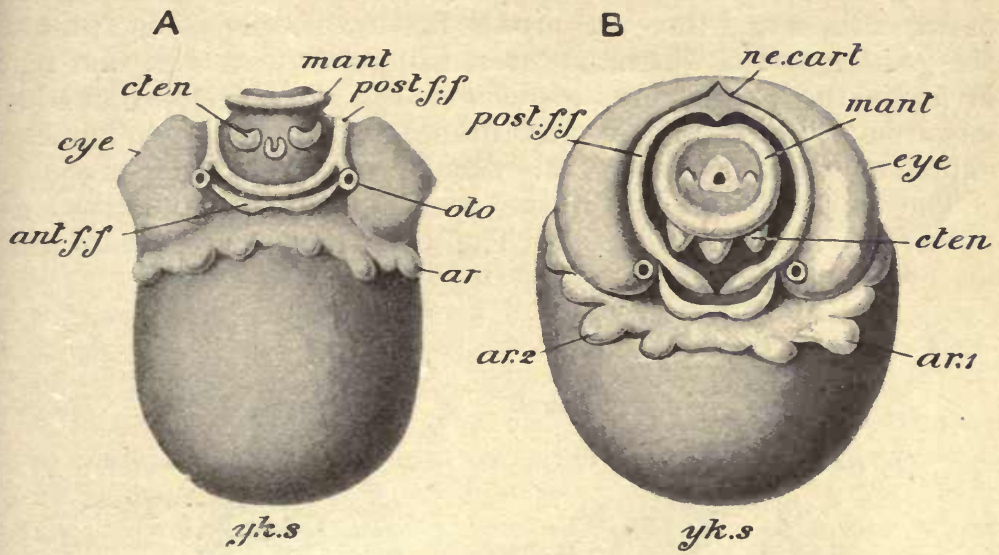

FIG. 701. - Two later stages in the development of Ioligo. A, from the funuel side. B, obliquely from above. Letters as in preceding figures; ne. cart. nuchal cartilage. (After Korschelt and Heider.)
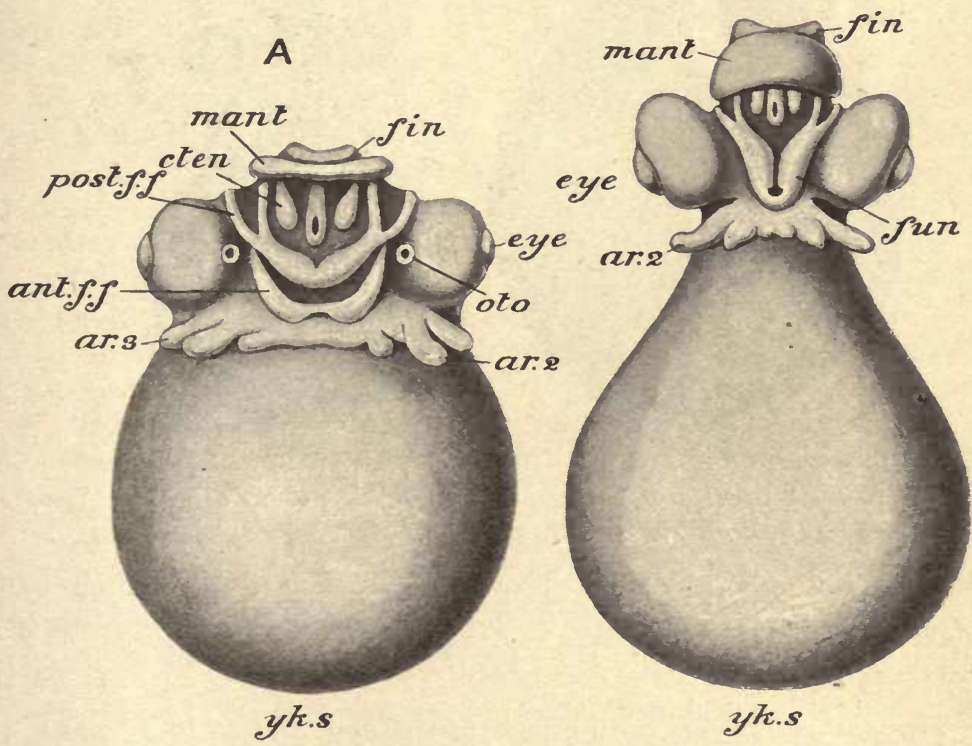

FIG. 702.-Two stages in the development of Ioligo, later than those represented in Fig. 701. From the anal or funnel side. Letters as in preceding figure; in additíon, fin, fins. (After Korschelt and Feider.)

After the complete enclosure of the yolk by the blastoderm, the mouth (mo.) is developed as an oval depression between the rudi- 
ments of the eyes. Immediately in front of the edge of the mantle appear two short ridges, the beginnings of the gills (cten.), and a pair of folds - the posterior funnel-folds (post.f.f.) - which are formed between these and the eyes, are the first rudiments of the funnel, the greater part of which, however, is formed from a second pair of folds-the anterior funnel-folds (ant. $f . f$.) -developed further forwards. Behind the anterior funnel-folds appear two pit-like depressions, which subsequently develop into the statocysts.

The elevations on which the eyes (eye) are situated become more and more prominent. The eyes themselves are formed
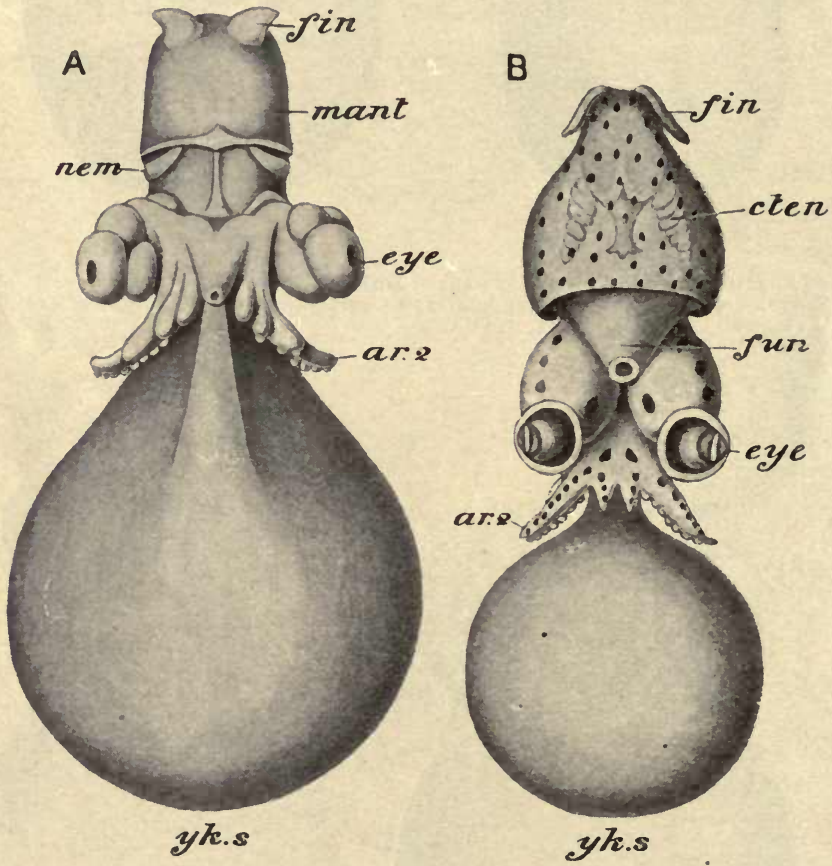

Fig. 703. - Tivo late stages in the development of Ioligo, seen from the funnel side. Letters as in preceding figures. (After Korsehelt and Heider.)

from a part only of these elevations; each is a pit which subsequently becomes closed to form a vesicle-the optic vesicle: later an ingrowth of the ectoderm over this gives rise to the lens.

The embryo covers only a part of the egg, and as it develops, it withdraws itself more towards the animal pole, at which the germinal disc was originally situated -a constriction, which soon hecomes very deep, separating it off from the rest of the egg; the latter, consisting of the greater part of the yolk enclosed in a thin layer of blastoderm, forms a rounded appendage of the embryo-the 
yolk-sac (yk. s.). The yolk-sac undergoes contractions, which are due to the action of contractile cells in the thin mesoderm lining it, and by this means the yolk is forced into the interior of the body of the embryo.

The anus appears as an aperture situated on a little papilla-the anal papilla. A row of cilia, which are developed in the neighbourhood of the mouth in some forms, perhaps represent the velum or pre-oral circlet of other molluscan embryos. The mantle now increases in extent, and its margins become more prominent: The anterior funnel-folds grow out and unite in the middle line; and these, with the posterior folds, go to form the completed funnel together with the "neck-muscles." For a time the edges of the two folds which form the funnel remain free; eventually they coalesce into a complete tube.

The edges of the mantle grow out into prominent folds to form the mantle-cavity, into which the gills are drawn. Lateral outgrowths have already given rise to the rudiments of the fins. The arms grow out into more and more prominent processes on which the suckers are developed, the second pair-the prehensile arms (ar. 2)-soon becoming distinguishable from the rest by their greater length.

As the embryo increases in size, the yolk is gradually absorbed, and the yolk-sac decreases in bulk, until, when the embryo leaves the egg, it has almost completely disappeared.

Distribution.- The Cephalopoda are all marine, and range from tidal limits to a considerable depth. A large number (Loligo, etc.) are pelagic and move together in great shoals. Sepia lives chiefly between stones and in rock-fissures in the littoral zone, and often burrows in sand. Octopus constructs a den or shelter of stones to which it always returns after excursions in search of food. Cephalopods are, nearly without exception, carnivorous. In length they range from an inch or two to as much as fifty feet-the gigantic members of the group, such as Architeuthis, being by a long way the largest of invertebrate animals. Like the other classes of Mollusca they are most abundant in tropical and warm-temperate seas.

If the Ammonites are to be included among the Tetrabranchiata, that sub-class was most abundantly represented during the Mesozoic period. The nautiloid Tetrabranchiata were most abundant in the Palæozoic epoch, during which there lived a great variety of forms of this group, the shell being straight (Orthoceras), or curved (Phragmoceras), or in a flat spiral with the turns not in contact, or in a helix, or a flat close spiral (Nautilus and others). The earliest representatives of the Nautiloids are found in rocks of Cambrian age; they are comparatively scarce in the Mesozoic epoch and in the Tertiary, and are represented at the present day only by the genus 
Nautilus itself. The Ammonites are mainly Mesozoic, the representatives found in the earlier rocks (from the Upper Silurian onwards) being few in number and simpler in structure than the more typical later forms. The oldest fossil representatives of undoubted Dibranchiata belong to the extinct order of the Belemnites, which flourished in the Mesozoic period from the Trias to the Cretaceous. and survived in scanty number into the Tertiary. Unlike the Tetrabranchiata, the Dibranchiata would appear to have reached their maximum at the present day.

The mutual relationship of the various groups of Cephalopoda are indicated, as nearly as the information at our disposal will allow, in the following diagram (Fig. 704).

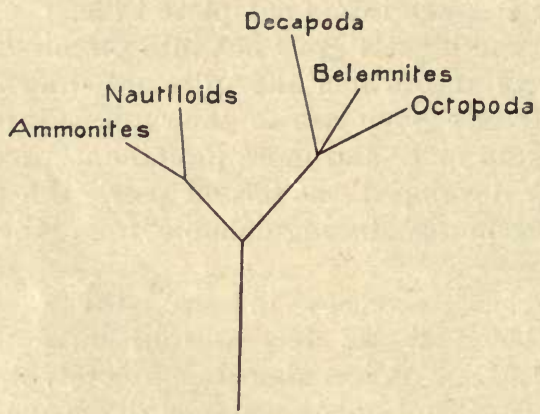

Fig. 704.-Diagram to illustrate the relationships of the groups of Cephalopoda.

\section{General Remarks on the Mollusca.}

The Mollusca, like the Arthropoda, form an extremely welldefined phylum, none of the adult members of which approach the lower groups of animals in any marked degree. There are, however, clear indications of affinity with "Worms," especially in the frequent occurrence of a trochophore stage in development, in the presence of nephridia, and in the occurrence, in Amphineura and some of the lower Gastropoda, of a ladder-like nervous system resembling that of some Turbellaria and of the most worm-like of Arthropods-Peripatus. The head-kidneys or primitive nephridia of the molluscan and annelid trochophore are practically identical, and are probably homologous with the various types of nephridial tubes found in "Worms" from Platyhelminthes to Chætopoda.

If the octurrence of the trochophore be taken as a guide towards the ancestry of the Mollusca, it need not necessarily be regarded as leading back to the Annulata. In fact the presence of not more than a single pair of nephridia (and of ctendia) in all with 
the exception of Nautilus, would seem to indicate the derivation of the phylum from a group in which metamerism had not arisen. It will be readily recognised that the gap between the typical trochophore and certain forms of Turbellarian larvæ (Müller's larva) is not a very wide one, and might be covered by adaptation of the larval Flat-worm to a freer pelagic life. If we were to suppose that the most primitive Mollusca were derived from Turbellarian-like ancestors, the conversion of a larva of the type of Müller's larva into a larval form like the mollusean trochophore would also have to be postulated. This might involve a common platyhelminth origin for Annulata and Mollusca, with subsequent extreme divergencea divergence in which the respective trochophores would take part, though in a limited degree. The chief changes which the adult animal would have to undergo in order to assume the character of a primitive Mollusc on this supposition, would be-(1) The development of some kind of protective layer of hard material, perhaps composed at first of spicules in a thickend integument, on the dorsal surface-the rudiment of the shell; (2) The greater development of the muscular layers of the body-wall on the ventral side to give rise to a more efficient and specialised creeping organ than was possessed by the Turbellarian ancestor; (3) The development of specialised respiratory organs in the form of ctenidia-a change rendered necessary by the great reduction in the available respiratory area brought about by the development of the shell; (4) The formation of an anus and proctodæum; and (5) the development of a cœlome.

With regard to the relationships of the various classes of Mollusca, the following points are some of the most important to be borne in mind.

The lowest members of the phylum are undoubtedly the Protobranchia among Pelecypoda, and the Aplacophora among Amphineura. The latter take the lowest rank in virtue of the absence of both foot and shell, but the possession by some of a radula indicates a comparatively high degree of specialisation. On the other hand, while there is no indication of an odontophore, even in a rudimentary condition, in the Pelecypoda, the foot and shell are well developed even in Nucula and its allies. There is no actual evidence to show that the foot and shell have been lost by degeneration in the Aplacophora or the odontophore in Pelecypoda; and it would appear, therefore, that the two groups are to be derived independently from some primitive form.

The facts that the pelecypod shell, at its first appearance, is univalve, and that the foot of the Protobranchia is of the creeping type and their ctenidia plume-like, suggest the derivation of the class from a form resembling a simple type of Gastropod with no odontophore and with undisturbed bilateral symmetry. The Amphineura are also bilaterally symmetrical, with paired ctenidia, kidneys, 
and auricles, and the fact that these organs are also paired in the lower Gastropoda, seems to point to a common ancestor for Pelecypoda, Amphineura, and Gastropoda, which was bilaterally symmetrical, had a creeping foot, a simple shell, paired auricles, kidneys, and gills, and no odontophore.

While the leading feature in the evolution of the Pelecypoda has been the splitting of the mantle into two halves and the resulting bivalve shell, the most noticeable fact in that of Gastropoda, apart from the appearance of the odontophore, has been the torsion of the visceral mass, producing a characteristic asymmetry. In the Cephalopoda, on the other hand, the primitive bilateral symmetry is retained, and the most characteristic special feature of the group is the extraordinary modification of the foot into arms or tentacles, and funnel. The class is raised far above the remaining Mollusca by its wonderfully high organisation, especially of the nervous system and the eye, and there is nothing to indicate close relationship with any of the lower classes beyond the general conformity to the molluscan plan of organisation and the presence of an odontophore. The Cephalopods form, in fact, a singularly isolated group. Palæontology has not hitherto given any indication of their origin, and embryology is equally silent; the absence of a free larva, and the profound modification in development produced by the enormous mass of food-yolk, sharply separating them from all other members of the phylum. 
INDEX 



\section{INDEX}

All numbers refer to pages: words in italics are names of families, genera and species: words in thick type are names of higher divisions: words in small capitals are names of examples. Numbers in thick type are numbers of pages on which there are figures: an asterisk after a number indicates a definition of the term or of the group.

A

Abdomen, of A pus, 531 : Astacus, 541 : Periplaneta, 620, 622

Aboral, $376^{*}, 418$

Absorption, 34

Abyssal species, $8^{*}$

A cantharia, 60

Acanthin, $61^{*}$

Acanthocephala, $297^{*}$ : External characters, 312, 313 : Body-wall, 312 : Bodycavity, 313 : Proboscis," 313 : Vessels, 313 : Nervous-system, 313 : Excretory organs, 313, 315: Reproductive organs, 314, 315 : Development, 315

Acanthobdella, 518, 519

Acarida, $661^{*}, 665,667,668,669,672$, 673

Achromatin, $17^{*}$

Aciculum, $441^{*}$

Acineta, 99, 100, 101

A cola , 252, 267, 272

Acontia, 188*, 201

Acorn-shells, 565

Actoon, 745

Actinal, $376^{*}, 418$

Actinia, 193, 228

Actiniaria, 194* $, 196,197$

Actinobolus, 93, 94

Actinodactylella, 259, 260, 267, 273

Actinometra, 425

Actinomma asteracanthion, 61, 62

Actinophrys sol, 56, 57, 59

Actinospharium, $\mathbf{5 7}$

Actinostome, $376^{*}$

Actinotrocha, 358, 359, 503

Actinozoa, 128 : Example, 185 : Distinctive characters and classification, 193 : Systematic position of example, 196 :
General organisation, 196 : Budding, 197 : Structure of polypes, 199 : Enteric system, 201: Fixed and frec forms, 202: Dimorphism, 202: Skeleton, 202: Colour, 208 : Commensalism, 208: Distribution, 209

Actinula, $226^{*}, 229$

Adamsia palliata, 208, 209

Adductor impressions, 682, 683, 694, $697,698,699$

Adductor muscles, 683, 697, 698

Adhesive cells, of Hormiphora, 215*:

Turbellaria, 264

Adipose tissue, $26^{*}, 27$

Adjustors, 364*

Adradius, 139

Adrectal gland, 744

Aiginopsis, 155, 156

Áginura, 155

Aiquorea, 143

Affinities-See Relationships

Agalma, 163

Agamobium, 140*, 176

Aggressive characters, in Crustacea, 601 Air-sacs, of Insects, 642

Albertia, 335

Alciopida, 488

Alcippe, 565, 579

Alcyonacea, 195, 197, 198, 199, 203

Alcyonaria, 195*, 196, 197, 199, 200, 201, 202, 203, 227

Alcyonidae, 208

¿ Alcyonidium, 348

Alcyonium, 195, 203

Alecithal, 219*

Alimentary canal-See Digestive system Alimentary system-See Digestivesystem Allolobophma antipa, 481

Alphers, 602 
Alpine forms, $8^{*}$

Alternation of generations-See Metagenesis.

Alveolus, of Sea-urchin, 397

Ambulacral area, 395

Ambulacral grooves, $376^{*}$

Ambulacral ossicles, 378, 381, 419

Ambulacral pores, 378, 396

Ambulacral ridges, 380

Ambulacral spines, 376,387

Ambulacral system, of Asterias, 383 : Echinus, 396, 399 : Sea-cucumber, 402 , 403: Antedon, 408: Echinodermata, 415

Amitotic division, $19^{*}$

Ammonites, 790, 795, 803, 804

Amnion, of Peripatus, 612: Periplaneta, 631 : Scorpion, 659

Амава, 10, 11, 12, 13, 14 : Pseudopods, 47: Endosarc, 46 : Ectosare, 46 : Contractile vacuole, 46: Encystation, 46: Fission, 46: Systematic position, 48

Amæbida, 48

Amœbocytes, 382, 399

Amobophyra, 233

Amobula, of Didymium, 66, 67: of Gregarina, 82, 83

Amphiblastula, $124^{*}, 125$

Amphidiscs, $121^{*}, 122$

Amphilina, 262, $287^{\circ}$

Amphineura, 680, 712* : Distinctive characters and classification, 712: General organisation, 713 : External features, 713, 714: Ctenidia, 713: Alimentary system, 715: Body cavity, 715: Vascular system, 715: Nervous system, 715, 717: Reproductive and renal organs, 718, 719: Development, 718, 720 : Ethology, distribution, \&c., 720

Amphinomida, 467

Amphipoda, 568* ${ }^{*}, 583,584,585,586,593$, 596,603

Amphiptyches, 262, 263

A mphistomum, 257

Amphitretus pelagicus, 794

Amphiura, 232

Amphiura squamata, 429

Ampullæ, 158*, 378*, 399

Ampullaria, 749

Amusinem, 702, 704

Anal filament, 663

Anal glands, of Periputus, 610

Anal respiration, 596

Anal spot, of Paramacium, 90

Anaspidacea, $566^{*}, 581,582,596,603$

Anaspides, 566

Anatomy, 3*

Anchors, 177

Anguillula, 305

Anisopoda, 584*

Ankylostoma duodenale, 306
Annulata, 439*: General remarks on, 523 : Relationships, 525

Annuli of Leech, 506

Anodonta, 680 : Shell, 682 : Body, 684 : Muscles, 684': Colome, 684 : Digestive organs, 684, 685 : Gills, 685 ; Excretory organs, 689 : Circulatory system, 689 , 690 : Nervous systeh, 690 : Sensory organs, 691: Reproductive organs, 691: Development, 692, 693, 694: Systematic position, 696

Anodonta, 696, 697, 698, 704

Anomia, 695, 697, 698

Anomura, 569* 588, 589

Anopheles, 87

Anoplophyra, 94

Anostraca, 563\%, 571

ANTEDON ROSACEA, 405 : General externa features, 405, 406: Ossicles, 406 Colome, 407: Enteric canal, 407: Ambulacral system, 408: Nervou: system, 408: Perihæmal and hæmas system, 409 : Sacculi, 409: Reprorlucl tive organs, 409 : Metamorphosis, 409 432, 433 : Systematic position, 414: Development, 432

Antenna, of Astacus, 544-See also Appendages

Antennary gland, of Astacus, 551, 552

Antennary glands, 55], 596

Antennule, of Astucus, 544-See also Appendages

ANTHENEA FLAVESCLNS，384, 386, 387, 388

Anthomedusæ, 141*, 143, 144, 149

Anthophysa, 576

Anthosoma, 575, 576

Anthura, 568

Antimeres, $42 * 415$

Antipatharia, 195*, 201, 202, 203, 204, 210

Antipathes, 201

Antispadix, 780

Ant-lions, 633

Ants, 636, 652

Aorta-See Vascular system

Aphides, 647

Aphis rosa, 633

Aphrodite, 475

Aphroditea, 474, 475

Apical plate, of Trochosphere, 323

Apical system of plates, 393, 397

Apis mellifica, 637, 652

Aplacophora, 713*, 715, 717, 718, 720, 721,805

Aplysia, 738, 739, 740, 746

Aplysiida, 734

Apoda (Holothuroidea), 413* $, 426,428$

Apodiidae, 569, 570*

Apopyle, 108, 109, $110^{*}$

Appendages, of Rotifera, 330 : Apus, 529, 530: Astacus, 542, 543, 544: Crustacea, 570: Peripatus, 607, 608: Peri- 
planeta, 621 : Insecta, 637 : Scorpion, 655

Apseudes, 567

Aptera, $632 *, 640,677$

Aptychus, 795*

APUs, 526: External characters, 527, 528: Appendages, 529, 530: Bodywall, 531 : Muscular system, 532 : Digestive organs, 532, 533: Bodycavity, 533 : Circulatory system, 533 : Respiration, 534: Renal organ, 534, 535: Nervous system, 534, 536: Organs of sense, 536, 537 : Reproductive organs, 537: Development, 537, 538 : Systematic position, 569

A pus, 534, 535, 537, 538

A pus cancriformis, 528, 536

A pus glacialis, 529

Aquatic pupa, 651

Arachnida, 526, 653*, 677: Example, 653 : Distinctive characters and classification, 660: General organisation, 662 : External form, 662-667: Endostcrnite, 667: Coxal glands, 667 : Alimentary system, 667 : Heart, 668 : Organs of respiration, 668: Nervous system, 670 : Sense-organs, $670:$ Reproductive apparatus, 671 : Mode of life, 672: Geological history, 673: Appendix, 673

Arachnidium, 664

Araneida, $661^{*}, 664,667,669,670$

A rbacia punctulata, pedicellaria, 422

Arca, 695, 697, 701, 702, 704, 706, 711

Arcella, 49, 50

Archæocytes, 122

Archenteron $23^{*}$

Archi-Annelida, 439, 503*, 504, 505, 524

Archi-cerebrum, 55

Archi-cerebrum, of Periplaneta, 631

Archi-Chætopoda, $465^{*}, 477$

Archigetcs, 262, 263, 287

Architeuthis, 803

Argiope, 366

Argonauta argo, 793, 796

Argonauts, $790,791,796$

Argulus, 565, 576, 577

Arhynchobdellida, 516*

Aricia, 479

Aristotle's lantern, 397

Ark-shell, 702

Armadillidium, 586, 587

Armadillo, 524, 546

Armata, 496*, 497, 498, 499, 502

Arrow worms, 297

Artemia, 563, 571

Arthrobranchiæ, 551

Arthropoda, 526* : Affinities of airbreathing, 677

Arthrostraca, 583*

Articulata, $366^{*}, 367,368,369$ : Shell, 398

Ascaridce, 304*
ASCARIS LUMBRICOIDES, 297 : External characters, 297: Body-wall, 298: Digestive organs, 299, 300 : Cœlome, 301 : Excretory system, 301 : Nervous system, 301, 302: Reproductive organs, 302, 303 : Development, 303 : Systematic position, 304

Ascaris megalocephala, 297

Ascaris nigrovenosa, 308, 309, 310

Ascaris suilla, 297

Ascetta, 116

Ascon, 117, 118*

Ascopodaria, 354

Asellus, 568, 583, 585

Asexual reproduction, 40: in Amœba, 46: Heliozoa, 59: Radiolaria, 63: Euglena, 69: Flagellata, 74 : Choanoflagellata, 78: Dinoflagellata, 79: Cystoflagellata, 79: Sporozoa, 80: Coccidiidea, 84: Hæmosporidean Myxosporidea, 87 : Paramøciuाn, 90 Ciliata, 98: Tentaculifera, 101. Sponges, 121 : Actinozoa, 197 : Platyhelminthes, 283: Bugula, 346 : Chætopoda, 486

Aspergillum, 696, 701, 702

Aspidobranchia, 733*

Aspidochirotæ, 415

Aspidocotylea, 253*, 260, 273

Aspidogaster, 284

Asplanchna, 328, 330, 331

Astacopsis, 569

A stacus, 569

Astacus floviatilis, 539, External characters, 540: Abdomen, 541, Thoracic region, 541: Head, 542: Appendages, 542, 543 : Articulations, 545: Body-wall, 546: Muscular system, 546, 547: Digestive organs, 548 : Respiratory organs, 549, 550 : Excretory organs, 551, 552: Circulatory organs, 551, 552, 553, 554: Nervous system, 555 : Sensory organs, 556 : Reproduction, 556, 557 : Development, 557, 558, 559, 560, 561 : System. atic position, 570

Astasiopsis, 71

Asterias RUBExs, 375 : General external features, 375, 376, 377: Transverse section of an arm, 378: Vascular and nervous systems, 379 : Structure of the disc, 380 : Body-wall and coelome, 381 : Digestive system, 382: Ambulacral system, 383: Reproductive system, 386 : Systematic position, 414

Asteriidae, $414^{*}$

Asterina, development, 388, 389, 390, $391,392,393$

Asterina gibbosa, 429, 432

Asteroidea, Example, 375: Development, 388 : Distinctive characters and classification, 410: Apical system, 417: Modifications of form, 418, 419 : 
Cœlome, 425: Ambulacral system, 425: Blood-vascular system, 426 : Hæmal system, 427: Axial organ, 428: Enteric canal, 429: Nervous system, 429: Reproductive organs, 429 : Development, 430 : Ethology, 434 Asthenosoma, 421

Astracoidea, 570*

Astrcea, 197, 198, 207

Astropecten, 419, 420

Astrophyton, 421

Astrosphere, 17, 18*

Atlanta peronii, 741

Atrium, 249, 346

Atrochal, $486^{*}$

Attraction-sphere, $18^{*}$

Auditory organs, 39,645

Aulactinium actinastrum, 62

Aulostoma, 516, 522

AUrelia AURITA, External characteristics, 168, 169: Digestive-cavity and canal system, 170, 171: Cell-layers, 170: Gonads, 171: Gastric filaments, 172: Muscular and nervous systems, 172 : Sense-organs, 172 : Development and life-history, 173, 174, 175: Systematic position, 177

Auricle, of heart, $36^{*}$

Auricles, of Sea-urchin, 397 : of Ctenophora, 223*

Auricularia, 404, 413, 431, 432

Australian region, $9^{*}$

Autolytus cornutus, 487

Avicularium, 341, 342, 352*

Axes, 42*

Axial fibre, 92, 96, 97

Axial nerve, 409

Axial organ, 384, 400, 428

Axial sinus, 380

Axis-cylinder, 29*, 30

B

B ALANUS, 565, 577, 579

Barnacles, 3, 526, 565, 577, 579, 594, 596

Barrier reef, 210

Basal plate, of coral, 205*, 206

Batteries, $161^{*}$

Bdelloida, 328*, 330, 334

Bdelloura, 284

Bear-animalcules, 673

Bee-parasites, 651

Bees, 526, 619, 636, 647, 652

Beetles, 525, 619, 635, 640

Belemnites, 790, 795, 796, 805

Benthos, $8^{*}$

Berenice, 150

Beroë, 224, 228

Beroiida, $221^{*}, 224$

Bicellariida, $348^{*}$

Bicellular glands, $493^{*}$
Bilateral symmetry, 41, 43*

Bile, $34^{*}$

Binomial nomenclature, $1^{*}$

Biology, $1^{*}$

Bionomics, $9^{*}$

Bipalium, 255

Bipinnaria, 411, 432

Bird-lice, 651

Bird's-Head Coralline, 341

Birgus, 569, 589, 595, 603

Birth-opening, 245*

Bivium, 377, 416 : of Sea-cucumber, 401

Black coral, 195*, 202, 210

Blastocole, $23^{*}$

Blastoidea, $414^{*}, 435,437$

Blastomeres, 22 *

Blastopore, 22, 23*

Blastosphere, $23^{*}$

Blastostyle, of Obelia, 129*, 130 : Lepto

linæ, 151 : Porpita, 165, 166

Blastula, 23*

Blatta-See Periplaneta

Blattida, 636,653

Blepharoblast, $70^{*}, 72$

Blood, 29*, 34*

Blood-corpuscles, 30

Blood-vascular system-See V'ascular system

Blood-vessels, 34

Blow-flies, 635

Blue coral, 195

Bodotria, 567

Body-cavity - See Colome

Body-wall, of Sea-anemone, 185 : Hormiphora, 215: Liver-fluke, 241: Platy. helminthes, 262: Nemertinea, 290: Ascriris, 298: Nematoda, 305: Chætognatha, 316: Brachionus rubens, 325: Bugula, 343 : Ectoprocta, 351 : Mayellania, 362: Asterias, 381 : Sea-cucumber, 402: Nereis, 443 : Earthworm, 456 : Chætopoda, 473 : Sipunculus, 493 : Gephyrea, 497: Hirudo, 508: Apus, 531 : Astacus, 546 : Crustacen, 594 : Peripatus, 608: Myriapoda, 617 : In secta, 636

Bojanus, organs of, 689

Bolina hydatina, 224

Bone, 25, 27* 28

Bone-corpuscles, $27^{*}$

Bonellia, 496, 497, 499, 500, 501

Book-gills, 671

Book-lungs, $65 \overline{7}, 668$

Book-scorpions, 662

Bopyrini, 586

Bopyrus, 568

Botany, $1^{*}$

Bot-fly, 635, 651

Bothridia, 262*

Bothriocephalus, 261, 279, 285

Bothriocephalus latus, 285

Botryoidal tissue, of Leech, 509

Bougainvillea, 143, 144, 152 
Brachial disc, of Discomedusæ, 183

Brachial ossicles, 407

Brachiolaria, 411, 432

Brachionida, 329*

Brachionus, 324, 325, 326, 328, 329

BRACHIONUS RUBENS: External characters, 323, 324 : Body-wall, 325 : Digestive organs, 325: Colome, 325: Excretory system, 326 : Nervous system, and sense organs, 326 : Reproduction and development, 326,327 : Systematic position, 329

Brachiopoda, 340, 360* : Example, 360 : Distinctive characters and classification, 366 : Systematic position of example, 367 : General organisation, 367 : Shell, 367, 368 : Peduncle, 367 : Lophophore, 368 : Muscular system, 368 : Enteric canal, 369: Heart, 369 : Nephridia, 369 : Nervous system, 369 : Gonads, 369 : Development, 370, 371 : Distribution, 371

Brachyurs, 569* $589,590,591,601$

Bract, $160,161^{*}, 531$

Brain, 38

Branchellion, 516, 517, 519, 5222 。

Branchiæ, 35* : of Asterias, 376: Seaurchin, 395: Polychæta, 471 : Oligochrta, 473: Branchellion, 519: Astacus, 549; Crustacea, 593: Anodonta, 686, 687, 688: Pelecypoda, 702: Triton, 725 : Gastropoda, 742

Branchial formula, of Astacus, \&c., 551

Branchiopoda, 563*, 569, 570, 571, 593, $594,596,597,598,602,603,677$

Branchipus, 563, 570, 571

Branchiura, 565* 573,577

Brine-shrimp, 571

Brisingidae, 418

Brood-pouch, 370, 531

Brood-cavity, 101

Brown body, 346

Buccal cavity, 32

Buccinum undatum, 722

Budding, 40*, 41-See Asexual reproduction

Budding, in Turbellaria, 257

Buffon, 5

Bugs, 634, 640, 651

Bugula, 347

BUGUla avicularia, 341, 342 : Bolywall, 343 : Colome, 343 : Alimentary canal, 343: Nervous system, 343: Excretory organs, 343 , Reproductive organs, 344: Developntent, 344, 345, 346 : Systematic position, 348

Bugula plumosa, 346

Bursa copulatrix, 270, 271, 272, 273, 647

Busycon, 749

Buturs, 653: External features, 654, 655 : Digestive system, 656, 657, 658 : Circulatory organs, 656,657 : Organs of respiration, 657 : Nervous system, 657,
658 : Organs of special sense, 658 : Re productive organs, 658 : Development, 659

Butterflies, 526, 619, 635

Byssus, 694, 702

Byssus-gland, 694, 702

Byssus, provisional, 693

\section{C}

Uaddis-flies, 633

Cake-urchins, $412^{*}, 417,423$

Calcarea, 112*, 120, 122, 123

Calcareous spicules, of Sponges, 107, 108, 120,122

Calciferous glands, 458

Callianira, 218, 219, 222

Callitiara, 150

Calocalanus, 574

Calotte $2: 30^{*}, 344$

Calymma, of Radiolaria, 60

Calyptoblastea $143^{*}$

Cambarue, 569

Cambrian, 7

Campanulariidae, 142 *

Canaliculæ (bone), 27 *

Canals, Haversian, 27*, 28 : incurrent, radial or flagellate, excurrent of Sponge, 107, 108, 109*, 117 : of Medusa, 135,136

Canal system of Sponges, 117, 118

Cancer, 569, 590

Cannostomæ, 180

Capillaries, $35^{*}$

Capillary vessels, 238

Capillitium, of Mycetozoa, 66, 67

Caprella, 568, 586

Capria, 178

Capsulogenous glands, 457

Carabus auratus, 641

Carapace, Apus, 527: Astacus, 540: of Scorpion, 654

Carboniferous, 7

Cardiac sac, of Polychrta, 476

Cardinal process, $360^{*}$

Cardium, 696, 698, 701, 711

Jarina, of Cirripedia, 578

Carinaria mediterranea, 739

Jarp-lice, 565

Carpoidea, 414*, 435

Cartilage, 25, 26* : Hyaline, 26, 27 : Fibrous, 26, 27: Yellow elastic, 26: Calcified, 27

Caryophyllaus, 262, 263, 287

Cassiopeia, 184

Caudal spine, 666

Caudal styles, of Apus, 527

Caudal vesicle, $248^{*}, 280$

Cell, animal, 14, 16*

Cell, 14, 16*, 17: Forms, 23 : Ciliater, 23, 45 : Flagellate, 24, 45 : Amoeboid, 45: Encysted, 45 
Cell-colony, $50^{*}, 66$

Cell-division, 17, 18

Cell-plate, 17, 19*

Cell-wall, $16^{*}$

Cellepora, 347

Cellulose, 14, 64, 67, 69, 72, 73, 78

Cement glands, 270,326

Centipedes, 526, 614, 615

Central capsule (Antedon), 409

Central capsule of Radiolaria, 60

Central nervous system of Medusi, 151

Centro-dorsal ossicle, of Antedon, 406

Centrolecithal, 537, 219*

Centrosome, 17*

Cephalic apodeme, of Apus, 533 : Astacus, 542

Cephalopoda, 680, 759*. Examiples, 759, 776 : Distinctive characters d.ıl classification, 789: Systematic position of the examples, 790: General organisation, 790: External features, 790: Shell, 793: Internal skeleton, 796 : Gills, 797: Osphradia, 797: Coelome, 797: Alimentary organs, 797 : Heart and vascular system, 797: Nervous system and sense-organs, 797 : Nephridia, 798 : Sexes, 798 : Development, 798, 799, 800, 801, 802 : Distribution, \&c., 803 : Relationships, 804

Cephalopodium, 761

Cephalothorax, of Astacus, 541

Cerata, 744

Ceratella, 146

Ceratella fusca, 145

Ceratites nodosus, 795

Ceratium, 79

Ceratosa, 126

Cercaria, 244, 245*

Cerci, 623

Cerebral organ, $294^{*}, 495$

Cerianthus, 200, 202

Cervical fold, of Apus, 527

Cervical glands, 306

Cervical groove, of Astacus, 541

Cervical sclerites, 622

Cestida, $221^{*}, 223$

Cestoda, 253* 261, 262, 263, 264, 266, $268,269,270,273,279,280,281,282$, 285, 286, 287, 288: Example, 245

Cestus veneris, 223

Cetonia aurata, 316

Chrta, 440-See Seta

Chcetoderma, 71:3, 715, 716, 717, 718

Chatogasta, 486

Chætognatha, 297* 316 : External characters, 316: Body-wall, 316 : Fnteric canal, 317: Ccelome, 317: Nervous system, 317: Sensory organs, 318 : Reproduction, 318: Development, 318

Chotonotus, 336

Chatopterus, 469, 481

Chatosoma, 319
Chatosomidae, 319*

Chatopoda, 439: Examples, 440, 454 Distinctive characters and classification, 464: Systematic position of examples, 466: General organisation, 467: General form, 467, 468: Parapodia and setæ, 468, 469, 470: Branchix, 471: Body-wall, 473: Colome, 474: Enteric canal, 475: Bloodvessels, 476: Nervous system, 476 Organs of special sense, 478: Orgins of excretion, 479, 480, 481 : Phosphorescence, 481: Reproductive organs, 481 : Development, 483, 485: Asexua reproduction, 486, 487: Mode of life, \&c., 488: Appendix, 489

Chatosomide, 319

Chalk, 56

Charybdiea marsupialis, 181

Cheilostomata, $347^{*}, 348,349,351,352$, 354

Chelæ, 543*

Cheliceræ, 653* $662,664,665,666$

Chelifer bravaisii, 662

Chelipeds, 543*

Chilaria, 666

Chilina, 745

Chilognatha, $615^{*}, 617,618$

Chilopoda, 615* $616,617,618$

Chironomus, 644

Chitin, 31, 46*

Chiton, 680, 713, 714, 715, 717, 718, 719, 720

Chitonellus, 714

Chlamydomyxa, 64, 65

Chloriemida, 475

Chloragen cells, 458

Chlorophyll, 14, 58, 65, 68, 72, 78, 119

Choanocytes, 107, 108, 109*, 111

Choanoflagellata, $69^{*}$ : General structure, 77: Collar, 77: Colonies, 77, 78: Reproduction, 78

Chotopterus, 469

Chondracanth $118,564,575,576$

Chordotonal organ, 645

Chorion, of Cephalopoda, 798 : of Insects, 628

Choristida, 126

Chromatin, $16^{*}, 17$

Chromatophores, Actinospharium, 57, 58 Chlamydomyxa, 65 : Flagellata, 71, 72: Dinoflagellata, 78, 79: Sepia, 762: Cephalopoda, 793

Chromosome, 17, 18*

Chrysalis, 651

Cicada, 634, 646

Cicatrix, 776

Cidaris, 426

Cilia, 23*

Ciliary flames, 269

Ciliary process, 771

Ciliata, $91^{*}$ : Form of body, 92, 93, 94. 95, 96, 97, 98: Stalk, 92, 96, 97 
Arrangement of cilia, 92, 94, 96 : Undulating membranes, 92, 94: Meganucleus, 93, 94 : Micronuclei, 93, 94 : Contractile vacuole, 93, 94, 96, 97 : Non-contractile vacuoles, 93, 94: Trichocysts, 93, 94: Digestive apparatus, 94, 95 : Skeleton, lorica, 94, 95, 96: Operculum, 95, 96 : Colonies, 94, 95, 97 : Reproduction, 96, 97, 98 : Conjugation, 99

Ciliated chamber's, 118

Cinclides, 188, 201

Circulation-See Vascular system

Circulatory system - See Vascular system Cirri, 405*, 439

Cirripedia, 565* , 593, 594, 596, 597, 598, 601,602

Cirrus, 240

Cirrus sac, 249

Cistella, 366, 368, 369, 370, 371

Cladocera, 564*, 572, 573, 596, 598

Cladophiuræ, $411^{*}$

Class, 4 *

Classification, $3^{*}, 5$ : of Rhizopoda, $4 \overline{7}$ : Mastigophora, 69 : Sporozoa, 81 : Infusoria, 91 : Porifera, 112 : Hydrozoa, 140: Scyphozoa, 176: Actinozoa, 193: Ctenophora, 220: Platyhelminthes, 251 : Nemertinea, 295: Nematoda, 303: Rotifera, 327: Polyzoa, 347: Brachiopoda, 366 : Echinodermata, 410 : Chætopoda, 464: Gephyrea, 495 : Hirudinea, 515: Crustacea, 561 : Insecta, 631 : Arachnida, 660 : Pelecypoda, 694: Amphineura, 712 : Gastropoda, 732: Cephalopoda, 789

Clathrina, 116, 120, 122, 124, 126

Clathrina blanca, 124,

Clathrozoon, 146

Clathrulina, 58

Clavatella, 145

Clavulæ, $417^{*}$

Cleaning foot, 581

Clepsine, 516, 517, 518, 519, 521, 522

Cliona, 122, 126

Clitellum, 455, 468, 509

Cloaca, 249

Clypeaster subdepressus, 423

Clypeastridea, $412^{*}, 417,423$

Clypeus, $620^{*}, 637$

Cnidoblast, $133^{*}$

Cnidocil, 133, 134*

Coccida, 647

Coccidiidea, $81^{*}$ : Characteristic features, $83,84,85$

Coccidium, 83, 84, 85

Coccoon, 463, 483

Cockchafer, 643

Cockles, 680, 696, 698

Cockroach - See Periplaneta

Cockroaches, 526, 619, 633, 636, 638, $648,653,656,658$

Cocoa-nut crab, 569
Codonella, 94

Cœlenterata, Classes, 128: Examples, 128, 168, 185, 211 : Relationships, 226229: Appendix, 230: Relationships to Sponges, 228

Coeliac canal, 407

Cielome and body-cavity, of Ascaris, 301 : Nematoda, 307 : Acanthocephala, 313: Chætognatha, 317 : Bugula, 343: Endoprocta, 354: Phoronis, 356 : Mugellania, 365: Asterias, 381 : Sea urchin, 399: Sea-cucumber, 403: Antedon, 407: Echinodermata, 425: Nereis, 442 : Chætopoda, 474: Sipnnculus, 493: Gephyrea, 498: A pus, 533 : Crustacea, 593 : Peripatus, 608: Insecta, 640: Anodonta, 684: Amphineura, 715 : Sepia, 766 : Cephalopoda, 797

Coelomoducts, 439, 480

Coeloplana, 225, 286

Cœnenchyma, 207*

Cuenosarc, 131*

Coleoptera, 635* , 638, 640, 645, 647, 649, 653

Collar of choanoflagellata, 77

Collared cells, 107, 108, 109*

Collared monads, 77, 78

Collencytes, 107, $111^{*}$

Colleterial glands, 628

Collozoum, 61, 63

Colochiros, 401 : General external features, 401: Structure of body-wall, 402: Ambulacral system, 402 : Nervering, 402: Perihæmal and hæmal systems, 402 : Colome, 403 : Enteric canal, 403, 404: Reproductive organs, 404: Development, 404: Systematic position, 414

Colony, 40*: of Foraminifera, 51 : Heliozoa, 59: Radiolaria, 61: Flagellata, 73 : Choanoflagellata, 78 : Ciliata, 97: Tentaculifera, 101: Obelia, 129 : Leptolinæ, 143 : Actinozoa, 197 : Polyzoa, 340 : Bugula, 341 : Ectoprocta, 348 : Endoprocta, 355

Colpoda, 98

Columella, of Coral, 205* : of Triton, 722

Column, 185*

Comatula, 434, 435

Comatulida, 415

Comb-jellies, 128

Combs, of Hormiphora, 211* 212

Comb-ribs, $213^{*}$

Commensalism, in Sponges, 126: $\mathrm{Hy}$ dractinia, 144: Actinozoa, 208 : Platyhelminthes, 284: Chætopoda, 488: Crustacea, 602

Complemental males, 597

Conchiolin, 683*

Conchostraca, 563*, 569

Condylostoma, 94 
Cone, of Coronata, 179

Cones, 733

Conjugation of $A m s e b a, 47$ : Foraminifera, 56: Heliozoa, 60: Cystoflagellata. 79 : Ciliata, 99: Flagellata, 75: Paramoecium, 90

Connective tissue, 25*: Gelatinous, 25, 26 : Fibrous, 25 : Retiform, 25, 26

Connective tissue cells of Sponges, 111

Contractile vacuole, $11 *, 13,47,66,68$, 77, 88, 89

Contractility of muscles, $28,37^{*}$

Conus, 749

Convoluta, 255, 265

Copepoda, 564* $573,593,594,596,597$, 598

Coral, Aporose, 207* : Black, 195 : Blue, 195: Fossil, 210: Organ pipe, 195 : Perforate, 207* : Red, 195, 210: Reefbuilding, 210 : Stony, 195

Coral limestones, 210

Coral reefs, 159, 210

Corallines, 340

Corallite, 128, 205*

Corallium, 195, 197, 198, 203, 208, 210

Corallum, 205*

Cordylophora, 167

Cornea, 771 : false, 771

Corona, of Polyzoa, 344: Sea-urchin, 396

Coronary groove, $176,179 *$

Coronata, $176^{*}, 179,180$

Corpuscles, 30 : amceboid, 30 : Miescher's or Rainey's, 88

Cortex, of Actinospharium, 57, 58 : Monocystis, 80: Paramacium, 88, 89 : Sponges, 111, 119

Corymorpha, 145, 146

Couplers, 573

Covered-budded Hydroids, 143

Cowries, 733

Coxa, 622

Coxal glands, Peripatus, 609: Scorpion, 658 : Árachnida, 667

Crabs, 526, 569, 587, 590, 593, 595, 596, 599,603

Crane-flies, 635

Crangon, 569, 588

Crania, 366, 368

Crayfish, 539-See Astacus

Crayfishes, 526, 569, 587, 596, 600

Cretaceous, 7

Crickets, 636

Crinoidea, example, 405: Distinctive characters and classification, 413 : Apical system, 417: Modifications of form, 424: Colome, 425: Ambulacral system, 426: Blood-vascular system, 427 : Hæmal system, 427 : Axial organ, 428: Enteric canal, 428: Nervous system, 429: Reproductive organs, 429 : Development, 430: Ethology, 434
Crioceris, 635

Crisia, 347

Crista acustica, $772^{*}$

Cristatella, 348, 350

Crop, 32

Crown, of Coronata, 179

Crustacea, 526, 678 : Example $a, 526$ Example $b, 539$ : Distinctive characters and classification, 561: Systematic position of the examples, $569:$ General organisation, 570 ; External characters and structure of appendages, 570: Texture of the exoskeleton, 593: Bodycavity, 593 : Enteric canal, 593: Re spiratory organs, 594: Heart, 596 : Fixcretory organs, 596: Nervous system, 596: Sense-organs, 597: Reproduction, 597 : Development, 597 Ethology, 600 : Affinities and mutual relationships, 602: Appendix, 604

Cryptocephala, $465^{*}, 468,475,476,484$, 488

Cryptomonas, 71

Cryptoniscus, 586, 587

Cryptozonia, $411^{*}, 414$

Crystalline style, 685: of Gastropoda, 744

Ctenaria, 150, 227

Ctenidium, 685, 687, 688, 702: of 'T'riton, 725 : of Gastropoda, 742

C'tenodrilus, 503, 504

Ctenophora, 128, 211: Example, 211: Distinctive characters and classification, 220: Systematic position of the example, 221 : General organisation, 222, 223, 224: Appendix, 225: Relationships, 226

Ctenoplana, 225, 286

Ctenopteryx, 791

Ctenostomata, 348*, 350, 351, 353

Cubomedusæ, $176^{*}, 180,181$

Cucumaria planci, 401

Cucumaria -See Colochirus

Culex, 586, 634, 638

Cuma, 567

Cumacea, 567* , 583, 594, 603

Cunarcha, 154

Cunina, 167

Cunina parasitica, 156

Cup-coral, 205

Cursoria, 636

Cursoria, 636

Cuspidaria, 696

Cuticle, $31^{*}$

Cuttle-fish, 680, 759, 790

Cuvier, 3

Cuvieran organs, 428

Cyamus, 568, 586

Cyanea arctica, 182

Cyclas, 710

Cyclidium, 94

Cyclops, 564, 573, 574, 596, 597

Cyclostomata, $347^{*}, 349,352,354$ 
Cydippe, 210

Cydippida, 221 *, 222

Cymothoa, 586, 597

Cynipida, 647

Cypræa, 739

Cypris, 564, 572, 573, 596

Cypris stage, of Cirripedes, 565, 598

Cyst, Amoeba, 46* , 47: Didymium, 67 ; Euglena, 68, 69: Monocystis, 80

Cysticercoid, $2 \times 0^{*}$

Cysticercus, 251, 280, 281*

Cysticercus cellulosa, 285,286

Cystoflagellata, $69^{*}$ : Characteristic features, 79

Cystoidea, $413^{*}, 435,437$

Cythere, 564, 572

Cytoplasm, 16*

\section{1)}

D ACTYLOPORES, of Millepora, 156* Stylaster, 159

Dactylozooids, 149*, 163: of Millepora, 158: Stylaster, 158: Halistemma, 160, 161

Daddy long-legs, 635

Dahlia wartlet, 185

Dallingeria, 71

Dalmanites socialis, 605

Daphnia, 564, 572

Darwin, 6

Dasychone, 478

Daughter-cell, 18*

Daughter-chromosomes, 18 *

Danghter-cysts, 282

Daughter-nucleus, $18^{*}$

Daughter-segments, 16

Day-flies, 641, 653

Dead men's fingers, 195, 203

Decapoda, 568* $570,587,594,596,597$, $599,602,603$

Decapoda (Cephalopoda), 790* 791, 804

Jegeneration, in Copepoda, 575 : in Cirripedia, 579: in Isopoda, 586

Deiopea, 223

Deltidium, $360^{*}$

Deltoid plates, 414

Demospongia, $112^{*}$

Dendrochirotæ, $415^{*}$

Dendrocolum-See Planaria

Dendrocometes, 100

Dendrophyllia, 207

Dendrosoma, 100, 101

Dentalium, 756, 757

Denticles, 442

Depastrida, 178

Derm (dermis), $31^{*}$

Dermal branchiæe of Echinoidea, 395 .

Dermal branchiæ, 376, 395, 425
Dermal cortex, 108, $111^{*}, 119$

Dermal pores, 376

Dermaptera, 636

Dermis, $31^{*}$

Desmoscolecida, 320, 321

Desmoscolex, 320

Destructive metabolism, $13^{*}$

Deutomerite of Gregarina, 82*, 83

Development, of Sycon, 124, 125 : Sponges, 122: Obelia, 139: Leptolina, 152: Trachylince. 155: Aurelia, 173, 174, 175: Sea-anemone, 192: Hormiphora, 217: Dicyemida, 231: Rhopalura, 233: Planaria, 239 : Liver-Fluke, 243: Tania, 250 : Platyhelminthes, 273-282: Nemertinea, 294, 295 : Ascaris, 303 : Nematoda, 308, 309: Chrtognatha, 318 : Brachionus rubens, 326 : Rotifera, 334 : Bugula, 344 : Ectoprocta, 352 : Fndoprocta, 355 : Phoronis, 358, 359 : Brachiopoda, 370: Asterina, 388, 389 : Sea-urchin, 400 : Sea-cucumber, 404 : Antedon, 432 : Echinodermata, 430 : Nereis, 450, 451, 453 : Lumbricus, 463, 464: Chætopoda, 483: Gephyrea, 500 : Hirudo, 515: Hirudinea, 520, 521 : Apus, 538: Astacus, 557: Crustacea, 597 : Peripatus, 611, 612, 613 : Myriapoda, 618: Periplanta, 628: Scorpion, 659: Anodonta, 692: Pelecypoda, 708: Amphineura, 718: Gastropoda, 749 : Scaphopoda, 757 : Cephalopoda, 798

Devonian, 7

Diastylis, 567, 583

Diatomin, $78^{*}$

Dibothriocephalus, 273

Dibranchiata, $789^{*}, 792,793,794,795$, $797,798,804$

Diceras, 700

Dichyocysta, 95

Dicyclica, $413^{*}$

Dicyema, 230, 231

Dicyemida, 230, 231

Dilinium, 93, 94

DIDYMIUM DIFFORME, 66,67

Differentiation, $23^{*}$

Diffugia, 49, 50

Digenetica, 252* 257, 259, 266, 268, 272, $273,277,284,286,287$

Digestion, intracellular, 33

Digestive glands, 33

Digestive system, 32 *

Digestive system, of Paramacium, 90: Aureliu, 170, 171; Sea-anemone, 187 : Hormiphora, 213: Planaria, 236, 237: Liver-Fluke, 241: Platyhelminthes, 265, 266, 267: Nenuertinea, 290: $A$ scaris, 299, 300: Nemałoda, 305, 306 : Chætognatha, 317 : Brachionus rubens, 325: Rotifera, 333: Bugula, 343: Ectoprocta, 351: Endoprocta, 354: 
Phoronis, 357 : Magellania, 362 : A8terias, 382 : Sea-urchin, 399, 400 : Seacucumber, 403, 404: Antedon, 407: Fchinodermata, 428: Nereis, 442: Lumbricus, 457: Chætopoda, 475: Sipunculus, 493, 494: Gephyrea, 498 : Hirudo, 409, 410: Hirudinea, 518: Apus, 532, 533: Astacus, 548 : Crustacea, 593: Peripatus, 608: Myriapoda, 617: Periplaneta, 624, 625 ; Insecta, 640, 641 : Scorpion, 656, 657, 658: Arachnida, 667 : Anodonta, 684, 685: Pelecypoda, 704: Anıphineura, 715 : Triton, 726, 727, 728: Gastropoda, 744 : Scaphopoda, 757 ; Sepia, 763 : Nautilus, 786 ; Cephalopoda, 797

Dimorpha, 70, 71

Dimorphism, in Foraminifera, 56: in Radiolaria, 63: in Flagellata, 76 : in Ciliata, 97, 98

Dimorphism, sexual, $40^{*}$

Dimorphograptus, 167

Dimyaria, 290

Dinobryon, 71, 74

Dinoflagellata, $69^{*}$ : Characteristic features, 78, 79

Dinophilea, 336, 337

Dinophilus, 524, 337

Dicecious, $40^{*}$, $139^{*}$

Diophrys, s2, 94

Diphyes, 164, 165

Dipleurula, $432^{*}$

1)iplomita, 71

Diplopoda, $615^{*}, 617,618$

Diplozoon, 277

Diptera, 634", 638, 639, 640, 644, 646, $647,649,651,653$

Directive mesenteries, of Sea-anemone, 189

Discina, 189, 366, 368

Disc, $185^{*}$

Disc-jellies, 182

Discoidal segmentation, 659

Discomedusæ, $176^{*}, 182,183,189$

Discorbina, 53

Discosoma, 208, 210

Discrelium lanceolatum, 285

Dissepiments, $205^{*}$

Distinctive characters-See Classification

Distomida, 253*, 254

Distomum, 265

Distomom hepaticoM, 240 - See Fasciola hepatica

Distomum rathousii, 285

Distribution, 8: of Sponges, 126 : Hydrocorollinæ, 159 : Scyphozoa, 184: Actinozoa, 209: Ctenophora, 224 : Platyhelminthes, 283 : Ectoprocta, 353 : Brachiopoda, 371 : Gephyrea, 502; Hirudinea, 524: Unychophora, 612: Pelecypoda, 710: Amphineura, 720: Gastropoda, 755 : Cephalopoda, 803

Distribution, geographical, 8
Distribution, geological, $8^{*}$ : of Foraminifera, 56 : Sponges, 126 : Corals, 210 : Brachiopoda, 371 : Chxtoporla, 488: Insecta, 652: Arachnida, 673 : Cephalopoda, 803

Distribution, vertical, $8^{*}$

Divaricators, 364

Dochmius duodenalis, 306

Docoglossa, 733*, 756

Doctrine of descent, 6

Donax, 706

Doris, 734, 742

Doris (Archidoris) tuberculata, 739

Dorsal, $42^{*}$

Dorsal cirri, of Autedon, 405

Dorsal organ, of $A$ pus, 527

Dorsal pores, of Earthworm, $45 \tilde{5}$

Dragon flies, 633, 644

Drepanophorus, 289

Drilophaga, 335

Dromia, 601

Ductus ejaculatorius, 302

Dytiscus, 645

\section{E}

E

1) $\mathrm{AR}, 39^{*}$

Ear-shells, 733

Earthworm-See Lumbricus

Earthworms, 439

Karwigs, 636

E.edyses, of A pus, 539

Echiniidle, $414^{*}$

Echinoderes, 319,320

Wchinoderidu, 319, 320

Echinodermata, $375^{*}$ : Examples, 375 , 393, 401, 405: Iistinctive characters and classification, 410: Systematic position of examples, 414 : General organisation, 415: General form and symmetry, 415 : Systems of plates, 417 : Modifications of form, 418: Cœlome, 425 : Ambulacral system, 425: Bloodvascular system, 426 : Hremal system, 427 ; Axial organ, 428 : Enteric canal, 428 : Nervous system, 429 : Sexes, 429 : Development and metamorphosis, 430 : Echinopædium, 432: Ethology, \&c., 434 : Self-mutilation and regeneration, 435: Affinities, 436

Echinoidea, example, 393: Distinctive characters and classification, 412 : Apical system, 417: Modifications of form, 421 : Dermal branchiæe, 425: Stewart's organs, 425: Ambulacral system, 426: Blood-vascular system, 427 : Hæmal system, 427 : Axial organ, 428: Enteric canal, 428: Nervous system, 429: Reproductive organs, 429: Development, 430: Ethology, 434 Echinopredium, $432^{*}$

E'chinorhynchus, 312, 313, 314, 315 
Echints, 393 : General external features, 393, 394, 395 : Corona, 396 : Aristotle's lantern, 397 : Nervolls system, 398 : Ambulacral system, 399: Enteric canal, 399, 400 : Colome, 399 : Blood-vascular system, 400: Reproductive organs, 400: Development, 400: Systematic position, 414

Echiuroidea, $496^{*}, 497,498,499,502$

Echiurus, 496, 497, 500, 501

Ectobranchiata, $415^{*}$

Ectocyst, $341^{*}$

Ectoderm, 23*-See Body-wall

Ectoprocta, $347^{*}, 348$ : Structure of borlywall, 351 : Alimentary canal, 351 : Nervous system, 351 : Nephridia, 352 : Avicularia, 352: Vibracula, 352: Reproduction and development, 352: Ethology and distribution, $\mathbf{3 5 3}$

Ectosare, 46

Edriasteroidea, $414^{*}, 435$

Edwardsia, 200, 202, 227, 229

Eggs-See Development

Eimeria, 84

Ejaculatory duct, 243

Elasipoda, $412^{*}, 428,434$

Eledone moschata, 798

Elephant's tusk shells, 756

Eleutherozoa, $410^{*}$

Elk-born coral, 156, 157

Elytra, of Cockroach, 622: Polychieta, 469 : Coleoptera, 635, 640

Embryology, $3^{*}$-See Development

Empis, 644

Emulsions, 34

Encystation, 45*, 46, 59

Endites, 530

Endocyst, 341*

Endoderm, 23*

Endoderm-disc, of Astacus, 558

Endoderm lamella, $136^{*} \iota$

Endogastric, $777^{*}$

Endophragmal system, of $A$ stacus, $54 \cdot 2$

Endopodite, 538*

Endoprocta, 340, 348*, 354: Vestibule, 354: Nephridia, 354: Cloaca, 354: Ganglion, 354: Testes and ovaries, 354 : Foot-gland, 354 : Development, 355

Endosare, 46

Endoskeleton, $31^{*}$

Endosternite, 655, 667

End-sac, 534

Enteric canal-See Digestive system

Enterocœle, 389

Enterozoa, 105

Entobranchiata, $415^{*}$

Entoralua, 711

Environment, $9^{*}$

Eocene, 7

Eolis, 734, 742, 744

Epeira diadema, 664

Ephelota, 100, 101
Ephemera, 633

Ephemerida, 641, 653

Ephippium, 597

Ephyropsis, 179, 180

Ephyrula, of A urelia, $175^{*}$

Epiblast, 23*

Epibolic gastrulation, 275

Epiboly, 218*

Epicranium, 620*, 637

Epidermis, 24, 31-See Body-wall

Epimerite of Gregarinida, 82, 83*

Epineural canals, 398*

Epipharynx, 641

Epiphragm, 738

Epiphysis, of Sea-urchin, 398

Epipodite, 544*

Epipodium, 740

Epistoma, of Astucus, 542

Epistome, 340, 351, 356

Epistylis plicatilis, 92, 94

Ejpistylis umhellaria, 93

Epitheca, 205*

Epithelia, 24*: Non-stratified, 24* : Stratified, 24*

Epithelium, enteric, $33^{*}$

Equatorial plate, $18^{*}$

Equivalve, 699*

Ergasilus, 564, 575, 576

Estheria, 564, 571

Ethiopian, 9*

Ethology, $9^{*}$ : of Platyhelminthes, 283: Rotifera, 335 : Ectoprocta, 353: Echinodermata, 434: Chætopoda, 488: Hirudinea, 522: Crustacea, 600 : Insecta, 651: Arachnida, 672: Pelecyporla, 710: Amphineura, 720 : Gastroporla, 755

Euchlanis, 328

Euchlora, 222

Eucirripedia, $565^{*}, 577,579$

Eucope, development, 152

Eucopepoda, 564* , 573, 574, 575, 602

Endendrium, 140

Euglena viridis, 67, 68: Systematic position, 70

Eulamellibranchiata, $696^{*}, 697,698,711$, 712

Eucarida, 568*, 587

Eucopella, 134

Englenidax, 70

Euglenoidea, 70

Eumalacostraca, $566^{*}, 581$

Euphausia, 568, 595, 599

Euphausiacea, 568*, 587, 594, 596, 599, 603

Euphrosyne, 468

Euphyllopoda, 596

Euplectella, 120

Ëupomatus, 485

Euryalida, 417, 420, 421

Eurypterida, $662^{*}, 666,668,673,678$

Eurypterus fischeri, 668

EUSCORPIO-See BUTHUS 
Euscorpius italicus, 659,671, 672

Euspongia, 115, 121

Euthyneura, 734*, 737, 742, 745, 747, 748

Evolution, 6

Examples, of Rhizopoda, 46 : Mycetozoa, 66: Mastigophora, 67 : Sporozoa, 80 : Infusoria, 88: Porifera, 105: Hydrozoa, 128: Scyphozoa, 168: Actinozoa, 185: Ctenophora, 211: Platyhelminthes, 236, 240, 245: Nematoda, 297 : Rotifera, 323: Polyzoa, 342: Branchioporla, 360: Asteroidea, 375: Echinoidea, 393: Crinoidea, 405: Chætopoda, 440, 454: Gephyrea, 492: Crustacea, 526, 539: Insecta, 619: Arachnida, 653 : Pelecyporla, 680: Gastropola, 721 : Cephalopora, 759 776 : Holothuroidea, 401

Excretion, 14*, 37

Excretory pores, 213, 290, 298

Excretory system, of Leptolinix, 151: Planaria, 238: Liver-Fluke, 241 : Trenia, 248: Platyhelminthes, 269: Nemertinea, 292: Ascraris, 301: Echinorhynchus, 313 : Bruchionus rubens, 326 : Rotifera, 333 : Ectoprocta, 352 : Endoprocta, 354: Phoronis, 356: Magel. lania, 365: Brachiopoda, 369: Nereis, 448 : Lumbricus, 460, 461 : Chietopoda, 479, 480: Siminculus, 495 : Gephyrea, 499: Hirudo, 510, 512: Hirudinea, 519, 520: Apus, 534, 535: Astacus, 551, 552: Crustacea, 596: Peripatus, 610: Periplaneta, 625: Insecta, 641: Scorpion, 656 : Arachnida, 667: Anodonta, 689: Pelecypoda, 705 : Amphineura, 718, 719 : Triton, 729 : Gastroporla, 747: Scaphopoda, 757: Sepia, 772, 773 : Nautilus, 786 : Cephalopoda, 798

Excurrentcanals, of Sponges, 108, 109*

Exhalant siphon, 681

Exites, 531

Exogastric, $777^{*}$

Wxopodite, $538^{*}$

Fxoskeleton, $31^{*}$--See Body-wall

External features, of Sycon, 105, 106: of Porifera, 114, 115, 116 : Obelia, 130 : Aurelia, 168, 169 : Sea-anemone, 185, 186 : Actinozoa, 196 : Hormiphora, 211, 212: Planaria and Dendrocoelum, 236 : Liver-Fluke, 240, 242: Toenia solium, 245: Platyhelminthes, 254 : Nemertinea, 288: Ascaris lumbricoides. 297 : Nematoda, 305 : Echinorhynchus, 312: Chætognatha, 316: Brachionus rubens, 323, 324: Rotifera, 330: Bugula avicularia, 341, 342 : Ectoprocta, 349 , 350 : Endoprocta, 354 : Phoronis, 356 : Magellania, 362 : Brachiopoda, 366 : Asterias rubens, 375, 376, 377 : Anthenea flavescens, 386; Sea-urchin, 393, 394,
395: Sea-cucumber, 401: Anterlon rosacea, 405, 406 : Echinodermata, 415 : Asteroidea, 418: Ophinroidea, 415: Echinoidea, 421 : Holothuroidea, 416 : Crinoidea, 424: Nereis, 440, 441 : Lumbricus, 4.74 : Chetoporla. 467, 468 : Sipnenculus nudıs, 492, 493 : Gephyrea, 496: Archi-annelida, 503: Hirudo, 506. 507, Hirudinea, 517: A pus, 527 : Astacus, 540: Crustacea, 570: Peripatus, 607, 608: Myriapoda, 615: Periplaneta, 619, 620 : Insecta, 636 : Scorpion, 654, 655: Arachnicla, 662 : Anodonta, 684: Pelecypola, 696: Amphineura, 713, 714: 'Triton, 723: Gastropoda, 735: Scaphopoda, 756 : Sepia, 759, 760: Nautilux, 777: Cephalopoda, 790

Ex-umbrella, 134*

Eyes, 39* : of Euglena, 68: Medusae, 155): Planaria, 236 : Platyhelminthes, 268: Nemertinea, 294: Nematoda, 307 : Chretognatha, 318 : Brachionus, 326 : Rotifera, 334: Dinophilus, 337 : Brachiopoda, 369 : Asterias, 377: Nereis, 447 : Chretoporla, 478 : Hirudo, 514: Apus, 536, 537: Astacus, 556: Crustacea, 597: Periplaneta, 627: Insecta, 644, 645: Arachnida, 670: Pelecypoda, 707: Chiton, 717 : T'rilon, 731 : Gastropoda, 746: Sepia, 771: Nantilus, 787

F

\section{$H_{A B R I C I A, 478}$}

Facets, $556^{*}$

Facial suture, $605^{*}$

Fæces, 33*

Falciform young - See Sporozoites

Family, $4^{*}$

Fasciola hepatica, 240: General feitures, 240, 242: Body wall, 241: Digestive system, 241: Water-vessels, 241: Nervous system, 241: Reproductive organs, 242, 243: Development, 243, 244: Systematic position, 254 Fasciolina, 254

Fat, $26^{*}, 27$

Fat body, of Periplaneta, 624

Fauna, $8^{*}$

Feather-star-See Antedon rosucea

Feeding, method of, $A m a b a, 12,46$ Actinophrys, 57: Chlamydomyxa, 65 : Euglena, 68, 69: Flagellata, 72 : Choanoflagellata, 78: Monocystis, 80 : Tentaculifera, 101

Femur, of Cockroach, 622

Fenestræ, of Periplaneta, 621 *

Ferment, 12 *

Fertilisation-See Impregnation 
Fever, parasite of, quartan, 86 : Tertian, 86

Fibres, nerve, $29^{*}, 30$

Fibro-cartilage. 26*, 27

Filaria bancrofti, 312 .

Filaria medinensis, 305, 312

Filariasis, 312

Filibranchia, 695* $697,698,711,712$

Fimbriæ, of Fresh-water Mussels, 681

Fire-flies, 645

Fission, 14, 40*, 46, 50, 59, 65, 69, 73, 74, $78,79,81,90,101$

Fissurella, 737, 745

Five-chambered organ, 409*

Fixed cheek of Trilobites, 605 *

Flabellum, 205, 206, 207

Flagella of Copepods, 565*

Flagellata, 69*: Cell body, 70, 71 : Flagella, 70, 71: Modes of nutrition, 72: Skeleton, 73: Colonies, 73: Asexual multiplication, 74: Sexual reproduction, 75

Flagellate canals, of Sponges, 107, 108, $109^{*}, 118$

Flagellate cells, 108, 109

Flagellula, 50, $51^{*}, 56,58,60,63,65,66$, $69,78,79$

Flagellum, $24^{*}, 68,69$

Flagellum, of Astacus, 544

Flame-cells, 238, 269

Flat-worms - See Platyhelminthes

Fleas, 635, 640, 651

Flies, 526, 619

Float, $159^{*}, 160$

Floscularia, 328, 330, 331

Flustra, 347

Folliculina, 94

Food-yolk, 30

Food vacuole, of Actinophrys, 57 : Paramucium, 90*

Foot, of Anodonta, 681: Pelecypoda, 701: Amphineura, 713: Triton, 724: Gastropods, 738, 739: Sepia, 760: Nautilus, 777 : Cephalopoda, 791

Foot-gland, 354

Foraminifera, 48* : General structure, 50, 51: Skeleton, 51, 52, 53 : Protoplasm, 54: Dimorphism, 55, 56* : Reproduction, 56 : Distribution, 56

Formica rufa, 652

Fossettes, 591*

Fossils, 7*

Fossula, 210

Fredericella, 348

Fresh-water Crayfish, 539

Fresh-water Mussel-See Anodonta

Fresh-water Sponges, 126

Fresh-water Worms, 439

Fritillaria, 233

Frondicularia, 53

Frontal suture, $605^{*}$

Fulcrum (of Rotifera), 325

Fungia, 205
Funic, $343^{*}$

Funiculus, 343, 35̃

Funnel, 792

Funnel folds, 792

\section{G}

G

Galathea, 569

Galeodes, 663, 661

Galls, 647

Gall-flies, 636, 647

Gall-insects, 647

Gametes, of Flagellata, 74, 75* : Sporozoa, 80,81

Gametocytes, $\mathbf{8 0}, \mathbf{8 6}$

Gammarus, 584, 568, 583

Gamobium, 140* 176

Gamogenesis, 21*

Ganglia, 38, 242

Gastral cortex, 108, $111^{*}, 119$

Gastric filaments, of Aurelia, 172*

Gastric mill, 593: of Astacus, 549

Gastric ostium, 110*

Gastric ridges, of A urelia, $173^{*}$ : pouches, $170^{*}$

Gastrolith, 549*

Gastrophilus equi, 634

Gastropoda, 680, 721* : Example, 721 : Distinctive characters and classification, 732: Systematic position of example, 734 : General organisation, 735 : External features, symmetry, \&c., 735 : Shell, 737 : Foot, 738, 739: Head, 740 : Mantle, 740: Respiratory organs, 742: Osphradium, 744: Digestive organs, 744: Heart, 744: Nervous system, 745: Organs of special sense, 746 : Nephridia, 747 : Reproduction, 748: Development, 749, 751, 752, 753, 754, 755 : Ethology and distribution, 755: Relationships, 756: Appendix, 756

Gastropores, of Millepora, 156*

Gastrotricha, 323, 335*, 336

Gastrozooids, 163, 202

Gastrula, 23*

Gastrulation, 22, 23*

Gelasimus, 591, 602

Gemmules, 112, 121*

Genæ, 621*

Generic, 1*

Genital cloaca, $248^{*}$ : rachis, 385,430 : stolon, 385, 430 : plates, 396, 397 : bursæ, 430 : operculum, $655^{*}$

Genital system-See Reproductive system Genus, 1* $^{*}$

Geological distribution-See Distribution, geological

Geoplanidae, 253

Gephyrea, 439, 491* : Example, 492 : Distinctive characters and classification, 
495 : Systematic position of the examples, 496: General organisation, 496 : Body-wall, 496 : Colome, 498 : Alimentary canal, 498: Vascular system, 498: Nervous system, 498 : Eyes, 498: Nephridia, 499: Reproductive organs, 499: Sexual dimorphism, 499: Development, 500, 501: Distribution, affinities, \&c., 502, 524

Germarium, 270, 272, 239*

Germinal bands, of Clepsine, 521: of Periputus, 612

Germinal disc, 798

Germinal layers, $23^{*}$

Germinal spot, $19^{*}, 30$

Germinal vesicle, $19^{*}, 30$

Germ-vitellarium, 270

Giant Clam, 710

Giant fibres, 477

Giant nerve-cells, 478

Gigantorhynchus, 312, 313, 314, 315

Gill-cover, of Astacus, 541

Gills, 35*-See Respiration

Gizzard, 32

Glabella, $605^{*}$

Glands, 25*

Glands, Multicellular, 25* : Unicellular, $25^{*}$ : Ducts, 25 : Salivary, $34^{*}$

Glass-crab, 599

Glass-rope sponge, 120, 127

Glenodinium, 79

Globigerina, 53, 56

Glochidium, 693

Glomeris, 282

Glossiphonia, 516

Glossocodon, 154

Glow-worms, 645

Glycera, 472, 474

Glycerida, 476

Gnathobase, 531

Gnathobdellida, $516^{*}, 517,518,522$

Gnats, 635

Goblet-shaped bodies, 460

Goblet-shaped organs, of Leech, 514

Gonads-See Reproductive system

Gonapophyses, 628*

Gonocœle, 783*

Gonodactylus, 569

Gonodendra, $163^{*}$

Gonopodaria, 354

Gonopore, 298

Gonotheca, of Obelia, 129*, 130

Gonozooids, 202*

Gordius, 304, 306, 308

Gorgonacea, 195*, 203, 204

Gorgonia, 197, 204

Granule glands, 270, 271

Grapsus, 569

Graptolithida, 142*, 166, 167

Grasshoppers, 619, 633, 636, 645

Green gland, of Astacus, 551

Gregarina, 82, 83

Gregarina blattarum, 82
Gregarina dujardini, 82

Gregarina gigantea, 82, 83

Gregarinida, 81* : Characteristic features, 82,83

Gressoria, 636

Gromia, 51, 52, 56

Guard, $795^{*}$

Guard-polypes, $149^{*}$

Guinea-worm, 305, 312

Gula, 637*

Gullet, 32-See Digestive organs

Gunda segmentata, 255, 256, 291, 295

Gustatory organ, $39^{*}$

Gyge, $\mathbf{5 8 7}$

Gymnoblastea, 143 *

Gymnolæmata, $347^{*}, 348,351,352$

Gyractis, 200, 201

Gyrocotyle, 262, 263, 287

Gyrodactylida, 259

Gyrodactylus, 277, 258

$\mathrm{H}$

$\mathrm{H}$ ABITS-See Ethology

Hamadip 8 , 516, 522

Hæmal system, 380

Hæmatochrome, 68, 72

Hamatococcus, 72, 73

Himocele, of Crustacea, 526, 593* : Peripatus, 624: Insecta, 640

Hæmocyanin, 555

Hæmoflagellata, 72

Hæmoglobin, 30, 36

Hamopsis vorax, 522

Hæmosporidea, $81^{*}$ : Characteristic features, 85,86

Halicystus, 177

Haliotidae, 733

Haliotis, 737, 744, 745

Halistemma, 159, 161, 162, 168

Halistemma tergestinum, 160, 163

Halteres, 640

Hamingia, 500

Hartea, 195, 202, 197

Harvest-men, 661, 665

Hastigerina, 54, 55

Haversian canals, $27^{*}, 28$

Head, 43*

Head-germs, 522*

Head-kidneys, 452

Head-lobes, of Astucus, 558: of Fasciola, 240 *

Heart, $36^{*}$

Heart-See Vascular system

Heart-urchins, $412^{*}, 417,422$

Hectocotylisation, 760, 792

Heliozoa, 48* : General structure, 56, 57, 58: Colonies, 59: Skeleton, 58, 59: Reproduction, 59: Conjugation, 59,60

Heliopora, 195, 203, 208, 209, 210 


\section{INDEX}

Helix, 748, 749

Helix nemoralıs, 742

Hemipneustes radiatus, 423

Hemiptera, $633^{*}, 638,653$

Hemisomes, $43^{*}$

Hepatic cæca, 407

Hepato-pancreas, 34, 549

Hermaphrodite, 39 *

Hermit-crabs, 569, 588, 589

Hermit-crabs and Hydractinia, 144

Herpobdella, 516, 519, 520, 522

Herpobdellida, 516*

Hesionida, 475

Heterocœla, $112^{*}$

Heterocotylea-See Monogenetica, 252

Heterocyemidu, 230, 232

Heterogamy, $41^{*}$

Heterogeny, 277* $287,310^{*}$

Heteromita, 71, 72, 75, 76

Heteromyaria, 698*

Heteronemertini, $296^{*}$

Heteronereis, 440, 450

Heteropoda, $733^{*}, 738.740,756$

Heterotrichous, 92*, 94

Hexacanth embryo, of Tenia, 250 : Cestodes, 280

Hexactinellida, $112^{*}, 120,123,126$

Hexactinice, 196, 200

Hexarthra, 328, 330, 332, 335

Hinge-ligament, 682

Hinge-line, 361, 682

Hinge-teeth, 361, 683

Hippa, 569, 589, 603

Hippurites, 700, 701

Hirudinea, 439, 506: Example, 506: Distinctive characters and classification, 515: General organisation, 517 : Form and size, 517: Setæ, 518: Proboscis, 518 : Enteric canal, 518 : Blondressels, 518: Respiratory organs, 519 : Nephridia, 519, 520: Nervous system, 520: Sense-organs, 520: Reproductive organs, 520: Development, 520, 521 : Habits, distribution, \&c., 522, 524

Hirudinida, $517^{*}$

Hirudo, 520, 522

Hirudo australis, 506, 510, 511

Hirudo medicinalis, 506: External characters, 506, 507: Body-wall, 508, Muscular system, 508, 509: Alimentary organs, 509, 510: Excretory system, 510, 511, 512: Vascular system, 512, 513: Nervous system, 513: Senseorgans, 514: Reproductive organs, 514: Development, 515: Systematic position, 517

HiRudo quinquestriata-See H. MediCINALIS

Hirudo sanguisuga, 522

Histology, $3^{*}$

Histriobdellea, 336, 338, 339, 524

Holarctic, 9*

Holoblastic, $22^{*}$
Holophytic, 65* $69,72,78$

Holothuria - See Sea-cucumber

Holothurian-See Colochirus

Holothuroidea : Example, 401 : Distinctive characters and classification, 412: Feneral forn, 416: Moditications of form, 423: Colome, 425: Ambulacral system of vessels, 426 : Blond-vascular system, 426 : Hæmal system, 427 : Axial organ, 428: Enteric canal, 428: Respiratory trees, 428: Cuvierian organs, 428: Nervous system, 429 : Reproductive organs, 429: Development, 430 : Ethology, 434

Holotrichous, $89,92^{*}, 94$

Holozoic, 65*, 69

Homaloguster, 257

Homarus, 569, 599

Homocœla, $112^{*}$

Hood, 779*

Hook-headed worms, 297

Hoplocarida, 569*

Hormiphora PLUMosa, 211: External characters, 211, 212: Enteric system, 213, 214 : Cell-layers, 215: Nervous system, 216 : Sense-organs, 216: Reproductive organs, 216 : Development, $217,218,219,220$ : Systematic position, 221

Host, of parasite, 84 : intermediate, 87

House-flies, 619, 635

Hyaline cartilage, $26^{*}, 27$

Hyalonema, 120, 127

Hyalosphenia, 49

Hybrids, 2*

Hydatids, 282*

Hydatina, 328, 331

Hydra, 133, 147, 148, 1533, 167, 168

Hydractinia, 144, 145, 149, 167

Hydranths, of Obelia, 129*, 148, 187

Hydrocoele, 390

Hydrocorallina, $142^{*}, 156,157,158,159$, 167

Hydrocorallinæ, distribution, 159

Hydrortena, 227

Hydroids, naked-budded, $143^{*}$ : coveredbudded, $143^{*}$

Hydrophilus, 650

Hydrophyllia, of Halistemma, $160^{*}, 160$

Hydrorhiza, 128*

Hydrospires, $414^{*}$

Hydrotheca, of Obelia, 129*, 130, 167

Hydrozoa, 128: Example, 128: General structure and classification, 140: Alternation of generations, 141 : Systematic position of example, 142: General remarks on, 167

Hydrula, 139*, 140, 153, 229

Hymenoptera, $636^{*}, 638,642,646,649$, 653

Hyperia, $56 \mathrm{~S}$

Hypoblast, $23^{*}$

Hypodermic impregnation, 334, 5:20 
Hypopharynx, 641

Hypostome, of Obelia, 129*

Hypotrichous, 92*, 94

\section{I}

I chneumons, 636, 651

Ichthyophthirius, 102

Idmonea, 347

Idotea, 568

Illoricata, $328^{*}$

Imaginal discs, 651

Imago, 649

Impregnation, 19*, 20

Impregnation, hypodermic, 309

Inarticulata, $366^{*}, 367,368,369$

Incurrent canals, of Sponges, 107, 108*, 109,117

Incus (of Rotifera), 325

Individualdifferences, in $\mathrm{N}$ ereis dumerilii, 449

Individual of the first order, $168^{*}$

Individual of the second order, 168*

Individual of the third order, $168^{*}$

Individual variations, $2^{*}, 113$

Individuation, in Hydrozoa, 167

Inequilateral, 699*

Inermia, 496*, 497, 498, 499, 502

Infundibula, of A urelia, $173^{*}$

Infundibular canal, $213^{*}$

Infusoria, 46* : Example, 88: Classification, 91: Systematic position of example, 91

Infusoriform embryos, of Dicyemida, $230,231^{*}$

Inhalant pores, 106,117

Inhalant siphon, 681

Ink-sac, of Sepia, 765

Insecta, 526, 619*, 677, 678: Example, 619: Distinctive characters and classification, 631 : Systematic position of the example, 636: General organisation, 636: Exoskeleton, 636: Head, 637: Thorax, 637 : Abdomen, 637: Appendages of head, $637,638,639$ : Appendages of thorax, 639: Abdomen, 640: Hæmocœle, 640: Fat body, 640: Digestive system, 640, 641: Malpighian tubes, 641: Tracheal system, 642: Blood-vascular system, 642: Nervous system, 643, 644; Organs of special sense, 644, 645: Luminous organs, 645 ; Production of sounds, 645: Reproductive organs, 646: Eggs, 647: Development, 648, 649, 650: Metamorphosis, 649: Mode of life, 65l : Distribution in time, 652

Integripalliata, 696*

Integripalliate, $699^{*}$

Integument-See Body-wall

Inter-filamentar junctious, 686

Inter-lamellar junctions, 686
Inter-mesenteric chambers, $187^{*}$

Internodes, of Siphonophora, 161

Inter-radius, 138, 139*

Interstitial cells, of Obelia, 132

Intertentacular tube, 352

Intestinal cæeca, 383, 388

Introvert, of Polyzoa, 341 : Sipunculus, 493: Gephyrea, 496: T'riton, 724: Gastropoda, 744

Invagination, $23^{*}$

Iridocytes, $762^{*}$

Iris, 39, $771^{*}$

Isomyaria, $698^{*}$

Isopoda, $567^{*}, 583,584,585,586,587$, $593,596,597,603,677$

Isopteryx, $645^{*}$

Isopteryx apicalis, 645

\section{J}

$\int_{A w S, 32}$

Jelly-fish, Common (Aurelia aurita), 168 Jelly-fishes, 128

Jurassic, 7

K

$\mathrm{K}$ ARYOGAMY, 59

Karyokinesis, 16, 17

Keber's organ, 689, 706

Kidneys-See Excretory system

King-Ürabs, 653, 662, 666

Kingdom, $5^{*}$

Koonunga, 566

Kraussina, 368

Krohnia, 316

$\mathrm{L}$

I JABIAL PALPI, of Pelecypoda, 685

Labial palps, 622

Labium, 622

Labrum, 620

Labrum, of Apus, 529 : of Astacus, 54: : of Scorpion, 655 : of Periplaneta, 620

Labyrinthula, 65, 66

Lacinia, 622

Lacrymaria, 94

Lacteals, 34

Lacunæ (bone), 27 *

Lacunar system, 380

Lagena, 53

Lamellæ (of bone), 27,28

Lampetia, 217, 222

Lamp-shells, 360

Lancet plate, 414

Land-snails, 734

Lantern cœlome, 400 
Laomedea, 140

Lappets, of Sea-anemone, 187

Larva, of Desor, 295

Larval membranes, of Periplaneta, 630*

Larval organ, 391

Latreillia, 591

Laurentian, 7

Laurer's canal, 243

Lxverania, 86

Leaf-insects, 633, 636

Leda, 695

Leech-See Hirudo

Leeches-See Hirudinea

Lemnisci, 313*

Lemnobdella-See Hirudo

Lens, 39-See Eye

Lерas, 565, 577, 578

Lepas anatifera, 578

Lepas fascicularis, 598

Lepidoptera, $635^{*}, 639,647,649,651$, 653

Lepidurus-See Apus

Lepidurus kirkii, 528, 532

Lepisma, 632

Leptochelia, 567

Leptochone, 478

Leptodiscus, 79

Leptodora, 564, 572, 598

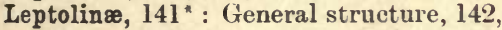
143, 144, 145: Perisarc, 143 : Medusæ, 143, 144, 145 : Cœnosarc, 143 : Reproductive zooids, 149 : Development, 152

Leptomedusæ, $141^{*}, 143,144,149$

Leptostraca, $566^{*}, 580,581,595,596,601$, 603

Lernaea, 564, 575, 576

Lesteira, $\mathbf{5 7 6}$

Leucilla convexa, 117

Lencocytes, 30

Leucodore, 209

Lice, 634, 640, 651

Ligula, 262, 288

Ligula, 622*

Lima, 696, 702

Limax, 739, 743

Limnceus, 746

Limnetis, 564, 571

Limnobdella-see Hirudo

Limnocnida, 167

Limnocodium, 167

Limpets. 721, 733, 743-See Patella

Limulus, 666, 667, 670, 671

Lineus, 232

Lingua, 641

Lingual ribbon, 726

Linguatulida, $673,674^{*}, 675,678$

Lingula, 366, 367, 368, 369, 371

Linnæus, 1, 3

Liquid tissues, 29

Lithite, $136^{*}$

Lithites, of Aurelia, 173

Lithobius forficatus, 615

Lithocircus annularis, 60
Lithocysts, $135^{*}$

Littoral forms, $8^{*}$

Liver, $34^{*}$

Liver-Fluke-See Fasciola hepatica

Liver-pancreas, 34

Lobata, $221^{*}, 223,224$

Lobosa, 48*, 49 : General structure, 48, 49 : Skeleton, 49

Lobsters, 569, 587, 599

Locomotion, of Amreba, 11, 46 : Heliozoa, 57: Euglena, 68: Flagellata, 72: Choanoflagellata, 78: Sporozoa, 80 :

Paramocium, 90: Tentaculifera, 101

Locomotor rods, 319

Locusta, 633

Locusts, 526, 633, 636, 646

Lohmanella, 233

Loligo, 796, 798, 800, 801, 802, 803

Loligo vulgaris, 792

Lophogaster, 567

Lophomonas, 93, 94

Lophophore, 340, 341, 362, 368

Lophopus, 351

Lorica, of Ciliata, 96: Flagellata, 71, 73: Choanoflagellata, 77, 78: Tentaculifera, 101 : Rotifera, 324

Loricata, $328^{*}, 329$

Loxosoma, 348, 354

Lucernarida--See Stauromedusæ, 176

Lucernaria, 176, 177, 178

Lucifer, 569, 599

Lucina, 703

Lumbricida, 467*

LUMBRicus, 454: General external features, 454: Body-wall, 456: Setæ, 454, 457 ; Setigerous sacs, 457 : Enteric canal, 457: Vascular system, 458: Nervous system, 459: Organs of excretion, 460, 461 : Reproductive organs, 461, 462: Development, 463, 464; Systematic position, 466

Lumbricus rubellus, 483

Lumbricus trapezoides, 483

Luminous organs, 645

Lung, $35^{*}$ : of Scorpion, 657 : of Pulmonata, 743

Lymph, 29*

Lysophiuræ, $411^{*}$

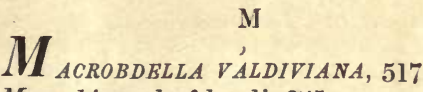

Macrobiotus hufelandi, 675

Macula acustica, 772

Macrura, 568* $601,602,603,670$

Madrepora, 207

Madreporaria, 194* $197,198,200,205$, $207,210,227$

Madrepores, 207, 210

Madreporic canal, 383, 399, 402

Madreporite, of Starfish, 376, 419 : of Sea-urchin, 397 
MagellaANIA, Shell, 360, 361: Body, 362: Mantle-lobes, 362: Mantle-cavity, 362: Lophophore, 362, 363 ; Foodgroove, 362: Digestive organs, 362 : Body-wall, 363: Muscular system, 364: Colome, 365 : Blood system, 365 : Excretory organs, 365 : Nervous system, 365 : Reproductive organs, 366 : Position of example, 367

Magellania flavescens, 360

Maggot, $649^{*}$

Maia, 569, 596, 600

Malacocotylea, 252-See Digenetica

Malacostraca, $565^{*}, 570,579,593,594$, $596,597,601$

Malaria parasites, 86

Malleus (of Rotifera), 325

Malpighian tubes, 617,625

Malpighian tubes, of Scorpion, 656: of Arachnida, 668 : of Tardigraila, 675

Mandibles-See Appendages

Manducation, 434*

Mantida, 636

Mantle lobes, 362

Mantle, of Anodonta, 684 : Pelecypoda, 698: Amphineura, 713: Triton, 725: Gastropoda, 740 : Scaphopoda, 757 : Sepia, 762 : Nautilus, 781

Manubrium, of Medusa, 129: of Obelia, 130

Marginal lappets, of Aurelia, 168*, 169, 179

Marginal sense-organs, $135^{*}$

Marginal tentacles, of Aurelia, 168*, 169

Marine Annelids, 439

Mastax of Rotifera, 325

Mastigamaba, 70, 71

Mastigophora, $45^{*}$ : Example, 67 : Classification, 69: General organisation, 69: Systematic position of the exanuple, 70

Matrix of connective tissue, $25^{*}$

Maturation, $19^{*}, 20$

Maxilla-See Appendages

Maxillary palp-Seo Appendages

Maxillipeds - See Appendages

Maxillutæ, $638^{*}$

May-flies, 633

Measly pork, 310

Medulla, of Actinospharium, 57, 58: Monocystis, 80: Paramocium, 88, 89

Medullary sheath, of Nerve-fibre, 29*, 30

Medusa-buds, of Obelia, 129

Medusæ, of Obelia, 134, 135

Megadrili, 466* 467

Megagametes, of Flagellata, $76^{*}$ : Sporozoa, 84, 85, 86 : Ciliata, 99

Megagametocyte, 84,85

Megalæsthetes, 717

Megalopa stage, 599

Megameres, of Ctenophora, 217, 218 : of Polyclad, 274,
Meganucleus, of Ciliata, 93: Pard. mocium, 88, 89: Dinoflagellata, 78

Megaspheric forms, 55,56

Megaspores, of Radiolaria, 63

Megazooid, of Vorticella. 96,98

Meleagrina, 696, 710

Meleayrina margaritifera, 711

Melicerta, 328, 330, 331

Melolontha, 643

Membranes, 24

Membranipora, 347

Mentum, 622

Meridional canal, 213

Meroblastic, 22*

Merogony, $21^{*}$

Merozoa, 253*-See Polyzoa

Merozoite, $84^{*}, 85,86$

Mesenchyme, 389

Mesenteric filaments, of Flabellum, 206: of Sea-anemone, $187^{*}$

Mesenteries, of Sea-anemone, $187,182^{*}$

Mesenteries, development of, in Seaanemones, 192, 193: Arrangement in Actinozoa, 200

Mesenteron, 187

Mesoblast, 23*

Mesocœle, 356*

Mesoderm, 23*, 219*

Mesoderm bands, of Periplaneta, 631

Mesoglnea, 110*, 111 : of Obelia, 131*

Mesonemertini, 296*

Mesopodium, 725, 739

Mesosoma, 654*

Mesostomum, 272

Mesothorax, 622

Mesotrochal, $486^{*}$

Mesozor, 230

Messmateism-See Commensalisin

Metabolism, 13*

Metacœle, 356*

Metacrinus interruptus, 424

Metagenesis, $40^{*}$ : of Foraminifera, 56 : Obelia, 139: Leptolinæ, 152: Trachylinæ, 156: Aurelia, 173: LiverFluke, 243: Tania, 250: Platyhelminthes, 273: Nematoda, 310

Metameres, $43^{*}, 523$

Metamerism, 523

Metamorphosis, of Trachylinæ, 155: Seaanemone, 193 : Platyhelminthes, 273 : Nemertinea, 295: Phoronis, 359 : Asterina, 388: Antedon, 432: Echinodermata, 430 : Chætopoda, 487 : Apnis, 537 : Crustacea, 597 : Insecta, 649

Metamorphosis, retrogressive-See Degeneration

Metanauplius, 539

Metanemertini, 296*

Metapodium, 725, 739

Metathorax, 622

Metazua, 19* 105

Metentera, 187 *

Micræsthetes, 717 
Microdrili, $466^{*}$

Microgametes, of Flagellata, $76^{*}:$ Sporozoa, 84, 85, 86 : Ciliata, 99

Microgainetocyte, 84,85

Microgromia, 50,56

Microhydra, 148, 167

Micromeres, of Ctenophora, 217, 218

Micromeres, of Polyclad, 274

Micronucleus, of Ciliata, 93: of Dinoflagellata, 78

Micronucleus, of Paramæcium, 88, 89

Micropyle, 20

Micropyle, of Cephalopoda, 798

Microspheric forms, 55,56

Microspores, of Radiolaria, 63

Microstomum, 282, 283

Microzooid of Vorticella, 96, 98

Miescher's corpuscles, 88*

Migration, 285*

Miliola, 52

Millepora, 156

Millepora alcicornis, 157, 158

Millipedes, 526, 614, 615

Minyas, 202, 226

Miocene, 7

Miracidium, 244*

Mites, 526, 653, 661, 665, 668, 673

Mitosis, $16^{*}, 17$

Mitotic division-See Mitosis

Modinla, 698, 702

Mollusca, 680* : General remarks, 804

Molluscoida, 340*

Molluscoida, mutual relationships of the classes, 372

Mongrels, $2^{*}$

Moniezia, 288

Monocyclica, $413^{*}$

Monocystis AGILIS, 80, 81 : Systematic position, 82

Monocious, 39 *

Monogenetica, 252* , 258, 259, 265, 268, $269,272,273,277,284,286,287$

Monomyaria, 698*

Monosiga, 77

Monotus, 255

Monozoa, 253*

Morphology, $3^{*}$

Morula, 22*

Moruloidea, $230^{*}$

Mosquitoes, 635, 651

Mother-cyst, 282

Mother-of-pearl, 684

Moths, 635

Mouth papillæ, $376^{*}$

Movable cheek, 605*

Mulberry body, 22*

Multicellular, $19^{*}$

Multicellular gland, 25*

Müller's larva, 275

Multicilia, 93, 94

Multiple fission, of Euglena, 69

Murex, 744, 747
Muscle, striated, $28^{*}, 29$ : non-striated, $28^{*}, 29$

Muscle processes, Hydra, 148

Muscles, $37^{*}$

Muscular fibres of Sponges, 111

Muscular system, of Aurelia, 172 : Seaanemone, 186, 188: Magellunia, 364: Brachiopoda, 368: Hirudo, 508, 509: Apus, 532: Astacus, 546, 547: Crustacea, 593: Periplaneta, 624: Anodonta, 684: Pelecypoda, 697

Muscular tissue, 28*, 29

Mushroom coral, 205

Mussels, 680

Mya, 696

Mya arenaria, 698

Mycetozoa, 45* : Example, 66, 67 : Sporangium, 66, 67: Capillitium, 66, 67: Sporcs, 66, 67 : Flagellula, 66, 67: Plasmodium, 66, 67: General remarks, 67 : Protomyxa, 67

Myomeres, of Apis, 532

Myosoma, 354

Myriapoda, 526, 614* $, 677,678$ : Distinctive characters and classification, 614 : General organisation, 614: External features, 615 : Integument and Bolly wall, 617: Alimentary canal, 617 Heart, 617 : Respiratory system, 617 : Nervous system 617 : Reproduction, 618 : Ovum, 618 : Fossil remains, 618

Myriothela, 145, 146, 148

Mysidacea, $567^{*}, 581,582,594,597,599$, 603

Mysis, 567,582

Mytilus, 695, 697, 702, 703, 708

Mytilus edulis, 697, 702

Mytilus latus, 697

Myxidium lieberkïhnii, $\mathbf{8 7}$

Myxobolus mülleri, 87

Myxospongiæ, 120, 126

Myzosporidea, $87^{*}$ : features, 87

Myzostoma, 489, 490, 491

Myzostomida, 489*, 490, 491

Characteristic

$\mathrm{N}$

$\mathrm{N}$

ACRE, $684^{*}$

Naiidomorpha, 473

Nais, 486

Narcomedusæ, 142*, 154, 15 .

Natural History, 1*

Nauplius, 538, 539

Nausithöe, 180

Nautiloids, 680, 790

Nautilus macromphalus, 779,789

NaUtilus pompilius, 776 : Shell, 776 : External characters of soft parts, 777 : Mantle and mantle-cavity, 781: Enteric canal, 783 : Colome, 783: Heart and circulation, 783 : Renal organs, 786: Nervous system, 787 : Sense- 
organs, 787 : Reproductive organs, 787,788 : Systematic position, 790

Neartic region, 9 *

Nebalia, 566, 579, 581

Neck, of cockroach, 620

Nectocalyx, 159*

Necturus, 304

Needham's sac, $774^{*}, 788$

Nekton, $8^{*}$

Nematomorpha, 304* $, 305,307$

Nemathelminthes, $297^{*}$ : Appendix, 319 : Atfinities and relationships, 320

Nematocyst, 78, 79, 87, 95, 133*

Nematoda, 297* : Example, 297: External characters, 305: Body-wall, 305 : Enteric canal, 305, 306: Coelome, 307 : Excretory canal, 307: Nervous system, 307: Eye-spots, 307: Reproductive organs, 307: Development, 308, 309 : Life-history, 310

Nenıatogene, 231 *

Nematoidea, $304^{*}, 307$

Nematomorpha, $304^{*}, 305,307$

Nemertinea, 288, 295* : General features, 288, 289, 291, 293 : Body-wall, 290 : Alimentary canal, 290 : Blood-vessels, 291, 293 : Excretory vessels, 292, 293 : Nervous system, 291,292, 293: Cerebral organs, 291, 294 : Eyes, 294 : Statocysts, 295: Reproductive system, 295 : Development, 294, 295: Distinctive characters and classification, 295

Neomenia, 713, 714, 717, 718

Neotropical region, $9^{*}$

Nephelis, 516

Nephridiopore, 448, 455, 506

Nephridium, 439, 448* 460, 461, 479, $495,511,689$

Nephridium, provisional, 481

Nephrostome, 365, 448, 461

Nereida, $466^{*}, 488$

Nereidiformia, 466

Nereis, External features, 440, 441 : Enteric canal, 442, Coelome, 442: Bodywall, 443 : Vascular system, 444 : Nervous system, 446 : Sense-organs, 447: Excretory ergans, 448 : Reproductive organs, 449 : Individual variation, 449 : Development, 450, 451, 452, 453 : Systematic position, 466

Nerve-cell, $29^{*}, 30$

Nerve-fibres, $29^{*}, 30$

Nerve ganglia, 38

Nerve pentagon, 379

Nervous system, $14,29,37^{*}, 38$ : Obelia, 132 : Leptolinæ, 151: Aurelia, 172: Tealia,192: Hormiphora,216: Planaria, 238 : Fasciola, 241 : Tania, 248 : Platyhelminthes, 266, 267: Nemertinea, 291, 292, 293: Ascaris, 301, 302: Nematoda, 307 : Acanthocephala, 313 : Chætognatha, 317 : Brachionus, 326 : Rotifera, 334: Dinophilea, 337: Gastro- tricha, 336 : Polyzoa, 351 : Endoprocta, 354 :-Phoronis, 357 : Magellania, 365 : Brachiopoda, 369 : Starfish, 379: Seaurchin, 398 : Holothurian, 402: Antedon, 408: Echinodermata, 429 : Nereis, 446 : Lumbricus, 459: Chætopoda, 476: Myzostomida, 490 : Sipunculus, 494: Gephyrea, 498: Archiannelida, 504: Hirudo, 513; Hirudinea, 520: Apus, 534, 536: Astacus, 555 : Crustacea, 596 : Peripatus, 609: Myriapoda, 617, 626, 627 : Periplaneta, 626, 627 : Insects, 643, 644: Scorpio, 657, 658: Arachnida, 670: Mussels, 690: Pelecypoda, 706 : Amplineura, 715, 717: Triton, 729: Gastropoda, 745: Scaphopoda, 757 : Rhodope, 758: Sepia, 767: Nautilus, 787: Cephalopoda, 797

Nervous tissue, 29

Nervures, 639*

Neuraxis, $29^{*}, 30$

Neurilemma, 29*, 30

Neuronephroblast, 521 *

Neuropodium, $441^{*}, 468$

Neuroptera, $633^{*}, 640,647,6.5$

New Zealand region, $9^{*}$

Nicothöe, 576

Nidamental gland, $775^{*}$

Noah's Ark shell, 695

Noctiluca, 79

Nodes, of Siphonophora, 161

Nodosaria, 53

Nomenclature, binomial, $1^{*}$

Non-contractile vacuoles, $11^{*}, 60$

Noteus, 331

Notholca, 331

Notommata werneckii, 335

Notopodium, 441, 468

Notostraca, $563^{*}$

Nuchal cartilage, 763

Nuchal organs, $447^{*}, 448$

Nuclear membrane, $16^{*}, 17,80$

Nuclear spindle, $17^{*}$

Nuclearia, 58

Nucleolus, $16^{*}, 19,80$

Nucleus, $11^{*}, 16,19$

Nucula, 695, 698, 701, 702, 703, 706, 707, 710

Nudibranchia, $734^{*}, 738,742,744,756$

Nummulites, 53

Nyctiphanes, 568

Nyctotherus, 92, 94, 95

Nymphon hispidium, 674

\section{0}

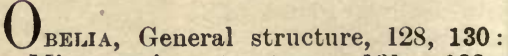
Microscopic structure, 131, 132 : Medusæ, 134, 135, 136 : Comparison of polype with medusa, 136, 137, 138 : 
Reproduction, 139 : Development, 139 : Systematic position, 142

Occlusor muscle, 350

Ocelli, 143, 173

Octopoda, 680, 790* $791,793,797,804$

Octopus, 791, 793, 796

Octorchandra, 150

Ocular plates, 396, 397

Odentophora, $676^{*}, 678$

Odontophore, $721,726,727$

(Esophagus-See Digestive system .

Oikomonas, 71, 72

Olfactory organs, $39^{*}$ : pits, $173^{*}$

Oligochæta, 465* 468, 469, 471, 473, 474, $475,478,479,481,482,483,486,488$, 489

Olynthus, 116

Ommatidium, $536^{*}, 537$

Onchidium, 746

Oniscus, 568, 583, 587

Onychophora, 526, 607* $677,678-$ See Peripatus

Oæcium, 341

Ookinete, $87^{*}$

Dosperm, 21 *

Oostegites, $585^{*}$

Oostegopod, 531*

Ootype, $243,270,271$

Opalina, 93, 98, 99

Jpalinopsis, 94

Operculnm Radiolaria, 61, 62 : Ciliata, 95, 96 : Gastropoda, 723* 724,739

Ophioglypha, 420

Ophiuroidea, Distinctive characters and classification, $411^{*}$ : General form and symmetry, 415: System of plates, 418: Modifications of form, 420, 421: Colome, 425: Ambulacral system, 426; Blood-vascular system, 426: Hæmal system, 427 : Axial organ, 428: Enteric canal, 428: Nervous system, 429: Reproductive organs, 429: Development, 430 : Ethology, \&c., 434

Ophrydium, 95, 97

Ophryodendron, 100

Ophryoglena, 94

Opisthobranchia, $734^{*}, 738,744,745^{\prime}$ 755

Opisthogoneata, $615^{*}, 619,677$

Opisthorchis sinensis, 285

Opossum-Shrimp, 523

Optic gland, $7 \% 2$

Optic vesicle, 802

Oral, 376* : Arms, 170*

Orchestia, 583, 594

Order, $4^{*}$

Organ of Bojanus, 689* : cf. Owen, 780*

Organic evolution-See Evolution

Organism, $1^{*}$

Organ-pipe Coral, 195

Organs, $31^{*}$

Oriental region, $9^{*}$
Orobdella, 516

Orthoceras, 803

Orthonectidce, 230, 232, 233, 234

Orthoptera, 632*, 638, 640, 653

Othoptera genuina, 636

Oscarella, 117

Oscaria, 115

Oscular sphincter, $111^{*}$

Osculum, 106*

Osphradium, 691, 707, 731, 797

Ossicles, 375

Ostia, 106*, 109, 533*

Ostium, 187*

Ostracoda, 564*, 572, 573, 593, 594, 597, $598,601,602$

Ostrea, 696, 698, 701, 706, 708, 709

Otocyst, $39^{*}, 478$

Ovariole, 628*, 646

Ovary, $39^{*}$

Ovicells, 352

Oviduct, $40^{*}$

Oviparous, $40^{*}$

Ovipositor, 610

Ovum, 19*, 30

Owen, organ of, 780

Oxygen, oxidation, 36

Oxeole spicules, $108,110^{*}$

Oxyuris, 307

Oysters, 680, 696

\section{$\mathbf{P}$}

P

ACHYCHALINA, 121

Prdogenesis, $41^{*}, 224^{*}, 647$

Pagumes, 569, 589

Palcemon, 569, 588

Palamonetes, 593

Palrar'ctic region, $9^{*}$

Palæodictyoptera, 652

Palæontology, $6^{*}$

Palinurus, 569, 587, 595, 599, 602

Pallial line, 683*

Pallial groove, 344

Pallial sinus, 365

Pallial muscles, 684

Pallial complex, 735

Pallium-See Mantle

Palpus, 470

Paludicella, 348, 353

Paludina, 754

Palus, 205*

Palythoa, 127, 197

Pancreas, $34^{*}$

Pancreatic juice, $34^{*}$

Pandorina, 72, 73, 74, 75

Papula, 424*

Parapodium, 439, 440, 441, 468, 739

Paragastric cavity, $106^{*}, 108$

Paragnatha, 530*, 548

Paramocida, 91

Paramecium, 88, 89, 90 : Systematic position, 91 
Paramithrax, 601

Paramitome, 15*

Paramylum, 65*

Paranaspides, 566, 582

Paranephrops, 569

Parasitism, Protozoa, 72, 81, 82, 85, 87, 95: Hydrozoa, 167: Mesozoa, 230: Rotifera, 335

Parenchyma, 2:36* $, 264,351$

Parenchyma muscle, 265

Parenchymula, 124*

Parthenogenesis, $21^{*}, 40^{*}, 287,647$

Parthenogonidium, $75^{*}$

Parthenope, 591

Patella, 737, 743, 745, 746, 747, 749, 751, $752,753,754$

Paraseison asplanchnus, 329

Parazoa, 105

Patellida, 733

Pauropoda, 614*, 618

Pauropus, 614, 615, 617

Paxilla, 414

Peachia, 202

Pea-crab, 602

Pearl-mussel, $; 10$

Pearl-oyster, 696

Pearls, 711

Pébrine, $87^{*}$

Pecten, 696, 697, 700, 708, 710

Pectines, 655 *

Pectinibranchia, $733,{ }^{*} \mathbf{7 4 4}$

Pedal gland, $725^{*}$ : Lobes, 179

Pedalion, 328, 330, 332, 335

Pedata, $413^{*}, 434$

Pedicellaria, 377, 381, 387, 394, 417

Pedicellina, 348, 354, 355

Pedipalpi, 653

Pedipalpida, $660^{*}, 662,663,66 \mathrm{~s}, 670$, 673

Peduncle, $205^{*}$

Pelagia, 184

Pellicle, 88

Pelomyxa, 49

Pelagic, $8^{*}$

Pelagohydra, 148

Pelecypoda, 680: Example, 680: Distinctive Characters and classification, $694^{*}$ : General organisation, 696 : Adductor nuscles, 697 : Shell, 697 : Siphons, 698: Foot, 701: Byssus gland, 702: Gills, 702, 704: Digestive organs, 704: Excretory organs, 705: Circulatory organs, 706 : Nervous system, 706 : Sense organs, 707: Reproduction and development, 708, 709, 710: General remarks, 710: Mutual relationship, 712

Pelmatozoa, $413^{*}, 434$

Peltogaster, 565, 5ั79

Pen, 795*

Penceus, 569, 599

Penial setæ, 298, 304

Pennatula, 196, 197, 198, 199, 204, 208
Pennatulacea, 195*, 197, 199, 204

Pentacrinoid larva, 410, 433

Pentacrinus, 410

Pentastomida - Sce Linguatulida

Pentastomum tanioides, 674

Peptones, 34

Peptonephridia, 481*

Peracarida, 566* 601

Perforate corals, $207^{*}$

Pericardial sinus, 533*

Perichondrium, $27^{*}$

Pericolpa, 179, 180

Perihæmal system, $380^{*}$

Periosteum, 28*

Periostracum, 683*

Peripates, 526, 607 : External features, 607, 608: Body-wall and body-cavity, 608: Enteric canal, 608: Circulatory system, 609 : Organs of respiration, 609: Coxal and slime glands, 609: Nervous system, 609 : Nephridia, 610 : Reproductive organs, 610: Develop ment, 611, 612, 613: Distribution, 612: Relationship, 612

Peripatus capensis, 607, 608, 609, 610, 613

Peripa'us nova-zealandia, 611

Periphylla, 179

Periplaneta americana, 619, 620: Head, 620, 621 : Neck, 622 : Thorax, 622 : Abdomen, 622: Respiratory movements, 623 : Muscles, 624 : Hæmocuele, 624: Digestive system, 624, 625: Renal organs, 625: Heart, 625: Respiration, 626, 627 : Nervous system, 626,627 ; Organs of special sense, 627: Reproductive organs, 627,628 : Development, 628, 629, 630 ; Systematic position, 636

Periplaneta orientalis, 620, 621

Periproct, 394 *

Perisarc, $130,131^{*}$

Perisaltic movements, $36^{*}, 445$

Peristome, 92, 95, 96. 185** $380^{*}, 394^{*}$

Peristomium, $441^{*}, 455,469$

Peritoneum, $442^{*}$

Peritrichous, $92^{*}, 96$

Perivisceral cavity, $442^{*}$

Periwinkles, 680

Peromedusæ, 180

Per-radius, 139*

Petaloid ambulacra, 422

Petasus, 154

Petrarca, 565, 579

Phacellæ, 172*

Phacops fecundus, 605

Phæodium, 61, 62

Phalangida, $661^{*}, 665,667,669,670$

Phanerocephala, $465^{*}, 466,476,484$, 488

Phanerozonia, 411*

Pharynx - See Digestive system

Phasmida, 636, 653 
Pheronema carpenteri, 120, 123

Philodina, 328, 331, 334

Pholas, 696, 700, 711

Phoronida, 340, 355*

Phoronis, 355, 356, 357, 358, 359

Phragmoceras, 803

Phragmocone, 795*, 796

Phreatoicus, 568

Phronima, 585, 586

Phrynus, 663

Phylactolæmata, $348^{*}, 349,351,352,353$

Phyllocarida, 566* , 580, 581, 594, 596, 601, 603

Phyllodoce paretti, 479

Phyllosoma, 600

Phylogeny, 8*

Phylum, 5* $43^{*}$

Physalia, 163

Physiology, $9^{*}$

Pieris, 635

Pigment, 68, 119

Pilema, 183

Pilidium, 295

Pill-bug, 586

Pinna, 696, 702

Pinnotheres, 602

Pinnules, 407*

Piscicola, 516, 522

Placophora, $713^{*}, 715,716,717,718$, 720,721

Planaria, 236: General features, 236, 237, 240: Digestive system, 236, 237 : Water vessels, 237, 235: Nervous system, 237, 238: Reproductive sysm tem, 238, 239: Systematic position, 253

Planarida, 253

Plankton, $8^{*}$

Planorbulina, 53

Plant lice, 634, 647

Planula, 139*, 140, 153, 173, 192

Plasma, of blood, 30

Plasmodium, $30^{*}, 66,67$

Platoum sterco eum, 51

Platyctenea, 226*

Platyhelminthes, 235, 251* : Examples, 236, 240, 245: Systematic position, 253: General external features, 254: Integument and muscular layers, 262, 264: Parenchyma, 264, 265 : Alimentary systems, 265, 266, 267 : Nervous system, 266, 267: Water vascular system, 269: Reproductive organs, 270, 271, 272: Development, 273, 274, 275, 276, 277, 278, 280, 281, 282, 379 : Asexual reproduction, 283 : Distribution occurrence and relationships, 283 : Appendix, 288

Platypods, 733*, 734

Pleopod, 542*

Pleurobrachia, 211

Pleurobrachiida, 221

Pleurobranchia, 550, 551*
Pleuron, 541*

Pleurophyllidia, 742, 743

Ploima, 328*, 329, 330

Plumatella, 348, 349

Plumularia, 149

Pluteus, 400, 411, 412, 431, 432

Pneumatophore, 159*

Podical plates, $623^{*}$

Podobranchia, 550*

Podomere, 526*

Podophrya, 99, 100

Podura, 632, 633

Polar body, 19* 20

Polar plates, 216*

Polian vesicle, 383*, 399, 402, 426

Pollicipes, 579

Polyarthra, 328, 330, 331

Polycelis, 255

Polychæta, 465*, 470, 471, 474, 478, 479, $480,481,483,484,485,486,488,489$

Polycladida, $252^{*}, 255,256,257,263$, $264,266,267,268,271,273,274,287$

Polycolpa, 154

Polydora, 488

Polygordiidae, 503

Polygordius lacteus, 504

Polygordius neapolitanus, 503, 504, 505

Polykrikos, 78, 79

Polymorphism, 141*

Polynesian region, $9^{*}$

Polynöe, 469, 484

Polynöe extenuata, $\mathbf{4 7 1}$

Polynöe setosissima, 467

Polynoidae, 467, 481

Polyoca, 77, 78

Polyophthalmus, 479

Polype, 129*, 148

Polyphemus, 564, 572

Polyphyletic, 286*

Polypodium, 167

Polyspermy, 21*

Polystomatous, $183^{*}$

Polystomea, 257, 258

Polystomella, 55

Polystomum, 258, 277, 284

Polytrochal, 486*

Polyzoa, 340* : Example, 341 : Distinctive characters and classification, 347 :

See Ectoprocta and Endoprocta

Polyzor (Cestoda), 253*

Pontellina mediterranea, 600

Pontobdella, 516, 517, 518, 520

Porcellana, 569

Pore-membrane, 108, 109*

Porifera, Example, 105: Distinctive characters and classification, 111: General form and mode of growth, 114 : Leading modifications of structure, 116: Histology, 119: Skeleton, 120: Reproduction, 120: Development, 122: Distribution, affinities, \&c., 126,228

Porocyte, 109*, 124 
Poromya, 696, 785

Porpita, 165, 166

Port-hole-See Cinclis

Portuguese Man-of-War, 163

Portunus, 591

Post-abdomen of Scorpion, 654

Potamobiida, 570 *

Poterion, 115

Præ-abdomen of Scorpion, 654

Prawns, 587, 588

Priapulida, 496

Priapulus, 497, 498, 499

Primary axis, $41^{*}, 42$

Prismatic layer, 684

Proboscis, 237

Prodandrous, $307^{*}$

Proglottides, $247^{*}$

Progoneata, $614^{*}, 619,677$

Pro-legs, 649

Proneomenia, 713, 717

Pronucleus, active and stationary, 91

Pronucleus, male and female, 19*, 20

Pro-ostracum, 795*

Propodium, 725, 739

Prorocentrum, 78, 79

Prorodon, 94, 95

Proscolex, 250*

Prosiphon, $741^{*}$

Prosobranchia-See Streptoneura

Prosocœle, 356*

Prosoma, 654

Prosopyle, 108, 109*

Prostate, 270, 271, 483

Prostomium, 440* 455, 469

Protamieba, 49

Protandrous, $307^{*}$

Protective characters, 601

Proteid, $15^{*}$

Proteolepas, 565, 579

Proterospongia, 77, 78, 127

Proteus Animalcule, 10

Prothorax, 622

Protobranchia, 695* 697 , 698, 702, 705, 711, 712, 805

Protoconch, $776^{*}, 794$

Protodrilus, 504, 524

Protohydra, 148

Protomerite, $82^{*}, 83$

Protomyxa, 67

Protonemertini, 295*

Protonephridial system, 236*, 269

Protoplasm, 11* $14,15^{*}, 16$

Protopodite, 538*, 542

Prototroch, 322, 451

Protozoa, 45

Protozoæa, 599

Protractor muscle, 683

Proventriculus, 624

Psammoclema, 115

Pseudo-gastrula, 124*

Pseudo-lamellibranchia, 695* 697,698 , $700,711,712$

Pseudo-manubrium, 155*
Pseudo-metamerism, 255*

Pseudopod, 10*, 45* : of Amoeba, 46

Pseudo-scorpionida, 660* 662, 668, 670

Psolus, 423

Psorosperms, 87

Pteropoda, 734* 741,756

Pterotrachea, 742

Pulmonary sac, $743^{*}$

Pulmonata, $734^{*}, 738,740,743,744,746$, $748,749,750,755,756$

Pulsellum, 756

Pupa (of Cirripedia), 598

Pupa (of Insects), 649

Purpura, 744

Pycnogonida, $673^{*}, 674,678$

Pygidium, 605*

Pyloric creca, 382

Pyrenoids, 73

Pyriform organ, 344

Pyriform sac, 787

Pyrula, 749

Pyxicola, 95

Q

UADRULA, 49

R

Rachis, 302*

Radial Canals, 107, 108, 109*, 118

Radial Symmetry, $42^{*}$

Radiata, 436

Radii, orders of, 139

Radiolaria, 48*: General structure, 60 : Central capsule, 60 : Skeleton, 61: Colonial forms, 61 : Reproduction, 63 : Symbiosis, 63

Radula, $726^{*}, 728$

Radular, sac, $726^{*}, 782$

Rainey's corpuscles, $88^{*}$

Raphidiophrys, 58

Razor-fish, 711

Receptacula ovorum, 462

Red coral, 195

Redia, $245^{*}$

Regularia, $412^{*}, 415$

Relationship, $6^{*}$

Relationships, of Protozoa, 102 : Sponges, 127, 228: Colenterata, 226 : Platyhelminthes, 283 : Nemathelminthes, 320 : Rotifera, 335 : Dinophilea, 336 : Molluscoida, 372: Echinodermata, 436, 502 : Annulata, 524, 602 : Air-breathing Arthropoda, 676: Pelecypoda, 712 : Cephalopoda, 804 : Mollusca, 804 Relationships, diagrams of : of Protozoa, 103 : Colenterata, 229: Platyhelminthes, 287: Echinodermata, 437: Annulata and Trochelminthes, 525 : 
Crustacea, 604: Arthropoda, 678: Pelecypoda, 712: Gastropoda, 756 : Cephalopoda, 804

Renal organs-See Excretory system

Reproduction, Reproductive System, 14, 30, 39 : Amaba, 14, 46 : Foraminifera, 56: Heliozoa, 59: Radiolaria, 63: Mycetozoa, 67 : Euglena, 69: Flagellata, 74: Choanoflagellata, 78: Dinoflagellata, 79: Cystoflagellata, 79 : Monocystis, 80: Gregarinida, 83: Coccidiidea, 84: Hæmosporidea, 86 : Paramoecium, 90: Ciliata, 97: Tentaculifera, 101: Porifera, 120: Obelia, 139 : Leptolinæ, 149 : Trachylinæ, 155 : Hydrocorallina, 158: Siphonophora, 162, 164, 166 : Aurelia, 171: Tealia, 192: Actinozoa, 197: Hormiphora, 216: Mesozoa, 231, 234 : Planaria, 238, 239: Fasciola, 242, 243 : Taniu, 248, 249, 250 : Platyhelminthes, 270 Nemertinea, 295: Ascaris, 302, 303: Nematoda, 307 : Acanthocephala, 314, 315 : Chætognatha, 318: Brachionus, 326: Rotifera, 334: Dinophilea, 337 : Bugula, 344 : Ectoprocta, 352 : Endoprocta, 355: Phoronis, 357: Magellania, 366: Brachiopoda, 369: Starfish, 386: Sea-urchin, 400: Holothurian, 404: Anterlon, 409: Echinodermata, 429: Nereis, 449: Lumbricus, 461, 462, 481 : Chætopoda, 48I : Myzostomida, 490, 495: Sipunculus, 495 : Gephyrea, 499: Archi-annelida, 504: Hirudo, 514: Hirudinea, 520: A pus, 537 : Astacus, 556: Crustacea, Peripatus, 610: Myriapoda, 618: Periplaneta, 627, 628 : Insects, 646 : Scorpion, 655: Arachnida, 671 : Mussel, 691: Pelecypoda, 708: Amphineura, 718, 719: Triton, 732: Gastropoda, 748 : Scaphopoda, 757 : Rhodope, 758 : Sepia, 773, 775: Nautilus, 787, 788: Cephalopoda, $79 \mathrm{~s}$

Requienia, 700, 701

Reservoir (Euglena), 68

Respiration, 13*, 35

Respiratory organs, $35^{*}$ : Starfish, 376 : Sea-urchin, 399: Holothurian, 404: Chrtopoda, 471: Hirudinea, 502: A pus, 534: Astacus, 549, 550: Crustacea, 594: Peripatus, 609: Myria. poda, 617: Periplaneta, 626, 627: Insects, 641, 642: Scorpion, 657: Arachnida, 668 : Mussel, 685 : Pelecypoda, 702, 704: Triton, 725: Gastropoda, 742: Sepia, 766: Nautilus, 781 : Cephalopoda, 797

Respiratory trees, 404, 428

Retina, 39

Retinophore, $732^{*}$

Retinula, 536* 537 , 5๊ 6

Retractor muscles, 683
Rhabdites, 263

Rhabditis-form, 310

Rhabdocœlida, 252*, 255, 257, 263, 265, 256, 267, 268, 269, 271, 272, 273, 276, 283, 284

Rhabdogaster, 319

Rhabdome, $563^{*}, 537$

Rhabdonema nigrovenosum, 308, 310

Rhagon-type of Sponge, 118*

Rhinophore, $787^{*}$

Rhipidodendron, 71, 73, 74

Rhipidoglossa, 733*, 756

Rhizocephala, 565*, 579, 580

Rhizopoda, 45*: Example, 46 : Classification and general organisation, 47

Rhizostomæ, $177^{*}, 182,183,227$

Rhizota, 328*, 330, 333

Rhodope, 758

Rhombogene, 232

Rhopalura, 232

Rhynchobdellida, 516* , 517, 5̄18, 519, 520

Rhynchodemus, 255*

Rhynchonella, 366, 369

Rhynchota, 100

Rhyncocole, 289 *

Ring-vessel, 270*, 283

Rock-lobster, 569

Rock systems, 7

Rocks, igneous and aqueous, $7^{*}$

Rosette, $407^{*}$

Rosette (Earthworm) 461

Rosette plate, 418

Rostellum, 246*

Rostrum, 542*

Rotalia, 52

Rotation, sense of, 39

Rotifer, 328

Rotifera, $323^{*}$ : Distinctive characters and classification, 327* : External characters, 330: Digestive organs, 333 : Excretory system, 333 : Nervous system and Sense-organs, 334: Reproduction and Development, 344 : Ethology, 335 : Affinities, 335

Rotulæ, 398*

Round-worms, 297-See Nemathelminthes

Rugosa, $210^{*}$

\section{S}

Sabella, 478

Saccammina, 53

Saccocirrus, 465, 477

Sacculi, $409^{*}$

Sacculina, 565, 579, 580, 599

Sagartia, 190, 191

Sagitta, 316, 317, 318

Sail, of Siphonophora, 163, 166

Salinella, 233, 234 
Saliva, 34*

Salivary glands, $34^{*}$

Salivary receptacle, 624

Salmacina, 486

Salpingoeca, 77

Saltatoria, 636

Sand-hopper-See Orchestia, 568

Saprophytic, 69*, 72

Sarcocystidea, $81^{*}, 88$

Sarcocystis, 88

Sarcolemma, 28*

Sarcophaga, 644

Sarcoptes scabiai, 665

Sarsia, 145

Scale-insects, 634, 647

Scallop, 696

Scaphoda, $756^{*}, 757$

Scaphopoda, $680,756^{*}, 757$

Schistosomum hamatobium, 285

Schistosomum japonicum, 285

Schizogony, $84^{*}, 85,86$

Schizopathes, 202

Schizopod-stage, 599

Schizopoda, 601

Scirtopoda, 328*, 330

Sclerite, $562^{*}$

Scleroblast, $110^{*}, 120,124$

Sclerotic, 771

Scolex, 246* 280

Scolopendra, 615

Scolopcndrella, 615, 617

Scorpion-See BUTHU's.

Scorpions, 526, 653

Scorpionida, $660^{*}, 673,676,677$

Scorpion-spiders, 632

Scrobicularia piperata, 699

Scuta, 578

Scutariella, 259

Scutigera, 615, 617

Scutigerella, 615

Scyllarus, 587, 589

Scyllis ramosa, 487

Scyphistoma-See Scyphula

Scyphozoa, 128: Example, 168: Structure and classification, 176: Systematic position of example, 177: Additional remarks on, 184

Scyphula, 174*, 175, 229

Sea-anemones, 128, 185, 194, 196, 200See Tealia

Sea-blubbers, 182

SeA-cucumber, External features, 401 : Structure of body-wall, 402: Ambulacral system, 402: Nervous-vascular systems, 402: Colome, 403: Enteric canal, 403, 404 : Reproductive organs, 404: Development, 404 : Systematic position, 414

Sea-cucumbers, 412-See Holothuroidea

Sea-fans, 195

Sea-firs (Sertularians), 143

Sea-hares, 721
Sea-mats, 340 -See Polyzoa

Sea-mice, 475

Sea-mussel, 695

Sea-pens, 196

Sea-urchin, External features, 393, 394, 395 : Corona, 396 : Aristotle's lantern, 397: Nervous system, 398: Ambulacral system, 399 : Enteric canal, 399, 400: Colome, 399: Blood-vascular system, 400: Reproductive organs, 400 : Development, 400: Systematic position, 414

Sea-urchins, 412*-See Echinoidea

Secondary axis, $41^{*}, 42$

Secretion, 25*

Segment, 43*

Segmental organ-See Nepredium

Segmentation of oosperm, $22^{*}$

Segmentation-cavity-See Blastocole

Segmentation-nucleus, 20 *

Seison, 335

Seisonida, 329

Selenaria, 347

Selenariida, 349, 352

Self-mutilation, 435

Semostomæ, $177^{*}, 182,227$

Sense-organs, $39^{*}$ : Obelia, 135 : Trachylinæ, 154: Aurelia, 172: Cubomedusæ, 182: Hormiphora, 216: Ctenoplana, 225 : Platyhelminthes, 268: Nemertinea, 294: Chætognatha, 318: Brachionus, 326 : Rotifera, 334 : Phoronis, 357 : Starfish, 377 : Sea-urchin, 395: Nereis, 447 : Chætopoda, 438: Sipunculus, 454: Hirudo, 514 : Hirudinea, 520 : A pus, 536 : Astacus, 55̃6 : Crustacea, 597: Peripatus, 609: Periplaneta, 627: Insects, 644, 645 : Scorpio, 658: Arachnida, 670: Mussel, 691: Pelecypoda, 707: Amphineura, 715 : Triton, 731: Gastropoda, 746\% Rhodope, 758: Sepia, 769, 771: Nautilus, 787 : Cephalopoda, 797-See also Eyes, Auditory organs, Olfactory organs, Gustatory organs, Tactile organs, Osphradia

Sense papillæ, 302, 493

Sepia cultrata, 759

SePIA, External features, 759, 760 : Shell, 761, 762: Chromatophores, 762: Mantle-cavity, 762: Internal skeleton, 763: Alimentary system, 763: Ink-sac, 765: Vascular system, 765: Colome, 766: Ctenidia, 766, 767: Nervous system, 767 : Sensory organs, 769, 771: Excretory organs, 772, 773 : Reproductive organs, 773, 775: Systematic position, 790

Sepiida, $790^{*}$

Septal funnel, $173^{*}$

Septal neck, $776^{*}$

Septibranchia, $696^{*}, 697,698$ 
Septum, 205*

Serialaria, 348

Serosa, 631, 659

Serpula, 472, 476, 477, 488

Serpulidae, 468, 472, 485, 486

Sertularians, 143

Seta, 439, 440, 441

Seta, provisional, 452

Setigerous sac, $441^{*}, 452,453,457$

Sexual dimorphism, $40^{*}$

Sexual generation, 140

Shell-gland, 243, 529, 692, 800

Shell-gland (Apus), 534, 535* : (Crustacea), 596

Shell, Magellania, 360, 361 : Brachiopoda, 367, 368 : Mussel, 682 : Pelecyporla, 697: Chiton, 714: Triton, 721, 722, 723: Gastropoda, 737 : Scaphopoda, 756: Sepia, 761, 762: Nautilus, 776, Cephalopoda, 793

Shelly loop, 360

Ship-worm, 696

Shrimp, 526, 569, 587

Sicula, $167^{*}$

Sigaretus, 740

Silicispongiæ, 126*

Silverfish, 632

Sinupalliata, 696", 698, 707

Sinupalliate, $699 *$

Sinus, 554

Siphon, 399, 428, 498, 723

Siphonal process, 722

Siphoniata, 711

Siphonodentalium, 756

Siphonoglyphe, 187*

Siphonophora, $142^{*}, 159,160,162,163$, $164,165,166,226$

Siphonozooid, 202*

Siphons, inhalant and exhalant, $681^{*}, 698$

Siphuncle, $776^{*}$

Sipunculidae, 496, 498, 502, 503

Sipunculoidea, $496^{*}, 497,498,499,502$

Sipunculds NUDUS, General external features, 492, 493 : Body-wall, 493 : Cœlome, 493: Blood-vascular system, 493: Alimentary canal, 493, 494 : Nerrous system, 494: Nephridia and gonads, 495 : Systematic position, 496

S'keleton, $31^{*}$ : Lobosa, 49 : Foraminifera, 51, 53: Heliozoa, 59: Radiolaria, 61: Mastigophora, 73 : Ciliata, 96 : Porifera, 120: Actinozoa, 202 : Sepia, 763 : Cephalopoda, 796-See also Shell and Body-wall

Skin, 31*-See Body-wall

Slime glands, 608

Slugs, 680,734

Snails, 680, 734

Solarium, 77

Solecurtus strigillatus, 700

Solen, 711

Solenocytes, 338,479
Solenogastres, $713^{*}, 715,717,718,720$, 721,805

Solenomya, 695

Solpugida, $661^{*}, 662,663,667,668,670$

Somatoblast, 450

Somatopleure, 631 *

Spadella, 316

Spadix, $780^{*}, 781$

Spatangoidea, $412^{*}, 417,422$

Species, $1^{*}, 113^{*}$

Specific name, $2^{*}$

Sperm, 20, 30*

Spermary, 39*

Spermatidia, 344

Spermatozoon-See Sperm

Spermiduct, $40^{*}$

Spermotheca, 308*

Sphæridia, 395*, 417

Sphæroma, 568

Spharophrya, 100

Spicules, 32*, 203

Spiders, 526, 653

Spinnerets, 664

Spinning-glands, 664

Spiuules, $240^{*}$

Spireme, $18^{*}$

Spirifera, 664,

Spiroloculina, 53

Spirorbis, 489

Spirorbis laevis, 484

Spimula, 790, 794, 795

Splanchnopleure, $631^{*}$

Spongelia, 121

Spongilla, 118

Spongillidae, 121, 126

Spongin, 112, 120*, 121

Spongin-blasts, $120^{*}$

Sporangium (Mycetozoa), 66, 67

Spore, 40, 67, 75, 80, 81, 88

Spore formation, $40,56,59,60,63,65$, $66,67,75,79,80,87,89,98$

Sporocyst $245^{*}$

Sporogony, $84^{*}, 85$

Sporosac, $151^{*}$

Sporozoa, $46^{*}$ : Example, 80 : Classification and general organization, 81

Sporozoites, $80,81,83,84,85,86,88$

Springtails, 632, 640

Squame, $544^{*}$

Squammulina, 51, 52

Squids, 790

Squilla, 592

Stocharthrum, 232

Statoblasts, 353

Statocones, $707^{*}, 787$

Statocysts, 268, 295, 691, 707, 798

Statolith, 268, 707*

StaRfish, External characters, 375, 376, 377: Transverse action of arm, 378: Vascular and nervous systems, 379 : Structure of disc, 380 : Body-wall and cœlome, 381 : Digestive system, 382 : 
Ambulacral system, 383: Reproductive system. 386 : Development, 388, 389, 390, 391, 392, 393 : Systematic position, 414

Starfishes, 374-See Asteroidea

Stauromedusæ, $176^{*}, 177,178,227$

Stenocyphus, 178

Stentor, 92, 94

Stephanoceros, 328, 330, 331

Sternaspis, 469, 471, 475, 477, 481

Sternaspis spinosa, 471

Sternum, 541*

Stewart's organs, 425 *

Stichopoda, 415

Stichotricha, 95

Stick-insects, 633, 636

Stigma (Kuglena), 68

Stigmata, 609, 623, 642, 655

Stinging capsule-See Nematocyst

Stolon, $196^{*}$

Stomach-See Digestive system

Stomatogastric nerves, 447

Stomatopoda, 569*, 592, 593, 596

Stomidium, $201^{*}$

Stomodæal canal, 213

Stomodæum, 173*

Stone-canal, 383*, 399

Stony-corals, 128, 195202

Stratiodrilus tasmanicus, 338

Strepsiptera, 640, 651,

Streptophiuræ, $411^{*}$

Streptoneura, $733^{*}, 735,736,739,742$, $744,745,746,748,750,755$

Strichotricha, 95

Strobila, 246* 247,262

Strongylocentrotus, 393, 395, 397, 414See Sea-urchin

Strongylostoma, 615

Strongylus, 305

Stylarioides, 488

Stylaster, 157, 158, 159

Style, 158*

Stylonychia, 99

Subcortical cavity of sponges, $119^{*}$

Subdermal cavity of sponges, 118, 119

Sub-genital pit (Aurelia), 170*

Sub-genital portico, $183^{*}$

Sub-kingdom-See Phylun

Sub-mentum, 622

Sub-radius, 139*

Sub-tentacular canal, 407

Sub-umbrella, $135^{*}$

Succession of Life in time, 7

Sucker (Sepia), 760*

Sucking-disc, 417

Suctorial mouths (Rhizostomæ), 183 : Discomedusæ, 183

Summer eggs, 597

Supplemental skeleton, 53, 54*

Supporting lamella-See Mesogløa

Swimming-bell-See Nectocalyx

Swimming ovaries, 315
Swimming-plate, $213^{*}$

Sycantha, 113

Sycetta, 113

Sycettidae, 113*, 172

Sycon : External characters, 105, 106, 107: Microscopic structure, 108, 109 : Systematic position, 112: Development, 124, 125

Sycon raphanus, 125

Sycon-type of sponge, 117, $118^{*}$

Syllida, 475, 487, 488

Syllis ramosa, 487

Symbiosis, $63^{*}$

Symmetry, 41*, 42 : Polype and Medusa, 138: Tealia, 189, 192

Symphyla, $615^{*}, 617,619,677$

Synapta, 424, 434, 438

Synaptida, 429

Synapticula, $205^{*}$

Syncarida, 566* 581

Syn-cerebrum, 555*, 631

Syncoryne, 145

Syncrypta, 71

Syncytium, 298*

Syngnatha, $615^{*}, 616,617,618$

\section{$\mathrm{T}$}

\section{'}

Tabula, 157* , 205, 208

Tactile cones, $260^{*}, 268$

Tactile organs, 39

Tania conurus, 252

Tania crassicollis, 285

Tania cncumerina, 270

Taniada, 254

Tania echinococcus, 261, 262, 282, 286

Tania mediocanellata, 285

T'onia saginata, 285

'T'ania serialis, 282

Tania serrata, 285

TANIA solium, General features, 245, 246, 247: Nervous system, 248: Excretory organs, 248 : Reproductive organs, 248, 249, 250 : Development, 250, 251: Systematic position, 254

Tæniole, $173^{*}$

Talitrus, 583

Tanaidacea, $567^{*}, 583,584,585,603$ : Tanais, 567, 583

Tape-worm-See Tænia and Cestoda

Tardigrada, $673,675^{*}, 678$

TEALIA : External characters, 185, 186 : Enteric system, 187, 188, Cell layers, 188 : Muscular system, 186, 188 : Symmetry, 189: Microscopic structure, 190, 191: Nervous system, 192: Reproductive organs, 193: Development, 192, 193 : Systematic position, 196 
Tealider, 196*

Tectibranchia, $734^{*}, 756$

Teloblast, $464^{*}$

Telolecithal, $219^{*}$

Telotrochal, $486^{*}$

Telson, $541^{*}$

Temnocephala, 259, 260, 269, 277, 278, 279

Temnocephalea, 253* , 259, 260, 269, 273, 278, 279, 284, 287

Tendon, $37^{*}$

Tentacle sheath, $210^{*}$ : Ronts, $155^{*}$

Tentacles, 32*, 91

Tentacular canal, 213

Tentaculifera, 91* : Body and tentacles, 99, 100 : Nucleus, contractile vacuoles, shell, colonies, reproduction, 100,101

Tentaculocyst, $155^{*}, 172$

Tentorium, $626^{*}$

Terebella, 474

Terebellida, 472, 474

Terebra, 738

Terebratula, 366, 368

Terebratulida, $367^{*}$

Terebratulina, 372

Teredo, 696, 701

Terga, 578*

Tergum, $541^{*}$

Termites, 633

Tessera, 177, 178

Testis-See Spermary

"ethys, 734

Tetrabranchiata, $790^{*}, 792,795,797,798$, 803, 804

Tetramita, 71

Tetrarhynchus, 261, 262

Tetrastemma, 291

Thalassoplancta, 61

Thallasema, 499

Theca, 205*

Thomson, J. Vaughan, 3

Thorax, of Apus, 531 : Astacus, 541 Periplancta, 622 : Solpugida, 664

Thread-worms, 297-See Nemathelminthes

Thysanopoda, 568

Thysanozoon, 255

Thysanura, 640

Thuricola, 95

Ticks, 653, 661-See Acaricla

Tiedemann's vesicle, $383^{*}, 426$

Tintinnidium, 94

Tissues, 23*

Tomopteris, $4 \mathrm{ss}$

Tooth-shells, 680_-See Scaphopoda

Tracheæ, $35^{*}, 609,743$

Tracheal gills, 642,643

Tracheliastes, 516

Trachelius, 94

Trachelomonas, 71

Trachylinæ, 142* : General structure, 153: Sense organs, 154: Tentacles,
155: Reproductive organs, 154, 155 :

Development, 155, 156

Trachymedusæ, 142*, 154, 155, 156

Translation, $285^{*}$

Trap-door Spider, 672

Trapezia, 209

Trematoda, 252* $257,258,259,260,264$, $265,266,267,268,269,270,272,273$, 277. 278, 279, 284, 285, 286, 287, 288 : Example, 240

Treptoplax, 233

Triarthrus beckii, 606

Trichina, 310, 311

Trichinella, 306, 311

Trichiniasis, 312

Trichocyst, 89, 93

Trichoplax, 233

Trichostomata, 91

Tricladida, 252*, 253, 255, 257, 263, 264,

$267,268,269,271,275,276,283,284$

T'ridacna gigas, 710

Trigger-hair-See Cnidocil

Trigonia, 695, 701, 710, 711

Trilobita, 604, 605, 606, 677

Trimorphism, 129*

Trimyaria, 290

Tristicothata, 319

Triton NODIFEREs, 721 : Shell, 721, 722, 723 : External features of soft parts, 723 : Foot, 724; Visceral spiral, 725: Mantle, 725; Ctenidium, 725; Osphradium, 726 : Digestive system, 726, 727, 728: Vascular system, 729: Excretory system, 729 : Nervous system, $729,730,731$ : Sensory organs, 731: Reproductive organs, 732 : Systematic position, 734

Tritonida, 733, 734*, 745

Trivium, $377^{*}, 416$

Trochal disc, 324

Trochelminthes, $322^{*}$ : Appendix, 336

Trocheta, 516

Trochophore-See Trochosphere

Trochosphara, 328, 332,335

Trochosphærida, 328 *

Trochosphere, 322, 440

Trochus, 733, 747

Trombidium fuliginosum, 665

Trophozoite, 80

Trypanosomes, 71, 72

Tube-feet, $377^{*}, 376,395,401$

Tubifex, 473, 4\$3

Tubificide, 488

Tubipora, 195, 198, 199, 203, 208, 210

Tubularia, 145, 153

Tubularice, 143

Turbellaria, 252* , 255, 256, 257, 262, $263,264,265,266,267,268,269,271$, 272, 273, 274, 275, 276, 284, 286 : Example, 236

Turbo, 733, 747

Typhlosole, 458* 475,685 


\section{$\bigcup_{L M A K I D E, 177^{*}}$}

Umbo, 683

Umbrella, 168*

Uncus, $325^{*}$

Undulating membrane, $72^{*}, 92,94$

Unicellular, 19*

Unicellular gland, 25*

Unio, 680, 696-See Anodonta

Unio margaritifer, 680, 711

Unionida, 696

Unisexual, $40^{*}$

Urea, 36

Uric acid, 13, 36

Urinary organs, 37 *

Urnatella, 354

Urns, $493^{*}$

Uropod, $542^{*}$

Uterine bell, 313

Uterus, $40^{*}$
Vermetes, 733

Vermetus, 755, 756

Vermiform embryos, 231

Vermilia cospitosa, 468

Vertebral column, 4

Vestibule, 95

Vibracula, 349, 352*

Vibratile corpuscles, 399

Virgula, $167^{*}$

Visceral spiral, of Triton, 725

Vitellaria, 270

Vitelline glands-See Yolk-glands

Vitelline membrane, 217

Vitreous body, 536*

Viviparous, $40^{*}$

Voluta, 749

Volvox, 72, 73, 75, 76, 77

Vortex, 265

Vorticella, 92, 93, 95, 96, 97, 98

Vulsella, 698

\section{V}

$\mathrm{V}$

ACUOLE, contractile, $11^{*}, 13,47,66$ $68,86,88,93,96,101$ : non-contractile, $11^{*}, 60,93,94$

Valenciennes, organ of, $781^{*}$

Valvulate, $387^{*}$

Van der Hoefen, organ of, $780^{*}$

Variation, individual, 2*, 113

Variety, 2*, $114^{*}$

Vascular system, 34: Nemcrtinea, 291 : Acanthocephala, 313: Phoronis, 357: Magellania, 365: Brachiopoda, 369 : Starfish, 379: Holothurian, 426: Antedon, 409: Echinodermata, 426: Nereis, 444: Lumbricus, 458: Sipunculus, 493: Gephyrea, 498: Archiannelida, 504: Hirudo, 512: Hirudinea, 518: Apus, 533 : Astacus, 551 : Crustacea, 596: Peripatus, 609: Periplaneta, 625: Insects, 642: Scorpio, 656: Arachnida, 668: Mussel, 689: Pelecypoda, 706: Amphineura, 715: Triton, 729: Gastropoda, 744 : Scaphopoda, 757 : Sepia, 765 : Nautilus, 783 ; Cephalopoda, 797

Vas deferens-See Spermiduct

Vegetal pole, 751

Velarium, $170^{*}, 182$

Velella, 166

Veliger, 709, 710*, 751

Velum, 135*, 136, 170, 710, 752

Ventral, $42^{*}$

Ventricle, $36^{*}$

Venus gnidia, 699

Venus's Flower-basket, 120

Venus's Girdle, 223

Vermes, 235

\section{W}

\section{$W$}

Wallace's line, $9^{*}$

Wandering cells, 111

Wasps, 636, 647, 652

Water-bugs, 634

Water-flea, 526, 564

Water-pores, $408^{*}$, 426

Water-sac, 536

Water-tubes, $408^{*}, 426,686$

Water-vascular system, 236*

Whale-louse, 586

Wheel-animalcules-See Rotifera

Wheel-organ, 324

Whelks, 680, 733

White body, 772

White substance of Schwann, 29*

Winter eggs, 327,597

Wood-louse, 526, 568-See Oniscus

\section{$\mathrm{X}$}

Xiphosura, $661^{*}, 666,667,668,669$, $673,677,678$

\section{Y}

Y ellow Cells-See Zoochlorella.

Yellow elastic cartilage, $26^{*}$

Yoldia, 695

Yolk, 19*

Yolk-glands, 236*

Yolk, epithelium, 799 : reservoir, 243

Yolk-sac, 803 
Z

$Z_{I L L A C A L L O P H Y L L A,} 670$ Zoæa, 599*

Zoantharia, $194^{*} 227$

Zoonthus, 196, 197, 200, 201

Zoœeium, 341
Zoochlorella, 60, 63, 208, 341

Zoo-geographical Regions, 8

Zooid, $40^{*} 50,75,283$

Zoology, $1^{*}$

Zoophyte, 128

Zoothamnium, 97

Zygophiuræ, 411*

Zygote, $74,75^{*}$ 80, 81 


BOOK MUST BE CHARGED OUT WITH CASE 
$40902 / 6661$ si wiw: 
Fing

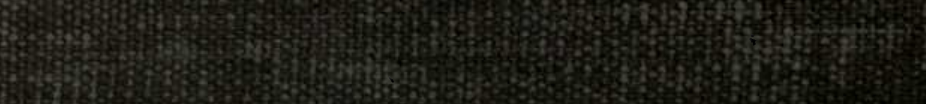

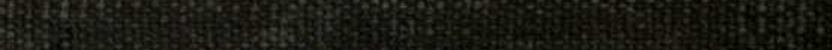
30.5

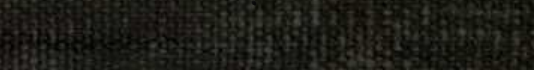

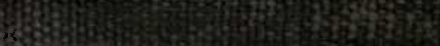
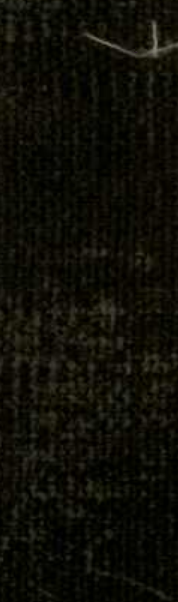